\title{
RCRA Facility Investigation/Remedial Investigation Report for the Grace Road Site (631-22G)
}

by

E. Palmer

Westinghouse Savannah River Company

Savannah River Site

Aiken, South Carolina 29808

A document prepared for REGULATORY REQUIREMENT at , , from ${ }_{4}<$.

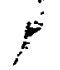

DOE Contract No. DE-AC09-89SR18035

This paper was prepared in connection with work done under the above contract number with the U.S.

Department of Energy. By acceptance of this paper, the publisher and/or recipient acknowledges the U.S. Government's right to retain a nonexclusive, royalty-free license in and to any copyright covering this paper, along with the right to reproduce and to authorize others to reproduce all or part of the copyrighted paper. 
Grace Road Site

WSRC-RP-95-93, Rev. 1.1

RFI/RI Report

February 1996

\section{DISCLAIMER}

\section{DISCLAIMER}

This report was prepared as an account of work sponsored by an agency of the United States Government. Neither the United States Government nor any agency thereof, nor any of their employees, makes any warranty, express or implied, or assumes any legal liability or responsibility for the accuracy, completeness, or usefulness of any information, apparatus, product, or process disclosed, or represents that its use would not infringe privately owned rights. Reference herein to any specific commercial product, process, or service by trade name, trademark, manufacturer, or otherwise does not necessarily constitute or imply its endorsement, recommendation, or favoring by the United States Government or any agency thereof. The views and opinions of authors expressed herein do not necessarily state or reflect those of the United States Government or any agency thereof.

This report has been reproduced directly from the best available copy.

Available to DOE and DOE contractors from the Office of Scientific and Technical Information, P.O. Box 62, Oak Ridge, TN 37831; prices available from (615) 576-8401.

Available to the public from the National Technical Information Service, U.S. Department of Commerce, 5285 Port Royal Road, Springfield, VA 22161. 


\section{DISCLAIMER}

\section{Portions of this document may be illegible in electronic image products. Images are produced from the best available original document.}




\section{CERTIFICATION PAGE}

\section{Revision 1.1 \\ RFI/RI REPORT FOR THE \\ GRACE ROAD SITE (631-22G)}

"I certify under the penalty of law that this document and all attachments were prepared under my direction or supervision in accordance with a system designed to assure that qualified personnel properly gather and evaluate the information submitted. Based on my inquiry of the person or persons who manage the system, the information submitted is, to the best of my knowledge and belief, true, accurate, and complete. I am aware that there are significant penalties for submitting false information, including the possibility of fines and imprisonment for knowing violations.

Date: $3 / 22 / 96 \quad$ Signature: Title:

Date: $3 / 26 / 96$

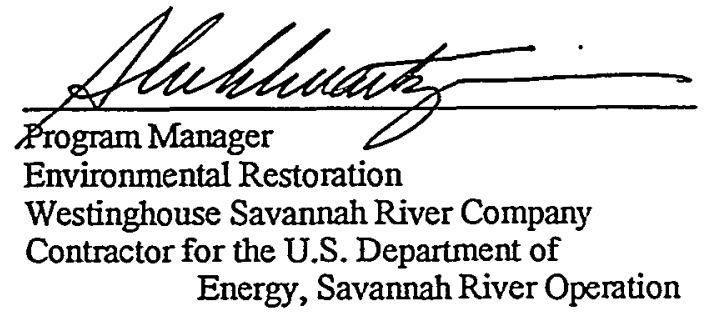

Energy, Savannah River Operation

Signature: Title:

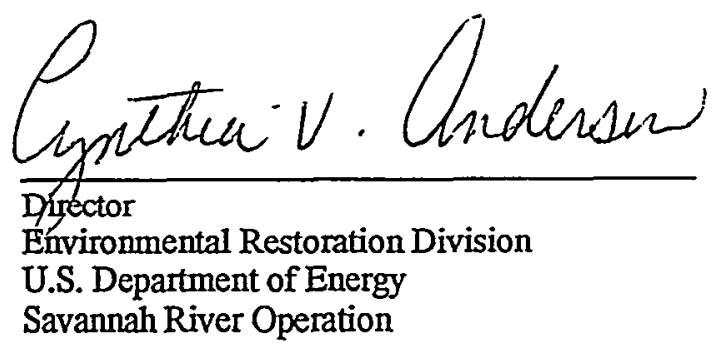


Document: WSRC-RP-95-93, Rev. 1

Title: $\quad$ RFI/RI Report for the Grace Road Site (631-22G)( (U)

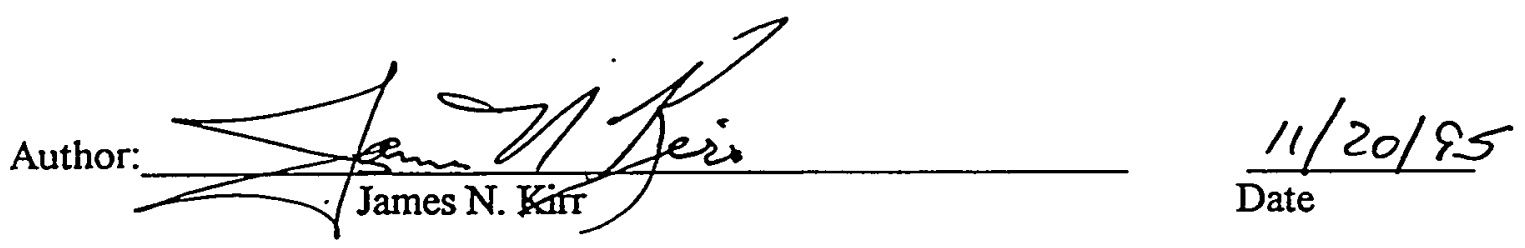

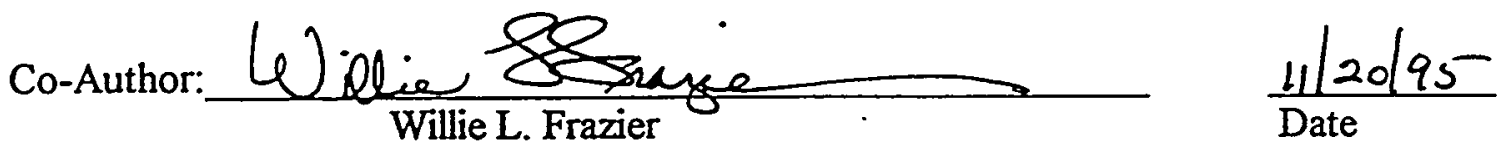

Approval:

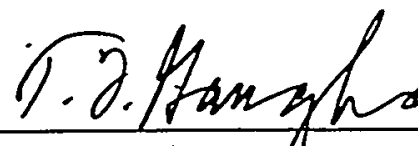

Thomas F. Gaughan, Mager, Site Characterization

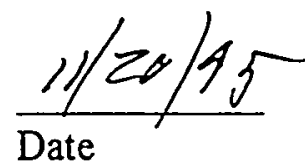

Site Geotechnical Services Department 


\section{TABLE OF CONTENTS}

EXECUTIVE SUMMARY. ES-1

1.0 INTRODUCTION $1-1$

1.1 Purpose $1-1$

1.1.1 SRS RCRA 3004(u)/CERCLA Program Description $1-1$

1.2 Site Background 1-3

1.2.1 Site Description $1-3$

1.2.2 Site History $1-6$

1.3 Previous Investigations $1-8$

1.3.1 Preliminary Unit Evaluation $1-10$

1.3.1.1 Existing Literature and Information. $1-10$

1.3.1.2 RFI/RI Literature Review Checklist 1-10

1.3.1.3 RFI/RI Site Reconnaissance 1-10

1.3.1.4 Preliminary Unit Evaluation Results 1-11

1.3.1.4.1 Waste Characteristics 1-11

1.3.1.4.2 Unit/Disposal Area Characteristics. 1-11

1.3.2 Unit Screening $1-12$

1.3.2.1 Evaluation of Unit Screening Options. $1-12$

1.3.2.2 Unit Screening/Confirmation Soil Analysis

Sampling 1-13

1.3.2.2.1 Soil Borings. $1-13$

1.3.2.2.2 Soil Borings Analysis. $1-15$

1.3.2.3 Unit Screening-Geophysical Investigation $1-21$

1.3.2.4 Unit Screening Conclusions. 1-23

1.4 Report Organization

2.0 STUDY AREA INVESTIGATION.......................................................... 2-1

2.1 Surface Feature Investigation ....................................................... 2-1

2.2 Coñtaminant Source Investigation ................................................. 2-1

2.3 Meteorological Investigation............................................................ 2-1

2.4 Surface Water and Sediment Investigation ......................................... 2-1

2.5 Soil/Vadose Zone Investigations ....................................................... 2-3 
2.5.1 Ground Penetrating Radar Investigation ................................... 2-3

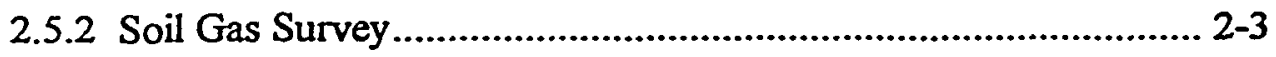

2.5.3 Conclusions from GPR and Gas Survey ...................................... 2-7

2.5.4 Gross Alpha and Non-Volatile Beta Analysis .............................. 2-7

2.5.5 Identification of Human Health Chemicals of Potential

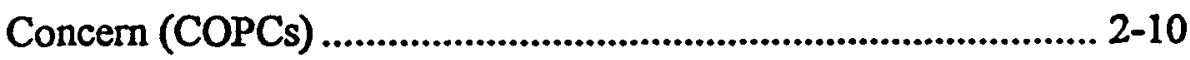

2.5.5.1 Rationale and Criteria for Selection of COPCs ............. 2-10

2.5.5.2 Risk-Based Concentrations (RBCs)............................. 2-10

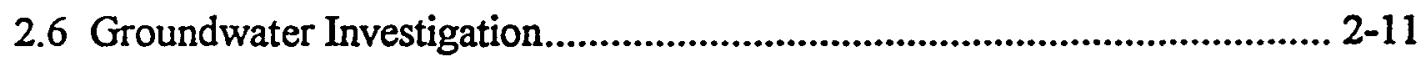

2.7 Human Population Surveys .............................................................. 2-12

3.0 PHYSICAL CHARACTERISTICS OF THE STUDY AREA ............................ 3-1

3.1 Surface Features ......................................................................................... 3-1

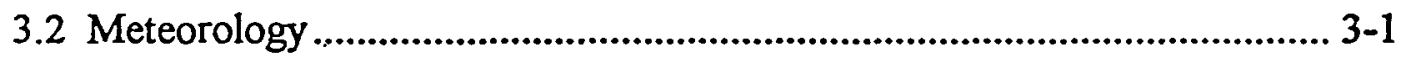

3.2.1 Regional Climatology .................................................................. 3-1

3.2.2 Temperature and Humidity ........................................................... 3-1

3.2.3 Precipitation and Evapotranspiration.......................................... 3-2

3.2.4 Wind ................................................................................... 3-3

3.2.5 Thunderstorms ........................................................................... 3-3

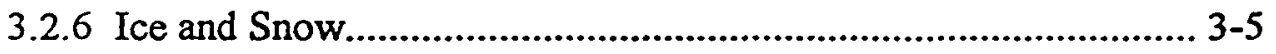

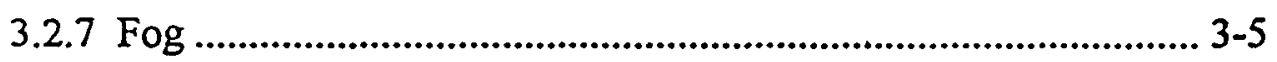

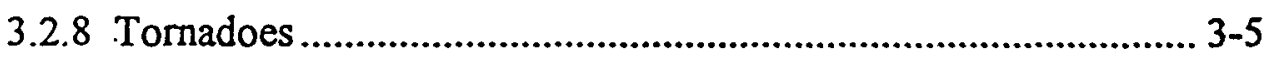

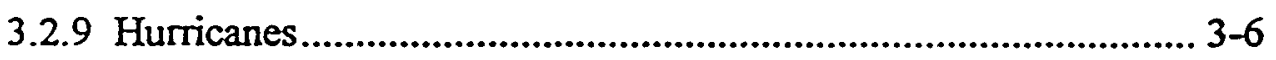

3.3 Surface Water Hydrology................................................................... 3-6

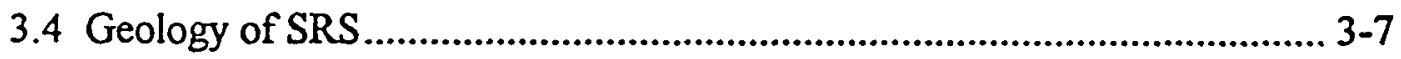

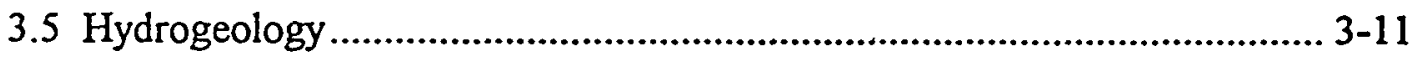

3.6 Geology and Hydrology of the Grace Road Site...................................... 3-13

3.7 Demography and Land Use .................................................................... 3-14

3.7.1 Demographics .......................................................................... 3-14

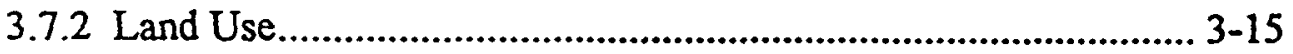

4.0 NATURE AND EXTENT OF CONTAMINATION .......................................... 4-1

4.1 Results of Data Usability Evaluation ................................................... 4-1

4.2 Results of Site Characterization........................................................... 4-1 
5.0 CONTAMINANT FATE AND TRANSPORT

5.1 Exposure Points

5.2 Exposure Routes and Potential Receptors

5.3 Exposure Point Concentrations ...........................................................5

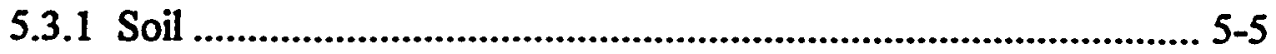

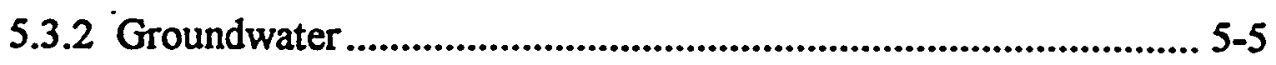

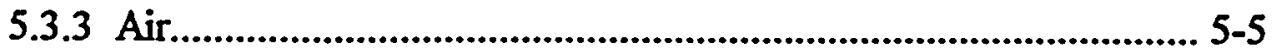

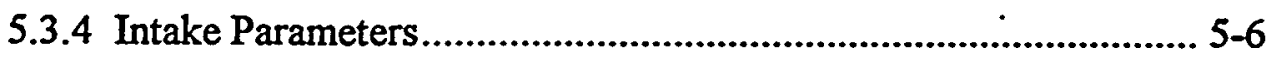

5.3.5 Equations for Exposure to Soil................................................ 5-7

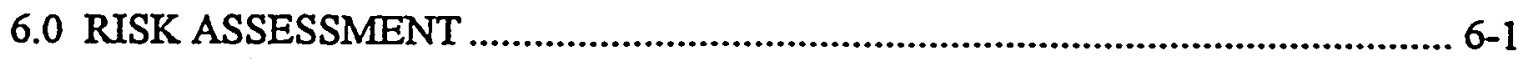

6.1 Human Health Assessment ..................................................................... 6-1

6.1.1 Exposure Assessment ............................................................ 6-1

6.1.1.1 Future Land Use Scenarios ........................................... 6-1

6.1.1.2 Identification Of Human Health Exposure Pathways..... 6-1

6.1.2 Toxicity Assessment ................................................................ 6-2

6.1.2.1 Non-Radiological Toxicity ........................................ 6-3

6.1.2.1.1 Carcinogens................................................... 6-3

6.1.2.1.2 Noncarcinogens............................................. 6-5

6.1.2.2 Chemicals With No EPA Toxicity Values..................... 6-7

6.1.3 Risk Characterization ............................................................ 6-8

6.1.3.1 Current Land Use...................................................... 6-10

6.1.3.2 Future Land Use ..................................................... 6-10

6.1.3.2.1 Carcinogenic Risk........................................ 6-10

6.1.3.2.2 Noncarcinogenic Hazard................................. 6-10

6.1.3.2.3 Chemicals of Concern.................................... 6-11

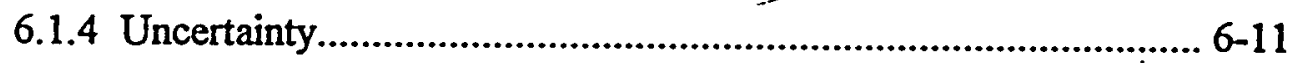

6.1.4.1 Chemicals of Potential Concern................................... 6-12

6.1.4.2 Exposure Assessment................................................. 6-12

- 6.1.4.3 Toxicity Assessment.................................................... 6-13

6.1.4.4 Risk Characterization .................................................... 6-13

6.2 Ecological Assessment ........................................................................ 6-14 
7.0 SUMMARY AND CONCLUSION

7.1 Summary $7-1$

7.2 Conclusion 7-1

REFERENCES REF-1

APPENDIX A - Technical Memoranda on Field Activities

APPENDIX B - Analytical Data and QA/QC Evaluation Results

APPENDIX C - Risk Assessment Methods 
Grace Road Site

RFI/RI Report
WSRC-RP-95-93, Rev. 1.1

February 1996

\section{LIST OF FIGURES}

Number

Title

Page

1-1 Location of the Grace Road Site in Relation to Major SRS Facilities $1-4$

1-2 Topographic Map Showing the Location of the Grace Road Site $1-5$

1-3 Site Configuration and Debris Locations $1-7$

1-4 Soil Gas Screening Locations, Grace Road Site. $1-9$

1-5 Soil Boring Locations. $1-14$

1-6 EM-31 Survey Grid and Results

2-1 Topographic Map of Grace Road Site. 2-2

2-2 GPR Survey Lines, $100 \mathrm{MHz}$ Antenna .. $2-4$

2-3 GPR Survey Lines, $300 \mathrm{MHz}$ Antenna 2-5

2-4 Soil Gas Survey Sample Locations $2-6$

2-5 Location of Radiological Soil Samples. 2-8

3-1 Annual Wind Rose for $\mathrm{H}$ Area $3-4$

3-2 Comparison of Chronostratigraphic, Lithostratigraphic, and Hydrostratigraphic Units at SRS. 3-8 $-$

3-3 Hydrogeologic Nomenclature for the SRS Region

5-1 Conceptual Model of Exposure Pathways 


\section{LIST OF TABLES}

Number Title

Page

1-1 Grace Road Soil Sampling Parameters.................................................. 1-16

1-2 Soil Chemical Analysis Results ........................................................... 1-18

1-3 Comparison of Unit Specific Soil Concentration to Two Times Background Concentrations and Risk-Based Concentrations (RBC)

1-4 Analytical Results from Well DP-6

2-1 Analysis of Soil Samples for Gross Alpha and Non-Volatile Beta $2-9$

4-1 Comparison of Unit Specific Soil Concentration to Two Times Background Concentrations and Risk-Based Concentrations (RBC)

6-1 Carcinogenic/Non-Carcinogenic Results for $3.2 \mathrm{mg} / \mathrm{kg}$ Arsenic 6-11 


\section{LIST OF ACRONYMS}

ABS

AF

ARAR

ATSDR

BRA

BW

CERCLA

CEC

$\mathrm{COC}$

$\mathrm{COPC}$

DOE

EIS

ECAO

ED

$\mathrm{EF}$

EM

EPA

ET

FFA

FI

FS

GPR

HEAST

HII

HQ

HWMF

IRIS

IRo

IRi

LOAEL

MF
Dermal Absorption Factor

Adherence Factor

Applicable or Relevant and Appropriate Requirements

Agency for Toxic Substances and Disease Registry

Basic Risk Assessment

Body Weight

Comprehensive Environmental Response, Compensation, and

Liability Act

Cation Exchange Capacity

Chemical of Concern

Chemicals of Potential Concern

Department of Energy

Environmental Impact Statement

Environmental Criteria and Assessment Office

Exposure Duration

Exposure Frequency

Electromagnetics

Environmental Protection Agency

Exposure Time

Federal Facility Agreement

Fraction Ingestion

Feasibility Study

Ground Penetrating Radar

Health Effects Assessment Summary Tables

Hazard Index

Hazard Quotient

Hazardous Waste Management Facility

Integrated Risk Information System

Incidental Soil Ingestion Rate

Inhalation Rate

Lowest Observed Adverse Effect Level

Modifying Factor 
Grace Road Site

RFI/RI Report

WSRC-RP-95-93, Rev. 1.1

February 1996

NEPA

NOAEL

NPL

NWS

ORNL

$\mathrm{PAH}$

PRG

PIP

RAGS

RBCs

RCRA

QA

QC

RFA

$\mathrm{RfC}$

$\mathrm{RfD}$

RFI

$\mathrm{RI}$

RME

SA

SCDHEC

SF

SRFS

SRL

SRP

SRS

SRTC

SVOC

SWMU

TAL

TCL

TES
LIST OF ACRONYMS (continued)

National Environmental Policy Act

No Observed Adverse Effect Level

National Priority List

National Weather Service

Oak Ridge National Laboratory

Polycyclic Aromatic Hydrocarbon

Preliminary Remediation Goals

Public Involvement Plan

Risk Assessment Guidance for Superfund

Risk-Based Concentrations

Resource Conservation and Recovery Act

Quality Assurance

Quality Control

RCRA Facility Assessment

Reference Concentrations

Reference Dose

RCRA Facility Investigation

Remedial Investigation

Reasonable Maximum Exposure

Skin Surface Area

South Carolina Department of Health and Environmental Control

Slope Factor

Savannah River Forest Service

Savannah River Laboratory (now SRTC)

Savannah River Plant (now SRS)

Savannah River Site

Savannah River Technology Center

Semi-Volatile Organic Compound

Solid Waste Management Unit

$=$

Target Compound List

Threatened, Endangered, and Sensitive 
Grace Road Site

RFI/RI Report

TIC

TOC

UF

VOC

WSRC
WSRC-RP-95-93, Rev. 1.1

February 1996

\section{LIST OF ACRONYMS (continued)}

Tentatively Identified Compounds

Total Organic Carbon

Uncertainty Factor

Volatile Organic Compound

Westinghouse Savannah River Company 
Grace Road Site

RFI/RI Report
WSRC-RP-95-93, Rev. 1.1

February 1996

THIS PAGE INTENTIONALLY LEFT BLANK

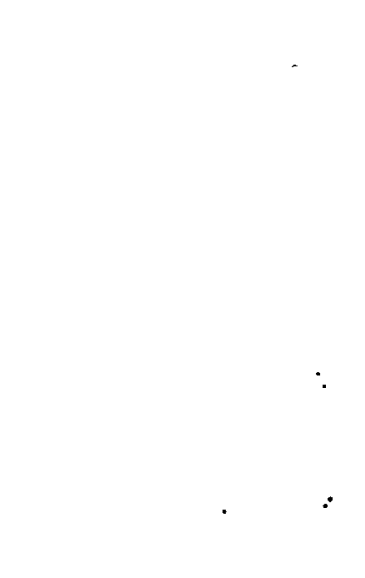

xii 


\section{EXECUTIVE SUMMARY}

Grace Road Site is located 1.3 kilometers ( 0.8 miles) south of B-Area and about 244 meters ( 800 feet) east of the intersection of Grace Road with SRS Road 2. The unit is roughly rectangular in shape, has a northwest-southeast orientation, and runs parallel to Grace Road. Approximate dimensions of the unit are 396.3 meters (1,300 feet) southeast to northwest and 97.6 meters (320 feet) southwest to northeast, and it covers an area of about 3.8 hectares ( 9.6 acres). Waste at the unit consisted of drums and cans in a variety of sizes (over 50 were uncovered during unit clearing activities), car seat springs, car and truck parts, old foundations, bricks, shingles, and slabs of concrete. The waste was on the surface or partially buried in scattered locations throughout the unit. Grace Road area was previously a tenant-operated farm and later served as the laydown yard for materials used in the construction of B-Area.

In March/April 1988, 41 soil samples and 41 soil gas samples were taken from Grace Road Site. Nine of the soil samples showed low levels of chlorocarbons suggesting the possible release of chlorinated solvents at those locations. Five of the gas samples contained elevated levels of light hydrocarbons (methane) which were suspected to indicate the presence of decaying vegetal matter or small quantities of oil. Several different solvents and daughter products, including tetrachloroethylene, trichloroethylene, dichloromethane, and trans-1,2-dichloroethylene, were detected. Slightly elevated concentrations of light hydrocarbons indicate that limited amounts of parent hydrocarbons such as oil may have been disposed of at this location. Because of these findings, it was suspected that small amounts of chlorinated solvents were disposed of at Grace Road Site. Based on these preliminary evaluation results, a Unit Screening Plan consisting of a Geonics@ EM-31 electromagnetic conductivity survey and an analysis of soil borings from 10 new locations was formulated.

Unit Screening data indicate that hazardous substance concentrations appear to be at or below background concentrations or below risk-based concentration levels: A comparison of Unit Screening soil data with EPA risk-based concentration levels indicates that, with the exception of arsenic, no concentrations of any hazardous substance (including 
laboratory artifacts) exceeds available risk-based standards. Arsenic levels were calculated for possible carcinogenic risk and found to be within acceptable limits.

No EPA risk-based action levels exist for gross alpha or non-volatile beta in soils. The maximum soil concentration of non-volatile beta at Grace $\operatorname{Road}(13 \mathrm{pCi} / \mathrm{g}$ ) is well within the reported range at three sampled sites and throughout SRS. The highest reported gross alpha value, $30 \mathrm{pCi} / \mathrm{g}$, is slightly above the SRS-wide range of values $(3.9-20 \mathrm{pCi} / \mathrm{g})$. Additional soil samples collected in August 1994 and analyzed for gross alpha and nonvolatile beta confirmed that activity in the soil is indicative of naturally occurring materials.

Ground water analytical results from water table well IDP-6 immediately east of Grace Road, show no constituents which exceed EPA MCLs. Any wastes would have to have been at Grace Road Site for a sufficiently long enough time to allow for any potential contaminant to travel down to the water table and the well.

Based on comparisons with unit-specific background concentrations and risk-based concentration levels, there is no indication of potential threat to human health caused by. hazardous substance disposal at Grace Road Site.

Approximately 21 locations with anomalous readings were detected by the EM-31 terrain conductivity survey. Most of these are limited in areal extent and many were associated with visible metal debris. The two areas with the greatest areal extent are located in the southeast end of the unit. Three soil borings in close proximity to these anomalies, and soil analytical results from those borings indicate that, if anything is buried in the area, its impacts are negligible.

A GPR survey conducted in March/April 1994 obtained data that indicate the sediment in the area is mostly undisturbed. However, disturbed soil is present around some former farm building locations and may indicate excavations in removing those buildings and/or buried debris.

An additional soil gas survey conducted in March 1994 shows the levels of volatiles and diesel range organics observed are very low with most below minimum detection levels. 
Light hydrocarbons and mercury levels are indicative of background concentrations in the SRS area.

An analysis of data collected as a result of the studies performed at Grace Road indicates that there is limited contamination at that unit. The only COPCs identified in the risk assessment that exceeded a risk of 1.0E-06 was arsenic and it is less than 3.80E-05, which is a slight risk of concern. Arsenic detected in the unit specific samples had a maximum concentration of $3.2 \mathrm{mg} / \mathrm{kg}$. SRS site wide values for arsenic range from $<0.5$ $\mathrm{mg} / \mathrm{kg}$ to $15.2 \mathrm{mg} / \mathrm{kg}$ with a median of $<2.0 \mathrm{mg} / \mathrm{kg}$. It should also be noted that arsenic was a component of agricultural chemicals in the period before SRS existed. Since the Grace Road area was a farm prior to SRS, the detected values may be a result of pre-1950 farming activities.

In addition, it is also reasonable to conclude that, since the contamination is limited, the unit presents no significant ecological risk.

Based on the review of the analytical data and the screening techniques used to evaluate all the. chemicals of potential concern at Grace Road, it is recommended that no further remedial action be performed at this unit. 
Grace Road Site

RFI/RI Report
WSRC-RP-95-93, Rev. 1.1

February 1996

\section{THIS PAGE INTENTIONALLY LEFT BLANK}




\subsection{INTRODUCTION}

\subsection{Purpose}

This report summarizes the activities and documents the results of a Resource Conservation and Recovery Act (RCRA) Facility Investigation/Remedial Investigation (RFI/RD) conducted at Grace Road Site on the Savannah River Site (SRS) near Aiken, South Carolina. The RFI/RI was conducted in stages, beginning with a preliminary unit evaluation in November 1988, followed by a screening phase, and culminating in a field assessment stage in March/April 1994.

The primary objective of the Remedial Investigation/Feasibility Study (RI/FS) is "to gather data sufficient to support an informed risk management decision regarding which remedy appears to be most appropriate for a given site" (EPA, 1988). In order to accomplish this, it is necessary to define the nature and extent of the contamination, determine the fate and transport of the contaminants, complete a baseline risk assessment (BRA), and identify remedial action objectives. In the event that a FS is required, this information is then used to identify potential remedial action alternatives and potential applicable or relevant and appropriate requirements (ARARs). 'To achieve the goals of this RI, both historical data and data collected during the 1994 characterization were used.

This introductory section presents background information about the unit, outlines the regulatory requirements guiding the investigation, the history of the unit, and previous investigations at the unit.

\subsubsection{SRS RCRA 3004(u)/CERCLA Program Description}

The United States Department of Energy (DOE) Savannah River Field Office manages waste materials regulated under the Resource Conservation and Recovery Act (RCRA). In particular, certain activities require RCRA operating or post closure permits. Savannah River Site (SRS) has received a RCRA permit from the South Carolina Department of Health and Environmental Control (SCDHEC). Part $V$ of the permit mandates that SRS establish and implement a RCRA Facility Investigation (RFI) Program to fulfill 
requirements of RCRA Section 3004(u). Section V.A.1 of the permit lists solid waste management units (SWMUs) that were identified by the United States Environmental Protection Agency (EPA) Region IV through the RCRA Facility Assessment (RFA) process and therefore subject to the RFI process.

On December 21, 1989, SRS was included on the National Priority List (NPL). A unit included on the NPL falls under the jurisdiction of the Comprehensive Environmental Response, Compensation, and Liability Act (CERCLA). This inclusion created the need to integrate the established RFI Program with CERCLA RI requirements. In accordance with Section 120 of CERCLA, DOE has negotiated a Federal Facility Agreement (FFA) with EPA and SCDHEC to integrate remedial activities at SRS into one comprehensive strategy which fulfills these dual regulatory requirements.

SWMUs listed in the RCRA permit and the FFA Appendix are required to be further investigated to determine their actual or potential impact on the environment. Those listed unites are called RCRA/ CERCLA Remedial Investigation Units in this document. Grace Road Site is one of the RCRA/CERCLA units mandated for further investigation.

In order to conduct the RI/FS, SRS developed the RFI/RI Program Plan (WSRC [Westinghouse Savannah River Company], 1993) (originally titled the RFI Program Plan) to provide guidance and delineate standard procedures. It also provides a reasonable and cost effective approach while allowing SRS flexibility in the investigation of each RCRA/CERCLA unit. The plan specifies generic methods and procedures to be used for each unit investigation. Because of continuing change in the status of facilities at SRS, the program plan is periodically reviewed and modified to keep it current.

The RFI/RI Program Plan (WSRC, 1993), in conjunction with Guidance for Conducting Remedial Investigations and Feasibility Studies (FS) Under CERCLA, Interim Final (EPA, 1988), were used as the primary sources of guidance for work plan preparation. These references are jointly referred to as RFI/RI guidance documents. The work plan for Grace Road Site conformed to the requirementš specified in the RFI/RI guidance documents. 
Sections 113 and 117 of CERCLA lists the requirements for public participation in the restoration process. These requirements include the establishment of an Administrative Record File that documents the selection of cleanup alternatives and provides for public review and comment on those alternatives. The DOE document, Public Involvement, $A$ Plan for the Savannah River Site (DOE, 1994), is designed to facilitate public involvement in the decision-making processes for permitting, closure, and selection of remedial alternatives. The Public Involvement Plan (PIP) addresses the requirements of CERCLA, RCRA, and the National Environmental Policy Act (NEPA).

Unit-specific documents, such as work plans and RIs, are a part of the Administrative Record File and available to the public. Information repositories have been established at the DOE Public Reading Room located at the Gregg-Graniteville Library of the University of South Carolina in Aiken, South Carolina and the Thomas Cooper Library located at the University of South Carolina in Columbia, South Carolina. A notice will be published in local newspapers when information is compiled regarding the investigation of Grace Road Site. Additional repositories may be added and/or locations changed to better meet the needs of the public.

The investigation of Grace Road Site also included preparation of a baseline risk assessment (BRA). The BRA was conducted using data generated during the RFI/RI assessment and analyzed risk posed by the unit to both human health and the environment.

\subsection{Site Background}

\subsubsection{Site Description}

Grace Road Site is located 1.3 kilometers ( 0.8 miles) south ó B-Area and about 244. meters ( 800 feet) east of the intersection of Grace Road with SRS Road 2. Figure 1-1 shows the location of the Grace Road Site in relation to major SRS facilities, and Figure 1-2 is a portion of the SRS topographic map showing the unit setting in relation to its immediate surroundings. The unit is roughly rectangular in shape, has a northwestsoutheast orientation, and runs parallel to Grace Road. Approximate dimensions of the unit are 396.3 meters (1,300 feet) southeast to northwest, 97.6 meters ( 320 feet) 


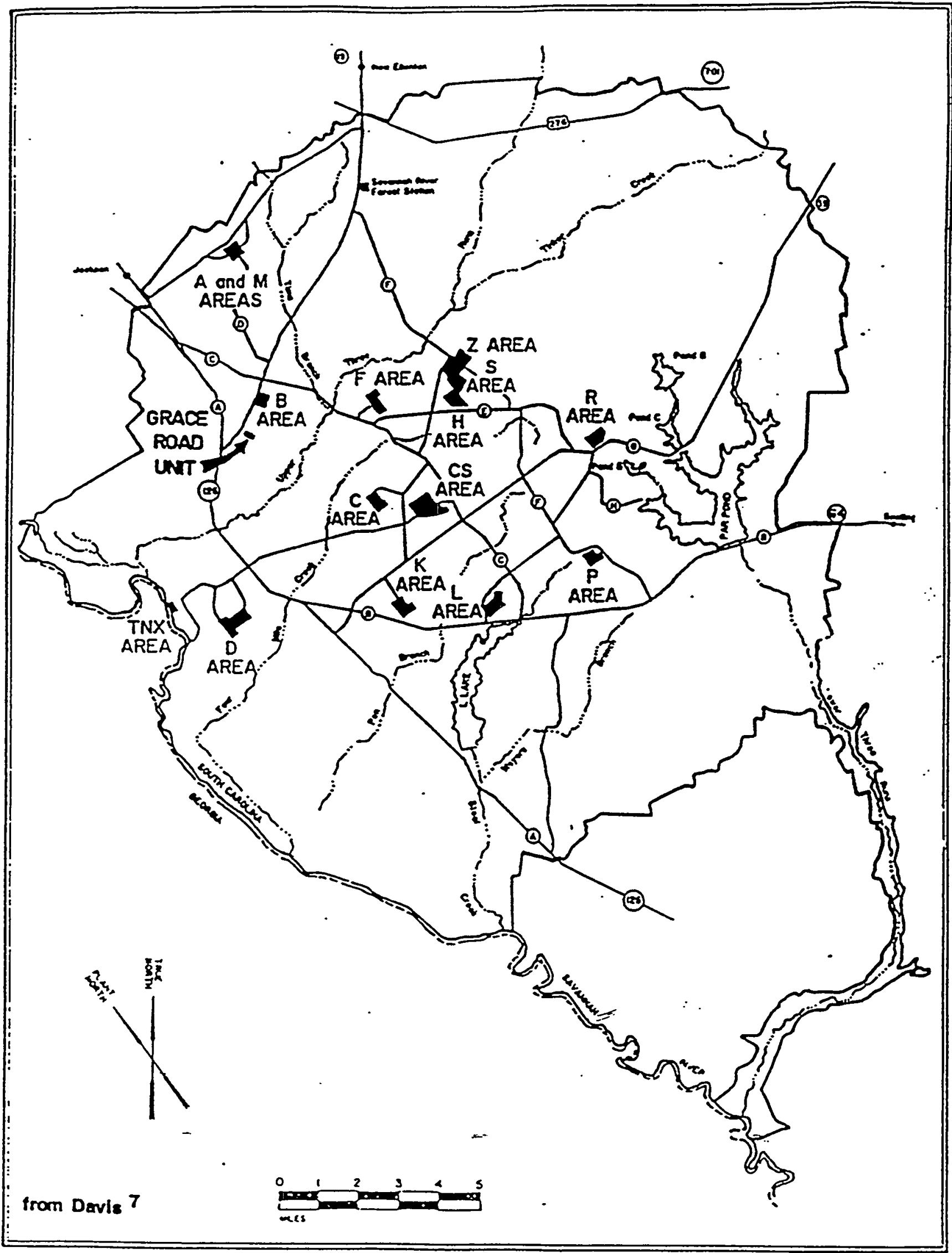

Figure 1-1 Location of the Grace Road Site in Relation to Major SRS Facilities. 
Grace Road Site

RFI/RI Report

WSRC-RP-95-93, Rev. 1.1

February 1996

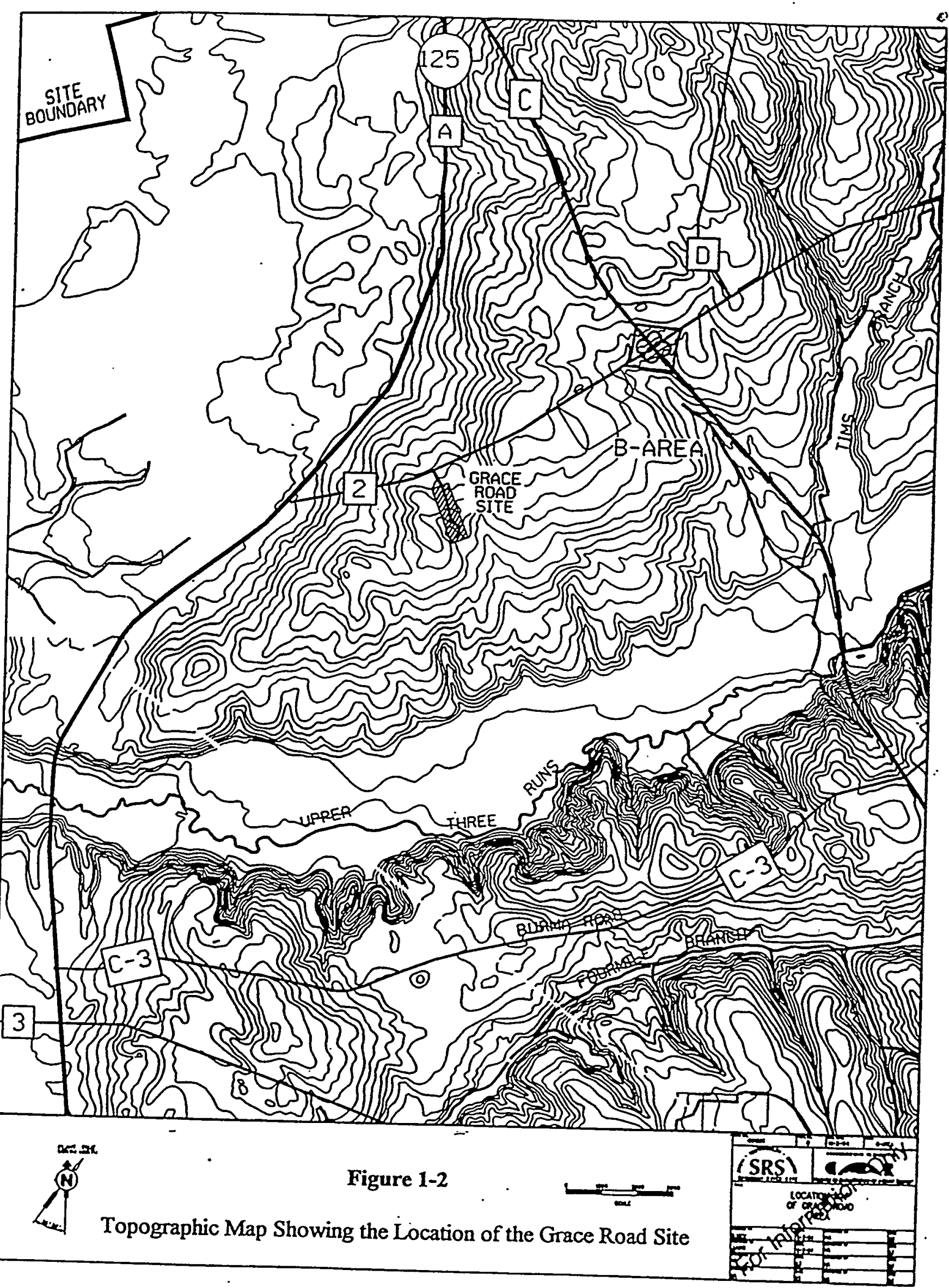


southwest to northeast and it covers an area of about 3.8 hectares ( 9.6 acres). Figure 1-3 shows the unit configuration and the location of rubble and concrete pads found at the unit.

Waste at the unit consisted of drums and cans in a variety of sizes (over 50 were uncovered during unit clearing activities), car seat springs, car and truck parts, old foundations, slabs of concrete, bricks, shingles and other debris. The waste was on the surface or partially buried in scattered locations throughout the unit.

Most of the unit is covered in tall grasses and weeds with numerous pine trees and low shrubs. The area slopes to a shallow ravine on the south side of the unit. The nearest surface water is Upper Three Runs Creek approximately 2.4 kilometers (1.5 mile) east of the unit.

\subsubsection{Site History}

Prior to the establishment of SRS, Grace Road Site was part of a tenant-operated farm owned by Mrs. Elise Grace. The farm consisted of about 217.6 hectares (544 acres) of which 92 hectares ( 230 acres) were under cultivation, and the remaining 125.6 hectares (314 acres) were in woodlands and swamp lands. Transfer records of this land to the $U$. S. Government in January 1951 indicate that this land had been a farm (part of the Red Hill Plantation) since the late 1890 's.

Buildings on the farm consisted of a main house, dog kennel, machine shed, oil house, two cottages, two turkey houses, two barns, garage, cook house, two=story barn, water tower with meat house, storage shed, grain storehouse, hay storage barn and an outhouse (privy). The majority of the buildings had a foundation of bricks, concrete or tile blocks. Several buildings, including the dog kennels and turkey houses, had concrete slab floors. The water tower also had massive concrete blocks that were used to hold treated timber stanchions that supported the water tank. Photographs of the farm show at least two. gasoline powered tractors in the machine sheđ; a truck and other assorted fäm machinery.

After purchase by the Government, the area in and around the farm was utilized as a laydown yard for materials used in the construction of the B-Area. The length of time that 


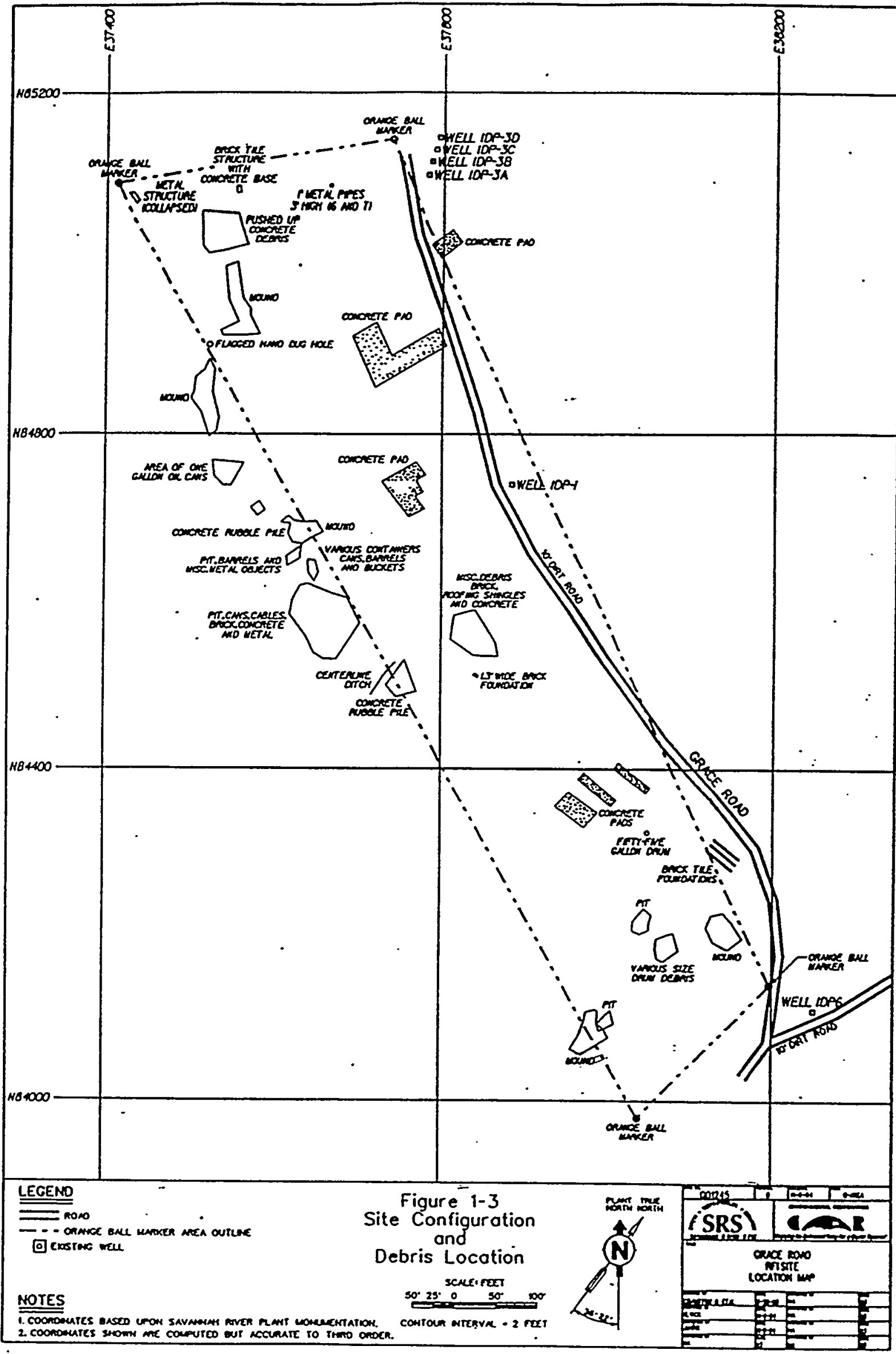


it was utilized for this purpose is unknown, but is estimated to be two to three years. There are no records to indicate that this unit has been used for any other purpose since it was closed as a laydown yard in the mid-1950s. There is no documented information available regarding past hazardous or non-hazardous waste disposal activities at Grace Road unit nor is there evidence of any recent activity or that any disposal activity was widespread.

\subsection{Previous Investigations}

The first step towards determining if a hazardous substance has been released from a RCRA/CERCLA unit consists of a Preliminary Unit Evaluation. The primary goal of the preliminary evaluation is to determine what hazardous substances, if any, were disposed of or released from the unit. In March/April 1988, 41 soil samples and 41 soil gas samples were taken from Grace Road Site. Figure 1-4 is a general sketch of the unit showing the location of the sample points. Nine of the soil samples contained low levels of chlorocarbons (see Figure 1-4) suggesting the possible release of chlorinated solvents at those locations. Five of the gas samples contained elevated levels of light hydrocarbons . (methane) which were suspected to indicate the potential presence of decaying vegetal matter or small quantities of oil. Several different solvents and daughter products, including tetrachloroethylene, trichloroethylene, dichloromethane, and trans-1,2dichloroethylene, were detected. Slightly elevated concentrations of light hydrocarbons indicate that limited amounts of parent hydrocarbons such as oil may have been disposed of at this location (Looney et al, 1988). Because of these findings, it was suspected that small amounts of chlorinated solvents were disposed of at Grace Road Site.

Based on the preliminary evaluation results, a Unit Screening Plan for Grace Road Site was formulated. The following sections describe the Preliminary Unit Evaluation and Unit Screening procedures and results. Chapter 2 describes the activities conducted to complete the Unit Assessment. 


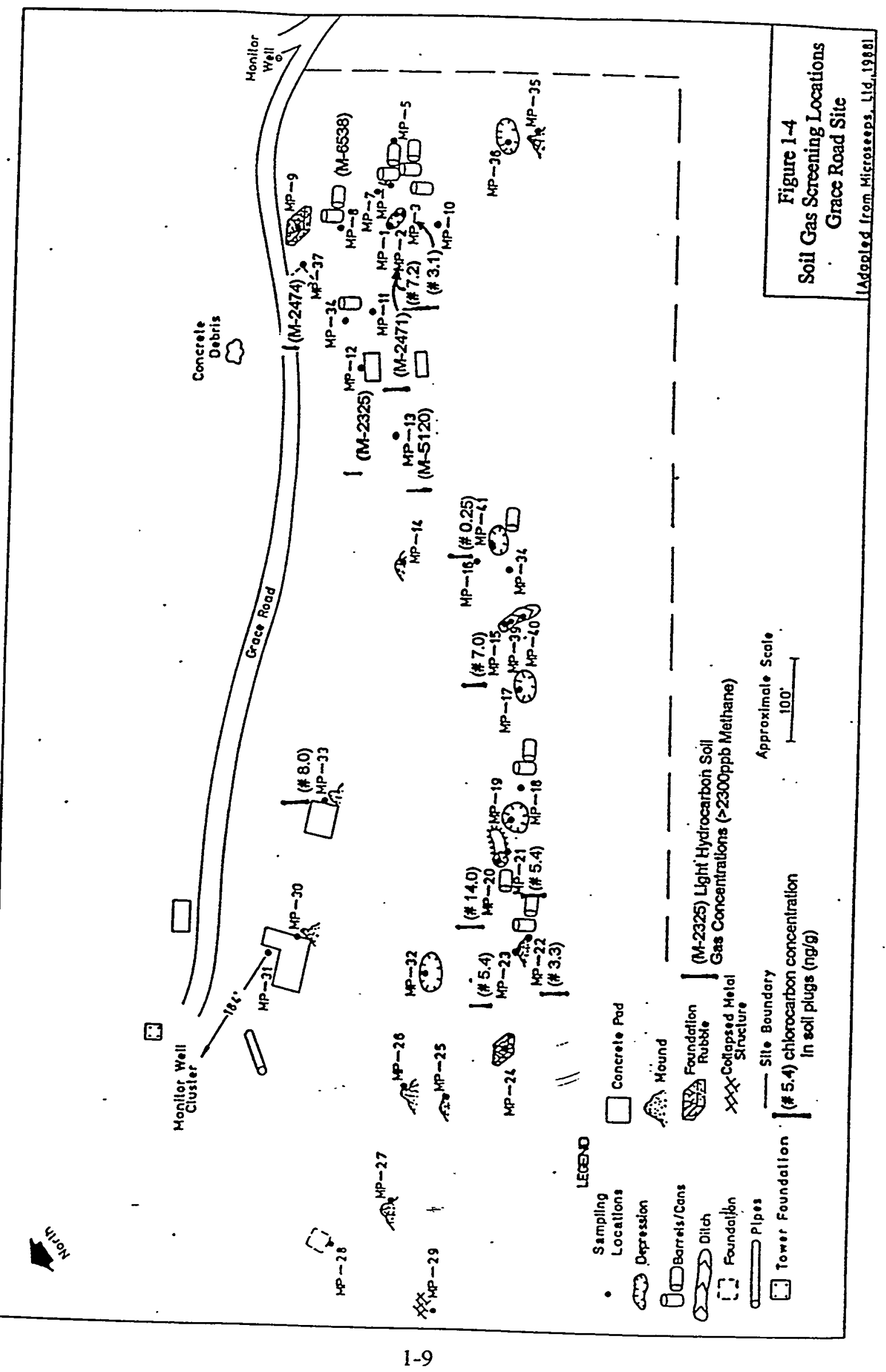




\subsubsection{Preliminary Unit Evaluation}

A review of existing literature and information from Grace Road, conducted in accordance with the RFI/RI Program Plan (WSRC, 1993), included:

- Physical and chemical characteristics of the waste (hazardous and non-hazardous) known to be managed at the unit;

- Unit/disposal area characteristics;

- General hydrogeologic conditions of the unit and the current environmental setting;

- Any previous sampling and/or monitoring data for the unit.

\subsubsection{Existing Literature and Information}

Existing information specifically pertaining to the Grace Road unit was reviewed. A complete list of the documents reviewed is included in Section 5.2.1 of the RFI/RI Plan for the Grace Road Unit, WSRC-RP-90-1250 (WSRC, 1990).

\subsubsection{RFI/RI Literature Review Checklist}

This checklist presents RFI data and information that are specific to each individual environmental medium. Copies of the completed checklist appear in Appendix D.1 of the RFI/RI Plan for the Grace Road Unit, WSRC-RP-90-1250 (WSRC, 1990).

\subsubsection{RFI/RI Site Reconnaissance}

Following a review of all existing data and information, a unit reconnaissance was performed to further assess the following field conditions:

- environmental setting;

- source characterization (RCRA/CERCLA unit conditions and waste characteristics);

- waste release potential (for each environmental medium).

The completed "Site Reconnaissance Field Data Sheet" lists unit-specific factors considered during the actual reconnaissance. This data sheet was completed in full to 
provide appropriate written documentation of field observations. Following the unit reconnaissance, the Literature Review Checklist was updated to include information and data collected from the field visit.

\subsubsection{Preliminary Unit Evaluation Results}

Information regarding current unit conditions is listed on the Literature Review Checklist and the Unit Reconnaissance Field Data Sheet which can be found in Appendix D.1 of the RFI/RI Plan for the Grace Road Unit, WSRC-RP-90-1250 (WSRC, 1990). That information is summarized in Sections 1.3.1.4.1 through 1.3.1.4.2.

\subsection{Waste Characteristics}

Waste at Grace Road Site consisted of drums and cans in a variety of sizes, car seat springs, car and truck parts, old foundations, bricks, shingles and slabs of concrete. Based on a soil plug survey (Looney et al., 1988), it is suspected that small amounts of chlorinated solvents were disposed of at Grace Road Site. Tetrachloroethylene, trichloromethane, dichloromethane, and trans-1,2-dichloroethylene and their daughter products were detected although the concentrations are low in comparison to major disposal units at SRS. Slightly elevated concentrations of light hydrocarbons indicate that limited amounts of parent hydrocarbons such as oils may have been disposed of at this location. Several of the drums removed from the unit were marked as oil and grease.

\subsection{Unit/Disposal Area Characteristics}

Grace Road Site boundaries were surveyed in June 1990, and the corners marked with orange balls and metal posts. Dimensions of the unit are 396.3 meters $(1,300$ feet) southeast to northwest and 97.6 meters (320 feet) northeast to southwest. Wastes were left on the surface or partially buried in scattered locations throughout the unit. Most of the unit is covered in tall grasses and weeds with pine trees and low shrubs. There is evidence of deer and small animal habitation irithe vicinity. 


\subsubsection{Unit Screening}

A Unit Screening program was performed at Grace Road because the Preliminary Unit Evaluation indicated a possible receipt of hazardous substances. The primary goal of the Unit Screening phase is to identify if a release of hazardous substances did occur and areas potentially impacted by a hazardous substance release. The RFI/RI Program Plan (WSRC, 1993) specifies that Unit Screening investigative activities include one or more of the following geophysical and/or geochemical techniques:

- Geophysical investigations such as ground penetrating radar (GPR) or electromagnetics (EM);

- Soil gas analysis; and/or

- Confirmation soils analysis.

The three options listed above were first evaluated regarding their applicability at Grace Road Site and a program was then designed and implemented. In addition, recommendations developed by the Savannah River Laboratory ([SRL] now called the Savannah River Technology Center [SRTC]) (Characterization Recommendations for Waste Sites at the Savannah River Plant [Gordon, et al., 1987]), for Grace Road Site were reviewed and incorporated into the Unit Screening program.

\subsubsection{Evaluation of Unit Screening Options}

A confirmatory soil sampling analysis program and a surface geophysical survey using the electromagnetic terrain conductivity meter (EM-31) were selected for use at Grace Road. The EM-31 was selected over ground penetrating radar (GPR) because at the time the unit was inaccessible to the heavier GPR equipment. A soil gas survey had previously been performed (see Looney et al, 1988).

A surface geophysical investigation was performed in March 1990 to determinie where tanks, drums, or other metallic debris may have been buried. The EM-31 allows continuous readings along survey lines thus increasing the potential for detecting subsurface anomalies. 
Confirmation soils sampling served as a screening for semi-volatile and volatile organic compounds, metals, and radioactivity. In addition, Appendix IX parameters were also analyzed. Results from the soil gas survey conducted by Looney et al (1988) and the location of the debris/rubble were used to select soil sample locations. The analytical data provided an indication of whether concentrations greater than background levels are present.

\subsubsection{Unit Screening/Confirmation Soil Analysis Sampling}

The goal of the unit screening effort was to evaluate whether a hazardous substance release had occurred and to identify any chemical constituents involved. Chemical parameters which indicate the presence and magnitude of a hazardous waste or hazardous substance release were selected for analysis. For screening at Grace Road Site, the following chemical parameters were analyzed at selected locations:

- Full EPA Appendix IX (40 CFR, Part 264) scan;

- Radionuclide indicators (gross alpha, non-volatile beta);

- Total metals (for the eight EP-toxicity metals: $\mathrm{As}, \mathrm{Ba}, \mathrm{Cd}, \mathrm{Cr}, \mathrm{Pb}, \mathrm{Hg}, \mathrm{Se}, \mathrm{Ag}$ );

- Volatile organics (EPA Method 8240);

- Semi-Volatile Organics (EPA Method 8270); and

- General indicator parameters (soil pH, TOC, CEC, Specific Conductance).

Chemical parameters were selected to obtain information on waste disposal history (waste oils, volatile organics, semi-volatile organics, and metals), waste characterization (Appendix DX, radionuclide indicators), constituents in excess of background, or to provide sufficient information to indicate constituent absence.(Appendix $\mathrm{LX}$, radionuclide indicators).

\subsection{Soil Borings}

Because the unit encompasses a large area where various types of activities occurred, soil sampling was conducted at 14 different locations.(see Figure 1-5). In March 1990 a Geonics ${ }^{\circledR}$ EM-31 electromagnetic conductivity meter was used to investigate the unit and 
Grace Road Site

WSRC-RP-95-93, Rev. 1.1

RFI/RI Report

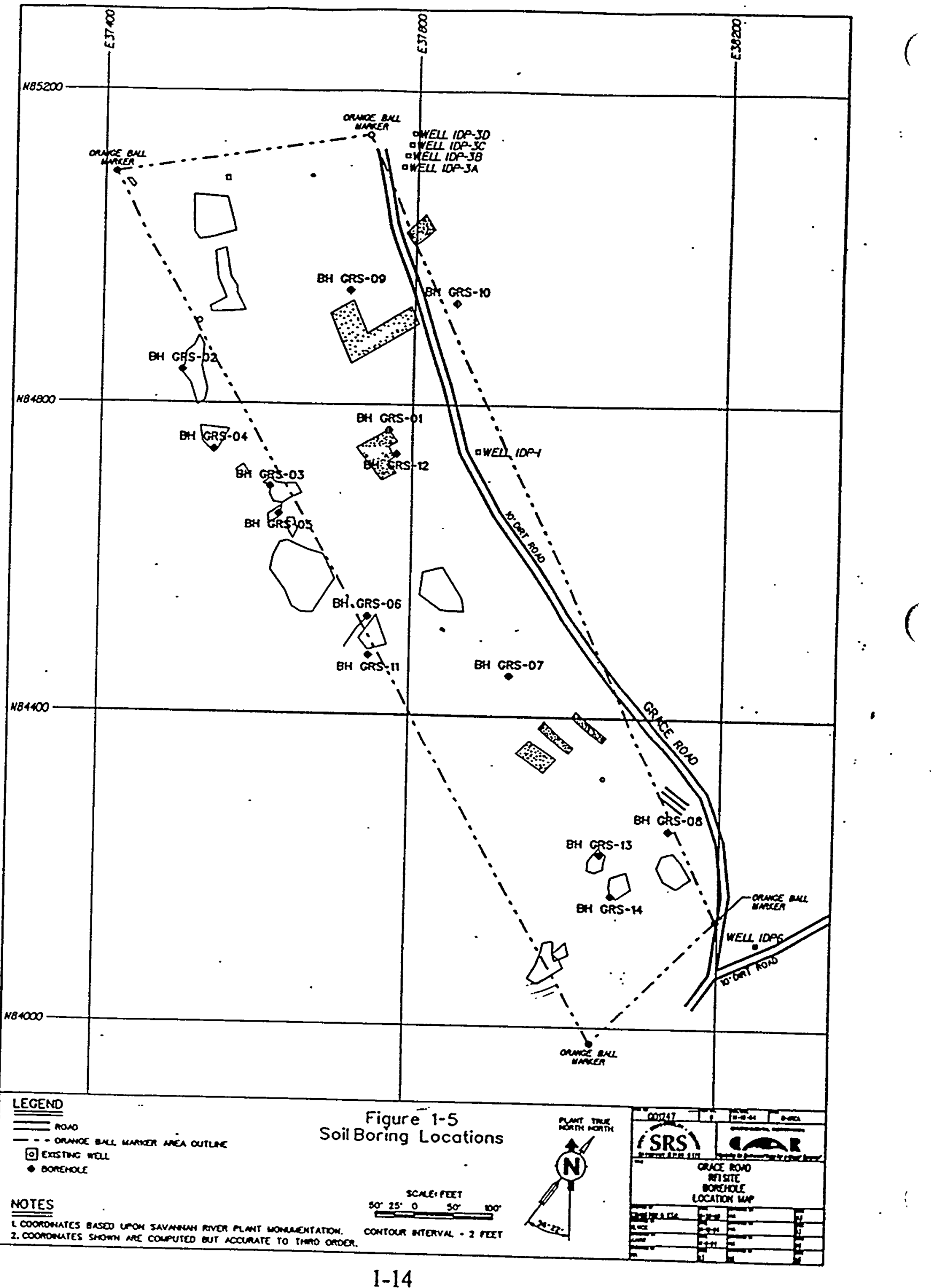


determine if there were any objects buried beneath the surface that might indicate a need for future sampling or removal. The grassy portion of the unit was gridded and transects run in areas where no metal objects had been previously identified. Some anomalies were observed during the survey.

Ten hand auger boring locations were selected in the portions of the unit which had the highest density of waste material based on the unit inspection and soil gas survey. These were supplemented by four shallow hollow stem auger borings (GRS 7-10). Three of these are located close to the northeastern boundary of the unit, and the fourth boring (GRS-10) is off unit to provide information on background conditions.

A stainless steel hand auger was used to collect shallow soil samples. At each location a sample was collected from intervals of 0.6-0.9 meters (2-3 feet) and 1.5-1.8 meters (5-6 feet) from the ground surface. The soil borings were drilled using hollow stem auger techniques. Split-spoon soil samples were collected at depths of 0.9-1.5 meters (3-5 feet), 3.1-3.7 meters (10-12 feet) and 5.5-6.1 meters (18-20 feet).

The soil samples were analyzed for the constituents listed in Table 1-1. Sample identification nomenclature and quality control field samples are also listed in that table.

\subsection{Soil Borings Analysis}

The primary constituents detected in soils collected from Grace Road Site were metals, volatile organic compounds, and semi-volatile organic compounds. Metals found in concentrations greater than analytical method detection limits are arsenic, barium, cadmium, chromium, mercury, lead, selenium, tin, vanadium and zinc. The concentrations are shown in Table 1-2.

A few volatile and semi-volatile organic constituents were detected in at least one soil sample. Bis(2-ethylhexyl)phthalate was detected in concentrations ranging from 410 to $6,800 \mu \mathrm{g} / \mathrm{kg}(\mathrm{ppb})$. Phthalate species are used as plasticizers for cellulose, gtass, plastic, and rubber products. Other substances detected, such as acetone, xylene, and methylene chloride are commonly used as solvents. 
Grace Road Site

WSRC-RP-95-93, Rev. 1.1

RFI/RI Report

February 1996

TABLE 1-1

GRACE ROAD SOIL SAMPLING PARAMETERS

\begin{tabular}{|c|c|c|c|c|c|c|}
\hline Facility & $\begin{array}{c}\text { Borehole } \\
\text { Number(s) } \\
\end{array}$ & $\begin{array}{c}\text { Sample } \\
\text { Number(s) } \\
\end{array}$ & $\begin{array}{c}\text { Sample } \\
\text { Type } \\
\end{array}$ & $\begin{array}{l}\text { Sampling } \\
\text { Method }\end{array}$ & $\begin{array}{c}\text { Depth in } \\
\text { meters (feet) }\end{array}$ & $\begin{array}{c}\text { Analyses } \\
\text { Performed }\end{array}$ \\
\hline GRS & 01 & 01 & $\mathrm{~S}$ & HA & $0.6-0.9(2-3)$ & $1,2,3$ \\
\hline GRS & 01 & 02 & $S$ & $\mathrm{HA}$ & $1.5-1.8(5-6)$ & 1,2 \\
\hline GRS & 02 & 01 & $\mathbf{S}$ & HA & $0.6-0.9(2-3)$ & 1,2 \\
\hline GRS & 02 & 02 & $\mathbf{S}$ & $\mathrm{HA}$ & $1.5-1.8(5-6)$ & 1,2 \\
\hline GRS & 03 & 01 & $\mathbf{S}$ & $\mathrm{HA}$ & $0.6-0.9(2-3)$ & $1,2,6$ \\
\hline GRS & 03 & 02 & $S$ & $\mathrm{HA}$ & $1.5-1.8(5-6)$ & $1,2,6$ \\
\hline GRS & 04 & 01 & $\mathbf{S}$ & $\mathrm{HA}$ & $0.6-0.9(2-3)$ & $1,2,3,4$ \\
\hline GRS & 04 & 02 & $\mathbf{S}$ & $\mathrm{HA}$ & $1.5-1.8(5-6)$ & 1,2 \\
\hline GRS & 05 & 01 & $\mathbf{S}$ & HA & $0.6-0.9(2-3)$ & $1,2,3$ \\
\hline GRS & 05 & 02 & $S$ & HA & $1.5-1.8(5-6)$ & 1,2 \\
\hline GRS & 05 & 02 & $S$ & $\mathrm{HA}$ & $1.5-1.8(5-6)$ & 1,2 \\
\hline GRS & 06 & 01 & $S$ & $\mathrm{HA}$ & $0.6-0.9(2-3)$ & $1,2,3,4$ \\
\hline GRS & 06 & 02 & $S$ & $\mathrm{HA}$ & $1.5-1.8(5-6)$ & 1,2 \\
\hline GRS & 07 & 01 & $\mathrm{~S}$ & SS & $0.9-1.5(3-5)$ & $1,2,3$ \\
\hline GRS & 07 & 02 & $S$ & SS & $3.2-3.7(10-12)$ & 1,2 \\
\hline GRS & 07 & 03 & $S$ & SS & $5.5-6.1(18-20)$ & 1,2 \\
\hline GRS & 08 & 01 & $S$ & SS & $0.9-1.5(3-5)$ & $4,5,6$ \\
\hline GRS & 08 & 02 & $S$ & SS & $3.2-3.7(10-12)$ & $1,2,6$ \\
\hline GRS & 08 & 03 & $S$ & SS & $5.5-6.1(18-20)$ & $1,2,6$ \\
\hline GRS & 09 & 01 & $S$ & SS & $0.9-1.5(3-5)$ & $1,2,3$ \\
\hline GRS & 09 & 02 & $S$ & SS & $3.2-3.7(10-12)$ & 1,2 \\
\hline GRS & 09 & 03 & $S$ & SS & $5.5-6.1(18-20)$ & 1,2 \\
\hline GRS & 10 & 01 & $S$ & SS & $0.9-1.5(3-5)$ & $1,2,3,4,6$ \\
\hline GRS & 10 & 02 & $S$ & SS & $3.2-3.7(10-12)$ & $1,2,4,6$ \\
\hline GRS & 10 & 03 & $\mathbf{S}$ & SS & $5.5-6.1(18-20)$ & $1,2,4,6$ \\
\hline GRS & 11 & 01 & $S$ & $\mathrm{HA}$ & $0.6-0.9(2-3)$ & $1,2,3$ \\
\hline GRS & 11 & 02 & $S$ & $\mathrm{HA}$ & $1.5-1.8(5-6)$ & 1,2 \\
\hline GRS & 12 & 01 & $S$ & $\mathrm{HA}$ & $0.6-0.9(2-3)$ & $1,2,6$ \\
\hline GRS & 12 & 02 & $\mathbf{S}$ & $\mathrm{HA}$ & $1.5-1.8(5-6)$ & $1,2,6$ \\
\hline GRS & 13 & 01 & $S$ & $\mathrm{HA}$ & $0.6-0.9(2-3)$ & $1,2,3$ \\
\hline GRS & 13 & 02 & $S$ & $\mathrm{HA}$ & $1.5-1.8(5-6)$ & 1,2 \\
\hline GRS & 14 & 01 & $S$ & $\mathrm{HA}$ & $0.6-0.9(2-3)$ & $1,2,3$ \\
\hline GRS & 14 & 02 & $S$ & $\mathrm{HA}$ & $1.5-1.8(5-6)$ & 1,2 \\
\hline GRS & 08 & 03E & $\mathrm{R}$ & SS & N/A & $1,3,4$ \\
\hline GRS & 13 & $01 \mathrm{E}$ & $\mathrm{R} \div$ & $\mathrm{HA}$ & N/A $\quad \Rightarrow$ & $1,3,4$ \\
\hline GRS & $04^{\circ}$ & $01 \mathrm{~A}$ & $\mathrm{D}$ & $\mathrm{HA}$ & $0.6-0.9(2-3)$ & $1,2,3$ \\
\hline GRS & 07 & $01 \mathrm{~A}$ & $\mathrm{D}$ & SS & $0.9-1.5(3-5)$ & $1,2,3$ \\
\hline
\end{tabular}

Table 1-1 continued on next page 
TABLE 1-1 (Continued)

GRACE ROAD SOIL SAMPLING PARAMETERS

\begin{tabular}{|l|c|c|c|c|c|c|}
\hline Facility & $\begin{array}{c}\text { Borehole } \\
\text { Number(s) }\end{array}$ & $\begin{array}{c}\text { Sample } \\
\text { Number(s) }\end{array}$ & $\begin{array}{c}\text { Sample } \\
\text { Type }\end{array}$ & $\begin{array}{c}\text { Sampling } \\
\text { Method }\end{array}$ & $\begin{array}{c}\text { Depth } \\
\text { in meters (feet) }\end{array}$ & $\begin{array}{c}\text { Analyses } \\
\text { Performed }\end{array}$ \\
\hline & & & \multicolumn{2}{|c|}{} & & \\
GRS & 00 & 01C & \multicolumn{2}{|c|}{ FIELD BLANK } & N/A & 1 \\
GRS & 00 & 01B & TRIP BLANK & N/A & 1 \\
GRS & 14 & 01C & FIELD BLANK & N/A & 1 \\
GRS & 14 & 01B & TRIP BLANK & N/A & 1 \\
\hline GRS & 05 & 01D & SPLIT & HA & $0.6-0.9(2-3)$ & $1,2,3$ \\
GRS & 08 & 02D & SPLIT & SS & $3.2-3.7(10-12)$ & 1,2 \\
GRS & 10 & 03D & SPLIT & SS & $5.5-6.1(18-20)$ & 1,2 \\
\hline
\end{tabular}

\section{ABBREVIATIONS}

S - Soil Sample

R - Rinsate

D - Duplicate

BG - Background sample

HA - Hand Auger

SS - Split Spoon

\section{ANALYSES}

1. Volatile Organic Compounds (EPA Method 8240)

2. Semi-Volatile Organic Compounds (EPA Method 8270)

3. Total Metals (for eight EP-toxicity metals: $\mathrm{As}, \mathrm{Ba}, \mathrm{Cd}, \mathrm{Cr}, \mathrm{Pb}$, $\mathrm{Hg}, \mathrm{Se}, \mathrm{Ag}$ )

4. Radionuclide indicators (gross alpha, non-volatile beta)

5. EPA Appendix IX (40 CFR, Part 264) Scan

6. General Indicator Parameters (soil pH, TOC, CEC, Specific Conductivity 
Grace Road Site

WSRC-RP-95-93, Rev. 1.1

RFI/RI Report

February 1996

Table 1-2

Soil Chemical Analysis Results (Organics in mg/kg)

\begin{tabular}{|c|c|c|c|c|c|c|c|c|c|}
\hline $\begin{array}{l}\text { GRS } \\
\text { Sample } \\
\text { Number }\end{array}$ & DEFP & $\begin{array}{c}\text { Carbon } \\
\text { Disulfide }\end{array}$ & DDT & Styrene & Acetone & Toluene & $\begin{array}{c}\text { Di-n- } \\
\text { BP }\end{array}$ & TCE & Xylene \\
\hline $01-01$ & $\mathrm{U}$ & $\mathrm{U}$ & $\mathrm{U}$ & $\mathrm{U}$ & $\mathrm{U}$ & $\mathrm{U}$ & $\mathrm{U}$ & $\mathrm{U}$ & $\mathrm{U}$ \\
\hline $02-01$ & $\mathrm{U}$ & $\mathbf{U}$ & $\mathrm{U}$ & $\mathrm{U}$ & $\mathrm{U}$ & $\mathrm{U}$ & $\mathrm{U}$ & $\mathrm{U}$ & $\mathrm{U}$ \\
\hline $03-01$ & $\mathrm{U}$ & $0.002 \mathrm{~J}$ & $\mathrm{U}$ & $\mathrm{U}$ & $\mathbf{U}$ & $\mathbf{U}$ & $\mathbf{U}$ & 0.0025 & $\mathrm{U}$ \\
\hline 04-01 & $\mathrm{U}$ & $\mathrm{U}$ & $\mathrm{U}$ & $\mathrm{U}$ & $\mathrm{U}$ & $\mathrm{U}$ & $\mathrm{U}$ & $\mathrm{U}$ & $\mathrm{U}$ \\
\hline 04-01A & $\mathrm{U}$ & $\mathrm{U}$ & $\mathrm{U}$ & $\mathrm{U}$ & $\mathrm{U}$ & $\mathrm{U}$ & $\mathrm{U}$ & $\mathbf{U}$ & $\mathrm{U}$ \\
\hline $05-01$ & $\mathrm{U}$ & $\mathrm{U}$ & $\mathrm{U}$ & $\mathrm{U}$ & $\mathrm{U}$ & $\mathrm{U}$ & $49 \mathrm{~J}$ & $\mathrm{U}$ & $\mathrm{U}$ \\
\hline $06-01$ & $\mathbf{U}$ & $\mathrm{U}$ & $\mathrm{U}$ & $\mathrm{U}$ & $\mathbf{U}$ & $\mathrm{U}$ & $42 \mathrm{~J}$ & $\bar{U}$ & $\bar{U}$ \\
\hline $07-01$ & 1.4 & $\mathrm{U}$ & $\mathrm{U}$ & $\mathrm{U}$ & $0.002 \mathrm{~J}$ & $\mathrm{U}$ & $\mathrm{U}$ & $\mathrm{U}$ & $\mathrm{U}$ \\
\hline $07-01 \mathrm{~A}$ & $\cdot 1.9$ & $\mathrm{U}$ & $\mathrm{U}$ & $\mathrm{U}$ & $0.002 \mathrm{~J}$ & $0.001 \mathrm{~J}$ & $\mathrm{U}$ & $\mathrm{U}$ & $\mathrm{U}$ \\
\hline $07-02$ & $0.088 \mathrm{~J}$ & $U$ & $U$ & $\mathrm{U}$ & U & $\mathrm{U}$ & $\mathrm{U}$ & $\mathrm{U}$ & $\mathrm{U}$ \\
\hline $07-03$ & 4.2 & $\mathrm{U}$ & $\mathrm{U}$ & $\underline{U}$ & $\mathrm{U}$ & $0.003 \mathrm{~J}$ & $\mathbf{U}$ & $\mathrm{U}$ & 0.004 \\
\hline $08-01$ & 2.8 & $\mathrm{U}$ & $0.0063 \mathrm{~J}$ & $\mathrm{U}$ & $\mathrm{U}$ & $\mathrm{U}$ & $\mathrm{U}$ & $\mathrm{U}$ & $\mathrm{U}$ \\
\hline $08-02$ & 6.8 & $\mathrm{U}$ & $\mathrm{U}$ & $\mathrm{U}$ & $\mathrm{U}$ & $\mathrm{U}$ & $\mathrm{U}$ & $\mathrm{U}$ & $\mathrm{U}$ \\
\hline $08-03$ & 3.7 & $\mathrm{U}$ & $\mathrm{U}$ & $\mathrm{U}$ & $\mathrm{U}$ & $\mathrm{U}$ & U & $\mathrm{U}$ & $U$ \\
\hline $09-01$ & 1.0 & $\mathrm{U}$ & $\mathrm{U}$ & $\mathrm{U}$ & $\mathrm{U}$ & $\mathrm{U}$ & $\mathrm{U}$ & $\mathrm{U}$ &.$U$ \\
\hline $09-02$ & 4.8 & $\mathbf{U}$ & $\mathbf{U}$ & $\mathrm{U}$ & $\mathbf{U}$ & $\mathrm{U}$ & $\mathrm{U}$ & $\mathrm{U}$ & $\mathrm{U}$ \\
\hline $09-03$ & 4.7 & $\mathrm{U}$ & $\mathbf{U}$ & $\mathrm{U}$ & $0.002 \mathrm{~J}$ & $\mathrm{U}$ & $\mathrm{U}$ & $\mathrm{U}$ & $\mathrm{U}$ \\
\hline $10-01$ & 1.3 & $\mathbf{U}$ & $\mathrm{U}$ & $\mathrm{U}$ & $\mathrm{U}$ & $\mathrm{U}$ & $\mathrm{U}$ & $\mathrm{U}$ & $\mathrm{U}$ \\
\hline $10-02$ & 6.9 & $\mathrm{U}$ & $\mathrm{U}$ & $U$ & $\mathbf{U}$ & $\mathrm{U}$ & $\mathrm{U}$ & $\mathrm{U}$ & $\mathrm{U}$ \\
\hline $10-03$ & 5.9 & $\mathrm{U}$ & $\mathrm{U}$ & $\mathrm{U}$ & $\mathrm{U}$ & $\mathrm{U}$ & $\mathrm{U}$ & $\mathrm{U}$ & $\mathrm{U}$ \\
\hline $11-01$ & $U$ & $\mathrm{U}$ & $\mathrm{U}$ & $\mathrm{U}$ & $\mathrm{U}$ & $\mathrm{U}$ & $\mathrm{U}$ & $0.003 \mathrm{~J}$ & $\mathrm{U}$ \\
\hline $12-01$ & $\mathrm{U}$ & $\mathrm{U}$ & $\mathrm{U}$ & $\mathrm{U}$ & $\mathrm{U}$ & $\mathrm{U}$ & $53 \mathrm{~J}$ & $\mathrm{U}$ & $\mathrm{U}$ \\
\hline $13-01$ & $\mathrm{U}$ & $\mathrm{U}$ & $\mathrm{U}$ & $\mathrm{U}$ & $\mathrm{U}$ & $\mathrm{U}$ & $49 \mathrm{~J}$ & 0.007 & $\mathrm{U}$ \\
\hline 14-01 & $\mathrm{U}$ & $\mathrm{U}$ & $\mathrm{U}$ & $0.004 \mathrm{~J}$ & $\mathrm{U}$ & $\mathrm{U}$ & $42 \mathrm{~J}$ & 0.006 & $\mathrm{U}$ \\
\hline Minimum & $0.088 \mathrm{~J}$ & $0.002 \mathrm{~J}$ & $0.0063 \mathrm{~J}$ & $0.004 \mathrm{~J}$ & $0.002 \mathrm{~J}$ & $0.001 \mathrm{~J}$ & $42 \mathrm{~J}$ & 0.0025 & 0.004 \\
\hline Maximum & 6.9 & $0.002 \mathrm{~J}$ & $0.0063 \mathrm{~J}$ & $0.004 \mathrm{~J}$ & $0.002 \mathrm{~J}$ & $0.003 \mathrm{~J}$ & $53 \mathrm{~J}$ & 0.007 & 0.004 \\
\hline
\end{tabular}

Table 1-2 continued on next page 
Grace Road Site

WSRC-RP-95-93, Rev. 1.1

RFI/RI Report

February 1996

Table 1-2 (continued)

Soil Chemical Analysis Results (Metals in $\mathrm{mg} / \mathrm{kg}$ )

\begin{tabular}{|l|c|c|c|c|c|c|c|c|c|c|}
\hline $\begin{array}{l}\text { GrS } \\
\text { Nample }\end{array}$ & As & Ba & Cd & Cr & Hg & Pb & Se & Sn & V & Zn \\
\hline $01-01$ & $\mathrm{U}$ & $\mathrm{U}$ & 1.8 & 5.7 & $\mathrm{U}$ & $\mathbf{0 . 9}$ & $\mathrm{U}$ & $\mathrm{U}$ & $\mathrm{U}$ & $\mathrm{U}$ \\
\hline $02-01$ & $\mathrm{U}$ & $\mathrm{U}$ & $\mathrm{U}$ & $\mathrm{U}$ & $\mathrm{U}$ & $\mathrm{U}$ & $\mathrm{U}$ & $\mathrm{U}$ & $\mathrm{U}$ & $\mathrm{U}$ \\
\hline $03-01$ & $\mathrm{U}$ & $\mathrm{U}$ & $\mathrm{U}$ & $\mathrm{U}$ & $\mathrm{U}$ & $\mathrm{U}$ & $\mathrm{U}$ & $\mathrm{U}$ & $\mathrm{U}$ & $\mathrm{U}$ \\
\hline $04-01$ & $\mathrm{U}$ & $\mathrm{U}$ & 1.2 & 5.7 & $\mathrm{U}$ & 0.95 & $\mathrm{U}$ & $\mathrm{U}$ & $\mathrm{U}$ & $\mathrm{U}$ \\
\hline $04-01 \mathrm{~A}$ & $\mathrm{U}$ & $\mathrm{U}$ & 1.2 & 5.3 & $\mathrm{U}$ & 1.1 & $\mathrm{U}$ & $\mathrm{U}$ & $\mathrm{U}$ & $\mathrm{U}$ \\
\hline $05-01$ & $\mathrm{U}$ & $\mathrm{U}$ & 1.2 & 8.0 & $\mathrm{U}$ & 2.1 & $\mathrm{U}$ & $\mathrm{U}$ & $\mathrm{U}$ & $\mathrm{U}$ \\
\hline $06-01$ & $\mathrm{U}$ & $\mathrm{U}$ & 1.2 & 14.9 & 0.14 & 5.1 & $\mathrm{U}$ & $\mathrm{U}$ & $\mathrm{U}$ & $\mathrm{U}$ \\
\hline $07-01$ & 3.2 & $\mathrm{U}$ & $\mathrm{U}$ & 28.8 & $\mathrm{U}$ & 8.9 & $\mathrm{U}$ & $\mathrm{U}$ & $\mathrm{U}$ & $\mathrm{U}$ \\
\hline $07-01 \mathrm{~A}$ & 2.7 & $\mathrm{U}$ & $\mathrm{U}$ & 29.0 & $\mathrm{U}$ & 8.1 & $\mathrm{U}$ & $\mathrm{U}$ & $\mathrm{U}$ & $\mathrm{U}$ \\
\hline $07-02$ & $\mathrm{U}$ & $\mathrm{U}$ & $\mathrm{U}$ & $\mathrm{U}$ & $\mathrm{U}$ & $\mathrm{U}$ & $\mathrm{U}$ & $\mathrm{U}$ & $\mathrm{U}$ & $\mathrm{U}$ \\
\hline $07-03$ & $\mathrm{U}$ & $\mathrm{U}$ & $\mathrm{U}$ & $\mathrm{U}$ & $\mathrm{U}$ & $\mathrm{U}$ & $\mathrm{U}$ & $\mathrm{U}$ & $\mathrm{U}$ & $\mathrm{U}$ \\
\hline $08-01$ & 2.6 & $\mathrm{U}$ & $\mathrm{U}$ & 29.6 & $\mathrm{U}$ & 8.4 & $\mathrm{U}$ & 32.5 & 61.8 & 7.0 \\
\hline $08-02$ & $\mathrm{U}$ & $\mathrm{U}$ & $\mathrm{U}$ & $\mathrm{U}$ & $\mathrm{U}$ & $\mathrm{U}$ & $\mathrm{U}$ & $\mathrm{U}$ & $\mathrm{U}$ & $\mathrm{U}$ \\
\hline $08-03$ & $\mathrm{U}$ & $\mathrm{U}$ & $\mathrm{U}$ & $\mathrm{U}$ & $\mathrm{U}$ & $\mathrm{U}$ & $\mathrm{U}$ & $\mathrm{U}$ & $\mathrm{U}$ & $\mathrm{U}$ \\
\hline $09-01$ & $\mathrm{U}$ & $\mathrm{U}$ & $\mathrm{U}$ & 3.9 & $\mathrm{U}$ & 2.0 & $\mathrm{U}$ & $\mathrm{U}$ & $\mathrm{U}$ & $\mathrm{U}$ \\
\hline $09-02$ & $\mathrm{U}$ & $\mathrm{U}$ & $\mathrm{U}$ & $\mathrm{U}$ & $\mathrm{U}$ & $\mathrm{U}$ & $\mathrm{U}$ & $\mathrm{U}$ & $\mathrm{U}$ & $\mathrm{U}$ \\
\hline $09-03$ & $\mathrm{U}$ & $\mathrm{U}$ & $\mathrm{U}$ & $\mathrm{U}$ & $\mathrm{U}$ & $\mathrm{U}$ & $\mathrm{U}$ & $\mathrm{U}$ & $\mathrm{U}$ & $\mathrm{U}$ \\
\hline $10-01$ & $\mathrm{U}$ & $\mathrm{U}$ & $\mathrm{U}$ & 4.2 & $\mathrm{U}$ & 1.4 & $\mathrm{U}$ & $\mathrm{U}$ & $\mathrm{U}$ & $\mathrm{U}$ \\
\hline $10-02$ & $\mathrm{U}$ & $\mathrm{U}$ & $\mathrm{U}$ & $\mathrm{U}$ & $\mathrm{U}$ & $\mathrm{U}$ & $\mathrm{U}$ & $\mathrm{U}$ & $\mathrm{U}$ & $\mathrm{U}$ \\
\hline $10-03$ & $\mathrm{U}$ & $\mathrm{U}$ & $\mathrm{U}$ & $\mathrm{U}$ & $\mathrm{U}$ & $\mathrm{U}$ & $\mathrm{U}$ & $\mathrm{U}$ & $\mathrm{U}$ & $\mathrm{U}$ \\
\hline $11-01$ & $\mathrm{U}$ & $\mathrm{U}$ & 1.3 & 13.3 & 0.14 & 3.9 & $\mathrm{U}$ & $\mathrm{U}$ & $\mathrm{U}$ & $\mathrm{U}$ \\
\hline $12-01$ & $\mathrm{U}$ & $\mathrm{U}$ & $\mathrm{U}$ & $\mathrm{U}$ & $\mathrm{U}$ & $\mathrm{U}$ & $\mathrm{U}$ & $\mathrm{U}$ & $\mathrm{U}$ & $\mathrm{U}$ \\
\hline $13-01$ & $\mathrm{U}$ & $\mathrm{U}$ & 1.2 & 22.9 & 0.15 & 48.1 & 1.3 & $\mathrm{U}$ & $\mathrm{U}$ & $\mathrm{U}$ \\
\hline $14-01$ & $\mathrm{U}$ & 48.4 & 1.7 & 18.1 & $\mathrm{U}$ & 5.1 & $\mathrm{U}$ & $\mathrm{U}$ & $\mathrm{U}$ & $\mathrm{U}$ \\
\hline & & & & & & & & & & \\
\hline Minimum & 2.6 & 48.4 & 1.2 & 3.9 & 0.14 & 0.9 & 1.3 & 32.5 & 61.8 & 7.0 \\
\hline Maximum & 3.2 & 48.4 & 1.8 & 29.6 & 0.15 & 48.1 & 1.3 & 32.5 & 61.8 & 7.0 \\
\hline $19611-2$ & $\mathrm{n}$ & & & & & & \\
\hline
\end{tabular}

Table 1-2 continued on next page 
Table 1-2 (continued)

NOTE:

$\mathrm{U}=$ Compound was analyzed for but not detected

$\mathrm{J}=$ Estimated detected value

DEHP = Bis(2-Ethylhexyl)Phthalate

Di-n-BP = Di-n-butyl Phthalate

$\mathrm{TCE}=$ Trichloroethylene

As $=$ Arsenic

$\mathrm{Ba}=$ Barium

$\mathrm{Cd}=$ Cadmium

$\mathrm{Cr}=$ Chromium

$\mathrm{Hg}=$ Mercury

$\mathrm{Pb}=$ Lead

$\mathrm{Se}=$ Selenium

$\mathrm{Sn}=\mathrm{Tin}$

$\mathrm{V}=$ Vanadium

$\mathrm{Zn}=$ Zinc

Only those compounds which showed a detection level are noted in this table.

Methylene Chloride was detected in the sample blanks

Radionuclide indicators were analyzed for in the following samples:

\begin{tabular}{ccc} 
Sample Number & Gross Alpha (pCi/g) & Nonvolatile Beta (pCi/g) \\
\cline { 2 - 3 } GRS-04-01 & 11 & 13 \\
GRS-06-01 & 5 & 7 \\
GRS-08-01 & 22 & 10 \\
GRS-10-01 & 14 & 0 \\
GRS-10-02 & 21 & 7 \\
GRS-10-03 & 30 & 4 \\
\hline
\end{tabular}

Acetone and the chlorinated solvent methylene chloride were detected in concentrations ranging from 10 to $99 \mu \mathrm{g} / \mathrm{kg}$ and 6 to $33 \mu \mathrm{g} / \mathrm{kg}$, respectively. One of the laboratory method blanks detected methylene chloride at $10 \mu \mathrm{g} / \mathrm{kg}$ and acetone at $21 \mu \mathrm{g} / \mathrm{kg}$. Because of the presence of these commonly documented laboratory artifacts in the method blanks, low to moderate concentrations of them in the soil samples may be suspect.

In some instances, analytes are detected at different intervals. The concentration level of the analytes, with the exception of arsenic and lead, at different intervals is very low and insignificant in terms of having an effect on human health and the environment. The concentration level of arsenic at different intervals ranges from 2.6 to $3.2 \mathrm{mg} / \mathrm{kg}$ and for lead, the range is 0,9 to $48.1 \mathrm{mg} / \mathrm{kg}$. 
Radionuclide indicators (gross alpha, non-volatile beta) were analyzed in six soil samples. Gross alpha was detected at levels of 5 to $30 \mathrm{pCi} / \mathrm{g}$ and non-volatile beta detected at levels of 0 to $13 \mathrm{pCi} / \mathrm{g}$.

Information on the soil collection procedures and analysis methods can be found in Section 3.0 and 6.0 of the RFI/RI Work Plan for Grace Road (WSRC-RP-90-1250) (WSRC, 1990). Data on the QC/QA procedures and results are contained in Appendix B of this report.

\subsubsection{Unit Screening-Geophysical Investigation}

A surface geophysical survey was conducted at Grace Road Site in March and April 1990 to screen the area for the presence of buried metal objects such as drums, cans, cables, or underground storage tanks. The survey was conducted over a 750 -foot by 200 -foot area using the Geonics $\otimes$ EM-31 terrain conductivity instrument. Using the EM-31 permitted continuous screening along the survey path to a depth of approximately 3.05 to 4.9 meters (10 to 16 feet), thus increasing the potential for detection of subsurface geo-electrical anomalies (which can be interpreted as objects).

The EM-31 survey grid was laid out over a portion of Grace Road Site which was least encumbered by vegetation and surface debris containing metal. Two baselines, trending southeast and southwest, and oriented 90 degrees to each other, were established. Both lines had stations established at 15.2 meter (50-foot) intervals and were labeled 1 to 16 along the 228.7 meter ( 750 foot) southeast trending boundary line (paralleling Grace Road), and $A$ to $E$ along the 61 meter ( 200 foot) southwest trending boundary line. A transit and tape measure were used to set up the 80 stations resulting from the intersection of these two sets of boundary line increments. These stations were staked and labeled A-1 to E-16 (see Figure 1-6).

Surveys were conducted in two orientations: first along all five southeast-oriented lines across the length of the grid (lines $A$ to $E$ ); and second along all 16 southwest-oriented lines across the width of the grid (lines 1 to 16). Ground conductivity readings at each node were recorded. Some areas of the unit are heavily wooded which prevented surveying according to a regular grid. Clearings within wooded areas were surveyed as 
Grace Road Site

WSRC-RP-95-93, Rev. 1.1

RFI/RI Report

-February 1996

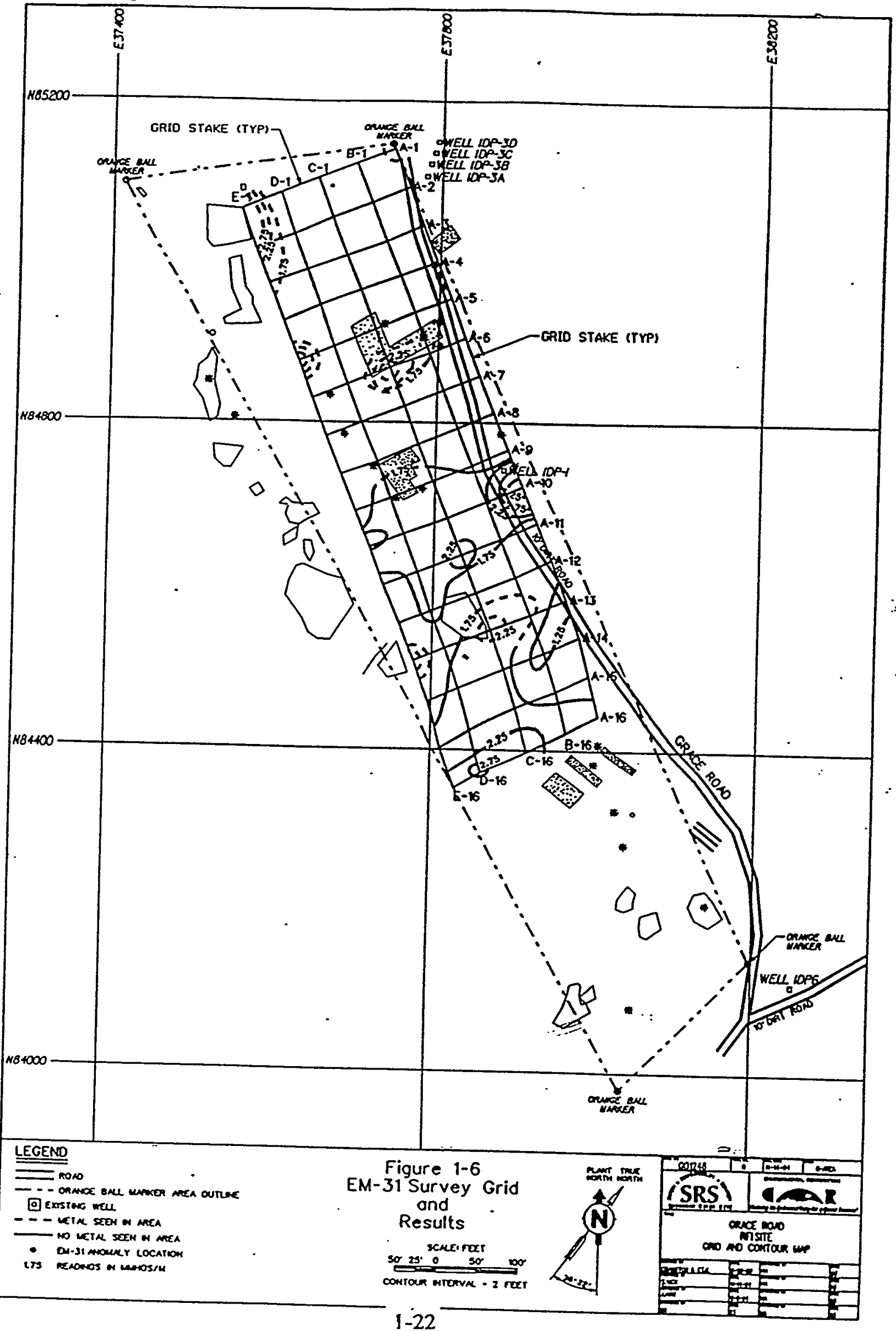


systematically as possible. Any anomalies noted between stations were plotted on the grid base map and flagged in the field (see Figure 1-6).

Two concrete pads which possibly contained reinforcing rod (between C-5 and C-6 and between D-8 and C-9), auto parts at C-13, bridge rubble at E-13, metal at E-5 and C-6, and construction debris at E-1 and E-2 encountered within the gridded area caused some interference.

\subsubsection{Unit Screening Conclusions}

Based on the Unit Screening data, hazardous substance concentrations appear to be at or below background concentrations or below risk-based concentration (RBC) levels. The soil chemical analysis results were compared to two times the average background concentration and risk-based concentration levels developed by the EPA Region III. The results are shown in Table 1-3. The only constituents which exceed the RBC and background levels are arsenic and non-volatile beta.

A comparison of Unit Screening soil data with EPA risk-based concentration levels indicates that, except for arsenic, no concentrations of any hazardous substance (including laboratory artifacts) exceeds available risk-based standards. Due to arsenic at levels significantly above risk levels, there is indication of human health risk from the soils.

Table 1-4, ground water analytical results from water table well DP-6 immediately east of Grace Road, provides the level of constituents found in the ground water samples. Any wastes would have to have been at Grace Road Site for a sufficiently long enough time to allow for any potential contaminant to travel down to the water table and to the well. High $\mathrm{pH}$ and alkalinity values for these ground water samples were caused by grout contamination in the well. Soil sampling site GRS- 08 with the second highest gross alpha and non-volatile beta, and bis(2-ethylhexyl) phthalate is 22.9 meters (75 feet) upgradient from well IDP-6. Soil sampling site GRS-13 with the highest lead and mercury and second highest chromium measurements is 61 meters (200 feet) upgradient from well DP6. 
Grace Road Site

WSRC-RP-95-93, Rev. 1.1

RFI/RI Report

February 1996

Table 1-3

\begin{tabular}{|c|c|c|c|c|}
\hline $\begin{array}{r}\text { COI } \\
\text { CONCENTI }\end{array}$ & $\begin{array}{r}\text { COMPARISC } \\
\text { ICENTRATION } \\
\text { UATIONS AND } \\
\end{array}$ & $\begin{array}{l}\text { N OF UNIT SPECIFIC SO } \\
\text { TO TWO TIMES BACKG } \\
\text { RISK -BASED CONCENTI }\end{array}$ & $\begin{array}{l}\text { L } \\
\text { ROUND } \\
\text { ATIONS (RB }\end{array}$ & \\
\hline Contaminant (units) & $\begin{array}{c}\text { Maximum } \\
\text { Concentration }\end{array}$ & $\begin{array}{l}\text { Average Background Soil } \\
\text { Concentration (GRS-10) }\end{array}$ & $\begin{array}{c}\text { Two Times } \\
\text { Background }\end{array}$ & $\begin{array}{c}\text { RBC Value* } \\
(\mathrm{mg} / \mathrm{kg})\end{array}$ \\
\hline $\begin{array}{l}\text { Bis(2-ethylhexyl) } \\
\text { Phthalate (mg/kg) }\end{array}$ & 6.8 & 4.7 & 9.4 & 46 \\
\hline Carbon Disulfide (mg/kg) & $0.002 \mathrm{~J}$ & ND & ND & 7800 \\
\hline $\mathrm{DDT}(\mathrm{mg} / \mathrm{kg})$ & $0.0063 \mathrm{~J}$ & $\mathrm{ND}$ & $\mathrm{ND}$ & 1.9 \\
\hline Styrene (mg/kg) & $0.004 \mathrm{~J}$ & $\mathrm{ND}$ & ND & 16000 \\
\hline Acetone (mg/kg) & $0.002 \mathrm{~J}$ & ND & $\mathrm{ND}$ & 7800 \\
\hline Toluene (mg/kg) & $0.003 \mathrm{~J}$ & $\mathrm{ND}$ & $\mathrm{ND}$ & 16000 \\
\hline $\begin{array}{l}\text { Di-n-Butyl Phthalate } \\
(\mathrm{mg} / \mathrm{kg})^{-}\end{array}$ & $53 \mathrm{~J}$ & ND & ND & 7800 \\
\hline $\begin{array}{l}\text { Trichloroethylene } \\
(\mathrm{mg} / \mathrm{kg})\end{array}$ & 0.007 & $\mathrm{ND}$ & ND & .58 \\
\hline Xylene (mg/kg) & 0.004 & $\mathrm{ND}$ & $\mathrm{ND}$ & 160,000 \\
\hline Arsenic (mg/kg) & 3.2 & ND & ND & 0.37 \\
\hline Barium (mg/kg) & 48.4 & $\mathrm{ND}$ & ND & 5500 \\
\hline Cadmium (mg/kg) & 1.8 & $\mathrm{ND}$ & $\mathrm{ND}$ & 39 \\
\hline Chromium (VI) (mg/kg) & 29.6 & 4.2 & 8.4 & 390 \\
\hline Mercury (mg/kg) & 0.15 & $\mathrm{ND}$ & $\mathrm{ND}$ & 23 \\
\hline Lead (mg/kg) & 48.1 & 1.4 & 2.8 & $400^{* *}$ \\
\hline Selenium (mg/kg) & 1.3 & $\mathrm{ND}$ & $\mathrm{ND}$ & 390 \\
\hline $\operatorname{Tin}(\mathrm{mg} / \mathrm{kg})$ & 32.5 & ND & $\mathrm{ND}$ & 47000 \\
\hline Vanadium (mg/kg) & 61.8 & ND & $\mathrm{ND}$ & 550 \\
\hline Zinc (mg/kg) & 7.0 & $\mathrm{ND}$ & $\mathrm{ND}$ & 23000 \\
\hline Gross Alpha (pCi/g) & 22.0 & 21.7 & 43.4 & - \\
\hline $\begin{array}{l}\text { Non-volatile Beta } \\
\text { (pCi/g) }\end{array}$ & 13.0 & 3.7 & 7.4 & - \\
\hline
\end{tabular}

Table 1-3 continued on next page 


\section{Table 1-3 (continued)}

* EPA Region III, Risk-Based Concentration Table, January-June 1995, dated March 7, 1995 $\mathrm{j}=$ estimated value

** The screening level for lead in soil is $400 \mathrm{mg} / \mathrm{kg}$ for residential land use. This value is described in OSWER Directive \# 9355.4-12, Revised Internal Soil Lead Guidance for CERCLA Sites and RCRA Corrective Action Facilities, dated July 14, 1994 and issued by Elliott $P$. Lewis of the USEPA. The screening level for lead was calculated using the USEPA new integrated exposure uptake biokinetic model with default parameters.

Comparisons with unit-specific and risk-based concentration levels show that, with the exception of arsenic, there is no indication of any environmental impact caused by hazardous substance disposal at Grace Road Site.

Approximately 21 locations (see Figure 1-6 for locations) with anomalous readings were detected by the EM-31 terrain conductivity survey. Three anomalous EM negative readings occurred near borehole GRS-02; one on top of an adjacent mound and one on either side of the mound, Probing in the area with a shovel found no evidence of buried metal objects at a depth of 2 to 3 feet below ground surface. Two negative readings occurred in the vicinity of three small rectangular-shaped concrete pads in the southern part of the unit. Six other negative reading in the southern part were local isolated features with limited scope and magnitude. Probing with a shovel 2 to 3 feet beneath the surface found no evidence of buried metal debris. The remainder of the anomalies are limited in areal extent and were associated with visible metaldebris. Analytical results from three soil borings in close proximity to anomalies in the southern sector indicate that, if anything is buried in the area, its impacts are negligible. 
Grace Road Site

WSRC-RP-95-93, Rev. 1.1

RFI/RI Report

February 1996

\begin{tabular}{|c|c|c|}
\hline \multicolumn{3}{|c|}{$\begin{array}{l}\text { Table } 1-4 \\
\text { Analytical Results from Well DP-6 }\end{array}$} \\
\hline Parameter & Units & Value \\
\hline Conductivity & $\mu \mathrm{S} / \mathrm{cm}$ & 55.00 \\
\hline $\mathrm{pH}$ & units & 5.3 \\
\hline Total Dissolved Solids & $\mathrm{mg} / \mathrm{L}$ & 45.00 \\
\hline Total Organic Carbon & $\mathrm{mg} / \mathrm{L}$ & $<1.00$ \\
\hline Total Inorganic Carbon & $\mathrm{mg} / \mathrm{L}$ & 5.00 \\
\hline Total Carbon & $\mathrm{mg} / \mathrm{L}$ & 6.00 \\
\hline Chloride & $\mathrm{mg} / \mathrm{L}$ & 2.20 \\
\hline Silica & $\mathrm{mg} / \mathrm{L}$. & 9.60 \\
\hline Fluoride & $\mathrm{mg} / \mathrm{L}$ & $<0.10$ \\
\hline Nitrite Nitrogen & $\mathrm{mg} / \mathrm{L}$ & $<0.01$ \\
\hline Total Phosphate (as P) & $\mathrm{mg} / \mathrm{L}$ & $<0.05$ \\
\hline Nitrate (as Nitrogen) & $\mathrm{mg} / \mathrm{L}$ & 1.92 \\
\hline Sulfate & $\mathrm{mg} / \mathrm{L}$ & $<5.00$ \\
\hline Total Phenolics & $\mathrm{mg} / \mathrm{L}$ & $<0.005$ \\
\hline Mercury & $\mathrm{mg} / \mathrm{L}$ & $<0.0002$ \\
\hline Chromium & $\mathrm{mg} / \mathrm{L}$ & $<0.004$ \\
\hline Potassium & $\mathrm{mg} / \mathrm{L}$ & 0.658 \\
\hline Nickel & $\mathrm{mg} / \mathrm{L}$ & $<0.004$ \\
\hline Calcium & $\mathrm{mg} / \mathrm{L}$ & 3.66 \\
\hline Lead & $\mathrm{mgL}$ & $<0.006$ \\
\hline Cadmium & $\mathrm{mg} / \mathrm{L}$ & 0.002 \\
\hline Sodium & $\mathrm{mg} / \mathrm{L}$ & 4.06 \\
\hline Magnesium & $\mathrm{mg} / \mathrm{L}$ & 0.635 \\
\hline Uranium & $\mathrm{mg} / \mathrm{L}$ & $<1.00$ \\
\hline
\end{tabular}




\subsection{Report Organization}

This RFI/RI Report, following EPA Guidance (EPA, 1988), consists of seven chapters for describing the rationale for the investigation, unit characterization activities conducted, data evaluation, risk assessment, and data assessment. Chapter 1 provides background information summarizing the history of the unit, and the purpose and objectives of this investigation. Chapter 2 describes the objectives of the EPA-approved work plan.

Chapter 3 describes the physical characteristics of the unit including surface topography, hydrogeology, soil types, demography and land use, and ecology. Chapter 4 presents an evaluation of the contamination present at Grace Road Site. Chapter 5 evaluates the fate and transport mechanisms applicable to the contaminants detected, and Chapter 6 presents a summary of the human health and ecology baseline risk assessment. Chapter 7 summarizes the results of the RCRA/CERCLA Unit Assessment along with conclusions derived from data analysis. 
Grace Road Site

WSRC-RP-95-93, Rev. 1.1

RFI/RI Report

February 1996

THIS PAGE INTENTIONALLY LEFT BLANK 


\subsection{STUDY AREA INVESTIGATION}

\subsection{Surface Feature Investigation}

The general topography of Grace Road Site has been mapped and the surface drainage delineated. Figure 2-1 is a topographic map of the unit and Chapter 3.0 provides additional information on area physiography. There is little evidence to suggest this area contains a waste unit.

\subsection{Contaminant Source Investigation}

The general history of this area does not provide any information about its use as a waste unit. However, a unit screening program was required since the preliminary unit evaluation indicated the possible disposal of hazardous substances. The unit screening is discussed in Section 1.3.2.

\subsection{Meteorological Investigation}

As a nuclear facility, SRS maintains a system of seven meteorological towers located adjacent to each production area and at the WJBG-TV tower about 15 kilometers ( 9 miles) north of the unit. In addition, the National Weather Service maintains a station at Augusta's Bush Field airport approximately 26 kilometers ( 16 miles) northwest of this area.

Data from these sources have been compiled and published in numerous documents including the EIS for continued operation of K-, L-, and P-Keactors (DOE, 1990). Meteorological data are summarized in Section 3.2 No ambient air sampling was performed because Grace Road Site is inactive with no potential for impact to the air.

\subsection{Surface Water and Sediment Investigation}

Surface water/sediment sampling was not conducted because the nearest surface water is 


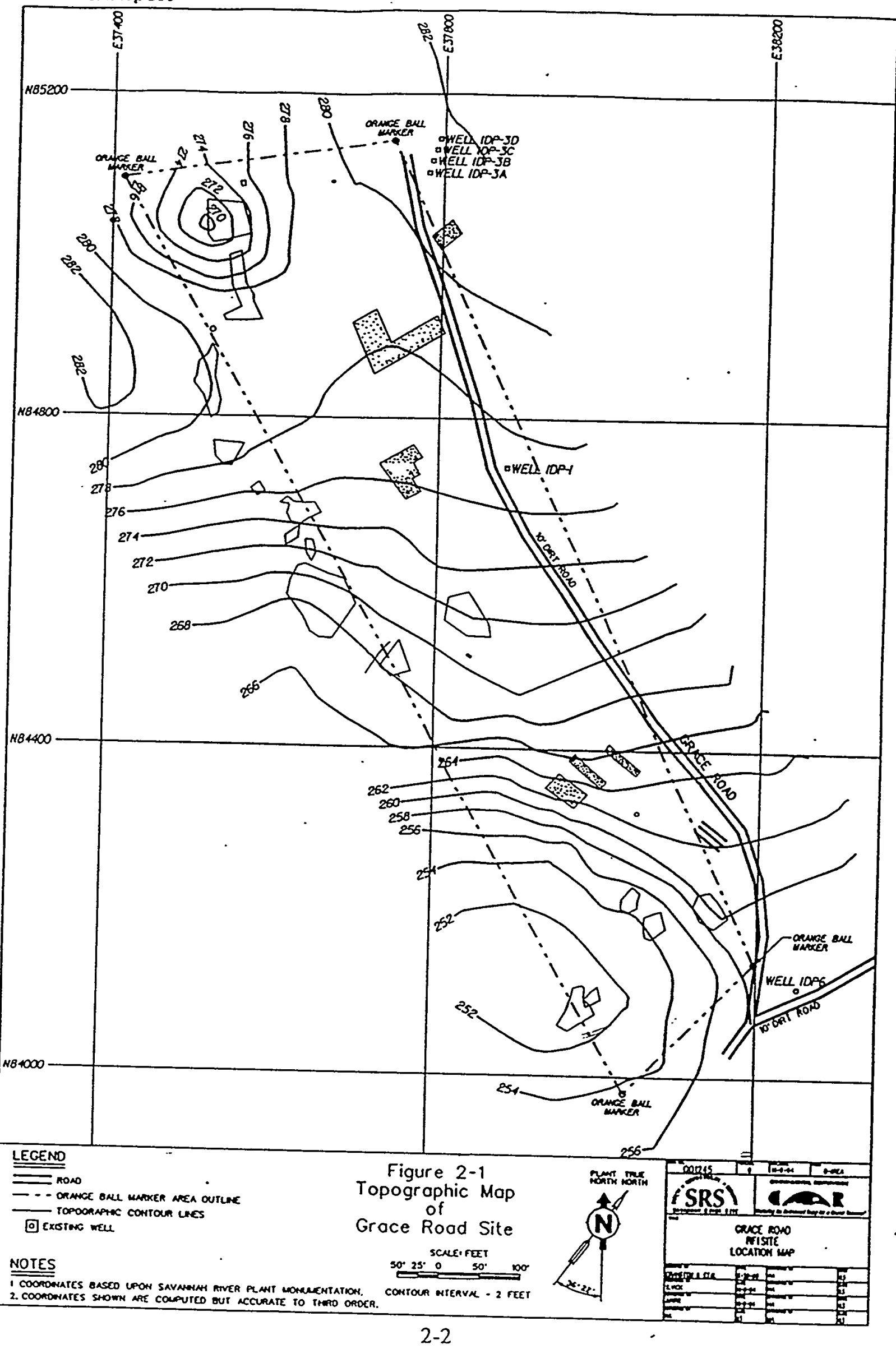


Upper Three Runs Creek approximately 2.4 kilometers ( 1.5 miles) east of the unit.

\subsection{Soil/Vadose Zone Investigations}

\subsubsection{Ground Penetrating Radar Investigation}

GPR was run at Grace Road Site for the purpose of identifying evidence of potential excavation and determining areas where buried waste and other debris may be found. A total of 53 lines totaling 12,879 feet were acquired in March and April 1994. The location of GPR lines acquired with a $100 \mathrm{MHz}$ antenna are shown in Figure 2-2, and lines surveyed with a $300 \mathrm{MHz}$ antenna are shown in Figure 2-3. A grid was laid out in approximately an east-west, north-south direction to determine the location of the GPR transects. Except for the trees and brush, surface conditions at the unit were considered good for GPR.

\subsubsection{Soil Gas Survey}

An additional soil gas survey was completed at the Grace Road unit in March 1994. The objective of that survey was to determine the presence and extent, or absence, of contamination in soils in and around the unit. A total of 85 sample locations were established and samples collected at each location (see Figure 2-4).

Species monitored for this survey were: light hydrocarbons $\left(\mathrm{C}_{1}-\mathrm{C}_{4}\right)$; gasoline range normal paraffins $\left(\mathrm{C}_{5}-\mathrm{C}_{10}\right)$; gasoline range aromatic hydrocarbons, (BTEX); diesel range hydrocarbons $\left(\mathrm{C}_{11}-\mathrm{C}_{17}\right)$; selected organics; and mercury. Samples were collected from a depth of 46 centimeters (18 inches).

Tabular results of the soil volatile organic compound (VOC) survey are provided in Appendix A.3. In general, the levels of volatile organics detected in this survey are very low with most below minimum detection levels. Low observed levels of chloroform in several samples is of the same order as that observed in the blanks and may result from chloroform present in the available distilled water. The level of light hydrocarbons is also 


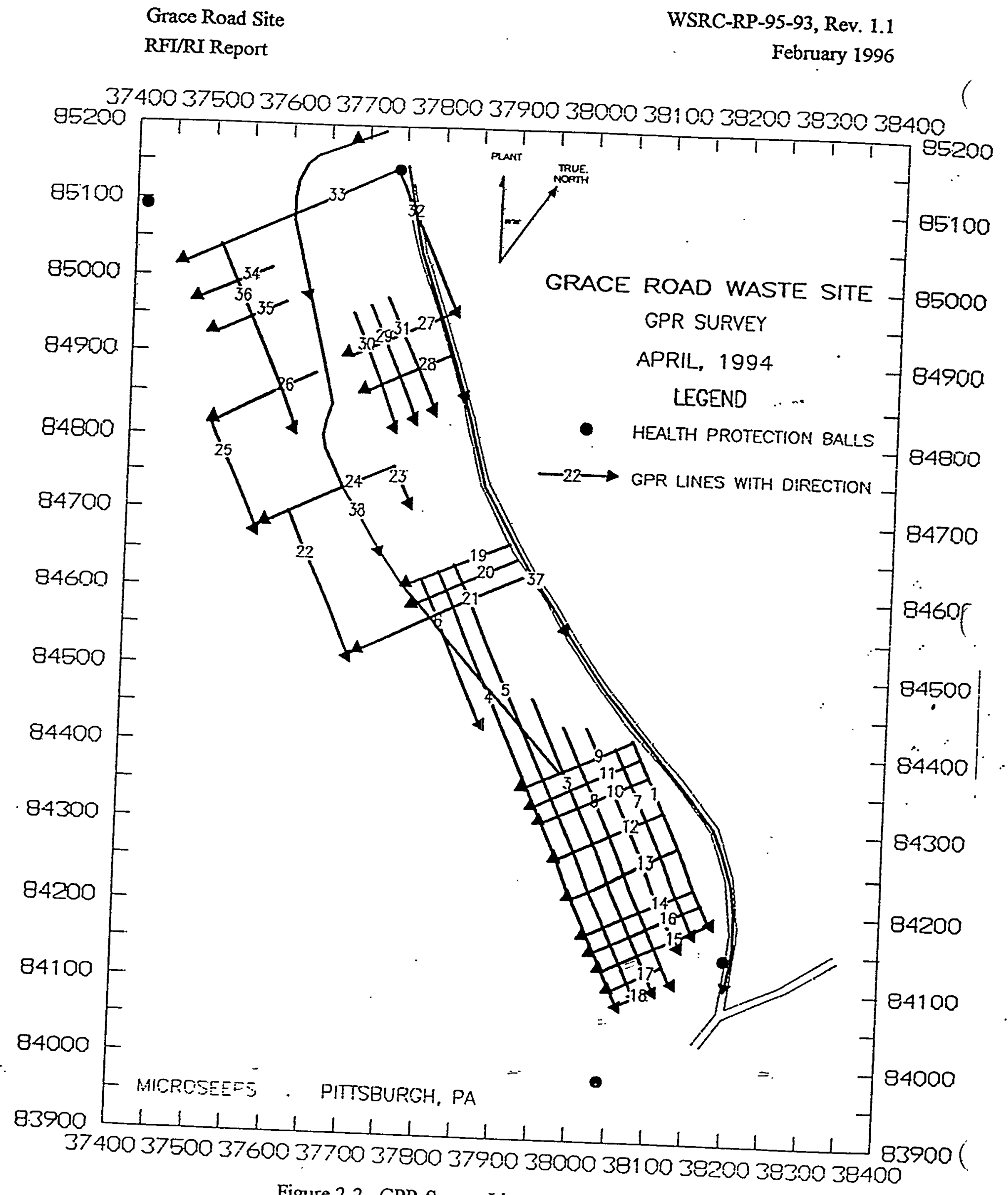

Figure 2-2 GPR Survey Lines, 100MHZ Antenna 
Grace Road Site

RFI/RI Report

WSRC-RP-95-93, Rev. 1.1

February 1996

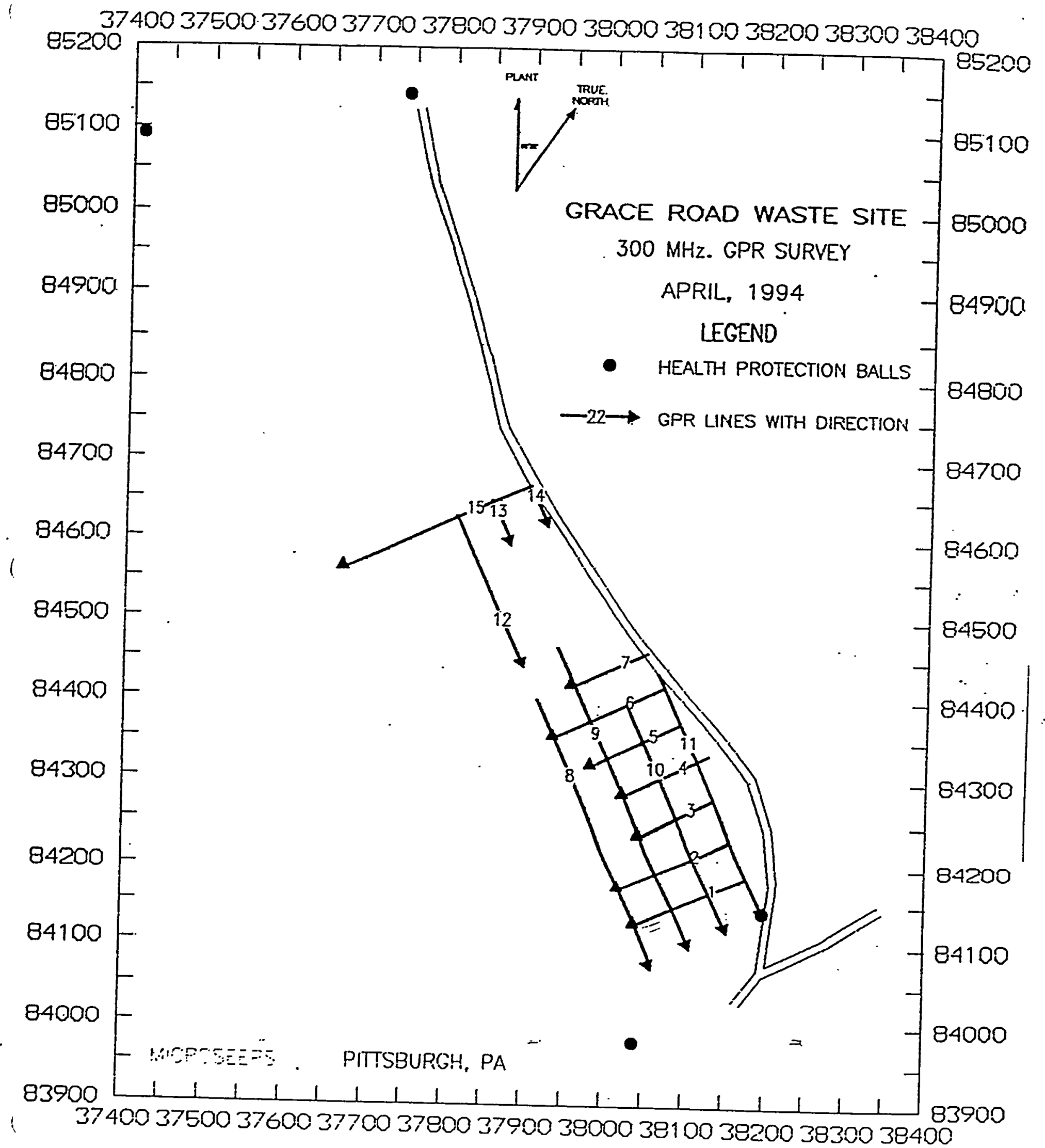

Figure 2- 3 GPR Survey Lines, 300MHZ Antenna 
Grace Road Site

WSRC-RP-95-93, Rev. 1.1

RFI/RI Report

February 1996

37400375003760003770037800379003800038100382003830038400 15200

$\begin{array}{lllllllll}1 & 1 & 1 & 1 & 1 & 1 & 1\end{array}$

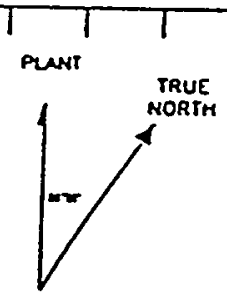

8520

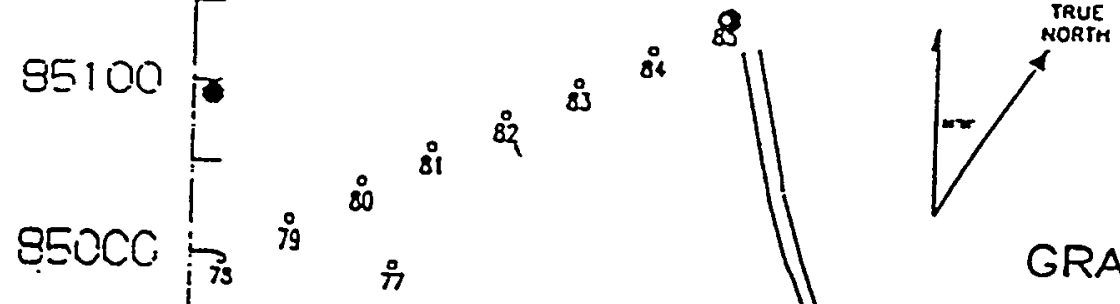

:

SACO:-

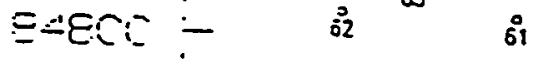

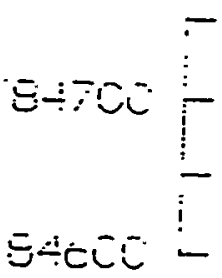

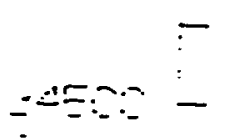

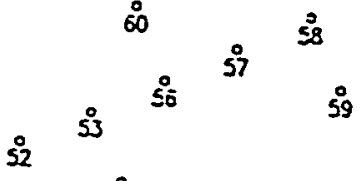

זั

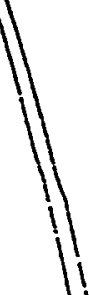

SOIL GAS SL'RVEY

MARC:- 1פG-1

85100

-

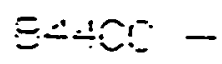

$\stackrel{4}{s}$

is is 4 is 4 is

- HENLIH FRCTELTION EALIS

3.9c0

$\Xi \div 9 C$

$-$

$\Xi \div-x i-$

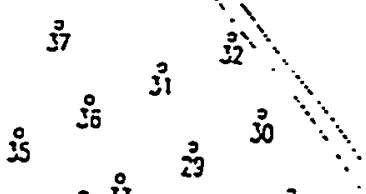

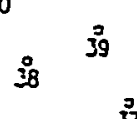

s-TC

si

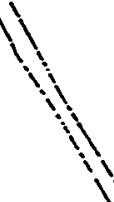

8

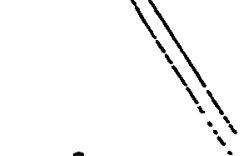

$\overbrace{3}^{3}$

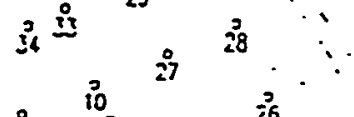

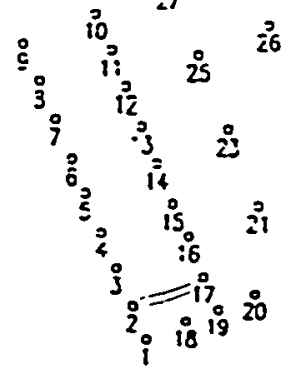

SAMPLE LOCATION MAP

84000

$-$

$\equiv \div: 00-$
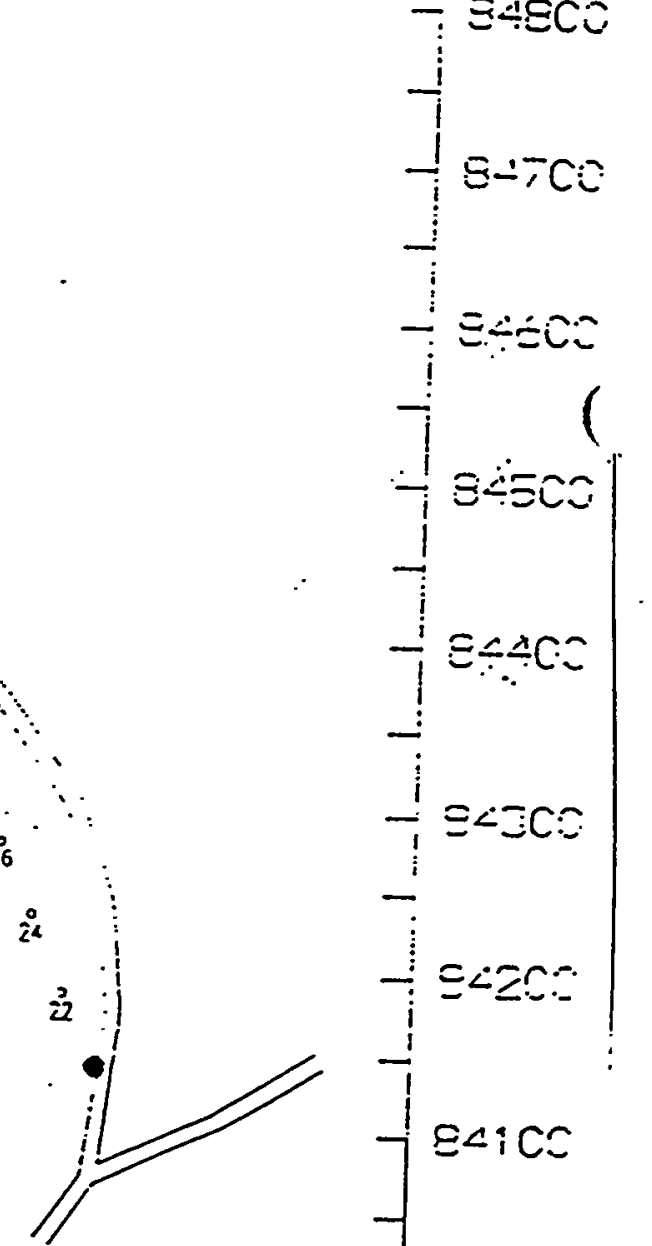

$\equiv \div 200-$

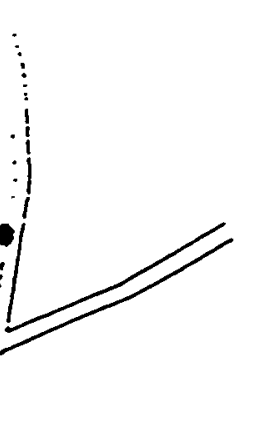

L MICRCSEEPS

PITTSEURGH, PA

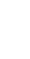

$\subseteq$

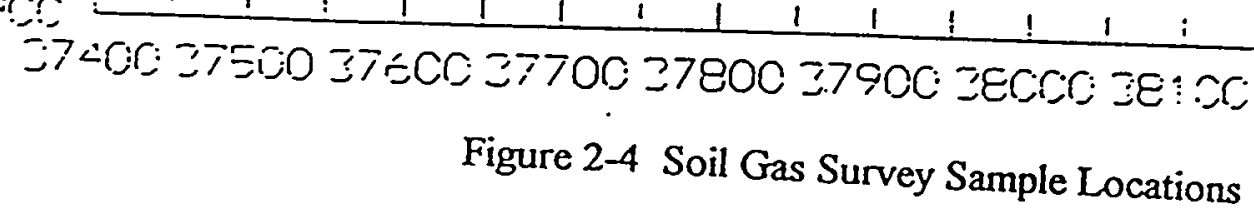


Grace Road Site

WSRC-RP-95-93, Rev. 1.1

RFI/RI Report

February 1996

very low. Mercury concentrations are in the range observed in background samples at SRS although the level at site \#1 is at the extreme of that range.

\subsubsection{Conclusions from GPR and Gas Survey}

The GPR survey was able to obtain good quality data with several of the plots having strong refractions indicating buried metallic objects. The data also indicated that the most of the sediment in the area is undisturbed except around some of the old farm buildings. The disturbed soil is either from the original building construction or from the removal of the slabs and blocks.

The levels of volatiles and diesel range organics observed in the survey are very low with most below minimum detection levels. Levels of light hydrocarbons and mercury are indicative of background concentration in the SRS area.

\subsubsection{Gross Alpha and Non-Volatile Beta Analysis}

As noted in Section 1.3.2.2.2, one gross alpha value of $30 \mathrm{pCi} / \mathrm{g}$ was found in the 5.5-6.1 meter (18-20 foot) sample taken from borehole GRS-10. While this value was only slightly above the SRS-wide range, it was decided to collect additional samples from Grace Road and test them for gross alpha and non-volatile beta.

A total of seven additional sample locations were selected, GRS-15 through GRS-21. Two of the locations, GRS 15 and GRS-16 were located adjacent to borehole locations GRS 10 and GRS-08 respectively. In August 1994 samples were collected from various depths at those locations. Samples from GRS-17 through GRS-21 were collected from the 0-0.3 meter (0-1 foot) range. Samples GRS-22 and GRS-23 were composite samples collected from L-Area and intended to serve as background samples. Figure 2-5 shows the locations for the gross alpha and non-volatile beta samples at Grace Road.

Table 2-1 shows the results of the analyses on the Grace Road samples. The findings represent what would be found in normal backgrounds for SRS soils. Fay and Pickett (1987) documented background levels for uranium and thorium decay chains and for K-40 in the top 30.5 centimeters ( 12 inches) of SRS soils. Their average findings were that the 
Grace Road Site

WSRC-RP-95-93, Rev. 1.1

RFU/RI Report

February 1996

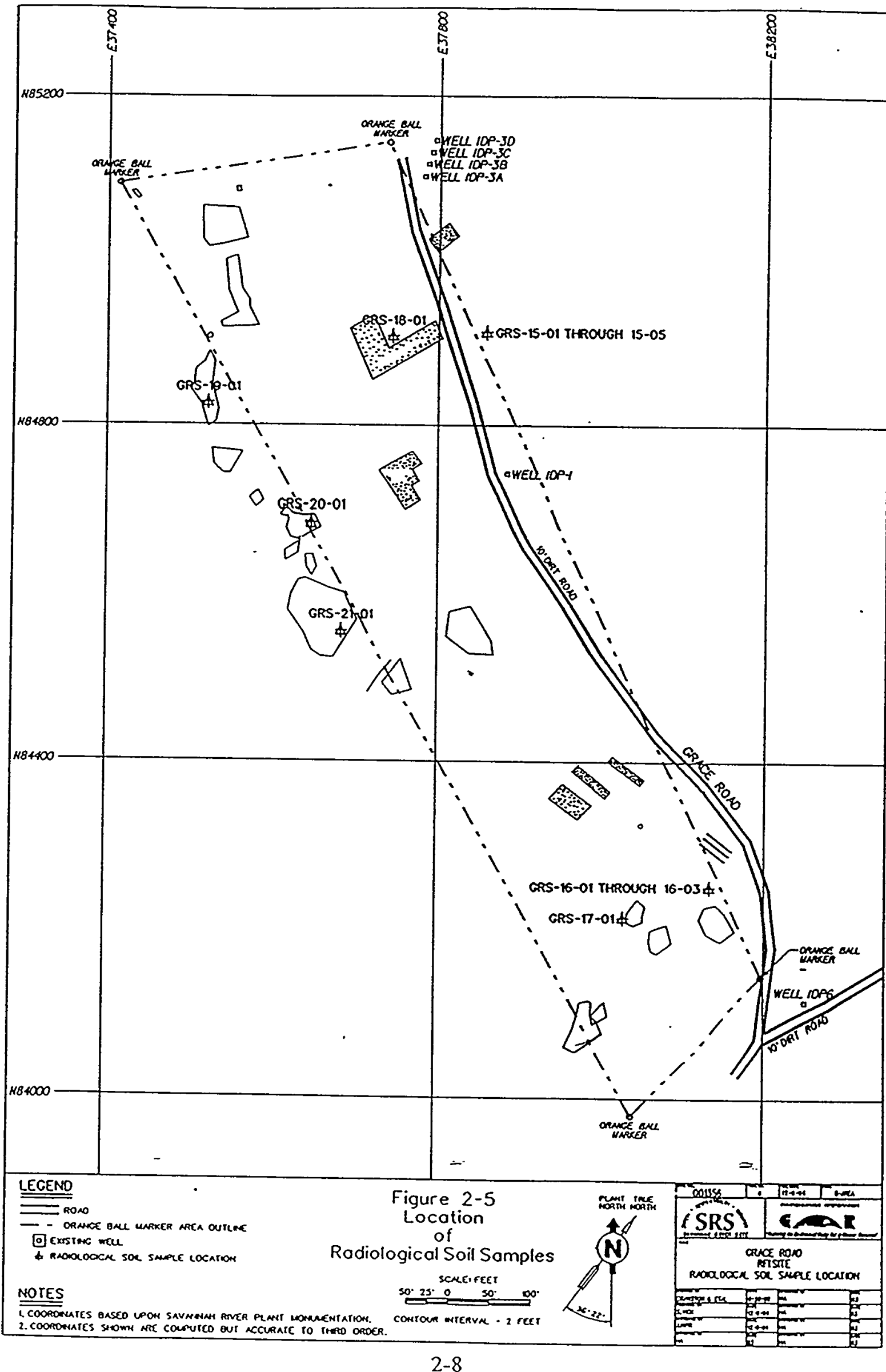


Table 2-1

Analysis of Soil Samples for Gross Alpha and Non-Volatile Beta

\begin{tabular}{|c|c|c|c|c|}
\hline $\begin{array}{c}\text { Sample } \\
\text { Identification }\end{array}$ & $\begin{array}{c}\text { Depth in } \\
\text { meters (feet) }\end{array}$ & $\begin{array}{c}\text { Gross Alpha* } \\
\text { (pCi/g) }\end{array}$ & $\begin{array}{l}\text { Non-Volatile } \\
\text { Beta* (pCi/g) }^{\circ}\end{array}$ & NOTE \\
\hline GRS-15-01 & $0-0.3(0-1)$ & $<5$ & $<5$ & near borehole GRS-10 \\
\hline GRS-15-02 & $1.5 \quad(5)$ & $<5$ & $<5$ & near borehole GRS- 10 \\
\hline GRS-15-03 & $3.05 \quad(10)$ & 13.30 & 5.73 & near borehole GRS-10 \\
\hline GRS-15-04 & $4.6 \quad(15)$ & 14.50 & 5.10 & near borehole GRS-10 \\
\hline GRS-15-05 & $(20)$ & 8.53 & 8.02 & near borehole GRS-10 \\
\hline GRS-16-01 & $0-0.3 \quad(0-1)$ & 5.04 & 5.26 & near borehole GRS-08 \\
\hline GRS-16-02 & $1.5 \quad(5)$ & $<5$ & $<5$ & near borehole GRS-08 \\
\hline GRS-16-03 & $3.05 \quad(10)$ & 8.83 & 5.35 & near borehole GRS-08 \\
\hline GRS-17-01 & $0-0.3 \quad(0-1)$ & $<5$ & $<5$ & surface sample \\
\hline GRS-18-01 & $0-0.3 \quad(0-1)$ & $<5$ & 5.13 & surface sample \\
\hline GRS-19-01 & $0-0.3 \quad(0-1)$ & $<5$ & $<5$ & surface sample \\
\hline GRS-20-01 & $0-0.3 \quad(0-1)$ & $<5$ & $<5$ & surface sample \\
\hline GRS-21-01 & $0-0.3 \quad(0-1)$ & $<5$ & $<5$ & surface sample \\
\hline GRS-22-01 & $0-0.3 \quad(0-1)$ & $<5$ & $<5^{\circ}$ & background sample \\
\hline GRS-23-01 & $0-0.3 \quad(0-1)$ & $<5$ & $<5$ & background sample \\
\hline
\end{tabular}

* Reporting Limit and Method Detection Limit $=5 \mathrm{pCi} / \mathrm{g}$ 
uranium series contributed $9.87 \mathrm{pCi} / \mathrm{g}$ and the thorium series contributed $9.4 \mathrm{pCi} / \mathrm{g}$. Potassium -40 contributed only $0.52 \mathrm{pCi} / \mathrm{g}$. Therefore, the average normal background in SRS soils is considered to be approximately $20 \mathrm{pCi} / \mathrm{g}$ (alpha plus beta-gamma). The sum of the "positive" activities in the Grace Road samples are very close to the total background level which would indicate that activity in those samples is indicative of naturally-occurring (primordial) material.

\subsubsection{Identification of Human Health Chemicals of Potential Concern (COPCs)}

All chemicals shown in Table 1-2, both organic and inorganic, are considered chemicals of potential concern for this unit.

\subsubsection{Rationale and Criteria for Selection of COPCs}

After combining analytical data and eliminating those analytes not detected in any samples, the data were evaluated on the basis of quality with respect to sample quantitation limits, laboratory qualifiers and codes, and blanks.

\subsubsection{Risk-Based Concentrations (RBCs)}

Risk-based concentrations (RBCs) developed by EPA Region III were used to screen the chemicals of potential concern from the list. The table contains reference doses and carcinogenic potency slopes (obtained from Integrated Risk Information System [IRIS] through January 1, 1995, and Health Effects Assessment Summary Tables [HEAST] through March 1994, the Superfund Health Risk Technical Support Center, and other EPA sources) for nearly 600 chemicals. These toxicity constants have been.combined with "standard" exposure scenarios to calculate RBCs - chemical concentrations corresponding to fixed levels of risk (i.e., a hazard quotient of 1 , or a lifetime cancer risk of $1.0 \mathrm{E}-06$, whichever occurs at a lower concentration) in water, air, fish tissue, and soil. The RBCs are very similar to preliminary remediation goals which are concēntration goals for individual chemicals for a specific medium and land use combinations at CERCLA units. 
As a result of using the RBCs to screen the chemicals detected in the soil samples, only two chemicals remain to be studied further, arsenic and lead. As shown in tables 1-2 and $1-3$, the maximum arsenic value detected was $3.2 \mathrm{mg} / \mathrm{kg}$ which exceeded the carcinogenic value of $0.37 \mathrm{mg} / \mathrm{kg}$ for the residential adult/child scenario.

The screening level for lead in soil is $400 \mathrm{mg} / \mathrm{kg}$ for residential land use. This value is described in OSWER Directive \# 9355.4-12, Revised Internal Soil Lead Guidance for CERCLA Sites and RCRA Corrective Action Facilities, dated July 14, 1994 and issued by Elliott $P$. Lewis of the USEPA. The screening level for lead was calculated using the USEPA new integrated exposure uptake biokinetic model with default parameters.

Based on the Report on Geochemical and Physical Properties of Soils and Shallow Sediments at SRS, WSRC-RP-90-1031 (Looney et al, 1990), the maximum concentration level for arsenic in Blanton soils is $7.05 \mathrm{mg} / \mathrm{kg}$ and the median value is $2.6 \mathrm{mg} / \mathrm{kg}$. SRS site wide values for arsenic range from $<0.5 \mathrm{mg} / \mathrm{kg}$ to $15.2 \mathrm{mg} / \mathrm{kg}$ with a median of $<2.0$ $\mathrm{mg} / \mathrm{kg}$. Note that arsenic was used as a component of agricultural chemicals in the period before SRS existed and that Grace Road was a farm. Thus, a few of the detected values may be a result of farming activities prior to 1950. If farming did cause the arsenic problem, and it was not used on SRS since the early 1950s, then it is reasonable to assume that the arsenic level is migrating to the deeper soils on SRS. Since arsenic was not eliminated from the screening process, calculations were performed to determine the risk for the on-unit resident scenario. The calculation of each scenario is described in Section 5.0 and Appendix $\mathrm{C}$ of this report.

\subsection{Groundwater Investigation}

It was recommended that groundwater samples not be obtained at this time. This recommendation was based on the facts that the expected depth to the water table is approximately 24.4 meters ( 80 feet) below land surface and that only minimal contamination was found near the surface at this unit. In addition, as noted in section 3.6, the unit sits on a ridge top indicating that the unit appears to be in the middle of a recharge zone, with groundwater flow south and east from it. Figure 1-2 shows the topography of the unit and its proximity to the local drainage systems. Ground water analytical results 
from water table well IDP-6 (see Section 1.3.2.4) immediately east of Grace Road, show no detection of mercury, total chromium, lead, cadmium, or uranium (an alpha emitter).

Based on the information known about the area, the probability of groundwater contamination at Grace Road Site is extremely low because of the following :

1) The unit sits on top of a ridge between two drainage systems which indicates that this area is a recharge zone for the water table aquifer. The only contamination that can occur in that aquifer would have to be derived from the ground surface above the aquifer or carried in solution with the rain falling on that area. Given that minimal contamination was found at the unit, the probability of water table aquifer contamination is minimal. Tests of water samples from well IDP-6, which is hydraulically down gradient from the unit, showed no signs of contamination.

2) Groundwater in the deeper aquifers has a general flow direction from northwest to southeast. There are no known sources of contamination northwest of Grace Road Site. The nearest contaminated sites are: M-Area, A-Area Burning Rubble Pit, and the Miscellaneous Chemical Basin-Metals Burning Pit unit. However, since these units lie 5 to 8 miles due north of Grace Road and the groundwater flow direction is to the . southeast, there is an extremely low probability that their contamination will reach Grace Road.

\subsection{Human Population Surveys}

There is no resident population on or in close proximity to Grace Road Site. The closest worker population to the unit is located in B-Area, 1.3 kilometers (0.8 miles) north of the unit (see Figure 1-2). Grace Road Site is accessible from SRS Road 2 to SRS workers and other authorized visitors. At present, the unit is outside the controlled access secured zone, but within the SRS security area and requires authorization for access. In addition, the area has been marked and labeled by the Environmental Restoration Department. 


\subsection{PHYSICAL CHARACTERISTICS OF THE STUDY AREA}

\subsection{Surface Features}

There are no structures of any type located at or near the unit; the only nearby man-made feature is Grace Road which runs through the unit and intersects with SRS Road 2. The topography of the unit slopes to the east from an elevation of about 84.8 meters (278 feet) above mean sea level (msl) at its westem end to about 76.2 meters ( 250 feet) at the eastern end (see Figure 2-1).

\subsection{Meteorology}

Climatology and meteorology of SRS are based on data collected on the unit, at Bush Field in Augusta, Georgia (DOE, 1990), and from the H-Area tower and A-Area shelter (DOE, 1991).

\subsubsection{Regional Climatology}

The SRS region has a temperate climate with short, mild winters and long, humid summers. The region is subject to continental influences, but is protected from the more severe winters in the Tennessee Valley by the Appalachian Mountains to the north and northwest, and is often influenced by warm and moist maritime air masses throughout the year. Gently rolling hills with no unusual topographic features that would significantly influence the general climate characterize the unit and the surrounding area.

\subsubsection{Temperature and Humidity}

The annual-average temperature at $\mathrm{SRS}$ is $18^{\circ} \mathrm{C}\left(64.4^{\circ} \mathrm{F}\right)$. Monthly averages range from $7^{\circ} \mathrm{C}\left(44.6^{\circ} \mathrm{F}\right)$ in January to $27^{\circ} \mathrm{C}\left(80.6^{\circ} \mathrm{F}\right)$ in July. The annual average relative humidity is 66 percent. The lowest monthly average daily minimum ( 36 percent) occurs in April, and the highest monthly average daily maximum (98 percent) occurs in August. 


\subsubsection{Precipitation and Evapotranspiration}

The average annual precipitation at SRS is 122 centimeters (48 inches). Precipitation is distributed fairly evenly throughout the year, with the highest precipitation in summer $(36.1 \mathrm{~cm} ; 14.2 \mathrm{in})$. Average precipitation totals for the fall months are less than the average totals for the other seasons, accounting for about 18 percent of the average annual total. For Augusta, precipitation totals greater than 0.025 centimeters $(0.01$ inches) occur, on average, about 107 days per year.

The average number of days per month with measurable precipitation range from about six in October to about 12 in July. Monthly precipitation extremes in Augusta range from a maximum of about 30 centimeters (11.8 inches), recorded in March 1980, to a trace, observed in October 1959.

The greatest observed rainfall for a 24-hour period was about 15 centimeters (5.9 Inches) in August 1964. Hourly observations from Augusta indicate that the rainfall rate is usually less than the 1.3 centimeters ( 0.5 inches) per hour, although rates greater than 1.3 centimeters per hour can be expected during spring and summer thunderstorms. The calculated 24-hour/100 year rainfall at SRS is 20.8 centimeters ( 8.2 inches; DOE, 1990).

Although snow can fall from October through March, the average annual snowfall is only 3.0 centimeters ( 1.2 inches). Large snowfalls are rare. The National Weather Service (NWS) station in Augusta, Georgia, observes maximum total snowfalls for 24-hour and monthly periods. From 1951 to 1987, the maximum snowfall in the SRS area was 36 centimeters (14.2 inches), recorded in February 1973 (DOE, 1990).

Evapotranspiration in the southeastern Coastal Plain physiographic province is approximately $88.9 \mathrm{~cm} / \mathrm{yr}$ ( 35 inches/yr), which is 73 percent of the reported average annual precipitation (DOE, 1990). The remaining precipitation represents the amount available to surface streams for overland flow and to groundwater for recharge. 


\subsubsection{Wind}

Wind data measured at the 6 meter (20 feet) level at the H-Area tower for the period 1982-1986 indicate that there is no predominant wind direction at SRS. The maximum directional frequency is from the northeast (about 9 percent of the time). Figure 3-1 shows an annual wind rose for H-Area, which is near the center of SRS. These data indicate that the observed wind directions tend to favor the southwest and northeast quadrants ( 28 and 30 percent of the time respectively) in relation to the northwest ( 20 percent) and the southeast ( 22 percent) quadrants. For all data, winds from the northeast sector occurred most frequently (nearly 10 percent of the time). Winds from direction sectors in the southwest quadrant also occurred with a relatively high frequency ( 7 to 8 percent of the time).

The average wind speed, from onsite data, from 1982 to 1986 was $3.25 \mathrm{~m} / \mathrm{s}(7.3 \mathrm{mph})$. Hourly wind speeds less than 2 meters per second occurred about 9 percent of the hours. For about half of the hours, wind speeds were less than $4 \mathrm{~m} / \mathrm{s}(9 \mathrm{mph})$. From 1975 to 1979 onsite data, the average speed was greatest during the winter $(3.35 \mathrm{~m} / \mathrm{s} ; 7.5 \mathrm{mph})$ and least during the summer $(2.48 \mathrm{~m} / \mathrm{s} ; 5.5 \mathrm{mph})$.

Winter storms in the SRS area occasionally bring strong and gusty winds with speeds as high as $32 \mathrm{~m} / \mathrm{s}$ (72 mph). On occasion, SRS instruments have recorded winds as high as $39 \mathrm{~m} / \mathrm{s}(87 \mathrm{mph}$ ) during winter storms. Thunderstorms can generate winds as high as 18 $\mathrm{m} / \mathrm{s}(40 \mathrm{mph}$ ) and even stronger gusts. During the history of the Site, only Hurricane Gracie, in September 1959, had onsite winds stronger than $34 \mathrm{~m} / \mathrm{s}$ (76 mph; DOE 1990).

\subsubsection{Thunderstorms}

On average, 56 thunderstorm days occur per year on the site. Summer thunderstorms occur primarily during the late afternoon and evening; they can be accompanied by strong winds, heavy precipitation, or, less frequently, hail (DOE 1990). On average, based on observations in a one-degree square of latitude and longitude, hail occurred once every two years. 
Plot and Table Indicate Direction From Which the Wind Blows

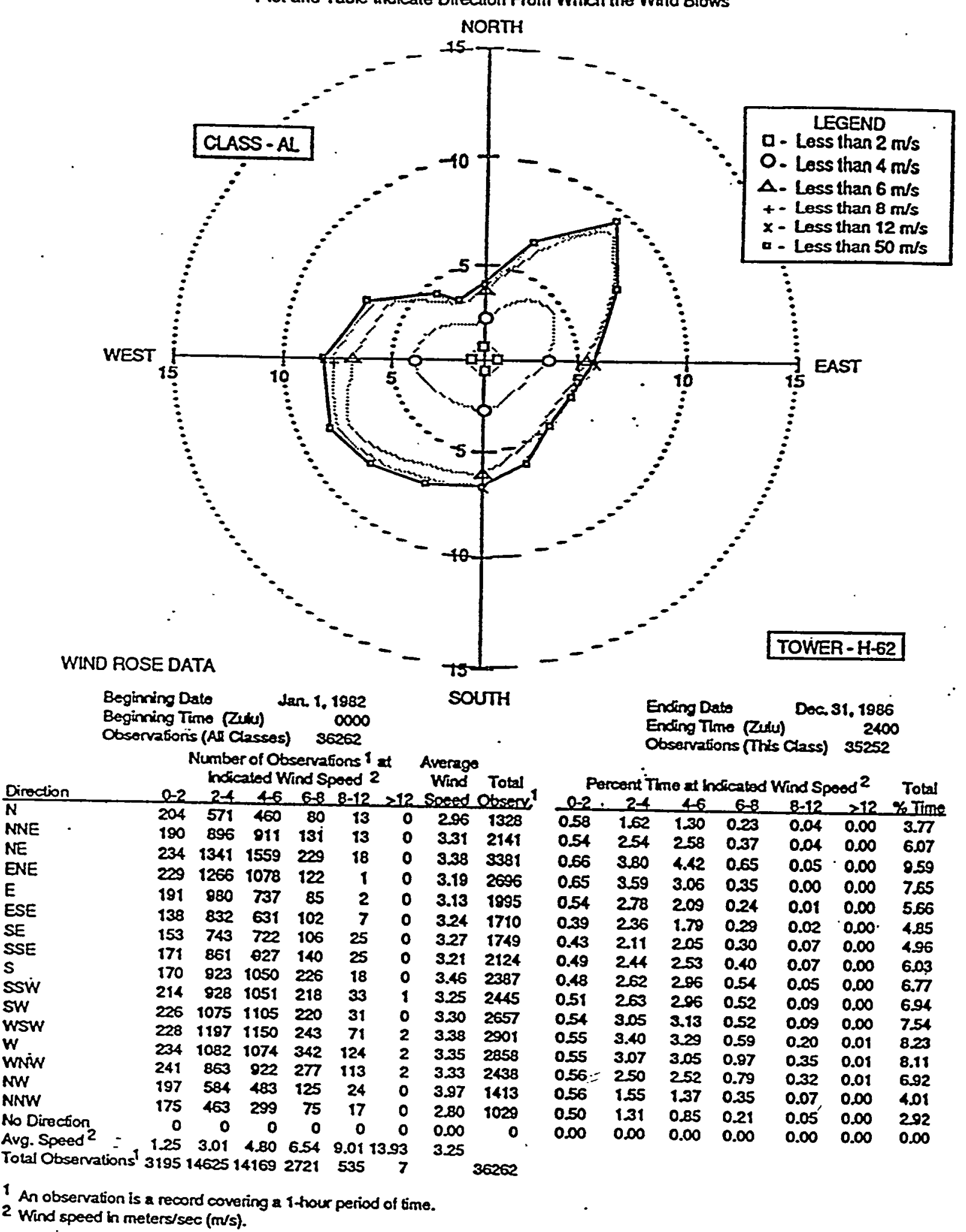

Source: $\quad$ DOE, 1990

Annual Wind Rose of Hourly Averaged Winds from H-Area Tower, 1982-86.

(Directions Are Sectors from Which Wind Blows.)

Figure 3-1. Annual Wind Rose for H Area 


\subsubsection{Ice and Snow}

Winter storms that produce more than 2.5 centimeters ( 1 inch) of ice or snow are rare. Snowfalls of 2.5 centimeters or greater occur once every five years on the average. Any accumulation of snow rarely lasts longer than three days.

For a nine year period of record, storms resulting in an accumulation of ice on exposed surfaces occurred in the SRS vicinity an average of about once every two years. Based on a 50-year period of record (1920 to 1969), a 1.3 centimeter ( 0.5 -inches) accumulation of ice would occur once every 25 years (DOE 1990).

\subsubsection{Fog}

Heavy fog, reducing visibility to less than 0.4 kilometer, occurred at Augusta on a average of about 28 days per year (1951 to 1969). Occurrences averaged about three days per month during the fall and winter and slightly more that one day per month during spring and summer. Most of the heavy fog observed at Augusta is caused by its proximity to the Savannah River.

\subsubsection{Tornadoes}

The estimated probability of a tornado striking a point at SRS is 7.0E-5/yr. (DOE, 1991). About half the total number of observed tornadoes, and most of the tornadoes resulting in severe or devastating damage, occurred in March, April, and May. However, tornadoes have been observed in the SRS area in every month of the year. Investigations of tornadoes occurring near the Site in 1975 and 1976 indicated wind speeds between about $45 \mathrm{~m} / \mathrm{s}(100 \mathrm{mph})$ and $78 \mathrm{~m} / \mathrm{s}$ (174 mph; DOE, 1990).

Since operations began at SRS in 1953, six tornadoes have been confirmed on or near SRS. Nothing more than light damage was reported from any of these storms, with the sole exception of a tornado in October 1989. That tornado caused considerable damage to timber resources in an unpopulated wooded area of SRS (DOE, 1991). 


\subsubsection{Hurricanes}

Thirty-six hurricanes caused damage in South Carolina from 1700 to 1987 . From 1899 to 1980, 13 hurricanes were experienced in South Carolina and Georgia, for an average frequency of about one hurricane every six years; however; the observed interval between hurricane occurrence has ranged from two months to 27 years. Three of these hurricanes were classified as major. Instruments at SRS measured winds associated with Hurricane Gracie, which passed to the north of the Site on September 29, 1959, as high as $34 \mathrm{~m} / \mathrm{s}$ (76 mph). No other hurricane-force wind has been measured on the Site. On September 22, 1989, Hurricane Hugo struck the coast of South Carolina near Charleston; sustained winds recorded at SRS were $17 \mathrm{~m} / \mathrm{s}(38 \mathrm{mph})$.

Because the Site is approximately 161 kilometers ( 100 miles) inland, winds associated with tropical weather systems usually have diminished below hurricane force (sustained speeds of 121 kilometers per hour $(75 \mathrm{mph})$ or greater). Extreme rainfall and tornadoes, which frequently accompany tropical weather systems, usually have the most significant impact on facility operations (DOE 1990).

\subsection{Surface Water Hydrology}

Grace Road Site is situated on soil mapped as part of the Blanton Series. The Blanton Series consists of somewhat excessively drained, moderately permeable soils that formed in sandy and loamy sediments of the Coastal Plain. These soils are on low ridges and side slopes and in broad swales adjacent to the low side slopes. Any surface water drainage from the unit would be generally to the southeast. Based on the soil description and the absence of perennial or intermittent streams in close proximity to the unit, it is unlikely that hazardous constituents would be carried from the unit by surface water runoff. The nearest surface water body to the Grace Road Site is Upper Three Runs Creek approximately 2.4 kilometers ( 1.5 miles) east of the unit. Based on the apparent lack of surface water runoff, Upper Three Runs Creek should not be affected by Grace Road Site. 


\subsection{Geology of SRS}

The subsurface geology underlying SRS includes crystalline basement rock and Triassic sediments which are overlain by Cretaceous and younger Coastal Plain sediments (Price, 1988). The crystalline basement rock is composed of chlorite-hornblende schist, horriblende gneiss, and lesser amounts of other types of crystalline rock. This bedrock is buried beneath approximately 274.4 meters ( 900 feet) of sediments. The Triassic sediments occur in the southem portion of SRS. Sediments of the Triassic bedrock are composed of poorly sorted conglomerate, sandstone and mud-rock, and are generally dark red in color. The stratigraphic units of the Coastal Plain, from oldest to youngest, are the Cape Fear Formation, the Lumbee Group, the Black Mingo Group, the Orangeburg Group, the Barnwell Group, and the Upland Unit (Figure 3-2).

The Cape Fear Formation is composed of Upper Cretaceous, poorly sorted, silty to clayey quartz sands and interbedded clays. The Cape Fear thickens across SRS, ranging from 9.2 meters ( 30 feet) at the northwest SRS site boundary to more than 54.9 meters (180 feet) at the southeast SRS site boundary.

The Lumbee Group (Upper Cretaceous) is subdivided into the Middendorf and the Black Creek Formations, and the Steel Creek Member of the Peedee Formation. The lower contact between the Middendorf and the Cape Fear Formations is a sharp erosional unconformity. The Middendorf Formation is dominantly made up of medium to coarse sand with some thin kaolinitic clay lenses. The Black Creek Formation is generally comprised of interbedded sands, silts and clays. The Peedee Formation (Steel Creek Member) contains poorly to well-sorted, silty-to-clayey sands in the lower section and interbedded clayey-sands in the upper section. The thickness of the Lumbee Group is from 115.9 meters ( 380 feet) to more than 213.4 meters ( 700 feet) from the northwestern SRS boundary to the southeastern SRS boundary, respectively.

The Lumbee Group formations are overlain unconformably by the Paleocene to Eocene aged Black Mingo Group. Locally, the Black Mingo Group Formations dip to the southeast, and range in thickness from 21.3 meters ( 70 feet) at the northwestern boundary of the SRS to approximately 45.7 meters ( 150 feet) near the southeastern boundary. The Black Mingo Group at the SRS is comprised of, from older to younger, the Ellenton 


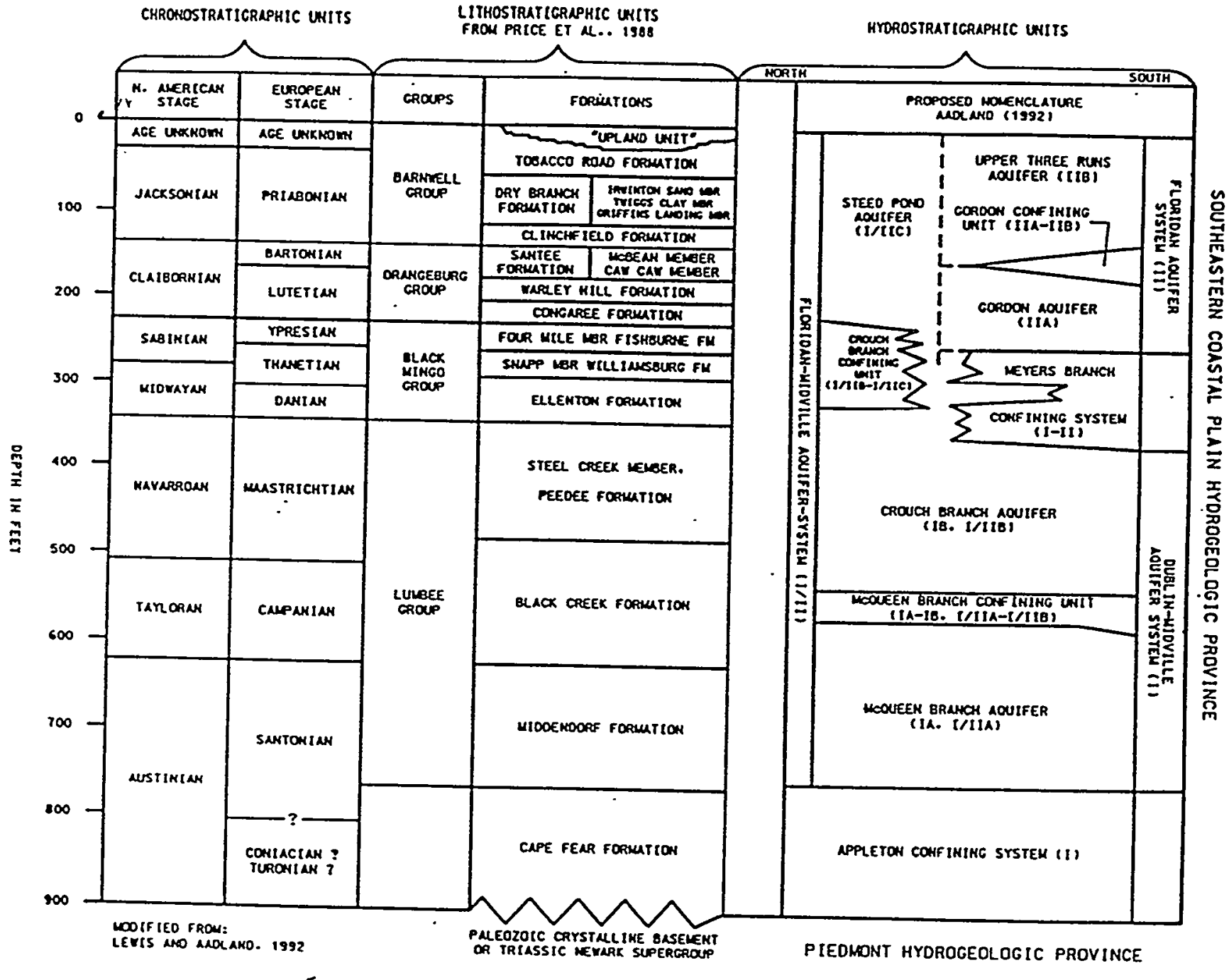

Figure 3-2 Comparison of Chronostratigraphic, Lithostratigraphic, and Hydrostratigraphic Units at SRS 
Formation (Early Paleocene), the Snapp Member of the Williamsburg Formation (Late Paleocene), and the Fourmile Member of the Fishburne Formation (Early Eocene).

The Ellenton Formation consists mostly of gray, poorly sorted, micaceous, lignitic, silty and clayey quartz sand interbedded with gray clays. The Ellenton is approximately $\mathbf{1 2 . 2}$ meters ( 40 feet) thick at the northwestern SRS boundary and thickens to approximately 30.5 meter (100 feet) at the southeastern SRS boundary.

The Snapp Member of the Williamsburg Formation is typically comprised of silty, medium to coarse grained quartz sand interbedded with clay. The Williamsburg thickens from 9.2 meters ( 30 feet) at the northwestern SRS boundary to 15.2 meters ( 50 feet) at the southeastern SRS boundary. The Fourmile Member of the Fishburne Formation consists of tan, orange, yellow, brown, and white clayey and micaceous sand (Fallaw, 1991). The Fishburne has been traced from well PBF-3 into the A/M area, the only locale of known distribution at SRS. The Fourmile section is 4.6 meters ( 15 feet) to 22.9 meters (75 feet) thick in this area.

The Black Mingo Group is overlain by the Middle Eocene age Orangeburg Group. The Orangeburg Group outcrops at many areas of low elevation around the SRS. The thickness of the Group ranges from approximately 30.5 meters (100 feet) at the northwestern SRS boundary to 48.8 meters ( 160 feet) at the southeastern boundary. The Orangeburg Group is made up of the lower Middle Eocene Congaree Formation and the upper Middle Eocene Santee Limestone Formation.

The Congaree Formation consists of yellow, orange, tan, gray and greenish gray, well sorted, quartz sands with thin clay laminae. The top section of the Congaree Formation is cemented with silica in may areas of the SRS and is slightly calcareous in other areas. The Congaree Formation is approximately 18.3 meters (60 feet) thick at the northwestern SRS boundary and thickens to approximately 25.9 meters ( 85 feet) near the southeastern SRS boundary.

The Warley Hill Formation is a fine-grained, often glauconitic sandstone occurring at or near the base of the Santee Formation at SRS (Aadland et al, 1992). The Warley Hill Formation and the Caw Caw Member of the overlying Santee Formation make up what 
has been informally referred to in many SRS reports as the "green clay". The "green clay" is composed of glauconitic, silty and clayey quartz sands and silty clays. Clay beds in this interval are laterally discontinuous in the northernmost SRS areas (Lewis and Aadland, 1992). The overlying Santee Formation is subdivided into the Caw Caw and the McBean Members, and varies in lithology from carbonates and calcareous quartz sands to quartz and glauconitic sands and clays. The uppermost member, McBean, consists of limestone overlain by quartz sand. The thickness of the formation ranges from 12.2 meters ( 40 feet) to more than 24.4 meters ( 80 feet) from the northwestern SRS boundary to the southeastern SRS boundary, respectively.

The Orangeburg Group is overlain by the Late Eocene deposits of the Barnwell Group. The Barnwell Group at the SRS is subdivided into the Clinchfield, Dry Branch, and Tobacco Road Formations. The Clinchfield Formation is present at some SRS locations, but it is not continuous enough to be correlated across the site. The quartz sand of the Clinchfield Formation has been identified at the SRS only when the contrasting carbonates of both the Griffins Landing Member of the Dry Branch Formation (stratigraphically above) and the McBean Member of the Santee Formation (stratigraphically below) are present, with the sand between them. The Dry Branch Formation is divided into (from oldest to youngest) the Griffins Landing, Twiggs Clay, and Irwinton Sand Member. This formation outcrops in several places across the SRS and ranges in thickness from 15.2 meters ( 50 feet) from the northwest boundary to 24.4 meters ( 80 feet) near the southeastern SRS boundary.

The Griffins Landing Member of the Dry Branch Formation is composed of carbonates that occur sporadically at the SRS. The Irwinton Sand Member.contains tan, yellow, and orange, moderately sorted quartz sand with interlaminated clays. The Twiggs Clay Member of the Dry Branch Formation is informally called the "tan clay." This clay lithofacies, which is tan, light gray, and brown, varies in thickness up to 3.7 meters (12 feet) at the SRS, but is not laterally continuous.

The Tobacco Road Formation, which overlies the Dry Branch Formation, consists of red, fine to coarse, moderately to poorly sorted, clayey quartz sand with minor clay laminae. The Tobacco Road outcrops at the surface at many locations throughout the site and ranges in thickness from 6.1 to 9.1 meters (20 to 30 feet). 
The Upland Unit (previously mapped at SRS as the Hawthorn Formation) is composed of poorly sorted, clay-to-silty sands, with lenses and layers of pebbly and cobbly quartz sands with extreme lateral and vertical variation. (Price, 1988). The presence of the Upland Unit in this area is inconsistent, occurring predominantly at higher elevations around SRS.

\subsection{Hydrogeology}

A multilayer hydrologic system exists in the Coastal Plain sediments at the SRS. Confining units within the system are interspersed with more transmissive units. Aadland and Bledsoe (1990) developed an alpha numeric hydrostratigraphic nomenclature system for . SRS and Aadland et al (1992) introduced proposed names for the systems, units and zones (Figure 3-2). Within this nomenclature, the basement complex underlying the Coastal Plain deposits is referred to as the Piedmont Hydrogeologic System. This system is overlain by three aquifer systems of Coastal Plain deposits, the Midville, the Dublin, and the Floridan Aquifer Systems, separated by the Allendale and Meyers Branch Confining Systems. These Aquifer systems are separated from the lower Piedmont Hydrogeologic .: System by the Appleton Confining System. The Appleton Confining System is composed of poorly sorted, clayey sediments of the Cape Fear Formation. Confining clay sediments of the middle Black Creek Formation make up the Allendale Confining Unit which separates the Midville and Dublin Aquifers. The Midville Aquifer is composed of sands of the Middendorf Formation and the lower sands of the Black Creek and lower Peedee Formations. As the confining systems and units thin and become discontinuous in a northerly direction at the SRS, the Midville and Dublin Aquifer Systems are combined to form a single Dublin-Midville Aquifer System. Figure 3-3 is a graphic illustration of the hydrogeologic nomenclature for the SRS region. The reader is referred to Aadland et al (1992) for the most recent description of SRS hydrogeology:

The Floridan Aquifer System is divided into the Gordon Aquifer and the Upper Three Runs Aquifer by the Gordon Confining Unit. Congaree/Fourmile sands comprise the Gordon Aquifer, and sands of the Santee Limestone and Tobacco Road Formation comprise the Upper Three Runs Aquifer. Warley Hill and Caw Caw clays (including the "green clay") comprise the Gordon Confining Unit. Northwest of Upper Three Runs 
Grace Road Site

RFI/RI Report
WSRC-RP-95-93, Rev. 1

November 1995

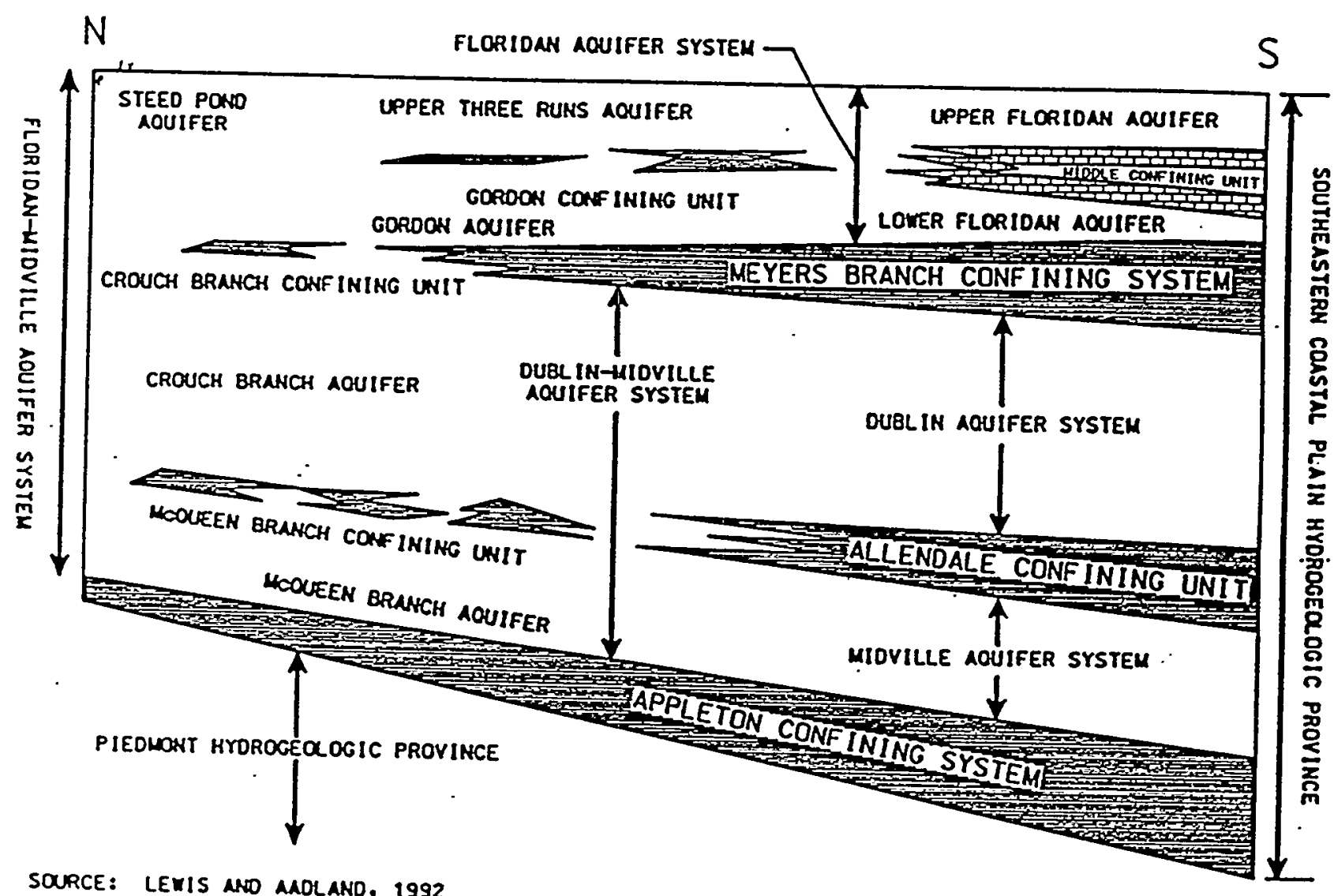

Figure 3-3 Hydrogeologic Nomenclature for the SRS Region 
Creek (UTRC), the individual confining beds separating the two Aquifer Systems become thin and discontinuous.

\subsection{Geology and Hydrology of the Grace Road Site}

The amount of detailed geologic and hydrogeologic information on the Grace Road area is rather limited. Investigations of the area indicate the geologic units underlying Grace Road Site from the shallowest to about 30.5 meters (100 feet) depth are a clayey sand unit, approximately 6.7 meters ( 22 feet) thick, a sand unit, approximately 15.2 meters (50 feet) thick, a clayey sand and silty sand unit approximately 1.2 to 3.7 meters ( 4 to 12 feet thick), and a calcareous zone approximately 1.8 meters ( 6 feet) thick. These overlie the McBean Formation which is approximately 7.9 meters ( 26 feet) thick, the Green Clay, 3.7 meters ( 12 feet) thick, the Congaree Formation, 17.1 meters ( 56 feet) thick, the Ellenton Formation, 47.6 meters ( 156 feet) thick, and the Black Creek Formation.

The upper soil layers at the unit consist mainly of fine- to medium-grained sands mixed with small amounts (5-20\%) of silt and clay and containing traces of lignite. In a few isolated patches clay dominates on the surface. Clay content in the soils increases with depth, especially below 1.8 to 3.05 meters (6-10 feet). From a depth of approximately 3.05 meters ( 10 feet) downward to the lowest sampling depth ( 6.1 meters [ 20 feet]) the sediment is a hard compact red clay.

There are numerous monitoring wells in the vicinity of Grace Road Site although only the IDP-3 well cluster is located 6.1 to 12.2 meters (20 to 40 feet) northeast of the unit and well DP- 6 is located 12.2 meters ( 40 feet) southeast of the unit. (see Figure 1-3). The water table elevations in these monitoring wells indicate that the water table is just above 61 meters (200 feet) mean sea level or approximately 24.4 meters ( 80 feet) below land surface at the Grace Road Site. The ridge top location indicates that the unit appears to be in the middle of a recharge zone, and groundwater flows south and east from it. Discharge of groundwater is into Upper Three Runs Creek (see topographic map in Figure 1-2). 
Data from well cluster IDP-3 showed the water table was encountered more than 22.9 meters ( 75 feet) from the surface. There is a negative vertical gradient between the wells which produce water from the McBean and Congaree Formations (Well IDP-3C and IDP$3 \mathrm{~B}$, respectively). A positive vertical gradient between the wells producing from the Congaree and Black Creek Formations (Wells IDP-3B and IDP-3A) indicates that there is a confining unit between these two formations. The most likely confining unit is a green clay layer approximately ten feet thick which occurs at approximately 45.7 meters (150 feet) above mean sea level.

\subsection{Demography and Land Use}

\subsubsection{Demographics}

SRS is located approximately 40 kilometers ( 25 miles) southeast of Augusta, Georgia, and 32 kilometers ( 20 miles) south of Aiken, South Carolina. According to 1990 census data (Rand McNalley, 1992) the average population densities (in people per square mile) for the surrounding South Carolina counties are 111 for Aiken County, 36 for Barnwell County, and 28 for Allendale County. For the surrounding Georgia counties the densities are 228 for Columbia County, 524 for Richmond County, 25 for Burke County and 21 for Screven County. The population within an 80.5 kilometer ( 50 mile) radius of SRS is 634,784 people.

The estimated population for the area in the year 2000 is projected to be 852,000 (Rand McNalley, 1992). This estimate was calculated utilizing the 1970 to 1980 growth rate of each county in an 80.5 kilometer ( 50 miles) radius, assuming that the same rate of growth would continue into the future. The calculation assumed that the population would remain constant for counties that experienced a negative population growth between 1970 and 1980.

Calibrated demographic data is available for the six-county area that provides 90 percent of the SRS work force (DOE, 1994). These are Aiken, Allendale, Bamberg and Barnwell Counties in South Carolina and Richmond and Columbia Counties in Georgia. The population in those six counties increased 13 percent between 1980 and 1990, from 
376,000 to 425,607 and is expected to increase to 474,820 by the year 2000 (DOE, 1992). A disproportionate share of the six county population increase was concentrated in Columbia County where the population increased more that 55 percent to 66,031 between 1980 and 1991.

\subsubsection{Land Use}

Less than 5 percent of the existing land in the area surrounding SRS is devoted to urban and developed uses (DOE, 1990). Most of the urbanized development in the area has occurred in and around the cities of Augusta, Georgia and Aiken, South Carolina. Agriculture accounts for $2 \dot{4}$ percent of total land use; forests, wetlands, water bodies, and unclassified land that is predominantly rural accounts for about 70 percent of the total land use. A projected 2 percent increase in the development of urban land surrounding SRS is expected by the year 2000 .

Less than 5 percent of the total SRS land area is used by facilities engaged in the production of special nuclear materials. Reservoirs and ponds comprise approximately 13 square kilometers ( 5 square miles) of SRS. The remainder of the $780+$ square kilometer $(300+$ square miles) area is undeveloped. 
Grace Road Site

WSRC-RP-95-93, Rev. 1

RFI/RI Report

November 1995

THIS PAGE INTENTIONALLY LEFT BLANK 


\subsection{NATURE AND EXTENT OF CONTAMUNATION}

\subsection{Results of Data Usability Evaluation}

The field investigation for Grace Road Site was conducted prior to the establishment of a formal data validation program at SRS. Data collected were subjected to the QA/QC standards in use at that time and considered to be acceptable by EPA Level III criteria. Information on the QA/QC methods employed during the investigation are provided in the RFI/RI Plan for the Grace Road Unit, WSRC-RP-90-1250 (WSRC, 1990). Additional information on the QA/QC procedures used for this unit are provided in Appendix B.

\subsection{Results of Site Characterization}

Data collected during Preliminary Unit Evaluation (see Section 1.3.1), Unit Screening (see Section 1.3.2), and field investigations (see Section 2.5) indicate there is no contamination which is considered significant at Grace Road. The only COPCs which had slightly elevated levels were arsenic and lead. Arsenic contamination is attributable to farming activities conducted at Grace Road prior to SRS. An evaluation of the lead levels indicates that exposure to the amount found at Grace Road does not pose a risk to human health. Table 4-1 provides a comparison of the maximum contaminants found at Grace Road compared to two times the average background and the risk-based concentrations. 
Grace Road Site

RFI/RI Report
WSRC-RP-95-93, Rev. 1.1

February 1996

Table 4-1 COMPARISON OF UNIT SPECIFIC SOIL

CONCENTRATION TO TWO TIMES BACKGROUND

CONCENTRATIONS AND RISK -BASED CONCENTRATIONS (RBC)

\begin{tabular}{|c|c|c|c|c|}
\hline Contaminant (units) & $\begin{array}{c}\text { Maximum } \\
\text { Concentration }\end{array}$ & $\begin{array}{l}\text { Average Background Soil } \\
\text { Concentration (GRS-10) }\end{array}$ & $\begin{array}{l}\text { Two Times } \\
\text { Background }\end{array}$ & $\begin{array}{c}\text { RBC Value* } \\
\text { (mg/kg) }\end{array}$ \\
\hline $\begin{array}{l}\text { Bis(2-ethylhexyl) } \\
\text { Phthalate (mg/kg) }\end{array}$ & 6.8 & 4.7 & 9.4 & 46 \\
\hline Carbon Disulfide (mg $/ \mathrm{kg}$ ) & $0.002 \mathrm{~J}$ & ND & ND & 7800 \\
\hline $\mathrm{DDT}(\mathrm{mg} / \mathrm{kg}$ ) & $0.0063 \mathrm{~J}$ & ND & ND & 1.9 \\
\hline Styrene (mg/kg) & $0.004 \mathrm{~J}$ & ND & ND & 16000 \\
\hline Acetone (mg/kg) & $0.002 \mathrm{~J}$ & $\mathrm{ND}$ & ND & 7800 \\
\hline Toluene $(\mathrm{mg} / \mathrm{kg})$ & $0.003 \mathrm{~J}$ & ND & $\mathrm{ND}$ & 16000 \\
\hline $\begin{array}{l}\text { Di-n-Butyl Phthalate } \\
(\mathrm{mg} / \mathrm{kg})\end{array}$ & $53 \mathrm{~J}$ & ND & ND & $\begin{array}{l}7800 \\
\because\end{array}$ \\
\hline $\begin{array}{l}\text { Trichloroethylene } \\
(\mathrm{mg} / \mathrm{kg})\end{array}$ & 0.004 & ND & ND & 58 \\
\hline Xylene (mg/kg) & 0,007 & $\mathrm{ND}$ & ND & 160,000 \\
\hline Arsenic (mg/kg) & 3.2 . & ND & ND & 0.37 \\
\hline Barium (mg/kg) & 48.4 & ND & ND & 5500 \\
\hline Cadmium $(\mathrm{mg} / \mathrm{kg})$ & 1.8 & ND & ND & 39 \\
\hline Chromium (VI) (mg/kg) & 29.6 & 4.2 & 8.4 & 390 \\
\hline Mercury (mg/kg) & 0.15 & ND & ND & 23 \\
\hline Lead $(\mathrm{mg} / \mathrm{kg})$ & 48.1 & 1.4 & 2.8 & $400^{* *}$ \\
\hline Selenium $(\mathrm{mg} / \mathrm{kg}$ ) & 1.3 & ND & ND & 390 \\
\hline Tin (mg/kg) & 32.5 & ND & ND & 47000 \\
\hline Vanadium (mg/kg) & 61.8 & ND & ND & 550 \\
\hline Zinc (mg/kg) & 7.0 & ND & $\mathrm{ND}$ & 23000 \\
\hline Gross Alpha (pCi/g) & 22.0 & 21.7 & 43.4 & - \\
\hline $\begin{array}{l}\text { Non-volatile Betä } \\
(\mathrm{pCi} / \mathrm{g})\end{array}$ & 13.0 & 3.7 & $=7.4$ & - \\
\hline
\end{tabular}

Table 4-1 continued on next page 


\section{Table 4-1 (continued)}

* EPA Region III, Risk-Based Concentration Table, January-June 1995, dated March 7, 1995

$\mathrm{j}=$ estimated value

** The screening level for lead in soil is $400 \mathrm{mg} / \mathrm{kg}$ for residential land use. This value is described in OSWER Directive \# 9355.4-12, Revised Internal Soil Lead Guidance for CERCLA Sites and RCRA Corrective Action Facilities, dated July 14, 1994 and issued by Elliott $P$. Lewis of the USEPA. The screening level for lead was calculated using the USEPA new integrated exposure uptake biokinetic model with default parameters. 
Grace Road Site

RFI/RI Report
WSRC-RP-95-93, Rev. 1.1

February 1996 


\subsection{CONTAMINANT FATE AND TRANSPORT}

Contaminants may migrate in environmental media following release from secondary sources by any of several release or transport mechanisms, including:

- suspension and dispersal by the wind of particulate contaminants or contaminants adsorbed to surface soil particles (fugitive dust generation);

- direct volatilization of volatile organic compounds from surface soil to air

- uptake of soil contaminants by vegetation;

- transport of soil contaminants by storm water runoff to surface water and sediments;

- leaching of deep soil contaminants into groundwater.

In addition to the exposure pathways which result from the above secondary release and transport mechanisms, potential receptors also may be exposed directly to contaminants present in surface soils.

\subsection{Exposure Points}

Exposure points are locations where receptors can potentially come into contact with contaminated media. If a probable route of intake into the receptor is available at the point of exposure, an exposure pathway is complete. As shown in Figure 5-1 there are five media (pathways) through which exposure to unit-related chemicals may occur: air (dust and vapor), soil, groundwater, surface water, and sediments.

Surface soil or deep soils that have been excavated and redistributed onto the surface are potential exposure points for constituents of concern ( $\mathrm{COCs}$ ) in soils. Exposure points for chemicals in groundwater and chemicals that may infiltrate/percolate from deep soils to groundwater exist during future household use of groundwater. Under current land use conditions there are no direct exposure points to groundwater for receptors.

Surface water and sediments become exposure points for chemicals in soil through erosion and transport by wind and water. Chemicals in groundwater also may migrate to surface water and sediments where they may be contacted by potential receptors. 
Grace Road Site

RFI/RI Report

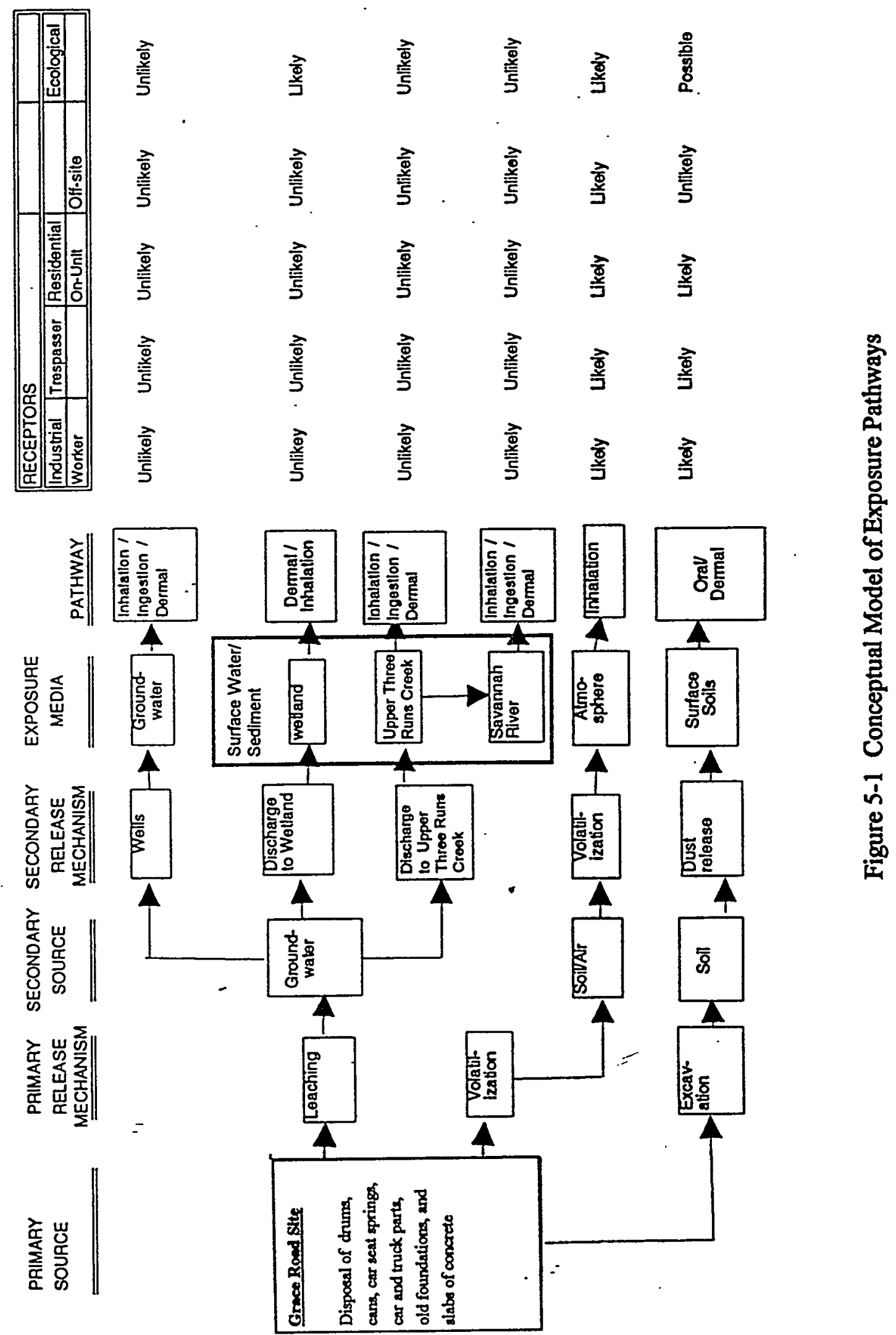

WSRC-RP-95-93, Rev. 1

November 1995

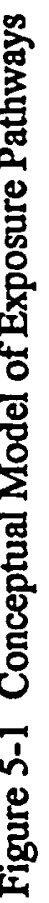


COCs present in surface soil, or in deep soils that have been excavated and redistributed onto the surface around Grace Road could result in airborne dispersion of particulate chemicals or chemicals adsorbed to surface soil particulates (windblown dust). Exposure points exist where chemicals are suspended in the air or from the volatilization of chemicals from surface soils and/or redistributed deep soils.

\subsection{Exposure Routes and Potential Receptors}

An exposure route is the means by which a receptor comes in contact with a contaminated medium. The most common exposure routes are inhalation, ingestion, dermal contact, or external radiation. Probable exposure routes (i.e., potentially complete pathways) were identified for each receptor for each medium based on anticipated activities at the exposure point (see Figure 5-1).

Potential receptors are those individuals that may come into contact with potentially contaminated media associated with Grace Road. There is no worker activity at Grace Road so there are no scenarios or possible receptors under current land use conditions. The possible receptor under hypothetical future land use conditions is an on-site resident or worker.

SRS employees and subcontractors were not considered to be potential receptors at Grace Road. These persons were assumed to be individuals who visit the unit on a infrequent basis, are involved in sampling activities, or are present in the area when sampling activities are being performed. In addition, SRS employees and subcontractors are required to use personal protective equipment which would preclude their exposure to unit-related chemicals.

Current populations that potentially could be receptors for exposure to chemicals associated with Grace Road include trespassers and residents living outside the SRS boundaries.

A trespasser was considered to be a potential receptor because of the proximity of the unit to Highway 125 , a public road. However, an intruder in the area would likely be detected 
by SRS security, thereby minimizing the possibility of trespassing as well as the frequency and duration of exposure of a trespasser to unit-related contaminants. Because the exposure parameters used for the trespasser are less conservative than the parameters that would be used for an on-unit resident, risks calculated for the on-unit resident can be assumed to be greater than risks for a trespasser.

Residents living outside SRS boundaries could potentially be exposed to unit-related chemicals through inhalation of airborne chemicals in the form of vapor or dust (suspended particulates). However, the potential for transport of significant concentrations to residences beyond the SRS boundary is negligible considering their distance from the unit is at least $11.2 \mathrm{~km}$ (7 mi.). Because of this, exposure via the air pathway was not quantified for off-site residents.

Incidental ingestion of, or dermal contact with, contaminants in surface water and sediments are incomplete pathways for the off-site resident since there is no surface water or sediments near the unit. Additional incomplete pathways would include ingestion of, dermal contact with, and inhalation of volatile organic compounds (VOCs) from groundwater. However, the deeper soil boring analyses indicated that the groundwater is not contaminated as a result of the potential source(s). Also, dilution and degradation of unit-related chemicals during transport, in conjunction with the travel time required for chemicals to migrate beyond SRS boundaries to a likely exposure point, would significantly reduce the potential for risk from these pathways relative to other principal pathways. Therefore, these pathways were not quantitatively evaluated for off-site residents.

Future land use planning at SRS will likely designate certain areas as industrial and prohibit residential development through deed restrictions. However, Grace Road is located apart from the B-Area complex and could possibly be converted to residential use in the future. In the event this occurs, an on-unit resident may potentially become exposed to chemicals in soil through ingestion, dermal contact, and inhalation of contaminated dust or volatile chemicals.

Because institutional controls preventing the excavation of contaminated soils cannot be guaranteed, the future residential scenario also assumes the possible excavation of soils 
from depths of 0 to $1.0 \mathrm{~m}$ ( 0 to $3 \mathrm{feet}$ ) and subsequent spreading of those soils on the surface. One meter is considered a reasonable depth for a residential contractor to excavate during typical home building. A future resident potentially could be exposed to either surface soils or the redistributed soils through the exposure routes described above.

\subsection{Exposure Point Concentrations}

The maximum concentration value was used as the exposure point concentration in lieu of the reasonable maximum exposure (RME). The RME is normally used as it is a conservative exposure case that is still within the range of possible exposures.

\subsubsection{Soil}

Soil sampling data were used to estimate COC exposure point concentrations in surface soil for the potential future residential scenarios.

\subsubsection{Groundwater}

Groundwater data were not collected for the Grace Road assessment. This recommendation was based on the facts that the expected depth to the water table is approximately 24.4 meters ( 80 feet) below land surface and that only minimal contamination was found near the surface.

\subsubsection{Air}

Exposure point concentrations for airborne particulates were derived for the future land use scenario through the use of a model. Particulate suspension was modeled following Milham et al (1975), who studied resuspension of plutonium from an old field that was converted tō agriculture specifically for the purposes of the study. Since none of the scenarios considered in this risk assessment involve the high degree of soil disturbance associated with agriculture, the value chosen is conservative and site specific. Chemical concentrations in soil particulates were assumed to equal surface soil concentrations. 


\subsubsection{Intake Parameters}

The values assumed for the RME exposure parameters, and the guidance they are based on, are presented in Table C-3 in Appendix C. Exposure time, frequency, and duration estimate the total time a receptor may be exposed to a contaminated medium. Exposure time (ET) is the number of hours per day that a receptor is present at a specific exposure point. Exposure frequency (EF) is the number of days per year that the exposure occurs, and exposure duration (ED) is the number of years over which exposure occurs.

For the future residential scenario, an ED of 30 years was assumed, representing the national upper-bound (90th percentile) time spent at one residence (EPA, 1991a).

Residential ED was apportioned between adult and child, with 24 years as an adult and 6 years as a child. An EF of 350 days/year (EPA, 1991a) was assumed. ETs assumed for inhalation of VOCs from soil were 24 hours/day for an adult and child based on average time spent at home as reported in time use studies (EPA, 1993). Vapor concentrations indoors and outdoors were assumed to be equal.

Incidental soil ingestion rates (IRo) were based on EPA guidance (EPA, 1991a). For the future on-unit residential receptor, an upper-bound soil IRo of $200 \mathrm{mg} / \mathrm{d}$ was assumed for children 1-6 years of age and $100 \mathrm{mg} / \mathrm{d}$ for older children and adults (EPA, 1991a).

An inhalation rate (IRi) of $0.83 \mathrm{~m}^{3} / \mathrm{hr}$ (i.e., $20 \mathrm{~m}^{3} / \mathrm{d} / 24 \mathrm{hr} / \mathrm{d}=0.83 \mathrm{~m}^{3} / \mathrm{hr}$ ) for outdoor activities was used for adult receptors in the future residential scenario (EPA, 1991a). This value represents the reasonable upper-bound $\mathrm{IRi}$ over an entire day for indoor and outdoor activities, including periods of rest and light, moderate and heavy activity. The corresponding IRi for children (used for the future residential scenario) is $1.0 \mathrm{~m}^{3} / \mathrm{hr}$ (EPA, 1990).

The values used for body weight (BW) were the average weight over the exposure period: $70 \mathrm{~kg}$ for adult and $15 \mathrm{~kg}$ for child (EPA, 1991a). In accordance with EPA guidance (EPA, 1991a), an adult body weight was used to calculate intake for older children and adults. 
Skin surface areas (SA) used for resident exposure to soil were 50th percentile values for the body parts representing the RME: head, hands, forearms and lower legs. Fiftieth percentile, instead of 95 th percentile, values were used because 95 th percentile values are misrepresentative of the surface area of individuals of average weight (e.g., $70 \mathrm{~kg}$ ) (EPA, 1989). It was assumed that the resident adult or child will wear a short-sleeve shirt, shorts, and shoes while gardening, working, or playing outdoors at home, with about 25 percent of the total skin area exposed. This equates to an $S A$ of $5000 \mathrm{~cm}^{2}$ for the adult and $1800 \mathrm{~cm}^{2}$ for the child (EPA, 1992).

For soil ingestion by the future on-unit resident, the value assumed for fraction ingestion (FI) from a contaminated source was 1.0. This conservative estimate assumes that all soil ingested was from the contaminated source.

The same FI values were used for the future child. The adherence factor (AF) assumed for soil is $0.2 \mathrm{mg} / \mathrm{cm}^{2}$, which represents the lower end of the range believed to be typical for environmental exposures (EPA, 1992). This value was selected because of the sandy nature of surface soil at the unit.

The dermal absorption factor (ABS) of 0.1 percent was used for arsenic since it is an inorganic COC (EPA, 1992).

\subsubsection{Equations for Exposure to Soil}

For future land use conditions, the equations for soil exposure were used for one soil exposure scenario: exposure to chemicals in soils regardless of its location below the surface. 
Grace Road Site

RFI/RI Report
WSRC-RP-95-93, Rev. 1 November 1995

THIS PAGE INTENTIONALLY LEFT BLANK 


\subsection{RISK ASSESSMENT}

\subsection{Human Health Assessment}

\subsubsection{Exposure Assessment}

One consideration in evaluating Grace Road unit is the determination of the environmental fate and transport of the COPCs identified in Section 2.5.5 and the potential pathways by which human population could be exposed to analytes at or originating from this unit. In identifying primary pathways of exposure, current and future land uses of the unit and surrounding area were considered. This section describes exposure scenarios, develops information on exposure pathways, estimates the concentration of COPCs at points of human exposure, and determines receptor intakes. Reasonable maximum exposure (RME) estimates are presented chemical intakes within each scenario. The uncertainties of the exposure assessment are described in Section 6.1.4.

\subsubsection{Future Land Use Scenarios}

Although future use of the area surrounding Grace Road is expected to remain industrial, it could potentially change from industrial to residential. For this reason and because an on-unit residential scenario is more conservative and since there is no unit worker activity, only a hypothetical on-unit resident was evaluated for the current and future use scenarios.

\subsubsection{Identification Of Human Health Exposure Pathways}

A complete exposure pathway consists of the following four elements: (1) a source and mechanism of contaminant release to the environment, (2) an environmental transport medium for the released contaminants, (3) a point of contact with the contaminated medium, and (4) a route of entry of the contaminant into the receptor at the exposure point. An integration of sources, releases, fate and transport mechanisms, exposure points, and exposure routes were integrated in order to determine the complete exposure pathways that exist at the unit. If any of these elements were missing, the pathway was incomplete and was not considered in the baseline risk assessment (BRA) for Grace Road. 
A conceptual site model (CSM) was developed to illustrate the potential exposure pathways at Grace Road (Figure 5-1). One land use scenario was considered: future land use (residential). Potential human receptors addressed were hypothetical future on-unit residents.

Figure 5-1 depicts the potential contaminant sources, release and transport mechanisms, exposure pathways, and receptors for Grace Road. A complete exposure pathway exists when a receptor could be exposed to a contaminant source through one of the potential exposure routes. Even though a complete exposure pathway may exist for a particular receptor, quantification of risk for the pathway may not be practicable because of the lack of toxicity factors, or may not be warranted because of the expected minimal contribution to risk relative to other major pathways.

\subsubsection{Toxicity Assessment}

The objective of the toxicity assessment is to weigh available evidence regarding the potential for each $\mathrm{COC}$ to cause adverse health effects in exposed individuals and to provide, where possible, an estimate of the relationship between the extent of exposure and the severity of adverse effects (EPA, 1989).

Toxicity information used in this evaluation was obtained from the Integrated Risk Information System (IRIS; EPA, 1995a). If values were not available from IRIS, the Health Effects Assessment Summary Tables (HEAST; EPA, 1994) were consulted. Toxicological data for the $\mathrm{COC}$ (arsenic), including both carcinogens and noncarcinogens, are presented in Table C-2 in Appendix C.

In order to assess toxicity via the dermal absorption route of intake, oral toxicity factors based on administered doses were adjusted to express toxicity on the basis of absorbed dose. Oral absorption efficiencies (percent absorbed by the gastrointestinal tract following oral intake) were identified for each COC and used to modify toxicity values as follows: for carcinogens, the oral slope factor was divided by the oral absorption efficiency to derive an adjusted slope factor; for noncarcinogens, the oral reference dose was multiplied by the oral absorption efficiency. If an appropriate oral absorption efficiency value was 
not identified, the following default values recommended by EPA Region IV were used: 80 percent for volatile organic compounds (VOCs), 50 percent for semi-volatile organic compounds (SVOCs), and 20 percent for inorganics. Table C-2 (Appendix C) presents the adjusted slope factors (SFs) and reference doses (RfDs) for all COCs for which oral toxicity values were available.

\subsubsection{Non-Radiological Toxicity}

\subsection{Carcinogens}

For risk assessment, EPA subscribes to the "non-threshold" theory of carcinogenesis, which proposes that there is essentially no level of exposure to a carcinogen that does not pose a finite probability of generating a carcinogenic response. This theory assumes that a small number of molecular events can evoke changes in a single cell that may lead to uncontrolled cellular proliferation and eventually to cancer (EPA, 1989). Therefore, no dose is thought to be risk free and, in evaluating cancer risk, an effect threshold cannot be estimated. As a result, EPA takes a probabilistic approach to the evaluation of the carcinogenicity of chemicals. This two-step evaluation includes the assignment of a weight-of-evidence classification to each chemical based on the strength of evidence that it is a human carcinogen, and the calculation of a slope factor for those chemicals that are possible, probable, or proven human carcinogens based on their weight-of-evidence (EPA, 1989).

The EPA weight-of-evidence classification system involves characterizing a chemical's carcinogenicity based on the availability of animal, human, and other supportive data. Based on the strength of the evidence that a chemical produces carcinogenic effects in humans, it is assigned to one of the following classes (EPA, 1989):

- Group A - Human Carcinogen - this category indicates that there is sufficient evidence from epidemiological studies to demonstrate carcinogenicity in humans;

- Group B - Probable Human Carcinogen - this category is subdivided into Group B1 and Group B2:

- Group B1 indicates that limited data are available suggesting carcinogenicity in 

humans;

- Group B2 indicates that there is sufficient evidence of carcinogenicity in animals and inadequate or no evidence in humans;

- Group C - Possible Human Carcinogen - this category indicates that there is limited evidence of carcinogenicity in animals and inadequate or no evidence in humans;

- Group D - Not Classifiable - this category indicates that there is inadequate or no data by. which to classify a chemical as a human carcinogen;

- Group E - Evidence of Human Noncarcinogenicity - this category indicates that in an adequate number of studies, there was no evidence of carcinogenicity;

The slope factor (SF) is a plausible upper-bound estimate of the probability of a carcinogenic response per unit intake of a chemical over a lifetime. It is usually the upper 95th percent confidence limit of the slope of the dose-response curve and is expressed as the reciprocal of milligram $(\mathrm{mg})$ of chemical intake per kilogram $(\mathrm{kg})$ of body weight per day $\left[(\mathrm{mg} / \mathrm{kg} \text {-day })^{-1}\right]$. The SF is used in risk assessments to estimate an upper-bound lifetime probability of an individual developing cancer as a result of exposure to a particular level of a potential carcinogen. SFs are accompanied by the weight-of-evidence classification to indicate the strength of the evidence that the chemical is a human carcinogen (EPA, 1989).

Oral SFs are reported as risk per unit dose $\left[(\mathrm{mg} / \mathrm{kg} \text {-day })^{-1}\right]$. Toxicity values for carcinogenic effects also may be reported as unit risk. Unit risk expresses risk from a substance per concentration of that substance in the medium where human contact occurs. For example, inhalation SFs are usually reported as risk per unit concentration in air $\left[\left(\mu \mathrm{g} / \mathrm{m}^{3}\right)^{-1}\right]$.

Because carcinogenic toxicity values calculated for the dermal route of intake are rare, EPA recommends that an oral slope factor for a chemical be modified based on its gastrointestinal absorption efficiency in order to derive a slope factor based on absorbed dose for use in evaluating dermal absorption (EPA, 1989). Oral absorption efficiency factors and adjusted slope factors are listed in Table C-2 (Appendix C). 
Arsenic is a COPC classified as Group A carcinogen. Exposure to this chemical may result in tumors in the lungs and bronchial system. Carcinogenic toxicity data for arsenic are summarized in Table C-2 (Appendix $C$ ) and includes weight-of-evidence classifications, slope factors (SFs), routes of exposure, types of cancer and target organs.

\section{Arsenic}

There is clear evidence that chronic oral exposure to elevated levels of arsenic increases the risk of skin cancer. The most common lesions are squamous cell carcinomas, but multiple basal cell carcinomas may occur. Dermal lesions of this sort can be surgically removed, but they may be fatal if left untreated. A number of studies suggest that the incidence of some types of internal malignancies may also be increased by chronic exposure to arsenic, but the data are inadequate to draw firm conclusions (ATSDR, 1993a). As mentioned earlier, arsenic is a class A carcinogen based on observations of increased lung cancer mortality in populations exposed primarily through inhalation, and on increased skin cancer incidence in several populations consuming drinking water with high arsenic concentrations.

IRIS (EPA, 1995a) lists nine studies that show the association between inhalation of arsenic and lung cancer, four that show the link between drinking water contaminated with arsenic and increased skin cancer, and four that show a link between arsenic consumption and increased cancer of various organs.

\subsection{Noncarcinogens}

For many noncarcinogenic toxicity effects, protective mechanisms may exist that must be overcome before an adverse effect is manifested. As a result, a range of exposures from zero to some finite threshold value may be tolerated by an organism without any expression of adverse effects. In developing toxicity values to evaluate noncarcinogenic effects, the EPA approach is to identify the upper bound of this tolerance range (i.e., the maximum sub threshold level). For most chemicals, this level can only be estimated, so uncertainty factors and modifying factors are applied to this estimated level in order to derive a reference dose (RfD) for evaluation of noncarcinogens (EPA, 1989). 
An $\mathrm{RfD}$ reported as an intake (in $\mathrm{mg} / \mathrm{kg}$-day) is the toxicity value used most often in evaluating noncarcinogenic effects. Also used to evaluate noncarcinogenic effects are reference concentrations ( $\mathrm{RfCs}$ ), which are reported as a concentration in air or water (in $\mathrm{mg} / \mathrm{m}^{3}$ or $\mathrm{mg} / \mathrm{l}$ ). Derivation and/or conversion from an $\mathrm{RfC}$ (concentration) to an $\mathrm{RfD}$ (dose) is not recommended by EPA. Table C-2 (Appendix C) presents the RfD, confidence level, routes of exposure, critical effects, and uncertainty and modifying factors for arsenic.

RfDs are developed and verified by EPA, and are defined as "an estimate of a daily exposure level for the human population, including sensitive subpopulations, that is likely to be without an appreciable risk of deleterious effects during a lifetime" (EPA, 1989). $\mathrm{RfDs}$ are preferably based on the highest concentration of a chemical tested at which no adverse effects were demonstrated in animal experiments (the NOAEL, or no observed adverse effect leyel). To calculate an RfD, the NOAEL is divided by uncertainty and modifying factors. If a NOAEL is not available for a chemical, a LOAEL (lowest observed adverse effect level) may be divided by additional factors for use as an RfD (EPA, 1989).

RfDs are reported with their uncertainty factors (UFs). The UFs generally consist of . multiples of 10 , with each factor representing a specific area of uncertainty inherent in the extrapolation from available data. The use of UFs helps to ensure that the potential for adverse noncarcinogenic effects is not underestimated, even for sensitive subpopulations, during the derivation of $\mathrm{RfD} / \mathrm{RfC}$. Because there are few, if any, noncarcinogenic toxicity values for dermal exposure, oral values are frequently used to assess risks from dermal exposure. The EPA has recommended that oral RfDs based on administered dose be modified using an absorption factor so that they represent absorbed dose (EPA, 1989). Oral absorption efficiency (percent absorbed) values were identified (using various sources listed in Table C-2 (Appendix C) for arsenic.

\section{Arsenic}

Inorganic arsenic (arsenic combined with oxygen, chlorine, or sulfur) is more toxic than organic arsenic (arsenic combined with carbon and/or hydrogen) (ATSDR, 1993a). The 
RfD for chronic oral exposure to arsenic is based on studies (Tseng, 1977; Tseng et al., 1968) of over 40,000 residents of Taiwan who consumed well water contaminated with arsenic at levels that ranged from 0.001 to $0.017 \mathrm{mg} /$. Consumption of contaminated water resulted in increased incidence of hyperpigmentation and keratosis that also increased with dose, increased incidence of skin lesions. The LOAEL (lowest observed : adverse affect level) was estimated at $0.17 \mathrm{mg} / \mathrm{l}(0.014 \mathrm{mg} / \mathrm{kg} /$ day $)$, and the NOAEL at $0.009 \mathrm{mg} / \mathrm{l}(0.0008 \mathrm{mg} / \mathrm{kg} /$ day). A UF of 3 and a Modifying Factor (MF) of 1 were used, resulting in a chronic oral RfD, reported in IRIS, of $3 E-04 \mathrm{mg} / \mathrm{kg} /$ day. Confidence in the oral $\mathrm{RfD}$ is medium. HEAST reports a subchronic RfD of $1.0 \mathrm{E}-03 \mathrm{mg} / \mathrm{kg} / \mathrm{day}$, using a UF of 1 , based upon the same study.

The chronic inhalation RfC for arsenic is $2.9 \mathrm{E}-04 \mathrm{mg} / \mathrm{m}^{3}(8.3 \mathrm{mg} / \mathrm{kg} /$ day $)$, as developed by Oak Ridge National Laboratory (ORNL). The RfC is currently in draft stage and has not been adopted by the Environmental Criteria and Assessment Office (ECAO) of the EPA. The RfC based primarily on studies by Perry et al. (1948) and Watrous and McCaughey (1945), who studied the effects of occupational exposures of sodium arsenate and arsenilic acid dusts on industrial workers. The critical effects noted were skin abnormalities, including hyperpigmentation and hyperkeratosis. A UF of 100 and MF of 1 were used. Confidence in the $\mathrm{RfC}$ was rated as medium.

\subsubsection{Chemicals With No EPA Toxicity Values}

Lead

Lead, a naturally occurring blue-gray metal, is found in small quantities in the earth's crust and in all compartments of the biosphere. Both natural and anthropogenic processes are responsible for the distribution of lead throughout the environment, with gasoline combustion acting as a major source of lead found in the atmosphere and the earth's surface from atmospheric deposition (ATSDR, 1993b).

The toxicological database for lead is unusual in that it contains a great deal of dose-effect data for humans. These data come primarily from studies of occupationally exposed groups and the general population. Most of the exposure or dose data are in terms of internal exposure, usually measured as levels of lead in the blood. Human exposure to 
lead occurs through a combination of inhalation and oral exposure, with inhalation contributing a greater proportion of the dose for occupationally exposed groups and the oral route contributing a greater proportion of the dose for the general population. In all work areas, the major route of lead exposure is by inhalation and ingestion of lead-bearing dusts and fumes. Airborne dust settles onto food, water, clothing, and other objects and may subsequently be transferred to the mouth.

The effects of lead are the same regardless of entry route into the body and are correlated with internal exposure as blood lead level (ATSDR, 1993b).

Human epidemiological studies have been inadequate in determining the carcinogenic effects of lead. Although several studies in occupational cohorts exposed to lead and lead compounds have been conducted, the available human evidence is considered to be inadequate to refute or demonstrate potential carcinogenicity for humans from lead exposure (EPA, 1991a).

The carcinogenic potential of lead salts, primarily phosphate and acetates, administered by the oral route or be injection has been demonstrated in rats and mice by more than 100 investigators. The most characteristic cancer response is bilateral renal carcinoma. Most of the investigations found a carcinogenic response only at the highest dose (EPA, 1991a).

The endpoints most sensitive to low-level exposure to lead are neurobehavioral deficits and growth retardation in young children and hypertension in middle-aged men. Effects on heme synthesis also occur at very low exposure levels (ATSDR, 1993b). Lead is classified as a probable human carcinogen, B2 weight of evidence, probable human carcinogen, inadequate or no human data.

\subsubsection{Risk Characterization}

A current on unit worker scenario was not performed because no worker activity is conducted in the area. The overall human health risk from exposure to unit-related chemicals is discussed in the following paragraphs. 
Cancer risks are estimated as the incremental probability of an individual developing cancer over a lifetime as a result of pathway-specific exposure to carcinogenic contaminants. The risk to an individual resulting from exposure to nonradioactive chemical carcinogens is expressed as the increased probability of a cancer occurring over the course of a 70 year lifetime. Cancer risks are related to the EPA target range of 1E-04 to 1E-06 for incremental cancer risk at NPL sites. Risk levels at or above 1E-04 are generally considered significant. In order to account for simultaneous exposure to multiple carcinogens through a given pathway, the risks calculated for each individual carcinogen in that medium were summed to obtain an estimate of the total cancer risk for the pathway.

The potential for excess cancer risk in humans is calculated by using the maximum daily intake since there was only one detect for arsenic over a lifetime and multiplying by a chemical-specific SF. The SF converts estimated daily intake directly to the incremental risk of an individual developing excess cancer (EPA, 1989). Risks could be estimated only for those COCs with a toxicity value currently available from IRIS or HEAST. The carcinogenic risk estimate is generally an upper-bound estimate because the SF is typically derived as the upper 95 percentile confidence limit of the probability of response based on experimental animal data (EPA, 1989). Thus, EPA is reasonably confident that the "true risk" will not exceed the risk estimate derived through use of the SF and is likely to be less than that predicted (EPA, 1989).

The potential for adverse noncarcinogenic health effects is evaluated as the ratio of the daily intake for the exposure period over the RfD. This ratio of exposure is called a hazard quotient (HQ). HQs for each COC are summed to obtain an HI for the specific pathway. A HI greater than one has been defined as the level of potential concern for adverse noncarcinogenic health effects (EPA, 1989). The greater the ratio (intake/RfD), the greater the level of concern (EPA, 1989). The HI allows an assessment of exposure to multiple chemicals. A HI that exceeds one (1) indicates a potential for adverse health effects from exposure to multiple COCs. 


\subsubsection{Current Land Use}

Under the current land use scenario, there was no determination of carcinogenic risks and noncarcinogenic hazards from nonradioactive chemicals because no worker activity is being performed in the area.

\subsubsection{Future Land Use}

Under the future land use scenario, carcinogenic risks and noncarcinogenic hazards associated with nonradioactive COCs were calculated for exposure of the future on-unit resident (adult and child) to surface soils and air

\subsection{Carcinogenic Risk}

Estimates of the total risk by pathway for nonradioactive carcinogens are summarized for the future residential scenario in Table C-1 of Appendix C. Several of the exposure pathways have an estimated total risk of 3.8E-05.

Total cancer risk for ingestion of soil on the Grace Road Site was 8.8E-06. Estimated risks were 4.7E-08 below the EPA point of departure of 1E-06 for dermal contact with soils at the unit. Total cancer risk for inhalation of particulates from soils at Grace Road is 2.9E-05 which is above the EPA point of departure of $1 \mathrm{E}-06$, but within the $1 \mathrm{E}-04$ to $1 \mathrm{E}$ 06 range of concern.

\subsection{Noncarcinogenic Hazard}

Table 6-1 (and Table C-1 in Appendix C) provides a summary of HIs by pathway for the future residential scenario. HIs for the soil pathways were calculated for adulthood and childhood exposures combined and for childhood exposure only. All of the exposure pathways for the on-unit resident had HIs less than one. 


\subsection{Chemicals of Concern}

Cancer risk levels at or above $1 \mathrm{E}-06$ are generally considered significant by EPA. Those COPCs determined to contribute significantly to a pathway having a total cancer risk of greater than 1E-06 were identified as COCs. Exposures to soils for Grace Road and to airborne soil particulates have nonradiological risk levels greater than 1E-06.

An HI of one (1) or greater has been defined as the level of concern for potential adverse noncarcinogenic health effects. Those COPCs that were determined to contribute significantly to a pathway having an $\mathrm{HI}$ of one or greater were identified as COCs. None of the COCs had HIs of one (1) or greater for the future on-site residential scenario.

A summary of the risk presented by the chemical of concern, arsenic with a maximum concentration of $3.2 \mathrm{mg} / \mathrm{kg}$, for Grace Road is provided in Table 6-1. Additional information of the compilation of data and the calculations necessary to derive that table are provided in Appendix $\mathrm{C}$.

Table 6-1

Carcinogenic/Non-Carcinogenic Results for $3.2 \mathrm{mg} / \mathrm{kg}$ Arsenic

\begin{tabular}{|l|c|c|c|}
\hline \multirow{2}{*}{ Pathway } & Carcinogenic Risk & \multicolumn{2}{|c|}{ Non-Carcinogenic Risk } \\
\cline { 3 - 4 } & $\begin{array}{c}\text { Adult and Child } \\
\text { (Unitless) }\end{array}$ & $\begin{array}{c}\text { Adult and Child } \\
\text { (Unitless) }\end{array}$ & $\begin{array}{c}\text { Child only } \\
\text { (Unitless) }\end{array}$ \\
\hline Dermal Contact & $4.7 \mathrm{E}-08$ & $3.9 \mathrm{E}-04$ & $2.6 \mathrm{E}-04$ \\
Ingestion & $8.8 \mathrm{E}-06$ & $1.5 \mathrm{E}-01$ & $1.4 \mathrm{E}-01$ \\
Inhalation & $2.9 \mathrm{E}-05$ & $5.5 \mathrm{E}-02$ & $4.7 \mathrm{E}-02$ \\
\hline Total Risk & $3.8 \mathrm{E}-05$ & $2.0 \mathrm{E}-01$ & $1.9 \mathrm{E}-01$ \\
\hline
\end{tabular}

\subsubsection{Uncertainty}

The evaluation of chemical risks to human health presented in this baseline risk assessment was, by necessity, based on a number of assumptions. In addition, many uncertainties are inherent to the risk assessment process. Uncertainties associated with key site-related 
variables and major assumptions used in this assessment in order to address their potential impact on the results are discussed in the following section.

\subsubsection{Chemicals of Potential Concern}

Sampling data collected at any unit are inevitably a limited subset of the nearly unlimited quantity of data that potentially could be collected. Because of this the results may be an underestimation of risk. On the other hand, samples were not collected randomly and may be biased toward overestimation of chemical concentrations representative of the unit.

Uncertainty also is inherent in the selection of COPCs. Uncertainty in contaminant identification is considered low because sampling protocol generally targets appropriate analytes based on historical information and guidance. Reasonable certainty also is assumed because of the sample data validation and quality assurance/quality control procedures applied to sample analysis and data evaluation. Eliminating contaminants in the COPC screening process can lead to lower estimates of potential health effects than inclusion of all analytes. However, other than essential human nutrients, the only chemicals excluded from the risk assessment were those detected at concentrations less than two times mean background concentrations.

\subsubsection{Exposure Assessment}

Factors that can contribute to uncertainty in the exposure assessment include identification of exposure pathways; assumptions for scenario development, intake parameters, exposure pathways; and derivation of exposure point concentrations.

The identification of potential exposure pathways and receptors was based on site-specific, reasonable, current and hypothetical future residential land uses. The residential scenario may overestimate potential risk for the future land use scenario if the Grace Road remains industrial.

Values assumed for exposure parameters (e.g., inhalation rate and exposure frequency) used in calculations of intakes were based primarily on EPA guidance (EPA, 1990 and 1991b). These assumptions might result in underestimating or overestimating the intakes 
calculated for specific receptors, depending on the accuracy of the assumptions relative to actual unit conditions and uses. In the case of dermal exposure, there is uncertainty associated with the conversion from an administered intake to an absorbed dose because of uncertainty associated with the conversion factors.

Exposure point concentrations for airborne particulates were derived for the future land use scenario through the use of a model. The suspension of particulates was modeled following Milham et al. (1975), who studied resuspension of plutonium from an old field converted to agriculture specifically for the purposes of the study. Since none of the scenarios considered in this risk assessment involve the high degree of soil disturbance associated with agriculture, the value chosen is conservative and unit specific.

\subsubsection{Toxicity Assessment}

Uncertainty is inherent in the toxicity values utilized in evaluating the carcinogenic and noncarcinogenic risks. Such uncertainty is chemical-specific and is incorporated into the toxicity value during its development. For example, an uncertainty factor may be applied for interspecies and intrahuman variability, for extrapolation from subchronic to chronic exposures, or for epidemiological data limitations. Application of uncertainty factors is expected to overestimate risks.

Toxicity information was not available for dermal exposure; hence, several assumptions were made which may overestimate or underestimate risk.

\subsubsection{Risk Characterization}

Uncertainties in the toxicity assessment are compounded under the assumption of dose additivity for multiple substance exposure. This assumption ignores possible synergisms and antagonisms among chemicals. The assumption that al the toxic and carcinogenic effects are additive could result in the underestimation of risk because concurrent exposure to several contaminants might have synergistic toxic effects (e.g., exposure to two metals concurrently might induce a greater toxic effect than indicated by simply adding the toxic effects from each metal). However; summing risks for chemicals having 
various weight-of-evidence classifications as well as toxic effects to different target organs and ignoring possible antagonistic effects of some chemicals may overestimate risks.

\subsection{Ecological Assessment}

The ecological information base for Grace Road Unit consists of a unit-specific threatened, endangered and sensitive species survey and a unit-specific ecological reconnaissance. Additional information is contained in the existing unit history, preliminary unit evaluation, and unit characterization data. These informational sources form the basis for this characterization. That information can be summarized as follows:

- There is no evidence of vegetation stress or ecological impact related to the unit;

- There are no threatened or endangered species known to exist at or in the vicinity of the unit;

- Review of the unit characterization data indicates that there are no constituents in the physical media analyzed at Grace Road which are significantly different from the unit specific background condition.

Based on the physical and analytical data obtained for this unit, there appears to be no compelling evidence that waste materials were managed or disposed at Grace Road. Therefore, it is reasonable to conclude that the unit, as it is currently characterized, presents no significant ecological risk. A further detailed ecological risk assessment and characterization would not be warranted at this time. 


\subsection{SUMMARY AND CONCLUSION}

\subsection{Summary}

An analysis of data collected as a result of the studies performed at Grace Road indicates that - there is no significant risk due to known contamination at that unit. Of the COPCs identified in the risk assessment (see Table 1-3), only arsenic exceeded a risk of 1.0E-06 and it is calculated to be 3.8E-05. Arsenic detected in the unit specific samples had a maximum concentration of 3.2 $\mathrm{mg} / \mathrm{kg}$. The calculated risk for arsenic is summarized in Table C-1 in Appendix C. The equations and input parameters used to determine the risk/hazard are also located in Appendix C.

The SRS site wide values for arsenic range from $<0.5 \mathrm{mg} / \mathrm{kg}$ to $15.2 \mathrm{mg} / \mathrm{kg}$ with a median of $<$ $2.0 \mathrm{mg} / \mathrm{kg}$. It should also be noted that arsenic was used as a component of agricultural chemicals in the period before SRS existed. Thus, the detected values may be a result of farming activities in the 1930's and 40's.

In addition, it is also reasonable to conclude that there is no significant ecological risk due to known contamination at the unit.

\subsection{Conclusion}

Based on the review of the analytical data and screening techniques used to evaluate all the chemicals of potential concern at Grace Road, it is recommended that no further remedial action be performed at this unit. 
Grace Road Site

RFI/RI Report
WSRC-RP-95-93, Rev. 1.1

February 1996

\section{THIS PAGE INTENTIONALLY LEFT BLANK}


Grace Road Site

RFI/RI Report
WSRC-RP-95-93, Rev. 1

November 1995

\section{REFERENCES}

Aadland, R. K., and H. W. Bledsoe, Classification of Hydrostratigraphic Units at Savannah River Site, South Carolina, WSRC-RP-90-987, Westinghouse Savannah River Company, Savannah River Laboratory, Aiken, SC. (1990).

Aadland, R. K., A. D. Smits, and P. A. Thayer, Geology and Hydrostratigraphy of the AM-Area, Savannah River Site (SRS), South Carolina (U). WSRC-RP-92-440, Westinghouse Savannah River Company, Savannah River Site, Aiken, SC. (1992).

ATSDR (Agency for Toxic Substances and Disease Registry), Toxicological Profile for Arsenic, United States Department of Health and Human Services, ATSDR/TP-92/02 Public Health Service, Atlanta, GA (1993a).

ATSDR (Agency for Toxic Substances and Disease Registry), Toxicological Profile for Lead, United States Department of Health and Human Services, ATSDR/TP-92/12 Prepared by Clement International Corporation for the U. S. Public Health Service, Atlanta, GA (1993b).

Conner and Shacklette, Background Geochemistry of Some Rocks, Soils, Plants, and Vegetables in the Conterminous United States. U.S. Geological Survey Professional Paper 574-F, Government Printing Office, Washington, DC (1975).

DOE, Continued Operation of $K-, L-$, and P-Reactors Savannah River Site: Final Environmental Impact Statement, DOE/EIS-0147, U. S. Department of Energy, (1990).

DOE, Public Involvement, A Plan for the Savannah River Site (1994).

EPA, Guidance for Conducting Remedial Investigations and Feasibility Studies Under CERCLA, Interim Final. EPA/540/G-89-004, U.S. Environmental Protection Agency, Washington, DC. (1988).

EPA, Risk Assessment Guidance for Superfund (RAGS) Volume I - Human Health Evaluation Manual (Part A) EPA/540/1-89/002, Office of Emergency and Remedial Response, U. S. Environmental Protection Agency, Washington, DC., (1989). 
Grace Road Site

RFI/RI Report
WSRC-RP-95-93, Rev. 1

November 1995

EPA, Exposure Factors Handbook, EPA/600/8-80/043, Office of Health and Environmental Assessment, Washington, DC., U. S. Environmental Protection Agency, Washington, DC., (1990).

EPA, Risk Assessment Guidance for Superfund (RAGS), Human Health Evaluation Manual, Part B: "Development of Risk-based Preliminary Remediation Goals" (OSWER . Directive 9285.7-01B), Office of Solid Waste and Emergency Response, U.S. Environmental Protection Agency, Washington, DC., (1991a).

EPA, Risk Assessment Guidance for Superfund: Volume I - Human Health Evaluation Manual. Supplemental Guidance "Standard Default Exposure Factors" - Interim Final, OSWER Directive 9285.6-03, Office of Emergency and Remedial Response Toxics Integration Branch, U.S. Environmental Protection Agency, Washington, DC., (1991b).

EPA, Dermal Exposure Assessment: Principles and Applications, Interim Report. EPA 600/891-011B, Office of Research and Development, U.S. Environmental Protection Agency, Washington, DC., (1992).

EPA, Superfund's Standard Default Exposure Factors for the Central Tendency and Reasonable Moximum Exposure, U.S. Environmental Protection Agency, Cincinnati, OH, (1993).

EPA, Health Effects Assessment Summary Tables (HEAST), FY 1994, EPA 540-R-94-020, Office of Research and Development, Office of Emergency and Remedial Response, U.S. Environmental Protection Agency, Cincinnati, OH, (1994)

EPA, The Integrated Risk Information System (IRIS), Environmental Criterion Assessment Office, Office of Research and Development, U.S. Environmental Protection Agency, Cincinnati, OH, (1995a).

EPA, Risk-Based Concentration Table, January-June 1995, U. S. Environmental Protection Agency, Region III, Technical Support Section, Philadelphia, PA (1995b).

Fallaw, W. C., Subsurface Stratigraphy and Structure of AM-Area at the Savannah River Site, Aiken County, South Carolina, WSRC-RP-91-0830, Westinghouse Savannah River 
Company, Aiken, SC. (1991).

Fay, W. M. and J. B. Pickett, Documentation of 1982 Soil Analyses to Determine the Natural Background Radioactivity in SRP Surface Soils, DPST-87-260, E. I. du Pont de Nemours and Company, Savannah River Plant, Aiken, SC, (1987).

Gordon, D. E., W. F. Johnson, D. S. Kabeck, B. B. Looney, R. L. Nichols, and C. B. Shedrow, Characterization Recommendations for Waste Sites at the Savannah River Plant, DPST87-667, (1987).

Lewis, S. E., and R. K. Aadland, Hydrogeologic Setting of the AM-Area: Framework for Groundwater Transport (U). WSRC-TR-92-355, Volume 1, Westinghouse Savannah River Company, Savannah River Site, Aiken, SC. (1992)

Looney, B. B., V. Price, D. J. Master, and C. A. Boe, Soil Gas Survey at Selected Potential Waste Sites at the Savannah River Plant, DPST-88-619, E. I. du Pont de Nemours and Company, Savannah River Laboratory, Aiken, SC, (1988).

Looney, B. B., C. A. Eddy, M. Ramdeen, J. Pickett, V. Rogers, M. T: Scott and P. A. Shirley, Geochemical and Physical Properties of Soils and Shallow Sediments at the Savannah River Site, WSRC-RP-90-1031, Westinghouse Savannah River Company, Savannah River Site, Aiken, South Carolina, (1990).

Milham, R. C., J. F. Schubert, J. R. Watts, A. L. Boni and J. C. Corey, Measured Plutonium Resuspension and Resulting Dose for Agricultural Operations on an Old Field at the Savannah River Plant in the Southeastern United States, DP-MS-75-29, IAEA-SM199/83, Int. Symp. on Transuranium Nuclides in the Environment, San Francisco, CA, (1975).

Perry, K., R. G. Bowler, H. M. Buckell, H. A. Druett and R. S. F. Schilling, Studies in the Incidence of Cancer in a Factory Handling Inorganic Compounds of Arsenic, II Clinical and Environmental Investigations, Br. J. Ind. Med. 5:6, (1948). 
Grace Road Site

RFI/RI Report
WSRC-RP-95-93, Rev. 1

November 1995

Price, V., Subsurface Hydrogeology, in Stephenson, A., 2 ed., Final Safety Analysis Report for DWPF (Drafi). DPSTSA-200-10, E. I. du Pont de Nemours and Company, Savannah River Plant, Aiken, SC (1988).

Rand McNalley, Commercial Atlas and Marketing Guide, 123 rd edition, Skokie, Illinois, (1992).

Tseng, W. P., H. M. Chu, S. W. How, J. M. Fong, C. S. Lin and S. Yeh, , Prevalence of Skin Cancer in an Endemic Area of Chronic Arsenicism in Taiwan, J. Natl. Cancer Inst. 40:453-463, (1968).

Tseng, W. P., Effects and Dose-response Relationships of Skin Cancer and Blackfoot Disease with Arsenic, Environ. Health Perspect. 19:109-119, (1977).

Watrous, R. M. and M. B. McCaughey, Occupational Exposure to Arsenic In the Mamufacture of Arsphenamine and Related Compounds, Ind. Med. 14:639-346, (1945).

WSRC (Westinghouse Savannah River Company), RCRA Facility Investigation/Remedial Investigation Plan for the Grace Road Unit (U), WSRC-RP-90-1250, Rev. 0, Westinghouse Savannah River Company, Aiken, SC, (1990).

WSRC (Westinghouse Savannah River Company), RCRA Facility Investigation/Remedial Investigation Program Plan. WSRC-RP-89-994, Rev. 1, Westinghouse Savannah River Company, Aiken, SC. (1993). 
Grace Road Site

RFI/RI Report
WSRC-RP-95-93, Rev. 1

November 1995

\section{APPENDIX A}

\section{TECHNICAL MEMORANDA ON FIELD ACTIVITIES}

Appendix A.1 Ground Penetrating Radar Survey

Appendix A.2 Gross Alpha and Non-Volatile Beta Analyses

Appendix A.3 Soil Gas Survey Analyses 
Grace Road Site

WSRC-RP-95-93, Rev. 1

RFI/RI Report

November 1995

sis

\section{THIS PAGE INTENTIONALLY LEFT BLANK}

A-2 
Grace Road Site

RFI/RI Report
WSRC-RP-95-93, Rev. 1

November 1995

APPENDIX A.1

GROUND PENETRATING RADAR SURVEY

A.1-1 
Grace Road Site

RFI/RI Report
WSRC-RP-95-93, Rev. 1 .

November 1995

\section{THIS PAGE INTENTIONALLY LEFT BLANK}


Final Report

\section{GROUND PENETRATING RADAR INVESTIGATIONS}

at the

GRACE ROAD WASTE SITE

SAVANNAH RIVER SITE

Apri1, 1994

G.Boyd Sexton

Robert J. Pirkle

Task 49

Subcontract *A38232N

A. 1-3 
Ground penetrating radar surveys were executed at the Grace Road Waste Site on March 20 and April 12, 1994 for the purpose of determining the presence of buried waste and debris and to identify the areas of excavation if present. Historical information indicates the presence of foundations, buildings and large amounts of debris at various locations within the waste site.

A total of 53 lines of GPR data, totaling 12,879 feet, were acquired. Fifteen lines totaling 3218 feet were acquired March 20, 1994. Thirty-eight additional lines totaling 9661 feet were acquired April 12, 1994. Much of the data suggests the presence of undisturbed sediments, but as expected around old building pads metallic objects and other debris were indicated. 
Executive Summary

1

List of Figures

iii

List of Tables

$\mathbf{v i}$

I. Introduction

1

II. Background

2

III. Acquisition and Processing Parameters

2

IV. Results

3

v. Conclusions 


\section{IIST OF FIGURES}

Figure 1. GPR Line Location Map $100 \mathrm{MHz}$ Data

Figure 1A. GPR Line Location Map $300 \mathrm{MHz}$ Data

Figure 2. Sample GPR Section With Illustrated Computerized Interpretation

Figure 3. GPR Line $\$ 1100 \mathrm{MHz}$. Data

Figure 4. GPR Line $\$ 2100 \mathrm{MHz}$. Data

Figure 5. GPR Line $\$ 3100 \mathrm{MHz}$. Data

Figure 6. GPR line $\$ 4100 \mathrm{MHz}$. Data

Figure 7. GPR Line $\$ 5100 \mathrm{MHz}$. Data

Figure 8. GPR Line $\$ 6100 \mathrm{MHz}$. Data

Figure 9. GPR Line $\$ 7100 \mathrm{MHz}$. Data

Figure 10. GPR Line $\neq 8100 \mathrm{MHz}$. Data

Figure 11. GPR Line $\neq 9100 \mathrm{MHz}$. Data

Figure 12. GPR Line $\neq 10100 \mathrm{MHz}$. Data

Pigure 13. GPR Line \$11 $100 \mathrm{MHz}$. Data

Figure 14. GPR Line $\$ 12100 \mathrm{kHz}$. Data

Figure 15. GPR Line $\$ 13100 \mathrm{MHz}$. Data

Figure 16. GPR Line $14100 \mathrm{MHz}$. Data

Figure 17. GPR Line $\$ 15100 \mathrm{kHz}$. Data

Figure 18. GPR Line $\$ 16100 \mathrm{MHz}$. Data

Pigure 19. GPR Line $\$ 17100 \mathrm{MHz}$. Data

Figure 20. GPR Line $\$ 18100 \mathrm{kHz}$. Data

Figure 21. GPR Line $\$ 19100 \mathrm{MHz}$. Data

Figure 22. GPR Line $\$ 20100 \mathrm{MHz}$. Data

Figure 23. GPR Line $\$ 21100 \mathrm{MHz}$. Data

$$
\text { A. 1-7 }
$$


$\frac{\text { IIST OF FIGURES }}{\left(\text { con't.) }^{\prime} \text { ) }\right.}$

Figure 24. GPR Line \$22 $100 \mathrm{MHz}$. Data Figure 25. GPR Iine $\$ 23100 \mathrm{MHz}$. Data Figure 26. 'GPR Iine $\$ 24100 \mathrm{MHz}$. Data Figure 27. GPR Iine $\$ 25100 \mathrm{MHz}$. Data Figure 28. GPR Line $\$ 26100 \mathrm{MHz}$. Data Figure 29. GPR Line $\$ 27100 \mathrm{MHz}$. Data Figure 30. GPR Line $\$ 28100 \mathrm{MHz}$. Data Figure 31. GPR Line $\$ 29100 \mathrm{MHz}$. Data Figure 32. GPR Line $\$ 30100 \mathrm{MHz}$. Data Figure 33. GPR Line $\$ 31100 \mathrm{MHz}$. Data Figure 34. GPR Iine $\$ 32100 \mathrm{MHz}$. Data Figure 35. GPR Line $\$ 33100 \mathrm{MHz}$. Data Figure 36. GPR Line $\$ 34100 \mathrm{MHz}$. Data Figure 37. GPR Line $\$ 35100 \mathrm{MHz}$. Data Figure 38. GPR Line $\$ 36100 \mathrm{MHz}$. Data Figure 39. GPR Line $\$ 37100 \mathrm{MHz}$. Data Figure 40. GPR Line $\$ 38100 \mathrm{MHz}$. Data Figure 41. GPR Line $\neq 1300 \mathrm{MHz}$. Data Figure 42. GPR Iine $\$ 2300 \mathrm{MHz}$. Data Figure 43. GPR Line $\$ 3300 \mathrm{MHz}$. Data Figure 44. GPR Iine \$4. $300 \mathrm{MHz}$. Data Figure 45. GPR Line $\neq 5300 \mathrm{MHz}$. Data Figure 46. GPR Iine $\$ 6300 \mathrm{MHz}$. Data Figure 47. GPR Iine $\$ 700 \mathrm{MHz}$. Data Figure 48. GPR Iine $\$ 8300 \mathrm{MHz}$. Data

$$
\text { A. } 1-8
$$


$\frac{\text { LIST OF FIGURES }}{\text { (con't.) }}$

Figure 49. GPR Iine $\$ 9300 \mathrm{MHz}$. Data Figure 50. GPR Line $\$ 10300 \mathrm{MHz}$. Data Figure 51. GPR Iine $\$ 11300 \mathrm{MHz}$ : Data Figure'52. GPR Iine $\$ 12300 \mathrm{MHz}$. 'Data Figure 53. GPR Line $\$ 13300 \mathrm{MHz}$. Data Figure 54. GPR Iine $\$ 14300 \mathrm{MHz}$. Data Figure. 55. GPR Line $\$ 15300 \mathrm{MHz}$. Data 
Table 1. Coordinates, Bearings, and Distances of GPR Lines

Table 2. Acquisition and Processing Parameters 


\section{INTRODUCTION}

Ground Penetrating Radar (GPR) surveys were run at the Grace Road Waste site for the purpose of identifying evidence of excavation and determining areas where buried waste and other debris may be found. A total of 53 lines totaling 12,879 feet were acquired on March 20 and April 12, 1994, as shown on Figures 1 and $1 \mathrm{~A}$ and listed on Table 1. Iines acquired with a $100 \mathrm{MHz}$ antenna, 200 ns range are shown on Figure 1 and lines acquired with the 300 MHz. antenna are shown on Figure $1 A$.

The line spacing interval varies from 25 feet to 50 feet dependent upon obstacles encountered during the survey. A grid was laid out in approximately north-south, east-west directions to determine locations of GPR transects. All lines are plotted in the same direction to aid in visualization and interpretation even though acquired differently in the field. The acquisition direction for each line is indicated on the base map (Figure 1). 
II. BACKGROUND AND OBSERVATIONS

The Grace Road Waste Site is approximately $1100 \times 350$ feet in size. The site is sporadically overgrown with dense trees and brush which hinder GPR data acquisition. The surface condition of the site was generally good for GPR Investigations except for the trees and brush.

The weather during field acquisition was warm and there was no rainfall. Personnel on-site during data acquisition were Boyd Sexton and Ken Grace.

III. ACOUISITION AND PROCESSING PARAMETERS

The equipment and software used in the acquisition and processing of the GPR data are listed on Table 2. 


\section{RESULTS}

The results of the GPR surveys at the Grace Road Waste site are shown on Figures 3 - 55. A sample GPR section with labeled interpretive information is shown on Figure 2. A typical signature of an area of disturbed soil is labeled so that similar anomalous areas on the actual lines presented on Figures 3 - 55 can be easily recognized. The horizontal scale, vertical time scale, and maximum depth of penetration are also shown on each line. The radar velocity in this area is 6-7 ns per foot.

The $100 \mathrm{MHz}$ data on Lines 1 - 38 (Figures 3-40) are displayed in the north-south, east-west direction and the $300 \mathrm{MHz}$ data on Lines 1 - 15 (Figures 41-55) are displayed in the same format. An instrument problem occurred on March 20 as Iine 15 was being acquired. Evidence of the problem is apparent on the displayed section. The problem was corrected and the survey was completed on April 12 .

The exact location of each individual line is displayed on the base maps (Figures 1 and $1 A$ ). In the field, successive lines were acquired in alternating directions, however alternating lines were reversed in processing to aid in viewing and interpretation.

Using the most shallow continuous reflector as the presence of undisțurbed or natural soil, the area and depth of excavation can be judged on each section. The same interpretive reasoning can be applied to both 100 and $300 \mathrm{MHz}$ data. Several prominent refractions, characterized by high amplitude hyperbolas are indicative of buried metallic objects. 
v. CONCLUSIONS

The areas surveyed yielded good data quality. The $100 \mathrm{MHz}$. data yielded deeper penetration than the $300 \mathrm{MHz}$ data. Several plots have strong refractions indicating buried metallic objects. Disturbed soil is present around some of the old building pads and may indicate excavation in removing the buildings and/or buried debris. 
TABLE 1

GRACE ROAD WASTE SITE

TABLE OF COORDINATES, BEARINGS, AND DI8TANCES

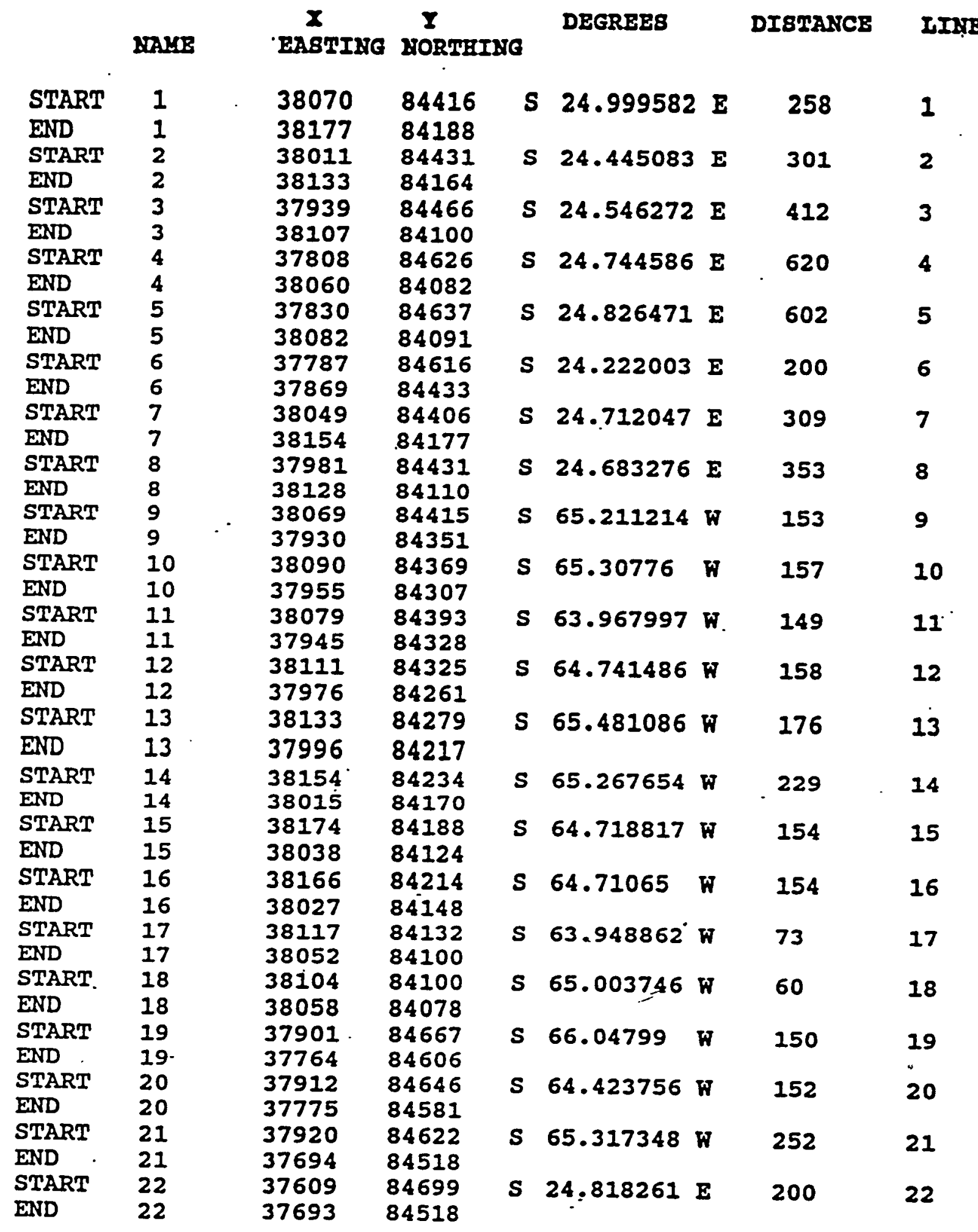

A. 1-1.5 
SAMB

TABLE $\neq 100 \mathrm{MHz}$ Data (Con't) EABTING NORTHING

DISTANCE

IINE

•.

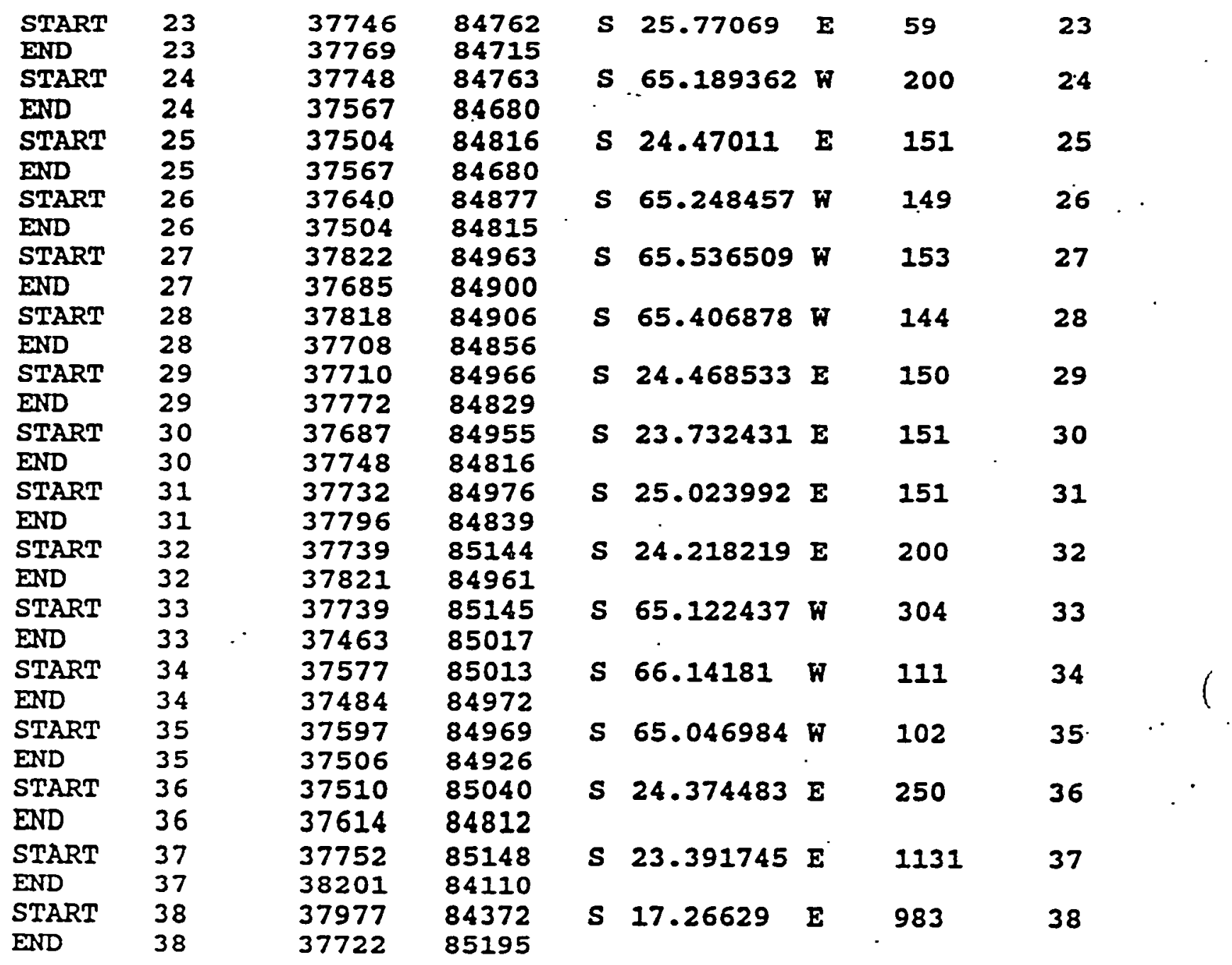

WAME EASTING MORTHING

TABLE $\$ 1300$ YHR DATA

$Y$ DEGREES DISTANCE IINE

$\begin{array}{lllll}\mathbf{X} & \mathbf{Y} & \text { DEGREES DISTANCE } & \\ \text { EASTING } & & \end{array}$

\begin{tabular}{|c|c|c|c|c|c|c|c|}
\hline START & 1 & 38176 & 84189 & $\mathbf{s}$ & 65.363936 & พ & 181 \\
\hline $\begin{array}{l}\text { END } \\
\text { START }\end{array}$ & $\frac{1}{2}$ & $\begin{array}{l}38038 \\
38153\end{array}$ & $\begin{array}{l}84126 \\
84235\end{array}$ & $\mathbf{s}$ & 65.075532 & w & \\
\hline END & 2 & 38017 & 84172 & 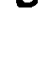 & גבכבנטיתם & $\boldsymbol{W}$ & 214 \\
\hline START & 3 & 38134 & 84280 & $\mathbf{s}$ & 64.797668 & พ & 212 \\
\hline END & 3 & 38043 & 84237 & & & & \\
\hline START & 4 & 38127 & 84333 & $\mathbf{s}$ & 64.296991 & พ & 181 \\
\hline END & 4 & 38023 & 84283 & & & & \\
\hline START & 5 & 38091 & 84372 & $\mathbf{s}$ & 64.022653 & พ & 130 \\
\hline END & 5 & 37983 & 84319 & & & & \\
\hline
\end{tabular}


NDAMS

TABLE $1300 \mathrm{MHz}$ Data (COn't)
$x$
EABTING YORTHING

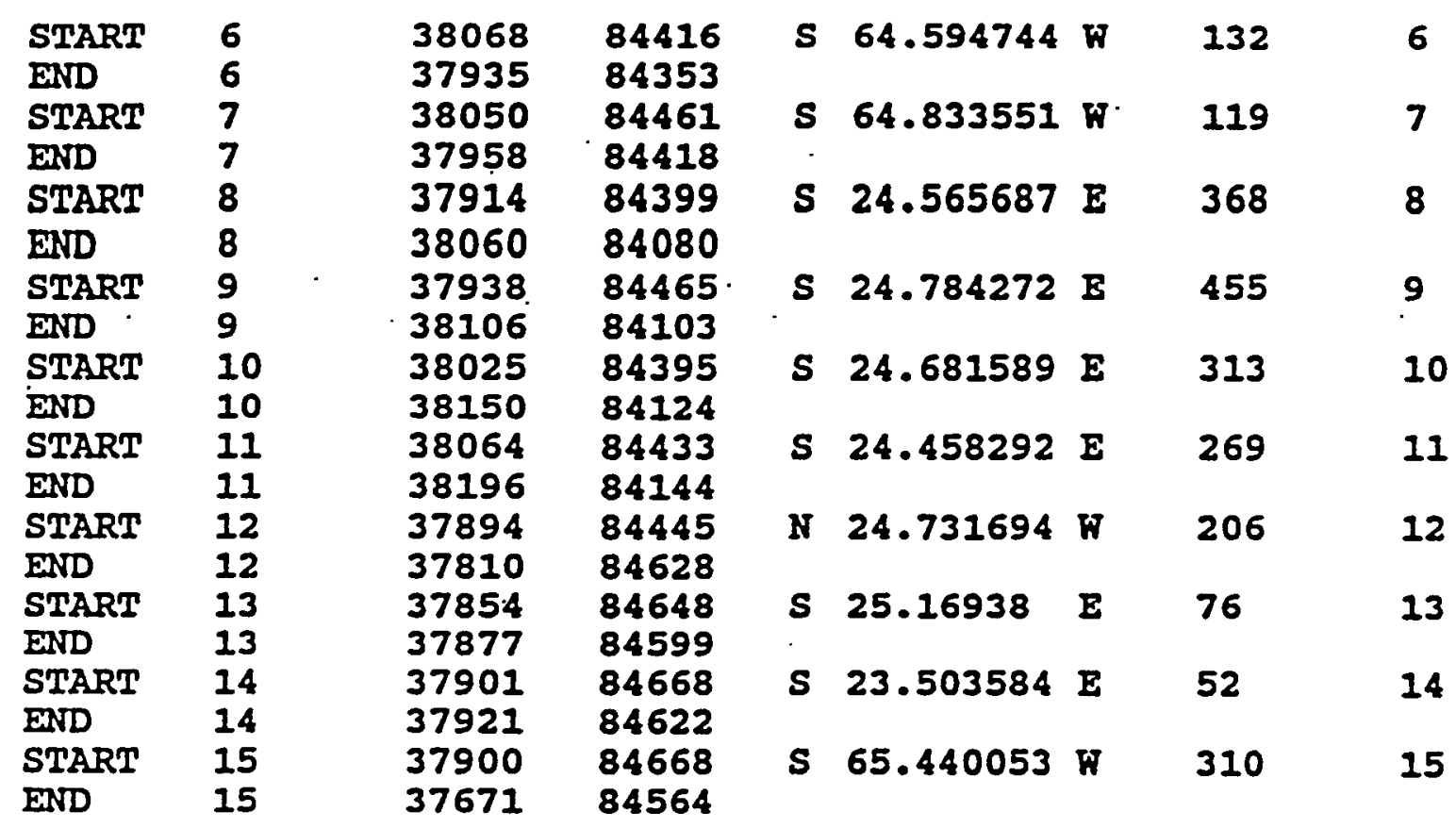

TOTAL FOOTAGE $100 \mathrm{kHz}$ Data

9661

TOTAL FOOTAGE $300 \mathrm{MHZ}$ DATA

3218

TOTAL FOOTAGE IN SURVEY

12879 
Table 2

Acquisition and Processing Parameters

at the

Grace Road Taste site '

Date Data Acquired

Instrument Type

Antenna Type

Calibration Numbers

Post Processing Software

Range

start Position

End Position

Survey Wheel

Scans/Foot

Samples/Scan

Transmit pulse Rate

Vertical. IIR Iow Pass Filter

Vertical IIR High Pass Filter

Horizontal Low Pass Filter

Scans/Meter

Marks/Meter (on plot)
$3 / 20 / 94 \& 4 / 12 / 94$

GSSI (SIR 10) S/N 1158

$100 \& 300 \mathrm{MHZ}$

Supplied by GSSI PG-90-177

Version 2.05

Max 141

Min 20

Diff 121

GSSI (RADANIII)

$200 \& 150$ ns

$-10 \mathrm{~ns}$

$180 \& 140$ ns

129.74 ticks/foot

4

512

$50 \mathrm{KHz}$

$N=2 \quad F=80$

$N=2 \quad F=11$

$\mathrm{TC}=0$

13.1234

approximately 5 meters/mark 
Figure

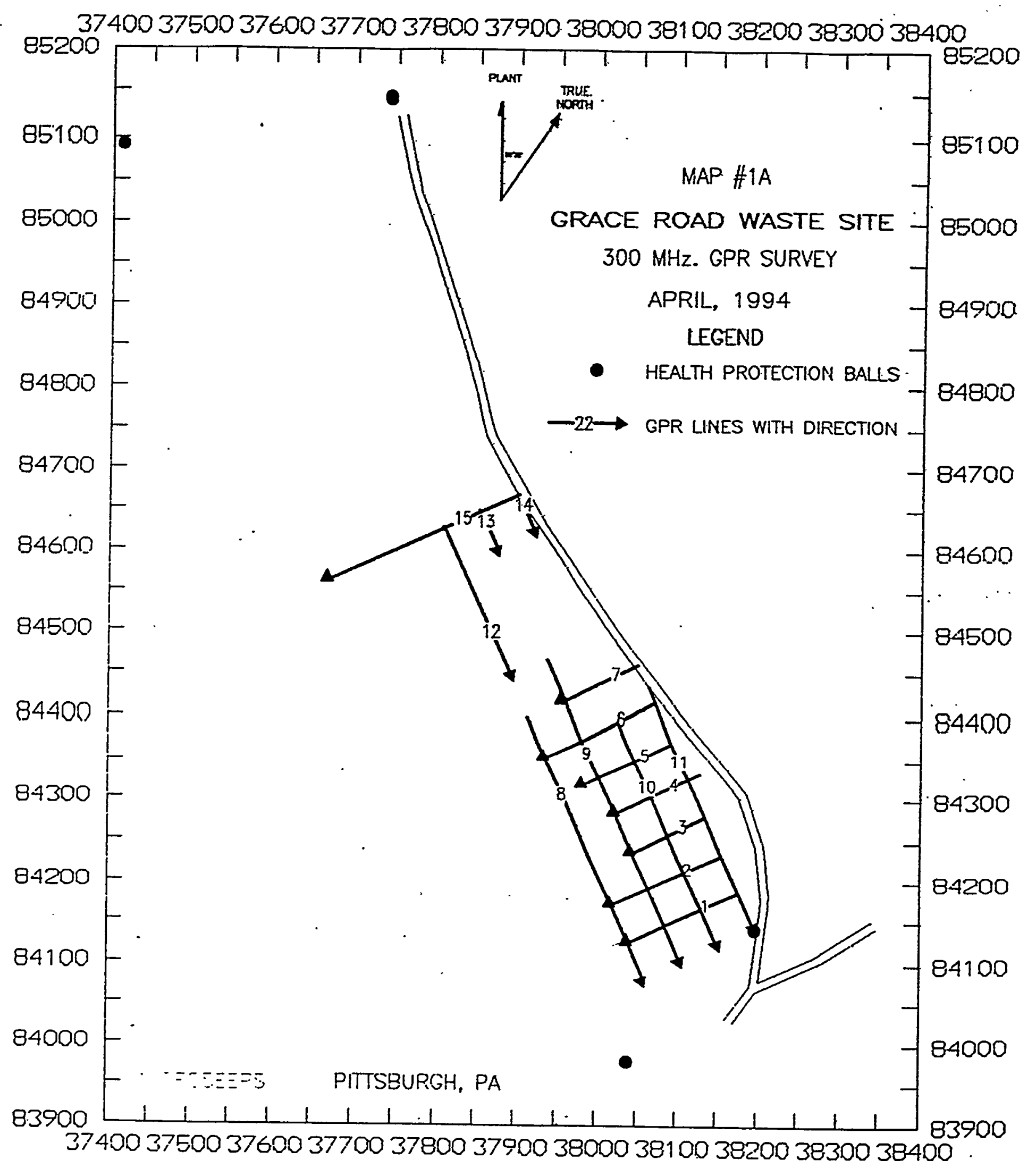


Figure 1

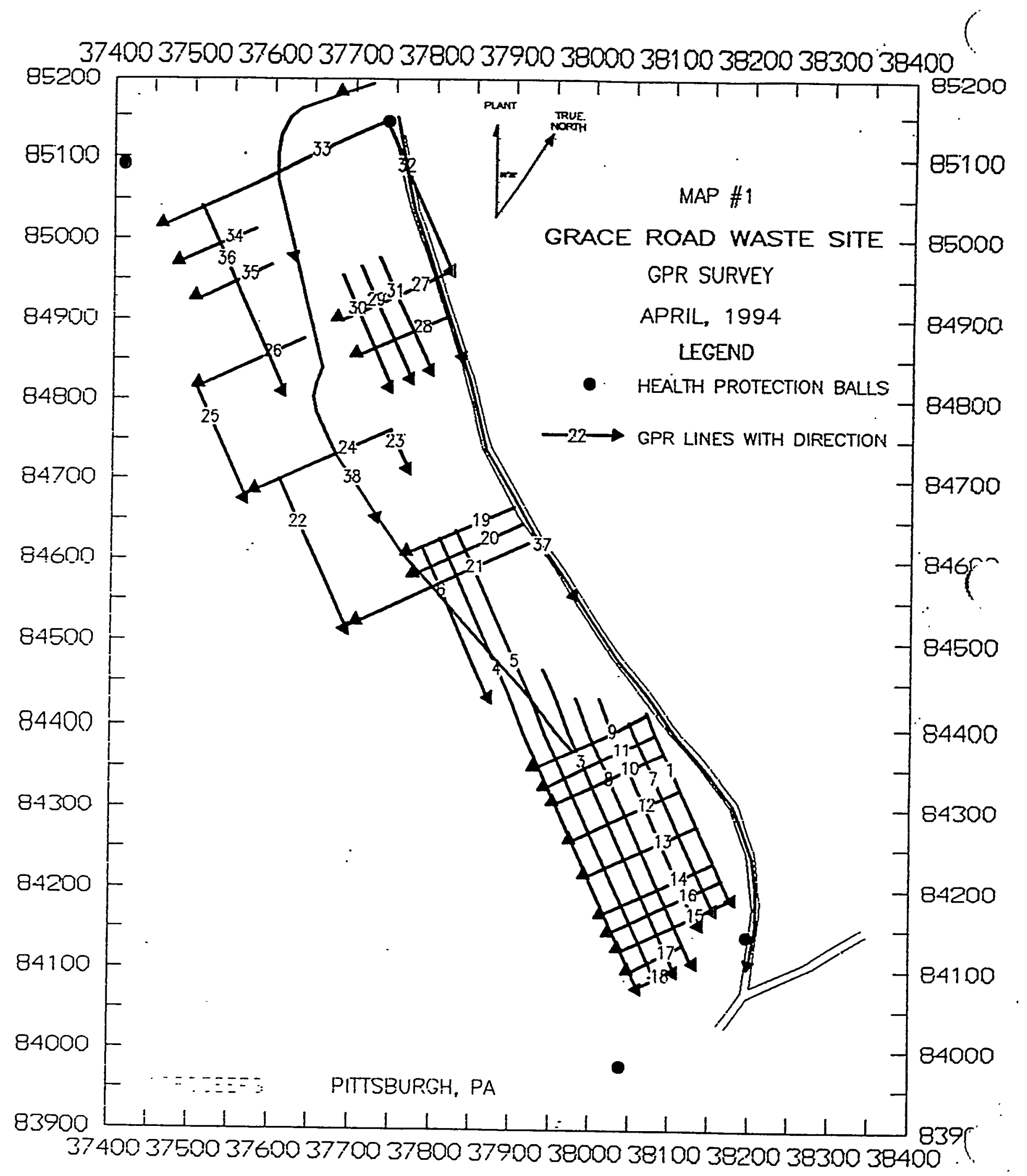




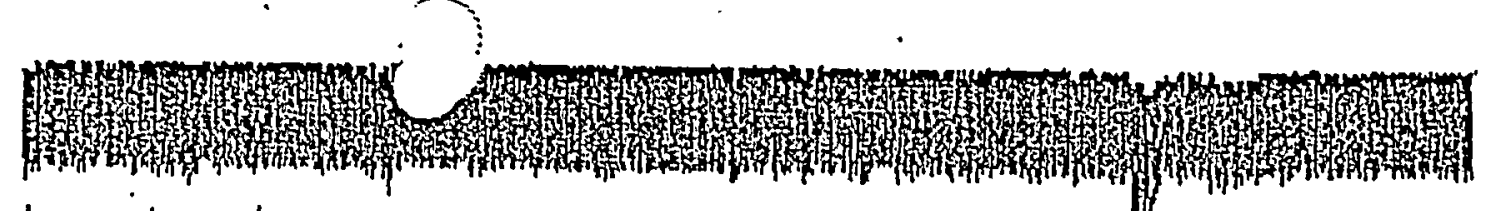

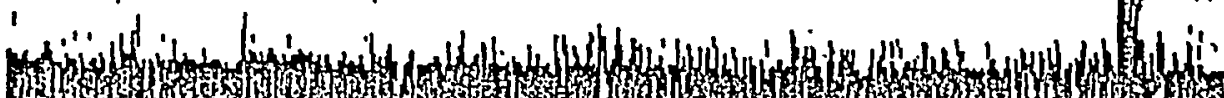

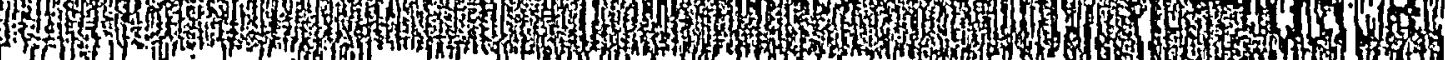

Ad

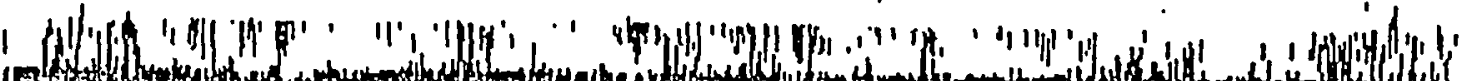
(6.2. for

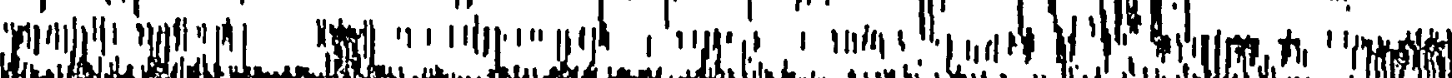

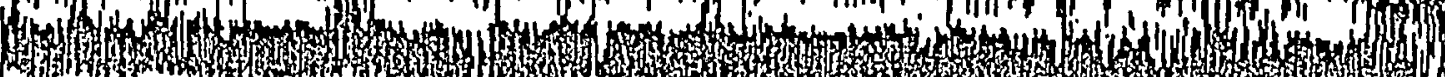
(n)

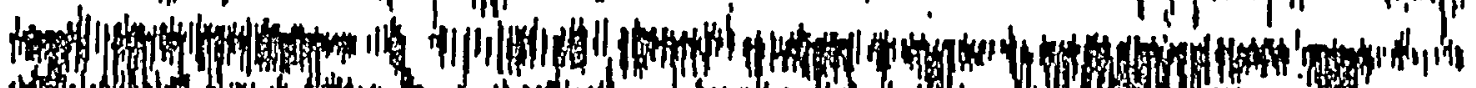

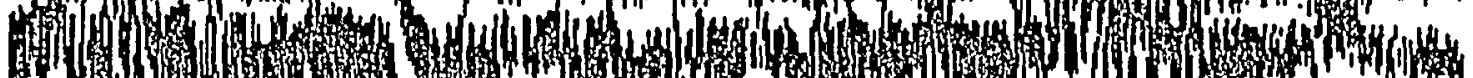

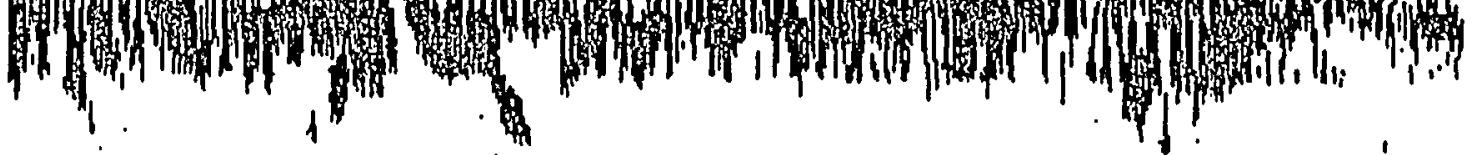

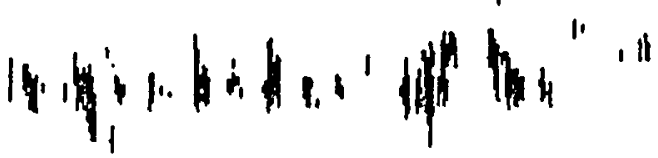

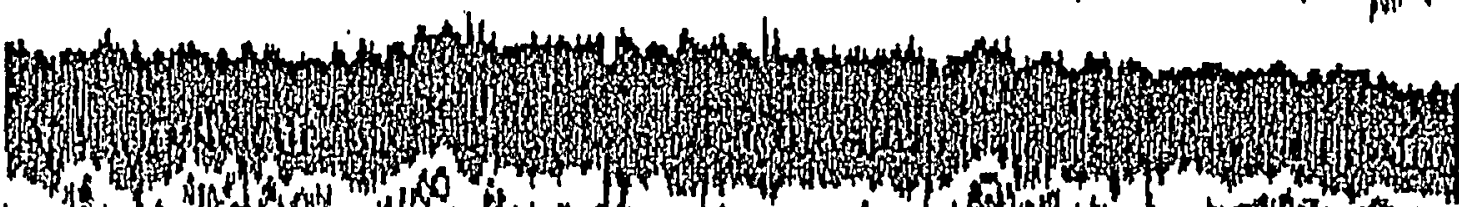
1. -7.

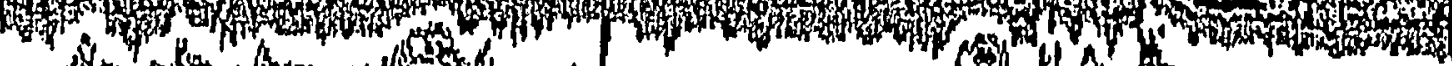

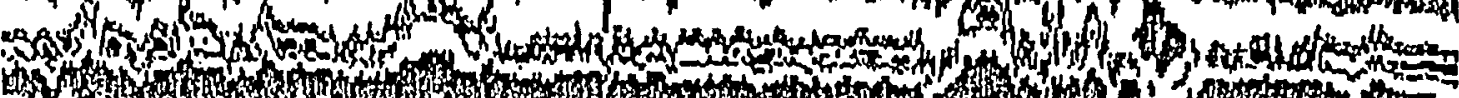
4. 4 (6)

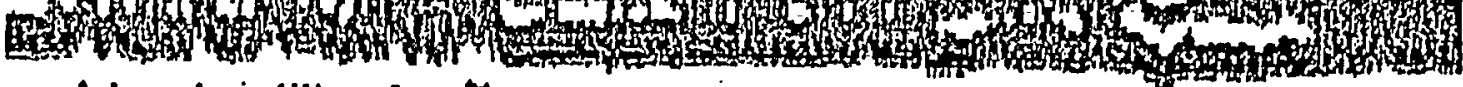

ox

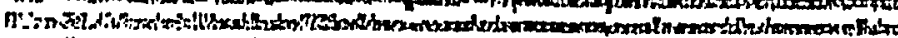

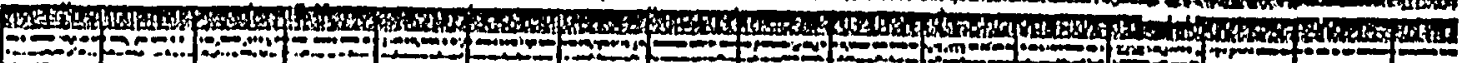

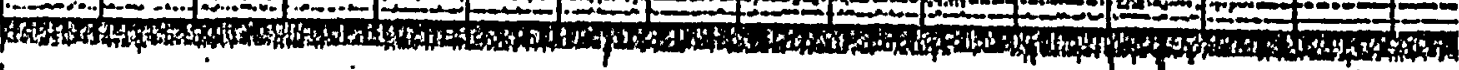
werly
象兽

Sint

$4+4$ (t)

on

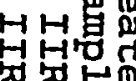

10

E.

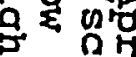

id

然芯

约

Zil

IIN

$N \rightarrow \infty$

用 II

北出设

w

苞。

赑

吕总

0

㗪

急

H

葛

i

No

昆

。

H艹

宫

.

옹

品

翠. 
IER Created Apr 12,1994 11:20 Modified Apr 21,1994 15:27

512 samples/scan 49.984 scans/sec position: $-20 \mathrm{~ns}$ range: $200 \mathrm{~ns}$ Vert. IIR low pass $\mathrm{N}=2 \mathrm{~F}=40$

Vert. IIR high pass $\mathrm{N}=2 \mathrm{~F}=5$

GRACE ROAD WASTE SITE

SAVANNAE RIVER SITE
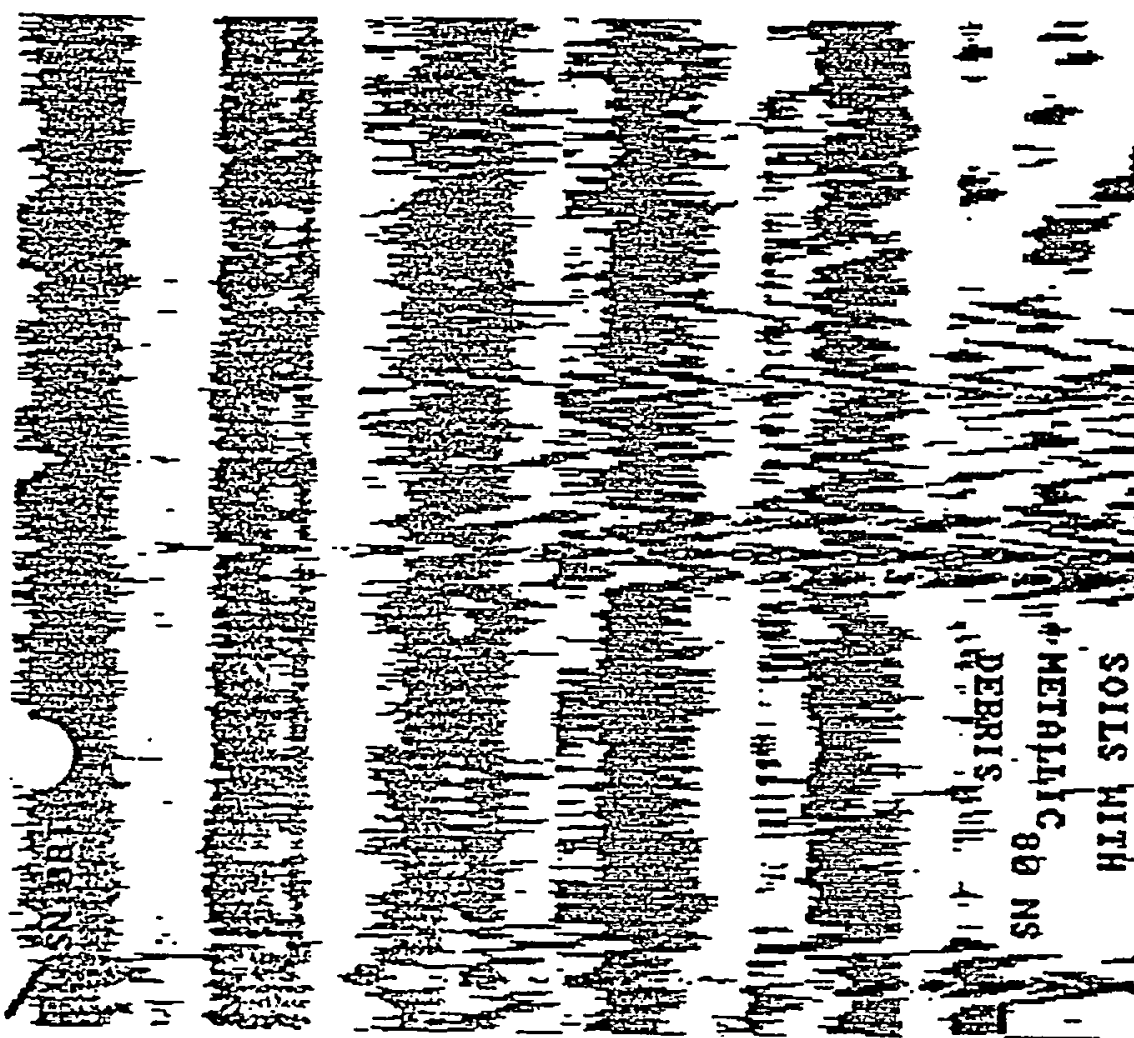

sis

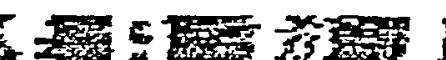

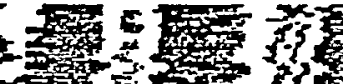
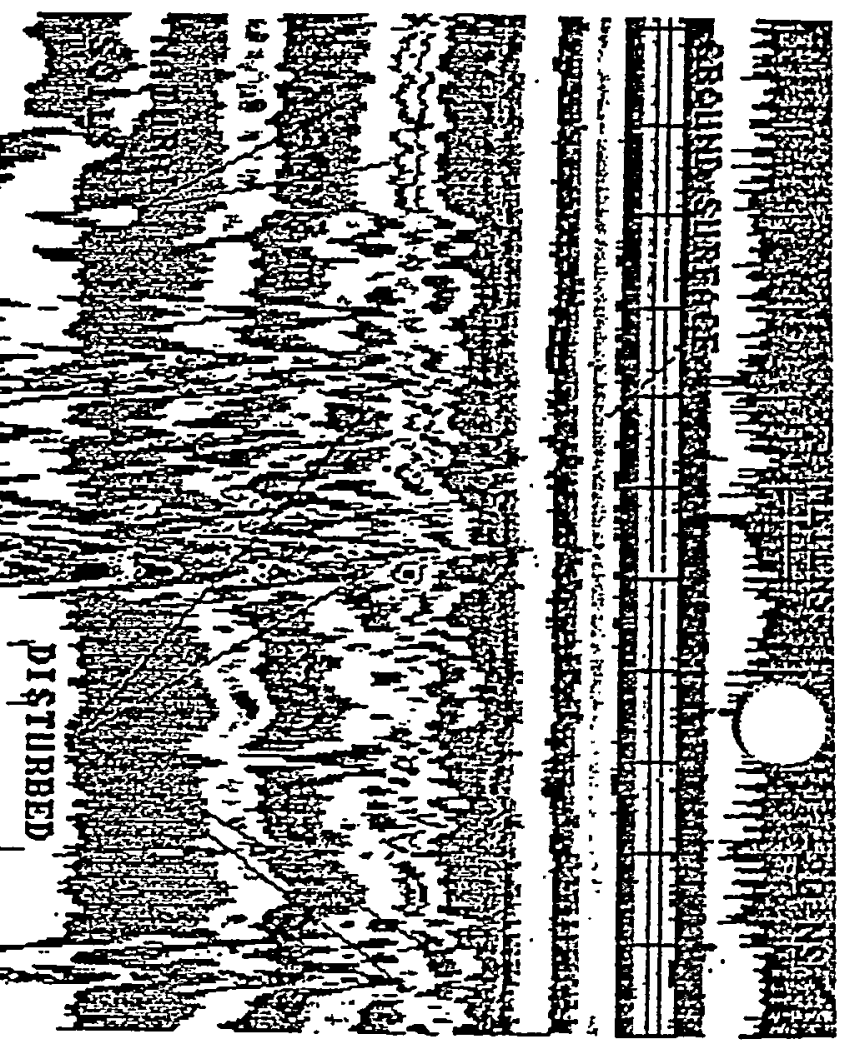
L ᄂtaled Apr $1<, 19 y 4$ 08:59 Modzrzed Apr 12,1994 $15: 16$

512 samples/scan 49.984 scans/sec position: -20 ns range: 200 ns

Vert. IIR IOW pass $\mathrm{N}=2 \mathrm{~F}=40$

Vert. IIR high pass $\mathrm{N}=2 \mathrm{~F}=5$

GRACE ROAD WASTE SITE

Figure 4

IVANNAE RIVER SITE
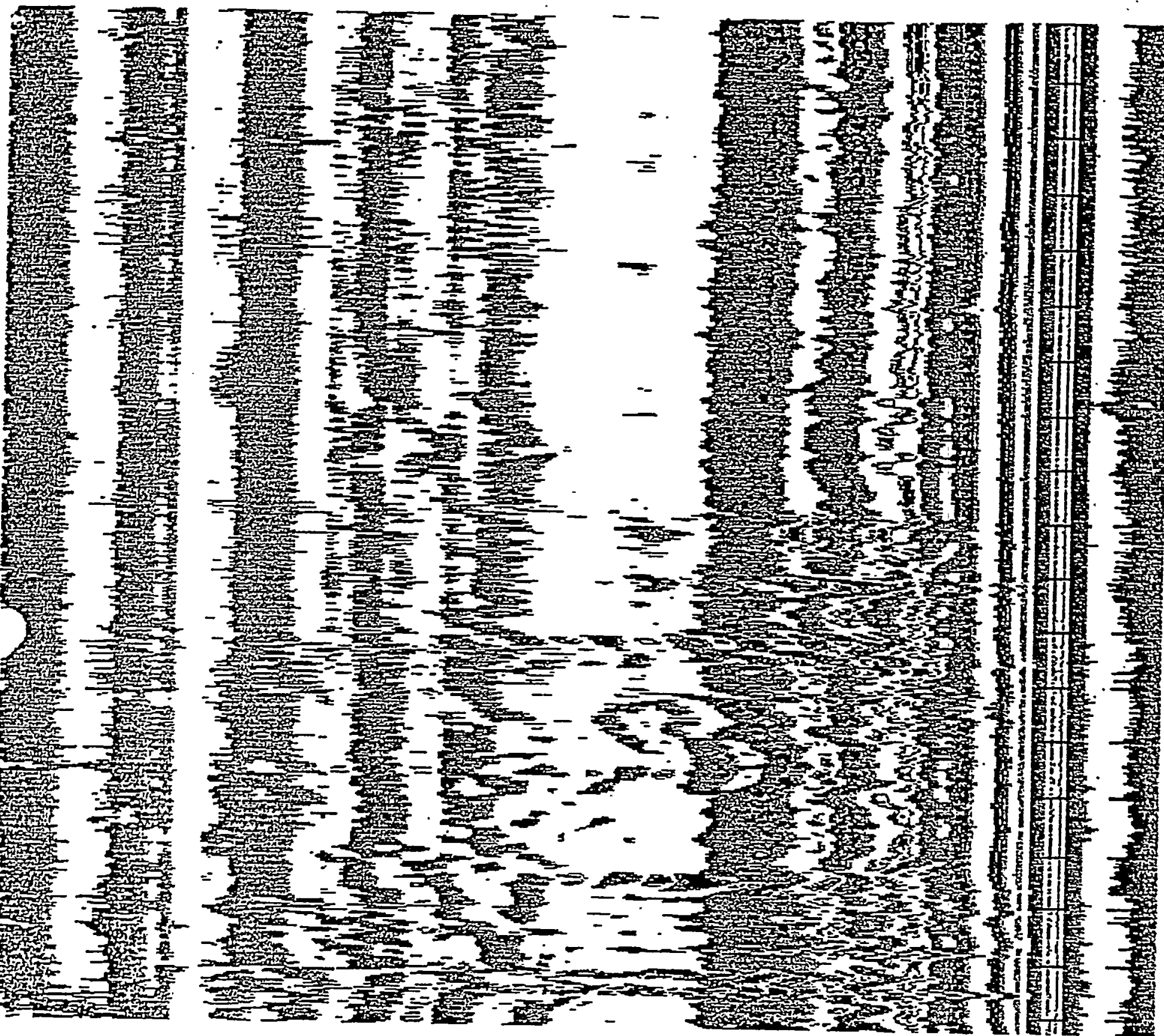
((6)

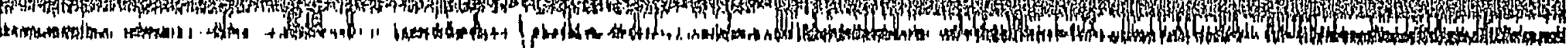

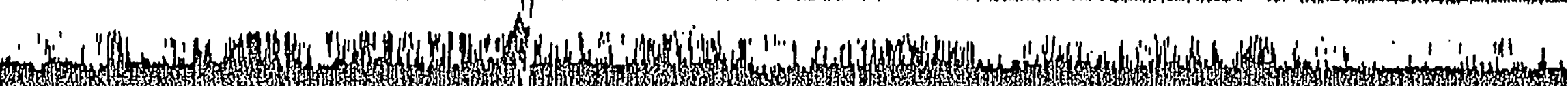

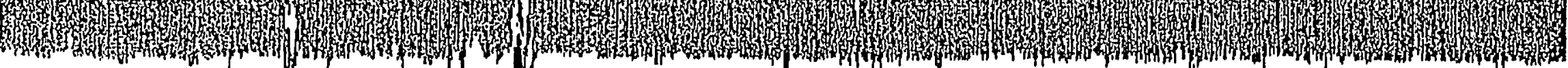
"le "li

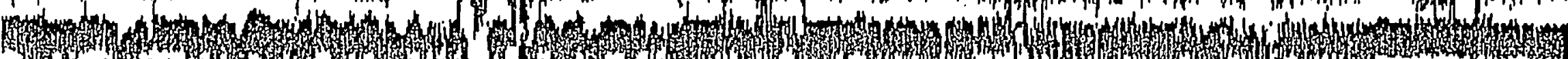

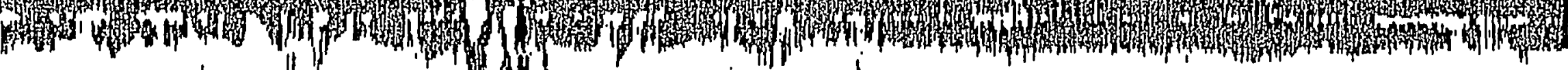

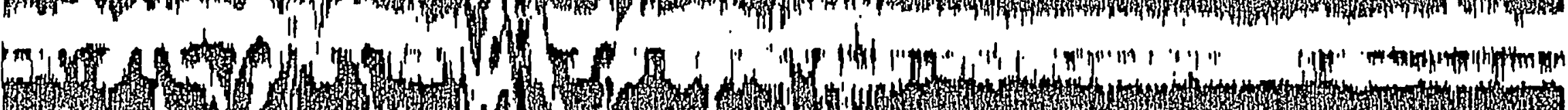

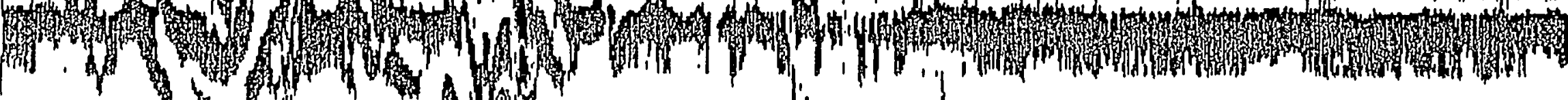

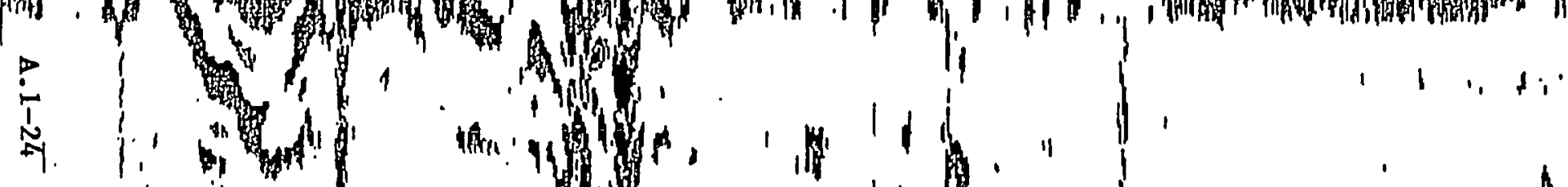

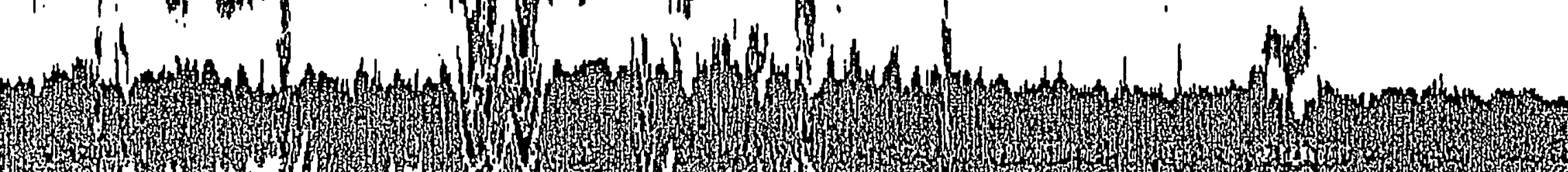

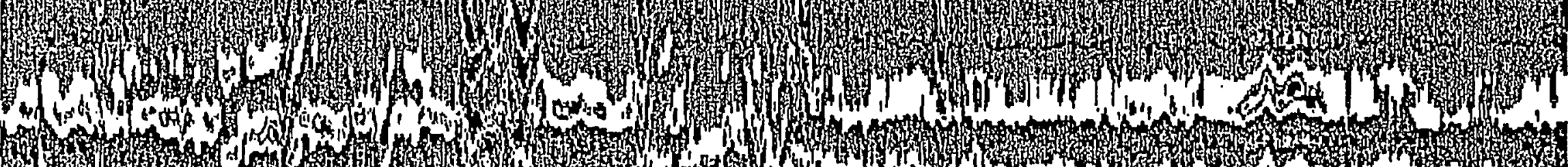
60.

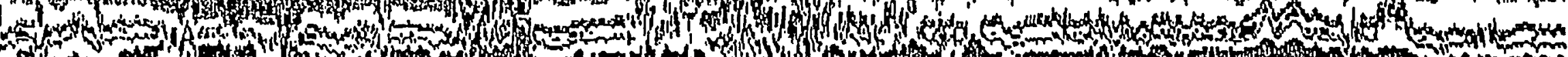

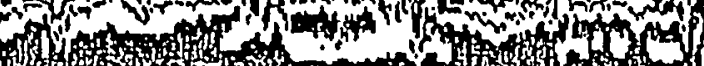
W6 -

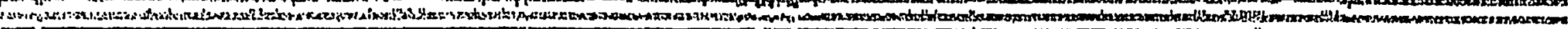
Q -

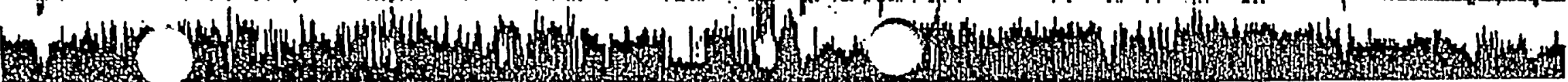

为 11

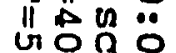

咢

点

量各

血

'员

on

雚驾

品

占茴

8

is

ณ

!

N

응

品 


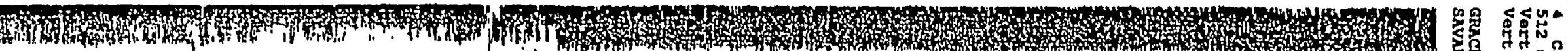

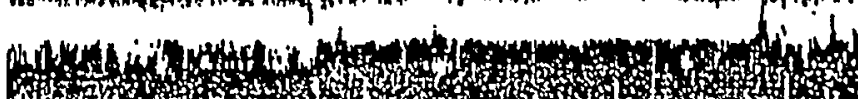

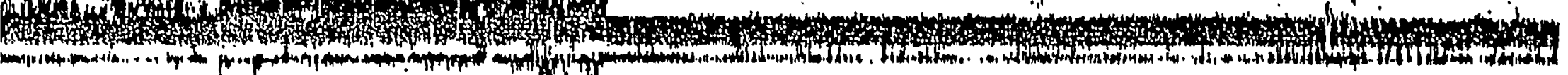

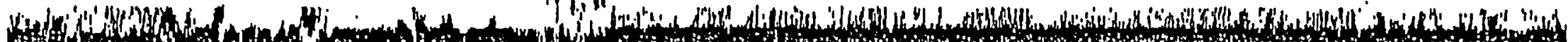

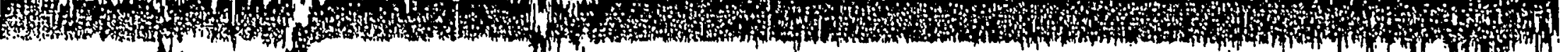

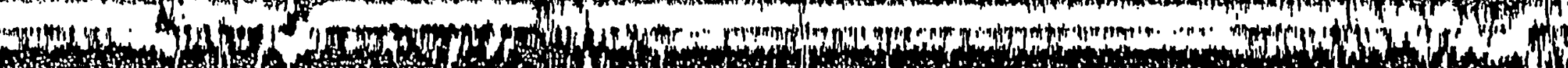
(W)

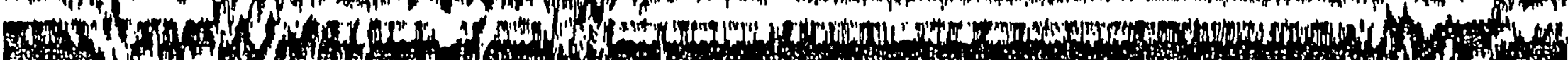
(1) Iifi

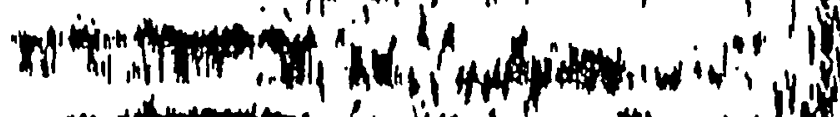
8)

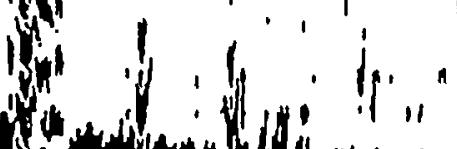
W.

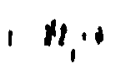
(n) 


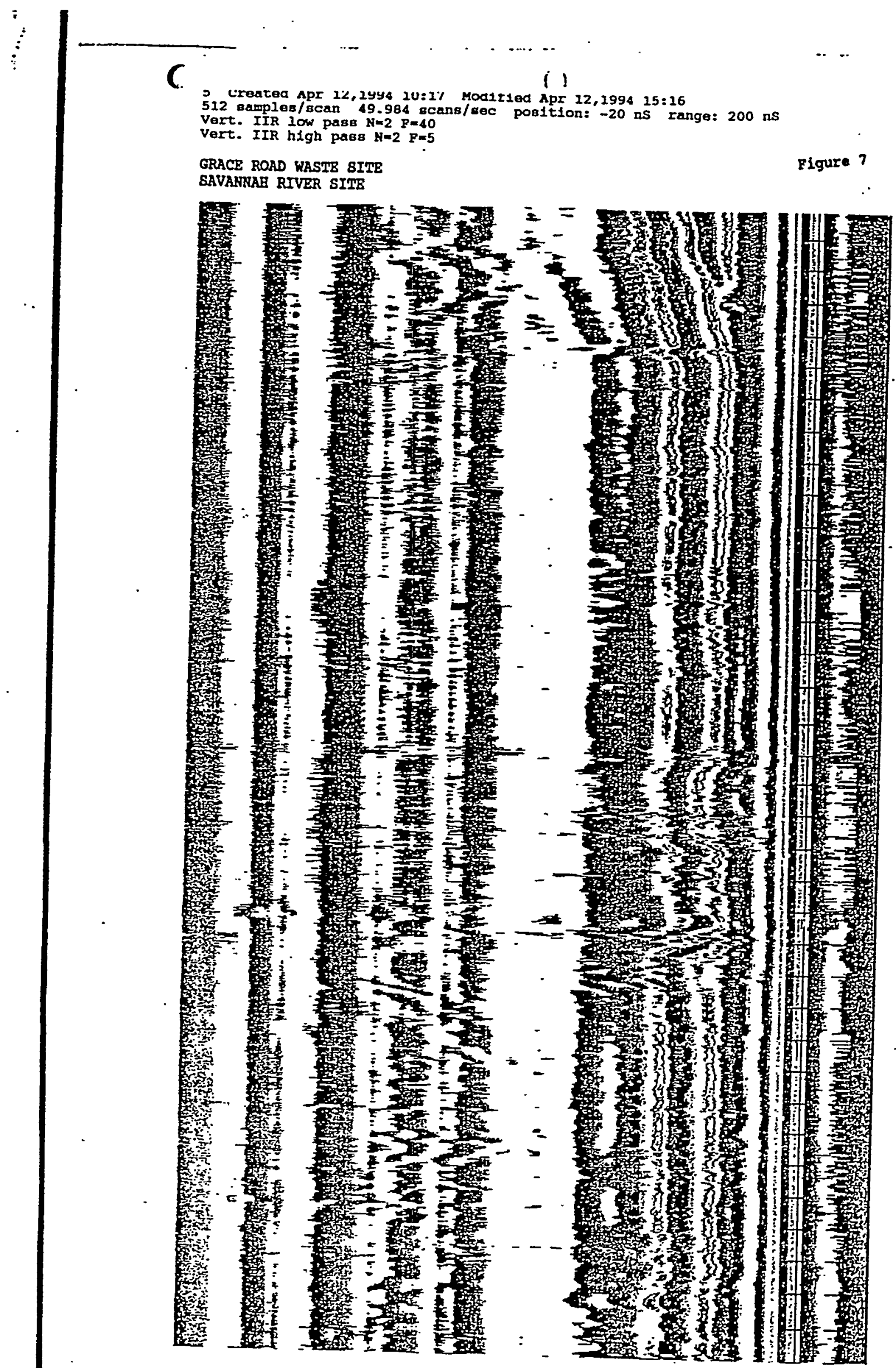


6 Created Apr 12,1994 10:29 Modified Apr 12,1994 15:17

512 samples/scan 49.984 scans/sec position: -20 ns range: 200 ns

Vert. IIR low pass $\mathrm{N}=2 \mathrm{~F}=40$

Vert. IIR high pass $N=2 \quad F=5$

FRACE ROAD WASTE SITE

Figure 8

IVANNAB RIVER SITE
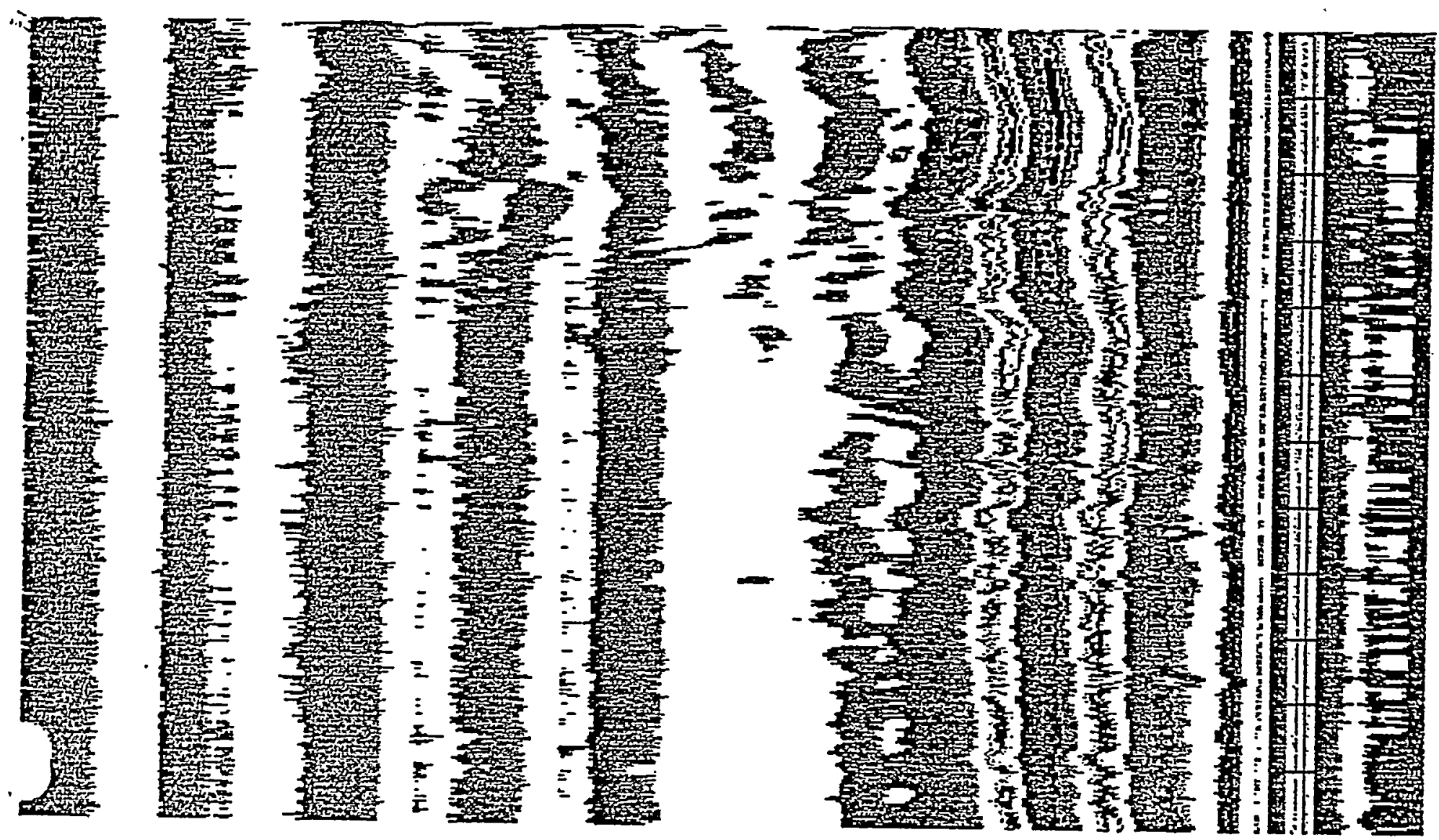
7 Created Apr 12,1994 10:32 Modified Apr 12,1994 15:17

512 samples/scan 49.984 scans/sec position: -20 ns range: 200 ns Vert. IIR low pass $\mathrm{N}=2 \mathrm{~F}=40$

Vert. IIR high pass $\mathrm{N}=2 \mathrm{~F}=5$

GRACE ROAD WASTE SITE

Figure 9

IVANNAB RIVER SITE
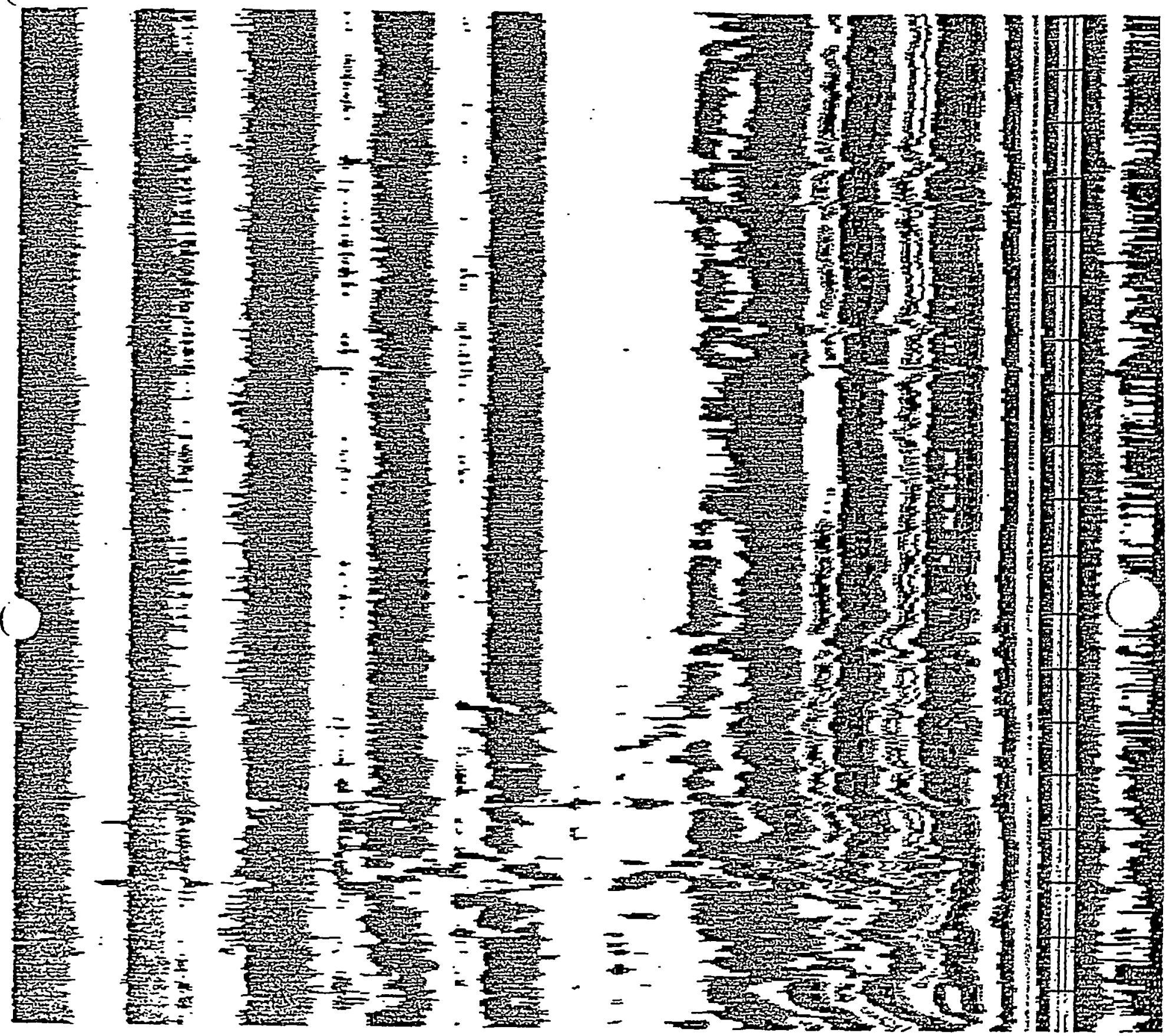
8 Created Apr 12,1994 10:38 Modified Apr 12,1994 15:17

512 samples/scan 49.984 scans/sec position: -20 ns range: 200 nS

Vert. IIR low pass $N=2 \quad F=40$

Vert. IIR high pass $N=2 \quad F=5$

नRACE ROAD WASTE SITE

Figure 10 VANNAB RIVER SITE

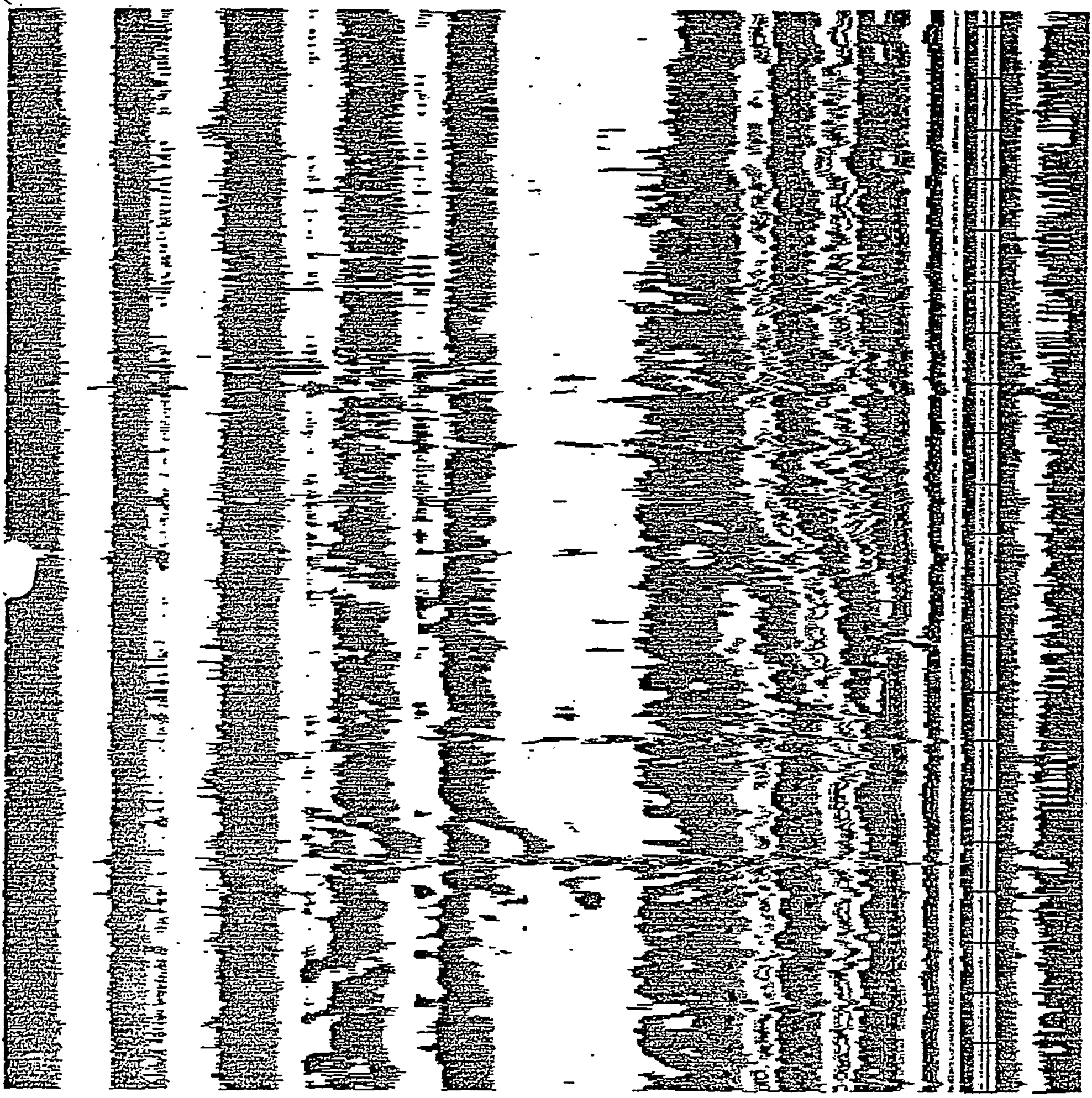


9 Created Apr 12,1994 10:47 Modified Apr 12,1994 15:17

512 samples/scan 49.984 scans/sec position: -20 ns range: $200 \mathrm{~ns}$ Vert. IIR low pass $\mathrm{N}=2 \mathrm{~F}=\mathbf{4 0}$

Vert. IIR high pass $\mathrm{N}=2 \mathrm{~F}=5$

GRACE ROAD WASTE SITE

Figure 11

VANNAB RIVER SITE
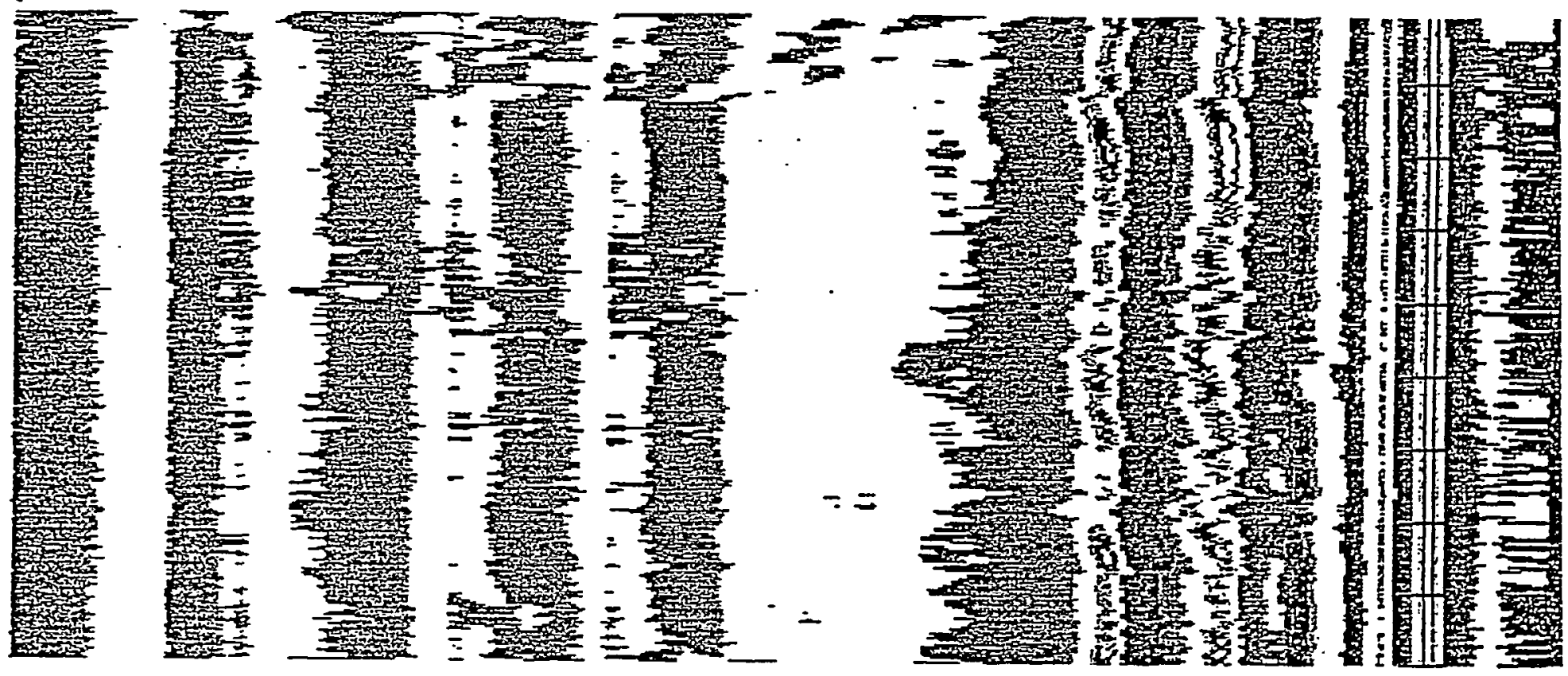
10 Created Apr 12,1994 10:50 Modified Apr 12,1994 15:17

512 samples/scan 49.984 scans/sec position: -20 ns range: 200 ns

Vert. IIR low pass $\mathrm{N}=2 \mathrm{~F}=40$

Vert. IIR high pass $\mathrm{N}=2 \mathrm{~F}=5$

GRACE ROAD WASTE SITE

Figure

12

VANNAE RIVER SITE
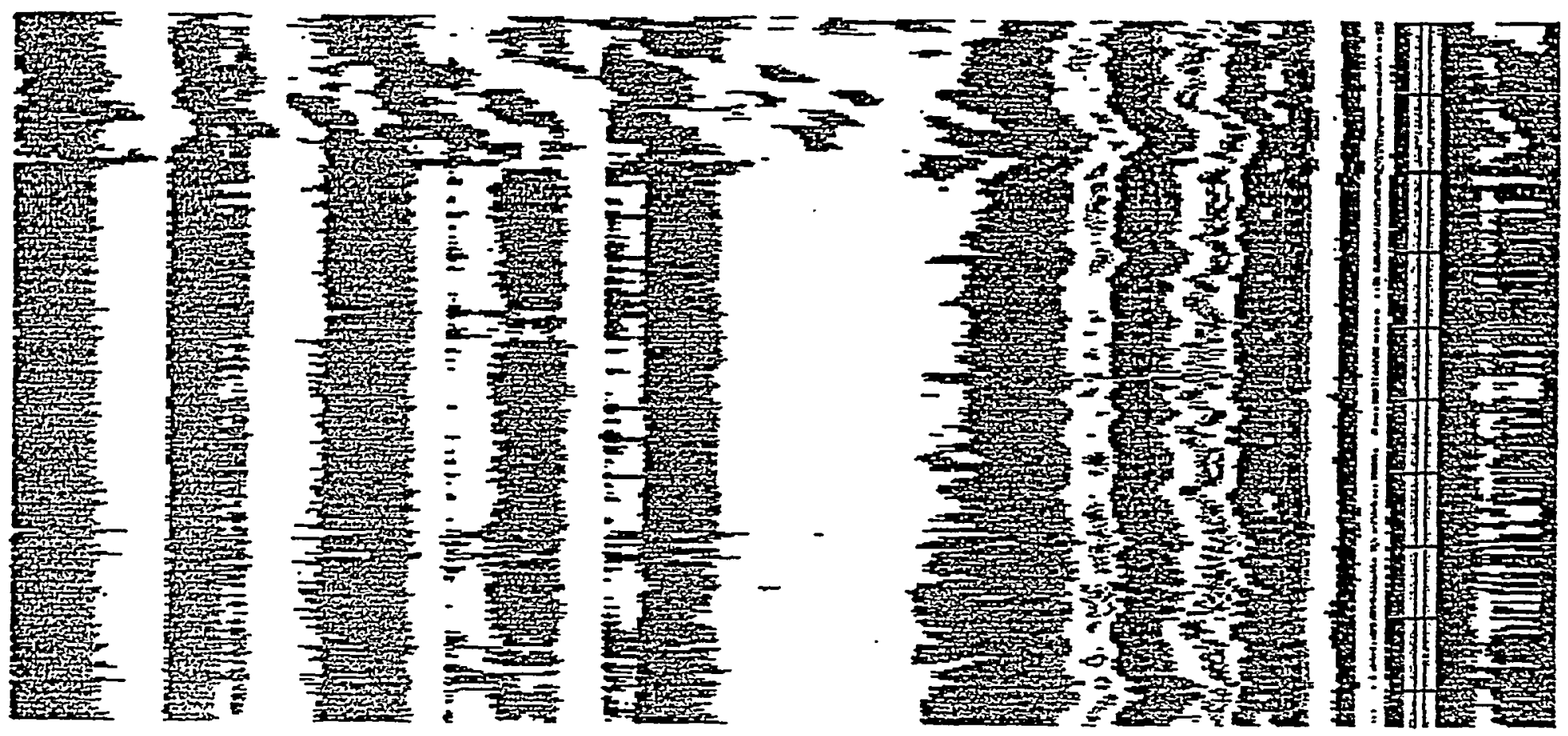
11 Created Apr 12,1994 10:52 Modified Apr 12,1994 15:18

512 samples/scan 49.984 scans/sec position: -20 ns range: $200 \mathrm{~ns}$

Vert. IIR Iow pass $\mathrm{N}=2 \mathrm{~F}=40$

Vert. IIR high pass $N=2, F=5$

GRACE ROAD WASTE SITE

Figure 1 $+1$
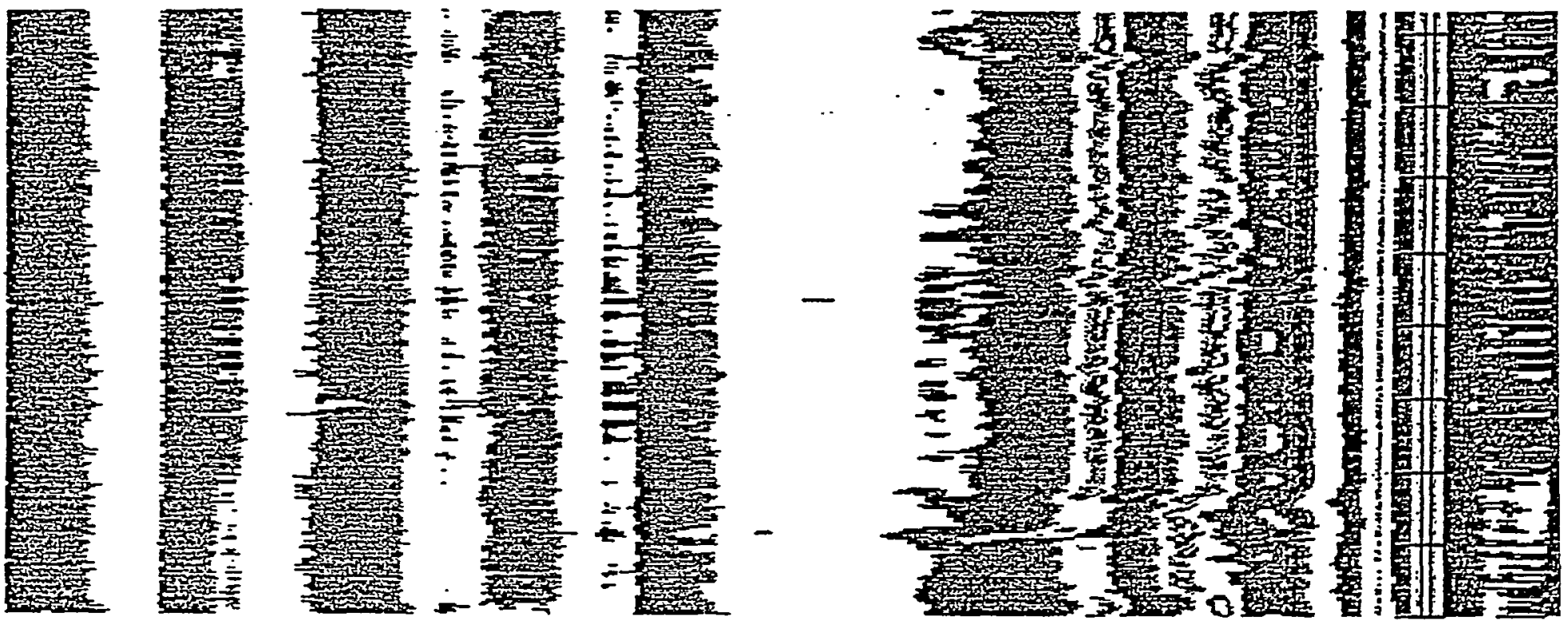

13 Created Apr 12,1994 10:57 Modified Apr 12,1994 15:18

512 samples/scan 49.984 scans/sec position: -20 ns range: 200 ns Vert. IIR low pass $\mathrm{N}=2 \quad \mathrm{~F}=40$

Vert. IIR high pass $\mathrm{N}=2 \mathrm{~F}=5$

GRACE ROAD WASTE SITE

Figure 1

\section{(. VANNAE RIVER SITE}
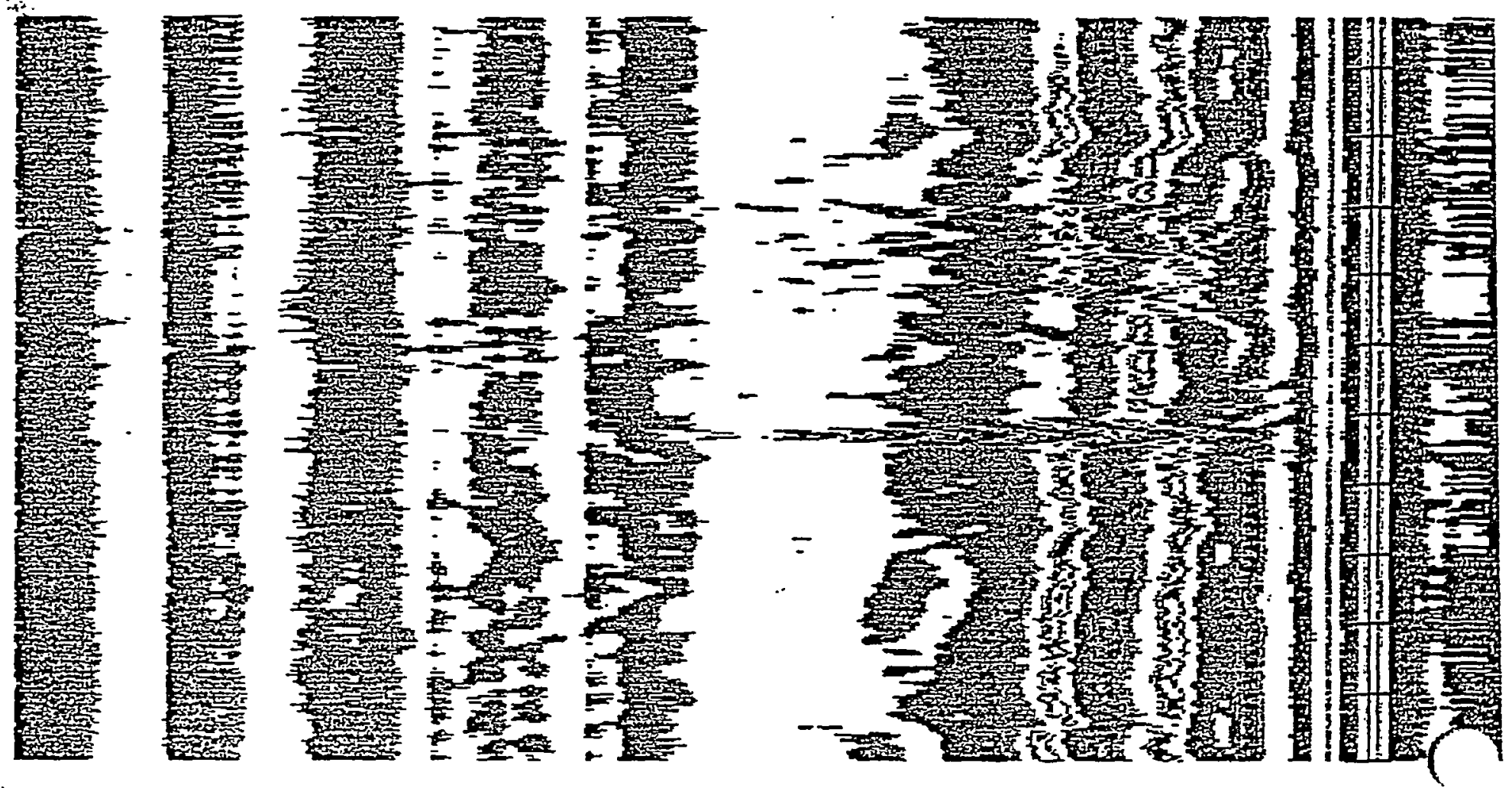
14 Created Apr 12,1994 10:59 Modified Apr 12,1994 15:18

512 samples/scan 49.984 scans/sec position: -20 is range: 200 is Vert. IIR low pass $\mathrm{N}=2 \quad \mathrm{~F}=40$

Vert. IIR high pass $N=2, F=5$

GRACE ROAD WASTE SITE

Figure 1.

- VANNAB RIVER SITE

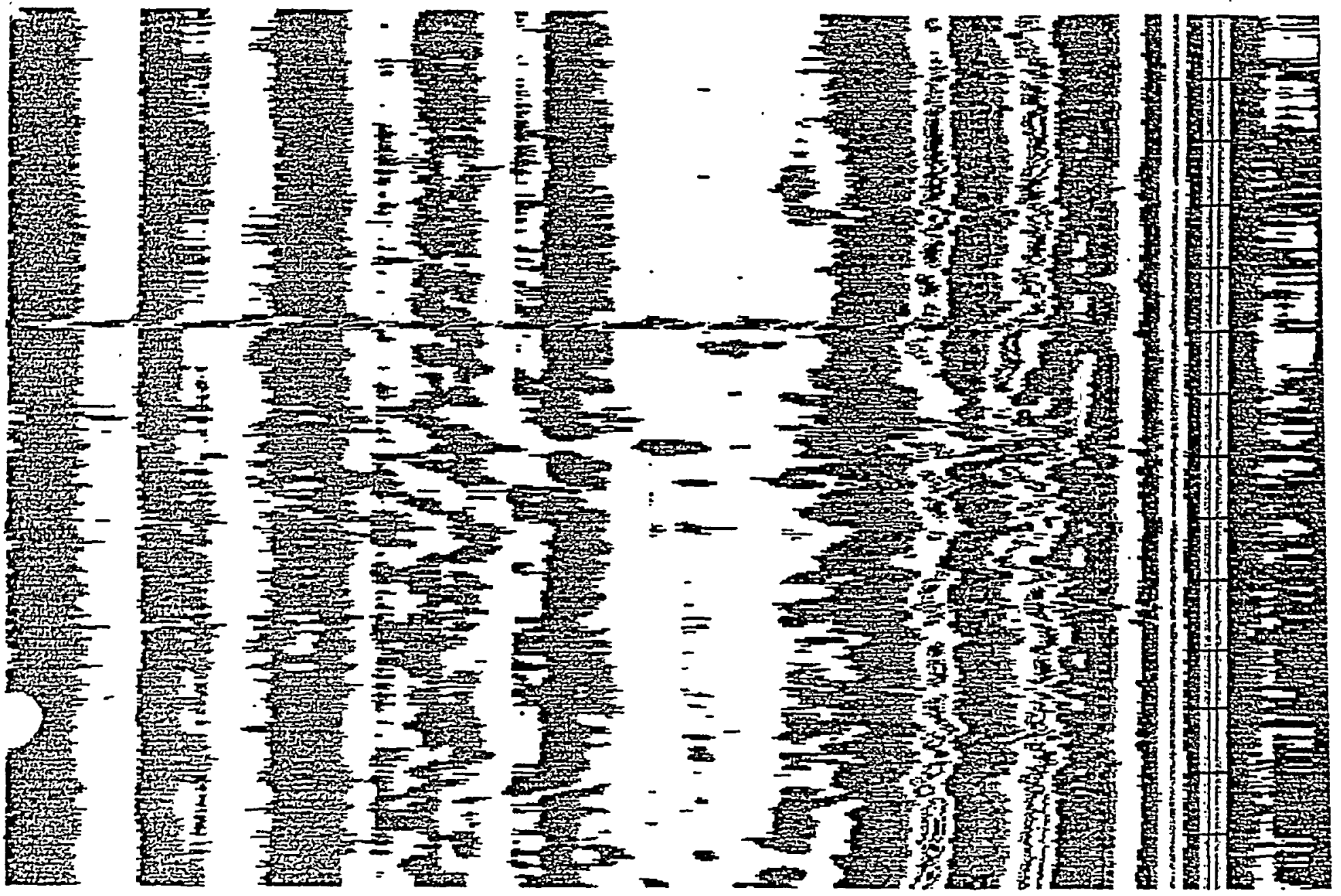


15 Created Apr 12,1994 11:02 Modified Apr 12,1994 15:18

512 samples/scan 49.984 scans/sec position: -20 ns range: 200 ns Vert. IIR low pass $\mathrm{N}=2 \mathrm{~F}=40$

Vert. IIR high pass $\mathrm{N}=2 \mathrm{~F}=5$

GRACE ROAD WASTE SITE

AVANNAB RIVER SITE
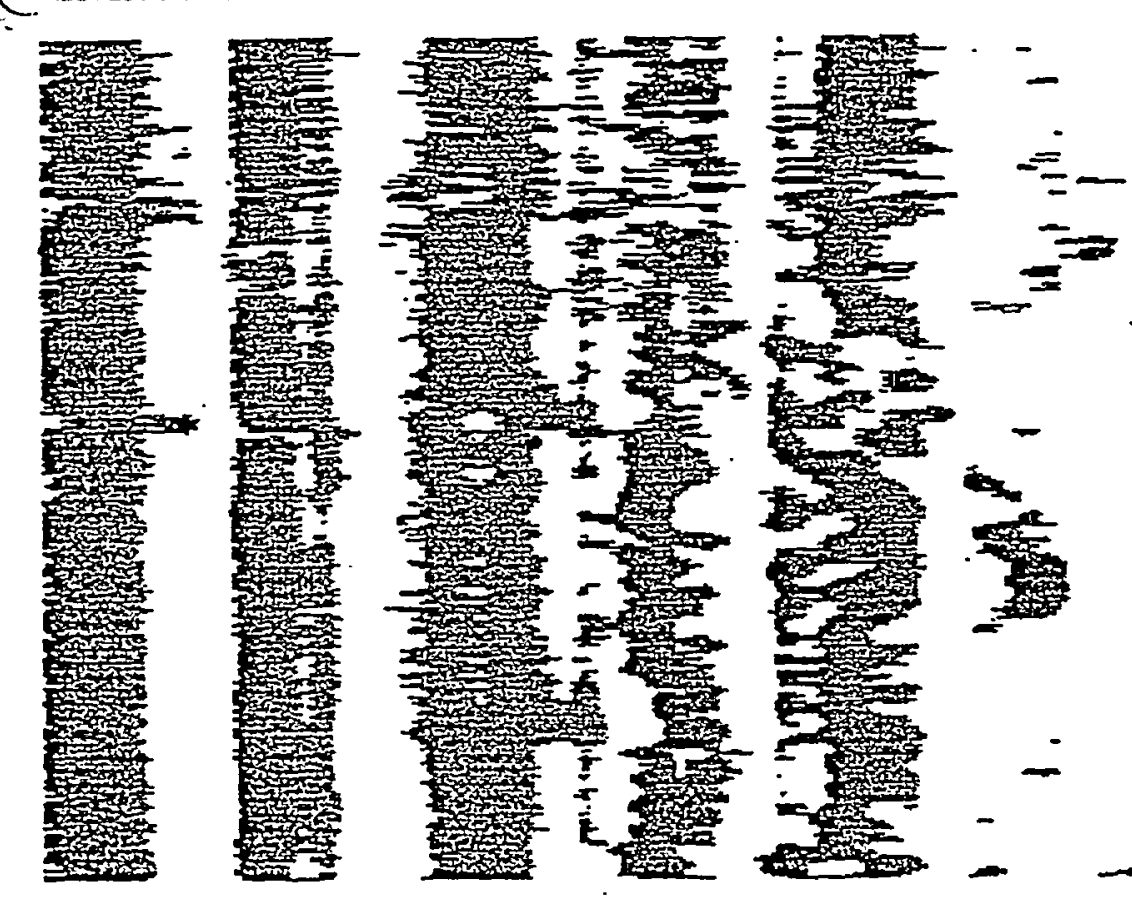
-
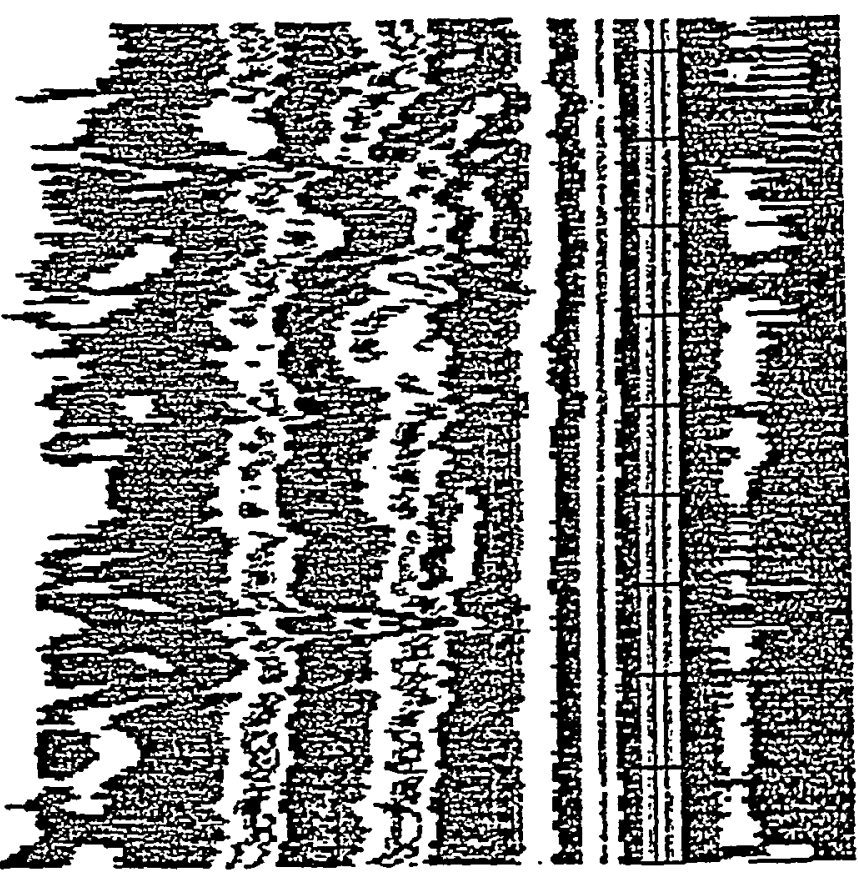
16 Created Apr 12,1994 11:04 Modified Apr 12,1994 15:18

512 samples/scan 49.984 scans/sec position: -20 ns range: 200 ns

Vert. IIR low pass $\mathrm{N}=2 \quad \mathrm{~F}=40$

Vert. IIR high pass $\mathrm{N}=2 \mathrm{~F}=5$

GRACE ROAD WASTE SITE

Figure 18

IAVANNAE RIVER SITE

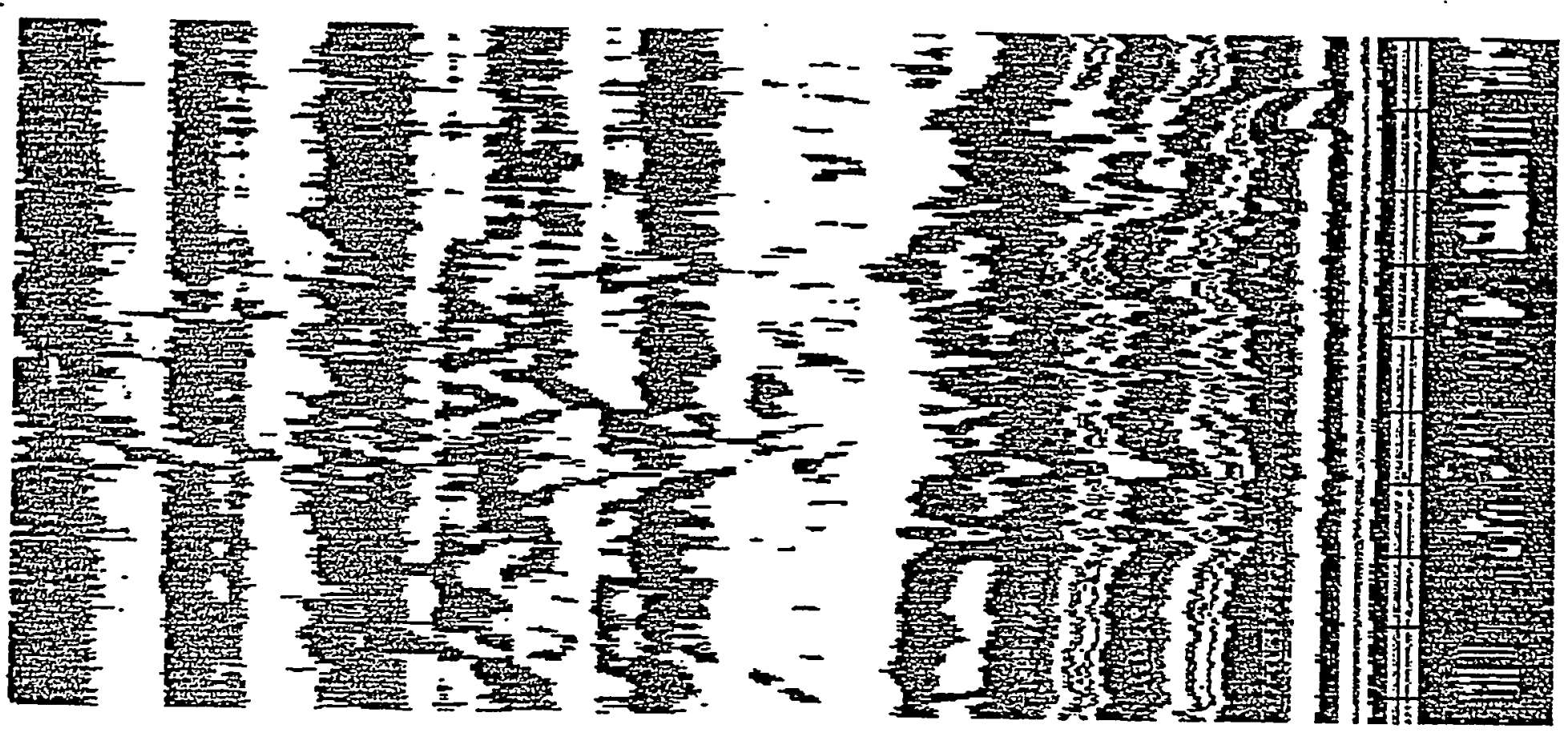


17 Created Apr 12,1994 11:05 Modified Apr 12,1994 15:18

512 samples/scan 49.984 scans/sec position: -20 ns Iange: $200 \mathrm{~ns}$
Vert. IIR low pass $N=2 \mathrm{~F}=40$

vert. IIR high pass $N=2 \quad F=5$

\section{GRACE ROAD WASTE SITE}

JAVANNAE RIVER SITE
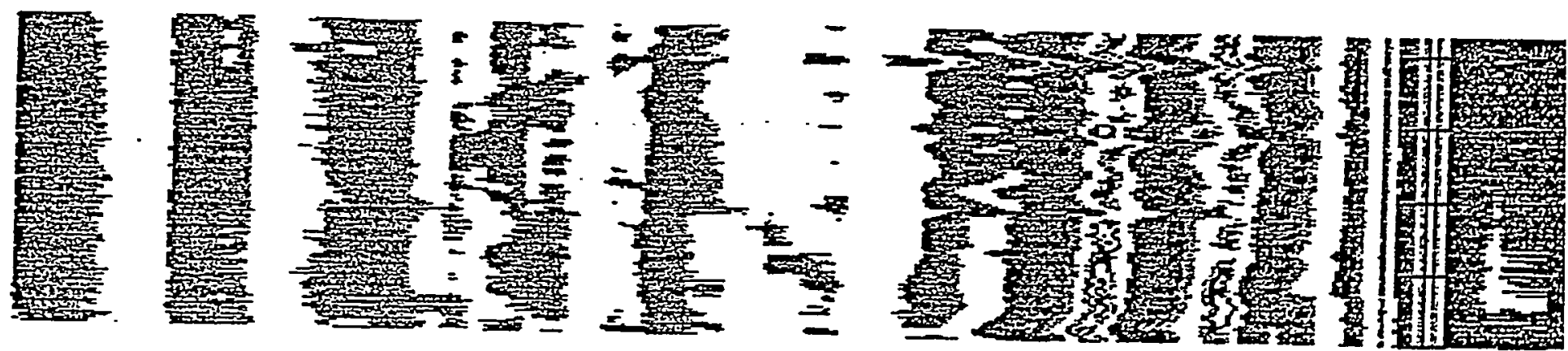

Figure् 
18 Created Apr 12,1994 11:07 Modified Apr 12,1994 15:18

512 samples/scan 49.984 scans/sec position: -20 ns range: $200 \mathrm{~ns}$
Vert. IIR low pass $\mathrm{N}=2 \mathrm{~F}=40$

Vert. IIR high pass $\mathrm{N}=2 \mathrm{~F}=5$.

TRACE ROAD WASTE SITE

की AVANNAB RIVER SITE

Figure 20
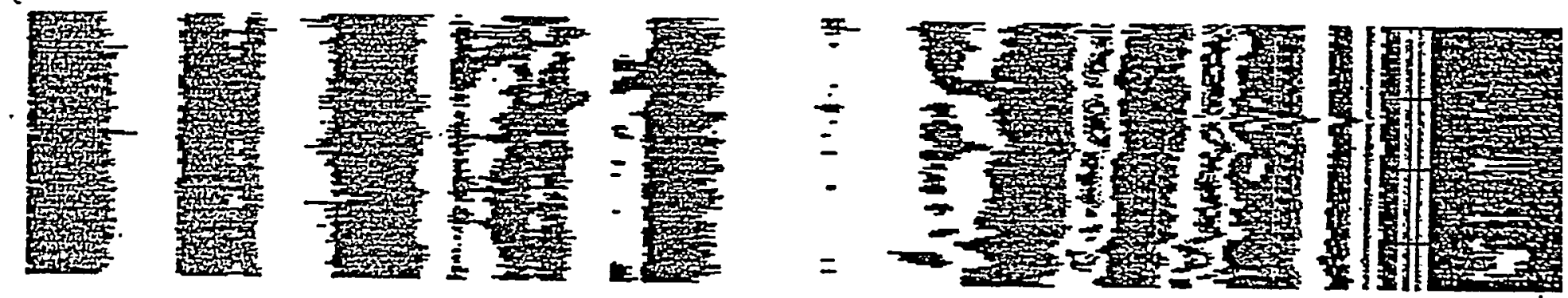
19 Created Apr 12,1994 11:13 Modified Apr 12,1994 15:18

512 samples/scan 49.984 scans/sec position: $-20 \mathrm{~ns}$ range: $200 \mathrm{~ns}$
Vert. IIR low pass $\mathrm{N}=2 \mathrm{~F}=40$

Vert. IIR high pass $\mathrm{N}=2 \quad \mathrm{~F}=5$

TRACE ROAD WASTE SITE

AVANNAH RIVER SITE
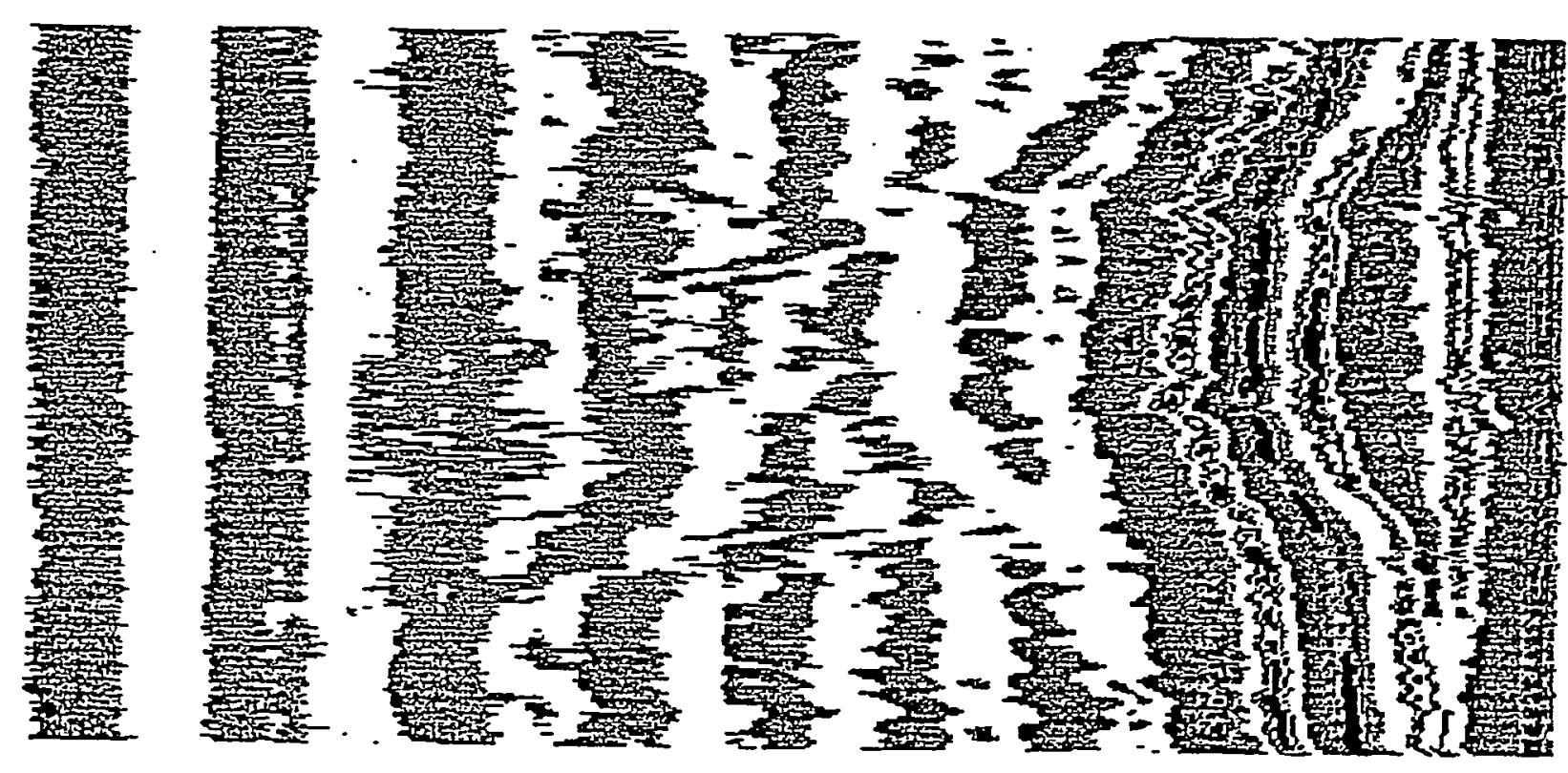

$\equiv=$

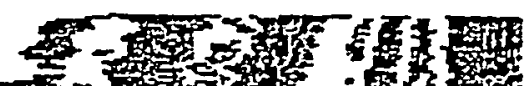

Figure $21^{\text {t. }}$ (

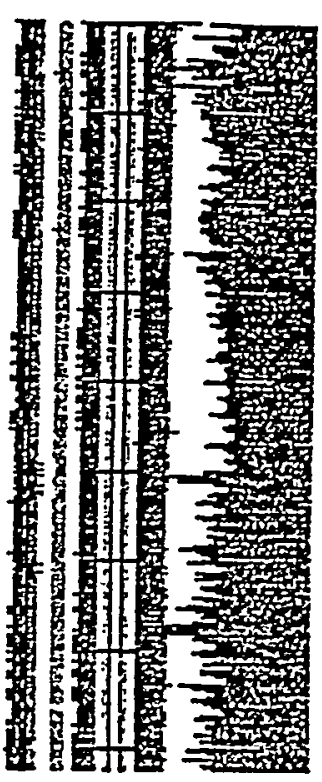


20 Created Apr 12,1994 11:15 Modified Apr 12,1994 15:19

512 samples/scan 49.984 scans/sec position: $-20 \mathrm{nS}$ range: $200 \mathrm{nS}$ Vert. IIR low pass $N=2 \quad F=40$

Vert. IIR high pass $\mathrm{N}=2 \mathrm{~F}=5$

IRACE ROAD WASTE SITE

Figure 22

( IAVANNAE RIVER SITE

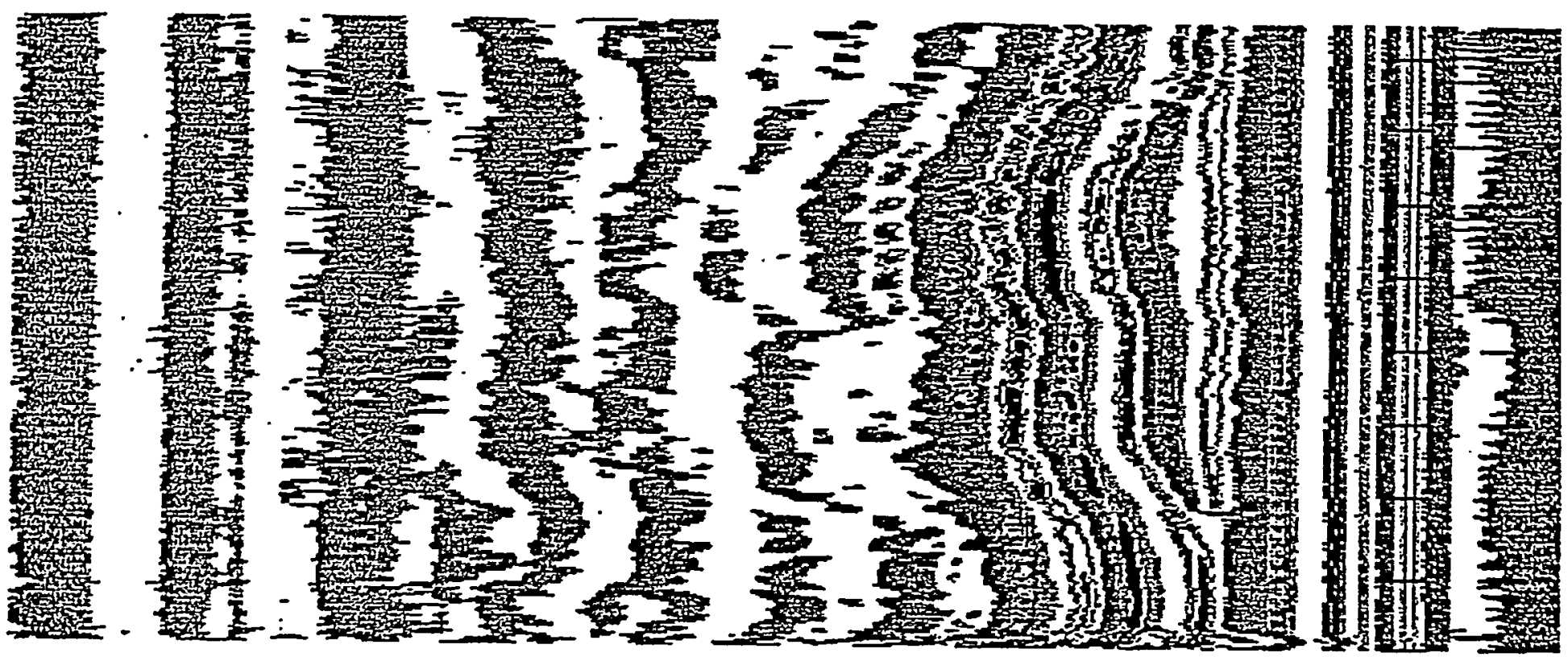


21 Created Apr 12,1994 11:17 Modified Apr 12,1994 15:19.

512 samples/scan 49.984 scans/sec position: -20 ns range: $200 \mathrm{~ns}$

Vert. IIR low pass $N=2 . F=40$

Vert. IIR high pass $\mathrm{N}=2 \quad \mathrm{~F}=5$

GRACE ROAD WASTE SITE

Figure 23

IAVANNAH RIVER SITE
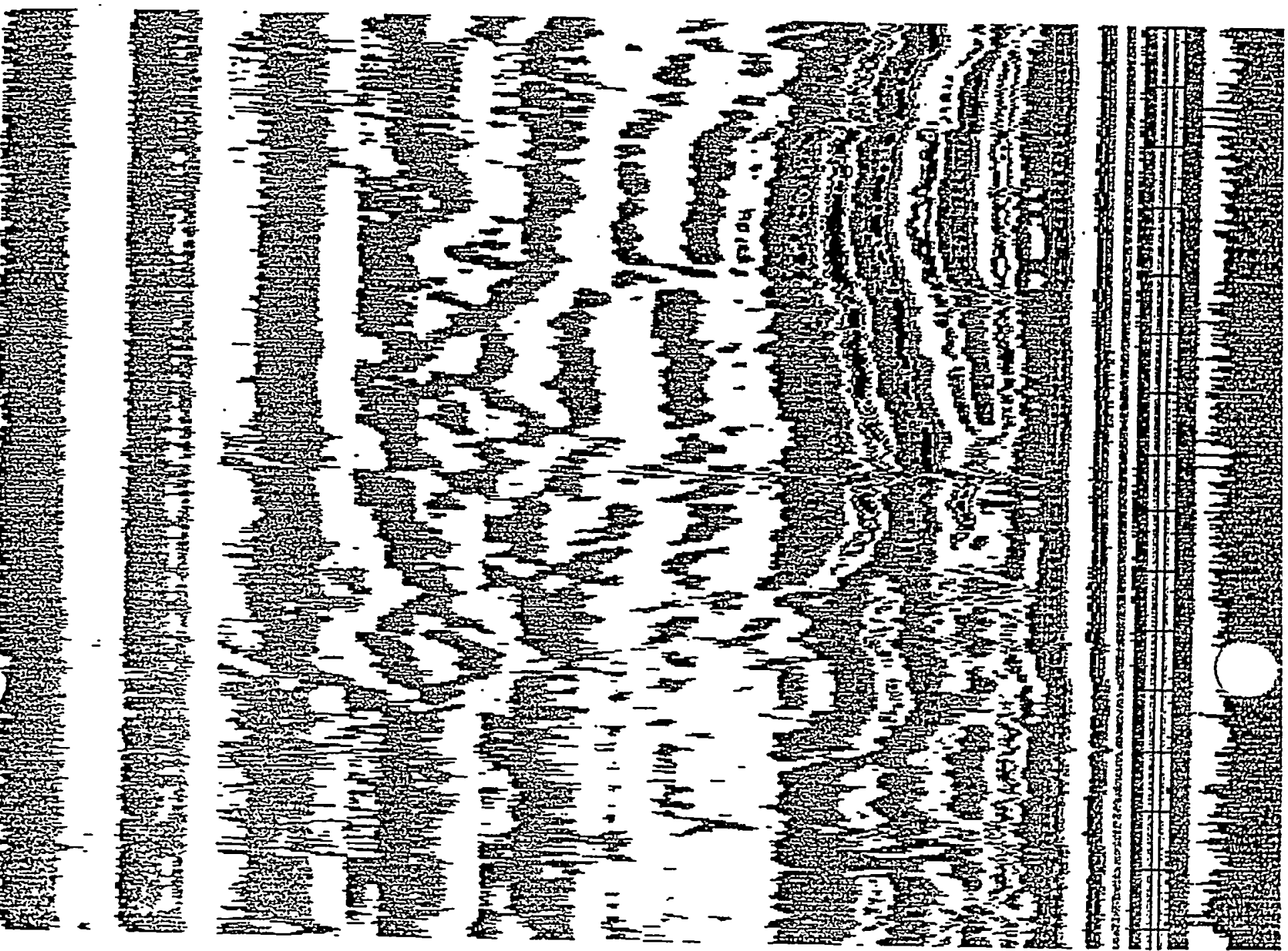
23 Created Apr 12,1994 11:23 Modified Apr 12,1994 15:19

512 samples/scan 49.984 scans/sec position: -20 ns range: $200 \mathrm{nS}$

Vert. IIR high pass $N=2 \quad F=5$

GRACE ROAD WASTE SITE

( AVANNAE RIVER SITE

Figure 25
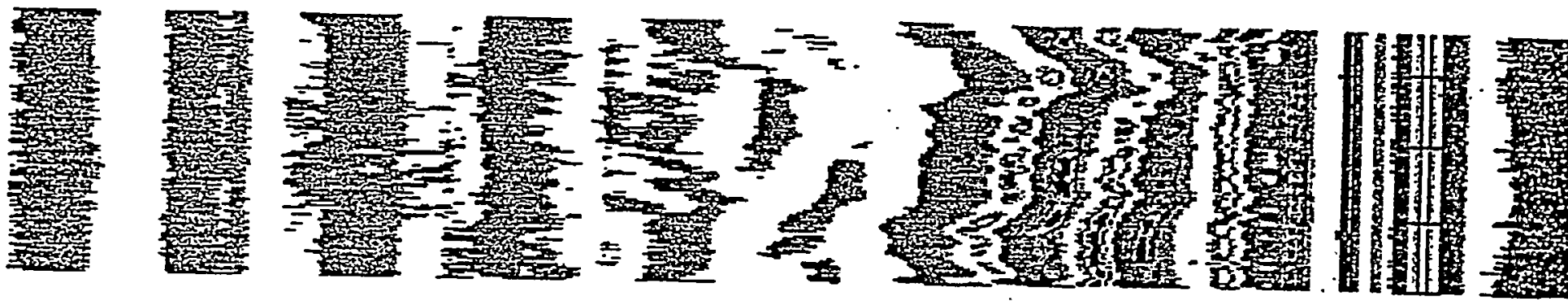
24 Created Apr 12,1994 11:24 Modified Apr 12,1994 15:19. •

512 samples/scan 49.984 scans/sec position: -20 ns range: 200 ns
Vert. IIR low pass $N=2 \quad F=40$

Vert. IIR high pass $\mathrm{N}=2 \quad \mathrm{~F}=5$

GRACE ROAD WASTE SITE

Figure $\$ 2.6$

AVANNAB RIVER SITE

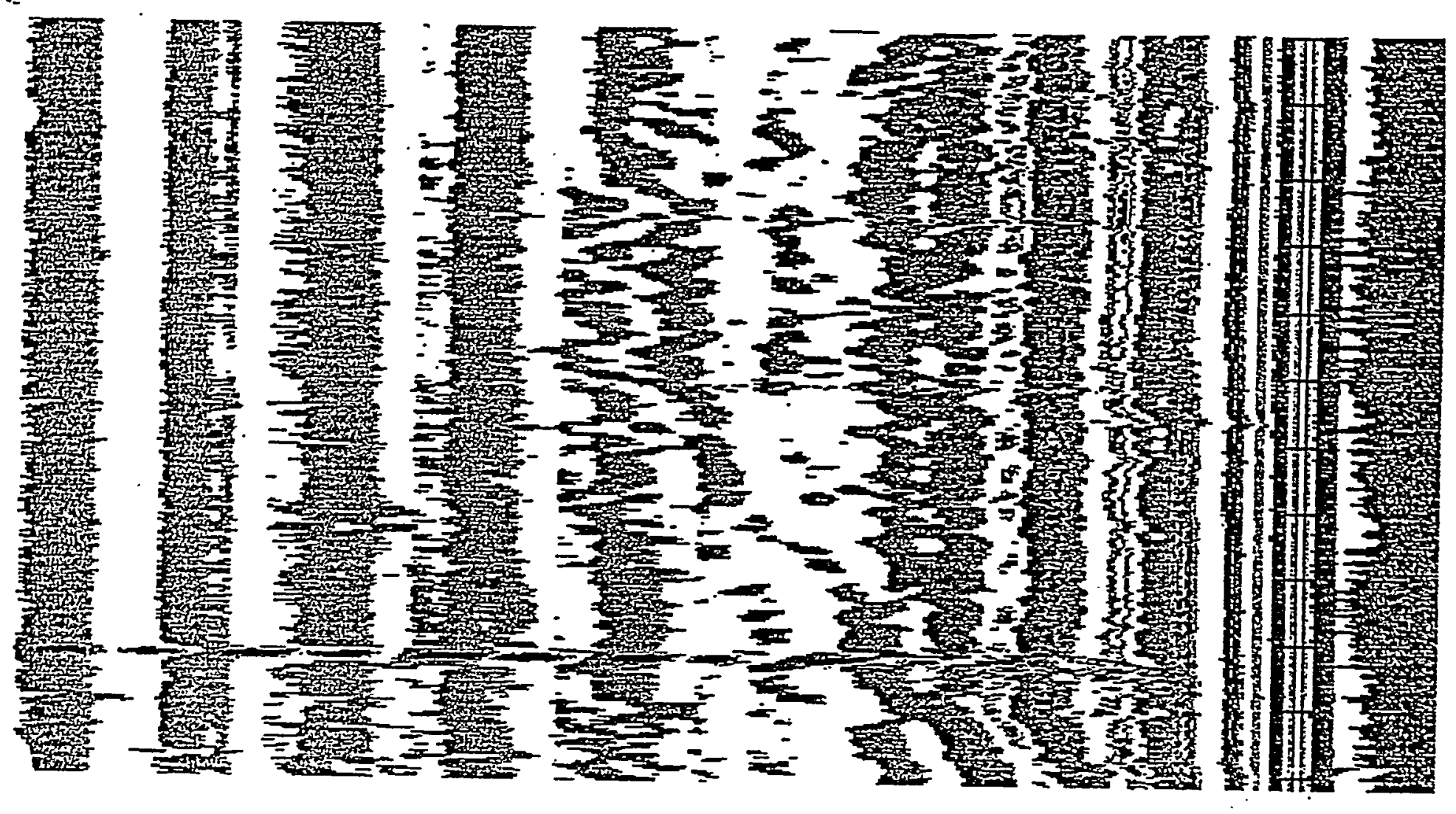


25 Created Apr 12,1994 11:27 Modified Apr 12,1994 15:19

512 samples/scan 49.984 scans/sec position: -20 ns range: 200 nS

Vert. IIR low pass $N=2 \quad F=40$

Vert. IIR high pass $N=2 \quad F=5$

GRACE ROAD WASTE SITE

Figure 27 AVANNAE RIVER SITE
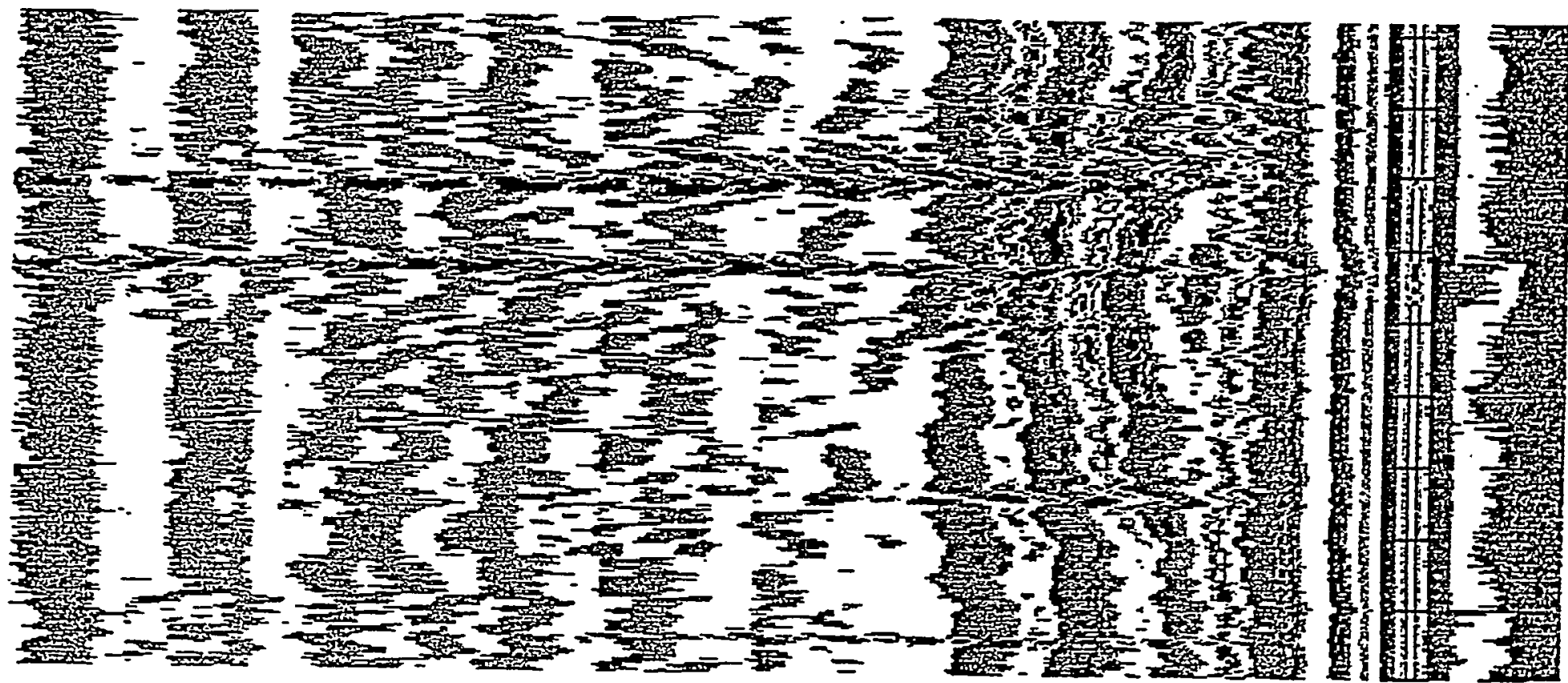


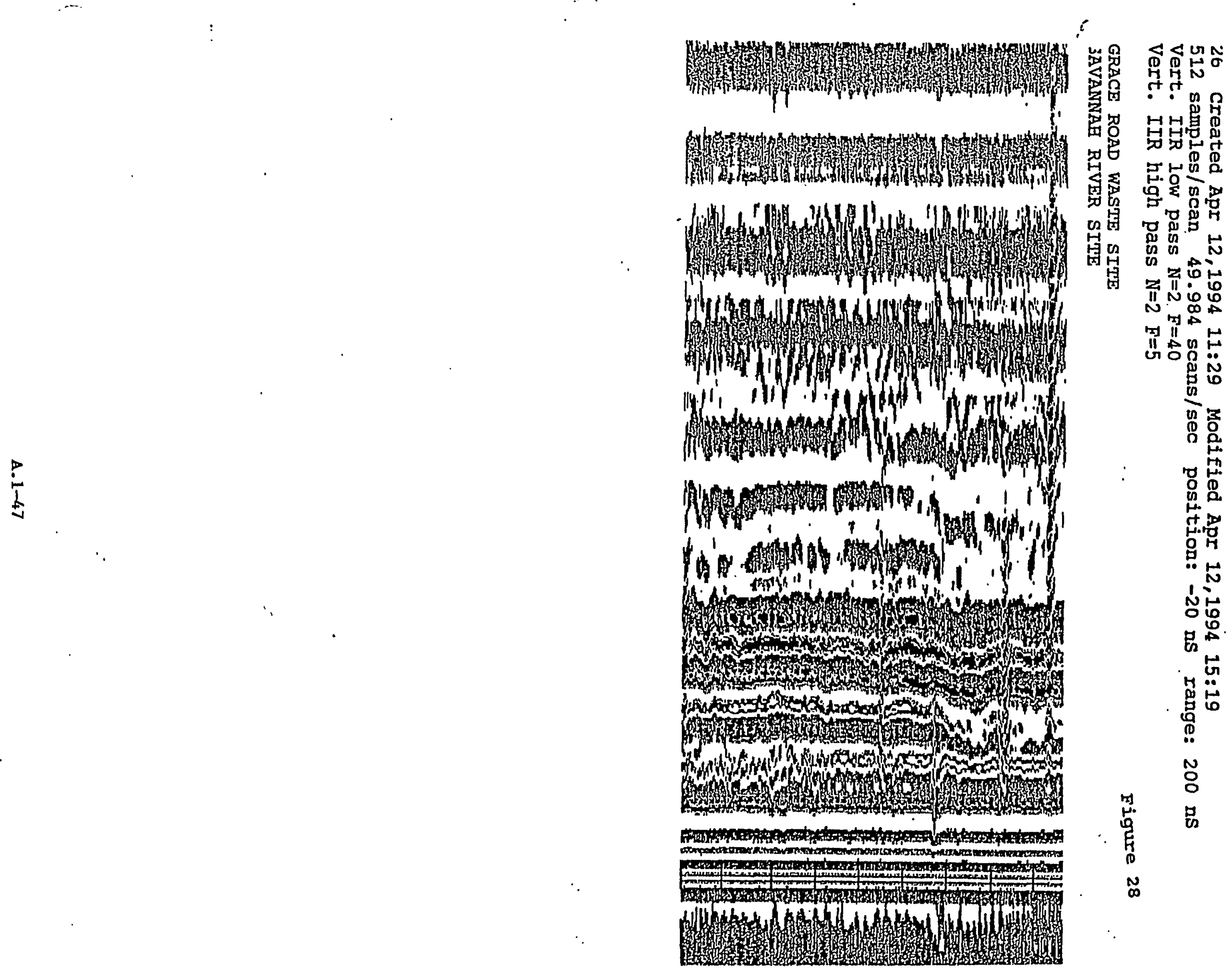


28 Creared Apr 12, 1994 11:35 Modified Apr 12,1994 15:20

512 samples/scan 49.984 scans/sec position: $-20 \mathrm{nS}$ range: $200 \mathrm{nS}$
Vert. IIR low pass $\mathrm{N}=2 \mathrm{~F}=40$

Vert. IIR high pass $N=2 \quad F=5$

GRACE ROAD WASTE SITE

AVANNAB RIVER SITE
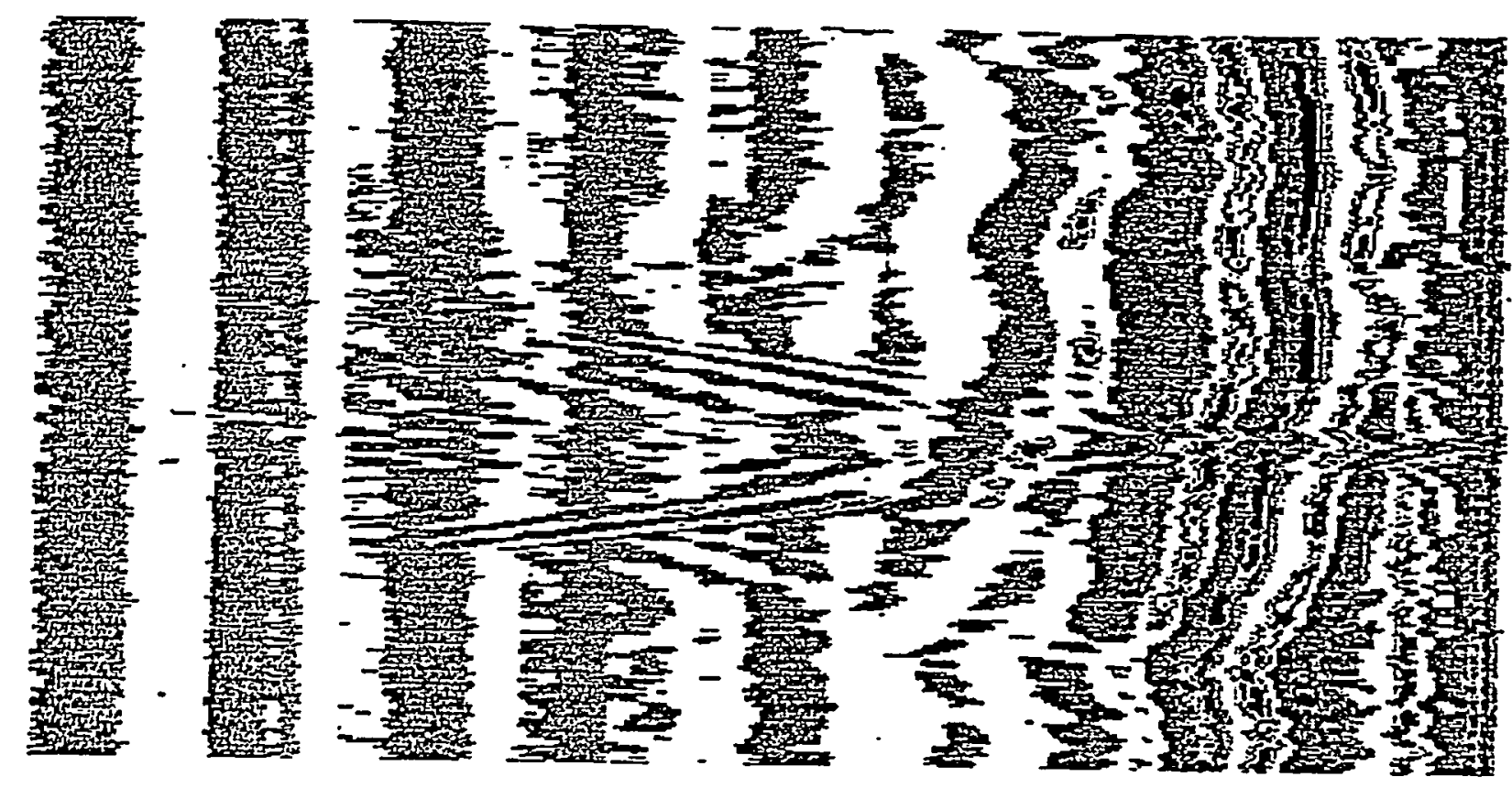

Figure 30

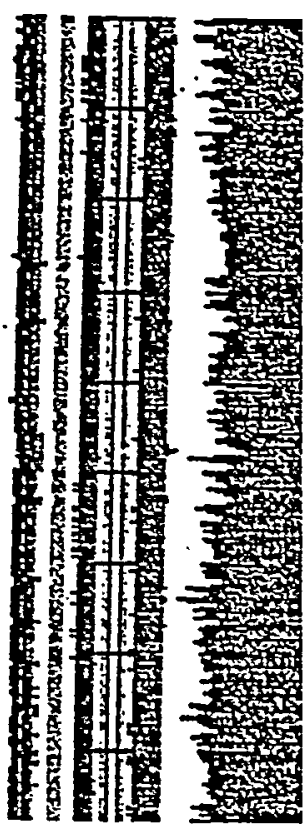




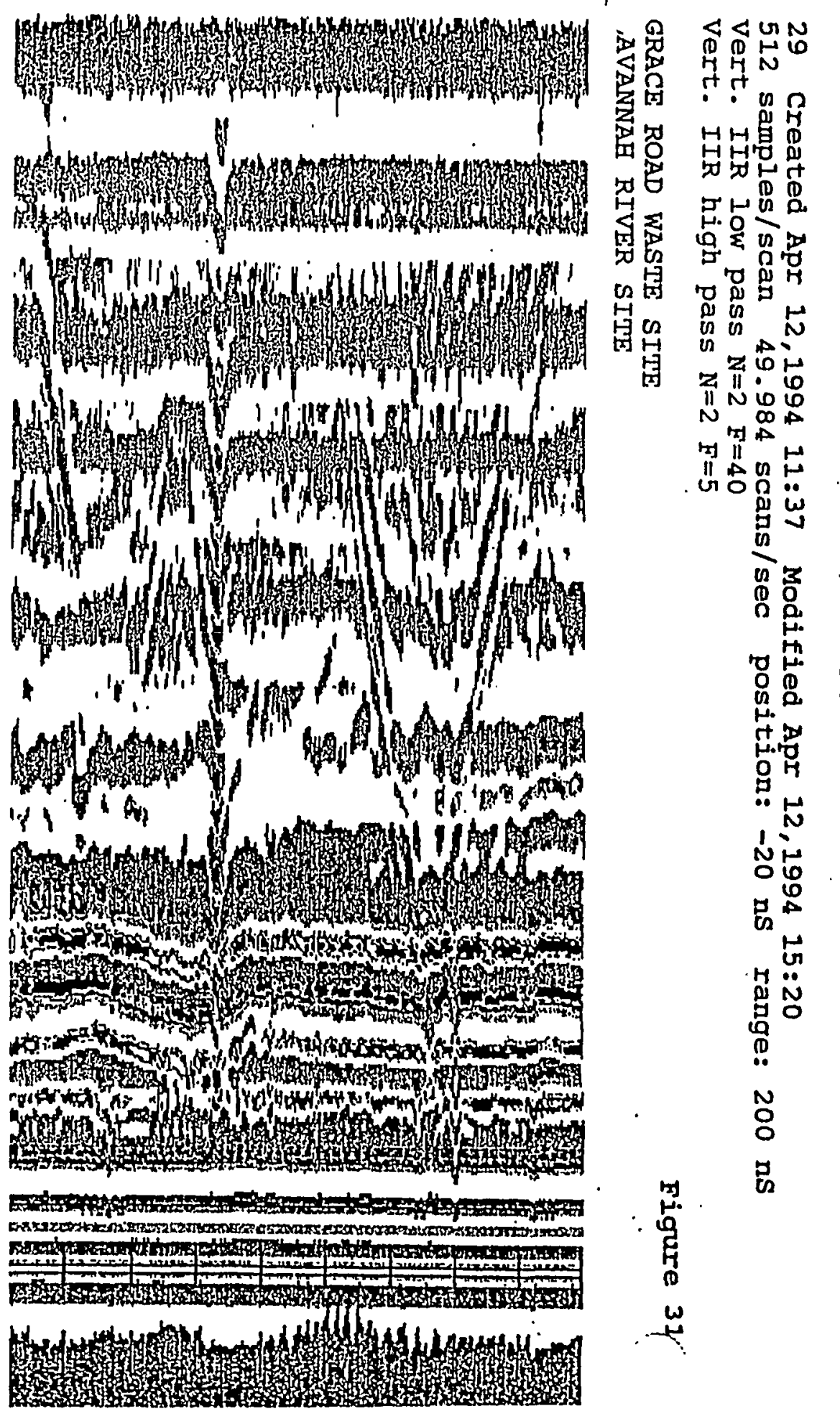


su Created Apr 12,1994 11:39 Modified Apr 12,1994 15:20

512 samples/scan 49.984 scans/sec position: -20 ns range: 200 ns Vert. IIR low pass $\mathrm{N}=2 \mathrm{~F}=40$

Vert. IIR high pass $\mathrm{N}=2 \mathrm{~F}=5$

GRACE ROAD WASTE SITE

Eigure 32

IAVANNAH RIVER SITE
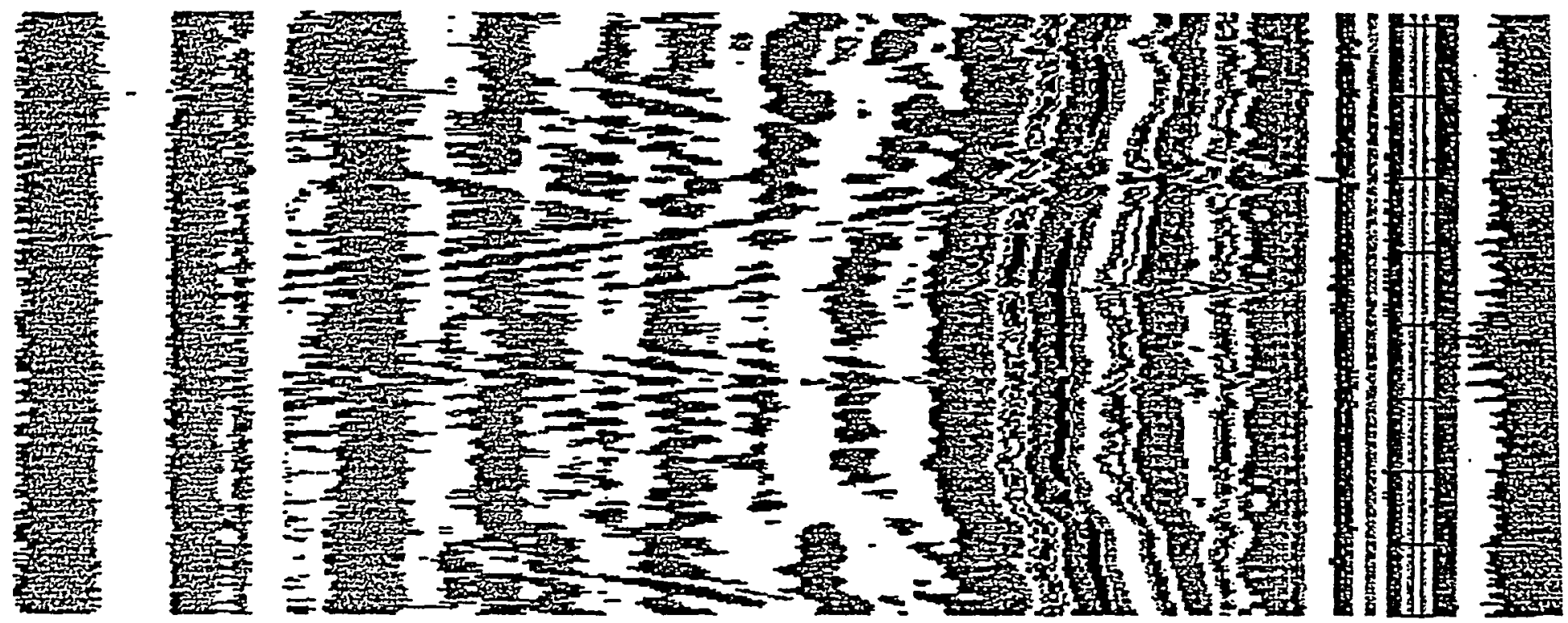
I1 Created Apr 12,1994 11:40 Modified Apr 12,1994 15:20

512 samples/scan 49.984 scans/sec position: $-20 \mathrm{~ns}$ range: $200 \mathrm{nS}$
Vert. IIR low pass $\mathrm{N}=2 \mathrm{~F}=40$

Vert. IIR high pass $N=2 \quad F=5$

GRACE ROAD WASTE SITE

IAVANNAE RIVER SITE

Figure (

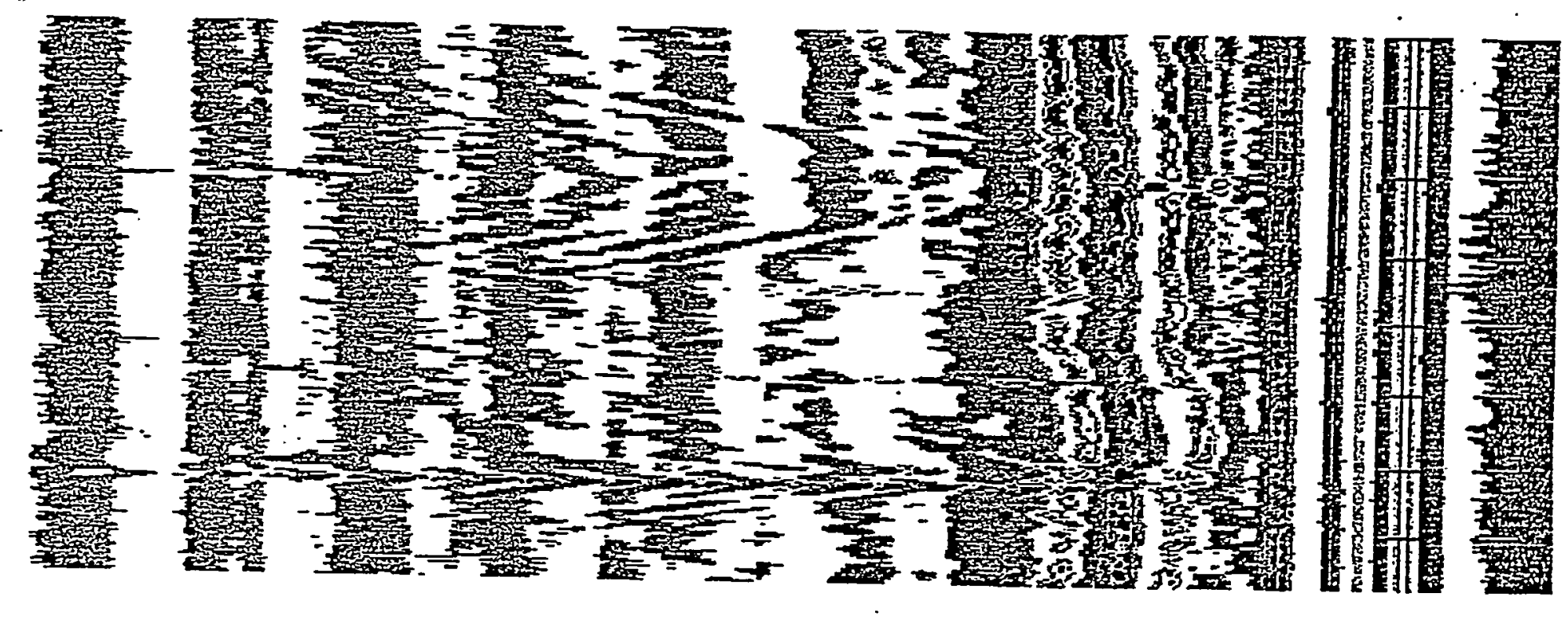



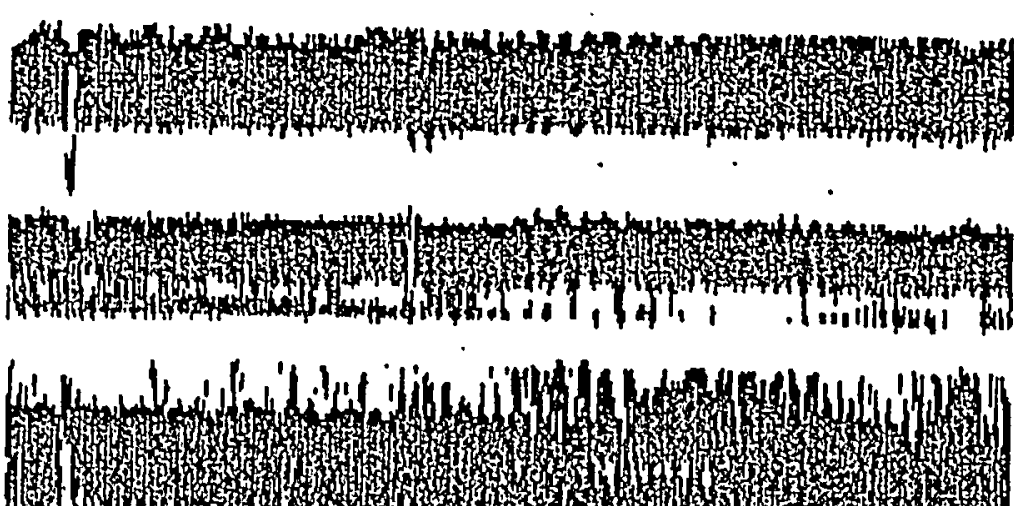

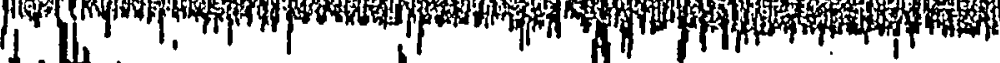

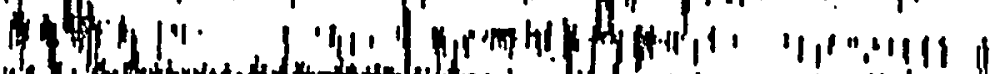

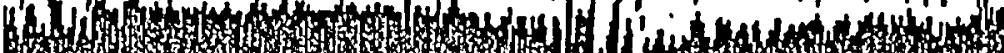

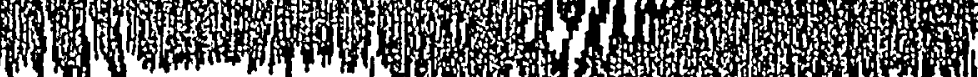
on

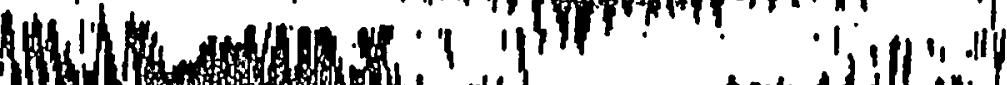
$13+y^{2}$

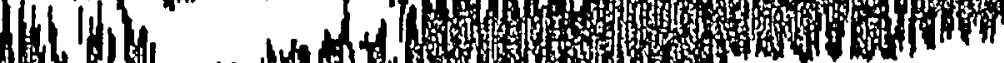
Histis

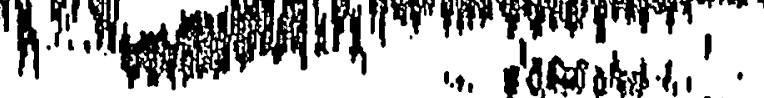
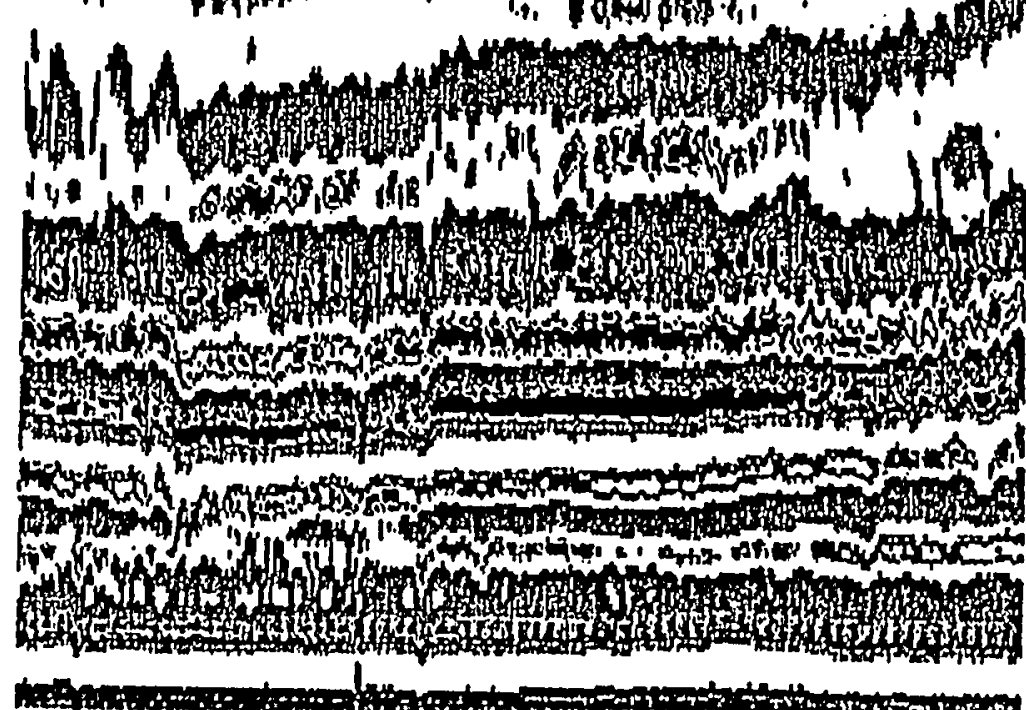

moxpe -

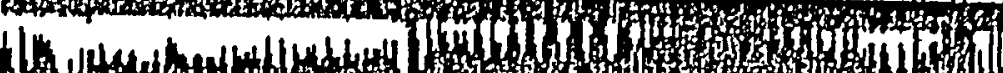

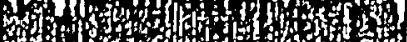

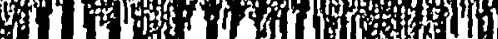

骂

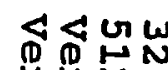

H

- $\infty$

붑붕

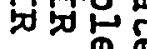

四员员

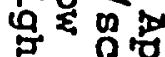

实

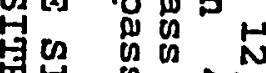

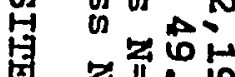

दा

$\sim N$

为战

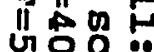

兽

品

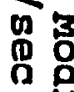

㝒

员

莫嵒

$\because N$

$N$

స్ํํำ

品

in

范

ด

..

옹

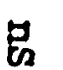

先 
33 Created Apr 12,1994 11:44 Modified Apr 12,1994 15:20

512 samples/scan 49.984 scans/sec position: -20 ns range: $200 \mathrm{~ns}$
Vert. IIR low pass $\mathrm{N}=2 \mathrm{~F}=40$

Vert. IIR high pass $\mathrm{N}=2 \quad \mathrm{~F}=5$

GRACE ROAD WASTE SITE

AVANNAE RIVER SITE

Figure. ? $^{-}$

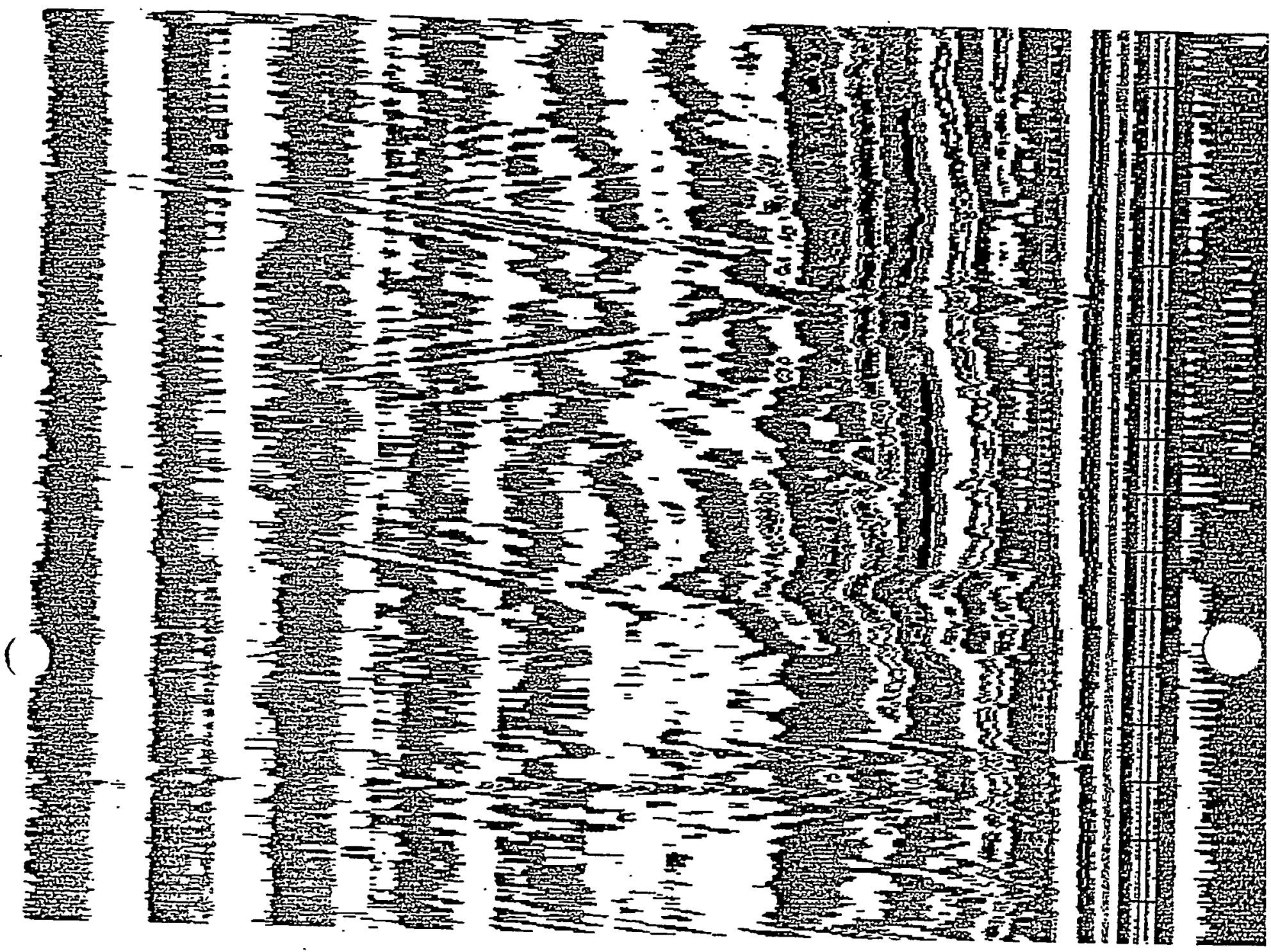


54 Created Apr 12,1994 11:48 Modified Apr 12,1994 15:20

Vert. IIR Iow pass $N=2 \quad \mathrm{~F}=40$ s

Vert. IIR high pass $N=2 \quad \dot{F}=5$

. GRACE ROAD WASTE SITE

IAVANNAE RIVER SITE

Figure 36
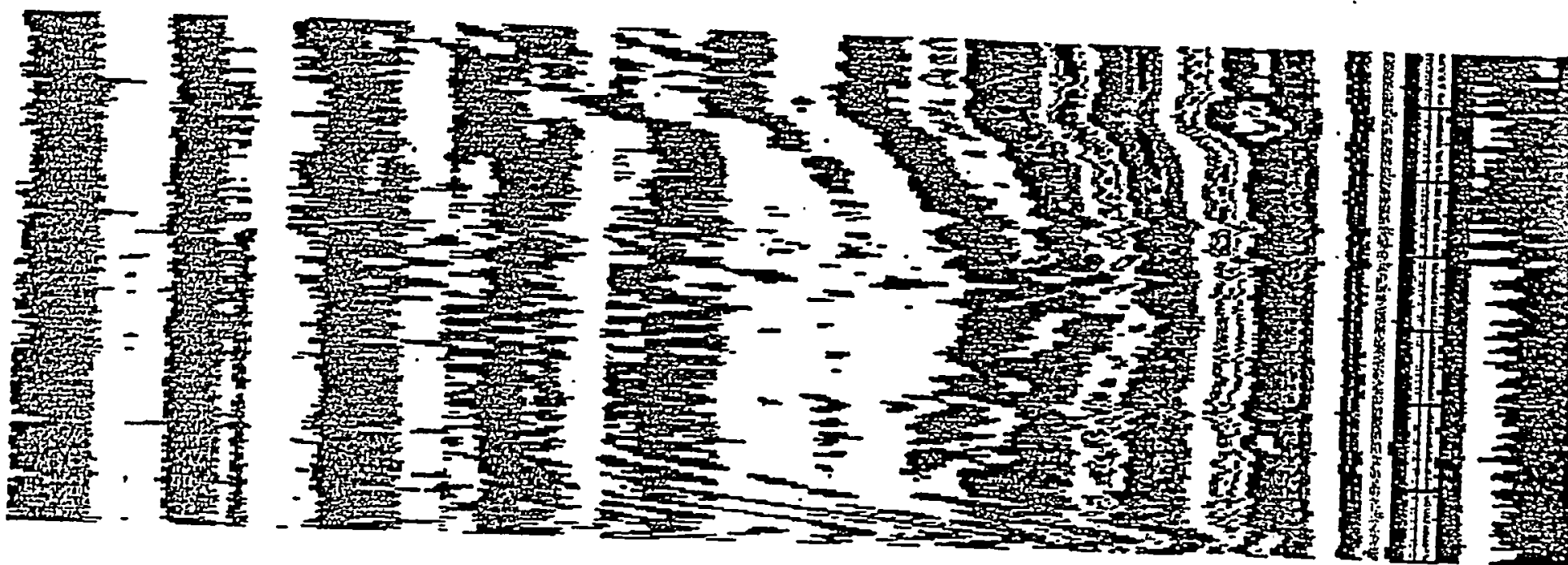
35 Created Apr 12,1994 11:49 Modified Apr 12,1994 15:20

512 samples/scan 49.984 scans/sec position: $-20 \mathrm{nS}$ Iange: $200 \mathrm{nS}$
Vert. IIR Iow pass $N=2 \quad F=40$

Vert. IIR high pass $N=2 \quad F=5$

GRACE ROAD WASTE SITE

AVANNAB RIVER SITE
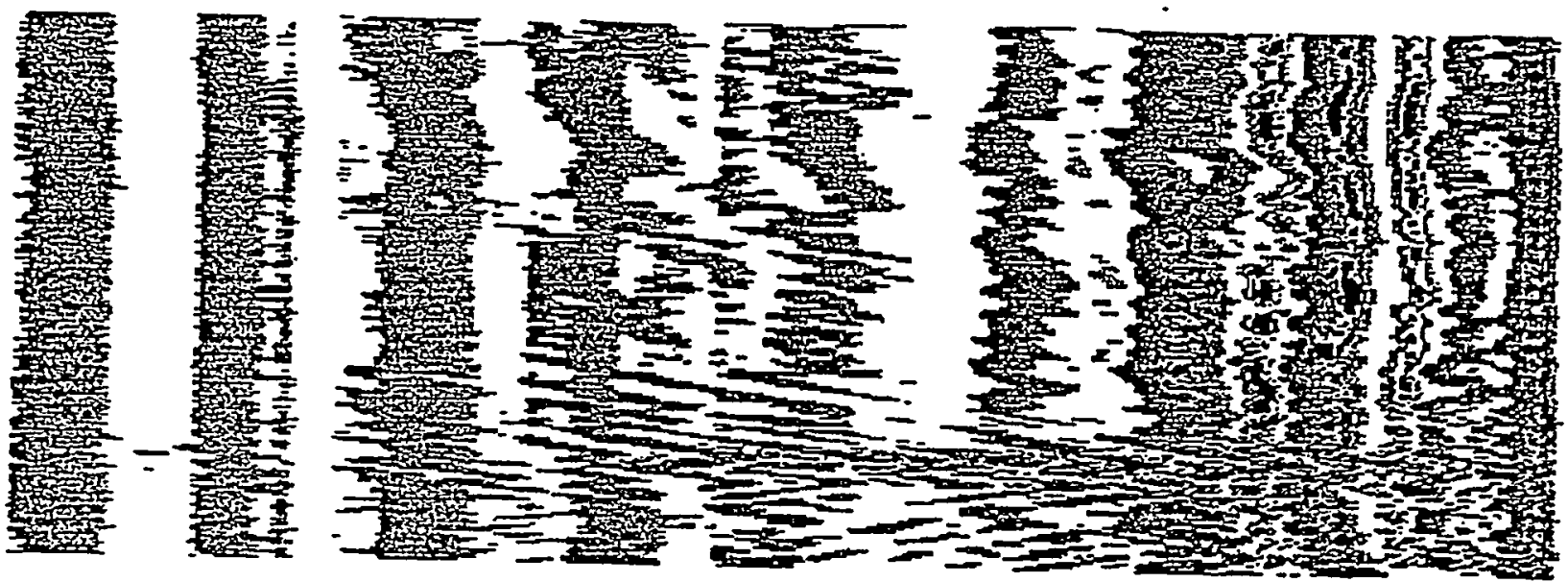

Figure

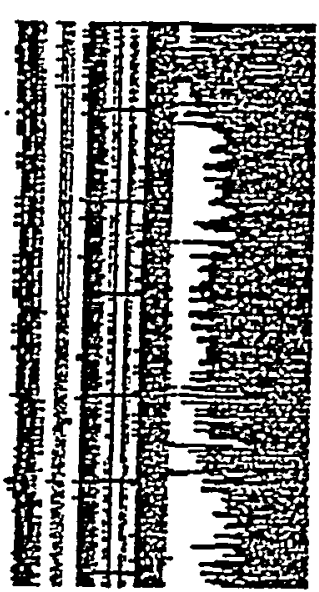


36 Created Apr 12,1994 11:53 Modified Apr 12,1994 15:21. .

512 samples/scan 49.984 scans/sec position: -20 ns range: $200 \mathrm{~ns}$

Vert. IIR low pass $\mathrm{N}=2 \quad \mathrm{~F}=40$

Vert. IIR high pass $\mathrm{N}=2 \mathrm{~F}=5$

GRACE ROAD WASTE SITE

Figue 38

IAVANNAB RIVER SITE

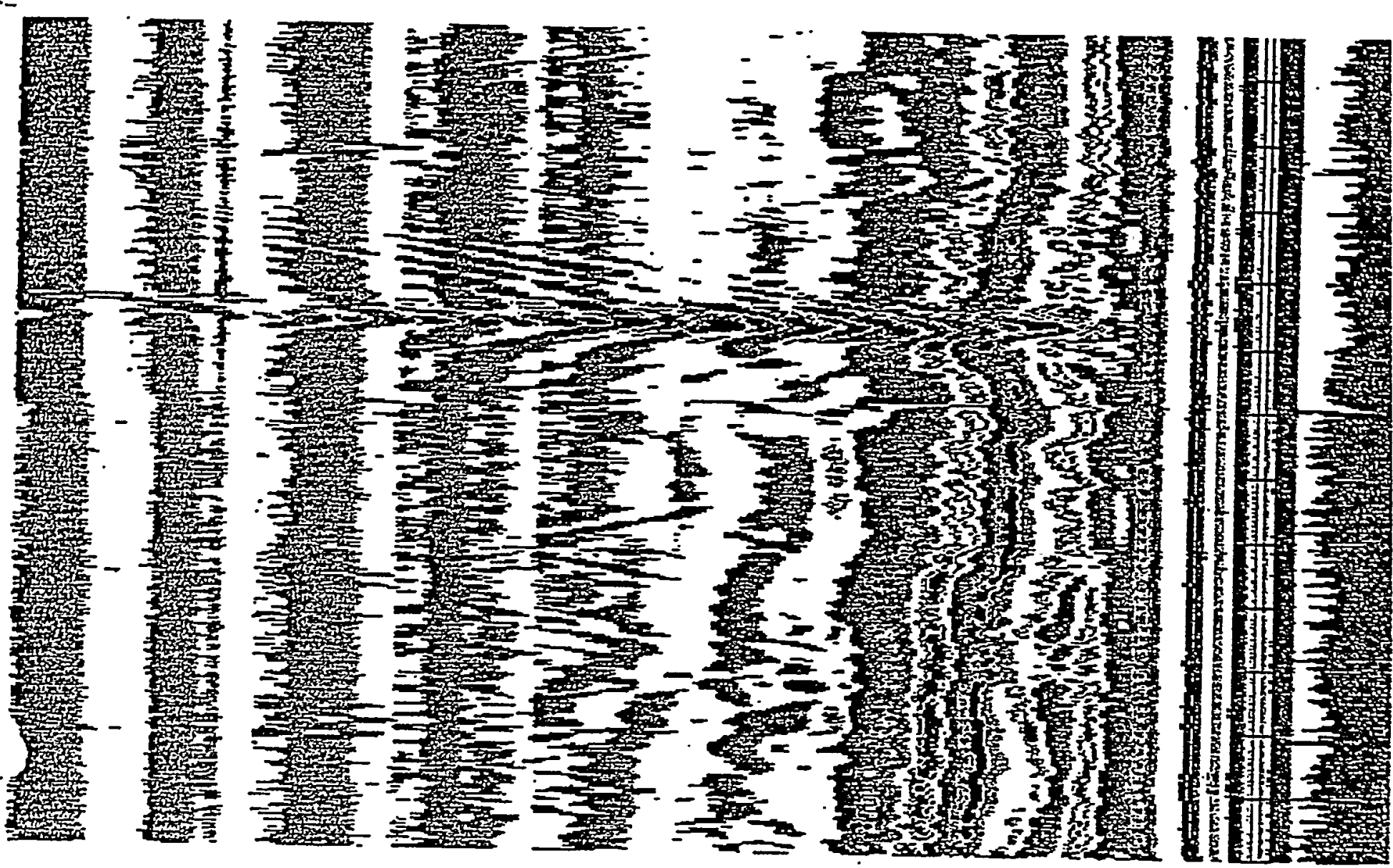


37 Created Apr 12,1994 11:58 Modified Apr 12,1994 15:21

512 samples/scan 49.984 scans/sec position: -20 ns range: $200 \mathrm{~ns}$

Vert. IIR low pass $N=2 \quad F=40$

Vert. IIR high pass $\mathrm{N}=2 \mathrm{~F}=5$

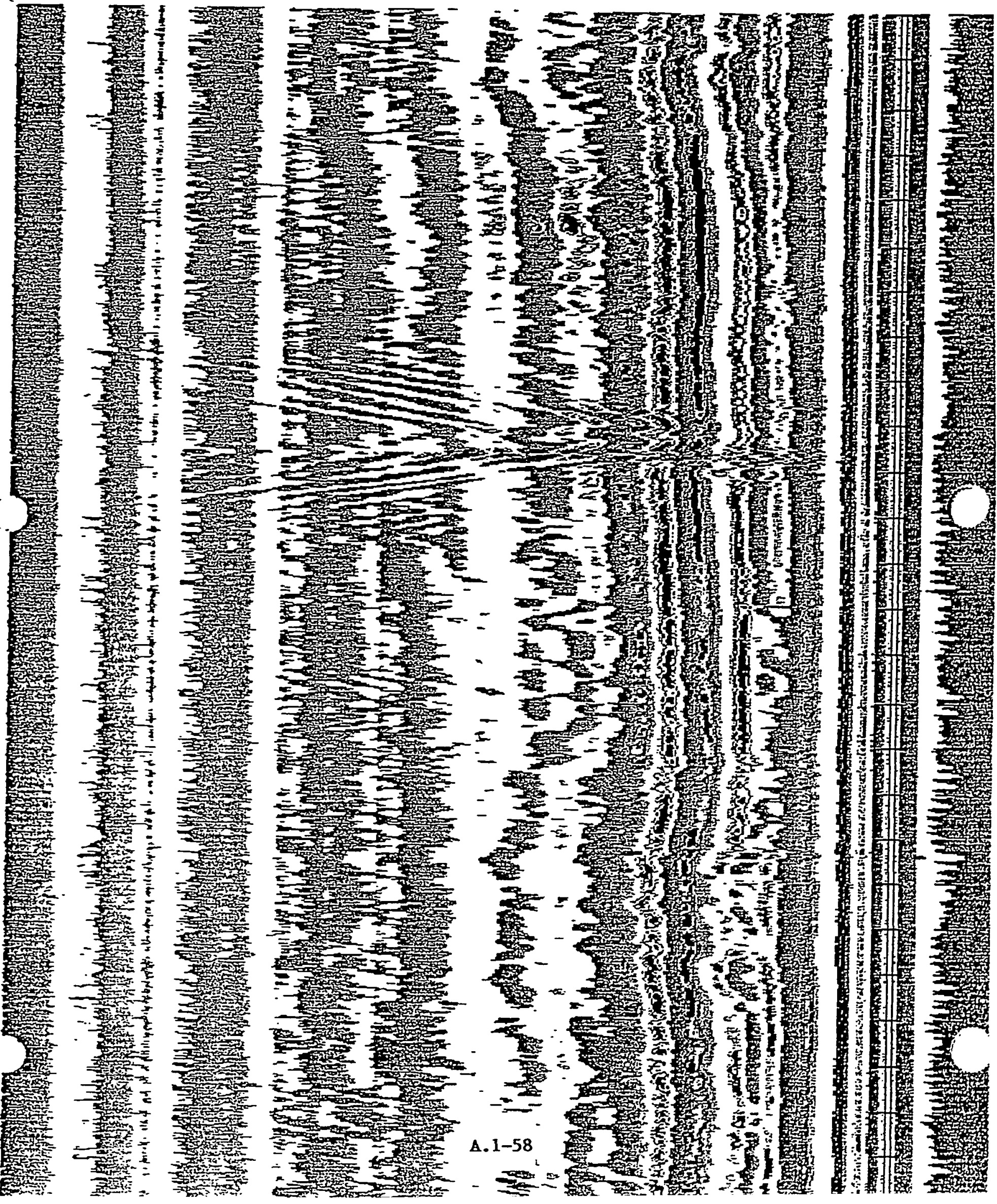


Figure 39 continued

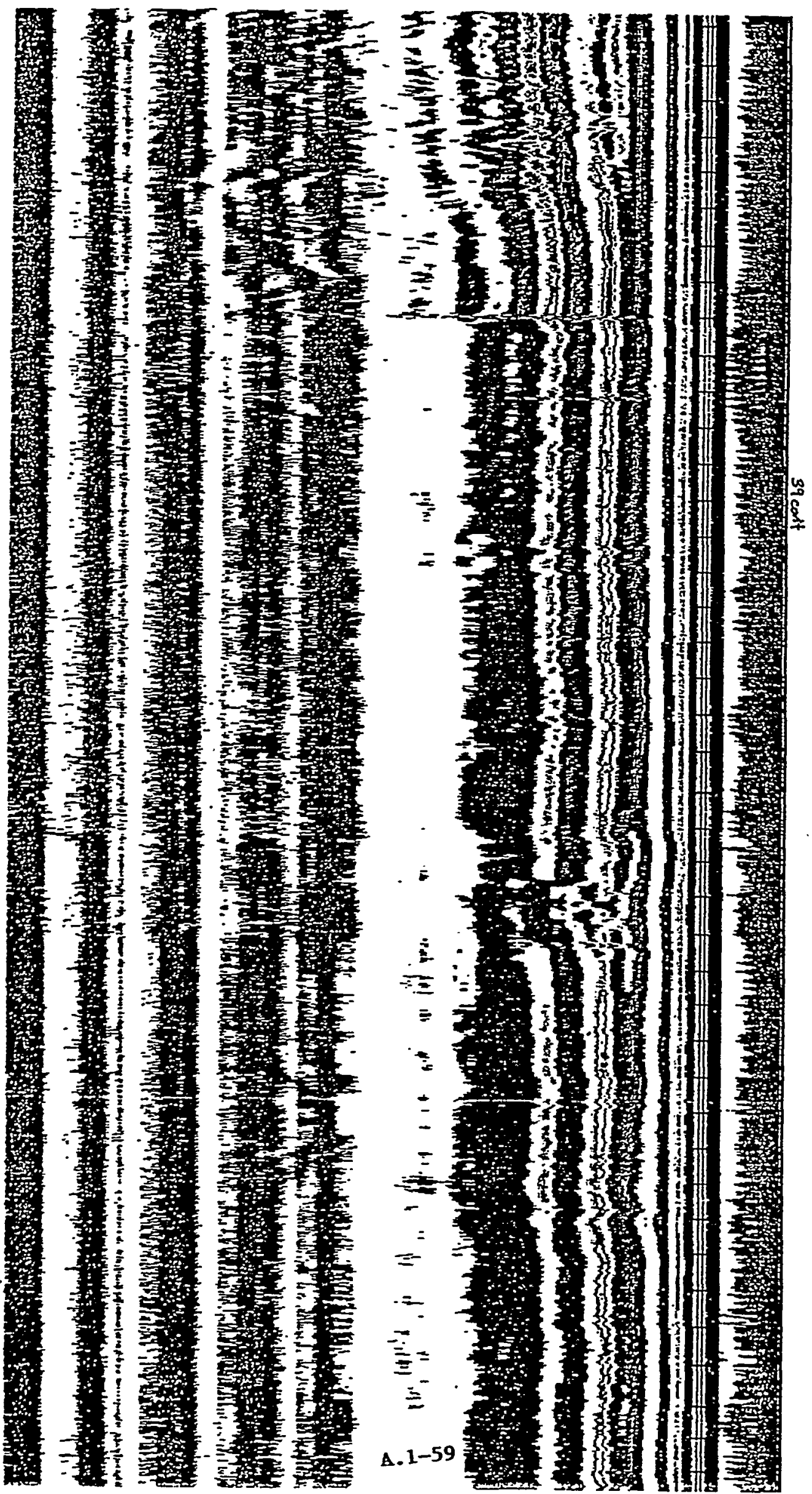


38 Created Apr 12,1994 12:09 Modified Apr 12,1994 15:21

512 samples/scan 49.984 scans/sec position: -20 ns range: 200 ns

Vert. IIR high pass $N=2 \quad F=5$

GRACE ROAD WASTE SITE

AVANNAH RIVER SITE

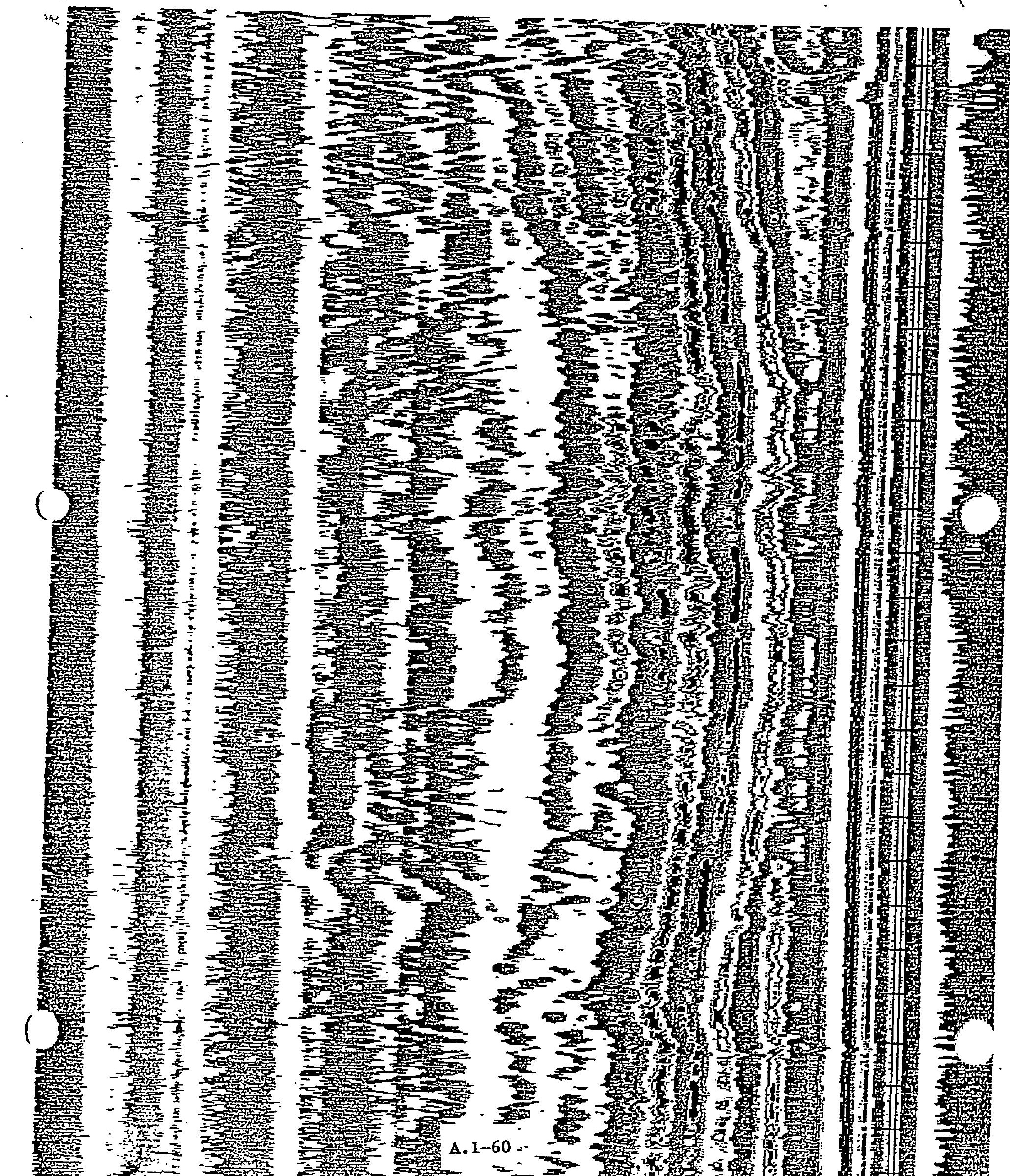


6.6.

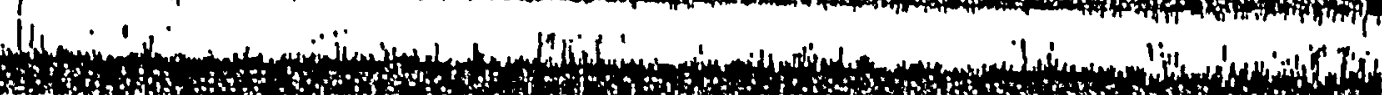

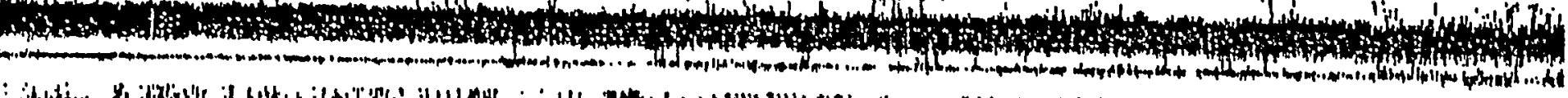

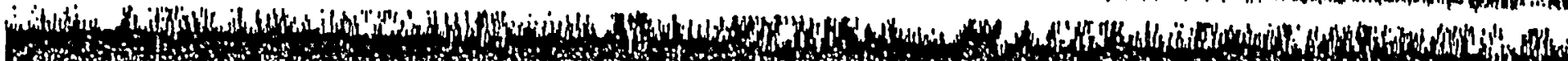

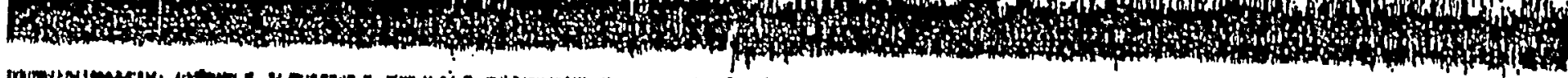

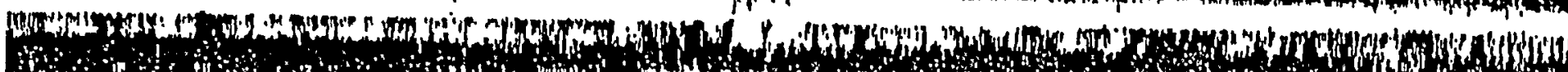

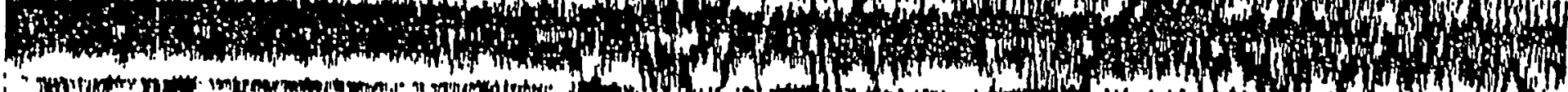

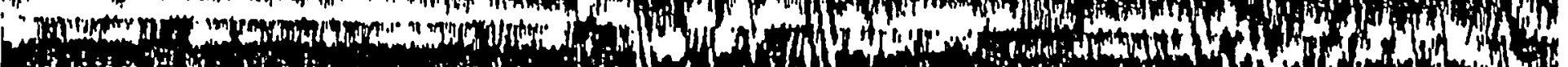

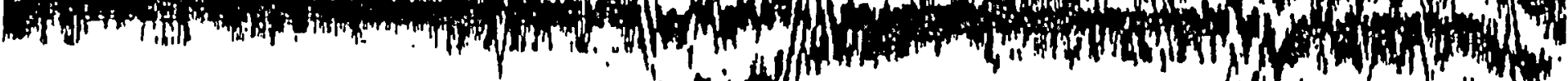

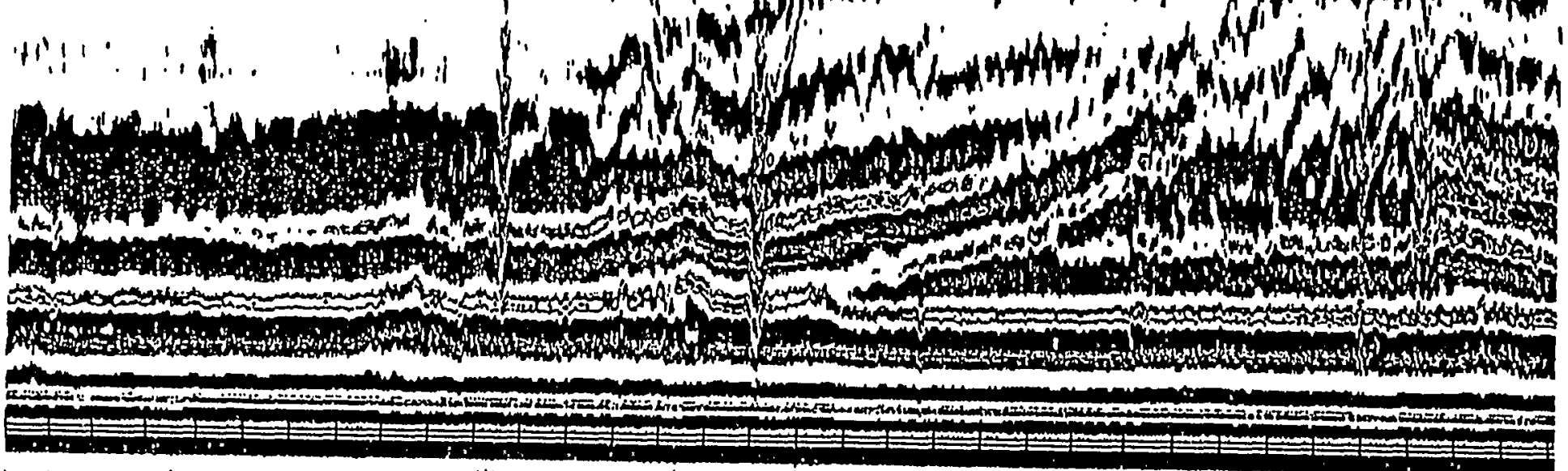

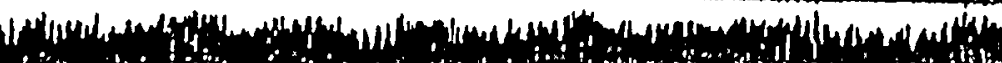
L 
1 Created Mar 20,1994 09:26 Modified Mar 20,1994 18:04

512 samples/scan 30.0019 scans/sec position: $-10 \mathrm{~ns}$ range: $150 \mathrm{~ns}$

Vert. IIR low pass $\mathrm{N}=2 \quad \mathrm{~F}=80$

Vert. IIR high pass $N=2 \quad F=11$

GRACE ROAD WASTE SITE

Figure 4

AVANNAH RIVER SITE
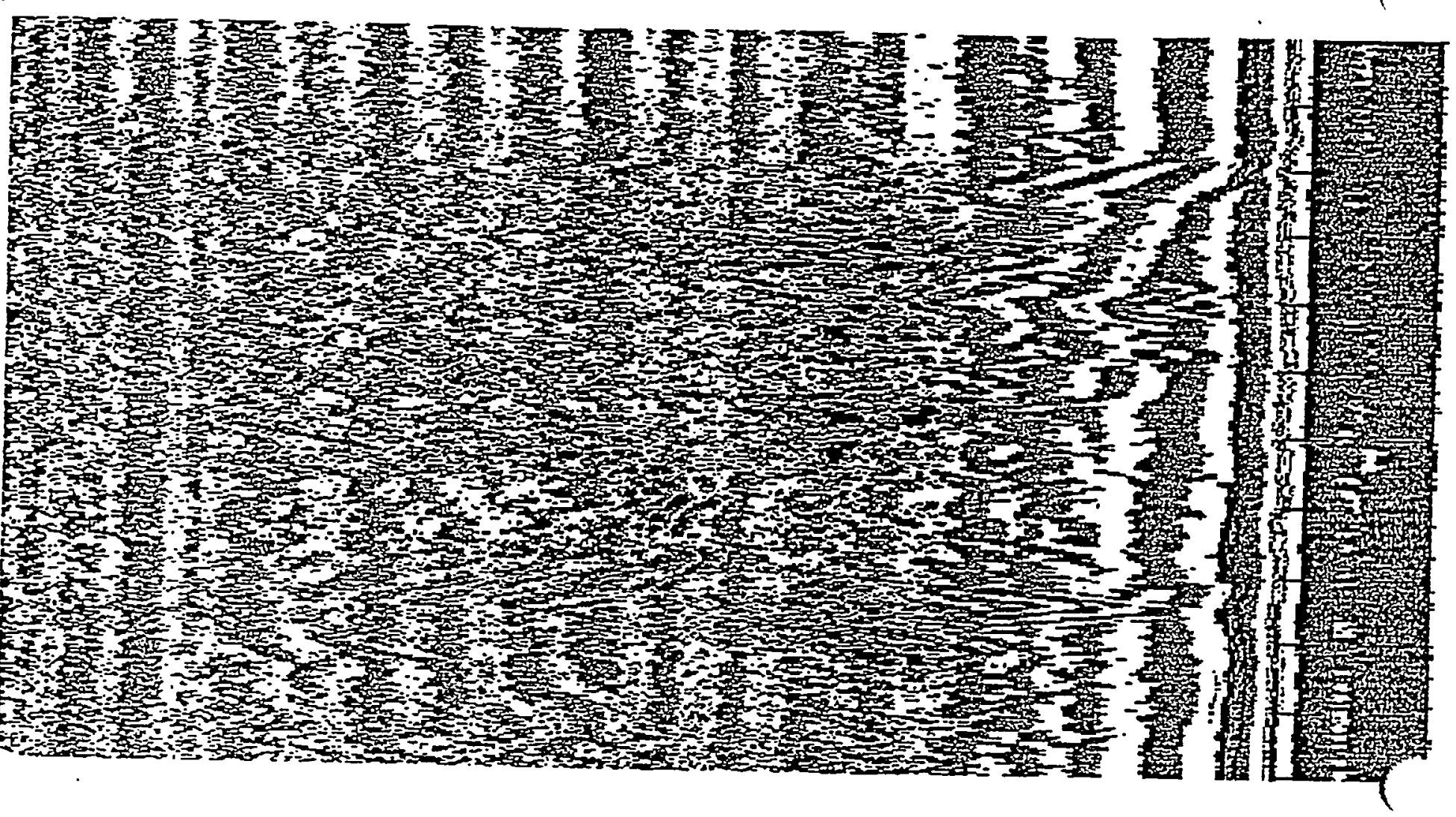
2 Created Mar 20,1994 09:29 Modified Mar 20, 1994 18:04

512 samples/scan 30.0019 scans/sec position: -10 ns range: 150 ns

Vert. IIR low pass $\mathrm{N}=2 \mathrm{~F}=80$

Vert. IIR high pass $N=2 \quad F=11$

GRACE ROAD WASTE SITE

IAVANNAB RIVER SITE

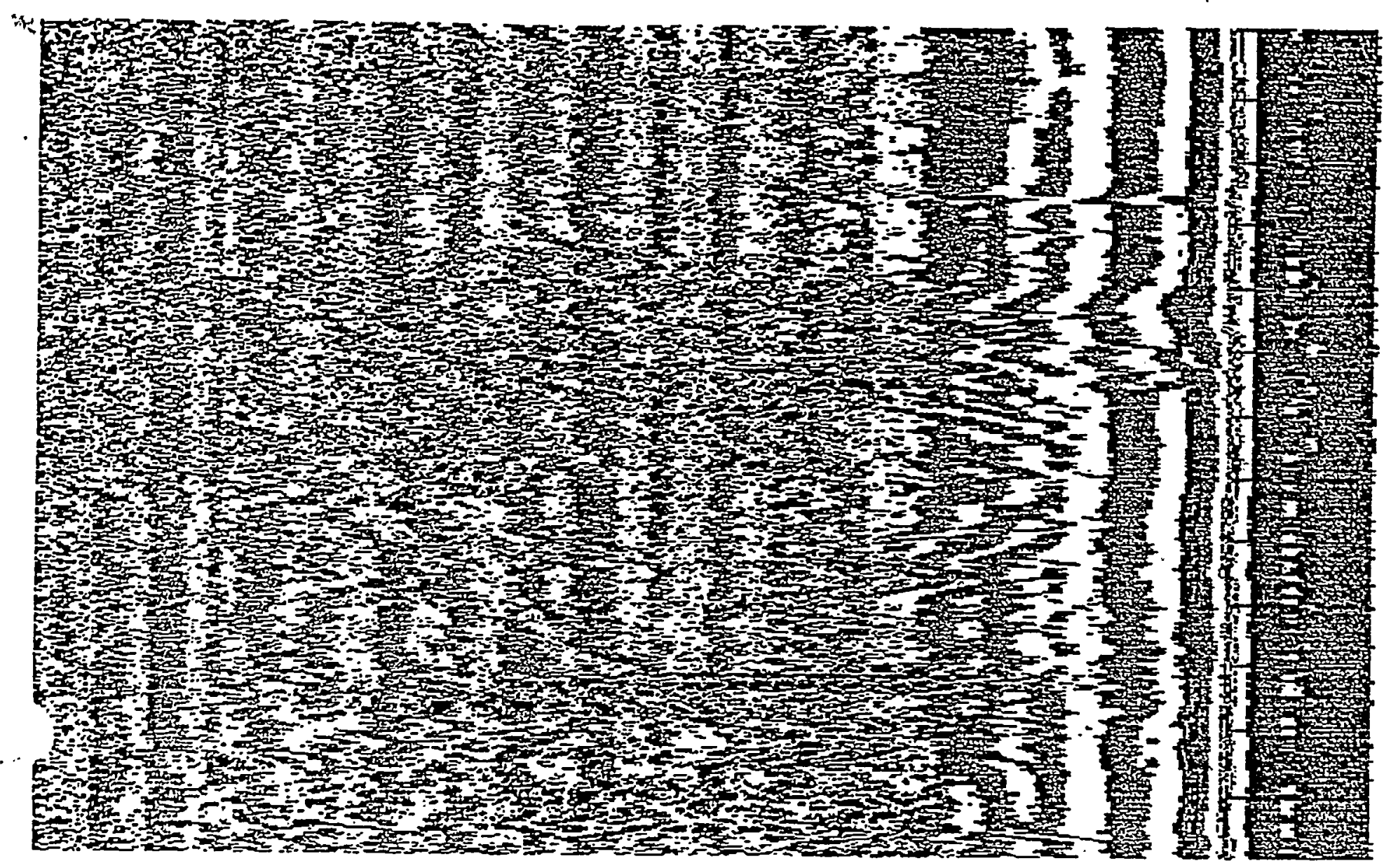


3 Lreacea Mar 20,1994 09:31 Modified Mar 20,1994 18:04

512 samples/scan 30.0019 scans/sec position: -10 ns range: $150 \mathrm{~ns}$

Vert. IIR low pass $\mathrm{N}=2 \quad \mathrm{~F}=80$

Vert. IIR high pass $\mathrm{N}=2 \mathrm{~F}=11$

GRACE ROAD WASTE SITE

IAVANNAE RIVER SITE

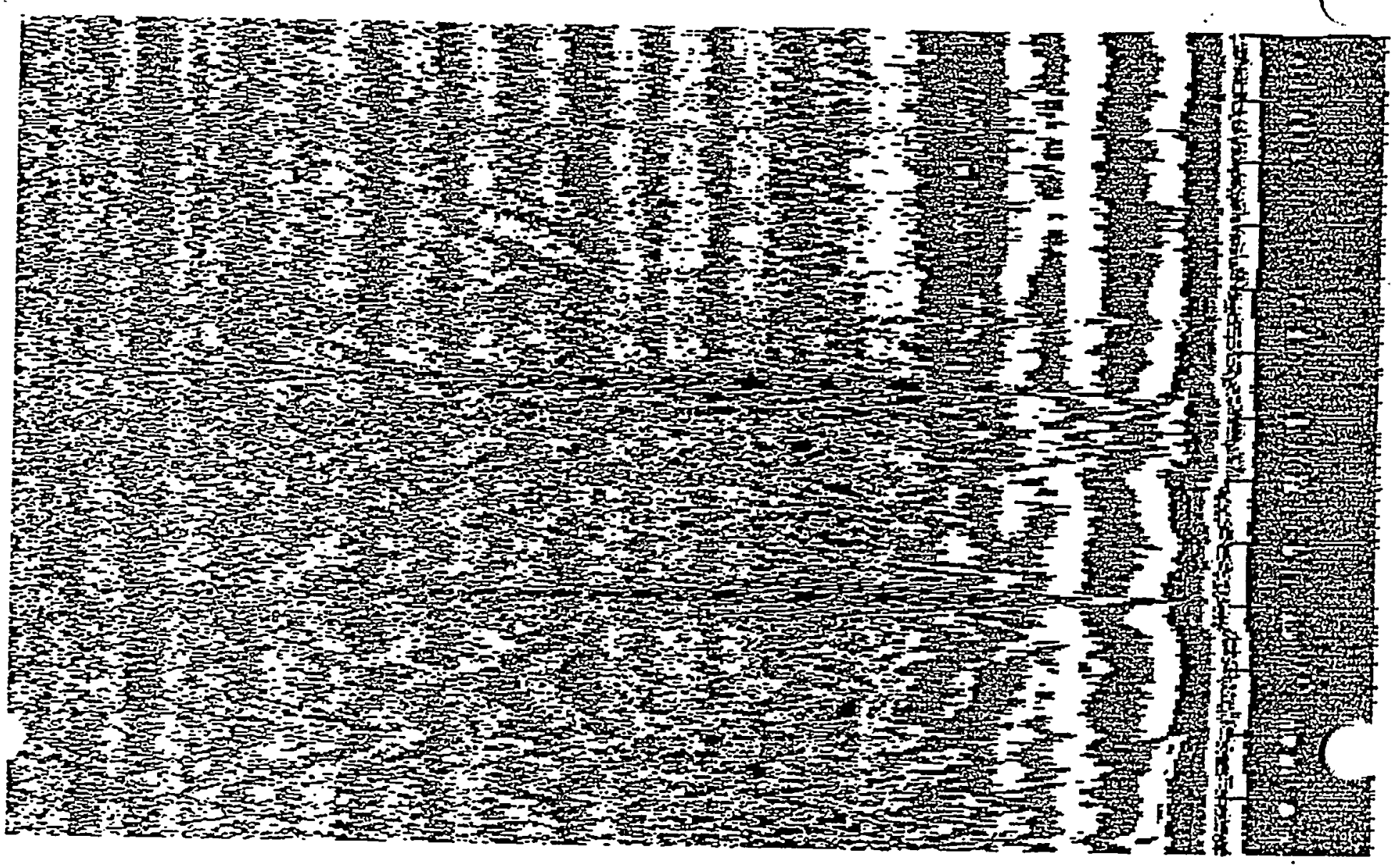


4 Created Mar 20,1994 09:33 Modified Mar 20,1994 18:05

512 samples/scan 30.0019 scans/sec position: -10 as range: $150^{\circ}$ ns

Vert. IIR high pass $\mathrm{N}=2 \mathrm{~F}=11$

GRACE ROAD WASTE SITE

Figure 44

IAVANNAB RIVER SITE

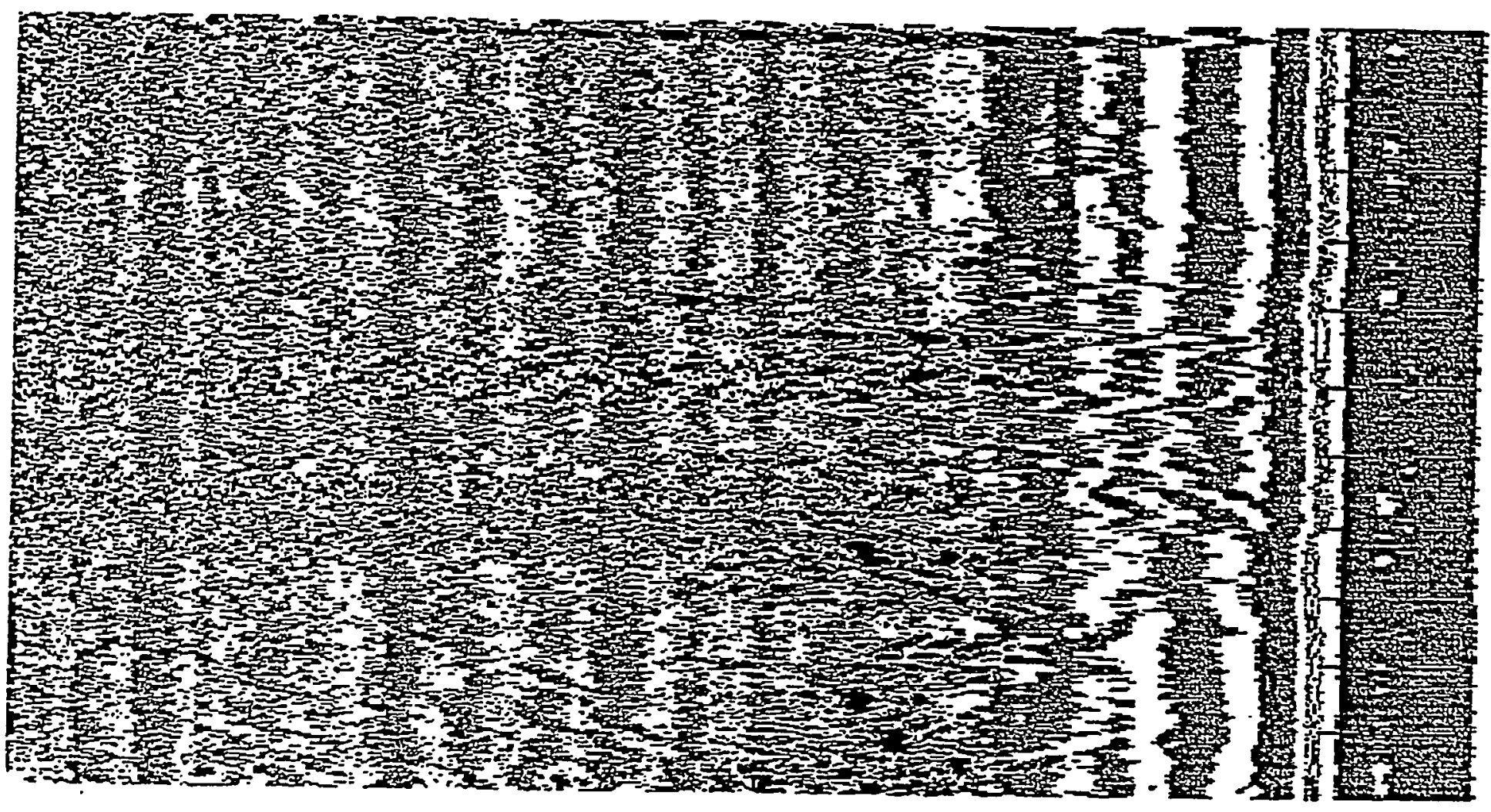

A. 1-65 
5 Created Mar 20,1994 09:35 Modified Mar 20,1994.18:05

512 samples/scan 30.0019 scans/sec position: $-10 \mathrm{~ns}$ range: $150 \mathrm{~ns}$

Vert. IIR liss $\mathrm{N}=2 \quad \mathrm{~F}=80$

GRACE ROAD WASTE SITE

Figure

JAVANNAB RIVER SITE

o.
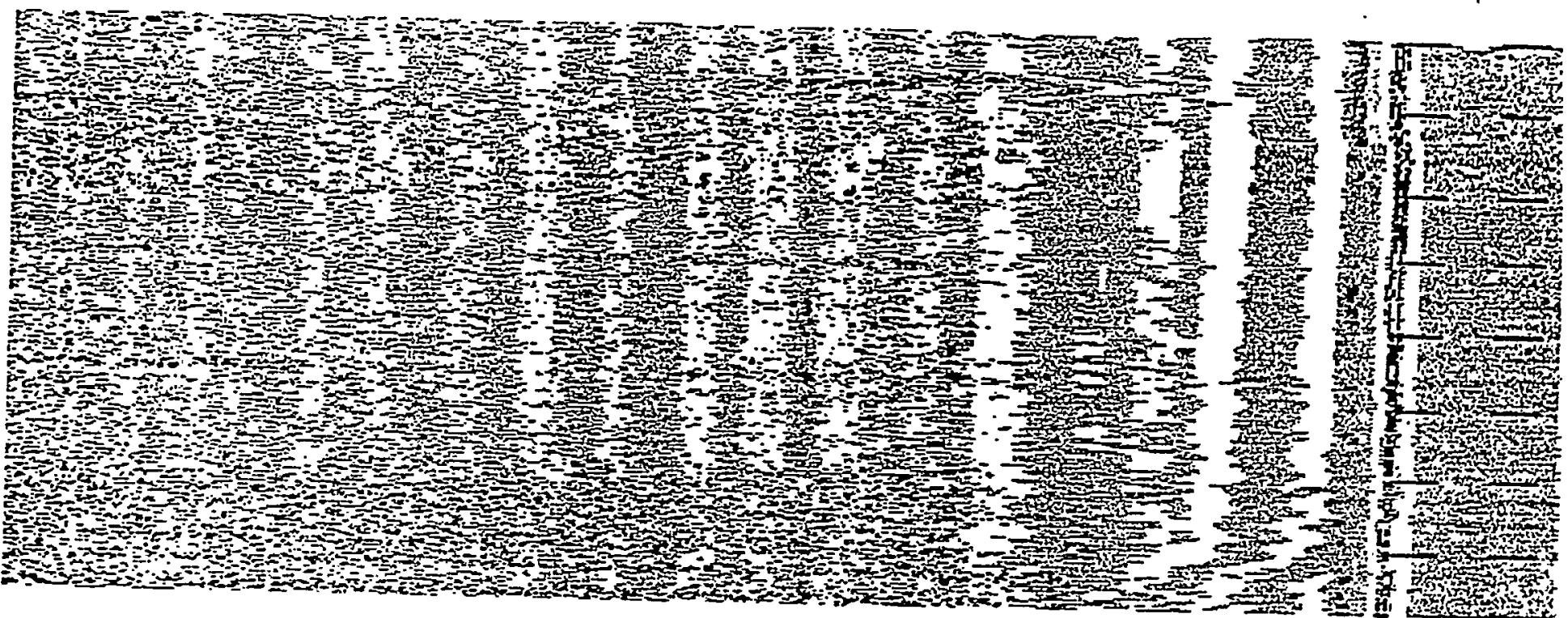
6 Created Mar 20,1994 09:37 Modified Mar 20,1994 18:05 512 samples/scan 30.0019 scans/sec position: -10 ns range: $150 \mathrm{nS}$
Vert. IIR low pass $\mathrm{N}=2 \mathrm{~F}=80$

Vert. IIR high pass $N=2 \quad F=11$

GRACE ROAD WASTE SITE

Figure 4!

AVANNAE RIVER SITE

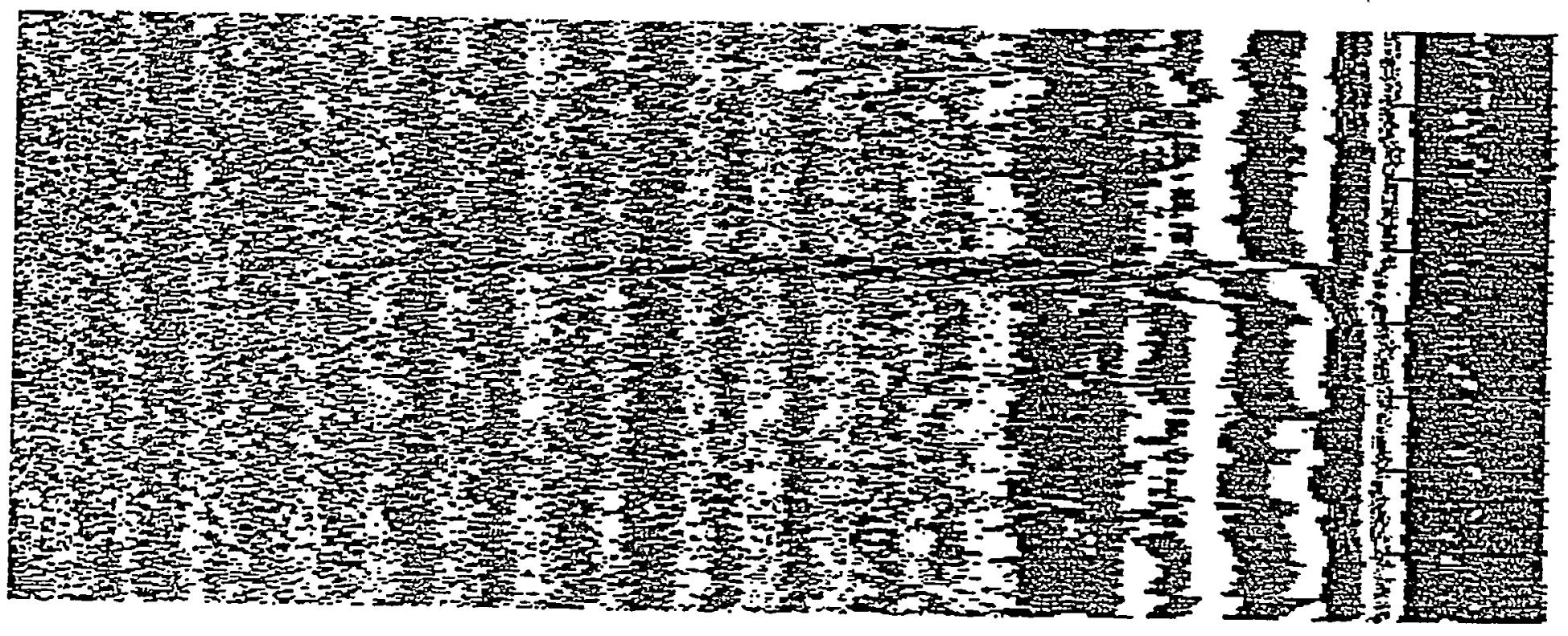

A. 1-67 
7 Created Mar 20,1994 09:38 Modified Mar 20,1994 18:05

512 samples/scan 30.0019 scans/sec position: $-10 \mathrm{nS}$ range: $150 \mathrm{~ns}$.
Vert. IIR Iow pass $\mathrm{N}=2 \mathrm{~F}=80$

Vert. IIR high pass $N=2 \quad F=11$

FRACE ROAD WASTE SITE

AVANNAH RIVER SITE

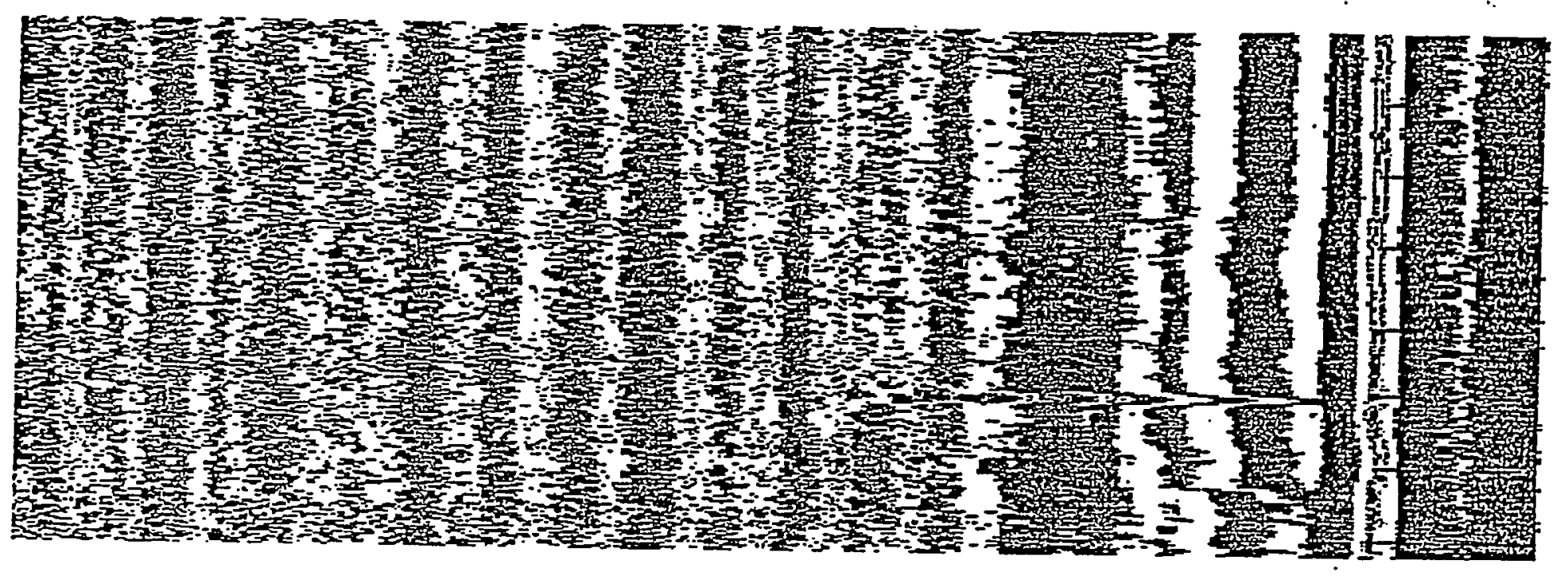

Figure

AVANMAH RIVER SITE 
8 Created Mar 20,1994 09:44 Modified Mar 20,1994 18:05

512 samples/scan 30.0019 scans/sec position: -10 ns range: 150 ns

Vert. IIR low pass $\mathrm{N}=2 \mathrm{~F}=80$

Vert. IIR high pass $\mathrm{N}=2 \mathrm{~F}=11$

Figure 48

GRACE ROAD WASTE SITE

SAVANNAH RIVER SITE

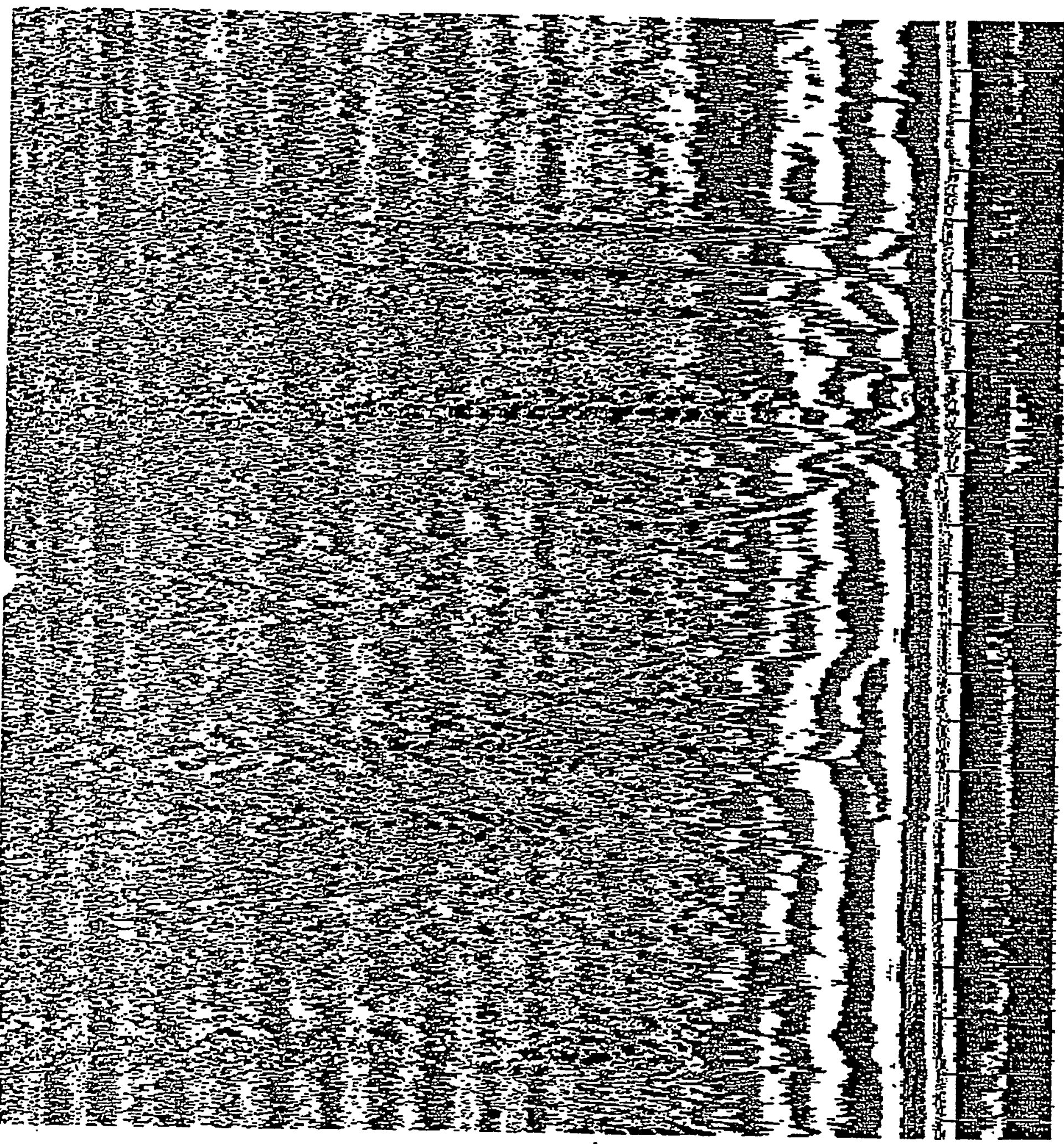


512 samples/scan, 1374 Uy:40 Modiried Mar 20,1994 18:05

vert. IIR Iow pass

Vert. IIR high

GRACE ROAD WASTE SITE

Figure

SAVANNAB RIVER SITE

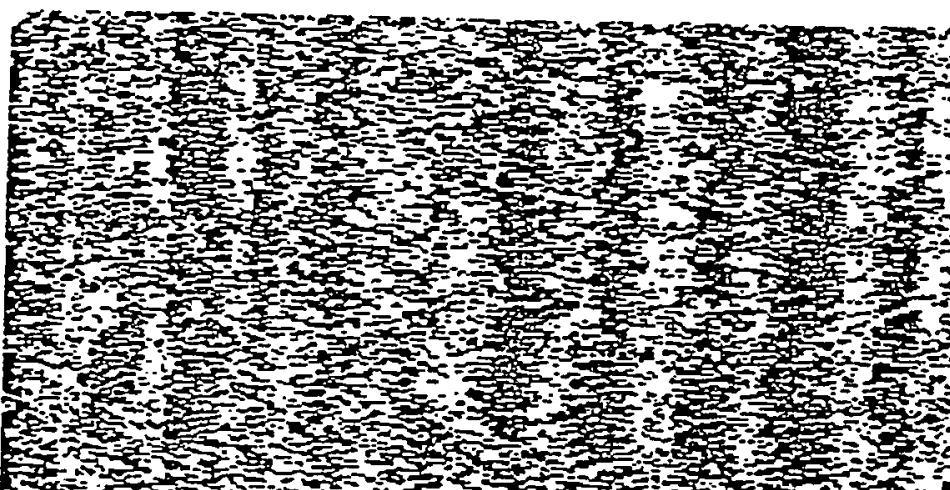

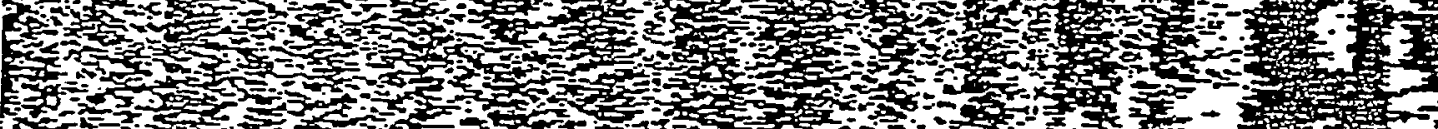
on (30)

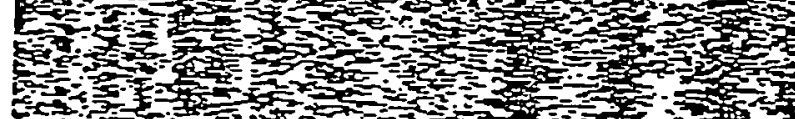

Non,

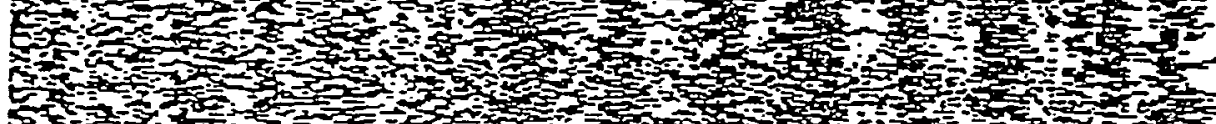

(1)

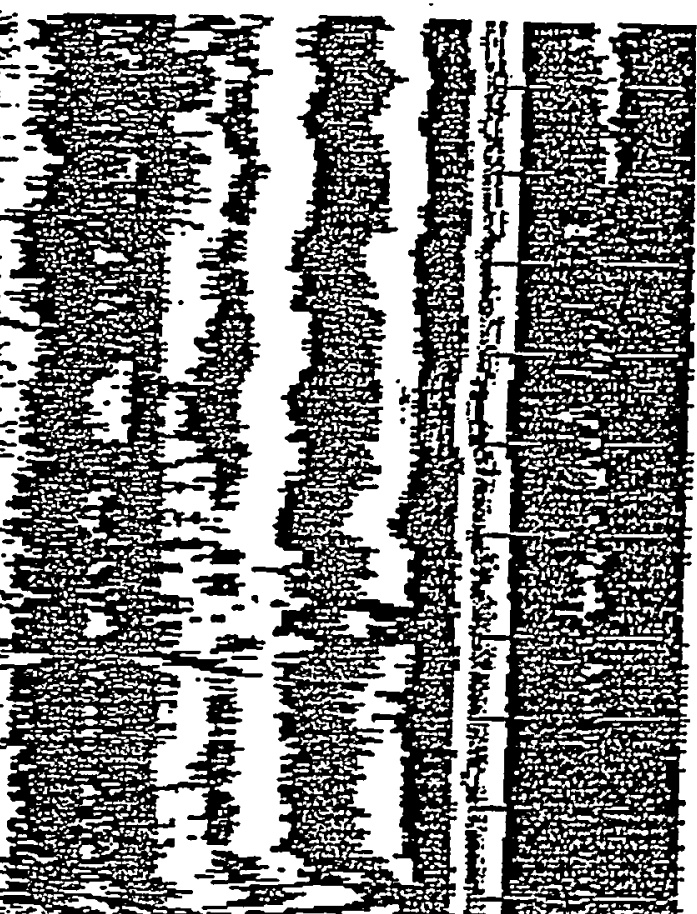

(1)

(1)

(1)

(1)

(1)

(1)

(1)

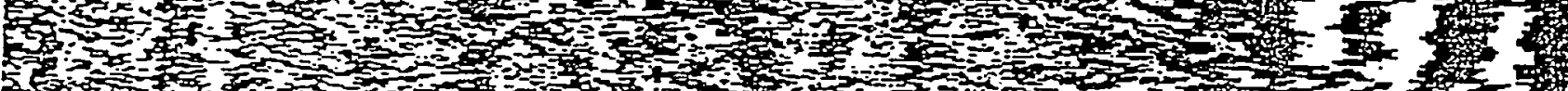

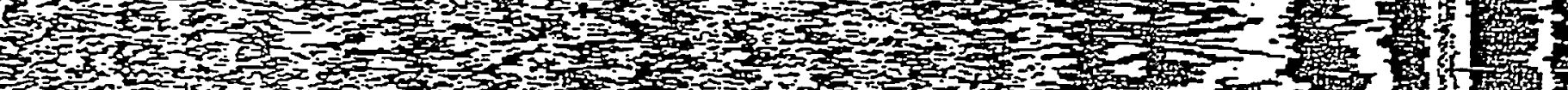
KE: tow (3) 5 
LU Lrearea Mar 20,1994 09:52 Modified Mar 20,1994 18:05

512 samples/scan 30.0019 scans/sec position: $-10 \mathrm{~ns}$ range: $150 \mathrm{~ns}$

Figure!

GRACE ROAD WASTE SITE

AVANNAB RIVER SITE

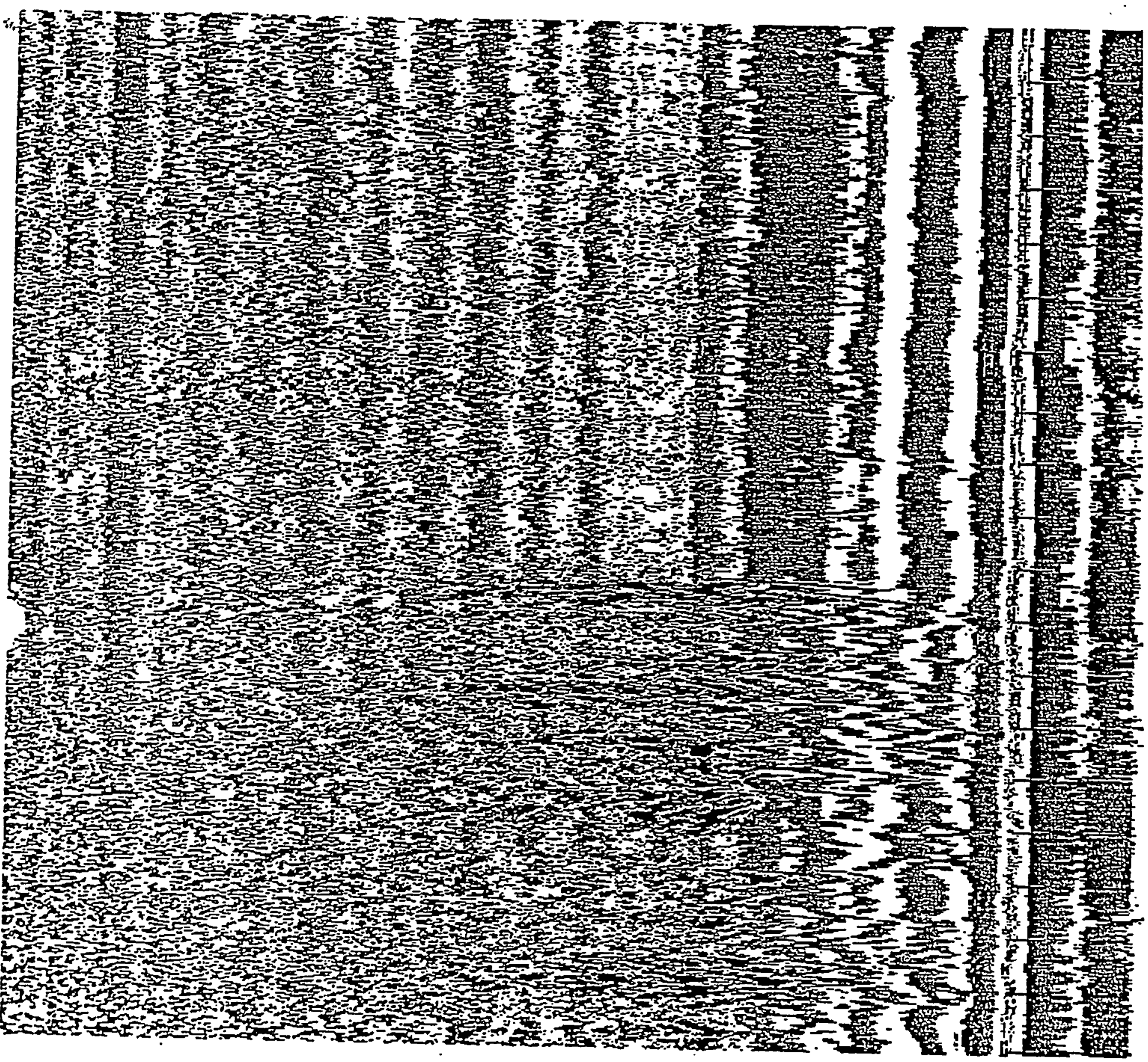


11 Created Mar 20,1994 09:55 Modified Mar 20,1994 18:06

512 samples/scan 30.0019 scans/sec position: $-10 \mathrm{~ns}$ range: $150 \mathrm{~ns}$
Vert. IIR low pass $N=2 \mathrm{~F}=80$

Vert. IIR low pass $N=2 \quad F=80$

vert. IIR high pass $N=2 \quad F=I I$

GRACE ROAD WASTE SITE

Figure 5:

IAVANNAB RIVER SITE

ins

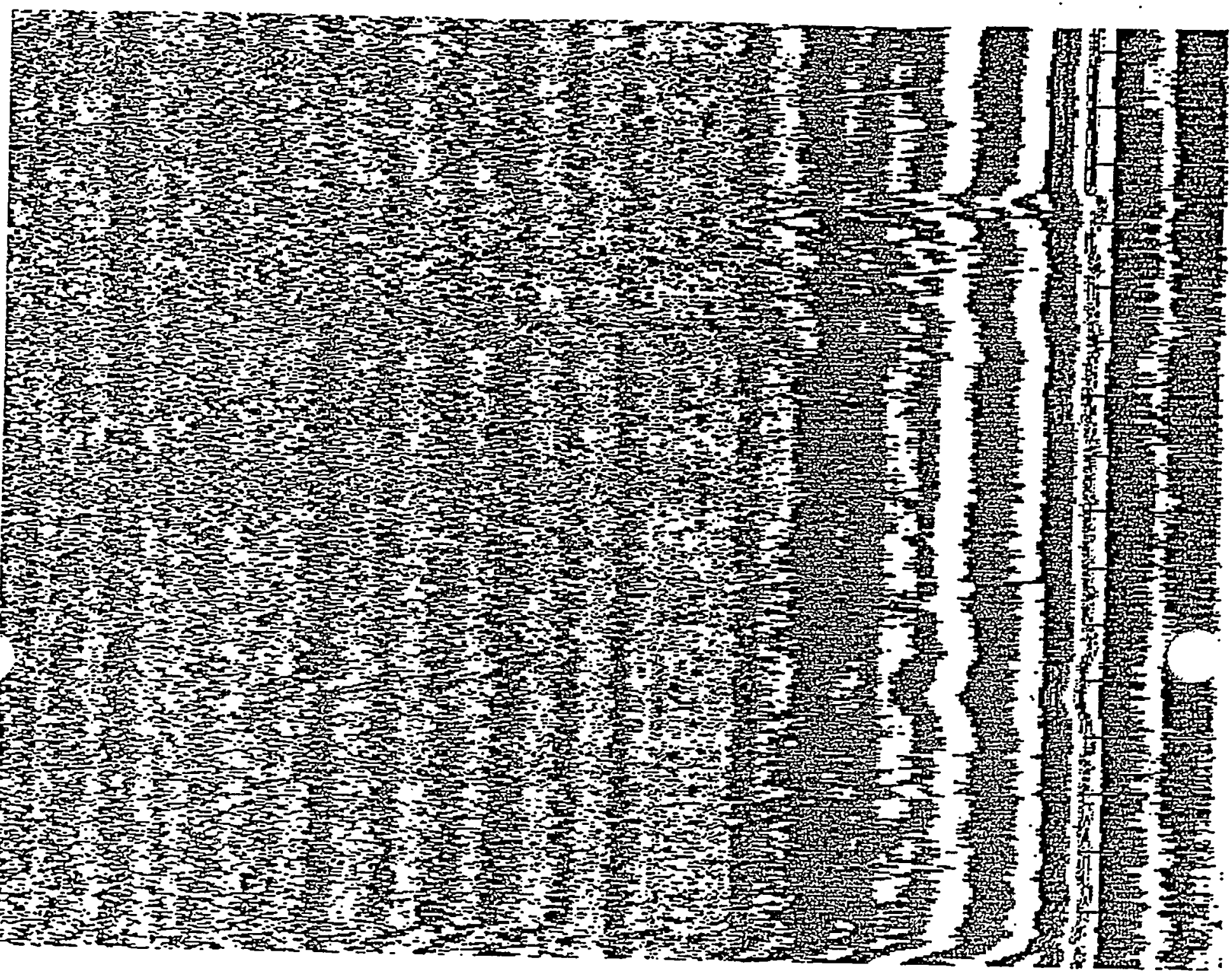


12 Created Mar 20,1994 09:59 Modified Mar 20,1994 18:06

$512 \mathrm{samples} / \mathrm{scan} 30.0019$ scans/sec position: $-10 \mathrm{nS}$ range: $150 \mathrm{~ns}$

Vert. IIR low pass $\mathrm{N}=2 \mathrm{~F}=80$

Vert. IIR high pass $\mathrm{N}=2 \mathrm{~F}=11$

GRACE ROAD WASTE SITE

Figure 52

JAVANNAE RIVER SITE

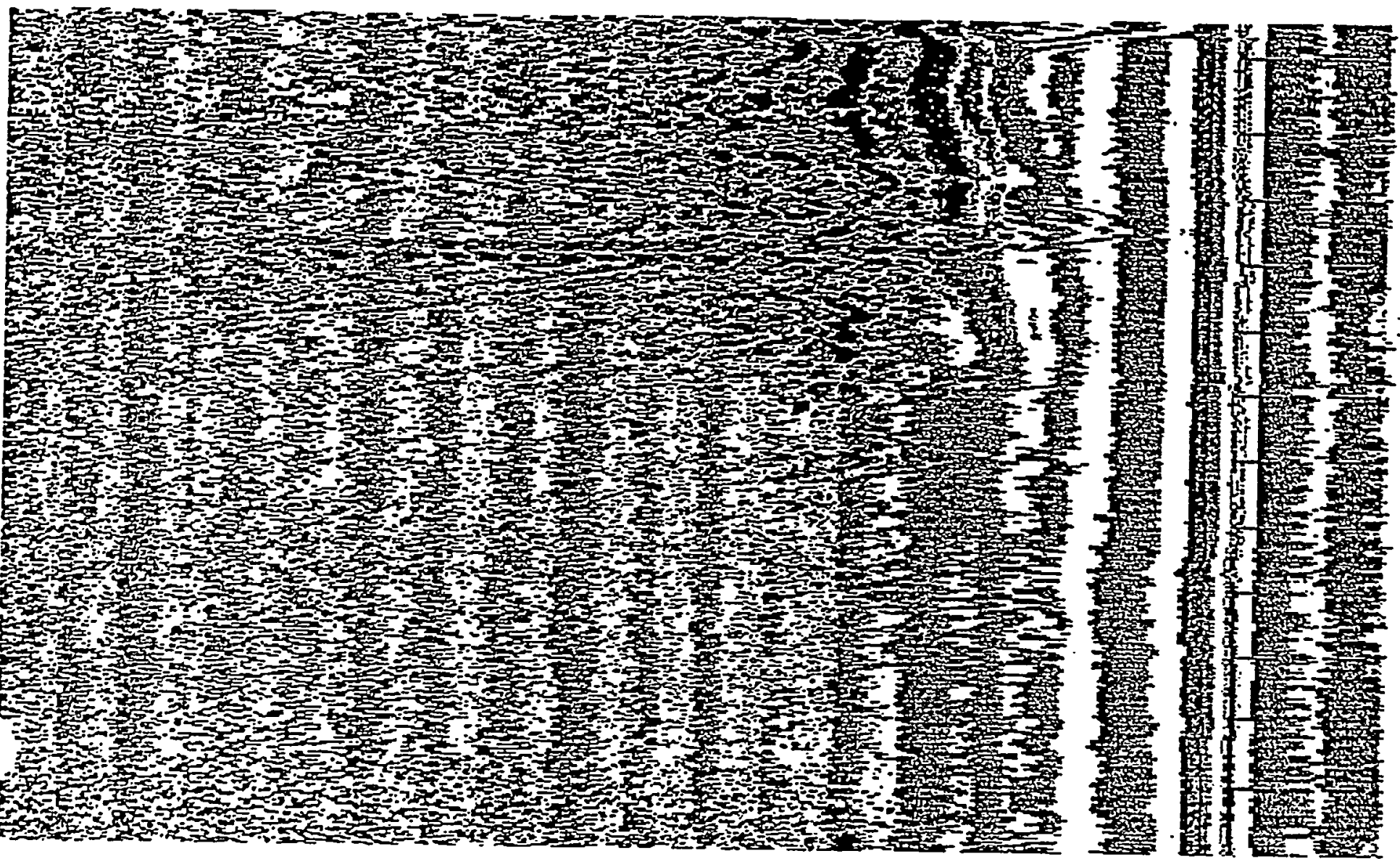


13 Created Mar 20,1994 10:02 Modified Mar 20,1994 18:06

512 samples/scan 30.0019 scans/sec position: -10 ns range: 150 nS
Vert. IIR low pass $N=2 \quad F=80$

Vert. IIR high pass $N=2 \quad F=11$

GRACE ROAD WASTE SITE

AVANNAE RIVER SITE

Figyro.

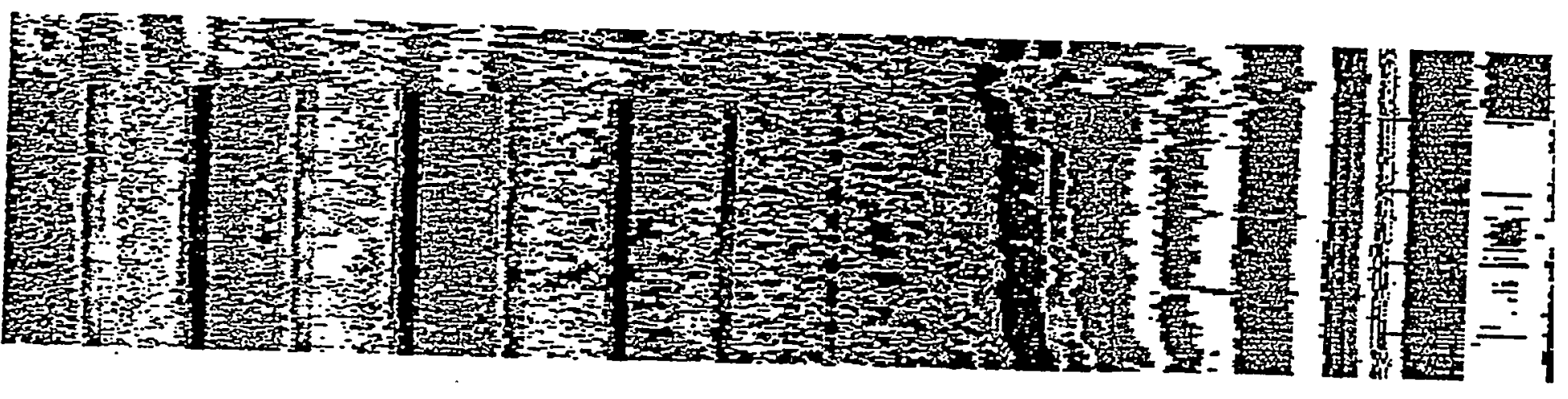


14 Created Mar 20,1994 10:07 Modified Mar 20,1994 18:06

512 samples/scan 30.0019 scans/sec position: $-10 \mathrm{~ns}$ range: $150 \mathrm{~ns}$ Vert. IIR Iow pass $N=2 \quad F=80$

Vert. IIR high pass $\mathrm{N}=2 \mathrm{~F}=11$

GRACE ROAD WASTE SITE

IVANNAE RIVER SITE

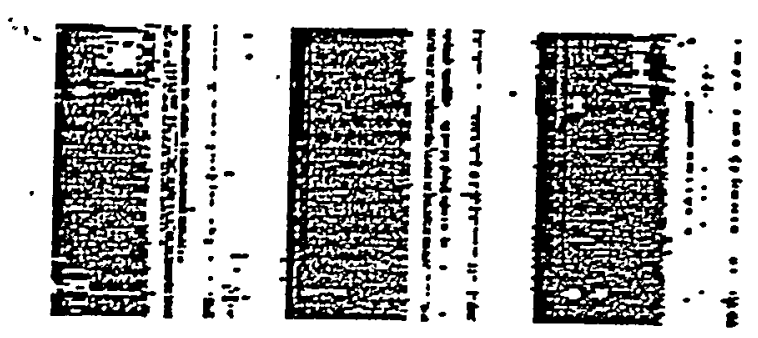

Figure 5
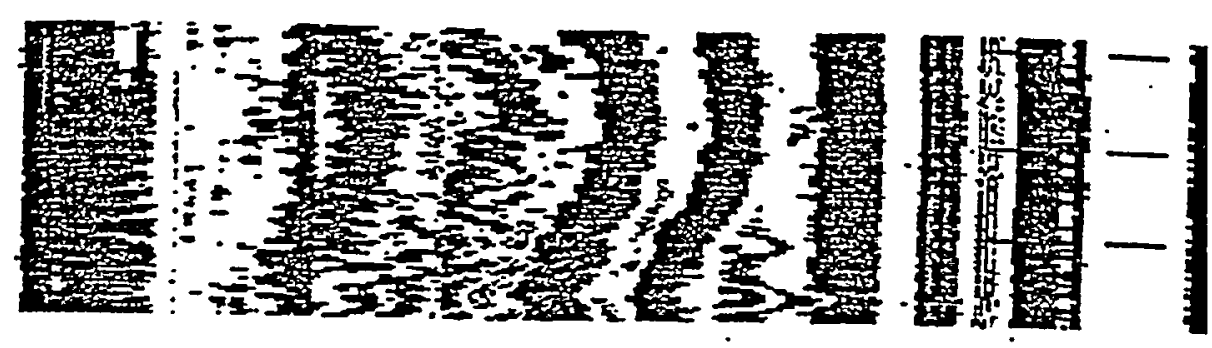
15 Created Mar 20,1994 10:09 Modified Mar 20,1994 18:06

512 samples/scan 30.0019 scans/sec position: -10 ns range: 150 ns

Vert. IIR low pass $\mathrm{N}=2 \quad \mathrm{~F}=80$

Vert. IIR high pass $\mathrm{N}=2 \mathrm{~F}=11$

GRACE ROAD WASTE SITE

Figerf 5!

IAVANNAE RIVER SITE

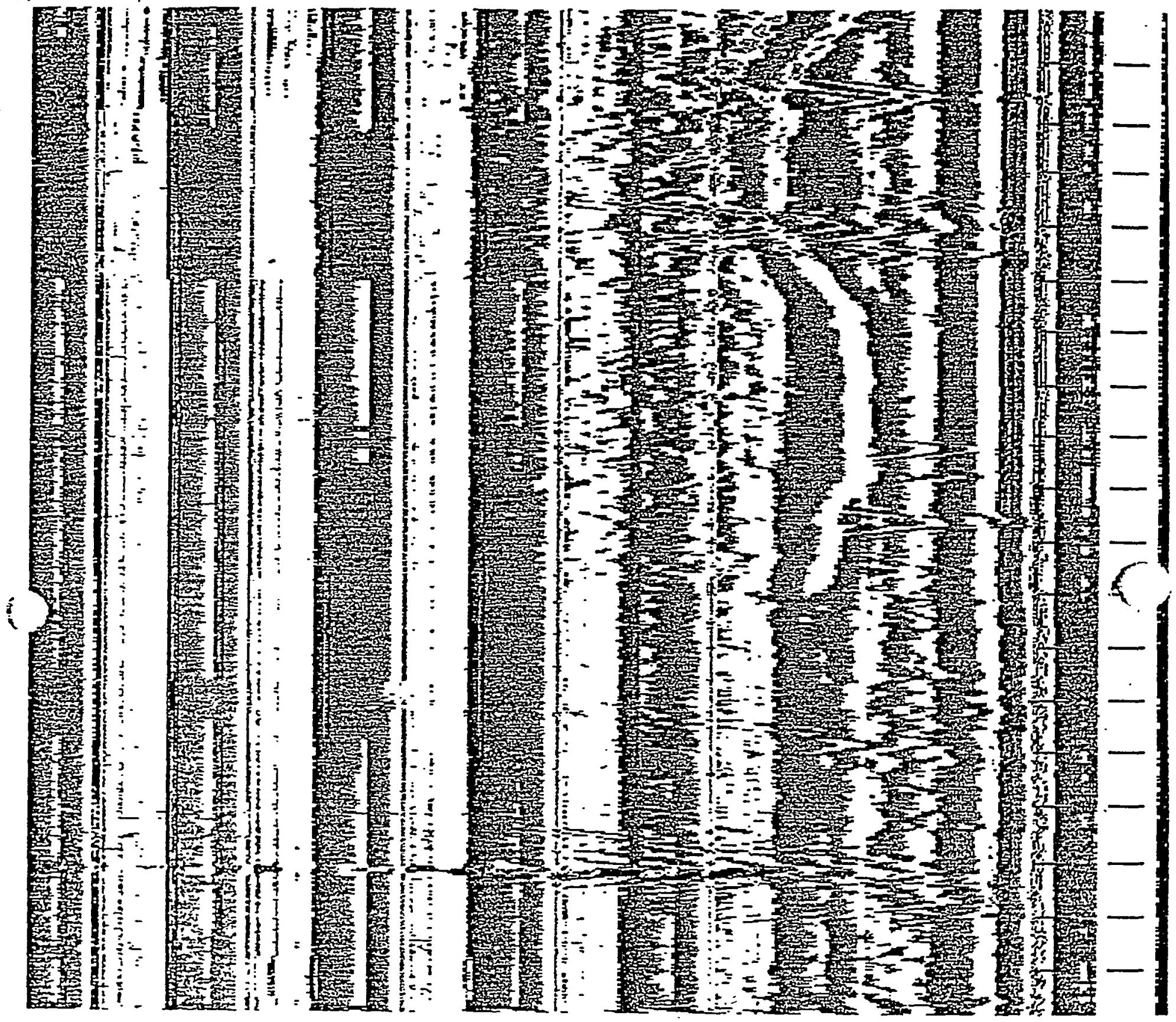


Grace Road Site

RFI/RI Report
WSRC-RP-95-93, Rev. 1

November 1995

APPENDIX A.2

GROSS ALPHA AND NON-VOLATILE BETA ANALYSES 
Grace Road Site

RFI/RI Report
WSRC-RP-95-93, Rev. 1

November 1995

. THIS PAGE INTENTIONALLY LEFT BLANK 


\title{
DON'T JUST SAY IT -- WRITE IT
}

WESTINGHOUSE SAVANNAH RNER COMPANY

Environmental Restoration-WASTE SITES

INTEROFFICE MEMORANDUM SW-ER-94-0161

MAY 12, 1994

TO: JOHNKUHNS

FROM: HOWARD HICKEY, 992-4W, 644-1802

SANDRA SMITH, 992-4W, 644-1815

RE: DESCRIPTION OF ADDTTONAL SAMPUNG AT GRACE ROAD WASTE UNTT

PER OUR CONVERSATIONS AND WALKDOWN, THE FOLLOWING IS A DESCRIPTION OFTHE ADDITIONAL SAMPLNG AND ANALYSES FOR THE GRACEROAD WASTE UNT, PER DHEC COMMENTS:

\begin{abstract}
ADDITIONAL SAMPLING WILL BE DONEAT THE GRACE ROAD WASTE UNIT USING A HAND AUGER AT THE TWO SAMPLELOCATIONS IDENTIFED ON THE ATTACHED MAP. ADDITIONAL SAMPUNG WILL ALSO BE DONEAT FIVE DISCRETE SURFACE SAMPUNG LOCATIONS THAT WILL BE IDENTIFIED AT A LATER DATE.
\end{abstract}

THE SAMPLES WILL BE ANALYZED BY GEL, AN OFFSITE LABORATORY. THE ANALYSES WILL CONSIST OF APPROXIMATELY 15 GROSS ALPHA AND NON-VOLATILE BETA ANALYSIS. TWO SOIL SAMPLES WILL BE PROVIDED TO BE USED FOR BLANKS. FOR ESTIMATING PURPOSES ONLY, FIVE ALPHA SCANS WILLBEDONE.

ATTACHED YOU WIL FIND A CHART LISTING THE SAMPLELOCATIONS, IDENTIFICATION NUMBERS, DEPTH AND ANALYSES TO BE PERFORMED. ALSO ATTACHED IS A LSTING OF THE NATURALY OCCURRING ALPHA EMITTERS TO BEANALYZED FOR, IF THEALPHA SCANS ARENECESSARY.

PLEASE SEND US A COST ESTIMATE FOR THE WORK ON THIS PROJECT AS SOONAS YOU REVIEW THE ABOVE INFORMATION AND THE ATTACHED CHARTS AND MAP.

C. - ER FILES, 992-4W 
I 
NATURALLY OCCURRING ALPHA EMITTERS

\begin{tabular}{ll} 
THORIUM SERIES & URANIUM SERIES \\
\hline THORIUM 232 & URANIUM 238 \\
THORIUM 228 & URANIUM 234 \\
RADIUM 224 & THORIUM 230 \\
RADON 220 & RADIUM 226 \\
POLONIUM 216 & RADON 222 \\
ASTATINE 216 & POLONIUM 218 \\
BISMUTH 212 & ASTATINE 218 \\
POLONIUM 212 & BISMUTH 214 \\
& POLONIUM 214
\end{tabular}




\section{GRACE ROAD ADDITIONAL SAMPLING AND ANALYSES}

\begin{tabular}{|c|c|c|c|}
\hline LOCATION & SAMPLEID & DEPTH & ANALYSES \\
\hline $\begin{array}{l}\text { GRS } 10 \\
\text { GRS } 10 \\
\text { GRS } 10 \\
\text { GRS } 10 \\
\text { GRS } 10\end{array}$ & $\begin{array}{l}\text { GRS-15-01 } \\
\text { GRS-15-02 } \\
\text { GRS-15-03 } \\
\text { GRS-15-04 } \\
\text { GRS-15-05 }\end{array}$ & $\begin{array}{l}0-1 \text { FT. } \\
-5 \text { FT. } \\
-10 \text { FT. } \\
-15 \text { FT. } \\
-20 \text { FT. }\end{array}$ & $\begin{array}{l}\text { GROSS ALPHANON-VOLATILE BETA } \\
\text { GROSS ALPHANON-VOLATILE BETA } \\
\text { GROSS ALPHANON-VOLATILEBETA } \\
\text { GROSS ALPHANON-VOLATILE BETA } \\
\text { GROSS ALPHANON-VOLATILE BETA }\end{array}$ \\
\hline $\begin{array}{l}\text { GRS } 08 \\
\text { GRS } 08 \\
\text { GRS } 08 \\
\text { GRS 08 } \\
\text { GRS 08 }\end{array}$ & $\begin{array}{l}\text { GRS-16-01 } \\
\text { GRS-16-02 } \\
\text { GRS-16-03 } \\
\text { GRS-16-04 } \\
\text { GRS-16-05 }\end{array}$ & $\begin{array}{l}0-1 \mathrm{FT} . \\
-5 \mathrm{FT} . \\
-10 \mathrm{FT} . \\
-15 \mathrm{FT} . \\
-20 \mathrm{FT} .\end{array}$ & $\begin{array}{l}\text { GROSS ALPHANON-VOLATILE BETA } \\
\text { GROSS ALPHANON-VOLATILE BETA } \\
\text { - GROSS ALPHANON-VOLATILE BETA } \\
\text { GROSS ALPHANON-VOLATILE BETA } \\
\text { GROSS ALPHANON-VOLATILE BETA }\end{array}$ \\
\hline $\begin{array}{l}\text { DISCRETE \#1 } \\
\text { DISCRETE \#2 } \\
\text { DISCRETE \#3 } \\
\text { DISCRETE \#4 } \\
\text { DISCRETE \#5 }\end{array}$ & $\begin{array}{l}\text { GRS-17-01 } \\
\text { GRS-18-01 } \\
\text { GRS-19-01 } \\
\text { GRS-20-01 } \\
\text { GRS-21-01 }\end{array}$ & $\begin{array}{ll}0-1 & \text { FT. } \\
0-1 & \text { FT. } \\
0-1 & \text { FT. } \\
0-1 & \text { FT. } \\
0-1 & \text { FT. }\end{array}$ & $\begin{array}{l}\text { GROSS ALPHANON-VOLATILE BETA } \\
\text { GROSS ALPHANON-VOLATILE BETA } \\
\text { GROSS ALPHANON-VOLATILE BETA } \\
\text { GROSS ALPHANON-VOLATILE BETA } \\
\text { GROSS ALPHANON-VOLATILE BETA }\end{array}$ \\
\hline $\begin{array}{l}\text { SOIL BLANK \#1 } \\
\text { SOIL-BLANK \#2 }\end{array}$ & $\begin{array}{l}\text { GRS-22-01 } \\
\text { GRS-23-01 }\end{array}$ & & $\begin{array}{l}\text { GROSS ALPHANON-VOLATILE BETA } \\
\text { GROSS ALPHANON-VOLATILE BETA }\end{array}$ \\
\hline
\end{tabular}

"If gross alpha exceeds $20 \mathrm{pCi} / \mathrm{g}$, then perform alpha scan.

-Expected range for gross alpha/non-volatile beta is 0-25 pCi/g. 


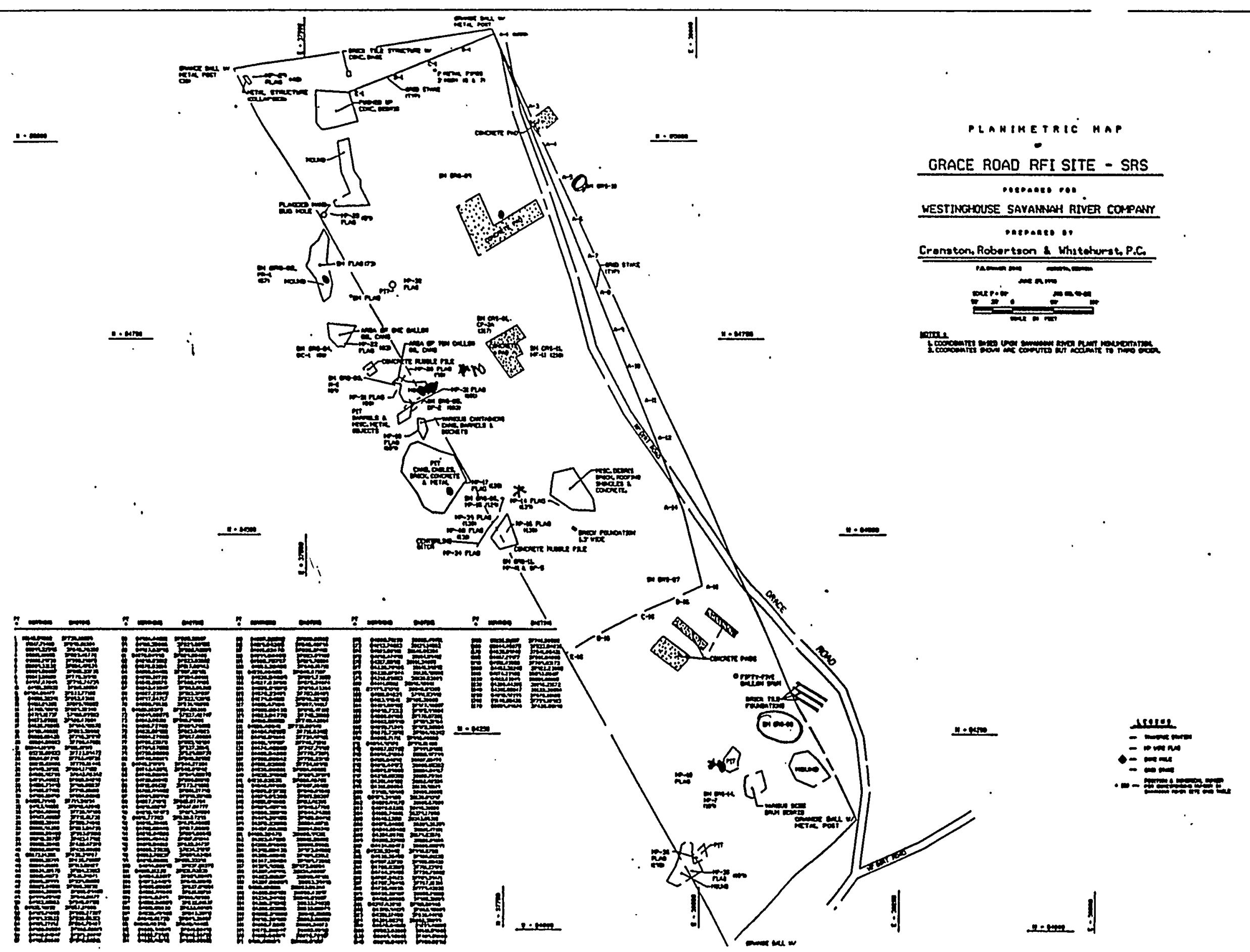




\section{SMP}

SAMPLE MANAGEMENT PROGRAM

\section{WESTINGHOUSE SAVANNAH RIVER COMPANY \\ INTER-OFFICE MEMORANDUM}

SMP-JCC-94109cF

Date: $\quad$ September 29, 1994

TO: $\quad$ Sandra B. Smith, 992-4W

From: $\quad$ JDC Crawford, 730-1M, 5-7482

Job $94109 c$

Attached are the results reported by General Engineering Laboratories (GEL) for the analysis of your samples for Job $94109 \mathrm{c}$.

Your hard copy report is considered the official record. It is your responsibility to file this report in the appropriate place for your future reference. The Sample Management Program will store a copy of this report for one year.

The Sample Management Program has not interpreted any data found in this report for regulatory purposes. You should contact your Environmental Coordinator or technical support personnel for any regulatory interpretations of the data.

Attachments:

SMP Job Log Sheet

Report

Approved By:

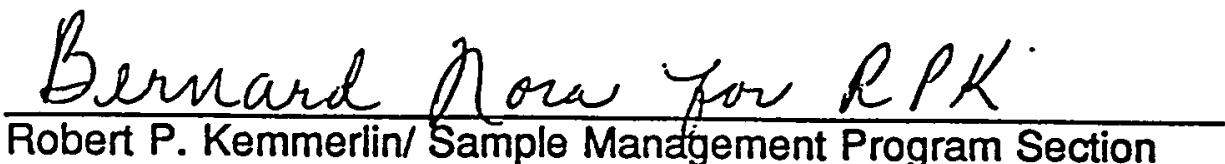


..

A. 2-10 
"JOB\# 94109 JOB LOG SHEET (U) Rov.6, 6-29.93 DATE 4/26/94

\begin{tabular}{|c|c|c|c|c|c|}
\hline CoNTH & 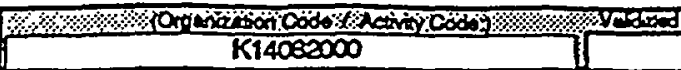 & $\mathrm{DEP} A \mathrm{M}$ MENT & APORESS & $0100 \mathrm{NE}$ & BEEPE \\
\hline Customer & 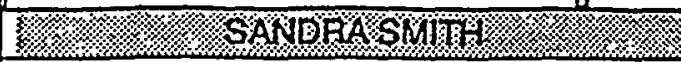 & ER & 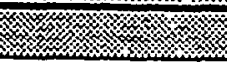 & (7815 & $\sqrt[3 \times \infty]{2 \times \infty}$ \\
\hline late & TOWALE TIEKEY & ER & & & 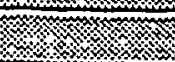 \\
\hline ryUUCON / HPO & 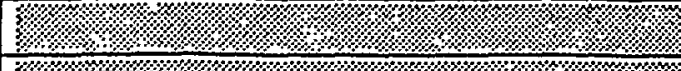 & 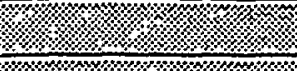 & & & 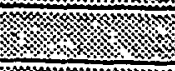 \\
\hline HMTR & 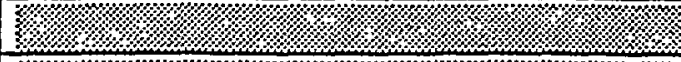 & 2. & & & 18 \\
\hline Industrial Hyg. & \% & 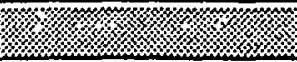 & & & (1) \\
\hline Results To: & 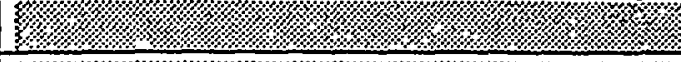 & 4. & 然 & & 2. \\
\hline 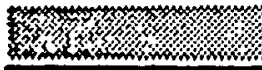 & 4 & (3) & ख & & \\
\hline
\end{tabular}

Doscription SO]| SAAUR:E

of Material:

History

GRACERTABSSIE

of Material:

Location of Material:

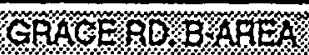

XCLEAN AREA $\square$ RCA

SizeNolume:

Condition of:

Info:

TRE

sibis

or

SaTEULTE abea $\square$ STAGing area

Material Destination: $\square$ POWER $\square$ WMO $\square$ CIF XOTHER:\%

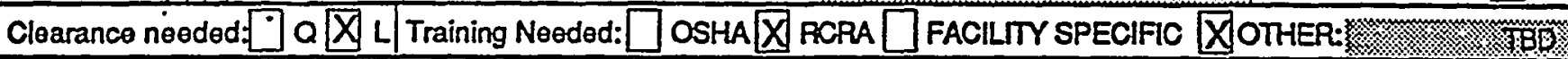

SpecialProtoctivo equipment/dotting requirements:

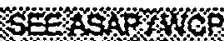

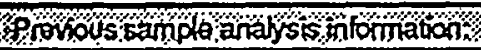

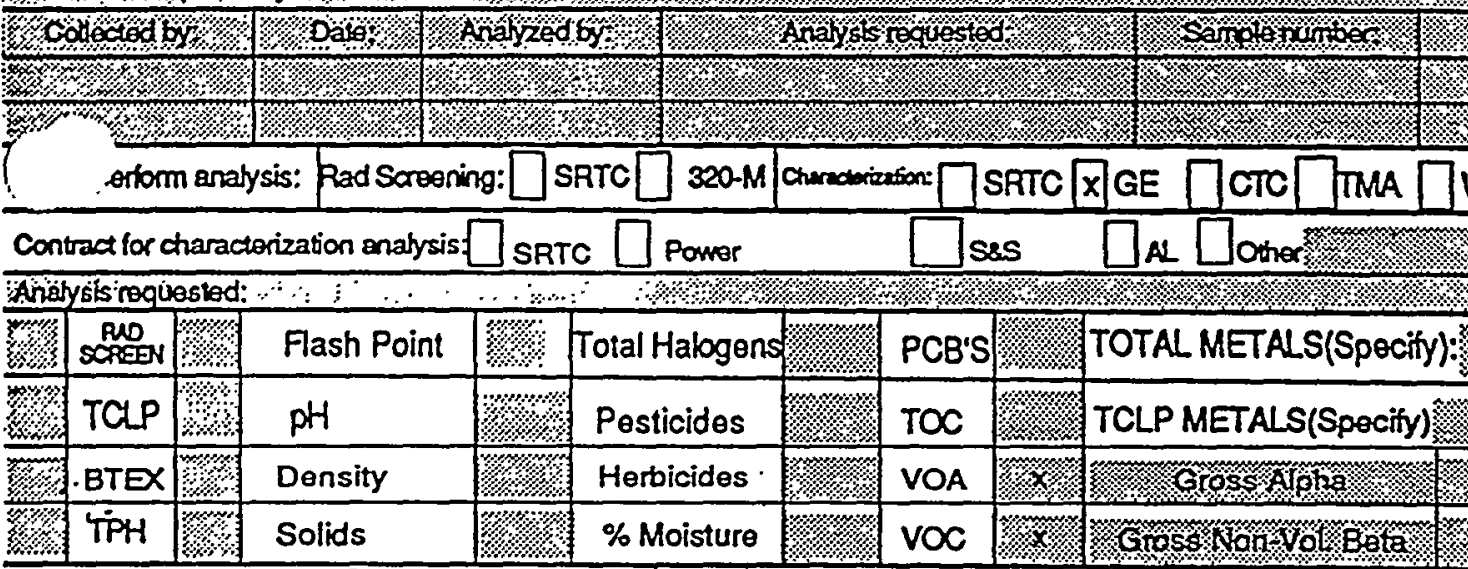

20 Day Clockinvolvad? D YES \ NO

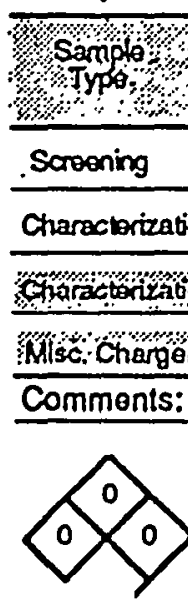

.

.

Mamber?

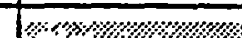

\% $715194 \%$

*11594

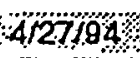

\begin{tabular}{|c|c|c|}
\hline \%anplog & \% subuitod & sionging \\
\hline $11 \%$ & & NYG \\
\hline YSTGQP & 17194 & GDP \\
\hline$G Q P$ & $814 \times 94$ & SWO \\
\hline & & \\
\hline
\end{tabular}

\section{DATEEND:}

\begin{tabular}{|c|c|c|c|}
\hline onding & pentiog & 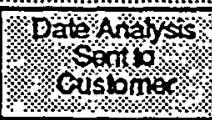 & \% \\
\hline & & & \\
\hline $6 x_{15}$ & xye\% & $907 \% 494$ & $10 \mathrm{O}$ \\
\hline 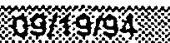 & wao $\alpha$ & 09299 & \%, \\
\hline 09$) \geq 104$ & $\alpha \%$ & 8 & mbeQ \\
\hline
\end{tabular}

- a Approved by:

(Printod nam:)

Focord Validatad by:

(Prinbod nams:
(Simnarue)

(Sipnturo)

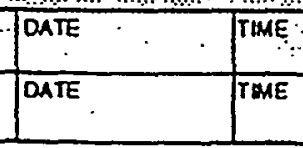




\section{Explanation of Terms and Symbols}

The following terms and symbols may be used in reports of analytical data:

\section{Quantities}

BTU Units of heat content expressed in British Thermal Units

"C or ${ }^{\circ} \mathrm{F} \quad$ Units of temperature expressed in degrees Celsius or degrees Eahrenheit

$<\quad$ Indicates the measured value isfess than the reported value

$>\quad$ Indicates the measured value is grenter than the reported value

NTU Units of turbidity expressed as Nephelometric Turbidity Units

\section{Concentrations}

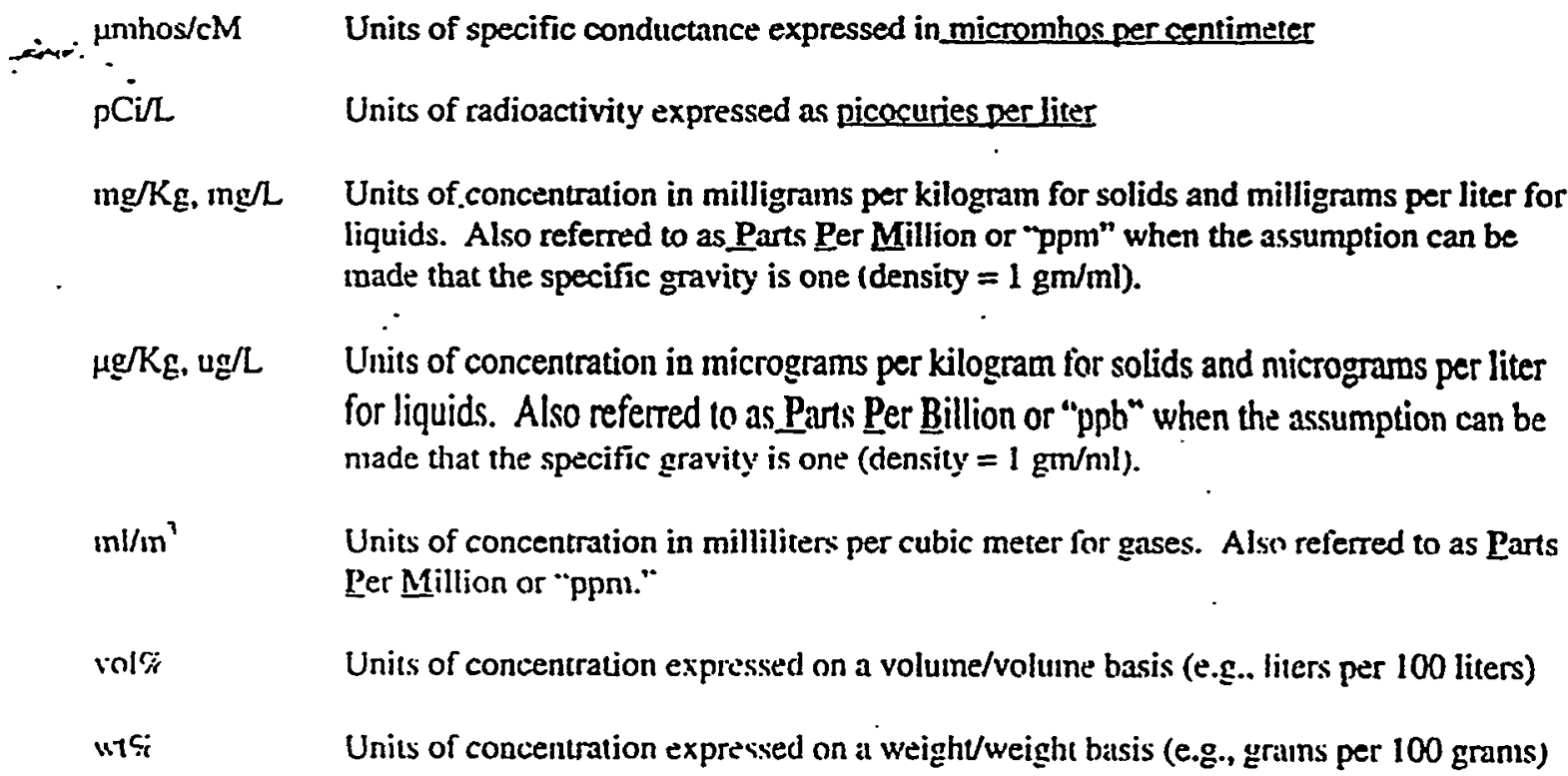

\section{Bacteriological}

MPN Units of bacteriological results expressed as Most Brobable Number

TNTC Units of bacteriological results expressed as Toio Numerous To Count

\section{WARRANTY AND LINIITATIONS OF LIABHLITY}

TIE AC CURACY OE ALL ANALYTICAL RESULTS IS WARRANTED FOR THE SAMPII: AS IT IS RECLIVHI) BY TUF I.ABORATORY. WARRANTY IOOR THE INTEGRITY OF THE SAIIII.E BEGINS AT THE TIMII IT IS PLACED IN THE POSSESSION OF AUTHORIZED GENERAL. ENGINIEERING I.ABCRRATORIISS PERS(INNIEL. ALI. OTHER WARRANTIIS. FXPRESSED OR IMPI.ITD. ARE

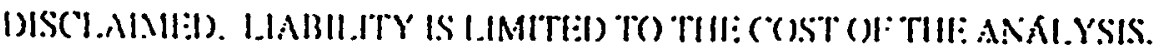


Case Narrative - Westinghouse Savannah River Site

September 20, 1994

Job No. 94109 c

page 1 of 1

\section{CASE NARRATIVE REPORT for \\ Westinghouse Savannah River Site Subcontract No. AB23504N \\ 94109c}

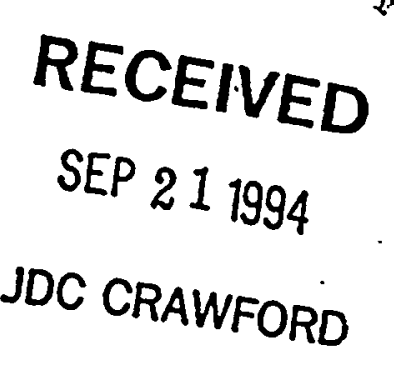

September 20, 1994

Laboratory Identification:

General Engineering Laboratories, Inc.

For Samples Received:

August 17, 1994

Sample Identification:

GRS-15-01

GRS-15-02

GRS-15-03

GRS-15-04

GRS-15-05

Lab Number: 9408337-01

Lab Number: 9408337-02

Lab Number: 9408337-03

- Lab Number: 9408337-04

Lab Number: 9408337-05

Methods Used

Gross Alpha

Nonvolatile Beta

\section{Qualitv Control Issues}

1. Samples were received in certified bottles provided by SRS, not certified bottles provided by GEL.

2. The temperature of the samples at arrival was $6^{\circ} \mathrm{C}$. There are no cited preservation requirements for radiochemistry in a soil matrix.

3. There are no bottle identification numbers entered in LIMS.

4. Results are reported on a dry weight basis.

5. Samples were scanned improperly.

Sample Dispošal

GEL will dispose of all remaining sample residue in the proper manner in accordance with all applicable regulations and statutes.

fc: wstc00194.081794.94109c

$$
\text { A. 2-13 }
$$




\section{GENERAL ENGINEERING LABORATORIES}

Meeting today's needs with a vision for tomorrow.

$\begin{array}{lr}\text { Labaratary Certincalloos } & 0 . \\ \text { FL. } & \text { E87156r87204 } \\ \text { NC } & 233 \\ \text { SC } & 10120 \\ \text { IN } & 02934 \\ \text { VA } & 00151 \\ \text { WI } & 99985779\end{array}$

CERTIFICATE OF ANALYSIS

$\begin{array}{ll}\text { Client: } & \text { Westinghouse Savannah River Co. } \\ & \text { Bldg. 730-1M, Cube 195 } \\ & \text { PO Box 616 } \\ & \text { Aiken, South Carolina 29803-6105 } \\ \text { Contact: } & \text { Ms. Janet Crawford }\end{array}$

Project Description: Hazardous Waste Contract

œ. WSRCO0194

Report Date: September 07, 1994

\section{RECEIVED}

\author{
SEP 211994
}

JDC CRAWFORD

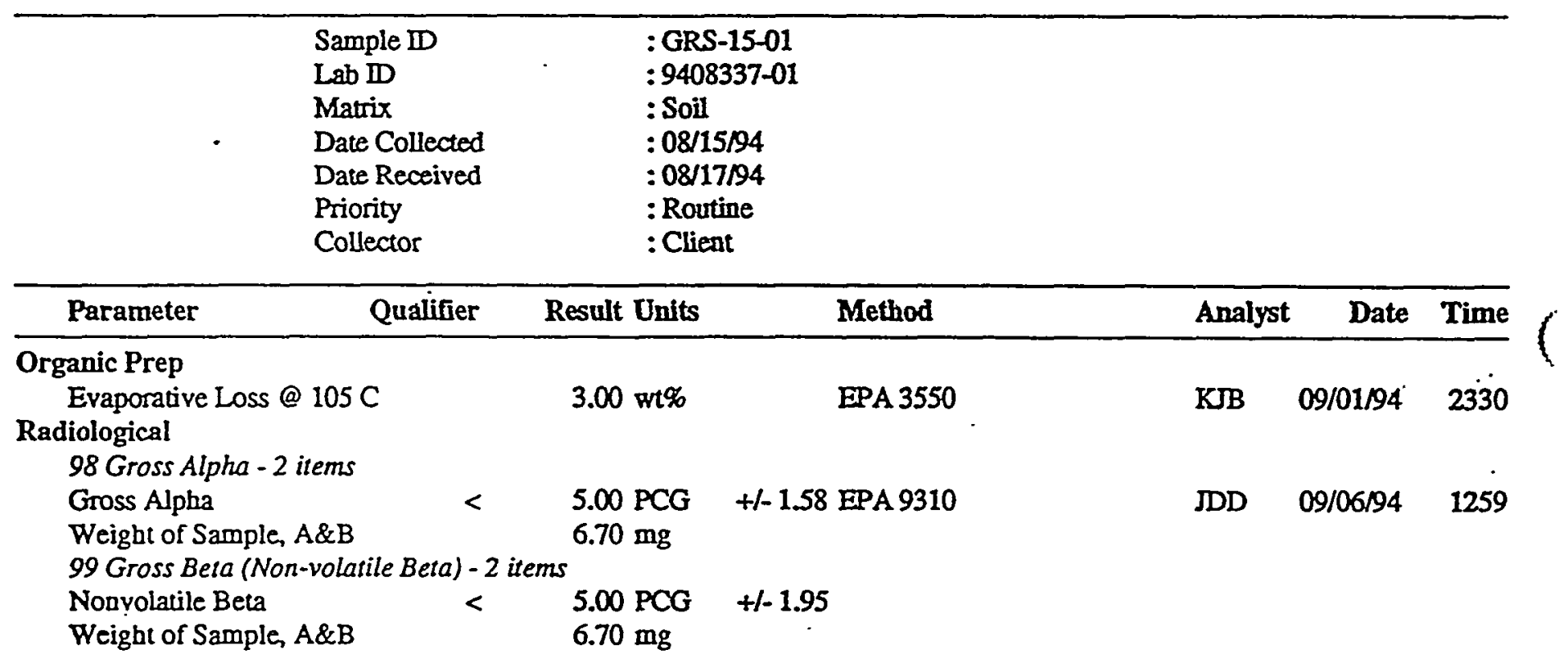

Data reported in mass/mass units is reported as 'dry weight'.

This data report has been prepared and reviewed in accordance with General Engineering Laboratories standard operating procedures. Please direct any questions to your.Project Manager, Edie Kent at (803) 769-7385.

- Babun Aufoon

Analytical Report Specialist 


\section{GENERAL ENGINEERING LABORATORIES}

Meeting today's needs with a vision for tomorrow.

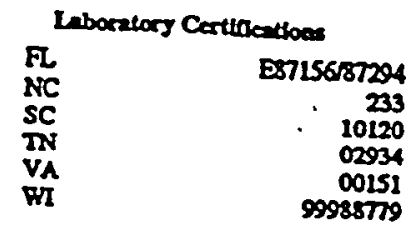

CERTIFICATE OF ANALYSIS
Client Westinghouse Savannah River Co. Bldg. 730-1M, Cube 195
PO Box 616
Contact Ms. Janet Crawford

Project Description: Hazardous Waste Contract
RECEIVED

SEP 211994.

JDC CRAWFORD

Repart Date: September 07, 1994

$\begin{array}{ll}\text { Sample ID } & \text { : GRS-15-02 } \\ \text { Lab ID } & : 9408337-02 \\ \text { Matrix } & : \text { Soil } \\ \text { Date Collected } & : 08 / 15 / 94 \\ \text { Date Received } & : 08 / 17 / 94 \\ \text { Priority } & : \text { Routine } \\ \text { Collector } & : \text { Client }\end{array}$

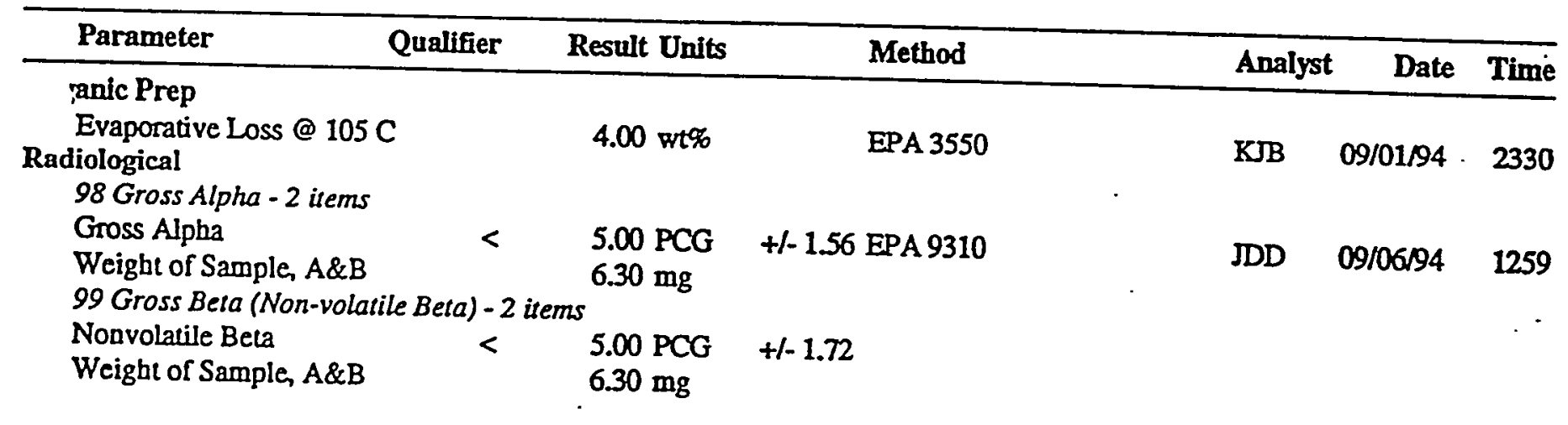

Data reported in mass/mass units is reported as 'dry weight'.

This data report has been prepared and reviewed

in accordance with General Engineering Laboratories

standard operating procedures. Please direct

any questions to your Project Manager, Edie Kent at (803).769-7385.

deratan A. Sejon

Analytical Report Specialist 


\section{GENERAL ENGINEERING LABORATORIES}

Meeting today's needs with a vision for tomorrow.

Client: Westinghouse Savannah River Co. Bldg. 730-1M, Cube 195

PO Box 616

Aiken, South Carolina 29803-6105

Contact: Ms. Janet Crawford

RECEIVED

SEP 211994

Project Description: Hazardous Waste Contract

\section{JDC CRAWFORD}

œ: WSRC00194

Report Date: September 07, 1994

Page 1 of 1

$\begin{array}{ll}\text { Sample ID } & : \text { GRS-15-03 } \\ \text { Lab ID } & : 9408337-03 \\ \text { Matrix } & : \text { Soil } \\ \text { Date Collected } & : 08 / 15 / 94 \\ \text { Date Received } & : 08 / 17 / 94 \\ \text { Priority } & : \text { Routine } \\ \text { Collector } & : \text { Client }\end{array}$

\begin{tabular}{|c|c|c|c|c|c|}
\hline Parameter & Result Units & Method & Analyst & Date & Time \\
\hline \multicolumn{6}{|l|}{ Organic Prep } \\
\hline $\begin{array}{l}\text { Evaporative Loss @ } 105 \mathrm{C} \\
\text { Radiological } \\
98 \text { Gross Alpha - } 2 \text { items }\end{array}$ & $15.0 \mathrm{wt} \%$ & EPA 3550 & $\mathrm{KJB}$ & $09 / 01 / 94$ & 2330 \\
\hline $\begin{array}{l}\text { Gross Alpha } \\
\text { Weight of Sample, A\&B } \\
99 \text { Gross Beta (Non-volatile Beta) - } 2\end{array}$ & $\begin{array}{l}13.3 \mathrm{PCG} \\
34.1 \mathrm{mg} \\
m s\end{array}$ & $+/-3.48$ EPA 9310 & JDD & $09 / 06 / 94$ & 1259 \\
\hline $\begin{array}{l}\text { Nonvolatile Beta } \\
\text { Weight of Sample, A\&B }\end{array}$ & $\begin{array}{l}5.73 \mathrm{PCG} \\
34.1 \mathrm{mg}\end{array}$ & $+1-2.33$ & & . & \\
\hline
\end{tabular}

Data reported in mass/mass units is reported as 'dry weight'.

This data report has been prepared and reviewed. in accordance with General Engineering Laboratories standard operating procedures. Please direct any questions to your Project Manager, Edie Kent at (803) 769-7385.

Baban A.Srexn

Analytical Report Specialist 


\section{GENERAL ENGINEERING LABORATORIES}

Meeting loday's needs with a vision for tomorrow.

CERTIFICATE OF ANALYSIS

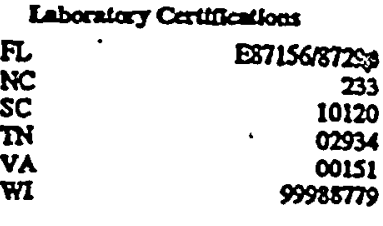

$\begin{array}{lll} & \text { Client } & \text { Westinghouse Savannah River Co. } \\ & & \text { Bldg. 730-1M, Cube 195 } \\ & \text { PO Box 616 } \\ & \text { Aiken, South Carolina 29803-6105 } \\ . \quad & \text { Contact: } & \text { Ms. Janet Crawford }\end{array}$

Project Description: Hazandous Waste Contract

œ: WSRC00194

Report Date: September 07, 1994

\section{RECEIVED}

\author{
SEP 211994
}

JDC CRAUNFur:

\begin{tabular}{|c|c|c|c|c|c|c|c|}
\hline$\cdot$ & $\begin{array}{l}\text { Sample ID } \\
\text { Lab ID } \\
\text { Matrix } \\
\text { Date Collected } \\
\text { Date Received } \\
\text { Priority } \\
\text { Collector }\end{array}$ & $\begin{array}{l}\text { : GR } \\
: 940 \\
\text { : Soil } \\
\text { :08/1 } \\
\text { :08/1 } \\
\text { : Rou } \\
\text { :Clie }\end{array}$ & $\begin{array}{l}-15-04 \\
337-04 \\
194 \\
194 \\
\text { ine } \\
t\end{array}$ & . & - & . & . \\
\hline Parameter & Qualifier & Result Units & Method & & Analyst & Date & Time \\
\hline \multicolumn{8}{|c|}{ Organic Prep } \\
\hline \multicolumn{2}{|c|}{$\begin{array}{l}\text { Evaporative Loss @ } 105 \text { C } \\
\text { _adiological }\end{array}$} & $10.0 \mathrm{wt} \%$ & \multicolumn{2}{|l|}{ EPA 3550} & KIB & $09 / 01 / 94$ & 2330 \\
\hline $\begin{array}{l}\text { Gross Alpha } \\
\text { Weight of Sample, }\end{array}$ & A\&B & $\begin{array}{l}14.5 \text { PCG } \\
33.9 \mathrm{mg}\end{array}$ & $+1-3.72$ EPA 9310 & $\cdot$ & JDD & $09 / 06 / 94$ & 1259 \\
\hline \multicolumn{2}{|c|}{$\begin{array}{l}\text { Nonvolatile Beta } \\
\text { Weight of Sample, A\&B }\end{array}$} & $\begin{array}{l}5.10 \mathrm{PCG} \\
33.9 \mathrm{mg}\end{array}$ & $+1-2.35$ & & & & \\
\hline
\end{tabular}

Data reported in mass/mass units is reported as 'dry weight'.

This data report has been prepared and reviewed

in accordance with General Engiricering Laboratories

standard operating procedures. Pleasedirect

any questions to your Project Manager, Edie Kent at (803) 769-7385.

Boubuan A. Souse

-Analytical Report Specialist 


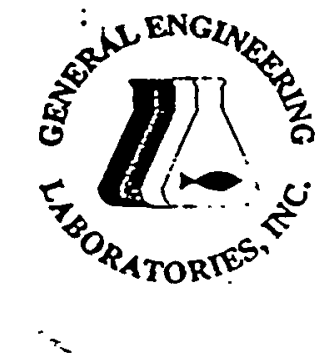

GENERAL ENGINEERING LABORATORIES

Meeting today's needs with a vision for tomorrow.

taboratary Oattibations

FL
NC
SC
IN
VA
WI

E87156r87294

10120

02934

00151

CERTIFICATE OF ANALYSIS

\section{Client: Westinghouse Savannah River Co. \\ Bldg. 730-1M, Cube 195 \\ PO Box 616 \\ Aiken, South Carolina 29803-6105 \\ Contact Ms. Janet Crawford}

Project Description: Hazardous Waste Contract

\section{RECEIVED}

\author{
SEP 211994
}

JDC CRAWFORD

Report Date: September 07, 1994

Page 1 of 1

\begin{tabular}{|c|c|c|c|c|c|c|}
\hline 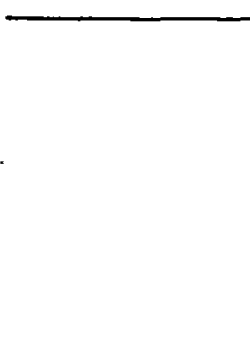 & $\begin{array}{l}\text { Sample ID } \\
\text { Lab ID } \\
\text { Matrix } \\
\text { Date Collected } \\
\text { Date Received } \\
\text { Priority } \\
\text { Collector }\end{array}$ & \multicolumn{2}{|c|}{$\begin{array}{l}\text { : GRS-15-05 } \\
\text { : } 9408337-05 \\
\text { : Soil } \\
\text { :08/15/94 } \\
\text { : 08/17/94 } \\
\text { : Routine } \\
\text { : Client }\end{array}$} & \multirow[b]{2}{*}{ Analyst } & \multirow[b]{2}{*}{ Date } & \multirow[b]{2}{*}{ Time } \\
\hline Parameter & Qualifier & Result Units & Method & & & \\
\hline \multicolumn{7}{|c|}{ Organic Prep } \\
\hline & Radiological & $10.0 \mathrm{wt} \%$ & EPA 3550 & $\mathrm{KJB}$ & $09 / 01 / 94$ & 2330 \\
\hline \multicolumn{2}{|c|}{$\begin{array}{l}98 \text { Gross Alpha - } 2 \text { items } \\
\text { Gross Alpha }\end{array}$} & $\begin{array}{l}8.53 \mathrm{PCG} \\
22.4 \mathrm{mg}\end{array}$ & $+/-2.80$ EPA 9310 & $\mathrm{JDD}$ & $09 / 06 / 94$ & 1259 \\
\hline $\begin{array}{l}99 \text { Gross Be } \\
\text { Nonvolatile } \\
\text { Weight of S }\end{array}$ & $\begin{array}{l}\text {-volatile Beta) - } 2 \\
\text { A\&B }\end{array}$ & \multicolumn{2}{|c|}{$\begin{array}{l}99 \text { Gross Beta (Non-volatile Beta) - } 2 \text { items } \\
\text { Nonvolatile Beta } \\
8.02 \text { PCG }\end{array}$} & & & $\therefore$ \\
\hline
\end{tabular}

Data reported in mass/mass units is reported as 'dry weight'.

This data repor has been prepared and reviewed

in accordance with General Engineering Laboratories

standard operating procedures. Please direct

any questions to your Project Manager, Edie Kent at (803) 769-7385.

Babent. Stim

Analytical Report Specialist 
Clleints Hastinghouse savannah Rlver Co. Bldg. 730-1H, Cube 295

Asken, SC 29803-6105

Contact: Ms. Sanet Crawford.

\section{RECEIVED}

SEP 211994

\section{JDC CRAWFORD}

sasple: 9408337-01 Cllent ID:

roject: WSRC00194

Manager: ExR

\author{
Matrix: 5011 \\ Sampled: 08/15/94
}

Descrlption: GRS-15-02 Received: 08/27/94 Page: 1

cates

DF surs.

Blank

RPD

ARe

10: Other Organic Teste Kethod: EPA 3550 Evaporative Loss $105 \mathrm{C} \quad 3.00$

C: Npha a Beta arose Alpha

Welght of sample, AfB

30. Nipha \& Bota Momolatile Beta tht of sample, AtB
Yethod, ERA 9310

$3.07 \mathrm{~J}$

$6.70 \mathrm{JB}$

Mothod: EPA 9310

2.57 J

$6.70 \mathrm{JB}$

Batch: 53156 Rum Analyat: IWB Dax: 09/01/94 TOA: 2330 oK $\begin{array}{lllll}\text { wtt } 1 & 1: 1 & 0.00 & 0.008\end{array}$ Batch: 53215 Run Analyet: J00 Dan: 09/06/94 man: 1259 or $\begin{array}{llllll}P C 6 & 5 & 100 & 1 i 1 & -0.123 & -7.96 t\end{array}$ 1.30 . Batch: 53215 Rum aralyats JDD DaAt 09/06/94 nas 1259 ox $\begin{array}{llllll}\text { PCO } & 5 & P C O & 1: 1 & 0.0270 & 2006\end{array}$ 1.30

lata reported in mass/mass units is reported as 'dry weight'.

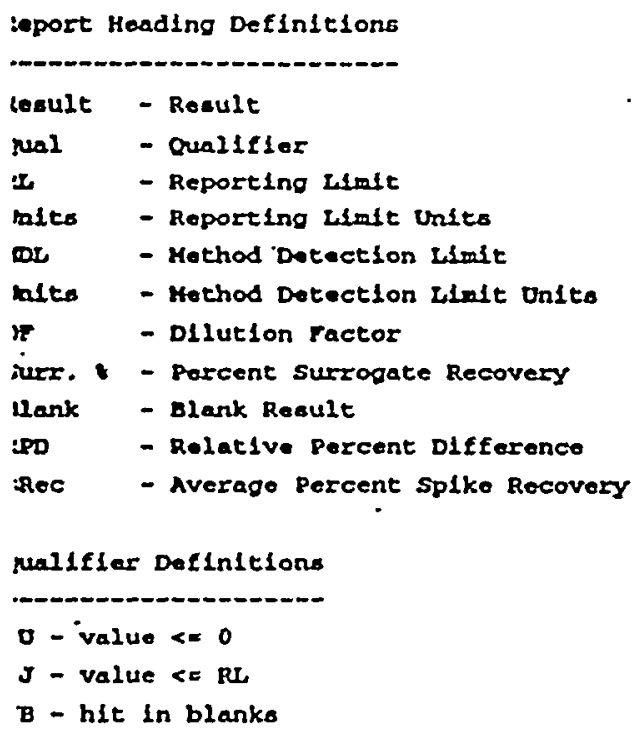




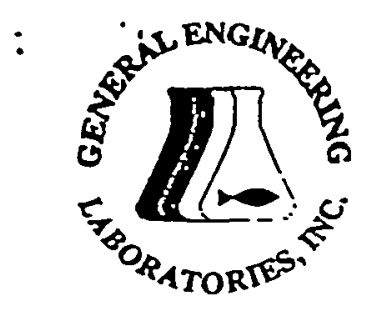

\section{GENERAL ENGINEERING LABORATORIES}

Meeting today's needs with a vision for tomorrow.

$2 x^{2}$

CERTIFICATE OF ANALYSIS

Iaboratary Cartifloation

II

$587156 / 87294$

rec

233

$s c$

2xi

$107 \sin ^{2}$

of

VA

Cllent: Westlnghouse savannah Rlver. Co. RECEIVED Bldg. 730-11, cibe 195 Aiken, Sc 29803-6105

contact: Hs. Janet Crawford

SEP 211994

JDC CRAWFORD Repart Date: 09/07/9.4

Semples 9408337-01 client ID:

7zoject: usro00194

Hanager: ExR

\author{
Naterixi sol1 \\ samplod: 08/15/94
}

Description: CRs-25-01

Recelved: 08/27/94

Page: 2

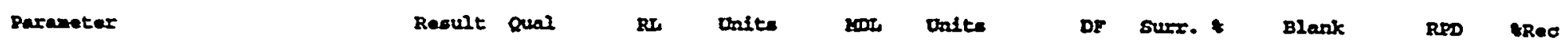

Thlo data repart has boen prepared and reviewod in accordanco with General Engineering Iaboratorles stentard operating procedures. Please direct uny questions to your Project Manager, Edie Kent at (803) 769-7385.

BabbanA. Susin

ualytical Report spoclalist 


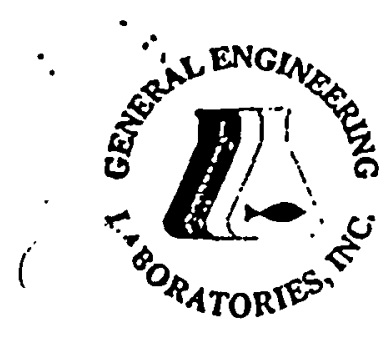

\section{GENERAL ENGINEERING LABORATORIES}

Meeting today's needs with a vision for tomorrow.

Cllents Westinghouse savannah River Co. BIdg. 730-11, Cube 195

Alken, se 29803-6105

samples 9108337-02 C11ent ID:

irofect: WSRC00194 Manager: ERT
Materixi sol1 Sampled: 08/15/94
Descripelons cors-25-02

Rocelived 08/17/94 Page: 1
30, Other Organic Teite Hathod: EPA 3550

Evaporative Loen a $105 \mathrm{C}$

30. Ilpha \& Beta Crase Nlpho

Welght of sample, ACB

30i Alpha \& Bota

- rolat1lo Beta

I the of sample, ASB

4.00

Method: EPA 9310

$\begin{array}{lll}9310 & & \\ 2.51 & 5 & 5\end{array}$

Method: EPA. 9310

$\begin{array}{rr}0.180 \\ 6.30 & 3\end{array}$

-

late repoxted in wass/mass unfts is roported as 'dry welght'.
Batch: 53156 Run Analyat: XwB DOA: 09/01/96 Tax: 2330 OK wt 1 wt $1.1 \quad 0.00 \quad 0.008$ satch: 53215 Run Analyst: JDD Dax: 09/06/94 man: 2259 OK

$\begin{array}{llllll}806 & 5 & -1.1 & -0.123 & -7.96 \% & 82.7 *\end{array}$ $0 \quad 0 \quad 1.1 \quad 0 \quad 1.30 \quad \ldots$ Batch: 53215 Rum Analyat: JDO Dax: 09/06/94 rax: 2259 ox"

$\begin{array}{llllll}P 00 & 5 & 1.1 & 0.0270 & 200 t & 75.68\end{array}$ $m \quad 0 \quad m \quad 1: 1$
N/C

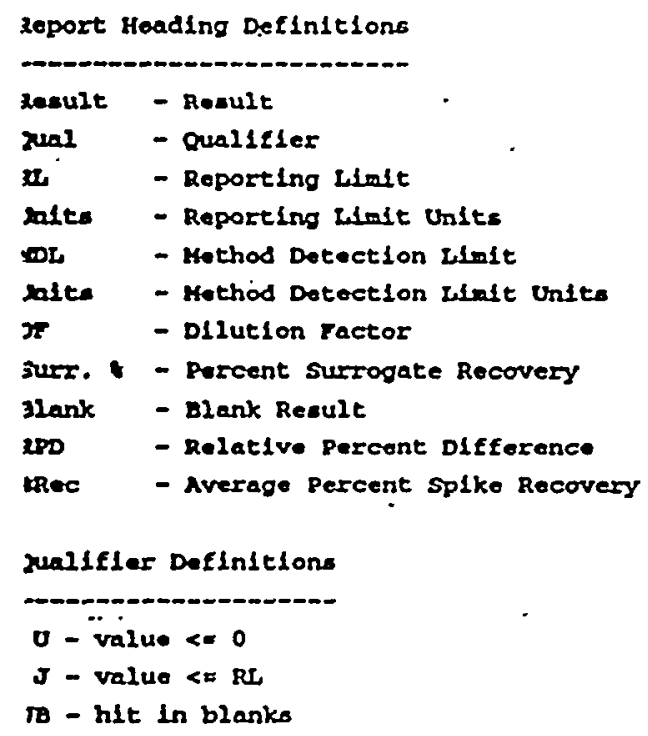


Cllent, WeatInghouse savannah RIver Co. Bldg. 730-11, Cube 195 Aiken, sc 29803-6105

Contect: Hs. Janet Crawford

\section{RECEIVED}

\author{
SEP 211994
}

Report Date: 09/07/94

\section{JDC CRAWFORD}

Dascription: CRS-15-02

Received: 08/17/94

\section{Pager 2}

zroject: KSRC00194 Manager: DIR Sampled: 08/15/94

Result gual

vin. Onite
RPD 


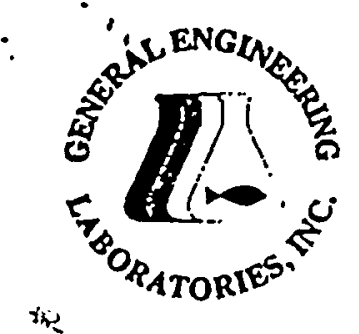

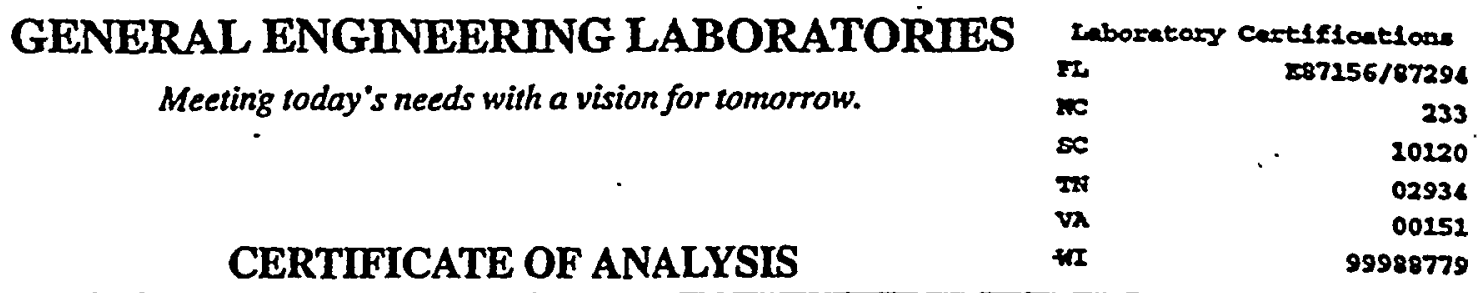

c1lent: Weatinghouse savannah plver co. RECEIVEC
Bldg. 730-1H, Cubb 195

Aiken, SC 29803-6105

Contact: Hs. Janet Crawford

SEP 211994

JDC CRAWFOR:

Repart Date: 09/07/96

Samples 9408337-03 Client ID:

Katrixi so11

sampled: $08 / 15 / 94$

Descelption: CRS-15-03

Received: 08/17/94 Rages 1

Profects KSRC00194 Manager: ERT

Result Qual

Ru tonste

sors conster

DF surr. t. Blank

RPD

Wee

\begin{tabular}{|c|c|c|c|c|c|c|c|c|c|c|c|c|}
\hline $30:$ & $\begin{array}{l}\text { Other organse Teste } \\
\text { Evaporative Loss e } 105\end{array}$ & $\begin{array}{l}\text { Method: } \\
\text { c }\end{array}$ & $\begin{array}{c}\text { EPA } 3550 \\
15.0\end{array}$ & & 1 & $\begin{array}{l}\text { Batch: } \\
\text { wt: }\end{array}$ & $\begin{array}{c}53156 \\
1\end{array}$ & $\begin{array}{l}\text { Rum Analyat: } \\
\text { wtt }\end{array}$ & $\begin{array}{l}\text { ET Dan: } \\
1: 1\end{array}$ & $\begin{array}{c}09 / 01 / 94 \text { 201: } 2330 \\
0.00\end{array}$ & $\begin{array}{l}0 x \\
0.002\end{array}$ & $n / c$ \\
\hline $30:$ & Nipha \& Beta & Method: & EPA 9310 & & & Batch: & $\mathbf{5 3 2 1 5}$ & Bun Analyat: & SDD DOA: & 09/06/96 IOA: 1259 & ox & \\
\hline & Oroes Npha & & 13.3 & & 5 & $P C 0$ & $\mathbf{5}$ & $\mathbf{P O C}$ & $-1: 1$ & -0.223 & $-7.96:$ & $82.7 *$ \\
\hline & Wolght of Sample, ACB & & $\begin{array}{c}34.1 \\
=82-0310\end{array}$ & SB & 0 & $=0$ & 0 & Dom & $1: 2$ & 1.30 & . & \\
\hline 802 & Mlpha Bota & Hethod: & $\begin{array}{c}2.73-9310 \\
5.73\end{array}$ & & 5 & 800 & $\begin{array}{c}33215 \\
5\end{array}$ & $\begin{array}{l}\text { Ros } \\
\text { PCo }\end{array}$ & $1: 1$ & $\begin{array}{c}09706 / 98 \text { Tan: } 1259 \\
0.0270\end{array}$ & $200 t$ & 75.64 \\
\hline & ith of Somple, ASB & & 34.1 & JB & 0 & $\mathbf{m g}$ & $\mathbf{0}$ & $\infty$ & $1: 1$ & 2.30 & & \\
\hline
\end{tabular}

sata reported in mass/mass units is reported as 'dry weight'.

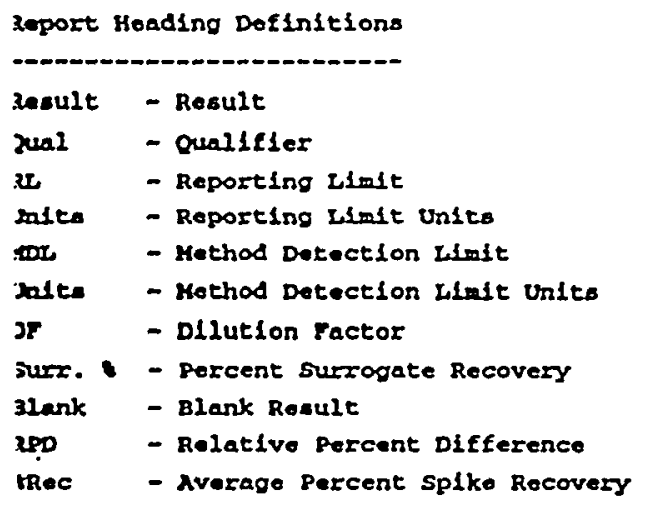

zealleler Dofinitions

0 - válue $<=0$

$J$ - value $<=R L$

iB - hite in blanks 
GENERAL ENGINEERING LABORATORIES

Meeting today's needs with a vision for tomorrow.

Meeing roday's needs with a vision for romorrow.

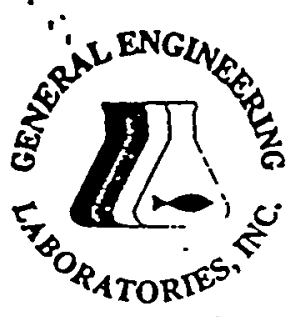

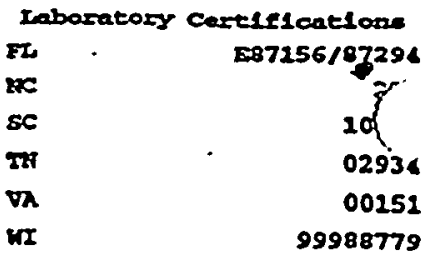

99988779
Cllent: Westlnghouse Savannah River co.

Bldg. 730-14, Cube 195

Aiken, SC 29803-6105

Contact: Ms. Janet Crawford

RECEIVED

SEP 211994

\section{JDC CRAWFORD}

Report Date: 09/07/94

Canple1 9408337-03 C11ent ID:

Hatrix: Sol1

Sampled: 08/25/94

roject: HSRC00194

Manager: Dak

Peranoter

Result Qual

Descoription: GR5-15-03

Roceived: $08 / 17 / 94$

Page: 2

Thl data report has been prepared and reviewed in eceordance with General Engineering Laboratories standard operating procedures. Please direct

wy questions to your Project kanager, Edle Rent at (803) 769-7385.

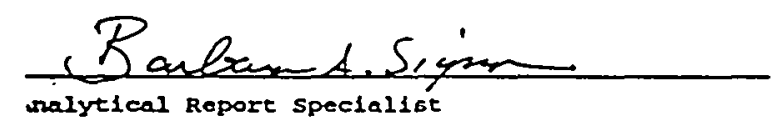




\section{Client: Westinghoure savannah Rlver co. REOEMUED
Bldg. 730-14, cubo 195 \\ Niken, SC 29803-6105}

Contact: Ms. Janet Crawford

SEP 211994

JDC CRAWFORO

Latrix: sol1

Somplod: 08/15/94
Description ces-15-04

Recelvedi 08/17/94

Report Datez 09/07/94

Saspler 9408337-04 cllent ID: Profects WSRC00194

Manager: $2 x$

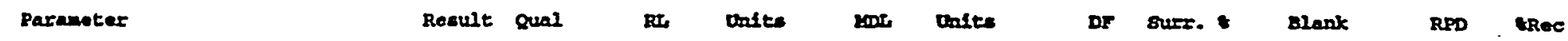

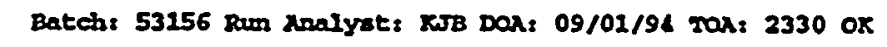

Other Oxganle Teits Method: EPA 3550

Hethod: EPA 9310

1

vet 1 wet 1,1

$0.00 \quad 0.008$

Batch: 53225 Run Analyst, JDD DOA: 09/06/94 IaA: 1259 OK

Oroses npha

14.5

33.9 JB

1,1

$-0.123$

$-7.96 t$

82.74

$\infty$

satchs 53215 Rum Aralyat: JDO DOAs 09/06/91 TaA: 1259 OK.

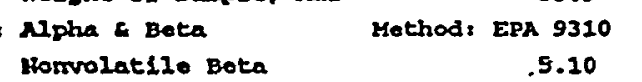

w-" Tht of somple, AfB

33.9 JB

0.0270

$2008 \quad 75.68$
$1: 1$

$1: 1$
1.30

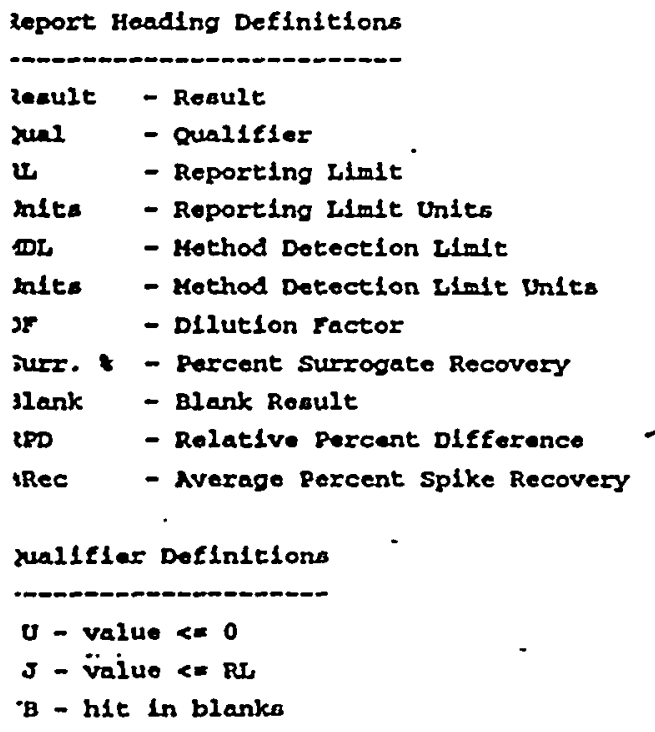




\section{GENERAL ENGINEERING LABORATORIES Laboratory certification \\ Meeting today's needs with a vision for tomorrow. \\ If \\ ac. \\ sc \\ Ixx \\ v \\ is \\ 287156/87294 ब. 232 iq $02 s$. \\ 00251

Report Date: 09/07/94

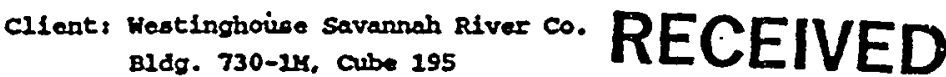

Miken, sc 29803-6105

Contact: Hs. Janet Crawtord

SEP 211994

JDC CRAWFORD

Hatrix: soil Deseription: Coss-15-01

Sampled: 08/25/94 Recelved: 08/17/94

Recelved: 08/17/94 Page: 2

Sample: 9408337-06 C11ent ID:

Hasager: ERT

RI

Onite.

2or.

unite

$\mathbf{D F}$

surr.

Blank - RPD

SRec

This data report has been prepared and reviewed

is accordance with Goneral Engineering Laboratories

itanderd operating proceduros. Please direct

uny questions to your Profect Manager, Edie Kent at (803) 769-7385.

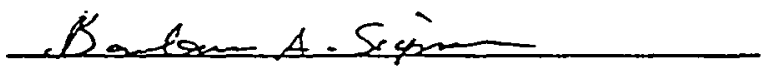

uralytical Report specialist 


\section{GENERAL ENGINEERING LABORATORIES}

Meeting today's needs with a vision for tomorrow.

$\begin{array}{lr}\text { Iaboratary Certiflaytlone } \\ \text { II } & 237156 / 87294 \\ \text { IC } & 10120 \\ \text { Sc } & 02936 \\ \text { Ix } & 00151 \\ \text { VI } & 99988779\end{array}$

Client: Westinghouse Savannah River co. BIdg. 730-1K, cube 195 Mken, SC 29803-6105

contact: Hs. Janet Crawtord

\section{RECEIVED}

SEP 211994 Report Dates 09/07/94 sample: 9108337-05 c11ent ID:

Project: HSRc00194

Manager: BrK

vaterix: 6011

Sampled: 08/15/94
JBG GRALIVROBATOS

Recelved; $08 / 17 / 98$

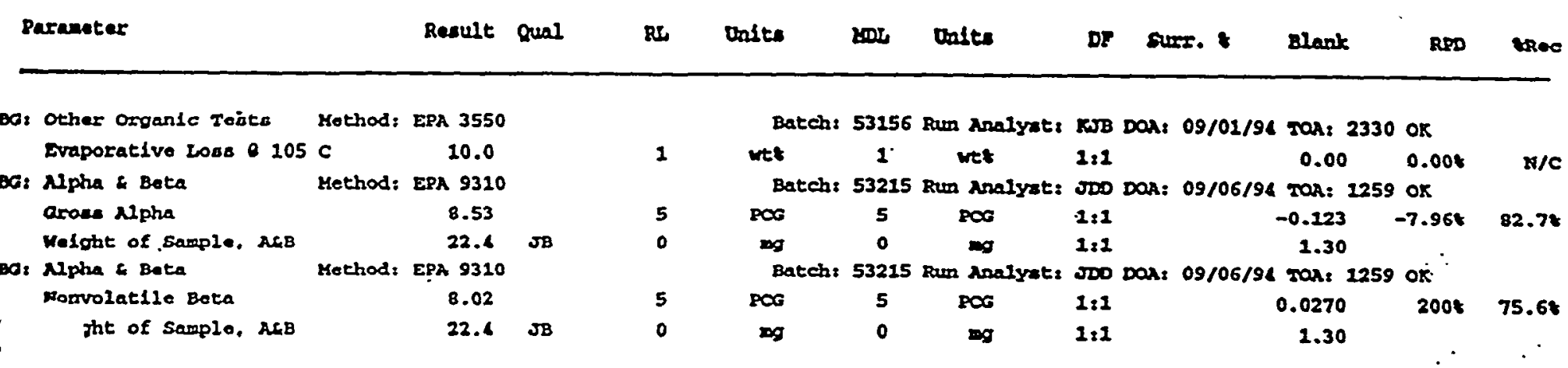

Date roported in mass/mass unlth is reported as 'dry weight'.

Roport Heading Definttlons

Renult - Reault

rual - Qualleler

aI - Reporting Llalt

Jolte - Reporelng Limlt units

wor - Kethod Datection Ilmit

arte - Method Detection Ilalt Units

DF. - Diluelon ractor

Surz. - Percent surrogate Recovery

elank - Blank Reault

2PD - Rolative percent Dlfference

unec - Average Percent Splke Recovery

pelleler Defintelon.

U - válue <e 0

$J$ - value <a RL

IB - hit in blanks 


\section{GENERAL ENGINEERING LABORATORIES}

Meeting today's needs with a vision for tomorrow.

CERTIFICATE OF ANALYSIS raboratory curteflontione it.

inc.

$8 \mathrm{C}$

ax

VA

ix
$287256 / 87294$
Client: Westinghouse Savannah River Co.

BIdg. 730-14, Cube 195

Aiken, sc 29803-6105

Contact: Hs. Janet Crauford
RECEIVED

SEP 211994

\section{JDC CRAWFORD}

Report Date: 09/07/94
Sapple: 9408337-05 CIlent ID:

Project: USRC00194 Kanager: ERK
Kateix: soll

Sampled: $08 / 25 / 96$
Description: GRS-15-05

Received: $08 / 17 / 94$ Rage: 2

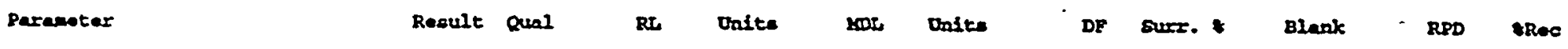

Inls data report has been prepared and reviewed

in accordance with General Dngineering Laboratorles

standard operating procodures. Please direct

iny quections to your Project Manager, Edie Kent at (803) 769-7385.

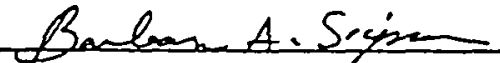

inalytical Report speclailat 


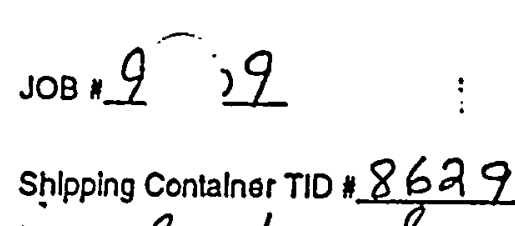

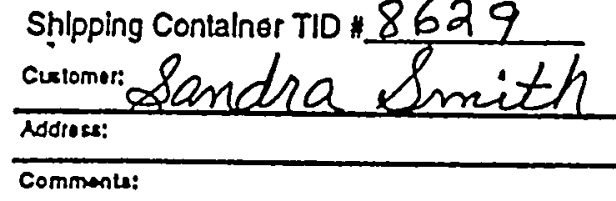

\section{crme}

CHAIN-OF-C TODY (U) Wosting house Sevansan River Compery, Savennah Rlver Sno Anabsical Laboratortos, Waslo Anabyets Group Nken, South Carolline
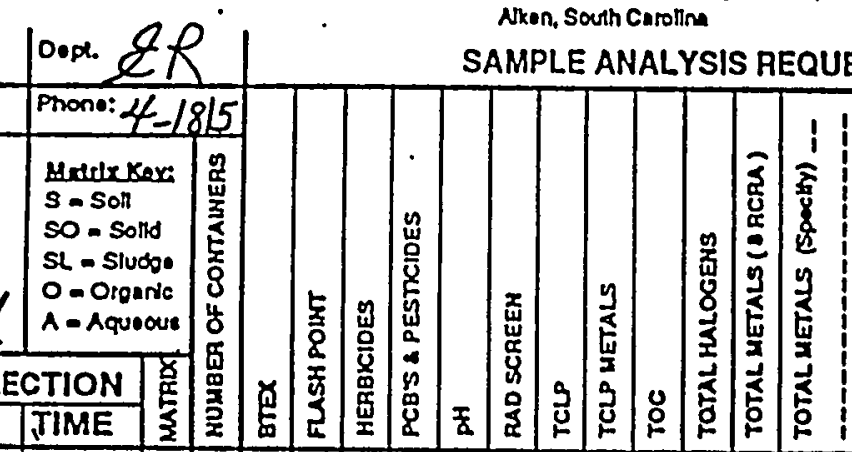

(REV. 1, 01-10-94)

Sample Destlnatlon: GEL

SAVANNAH RIVER SITE :

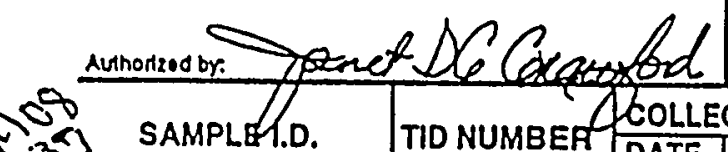

o GRS-15-01 5795 DATE TIME ₹

YSIS REQUESTED $3 / 15411305$

5796

5797

$\because \because G R S-15-0.3$

$.4 G R S-15-04$ $.05 R S-15-0 S$
5798

5799

sit

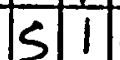

51

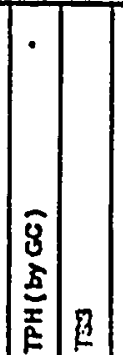

\begin{tabular}{c|c}
\hline & \\
\hline & \\
\hline$\cdot$ & \\
\hline$\cdot$ & \\
\hline$\cdot$ & \\
\hline$\cdot$ & \\
\hline$\cdot$ & \\
\hline
\end{tabular}

1 Rollnqulohod by: Dmo/ Timo Rocolved by:

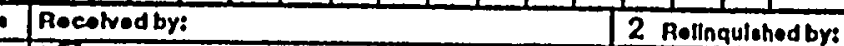
PAG $O F \perp: \therefore$ (Pini) H. W. Davidson ${ }^{\text {(sion }}$ Hew Dasadom 3 Rollinqulatiod by: (Pegini) Sh: Durion

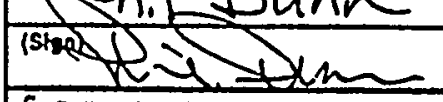
$8 / 12$ /94 (ard ard
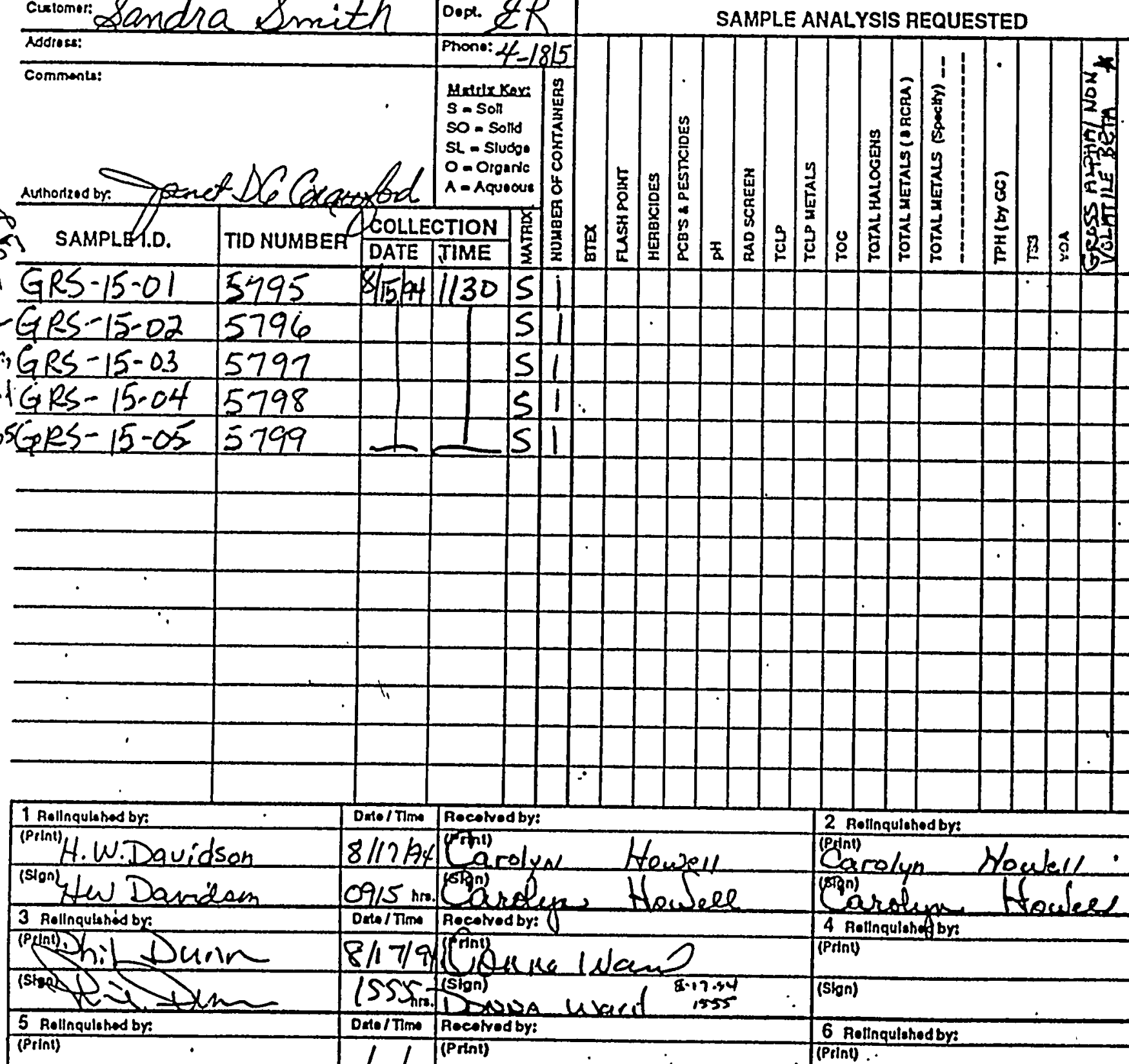
09/5 in. (5) Howeles, How Orta/Tim Recoived by: ()

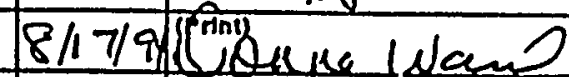
(525 (Sign)

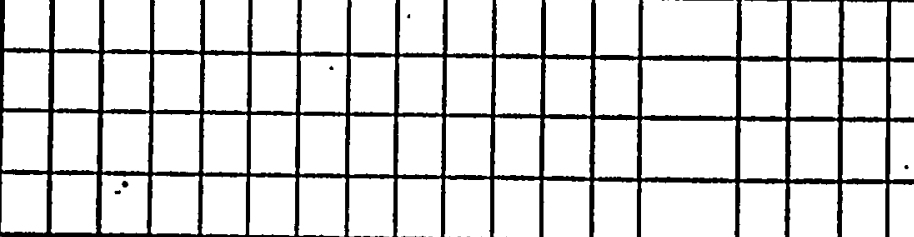

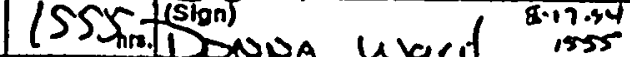
Dmel inmo Rocolved by:

\begin{tabular}{|l|l|l|}
\hline (Slon) & 1 & \\
\hline
\end{tabular} $11^{(\operatorname{Pr} r \sin )}$

\begin{tabular}{|l|l|}
\hline & 6 Rolinqulehod by: \\
\hline & (Pilni)
\end{tabular}

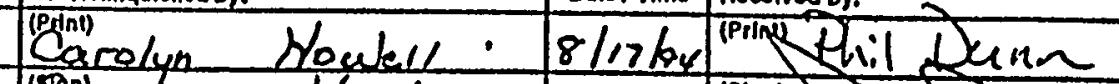
(onnolen Houles 4 Rotlnqularef br: $9: 17 \mathrm{hm}$ Detol Time Rocolved by: (Print) $1 /{ }^{(\text {Pinni) }}$ (Shon)

. $\frac{6 \text { Rollingu }}{(\text { Prini) }}$
\begin{tabular}{|c|c|} 
& hrse \\
\hline Detel Time & Rocolved by: \\
\hline$/ /$ & (Prlni)
\end{tabular} (Slgn) (5lon) 
3408337-01.01 - $500 \mathrm{~mL} / \mathrm{P}$

17-AuG-94 22:41:36 Regino Dessaure

31- itug-94 07:37:59 Mindy Lowe

31-AUG-94 15:00:53 Gary Low15

31-2uG-91 15:11:08 - K1shio Gibbs

31-Nut-94 16:56:14 Regina Dessaure

02-SEP-94 20:14:14 KLm Bennett

01-SEP-94 20:45:42 Kim-Bennett

02-5EP-94 07:23:18 Mindy Lowe

$1408337-02.01-500 \mathrm{ml} / \mathrm{P}$

17-nue-94 22:41:35 Regina Dessaure

31-2uK-94 07:37:59 MIndy Lowe

31-nuc-94 15:00:53 Gary Lovis

31-RUC-94 15:11:09 K1sisia Glbbs

31-RUG-94 16:56:13 Reglna Dessaure

01-SEP-94 20:44:14 K1m Bennett

01-SEP-94 20:45:42 K1m Bennett

02-SEP-94 07:23:19 Mindy Loue

108337-03.01 - $500 \mathrm{sil/P}$

17-AUG-94 22:41:37 Regina Dessaure

31-2UG-94 07:38:00 Mindy Lowe

31-NUG-94 15:00:53 Gary Lew1s

31-AUC-94 15:11:09 Kish1a Gibbs

31-xuG-94 16:56:12 Reglna Dessaure

01-5EP-94 20:44:14, K1m Bennetr

01-SEP-94 20:45:43 KIm Bennett

02-5EP-94 07:23:18 Mindy Lowe

108337-04.01 - $500 \mathrm{ml} / \mathrm{P}$

\begin{tabular}{|c|c|c|c|}
\hline $17-A U G-94$ & $22: 42: 36$ & Regind Dessaure & $\rightarrow$ Maln Cooler \\
\hline $31-\lambda \cup G-94$ & $07: 38: 00$ & MIndy Lowe & $\rightarrow$ Dellvery \\
\hline $31-\lambda U G-94$ & $25: 00: 54$ & Gary Lew 1 s & $\rightarrow$ Delivery \\
\hline 31-AUG-94 & $15: 11: 08$ & KIshia Glbbs & Main Coolex \\
\hline $31-\lambda 0 c-94$ & $16: 56: 13$ & Ragina Dessaure & $\rightarrow$ Delivery \\
\hline 01-SEP-94 & $20: 44: 12$ & K1m Bennett & Organic Prep \\
\hline 01-SEP-94 & $20: 15: 11$ & KIm Bennett & - Mala Cooler \\
\hline 02-SEP-94 & $07: 23: 38$ & Mindy Love & $\rightarrow$ Main Cooler \\
\hline \multicolumn{4}{|c|}{$108337-05.01-500 \times 1 / \mathrm{P}$} \\
\hline $17-A U G-94$ & $22: 41: 36$ & Regina Dessaure & Main Cooler \\
\hline $32-x u G-94$ & $07: 38: 00$ & Mindy Lowe & $\rightarrow$ Delivery \\
\hline $31-200-94$ & $15: 00: 52$ & Gary Ievis & Dellvery \\
\hline $31-2 u c-94$ & $15: 11: 09$ & Kishla Glbbs & $\rightarrow$ maln cooler \\
\hline $31-\lambda \cup G-94$ & $16: 56: 11$ & Regins Dessaure & $\rightarrow$ Del1very \\
\hline 01-SEP-94 & $20: 14: 14$ & KIn .Bennett & $\rightarrow$ Organic Prep \\
\hline $01-5 E P-91$ & $20: 45: 42$ & KL Bennett & $\rightarrow$ Main Cooler \\
\hline O2-5EP-91 & $07: 23: 19$ & MLndy Lowe & $\rightarrow$ Main Cooler \\
\hline
\end{tabular}

$\rightarrow$ Main cooler

$\rightarrow$ Dellvery

$\rightarrow$ Dellvory

$\rightarrow$ main cooler

$\rightarrow$ organic Prop

$\rightarrow$ Maln Cooler

$\rightarrow$ Maln cooler $\rightarrow$ Dellivery $\rightarrow$ Main cosler

$\rightarrow$ Delivery

$\rightarrow$ Delivery

$\rightarrow$ Main cooler

$\rightarrow$ Dellvery

$\rightarrow$ Organic Prep

$\rightarrow$ Main Cooler

$\rightarrow$ maln cooler 
0000000000

- 909090900

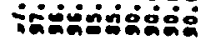

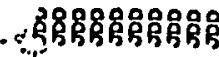

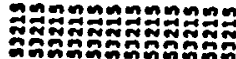

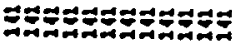

000000000000

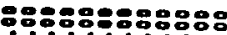

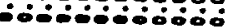

คำ

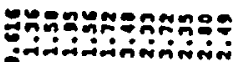

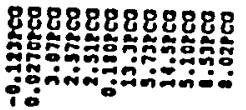

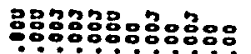

ivivinivinivivisivio

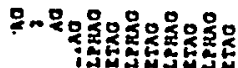

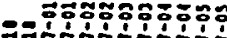

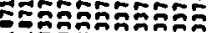

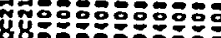

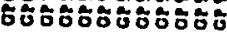

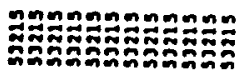

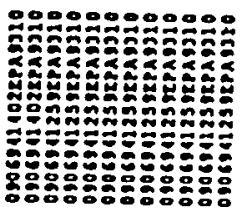

*.

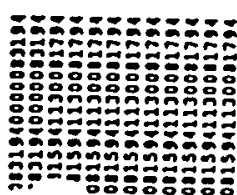

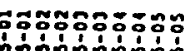

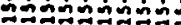

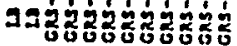




$$
z \varepsilon-\tau \cdot v
$$


Grace Road Site

RFI/RI Report
WSRC-RP-95-93, Rev. I

November 1995

APPENDIX A.3

SOIL GAS SURVEY RESULTS 
Grace Road Site

RFI/RI Report
WSRC-RP-95-93, Rev. 1 November 1995

\section{THIS PAGE INTENTIONALLY LEFT BLANK}


TABLE A-1

GRACE ROAD WASTE SITE (631-22G)

GAS CONCENTRATIONS IN SOIL (ng/g)

\begin{tabular}{|c|c|c|c|c|c|c|c|c|c|c|c|}
\hline $\begin{array}{c}\text { SAMPLE } \\
\text { NAME }\end{array}$ & PENTANE & HEXANE & HEPTANE & BENZENE & OCTANE & TOLUENE & NONANE & $\begin{array}{c}\text { ETHYL } \\
\text { BENZENE }\end{array}$ & $\begin{array}{c}\text { M\&P- } \\
\text { XYLENE }\end{array}$ & $\begin{array}{c}\text { 0- } \\
\text { XYLENE }\end{array}$ & DECANE \\
\hline $631-22 G-S 1$ & $<2$ & $<2$ & $<2$ & $<2$ & $<2$ & $<<2$ & $<2$ & $<2$ & $<2$ & $<2$ & $<2$ \\
\hline $631-22 G-\$ 2$ & $<2$ & $<2$ & $<2$ & $<2$ & $<2$ & $<2$ & $<2$ & $<2$ & $<2$ & $<2$ & $<2$ \\
\hline $631-22 G-53$ & $<2$ & $<2$ & $<2$ & $<2$ & $<2$ & $<2$ & $<2$ & $<2$ & $<2$ & $<2$ & $<2$ \\
\hline 631-22G-S4 & $<2$ & $<2$ & $<2$ & $<2$ & $<2$ & $<2$ & $<2$ & $<2$ & $<2$ & $<2$ & $<2$ \\
\hline 631-22G-S5 & $<2$ & $<2$ & $<2$ & $<2$ & $<2$ & $<2$ & $<2$ & $<2$ & $<2$ & $<2$ & $<2$ \\
\hline $631-22 G-\$ 6$ & $<2$ & $<2$ & $<2$ & $<2$ & $<2$ & $<2$ & $<2$ & $<2$ & $<2$ & $<2$ & $<2$ \\
\hline 631-22G-S7 & $<2$ & $<2$ & $<2$ & $<2$ & $<2$ & $<2$ & $<2$ & $<2$ & $<2$ & $<2$ & $<2$ \\
\hline 631-22G-S8 & $<2$ & $<2$ & $<2$ & $<2$ & $<2$ & $<2$ & $<2$ & $<2$ & $<2$ & $<2$ & $<2$ \\
\hline 631-22G-S9 & $<2$ & $<2$ & $<2$ & $<2$ & $<2$ & $<2$ & $<2$ & $<2$ & $<2$ & $<2$ & $<2$ \\
\hline $631-22 G-\$ 10$ & $<2$ & $<2$ & $<2$ & $<2$ & $<2$ & $<2$ & $<2$ & $<2$ & $<2$ & $<2$ & $<2$ \\
\hline 631-22G-S10A & $<2$ & $<2$ & $<2$ & $<2$ & $<2$ & $<2$ & $<2$ & $<2$ & $<2$ & $<2$ & $<2$ \\
\hline $631-22 G-S 11$ & $<2$ & $<2$ & $<2$ & $<2$ & $<2$ & $<2$ & $<2$ & $<2$ & $<2$ & $<2$ & $<2$ \\
\hline 631-22G-S12 & $<2$ & $<2$ & $<2$ & $<2$ & $<2$ & $<2$ & $<2$ & $<2$ & $<2$ & $<2$ & $<2$ \\
\hline 631-22G-S13 & $<2$ & $<2$ & $<2$ & $<2$ & $<2$ & $<2$ & $<2$ & $<2$ & $<2$ & $<2$ & $<2$ \\
\hline 631-22G-S14 & $<2$ & $<2$ & $<2$ & $<2$ & $<2$ & $<2$ & $<2$ & $<2$ & $<2$ & $<2$ & $<2$ \\
\hline 631-22G-S15 & $<2$ & $<2$ & $<2$ & $<2$ & $<2$ & $<2$ & $<2$ & $<2$ & $<2$ & $<2$ & $<2$ \\
\hline $631-22 G-S 16$ & $<2$ & $<2$ & $<2$ & $<2$ & $<2$ & $<2$ & $<2$ & $<2$ & $<2$ & $<2$ & $<2$ \\
\hline 631-22G-S17 & $<2$ & $<2$ & $<2$ & $<2$ & $<2$ & $<2$ & $<2$ & $<2$ & $<2$ & $<2$ & $<2$ \\
\hline $631-22 G-\$ 18$ & $<2$ & $<2$ & $<2$ & $<2$ & $<2$ & $<2$ & $<2$ & $<2$ & $<2$ & $<2$ & $<2$ \\
\hline 631-22G-S19 & $<2$ & $<2$ & $<2$ & $<2$ & $<2$ & $<2$ & $<2$ & $<2$ & $<2$ & $<2$ & $<2$ \\
\hline 631-22G-S20 & $<2$ & $<2$ & $<2$ & $<2$ & $<2$ & $<2$ & $<2$ & $<2$ & $<2$ & $<2$ & $<2$ \\
\hline 631-22G-S20A & $<2$ & $<2$ & $<2$ & $<2$ & $<2$ & $<2$ & $<2$ & $<2$ & $<2$ & $<2$ & $<2$ \\
\hline $631-22 G-S 21$ & $<2$ & $<2 \therefore$ & $<2$ & $<2$ & $<2$ & $<2$ & $<2$ & $<2$ & $<2$ & $<2$ & $<2$ \\
\hline 631-22G-S22 & $<2$ & $<2$ & $<2$ & $<2$ & $<2$ & $<2$ & $<2$ & $<2$ & $<2$ & $<2$ & $<2$ \\
\hline 631-22G-\$23 & $<2$ & $<2$ & $<2$ & $<2$ & $<.2$ & $<2$ & $<2$ & $<2$ & $<2$ & $<2$ & $<2$ \\
\hline 631-22G-S24 & $<2$ & $<2$ & $<2$ & $<2$ & $<2$ & $<2$ & $<2$ & $<2$ & $<2$ & $<2$ & $<2$ \\
\hline
\end{tabular}


TABLE A-1 (Continued)

GRACE ROAD WASTE SITE (631-22G)

GAS CONCENTRATIONS IN SOIL (ng/g)

\begin{tabular}{|c|c|c|c|c|c|c|c|c|c|c|c|}
\hline $\begin{array}{c}\text { SAMPLE } \\
\text { NAME }\end{array}$ & PENTANE & HEXANE & HEPTANE & BENZENE & OCTANE & TOLUENE & NONANE & $\begin{array}{c}\text { ETHYL } \\
\text { BENZENE }\end{array}$ & $\begin{array}{c}\text { M\&P- } \\
\text { XYLENE }\end{array}$ & $\begin{array}{c}0- \\
\text { XYLENE }\end{array}$ & DECANE \\
\hline $631-22$ G-S25 & $<2$ & $<2$ & $<2$ & $<2$ & $<2$ & i $<2$ & $<2$ & $<2$ & $<2$ & $<2$ & $<2$ \\
\hline 631-22G-S26 & $<2$ & $<2$ & $<2$ & $<2$ & $<2$ & $<2$ & $<2$ & $<2$ & $<2$ & $<2$ & $<2$ \\
\hline 631-22G-S27 & $<2$ & $<2$. & $<2$ & $<2$ & $<2$ & $<2$ & $<2$ & $<2$ & $<2$ & $<2$ & $<2$ \\
\hline $631-22 G-S 28$ & $<2$ & $<2$ & $<2$ & $<2$ & $<2$ & $<2$ & $<2$. & $<2$ & $<2$ & $<2$ & $<2$ \\
\hline 631-22G-\$29 & $<2$ & $<2$ & $<2$ & $<2$ & $<2$ & $<2$ & $<2$ & $<2$ & $<2$ & $<2$ & $<2$ \\
\hline 631-22G-S30 & $<2$ & $<2$ & $<2$ & $<2$ & $<2$ & $<2$ & $<2$ & $<2$ & $i<2$ & $<2$ & $<2$ \\
\hline $631-22 \mathrm{G}-\mathrm{S} 30 \mathrm{~A}$ & $<2$ & $<2$ & $<2$ & $<2$ & $<2$ & $<2$ & $<2$ & $<2$ & $<2$ & $<2$ & $<2$ \\
\hline $631-22$ G-S31 & $<2$ & $<2$ & $<2$ & $<2$ & $<2$ & $<2$ & $<2$ & $<2$ & $<2$ & $<2$ & $<2$ \\
\hline 631-22G-S32 & $<2$ & $<2$ & $<2$ & $<2$ & $<2$ & $<2$ & $<2$ & $<2$ & $<2$ & $<2$ & $<2$ \\
\hline 631-22G-S33 & $<2$ & $<2$ & $<2$ & $<2$ & $<2$ & $<2$ & $<2$ & $<2$ & $<2$ & $<2$ & $<2$ \\
\hline 631-22G-S34 & $<2$ & $<2$ & $<2$ & $<2$ & $<2$ & $<2$ & $<2$ & $<2$ & $<2$ & $<2$ & $<2$ \\
\hline 631-22G-S35 & $<2$ & $<2$ & $<2$ & $<2$ & $<2$ & $<2$ & $<2$ & $<2$ & $<2$ & $<2$ & $<2$ \\
\hline 631-22G-S36 & $<2$ & $<2$ & $<2$ & $<2$ & $<2$ & $<2$ & $<2$ & $<2$ & $<2$ & $<2$ & $<2$ \\
\hline 631-22G-\$37 & $<2$ & $<2$ & $<2$ & $<2$ & $<2$ & $<2$ & $<2$ & $<2$ & $<2$ & $<2$ & $<2$ \\
\hline 631-22G-S38 & $<2$ & $<2$ & $<2$ & $<2$ & $<2$ & $<2$ & $<2$ & $<2$ & $<2$ & $<2$ & $<2$ \\
\hline 631-22G-S39 & $<2$ & $<2$ & $<2$ & $<2$ & $<2$ & $<2$ & $<2$ & $<2$ & $<2$ & $<2$ & $<2$ \\
\hline $631-22 \mathrm{G}-\mathrm{S} 40$ & $<2$ & $<2$ & $<2$ & $<2$ & $<2$ & $<2$ & $<2$ & $<2$ & $<2$ & $<2$ & $<2$ \\
\hline 631-22G-S40A & $<2$ & $<2$. & $<2$ & $<2$ & $<2$ & $<2$ & $<2$ & $<2$ & $<2$ & $<2$ & $<2$ \\
\hline $631-22 G-S 41$ & $<2$ & $<2$ & $<2$ & $<2$ & $<2$ & $<2$ & $<2$ & $<2$ & $<2$ & $<2$ & $<2$ \\
\hline 631-22G-S42 & $<2$ & $<2$ & $<2$ & $<2$ & $<2$ & $<2$ & $<2$ & $<2$ & $<2$ & $<2$ & $<2$ \\
\hline $631-22 G-S 43$ & $<2$ & $<2$ & $<2$ & $<2$ & $<2$ & $<2$ & $<2$ & $<2$ & $<2$ & $<2$ & $<2$ \\
\hline $631-22 G-S 44$ & $<2$ & $<2$ & $<2$ & $<2$ & $<2$ & $<2$ & $<2$ & $<2$ & $<2$ & $<2$ & $<2$ \\
\hline 631-22G-S45 & $<2$ & $<2$ & $<2$ & $<2$ & $<2$ & $<2$ & $<2$ & $<2$ & $<2$ & $<2$ & $<2$ \\
\hline 631-22G-S46 & $<2$ & $<2$ & $<2$ & $<2$ & $<2$ & $<2$ & $<2$ & $<2$ & $<2$ & $<2$ & $<2$ \\
\hline 631-22G-S47 & $<2$ & $<2$ & $<2$ & $<2$ & $<2$ & $<2$ & $<2$ & $<2$ & $<2$ & $<2$ & $<2$ \\
\hline $631-22 G-S 48$ & $<2$ & $<2$ & $<2$ & $<2$ & $<2$ & $<2$ & $<2$ & $<2$ & $<2$ & $<2$ & $<2$ \\
\hline
\end{tabular}


TABLE A-1 (Continued)

GRACE ROAD WASTE SITE (631-22G)

GAS CONCENTRATIONS IN SOIL (ng/g)

\begin{tabular}{|c|c|c|c|c|c|c|c|c|c|c|c|}
\hline $\begin{array}{l}\text { SAMPLE } \\
\text { NAME }\end{array}$ & PENTANE & BEXANE & BEPTANE & BENZENE & OCTANE & TOLUENE & NONANE & $\begin{array}{c}\text { ETHYL } \\
\text { BENZENE }\end{array}$ & $\begin{array}{c}\text { M\&P- } \\
\text { XYLENE }\end{array}$ & $\begin{array}{c}0- \\
\text { XYLENE }\end{array}$ & DECANE \\
\hline $631-22 \mathrm{G}-\mathrm{S} 49$ & $<2$ & $<2$ & $<2$ & $<2$ & $<2$ & $\cdot<2$ & $<2$ & $<2$ & $<2$ & $<2$ & $<2$ \\
\hline 631-22G-S50 & $<2$ & $<2$ & $<2$ & $<2$ & $<2$ & $<2$ & $<2$ & $<2$ & $<2$ & $<2$ & $<2$ \\
\hline $631-22 \mathrm{G}-550 \mathrm{~A}$ & $<2$ & $<2$ & $<2$ & $<2$ & $<2$ & $<2$ & $<2$ & $<2$ & $<2$ & $<2$ & $<2$ \\
\hline $631-22 G-S 51$ & $<2$ & $<2$ & $<2$ & $<2$ & $<2$ & $<2$ & $<2$ & $<2$ & $<2$ & $<2$ & $<2$ \\
\hline 631-22G-S52 & $<2$ & $<2$ & $<2$ & $<2$ & $<2$ & $<2$ & $<2$ & $<2$ & $<2$ & $<2$ & $<2$ \\
\hline 631-22G-S53 & $<2$ & $<2$ & $<2$ & $<2$ & $<2$ & $<2$ & $<2$ & $<2$ & $<2$ & $<2$ & $<2$ \\
\hline 631-22G-S54 & $<2$ & $<2$ & $<2$ & $<2$ & $<2$ & $<2$ & $<2$ & $<2$ & $<2$ & $<2$ & $<2$ \\
\hline 631-22G-S55 & $<2$ & $<2$ & $<2$ & $<2$ & $<2$ & $<2$ & $<2$ & $<2$ & $<2$ & $<2$ & $<2$ \\
\hline 631-22G-S56 & $<2$ & $<2$ & $<2$ & $<2$ & $<2$ & $<2$ & $<2$ & $<2$ & $<2$ & $<2$ & $<2$ \\
\hline 631-22G-S57 & $<2$ & $<2$ & $<2$ & $<2$ & $<2$ & $<2$ & $<2$ & $<2$ & $<2$ & $<2$ & $<2$ \\
\hline 631-22G-S58 & $<2$ & $<2$ & $<2$ & $<2$. & $<2$ & $<2$ & $<2$ & $<2$ & $<2$ & $<2$ & $<2$ \\
\hline 631-22G-S59 & $<2$ & $<2$ & $<2$ & $<2$ & $<2$ & $<2$ & $<2$ & $<2$ & $<2$ & $<2$ & $<2$ \\
\hline $631-22 G-\$ 60$ & $<2$ & $<2$ & $<2$ & $<2$ & $<2$ & $<2$ & $<2$ & $<2$ & $<2$ & $<2$ & $<2$ \\
\hline $631-22 G-S 60 A$ & $<2$ & $<2$ & $<2$ & $<2$ & $<2$ & $<2$ & $<2$ & $<2$ & $<2$ & $<2$ & $<2$ \\
\hline $631-22 G-S 61$ & $<2$ & $<2$ & $<2$ & $<2$ & $<2$ & $<2$ & $<2$ & $<2$ & $<2$ & $<2$ & $<2$ \\
\hline 631-22G-S62 & $<2$ & $<2$ & $<2$ & $<2$. & $<2$ & $<2$ & $<2$ & $<2$ & $<2$ & $<2$ & $<2$ \\
\hline 631-22G-S63 & $<2$ & $<2$ & $<2$ & $<2$ & $<2$ & $<2$ & $<2$ & $<2$ & $<2$ & $<2$ & $<2$ \\
\hline 631-22G-S64 & $<2$ & $<2$ & $<2$ & $<2$ & $<2$ & $<2$ & $<2$ & $<2$ & $<2$. & $<2$ & $<2$. \\
\hline 631-22G-S65 & $<2$ & $<2$ & $<2$ & $<2$ & $<2$ & $<2$ & $<2$ & $<2$ & $<2$ & $<2$ & $<2$ \\
\hline 631-22G-S66 & $<2$ & $<2$ & $<2$ & $<2$ & $<2$ & $<2$ & $<2$ & $<2$ & $<2$ & $<2$ & $<2$ \\
\hline 631-22G-S67 & $<2$ & $<2$ & $<2$ & $<2$ & $<2$ & $<2$ & $<2$ & $<2$ & $<2$ & $<2$ & $<2$ \\
\hline 631-22G-S68 & $<2$ & $<2$ & $<2$ & $<2$ & $<2$ & $<2$ & $<2$ & $<2$ & $<2$ & $<2$ & $<2$ \\
\hline 631-22G-S69 & $<2$ & $<2$ & $<2$ & $<2$ & $<2$ & $<2$ & $<2$ & $<2$ & $<2$ & $<2$ & $<2$ \\
\hline 631-22G-S70 & $<2$ & $<2$ & $<2$ & $<2$ & $<2$ & $<2$ & $<2$ & $<2$ & $<2$ & $<2$ & $<2$ \\
\hline $631-22 G-S 70 A$ & $<2$ & $<2$ & $<2$ & $<2$ & $<2$ & $<2$ & $<2$ & $<2$ & $<2$ & $<2$ & $<2$ \\
\hline 631-22G-S71 & $<2$ & $<2$ & $\leq 2$ & $<2$ & $<2$ & $<2$ & $<2$ & $<2$ & $<2$ & $<2$ & $<2$ \\
\hline
\end{tabular}


TABLE A-1 (Continued)

GRACE ROAD WASTE SITE (631-22G)

GAS CONCENTRATIONS IN SOR (ng/g)

\begin{tabular}{|c|c|c|c|c|c|c|c|c|c|c|c|}
\hline $\begin{array}{l}\text { SAMPLE } \\
\text { NAME }\end{array}$ & PENTANE & HEXANE & HEPTANE & BENZENE & OCTANE & TOLUENE & NONANE & $\begin{array}{c}\text { ETHYL } \\
\text { BENZENE }\end{array}$ & $\begin{array}{c}\text { M\&P- } \\
\text { XYLENE }\end{array}$ & $\begin{array}{c}\text { 0- } \\
\text { XYLENE }\end{array}$ & DECANE \\
\hline 631-22G-S72 & $<2$ & $<2$ & $<2$ & $<2$ & $<2$ & $<2$ & $<2$ & $<2$ & $<2$ & $<2$ & $<2$ \\
\hline $631-22 G-S 73$ & $<2$ & $<2$ & $<2$ & $<2$ & $<2$ & $<2$ & $<2$ & $<2$ & $<2$ & $<2$ & $<2$ \\
\hline 631-22G-S74 & $<2$ & $<2$ & $<2$ & $<2$ & $<2$ & $<2$ & $<2$ & $<2$ & $<2$ & $<2$ & $<2$ \\
\hline 631-22G-S75 & $<2$ & $<2$ & $<2$ & $<2$ & $<2$ & $<2$ & $<2$ & $<2$ & $<2$ & $<2$ & $<2$ \\
\hline 631-22G-S76 & $<2$ & $<2$ & $<2$ & $<2$ & $<2$ & $<2$ & $<2$ & $<2$ & $<2$ & $<2$ & $<2$ \\
\hline 631-22G-S77 & $<2$ & $<2$ & $<2$ & $<2$ & $<2$ & $<2$ & $<2$ & $<2$ & $<2$ & $<2$ & $<2$ \\
\hline 631-22G-S78 & $<2$ & $<2$ & $<2$ & $<2$ & $<2$ & $<2$ & $<2$ & $<2$ & $<2$ & $<2$ & $<2$ \\
\hline 631-22G-S79 & $<2$ & $<2$ & $<2$ & $<2$ & $<2$ & $<2$ & $<2$ & $<2$ & $<2$ & $<2$ & $<2$ \\
\hline $631-22 G-S 80$ & $<2$ & $<2$ & $<2$ & $<2$ & $<2$ & $<2$ & $<2$ & $<2$ & $<2$ & $<2$ & $<2$ \\
\hline 631-22G-S80A & $<2$ & $<2$ & $<2$ & $<2$ & $<2$ & $<2$ & $<2$ & $<2$ & $<2$ & $<2$ & $<2$ \\
\hline 631-22G-S81 & $<2$ & $<2$ & $<2$ & $<2$ & $<2$ & $<2$ & $<2$ & $<2$ & $<2$ & $<2$ & $<2$ \\
\hline 631-22G-S82 & $<2$ & $<2$ & $<2$ & $<2$ & $<2$ & $<2$ & $<2$ & $<2$ & $<2$ & $<2$ & $<2$ \\
\hline $631-22 G-S 83$ & $<2$ & $<2$ & $<2$ & $<2$ & $<2$ & $<2$ & $<2$ & $<2$ & $<2$ & $<2$ & $<2$ \\
\hline 631-22G-S84 & $<2$ & $<2$ & $<2$ & $<2$ & $<2$ & $<2$ & $<2$ & $<2$ & $<2$ & $<2$ & $<2$ \\
\hline $631-22 G-\$ 85$ & $<2$ & $<2$ & $<2$ & $<2$ & $<2$ & $<2$ & $<2$ & $<2$ & $<2$ & $<2$ & $<2$ \\
\hline 631-22G-SB1 & $<2$ & $<2$ & $<2$ & $<2$ & $<2$ & $<2$ & $<2$ & $<2$ & $<2$ & $<2$ & $<2$ \\
\hline $631-22 G-S B 2$ & $<2$ & $<2$ & $<2$ & $<2$ & $<2$ & $<2$ & $<2$ & $<2$ & $<2$ & $<2$ & $<2$ \\
\hline 631-22G-SB3 & $<2$ & $<2$ & $<2$ & $<2$ & $<2$ & $<2$ & $<2$ & $<2$ & $<2$ & $<2$ & $<2$ \\
\hline $631-22 G-S B 4$ & $<2$ & $<2$ & $<2$ & $<2$ & $<2$ & $<2$ & $<2$ & $<2$ & $<2$ & $<2$ & $<2$ \\
\hline 631-22G-SB5 & $<2$ & $<2$ & $<2$ & $<2$ & $<2$ & $<2$ & $<2$ & $<2$ & $<2$ & $<2$ & $<2$ \\
\hline 631-22G-SB6 & $<2$ & $<2$ & $<2$ & $<2$ & $<2$ & $<2$ & $<2$ & $<2$ & $<2$ & $<2$ & $<2$ \\
\hline 631-22G-SB7 & $<2$ & $<2$ & $<2$ & $<2$ & $<2$ & $<2$ & $<2$ & $<2$ & $<2$ & $<2$ & $<2$ \\
\hline 631-22G-SB8 & $<2$ & $<2$ & $<2$ & $<2$ & $<2$ & $<2$ & $<2$ & $<2$ & $<2$ & $<2$ & $<2$ \\
\hline 631-22G-SB9 & $<2$ & $<2$ & $<2$ & $<2$ & $<2$ & $<2$ & $<2$ & $<2$ & $<2$ & $<2$ & $<2$ \\
\hline
\end{tabular}




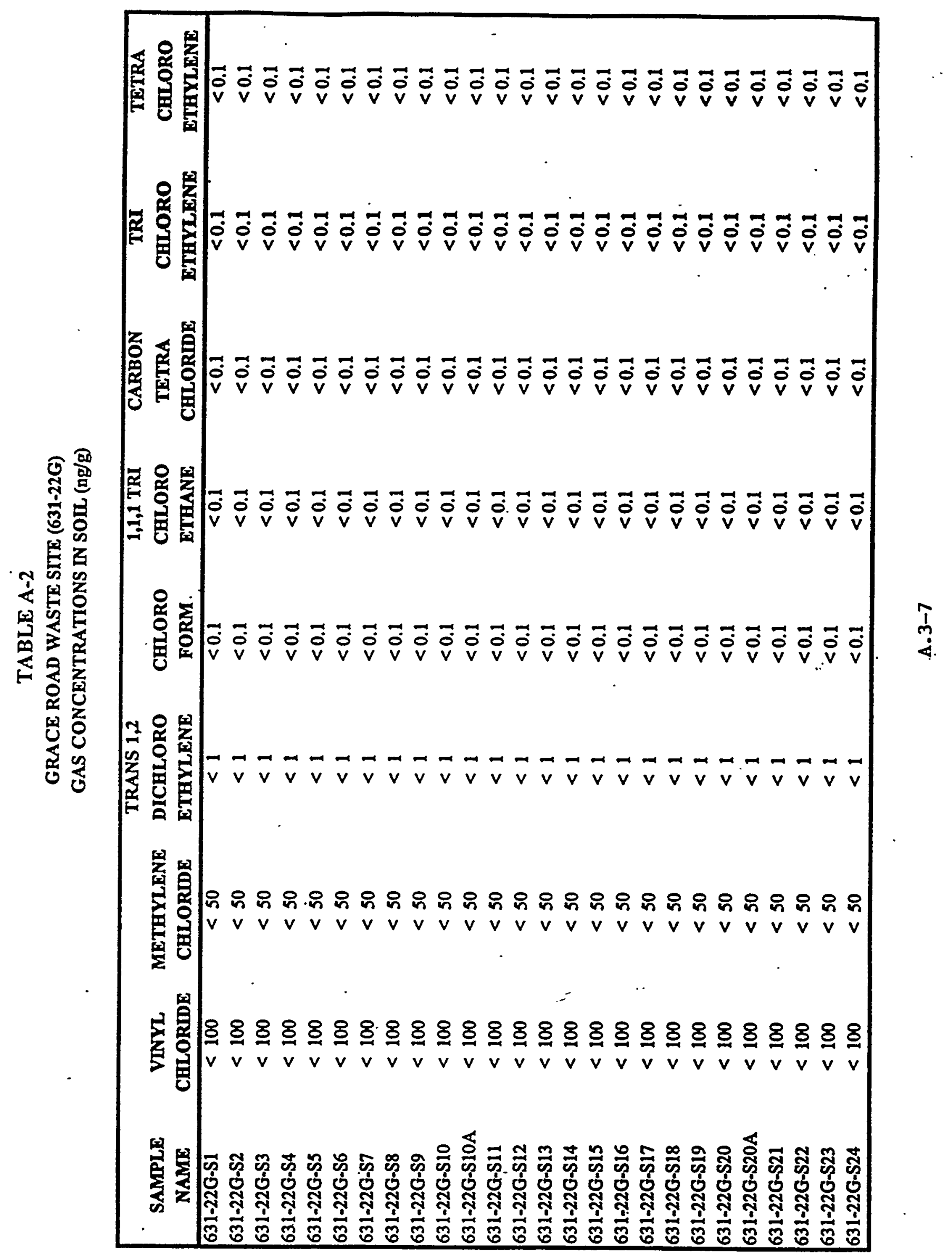




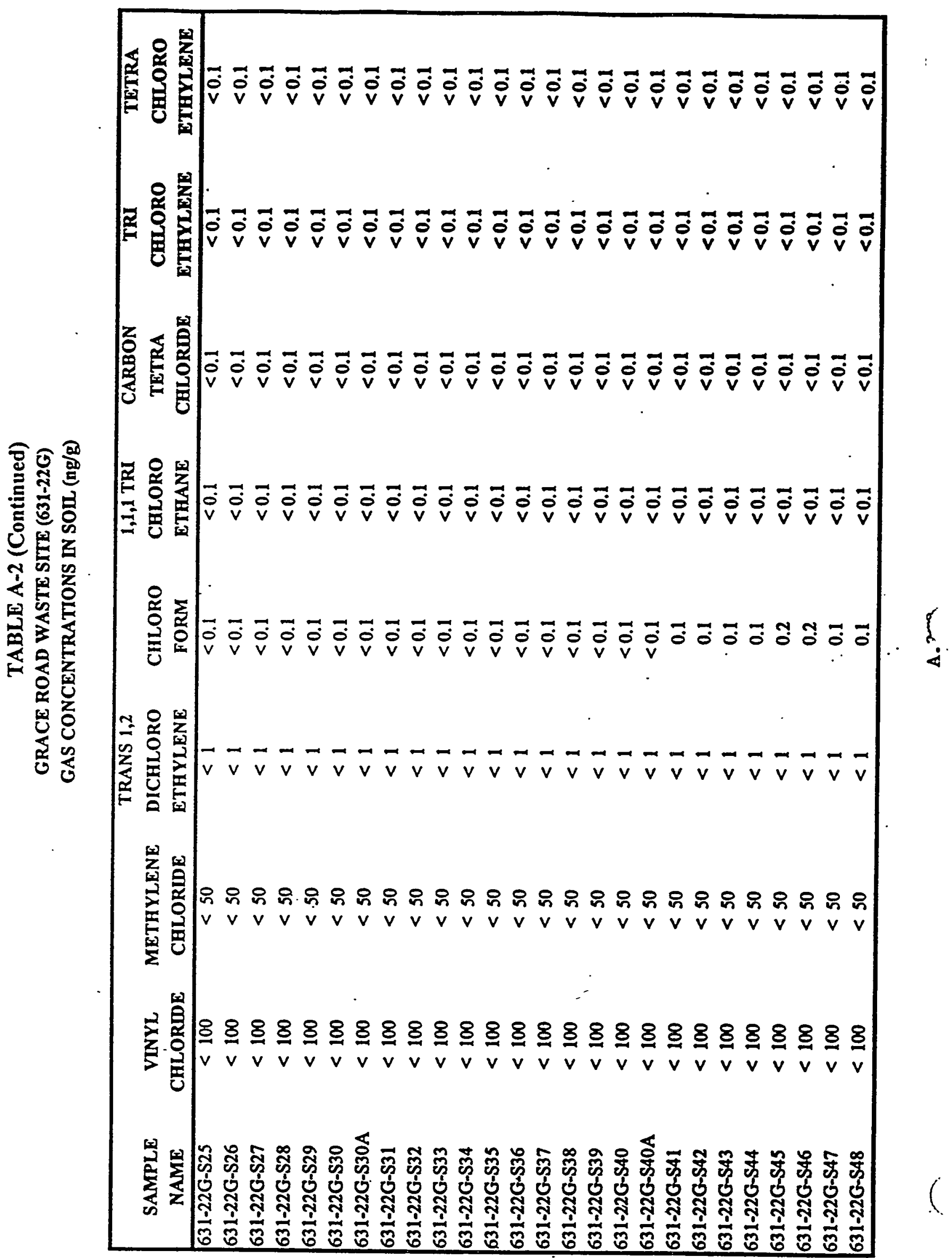




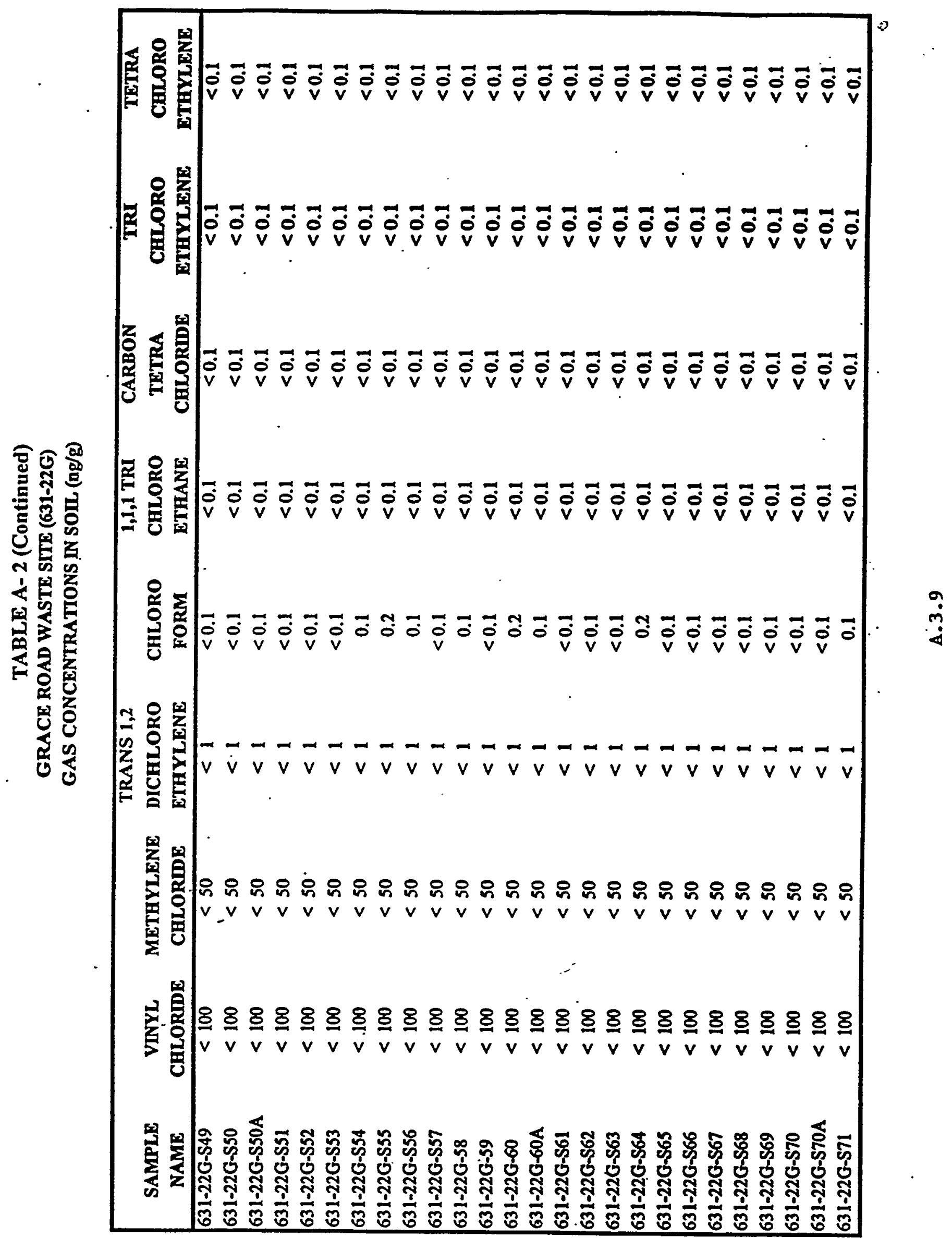




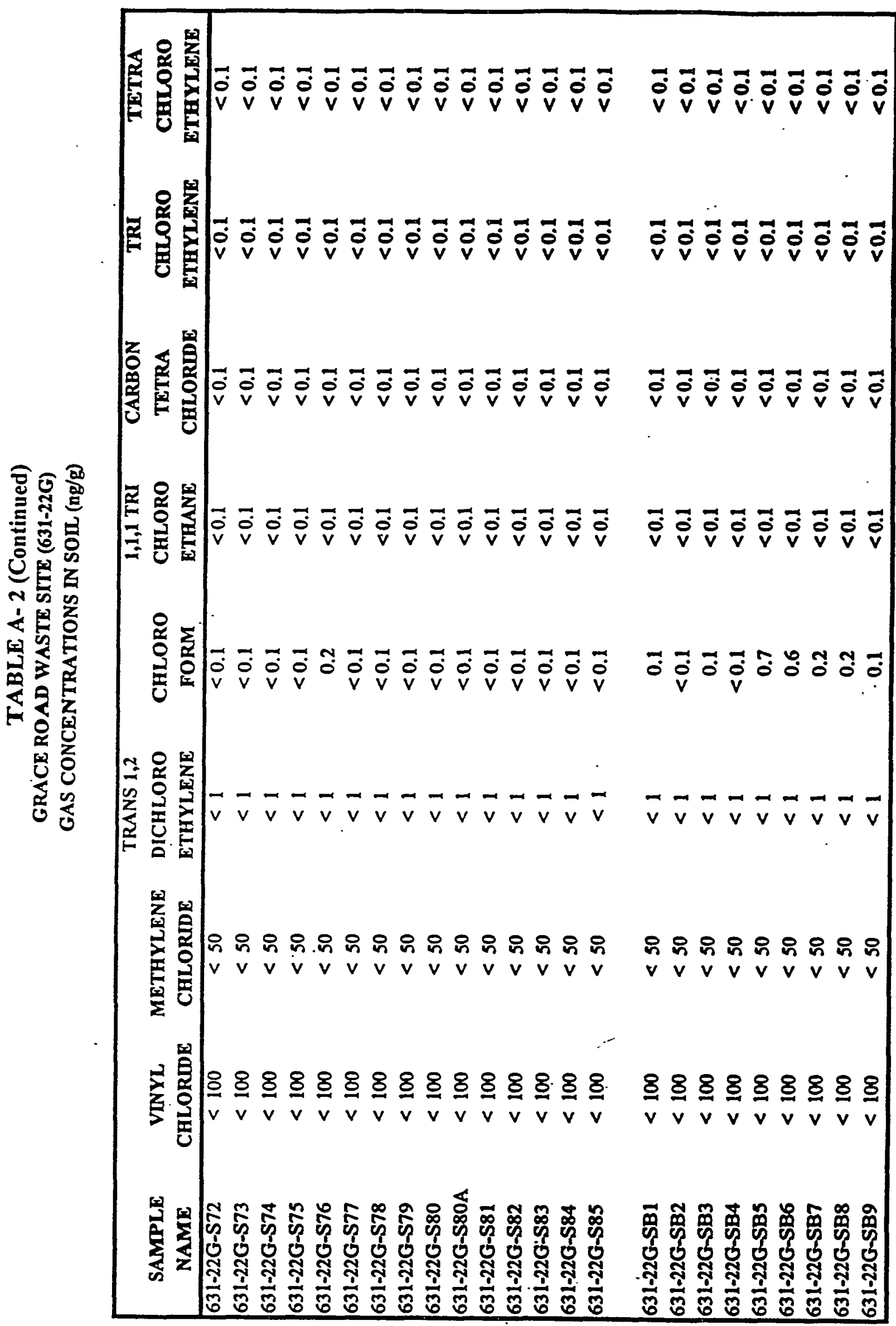


TABLE A-3

GRACE ROAD WASTE STTE (631-22G)

GAS CONCENTRATIONS IN SOIL (ng/g)

\begin{tabular}{|c|c|c|c|c|c|c|c|c|}
\hline $\begin{array}{l}\text { SAMPLE } \\
\text { NAME }\end{array}$ & METHANE & ETHANE & ETBYLENE & PROPACNE & PROPYLENE & I-BUTANE & N-BUTANE & MERCURY \\
\hline $631-22 \mathrm{G}-\mathrm{S} 1$ & 4.31 & $<0.05$ & 0.07 & $<0.05^{\circ}$ & 0.13 & $<0.05$ & $<0.05$ & 103 \\
\hline 631-22G-S2 & 4.57 & $<0.05$ & 0.22 & 0.27 & 0.60 & $<0.05$ & $<0.05$ & 11 \\
\hline 631-22G-S3 & 4.41 & $<0.05$ & 0.05 & $<0.05$ & $<0.05$ & $<0.05$ & $<0.05$ & 2 \\
\hline 631-22G-S4 & 3.70 & $<0.05$ & 0.06 & $<0.05$ & $<0.05$ & $<0.05$ & $<0.05$ & 5 \\
\hline 631-22G-S5 & 4.35 & $<0.05$ & 0.08 & $<0.05$ & $<0.05$ & 0.12 & $<0.05$ & 1 \\
\hline $631-22 G-\$ 6$ & 3.77 & $<0.05$ & $<0.05$ & 0.05 & $<0.05$ & $<0.05$ & $<0.05$ & $<1$ \\
\hline 631-22G-57 & 4.51 & $<0.05$ & 0.08 & $<0.05$ & $<0.05$ & $<0.05$ & $<0.05$ & 4 \\
\hline $631-22 G-58$ & 4.08 & $<0.05$ & 0.05 & $<0.05$ & $<0.05$ & $<0.05$ & $<0.05$ & 2 \\
\hline 631-22G-S9 & 4.52 & $<0.05$ & 0.06 & $<0.05$ & $<0.05$ & $<0.05$ & $<0.05$ & 1 \\
\hline $631-22 G-\$ 10$ & 96.34 & 0.07 & 0.19 & 0.23 & 0.41 & $<0.05$ & $<0.05$ & 4 \\
\hline $631-22 G-S 10 A$ & 4.77 & $<0.05$ & 0.09 & $<0.05$ & $<0.05$ & $<0.05$ & $<0.05$ & $<1$ \\
\hline $631-22 G-S 11$ & 4.00 & $<0.05$ & 0.05 & $<0.05$ & $<0.05$ & $<0.05$ & $<0.05$ & 2 \\
\hline 631-22G-S12 & 3.76 & $<0.05$ & 0.06 & $<0.05$ & $<0.05$ & $<0.05$ & $<0.05$ & $<1$ \\
\hline $631-22 \mathrm{G}-\mathrm{S} 13$ & 3.71 & $<0.05$ & 0.05 & 0.13 & $<0.05$ & $<0.05$ & $<0.05$ & $<1$ \\
\hline $631-22 \mathrm{G}-\mathrm{S} 14 \mathrm{R}$ & 0.67 , & $<0.05$ & $<0.05$ & $<0.05$ & $<0.05$ & $<0.05$ & $<0.05$ & $<1$ \\
\hline $631-22 G-S 15$ & 3.46 & $<0.05$ & $<0.05$ & $<0.05$ & $<0.05$ & $<0.05$ & $<0.05$ & $<1$ \\
\hline 631-22G-S16 & 4.10 & $<0.05$ & $<0.05$ & 0.12 & $<0.05$ & $<0.05$ & $<0.05$ & $<1$ \\
\hline 631-22G-S17 & 3.92 & $<0.05$ & 0.09 & 0.06 & $<0.05$ & 0.05 & $<0.05$ & $<1$ \\
\hline 631-22G-S18 & 3.91 & $<0.05$ & $<0.05$ & $<0.05$ & $<0.05$ & $<0.05$ & $<0.05$ & $<1$ \\
\hline 631-22G-S19 & 3.85 & $<0.05$ & $<0.05$ & $<0.05$ & $<0.05$ & $<0.05$ & $<0.05$ & $<1$ \\
\hline $631-22 G-\$ 20$ & 3.14 & $<0.05$ & $<0.05$ & 0.05 & $<0.05$ & $<0.05$ & 0.15 & $<1$ \\
\hline $631-22 G-S 20 A$ & 4.37 & $<0.05$ & 0.07 & $<0.05$ & $<0.05$ & $<0.05$ & $<0.05$ & $<1$ \\
\hline $631-22 G-S 21$ & 4.54 & $<0.05$ & 0.09 & $<0.05$ & $<0.05$ & $<0.05$ & 0.81 & 5 \\
\hline 631-22G-S22 & 4.72 & 0.05 & 0.15 & $<0.05$ & $<0.05$ & $<0.05$ & 1.13 & 2 \\
\hline 631-22G-S23 & 4.47 & $<0.05$ & 0.12 & $<0.05$ & $<0.05$ & $<0.05$ & $<0.05$ & 1 \\
\hline 631-22G-S24 & 4.43 & $<0.05$ & $<0.05$ & $<0.05$ & $<0.05$ & $<0.05$ & $<0.05$ & 6 \\
\hline
\end{tabular}

A. 3-11 
TABLE A-3 (Continued)

GRACE ROAD WASTE STTE (631-22G)

GAS CONCENTRATIONS IN SOIL (ng/g)

\begin{tabular}{|c|c|c|c|c|c|c|c|c|}
\hline $\begin{array}{l}\text { SAMPLE } \\
\text { NAME }\end{array}$ & METHANE & ETHANE & ETHYLENE & PROPANE & PROPYLENE & I-BUTANE & N-BUTANE & MERCURY \\
\hline $631.22 \mathrm{G}-\mathrm{S} 25$ & 4.16 & $<0.05$ & 0.07 & $<0.05$ & $<0.05$ & $<0.05$ & $<0.05$ & 1 \\
\hline 631-22G-S26 & 4.42 & $<0.05$ & $<0.05$ & $<0.05$ & $<0.05$ & $<0.05$ & $<0.05$ & 1 \\
\hline 631-22G-S27 & 4.86 & $<0.05$ & 0.06 & $<0.05$ & $<0.05$ & 0.06 & $<0.05$ & 5 \\
\hline 631-22G-S28 & 4.36 & $<0.05$ & 0.07 & $<0.05$ & 0.08 & $<0.05$ & $<0.05$ & 3 \\
\hline 631-22G-S29 & 4.73 & $<0.05$ & $\quad 0.05$ & $<0.05$ & $<0.05$ & $<0.05$ & $<0.05$ & 6 \\
\hline $631-22$ G-S30 & 4.02 & $<0.05$ & $<0.05$ & $<0.05$ & $<0.05$ & 0.11 & $<0.05$ & 10 \\
\hline $631-22 \mathrm{G}-\mathrm{S} 30 \mathrm{~A}$ & 4.02 & $<0.05$ & 0.05 & 0.06 & $<0.05$ & $<0.05$ & $<0.05$ & 6 \\
\hline $631-22 G-\$ 31$ & 4.60 & $<0.05$ & 0.07 & 0.11 & $<0.05$ & $<0.05$ & 1.05 & 7 \\
\hline $631-22 G-S 32$ & 4.64 & $<0.05$ & $<0.05$ & $<0.05$ & $<0.05$ & $<0.05$ & $<0.05$ & 6 \\
\hline 631-22G-S33 & 4.32 & $<0.65$ & 0.06 & $<0.05$ & $<0.05$ & $<0.05$ & $<0.05$ & 5 \\
\hline 631-22G-S34 & 5.02 & $<0.05$ & 0.09 & 0.12 & $<0.05$ & $<0.05$ & $<0.05$ & 9 \\
\hline 631-22G-S35 & 4.77 & $<0.05$ & 0.06 & $<0.05$ & $<0.05$ & $<0.05$ & $<0.05$ & 2 \\
\hline 631-22G-S36 & 4.53 & $<0.05$ & 0.05 & $<0.05$ & $<0.05$ & $<0.05$ & $<0.05$ & 1 \\
\hline $631-22 G-\$ 37$ & 4.31 & $<0.05$ & 0.05 & $<0.05$ & $<0.05$ & 0.08 & $\quad 0.69$ & 1 \\
\hline $631-22 G-S 38$ & 4.34 & $<0.05$ & 0.05 & $<0.05$ & 0.05 & $<0.05$ & 0.22 & 8 \\
\hline $631-22 G-\$ 39$ & 4.60 & $<0.05$ & $<0.05$ & $<0.05$ & $<0.05$ & $<0.05$ & $<0.05$ & 2 \\
\hline $63 L-22 G-\$ 40$ & 4.73 & $<0.05$ & $<0.05$ & $<0.05$ & $<0.05$ & $<0.05$ & $<0.05$ & 4 \\
\hline $631-22 \mathrm{G}-\mathrm{S} 40 \mathrm{~A}$ & 4.23 & $<0.05$ & $<0.05$ & $<0.05$ & $<0.05$ & $<0.05$ & $<0.05$ & 3 \\
\hline $631-22 G-S 41$ & 4.42 & $<0.05$ & $<0.05$ & $<0.05$ & $<0.05$ & 0.25 & 1.96 & 2 \\
\hline $631-22$ G-S42 & 4.53 & $<0.05$ & $<0.05$ & $<0.05$ & $<0.05$ & $<0.05$ & 0.42 & $<1$ \\
\hline $631-22 G-S 43$ & 4.38 & $<0.05$ & $<0.05$ & $<0.05$ & $<0.05$ & 0.12 & 0.68 & $<1$ \\
\hline 631-22G-S44 & 4.58 & $<0.05$ & 0.05 & $<0.05$ & $<0.05$ & $<0.05$ & 0.38 & $<1$ \\
\hline 631-22G-S45 & 4.64 & $<0.05$ & 0.06 & $<0.05$ & $<0.05$ & $<0.05$ & 0.86 & 6 \\
\hline $631-22 G-S 46$ & 4.39 & $<0.05$ & 0.05 & $<0.05$ & $<0.05$ & $<0.05$ & 1.03 & 2 \\
\hline $631-22 G-\$ 47$ & 4.40 & $<0.05$ & $<0.05$ & $<0.05$ & $<0.05$ & $<0.05$ & $<0.05$ & 1 \\
\hline 631-22G-S48 & 4.54 & $<0.05$ & $<0.05$ & $<0.05$ & $<0.05$ & $<0.05$ & 0.63 & 2 \\
\hline
\end{tabular}

A.? 
TABLE A-3 (Continued)

GRACE ROAD WASTE SITE (631-22G)

GAS CONCENTRATIONS IN SOIL (ng/g)

\begin{tabular}{|c|c|c|c|c|c|c|c|c|}
\hline $\begin{array}{l}\text { SAMPLE } \\
\text { NAME }\end{array}$ & METHANE & ETHANE & ETHYLENE & PROPANE & PROPYLENE & I-BUTANE & N-BUTANE & MERCURY \\
\hline 631-22G-S49 & 4.67 & $<0.05$ & 0.05 & $<0.05$ & $<0.05$ & $<0.05$ & $<0.05$ & 2 \\
\hline 631-22G-S50 & 4.54 & $<0.05$ & $<0.05$ & $<0.05$ & $<0.05$ & $<0.05$ & 0.72 & 1 \\
\hline $631-22 G-550 A$ & 4.57 & $<0.05$ & $<0.05$ & $<0.05$ & $<0.05$ & $<0.05$ & 0.31 & 2 \\
\hline $631-22 G-551$ & 4.63 & $<0.05$ & $<0.05$ & $<0.05$ & $<0.05$ & $<0.05$ & $<0.05$ & 1 \\
\hline 631-22G-S52 & 4.41 & $<0.05$ & $<0.05$ & $<0.05$ & $<0.05$ & $<0.05$ & $<0.05$ & 2 \\
\hline $631-22 G-S 53$ & 4.55 & $<0.05$ & $<0.05$ & $<0.05$ & $<0.05$ & $<0.05$ & $<0.05$ & 1 \\
\hline 631-22G-S54 & 4.49 & $<0.05$ & 0.07 & $<0.05$ & $<0.05$ & $<0.05$ & $<0.05$ & 1 \\
\hline 631-22G-S55 & 4.33 & $<0.05$ & 0.06 & $<0.05$ & $<0.05$ & $<0.05$ & 0.15 & $<1$ \\
\hline 631-22G-S56 & 4.66 & $<0.05$ & $<0.05$ & $<0.05$ & $<0.05$ & $<0.05$ & $<0.05$ & $<1$ \\
\hline 631-22G-S57 & 4.52 & $<0.05$ & $<0.05$ & $<0.05$ & $<0.05$ & $<0.05$ & $<0.05$ & l \\
\hline 63.1-22G-S58 & 4.45 & $<0.05$ & $<0.05$ & $<0.05$ & $<0.05$ & $<0.05$ & $<0.05$ & $<1$ \\
\hline 631-22G-S59 & 4.46 & $<0.05$ & $<0.05$ & $<0.05$ & $<0.05$ & $<0.05$ & 0.13 & $<1$ \\
\hline $631-22 G-560^{\circ}$ & 4.10 & $<0.05$ & $<0.05$ & $<0.05$ & $<0.05$ & $<0.05$ & 0.07 & $<1$ \\
\hline $631-22 G-560 A$ & 4.49 & $<0.05$ & $<0.05$ & $<0.05$ & $<0.05$ & $<0.05$ & $<0.05$ & $<1$ \\
\hline $631-22 G-S 61$ & 3.71 & $<0.05$ & 0.06 & $<0.05$ & 0.08 & $<0.05$ & $<0.05$ & 4 \\
\hline $631-22 G-S 62$ & 3.89 & $<0.05$ & 0.12 & $<0.05$ & $<0.05$ & $<0.05$ & 0.16 & 1 \\
\hline $631-22 \mathrm{G}-\mathrm{S} 63$ & 3.62 & $<0.05$ & $<0.05$ & $<0.05$ & $<0.05$ & $<0.05$ & $<0.05$ & 2 \\
\hline $631-22 G-\$ 64$ & 3.59 & $<0.05$ & 0.08 & $<0.05$ & $<0.05$ & $<0.05$ & $<0.05$ & 4 \\
\hline 631-22G-\$65 & 3.77 & $<0.05$ & 0.12 & $<0.05$ & $<0.05$ & $<0.05$ & $<0.05$ & 2 \\
\hline $631-22$ G-S66 & 3.63 & $<0.05$ & 0.05 & 0.06 & $<0.05$ & $<0.05$ & 0.22 & 2 \\
\hline $631-22 G-S 67$ & 3.86 & $<0.05$ & $<0.05$ & $<0.05$ & $<0.05$ & $<0.05$ & $<0.05$ & 1 \\
\hline 631-22G-S68 & 3.83 & $<0.05$ & $<0.05$ & $<0.05$ & $<0.05$ & $<0.05$ & $<0.05$ & 3 \\
\hline 631-22G-\$69 & 3.86 & $<0.05$ & $<0.05$ & $<0.05$ & $<0.05$ & $<0.05$ & $<0.05$ & 2 \\
\hline 631-22G-S70 & 3.85 & $<0.05$ & 0.07 & $<0.05$ & $<0.05$ & $<0.05$ & $<0.05$ & 2 \\
\hline 631-22G-S70A & 3.92 & $<0.05$ & $<0.05$ & $<0.05$ & 0.13 & 0.13 & 0.32 & 2 \\
\hline 631-22G-S71 & 3.86 & $<0.05$ & $<0.05$ & $<0.05$ & $<0.05$ & $<0.05$ & 0.11 & 4 \\
\hline
\end{tabular}


TABLE A-3 (Continued)

GRACE ROAD WASTE SITE (631-22G)

GAS CONCENTRATIONS IN SOIL (ng/g)

\begin{tabular}{|c|c|c|c|c|c|c|c|c|}
\hline $\begin{array}{c}\text { SAMPLE } \\
\text { NAME }\end{array}$ & METHANE & ETHANE & ETHYLENE & PROPANE & PROPYLENE & I-BUTANE & N-BUTANE & MERCURY \\
\hline $631-22 \mathrm{G}-\mathrm{S} 72$ & 3.79 & $<0.05$ & $<0.05$ & $<0.05$ & $<0.05$ & $<0.05$ & $<0.05$ & 11 \\
\hline 631-22G-S73 & 3.95 & $<0.05$ & 0.05 & $<0.05$ & $<0.05$ & $<0.05$ & 0.20 & 2 \\
\hline 631-22G-S74 & 3.74 & $<0.05$ & 0.08 & $<0.05$ & $<0.05$ & $<0.05$ & $<0.05$ & 21 \\
\hline $631-22 G-S 75$ & 3.61 & $<0.05$ & 0.06 & $<0.05$ & $<0.05$ & $<0.05$ & $<0.05$ & 2 \\
\hline $631-22 G-S 76$ & 3.73 & $<0.05$ & 0.19 & 0.11 & 0.14 & 0.20 & $<0.05$ & $<1$ \\
\hline 631-22G-S77 & 3.78 & $<0.05$ & 0.06 & 0.06 & $<0.05$ & $<0.05$ & $<0.05$ & $<1$ \\
\hline 631-22G-S78 & 3.58 & $<0.05$ & $<0.05$ & $<0.05$ & $<0.05$ & $<0.05$ & $<0.05$ & 1 \\
\hline 631-22G-S79 & 3.59 & $<0.05$ & 0.16 & $<0.05$ & $<0.05$ & $<0.05$ & $<0.05$ & $<1$ \\
\hline $631-22 G-S 80$ & 3.80 & $<0.05$ & $<0.05$ & $<0.05$ & $<0.05$ & $<0.05$ & 0.18 & $<1$ \\
\hline $631-22 G-580 A$ & 3.82 & $<0.05$ & $<0.05$ & $<0.05$ & $<0.05$ & $<0.05$ & $<0.05$ & 1 \\
\hline $631-22 G-S 81$ & 3.73 & $<0.05$ & $<0.05$ & $<0.05$ & $<0.05$ & $<0.05$ & $<0.05$ & $<1$ \\
\hline 631-22G-S82 & 3.41 & $<0.05$ & $<0.05$ & 0.05 & $<0.05$ & $<0.05$ & 0.08 & $<1$ \\
\hline $631-22 G-583$ & 3.75 & $<0.05$ & 0.14 & 0.14 & $<0.05$ & $<0.05$ & $<0.05$ & $<1$ \\
\hline 631-22G-S84 & 3.74 & $<0.05$ & $<0.05$ & $<0.05$ & $<0.05$ & $<0.05$ & $<0.05$ & $<1$ \\
\hline 631-22G-S85 & 3.53 & $<0.05$ & $<0.05$ & $<0.05$ & $<0.05$ & $<0.05$ & $<0.05$ & $<1$ \\
\hline $631-22 \mathrm{G}-\mathrm{SB} 1$ & 4.24 & $<0.05$ & $<0.05$ & $<0.05$ & $<0.05$ & $<0.05$ & $<0.05$ & NA \\
\hline 631-22G-SB2 & 4.25 & $<0.05$ & 0.28 & $<0.05$ & $<0.05$ & $<0.05$ & $<0.05$ & NA \\
\hline 631-22G-SB3 & 4.30 & $<0.05$ & 0.54 & 0.05 & $<0.05$ & $<0.05$ & $<0.05$ & NA \\
\hline $631-22 G-S B 4$ & 4.11 & $<0.05$ & 0.40 & 0.08 & $<0.05$ & $<0.05$ & $<0.05$ & NA \\
\hline 631-22G-SB5 & 4.16 & $<0.05$ & 0.37 & 0.13 & 0.15 & $<0.05$ & 0.39 & NA \\
\hline $631-22 G-S B 6$ & 4.27 & $<0.05$ & 0.35 & 0.12 & $<0.05$ & $<0.05$ & 0.37 & NA \\
\hline $631-22 G-S B 7$ & 3.39 & $<0.05$ & 0.37 & 0.07 & $<0.05$ & $<0.05$ & $<0.05$ & NA \\
\hline 631-22G-SB8 & 3.55 & $<0.05$ & 0.38 & 0.10 & $<0.05$ & $<0.05$ & $<0.05$ & NA \\
\hline $631-22 G-S B 9$ & 3.50 & $<0.05$ & 0.32 & $<0.05$ & $<0.05$ & $<0.05$ & $<0.05$. & NA \\
\hline
\end{tabular}


TABLE A-4

GRACE ROAD WASTE SITE (631-22G)

GAS CONCENTRATIONS IN SOIL (ng/g)

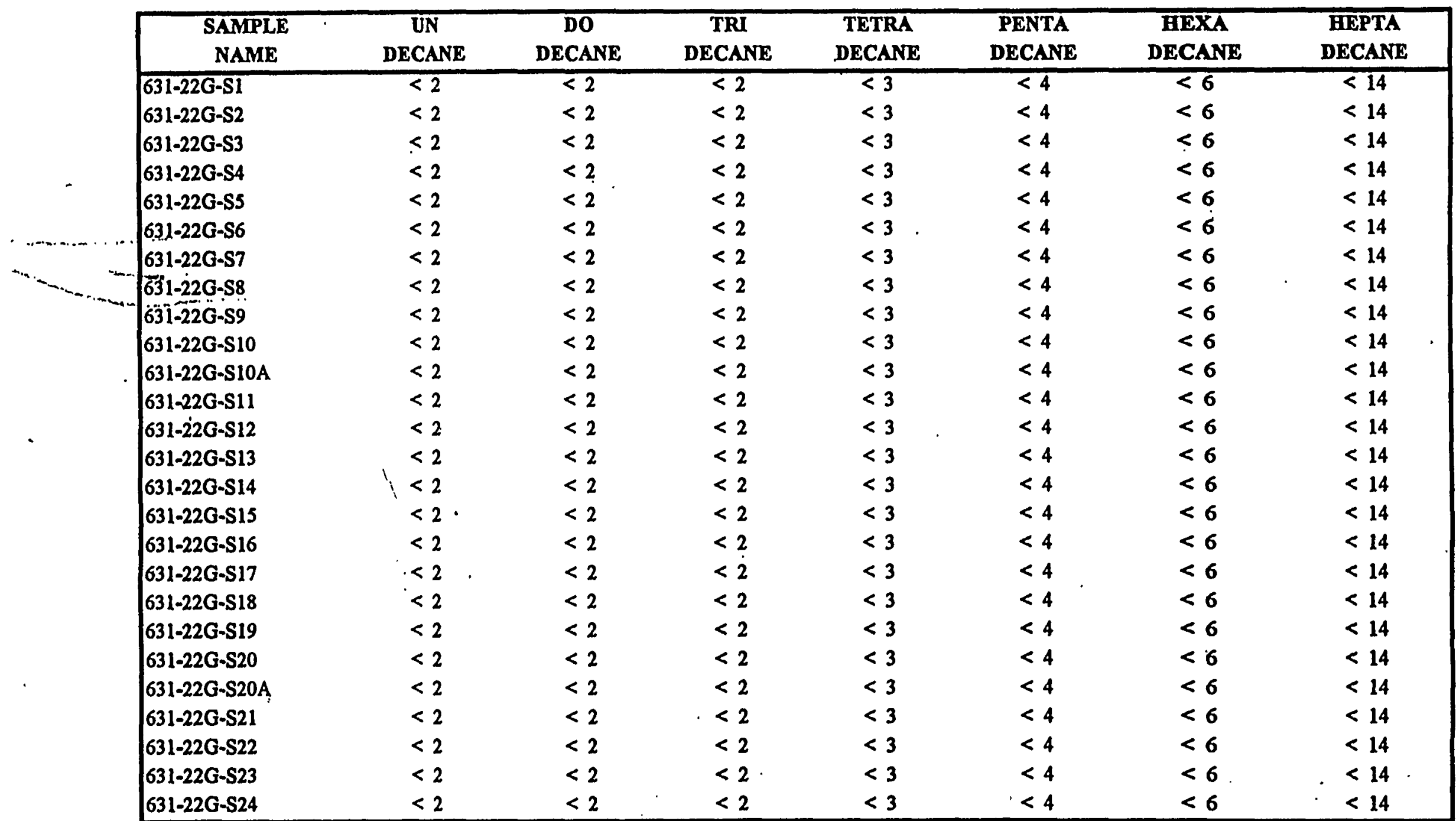


TABLE A-4 (Continued)

GRACE ROAD WASTE SITE (631-22G)

GAS CONCENTRATIONS IN SOIL (ng/g)

\begin{tabular}{|c|c|c|c|c|c|c|c|}
\hline $\begin{array}{c}\text { SAMPLE } \\
\text { NAME }\end{array}$ & $\begin{array}{c}\text { UN } \\
\text { DECANE }\end{array}$ & $\begin{array}{c}\text { DO } \\
\text { DECANE }\end{array}$ & $\begin{array}{c}\text { TRI } \\
\text { DECANE }\end{array}$ & $\begin{array}{c}\text { TETRA } \\
\text { DECANE }\end{array}$ & $\begin{array}{c}\text { PENTA } \\
\text { DECANE }\end{array}$ & $\begin{array}{c}\text { HEXA } \\
\text { DECANE }\end{array}$ & $\begin{array}{c}\text { HEPTA } \\
\text { DECANE }\end{array}$ \\
\hline 631-22G-S25 & $<2$ & $<2$ & $<2$ & $<3$ & $<4$ & $<6$ & $<14$ \\
\hline 631-22G-S26 & $<2$, & $<2$ & $<2$ & $<3$ & $<4$ & $<6$ & $<14$ \\
\hline 631-22G-S27 & $<2$ & $<2$ & $<2$ & $<3$ & $<4$ & $<6$ & $<14$ \\
\hline $631-22 G-S 28$ & $<2$ & $<2$. & $<2$ & $<3$ & $<4$ & $<6$ & $<14$ \\
\hline $631-22 G-\$ 29$ & $<2$ & $<2$ & $<2$ & $<3$ & $<4$ & $<6^{\circ}$ : & $<14$ \\
\hline $631-22 G-\$ 30$ & $<2$ & $<2$ & $<2$ & $<3$ & $<4$ & $<6$ & $<14$ \\
\hline $631-22 G-S 30 A$ & $<2$ & $<2$ & $<2$ & $<3$ & $<4$ & $<6$ & $<14$ \\
\hline 631-22G-S31 & $<2$ & $<2$ & $<2$ & $<3$ & $<4$ & $<6$ & $<14$ \\
\hline 631-22G-S32 & $<2$ & $<2$ & $<2$ & $<3$ & $<4$ & $<6$ & $<14$ \\
\hline 631-22G-S33 & $<2$ & $<2$ & $<2$ & $<3$ & $<4$ & $<6$ & $<14$ \\
\hline 631-22G-S34 & $<2$ & $<2$ & $<2$ & $<3$ & $<4$ & $<6$ & $<14$ \\
\hline 631-22G-S35 & $<2$ & $<2$ & $<2$ & $<3$ & $<4$ & $<6$ & $<14$ \\
\hline $631-22 \mathrm{G}-\$ 36$ & $<2$ & $<2$ & $<2$ & $<3$ & $<4$ & $<6$ & $<14$ \\
\hline 631-22G-S37 & $<2$ & $<2$ & $<2$ & $<3$ & $<4$ & $<6$ & $<14$ \\
\hline 631-22G-S38 & $<2$ & $<2$ & $<2$ & $<3$ & $<4$ & $<6$ & $<14$ \\
\hline 631-22G-S39 & $<2$ & $<2$ & $<2$ & $<3$ & $<4$ & $<6$ & $<14$ \\
\hline $631-22 G-S 40$ & $<2$ & $<2$ & $<2$ & $<3$ & $<4$ & $<6$ & $<14$ \\
\hline 63.1-22G-S40A & $<2$ & $<2$ & $<2$ & $<3$ & $<4$ & $<6$ & $<14$ \\
\hline $631-22 G-S 41$ & $<2$ & $<2$ & $<2$ & $<3$ & $<4$ & $<6$ & $<14$ \\
\hline $631-22 G-S 42$ & $<2$ & $<2$ & $<2$ & $<3$ & $<4$ & $<6$. & $<14$ \\
\hline 631-22G-S43 & $<2$ & $<2$ & $<2$ & $<3$ & $<4$ & $<6$ & $<14$ \\
\hline $631-22 G-S 44$ & $<2$ & $<2$ & $<2$ & $<3$ & $<4$ & $<6$ & $<14$ \\
\hline 631-22G-S45 & $<2$ & $<2$ & $<2$ & $<3$ & $<4$ & $<6$ & $<14$ \\
\hline 631-22G-S46 & $<2$ & $<2$ & $<2$ & $<3$ & $<4$ & $<6$ & $<14$ \\
\hline $631-22 G-S 47$ & $<2$ & $<2$ & $<2$ & $<3$ & $<4$ & $<6$ & $<14$ \\
\hline $631-22 \mathrm{G}-\$ 48$ & $<2$ & $<2$ & $<2$ & $<3$ & $<4$ & $<6$. & $<14$ \\
\hline
\end{tabular}

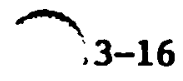


TABLE A-4 (Continued)

GRACE ROAD WASTE SITE (631-22G)

GAS CONCENTRATIONS IN SOUL (ng/g)

\begin{tabular}{|c|c|c|c|c|c|c|c|}
\hline $\begin{array}{c}\text { SAMPLE } \\
\text { NAME }\end{array}$ & $\begin{array}{c}\text { UN } \\
\text { DECANE }\end{array}$ & $\begin{array}{c}\text { DO } \\
\text { DECANE }\end{array}$ & $\begin{array}{c}\text { TRI } \\
\text { DECANE }\end{array}$ & $\begin{array}{c}\text { TETRA } \\
\text { DECANE }\end{array}$ & $\begin{array}{c}\text { PENTA } \\
\text { DECANE }\end{array}$ & $\begin{array}{c}\text { HEXA } \\
\text { DECANE }\end{array}$ & $\begin{array}{c}\text { HEPTA } \\
\text { DECANE }\end{array}$ \\
\hline 631-22G-S72 & $<2$ & $<2$ & $<2$ & $<3$ & $<4$ & $<6$ & $<14$ \\
\hline $631-22 G-573$ & $<2^{\prime}$ & $<2$ & $<2$ & $<3$ & $<4$ & $<6$ & $<14$ \\
\hline 631-22G-S74 & $<2$ & $<2$ & $<2$ & $<3$ & $<4$ & $<6$ & $<14$ \\
\hline 631-22G-S75 & $<2$ & $<2$ & $<2$ & $<3$ &.$<4$ & $<6$ & $<14$ \\
\hline $631-22 G-S 76$ & $<2$ & $<2$ & $<2$ & $<3$ & $<4$ & $<6$ & $<14$ \\
\hline 631-22G-S77 & $<2$ & $<2$ & $<2$ & $<3$ & $<4$ & $<6$ & $<14$ \\
\hline 631-22G-S78 & $<2$ & $<2$ & $<2$ & $<3$ & $<4$ & $<6$ & $<14$ \\
\hline 631-22G-S79 & $<2$ & $<2$ & $<2$ & $<3$ & $<4$ & $<6$ & $<14$ \\
\hline $631-22 G-S 80$ & $<2$ & $<2$ & $<2$ & $<3$ & $<4$ & $<6$ & $<14$ \\
\hline $631-22 G-S 80 A$ & $<2$ & $<2$ & $<2$ & $<3$ & $<4$ & $<6$ & $<14$ \\
\hline $631-22 G-S 81$ & $<2$ & $<2$ & $<2$ & $<3$ & $<4$ & $<6$ & $<14$ \\
\hline 631-22 G-S82 & $<2$ & $<2$ & $<2$ & $<3$ & $<4$ & $<6$ & $<14$ \\
\hline 631-22G-S83 & $<2$ & $<2$ & $<2$ & $<3$ & $<4$ & $<6$ & $<14$ \\
\hline $631-22 G-\$ 84$ & $<2$ & $<2$ & $<2$ & $<3$ & $<4$ & $<6$ & $<14$ \\
\hline $631-22 G-S 85^{\circ}$ & $i<2$ & $<2$ & $<2$ & $<3$ & $<4$ & $<6$ & $<14$ \\
\hline 631-22G-SB1 & $<2$ & $<2$ & $<2$ & $<3$ & $<4$ & $<6$ & $<14$ \\
\hline 631-22G-SB2 & $<2$ & $<2$ & $<2$ & $<3$ & $<4$ & $<6$ & $<14$ \\
\hline 631-22G-SB3 & $<2$ & $<2$ & $<2$ & $<3$ & $<4$ & $<6$ & $<14$ \\
\hline 631-22G-SB4 & $<2$ & $<2$ & $<2$ & $<3$ & $<4$ & $<6$ & $<14$ \\
\hline 631-22G-SB5 & $<2$ & $<2$ & $<2$ & $<3$ & $<4$ & $<6$ & $<14$ \\
\hline 631-22G-SB6 & $<2$ & $<2$ & $<2$ & $<3$ & $<4$ & $<6$ & $<14$ \\
\hline $631-22 \mathrm{G}-\mathrm{SB7}$ & $<2$ & $<2$ & $<2$ & $<3$ & $<4$ & $<6$ & $<14$ \\
\hline 631-22G-SB8 & $<2$ & $<2$ & $<2$ & $<3$ & $<4$ & $<6$ & $<14$ \\
\hline 631-22G-SB9 & $<2$ & $<2$ & $<2$ & $<3$ & $<4$ & $<6$ & $<14$ \\
\hline
\end{tabular}


TABLE A-4 (Continued)

GRACE ROAD WASTE SITE (631-22G)

GAS.CONCENTRATIONS IN SOIL (ng/g)

\begin{tabular}{|c|c|c|c|c|c|c|c|}
\hline $\begin{array}{l}\text { SAMPLE } \\
\text { NAME }\end{array}$ & $\begin{array}{c}\text { UN } \\
\text { DECANE }\end{array}$ & $\begin{array}{c}\text { DO } \\
\text { DECANE }\end{array}$ & $\begin{array}{c}\text { TRI } \\
\text { DECANE }\end{array}$ & $\begin{array}{c}\text { TETRA } \\
\text { DECANE }\end{array}$ & $\begin{array}{l}\text { PENTA } \\
\text { DECANE }\end{array}$ & $\begin{array}{c}\text { HEXA } \\
\text { DECANE }\end{array}$ & $\begin{array}{c}\text { HEPTA } \\
\text { DECANE }\end{array}$ \\
\hline 631-22G-S49 & $<2$ &.$<2$ & $<2$ & $<3$ & $<4$ & $<6$ & $<14$ \\
\hline 631-22G-S50 & $<2^{\prime}$ & $<2$ & $<2$ & $<3$ & $<4$ & $<6$ & $<14$ \\
\hline 631-22G-S50A & $<2$ & $<2$ & $<2$ & $<3$ & $<4$ & $<6$ & $<14$ \\
\hline $631-22 G-S 51$ & $<2$ & $<2$ & $<2$ & $<3$ & $\cdot<4$ & $<6$ & $<14$ \\
\hline 631-22G-S52 & $<2$ & $<2$ & $<2$ & $<3$ & $<4$ & $<6$ & $<14$ \\
\hline 631-22G-S53 & $<2$ & $<2$ & $<2$ & $<3$ & $<4$ & $<6$ & $<14$ \\
\hline 631-22G-S54 & $<2$ & $<2$ & $<2$ & $<3$ & $<4$ & $<6$ & $<14$ \\
\hline 631-22G-S55 & $<2$ & $<2$ & $<2$ & $<3$ & $<4$ & $<6$ & $<14$ \\
\hline 631-22G-S56 & $<2$ & $<2$ & $<2$ & $<3$ & $<4$ & $<6$. & $<14$ \\
\hline 631-22G-S57 & $<2$ & $<2$ & $<2$ & $<3$ & $<4$ & $<6$ & $<1.4$ \\
\hline 631-22G-58 & $<2$ & $<2$ & $<2$ & $<3$ & $<4$ & $<6$ & $<14$ \\
\hline 631-22G-59 & $<2$ & $<2$ & $<2$ & $<3$ & $<4$ & $<6$ & $<14$ \\
\hline $631-22 \mathrm{G}-60$ & $<2$ & $<2$ & $<2$ & $<3$ & $<4$ & $<6$ & $<14$ \\
\hline $631-22 \mathrm{G}-60 \mathrm{~A}$ & $<2$ & $<2$ & $<2$ & $<3$ & $<4$ & $<6$ & $<14$ \\
\hline $631-22 G-\$ 61$ & $1<2$ & $<2$ & $<2$ & $<3$ & $<4$ & $<6$ & $<14$ \\
\hline 631-22G-S62 & $<2$ & $<2$ & $<2$ & $<3$ & $<4$ & $<6$ & $<14$ \\
\hline 631-22G-S63 & $<2$ & $<2$ & $<2$ & $<3$ & $<4$ & $<6$ & $<14$ \\
\hline 631-22G-S64 & $<2$ & $<2$ & $<2$ & $<3$ & $<4$ & $<6$ & $<14$ \\
\hline 631-22G-S65 & $<2$ & $<2$ & $<2$ & $<3$ & $<4$ & $<6$ & $<14$ \\
\hline 631-22G-S66 & $<2$ & $<2$ & $<2$ & $<3$ & $<4$ & $<6$ & $<14$ \\
\hline 631-22G-S67 & $<2$ & $<2$ & $<2$ & $<3$ & $<4$ & $<6$ & $<14$ \\
\hline 631-22G-S68 & $<2$ & $<2$ & $<2$ & $<3$ & $<4$ & $<6$ & $<14$ \\
\hline 631-22G-\$69 & $<2$ & $<2$ & $<2$ & $<3$ & $<4$ & $<6$ & $<14$ \\
\hline $631-22 G-S 70$ & $<2$ & $<2$ & $<2$ & $<3$ & $<4$ & $<6$ & $<14$ \\
\hline $631-22 G-S 70 A$ & $<2$ & $<2$ & $<2$ & $<3$ & $<4$ & $<6$ & $<14$ \\
\hline 631-22G-S71 & $<2$ & $<2$ & $<2$ & $<3$ & $<4$ & $<6$ & $<14$ \\
\hline
\end{tabular}


Grace Road Site

RFI/RI Report
WSRC-RP-95-93, Rev. 1

November 1995

APPENDIX B

ANALYTICAL DATA AND QAVQ EVALUATION RESULTS

B-1 
Grace Road Site

RFI/RI Report
WSRC-RP-95-93, Rev. 1

November 1995

THIS PAGE INTENTIONALLY LEFT BLANK

$=$

B-2 


\section{Was

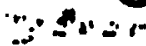 \\ $\infty$ is \\ $\because$}

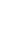

$$
\begin{aligned}
& \text { CI.IENT: WSRC-GRACE; ROAD } \\
& \text { RIE : 9003L865:3.\%: } \\
& \text { W.0. : : 0630-28-12\%-is }
\end{aligned}
$$

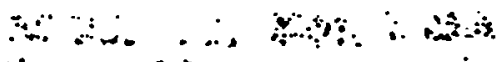

ROY

Iifonvilila Jiboratore it

\section{$\therefore \rightarrow 2$}

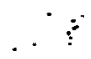

BAMPI

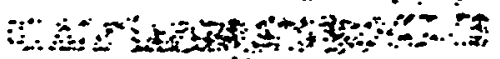

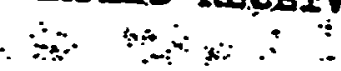

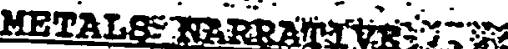

The following is a sunging

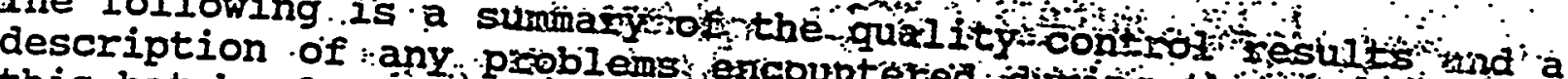

- this batch of samiles:

1. AIl sample=hiogaing

for water sample

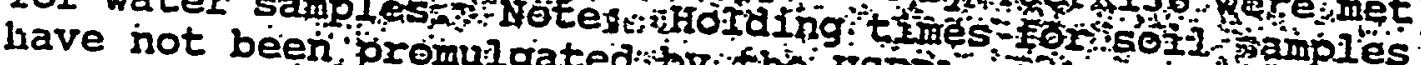

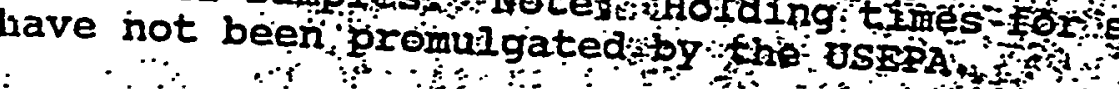

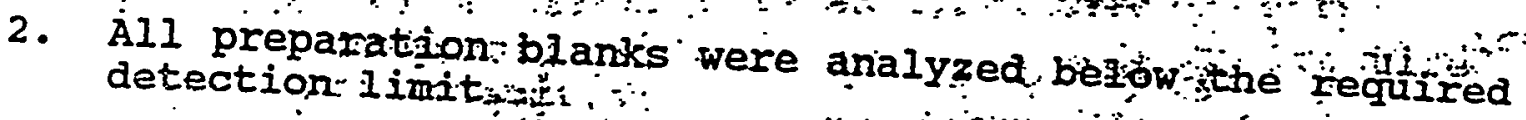

3. A.11 laboratory contio ?imits of: $80=2,20 \% \div ;$

$$
\text { .it : . }
$$

standards are wathine the controt
(1) limitsifac oliter and antinopidu to documented iteficities in ou to

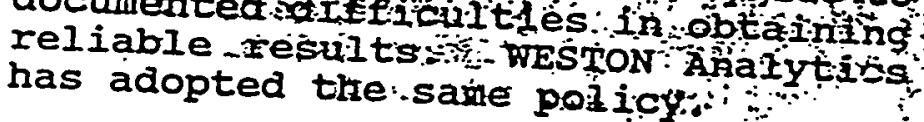

\section{4.}

All calibration verification tohecks were within the
required control limits of $90-100 \%$ ( $85-115 \%$ for fig).
Calibration verification is performed using independent
standards from Inorganic

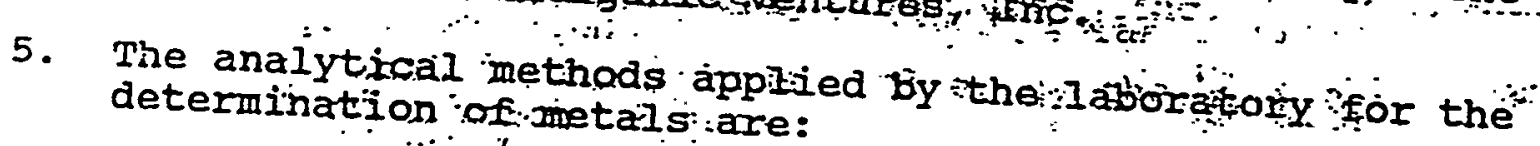

$H g:$
ICP SCNG

AII OEsier

EP Ieachates (excopt

NOTE: For sol-id sample : basis. $\quad$ s. 



\section{ROY F. WESTON, INC. \\ GLOSSARY OF TERMS - INORGANIC REPORTS}

DATA OUALIFIERS

$U$ - Indicates that the parameter was not detected at or above the reported limit. The associated numerical . value is the sample detection limit.

* - Indicates that the original sample result is griater than. $4 \mathrm{x}$ the spike amount added. The USEPA-CLP has determined that spike results on samples where this occurs may be unreliable and, therefore, the control limits are not applicable.

\section{ABBREVIATIONS}

MB - Method or preparation blank.

MS - Matrix Spike.

MSD - Matrix Spike Duplicate.

REP - Sample Replicate.

LC - Indicates a method LCS or Blank Spike.

NC - Not calculable, result below the detection limit.

\section{IABORATORY CHRONOIOGY AND HOIDTIME REPORT}

The test code listed indicates the specific analysis or preparation procedure employed. The codes may be interpreted as follows:

MAAW - Metals prep test for AA digestion; water matrix. MAAS - Metals prep test for AA digestion, soil matrix. MICW - Metals prep test for ICP digestion, water matrix. MICS - Metals prep test for ICP digestion, soil matrix.

M**iTO- This type of code indicates a total metal analysis (eg. MAGTO indicates an analysis for total silver).

$M * \star S O-$. This type of code indicates a soluble metal analysis. (eg. MAGso indicates an analysis for soluble silver).

$M * * E P-\quad T h i s$ type of code indicates an EPTOXICITY metals analysis (eg. MAGEP indicates an analysis for eptox silver).

I**TO- This type of code indicates a non-metallic total analysis. There is also a complimentary soluble analysis for each of these codes (eg. ICNTO indicates an analysis for total cyanide).

$A$ suffix of $-R$ or $-S$ following these codes indicates $a$ replicate or spike analysis respectively. 
ROY F. WESTON INC.

INORGANICS DATA SUMRARY REPORT 05/25/90

CLIENT: HSRC-GRACE ROAD

HORR ORDER: 0630-28-12-0000

\begin{tabular}{|c|c|}
\hline $\begin{array}{l}\text { SAMPLE } \\
\text { =E=I=E= }\end{array}$ & 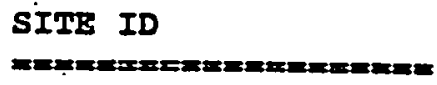 \\
\hline-002 & GRS-04-01 \\
\hline
\end{tabular}

-003 GRS-04-01A

$-005 \quad$ GRS-05-01

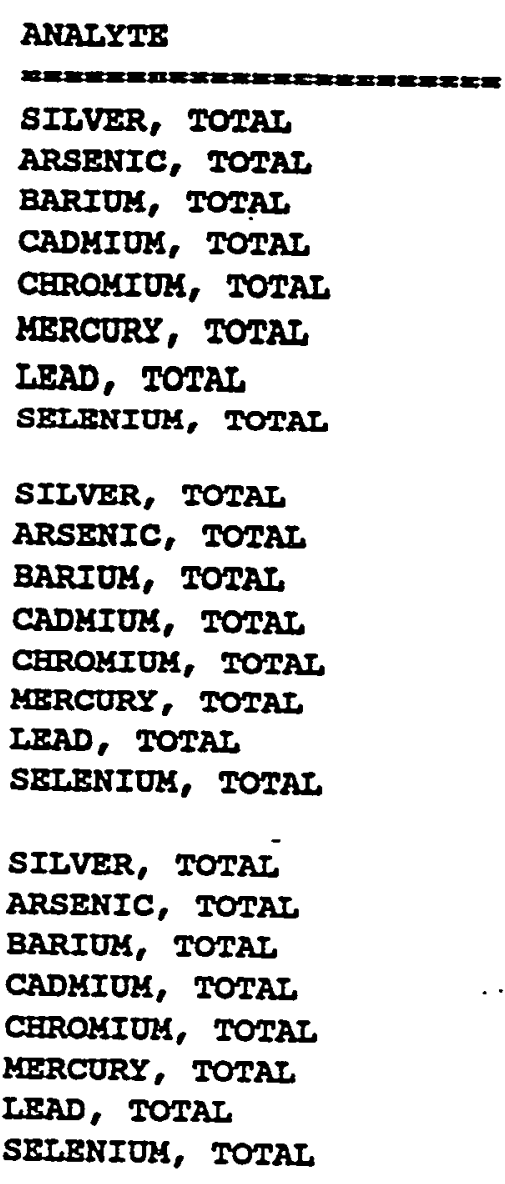

FESTON BATCH. $\%$. 9003L865

\begin{tabular}{|c|c|c|c|}
\hline $\begin{array}{l}\text { RESULT } \\
x=2=2=0\end{array}$ & & 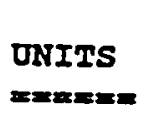 & $\begin{array}{l}\text { REPORTING } \\
\text { IIKIT } \\
\text { =E=m=m=m }\end{array}$ \\
\hline 1.9 & $\mathbf{u}$ & KG/RG & 1.9 \\
\hline 2.1 & $\mathbf{u}$ & $M G / K G$ & 2.1 \\
\hline 37.5 & $\mathbf{u}$ & $M G / R G$ & 37.5 \\
\hline 1.2 & & $M G / R G$ & 0.94 \\
\hline 5.7 & & $\mathrm{MG} / \mathrm{KG}$ & 1.9 \\
\hline 0.11 & $\mathbf{u}$ & MG/KG & 0.11 \\
\hline 0.95 & & MG/KG & 0.63 \\
\hline 1.1 & $\mathbf{u}$ & MG/KG & 1.1 \\
\hline 2.0 & $\mathbf{u}$ & MG/KG & 2.0 \\
\hline 2.3 & $\mathbf{u}$ & $M G / R G$ & 2.3 \\
\hline 40.1 & $\mathbf{u}$ & $M G / K G$ & 40.1 \\
\hline 1.2 & & $\mathrm{KG} / \mathrm{KG}$ & 1.0 \\
\hline 5.3 & & $\mathrm{MG} / \mathrm{KG}$ & 2.0 \\
\hline 0.12 & $\mathbf{u}$ & MG/RG & 0.12 \\
\hline $\begin{array}{l}1.1 \\
1.2\end{array}$ & $\mathbf{u}$ & $\begin{array}{l}\text { MG/KG } \\
M G / K G\end{array}$ & 0.70 \\
\hline 2.0 & $\mathbf{u}$ & $\mathbf{K G} / \mathbf{K G}$ & 2.0 \\
\hline 2.1 & $\mathbf{u}$ & $M G / R G$ & 2.1 \\
\hline 39.3 & $\mathbf{u}$ & MG/RG & 39.3 \\
\hline 1.2 & & MG/KG & 0.98 \\
\hline 8.0 & & $M G / K G$ & 2.0 \\
\hline 0.11 & $\mathbf{u}$ & $\mathrm{MG} / \mathrm{KG}$ & 0.11 \\
\hline $2 \cdot 1$ & & $\mathrm{MG} / \mathrm{KG}$ & 0.62 \\
\hline 1.0 & $\mathbf{u}$ & MG/KG & 1.0 \\
\hline
\end{tabular}


ROY F. FESTON IXC.

INORGANICS DATA SUMYARY REPORT 05/25/90

CLIENT: HSRC-GRACE ROAD

WORK ORDER: 0630-28-12-0000

SAMPIR

Ix=m-man

$-006$

SITE ID

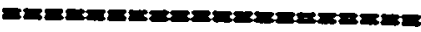

GRS-06-01

-007 GRS-11-01

$-008$

GRS-01-01
HESTON BATCA \&. 9003L865

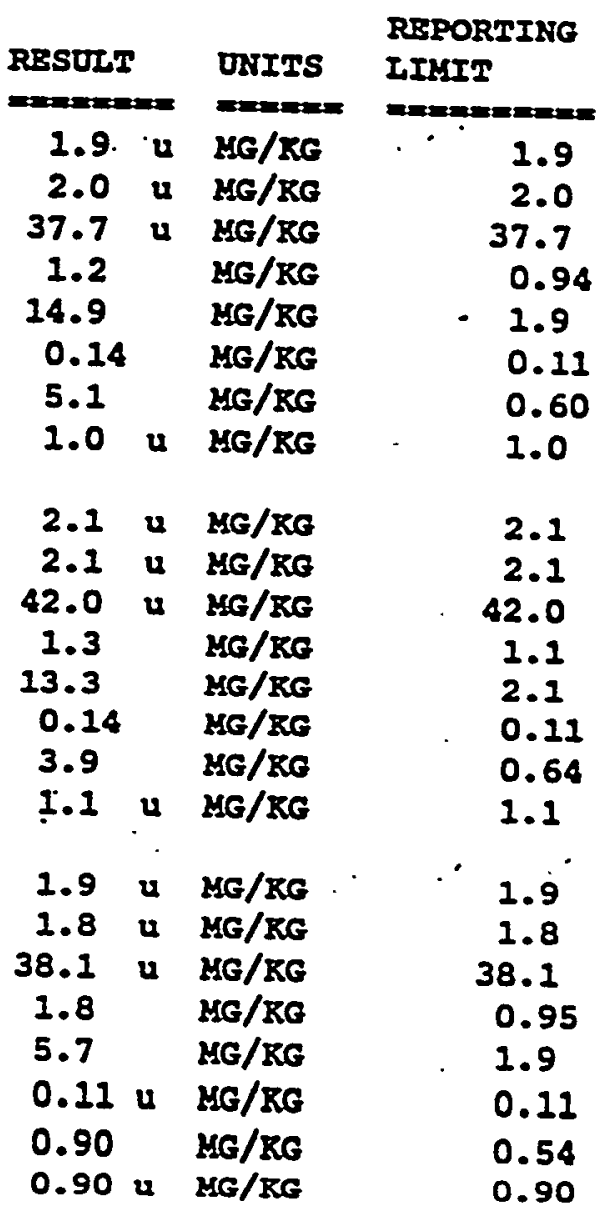


ROY F. FESTON INC.

INORGANICS DATA SUMAARY REPORT 05/25/90

CLIENT: WSRC-GRACB ROAD

WORR ORDER: 0630-28-12-0000

SAMPLE - SITE ID

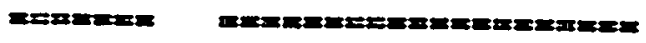

-010 GRS-13-01

-011 GRS-14-01

$-012$

\section{ANALYTB}

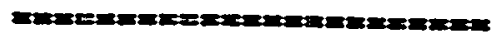

SIIVER, TOTAT

ARSERIC, TOTAL.

BARIUL, TOLAT

CADKIUK, TOLAT

CHROMIUM, TOTAT

MERCURY, TOTAT

IEAD, TOTAI.

SEIEKIUK, TOTAI

SIIVHR, TOTAI ARSENIC, TOTAX BARIUS, TOTAI CADMIOK, TOTAI CHROMIUK, TOTAL. MERCURY, TOTAI IEAD, TOTAL SETENIUK, TOTAT.

SILVtar, TOMAL' ARSENIC, TOTAY BARIUR! TOTAL CADMIUM, TOTAL CEROKIUM, TOTAI MBRCURY; TOIAL IEAD, TOTAL SELENIUK, TOTAT
WESTON BATCA 90032865

\begin{tabular}{|c|c|c|c|}
\hline \multicolumn{2}{|c|}{ RESULT } & $\begin{array}{l}\text { UNITS } \\
\text { =:m=m= }\end{array}$ & $\begin{array}{l}\text { REPORMING } \\
\text { IIXIT } \\
==\end{array}$ \\
\hline 2.0 & $\mathbf{u}$ & $\mathrm{MG} / \mathrm{KG}$ & 2.0 \\
\hline 2.3 & $\mathbf{u}$ & MG/KG & 2.3 \\
\hline 40.3 & $\mathbf{u}$ & $\mathrm{KG} / \mathrm{RG}$ & 40.3 \\
\hline 1.2 & & MG/RG & 1.0 \\
\hline 22.9 & & $M G / R G$ & 2.0 \\
\hline 0.15 & & MG/KG & 0.12 \\
\hline 48.1 & & LG/RG & 7.0 \\
\hline $1 \cdot 3$ & & MG/RG & 1.2 \\
\hline 2.1 & $\mathbf{u}$ & $\mathrm{MG} / \mathrm{KG}$ & 2.1 \\
\hline 2.2 & $\mathbf{u}$ & KG/KG & 2.2 \\
\hline 48.4 & & MG/RG & 43.0 \\
\hline 1.7 & & $M G / K G$ & 1.1 \\
\hline 18.1 & & MG/RG & 2.1 \\
\hline 0.12 & $\mathbf{u}$ & MG/RG & 0.12 \\
\hline 5.1 & & MG/KG & 0.65 \\
\hline 1.1 & $\mathbf{u}$ & MG/KG & \\
\hline 10.0 & $\mathbf{u}$ & $\nabla G / I$ & .10 .0 \\
\hline 10.0 & $\mathbf{u}$ & UG/L & 10.0 \\
\hline 200 & $\mathbf{u}$ & DG/L & 200 \\
\hline 5.0 & $\mathbf{u}$ & UG/I & 5.0 \\
\hline $\begin{array}{c}10.0 \\
0.20\end{array}$ & $\begin{array}{l}\mathbf{u} \\
\mathbf{u}\end{array}$ & $\begin{array}{l}U G / I \\
U G / I\end{array}$ & $\begin{array}{r}10.0 \\
0.20\end{array}$ \\
\hline 3.0 & $\mathbf{u}$ & UG/L & 3.0 \\
\hline 5.0 & $\mathbf{u}$ & $0 G / I$ & 5.0 \\
\hline
\end{tabular}


CLIENT: WBRC-GRACB ROAD

HORK ORDER: 0630-28-12-0000

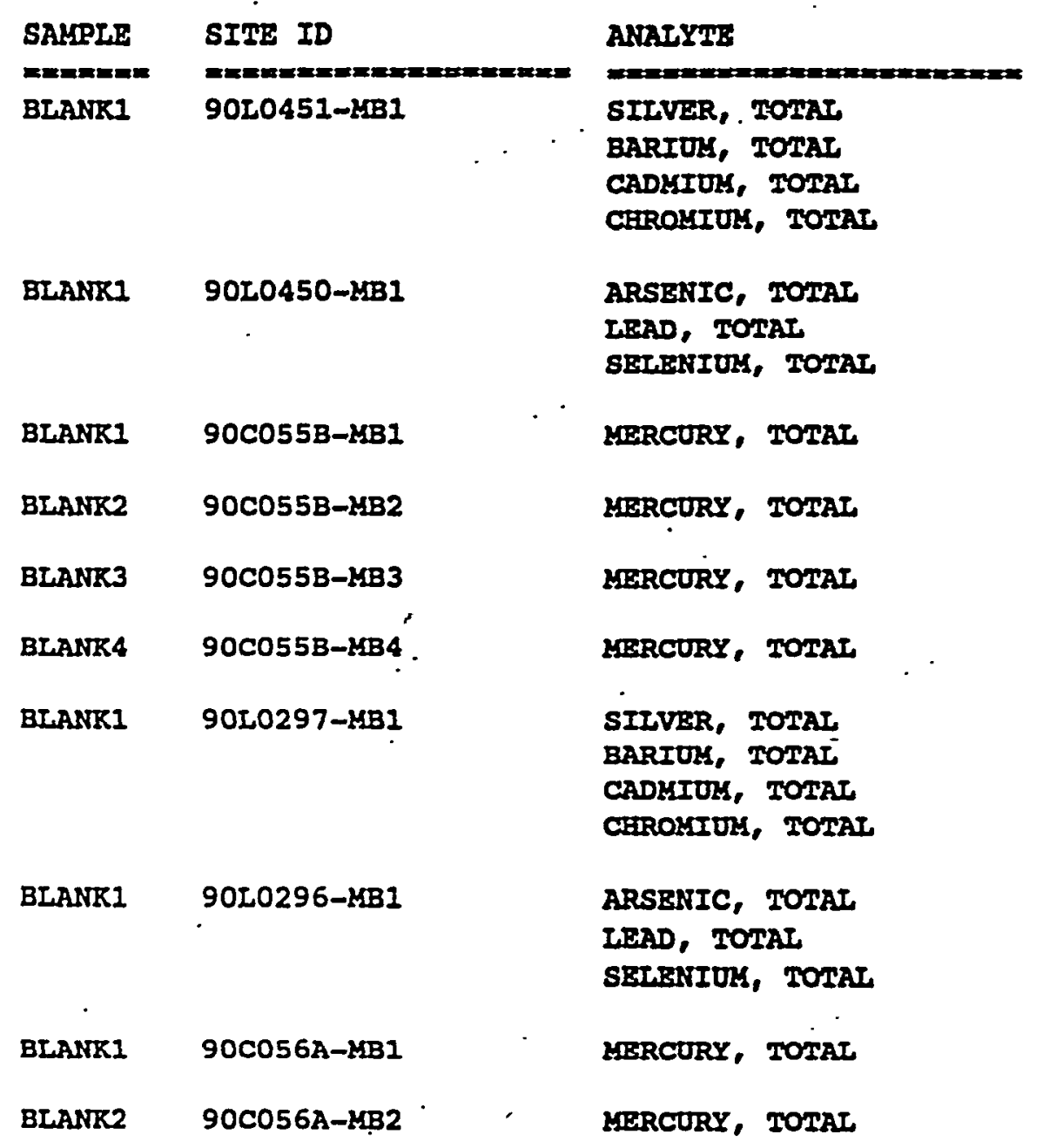

RESTON BATCA 9.0032865

\begin{tabular}{|c|c|c|c|}
\hline \multicolumn{2}{|c|}{ RESULT } & $\begin{array}{l}\text { UNIIS } \\
\text { =m:=m }\end{array}$ & $\begin{array}{l}\text { REBORIING } \\
\text { IIYIT } \\
=\end{array}$ \\
\hline 2.0 & $\mathbf{u}$ & ME/XG & 2.0 \\
\hline 40.0 & $\mathbf{u}$ & KG/KG & 40.0 \\
\hline 1.0 & $\mathbf{u}$ & MG/KG & 1.0 \\
\hline 2.0 & $\mathbf{u}$ & $\mathrm{NG} / \mathrm{KG}$ &.$\quad 2.0$ \\
\hline 2.0 & $\mathbf{u}$ & MG/KG & 2.0 \\
\hline 0.60 & $\mathbf{u}$ & ME/XE & 0.60 \\
\hline 1.0 & $\mathbf{u}$ & MG/KG & 1.0 \\
\hline 0.10 & $\mathbf{u}$ & YG/RG & 0.10 \\
\hline 0.10 & $\mathbf{u}$ & HG/KG & 0.10 \\
\hline 0.10 & $\mathbf{u}$ & $M G / K G$ & 0.10 \\
\hline 0.10 & $\mathbf{u}$ & $\mathrm{MG} / \mathrm{KG}$ & 0.10 \\
\hline 10.0 & $\mathbf{u}$ & $\nabla G / L$ & 10.0 \\
\hline 200 & $\mathbf{u}$ & $0 G / \tau$ & $\cdot 200 \cdot$ \\
\hline 5.0 & $\mathbf{u}$ & UG/I & 5.0 \\
\hline 10.0 & $\mathbf{u}$ & UG/I & .10 .0 \\
\hline 10.0 & $\mathbf{u}$ & $\nabla G / L$ & 10.0 \\
\hline 3.0 & $\mathbf{u}$ & UG/L & 3.0 \\
\hline 5.0 & $\mathbf{u}$ & $U G / L$ & 5.0 \\
\hline 0.20 & $\mathbf{u}$ & UG/I & 0.20 \\
\hline 0.20 & $\mathbf{u}$ & DG/L & 0.20 \\
\hline
\end{tabular}


ROY F. WESTON INC.

INORGANICS METHOD BLANK DATA SULRARY PAGE 05/25/90

CLIENT: WSRC-GRACE ROAD

WORR ORDER: 0630-28-12-0000

\section{SAYPLE}

$=x=m=$

BIANR3
SITE ID

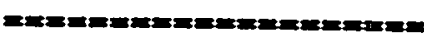

$90 C 056 A-K B 3$
ANALYTIE

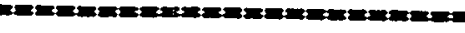

MERCURY, TOTAL
WESTON BATCA : 9003L865

\begin{tabular}{|c|c|c|}
\hline RESULI & DNITS & $\begin{array}{l}\text { REPORTING } \\
\text { LIMIT } \\
\text { =Em=m=m=m }\end{array}$ \\
\hline & $\mathrm{UG} / \mathrm{I}$ & 0.20 \\
\hline
\end{tabular}


ROY P. FESTON INC.

INORGANICS DUPLICATE SPIKB REPORT 05/25/90

CLIENT: WSRC-GRACE ROAD

WORR ORDER: 0630-28-12-0000

WBSTON BATCE

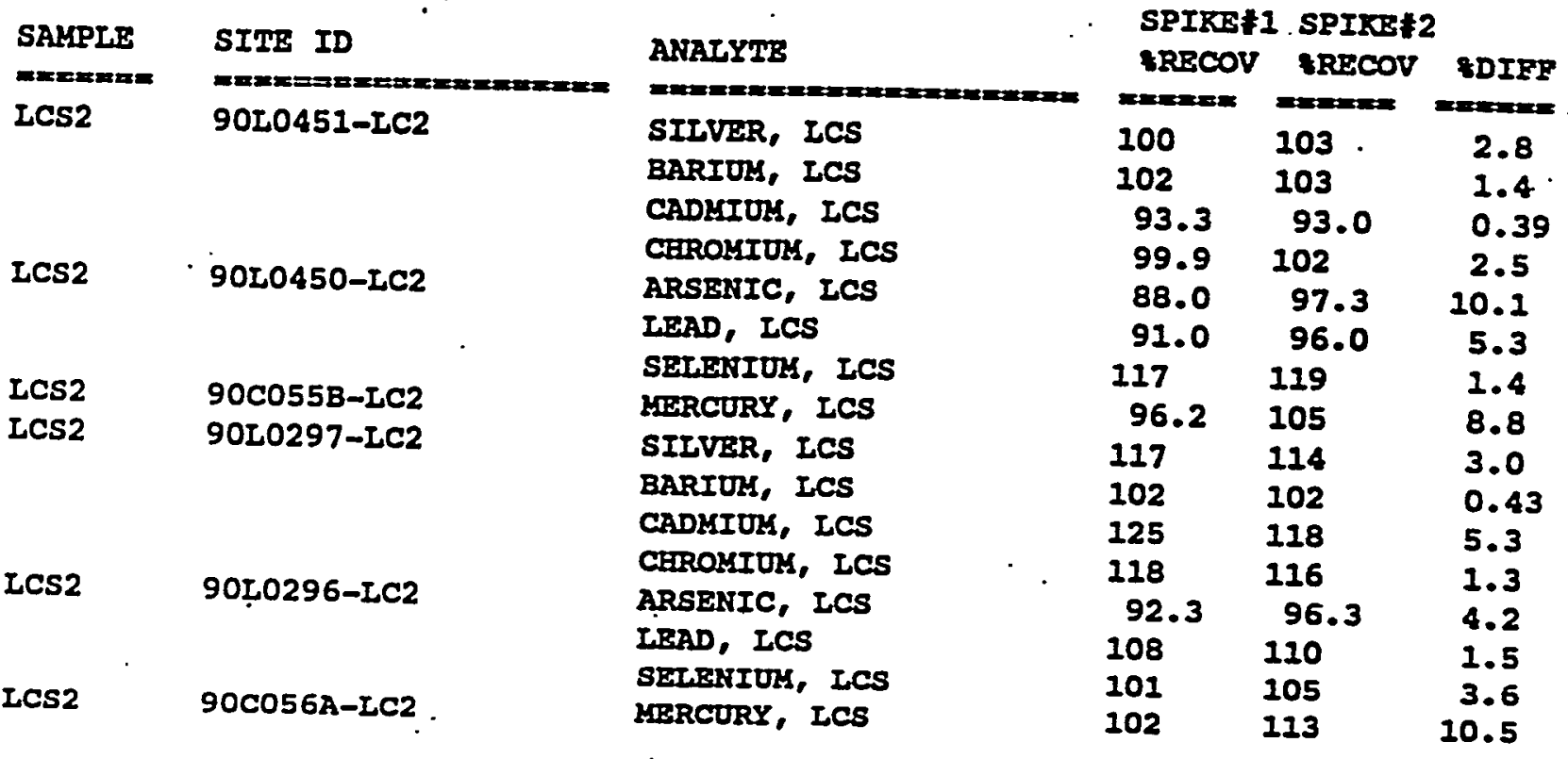


ROY E. HESTON INC.

INORGANICS IABORATORY CONTROL STANDARDS REPORT 05/25/90

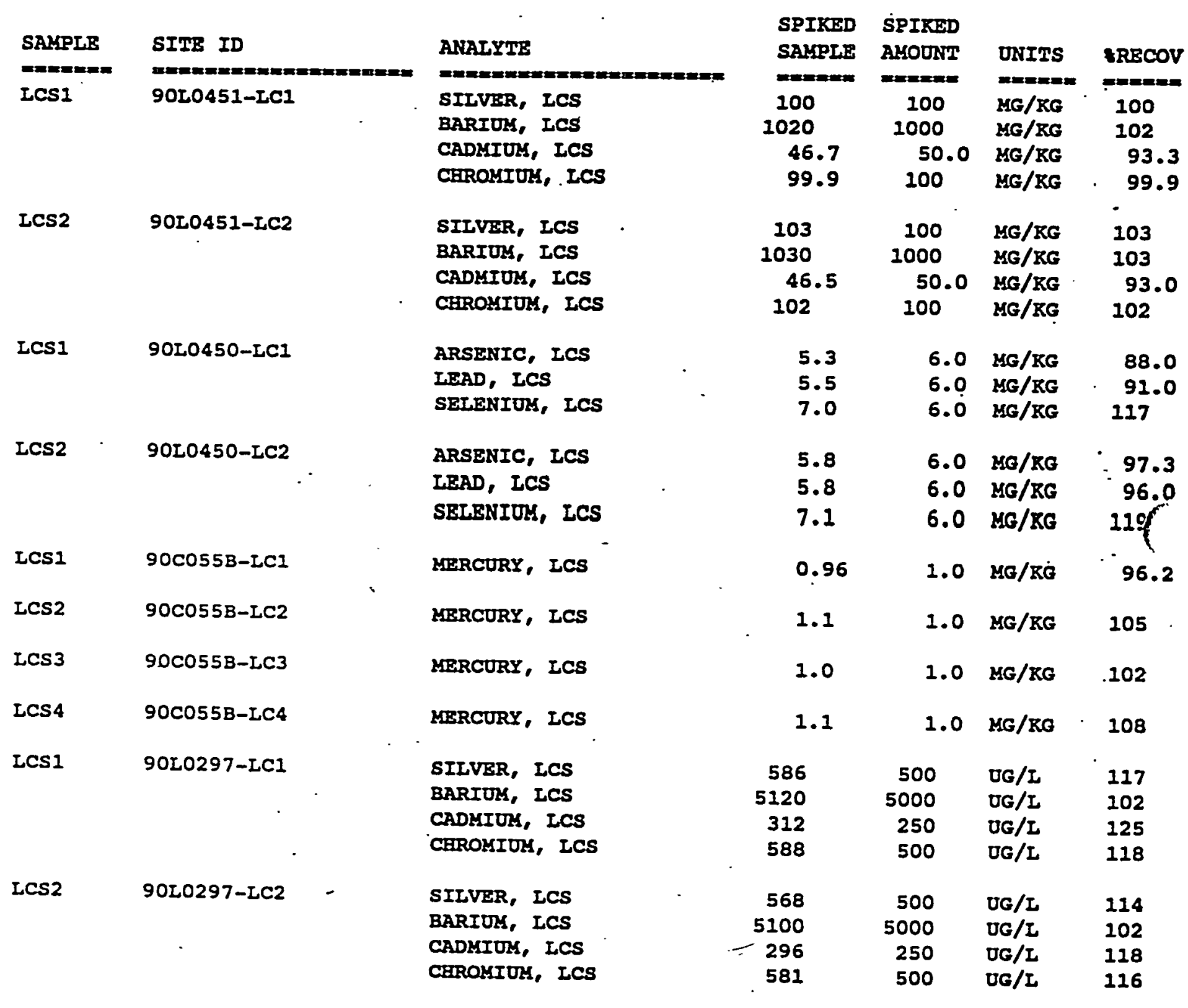


ROY $F$. FESTON INC.

INORGANICS IABORATORY CONTROL STANDARDS REPORT 05/25/90

\begin{tabular}{|c|c|c|c|c|c|c|c|}
\hline $\begin{array}{l}\text { SAMPLE } \\
m==m=m=\end{array}$ & 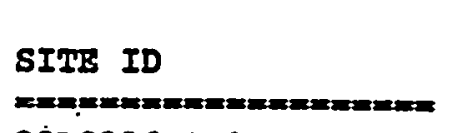 & 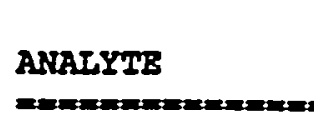 & $==$ & $\begin{array}{l}\text { SPIKKD. } \\
\text { SALPLE } \\
\text { S= }\end{array}$ & 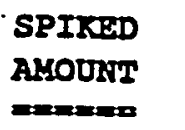 & $\begin{array}{l}\text { UNIFS } \\
\text { ==menam }\end{array}$ & \$RECOV \\
\hline LCSI & 90L0296-LC1 & $\begin{array}{l}\text { ARSENIC, ICS } \\
\text { LEAD, LCS } \\
\text { SELENIUM, LCS }\end{array}$ & & $\begin{array}{l}27.7 \\
32.4 \\
30.4\end{array}$ & $\begin{array}{l}30.0 \\
30.0 \\
30.0\end{array}$ & $\begin{array}{l}\nabla G / I \\
\nabla G / I \\
\nabla G / I\end{array}$ & $\begin{array}{l}92.3 \\
108 \\
101\end{array}$ \\
\hline LCS2 & 90L0296-LC2 & $\begin{array}{l}\text { ARSENIC, ICS } \\
\text { LEAD, ICS } \\
\text { SELENIUM, LCS }\end{array}$ & - & $\begin{array}{l}28.9 \\
32.9 \\
31.5\end{array}$ & $\begin{array}{l}30.0 \\
30.0 \\
30.0\end{array}$ & $\begin{array}{l}0 G / I \\
\nabla G / L \\
U G / L\end{array}$ & $\begin{array}{l}.96 .3 \\
110 \\
105\end{array}$ \\
\hline LCS1 & 90C056A-LC1 & MERCURY, ICS & & 2.0 & 2.0 & $\nabla G / L$ & 102 \\
\hline LCS2 & $90 C 056 A-I C 2$ & MERCURY, ICS & & 2.3 & 2.0 & UG/L & 113 \\
\hline ICS3 & $90 \operatorname{Co5} 6 \mathrm{~A}-\mathrm{LC} 3$ & MERCURY, ICS & & 2.3 & 2.0 & $\sigma G / L$ & 113 \\
\hline
\end{tabular}


Roy E. Weston, Inc. - ILonville Laboratory INORGANIC ANAIYTICAI DATA PACKAGE FOR WSRC-GRACE ROAD

DATE RECEIVED: 03/22/90

CLIENT ID /AKALYSTS
RFT * $\operatorname{2rctx}$
RFW LOT : :9003L865

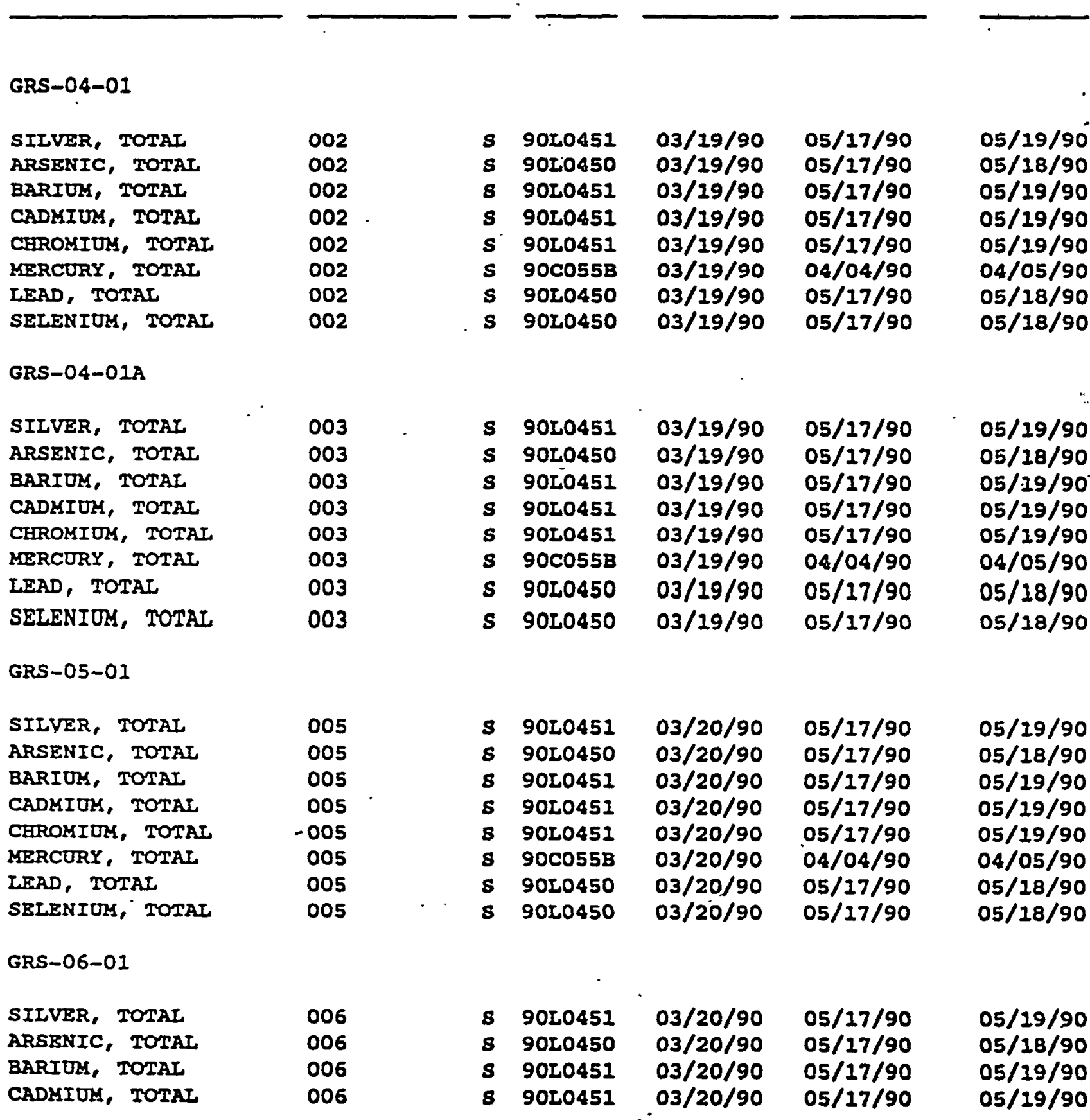


Roy. F. Weston, Inc. - Ilonplile Laboratory

INORGANIC ANALYTICAL DATA PACRAGE FOR

WSRC-GRACE ROAD

DATE RECEIVED: $03 / 22 / 90$

RET IOT :9003L865

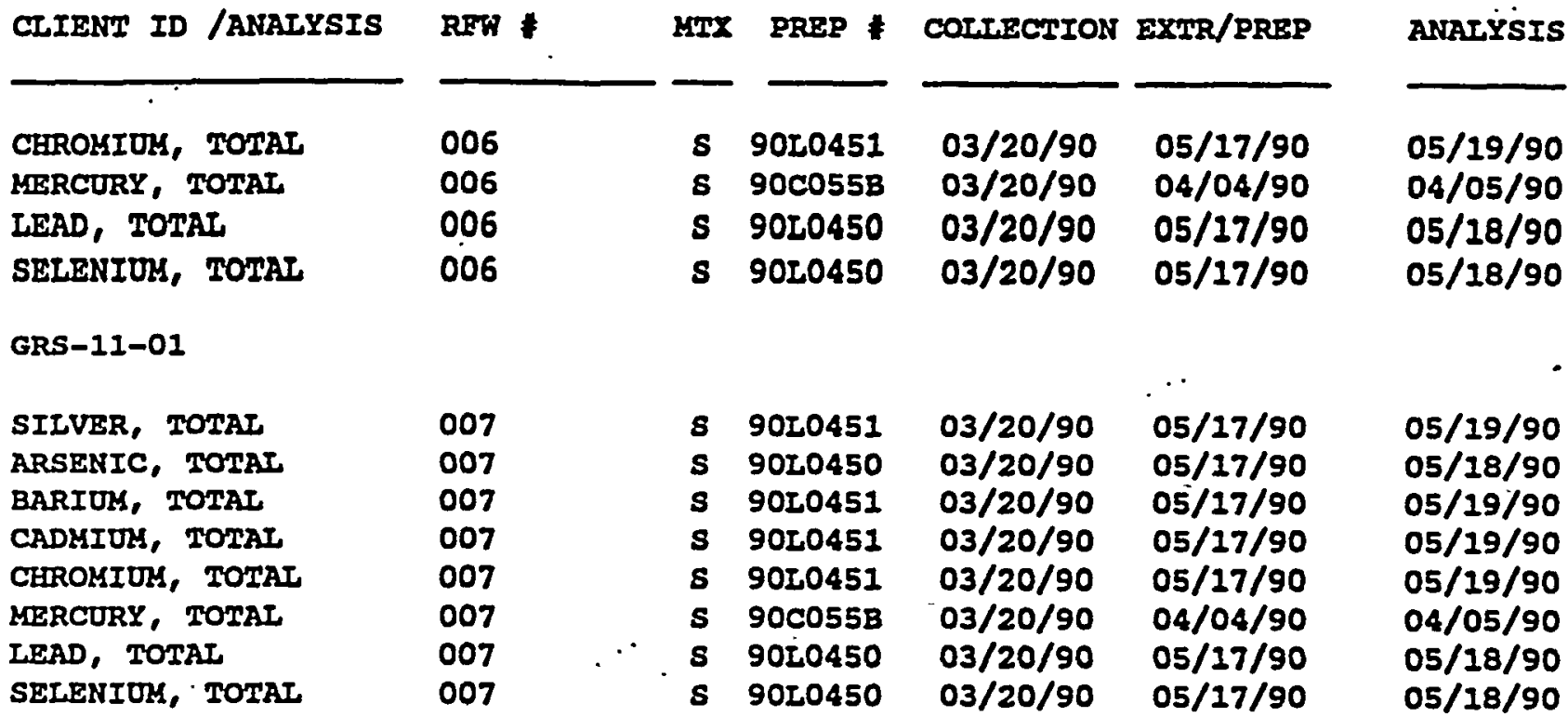

GRS-01-01

SILVER, TOTAI ARSENIC, TOTAL

008

008

BARIUM, TOTAL

CADMIUM, TOTAL

CHROMIUM, TOTAL

008

008

MERCURY, TOTAL

LEAD, TOTAL

SELENIUM, TOTAL

008

008

008

9020451

$03 / 20 / 90$

$05 / 17 / 90$

$05 / 17 / 90$

9010451 03/20/90

$05 / 17 / 90$

$901045103 / 20 / 90$

$05 / 17 / 90$

$03 / 20 / 90 \quad 05 / 17 / 90$

9020451

$03 / 20 / 90$

$90 L 0450 \quad 03 / 20 / 90$

$04 / 04 / 90$

$05 / 17 / 90$

$03 / 20 / 90$

$05 / 17 / 90$

$05 / 19 / 90$

$05 / 18 / 90$

$05 / 19 / 90$

$05 / 19 / 90$

$05 / 19 / 90$

$04 / 05 / 90$

$05 / 18 / 90$

$05 / 18 / 90$

GRS-13-01

SIIVER, TOTAL

ARSENIC, TOTAL

010

010

BARIOM, TOTAL

010

CADMIUI, TOTAL

010

CHROMIUM, TOTAI

010

MERCURY, TOTAI

010

LEAD, TOTAI

010

SELENIUM, TOTAL

010

9020451

90 0450

90L0451

90L0451

9020451

$90 \mathrm{C0} 55 \mathrm{~B}$

90 0450

9010450
$03 / 21 / 90$

$03 / 21 / 90$

$03 / 21 / 90$

$03 / 21 / 90$

$03 / 21 / 90$

$03 / 21 / 90$

$03 / 21 / 90$

$03 / 21 / 90$
$05 / 17 / 90$

$05 / 17 / 90$

$05 / 17 / 90$

$05 / 17 / 90$

$05 / 17 / 90$

$04 / 04 / 90$

$05 / 17 / 90$

$05 / 17 / 90$
$05 / 19 / 90$

$05 / 18 / 90$

$05 / 19 / 90$

$05 / 19 / 90$

$05 / 19 / 90$

$04 / 05 / 90$

$05 / 18 / 90$

$.05 / 18 / 90$

GRS-14-01

SILVER, TOTAL

011

89020451

$03 / 21 / 90$

$05 / 17 / 90$

$05 / 19 / 90$ 
Roy F. Weston, Inc. - ILonv11le Laboratory

INORGANIC ANALYTICAL DATA PACKAGE FOR

WSRC-GRACE ROAD

DATE RECEIVED: $03 / 22 / 90$

RFW LOT \$ $\$ 9003 L 865$

CLIENT ID /ANALYSIS RFH * KTTX PREP * COLCECTION EXTR/PREP

ANALYSIS

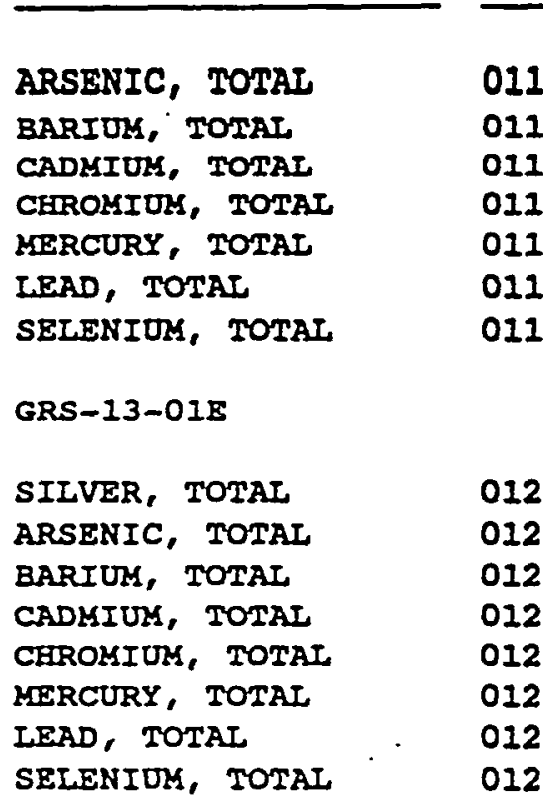

anteris

ARSENIC, TOTAI

011

011

$03 / 21 / 90$

$03 / 21 / 90$

$03 / 21 / 90$

$03 / 21 / 90$

$03 / 21 / 90$

$03 / 21 / 90$

$03 / 21 / 90$

$05 / 17 / 90$

$05 / 17 / 90$

$05 / 17 / 90$

$05 / 17 / 90$

$04 / 04 / 90$

$05 / 17 / 90$

$05 / 17 / 90$

$05 / 18 / 90$

$05 / 19 / 90$

$05 / 19 / 90$

$05 / 19 / 90$

$04 / 05 / 90$

$05 / 18 / 9 Q$

$05 / 18 / 90$

SELENIUM, TOTAL

012

012

012

012

012

012

012

$\begin{array}{ccc}W & 90 L 0297 & 03 / 21 / 90 \\ W & 90 L 0296 & 03 / 21 / 90 \\ W & 90 L 0297 & 03 / 21 / 90 \\ W & 90 L 0297 & 03 / 21 / 90 \\ W & 90 L 0297 & 03 / 21 / 90 \\ W & 90 C 056 A & 03 / 21 / 90 \\ W & 9010296 & 03 / 21 / 90 \\ W & 90 L 0296 & 03 / 21 / 90\end{array}$

$03 / 29 / 90$

$03 / 29 / 90$

$03 / 29 / 90$

$03 / 29 / 90$

$03 / 29 / 90$

$04 / 06 / 90$

$03 / 29 / 90$

$03 / 29 / 90$
$04 / 02 / 90$

$04 / 02 / 90$

$04 / 02 / 90$

$04 / 02 / 90$

$04 / 02 / 90$

$04 / 08 / 90$

$04 / 05 / 90$

04/04/90

LAB QC:

SILVER LABORATORY
ALUMINUM LABORTORY
ARSENIC LABORATORY
BORON LABORATORY
BARIUM LABORATORY
BERYIIIUM LABORATORY
CALCIUM LABORATORY
CADMIUM LABORATORY
COBALT LABORATORY
CHROMIUM LABORATORY
COPPER LABORATORY
IRON ILABORATORY
POTASSIOM LABORATORY
MAGNESIUM LABORATORY
MANGANESE LABORATORY
MOLYBDENUM LABORATOR
SODIUM IAABORATORY
NICXEL LABORATORY
LRAD LABORATORY

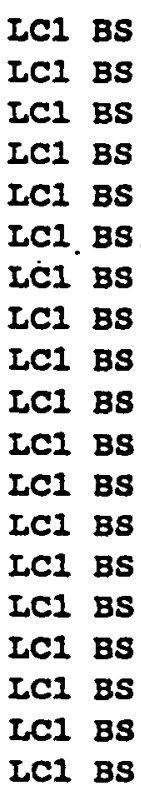

$\begin{array}{ll}\text { s } & 9010451 \\ \text { s } & 9010451 \\ \text { s } & 9010451 \\ \text { s } & 9010451 \\ \text { s } & 9010451 \\ \text { s } & 9010451 \\ \text { s } & 9010451 \\ \text { s } & 9020451 \\ \text { s } & 9010451 \\ \text { s } & 9010451 \\ \text { s } & 9010451 \\ \text { s } & 9010451 \\ \text { s } & 9010451 \\ \text { s } & 9010451 \\ \text { s } & 9010451 \\ \text { s } & 9010451 \\ \text { s } & 9010451 \\ \text { s } & 9010451 \\ \text { s } & 9010451\end{array}$

$05 / 19 / 90$

$05 / 19 / 90$

$05 / 19 / 90$

$05 / 19 / 90$

$05 / 19 / 90$

$05 / 19 / 90$

$05 / 19 / 90$

$05 / 19 / 90$

$05 / 19 / 90$

$05 / 19 / 90$

$05 / 19 / 90$

$05 / 19 / 90$

$05 / 19 / 90$

$05 / 19 / 90$

$05 / 19 / 90$

$05 / 19 / 90$

$05 / 19 / 90$

$05 / 19 / 90$

$05 / 19 / 90$ 
Roy F. Weston, Inc. - Ilonville Laboratory

INORGANIC ANALYTICAL DATA PACKAGE FOR WSRC-GRACB ROAD

DATE RECEIVED: $03 / 22 / 90$

RFW LOT *9003L865

\begin{tabular}{|c|c|c|c|c|c|c|c|}
\hline CLIENT ID / ANALYSIS & REW & $\neq$ & $\operatorname{sTr}$ & PRBP & COLTRETION & EXIR/PREP & ANAITYSIS \\
\hline ANTIMONY LABORATORY & LC1 & BS & $\mathbf{8}$ & 9020451 & $N / A$ & $05 / 17 / 90$ & $05 / 19 / 90$ \\
\hline SELENIUM IABORATORY & IC1 & BS & s & 9020451 & $N / A$ & $05 / 17 / 90$ & $05 / 19 / 90$ \\
\hline SILICON ·LABORATORY & LC1 & BS & $\mathbf{s}$ & 9020451 & $\pi / A$ & $05 / 17 / 90$ & $05 / 21 / 90$ \\
\hline TIN IABORATORY & LCI & BS & $\mathbf{s}$ & 9020451 & $N / \boldsymbol{A}$ & $05 / 17 / 90$ & $05 / 19 / 90$ \\
\hline TITANIUY LABORATORY & LCI & Bs & $\mathbf{s}$ & 9020451 & $N / A$ & $05 / 17 / 90$ & $05 / 19 / 90$ \\
\hline THATIIUM LABORATORY & LCI & BS & $\mathbf{s}$ & 9020451 & $n / A$ & $05 / 27 / 90$ & $05 / 19 / 90$ \\
\hline VANADIUY IABORATORY & IC1 & BS & $\mathbf{s}$ & 90L0451 & $\boldsymbol{N} / \boldsymbol{A}$ & 05/17/90 & $05 / 19 / 90$ \\
\hline ZINC LABORATORY & LC1 & BS & $\mathbf{s}$ & 9020451 & $N / A$ & 05/17/90 & $05 / 19 / 90$ \\
\hline SILVER IABORATORY & LC2 & BS & $\mathbf{s}$ & 9020451 & $N / A$ & $05 / 17 / 90$ & $05 / 19 / 90$ \\
\hline ALOMINOM LABORTORY & LC2 & BS & $\mathbf{s}$ & 9020451 & $N / A$ & $05 / 17 / 90$ & $05 / 19 / 90$ \\
\hline ARSENIC LABORATORY & IC2 & Bs & $\mathbf{s}$ & 9020451 & $N / 2$ & 05/17/90 & $05 / 19 / 90$ \\
\hline BORON LABORATORY & LC2 & BS & $\mathbf{s}$ & 9020451 & $N / \mathbf{A}$ & 05/17/90 & $05 / 19 / 90$ \\
\hline BARIOY IABORATORY & IC2 & Bs & $\mathbf{s}$ & 9020451 & $N / A$ & 05/17/90 & $05 / 19 / 90$ \\
\hline BERYLIIUM IABORATORY & LC2 & B8 & 8 & 9020451 & $n / A$ & $05 / 17 / 90$ & $05 / 19 / 90$ \\
\hline CALCIUY IABORATORY & LC2 & Bs & $\mathbf{s}$ & 9020451 & $N / A$ & $05 / 17 / 90$ & $05 / 29 / 90$ \\
\hline CADMIUM IABORATORY & LC2 & BS & $\mathbf{s}$ & 90น0451 & $N / A$ & $05 / 17 / 90$ & $05 / 19 / 90$ \\
\hline COBALT LABORATORY & IC2 & BS & $\mathbf{s}$ & 90L0451 & $N / A$ & $05 / 17 / 90$ & $05 / 19 / 90$ \\
\hline CHROMIUM LABORATORY & IC2 & BS & $\mathbf{s}$ & 9020451 & $N / A$ & $05 / 17 / 90$ & $05 / 19 / 90$ \\
\hline COPPER IABORATORY & LC2 & BS & $\mathbf{s}$ & 9020451 & $N / A$ & $05 / 17 / 90$ & $05 / 19 / 90$ \\
\hline IRON LABORATORY - & LC2 & BS & $\mathbf{s}$ & 90ז0451 & $N / A$ & $05 / 17 / 90$ & $05 / 19 / 90$ \\
\hline POTASSIUM IABORATORY & LC2 & BS & $\mathbf{s}$ & 9020451 & $N / A$ & $05 / 17 / 90$ & $05 / 19 / 90$ \\
\hline MAGNESIUM LABORATORY & LC2 & BS & $\mathbf{s}$ & 9020451 & $N / A$ & $05 / 17 / 90$ & $05 / 19 / 90$ \\
\hline MANGANESE ILABORATORY & IC2 & BS & $\mathbf{s}$ & 9020451 & $N / A$ & $05 / 17 / 90$ & $05 / 19 / 90$ \\
\hline MOLYBDENUM IABORATOR & LC2 & BS & $\mathbf{s}$ & 9020451 & $N / A$ & $05 / 17 / 90$ & $05 / 19 / 90$ \\
\hline SODIUY LABORATORY & LC2 & BS & $\mathbf{s}$ & 9020451 & $N / A$ & $05 / 17 / 90$ & $05 / 19 / 90$ \\
\hline NICKEI LABORATORY & LC2 & BS & $\mathbf{s}$ & 9020451 & $\mathrm{H} / \mathrm{A}$ & $05 / 17 / 90$ & $05 / 19 / 90$ \\
\hline LEAD LABORATORY & LC2 & BS & $\mathbf{s}$ & 9020451 & $N / A$ & $05 / 17 / 90$ & $05 / 19 / 90$ \\
\hline ANTIMONY LABORATORY & LC2 & Bs & $\mathbf{s}$ & 9020451 & $N / A$ & $05 / 17 / 90$ & $05 / 19 / 90$ \\
\hline SELENIUY LABORATORY & LC2 & BS & $\mathbf{s}$ & 9020451 & $N / A$ & $05 / 17 / 90$ & $05 / 19 / 90$ \\
\hline SILICON LABORATORY & LC2 & BS & $\mathbf{s}$ & 9020451 & $N / A$ & $05 / 17 / 90$ & $05 / 21 / 90$ \\
\hline TIN LABORATORY & LC2 & BS & $\mathbf{s}$ & 9020451 & $N / A$ & $05 / 17 / 90$ & $05 / 19 / 90$ \\
\hline TITANIUM LABORATORY & LC2 & Bs & $\mathbf{s}$ & 9010451 & $N / A$ & $05 / 17 / 90$ & $05 / 19 / 90$ \\
\hline THALCIOM LABORATORY & LC2 & BS & $\mathbf{s}$ & 9020451 & $N / A$ & $05 / 17 / 90$ & $05 / 19 / 90$ \\
\hline VANADIUA IABORATORY & LC2 & B8 & 8 & 9010451 & $\boldsymbol{N} / \mathrm{A}$ & $05 / 17 / 90$ & $05 / 19 / 90$ \\
\hline ZINC LABORATORY & LC2 & BS & $\mathbf{s}$ & 9020451 & $N / A$ & $05 / 17 / 90$ & $05 / 19 / 90$ \\
\hline SILVER, TOTAL & MBDI & & $\mathbf{s}$ & 9020451 & H/ג & $05 / 17 / 90$ & $05 / 19 / 90$ \\
\hline ALOMINUM, TOTAL & MB1 & & 8 & $90 \check{0451}$ & $N / A$ & $05 / 17 / 90$ & $05 / 19 / 90$ \\
\hline ARSENIC, TOTAI & MBI & & $\mathbf{s}$ & 9020451 & $N / A$ & $05 / 17 / 90$ & $05 / 19 / 90$ \\
\hline BORON, TOTAL & MBI & & $\mathbf{s}$ & 9020451 & $N / A$ & $05 / 17 / 90$ & $05 / 19 / 90$ \\
\hline BARIUK, TOTAL & HBBI & & $\mathbf{s}$ & 9020451 & H/A & 05/17/90 & $05 / 19 / 90$ \\
\hline
\end{tabular}


Roy F. Weston, Inc. - Lionville Laboratory

INORGANIC ANAIYTICAI DATA PACKAGE FOR WSRC-GRACB ROAD

DATE RECEIVED: $03 / 22 / 90$

RFW LOT : :9003L865

\begin{tabular}{|c|c|c|c|c|c|c|c|}
\hline CLIENT ID / ANALYSIS & RFW & $*$ & MTX & PREP & COLLECTION & EXTR/PREP & ANALYSIS \\
\hline BERYIIIUR, TOTAL & MBI & & $\mathbf{s}$ & $90[0451$ & $N / A$ & $05 / 17 / 90$ & $05 / 19 / 90$ \\
\hline CALCIUR, TOTAI & MBII & & $\mathbf{8}$ & 9020451 & $x / \lambda$ & $05 / 17 / 90$ & $05 / 19 / 90$ \\
\hline CADMIUY, TOTAL & MBI & & $\mathbf{s}$ & 9010451 & $N / A$ & $05 / 17 / 90$ & $05 / 19 / 90$ \\
\hline COBALT, TOTAI & MBI & & $\mathbf{s}$ & 90L0451 & $N / A$ & $05 / 17 / 90$. & $05 / 19 / 90$ \\
\hline CHROMIUM, TOTAL & MBI & & $\mathbf{s}$ & 9020451 & $N / A$ & $05 / 17 / 90^{\circ}$ & $05 / 19 / 90$ \\
\hline COPPER, TOTAI & MBI & & $\mathbf{s}$ & 9020451 & $H / A$ & $05 / 17 / 90$ & $05 / 19 / 90$ \\
\hline IRON, TOTAI & MBI & & $\mathbf{s}$ & 9010451 & $N / \lambda$ & $05 / 17 / 90$ & $05 / 19 / 90$ \\
\hline POTASSIUK, TOTAL & MBI & & $\mathbf{s}$ & 9010451 & $N / A$ & $05 / 17 / 90$ & $05 / 19 / 90$ \\
\hline MAGNESIUM, TOTAL & MBI & & $\mathbf{s}$ & 9020451 & $H / A$ & $05 / 17 / 90$ & $05 / 19 / 90$ \\
\hline MANGANESE, TOTAL & MBI & & $\mathbf{s}$ & 9020451 & $\mathbf{N} / \mathbf{A}$ & 05/17/90 & $05 / 19 / 90$ \\
\hline MOLYBDENUM, TOTAL & MB1 & & $\mathbf{s}$ & 9020451 & $N / B$ & $05 / 17 / 90$ & $05 / 19 / 90$ \\
\hline SODIUM, TOTAI & MBI & & $\mathbf{s}$ & 9020451 & $N / A$ & $05 / 17 / 90$ & $05 / 19 / 90$ \\
\hline NICKEL, TOTAL & MBI & & $\mathbf{s}$ & 9010451 & $N / A$ & $05 / 17 / 90$ & $05 / 19 / 90$ \\
\hline LEAD, TOTAL & MBI & & $\mathbf{s}$ & 9020451 & $N / A$ & $05 / 17 / 90$ & $05 / 19 / 90$ \\
\hline ANTIMONY, TOTAI & MBI & & $\mathbf{s}$ & 9010451 & $N / A$ & $05 / 17 / 90$ & $05 / 19 / 90$ \\
\hline SELENIUM, TOTAL & MB1 & & $\mathbf{s}$ & 9020451 & $N / A$ & $05 / 17 / 90$ & $05 / 19 / 90$ \\
\hline SILICON, TOTAI & MBI & & $\mathbf{s}$ & 9020451 & $N / A$ & $05 / 17 / 90$ & $05 / 21 / 90$ \\
\hline TIN, TOTAI & MBI & & $\mathbf{s}$ & 9020451 & $N / A$ & $05 / 17 / 90$ & $05 / 29 / 90$ \\
\hline TITANIUM, TOTAL & MB1 & & $\mathbf{s}$ & 9010451 & $N / A$ & $05 / 17 / 90$ & $05 / 19 / 90$ \\
\hline THAIIIUK, TOTAL & MBI & & $\mathbf{s}$ & 9020451 & $\mathbf{N} / \mathbf{A}$ & $05 / 17 / 90$ & $05 / 19 / 90$ \\
\hline VANADIUM, TOTAL & MBI & & $\mathbf{s}$ & 9020451 & N/A & $05 / 17 / 90$ & $05 / 19 / 90$ \\
\hline ZINC, TOTAL & MBI & & $\mathbf{s}$ & 9020451 & $N / A$ & $05 / 17 / 90$ & $05 / 19 / 90$ \\
\hline ARSENIC LABORATORY & IC1 & BS & $\mathbf{s}$ & 9020450 & $N / A$ & $05 / 17 / 90$ & $05 / 18 / 90$ \\
\hline LEAD IABORATORY & LCI & BS & $\mathbf{s}$ & 9020450 & $N / A$ & $05 / 17 / 90$ & $05 / 18 / 90$ \\
\hline SELENIUM LABORATORY & LCI & BS & $\mathbf{s}$ & 9020450 & $N / A$ & $05 / 17 / 90$ & $05 / 18 / 90$ \\
\hline ARSENIC LABORATORY & IC2 & Bs & $\mathbf{s}$ & 9020450 & $N / A$ & $05 / 17 / 90$ & $05 / 18 / 90$ \\
\hline LEAD IABORATORY & LC2 & BS & $\mathbf{s}$ & 9020450 & $N / A$ & $05 / 17 / 90$ & $05 / 18 / 90$ \\
\hline SELENIOH LABORATORY & LC2 & BS & $\mathbf{s}$ & 9020450 & $N / A$ & $05 / 17 / 90$ & $05 / 18 / 90$ \\
\hline ARSENIC, TOTAL & MBI & & $\mathbf{s}$ & $90 L 0450$ & $N / A$ & $05 / 17 / 90$ & $05 / 18 / 90$ \\
\hline LEAD, TOTAI & MBI & & $\mathbf{s}$ & $90 L 0450$ & $N / A$ & $05 / 17 / 90$ & $05 / 18 / 90$ \\
\hline SELENIOR, TOTAL & MBI & & s & 9020450 & $N / A$ & $05 / 17 / 90$ & $05 / 18 / 90$ \\
\hline FERCURY LABORATORY & LCI & BS & $\mathbf{8}$ & $90 C 055 B$ & $N / A$ & $04 / 04 / 90$ & $04 / 05 / 90$ \\
\hline MERCURY LABORATORY & LC2 & BS & $\mathbf{s}$ & $90 \mathrm{C0} 55 \mathrm{~B}$ & $N / A$ & $04 / 04 / 90$ & $04 / 05 / 90$ \\
\hline MERCURY IAABORATORY & LC3 & BS & $\mathbf{s}$ & $90 \mathrm{C055B}$ & $N / A$ & $04 / 04 / 90$ & $04 / 05 / 90$ \\
\hline MRRCURY TABORATORY & IC4 & BS & $\mathbf{s}$ & $90 \mathrm{CO55B}$ & $N / A$ & $04 / 04 / 90$ & $04 / 05 / 90$ \\
\hline MRRCURY, TOTAI & MB1 & & $\mathbf{s}$ & $90 \mathrm{C0} 55 \mathrm{~B}$ & $N / A$ & $04 / 04 / 90$ & $04 / 05 / 90$ \\
\hline MERCURY, TOTAL & MB2 & & $\mathbf{s}$ & $90 c 0558$ & $N / A$ & $04 / 04 / 90$ & $04 / 05 / 90$ \\
\hline MERCURY, TOTAL - & MB3 & & $\mathbf{s}$ & $90 C 055 B$ & $N / A$ & $04 / 04 / 90$ & $04 / 05 / 90$ \\
\hline MERCURY， TOTAI & 2AB4 & & $\mathbf{s}$ & $90 C 055 B$ & $N / A$ & $04 / 04 / 90$ & $04 / 05 / 90$ \\
\hline SILVBR IAABORATORY & LC1 & BS & พ & 9010297 & $N / A$ & $03 / 29 / 90$ & \\
\hline
\end{tabular}


Roy F. Weston, Inc. - Ilonville Laboratory INORGANIC ANALYTICAI DATA PACRAGB FOR HSRC-GRACB ROAD

DATB RECEIVEDs, 03/22/90

REW LOT $: 90032865$

\begin{tabular}{|c|c|c|c|c|c|c|c|}
\hline CLIENT ID /ANALYSIṢ & REW & 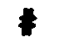 & LETX & PREP & COLIRETION & / EXTR/PREP & ANALYSIS \\
\hline BARIOU LABORATORY & LC1 & BS & $\boldsymbol{\omega}$ & 9020297 & $x / \lambda$ & $03 / 29 / 90$ & $04 / 02 / 90$ \\
\hline CALCIUU ILABORATORY & LCI & Bs & $\boldsymbol{W}$ & 9020297 & $\pi / \lambda$ & $03 / 29 / 90$ & $04 / 02 / 90$ \\
\hline CADMIUUA LABORATORY & LCI & BS & $\boldsymbol{m}$ & 9020297 & $N / \boldsymbol{A}$ & $03 / 29 / 90$ & $04 / 02 / 90$ \\
\hline CEROMIUM LABORATORY & LCI & BS & $w$ & 9020297 & $N / \AA$ & $03 / 29 / 90$ & $04 / 02 / 90$ \\
\hline COPPER IABORATORY & LC1 & BS & $\boldsymbol{w}$ & 90L0297 & $N / A$ & $03 / 29 / 90$ & $04 / 02 / 90$ \\
\hline IRON LABORATORY & LC1 & BS & $w$ & 9020297 & $\boldsymbol{N} / \mathbf{A}$ & $03 / 29 / 90$ & $04 / 02 / 90$ \\
\hline MANGANESE IABORATORY & LCI & BS & $\omega$ & 9020297 & $\pi / A$ & $03 / 29 / 90$ & $04 / 02 / 90$ \\
\hline SODIOMS IABORATORY & LCI & BS & $\boldsymbol{H}$ & 9020297 & $N / \pi$ & $03 / 29 / 90$ & $04 / 02 / 90$ \\
\hline NICKEL IABORATORY & LCI & BS & ต & 9020297 & $x / A$ & $03 / 29 / 90$ & $04 / 02 / 90$ \\
\hline ZINC IABORATORY & ICI & BS & $\mathbf{w}$ & 9020297 & $N / A$ & $03 / 29 / 90$ & $04 / 02 / 90$ \\
\hline SILVER IABORATORY & LC2 & BS & $\mathbf{w}$ & 9010297 & $N / A$ & $03 / 29 / 90$ & $04 / 02 / 90$ \\
\hline BARIUM IABORATORY & LC2 & BS & $\mathbf{w}$ & 9020297 & $N / A$ & $03 / 29 / 90$ & $04 / 02 / 90$ \\
\hline CALCIUM LABORATORY & LC2 & BS & ต & 9020297 & $N / A$ & $03 / 29 / 90$ & $04 / 02 / 90$ \\
\hline CADMIOM IABORATORY & LC2 & BS & $\mathbf{W}$ & 9020297 & $\boldsymbol{N} / \mathbf{A}$ & $03 / 29 / 90$ & 04/02/90 \\
\hline CHROMIOM IABORATORY & IC2 & BS & $\boldsymbol{w}$ & 9010297 & $N / A$ & $03 / 29 / 90$ & $04 / 02 / 90$ \\
\hline COPPER LABORATORY & LC2 & BS & $\boldsymbol{\omega}$ & 9020297 & $N / \mathbf{A}$ & $03 / 29 / 90$ & $04 / 02 / 90$ \\
\hline IRON LABORATORY .' & LC2 & BS & $\boldsymbol{W}$ & 9020297 & $N / A$ & $03 / 29 / 90$ & $04 / 02 / 90$ \\
\hline MANGANESB LABORATORY & LC2 & BS & $\mathbf{w}$ & 9020297 & $N / A$ & 03/29/90 & 04/02/90 \\
\hline SODIURS LABORATORY & LC2 & Bs & $\boldsymbol{\omega}$ & 9020297 & $N / A$ & $03 / 29 / 90$ & 04/02/90. \\
\hline NICKEL LABORATORY & LC2 & BS & $\boldsymbol{W}$ & 90L0297 & $N / \lambda$ & $03 / 29 / 90$ & $04 / 02 / 90^{\circ}$ \\
\hline ZINC IABORATORY & LC2 & BS & W & 9020297 & $N / A$ & $03 / 29 / 90$ & $04 / 02 / 90$ \\
\hline SILVER, TOTAI & MBI & & $\mathbf{W}$ & 90ז0297 & $N / A$ & $03 / 29 / 90$ & $04 / 02 / 90$ \\
\hline BARIUY, TOTAL & MB1 & & พ & 9020297 & $N / A$ & $03 / 29 / 90$ & $04 / 02 / 90$ \\
\hline CALCIUM, TOTAL & MB1 & & $\boldsymbol{พ}$ & 9020297 & $\mathbf{N} / \mathbf{A}$ & $03 / 29 / 90$ & 04/02/90 \\
\hline CADMIOM, TOTAI & MB1 & & $\boldsymbol{H}$ & 9020297 & $N / A$ & $03 / 29 / 90$ & $04 / 02 / 90$ \\
\hline CEROMIUM, TOTAL & MBI & & H & 90L0297 & $N / A$ & $03 / 29 / 90$ & $04 / 02 / 90$ \\
\hline COPPER, TOTAL & MBI & & $\boldsymbol{\omega}$ & 9020297 & $N / A$ & $03 / 29 / 90$ & $04 / 02 / 90$ \\
\hline IRON, TOTAL & MBI & & $\boldsymbol{H}$ & 9020297 & $N / A$ & $03 / 29 / 90$ & $04 / 02 / 90$ \\
\hline MANGANESE, TOTAL' & MB1 & & $\mathbf{W}$ & 90โ0297 & $N / A$ & $03 / 29 / 90$ & $04 / 02 / 90$ \\
\hline SODIUS, TOTAL & MBI & & ต & 9020297 & $N / A$ & $03 / 29 / 90$ & $04 / 02 / 90$ \\
\hline NICKEI, TOTAL & MBI & . & $\mathbf{w}$ & 9020297 & $N / A$ & $03 / 29 / 90$ & $04 / 02 / 90$ \\
\hline ZINC, TOTAL & MBI & & $\mathbf{W}$ & 9020297 & $N / A$ & $03 / 29 / 90$ & $04 / 02 / 90$ \\
\hline ARSENIC LABORATORY & ICI & BS & $\boldsymbol{\varphi}$ & 90L0296 & $N / \mathbf{A}$ & $03 / 29 / 90$ & $04 / 02 / 90$ \\
\hline LEAD LABORATORY & LCI & B8 & $\boldsymbol{\omega}$ & 9020296 & $N / A$ & $03 / 29 / 90$ & $04 / 05 / 90$ \\
\hline SELENIUM LABORATORY & LC1 & BS & $\mathbf{W}$ & 9020296 & $N / A$ & $03 / 29 / 90$ & $04 / 04 / 90$ \\
\hline ARSENIC LABORATORY & LC2 & BS & $\boldsymbol{\omega}$ & 9020296 & $N / A$ & $03 / 29 / 90$ & $04 / 02 / 90$ \\
\hline LEAD LABORATORY & LC2 & BS & $\boldsymbol{w}$ & 9020296 & $N / \lambda$ & $03 / 29 / 90$ & $04 / 05 / 90$ \\
\hline SELEENIOM LABORATORY & LC2 & BS & พ & 9020296 & $N / A$ & 03/29/90 & $04 / 04 / 90$ \\
\hline ARSENIC, TOTAL & KB1 & & พ & 9020296 & $N / A$ & $03 / 29 / 90$ & $04 / 02 / 90$ \\
\hline LEAD, TOTAL & MBI & & $\boldsymbol{W}$ & 9020296 & $N / A$ & $03 / 29 / 90$ & $04 / 05 / 90$ \\
\hline
\end{tabular}


Roy F. Weston, Inc. - Iionville Iaboratory INORGANIC ANALYTICAT DATA PACRAGB FOR

$x_{x}$ HSRC-GRACE ROAD

DATE RECEIVED: 03/22/90

RET LOT * :9003L865

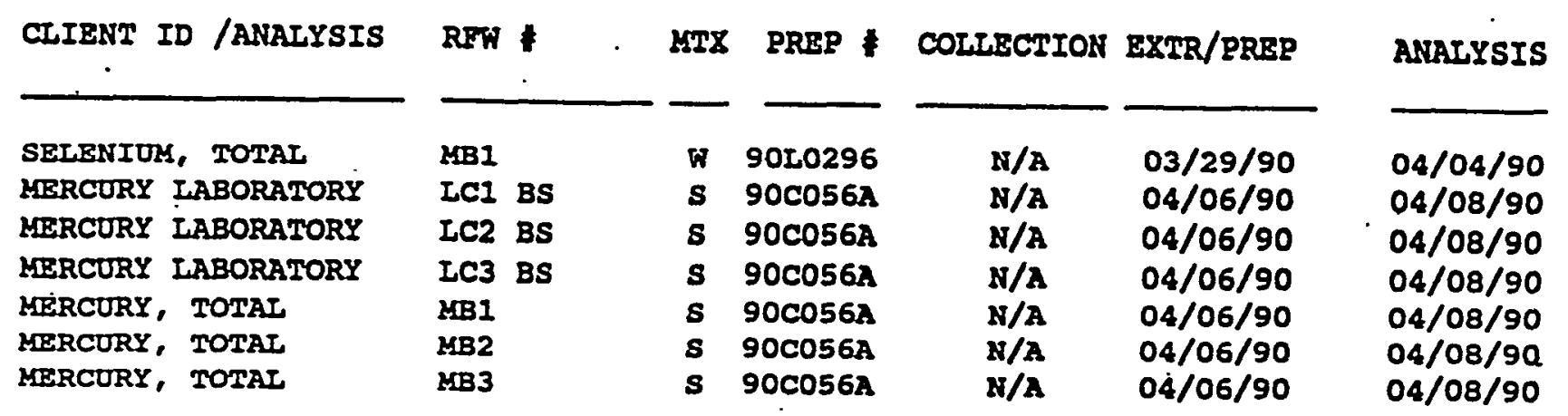




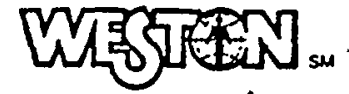

CLIENT: WSRC=GRACE ROAD

RFW \#: $9002 \overline{\mathrm{L}} 607$

พ.0. \#: 0630-28-12.
ROY F. WESTON, INC.

Lionville Laboratory

SAKPLES RECEIVED: $02-23-90$

\section{METALS NARRATIVE}

The following is a summary of the quality control results and a description of any problemis encountered during the analysis of this batch of samples:

1. All sample holding times as required by 40 CFR 136 were met for water samples. Note: Holding times for soil samples have not been promulgated by the USEPA.

2. All calibration verification checks were within the required control limits of 90-100\% (85-115\% for $\mathrm{Hg}$ ). Calibration verification is performed using independent standards from Inorganic Ventures, Inc.

3. All preparation blanks were analyzed below the required detection limit.

4. Laboratory control standards were within the control limits of $80-120 \%$ with the exception of silver (59\%) for site ID\# 90L0226-LCI. The blank spike reproducibility was outside the $20 \%$ window for silver at $58 \%$.

Note: The USEPA-CLP has dropped control Iimits for silver and antimony due to documented difficulties in obtaining reliable results. WESTON Analytics has adopted the same policy.

5. The analytical methods applied by the laboratory for the determination of metals are:

$\begin{array}{ll}\text { As: } & \text { EPA } 206.2 \\ \text { Se: } & \text { EPA } 270.2 \\ \mathrm{~Pb}: & \text { EPA } 239.2 \\ \text { T1: } & \text { EPA } 279.2\end{array}$
$\mathrm{Hg}$ :

ICP Scan: EPA 245.1

All Others:

EP Leachates (except $\mathrm{Hg}$ ) : 200.7 NOTE: For solid samples, all results are reported on a dry weight
basis.

$\int \frac{\tau-h-j \mu d}{\text { Jack R. Tuschall, Ph.D. }} \frac{\dot{4}-y-\xi u}{\text { Date }}$

Laboratory Manager

Lionville Analytical Laboratory 
B -22 
ROY F. WESTON, INC.

GLOSSARY OF TERMS - INORGANIC REPORTS

DATA OUALIFIERS

$U$ - Inficates that the parameter was not detected at or above the reported limit. The associated numerical value is the sample detection limit.

* - Indicates that the original sample result is greater than $4 \mathrm{x}$ the spike amount added. The USEPA-CLP has determined that spike results on samples where this limits are not applicable.

ABBREVIATIONS

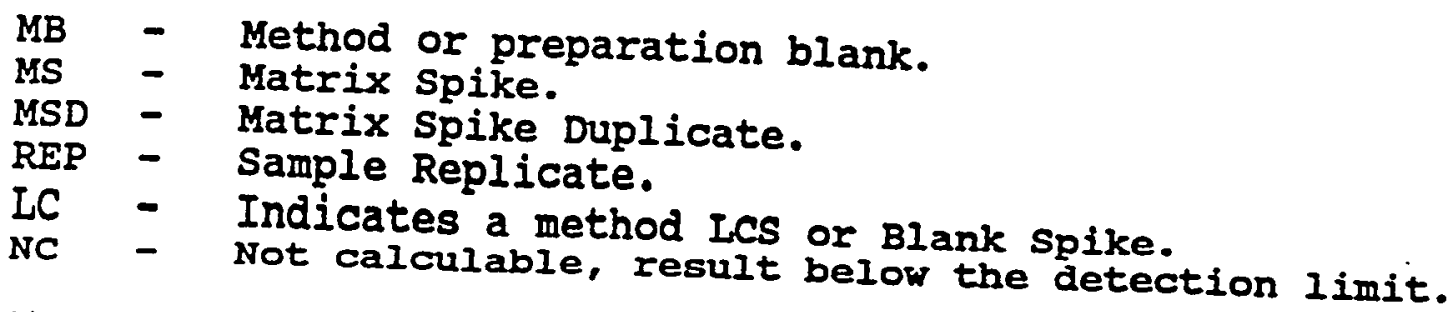

\section{LABORATORY CHRONOLOGY AND HOLDTIME REPORT}

The test code listed indicates the specific analysis or preparation procedure employed. The codes may be

MAAW - Metals prep test for AA digestion, water matrix.

MICW - Metals prep test for AA digestion, soil matrix.

MICS - Metals prep test for ICP digestion, water matrix. M**o- digestion, soil matrix.

M**TO- This type of code indicates a total metal analysis (eg. MAGTO indicates an analysis for total silver).

$M * \star S O-\quad T h i s$ type of code indicates a soluble metal analysis (eg. MAGSo indicates an analysis for soluble silver).

M**EP- This type of code indicates an EPTOXICITY metals analysis (eg. MAGEP indicates an analysis for eptox
silver).

I**TO- This type of code indicates 'a non-metallic total analysis. There is also a complimentary soluble analysis for each of these codes (eg. ICNTO indicates an analysis for total cyanide).

$A$ suffix of $-R$ or $-S$ following these codes indicates a replicate or spike analysis respectively. 
ROY F. WESTON INC.

INORGANICS DATA SUMMARY REPORT 04/09/90

CLIENT: WSRC-GRACE ROAD

WORK ORDER: 0630-28-12-0000

\begin{tabular}{|c|c|}
\hline $\begin{array}{l}\text { SAMPLE } \\
==\approx=E=\pi\end{array}$ & SITE ID \\
\hline & GR \\
\hline
\end{tabular}

$-001$

\section{ANALYTS}

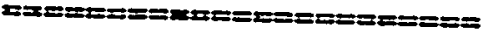

SILVER, TOTAT.

ARSENIC. TOTAL

BARIUK, TOTAL

BERYLIIUM, TOTAL

CADKIUK, TOTAL

COBALT, TOTAL

CHROMIUM, TOTAL

COPPER, TOTAT

MERCURY, TOTAL

NICKIS, TOTAL

LEAD, TOTAI

ANTIKONY, TOTAL

SELENIUM, TOTAI

TIN, TOTAL

THATIIUM, TOTAT,

VANADIUM, TOTAI

ZINC, TOTAI
WESTON BATCE : $9002 L 607$

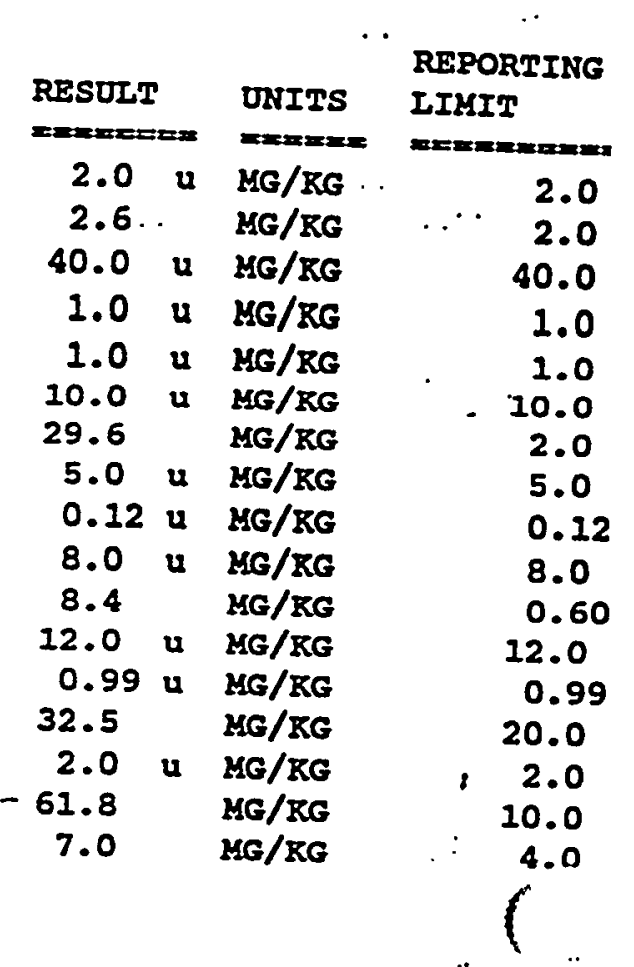


ROY F. WESTON INC.

IKORGANICS METEOD BIANT DATA SUMAARY PAGB 04/09/90

CLIENT: WSRC-GRACE ROAD"

WORK ORDER: 0630-28-12-0000

\begin{tabular}{|c|c|c|}
\hline $\begin{array}{l}\text { SAMPLE } \\
===\approx=\pi=\end{array}$ & 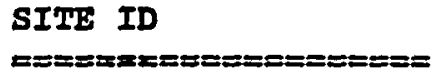 & 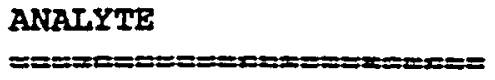 \\
\hline BLANK1 & $904,0226-M B 1$ & $\begin{array}{l}\text { SILVER, TOTAI } \\
\text { BARIUM, TOTAI } \\
\text { BERYIIIU,, TOTAI } \\
\text { CADKIU,, TOTAI } \\
\text { COBAIT, TOTAI } \\
\text { CHROKIU,, TOTAI } \\
\text { COPPER, TOTAI } \\
\text { NICKEL, TOTAI } \\
\text { ANTIKONY, TOTAI } \\
\text { TIN, TOTAI } \\
\text { VANADIUM, TOTAI } \\
\text { ZINC, TOTAI }\end{array}$ \\
\hline BLANKI & $90 L 0225-M B 1$ & $\begin{array}{l}\text { ARSENIC, TOTAL } \\
\text { LEAD, TOTAL } \\
\text { SELENIUM, TOTAL } \\
\text { TEALIIUM, TOTAL }\end{array}$ \\
\hline BIAANRI & $90 \mathrm{C} 042 \mathrm{~B}-\mathrm{MBI}$ & MERCURY, TOTALI \\
\hline BLANK2 & $90 C 042 B-M B 2$ & MERCURY, TOIAI \\
\hline BLANK3 & $90 \mathrm{CO42B}-\mathrm{MB} 3$ & MERCURY, TOTAI \\
\hline BLANK 4 & $90 C 042 B-M B 4$ & MERCURY, TOTAI \\
\hline
\end{tabular}

WESTON BATCA : 90021607

\begin{tabular}{|c|c|c|c|}
\hline \multicolumn{2}{|c|}{$\begin{array}{l}\text { RESULT } \\
==x=x=2=\end{array}$} & $\begin{array}{l}\text { UNITS } \\
\text { =I=x=E }\end{array}$ & 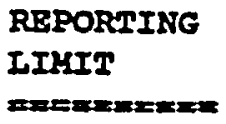 \\
\hline 2.0 & $\mathbf{u}$ & $M G / R G$ & 2.0 \\
\hline 40.0 & $\mathbf{u}$ & $M G / R G$ & 40.0 \\
\hline 1.0 & $\mathbf{u}$ & $M G / R G$ & 1.0 \\
\hline 1.0 & $\mathbf{u}$ & MG/RG & 1.0 \\
\hline 10.0 & $\mathbf{u}$ & $M G / R G$ & 10.0 \\
\hline 2.0 & $\mathbf{u}$. & $M G / R G$ & 2.0 \\
\hline 5.0 & $\mathbf{u}$ & $M G / R G$ & 5.0 \\
\hline 8.0 & $\mathbf{u}$ & KG/RG & 8.0 \\
\hline 12.0 & $\mathbf{u}$ & NG/RG & 12.0 \\
\hline 20.0 & $\mathbf{u}$ & $M G / R G$ & 20.0 \\
\hline 10.0 & $\mathbf{u}$ & MG/RG & 10.0 \\
\hline 4.0 & $\mathbf{u}$ & $M G / R G$ & 4.0 \\
\hline 2.0 & $\mathbf{u}$ & $\mathrm{MG} / \mathrm{RG}$ & 2.0 \\
\hline 0.60 & $\mathbf{u}$ & MG/RG & 0.60 \\
\hline 1.0 & $\mathbf{u}$ & MG/RG & 1.0 \\
\hline 2.0 & $\mathbf{u}$ & KG/KG & 2.0 \\
\hline 0.10 & u & MG/RG & 0.10 \\
\hline 0.10 & $\mathbf{u}$ & $M G / R G$ & 0.10 \\
\hline 0.10 & $\mathbf{u}$ & $M G / R G$ & 0.10 \\
\hline 0.10 & $\mathbf{u}$ & MG/KG & 0.10 \\
\hline
\end{tabular}


ROY F. WESTON INC.

INORGANICS DUPLICATE SPIKR REPORT 04/09/90

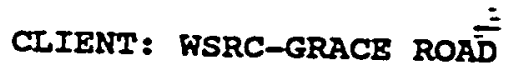

WORR ORDER: 0630-28-12-0000

WESTON BATCH * 9002L607

\begin{tabular}{|c|c|}
\hline $\begin{array}{l}\text { SARPLE } \\
x=====x\end{array}$ & 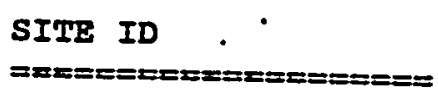 \\
\hline LCS2 & 90L0226-LC2 \\
\hline
\end{tabular}

LCS2 90L0225-LC2

LCS2

90C042B-LC2.

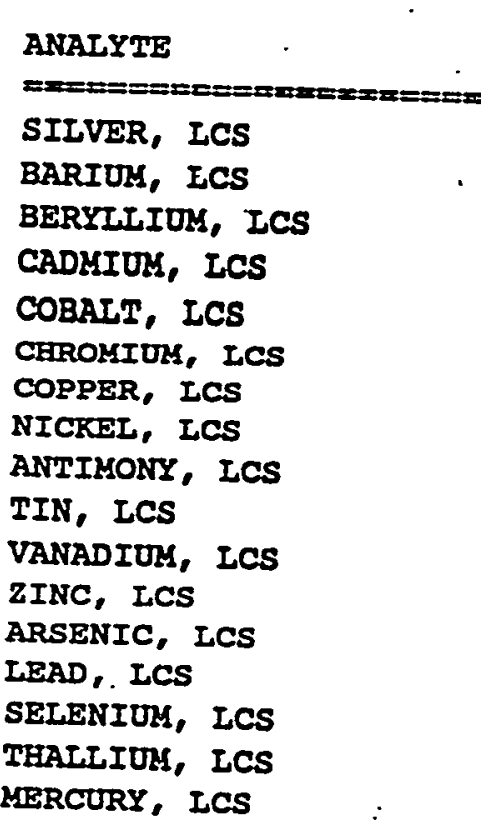

SPIKE\#1 SPIKE\$2

\$RECOV - \&RECOV YDIFF

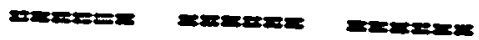

$59.0 \quad 107 \quad 57.6$

$102 \quad 101 \quad 0.74$

$107 \quad 108 \quad 1.3$

$106 \quad 106 \quad 0.30$

$104 \quad 104 \quad 0.029$

$\begin{array}{lll}112 & 111 & 0.50\end{array}$

$109 \quad 110 \quad 0.95$

$104 \quad 105 \quad 0.48$

$\begin{array}{lll}93.1 & 93.7 & 0.64\end{array}$

$\begin{array}{lll}92.6 & 93.0 & 0.41\end{array}$

$116 \quad 118 \quad 1.7$

$\begin{array}{lll}103 & 103 & 0.058\end{array}$

$\begin{array}{lll}94.0 & 97.7 & 3.8\end{array}$

$\begin{array}{lll}113 & 112 & 0.89\end{array}$

$111 \quad 113 \quad 1.5$

$107 \quad 109 \quad 1.2$

$86.2 \quad 90.4 \quad 4.8$ 
ROY F. FESTON INC.

IMORGANICS IABORATORY CONIROI STANDARDS REPORT 04/09/90

$-x_{x}$

$$
\doteq
$$

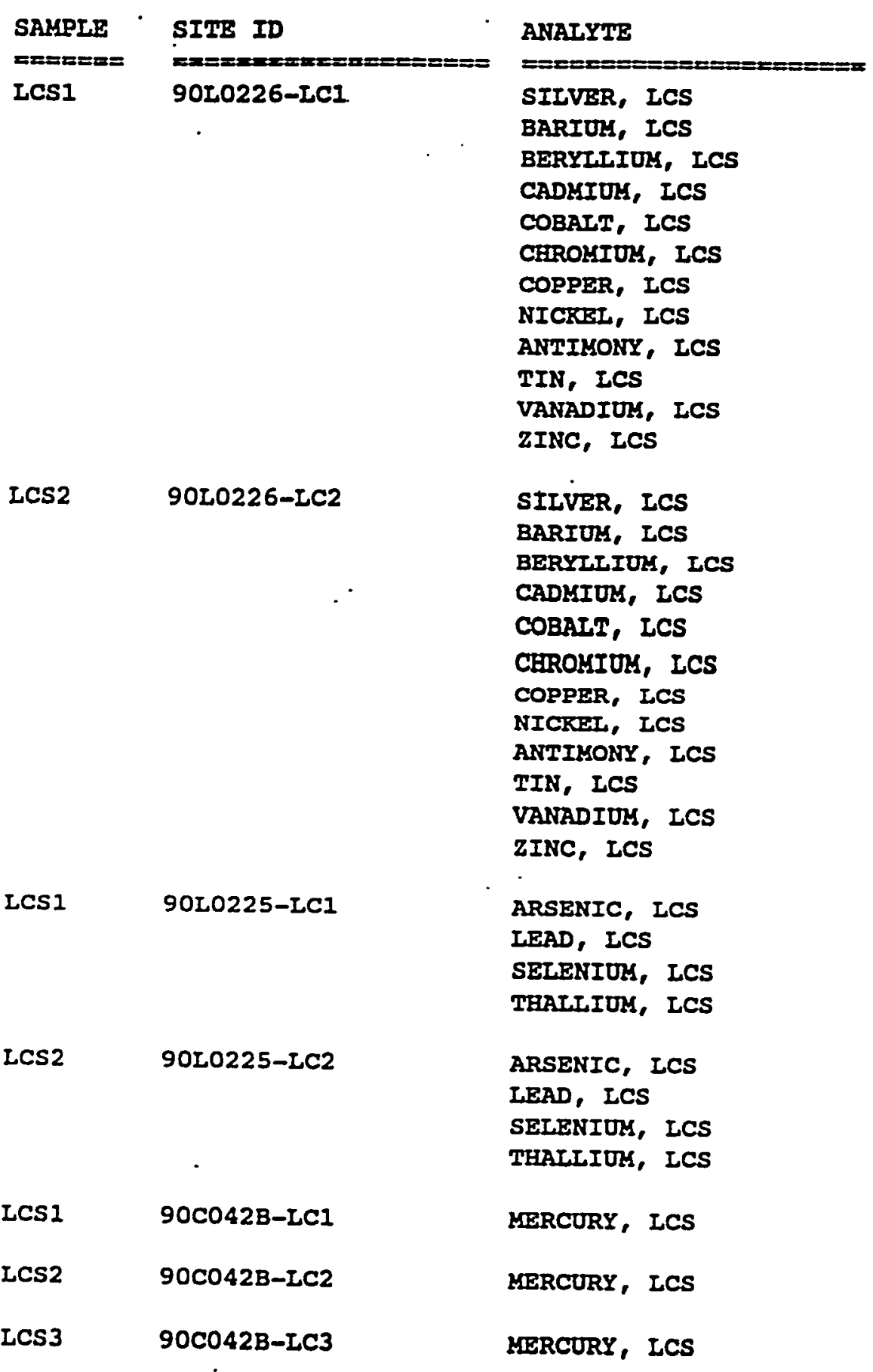

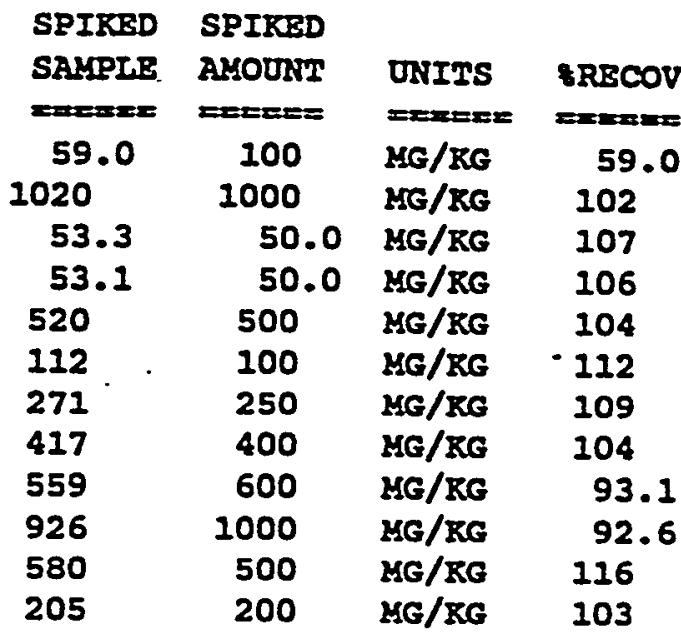

$107 \quad 100 \quad$ KG/KG $\quad 107$

$1010 \quad 1000$ MG/KG $: 101$

$54.1 \quad 50.0 \quad$ HG/KG $\quad 108$

$53.0 \quad 50.0$ MG/RG 106

$520 \quad 500 \quad \mathrm{MG} / \mathrm{KG} \quad 104$

$111 \quad 100 \mathrm{NG} / \mathrm{KG} . .111$

$274250 \quad \mathrm{MG} / \mathrm{RG} \cdot 110$

$419 \quad 400 \quad$ MG/KG 105

$562 \quad 600 \quad \mathrm{KG} / \mathrm{KG} \quad 93.7$

$930 \quad 1000 \quad \mathrm{KG} / \mathrm{KG} \quad 93.0$

$590 \quad 500 \quad$ MG/RG 118

$206200 \quad$ MG/KG 103

$5.6 \quad 6.0 \quad$ MG/KG $\quad 94.0$

$6.8 \quad 6.0 \quad \mathrm{MG} / \mathrm{RG} \quad 113$

$6.7 \quad 6.0 \mathrm{MG} / \mathrm{KG} \quad 111$

$6.4 \quad 6.0 \mathrm{MG} / \mathrm{KG} .107$
. $5.9 \quad 6.0$ MG/RG 97.7
$6.7 \quad 6.0 \quad$ MG/RG $\quad 112$
$6.8 \quad 6.0 \mathrm{MG} / \mathrm{KG} \quad 113$
$\begin{array}{lll}6.5 & 6.0 \mathrm{MG} / \mathrm{KG} & 109\end{array}$

$0.86 \quad 1.0 \mathrm{MG} / \mathrm{KG} \quad 86.2$

$0.90 \cdot \quad 1.0 \mathrm{MG} / \mathrm{KG} \quad 90.4$

$0.90 \quad 1.0 \mathrm{MG} / \mathrm{KG} \quad 90.4$ 
ROY $F$. WESTON INC.

INORGANICS IABORATORY CONTROL STANDARDS REPORT

$04 / 09 / 90$

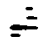

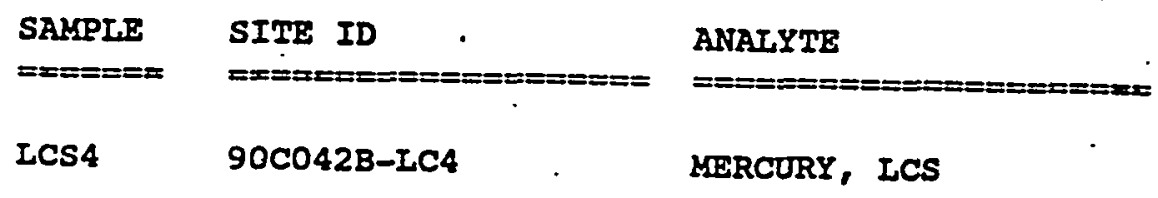

\begin{tabular}{|c|c|c|c|}
\hline SPIKBD & SPIKED & & \\
\hline SAMPIE & $\begin{array}{l}\text { AMOUNT } \\
\text { =m:=E= }\end{array}$ & $\begin{array}{l}\text { DNITS } \\
=x=0\end{array}$ & 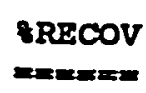 \\
\hline 0.95 & 1.0 & MG/RG & 94.6 \\
\hline
\end{tabular}




$$
\begin{aligned}
& \text { ROY F. Weston, Inc. - Iionville Laboratory } \\
& \text { INORGANIC ANALYTICAL DATA PACRAGB FOR } \\
& \text { WSRC-GRACE ROAD }
\end{aligned}
$$

DATE RECEIVED: 02/23/90

RFF LOT : $: 90025607$

CLIENT ID / ANALYSIS REW * HTX PREP * COLLECTION EXTR/PREP

ANALYSIS

\begin{tabular}{|c|c|c|c|}
\hline 001 & $\mathbf{s}$ & 9020226 & $02 / 21 / 90$ \\
\hline 001 & $\mathbf{s}$ & 90L0225 & $02 / 21 / 90$ \\
\hline 001 & $\mathbf{s}$ & 90̇_0226 & $02 / 21 / 90$ \\
\hline 001 & s & 9020226 & $02 / 21 / 90$ \\
\hline 001 & $\mathbf{s}$ & 9020226 & $02 / 21 / 90$ \\
\hline 001 & $\mathbf{s}$ & 90 .0226 & $02 / 21 / 90$ \\
\hline 001 & s & 9020226 & $02 / 21 / 90$ \\
\hline 001 & $\mathbf{s}$ & 9020226 & $02 / 21 / 90$ \\
\hline 001 & $\mathbf{s}$ & $90 \mathrm{CO} 42 \mathrm{~B}$ & $02 / 21 / 90$ \\
\hline 001 & $\mathbf{s}$ & 9020226 & $02 / 21 / 90$ \\
\hline 001 & $\mathbf{s}$ & 9020225 & $02 / 21 / 90$ \\
\hline 001 & $\mathbf{s}$ & 90L0226 & $02 / 21 / 90$ \\
\hline 001 & $\mathbf{s}$ & 9020225 & $02 / 21 / 90$ \\
\hline 001 & $\mathbf{s}$ & 90L0226 & $02 / 21 / 90$ \\
\hline 001 & $\mathbf{s}$ & 901.0225 & $02 / 21 / 90$ \\
\hline 001 & $\mathbf{s}$ & 90I0226 & $02 / 21 / 90$ \\
\hline 001 & $\mathbf{s}$ & 9010226 & $02 / 21 / 90$ \\
\hline
\end{tabular}

\begin{tabular}{l}
\hline . \\
GRS-08-01 \\
SILVER, TOTAL \\
ARSENIC, TOTAL \\
BARIUM, TOTAI \\
BERYLIIUM, TOTAI \\
CADMIUM, TOTAL \\
COBALT, TOTAL \\
CHROMIUM, TOTAI \\
COPPER, TOTAI \\
MERCURY, TOTAL \\
NICKEL, TOTAI \\
LEAD, TOTAL \\
ANTIMONY, TOTAL . \\
SELENIUM, TOTAI \\
TIN, TOTAL \\
THALIUM, TOTAL \\
VANADIUM, TOTAL \\
ZINC, TOTAL
\end{tabular}

$03 / 09 / 90$
$03 / 09 / 90$
$03 / 09 / 90$
$03 / 09 / 90$
$03 / 09 / 90$
$03 / 09 / 90$
$03 / 09 / 90$
$03 / 09 / 90$
$03 / 16 / 90$
$03 / 09 / 90$
$03 / 09 / 90$
$03 / 09 / 90$
$03 / 09 / 90$
$03 / 09 / 90$
$03 / 09 / 90$
$03 / 09 / 90$
$03 / 09 / 90$

$03 / 23 / 90$

$03 / 30 / 90$

$03 / 23 / 90$

$03 / 23 / 90$

$03 / 23 / 90$

$03 / 23 / 90$

$03 / 23 / 90$

$03 / 23 / 90$

$03 / 19 / 90$

$03 / 23 / 90$

$04 / 05 / 90$

$03 / 23 / 90$

$04 / 01 / 90$

$03 / 23 / 90$

$04 / 04 / 90$

$03 / 23 / 90$

$03 / 23 / 90$.

\begin{tabular}{|c|c|}
\hline SIIVER IABORATORY & LCI \\
\hline BARIUM LABORATORY & LCI \\
\hline BERYLIIUM ILABORATORY & LCI \\
\hline CADMIOR LABORATORY & LC1 \\
\hline COBALT LABORATORY & LCI \\
\hline CHROMIOM LABORATORY & LCI \\
\hline COPPER LABORATORY & LCI \\
\hline NICKCLL LABORATORY & ICI \\
\hline ANTIMONY LABORATORY & LC1 \\
\hline TIN LABORATORY & LC1 \\
\hline VANADIUM LABORATORY & LCI \\
\hline ZINC LABORATORY & LC1 \\
\hline SILVER LABORATORY & LC2 \\
\hline BARIUK LABORATORY & LC2 \\
\hline BERYLIIUM IABORATORY & LC2 \\
\hline CADKIOM IABORATORY & LC2 \\
\hline COBALT LABORATORY & LC2 \\
\hline
\end{tabular}

LAB QC:

\begin{tabular}{|c|c|}
\hline & 90工0226 \\
\hline$S$ & 9010226 \\
\hline & 9020226 \\
\hline $\mathbf{S}$ & 9020226 \\
\hline$S$ & 9020226 \\
\hline $\mathbf{S}$ & 9010226 \\
\hline $\mathbf{s}$ & 9020226 \\
\hline $\mathbf{s}$ & 9010226 \\
\hline $\mathbf{S}$ & 90L0226 \\
\hline $\mathbf{s}$ & $90 \mathrm{~L} 0226$ \\
\hline $\mathbf{s}$ & 9020226 \\
\hline $\mathbf{s}$ & $90 \Sigma 0226$ \\
\hline $\mathbf{s}$ & 9020226 \\
\hline $\mathbf{s}$ & 9020226 \\
\hline $\mathbf{s}$ & 90L0226 \\
\hline $\mathbf{s}$ & 90L0226 \\
\hline & 90L0226 \\
\hline
\end{tabular}

$N / A$
$N / A$
$N / A$
$N / A$
$N / A$
$N / A$
$N / A$
$N / A$
$N / A$
$N / A$
$N / A$
$N / A$
$N / A$
$N / A$
$N / A$
$N / A$
$N / A$

$03 / 09 / 90$

$03 / 09 / 90$

$03 / 09 / 90$

$03 / 09 / 90$

$03 / 09 / 90$

$03 / 09 / 90$

$03 / 09 / 90$

$03 / 09 / 90$

$03 / 09 / 90$

$03 / 09 / 90$

$03 / 09 / 90$

$03 / 09 / 90$

$03 / 09 / 90$

$03 / 09 / 90$

03/09/90

$03 / 09 / 90$

$03 / 09 / 90$

$03 / 23 / 90$
$03 / 23 / 90$
$03 / 23 / 90$
$03 / 23 / 90$
$03 / 23 / 90$
$03 / 23 / 90$
$03 / 23 / 90$
$03 / 23 / 90$
$03 / 23 / 90$
$03 / 23 / 90$
$03 / 23 / 90$
$03 / 23 / 90$
$03 / 23 / 90$
$03 / 23 / 90$
$03 / 23 / 90$
$03 / 23 / 90$
$03 / 23 / 90$ 
Roy B. Heston, Inc. - ILonville Laboratory

INORGANIC ANALYTICAI DATA PACKAGE FOR HSRC-GRACE ROAD

DATE RECEIVED: 02/23/90

RFW LOT :9002L607

\begin{tabular}{|c|c|c|c|c|c|c|c|}
\hline CLIBNT ID / ANALYSIS & RFP & * & MTX & PREP & COLIEETION & EXTR/PREP & ANALIYSIS \\
\hline CEROMIULY IABORATORY & LC2 & Bs & $\mathbf{s}$ & 9020226 & $\boldsymbol{N} / \mathbf{A}$ & $03 / 09 / 90$ & $03 / 23 / 90$ \\
\hline COPPER IAABORATORY & LC2 & BS & $\mathbf{s}$ & 9020226 & $N / A$ & $03 / 09 / 90$ & $03 / 23 / 90$ \\
\hline NICKRI IABORATORY & LC2 & BS & $\mathbf{s}$ & 9010226 & $N / A$ & $03 / 09 / 90$ & $03 / 23 / 90$ \\
\hline ANTIMONY LABORATORY & LC2 & BS & $\mathbf{s}$ & 9020226 & $\mathbf{N} / \mathbf{A}$ & $03 / 09 / 90$ & $03 / 23 / 90$ \\
\hline TIN LABORATORY & LC2 & BS & $\mathbf{s}$ & $90 \check{0} 0226$ & $N / A$ & $03 / 09 / 90$ & $03 / 23 / 90$ \\
\hline VANADIUH IABORATORY & LC2 & BS & $\mathbf{s}$ & 9020226 & $N / A$ & $03 / 09 / 90$ & $03 / 23 / 90$ \\
\hline ZINC IABORATORY & IC2 & BS & $\mathbf{s}$ & 9020226 & $N / A$ & $03 / 09 / 90$ & $03 / 23 / 90$ \\
\hline SIIVER, TOTAI & MB1 & & $\mathbf{S}$ & 90L0226 & $N / A$ & $03 / 09 / 90$ & $03 / 23 / 90$ \\
\hline BARIUK, TOTAT & MBI & & $\mathbf{s}$ & $90 L 0226$ & $N / A$ & $03 / 09 / 90$ & $03 / 23 / 90$ \\
\hline BERYLIIUM, TOTAI & MB1 & & $\mathbf{s}$ & 9010226 & $N / A$ & $03 / 09 / 90$ & $03 / 23 / 90$ \\
\hline CADMIUM, TOTAI & 2KBI & & $\mathbf{s}$ & 9010226 & $N / A$ & $03 / 09 / 90$ & $03 / 23 / 90$ \\
\hline COBALT, TOTAL & MBI & & $\mathbf{s}$ & $90 \mathrm{~L} 0226$ & $N / A$ & $03 / 09 / 90$ & $03 / 23 / 90$ \\
\hline CHROMIUS, TOTAI & MBI & & $\mathbf{s}$ & 9010226 & $N / A$ & $03 / 09 / 90$ & $03 / 23 / 90$ \\
\hline COPPER, TOTAL & MBI & & $\mathbf{s}$ & 9010226 & $\boldsymbol{N} / \mathbf{A}$ & $03 / 09 / 90$ & $03 / 23 / 90$ \\
\hline NICKEL, TOTAL & KB1 & & $\mathbf{s}$ & 9010226 & $N / A$ & $03 / 09 / 90$ & $03 / 23 / 90$ \\
\hline ANTIMONY, TOTAL & MBI & & $\mathbf{s}$ & 9010226 & $\mathbf{N} / \mathbf{A}$ & $03 / 09 / 90$ & $03 / 23 / 90$ \\
\hline TIN, TOTAI & MBI & & $\mathbf{s}$ & 9020226 & $\mathrm{~N} / \mathrm{A}$ & $03 / 09 / 90$ & $03 / 23 / 90$ \\
\hline VANADIUM, TOTAL .. & MBI & & $\mathbf{s}$ & $90 L 0226$ & $N / A$ & $03 / 09 / 90$ & $03 / 23 / 90$ \\
\hline ZINC, TOTAL & MBI & • & $\mathbf{s}$ & 9020226 & $N / A$ & $03 / 09 / 90$ & $03 / 23 / 90$ \\
\hline ARSENIC IABORATORY & LCI & BS & $\mathbf{s}$ & $90 \lcm{0225}$ & $N / A$ & $03 / 09 / 90$ & $03 / 30 / 90$ \\
\hline LEAD IABORATORY & LCI & BS & $\mathbf{s}$ & $90 L 0225$ & $N / A$ & $03 / 09 / 90$ & $04 / 05 / 90$ \\
\hline SELENIOM IABORATORY & LCI & BS & $\mathbf{s}$ & 90L0225 & $N / A$ & $03 / 09 / 90$ & $04 / 01 / 90$ \\
\hline TEALCIUM LABORATORY & LCI & BS & $\mathbf{s}$ & 9020225 & $N / A$ & $03 / 09 / 90$ & $04 / 04 / 90$ \\
\hline ARSENIC LABORATORY & LC2 & BS & $\mathbf{s}$ & $90 L 0225$ & $N / A$ & $03 / 09 / 90$ & $03 / 30 / 90$ \\
\hline LEAD ILABORATORY & IC2 & BS & $\mathbf{s}$ & $90 \longleftarrow 0225$ & $N / A$ & $03 / 09 / 90$ & $04 / 05 / 90$ \\
\hline SELENIUT IABORATORY & LC2 & BS & $\mathbf{s}$ & $90 L 0225$ & $N / A$ & $03 / 09 / 90$ & $04 / 01 / 90$ \\
\hline THALLIUM LABORATORY & LC2 & BS & $\mathbf{s}$ & 9010225 & $N / A$ & $03 / 09 / 90$ & $04 / 04 / 90$ \\
\hline ARSENIC, TOTAL & MBI & . & $\mathbf{s}$ & $90 L 0225$ & $N / A$ & $03 / 09 / 90$ & $03 / 30 / 90$ \\
\hline LEAD, TOTAL & MBI & & $\mathbf{s}$ & $90 L 0225$ & $N / A$ & $03 / 09 / 90$ & $04 / 05 / 90$ \\
\hline SELENIUM, TOTAL & MBI & & $\mathbf{s}$ & $90 L 0225$ & $N / A$ & $03 / 09 / 90$ & $04 / 01 / 90$ \\
\hline THAILIUM, TOTAL & MBI & & $\mathbf{S}$ & $90 \mathrm{~L} 0225$ & $N / A$ & $03 / 09 / 90$ & $04 / 04 / 90$ \\
\hline MERCURY LABORATORY & LCI & BS & $\boldsymbol{H}$ & $90 \mathrm{C042B}$ & $N / A$ & $03 / 16 / 90$ & $03 / 19 / 90$ \\
\hline KERCURY LABORATORY & IC2 & BS & $\boldsymbol{W}$ & $90 \mathrm{C042B}$ & $N / A$ & $03 / 16 / 90$ & $03 / 19 / 90$ \\
\hline MERCURY IABORATORY & $\mathrm{LC3}$ & BS & $\mathbf{W}$ & $90 C 042 B$ & $N / A$ & $03 / 16 / 90$ & $03 / 19 / 90$ \\
\hline MERCURY LABORATORY & LC4 & BS & $\boldsymbol{W}$ & $90 C 042 B$ & $N / A$ & $03 / 16 / 90$ & $03 / 19 / 90$ \\
\hline KERCURY, . TOTAL & MBI & & $\mathbf{W}$ & $90 \mathrm{C042B}$ & $. N / A$ & $03 / 16 / 90$ & $03 / 19 / 90$ \\
\hline MERCURY, TOTAI & MB2 & & $\%$ & $90 \mathrm{C042B}$ & $N / A$ & $03 / 16 / 90$ & $03 / 19 / 90$ \\
\hline MERCURY, TOTAL & MB3 & & $\mathbf{H}$ & $90 C 042 B$ & $N / A$ & $03 / 16 / 90$ & $03 / 19 / 90$ \\
\hline MERCURY, TOTAI & MB4 & & พ & $90 C 042 B$ & $N / \mathbf{A}$ & $03 / 16 / 90$ & $03 / 19 / 90$ \\
\hline
\end{tabular}




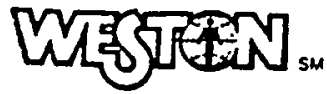

ROY F. FESTON, INC.

Lionvilie Laboratory.

CLIENT: WSRC-GRACE ROAD

RFW \#: $9002 \mathrm{~L} 606$

SAMPLES RECEIVED: $02-23-90$

W.O. \#: 0630-28-12

\section{METALS NARRATIVE}

The following is a summary of the quality control results and a this batch of samples:
this encountered during the analysis "of

1. All sample holding times as required by 40 CFR136 were met for water samples. Note: Holding times for soil samples
have not been promulgated by the USEPA.

2. All calibration verification checks were within the required control limits of $90-100 \%$ ( $85-115 \%$ for $\mathrm{Hg}$ ). standards from Inorganic is performed using independent

3. All preparation blanks were analyzed below the required
detection limit.

4. Laboratory control standards were within the control limits of $80-120 \%$ with the exception of silver (59\%) for site ID\# 90L0226-LCl. The blank spike reproducibility was
outside the $20 \%$ window for silver at $58 \%$.

Note: The USEPA-CLP has dropped control limits for silver and antimony due to documented difficulties in obtaining reliable results. WESTON Analytics has adopted the same policy. 5. The analytical methods applied by the laboratory for the
determination of metals are:
As: EPA 206.2
Se: EPA 270.2
$\mathrm{Pb}:$ EPA 239.2
T1: EPA 279.2

\author{
$\mathrm{Hg}$ : \\ ICP Scan: \\ EPA 200.7 \\ All Others: EPA 200.7 \\ EP Leachates (except $\mathrm{Hg}$ ): 200.7
}

NOTE: For solid samples, all results are reported on a dry weight
basis.

$/ \frac{\tau_{2} h \text { h K } K}{\text { Jack R. Tuschall, Ph.D. }}$
Laboratory Manager
Lionville Analytical Laboratory

pas.21/M2-606 
? 
ROY F. WESTON, INC.

\section{DATA QUAIIEIERS}

U - Indicates that the parameter was not detected at or above the reported Iimit. The associated numerical value is the sample detection limit.

* - Indicates that the original sample result is greater than $4 \mathrm{x}$ the spike amount added. The USEPA-CLP has determined that spike results on samples where this occurs may be unreliable and, therefore, the control limits are not applicable.

\section{ABBREVIATIONS}

MB - Method or preparation blank.

MS - Matrix Spike.

MSD - Matrix Spike Duplicate.

REP - Sample Replicate.

LC - Indicates a method ICS or Blank spike.

NC - Not calculable, result below the detection limit.

\section{IABORATORY CHRONOLOGY AND HOLDTIME REPORT}

The test code listed indicates the specific analysis or preparation procedure employed. The codes may be interpreted as follows:

MAAW - Metals prep test for AA digestion, water matrix. MAAS - Metals prep test for AA digestion, soil matrix. MICW - Metals prep test for ICP digestion, water matrix. MICS - Metals prep test for ICP digestion, soil matrix.

$M * *$ TO- This type of code indicates a total metal analysis (eg. MAGTO indicates an analysis for total silver).

M**SO- This type of code indicates a soluble metal analysis. (eg. MAGSO indicates an analysis for soluble silver).

$M * \star E P-$ This type of code indicates an EPTOXICITY metals analysis (eg. MAGEP indicates an analysis for eptox silver).

I**TO- This type of code indicates a non-metallic total analysis. There is also a complimentary soluble analysis for each of these codes (eg. ICNTO indicates an analysis for total cyanide).

$A$ suffix of $-R$ or $-S$ following these codes indicates a replicate or spike analysis respectively. 
ROY F. WESTON INC.

\section{INORGANICS DATA SURMARY REPORT 04/09/90}

CLIENT: WSRC-GRACE ROA $\overline{\bar{D}}$

HORR ORDER: 0630-28-12-0000

WESTON BATCH : $90021606^{\circ}$

\begin{tabular}{|c|c|c|}
\hline $\begin{array}{l}\text { SAMPLER } \\
=x====x=\end{array}$ & 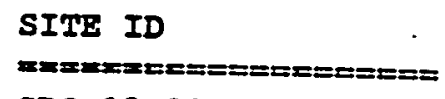 & 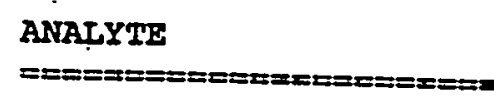 \\
\hline-001 & GRS-10-01 & $\begin{array}{l}\text { SILVER, TOTAL } \\
\text { ARSENIC, TOTAL } \\
\text { BARIUM, TOTAL } \\
\text { CADMIUK, TOTAL } \\
\text { CHROMIUM, TOTAL } \\
\text { MERCURY, TOTAL } \\
\text { LEAD, TOTAL } \\
\text { SELENIUM, TOTAI }\end{array}$ \\
\hline
\end{tabular}

\begin{tabular}{|c|c|c|c|}
\hline \multicolumn{2}{|c|}{$\begin{array}{l}\text { RESULT } \\
=E=E=E x\end{array}$} & $\begin{array}{l}\text { ONITS } \\
\text { =x=man= }\end{array}$ & 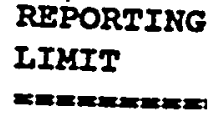 \\
\hline $\begin{array}{l}1.9 \\
1.9\end{array}$ & $\begin{array}{l}\mathbf{u} \\
\mathbf{u}\end{array}$ & $\begin{array}{l}\text { MG/RG } \\
M G / R G\end{array}$ & . $\quad 1.9$ \\
\hline 37.3 & $\mathbf{u}$ & $M G / R G$ & $\begin{array}{r}1.9 \\
37.3\end{array}$ \\
\hline 0.93 & $\mathbf{u}$ & $M G / K G$ & 0.98 \\
\hline 4.2 & & MG/KG & 1.9 \\
\hline $\begin{array}{l}0.11 \\
1.4\end{array}$ & $\mathbf{u}$. & $\begin{array}{l}\mathrm{MG} / \mathrm{RG} \\
\mathrm{MG} / \mathrm{RG}\end{array}$ & $\begin{array}{l}0.11 \\
0.5 \varepsilon\end{array}$ \\
\hline & $\mathbf{u}$ & $M G /$ & 0.97 \\
\hline
\end{tabular}


ROY $F$. WESTON INC.

INORGANICS DATA SURRARY REPORT 04/09/90

CLIENT: WSRC-GRACE ROAD

WORK ORDERz 0630-28-12-0000

WESTON BATCE \# 9002L606

\begin{tabular}{|c|c|c|}
\hline $\begin{array}{l}\text { SARPLE } \\
===\pi=m \pi m\end{array}$ & 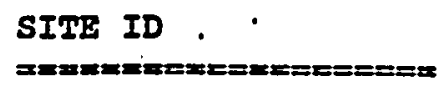 & 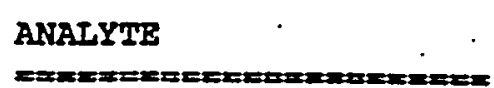 \\
\hline-004 & GRS-09-01 & $\begin{array}{l}\text { SILVER, TOTAL } \\
\text { ARSENIC, TOTAL } \\
\text { BARIUM, TOTAL } \\
\text { CADKIUM, TOTAI } \\
\text { CHROMIUM, TOTAI } \\
\text { MERCURY, TOTAL } \\
\text { LEAD, TOTAL } \\
\text { SEIENIUY, TOTAT }\end{array}$ \\
\hline
\end{tabular}

\begin{tabular}{|c|c|c|c|}
\hline \multicolumn{2}{|c|}{ 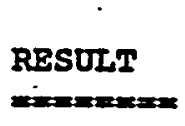 } & ONITS & $\begin{array}{l}\text { RBPORTING } \\
\text { LIMIT } \\
\text { =m=m }\end{array}$ \\
\hline 2.1 & $\mathbf{u}$ & MG/RG & 2.1 \\
\hline 1.5 & $\mathbf{u}$ & $M G / R G$ & 1.5 \\
\hline 41.7 & $\mathbf{u}$ & MG/RG & 41.7 \\
\hline 1.0 & $\mathbf{u}$ & $M G / R G$ & 1.0 \\
\hline 3.9 & & $M G / K G$ & 2.1 \\
\hline 0.11 & $\mathbf{u}$ & $\mathrm{KG} / \mathrm{RG}$ & 0.11 \\
\hline $\begin{array}{l}2.0 \\
0.77\end{array}$ & $\mathbf{u}$ & $\begin{array}{l}M G / R G \\
M G / R G\end{array}$ & $\begin{array}{l}0.46 \\
0.79\end{array}$ \\
\hline
\end{tabular}


ROY $F$. FESTON INC.

INORGANICS DATA SURRARY REPORT 04/09/90

CLIENT: HSRC-GRACE ROAD̄

WORR ORDER: 0630-28-12-0000

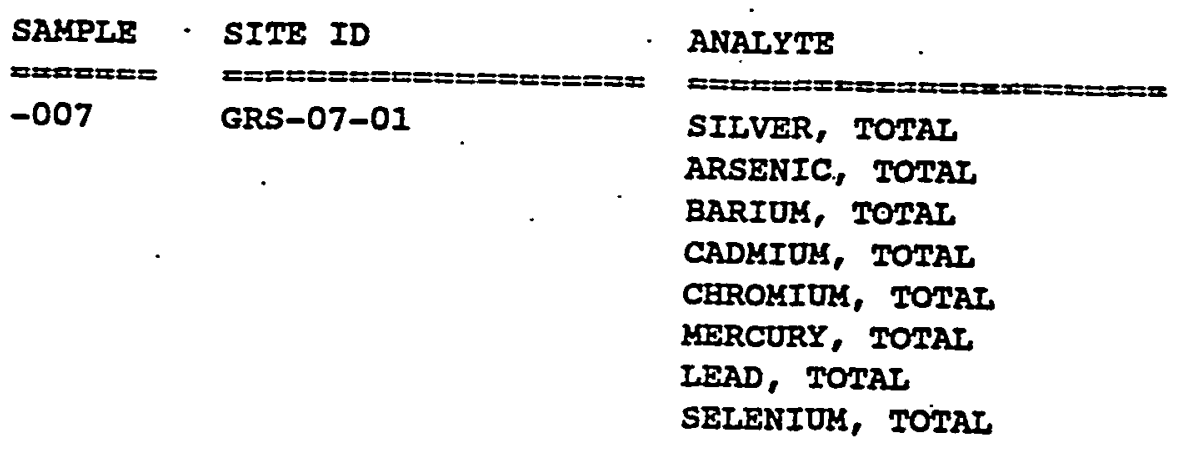

HESTON BATCE * 9002L606

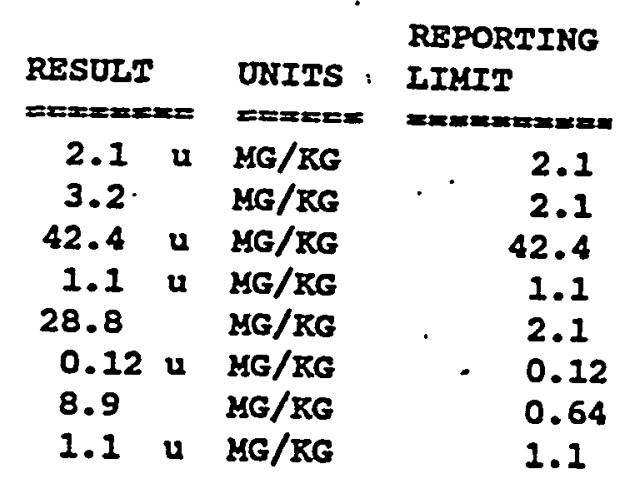


ROY F. WESTON INC.

INORGANICS DATA SURRARY RBPORT 04/09/90

CLIENT: WSRC-GRACB ROAD

FORR ORDER: 0630-28-12-0000

\begin{tabular}{|c|c|c|}
\hline 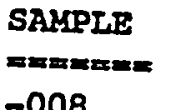 & SITE ID & 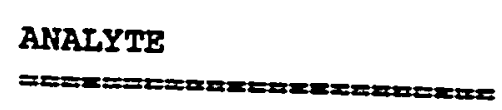 \\
\hline & GRS=07-01A & $\begin{array}{l}\text { SILVER, TOTAI } \\
\text { ARSENIC, TOTAI } \\
\text { BARIUM, TOTAI } \\
\text { CADMIUM, TOTAI } \\
\text { CHROMIUM, TOTAI } \\
\text { MGRCURY, TOTAL } \\
\text { LEAD, TOTAL } \\
\text { SELENIOY, TOTAI }\end{array}$ \\
\hline
\end{tabular}

WESTON BATCH * 9002L606

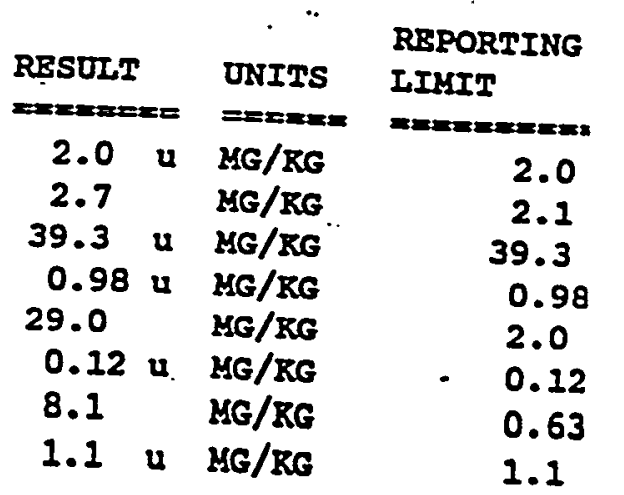


ROY F. HESTON INC.

INORGANICS DATA SUMMARY REPORT 04/09/90

CLIENT: WSRC-GRACE ROA $\overline{\bar{D}}$

WORR ORDER: 0630-28-12-0000

FESTON BATCH * 9002L606

\begin{tabular}{|c|c|c|}
\hline $\begin{array}{l}\text { SAMPIS } \\
\text { =Ex=E=E }\end{array}$ & 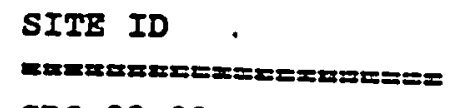 & 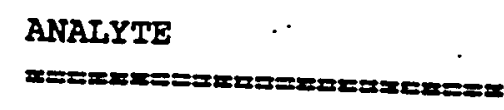 \\
\hline-014 & GRS-08-03E & $\begin{array}{l}\text { SILVGR, TOTAI } \\
\text { ARSENIC,-TOTAI } \\
\text { BARIUM, TOTAL } \\
\text { CADMIUY, TOTAI } \\
\text { CHROYIUM, TOTAL } \\
\text { MERCURY, TOTAL } \\
\text { LEAD, TOTAI } \\
\text { SELENIUM, TOTAI }\end{array}$ \\
\hline
\end{tabular}

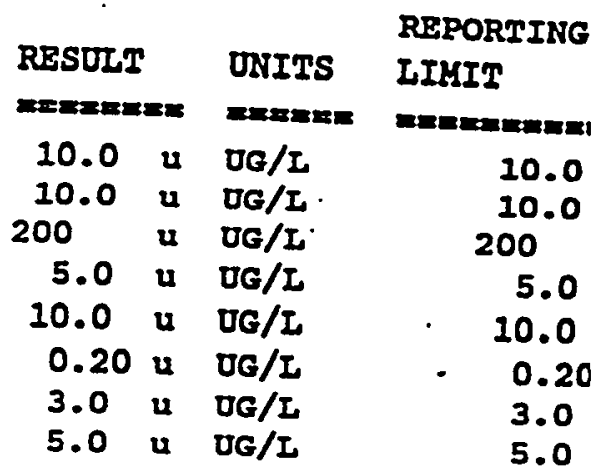

1 
ROY E. WESTON INC.

INORGANICS IIETHOD BLANR DATA SUMLARY PAGE 04/09/90

CLIENT: WSRC-GRACE ROA $\overline{\bar{D}}$

WORK ORDER\& 0630-28-12-0000

\begin{tabular}{|c|c|c|}
\hline $\begin{array}{l}\text { SAMPLE } \\
======2\end{array}$ & 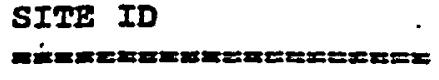 & 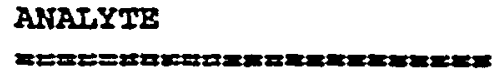 \\
\hline BLANRI & 90L0226-MB1 & $\begin{array}{l}\text { SILVER, TOTAI } \\
\text { BARIUM, TOTAI } \\
\text { CADMIUM, TOTAI } \\
\text { CEROMIUK, TOTAI }\end{array}$ \\
\hline BLANR1 & 90L0225-MBBI & $\begin{array}{l}\text { ARSENIC, TOTAL } \\
\text { LEAD, TOTAL } \\
\text { SELENIUM, TOTAL }\end{array}$ \\
\hline BLANKI & $90 C 042 B-M B 1$ & MERCURY, TOTAI \\
\hline BLANK2 & $90 \mathrm{CO42B-2BB2}$ & MERCURY, TOTAT \\
\hline BLANK 3 & $90 \mathrm{CO} 42 \mathrm{~B}-\mathrm{MB}$ & MERCURY, TOTAI \\
\hline BLANK4 & $90 \mathrm{CO42B}-\mathrm{MB} 4$ & MRRCURY, TOTAI \\
\hline BLANR1 & $9020219-K B 1$ & $\begin{array}{l}\text { SIIVER, TOTAI } \\
\text { BARIUM, TOTAI } \\
\text { CADKIUM, TOTAI } \\
\text { CHROKIUK, TOTAL }\end{array}$ \\
\hline BLANRI & $9010218-\mathrm{MBI}$ & $\begin{array}{l}\text { ARSENIC, TOTAI } \\
\text { LEAD, TOTAL } \\
\text { SELENIUA, TOTAL }\end{array}$ \\
\hline BLANR I & $90 \mathrm{CO} 42 \mathrm{~A}-\mathrm{MB1}$ & MERCURY, TOTAI \\
\hline BLANR2 & $90 C 042 A-M B 2$ & AERCURY, TOTAI \\
\hline
\end{tabular}

FESTON BATCH : $9002 L 606$

\begin{tabular}{|c|c|c|c|}
\hline \multicolumn{2}{|c|}{$=x$} & UNITS & $\begin{array}{l}\text { RBPORTING } \\
\text { IIKIT } \\
\text { anterem }\end{array}$ \\
\hline $\begin{array}{r}2.0 \\
40.0\end{array}$ & $\mathbf{u}$ & $\begin{array}{l}M G / R G \\
M G / R G\end{array}$ & $\begin{array}{r}2.0 \\
40.0\end{array}$ \\
\hline 1.0 & $\mathbf{u}$ & $M G / K G$ & 1.0 \\
\hline 2.0 & $\mathbf{u}$ & HG/RG & 2.0 \\
\hline $\begin{array}{l}2.0 \\
0.60\end{array}$ & $\mathbf{u}$ & $\begin{array}{l}M G / R G \\
M G / R G\end{array}$ & 2.0 \\
\hline 1.0 & $\mathbf{u}$ & HG/RG & 1.0 \\
\hline 0.10 & $\mathbf{u}$ & $\mathrm{MG} / \mathrm{RG}$ & 0.10 \\
\hline 0.10 & $\mathbf{u}$ & $\mathrm{MG} / \mathrm{KG}$ & 0.10 \\
\hline 0.10 & $\mathbf{u}$ & $\mathrm{MG} / \mathrm{RG}$ & 0.10 \\
\hline 0.10 & $\mathbf{u}$ & $\mathrm{MG} / \mathrm{KG}$ & 0.10 \\
\hline
\end{tabular}

10.0 บ UG/I 10.0

200 u UG/I 200

5.0 u UG/I $\quad \therefore 5.0$

10.0 u UG/L 10.0

10.0 U DG/I 10.0

3.0 u UG/I 3.0

5.0 u UG/L 5.0

0.20 u UG/I 0.20

0.20 u JG/I 0.20 
ROY F. WESTON INC.

INORGANICS METHOD BLANK DATA SUMAGARY PAGE 04/09/90

CLIENT: WSRC-GRACE ROAD

WORR ORDER: 0630-28-12-0000

SAMPLE

SITE ID

BLANR3

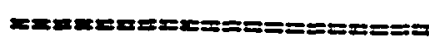

$90 C 042 \mathrm{~A}-\mathrm{MB} 3$

ANALYTE

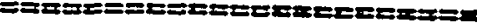

MERCURY, TOTAL

BLANK4 90C042A-MB4
WESTON BATCH \& 9002L606.

\begin{tabular}{clr} 
RESULT & UNITS & \multicolumn{2}{l}{ REPORTING } \\
LIMIT \\
0.20 u & UG/L & 0.20 \\
0.20 u & UG/L & 0.20
\end{tabular}


ROY F. FESTOR INC.

INORGANICS DUPLICATE SPIRB REPORT 04/09/90

CLIENT: WSRC-GRACE ROAD

WORK ORDER: 0630-28-12-0000

WBSTON BATCE * 9002L606

\begin{tabular}{|c|c|c|c|c|c|}
\hline SAMPLB. & SITR ID & 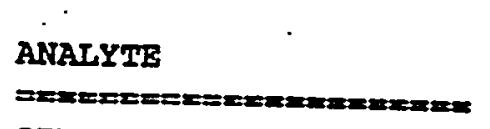 & $\begin{array}{l}\text { SPIKE\$1 } \\
\text { IRRCOV } \\
==1=\end{array}$ & 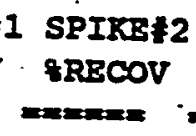 & \&DIFE \\
\hline LCS2 & 90L0226-LC2 & SILVER, ICS & 59.0 & 107 & 57.6 \\
\hline & . & $\begin{array}{l}\text { BARIUA, ICS. } \\
\text { CADKIOM, LCS }\end{array}$ & $\begin{array}{l}102 \\
106\end{array}$ & $\begin{array}{l}101 \\
105\end{array}$ & 0.74 \\
\hline LCS2 & 90L0225-LC2 & $\begin{array}{l}\text { CEROMIOM, LCS } \\
\text { ARSENIC, LCS } \\
\text { LEAD, ICS }\end{array}$ & $\begin{array}{l}112 \\
94.0 \\
113\end{array}$ & $\begin{array}{c}100 \\
111 \\
97.7 \\
112\end{array}$ & $\begin{array}{l}0.30 \\
0.50 \\
3.8 \\
0.89\end{array}$ \\
\hline LCS2 & $90 \mathrm{CO} 42 \mathrm{~B}-\mathrm{IC2} 2$ & SELENIOM, LCS & 111 & 113 & $\begin{array}{l}0.89 \\
1.5\end{array}$ \\
\hline $\operatorname{LCS} 2$ & $\begin{array}{l}90 C 042 \mathrm{~B}-\mathrm{LC2} \\
\text { 90L0219-LC2 }\end{array}$ & $\begin{array}{l}\text { MERCURY, ICS } \\
\text { SILVER, ICS }\end{array}$ & 86.2 & 90.4 & 4.8 \\
\hline & & BARIUM, LCS & $\begin{array}{l}127 \\
102\end{array}$ & $\begin{array}{l}119 \\
101\end{array}$ & $\begin{array}{l}6.0 \\
1.2\end{array}$ \\
\hline & & CADMIUM, ICS & 115 & 111 & 3.4 \\
\hline $\operatorname{LCs} 2$ & 90L0218-LC2 & $\begin{array}{l}\text { CEROKIUM, LCS } \\
\text { ARSENIC, ICS } \\
\text { IRAD }\end{array}$ & $\begin{array}{r}120 \\
87.0\end{array}$ & $\begin{array}{l}116 \\
90.7\end{array}$ & $\begin{array}{l}3.3 \\
4.1\end{array}$ \\
\hline LCS2 & $90 C 042 A-I C 2$ & $\begin{array}{l}\text { LEAD, ICS } \\
\text { SELENIUM, ICS } \\
\text { MERCURY, ICS }\end{array}$ & $\begin{array}{l}105 \\
110 \\
86.2\end{array}$ & $\begin{array}{l}104 \\
113 \\
90.4\end{array}$ & $\begin{array}{l}0.95 \\
2.7 \\
4.8\end{array}$ \\
\hline
\end{tabular}

$\$$ 
ROY F. WESTON INC.

INORGANICS LABORATORY CONIROL STANDARDS REPORT 04/09/90

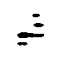

\begin{tabular}{|c|c|c|c|c|c|c|}
\hline $\begin{array}{l}\text { SAMPLE } \\
=E=====\end{array}$ & 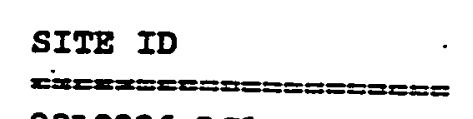 & 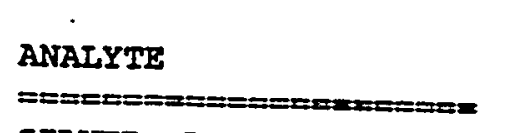 & $\begin{array}{l}\text { SPIKED } \\
\text { SALPLIB } \\
\text { =x=E== }\end{array}$ & $\begin{array}{l}\text { SPIKESD } \\
\text { AMOUNTT } \\
=2 x=\approx=\pi x\end{array}$ & $\begin{array}{l}\text { UNITS } \\
==2=x=x=\end{array}$ & RRECOV \\
\hline LCSI & 90L0226-IC1 & $\begin{array}{l}\text { SILVER, ICS } \\
\text { BARIUM, LCS } \\
\text { CADMIUM, LCS } \\
\text { CEROMIOM, LCS }\end{array}$ & $\begin{array}{l}59.0 \\
1020 \\
53.1 \\
112\end{array}$ & $\begin{array}{l}100 \\
1000 \\
50.0 \\
100\end{array}$ & $\begin{array}{l}M G / K G \\
M G / K G \\
M G / R G \\
M G / R G\end{array}$ & $\begin{array}{l}59.0 \\
102 \\
106 \\
112\end{array}$ \\
\hline LCS2 & 90L0226-IC2 & $\begin{array}{l}\text { SILVER, LCS } \\
\text { BARIUM, LCS } \\
\text { CADMIUM, LCS } \\
\text { CHROMIUM, LCS }\end{array}$ & $\begin{array}{c}107 \\
1010 \\
53.0 \\
111\end{array}$ & $\begin{array}{c}100 \\
1000 \\
50.0 \\
100\end{array}$ & $\begin{array}{l}\text { MG/RG } \\
M G / R G \\
M G / R G \\
M G / K G\end{array}$ & $\begin{array}{r}107 \\
101 \\
106 \\
111\end{array}$ \\
\hline LCS 1 & 90L0225-LCI & $\begin{array}{l}\text { ARSENIC, ICS } \\
\text { LEAD, ICS } \\
\text { SELENIUK, ICS } \\
\end{array}$ & $\begin{array}{l}5.6 \\
6.8 \\
6.7\end{array}$ & $\begin{array}{l}6.0 \\
6.0 \\
6.0\end{array}$ & $\begin{array}{l}M G / K G \\
M G / R G \\
M G / K G\end{array}$ & $\begin{array}{l}94.0 \\
113 \\
111\end{array}$ \\
\hline LCS2 & $90 L 0225-L C 2$ & $\begin{array}{l}\text { ARSENIC, LCS } \\
\text { LEAD, LCS } \\
\text { SELENIUM, ICS }\end{array}$ & $\begin{array}{l}5.9 \\
6.7 \\
6.8\end{array}$ & $\begin{array}{l}6.0 \\
6.0 \\
6.0\end{array}$ & $\begin{array}{l}M G / K G \\
M G / R G \\
M G / K G\end{array}$ & $\begin{array}{l}97.7 \\
112 \\
113\end{array}$ \\
\hline $\operatorname{LCS} 1$ & $90 \mathrm{CO} 42 \mathrm{~B}-\mathrm{LCl}$ & MERCURY, LCS & 0.86 & 1.0 & $M G / K G$ & $\therefore$ \& \\
\hline LCS2 & $90 \mathrm{CO} 42 \mathrm{~B}-\mathrm{LC} 2$ & MERCURY, LCS & 0.90 & 1.0 & MG/KG & 90.4 \\
\hline $\operatorname{LCs} 3$ & $90 \mathrm{CO} 42 \mathrm{~B}-\mathrm{LC} 3$ & MERCURY, LCS & 0.90 & 1.0 & MG/KG & 90.4 \\
\hline $\operatorname{LCs} 4$ & $90 \mathrm{CO} 42 \mathrm{~B}-\mathrm{LC} 4$ & MERCURY, LCS & 0.95 & 1.0 & $M G / K G$ & 94.6 \\
\hline $\operatorname{LCs} 1$ & 90L0219-LC1 & $\begin{array}{l}\text { SILVER, LCS } \\
\text { BARIUM, ICS } \\
\text { CADMIUM, LCS } \\
\text { CHROMIUM, ICS }\end{array}$ & $\begin{array}{r}633 \\
5100 \\
287 \\
599\end{array}$ & $\begin{array}{r}500 \\
5000 \\
250 \\
500\end{array}$ & $\begin{array}{l}\text { UG/L } \\
\text { UG/L } \\
\text { UG/L } \\
\text { UG/L }\end{array}$ & $\begin{array}{l}127 \\
102 \\
115 \\
120\end{array}$ \\
\hline $\operatorname{css} 2$ & 90L0219-LC2 & $\begin{array}{l}\text { SILVER, LCS } \\
\text { BARIUY, LCS } \\
\text { CADMIUM, LCS } \\
\text { CHROMIUM, ICS }\end{array}$ & $\begin{array}{r}596 \\
5040 \\
-\quad 278 \\
-\quad 580\end{array}$ & $\begin{array}{r}500 \\
5000 \\
250 \\
500\end{array}$ & $\begin{array}{l}U G / L \\
U G / L \\
U G / L \\
U G / L\end{array}$ & $\begin{array}{l}119 \\
101 \\
111 \\
116\end{array}$ \\
\hline
\end{tabular}


ROY F. HESTON INC.

INORGANICS IABORATORY CONTROL STANDARDS REPORT 04/09/90

$$
=
$$

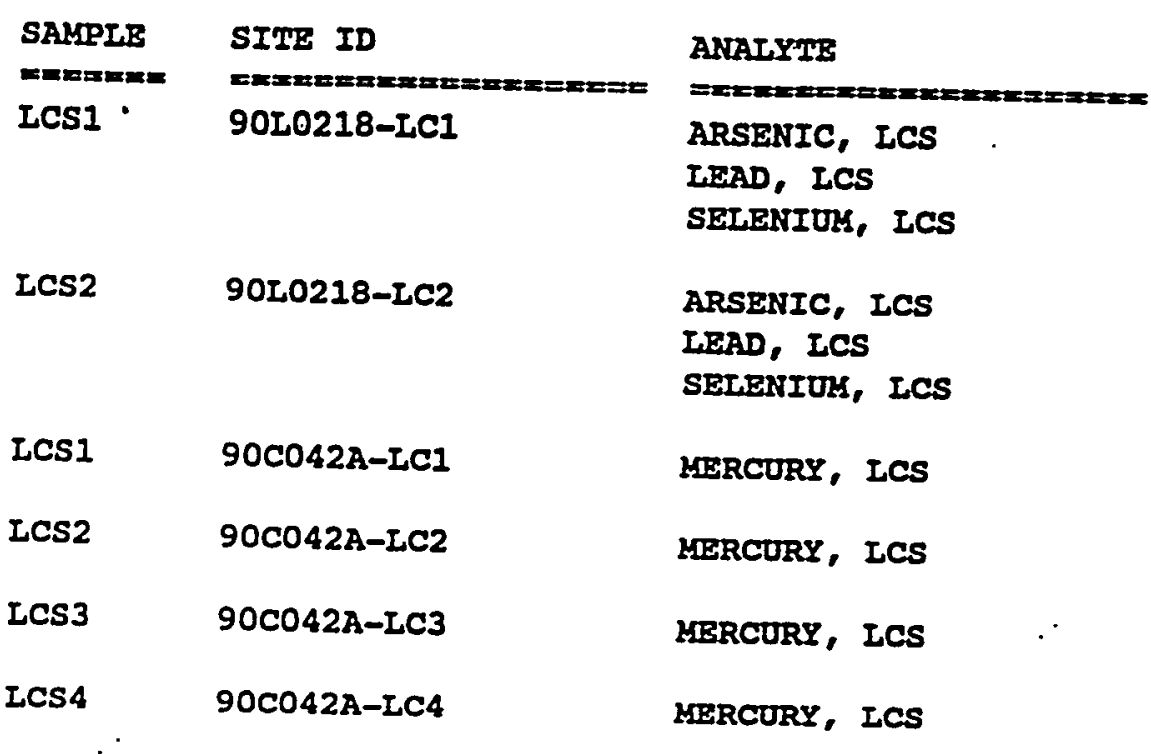

\begin{tabular}{|c|c|c|c|}
\hline SPIKED & SPIRED & & \\
\hline $\begin{array}{l}\text { SALIPLE } \\
\text { WE=E=E }\end{array}$ & $\begin{array}{l}\text { AMOUNT } \\
=X=== \pm\end{array}$ & $\begin{array}{l}\text { DNITS } \\
\text { =arements }\end{array}$ & $\begin{array}{l}\text { SRBCOV } \\
=2=1=1\end{array}$ \\
\hline 26.1 & 30.0 & $\nabla G / L$ & 87.0 \\
\hline 31.6 & 30.0 & UG/I & 105 \\
\hline 33.1 & 30.0 & $0 G / L$ & 110 \\
\hline 27.2 & 30.0 & UG/L & 90.7 \\
\hline 31.3 & 30.0 & UG/L & $\cdot 104$ \\
\hline 34.0 & 30.0 & UG/I & 213 \\
\hline 1.7 & 2.0 & $\tilde{U} / \mathbf{L}$ & 86.2 \\
\hline 1.8 & 2.0 & UG/I & 90.4 \\
\hline 1.8 & 2.0 & UG/I & 90.4 \\
\hline 1.9 & 2.0 & UG/L & 94.6 \\
\hline
\end{tabular}


DATE RECEIVED: $02 / 23 / 90$ CLIENT ID /ANALYSIS
REW
RFW LOT * :9002亡606

ANALYSIS

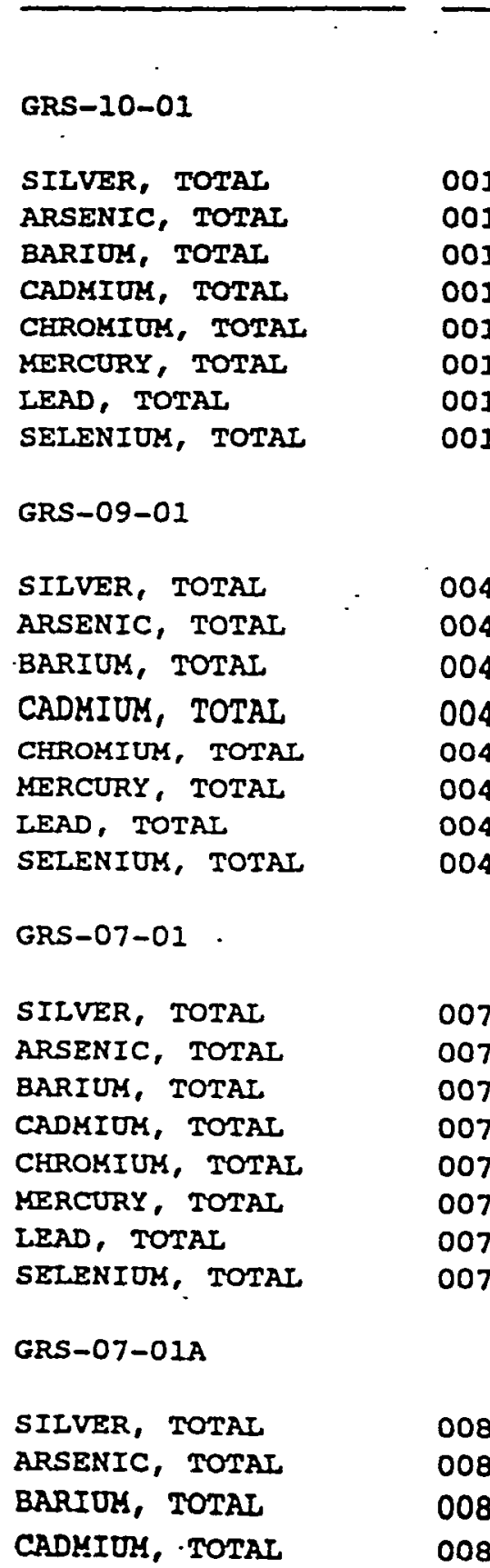

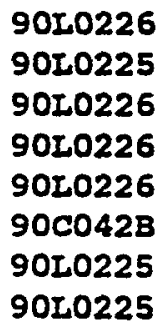

9020226 9020225 90L0226 9020226 9020226 $900042 B$ 9010225 90L0225
$02 / 20 / 90$ $02 / 20 / 90$ $02 / 20 / 90$ $02 / 20 / 90$ $02 / 20 / 90$ $02 / 20 / 90$ $02 / 20 / 90$ $02 / 20 / 90$
$03 / 09 / 90$ $03 / 09 / 90$ $03 / 09 / 90$ 03/09/90 $03 / 09 / 90$ $03 / 16 / 90$ $03 / 09 / 90$ $03 / 09 / 90$
$03 / 23 / 90$ $03 / 30 / 90$ $03 / 23 / 90$ $03 / 23 / 90$ $03 / 23 / 90$ $03 / 19 / 90$ $04 / 05 / 90$ $04 / 01 / 90$
$02 / 20 / 90$ $02 / 20 / 90$ $02 / 20 / 90$ $02 / 20 / 90$ $02 / 20 / 90$ $02 / 20 / 90$ $02 / 20 / 90$ $02 / 20 / 90$
$03 / 09 / 90$ $03 / 09 / 90$ $03 / 09 / 90$ $03 / 09 / 90$ $03 / 09 / 90$ $03 / 16 / 90$ $03 / 09 / 90$ $03 / 09 / 90$

$03 / 23 / 90$ $03 / 30 / 90$ $03 / 23 / 90$ $03 / 23 / 90$ $03 / 23 / 90$ $03 / 19 / 90$. 04/05/90 $04 / 01 / 90$

007

007

007

007

007

007

007

007

90LO226
90L.0225
90L0226
90L0226
90L0226
90C042B
90L0225
90L0225

$02 / 21 / 90$ $02 / 21 / 90$ $02 / 21 / 90$ $02 / 21 / 90$ $02 / 21 / 90$ $02 / 21 / 90$ $02 / 21 / 90$ $02 / 21 / 90$
$03 / 09 / 90$ $03 / 09 / 90$ $03 / 09 / 90$ $03 / 09 / 90$ $03 / 09 / 90$ $03 / 16 / 90$ $03 / 09 / 90$ 03/09/90
$03 / 23 / 90$ $03 / 30 / 90$ $03 / 23 / 90$ $03 / 23 / 90$ $03 / 23 / 90$ $03 / 19 / 90$ $04 / 05 / 90$ $04 / 01 / 90$
008
008
008
008 $\begin{array}{ll}\text { s } & 9020226 \\ s & 90 L 0225 \\ \text { s } & 90 L 0226 \\ \text { s } & 90 L 0226\end{array}$
$02 / 21 / 90$
$02 / 21 / 90$
$02 / 21 / 90$
$02 / 21 / 90$
$03 / 09 / 90$ $03 / 09 / 90$ $03 / 09 / 90$ $03 / 09 / 90$
$03 / 23 / 90$ $03 / 30 / 90$ $03 / 23 / 90$ $03 / 23 / 90$ 
Roy B. Heston, Inc. - ILonville Laboratory :

INORGANIC ANALYTICAL DATA PACRAGE FOR

WSRC-GRACE ROAD

DATB RECEIVED: 02/23/90

RFW LOT :9002L606

CLIENT ID /ANALYSIS

-

REN *

MTX PRBP *

COLTECTION EXTR/PREP

ANATYSIS

CEROYIUK, TOTAI

008

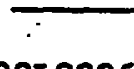

MRRCURY, TOTAL

008

S 90L0226

TEAD, TOTAL 008

SELENIUK, TOTAI $.008^{\circ}$

s 90C042B

s. 90 r.0225

s 90ז0225

$02 / 21 / 90$
$02 / 21 / 90$
$02 / 21 / 90$
$02 / 21 / 90$

$03 / 09 / 90$

$03 / 16 / 90$

$03 / 09 / 90$

$03 / 09 / 90$

$03 / 23 / 90$

$03 / 19 / 90$

$04 / 05 / 90$

$04 / 01 / 90$

GRS-08-03E

SILVER, TOTAT

ARSENIC, TOTAI

BARIUM, TOTAT

CADMIUM, TOTAI

CHROMIUM, TOTAL

MERCURY, TOTAL

LEAD, TOTAL

SELENIUM, TOTAI
014

014

014

014.

014

014

014

014
9020219

9020218

90工0219

90L0219

90L0219

$90 \mathrm{C042A}$

9020218

90 0218
$02 / 21 / 90$

$02 / 21 / 90$

$02 / 21 / 90$

$02 / 21 / 90$

$02 / 21 / 90$

$02 / 21 / 90$

$02 / 21 / 90$

$02 / 21 / 90$
$03 / 08 / 90$

$03 / 08 / 90$

$03 / 08 / 90$

$03 / 08 / 90$

$03 / 08 / 90$

$03 / 16 / 90$

$03 / 08 / 90$

$03 / 08 / 90^{\circ}$

IAB QC:

\begin{tabular}{|c|c|c|}
\hline SILVER IABORATORY & LCI & BS \\
\hline BARIUM LABORATORY & LCI & \\
\hline RYLLIUM LABORATORY & LC1 & \\
\hline CADMIUM IABORATORY & LCI & \\
\hline COBALT IABORATORY & LC1 & \\
\hline CHROMIUK IABORATORY & LC! & \\
\hline COPPER LABORATORY & LC1 & \\
\hline NICKEL IABORATORY & LC1 & \\
\hline ANTIMONY IABORATORY & LC1 & \\
\hline TIN IABORATORY & LC1 & \\
\hline VANADIUM LABORATORY & ICl & \\
\hline ZINC IABORATORY & IC1 & \\
\hline SILVER IABORATORY & LC2 & \\
\hline BARIUM IAABORATORY & LC2 & \\
\hline BERYLIIUY LABORATORY & LC2 & \\
\hline CADMIUM IABORATORY & IC2 & \\
\hline COBAIT IABORATORY & IC2 & \\
\hline CHROMIUM IABORATORY & LC2 & \\
\hline COPPER IABORATORY & LC2 & \\
\hline NICKEL IABORATORY & LC2 & \\
\hline MONY LABORAT & LC2 & \\
\hline TIN & IC2 & \\
\hline
\end{tabular}

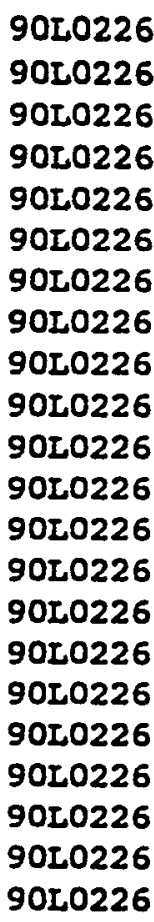

$N / A$
$N / A$
$N / A$
$N / A$
$N / A$
$N / A$
$N / A$
$N / A$
$N / A$
$N / A$
$N / A$
$N / A$
$N / A$
$N / A$
$N / A$
$N / A$
$N / A$
$N / A$
$N / A$
$N / A$
$N / A$
$N / A$

$03 / 09 / 90$

$03 / 09 / 90$

$03 / 09 / 90$

$03 / 09 / 90$

$03 / 09 / 90$

$03 / 09 / 90$

$03 / 09 / 90$

$03 / 09 / 90$

$03 / 09 / 90$

$03 / 09 / 90$

$03 / 09 / 90$

$03 / 09 / 90$

$03 / 09 / 90$

$03 / 09 / 90$

$03 / 09 / 90$

$03 / 09 / 90$

$03 / 09 / 90$

$03 / 09 / 90$

$03 / 09 / 90$

$03 / 09 / 90$

$03 / 09 / 90$

$03 / 09 / 90$
03/23/90: $03 / 23 / 90$ $03 / 23 / 9.0$ $03 / 23 / 90$ $03 / 23 / 90$ $03 / 23 / 90$ $03 / 23 / 90$ $03 / 23 / 90$ $03 / 23 / 90$ $03 / 23 / 90$ $03 / 23 / 90$ $03 / 23 / 90$ $03 / 23 / 90$ $03 / 23 / 90$ $03 / 23 / 90$ $03 / 23 / 90$ $03 / 23 / 90$ $03 / 23 / 90$ $03 / 23 / 90$ $03 / 23 / 90$ $03 / 23 / 90$ $03 / 23 / 90$ 
Roy E. Weston, Inc. - Lionville Laboratory

INORGANIC ANALYTICAL DATA PACKAGE POR WSRC-GRACE ROAD

DATE RECEIVED: $02 \not 23 / 90$

RFW LOT : $: 9002 L 606$

\begin{tabular}{|c|c|c|c|c|c|c|c|}
\hline CLIENT ID /ANALYSIS & RFW & $*$ & MTTX & PREP & COLLBCTION & EXTR/RRER & AMKAIYSIS \\
\hline VANADIUM LABORATORY & LC2 & BS & $\mathbf{s}$ & 9010226 & $N / A$ & $03 / 09 / 90$ & $03 / 23 / 90$ \\
\hline ZINC LABORATORY & IC2 & BS & $\mathbf{s}$ & $90 L 0226$ & $N / A$ & $03 / 09 / 90$ & $03 / 23 / 90$ \\
\hline SILVER, TOTAI & MBI & & $\mathbf{s}$ & 9010226 & $N / A$ & $03 / 09 / 90$ & $03 / 23 / 90$ \\
\hline BARIUM, TOTAI & MB1 & & $\mathbf{s}$ & 90L0226 & $N / A$ & $03 / 09 / 90$ & $03 / 23 / 90$ \\
\hline BERYIIIOM, TOTAI & MBI & & $\mathbf{s}$ & 90L0226 & $N / A$ & $03 / 09 / 90$ & $03 / 23 / 90$ \\
\hline CADMIUM, TOTAL & MBI & & $\mathbf{s}$ & 90ז0226 & $N / A$ & $03 / 09 / 90$ & $03 / 23 / 90$ \\
\hline COBALT, TOTAL & MBI & & $\mathbf{s}$ & $90 \check{0226}$ & $\pi / \pi$ & $03 / 09 / 90$ & $03 / 23 / 90$ \\
\hline CHROMIUM, TOTAL & MBI & & $\mathbf{s}$ & $90 \Sigma 0226$ & $N / A$ & $03 / 09 / 90$ & $03 / 23 / 90$ \\
\hline COPPER, TOTAL & MBI & & $\mathbf{s}$ & 9020226 & $N / A$ & $03 / 09 / 90$ & $03 / 23 / 90$ \\
\hline NICKEL, TOTAL & MBI & & $\mathbf{s}$ & 90工0226 & $N / A$ & $03 / 09 / 90$ & $03 / 23 / 90$ \\
\hline ANTIMONY, TOTAL & MBI & & $\mathbf{s}$ & 9020226 & $N / A$ & $03 / 09 / 90$ & $03 / 23 / 90$ \\
\hline TIN, TOTAL & MBI & & $\mathbf{s}$ & $90 \Sigma 0226$ & $N / A$ & $03 / 09 / 90$ & $03 / 23 / 90$ \\
\hline VANADIUM, TOTAL & MB1 & & $s$ & 90工0226 & $N / A$ & $03 / 09 / 90$ & $03 / 23 / 90$ \\
\hline ZINC, TOTAL & MBI & & $\mathbf{s}$ & 9020226 & $\mathrm{~N} / \mathrm{A}$ & $03 / 09 / 90$ & $03 / 23 / 90$ \\
\hline ARSENIC LABORATORY & ICI & BS & $\mathbf{s}$ & 9020225 & $\mathrm{~N} / \mathrm{A}$ & $03 / 09 / 90$ & $03 / 30 / 90$ \\
\hline LEAD LABORATORY & ICI & BS & $\mathbf{s}$ & 90工0225 & $N / A$ & $03 / 09 / 90$ & $04 / 05 / 90$ \\
\hline SELENIUM LABORATORY & LCI & BS & $\mathbf{s}$ & 9020225 & $N / A$ & $03 / 09 / 90$ & $04 / 01 / 90$ \\
\hline THALLIUM LABORATORY & LC1 & BS & $\mathbf{s}$ & 90L0225 & $N / A$ & $03 / 09 / 90$ & $04 / 04 / 90$ \\
\hline ARSENIC LABORATORY & LC2 & BS & s & 9020225 & $N / A$ & $03 / 09 / 90$ & $03 / 30 / 90$ \\
\hline LEAD LABORATORY & LC2 & BS & $\mathbf{s}$ & 9010225 & $\mathrm{~N} / \mathrm{A}$ & $03 / 09 / 90$ & 04/05/90 \\
\hline SELENIUM LABORATORY & LC2 & BS & $\mathbf{s}$ & $90 L 0225$ & $N / A$ & $03 / 09 / 90$ & $04 / 01 / 90$ \\
\hline THALIIUM LABORATORY & LC2 & BS & $\mathbf{s}$ & 9020225 & N/A & $03 / 09 / 90$ & $04 / 04 / 90$ \\
\hline ARSENIC, TOTAI & MBI & & $\mathbf{s}$ & 90L0225 & $N / A$ & $03 / 09 / 90$ & $03 / 30 / 90$ \\
\hline IEAD, TOTAL & MBI & & $\mathbf{s}$ & 90L0225 & $N / A$ & $03 / 09 / 90$ & $04 / 05 / 90$ \\
\hline SELENIUM, TOTAL & MBI & & $\mathbf{s}$ & $90 L 0225$ & $N / A$ & $03 / 09 / 90$ & $04 / 01 / 90$ \\
\hline THAILIUM, TOTAL & $\mathrm{MB1}$ & & $\mathbf{s}$ & 90L0225 & $N / A$ & $03 / 09 / 90$ & $04 / 04 / 90$ \\
\hline MERCURY IABORATORY & LCI & BS & W & $90 C 042 B$ & $N / A$ & $03 / 16 / 90$ & $03 / 19 / 90$ \\
\hline MERCURY LABORATORY & LC2 & BS & ต & $90 \mathrm{CO} 42 \mathrm{~B}$ & $\mathrm{~N} / \mathrm{A}$ & $03 / 16 / 90$ & $03 / 19 / 90$ \\
\hline MERCURY LABORATORY & LC3 & BS & $\boldsymbol{\varphi}$ & $90 \mathrm{CO} 42 \mathrm{~B}$ & $N / A$ & $03 / 16 / 90$ & $03 / 19 / 90$ \\
\hline MERCURY LABORATORY & IC4 & BS & พ & $90 \mathrm{C0} 42 \mathrm{~B}$ & $N / A$ & $03 / 16 / 90$ & $03 / 19 / 90$ \\
\hline MERCURY, TOTAI & $\mathrm{MBI}$ & & W & $90 \mathrm{C0} 42 \mathrm{~B}$ & $N / A$ & $03 / 16 / 90$ & $03 / 19 / 90$ \\
\hline MERCURY, TOTAL & MB2 & . & $\mathbf{W}$ & $90 C 042 B$ & $\mathrm{~N} / \mathrm{A}$ & $03 / 16 / 90$ & $03 / 19 / 90$ \\
\hline MERCURY, TOTAL & MB3 & & $\mathbf{W}$ & $90 \mathrm{CO} 42 \mathrm{~B}$ & $N / A$ & $03 / 16 / 90$ & $03 / 19 / 90$ \\
\hline MRRCURY, TOTAL & MB4 & & $\boldsymbol{W}$ & $90 \mathrm{C} 042 \mathrm{~B}$ & $N / A$ & $03 / 16 / 90$ & $03 / 19 / 90$ \\
\hline SILVER LABORATORY & ICI & BS & $\mathbf{w}$ & 9010219 & $\mathrm{~N} / \mathrm{A}$ & $03 / 08 / 90$ & $03 / 23 / 90$ \\
\hline BARIUM LABORATORY & ICI & BS & W & 9010219 & $B / A$ & $03 / 08 / 90$ & $03 / 23 / 90$ \\
\hline CADKIOK LABORATORY & ICI & BS & $\mathbf{W}$ & 9010219 & $\mathrm{N} / \mathrm{A}$ & $03 / 08 / 90$ & $03 / 23 / 90$ \\
\hline CEROMIUM LABORATORY & LCI & BS & W & 9010219 & N/A & $03 / 08 / 90$ & $03 / 23 / 90$ \\
\hline SILVER LABORATORY & LC2 & BS & W & 9020219 & $\mathrm{~N} / \mathrm{A}$ & $03 / 08 / 90$ & $03 / 23 / 90$ \\
\hline BARIOY LABORATORY & LC2 & BS & $\mathbf{H}$ & 9010229 & $N / A$ & $03 / 08 / 90$ & $03 / 23 / 90$ \\
\hline
\end{tabular}


Roy I. Weston, Inc. - Llonville Laboratory

INORGANIC ANALYTICAL DATA PACKAGE FOR

WSRC-GRACE ROAD

DATE RECEIVED: $02 \not 23 / 90$

RFF LOT :9002L606

\begin{tabular}{|c|c|c|c|c|c|c|c|}
\hline CLIENT ID / ANALYSIS & RFW & 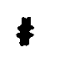 & HTXX & PREP & COITECTION & EXTR/PREP & ANALYSIS \\
\hline CADMIOM IAABORATORY & LC2 & BS & พ & $90 \check{02219}$ & $N / A$ & $03 / 08 / 90$ & $03 / 23 / 90$ \\
\hline CEROMIUM LABORATORY & LC2 & BS & พ & 9020219 & $N / A$ & $03 / 08 / 90$ & $03 / 23 / 90$ \\
\hline SILVER, TOTAL & MBI & & พ & 9020219 & $N / A$ & $03 / 08 / 90$ & $03 / 23 / 90$ \\
\hline BARIUM, TOTAY & KB1 & & ต & 9020219 & $N / \lambda$ & $03 / 08 / 90$ & $03 / 23 / 90$ \\
\hline CADMIUK, TOTAL & MB1 & & พ & 9020219 & $N / \lambda$ & $03 / 08 / 90$ & $03 / 23 / 90$ \\
\hline CHROMIUM, TOTAL & MBI & & $\boldsymbol{พ}$ & 9020219 & $N / A$ & $03 / 08 / 90$ & $03 / 23 / 90$ \\
\hline ARSENIC IABORATORY & LC1 & BS & พ & 9020218 & $N / A$ & $03 / 08 / 90$ & $03 / 30 / 90$ \\
\hline IEAD IABORATORY & LC1 & BS & พ & 9020218 & $N / A$ & $03 / 08 / 90$ & $04 / 05 / 90$ \\
\hline SELENIUM LABORATORY & ICI & BS & ต & 90L0218 & $N / A$ & $03 / 08 / 90$ & $04 / 02 / 90$ \\
\hline ARSENIC LABORATORY & $\mathrm{LC2}$ & BS & $\boldsymbol{H}$ & 9020218 & $N / A$ & $03 / 08 / 90$ & $03 / 30 / 90$ \\
\hline IEAD LABORATORY & LC2 & BS & $\mathbf{W}$ & $90 L 0218$ & $N / A$ & $03 / 08 / 90$ & $04 / 05 / 90$ \\
\hline SELENIUM LABORATORY & LC2 & BS & $\boldsymbol{H}$ & $90 L 0218$ & $N / A$ & $03 / 08 / 90$ & $04 / 02 / 90$ \\
\hline ARSENIC, TOTAL & MB1 & & พ & 9020218 & $\mathrm{~N} / \mathrm{A}$ & $03 / 08 / 90$ & $03 / 30 / 90$ \\
\hline I.EAD, TOTAL & MAB1 & & พ & 9010218 & $N / A$ & $03 / 08 / 90$ & $04 / 05 / 90$ \\
\hline SELENIUM， TOTAL & MBI & & พ & $90 \longleftarrow 0218$ & $N / A$ & $03 / 08 / 90$ & $04 / 02 / 90$ \\
\hline MERCURY LABORATORY & LCI & BS & พ & $90 \mathrm{CO} 42 \mathrm{~A}$ & $N / A$ & $03 / 16 / 90$ & $03 / 19 / 90$ \\
\hline MERCURY LABORATORY & LC2 & BS & พ & $90 C 042 A$ & $N / A$ & $03 / 16 / 90$ & $03 / 19 / 90$ \\
\hline MERCURY IABORATORY & IC3 & BS & พ & $90 C 042 A$ & $N / A$ & $03 / 16 / 90$ & $03 / 19 / 90$ \\
\hline MERCURY LABORATORY & LC4 & BS. & พ & $90 C 042 \pi$ & $\mathrm{N} / \mathrm{A}$ & $03 / 16 / 90$ & $03 / 19 / 90$ \\
\hline MERCURY, TOTAL & MBI & & ต & $90 \mathrm{CO} 42 \mathrm{~A}$ & $N / A$ & $03 / 16 / 90$ & $03 / 19 / 90$ \\
\hline MERCURY, TOTAI & $\mathbf{M B 2}$ & & $\boldsymbol{H}$ & $90 C 042 A$ & $N / A$ & $03 / 16 / 90$ & $03 / 19 / 90$ \\
\hline MERCURY, TOTAL & MB3 & & ต & $90 C 042 A$ & $N / A$ & $03 / 16 / 90$ & $03 / 19 / 90$ \\
\hline MERCURY, TOTAI & MB4 & & H & $90 C 042 A$ & $N / A$ & $03 / 16 / 90$ & $03 / 19 / 90$ \\
\hline
\end{tabular}




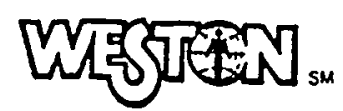

$$
\begin{aligned}
& \text { ROY F. WESTON, INC. } \\
& \therefore \quad \text { LiOnville Laboratory }
\end{aligned}
$$

CLIENT: WSRC-GRACE ROAD

RFF \#: 9002L607, GC/MS VOLATILE

SAMPLES RECEIVED: $02-23-90$

\section{NARRATIVE}

The set of samples consisted of one soil sample collected on
$02-21-90$.

The sample was analyzed according to criteria set forth in 03-05-90.

The following is a summary of the $Q C$ results accompanying these during their analysis:

1. Non-target compounds were not detected in
these samples.

2. All surrogate recoveries are within EPA QC
limits. 3. The blank contains Methylene chloride and
Acetone at levels less than $5 x$ the CRQL.

$\int \frac{\text { ZhhHed }}{\text { Jack R. Tuschall, Ph.D. }} \frac{4-9-9 u}{\text { Daboratory Manager }}$

Lionville Analytical Laboratory 


\section{1}

思

1 
Roy F. Weston, Inc. - Iionville Laboratory VOA ANALYTICAL DATA PACRAGE FOR

$\therefore$ $\therefore$ WSRC-GRACE ROAD

DATE RECEIVED: $02 / 23 / 90$

RFH LOT : $9002 L 607$.

CLIENT ID

RFW *

HTX PREP * COLTECTION EXTR/PREP

ANALYSIS

GRS-08-01

001

S 9OLVH036

$02 / 21 / 90$

N/A

$03 / 05 / 90$

LAB QC:

VBLR

MB1

S 90 LVพ036

N/A

$\ddot{i} / \mathbf{A}$

$03 / 05 / 90$ 


\section{DAT, QTATTIPISTS}

$U$ - Copoud was analyzed for but not detected. In associated

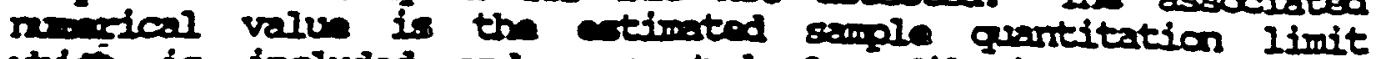

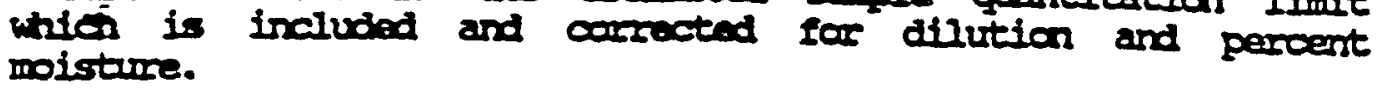

$J$ - Indicates an estimatad valu. Ints lag is used either when estimating a concutration for tartativaly identified

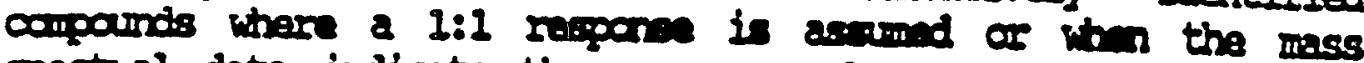
genctial data indiegte the pono of a coupord that mecte the idatification coltenis but the ravit is 10 thar the

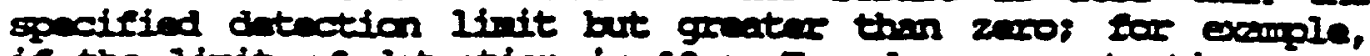
if the Iimit of datection is $10 \mathrm{vg} / \mathrm{L}$ and a cascontertion of 3 ugl is caloulated, it is suportend as 35 .

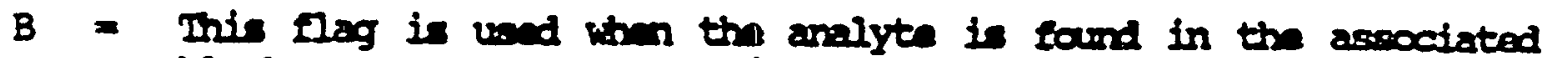
blank as wlil as in the ingle. indotes

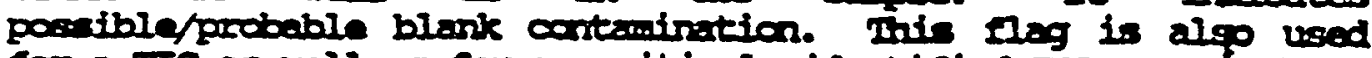

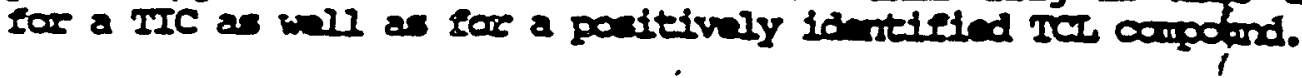

$E$ = Indicates that the conpound wa dencted bayold the calibeation zang and was abopunty analyzed at a difution.

$I=$ Intreserose.

$x$ - Additional qualifios und as sogind an splatond in the case narrativa.

$M$ - Rmult qualitativaly oafirend be not able to quantify.

\section{ABEREIDTTONS}

BS - Indicates blank spive in which ragunt grads water is spiked with the CP putix piting colution and carried through all

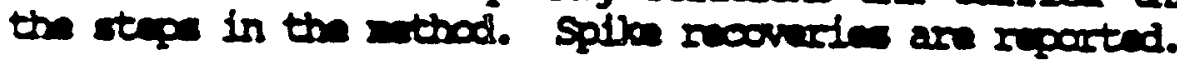

BSD = Indtater blan's pibe dupliate.

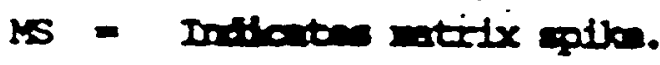

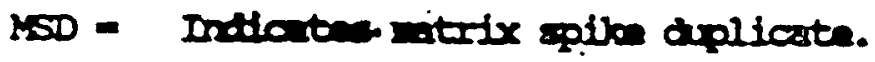

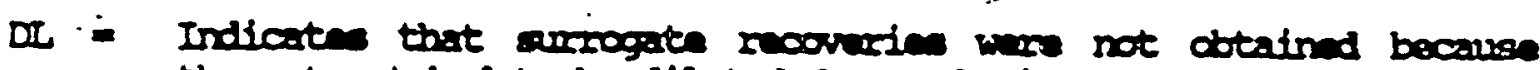
the extrect had to be diluted for analyats.

N - Not applicable.

DF - Dflution factor.

NR = Not requirud. 


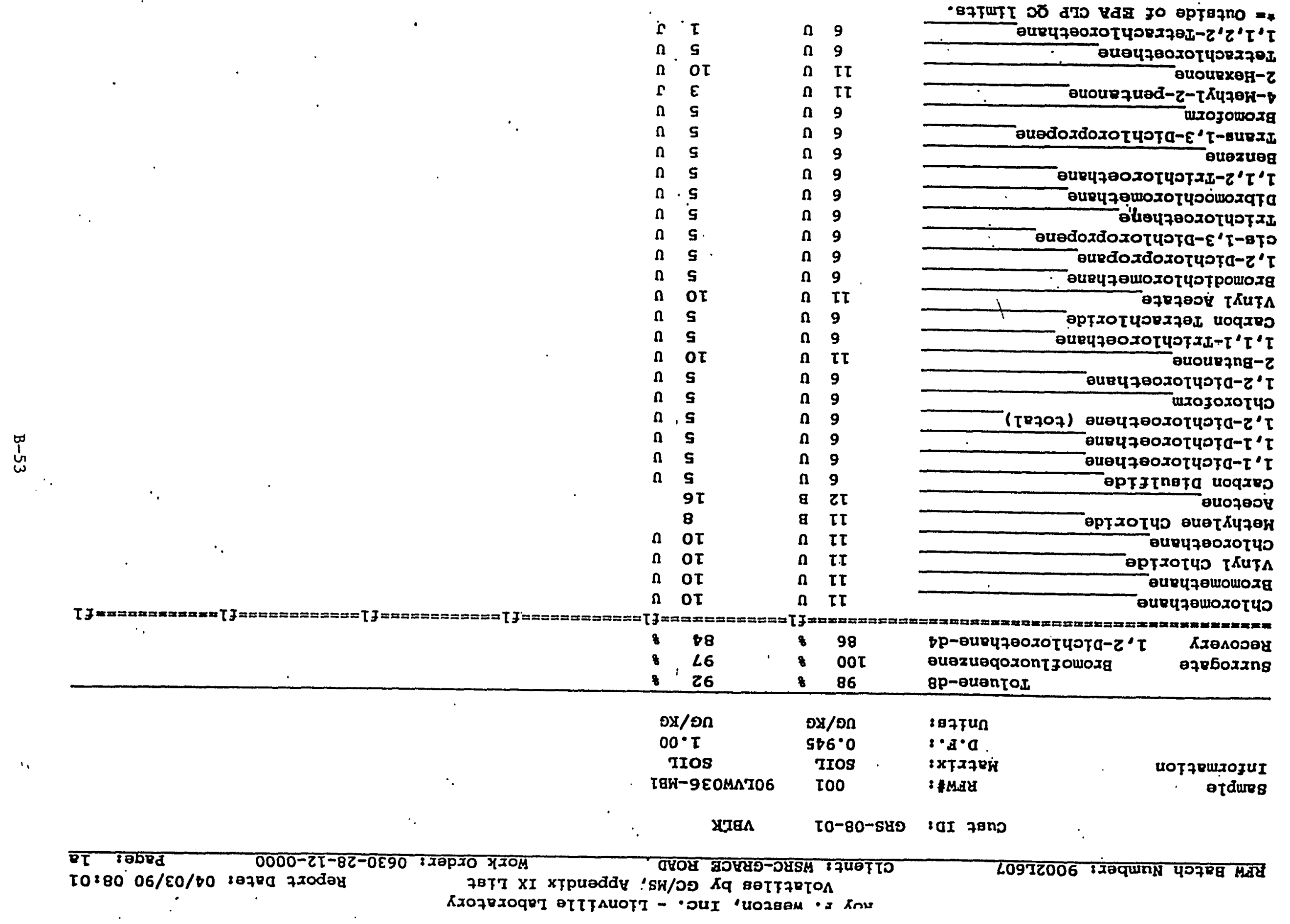




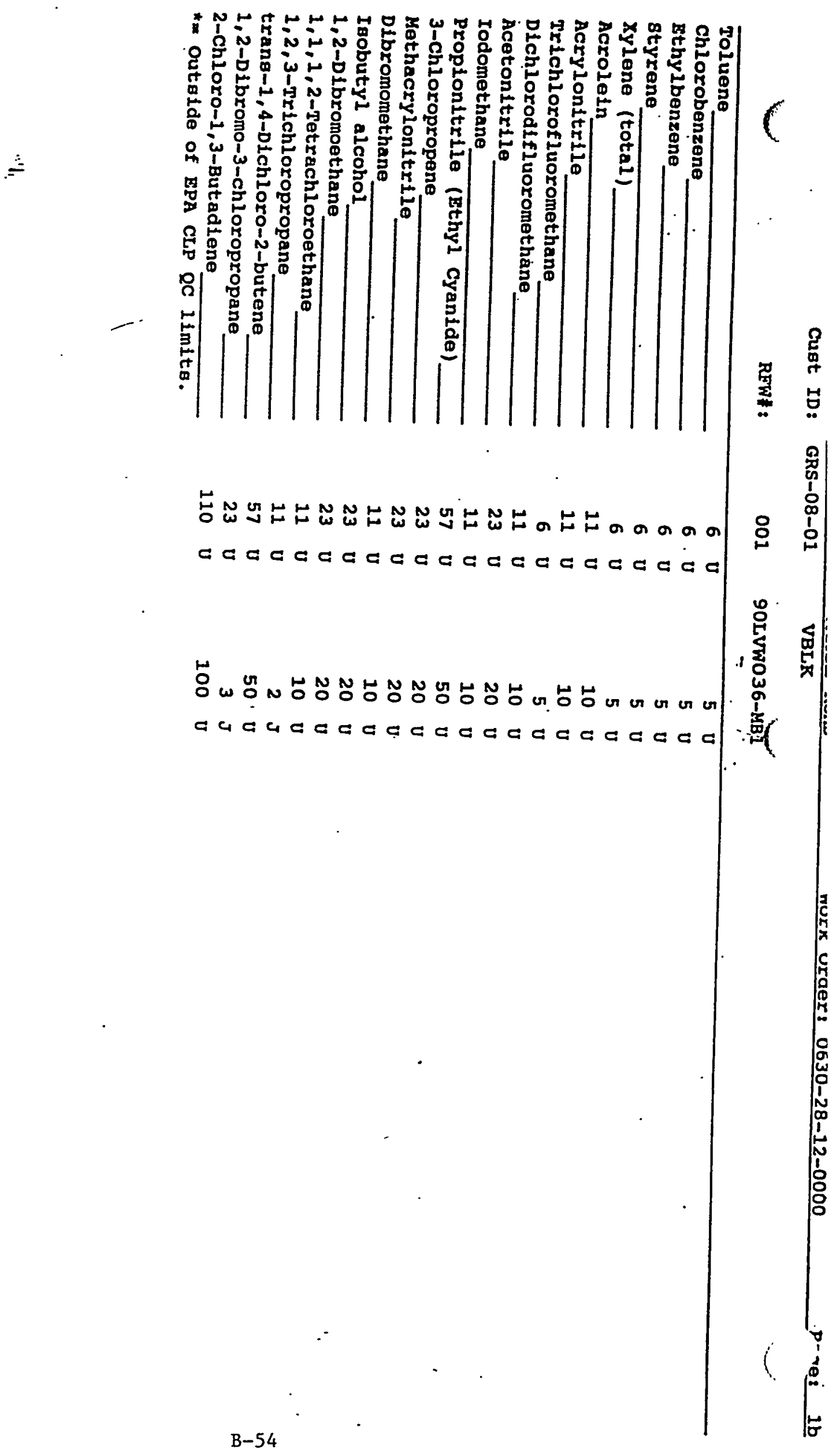




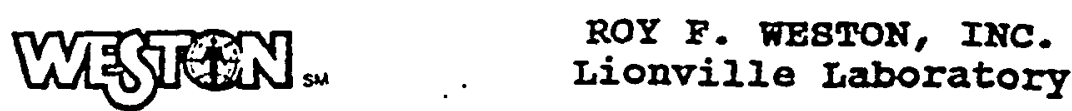

CLIENT: WSRC-GRACE ROAD

RFW \#: 9002L606, GC/MS SEMIVOIATIIE

SAMPLES RECEIVED: 02-23-90

พ.0. \# : $063 \overline{0}-28-12$

NARRATIVE

The set of samples consisted of twelve soil samples collected on 02-20, 21-90.

The samples were extracted on 02-28-90 and analyzed according to criteria set forth in SW 846 Method 8270 for TCL Semivolatile target compounds on 03-08,09,13-90.

The following is a summary of the QC results accompanying these sample results and a description of any problems encountered during their analysis:

1. Non-target compounds were detected in these samples.

2. Sample "GRS-10-03DL" required a five-fold dilution because it contained high levels of target compounds.

3. All. surrogate and blank spike recoveries are within EPA QC Iimits.

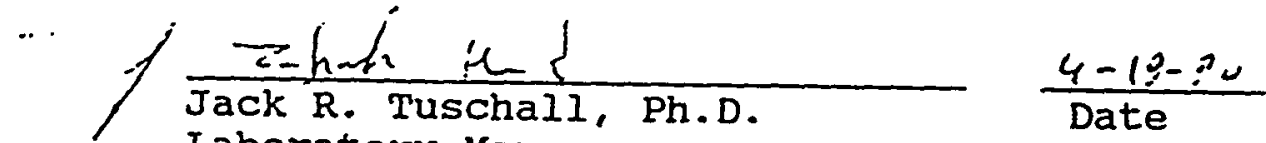

Laboratory Manager

Lionville Analytical Laboratory

$\operatorname{sma} / \mathrm{cn} / 02-606 \mathrm{~b} . \mathrm{cn}$
} 
落

N

7 
DATA CGATIFIERS

$U=$ campound was analyzed for but not detected. The associated remerical value is the estimated sample quantitation limit which is included and corrected for dilution and percent moisture.

. I = Indicates an estimated value. This flag is used either when estimating a concentration for tentatively identified compounds where a $1: 1$ respanse is assumed or when the mass spectral data indicate the presenoe of a compound that meets the identification criteria but the result is less than the specified detection limit but greater than zero; for example, if the limit of detection is $10 \mathrm{ug} / \mathrm{L}$ and a concentration of ? $\mathrm{ug} / \mathrm{L}$ is calculated, it is reported as $3 \mathrm{~J}$.

$B=$ This flag is used when the analyte is found in the associated blank as well as in the sample. It indicates possible/probable blank contamination. This flag is also used for a TIC as well as for a positively identified TCL campound.

$E=$ Indicates that the compound was detected beyond the calibration range and was subsequently analyzed at a dilution.

$I=$ Interference.

$A=$ Aidol condensation Product.

$x=$ Additional qualifiers used as required are explained in the case narrative.

NQ = Result qualitatively confirmed but not able to quantify.

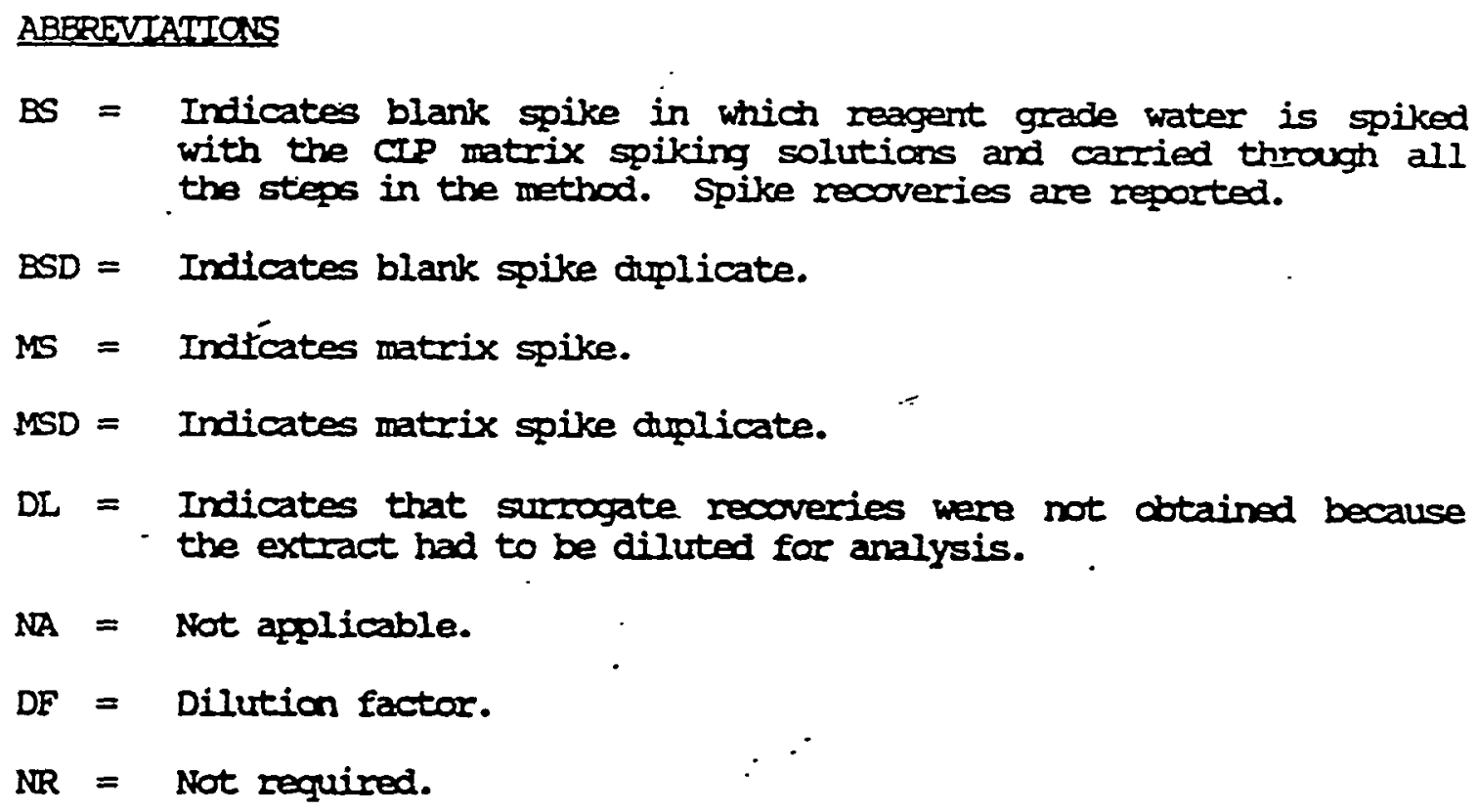
with the CIP matrix spiking solutions and carried through all the stieps in the method. Spike recoveries are reported.

BSD = Indicates blank spike ciplicate.

MS = Indtácates matrix spike.

MSD = Indicates matrix spike dplicate.

$D L=$ Indicates that surrogate recoveries were not obtained because the extract had to be diluted for analysis.

NA $=$ Not applicable.

$\mathrm{DF}=$ Dilution factor.

NR = Not required. 
\& $-$

Roy F. Weston, Inc. - Ilonville Laboratory

BNA ANALYTICAL DATA PACRAGE FOR WSRC-GRACE ROAD

DATE RECEIVED: $02 / 23 / 90$

REW LOT * :9002L606

CLIENT ID

GRS-10-01

GRS-10-02

GRS-10-03

GRS-10-03

GRS-09-01

GRS-09-02

GRS-09-03

GRS-07-01

GRS-07-01A

GRS-07-02

GRS $-07-03$

GRS $-08-02$

GRS $-08-03$

LAB QC:

SBLR
SBLR

SBLR
SBLR

\begin{tabular}{|c|c|c|c|}
\hline RFW : & & MTX & PREP \\
\hline 001 & & $\mathbf{s}$ & 9OLE0286 \\
\hline 002 & & $\mathbf{s}$ & 90LE0286 \\
\hline 003 & & $\mathbf{s}$ & 90LE0286 \\
\hline 003 & 01 & s & 9OLE0286 \\
\hline 004 & & $\mathbf{s}$ & 90I80286 \\
\hline 005 & & $\mathbf{s}$ & $90 \mathrm{LR} 0286$ \\
\hline 006 & & $\mathbf{s}$ & 90นR0286 \\
\hline 007 & & $\mathbf{s}$ & 90LE0286 \\
\hline 008 & & $\mathbf{s}$ & 90นE0286 \\
\hline 009 & & $\mathbf{s}$ & 90 LB0286 \\
\hline 010 & & $\mathbf{s}$ & 90LE0286 \\
\hline 012 & & $\mathbf{s}$ & 90180286 \\
\hline 013 & & $\mathbf{s}$ & 90 LE0286 \\
\hline
\end{tabular}

cos

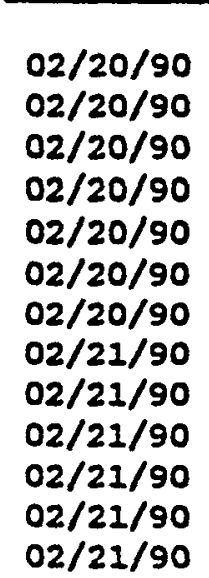

$02 / 28 / 90$

$02 / 28 / 90$

$02 / 28 / 90$

$02 / 28 / 90$

$02 / 28 / 90$

$02 / 28 / 90$

$02 / 28 / 90$

$02 / 28 / 90$

$02 / 28 / 90$

$02 / 28 / 90$

$02 / 28 / 90$

$02 / 28 / 90$

$02 / 28 / 90$

ANALYSIS

.

$03 / 08 / 90$

$03 / 08 / 90$

$03 / 08 / 90$

$03 / 09 / 90$

$03 / 09 / 90$

$03 / 09 / 90$

03/09/90

$03 / 09 / 90$

$03 / 13 / 90$

03/09/90

$03 / 09 / 90$

$03 / 09 / 90$

$03 / 09 / 90$
KB1

MB1 BS
S $90 L R 0286$

S 90 LE0286
$N / A$

N/A
$02 / 28 / 90$

$02 / 28 / 90$
$03 / 08 / 90$

$03 / 08 / 90$ 
ROY F. WESTOY, INC.

Lionville Laboratory

CLIENT: WSRC-GRACE ROAD

RFW : $\quad$ 9003L865, GC/MS

W.O. : 0630-28-12

VOLATILE

SAMPLES RECEIVED: 03-22-90

NARRATIVE

The set of samples consisted of three water samples and eleven soil samples collected on 03-19,20,21-90.

The samples were analyzed according to criteria set forth in CLP SOW 2/88 (rev. 5/89) for TCL Volatile target compounds on

The following is a summary of the $Q C$ results accompanying these during their analysis:

1. Non-target compounds were not detected in these samples.

2. All surrogate recoveries are within EPA QC
limits.

3. The blank contains common contaminants Methylene chloride and Acetone at levels less than $2 x$ the CRQL.

\footnotetext{
$\int \frac{\text { Chah Had }}{\text { Jack R. Tuschall, Ph.D. }} \frac{5-3-90}{\text { Daboratory Manager }}$

Lionville Analytical Laboratory

maj/kpmerno/03-865v.cn
} 
Roy F. Heston, Inc Lionville Laboratory

volatiles by GC/MS, HSL Ilat

Report Date: 04/27/90 11:39

RFW Batch Number: 9003L865 Client: WSRC-GRACE ROAD Fork Order: 0630-28-12-0000

Page: 1a

Cust ID\& GRS-02-01 GRS-04-01 GRS-04-01A GRS-03-01 GRS-05-01 GRS-06-01

Sample

Information

\begin{tabular}{|c|c|c|c|}
\hline RFW & 001 & 002 & 003 \\
\hline Matr $\{x\}$ & SOIL & SOIL & SOIL \\
\hline D.F. & 1.00 & 1.00 & 1.00 \\
\hline
\end{tabular}

004

SOIL

005

006

Units:

$\mathrm{ug} / \mathrm{Kg}$

$\mathrm{ug} / \mathrm{kg}$

$\mathrm{ug} / \mathrm{kg}$

0.996

Toluene-de

Surrogate

Bromofluorobenzene

Recovery

1,2-Dichloroethane-d4

$\begin{array}{l:l}89 & 1 \\ 96 & 113\end{array}$

Chloromethan

Bromomethane

vinyl chloride

Chloroethane

Methylene chloride

Acetone

Carbon Disulfide

1,1-Dichloroethene

1,1-Dichloroethane

I 19 1,2-Dichloroethene (total)

o chloroform

1,2-Dichloroethane

2-Butanone

1,1,1-Trichloroethane

Carbon Tetrachloride

vinyl, Acetate

Bromodichloromethane

1,2-Dichloropropane

cis-1, 3-Dichloropropene

Trichloroethene

Dibromochloromethane

1,1,2-Trichloroethane

Benzeno

Trans-1,3-D1chloropropene

Bromoform

4-Methyl-2-pentanone

2-Hexanone

Tetrachloroethene

1,1,2,2-Tetrachloroethane

*- Outbide of EPA CLP QC IImita.

\begin{tabular}{|c|c|c|}
\hline 12 & $\mathbf{U}$ & 11 \\
\hline 12 & $\mathbf{U}$ & 11 \\
\hline 12 & $\mathbf{U}$ & 11 \\
\hline 12 & $\mathbf{v}$ & 11 \\
\hline 11 & B & 17 \\
\hline 4 & JB & 12 \\
\hline 6 & $\mathbf{v}$ & 5 \\
\hline 6 & $\mathbf{v}$ & 5 \\
\hline 6 & $\mathbf{U}$ & 5 \\
\hline 6 & $\mathbf{U}$ & 5 \\
\hline 6 & $\mathbf{U}$ & 5 \\
\hline 6 & $\mathbf{U}$ & 5 \\
\hline 12 & $\mathbf{U}$ & 11 \\
\hline 6 & $\mathbf{U}$ & 5 \\
\hline 6 & $\mathbf{U}$ & 5 \\
\hline 12 & $\mathbf{U}$ & 11 \\
\hline 6 & $\mathbf{U}$ & 5 \\
\hline 6 & $\mathbf{U}$ & 5 \\
\hline 6 & $\mathbf{U}$ & 5 \\
\hline 6 & $\mathbf{U}$ & 5 \\
\hline 6 & $\mathbf{U}$ & 5 \\
\hline 6 & $\mathbf{U}$ & 5 \\
\hline 6 & $\mathbf{U}$ & 5 \\
\hline 6 & 0 & 5 \\
\hline 6 & $\mathbf{U}$ & 5 \\
\hline 12 & v & 11 \\
\hline 12 & $\mathbf{0}$ & 11 \\
\hline 6 & $\mathbf{v}$ & 5 \\
\hline 6 & $\mathbf{U}$ & 5 \\
\hline
\end{tabular}

$\begin{array}{rlrr}102 & 98 & 8 \\ 113 & 105 & 8 \\ 97 & 87 & 8\end{array}$

105
105

ug/Rg

$\mathrm{ug} / \mathrm{xg}$

89

$\begin{array}{rrrr}98 & 102 & \\ 102 & 107 & \\ 87 & 91 & \end{array}$

$===\approx=\mathrm{f}$

$===x=f$

$11 \mathrm{U}$

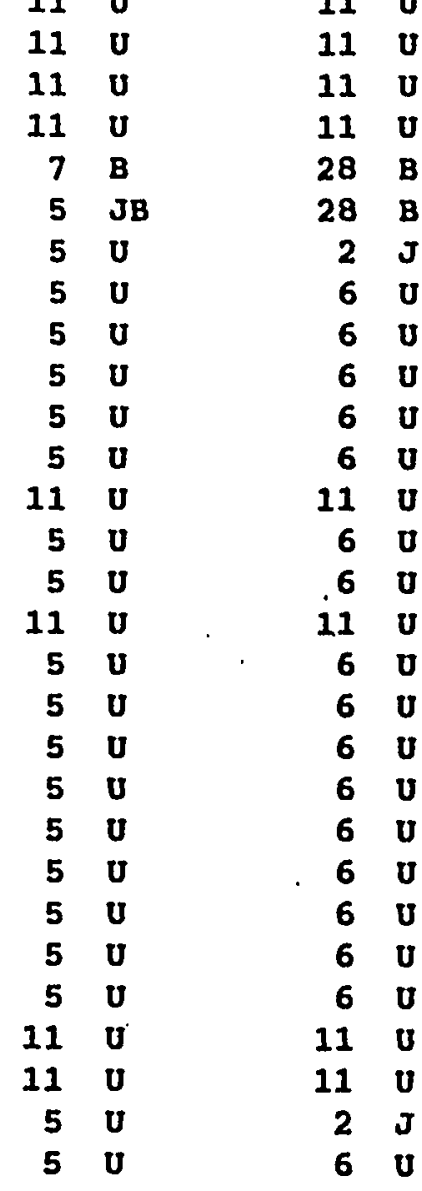

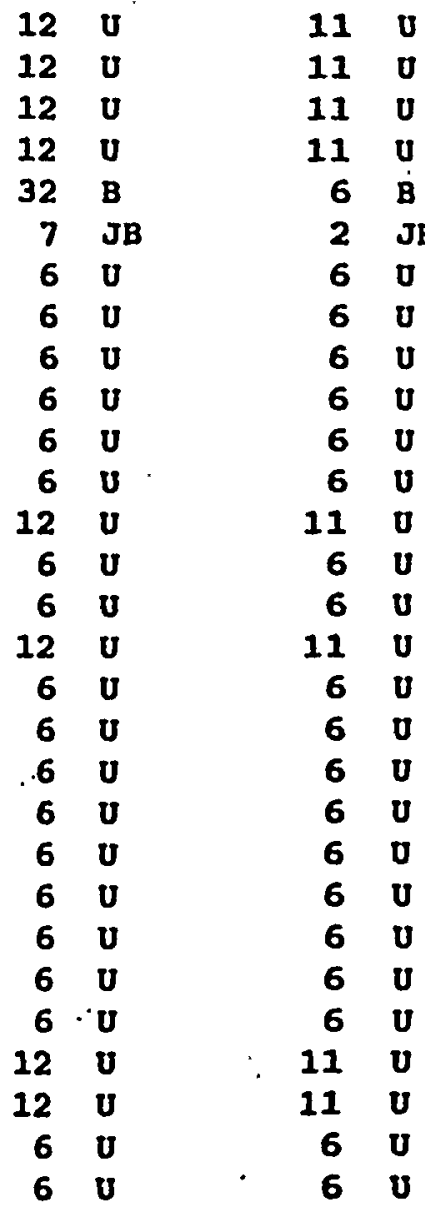


RFW Batch Number: 9003L865

Cllent: WSRC-GRACE ROAD

CuBt ID! GRS-02-01 GRS-04-01

Work Order: 0630-28-12-0000

GRS-04-01A GRS-03-01 GRS-05-01

Page: 2b

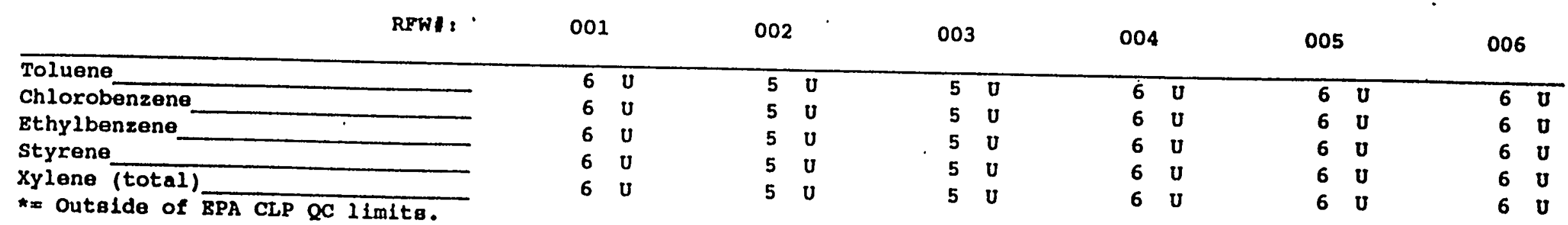


Roy F. Heston, Inc. Ionv1lle Laboratory

Volatiles by GC/MS, HSL Ilot

Report Date: 04/27/90 11:39

Cust ID: GRS-11-01 GRS-01-01 GRS-12-01 GRS-13-01 GRS-14-01

GRS-13-01E

Sample

Information .

RFW: 007

Matrix: SoIL

D.F.:

0.984

Units:

$\mathrm{ug} / \mathrm{kg}$

$\begin{array}{ll}\text { 008 } & 009 \\ \text { sOIL } & \text { sOII } \\ 0.984 & 0.984 \\ \mathrm{ug} / \mathrm{kg} & \mathrm{ug} / \mathrm{kg}\end{array}$

010

SOIL

0.994

011

sórt.

012

HATER

1.00

Toluene-d8

Surrogate

Bromofluorobenzene

Recovery

1,2-Dichloroethane-d4

103
105

108
97

Chloromethane

Bromomethane

vinyl Chloride

Chloroethane

Methylene Chloride

Acetone

Carbon Dlsulfido

1,1-Dichloroethene

to 1,1-Dichloroethane

of 1,2-D!chloroethene (total)

Chloroform

1,2-Dlchloroethane

2-Butanone

1,1,1-Trichloroethane

Carbon Tetrachloride

Vinyl Acetate

86 1 104 \& 113

91 2

90 ह

$\begin{array}{rrrr}110 & 103 & \\ 96 & 106 & \\ 90 & 77 & 7\end{array}$

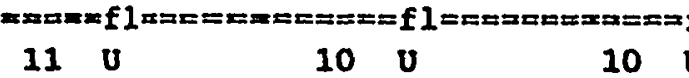

11.0

$11 \mathrm{v}$

$11 \mathrm{U}$

18 B

$6 \mathrm{JB}$

$5 \mathbf{U}$

$5 \mathbf{U}$

5 U

$5 \mathbf{v}$

5 U

5 U

110

5 U

50

$11 \mathrm{v}$

Bromodichloromethane

1,2-D1chloxopropane

c18-1, 3-Dichloropropene

Trichloroethene

Dlbromochloromethane

1, 1,2-Trichloroethane

Benzene

Trans-1, 3-DLchloropropene

Bromoform

4-Lethy 1-2-pentanone

2-Hexanone

Tetrachloroathene

1, 1,2,2-Tetrachloroethane

* Outbide of EPA CLP QC IImita.

$\begin{array}{rl}10 & \mathbf{U} \\ 10 & \mathbf{U} \\ 10 & \mathbf{U} \\ \mathbf{6} & \mathrm{B} \\ 9 & \mathrm{JB} \\ \mathbf{5} & \mathrm{U} \\ \mathbf{5} & \mathbf{U} \\ \mathbf{5} & \mathbf{U} \\ \mathbf{5} & \mathbf{U} \\ \mathbf{5} & \mathbf{U} \\ \mathbf{5} & \mathbf{U} \\ 10 & \mathbf{U} \\ \mathbf{5} & \mathbf{U} \\ \mathbf{5} & \mathbf{U} \\ 10 & \mathbf{U} \\ \mathbf{5} & \mathbf{U} \\ \mathbf{5} & \mathbf{U} \\ \mathbf{5} & \mathbf{U} \\ \mathbf{5} & \mathbf{U} \\ \mathbf{5} & \mathbf{U} \\ \mathbf{5} & \mathbf{U} \\ \mathbf{5} & \mathbf{U} \\ \mathbf{5} & \mathbf{U} \\ \mathbf{5} & \mathbf{U} \\ \mathbf{1 0} & \mathbf{U} \\ \mathbf{1 0} & \mathbf{U} \\ \mathbf{5} & \mathbf{U} \\ \mathbf{5} & \mathbf{U}\end{array}$

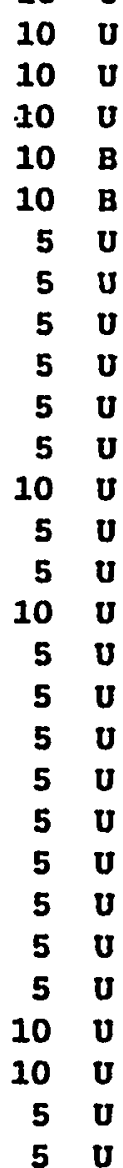

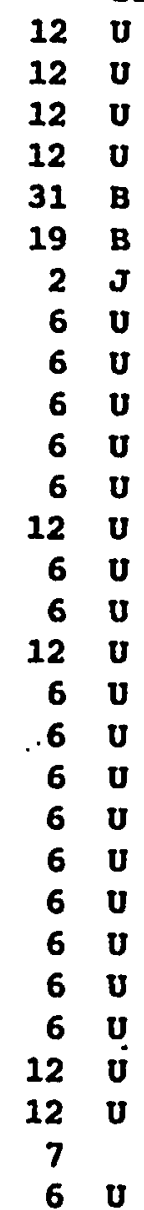

\begin{tabular}{|c|c|c|}
\hline 11 & $\mathbf{U}$ & 10 \\
\hline 11 & $\mathbf{U}$ & 10 \\
\hline 11 & $\boldsymbol{U}$ & 10 \\
\hline 11 & $\mathbf{U}$ & 10 \\
\hline 28 & $\mathbf{B}$ & 5 \\
\hline 7 & JB & 10 \\
\hline 3 & $\mathbf{J}$ & 5 \\
\hline 6 & $\mathbf{U}$ & 5 \\
\hline 6 & $\mathbf{U}$ & 5 \\
\hline 6 & $\boldsymbol{U}$ & 5 \\
\hline 6 & $\mathbf{U}$ &. \\
\hline 6 & $\mathbf{U}$ & 5 \\
\hline 11 & $\mathbf{U}$ & 10 \\
\hline 6 & $\mathbf{U}$ & 5 \\
\hline 6 & $\mathbf{U}$ & 5 \\
\hline 11 & $\mathbf{U}$ & 10 \\
\hline 6 & $\mathbf{U}$ & 5 \\
\hline 6 & $\mathbf{v}$ & 5 \\
\hline 6 & $\boldsymbol{U}$ & 5 \\
\hline 6 & $\mathbf{U}$ & 5 \\
\hline 6 & $\mathbf{U}$ & 5 \\
\hline 6 & $\mathbf{u}$ & 5 \\
\hline 6 & $\mathbf{U}$ & 5 \\
\hline 6 & $\boldsymbol{v}$ & 5 \\
\hline 6 & $\boldsymbol{U}$ & 5 \\
\hline 11 & $\boldsymbol{U}$ & $\therefore 10$ \\
\hline 11 & $\mathbf{U}^{\circ}$ & 10 \\
\hline $\begin{array}{l}6 \\
6\end{array}$ & & \\
\hline
\end{tabular}


RFW Batch Number: 9ं003L865 Cllent: WSRC-GRACE ROAD

Cust ID: GRS-11-01 GRS-01-01 GRS-12-01 GRS-13-01 GRS-13-01 Pager 2b

RFW: : 007 009

010

011

012

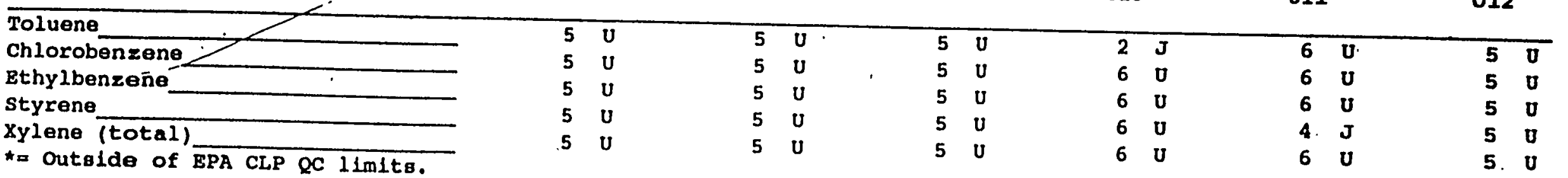


Roy F. Weston, Inc - Llonville Laboratory

Volatiles by GC/MS, HSL List

Report Dates 04/27/90.11:39

RFW Batch Number: 9003L865 Cllent: HSRC-GRACB ROAD Work Order: 0630-28-12-0000 Pages $3 a$

CuBt ID: GRS-14-01B GRS-14-01C VBLK VBLK $\because \quad$ VBLK

Samplẹ

Information

$\begin{array}{rr}\text { RFWI: } & 014 \\ \text { Matrix: } & \text { WATBR }\end{array}$

D.F.8 1.00

$u g / L$

015

90LVพO

Un1te:

1.00

SOIL

ug/I

$\mathrm{ug} / \mathrm{Kg}$

SOIL

1.00

WATER

Toluene-d8

surrogate Bromofluorobenzene

$\begin{array}{ll}94 & \\ 98 & \end{array}$

Recovery 1,2-Dichloroethane-d4

$\begin{array}{rl}101 & 8 \\ 107 & 8\end{array}$

Chloromethane

Bromomethane

Vinyl Chloride

Chloroethane

Methylene Chloride

Acetone

Carbon Dlaulfide

1, 1-Dlchloroethene

if 1,1-D1chloroethane

on 1,2-Dlchloxoethene (total.)

Chloroform

1,2-Dichloroethane

2-Butanone

1,1,1-Trichloroethane

Carbon Tetrachloride

Vinyl Acatate

Bromod lchloromethane

1,2-D1chloropropane

C18-1, 3-Dichloropropene

Irichloroethene

Dibromochloromethane

1,1,2-Trichloroethane

Benzene

Trans-1, 3-D1Chloropropene

Bromoform

4-Methy 1-2-pentanone

2-Hexanone

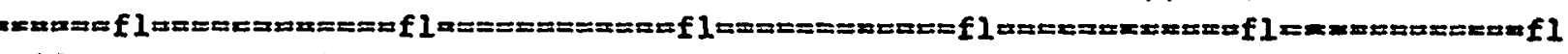

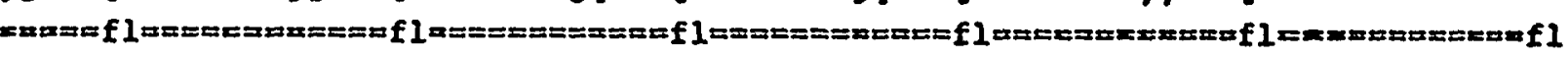

$\begin{array}{rrrrrr}92 & 96 & & 102 & \\ 93 & 100 & 8 & 109 \\ 84 & 94 & 8 & 77\end{array}$

Tetrachloroethene

$1,1,2,2-$ Tetrachloroethane

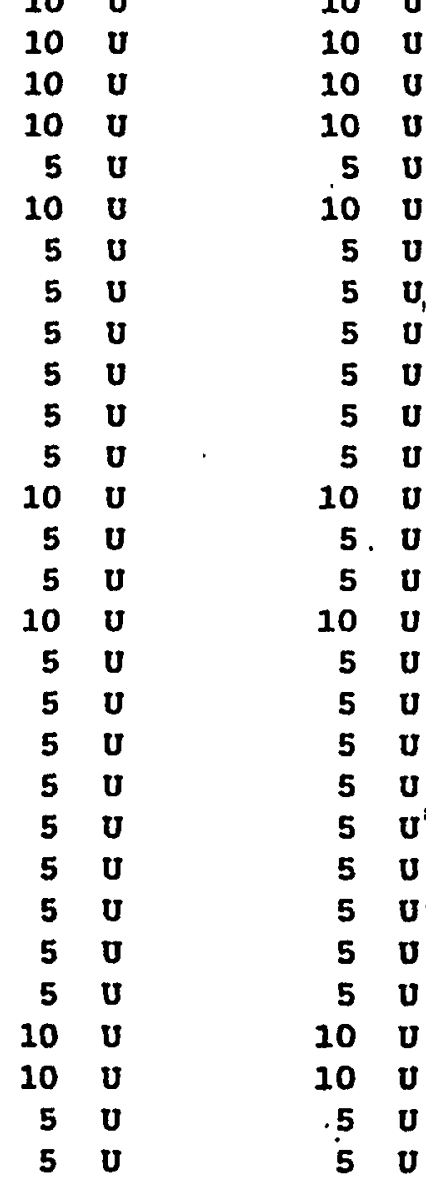

* Outalde of BPA CLP gC IImitB. 
VBLK

RFW: .

014

015

90LVW051-MB1

90LVพ054-MB1

90LVBO43-KB1

\section{Toluene}

Chlorobenzeno

Ethylbenzerie

Styrene

Xylene (total)

$\begin{array}{ll}5 & \mathrm{U} \\ 5 & \mathrm{U}\end{array}$

50

50

$\star=$ Outside of EPA CLP QC IImita.

5 U

$\begin{array}{ll}5 & \mathbf{U} \\ 5 & \mathrm{U} \\ 5 & \mathrm{U} \\ 5 & \mathrm{U} \\ 5 & \mathrm{U}\end{array}$

5 U

$5 \quad$ U

$\begin{array}{ll}5 & U \\ 5 & 0\end{array}$

50

$\begin{array}{ll}5 & \text { U } \\ 5 & \text { U }\end{array}$

$\begin{array}{lllll}\mathbf{U} & \mathbf{5} & \mathbf{U} & \mathbf{5} & \mathbf{U} \\ \mathbf{U} & 5 & \mathbf{U} & 5 & \mathbf{U} \\ \mathbf{U} & 5 & \mathbf{U} & 5 & \mathbf{U} \\ \mathbf{U} & 5 & \mathbf{U} & 5 & \mathbf{U} \\ \mathbf{U} & 5 & \mathbf{U} & 5 & \mathbf{U}\end{array}$


Roy F. Heston. Inc Lionville Laboratory

Semi.rolatiles ny GC/MS, HSL Llot

RFY! Batch Number: 9002L606 Cllent: iSRC-GRACE ROAD

Fork Order: 0630-28-12-0000

Cust ID: GRS-10-01

GRS-10-02

GRS-10-03

GRS-10-03

GRS-09-01

GRS-09-02

Sample

Information

$\begin{array}{rr}\text { RFWl: } & 001 \\ \text { Matrix: } & \text { SOIL } \\ \text { D.F.: } & 1.11\end{array}$

$002 \quad 003$

D.F.8

$\mathrm{ug} / \mathrm{kg}$

1.11

SOIL

$\mathrm{ug} / \mathrm{kg}$

1.11

$003 \mathrm{DL}$

SOIL

$\mathrm{ug} / \mathrm{kg} \quad \mathrm{ug} / \mathrm{Rg}$

$\begin{array}{cc}004 & 005 \\ \text { soIL } & \text { soIL } \\ 1.11 & 1.11 \\ \mathrm{ug} / \mathrm{Kg} & \mathrm{ug} / \mathrm{Kg}\end{array}$

\begin{tabular}{lr}
\hline Surrogate & N1trobenzene-d5 \\
Recovery & Pluorobiphenyl \\
& P-Terphenyl-d14 \\
Phenol-d5 \\
2-Fluorophenol \\
& $2,4,6-$ Tribromophenol
\end{tabular}

\section{Phenol}

bis(2-chloroethyl) ether

2-Chlorophenol

1,3-Dichlorobenzene

1,4-Dichlorobenzene

Benzyl alcohol

if 1,2-Dlchlorobenzene

73
84
95
83
82
72

$\begin{aligned} 78 & : \\ 86 & : \\ 108 & \\ 92 & \\ 98 & \end{aligned}$

\begin{tabular}{|c|c|c|c|}
\hline 70 & 8 & 52 & 8 \\
\hline 81 & 8 & 70 & 8 \\
\hline 93 & 8 & 68 & 8 \\
\hline 80 & 8 & 57 & 8 \\
\hline 82 & 8 & 69 & 8 \\
\hline 84 & 8 & 40 & 8 \\
\hline
\end{tabular}

81
88
98
90
92
92

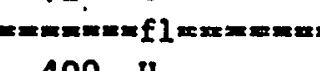

$450 \mathrm{U}$

$\begin{array}{ll}400 & U \\ 400 & U \\ 400 & U \\ 400 & U\end{array}$

$\begin{array}{llll}450 & U & 430 & U\end{array}$

$450 v$

$450 \mathrm{U}$

$450 \mathrm{U}$

$400 \mathrm{U}$

$400 \mathrm{v}$

$400 \mathrm{v}$

2-Methylphenol

b18 (2-Chlproisopropyl) ether

4-Mothylphonol

N-N1troso-D1-n-propylamine

Hexachloroethane

Nitrobenzene

Irophorone

2-Nitrophanol

2,4-Dimathylphenol

Bensolo acid.

bla (2-Chloroothoxy)methane

2,4-Dlchlorophenol

1,2,4-Trlchlorobenzene

Naphthalene

4-Chloroantilne

Bexachlorobutadiene

$400 \mathrm{U}$

$400 \mathrm{v}$

$400 \mathrm{U}$

$400 \mathrm{v}$

$400 \mathrm{v}$

$400 \mathrm{U}$

$400 \mathrm{U}$

$400 \mathrm{v}$

$400 \mathrm{U}$

$2000 \mathrm{v}$

$400 \mathrm{U}$

$400 \mathrm{U}$

$400 \mathrm{v}$

$400 \mathrm{O}$

$400 \mathrm{v}$

4000

4000

$400 \mathrm{U}$

$400 \mathrm{U}$

$450 \mathrm{U}$

$450 \mathrm{U}$,

$450 \mathrm{U}$

$450 \mathrm{U}$

$450 \mathrm{U}$

$450 \mathrm{U}$

$450 \mathrm{v}$

$450 \mathrm{U}$

$450 \mathrm{U}$

$450 \mathrm{U}$

$450 \mathrm{U}$

$450 \mathrm{U}$

2200 U

$450 \mathrm{U}$

$450 \mathrm{U}$

$450 \mathrm{U}$

$450 \mathrm{U}$

$450^{\circ} \mathrm{U}$

$450 \mathrm{U}$

$450 \mathrm{U}$

$450^{\circ} \mathrm{U}$

2-Kothylnaphthalene

Hexachlorocyclopentadlene

$450^{\circ} . \mathrm{U}$

$\begin{array}{rl}430 & U \\ 430 & U \\ 430 & U \\ 430 & U \\ 430 & U \\ 430 & U \\ 430 & U \\ 430 & U \\ 430 & U \\ 430 & U \\ 430 & U \\ 430 & U \\ 430 & U \\ 430 & U \\ 430 & U \\ 430 & U \\ 2200 & U \\ 430 & U \\ 430 & U \\ 430 & U \\ 430 & U \\ 430 & U \\ 430 & U \\ 430 & U \\ 430 & U \\ 430 & U\end{array}$

NA

NA

NA

NA

NA
NA
NA

NA
NA
NA

NA

NA

NA.

8

53
65
80
67
64.
64.

" = Outelde of BPA CLP QC IIm1ta.

. 
RFW:

$$
001
$$

002

003

$003 \mathrm{DL}$

004

005

2,4,6-Trichlorophenol

$2,4,5-T \times 1$ chlorophenol

2-Chloronaphthalene

2-Nitroaniline

Dimethylphthalate

Acenaphthylene

2, 6-Dinitrotoluene

3-N1troan111ne

Acenaphthene

2,4-Dinitrophenol

4-N1trophenol.

Dibenzofuran

2,4-Dinitrotoluene

Dlethylphthalate

$450 \mathrm{U}$

$\begin{array}{rl}400 & U \\ 2000 & U \\ 400 & U \\ 2000 & U\end{array}$

$\begin{array}{rr}2200 & U \\ 450 & U \\ 2200 & U\end{array}$

$400 \mathrm{U} \quad 450$

$400 \mathrm{U}$

$400 \mathrm{U}$

$2000 \mathrm{U}$

$400 \mathrm{U}$

$2000 \mathrm{v}$

$2000 \mathrm{U}$

$400 \mathrm{U}$

$400 \mathrm{U}$

$400 \mathrm{U}$

4-Chlorophenyl-phenylether

Fluorene

4-N1troaniline

4, 6-DIn1tro-2-mathylphenol

N-N1trogodiphenylamine (I)

4-Bromopheny 1-phenylether

Hexachlorobenzene

Pentachlorophenol

Phenanthrene

Anthracene

Di-n-Buty 1 phthalate

Fluoranthene

Pyrene

Buty lbenzy lphthalate

$3,3^{\circ}-\mathrm{D} 1 \mathrm{chl}$ orobenzldine

Benzo (a) anthracene

Chryeene

blo(2-Bthylhexyl) phthalate

D1-n-octyl phthalate

Benzo (b) fluoranthene

Benro (k) fluoranthene

Benzo (a) pyrene

Indeno $(1,2,3-c d)$ pyrene

DLbenzo $(a, h)$ anthracene

$400 \mathrm{U}$

$400 \mathrm{U}$

$2000 \mathrm{U}$

$2000 \mathrm{U}$

$400 \mathrm{~V}$

$400 \mathrm{U}$

$400 \mathrm{~V}$

$2000 \mathrm{v}$

$400 \mathrm{~V}$

$400 \mathrm{U}$

$400 \mathrm{U}$

$400 \mathrm{U}$

$400 \mathrm{U}$

$400 \mathrm{U}$

$800 \mathrm{U}$

$400 \mathrm{U}$

$400 \mathrm{U}$

1300

$400 \mathrm{U}$

$400 \mathrm{U}$

$400 \mathrm{U}$

$400 \mathrm{U}$

$400 \mathrm{U}$

$400 \mathrm{U}$

$400 \mathrm{U}$

$450 \mathrm{U}$

$450 \mathrm{U}$

$2200 \mathrm{U}$

$450 \mathrm{U}$

$2200 \quad U$

$2200 \mathrm{U}$

$450 \mathrm{U}$

$450 \mathrm{U}$

$450 \mathrm{U}$

$450 \mathrm{v}$

$450 \mathrm{U}$

$2200 \mathrm{U}$

$2200 \mathrm{U}$

$450 \mathrm{U}$

$450 \mathrm{U}$

$450 \mathrm{U}$

2200 U

$450 \mathrm{U}$

$450 \mathrm{U}$

$450 \mathrm{U}$

$450 \mathrm{U}$

$450 \mathrm{~V}$

$450 \mathrm{~V}$

$900 \mathrm{U}$

$450 \mathrm{U}$

$430 \mathrm{U}$

6900

$450 \mathrm{U}$

$450 \mathrm{U}$

$450 \mathrm{U}$

450 U

$\begin{array}{rr}430 & U \\ 2200 & U\end{array}$

NA
NA
NA
NA
NA
NA
NA

$\begin{array}{rlrr}390 & U & 440 & 0 \\ 2000 & U & 2200 & 0\end{array}$

$430 \mathrm{U}$

$2200 \mathrm{U}$

$\begin{array}{ll}430 & U \\ 430 & U\end{array}$

$430 \mathrm{U}$

$2200 \mathrm{U}$

430
$2200 \quad U$

$2200 \mathrm{U}$

$430 \mathrm{U}$

$430 \mathrm{U}$

$430 \mathrm{U}$

$430 \mathrm{~V}$

$430 \mathrm{~V}$

$2200 \mathrm{v}$

2200 U

$430 \mathrm{U}$

$430 \mathrm{v}$

$430 \mathrm{U}$

$2200 v$

$430 \mathrm{U}$

$430 \mathrm{U}$

$430 \mathrm{U}$

$430 \mathrm{U}$

$430 \mathrm{U}$

$430 \mathrm{U}$

$860 \mathrm{U}$

$430 \mathrm{U}$

$430 \quad \mathrm{~V}$

$430 \mathrm{U}$

$430 \mathrm{U}$

$430 \mathrm{U}$

$450.0 \quad 430 \mathrm{U}$

$450 \mathrm{U}$

$430 \mathrm{U}$

$390 \quad 0$

$2000 \mathrm{U}$

390 U

390 U

$390 \mathrm{U}$

$2000 \mathrm{~V}$

$390 \mathrm{U}$

$2000 \mathrm{v}$

$2000 \mathrm{~V}$

$390 \mathrm{U}$

$390 \quad 0$

390 . 0

$390 \mathrm{v}$

$390 \mathrm{v}$

$2000 \mathrm{v}$

$2000 \mathrm{U}$

$390 \mathrm{U}$

$390 \mathrm{U}$

390 v

$2000 t$

$390 \quad \mathbf{U}$

390 U

$390 U$

390 U

390 U

$390 \quad v$

790 U

390 U

$390 \mathrm{U}$

1000

5900

390

$390 \mathrm{v}$

390

390 U

390

390 u

390

$\begin{array}{rr}440 & 0 \\ 2200 & U\end{array}$

$440 \mathrm{~V}$

$440 \mathrm{~V}$

$440 \mathrm{~J}$

$2200 \mathrm{~V}$

$440 \mathrm{O}$

22000

22000

$440 \mathrm{~V}$

$440 \mathrm{~V}$

$440 \mathrm{~V}$

$440 \mathrm{~V}$

$440 \mathrm{~V}$

22000

$2200 \mathrm{v}$

$440 \mathrm{~V}$

$440 \mathrm{~V}$

$440 \mathrm{~V}$

$2200 \mathrm{U}$

$440 \mathrm{U}$

$440 \mathrm{~V}$

$440 \mathrm{~V}$

$440 \mathrm{U}$

$440 \mathrm{~V}$

$440 \mathrm{~V}$

$870 \mathrm{U}$

$440 \mathrm{~V}$

$440 \mathrm{U}$

4800

$440 \mathrm{~V}$

$440 \mathrm{U}$

$440 \mathrm{U}$

$440 \cdot U$

$440 \mathrm{U}$

$440 \mathrm{~V}$

(1) - Cannot be separated from DLphenylamine. *: Outside of EPA CLP gC limits.

NA

$440 \mathrm{v}$ 
Roy F. Weston, In Lionville Laboratory Semivolatiles \& GC/MS, HSL List Cl lent: FSRC-GRACE ROAD Work Order: 0630-28-12-0000

Report Date: 04/03/yo $11: 17$ REH Barch Number: 9002L606

\begin{abstract}
Cust IDI GRS-09-03
\end{abstract}

\begin{abstract}
GRS-07-01
\end{abstract}

\begin{abstract}
GRS-07-01A
\end{abstract}

007
soll
1.11

$\mathrm{ug} / \mathrm{kg}$

$\begin{array}{rc}\text { RFWl: } & 006 \\ \text { Matrix: } & \text { soIL } \\ \text { D.F.: } & 1.11 \\ \text { Units: } & \text { ug/Kg }\end{array}$

8 amplo

Information

Surrogate
Recovery

Surrogate
Recovery

008
soIL
1.11
ug/kg

54
66
79
67
67
61

$\begin{array}{ll}60 & 8 \\ 68 & 8 \\ 76 & 8 \\ 69 & 8 \\ 73 & 8 \\ 64 & 8 \\ 440=\end{array}$

GRS-07-02

009

SoIL

1.11
$\mathrm{ug} / \mathrm{kg}$
GRS-07-03

010

1.11

ug/Kg

GRS-08-02

$\begin{array}{llll}60 & 8 & 49 & 8 \\ 69 & 8 & 60 & 8 \\ 61 & 8 & 71 & 8 \\ 66 & 8 & 59 & 8 \\ 46 & 8 & 55 & 8 \\ 47 & 8 & 48 & 8 \\ 430 & 0\end{array}$

$\begin{array}{lll}65 & & \\ 76 & 79 \\ 94 & 89 \\ 82 & 81\end{array}$

\section{Phenol}

ble (2-Chloroethyl) ether

2-Chlorophenol

1, 3-DLChlorobenzene

1,4-Dichlorobenzene

Benxyl alcohol

if 1,2-D Lchlorobenzene

2-Methylphenol

bis (2-Chlorolsopropyl)ether 4-Mathylphenol

N-N1ttoso-D1-n-propylamine Hexachloroethane

N1trobenzene

Inophorone

2-N1trophenol

2,4-Dimathylphenol

Benrole acld!

b1. (2-Chloroethoxy) methane

2, 4-Dichlorophenol

1,2,4-Txlchiorobenzene

Naphthaleno

4-Chloroaniline

Bexachlorobutadiene

4-Chloro-3-methylphenol

2-Kothylnaphthalene

- Hexachlorocyclopentadlene

* = Outbide of IPA CLP QC $\overline{1 \operatorname{lm} 1 t 8 .}$

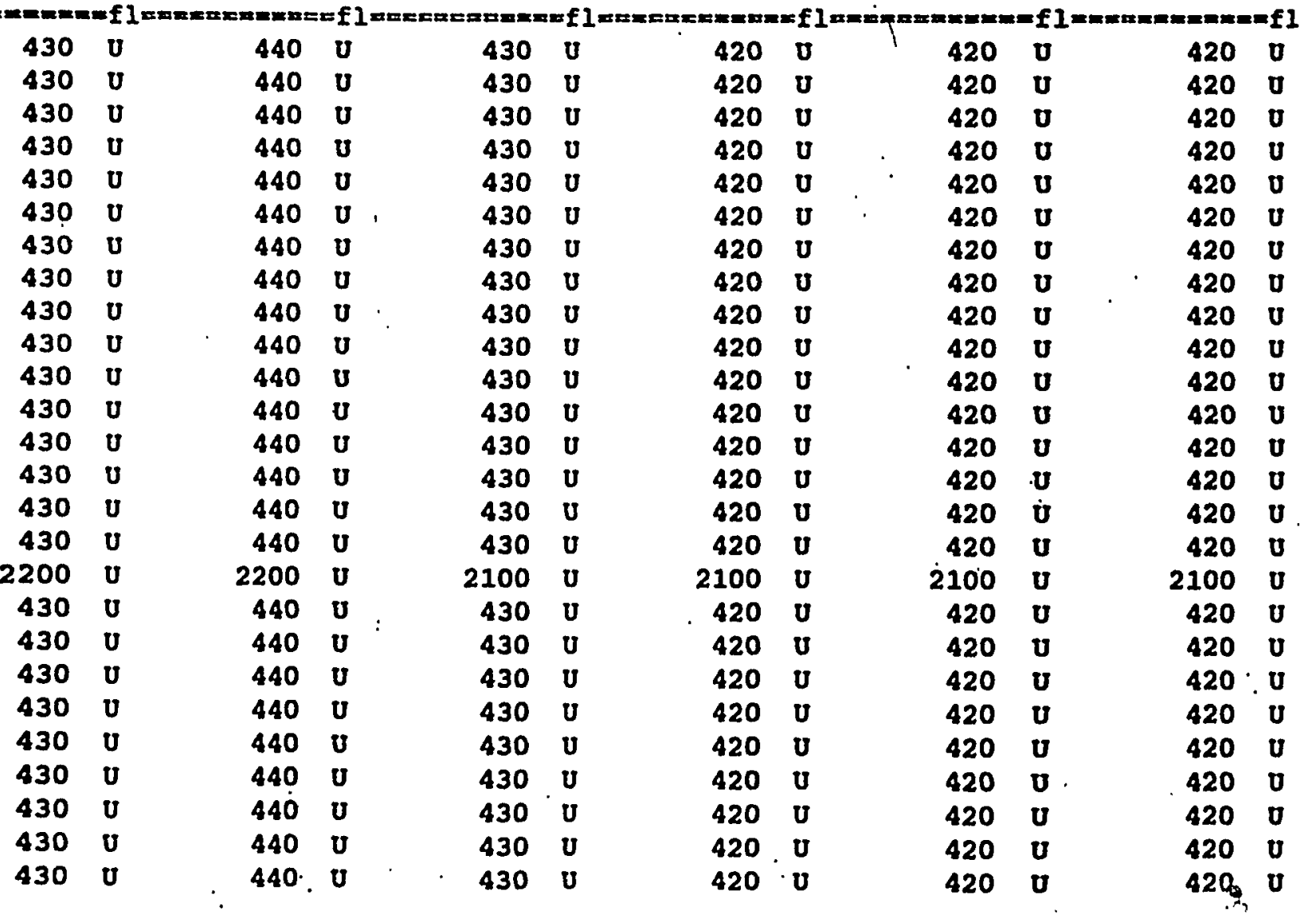

012

1.11

$\mathrm{ug} / \mathrm{kg}$

65

77

94

80

81

0

$20 \mathrm{v}$

$20 \mathrm{~V}$

$420 \mathrm{U}$

$20 \mathrm{U}$

20

$20 \mathrm{U}$

$20 \mathrm{U}$

$420 \mathrm{U}$

$100 \mathrm{~V}$

$420 \mathrm{U}$

0

0

20,0 
Rew Batch Number: 9002L606

Cllent: WSRC-GRACE ROAD

Hork Order: $0630-28-12-0000$

Page: 2b Cust ID: GRS-09-03 GRS-07-01 GRS-07-01A GRS-07-02

GRS-07-03

GRS-08-02

RPW:

006

008

009

010

012

2,4,6-Trlchlorophenol

2,4,5-Trichlorophenol

2-Chloronaphthalene

2-N1troaniline

Dimethylphthalate

Acenaphthylene

2,6-Dinitrotoluene

3-N1troan12 Lne

Acenaphthene

2, 4-Dinitrophenol

4-N1trophenol

Dlbenzofuran

2, 4-DLnitrotoluene

Diethylphthalate

4-Chlorophenyl-pheny lether.

Fluorene

4-k1troan11Lno

4,6-Dinitro-2-methylphenol

N-Nitronodiphenylamine (1)

if 4-Bromophenyl-phenylether

I Hexachlorobenzene

Pentachlorophenol

Phenanthrene

Anthracene

D1-n-Butylphthalate

Fluoranthene

Pyrene

Buty lbenzy Iphthalate

$3,3^{\circ}-$ Dichlorobenzldine

Benzo (a) dnthrpceno

Chrymene

b1o (2-sthylhexyl)phthalate

D1-n-octyl phthalate

Benzo (b) Eluoranthene

Benzo (k) Eluoranthene

Benro (a) pyrene

Indeno (1,2, 3-cd) pyrene

Dibenzo $(a, h)$ anthracene

$\begin{array}{rrrr}430 & U & 440 & U \\ 2200 & U & 2200 & U \\ 430 & U & 440 & U\end{array}$

$430 \mathrm{U}$

\begin{tabular}{|c|c|c|c|c|c|c|}
\hline 420 & $\mathbf{U}$ & & 420 & $\mathbf{0}$ & 420 & $\mathbf{0}$ \\
\hline 2100 & $\mathbf{v}$ & & 2100 & $\mathbf{v}$ & 2100 & $\mathbf{0}$ \\
\hline 420 & $\mathbf{v}$ & . & 420 & $\mathbf{0}$ & 420 & $\mathbf{0}$ \\
\hline 2100 & $\mathbf{0}$ & & 2100 & $\mathbf{0}$ & 2100 & 0 \\
\hline 420 & $\mathbf{v}$ & & 420 & $\mathbf{u}$ & 420 & 0 \\
\hline 420 & $\mathbf{v}$ & & 420 & $\mathbf{v}$ & 420 & $\mathbf{0}$ \\
\hline 420 & $\mathbf{u}$ & & 420 & $\mathbf{0}$ & 420 & $\mathbf{0}$ \\
\hline 2100 & $\mathbf{0}$ & 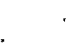 & 2100 & $\mathbf{0}$ & 2100 & $\mathbf{v}$ \\
\hline 420 & $\mathbf{U}$ & . & 420 & $\mathbf{u}$ & 420 & $\mathbf{0}$ \\
\hline 2100 & U & & 2100 & $\mathbf{v}$ & 2100 & $\mathbf{0}$ \\
\hline 2100 & $\mathbf{U}$ & & 2100 & $\boldsymbol{U}$ & 2100 & $\mathbf{U}$ \\
\hline 420 & $\mathbf{U}$ & & 420 & $\boldsymbol{U}$ & 420 & $\mathbf{u}$ \\
\hline 420 & $\mathbf{U}$ & $!$ & 420 & $\boldsymbol{v}$ & 420 & $\mathbf{U}$ \\
\hline 420 & $\mathbf{U}$ & & 420 & $\mathbf{v}$ & 420 & $\mathbf{U}$ \\
\hline 420 & $\mathbf{v}$ & & $420^{\circ}$ & $\mathbf{v}$ & 420 & $\mathbf{v}$ \\
\hline 420 & $\mathbf{v}$ & & 420 & $\mathbf{v}$ & 420 & $\mathbf{0}$ \\
\hline 2100 & $\mathbf{u}$ & & 2100 & $\boldsymbol{U}$ & 2100 & $\mathbf{v}$ \\
\hline 2100 & $\mathbf{v}$ & & 2100 & $\mathbf{v}$ & 2100 & $\mathbf{v}$ \\
\hline 420 & $\mathbf{U}$ & & 420 & $\mathbf{U}$ & 420 & $\mathbf{U}$ \\
\hline 420 & $\mathbf{v}$ & & 420 & $\boldsymbol{U}$ & 420 & $\mathbf{u}$ \\
\hline 420 & $\mathbf{v}$ & & 420 & $\boldsymbol{U}$ & 420 & $\mathbf{u}$ \\
\hline 2100 & $\mathbf{U}$ & & 2100 & $\mathbf{U}$ & 2100 & $\mathbf{U}$ \\
\hline 420 & $\boldsymbol{v}$ & & 420 & $\boldsymbol{U}$ & 420 & $\mathbf{0}$ \\
\hline 420 & $\mathbf{0}$ & & 420 & $\mathbf{U}$ & 420 & $\mathbf{0}$ \\
\hline 420 & $\mathbf{v}$ & & 420 & $\mathbf{v}$ & 420 & $\boldsymbol{0}$ \\
\hline 420 & $\mathbf{U}$ & & 420 & $\boldsymbol{v}$ & 420 & $\mathbf{0}$ \\
\hline 420 & $\mathbf{U}$ & $\therefore$ & 420 & $\boldsymbol{v}$ & 420 & $\mathbf{u}$ \\
\hline 420 & $\mathbf{U}$ & & 420 & $\mathbf{u}$ & 420 & $\mathbf{v}$ \\
\hline 830 & $\mathbf{U}$ & & 840 & $\mathbf{U}$ & 830 & $\mathbf{U}$ \\
\hline 420 & $\boldsymbol{U}$ & & 420 & $\boldsymbol{U}$ & 420 & $\mathbf{U}$ \\
\hline 420 & $\boldsymbol{U}$ & & 420 & $\boldsymbol{U}$ & 420 & $\mathbf{U}$ \\
\hline 88 & $\mathbf{J}$ & & 4200 & & 6800 & \\
\hline 420 & $\mathbf{U}$ & & 420 & $\boldsymbol{U}$ & 420 & $\boldsymbol{0}$ \\
\hline 420 & $\mathbf{v}$ & & 420 & $\mathbf{v}$ & 420 & $\mathbf{U}$ \\
\hline 420 & $\mathbf{0}$ & & 420 & 0 & 420 & $\mathbf{U}$ \\
\hline 420 & $\mathbf{U}$ & & 420 & u & 420 & $\boldsymbol{v}$ \\
\hline 420 & $\mathbf{U}$ & $\cdot$ & 420 & $\mathbf{v}$ & 420 & $\mathbf{v}$ \\
\hline 420 & $\mathbf{U}$ & . & 420 & $\mathbf{U}$ & 420 & $\mathbf{v}$ \\
\hline 420 & $\mathbf{U}$ & & 420 &. $\mathbf{0}$ & 420 & $\mathbf{v}$ \\
\hline
\end{tabular}

$2100 \mathrm{U}$

$2200 \mathrm{U}$

$430 \mathrm{U}$

$430 \mathrm{U}$

$430 \mathrm{~V}$

$2200 \mathrm{~V}$

$430 \mathrm{~V}$

$2200 \mathrm{v}$

$2200 v$

$430 \mathrm{U}$

$430 \mathrm{v}$

$430 \mathrm{~V}$

$430 \mathrm{U}$

$430 \mathrm{U}$

22000

$2200 \mathrm{U}$

$430 \mathrm{U}$

$430 \mathrm{~V}$

$430 \mathrm{U}$

$2200 \mathrm{U}$

$430 \mathrm{~V}$

$430 \mathrm{~V}$

$430 \mathrm{U}$

$430 \mathrm{~V}$

$430 \mathrm{U}$

$430 \mathrm{~V}$

$870 \mathrm{U}$

$430 \mathrm{U}$

$430 \mathrm{U}$

4700

$430 \mathrm{~V}$

$430 \mathrm{~V}$

$430 \mathrm{~V}$

$430 \mathrm{~V}$

$2200 \mathrm{U}$

$440 \mathrm{~V}$

$2100 \mathrm{v}$

$440 \mathrm{U}$

$440 \mathrm{U}$

$2200 \mathrm{v}$

$440 \mathrm{U}$

$2200 \mathrm{~V}$

$2200 \mathrm{U}$

$440 v$

$440 \mathrm{U}$

$440 \mathrm{U}$

$440 \mathrm{~V}$

$440 \mathrm{U}$

2200 U

2200 U

$440 \mathrm{U}$,

$440 \mathrm{~V}$

$440 \mathrm{v}$

$2200 v$

$440 \mathrm{U}$

4400

$440 \mathrm{U}$

$440 \mathrm{U}$

$440 \mathrm{U}$

$440 \mathrm{U}$

$880 \mathrm{U}$

$440 \mathrm{U}$

$440 \mathrm{U}$

1400

$440 \mathrm{~V}$

$440 \mathrm{U}$

$440 \mathrm{U}$

$440 \mathrm{U}$

$430 \mathrm{~V}$

4300

$440 \mathrm{U}$

$440 \mathrm{U}$

$430 \mathrm{U}$

$430 \mathrm{U}$

$430 \mathrm{U}$

$2100 \mathrm{U}$

$430 \mathrm{U}$

$2100 \mathrm{U}$

$2100 \mathrm{U}$

$430 U$

$430 \mathrm{~V}$

$430 \mathrm{~V}$

$430 v$

$430 \mathrm{U}$

$2100 \mathrm{U}$

$2100 \mathrm{v}$

2100
$430 \quad U$

$430 \mathrm{~V}$

4300

21000

$430 \mathrm{U}$
4300

430
430

$430 \mathrm{U}$

$430 \mathrm{U}$

$430 \mathrm{U}$

$430 \mathrm{U}$

$860 \mathrm{U}$

$430 \mathrm{U}$

430

1900

$430 \mathrm{U}$

$430 \mathrm{U}$

$430 \mathrm{U}$

$430 \mathrm{~V}$

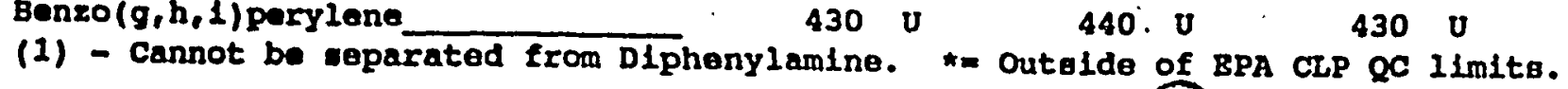

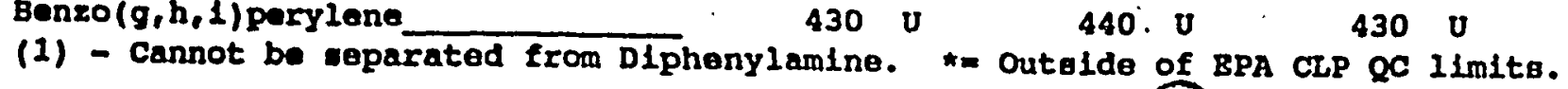

$430 \mathrm{U}$

$430 \mathrm{U}$ 


Cust ID: GR8-08-03 SBLK ' SBLK BS

\begin{tabular}{|c|c|c|c|c|}
\hline $\begin{array}{l}\text { 8amplo } \\
\text { Information }\end{array}$ & $\begin{array}{r}\text { RFW: } \\
\text { Matrix: } \\
\text { D.F. : } \\
\text { Unlta! }\end{array}$ & $\begin{array}{c}013 \\
\text { soIL } \\
1.11 \\
\text { ug/ Kg }\end{array}$ & $\begin{array}{c}\text { 90LB0286-MB1 } \\
\text { SOIL } \\
1.11 \\
\mathrm{ug} / \mathrm{Kg}\end{array}$ & $\begin{array}{c}\text { 90LE0286-MBI } \\
\text { SOIL } \\
1.11 \\
\text { ug } / \mathrm{Kg}\end{array}$ \\
\hline
\end{tabular}

\begin{tabular}{lr}
\hline 8urrogate & Nitrobenzene-d5 \\
Recovery & -Fluoroblphenyl \\
p-Terphenyl-d14 & Phenol-d5 \\
& 2-Fluorophenol \\
& $2,4,6-$ Tribromophenol
\end{tabular}

$\begin{array}{rlll}69 & 53 & \\ 85 & 61 & \\ 102 & 78 \\ 80 & 70 \\ 69 & 66 \\ 47 & 77\end{array}$

$\begin{array}{ll}49 & 8 \\ 61 & \\ 71 & 8 \\ 50 & 8 \\ 46 & 8\end{array}$

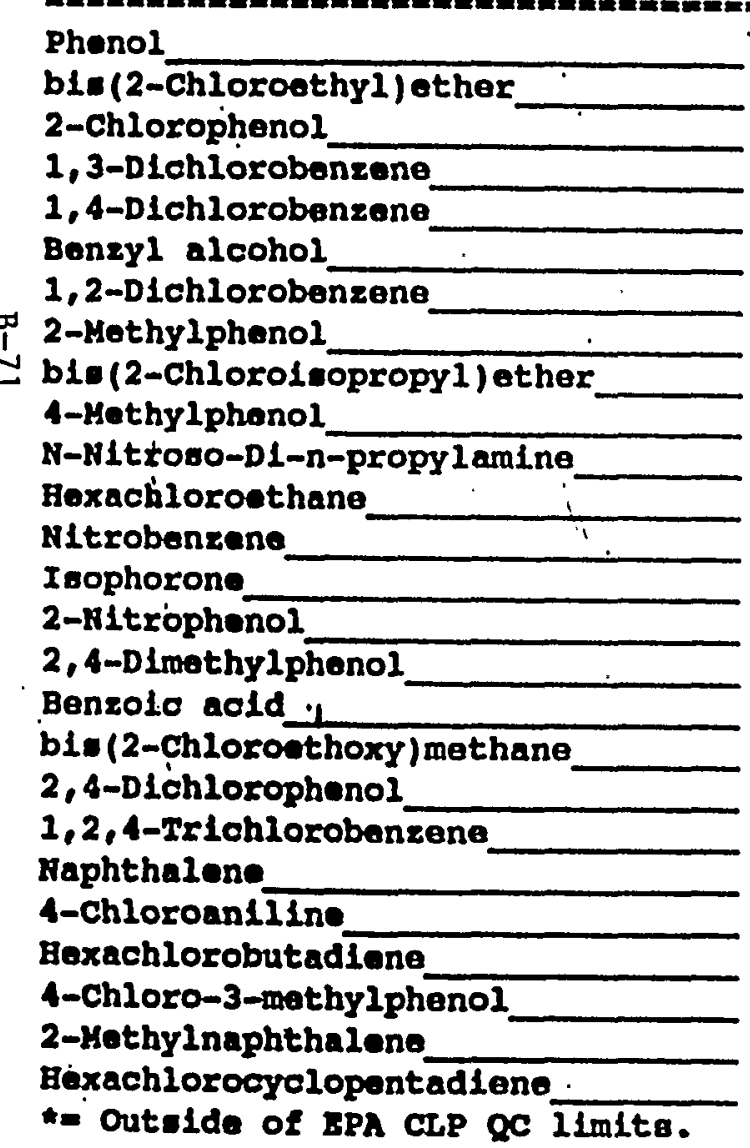

$420 \mathrm{~V}$

$\begin{array}{ll}420 & 0 \\ 420 & 0\end{array}$

$420 \mathrm{U}$

$420 \mathrm{v}$

$420 \mathrm{U}$

$420 \mathrm{U}$

$420 \mathrm{U}$

$420 \mathrm{U}$

$420 \mathrm{U}$

$420 \mathrm{v}$

420 U

$420 \mathrm{v}$

$420 \mathrm{U}$

$420 \mathrm{U}$

420 U

$420 \mathrm{U}$

21000

$420 \mathrm{U}$

$420 \mathrm{U}$

$420 \mathrm{U}$

$420 \mathrm{U}$

$420 \mathrm{U}$

$420 \mathrm{~V}$

$420 \mathrm{U}$

$420 \mathrm{U}$

420 U

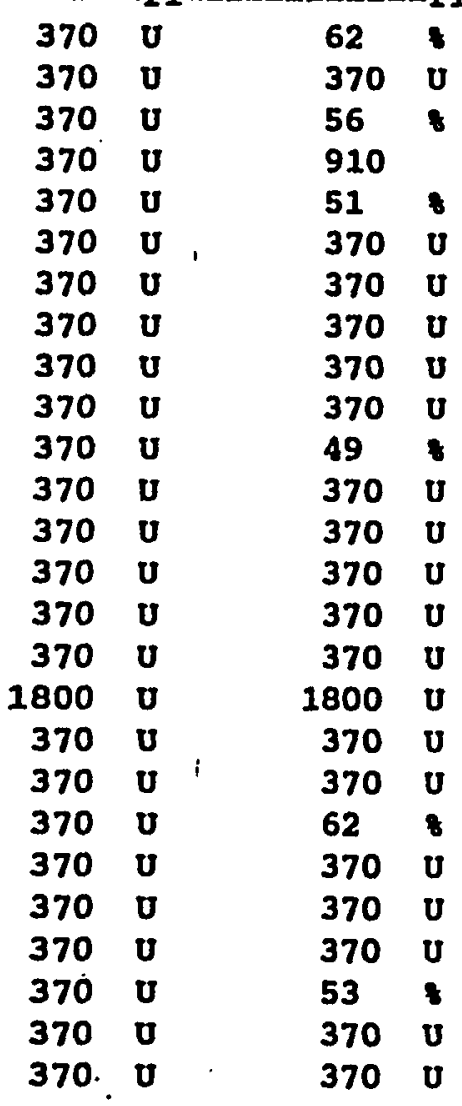


90LE0286-MBI 9OLE0286-MBI

2,4,6-Trichlorophenol 2,4,5-Trichlorophenol 2-Chloronaphthalene

2-Nitroendilne

Dimethylphthalate

Acenaphthylene

2,6-Din1trotoluene

3-Nitroaniline

Acenaphtheno

2, 4-Dinitrophenol

4-N1trophenol

Dibenzofuran

2,4-Dinitrotoluene

Dlethylphthalate

$420 \mathrm{U}$

Fluorene

\section{4-Nitroaniline}

4,6-Dinitro-2-methylphenol

N-N1trosodiphenylamine (1)

t 4-Bromophenyl-pheny lether

Hexachlorobenzene

Pentachlorophenol

Phenanthrene

Anthrdcene

D1-n-Butylphthalate

Fluoranthene

Pyrene

Butylbensylphthalate

3, 3'-D1Chlorobenzidine

Benzo (a) anthrqcene

Chrygene

b1s(2-sthylhaxyl) phthalate

D1-n-octyl phthalate

Benzo (b) fluoranthene

Benro (k) Eluoranthene

Benzo (a) pyrene

Indeno $(1,2,3-c \bar{d})$ pyrene

DIbenzo $(a, h)$ anthracene $-$

\begin{tabular}{|c|c|c|c|c|c|}
\hline & & $\begin{array}{c}90150286- \\
-\end{array}$ & & & \\
\hline 420 & U & 370 & $\mathbf{U}$ & 370 & \\
\hline 2100 & $U$ & 1800 & $\mathbf{U}$ & 1800 & \\
\hline 420 & 0 & 370 & $\mathbf{U}$ & 370 & \\
\hline 2100 & $\mathbf{U}$ & 1800 & $\mathbf{U}$ & 1800 & \\
\hline 420 & U & 370 & $\mathbf{U}$ & 370 & \\
\hline 420 & $\mathbf{u}$ & 370 & $\mathbf{U}$ & 370 & \\
\hline 420 & $\mathbf{U}$ & 370 & $\mathbf{U}$ & 370 & \\
\hline 2100 & $\mathbf{U}$ & 1800 & $\mathbf{U}$ & 1800 & \\
\hline 420 & $\mathbf{U}$ & 370 & $\mathbf{U}$ & 72 & 8 \\
\hline 2100 & $\mathbf{u}$ & 1800 & $\mathbf{U}$ & 1800 & \\
\hline 2100 & $\mathbf{U}$ & 1800 & $\mathbf{U}$ & 89 & 8 \\
\hline 420 & $\mathbf{v}$ & 370 & $\mathbf{v}$ & 370 & $\mathbf{U}$ \\
\hline 420 & $\mathbf{U}$ & 370 & $\boldsymbol{U}$ & 58 & \\
\hline 420 & $\mathbf{U}$ & 370 & $\mathbf{0}$ & 370 & $\mathbf{U}$ \\
\hline 420 & $\mathbf{U}$ & 370 & $\mathbf{U}$ & 370 & 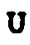 \\
\hline 420 & $\boldsymbol{U}$ & 370 & $\boldsymbol{U}$ & 370 & $\boldsymbol{U}$ \\
\hline 2100 & U & 1800 & $\mathbf{U}$ & 1800 & 0 \\
\hline 2100 & $\mathbf{U}$ & 1800 & $\boldsymbol{U}$ & 1800 & $\mathbf{U}$ \\
\hline 420 & $\mathbf{U}$ & 370 & $\mathbf{U}$ & 370 & $\mathbf{U}$ \\
\hline 420 & $\mathbf{U}$ & 370 & $\mathbf{U}$ & 370 & $\mathbf{U}$ \\
\hline 420 & $\mathbf{U}$ & 370 & $\mathbf{U}$ & 370 & U \\
\hline 2100 & $\mathbf{U}$ & 1800 & $\mathbf{U}$ & 68 & 8 \\
\hline 420 & $\mathbf{U}$ & 370 & $\mathbf{u}$ & 370 & $\mathbf{U}$ \\
\hline 420 & U & 370 & $\mathbf{U}$ & 370 & U \\
\hline 420 & $\mathbf{U}$ & 370 & $\mathbf{u}$ & 370 & v \\
\hline 420 & $\mathbf{U}$ & 370 & $\mathbf{u}$ & 370 & $\mathbf{v}$ \\
\hline 420 & $\mathbf{U}$ & 370 & $\mathbf{v}$ & 92 & 8 \\
\hline 420 & $\mathbf{U}$ & 370 & $\mathbf{U}$ & 370 & $\mathbf{U}$ \\
\hline 830 & $\mathbf{u}$ & 740 & $\mathbf{U}$ & 740 & $\mathbf{U}$ \\
\hline 420 & U & 370 & $\mathbf{U}$ & 370 & $\mathbf{U}$ \\
\hline 420 & U & 370 & $\mathbf{U}$ & 370 & $\mathbf{v}$ \\
\hline 3700 & & 370 & $\mathbf{U}$ & 370 & U \\
\hline 420 & $\mathbf{U}$ & 370 & $\mathbf{U}$ & 370 & $\mathbf{U}$ \\
\hline 420 & $\mathbf{v}$ & 370 & $\mathbf{U}$ & 370 & $\mathbf{u}$ \\
\hline 420 & $\boldsymbol{U}$ & 370 & $\mathbf{U}$ & 370 & $\dot{U}$ \\
\hline 420 & $\mathbf{U}$ & 370 & $\mathbf{U}$ & 370 & U \\
\hline 420 & $\mathbf{U}$ & 370 & $\mathbf{U}$ & 370 & $\boldsymbol{U}$ \\
\hline 420 & $\mathbf{U}$ & $370^{\circ}$ & $\mathbf{U}$ & 370 & U \\
\hline 420 & $\mathbf{v}$ & 370 . & $\mathbf{U}$ & 370 & $\mathbf{U}$ \\
\hline
\end{tabular}

(1) - Cannot be separated from Dlphenylamine. *= outside of EPA CLP QC IImits. 


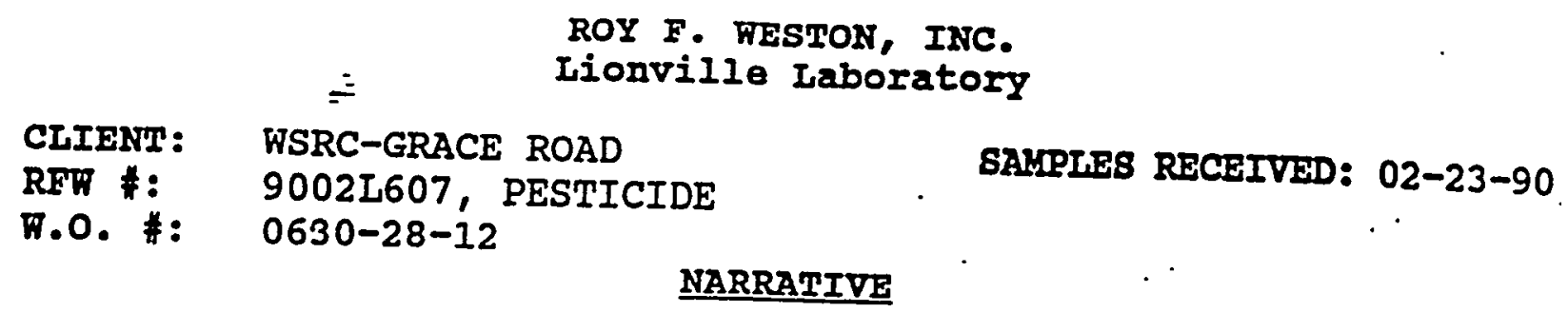

The set of samples consisted of one soil sample collected on
$02-21-90$.

The sample was extracted on $02-28-90$ and analyzed according to criteria set forth in the Contract Lab Program for Appendix IX
Pesticide and PCB target compounds on 04-08-90. The following is a summary of the $Q C$ results accompanying these sample results and a description of any problems encountered
during their analysis:

1. The following $D B C$ surrogate recoveries are outside EPA QC Limits:

SAMPLE

GRS-08-01

GRS-08-01 REP

GRS-08-01 MS

\section{\%RECOVERY}

203

219

195

The presence of interferes with recovery.
bis(2-Ethylhexyl) phthalate the quantitation of DBC

UPPER LIMIT

2. The blank spike recovery for aldrin (32\%) was below the EPA QC limit of $34 \%$.

150

150

150 
$\stackrel{\infty}{\stackrel{1}{\unlhd}}$

\section{i}


WESTECN.

GLOSBARY OF PEST/PCB DATA

DATX OUXÉIFIERS

0 - Indicates that the compounds was analyzed for but not detected. The minimum detection limit for the sample (not the method detection limit) is reported with the U (e.g., 10U).

$J$ - Indicates an estimated value. This flag is used in cases where a target analyte is detected at a level less than the lower quantification level. If the limit of quantification is $10 \mathrm{ug} / \mathrm{L}$ and a concentration of 3 $\mathrm{ug} / \mathrm{L}$ is calculated, it is reported as $3 \mathrm{~J}$.

B - This flag is used when the analyte is found in the associated blank as well as in the samplè. It indicates possible/probable blank contamination. This flag is also used for a TIC as well as for a positively identified TCL compound.

E - Indicates that the compound was detected beyond, the calibration range and was subsequently analyzed at a dilution.

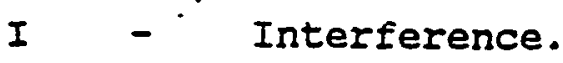

ABEREVIATIONB

88 - Indicates blank spike in which reagent grade water is spiked with the CLP matrix spiking solutions and carried through all the steps in the method. spike recoveries are reported.

B8D - Indicates blank spike duplicate.

Ms - Indicates matrix spike.

MSD - Indicates matrix spike duplicate.

DI - Indicates that recoveries were not obtained because the extract had to be diluted for analysis.

NA - Not applicable.

DF - Dilution factor.

NR - Not required. 
Roy F. Weston, Inc. - LLonv11le Laboratory

Pest1clde/PCBg by GC, Appendix IX Llat

Report Date: 04/24/90 $13: 49$

RFW Batch Number: 9002L607 Cl lent: WSRC-GRACE ROAD Work Order: $0630-28-12-0000$

CUBt IDI GRS-08-01 GRS=08-01 GRS-08-01 SBLR SBLR BS

\begin{tabular}{|c|c|c|c|c|c|c|}
\hline Sample & RFWI & 001 & 001 REP & $001 \mathrm{MB}$ & 90LE0286-MB1 & 90LE0286-MB1 \\
\hline Information & $\begin{array}{r}\text { Matrix: } \\
\text { D.F. : } \\
\text { Unlta: }\end{array}$ & $\begin{array}{l}\text { SOIL } \\
1.00 \\
\mathrm{ug} / \mathrm{Kg}\end{array}$ & $\begin{array}{r}\text { soIL } \\
1.00 \\
u g / \mathrm{Kg}\end{array}$ & $\begin{array}{r}\text { SOIL } \\
1.00 \\
u g / \mathrm{Kg}\end{array}$ & $\begin{array}{c}\text { sort } \\
1.00 \\
u g / \mathrm{kg}\end{array}$ & $\begin{array}{c}\text { soII } \\
1.00 \\
u g / \mathrm{kg}\end{array}$ \\
\hline
\end{tabular}

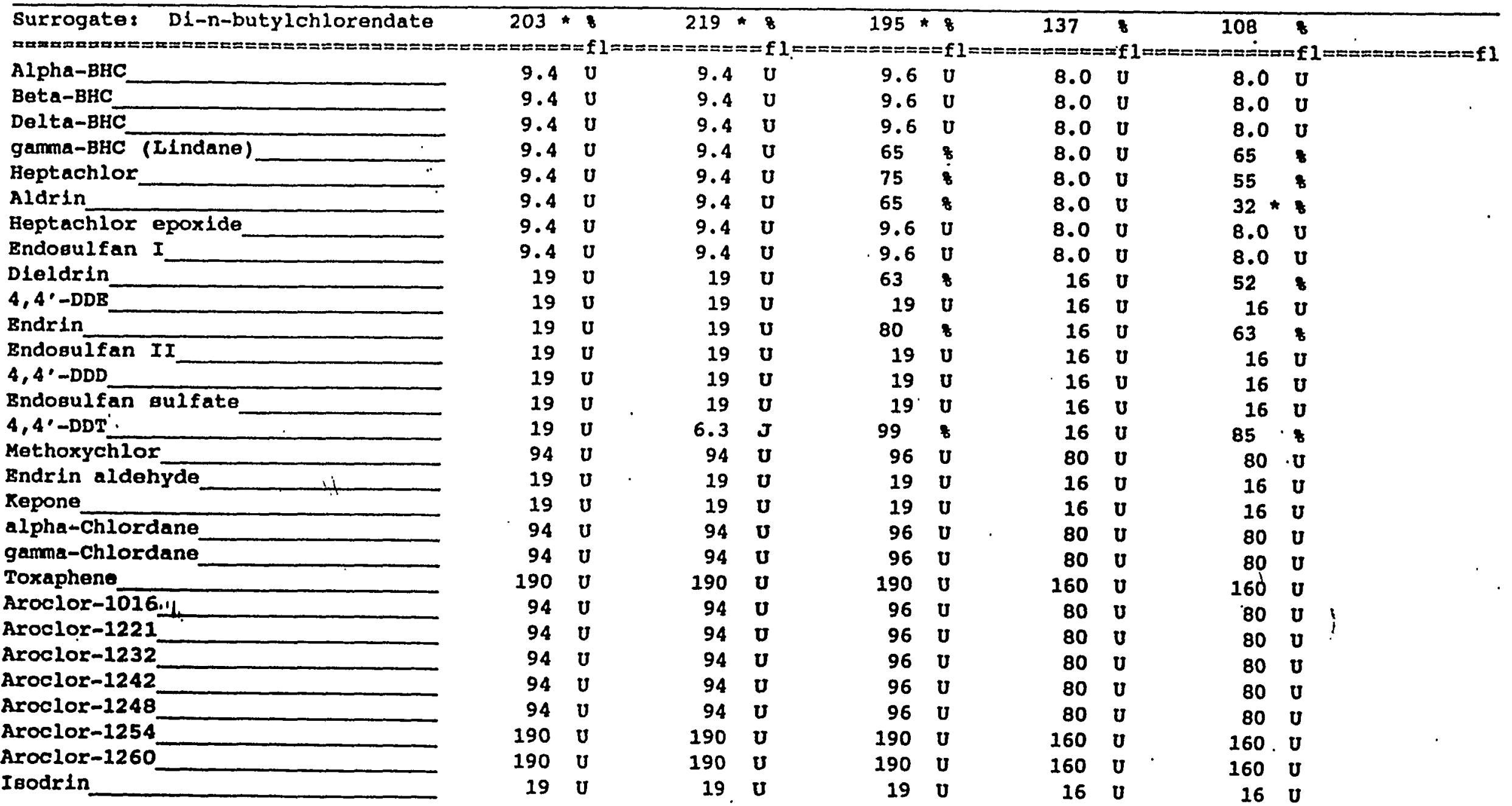

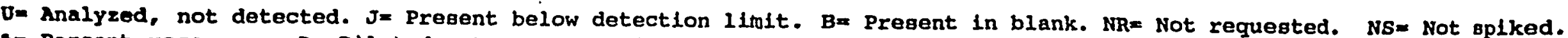

s= Percent recopery. D= Dlluted out. In Interference. NA= Not Applicable. $\star=$ Outside of EPA CLP QC in. 


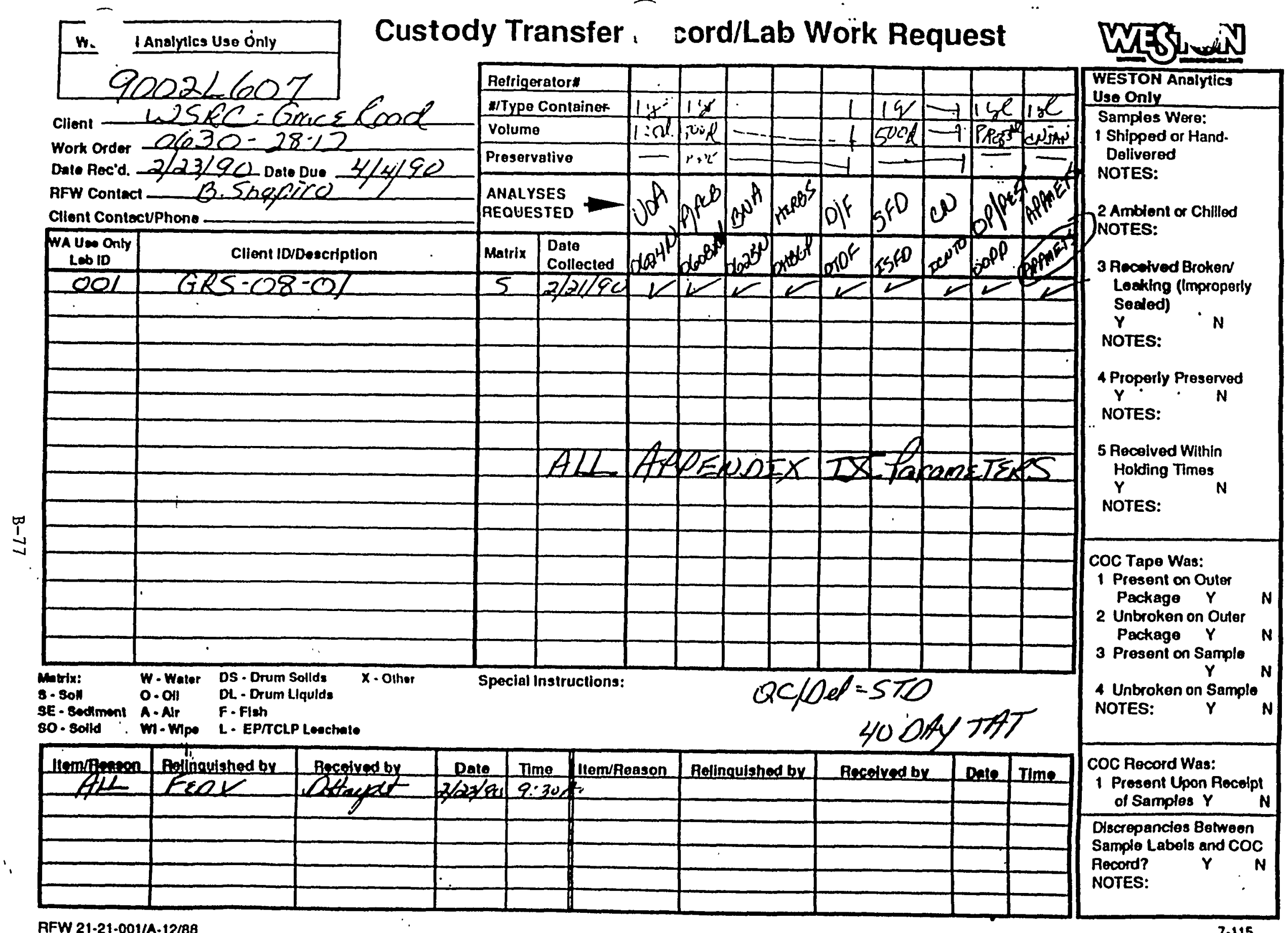




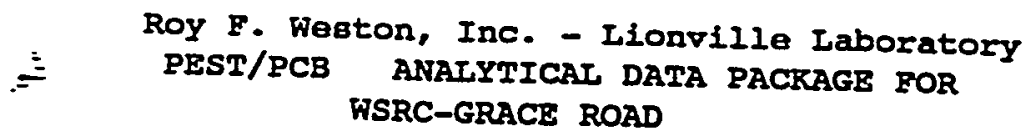

DATE RECEIVED: $02 / 23 / 90$

CLIENT ID

RFW \#

RFW LOT \$:9002L607

GRS-08-01

GRS-08-01

GRS-08-01

001

001 REP

001 MS

WSRC-GRACE ROAD

IAB QC:

SBILK

SBLK

MBI

$\begin{array}{lr}\text { S } & 90 L E 0286 \\ \text { S } & 90 L E 0286\end{array}$

N/A

N/A

$02 / 28 / 90$

$02 / 28 / 90$

$04 / 08 / 90$

$04 / 08 / 90$

ANALYSIS

$02 / 28 / 90$

$02 / 28 / 90$

$04 / 08 / 90$

$04 / 08 / 90$

$04 / 08 / 90$

$02 / 28 / 90$

- 
CLIENT: WSRC-GRACE ROAD

RFW *: 9002L607, SEMIVOLATILE

SAMPLES RECEIVED: $02 / 2 \dot{3} / 90$

พ.0. \#: $063 \overline{0}-28-12$

\section{NARRATIVE}

The set of samples consisted of 1 soil sample collected on

The sample was extracted on $02 / 28 / 90$ and analyzed according to criteria set forth in SW 846 Method 8270 for Appendix IX Semivolatile target compounds on $03 / 07,08 / 90$.

The following is a summary of the $Q C$ results accompanying these sample results and a description of any problems encountered
during their analysis:

1. Non-target compounds were detected in these samples.

2. All surrogate recoveries are within EPA QC
limits. 3. All soil blank and matrix spike recoveries criteria
are. met.

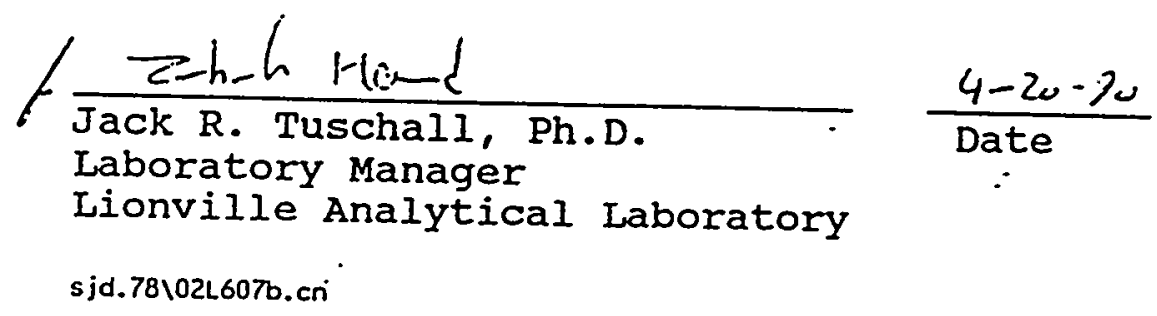




$$
\begin{aligned}
& \text { M } \\
& \text { DATA gUAITFIERS }
\end{aligned}
$$

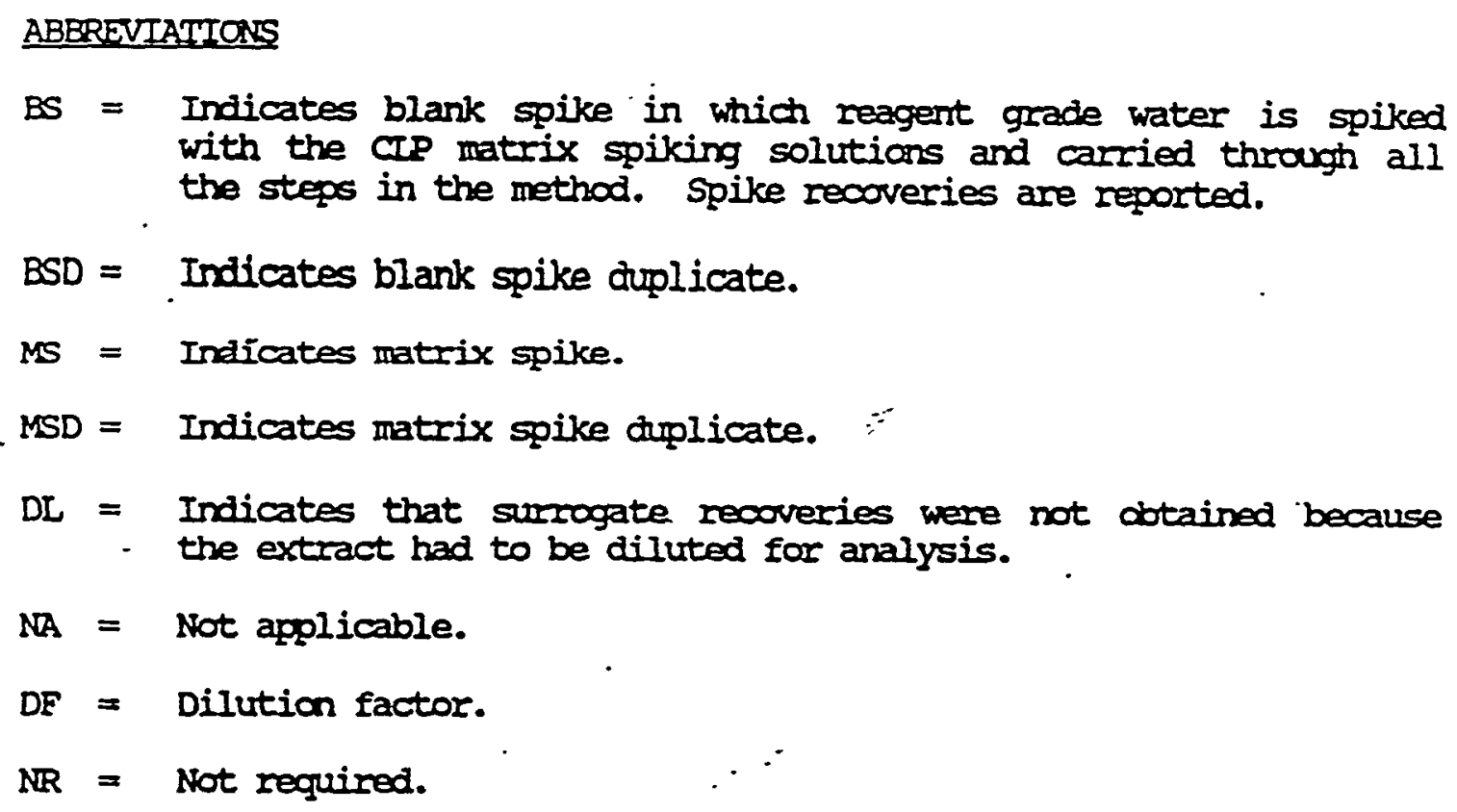

BS = Indicates blank spike in which reagent grade water is spiked with the CIP matrix spiking solutions and carried through all the steps in the method. Spike recoveries are reported.

$B S D=$ Indicates blank spike duplicate.

MS = Indicates matrix spike.

$\mathrm{DL}=$ Indicates that surrogate recoveries were not cbtained because - the extract had to be diluted for analysis.

$N A=$ Not apolicable.

DF = Dilution factor.

$\mathrm{NR}=$ Not required. 
Roy F. Weston, Inc. - LLonville Laboratory

Semivolatiles by GC/MS, Appendix IX List Cllent: WSRC-GRACE ROAD

Report Date: 04/09/90 12:45 Nork Order: $0630-28-12-0000$ Page: 1a

Cust ID: GRS-08-01 GRS-08-01 GRS-08-01 SBLR SBLR BS

sample

Information

$\begin{array}{rrr}\text { RFW : } & 001 & 001 \text { REP } \\ \text { Matrix: } & \text { SOIL } & \text { SOIL } \\ \text { D.F. } & 1.11 & 2.11 \\ \text { UnItB: } & u g / R g & u g / R g\end{array}$

001 MS 9OLE0286-MBI 90LE0286-MB1

SOIL SOIL SOIL

Units : $\quad \mu g / R g$

$\mathrm{ug} / \mathrm{Rg}$

1.11

1.11

1.11

$\mathrm{ug} / \mathrm{Kg}$

$\mathrm{ug} / \mathrm{Kg}$

\begin{tabular}{lr}
\hline & Nitrobenzene-d5 \\
Surrogate & 2-Fluoroblphenyl \\
Recovery & p-Terpheny l-d14 \\
& Phenol-d5 \\
& $2-$ Fluorophenol
\end{tabular}

2, 4,6-Tribromophenol

Phenol

bio (2-Chloroethyl)ether

2-Chlorophenol

1,3-D1chlorobenzene

1,4-Dichlorobenzene

Benryl alcohol

\% 1,2-Dichlorobenzene

is 2-Methylphenol.

b1s (2-Chlorol bopropyl)ether

4-Methylphenol

N-N1tro8o-D1-n-propylamine

Hexachloroethane

Nitrobensene

Isophorione

2-Nitrophenol

2,4-Dimethyl phenol

Benzolc acld.

b18 (2-Chloroethoxy) methane

2, 4-Dichlorophenol

1,2,4-Trichlorobenzene

Naphthalene

4-Chloroaniline

Hexachlorobutadiene

4-Chloro-3-methylphenol

2-Methylnaphthalene

Hexachlorocyclopentadieno

* Outs1de of BPA CLP gC IImIts.

$\begin{array}{ll}50 & 8 \\ 58 & 8 \\ 65 & 8 \\ 59 & 8 \\ 52 & 8 \\ 54 & 8\end{array}$

$\begin{array}{ll}51 & 8 \\ 59 & 8 \\ 72 & 8 \\ 59 & 8 \\ 51 & 8 \\ 51 & 8\end{array}$

$\begin{array}{ll}52 & 8 \\ 59 & 8 \\ 70 & 8 \\ 60 & 8 \\ 50 & 8 \\ 45 & 8\end{array}$

$\begin{array}{llll}53 & 8 & 49 & 8 \\ 61 & 8 & 61 & 8 \\ 78 & 8 & 71 & 8 \\ 70 & 8 & 59 & 8 \\ 66 & 8 & 50 & 8 \\ 77 & 8 & 46 & 8\end{array}$

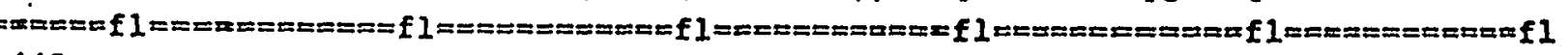

$440 \mathrm{~V}$

$440 \mathrm{U}$

$440 \mathrm{U}$

$440 \mathrm{U}$

$440 \mathrm{U}$

$440 \mathrm{U}$

$440 \mathrm{U}$

$440 \mathrm{U}$

$440 \mathrm{U}$

$440 \mathrm{U}$

$440 \mathrm{U}$

$440 \mathrm{U}$

$440 \mathrm{U}$

$440 \mathrm{~V}$

$440 \mathrm{U}$

$440 \mathrm{U}$

$2200 \mathrm{U}$

$440 \mathrm{U}$

$440 \mathrm{U}$

$440 \mathrm{U}$

$440 \mathrm{U}$

$440 \mathrm{U}$

$440 \mathrm{U}$

$440 \mathrm{~V}$

$440 \mathrm{U}$

$440 \mathrm{U}$

$\begin{array}{rl}440 & U \\ 440 & U \\ 440 & U \\ 440 & U \\ 440 & U \\ 440 & U \\ 440 & U \\ 440 & U \\ 440 & U \\ 440 & U \\ 440 & U \\ 440 & U \\ 440 & U \\ 440 & U \\ 440 & U \\ 440 & U \\ 2200 & U \\ 440 & U \\ 440 & U \\ 440 & U \\ 440 & U \\ 440 & U \\ 440 & U \\ 440 & U \\ 440 & U \\ 440 & U\end{array}$

$\begin{array}{cc}63 & 8 \\ 440 & U \\ 57 & 8 \\ 440 & U \\ 57 & 8 \\ 440 & U \\ 440 & U \\ 440 & U \\ 440 & U \\ 440 & U \\ 49 & 8 \\ 440 & U \\ 440 & U \\ 440 & U \\ 440 & U \\ 440 & U \\ 2200 & U \\ 440 & U \\ 440 & U \\ 68 & 8 \\ 440 & U \\ 440 & U \\ 440 & U \\ 59 & 8 \\ 440 & U \\ 440 & U\end{array}$

$370 \mathrm{U}$

370 U

$370 \mathrm{U}$

370 U

370 U

370 U

$370 \quad U$

370 U

370 U

370 U

370 v

370 U

370 U

370 U

370 U

$370 \mathrm{U}$

$1800 \mathrm{U}$

$370 \mathrm{U}$

$370 \mathrm{v}$

370 U

$370 \mathrm{v}$

370 U

$370 \mathrm{U}$

370 U

$370 \mathrm{U}$

$370 \mathrm{U}$

$\begin{array}{ll}62 & 8 \\ 370 & U \\ 56 & \\ 370 & U \\ 51 & 8 \\ 370 & U \\ 370 & U \\ 370 & U \\ 370 & U \\ 370 & U \\ 49 & 8 \\ 370 & U \\ 370 & U \\ 370 & U \\ 370 & U \\ 370 & U \\ 1800 & U \\ 370 & U \\ 370 & U \\ 62 & \text { : } \\ 370 & U \\ 370 & U \\ 370 & U \\ 53 & 8 \\ 370 & U \\ 370 & U \\ & \end{array}$


RFWI: $001 \quad 001$ REP

001 MS 90LE0286-MB1 90LE0286-MB1

2,4,6-Trichlorophenol

$2,4,5$-Trichlorophenol

2-Chloronaphthalene

2-Nitroaniline

Dimethylphthalate

Acenaphthylene

2,6-Dinitrotoluene

3-Nitroaniline

Acenaphthene

2, 4-Dinitrophenol

4-Nitrophenol

Dibenzofuran

2,4-Dinitrotoluene

Diethylphthalate

4-Chlorophenyl-phenylether

Eluorene

4-Nitroaniline

4, 6-Dinitro-2-methylphenol

N-Nitrosodiphenylamine (1)

it 4-Bromophenyl-phenylether

$\stackrel{\infty}{\omega}$ Hexachlorobenzene

Pentachlorophenol

Phenanthrene

Anthracen

D1-n-Butylphthalate

$440 \mathrm{U}$

$440 \mathrm{U}$

\begin{tabular}{|c|c|}
\hline 440 & $\mathrm{v}$ \\
\hline 2200 & $u$ \\
\hline 440 & $\mathbf{U}$ \\
\hline 2200 & $\mathbf{v}$ \\
\hline 440 & $\mathbf{U}$ \\
\hline 440 & U \\
\hline 440 & U \\
\hline 2200 & $\mathbf{u}$ \\
\hline 74 & 8 \\
\hline 2200 & $\mathbf{u}$ \\
\hline 82 & 8 \\
\hline 440 & $\mathbf{v}$ \\
\hline 60 & 8 \\
\hline 440 & $\mathbf{U}$ \\
\hline 440 & v \\
\hline 440 & $\mathbf{v}$ \\
\hline 2200 & $\mathbf{v}$ \\
\hline 2200 & $\mathbf{U}$ \\
\hline 440 & $\mathbf{u}$ \\
\hline 440 & U \\
\hline 440 & U \\
\hline 76 & 8 \\
\hline 440 & $\mathbf{u}$ \\
\hline 440 & U \\
\hline 440 & U \\
\hline 440 & $\mathbf{v}$ \\
\hline 92 & 8 \\
\hline 440 & $\mathbf{v}$ \\
\hline 870 & $\mathbf{U}$ \\
\hline 440 & $\mathbf{u}$ \\
\hline 440 & $\mathbf{U}$ \\
\hline 3200 & \\
\hline 440 & $\mathbf{U}$ \\
\hline 440 & $\mathbf{v}$ \\
\hline 440 & $\mathbf{U}$ \\
\hline 440 & $\mathbf{U}$ \\
\hline 440 & U \\
\hline 440 & $\mathbf{U}$ \\
\hline 440 & $\mathbf{U}$ \\
\hline 440 & 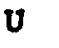 \\
\hline
\end{tabular}

\begin{tabular}{|c|c|c|c|}
\hline 370. & $\mathbf{U}$ & 370 & $\mathbf{u}$ \\
\hline 1800 & $\mathbf{U}$ & 1800 & $\mathbf{v}$ \\
\hline 370 & $\mathbf{u}$ & 370 & v \\
\hline .1800 & $\mathbf{U}$ & 1800 & $\mathbf{u}$ \\
\hline 370 & $\mathbf{U}$ & 370 & v \\
\hline 370 & $\mathbf{U}$ & 370 & $\mathbf{U}$ \\
\hline 370 & $\mathbf{U}$ & 370 & U \\
\hline 1800 & $\mathbf{u}$ & 1800 & v \\
\hline 370 & U & 72 & 8 \\
\hline 1800 & U & 1800 & $\mathbf{u}$ \\
\hline 1800 & $\mathbf{U}$ & 89 & 8 \\
\hline 370 & U & 370 & v \\
\hline 370 & v & 58 & 8 \\
\hline 370 & $\mathbf{v}$ & 370 & U \\
\hline 370 & v & 370 & v \\
\hline 370 & v & 370 & v \\
\hline 1800 & $\mathbf{v}$ & 1800 & u \\
\hline 1800 & $\mathbf{v}$ & 1800 & $\mathbf{u}$ \\
\hline 370 & $v$ & 370 & v \\
\hline 370 & $\mathbf{v}$ & 370 & $\mathbf{u}$ \\
\hline 370 & v & 370 & $\mathbf{v}$ \\
\hline 1800 & v & 68 & .8 \\
\hline 370 & U & 370 & ụ \\
\hline 370 & $\mathbf{U}$ & 370 & $\mathbf{U}$ \\
\hline 370 & $\mathbf{U}$ & 370 & $\mathbf{U}$ \\
\hline 370 & U & 370 & u \\
\hline 370 & $\mathbf{U}$ & 92 & 8 \\
\hline 370 & $\mathbf{u}$ & 370 & $\mathbf{U}$ \\
\hline 740 & $\mathbf{u}$ & 740 & U \\
\hline 370 & $\mathbf{U}$ & 370 & $\mathbf{u}$ \\
\hline 370 & $\mathbf{u}$ & 370 & $\mathbf{u}$ \\
\hline 370 & $\mathbf{U}$ & 370 & $\mathbf{U}$ \\
\hline 370 & $\mathbf{U}$ & 370 & $\mathbf{U}$ \\
\hline 370 & $\mathbf{U}$ & 370 & $\mathbf{U}$ \\
\hline 370 & $\mathbf{v}$ & 370 & $\mathbf{U}$ \\
\hline 370 & $\mathbf{u}$ & 370 & $\mathbf{u}$ \\
\hline 370 & $\mathbf{U}$ & 370 & u. \\
\hline 370 & $\mathbf{U}$ & 370 & U \\
\hline 370 & $\mathbf{v}$ & 370 & $\mathbf{v}$ \\
\hline 370 & U & 370 & v \\
\hline
\end{tabular}

$2200 \mathrm{U}$

$440 \mathrm{U}$

$2200 U$

$440 \mathrm{U}$

$440 \mathrm{U}$

$440 \mathrm{U}$

$2200 \mathrm{U}$

$440 \mathrm{U}$

$2200 U$

$2200 \mathrm{U}$

$440 \mathrm{U}$

$440 \mathrm{U}$

$440 \mathrm{~V}$

$440 v$

$440 \mathrm{U}$

2200 U

$2200 \mathrm{U}$

$440 \mathrm{U}$

$440 \mathrm{U}$

$440 \mathrm{U}$

2200 U

$440 \mathrm{U}$

$440 \mathrm{U}$

$440 \mathrm{~V}$

$440 \mathrm{U}$

$440 \mathrm{~V}$

$440 \mathrm{U}$

$870 \mathrm{U}$

$440 \mathrm{U}$

440 U

2800

$440 \mathrm{U}$

440 . U

$440 \mathrm{U}$

$440 \mathrm{U}$

440 U

$440 \cdot \mathrm{V}$

$440 \mathrm{U}$

$\begin{array}{rr}2200 & U \\ 440 & U\end{array}$

$2200 \mathrm{U}$

$440 \mathrm{U}$

$440 \mathrm{U}$

$440 \mathrm{U}$

$2200 \mathrm{U}$
440
$\mathrm{U}$

$\begin{array}{rr}440 & \mathrm{U} \\ 2200 & \mathrm{U}\end{array}$

$2200 \mathrm{U}$

$440 \mathrm{U}$

$440 \mathrm{U}$

$440 \mathrm{U}$

$440 \mathrm{U}$

$440 \mathrm{U}$

$2200 \mathrm{U}$

$2200 \mathrm{U}$

$440 \mathrm{U}$

440 'U

$440 \mathrm{U}$

$2200 v$

$440 \mathrm{U}$

$440 \mathrm{U}$

$440 \quad U$

$440 \mathrm{U}$

$440 \mathrm{U}$

$440 \mathrm{U}$

$890 U$

$440 \mathrm{U}$

$440 \mathrm{U}$

2800

$440 \mathrm{U}$

$440 \mathrm{U}$

nzo (b) fluoranthene

Benzo (a) pyrene

$440 \mathrm{U}$

$440 \mathrm{U}$

440 U

$440 \mathrm{U}$

$440 \%$ U

Benzo $(g, h, 1)$ perylene

1, 4-Dloxane

* Outside of EPA CLP QC IImits.

$440 \mathrm{v}$

$\because \because \because \because \cdots$ 
RFW :

$001 \quad 001$ REP

001 MS 90LE0286-MB1 9OLE0286-MB1

Methyl methacrylate

Pyridine

N-Nitrosodimethylamine

Ethyl methacrylate

2-Picoline

N-Nitrosomethylethylamine

Methyl methanesulfonate

N-Nitrosodiethylamine

Ethyl methanesulfonate

Aniline

Pentachloroethane

3-Methylphenol

N-Nitrosopyrrolldine

Acetophenone

N-N1trosomorpholine

o-Toluidine

N-Nitrosopiperidine

a, a-Dimethylphenethylamine

2,6-Dichlorophenol

Hexachloropropene

\& p-Phenylenediamine

N-Nitroso-di-n-butylamine

safrole

$1,2,4,5$-Tetrachlorobenzene

Isosafrolo

1,4-Naphthoquinone

$\begin{array}{llllllllll}440 & U & 440 & U & 440 & U & 370 & U & 370 & U \\ 440 & U & 440 & U & 440 & U & 370 & U & 370 & U \\ 440 & U & 440 & U & 440 & U & 370 & U & 370 & U \\ 440 & U & 440 & U & 440 & U & 370 & U & 370 & U\end{array}$

1,3-Dinitrobenzene

Pentachlorobenzene

1-Naphthylamine

2-Naphthylamine

$2,3,4,6$-Tetrachiorophenol

1,3,5-Trin1trobenzene

Diallate

Phenacet in

Diphenylamine

5-N1tro-0-toluldine

4-Aminoblphenyl

Pronamide

2-8ec-Buty1-4,6-din1trophenol

Pentachloron 1 trobenzene

** outride of BPA CLP QC 1imita.

$440 \mathrm{U}$

$440 \mathrm{U}$

$440 \mathrm{U}$

$440 \mathrm{U}$

$440 \mathrm{U}$

$440 \mathrm{U}$

$440 \mathrm{U}$

$440 \mathrm{v}$

$440 v$

$440 \mathrm{U}$

$440 \mathrm{v}$

$440 \mathrm{~V}$

2200

$440 \mathrm{U}$

$440 \mathrm{U}$

$440 \mathrm{U}$

$440 \mathrm{U}$

$440 \mathrm{U}$

$440 \quad U$

$440 \mathrm{U}$

$440 \mathrm{U}$

$440 \mathrm{U}$

$440 \mathrm{U}$

$440 \mathrm{~V}$

$440 \mathrm{~V}$

$440 \mathrm{U}$

$440 \mathrm{~V}$

$440 \mathrm{U}$

$440 \mathrm{U}$

$440 \mathrm{~V}$

$440 \mathrm{U}$

$440 \mathrm{U}$

$.440 \mathrm{~V}$

$440 \mathrm{U}$

22000

$2200 \mathrm{U}$

$440 \mathrm{U}$

$440 \mathrm{U}$

$440 \mathrm{U}$

$440 \mathrm{U}$

$440 \mathrm{U}$

$440 \mathrm{~V}$

$440 \mathrm{U}$

$440 \mathrm{U}$

$440 \mathrm{U}$

$440 \mathrm{U}$

$440 \mathrm{U}$

$440 \mathrm{U}$

$2200 U$

$440 \mathrm{U}$

$440 \mathrm{U}$

$440 \cdot U$

$440 \mathrm{U}$

$440 \mathrm{U}$

$440 \mathrm{~V}$

$440 \mathrm{U}$

$440 \mathrm{U}$

$440 \mathrm{U}$

$440 \mathrm{U}$

$440 \mathrm{U}$

$440 \mathrm{U}$

$440 \mathrm{U}$

$440 \mathrm{U}$

$440 \mathrm{U}$

$440 \mathrm{U}$

$440 \mathrm{U}$

$440 \mathrm{U}$

$440 \mathrm{U}$

$440^{\circ} \mathrm{U}$

$440 \mathrm{U}$

2200 . U

$2200 \mathrm{U}$

$440 \mathrm{U}$

$440 \mathrm{U}$

$440 \mathrm{U}$

$440 \mathrm{U}$

$440 \mathrm{U}$

$440 \mathrm{~V}$

$440 \mathrm{U}$

$440 \mathrm{U}$

$440 \mathrm{U}$

$440 \mathrm{U}$

$440 \mathrm{U}$

$440 \mathrm{U}$

$440 \mathrm{U}$

$2200 \mathrm{U}$

$440 \mathrm{U}$

$440 \mathrm{U}$

$440 \mathrm{U}$

$440 \mathrm{U}$

$440 \mathrm{U}$

$440^{\circ} \mathrm{U}$

$440 \mathrm{U}$

$440 \mathrm{U}$

$440 \mathrm{v}$

$440 \mathrm{U}$

$440 \mathrm{U}$

$440 \mathrm{U}$

$440 \mathrm{U}$

$440 \mathrm{U}$

$440 \mathrm{U}$

$440 \mathrm{U}$

$440 \mathrm{U}$

$440 \mathrm{U}$

440 U

440 U

$440 \mathrm{U}$

$2200 \mathrm{U}$

$2200 \mathrm{U}$

370 U . 370

$\begin{array}{llll}370 & U & 370 & U\end{array}$

370 U

$370 \mathrm{U} \quad 370 \mathrm{U}$

$370 \mathrm{U} \quad 370 \mathrm{U}$

$\begin{array}{llll}370 & U & 370 & U\end{array}$

$370 \mathrm{U} .370 \mathrm{U}$

$\begin{array}{llll}370 & U & 370 & U\end{array}$

$370 \mathrm{U} \quad 370 \mathrm{U}$

$\begin{array}{lll}370 & U & 370 \\ 370\end{array}$

$\begin{array}{lll}370 & U & 370 \\ \end{array}$

$370 \mathrm{U} \quad 370 . v$

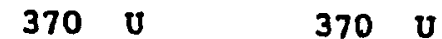

$1800 \mathrm{U} \quad 1800 \mathrm{U}$

370 U 370 U

370 U 370 U

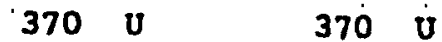

$370 \mathrm{U} \quad 370 \mathrm{U}$

$\begin{array}{lll}370 & U & 370 \\ \end{array}$

370 U

$370 \mathrm{v}$

370 U

$370 \mathrm{U}$

$370 \mathrm{U}$

$370 \mathrm{U}$

$370 \mathrm{U}$

$370 \mathrm{v}$

$370 \quad U$

370

$370 \mathrm{U}$

$370 \mathrm{U}$

370 U

370 U

370 U

$370 v$

1800

$1800 \mathrm{v}$

370 U

370 U

$370 \mathrm{U}$

$370 \mathrm{v}$

$370 \mathrm{U}$

370 v

$370 \quad 0$

$370 \quad U$

$370 \mathrm{U}$

$370 \mathrm{U}$

$370 \mathrm{U}$

$370 \mathrm{U}$

$370 \mathrm{v}$

370 v

370 U

370 v

1800

1800 
RFW L 7 Number: 9002L607

Cllent: WSRC-GRACE RL

$-$

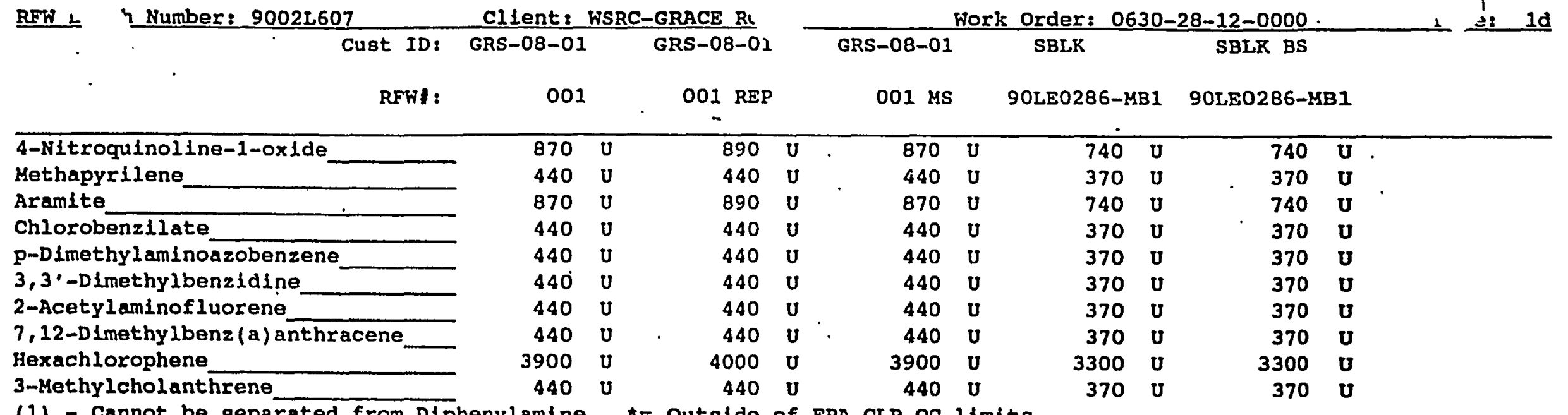

(1) - Cannot be separated from Diphenylamine. *z Outgide of EPA CLP QC 1imits. 
12

SEMIVOLATILE ORGANICS ANALYSIS SEEET

TENTATIVELY IDENTIFIED COMPOUNDS

Lab Name: Roy F. Weston, Inc. Work Order: 0630-28-12-0000

Client: WSRC-GRACE ROAD

Matrix:

SOIL

Sample wt/vol: $30.6(\mathrm{~g} / \mathrm{mr}) \mathrm{G}$

Iab Sample ID: 9002L607-001

Lab File ID?. M030710

Level: (low/med) IOW

Date.Received: $02 / 23 / 90$

8 Molsture: not dec. 17 dec.

Date Extracted: $02 / 28 / 90$

Extraction: (Sepr/cont/Sonc) sonc

Date Analyzed: $03 / 07 / 90$

GPC Cleanup: $\quad(Y / N) \underline{Y}$

pH: $\quad 7.0$

Dilution Factor: 1.11

Number TICs found: 4

CONCENTRATION UNITS:

(ug/I or ug/kg) ug/Rg

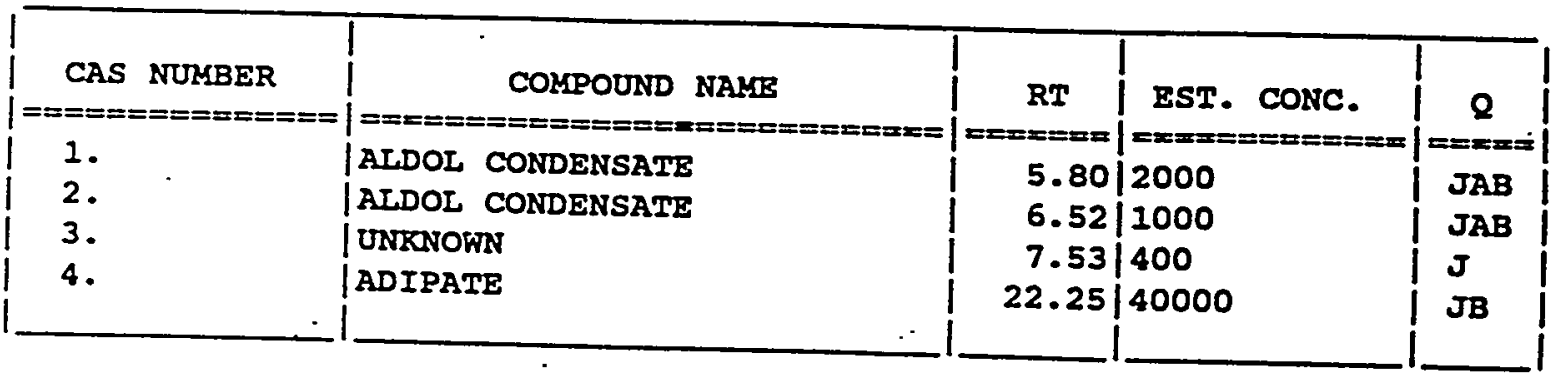


Lab Name: Roy F. Wegton, Inc. Work Order: 0630-28-12-0000

Client: WSRC-GRACE ROAD

Matrix:

Sample wt/vol:

$$
\text { sorL }
$$$$
30.0(\mathrm{~g} / \mathrm{mr}) \mathrm{G}
$$

Level. (Iow/med) LOF

\& Moisture: not dec.

17 dec.

Extraction: (SepE/Cont/Sonc) SONC

GPC Cleanup: $\quad(Y / N) \underline{Y}$ $\mathrm{pH}: \quad 7.0$
Lab sample ID: 9002L607-001 REP

Lab File ID: y030712

Date Received: $02 / 23 / 90$

Date Extracted: 02/28/90

Date Analyzed: $03 / 07 / 90$

Dilution Eactor: 1.11

Number TICg found: $\underline{5}$

CONCENTRATION UNITS:

(ug/L or $u g / k g)$ ug/ $/ \mathrm{gq}$

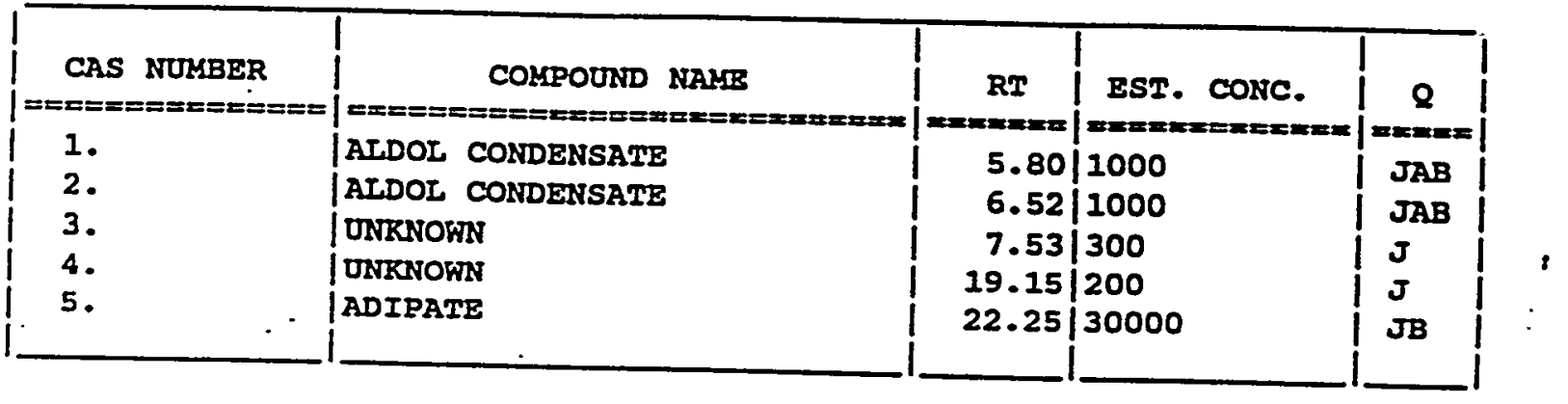




\section{. $1 F$ \\ SEMIVOLATIIE ORGANICS ANALYSIS SEEET}

TENTATIVELY IDENTIFIED COMPOUNDS

Lab Name: Roy F. Weston, Inc. Work Order: 0630-28-12-0000

| SBLK

Client: WSRC-GRACE ROAD

Matrix:

FOIL

Sample it/vol:

Lab Sample ID: 90Lg0286-MBI

Lab File ID: $\underline{\text { YO30804 }}$

Level: (low/med) LOW

Date Receiveds . $02 / 28 / 90$

\& Moisture: not dec.

o dec.

Extraction: (SepF/Cont/Sonc) soNC

GPC Cleanup: $(Y / N) \underline{Y}$

$\mathrm{pH}: \quad 7.0$

Date Extracted: $02 / 28 / 90$

Date Analyzed: $03 / 08 / 90$

Dilution Factor: 1.11

Number TICs found: 4

CONCENTRATION UNITS:

(ug/L or ug/ $\mathrm{kg}$ ) ug/ $\mathrm{Rg}$

\begin{tabular}{|c|c|c|c|c|}
\hline CAS NOMBER & COMPOUND NAME & RT & EST. CONC. & $\mathbf{Q}$ \\
\hline 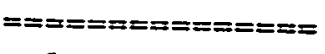 & 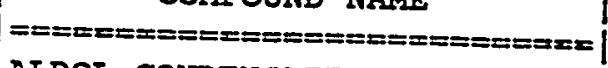 & $z=\Sigma x=x=0$ & $==2 x=1$ & $x=2 x=2$ \\
\hline 1 & ALDOL CONDENSATE & 5.60 & 600 & JA \\
\hline 2 & ATDOL CONDENSATE & 5.85 & 300 & JA \\
\hline 3. & ALDOI CONDENSATE & 6.55 & 300 & JA \\
\hline 4. & ADIPATE & 22.30 & 30000 & $\mathbf{J}$ \\
\hline
\end{tabular}




$$
\begin{gathered}
\text { ROY F. Wexton, InC. - ILONVille Laboratory } \\
\text { BNA ANALYTICAI DATA PACRAGE EOR } \\
\text { WSRC-GRACB ROAD }
\end{gathered}
$$

DATE RECEIVED: $02 / 23 / 90$

CLIENT ID

ב-

RPW *

GRS-0,8-01

GRS-08-01

GRS-08-01

LAB QC:

SBLR

001
MB1

LBI BS
RSW LOT :9002L607

MTXX PREP * COLIECTION EXTTR/PREP

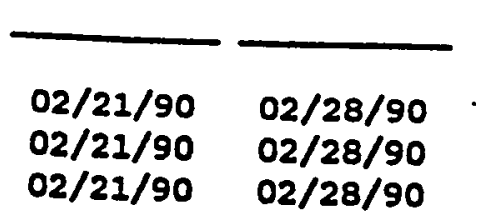

ANALYSIS

001 REP

001 us

S 90 LE0286

S $90 L B 0286$

S 90 LB0286

$02 / 21 / 90$

$02 / 28 / 90$

03/07/90

$03 / 07 / 90$

$03 / 07 / 90$

SBLK

S 90LE0286

$N / \boldsymbol{A}$

$N / A$

$02 / 28 / 90$

$02 / 28 / 90$

$03 / 08 / 90$

$03 / 08 / 90$ 
WIESTEN
Roy F. Weston, Inc.

Lionville Laboratory

Client: WSRC = Grace Road

RFW\#: 9002 5607 , Herbicides

Date Received: 02/23/90

W. O.\#: $0630-28-12$

The set of samples consisted of 1 soil sample collected on $02 / 21 / 90$.

The sample was extracted on $02 / 27 / 90$ and analyzed according to criteria set forth in Method 8150 for Chlorinated Phenoxy Acid

i. The blank spike recoveries are within laboratory control chart
warning limits.

2. No problems were encountered during these analyses. 


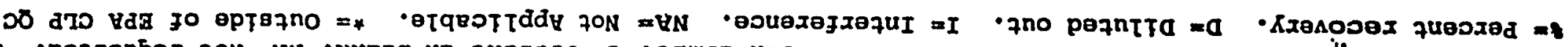

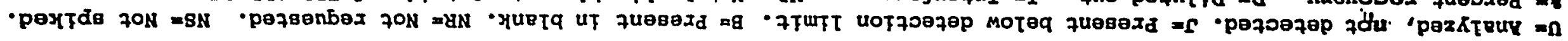

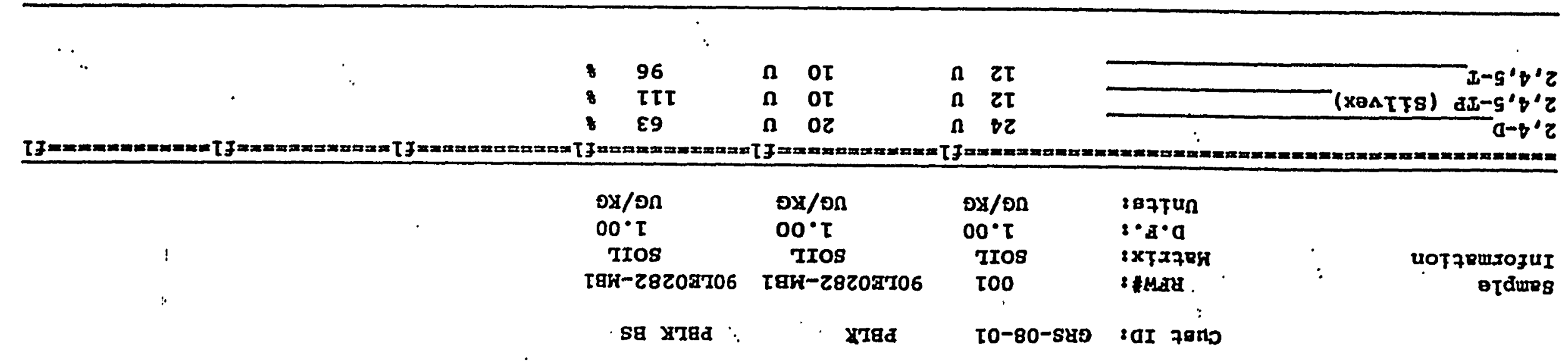



ANALYTICAI DATA PACRAGE FOR WSRC-GRACE ROAD

DATE RECEIVED: $02 / 23 / 90$ RFW LOT : $: 90021607$

CLIENT ID

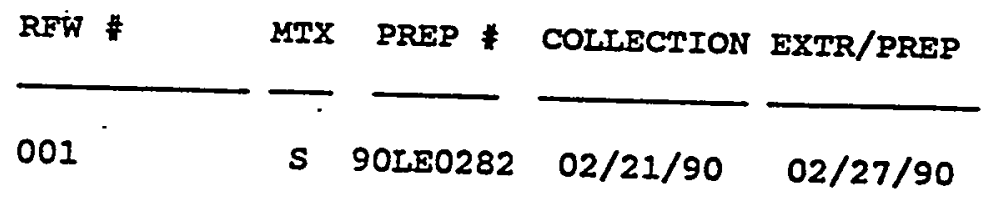

ANAIYSIS

GRS-08-01

s 90r:0282 02/21/90

2/27/90

$03 / 14 / 90$

LAB $\cdot Q C:$

PBIK

PBLK

MB1

MBI BS

$\begin{array}{ll}\text { S } & 90 \text { LEO282 } \\ \text { S } & 90 \text { LE0282 }\end{array}$

$N / A$

$02 / 27 / 90$

$02 / 27 / 90$

$03 / 14 / 90$

$03 / 14 / 90$ 


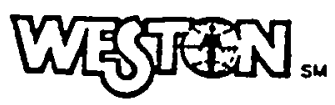

ROY F. HESTON, INC.

Lionville Laboratory

(2)

CLIENT: WSRC:

$\begin{array}{ll}\text { RFW \#: } & 9002 \mathrm{~L} 607, \text { PCDD/PCDF } \\ \text { W.O. \#: } & 0630-28-12\end{array}$

SAMPLES RECEIVED: $02 / 23 / 90$

\section{NARRATIVE}

The set of samples consisted of 1 soil sample collected on
$02 / 21 / 90$.

The samples were extracted on $03 / 01 / 90$ and analyzed according to target compound on $03 / 12 / 90$. sample results and a description of any problems encountered
during their analysis:

1. The $C-13$ HXCDD surrogate recovery for the

blank and the blank spike are below method 8280 QC limits. This is due to loss during
the clean-up process. 2. No other problems were encountered during the
analysis.

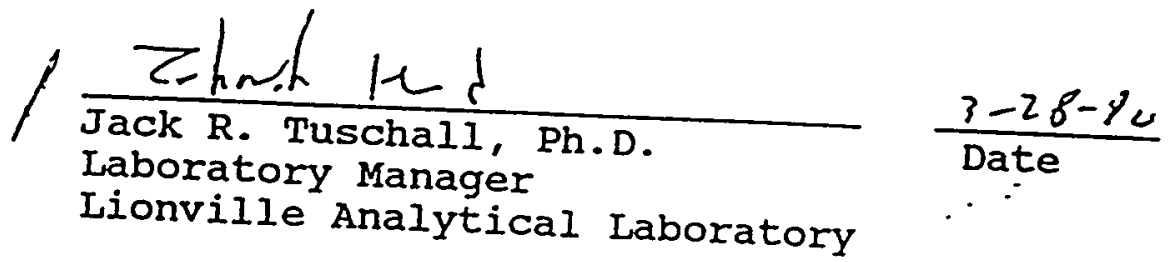


范 
Iab Nama: Roy P. Heston, Inc. Hork Order: 0630-28-12-0000

Client: WSRC-GRMCR ROAD

Matrix:

Sample wt/vol: $\quad 30.5(\mathrm{~g} / \mathrm{mr}) \mathrm{G}$

Level: (Low/med) LOW

* Molbturer not dec. 16 dec.

Extraction: (SepF/Cont/Sonc) SoNC

GPC Cleanup: $(\Psi / N) \underline{Y}$

Number TICB found: 5
Lab Sample ID: 9002L606-005

Iab File ID? J030810

Date Received: $02 / 23 / 90$

Date Bxtractedz 02/28/90

Date Analyzed: $\underline{03 / 09 / 90}$

Dilution Factor: 1.11

CONCENTRATION UNITS:

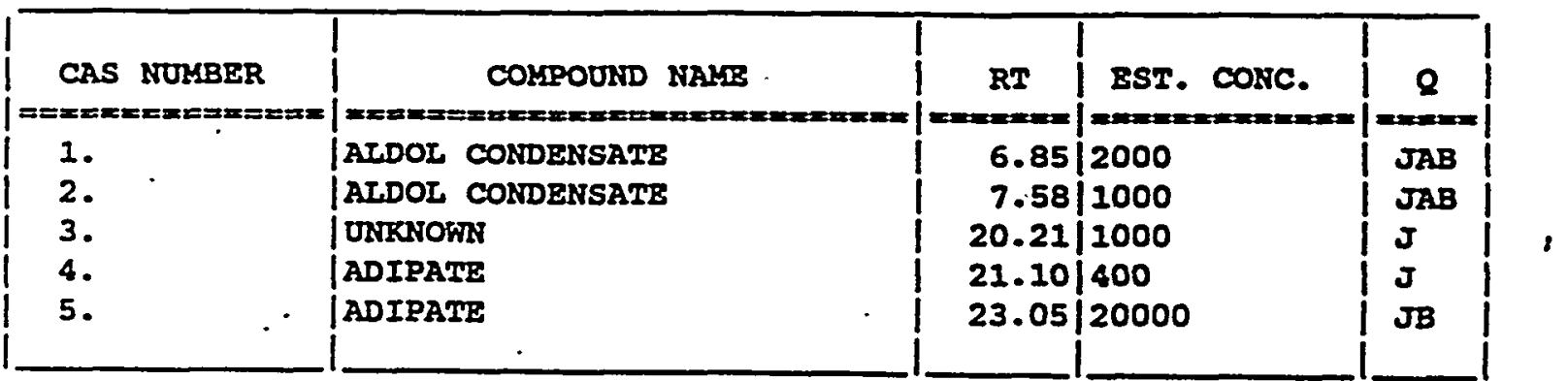


TENTATIVELY IDENTIFIED COMPOUNDS

Lab Name: Roy F. WeBton, Inc. Hork Orders 0630-28-12-0000

Cllent: MSRC-GRACE ROAD

MatrLx:

SoIL

Sample wt/vol: $30.2(\mathrm{~g} / \mathrm{mr}) \mathrm{G}$

Level: (Low/med) LOW

\&oisturer not dec. 8 dec.

Extraction: (Sepr/Cont/Sonc)

SONC.

GPC Cleanup: $\quad(Y / N) \underline{Y}$ $\mathrm{pH}: \quad 7.0$
Iab Sample ID: 9002I,606-001

Lab File ID: J030806

Date Received: 02/23/90

Date Extracteds 02/28/90

Date Analyzed: $03 / 08 / 90$

Dllution Factor: 1.11

Number TICB found: 5 CONCENTRATION UNITS: (ug/I or $u g / \mathrm{kg}$ ) ug/ $\mathrm{kg}$

\begin{tabular}{|c|c|c|c|c|}
\hline CAS NUMBER & COMPOUND NAKE & $\mathbf{R T}$ & EST. CONC. & $\mathbf{Q}$ \\
\hline 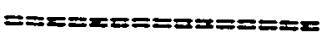 & 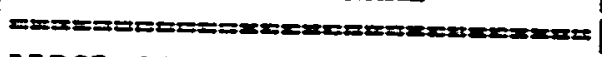 & $x \rightarrow=x=$ & 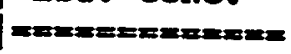 & $=m=0$ \\
\hline 1 & ALDOL CONDENSATE & 6.83 & 1000 & JAB \\
\hline 2. & ATDOL CONDENSATE & 7.57 & 1000 & JAB \\
\hline 3. & ATDOL CONDENSATE & 8.64 & 1000 & JA \\
\hline 4. & UNTRNOWN & 20.20 & 600 & $\boldsymbol{J}$ \\
\hline 5. & ADIPATE & 23.07 & 50000 & JB \\
\hline
\end{tabular}




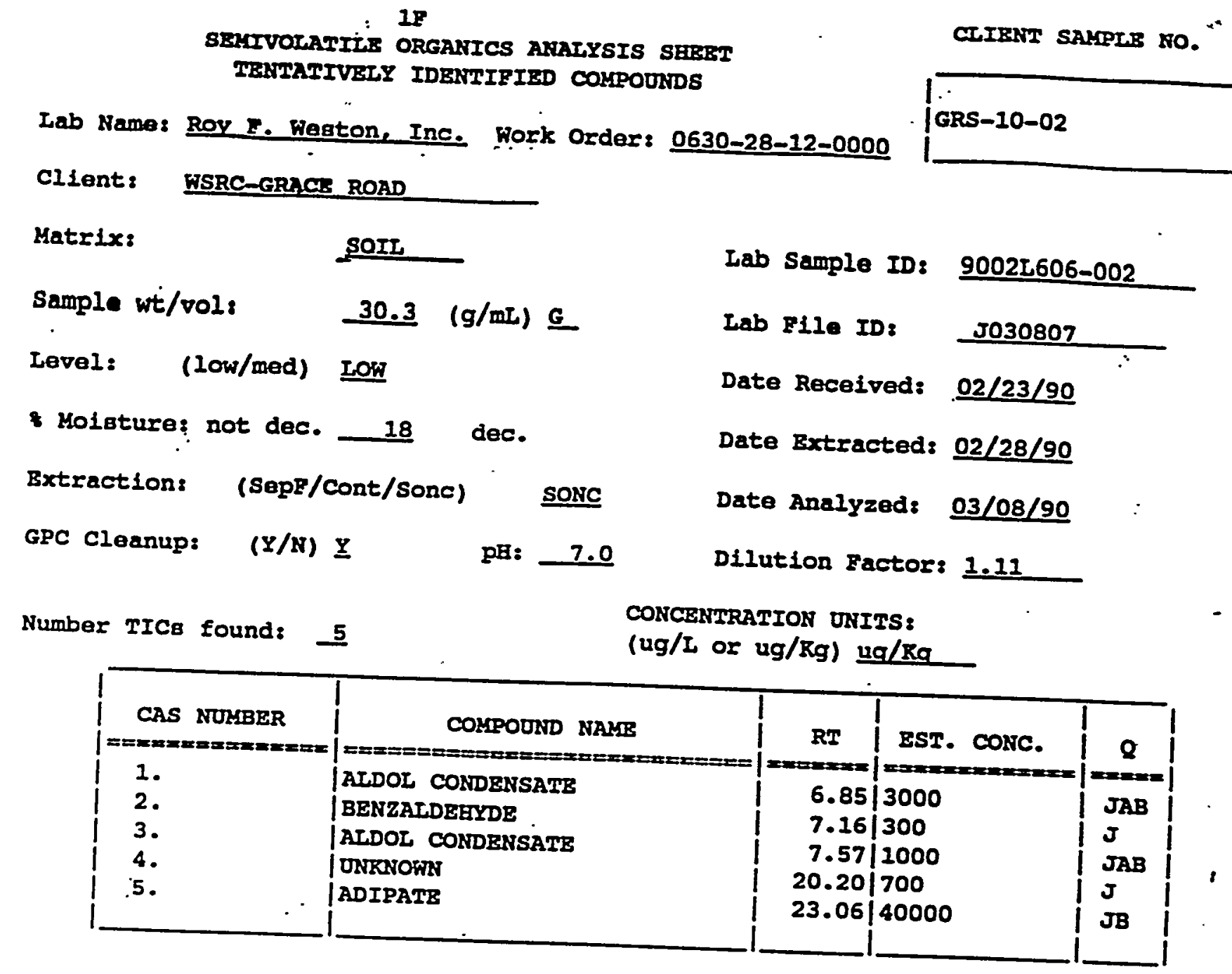


Lab Name: Roy $F$. Weston, Inc. Work Order: 0630-28-12-0000

Cllent: WSRC-GRACB ROAD

Hatrix:

SoIt

Sample wt/vol:

$30.4(\mathrm{~g} / \mathrm{mI}) \mathrm{G}$

Level: (low/med) LOW

\& Hoisture: not dec.

15 dec.

Extraction: (Sepr/cont/sonc) soNe

GPC Cleanup: $\quad(Y / N) \underline{Y}$

$$
\text { pH: } 7.0
$$

Iab Sample ID: 9002L606-003

Iab. File ID:

Date Received: $\underline{02 / 23 / 90}$

Date Extracted: 02/28/90

Date Analyzed: $\underline{03 / 08 / 90}$

Dilution Factor: 1.11

Number TICs found: 5

CONCENTRATION UNITS:

(ug/L or ug/Rg) uqg/Rg

\begin{tabular}{|c|c|c|c|c|}
\hline CAS NUMBER & COMPOUND NAYS & RT & EST. CONC. & $\mathbf{Q}$ \\
\hline 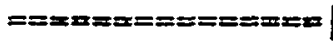 & | & $==\pi=2 x=\pi$ & 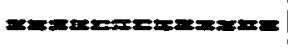 & 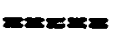 \\
\hline 1. & AIDOL CONDENSATE & 6.87 & 3000 & JAB \\
\hline 2 . & ATDOL CONDENSATE & 7.59 & 2000 & JAB \\
\hline 3. & ALDOL CONDENSATE & 8.67 & 1000 & $\mathbf{J}$ \\
\hline 4. & UNKNOWN & 20.22 & 800 & $\mathbf{J}$ \\
\hline 5. & ADIPATE & 23.06 & 30000 & JB \\
\hline
\end{tabular}




\section{. \\ SEHIVOLATILB ORGANICS ANALYSIS SHBET \\ TENTATIVELY IDENTIFIED COMPOUNDS}

Lab Name: Roy F. Weston, Inc. Work-Order: 0630-28-12-0000

Cllent: FSRC-GRACE ROAD

Matrix:

SOIL

Sample wt/vol:

$30.6(\mathrm{~g} / \mathrm{mL}) \mathrm{G}$

Level: . (low/med) LOW

\&olatures not dec.

Extraction: (Sepp/cont/sonc) SONC

GPC Cleanup: $\quad(\Psi / N) \underline{Y}$

$\mathrm{pH}: \quad \mathbf{7 . 0}$
CLIENT SAMPLE NO.

|GRS-09-01
Lab Sample ID: 9002L606-004

Iab Plle ID: J030809

Date Recelved: $02 / 23 / 90$

Date Extracted: $02 / 28 / 90$

Date Analyzed: 03/09/90

Dilution Factor: 1.11

Number TICB found: $\underline{5}$

CONCENTRATION UNITS:

(ug/t or $u g / R g$ ) ug $/ \mathrm{Rg}$

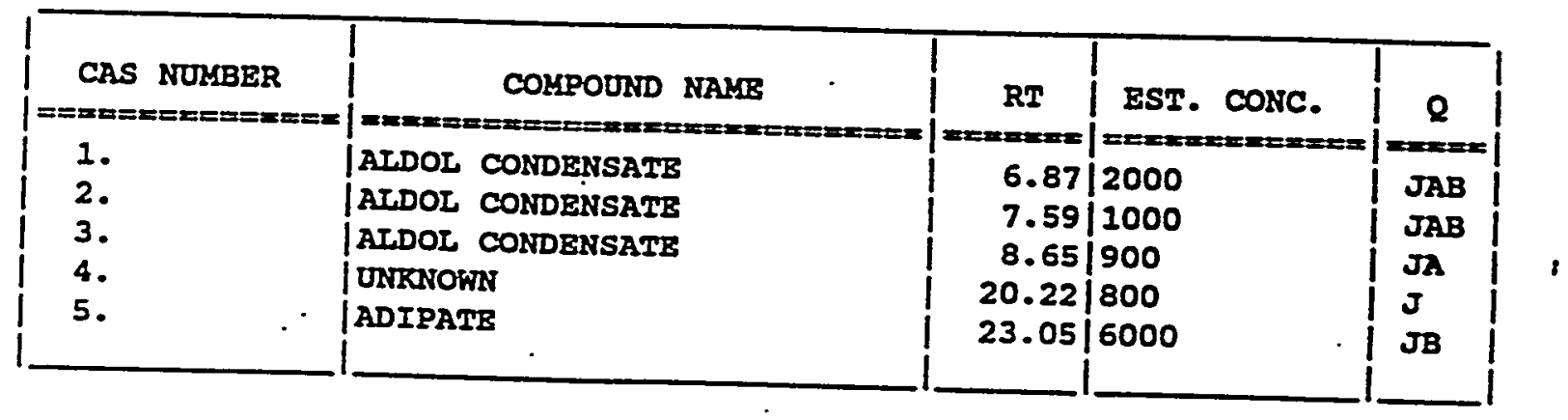


18

SEHTVOLATILB ORGANICS RNATYSIS SERET

TENTAYIVETY IDENTIEIED COMPOUNDS

Iab Wame: Roy P. Wegton, Inc. Work. Order: 0630-28-12-0000

Client: WSRC-GRACR ROAD

Matrix:

SOII

Sample it/vol: $30.6(\mathrm{~g} / \mathrm{mL}) \mathrm{G}$

Level: (low/med) 'LOW

\&oisture: not dec. 16 dec. Extraction: (SepF/Cont/Sonc) SONC

GPC Cleanup: $(Y / N) \underline{Y}$ $\mathrm{pH}=-7.0$
Lab Sample ID: 9002L606-006

Iab P11e ID: J030811

Date Received: . 02/23/90

Date Bxtracted: $02 / 28 / 90$

Date Analyzad: $03 / 09 / 90$

Dilution Factor: 1.11

Number TICs found: $\mathbf{5}$

CONCENTRATION ONITS:

(ug/L or ug/Rg) ug/Rg

\begin{tabular}{|c|c|}
\hline CAS NUMBER & COMPOUND NAME \\
\hline$==\Rightarrow x=2 x=x=x==0 x$ & | \\
\hline 1. & ALDOL CONDBNSATE \\
\hline 2 . & BENZATDEHYDE \\
\hline 3. & ALDOL CONDENSATB \\
\hline 4. & ONKNOWN \\
\hline 5. & ADIPATE \\
\hline & $\cdot$ \\
\hline
\end{tabular}

RT

$6.83 / 1000$

$7.16 / 300$

$7.57 \mid 400$

$20.20 / 500$

$23.03 / 200$ 
Lab Name: Roy F. Weston, Inc. Fork Order: 0630-28-12-0000

Client: WSRC-GRACE ROAD

Matrix:

SOIL

Sample wt/rol: $30.2(\mathrm{~g} / \mathrm{mL}) \mathrm{G}$

Level:

(low/med)

IOH .

\& Molstures not deç. 16 dec. Extraction: (SepF/cont/Sonc) sonc GPC Cleanup:. $\quad(Y / N) \underline{Y}$ $\mathrm{pH}:-7.0$
Lab Sample ID: 9002L606-007

Lab File ID: $\quad$ J030812

Date Recelved: $\underline{02 / 23 / 90}$

Date Extracted: $\underline{02 / 28 / 90}$

Date Analyzed: 03/09/90

Dilution Factor: 1.11

Number TICB found: 5

CONCENTRATION UNITS: (ug/L or ug/ $\mathrm{Rg}$ ) ug/ $/ \mathrm{kg}$

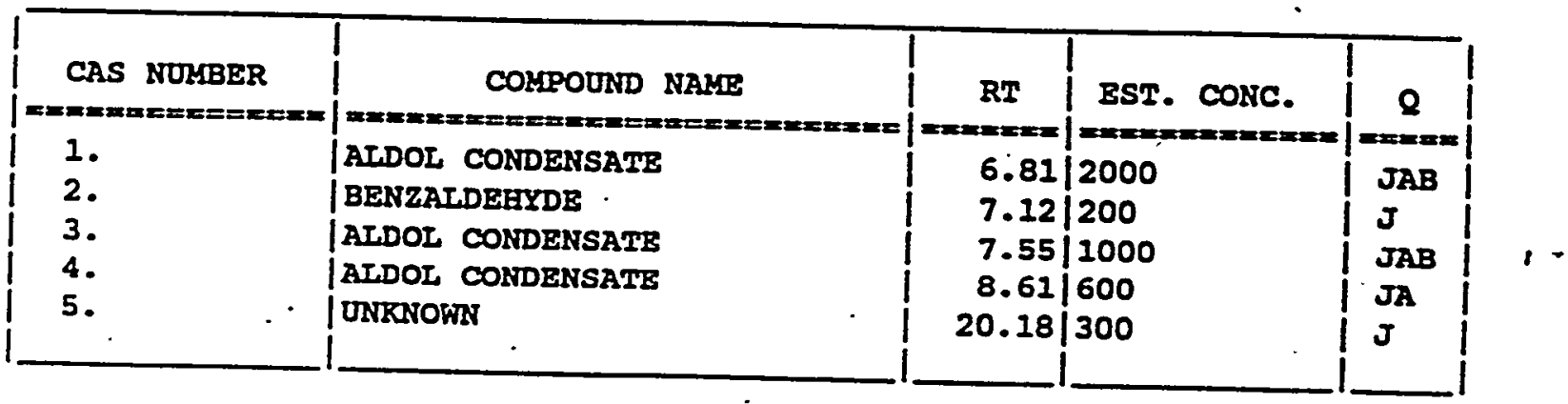


Lab Names Roy E. Heston, Inc. Fork Order: 0630-28-12-0000

Client: WSRC-GRICB ROAD

Natrix:

SOIL

Sample wt/vol:
Lab Sample ID: 9002L606-008

Iab File ID:

Date Received: $02 / 23 / 90$

Level: (low/med) IOW

\& Molsture: not dec. 16 dec.

Extraction: (SepF/Cont/Sonc) sonc

GPC Cleanup: (Y/N) $\underline{Y}$

$\mathrm{pH}: \quad 7.0$
Date Extracted: 02/28/90

Date Analyzed: $03 / 13 / 90$

Dilution Factor: 1.11
GRS-07-01A

Number TICs found: 5

CONCENTRATION UNITS:

\begin{tabular}{|c|c|c|c|c|}
\hline CAS NUMBBR & COMPOUND NAMB & RT & EST. CONC. & $\mathbf{Q}$ \\
\hline 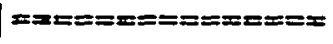 & le=se=" & $m=x=x=2$ & 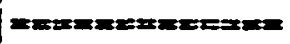 & $=m$ \\
\hline 1 & ALDOL CONDENSATB & 6.73 & 2000 & JAB \\
\hline 2. & ALDOL CONDENSATE & 7.47 & 1000 & JAB \\
\hline 3. & ALDOL CONDENSATE & 8.55 & 1000 & JA \\
\hline 4. & UNRNOWN & 10.07 & 400 & $\mathbf{J}$ \\
\hline 5. & UNKANOWN & 20.12 & 800 & $\mathbf{J}$ \\
\hline
\end{tabular}


Lab Nama: Roy E. Weaton. Inc. Work Order: 0630-28-12-0000

Client: HSRC-GRACR ROAD

Matrix:

Sample wt/rols

BOII

$$
30.1(g / m r) G
$$

Level: (low/med) IOX

\& Molsturei not dec. 11 dec.

Extraction: (Sepr/cont/Sonc) SONC

GPC Cleanup: $(Y / N) \underline{Y}$

pH:

7.0
Lab Sample ID: 9002L606-009

Lab File ID: J030903

Date Received: .02/23/90

Date Extracted: $02 / 28 / 90$

Date Analyzed: 03/09/90

Dilution Factor: 1.11

CONCENTRATION DNITS:

(ug/L or ug/kg) ug/ $\mathrm{kg}$

Number TICe found: 5

\begin{tabular}{|c|c|c|}
\hline RT & BST. COKC. \\
\hline 6.88 & 2000 \\
7.60 & 1000 \\
8.68 & 300 \\
$20.23 / 500$ \\
23.07 & 800 \\
\hline
\end{tabular}

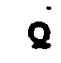

JAB

JAB

JA $\mathbf{J}$ JB 
Lab Name: Roy F. Weston. Inc. Work. Order: 0630-28-12-0000

Client: MSRC-GRACE ROAD

GRS-07-03

Matrix: soII

Sample wt/vol: $\quad 30.6(g / m \pi) ~ G$

Level.8 (low/med) Low .

8 Molsture: not dec. 13 dec. Extraction: (Sepg/Cont/Sonc) soNc GPC Cleanup: $\quad(Y / N) \underline{Y}$ $\mathrm{pH}: 7.0$
Lab Sample ID: 9002L606-010

Iab File ID: $\mathrm{J} 030905$

Dafe Received: $02 / 23 / 90$

Date Extracted: $02 / 28 / 90$

Date Analyzedz 03/09/90

Dilution Factor: 1.11

Number TICs found: 4

CONCBNTRATION UNITS:

(ug/L or ug/ $\mathrm{kg}$ ) ug/ $\mathrm{kg}$

\begin{tabular}{|c|c|}
\hline CAS NUMBER & COMPOUND NAKB \\
\hline 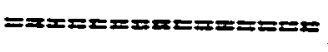 & 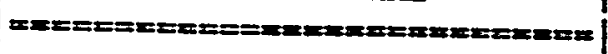 \\
\hline 1. & ALDOL CONDENSATB \\
\hline 2 . & ATDOL CONDENSATE \\
\hline 3. & AIDOL CONDENSATB \\
\hline 4. & DNKRYOWN \\
\hline
\end{tabular}

\begin{tabular}{|l|l|l|}
\hline RT & BST. CORC. & $\mathbf{Q}$ \\
\hline $6.86 \mid 2000$ & $\mathrm{JAB}$ \\
$7.58 \mid 900$ & $\mathrm{JAB}$ \\
$8.65 \mid 200$ & $\mathrm{JA}$ \\
$20.21 \mid 400$ & $\mathbf{J}$ \\
\hline
\end{tabular}


Lab Name: Roy F. Weston, Inc. Hork. Order: 0630-28-12-0000

|GRS-08-02

Client: WSRC-GRACB ROND

Matrix:

SOII

Sample wt/vol: $31.0(\mathrm{~g} / \mathrm{mL}) \mathrm{G}$

Level: (low/med) Low

8 Moisture: not dec. 14 dec.

Extraction: (SepR/Cont/Sonc) sonc

GPC Cleanup: $\quad(Y / N) \underline{Y}$ $\mathrm{pH}: \quad \mathbf{7 . 0}$
Lab Sample ID: 9002L606-012

Lab File ID: J030906

Date Receiveds $\underline{02 / 23 / 90}$

Date Extracted: 02/28/90

Date Analyzed: 03/09/90

Dilution Factor: 1.11

Number TICs found: 5

CONCENTRATION DNITS:

(ug/L or ug/Kg) ug/Rg

\begin{tabular}{|c|c|c|c|c|}
\hline $\begin{array}{c}\text { CAS NUMBER } \\
============ \pm=x\end{array}$ & 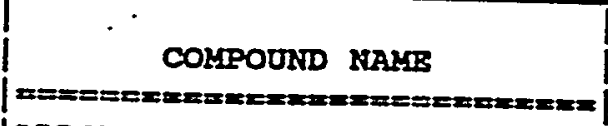 & 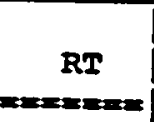 & | BST. CONC. & $=2$ \\
\hline $\begin{array}{l}1 . \\
2 . \\
3 . \\
4 . \\
5 .\end{array}$ & $\begin{array}{l}\text { |ALDOL CONDENSATE } \\
\text { |BENZALDEHYDE } \\
\text { |ALDOL CONDENSATB } \\
\text { ALDOL CONDENSATB } \\
\text { |UNKRONN }\end{array}$ & $\begin{array}{r}6.87 \\
7.18 \\
7.59 \\
8.67 \\
20.22\end{array}$ & $\mid \begin{array}{l}3000 \\
200 \\
1000 \\
1000 \\
500\end{array}$ & $\begin{array}{l}\text { J } \\
\text { JAB } \\
\text { JAB } \\
\text { JAB }\end{array}$ \\
\hline
\end{tabular}


BEATVOLATITB ORGANICS ANTIISIS SERBY

TENTATIVELY IDENTIEIED COMPOUNDS

Iab Name: Roy F. Fegton, Inc. Fork. Order: 0630-28-12-0000

GRS-08-03

Client: HSRC-GRACE RORD

Matrix:

SOIL

Sample wt/rol: $30.1(\mathrm{~g} / \mathrm{mr}) \mathrm{G}$

Level:. (low/med) LOW

- Molsturer not dec. 11 dec.

Extraction: (Sepr/Cont/Sonc) SONC

GPC Cleanup: $(Y / N) \underline{\underline{Y}}$

PE:

7.0
Lab Sample ID: 9002L606-013

Iab File ID: J030907

Date Received: $02 / 23 / 90$

Date Extracted: 02/28/90

Date Analyzed: 03/09/90

Dllution Factor: 1.11

Number TICs found: $\underline{5}$

CONCENLRATION ONITS:

(ug/L or ug/Rg) ug/ $\mathrm{kg}$

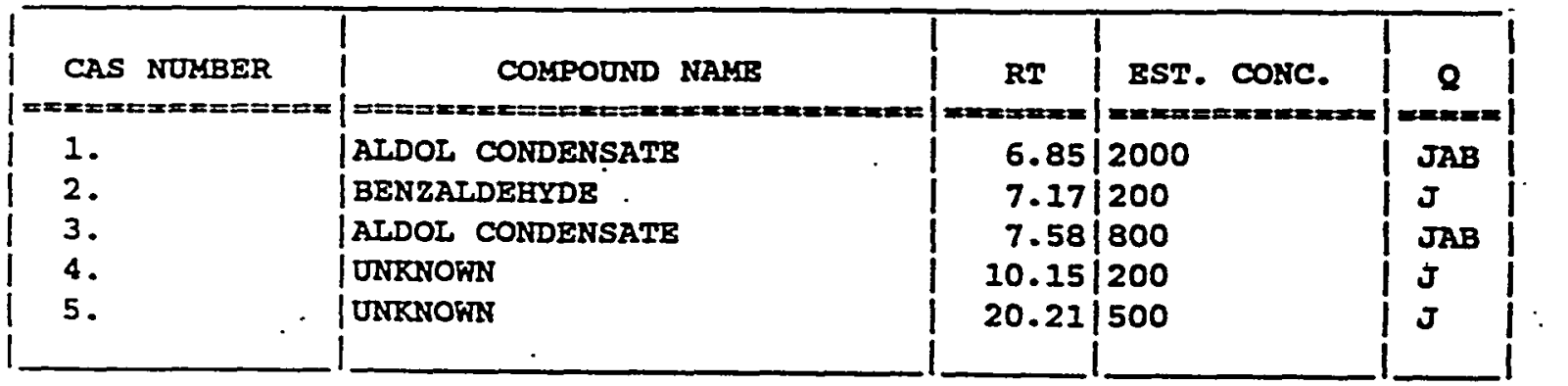


Lab Name: Roy F. Weaton. Inc. Fork Order: 0630-28-12-0000

Cllent: WSRC-GRACR ROAD

Matrixi

8OIL

Sample wt/rol: 30.0 $(\mathrm{g} / \mathrm{mL}) \mathrm{G}$

Level: (low/med) IOF

* Moleture: not dec. o dec.

Extraction: (Sepg/Cont/Sonc) sonc GPC Cleanup: $\quad(\Psi / N) \underline{\underline{Y}}$ pa: 7.0
Lab Sampla IDs 90LE0286-KB1

Iab F110 ID! $\quad$ 030804

Date Received: . 02/28/90

Date Bxtracted: $02 / 28 / 90$

Date Analyzed: $03 / 08 / 90$..

Dilution Factor: 1.11

Number TICs found: 4

CONCENTRATION UNITS:

(ug/L or ug/lgg) uq/ $\mathrm{hq}$

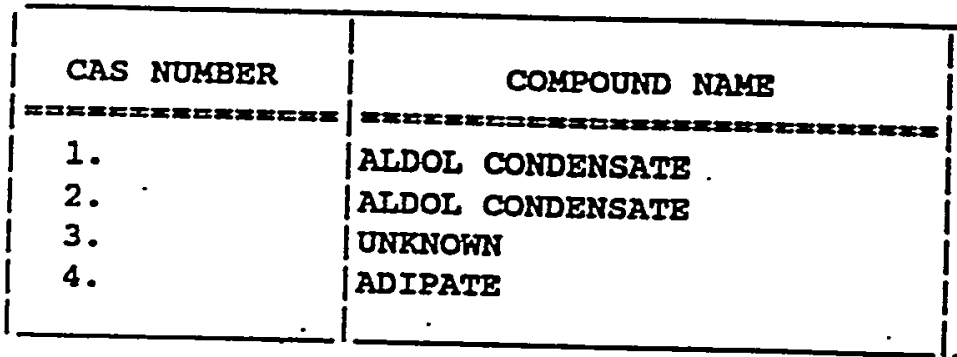

\begin{tabular}{|c|c|c|}
$\mid$ RT & EST. CONC. & $\mathbf{Q}$ \\
$5.85 \mid 300$ & $\mathrm{JA}$ \\
$6.55 \mid 400$ & $\mathrm{JA}$ \\
$7.57 \mid 200$ & $\mathrm{~J}$ \\
$22.30 \mid 30000$ & $\mathrm{~J}$ \\
\hline
\end{tabular}


Róy F. Weston, Inc. - Lionville Laboratory VOA ANALYTICAL DATA PACRAGE gOR WSRC-GRACE ROAD

DATE RECEIVED: $0 \overline{\overline{2}} / 23 / 90$

RFW LOT \$ $\$ 9002 L 606$

CLIENT ID

RFH * MTX PREP * COLLECTION EXTR/PREP

ANALYSIS

GRS-10-01

GRS-10-01

GRS-10-01

GRS-10-02

GRS-10-03

GRS-09-01

GRS-09-02

GRS-09-03

GRS-07-01

GRS-07-01A

GRS-07-02

GRS-07-03

GRS-08-02

GRS-08-03

GRS-08-03E

GRS-00-01B

GRS-00-01C

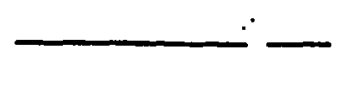

001 s 90LVF032

001 REP S 90LVW034

001 MS S $90 L \mathrm{LW036}$

002

003

004

005

006

007

00.8

009

010

012

013

014

015

016

902 Tw032

$90 L V * 032$

901 VTO32

90LVW032

90 LVพ032

90 LVR032

90LVพ032

90LVพ032

90LVพ036

90LVW033.

90LVस033

90LVR035

90 IVR035

90LVKo35

$02 / 20 / 90$

$02 / 20 / 90$

$02 / 20 / 90$

$02 / 20 / 90$

$02 / 20 / 90$

$02 / 20 / 90$

$02 / 20 / 90$

$02 / 20 / 90$

$02 / 21 / 90$

$02 / 21 / 90$

$02 / 21 / 90$

$02 / 21 / 90$

$02 / 21 / 90$

$02 / 21 / 90$

$02 / 21 / 90$

$02 / 21 / 90$

$02 / 21 / 90$

$N / A$

N/A

$N / A$

$\mathrm{N} / \mathrm{A}$

$\mathrm{N} / \mathrm{A}$

$\mathrm{N} / \mathrm{A}$

N/A

$N / A$

N/A

$N / A$

$N / A$

$N / A$

N/A

$N / A$

$\mathrm{N} / \mathrm{A}$

$\mathrm{N} / \mathrm{A}$

N/A

LAB QC:

VBLR

VBLIX

VBLX

VBLR

VBLR
MBI

MBI

MBI

MBI

MBI s 90LVw032

s 90LVW034

S 90 LVw036

S 90LVพ033

พ 90LVK035
$N / A$

$N / A$

N/A

N/A

$N / A$

$N / A$

$\mathrm{N} / \mathrm{A} \quad \mathrm{N} / \mathrm{A}$

N/A N/A
$02 / 28 / 90$

$03 / 02 / 90$

$03 / 05 / 90$

$02 / 28 / 90$

$02 / 28 / 90$

$02 / 28 / 90$

$02 / 28 / 90$

$02 / 28 / 90$

$02 / 28 / 90$

$03 / 01 / 90$

$03 / 01 / 90$

$03 / 05 / 90$

$03 / 01 / 90$

$03 / 02 / 90$

$03 / 02 / 90$

$03 / 02 / 90$

$03 / 02 / 90$

(




\section{W}

$$
\begin{aligned}
& \text { ROY F. WESTON, INC. } \\
& =\quad \text { Lionvilie Laboratory }
\end{aligned}
$$

CLIENT: WSRC-GRACE ROAD

RFW : 9002L606, GC/MS VOLATILE

SAMPLES RECEIVED: 02-23-90

H.O. : $0630-28-12$

\section{NARRATIVE}

The set of samples consisted of three water samples and twelve soil samples collected on $02-20,21-90$.

The samples were analyzed according to criteria set forth in SW 846 Method 8240 for TCL Volatile target compounds on

$02-28-90$ and $03-01,02,05-90$.

The following is a summary of the oc results accompanying these sample results and a description of any problems encountered
during their analysis:

1. Non-target compounds were not detected in these samples.

2. Three of sixty-six surrogate recoveries are outside the EPA QC limits. Samples with surrogate recoveries outside EPA CLP QC limits were inspected for data quality and reported. 3. All matrix spike recoveries are within EPA QC
limits.

4. The blanks contain Methylene chloride and Acetone at levels less than $5 x$ the CRQL. They also contained several other target compounds at levels equal to or less than the CRQL.

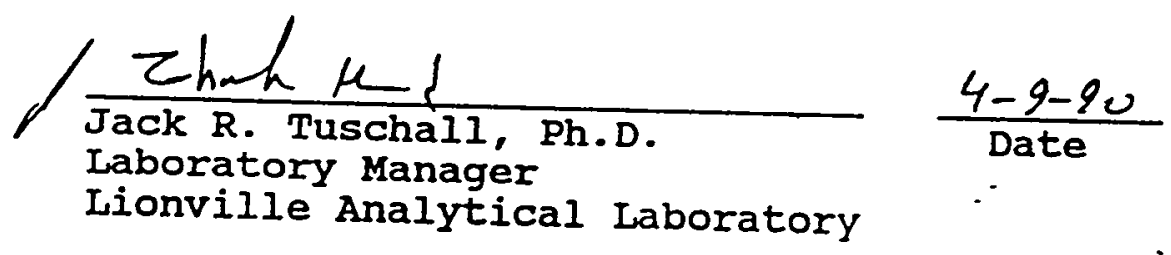


Roy F. Heston, Inc. Llonville Laboratory

Volatiles $b_{2}, C / M S, H S L$ List

Report Dates 04/02/90 $15: 02$

Cust ID: GRS-10-01 GRS-I0-01 GRS-10-01 GRS-10-02 GRS-10-03 GRS-09-01

Samplo

Information.

$\begin{array}{rr}\text { RFW : } & 001 \\ \text { Matrlx: } & \text { SOIL }\end{array}$

Matrix: SOTL

Unita: ug/Kg

001 REP

SOIL

1.06

$\mathrm{ug} / \mathrm{Kg}$
001 MS

soIL

1.00

ug/Rg

002
sOIL
1.00
$u g / \mathrm{Rg}$

105
110

878

100

103
99
100

89
93
77

105
103
110

12
$\mathrm{U}$

Chloromethane

Bromomethane

Vinyl Chloride

Chloroothane

Methylene Chlorlde

Acetone

Carbon DLsulfide

1,1-Dichloroethene

i) 1,1-Dichloroethane

i $1,2-D$ lchloroethene (total)

Chloroform

1,2-Dichloroethane

2-Butanone.

1,1,1-Trichloroethane

Carbon Tetrachloride

vinyl Acetato

Bromodlchloromethane

1,2-DLchloropropane

C1B-1, 3-Dlchioropropene

Trlchloroethenp

D1bromochloromethane

1, 1,2-Tx1chloroethane

Benzene

Trans-1, 3-Dlchloropropeno

Bromoform

4-Kethy 1-2-pentanone

2-Hexanone

Tetrachloroethene

1, 1,2,2-Tetrachlo roethano

* outside of BPA CLP ge IImita.

\begin{tabular}{|c|c|c|c|c|c|c|c|}
\hline 11 & $\mathbf{U}$ & 12 & $\mathbf{U}$ & 11 & $\mathbf{U}$ & 12 & $\mathbf{U}$ \\
\hline 11 & $\mathbf{U}$ & 12 & $\mathbf{U}$ & 11 & $\boldsymbol{U}$ & 12 & $\mathbf{U}$ \\
\hline 11 &. $\mathbf{U}$ & 12 & $\mathbf{v}$ & 11 & $\mathbf{U}$ & 12 & $\mathbf{U}$ \\
\hline 11 & $\boldsymbol{U}$ & 12 & $\mathbf{U}$ & 11 & $\mathbf{U}$ & 12 & U \\
\hline 14 & $\mathbf{B}$ & 8 & $\mathbf{B}$ & 6 & B & 28 & $\mathbf{B}$ \\
\hline 9 & JB & 9. & JB & 12 & $\mathbf{B}$ & 53 & B \\
\hline 1 & JB & 6 & $\mathbf{U}$ & 5 & $\mathbf{u}$ & 2 & $\mathbf{J B}$ \\
\hline 5 & $\boldsymbol{U}$ & 6 & $\mathbf{U}$ & 76 & 8 & 6 & $\mathbf{u}$ \\
\hline 5 & $\mathbf{U}$ & 6 & $\mathbf{U}$ & 5 & $\mathbf{U}$ & 6 & $\mathbf{U}$ \\
\hline 5 & $\mathbf{U}$ & 6 & $\mathbf{U}$ & 5 & $\boldsymbol{U}$ & 6 & $\mathbf{U}$ \\
\hline 5 & $\mathbf{U}$ & 3 & $\boldsymbol{J}$ & 5 & $\mathbf{U}$ & 6 & $\mathbf{U}$ \\
\hline 5 & $\mathbf{U}$ & 6 & $\mathbf{U}$ & 5 & $\mathbf{U}$ & 6 & $\boldsymbol{U}$ \\
\hline 11 & $\mathbf{U}$ & 12 & $\boldsymbol{U}$ & 11 & $\mathbf{u}$ & 12 & $\mathbf{v}$ \\
\hline 5 & $\mathbf{U}$ & 6 & $\mathbf{U}$ & 5 & $\mathbf{U}$ & 6 & $\mathbf{v}$ \\
\hline 5 & $\mathbf{U}$ & 6 & $\mathbf{U}$ & 5 & $\mathbf{U}$ & 6 & $\mathbf{U}$ \\
\hline 11 & $\mathbf{U}$ & 12 & $\mathbf{U}$ & 11 & $\mathbf{U}$ & 12 & $\mathbf{U}$ \\
\hline 5 & $\mathbf{u}$ & 6 & v & 5 & $\mathbf{U}$ & 6 & $\boldsymbol{U}$ \\
\hline 5 & $\mathbf{U}$ & 6 & $\mathbf{U}$ & 5 & $\mathbf{u}$ & 6 & $\boldsymbol{U}$ \\
\hline 5 & $\mathbf{U}$ & 6 & $\mathbf{U}$ & 5 & $\dot{\mathbf{u}}$ & 6 & $\mathbf{U}$ \\
\hline 5 & $\mathbf{U}$ & 6 & $\mathbf{U}$ & 107 & 8 & 6 & $\mathbf{U}$ \\
\hline 5 & $\mathbf{v}$ & 6 & $\mathbf{U}$ & 5 & $\mathbf{U}$ & 6 & $\mathbf{U}$ \\
\hline 5 & $\mathbf{u}$ & 6 & $\boldsymbol{U}$ & 5 & $\mathbf{U}$ & 6 & $\boldsymbol{U}$ \\
\hline 5 & $\boldsymbol{U}$ & 6 & $\mathbf{U}$ & 86 & 8 & 6 & $\mathbf{U}$ \\
\hline 5 & $\mathbf{U}$ & 6 & $\mathbf{U}$ & 5 & $\mathbf{U}$ & 6 & $\mathbf{U}$ \\
\hline 5 & $\boldsymbol{U}$ & 6 & $\mathbf{U}$ & 5 & $\mathbf{U}$ & 6 & $\mathbf{U}$ \\
\hline 11 & $\mathbf{U}$ & 12. & $\mathbf{U}$ & 11 & $\mathbf{U}$ & 12 & $\mathbf{U}$ \\
\hline 11 & $\boldsymbol{U}$ & 12 & $\mathbf{U}$ & 11 & $\mathbf{U}$ & 12 & $\mathbf{U}$ \\
\hline 5 & $\mathbf{U}$ & 6 & $\mathbf{U}$ & 5 & $\mathbf{U}$ & 6 & $\boldsymbol{U}$ \\
\hline 5 & $\mathbf{u}$ & $6^{\circ}$ & $\mathbf{v}$ & 5 & $\boldsymbol{U}$ & 6 & $\mathbf{U}$ \\
\hline
\end{tabular}

\begin{tabular}{|c|c|c|c|c|c|c|c|}
\hline 11 & $\mathbf{U}$ & 12 & $\mathbf{U}$ & 11 & $\mathbf{U}$ & 12 & $\mathbf{U}$ \\
\hline 11 & $\mathbf{U}$ & 12 & $\mathbf{U}$ & 11 & $\boldsymbol{U}$ & 12 & $\mathbf{U}$ \\
\hline 11 &. $\mathbf{U}$ & 12 & $\mathbf{v}$ & 11 & $\mathbf{U}$ & 12 & $\mathbf{U}$ \\
\hline 11 & $\boldsymbol{U}$ & 12 & $\mathbf{U}$ & 11 & $\mathbf{U}$ & 12 & U \\
\hline 14 & $\mathbf{B}$ & 8 & $\mathbf{B}$ & 6 & B & 28 & $\mathbf{B}$ \\
\hline 9 & JB & 9. & JB & 12 & $\mathbf{B}$ & 53 & B \\
\hline 1 & JB & 6 & $\mathbf{U}$ & 5 & $\mathbf{u}$ & 2 & $\mathbf{J B}$ \\
\hline 5 & $\boldsymbol{U}$ & 6 & $\mathbf{U}$ & 76 & 8 & 6 & $\mathbf{u}$ \\
\hline 5 & $\mathbf{U}$ & 6 & $\mathbf{U}$ & 5 & $\mathbf{U}$ & 6 & $\mathbf{U}$ \\
\hline 5 & $\mathbf{U}$ & 6 & $\mathbf{U}$ & 5 & $\boldsymbol{U}$ & 6 & $\mathbf{U}$ \\
\hline 5 & $\mathbf{U}$ & 3 & $\boldsymbol{J}$ & 5 & $\mathbf{U}$ & 6 & $\mathbf{U}$ \\
\hline 5 & $\mathbf{U}$ & 6 & $\mathbf{U}$ & 5 & $\mathbf{U}$ & 6 & $\boldsymbol{U}$ \\
\hline 11 & $\mathbf{U}$ & 12 & $\boldsymbol{U}$ & 11 & $\mathbf{u}$ & 12 & $\mathbf{v}$ \\
\hline 5 & $\mathbf{U}$ & 6 & $\mathbf{U}$ & 5 & $\mathbf{U}$ & 6 & $\mathbf{v}$ \\
\hline 5 & $\mathbf{U}$ & 6 & $\mathbf{U}$ & 5 & $\mathbf{U}$ & 6 & $\mathbf{U}$ \\
\hline 11 & $\mathbf{U}$ & 12 & $\mathbf{U}$ & 11 & $\mathbf{U}$ & 12 & $\mathbf{U}$ \\
\hline 5 & $\mathbf{u}$ & 6 & v & 5 & $\mathbf{U}$ & 6 & $\boldsymbol{U}$ \\
\hline 5 & $\mathbf{U}$ & 6 & $\mathbf{U}$ & 5 & $\mathbf{u}$ & 6 & $\boldsymbol{U}$ \\
\hline 5 & $\mathbf{U}$ & 6 & $\mathbf{U}$ & 5 & $\dot{\mathbf{u}}$ & 6 & $\mathbf{U}$ \\
\hline 5 & $\mathbf{U}$ & 6 & $\mathbf{U}$ & 107 & 8 & 6 & $\mathbf{U}$ \\
\hline 5 & $\mathbf{v}$ & 6 & $\mathbf{U}$ & 5 & $\mathbf{U}$ & 6 & $\mathbf{U}$ \\
\hline 5 & $\mathbf{u}$ & 6 & $\boldsymbol{U}$ & 5 & $\mathbf{U}$ & 6 & $\boldsymbol{U}$ \\
\hline 5 & $\boldsymbol{U}$ & 6 & $\mathbf{U}$ & 86 & 8 & 6 & $\mathbf{U}$ \\
\hline 5 & $\mathbf{U}$ & 6 & $\mathbf{U}$ & 5 & $\mathbf{U}$ & 6 & $\mathbf{U}$ \\
\hline 5 & $\boldsymbol{U}$ & 6 & $\mathbf{U}$ & 5 & $\mathbf{U}$ & 6 & $\mathbf{U}$ \\
\hline 11 & $\mathbf{U}$ & 12. & $\mathbf{U}$ & 11 & $\mathbf{U}$ & 12 & $\mathbf{U}$ \\
\hline 11 & $\boldsymbol{U}$ & 12 & $\mathbf{U}$ & 11 & $\mathbf{U}$ & 12 & $\mathbf{U}$ \\
\hline 5 & $\mathbf{U}$ & 6 & $\mathbf{U}$ & 5 & $\mathbf{U}$ & 6 & $\boldsymbol{U}$ \\
\hline 5 & $\mathbf{u}$ & $6^{\circ}$ & $\mathbf{v}$ & 5 & $\boldsymbol{U}$ & 6 & $\mathbf{U}$ \\
\hline
\end{tabular}

\begin{tabular}{|c|c|c|c|c|c|c|c|}
\hline 11 & $\mathbf{U}$ & 12 & $\mathbf{U}$ & 11 & $\mathbf{U}$ & 12 & $\mathbf{U}$ \\
\hline 11 & $\mathbf{U}$ & 12 & $\mathbf{U}$ & 11 & $\boldsymbol{U}$ & 12 & $\mathbf{U}$ \\
\hline 11 &. $\mathbf{U}$ & 12 & $\mathbf{v}$ & 11 & $\mathbf{U}$ & 12 & $\mathbf{U}$ \\
\hline 11 & $\boldsymbol{U}$ & 12 & $\mathbf{U}$ & 11 & $\mathbf{U}$ & 12 & U \\
\hline 14 & $\mathbf{B}$ & 8 & $\mathbf{B}$ & 6 & B & 28 & $\mathbf{B}$ \\
\hline 9 & JB & 9. & JB & 12 & $\mathbf{B}$ & 53 & B \\
\hline 1 & JB & 6 & $\mathbf{U}$ & 5 & $\mathbf{u}$ & 2 & $\mathbf{J B}$ \\
\hline 5 & $\boldsymbol{U}$ & 6 & $\mathbf{U}$ & 76 & 8 & 6 & $\mathbf{u}$ \\
\hline 5 & $\mathbf{U}$ & 6 & $\mathbf{U}$ & 5 & $\mathbf{U}$ & 6 & $\mathbf{U}$ \\
\hline 5 & $\mathbf{U}$ & 6 & $\mathbf{U}$ & 5 & $\boldsymbol{U}$ & 6 & $\mathbf{U}$ \\
\hline 5 & $\mathbf{U}$ & 3 & $\boldsymbol{J}$ & 5 & $\mathbf{U}$ & 6 & $\mathbf{U}$ \\
\hline 5 & $\mathbf{U}$ & 6 & $\mathbf{U}$ & 5 & $\mathbf{U}$ & 6 & $\boldsymbol{U}$ \\
\hline 11 & $\mathbf{U}$ & 12 & $\boldsymbol{U}$ & 11 & $\mathbf{u}$ & 12 & $\mathbf{v}$ \\
\hline 5 & $\mathbf{U}$ & 6 & $\mathbf{U}$ & 5 & $\mathbf{U}$ & 6 & $\mathbf{v}$ \\
\hline 5 & $\mathbf{U}$ & 6 & $\mathbf{U}$ & 5 & $\mathbf{U}$ & 6 & $\mathbf{U}$ \\
\hline 11 & $\mathbf{U}$ & 12 & $\mathbf{U}$ & 11 & $\mathbf{U}$ & 12 & $\mathbf{U}$ \\
\hline 5 & $\mathbf{u}$ & 6 & v & 5 & $\mathbf{U}$ & 6 & $\boldsymbol{U}$ \\
\hline 5 & $\mathbf{U}$ & 6 & $\mathbf{U}$ & 5 & $\mathbf{u}$ & 6 & $\boldsymbol{U}$ \\
\hline 5 & $\mathbf{U}$ & 6 & $\mathbf{U}$ & 5 & $\dot{\mathbf{u}}$ & 6 & $\mathbf{U}$ \\
\hline 5 & $\mathbf{U}$ & 6 & $\mathbf{U}$ & 107 & 8 & 6 & $\mathbf{U}$ \\
\hline 5 & $\mathbf{v}$ & 6 & $\mathbf{U}$ & 5 & $\mathbf{U}$ & 6 & $\mathbf{U}$ \\
\hline 5 & $\mathbf{u}$ & 6 & $\boldsymbol{U}$ & 5 & $\mathbf{U}$ & 6 & $\boldsymbol{U}$ \\
\hline 5 & $\boldsymbol{U}$ & 6 & $\mathbf{U}$ & 86 & 8 & 6 & $\mathbf{U}$ \\
\hline 5 & $\mathbf{U}$ & 6 & $\mathbf{U}$ & 5 & $\mathbf{U}$ & 6 & $\mathbf{U}$ \\
\hline 5 & $\boldsymbol{U}$ & 6 & $\mathbf{U}$ & 5 & $\mathbf{U}$ & 6 & $\mathbf{U}$ \\
\hline 11 & $\mathbf{U}$ & 12. & $\mathbf{U}$ & 11 & $\mathbf{U}$ & 12 & $\mathbf{U}$ \\
\hline 11 & $\boldsymbol{U}$ & 12 & $\mathbf{U}$ & 11 & $\mathbf{U}$ & 12 & $\mathbf{U}$ \\
\hline 5 & $\mathbf{U}$ & 6 & $\mathbf{U}$ & 5 & $\mathbf{U}$ & 6 & $\boldsymbol{U}$ \\
\hline 5 & $\mathbf{u}$ & $6^{\circ}$ & $\mathbf{v}$ & 5 & $\boldsymbol{U}$ & 6 & $\mathbf{U}$ \\
\hline
\end{tabular}

003
soIL
1.00
$u g / \mathrm{kg}$

004

sort.

1.00 ug/ Kg

108

108
105
112

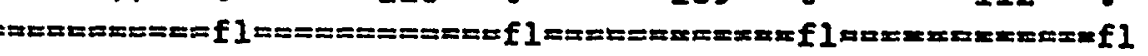

\begin{tabular}{rl}
12 & $U$ \\
12 & $U$ \\
12 & $U$ \\
12 & $U$ \\
33 & $B$ \\
23 & $B$ \\
3 & $J B$ \\
6 & $U$ \\
6 & $U$ \\
6 & $U$ \\
6 & $U$ \\
6 & $U$ \\
12 & $U$ \\
6 & $U$ \\
6 & $U$ \\
12 & $U$ \\
6 & $U$ \\
6 & $U$ \\
6 & $U$ \\
6 & $U$ \\
6 & $U$ \\
6 & $U$ \\
6 & $U$ \\
6 & $U$ \\
6 & $U$ \\
12 & $U$ \\
12 & $U$ \\
6 & $U$ \\
6 & $U$ \\
& $U$ \\
\hline
\end{tabular}

$11 \mathrm{U}$

$11 \mathrm{v}$

110

$11 \mathrm{U}$

$12 \mathrm{~B}$

17 B

$1 \mathrm{JB}$

50

5 U

5 U

50

5 U

110

5 U

5 U

$11 \mathrm{U}$

5 U

5 U

5 U

5 บ

5 ป

5 v

5 v

5 U

5 ป

11 U

- $11 \mathrm{~V}$

5 U

50 
RFWI

001

001 REP

001 MS

002

003

004

Chlorobenzene

Bthylbenzene

5 U

styrene

Xylene (total)

50

6

6 U

$\begin{array}{rr}86 & \\ 96 & 8 \\ 5 & 0 \\ 5 & 0 \\ 5 & 0\end{array}$

6 U

6 U

$6 \cdot \mathrm{U}$

6 U

6 U

$\begin{array}{llll}1 & J & 5 & 0 \\ 6 & U & 5 & 0 \\ 6 & 0 & 5 & 0 \\ 6 & 0 & 5 & 0 \\ 6 & U & 5 & 0\end{array}$

苦 
Roy F. Weston, Inc. ionville Laboratory volat lles by $c /$ MS, HSL List

Report Dates 04/02/90 15:02 RFW Batch Number: 9002L606 Cllent: WSRC-GRACE ROAD Work Order: 0630-28-12-0000 Page: 2a

Cust ID: GRS-09-02 GRS-09-03

sample

Information

$\begin{array}{rrr}\text { RFWl: } & 005 & 006 \\ \text { Matrlx: } & \text { soIL } & \text { SOIL } \\ \text { D.F. } & 1.00 & 1.00\end{array}$

$\cdot \cdot \cos$

GRS-07-01

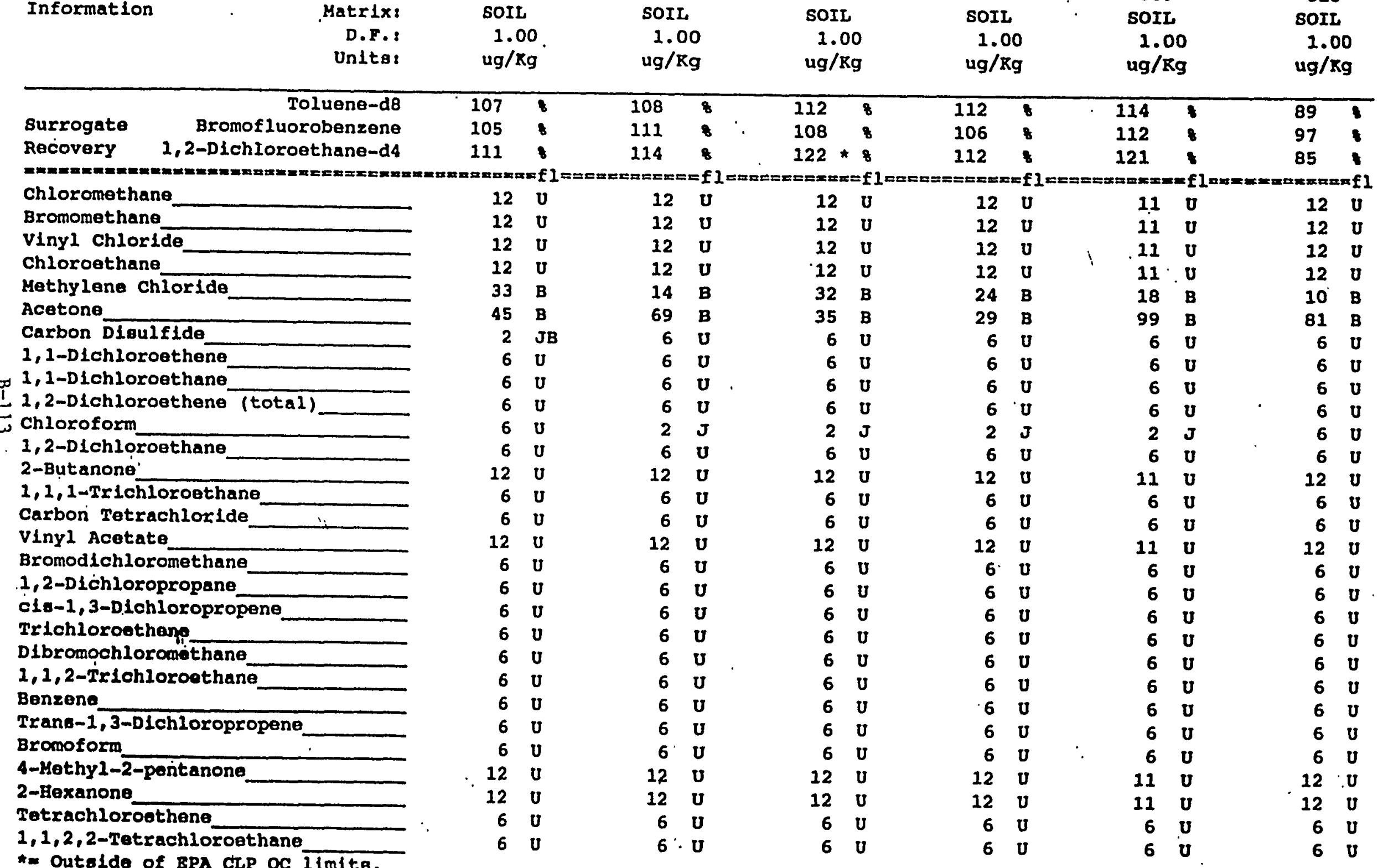


RFW :

005

$-006$

007

008

009

Chlorobenzene

$\begin{array}{ll}6 & U \\ 6 & U \\ 6 & U \\ 6 & U \\ 6 & U\end{array}$

$6 \mathrm{U}$

60

13

6 U

$6 \mathrm{U}$

6 U

$6 \mathrm{U}$

styrene

$6 \mathrm{U}$

$6 \mathrm{U}$

6 U

xylene (total)

* = Outbide of BPA CLP QC IImitB.

$\begin{array}{ll}6 & U \\ 6 & U\end{array}$

6 U

$6 \mathrm{U}$

60

6 U

$3 \mathrm{~J}$

6 U

$6 \mathrm{U}$

6 U

$\begin{array}{ll}6 & 0 \\ 6 & 0\end{array}$

$6 \mathrm{U}$

60

6 . U

$\stackrel{\infty}{1}$ 
Cust ID: GRS-08-02

Sample

Information

Hatrix: SOIL
D.P.:

Toluene-d8

Burrogate

Recovery

\section{Bromofluorobenzene} 1,2-Dichloroethane-d4

108

\section{Chloromethane}

Bromomethane

vinyl Chloride

Chloroethane

Mothylene Chloride

Acetone

Carbon Dlaulfide

1,1-Dichloroothene

\% 1,1-Dlchloroethane

Dichloroethene (total)

Chloroform

1,2-DLohloroethane

2-Butanoni.

1,1,1-irichloroethane

Carbon Tetrachloride

Vinyl Acetate

Bromodlchloromethane

1,2-Dlchloropropane

c1e-1, 3-Dlchloropropene

Trichloroethene

Dlbromochloromethane

1,1,2-Trlohloroethane

Benzene

Trans-1,3-D1chloropropene

Bromoform

4-Methy 1-2-pentanone

2-Hexanone

Tetrachloroethene

1, 1,2,2-Tetrachloroethane

* Outalde of IPA CLP QC IImita.

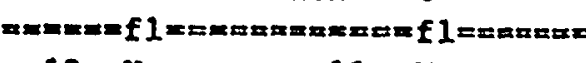

GRS-D8-03

GRS-08-03E

\begin{abstract}
013
\end{abstract}

\begin{abstract}
1.00
\end{abstract}
$\mathrm{ug} / \mathrm{Kg}$

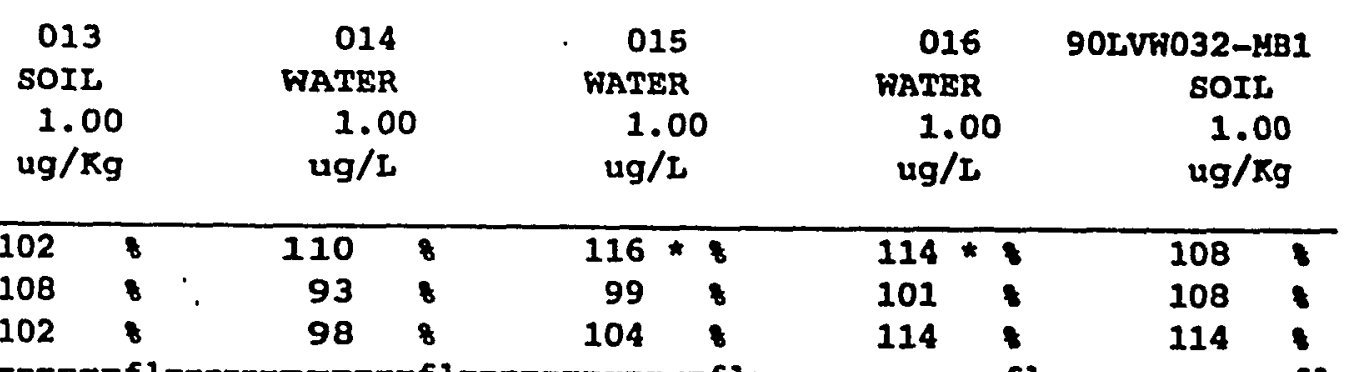

\begin{tabular}{|c|c|c|c|c|}
\hline 12 & $\mathbf{U}$ & 11 & $\boldsymbol{U}$ & 10 \\
\hline 12 & $\mathbf{U}$ & 11 & $\mathbf{U}$ & 10 \\
\hline 12 & $\mathbf{U}$ & 11 & $\mathbf{v}$ & 10 \\
\hline 12 & $\mathbf{U}$ & 11 & $\mathbf{U}$ & 10 \\
\hline 13 & B & 15 & B & 4 \\
\hline 49 & B & 61 & B & 11 \\
\hline 6 & $\mathbf{U}$ & 6 & $\mathbf{U}$ & 5 \\
\hline 6 & $\mathbf{U}$ & 6 & $\mathbf{U}$ & 5 \\
\hline 6 & $\mathbf{U}$ & 6 & $\mathbf{v}$ & 5 \\
\hline 6 & $\mathbf{v}$ & 6 & $\mathbf{v}$ & 5 \\
\hline 6 & $\mathbf{U}$ & 6 & $\mathbf{U}$ & 5 \\
\hline 6 & $\mathbf{v}$ & 6 & $\mathbf{U}$ & 5 \\
\hline 12 & $\boldsymbol{v}$ & 11 & $\mathbf{v}$ & 10 \\
\hline 6 & $\mathbf{U}$ & 6 & $\mathbf{U}$ & 5 \\
\hline 6 & $\mathbf{U}$ & 6 & $\mathbf{U}$ & 5 \\
\hline 12 & $\mathbf{v}$ & 11 & $\mathbf{U}$ & 10 \\
\hline 6 & $\mathbf{U}$ & 6 & $\mathbf{U}$ & 5 \\
\hline 6 & $\mathbf{U}$ & 6 & $\mathbf{U}$ & 5 \\
\hline 6 & $\mathbf{U}$ & 6 & $\mathbf{U}$ & 5 \\
\hline 6 & $\mathbf{U}$ & 6 & $\mathbf{U}$ & 5 \\
\hline 6 & $\mathbf{U}$ & 6 & $\mathbf{U}$ & 5 \\
\hline 6 & $\mathbf{v}$ & 6 & $\boldsymbol{U}$ & 5 \\
\hline 6 & v & 6 & $\mathbf{U}$ & 5 \\
\hline 6 & $\mathbf{U}$ & 6 & $\mathbf{U}$ & 5 \\
\hline 6 & U & 6 & $\mathbf{U}$ & 5 \\
\hline 12 & $\mathbf{U}$ & 11 & $\mathbf{U}$ & 10 \\
\hline 12 & $\mathbf{U}$ & 11 & 0 & 10 \\
\hline 6 & U & 6 & $\mathbf{U}$ & 5 \\
\hline 6 & $\mathbf{U}$ & 6 & U. & 5 \\
\hline
\end{tabular}

GRS-00-018 GRS-00-01C

vBLX

$10 \mathrm{U} \quad 10 \mathrm{U} \quad 10 \mathrm{U}$.

100

$10 \mathrm{U}$

$10 \mathrm{U}$

5 B

$10 \mathrm{U}$

5 U

5 U

50

5 U

50

5 U

$10 \mathrm{U}$

5 U

5 U

$10 \mathrm{U}$

5 U

5 U

5 U

5 U

5 U

5 U

50

50

5 U

$10 \mathrm{v}$

$10 \mathrm{U}$

$5 \mathrm{U}$
$10 \mathrm{U}$

$10 \mathrm{U}$

$10 \mathrm{U}$

4 JB

$45 \mathrm{~B}$

50

5 U

5 U

50

5 U

5 U

$10 \mathrm{U}$

$5 \mathrm{U}$.

5 U

$10 \mathrm{U}$

50

50

50

50

5 U

5 U

5 U

5 U

5 U

$10 \mathrm{~V}$

$10 \mathrm{U}$

$5 \mathrm{U}$

50
$10 \mathrm{U}$

$10 \mathrm{U}$

$10 \mathrm{~V}$

9

10

$1 \mathrm{~J}$

$5 \mathrm{v}$

50

50

$5 v$

$5 v$

$10 v$

$5 \mathrm{U}$

50

$10 \mathrm{~V}$

$5 \mathrm{v}$

5 v

5 U

50

5 v

50

50

5 U

50

$10 \mathrm{U}$

$10 \mathrm{U}$

50

5 U 
Roy F. Weston, Inc. - LLonville Laboratory

Volatlles by GC/MS, HSL Llat

Report Date: 04/0\%/90 15:02

Sample

Information

\begin{abstract}
Cugt ID: ' VBLK
\end{abstract}
VBLK

VBLK

VBLK

RFWI 90LVW034-MB1 90LVW036-MB1 90LVW033-MB1 9OLVK035-MB1

$\begin{array}{rcccc}\text { Matrix: } & \text { SOIL } & \text { SOIL } & \text { SOIL } & \text { WATER } \\ \text { D.F.1 } & 1.00 & 1.00 & 1.00 & 1.00 \\ \text { Units: } & \mathrm{ug} / \mathrm{Kg} & \mathrm{ug} / \mathrm{Kg} & \mathrm{ug} / \mathrm{Kg} & \mathrm{ug} / \mathrm{L}\end{array}$

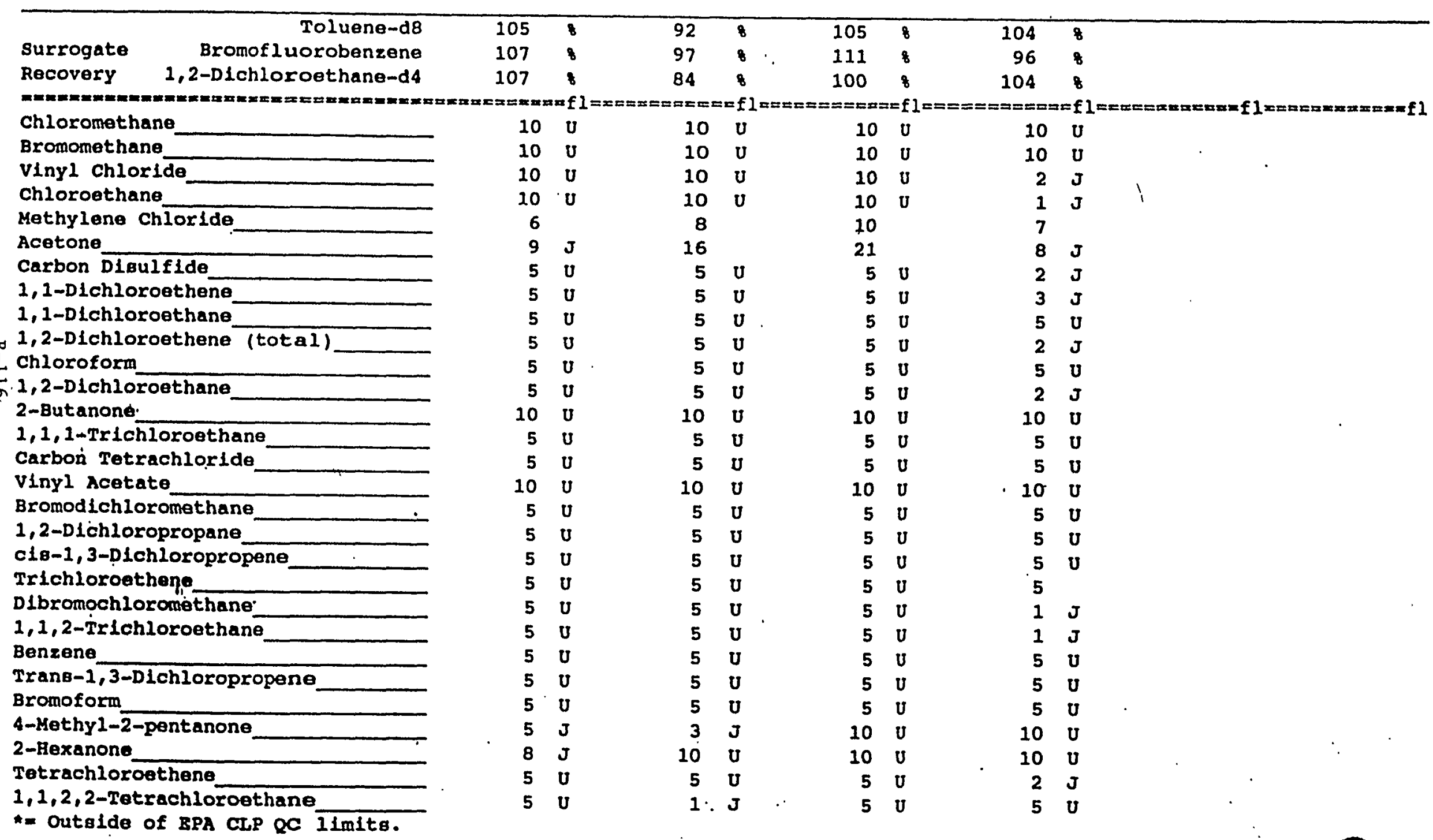




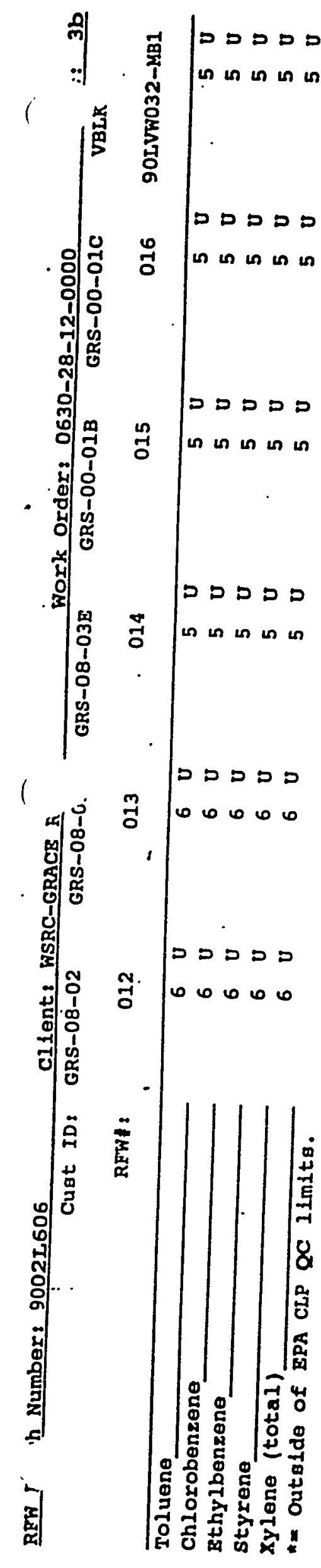


RFWH 90LVW034-MB1 90LVW036-MBI 90LVW033-MB1 90LVK035-MB1

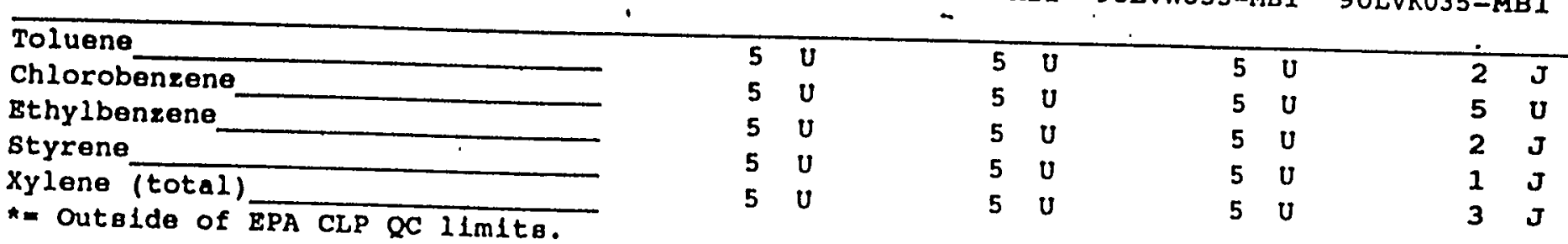


WESTON ANALYT $\triangle S$

DIOXINS/FURANS DATA SUMMARY

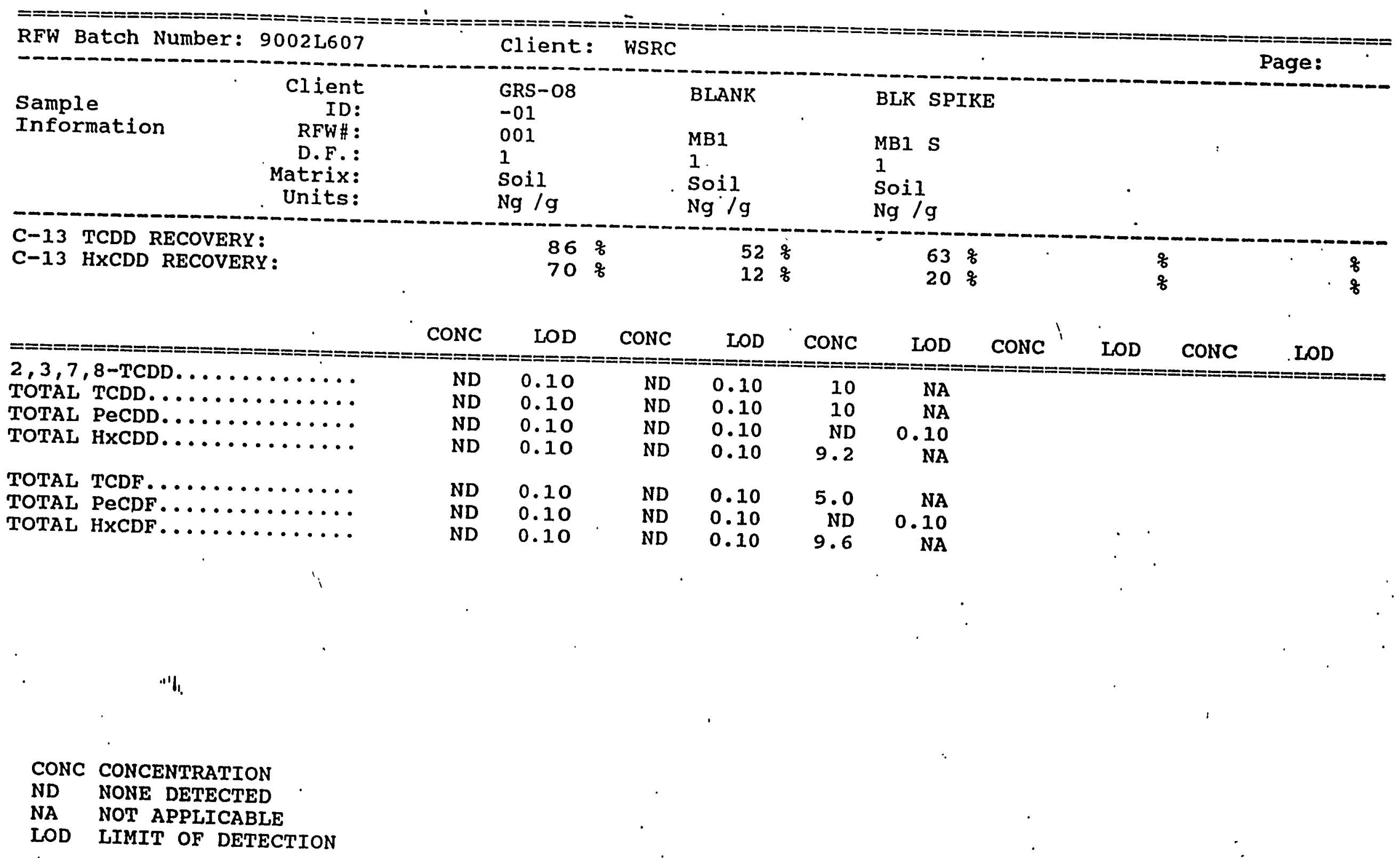




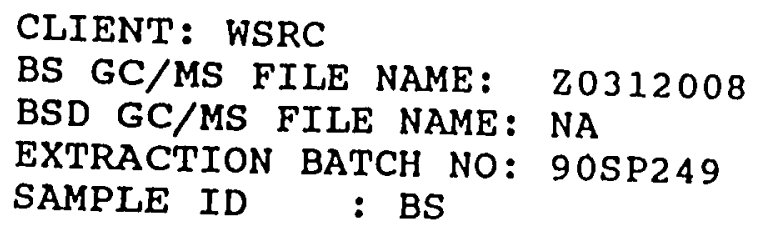

\begin{tabular}{|c|c|c|c|c|c|c|c|}
\hline $\begin{array}{l}\text { COMPOUND } \\
===========================0\end{array}$ & $\begin{array}{r}\text { AMOUNT } \\
\text { SPIKED } \\
\text { ng } \\
======\end{array}$ & $\begin{array}{l}\text { SAMPLE } \\
\text { WT./VOL. } \\
\quad \mathrm{g} \\
========\end{array}$ & $\begin{array}{l}\text { SPIKE } \\
\text { CONC. } \\
\text { Ng/g } \\
=======\end{array}$ & $\begin{array}{l}\text { CONC. } \\
\text { FOUND } \\
\mathrm{Ng} / \mathrm{g}\end{array}$ & $\begin{array}{l}\text { BLANK } \\
\text { CONC. }\end{array}$ & $\stackrel{q}{q}$ & \\
\hline $2,3,7,8-\mathrm{TCDD}$ & 50 & 5.0 & 10 & 10.0 & $=====$ & $=====$ & $====x=$ \\
\hline $1,2,3,6,7,8-\mathrm{HXCDD}$ & 50 & 5.0 & 10 & 9.2 & ND ${ }^{\prime}$ & 92 & . \\
\hline OCDD & NA & NA & NA & NA & NA & NA & \\
\hline $2,3,7,8-\mathrm{TCDF}$ & 50 & 5.0 & 10 & 5.0 & ND & 50. & \\
\hline $1,2,3,6,7,8-\mathrm{H} \times \mathrm{CDF}$ & 50 & 5.0 & 10 & 9.6 & ND & 96 & \\
\hline OCDF & $\mathrm{NA}$ & NA & $\cdot \mathrm{NA}$ & $\mathrm{NA}$ & NA & NA & \\
\hline
\end{tabular}


WESER

CLIENT: WSRC-GRACE ROAD

RFW : $9002 \mathrm{~T} \div 07$

พ.O. * : $0630=28-12$
ROY F. RESTON, INC.

Lionville Laboratory

SAMPLES RECEIVED: $02-23-90$.

\section{INORGANIC NARRATIVE}

The following is a summary of the quality control results and a description of any problems encountered during the analysis of
this batch of samples:

1. All sample holding times as required by 40 CFR 136 were met for water samples. Note: Holding times for soil samples have not been promulgated by the USEPA.

2. All preparation blanks were analyzed below the required

3. All calibration verification checks were within the required control limits of 90-100\%. Within the verification is performed using independent standards. 4. All laboratory control standards (blank spikes) were
within the control limits of $80-120 \%$.

5. ' All replicate results were within the $20 \%$ guidance limit.

6. The analytical methods applied by the laboratory, unless otherwise requested, for all inorganic analyses are Water and Methods for the Methods for the analysis of of Water and Wastewater 16 ed. Test Methods for cyanide. Sow 787 was followed for the analysis of

NOTE: For solid samples, all results are reported on a dry weight basis.

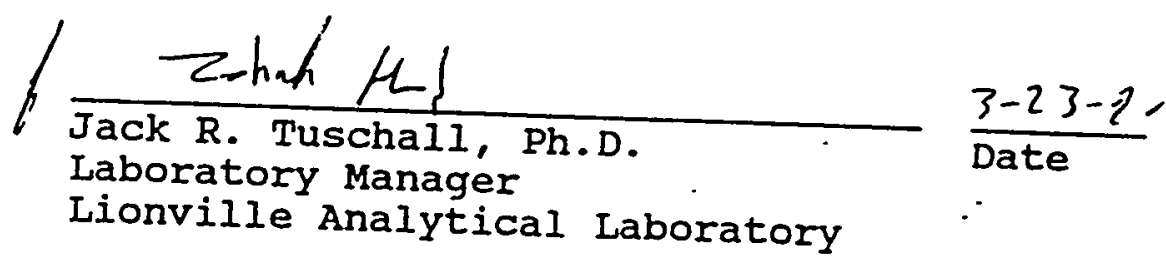


$\underset{N}{\stackrel{1}{N}}$

$$
-\infty
$$




\section{Roy F. Weston, Inc. - Lionville Laboratory $\doteq \quad$ INORGANIC ANALYTICAL DATA PACRAGR BOR WSRC-GRACE ROAD}

DATE RECEIVED: $02 / 23 / 90$

RFW LOT * :9002L607

CLIENT ID / ANALYSIS RFW * IMX PREP * COLIRCTION EXTR/PREP

ANALYSIS

GRS-08-01

8 SOLIDS

$\&$ SOLIDS

TOTAL CYANIDE

SULFIDE

001
001 REP
001
001

$\begin{array}{lll}\text { s } & 90 L \& 5040 & 02 / 21 / 90 \\ \text { s } & 90 L \& 5040 & 02 / 21 / 90 \\ \text { s } & 90 L C 095 & 02 / 21 / 90 \\ \text { s } & 90 L S D 022 & 02 / 21 / 90\end{array}$

$03 / 08 / 90$

$03 / 08 / 90$

$03 / 04 / 90$

$03 / 15 / 90$

$03 / 08 / 90$

$03 / 08 / 90$

$03 / 05 / 90$

$03 / 15 / 90$

IAB QC:

\begin{abstract}
TOTAL CYANIDE
TOTAL CYANIDE

TOTAL CYANIDE

TOTAL CYANIDE

TOTAL CYANIDE

TOTAL CYANIDE

TOTAL CYANIDE

TOTAL CYANIDE

TOTAL CYANIDE

SULFIDE

SULFIDE

SULFIDE

SULFIDE

SULFIDE

SULFIDE

SULFIDE

SULFIDE
\end{abstract}

CCB
CCB
CCV I
CCV I
ICB
ICV I
ICS I
ICS I
MB1
MB1
MB1 BS
MB2
MB2 BS
MB3
MB3 BS
MB4
MB4 BS

$\begin{array}{ll}W & 90 L C 095 \\ W & 90 L C 095 \\ W & 90 L C 095 \\ W & 90 L C 095 \\ W & 90 L C 095 \\ W & 90 L C 095 \\ W & 90 L C 095 \\ W & 90 L C 095 \\ W & 90 L C 095 \\ \text { S } & 90 L S D 022 \\ \text { S } & 90 L S D 022 \\ \text { S } & 90 L S D 022 \\ \text { S } & 90 L S D 022 \\ \text { S } & 90 L S D 022 \\ \text { S } & 90 L S D 022 \\ \text { S } & 90 L S D 022 \\ \text { S } & 90 L S D 022\end{array}$

\begin{abstract}
N/A
$N / A$

N/A

$N / A$

N/A

N/A

N/A

N/A

$N / A$

$N / A$

N/A

$N / A$

$N / A$

N/A

$N / A$

N/A.

$N / A$
\end{abstract}

$03 / 04 / 90$

$03 / 04 / 90$

$03 / 04 / 90$

$03 / 04 / 90$

$03 / 04 / 90$

$03 / 04 / 90$

$03 / 04 / 90$

$03 / 04 / 90$

$03 / 04 / 90$

$03 / 15 / 90$

$03 / 15 / 90$

$03 / 15 / 90$

$03 / 15 / 90$

$03 / 15 / 90$

$03 / 15 / 90$

$03 / 15 / 90$

$03 / 15 / 90$
$03 / 05 / 90$

03/05/90

$03 / 05 / 90$

03/05/90

$03 / 05 / 90$

03/05/90

03/05/90

$03 / 05 / 90$

$03 / 05 / 90$

$03 / 15 / 90$

$03 / 15 / 90$

$03 / 15 / 90$

$03 / 15 / 90$

$03 / 15 / 90$

$03 / 15 / 90$

$03 / 15 / 90$

$03 / 15 / 90$ 
ROY F. WESTON, INC.

GLOSSARY OF TERMS - INORGANIC REPORTS

\section{DATA QUAIIEIERS}

$U$ - Indifcates that the parameter was not detected at or above the reported limit. The associated numerical value is the sample detection limit.

* - Inaicates that the original sample result is greater than $4 x$ the spike amount added. The USEPA-CLP has determined that spike results on samples where this occurs may be unreliable and, therefore, the control limits are not applicable.

\section{ABBREVIATIONS}

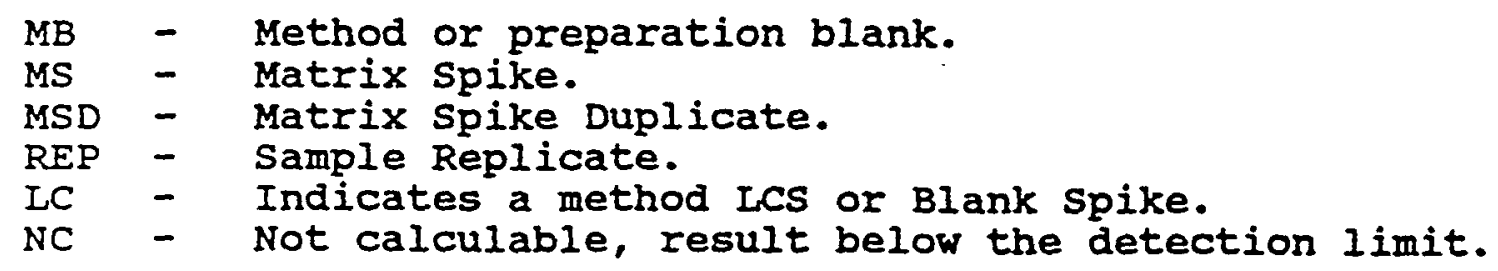

\section{LABORATORY CHRONOLOGY AND HOLDTIME REPORT}

The test code listed indicates the specific analysis or preparation procedure employed. The codes may be interpreted as follows:

MAAW - Metals prep test for AA digestion, water matrix. MAAS - Metals prep test for AA digestion, soil matrix. MICW - Metals prep test for ICP digestion, water matrix. MICS - Metals prep test for ICP digestion, soil matrix.

$M * *$ TO- This type of code indicates a total metal analysis (eg. MAGTO indicates an analysis for total silver).

$M * * S O-\quad T h i s$ type of code indicates a soluble metal analysis. (eg. MAGSO indicates an analysis for soluble silver).

M**EP- This type of code indicates an EPTOXICITY metals analysis (eg. MAGEP indicates an analysis for eptox silver).

$I * *$ TO- This type of code indicates a non-metaliic total analysis. There is also a complimentary soluble analysis for each of these codes (eg. ICNTO indicates an analysis for total cyanide).

A suffix of $-R$ or -5 following these codes indicates a replicate or spike analysis respectively. 
.. ROY F. HESTON INC.

INORGANICS DATA SUMRARY REPORT 03/19/90

CLIENT: WSRC-GRACE ROÄD

WESTON BATCH : 9002L607

WORR ORDER: 0630-28-12-0000

\begin{tabular}{|c|c|c|}
\hline SAMPLE : & SITE ID & 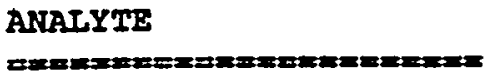 \\
\hline-001 & $\begin{array}{l}\text { GRS-08-01 } \\
:\end{array}$ & $\begin{array}{l}\text { \& SOLIDS } \\
\text { CYANIDE, TOTAI } \\
\text { SULEIDE }\end{array}$ \\
\hline
\end{tabular}

\begin{tabular}{clr} 
& & \multicolumn{2}{l}{ RBPORTING } \\
RESULT & UNITS & \multicolumn{2}{l}{ IIMIT } \\
\hline 83.6 & $\&$ & 0.1 \\
1.2 u & HG/KG & 1.2 \\
0.30 u & KG/RG & 0.3
\end{tabular}


- . ROY F. WESTON INC.

INORGANICS METEOD BLANK DATA SUMALARY PAGE 03/19/90

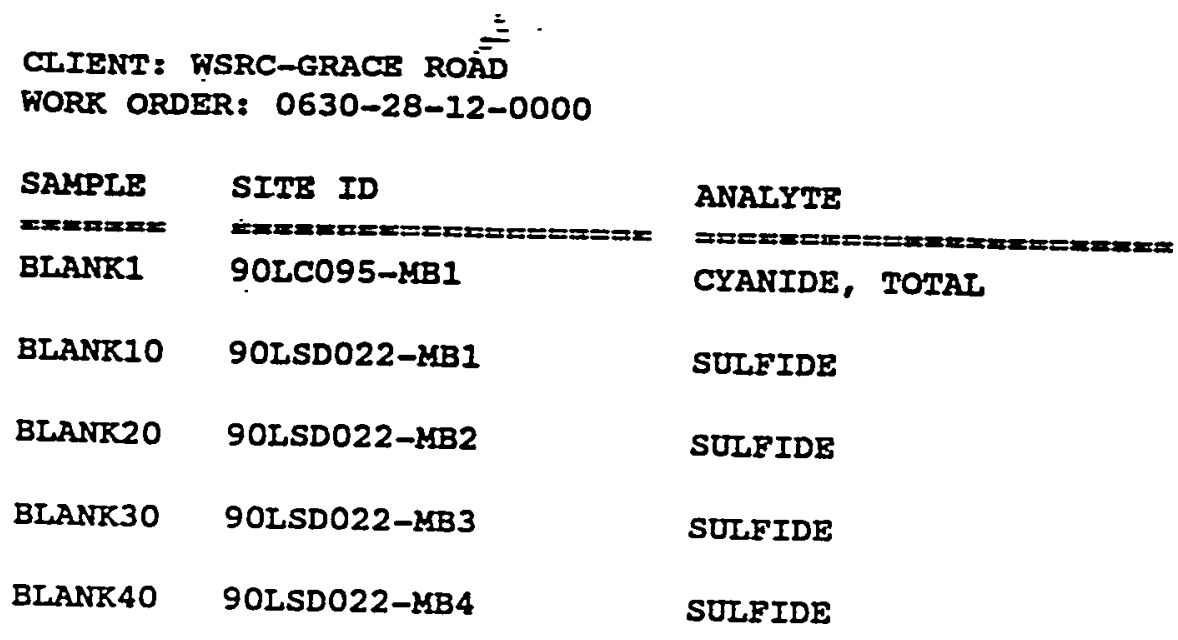

WESTON BATCE * 9002L607.

\begin{tabular}{|c|c|c|}
\hline 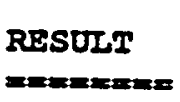 & 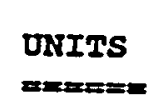 & $\begin{array}{l}\text { REPORTI } \\
\text { LIKIT } \\
\text { =EE }\end{array}$ \\
\hline $1.0 \mathrm{u}$ & $M G / R G$ & \\
\hline
\end{tabular}

0.25 u MG/RG 0.25

0.25 u $\mathrm{MG} / \mathrm{KG} \quad 0.25$

0.25 u $\mathrm{KG} / \mathrm{RG} \quad 0.25$

0.25 u KG/KG 0.25 
ROY F. FESTON INC.

INORGANICS ACCURACY REPORT 03/19/90

CLIENT: WSRC-GRACE ROAD

WORK ORDER: 0630-28-12-0000

WESTON BATCH : 9002L607

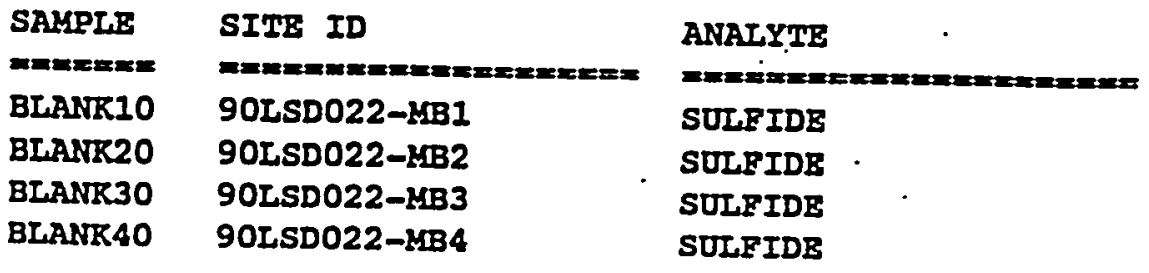

\begin{tabular}{|c|c|c|c|}
\hline $\begin{array}{l}\text { SPIKISD } \\
\text { SALPLE } \\
==2==-\end{array}$ & $\begin{array}{l}\text { INITIAI } \\
\text { RESULT } \\
\text { =EXE=IE= }\end{array}$ & 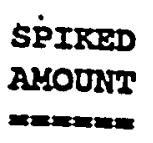 & RRBCOV \\
\hline & $0.25 \mathrm{u}$ & 1.0 & 104 \\
\hline 1.0 & $0.25 u$ & $1: 0$ & 105 \\
\hline $\begin{array}{l}1.1 \\
1.1\end{array}$ & $\begin{array}{l}0.25 u \\
0.25 u\end{array}$ & 1.0 & 109 \\
\hline
\end{tabular}


ROY F. WESTON INC.

\section{INORGANICS PRECISION REPORT 03/19/90}

its

CLIENT: HSRC-GRACB ROAD WORR ORDER: 0630-28-12-0000

SAMPLE SITE ID

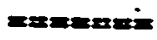

-OO1REP
ANALYTE

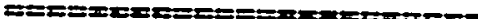
\& SOLIDS
WESTON BATCA : 9002L607

INITIAI RESULT REPLICATE \& DIPE

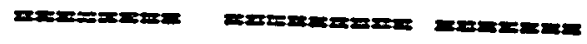

83.5 0.067 
ROY F. HESTON INC.

INORGANICS LABORATORY CONTROL STANDARDS REPORT 03/19/90

$$
\doteq
$$

\begin{tabular}{|c|c|c|}
\hline SALPLE & SITB ID & 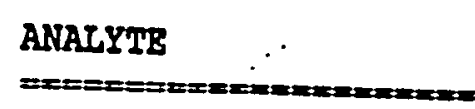 \\
\hline & 90LC095-LCS1 & CYANIDE, TOTAL LCS \\
\hline & 5-LCS2 & CYANIDE, TOTAL ICS \\
\hline
\end{tabular}

\begin{tabular}{rrrr} 
SPIKED & SPIKRD & & \\
SAMPLB & AHOUNT & ONITS & IRECOV \\
\hline 9.8 & 10.0 & HG/RG & 98.1 \\
9.8 & 10.0 & MG/RG & 98.4
\end{tabular}

I. 


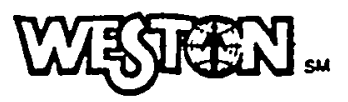

CLIENT: WSRC-GRACE RFT * : 9002 :607

T.O. \#: 0630-28-12
ROY $F$. WEBTON, IXC. Lionville Laboratory.

SAMPLES RECEIVED: $02 / 23 / 90$

\section{NARRATIVE}

The set of ${ }^{\circ}$ samples consisted of one soil sample collected on The samples were extracted on $02 / 27 / 90$ and analyzed according to criteria set forth in Method 8140 for Appendix IX Organophosphorus
Pesticide target compounds on $03 / 23 / 90$. Ail surrogate and blank spike recoveries are within QC limits. No problems were encounterd during sample analysis.

$$
\begin{aligned}
& \int \frac{\text { r.h.h }(-1 a-d}{\text { Jack R. Tuschall, Ph.D. }} \frac{3-29-z_{0}}{\text { Date }} \\
& \text { Laboratory Manager } \\
& \text { Lionville Analytical Laboratory }
\end{aligned}
$$


$\underset{⿱ 亠 䒑}{\stackrel{1}{\omega}}$

$-$ 


\begin{abstract}
DATR OVAIETERB
ס - Indicates that the compounds was analyzed for but not detected. The minimum detection Iimit for the sample (not the method detection limit) is reported with the $U$ (e.g., 10U).

3 - Indicates an estimated value. This flag is used in cases where a target analyte is detected at a level less than the lower quantification level. If the limit of quantification is $10 \mathrm{ug} / \mathrm{L}$ and a concentration-of 3 ug/ $L$ is calculated, it is reported as $3 J$.

B - This flag is used when the analyte is found in the associated blank as well as in the sample. It indicates possible/probable blank contamination. This flag is also used for a TIC as well as for a positiveİy
identified TCI compound.

B - Indicates that the compound was detected beyond the calibration range and was subsequently analyzed at a
dilution.

I - Interference.
\end{abstract}

ABBREVIATION8

B8 - Indicates blank spike in which reagent grade water is spiked with the CLP matrix spiking solutions and carried through all the steps in the method. Spike recoveries are reported.

B8D - Indicates blank spike duplicate.

4s - Indicates matrix spike.

MBD - Indicates matrix spike duplicate.

DI - Indicates that recoveries were not obtained because the extract had to be diluted for analysis.

NA - Not applicable.

Dr - Dilution factor.

NR - Not required. 
Roy P. Weston, Inc. - Ilonville Laboratory OPP ANALYTICAL DATA PACKAGE FOR MSRC-GRACE ROAD

DATE RECEIVED: $02 / 23 / 90$

CLIENT ID

GRS-08-01

GRS-08-01

IAB QC:

PBLK

PBLK

LAB ges
MBI

MBI. BS

\begin{tabular}{l} 
RFW \\
\hline 001 \\
001 REP
\end{tabular}

MTX PREP * COLIECTION EXTR/PREP

RFW LOT $: 9002 L 607$

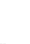

s

9OLE0283

90LE0283

$02 / 21 / 90$

$02 / 21 / 90$

$02 / 27 / 90$

$02 / 27 / 90$

ANALYSIS

$.03 / 23 / 90$

$03 / 23 / 90$

$N / A$

N/A
$02 / 27 / 90$

$02 / 27 / 90$
$03 / 23 / 90$

$03 / 23 / 90$

เ

S 90 LEO283 N/A $02 / 27 / 90 \quad 03 / 23 / 90$


Roy F. Heston, I1. ILonville Laboratiory

ORTHOPHOSEAATE PESTICIDES Cllent: WSRC-GRACE ROAD Work Order: 0630-28-12-0000

CILent: HSRC-GRACB ROAD.

\begin{abstract}
Cust ID\& GRS-08-01
\end{abstract}

\begin{tabular}{|c|c|c|c|c|c|}
\hline & Cust ID\& & GRS-08-01 & GRS $=08-01$ & PBLK & PBLR BS \\
\hline Sample & RPKI: & 001 & 001 REP & 90LE0283-MB1 & 90LE0283-MB1 \\
\hline Informat Ion & $\begin{array}{r}\text { Matrlx: } \\
\text { D.F.: } \\
\text { Unita: }\end{array}$ & $\begin{array}{l}\text { soIL } \\
0.500 \\
u g / \mathrm{Kg}\end{array}$ & $\begin{array}{l}\text { soIL } \\
0.500 \\
\text { ug/Rg }\end{array}$ & $\begin{array}{l}\text { soIt } \\
0.500 \\
\text { ug/ } / \mathrm{Rg}\end{array}$ & $\begin{array}{l}\text { sort } \\
0.500 \\
\text { ug/ Kg }\end{array}$ \\
\hline
\end{tabular}

PBLK

PBLR BS

\begin{tabular}{|c|c|c|c|c|c|c|c|c|c|}
\hline Burrogate: $\quad$ Ethion & 68 & 8 & 65 & 8 & 62 & 8 & 64 & 8 & \\
\hline \multicolumn{10}{|c|}{ 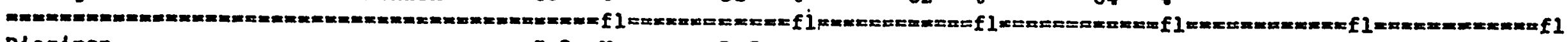 } \\
\hline Diazinon & 7.8 & $\mathbf{U}$ & B. 0 & $\mathbf{U}$ & 6.7 & $\mathbf{u}$ & 69 & 8 & \\
\hline Mothyl parathion & 7.8 & $\mathbf{U}$ & 8. 0 & $\mathbf{U}$ & 6.7 & $\mathbf{v}$ & 60 & 8 & \\
\hline $0,0,0$-Trlethyl phosphorothloat & 39 & $\mathbf{U}$ & 40 & $\mathbf{u}$ & 33 & $\mathbf{U}$ & 33 & U & \\
\hline Thlonazin (zinophos) & 39 & $\mathbf{U}$ & 40 & $\mathbf{u}$ & 33 & $\mathbf{U}$ & 33 & $\mathbf{u}$ & \\
\hline Phorate & 7.8 & $\mathbf{U}$ & 8.0 & $\mathbf{v}$ & 6.7 & $\mathbf{U}$ & 6.7 & $\mathbf{u}$ & $\cdot$ \\
\hline sulfotepp & 39 & $\boldsymbol{U}$ & 40 & $\mathbf{0}$ & 33 & $\mathbf{U}$ & 33 & $\mathbf{u}$ & \\
\hline Dlsulfoton & 7.8 & $\mathbf{u}$ & 8.0 & $\mathbf{v}$ & 6.7 & $\mathbf{u}$ & 6.7 & $\mathbf{u}$ & . \\
\hline Dimethoate & 39 & $\mathbf{U}$ & 40 & $\mathbf{U}$ & . $\mathbf{3 3}$ & $\mathbf{U}$ & 33 & $\mathbf{v}$ & \\
\hline Ethyl Parathion & 7.8 & $\mathbf{U}$ & 8.0 & $\mathbf{U}$ & 6.7 & $\mathbf{U}$ & 6.7 & $v$ & \\
\hline Eamphur (Famophos) & 78 & $\mathbf{U}$ & 80 & $\mathbf{U}$ & 67 & $\mathbf{U}$ & 67 & $\boldsymbol{U}$ & \\
\hline
\end{tabular}

$U=$ Analyzed, not detected. $J=$ Present below detection 1 imit. B= Present in blank. NR= Not requested. NS= Not splked. = Percent recovery. $D=$ Dlluted out. In Interference. NA= Not Applicable. *a Outelde of EPA CLP $Q C$ 
B-136 


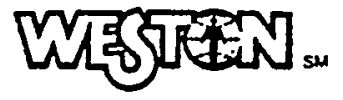

CLIENT: WSRC-GRACE ROAD

RFW : 9002 LE 606

พ.0. \#: 0630-28-12
ROY $F$. HESTON, INC.

Lionville Laboratory

SAMPLES RECEIVED: 02-23-90

\section{INORGANIC NARRATIVE}

The following is a summary of the quality control results and a description of any problems encountered during the analysis of this batch of samples:

1. All sample holding times as required by 40 CFR136 were met for water samples. Note: Holding times for soil samples have not been promulgated by the USEPA. 2. All preparation blanks were analyzed below the required
detection limit.

3. All calibration verification checks were within the required control limits of 90-100\%. Calibration verification is performed using independent standards.

4. The analytical methods applied by the laboratory, unless otherwise requested, for all inorganic analyses are derived from the USEPA Method for Chemical Analysis of Water and Wastes (USEPA 600/4-79-020), and Standard Methods for the Examination of Water and Wastewater 16 ed. Methods for the analysis of solid samples are derived from Test Methods for Evaluating Solid Waste (USEPA SW846).

NOTE: For solid samples, all results are reported on a dry weight basis.

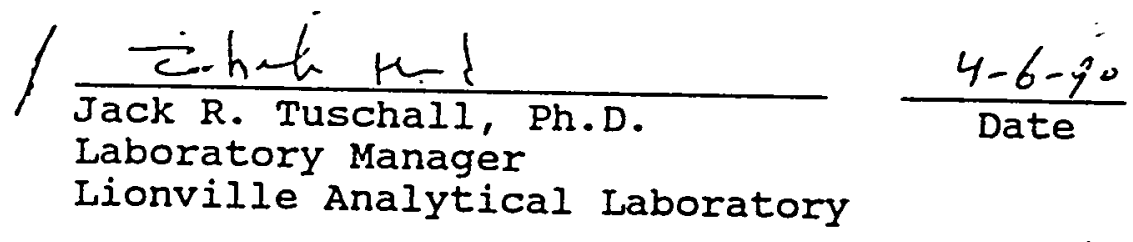

/PAS.21 


\section{ROY F. WESTON, INC. \\ GLOSSARY OF TERMS - INORGANIC REPORTS}

\section{DATA OUALIFIERS}

U - Indicates that the parameter was not detected at or above the reported limit. The associated numerical value is the sample detection limit.

* - Indicates that the original sample result is greater than $4 \mathrm{x}$ the spike amount added. The USEPA-CLP has determined that spike results on samples where this. occurs may be unreliable and, therefore, the control limits are not applicable.

\section{ABBREVIATIONS}

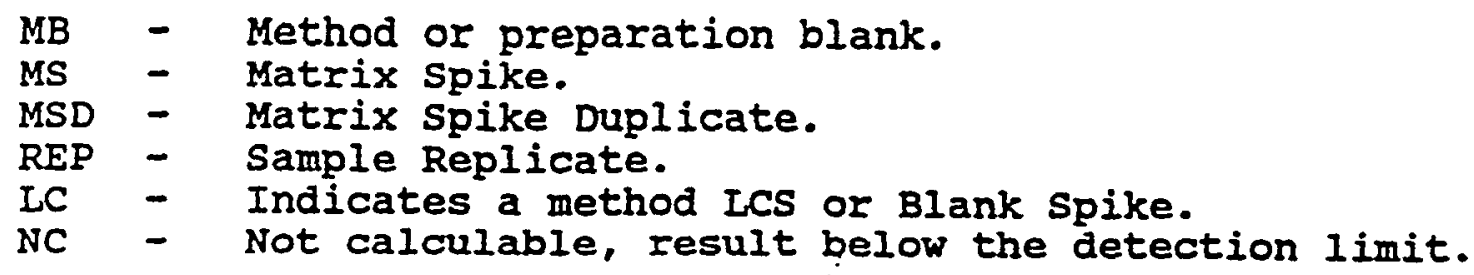

\section{LABORATORY CHRONOLOGY AND HOLDTIME REPORT}

The test code listed indicates the specific analysis or preparation procedure employed. The codes may be interpreted as follows:

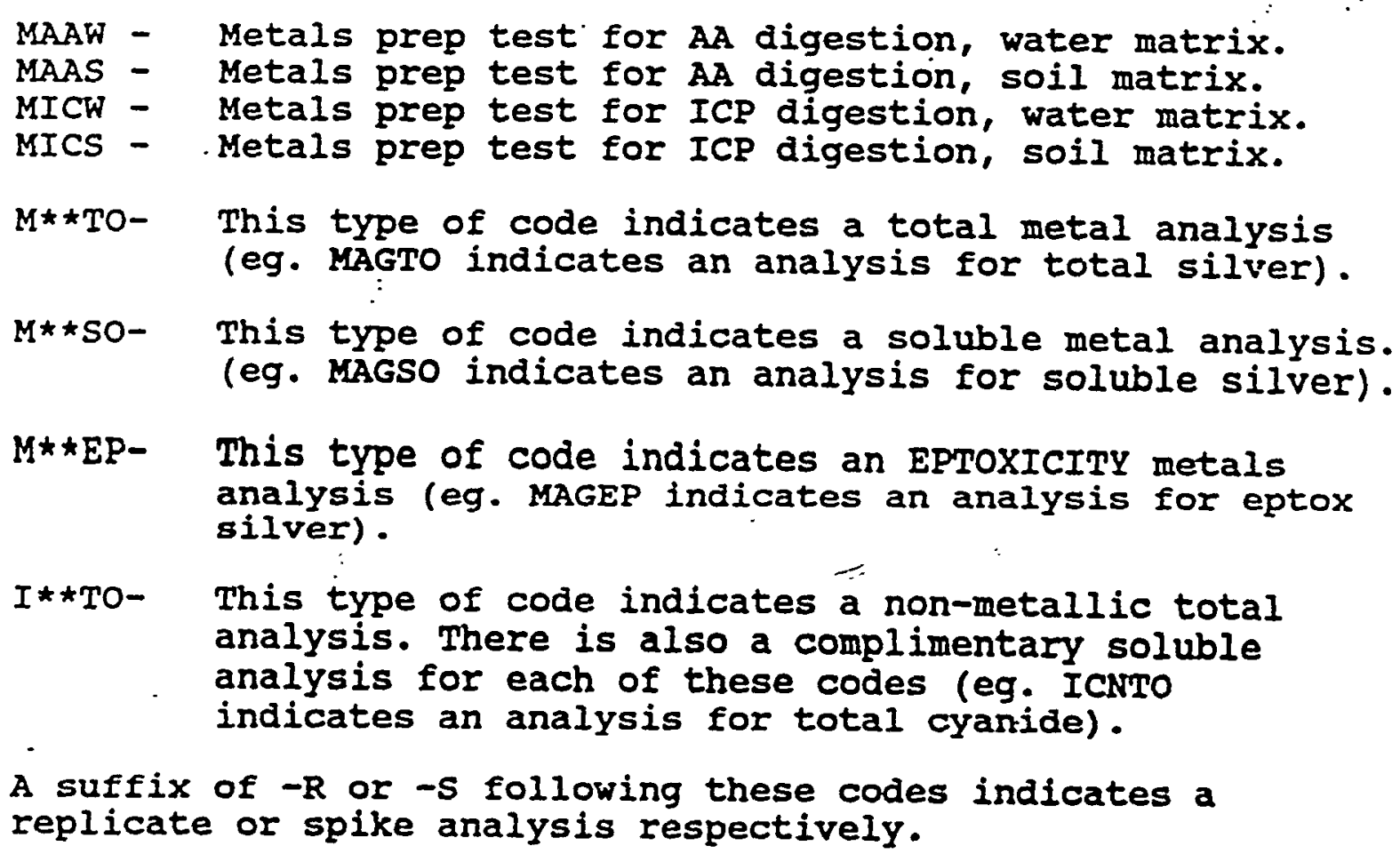

M**EP- This type of code indicates an EPTOXICITY metals analysis (eg. MAGEP indicates an analysis for eptox silver).

$I * *$ TO- This type of code indicates a non-metallic total analysis. There is also a complimentary soluble analysis for each of these codes (eg. ICNTO indicates an analysis for total cyanide).

A suffix of $-R$ or $-S$ following these codes indicates a replicate or spike analysis respectively. 
ROY F. WESTON INC.

INORGANICS DATA SUMRARY REPORT 03/30/90

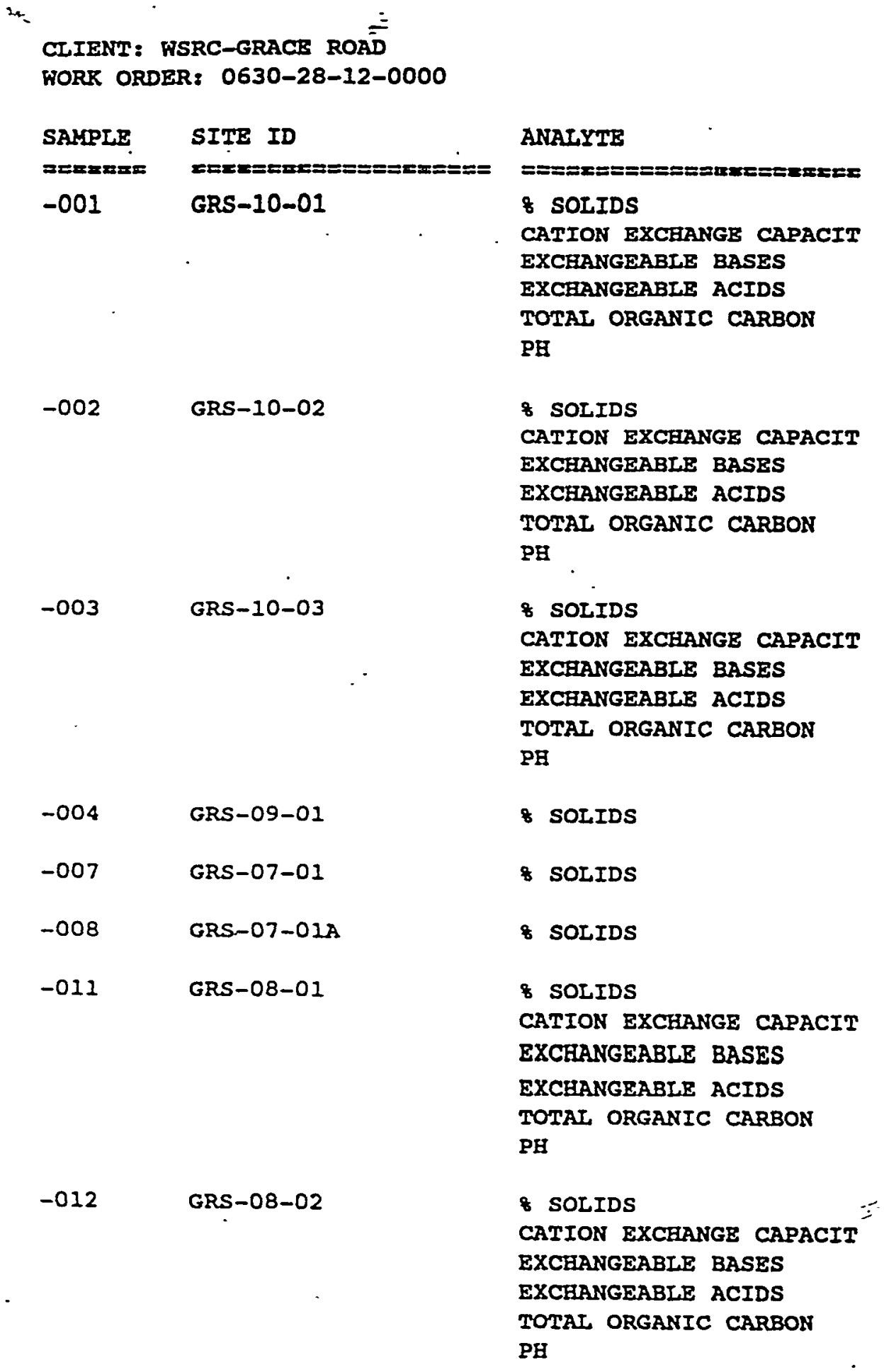

WESTON BATCE : $9002 L 606$

\begin{tabular}{|c|c|c|}
\hline & & REPORTING \\
\hline 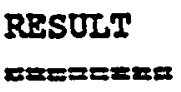 & $\begin{array}{l}\text { UNITS } \\
\text { yr===- }\end{array}$ & 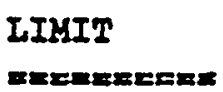 \\
\hline 94.8 & \& & 0.10 \\
\hline 0.94 & meg/ $100 \mathrm{~g}$ & 0.01 \\
\hline 0.34 & $\mathrm{meq} / 100 \mathrm{~g}$ & 0.01 \\
\hline 0.60 & $\mathrm{meg} / 100 \mathrm{~g}$ & 0.20 \\
\hline 513 & KG/KG & 27.0 \\
\hline 6.6 & pA ONITS & 0.10 \\
\hline
\end{tabular}

$\begin{array}{clc}88.3 & \text {. } & 0.10 \\ 5.9 & \mathrm{meq} / 100 \mathrm{~g} & 0.016 \\ 0.26 & \mathrm{meq} / 100 \mathrm{~g} & 0.016 \\ 5.6 & \mathrm{meq} / 100 \mathrm{~g} & 0.20 \\ 258 & \mathrm{MG} / \mathrm{KG} & 82.0 \\ 4.7 & \mathrm{pH} \text { UNITS } & 0.10\end{array}$

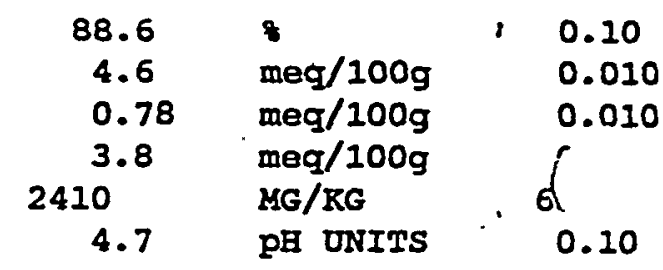

$\begin{array}{cll}93.8 & & 0.10 \\ 83.1 & 8 & 0.10 \\ 83.5 & 8 & 0.10 \\ 82.8 & 8 & 0.10 \\ 7.7 & \mathrm{meq} / 100 \mathrm{~g} & 0.010 \\ 0.92 & \mathrm{meq} / 100 \mathrm{~g} & 0.010 \\ 6.8 & \mathrm{meq} / 100 \mathrm{~g} & 0.20 \\ 873 & \mathrm{Mg} / \mathrm{kg} & 86.0 \\ 7.9 & \mathrm{pH} \text { JNITs } & 0.10\end{array}$

$\begin{array}{clc}91.8 & \text { \& } & 0.10 \\ 5.5 & \mathrm{meq} / 100 \mathrm{~g} & 0.010 \\ 0.51 & \mathrm{meq} / 100 \mathrm{~g} & 0.010 \\ 5.0 & \mathrm{meq} / 100 \mathrm{~g} & 0.20 \\ 454 & \mathrm{Mg} / \mathrm{RG} & 66.0 \\ 4.8 & \mathrm{pH} \mathrm{UNITS} & 0.10\end{array}$


ROY F. FESTON INC.

INORGANICS DATA SURRARY REPORT 03/30/90

CLIENT: WSRC-GRACE ROAD WORR ORDER: 0630-28-12-0000
ANALYTE

8 SOLIDS

CATION EXCHANGE CAPACIT

EXCEANGEABLE BASES

EXCEANGEABLE ACIDS

TOTAL ORGANIC CARBON

$\mathrm{PB}$
WESTON BATCH \& $9002 L 606$

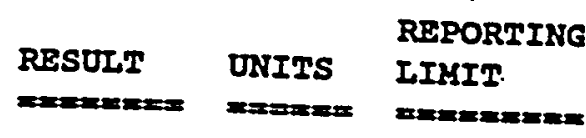

$\begin{array}{clc}91.8 & & 0.11 \\ 3.4 & \mathrm{meg} / 100 \mathrm{~g} & 0.0 \\ 0.23 & \mathrm{meq} / 100 \mathrm{~g} & 0.0 \\ 3.2 & \mathrm{meq} / 100 \mathrm{~g} . & 0.21 \\ 292 & \mathrm{MG} / \mathrm{Rg} & 60.0 \\ 5.0 & \mathrm{pH} \text { UNITs } & 0.11\end{array}$


ROY $F$. WESTON INC.

INORGANICS METHOD BLANR DATA SUMMARY PAGE 03/30/90

CLIENT: HSRC-GRACE ROAD

WORR ORDER: 0630-28-12-0000

\begin{tabular}{|c|c|c|}
\hline $\begin{array}{l}\text { SAMIPLE } \\
==2==\end{array}$ & 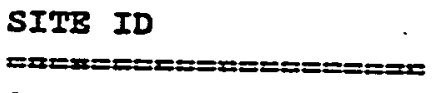 & 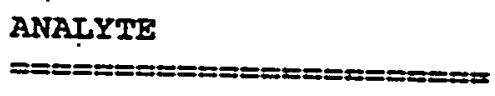 \\
\hline BLANR10 & 90ITZ002-KB1 & TOTAL ORGANIC CARBON \\
\hline BLANR20 & 90LTZ002-MB2 & AI ORGANIC CARBON \\
\hline
\end{tabular}

WESTON BATCH : 9002L606

\begin{tabular}{|c|c|c|}
\hline 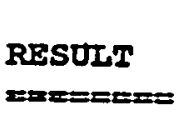 & $\begin{array}{l}\text { DNITS } \\
==2 \pi==x\end{array}$ & $\begin{array}{l}\text { REPORTING } \\
\text { IIMTT } \\
x===m=1=x=4\end{array}$ \\
\hline 20.0 & $\mathrm{MG} / \mathrm{KG}$ & 20.0 \\
\hline
\end{tabular}

20.0 U $M G / K G$

20.0 
ROY E. HESTON INC.

INORGANICS ACCURACY REPORT 03/30/90

CLIENI: WSRC-GRACE ROA

FORK ORDER: 0630-28-12-0000

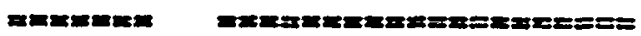

BLANK10 90LTZ002-LBI

BLANK20 90LTZ002-MB2

WESTON BATCA : 9002L606

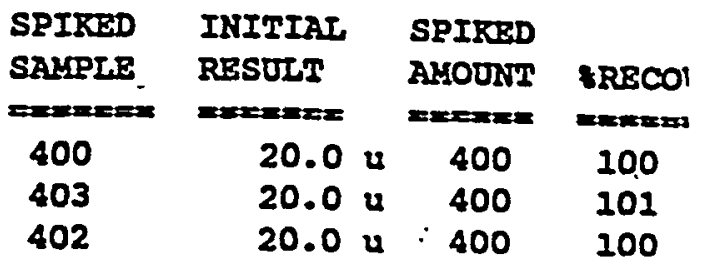


ROY F. WESTON INC.

INORGANICS DUPLICATE SPIKR REPORT 03/30/90

CLIENT: WSRC-GRACE ROA

WORR ORDER: 0630-28-12-0000

FESTON BATCE \# 9002L606

SAKRIRE

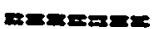

BLANR10

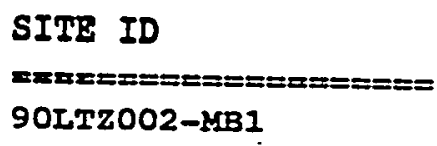

\section{ANALYTE}

TOTAL ORGANIC CARBON
SPIRE 1 . SPIKE\#2

\&RECOV \&RECOV \&DIFE

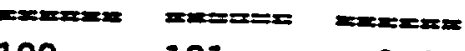


ROY F. HESTON INC.

INORGANICS PRECISION REPORT

$03 / 30 / 90$

CLIENT: WSRC-GRACE ROAD"

WORK ORDER: 0630-28-12-0000

WESTON BATCH \&: 9002L606

SAMPLE

m=m=ma:

-OOLREP

SITE ID

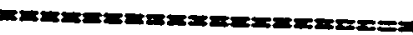

GRS-10-01

-OO7REP

-011REP
GRS-07-01

GRS-08-01
ANAIYTE

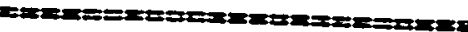

CATION EXCEANGE CAPACIT

BXCEANGEABLE BASES

EXCHANGEABLE ACIDS

\& SOLIDS

\& SOLIDS
INIIIAT

RESULT : REPLICATE \& DIFP

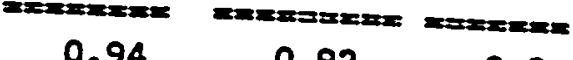

0.94

0.34

0.60

83.1

82.8

0.32

0.60

83. 1

82.9
2.2

6.1

0.00

0.099

0.10 


$$
\begin{aligned}
& \text { ROY F. Weston, InC. - LiOnville Laboratory } \\
& \text { INORGANIC ANAIYTICAI DATA PACKAGE FOR } \\
& \text { WSRC-GRACE ROAD }
\end{aligned}
$$

DATE RECEIVED: $02 / 23 / 90$ CLIENT ID / ANALYSIS
RFW \#
RFW LOT *:9002L606

MTX PREP * COLIECTION EXTR/PREP

GRS-10-01

$\begin{array}{ll}\text { 8 SOLIDS } & 001 \\ \text { GROSS ALPHA } & 001 \\ \text { GROSS BETA } & 001 \\ \text { CATION EXCHANGE CAPA } & 001 \\ \text { CATION EXCHANGE CAPA } & 001 \text { REP } \\ \text { CEC BASE } & 001 \\ \text { CEC BASE } & 001 \text { REP } \\ \text { CEC PREP } & 001 \\ \text { CEC TITRATION } & 001 \\ \text { CEC TITRATION } & 001 \text { REP } \\ \text { TOTAL ORGANIC CARBON } & 001 \\ \text { PH } & 001\end{array}$

GRS-10-02

$\begin{array}{ll}\text { 8 SOIIDS } & 002 \\ \text { GROSS ALPHA } & 002 \\ \text { GROSS BETA } & 002 \\ \text { CATION EXCHANGE CAPA } & 002 \\ \text { CEC BASE } & 002 \\ \text { CEC PREP } & 002 \\ \text { CEC TITRATION } & 002 \\ \text { TOTAL ORGANIC CARBON } & 002 \\ \text { PH. } & 002\end{array}$

GRS-10-03

$\begin{array}{lr}\text { \& SOLIDS } & 003 \\ \text { GROSS ALPHA } & 003 \\ \text { GROSS BETA } & 003 \\ \text { CATION EXCEANGE CAPA } & 003 \\ \text { CEC BASE } & 003 \\ \text { CEC PREP } & 003 \\ \text { CEC TITRATION } & 003 \\ \text { TOTAL ORGANIC CARBON } & 003 \\ \text { PH } & 003 \\ & \\ \text { GRS-09-01 } & \end{array}$
S 90L85040
$\mathbf{S}$
$\mathbf{s}$
s 90LCE002
s 9OLCE002
$s$
S 90LCE002
S 90LTZOO2
S 9OLPHO42

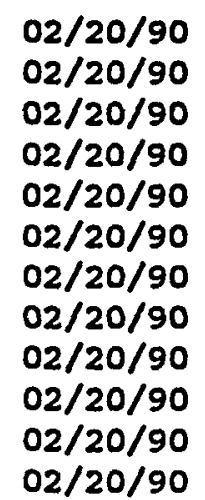

03/03/90

$03 / 15 / 90$

$03 / 15 / 90$

$03 / 12 / 90$

$03 / 12 / 90$

$03 / 08 / 90$

$03 / 08 / 90$

$03 / 02 / 90$

$03 / 08 / 90$

$02 / 20 / 90$ 
Roy F. Feston, Inc. - ILonville Iaboratory

INORGANIC ANAIYTICAL DATA PACRAGE FOR MSRC-GRACB ROAD

DATE RECEIVED: $02 \leq 23 / 90$

RFW LOT :9002L606

CLIENT ID /ANALYSIS

RFW * MTX PREP * COLLECTION EXTR/PREP

ANALYSIS

$\overline{+}$

GRS-07-01
7 SOLIDS
007
- SOLIDS
007 REP
90L85039
$02 / 21 / 90$
$03 / 07 / 90$
$03 / 08 / 90$
$03 / 07 / 90$
$03 / 08 / 90$
GRS-07-01A
8 sorIDs
008
s 90L:5039
$02 / 21 / 90$
$03 / 07 / 90$
03/08/90

GRS-08-01

8 SOLIDS 01

$\&$ SOLIDS

GROSS ALPHA

GROSS BETA

CATION EXCHANGE CAPA

CEC BASE

CEC PREP

CEC TITRATION

TOTAL ORGANIC CARBON $\mathrm{PH}$

GRS-08-02

8 SOLIDS

CATION EXCHANGE CAPA

CEC BASE

CEC PREP

CEC TITRATION

TOTAL ORGANIC CARBON

PH

011
011 REP
011
011
011
011
011
011
011
011

012

012

012

012

012

012

012

GRS-08-03

\& SOLIDS

CATION EXCHANGE CAPA

CEC BASE

CEC PREP

CEC TITRATION
013

013

013

013

013
90185039

90LCE002

90LCE002

$90 L C E 002$

90LTZOO2

90LPR042
$02 / 21 / 90$

$02 / 21 / 90$

$02 / 21 / 90$

$02 / 21 / 90$

$02 / 21 / 90$

$02 / 21 / 90$

$02 / 21 / 90$

$03 / 07 / 90$

$03 / 15 / 90$

$03 / 12 / 90$

$03 / 08 / 90$

$03 / 02 / 90$

$03 / 08 / 90$

$03 / 08 / 90$

$03 / 15 / 90$

$03 / 12 / 90$

$03 / 06 / 90$

$03 / 08 / 90$

$03 / 02 / 90$

$03 / 08 / 90$

$03 / 08 / 90$

$03 / 08 / 90$

$03 / 15 / 90$

$03 / 12 / 90$

$03 / 06 / 90$

03/08/90

$03 / 02 / 90$

$03 / 08 / 90$
S 90L\&5039
S 90LCE002
$02 / 21 / 90$
$02 / 21 / 90$
9OLCE002
$02 / 21 / 90$
$02 / 21 / 90$
$S$
S 90LCE002
$02 / 21 / 90$

$03 / 07 / 90$

$03 / 15 / 90$

$03 / 12 / 90$

$03 / 08 / 90$

$03 / 15 / 90$

$03 / 12 / 90$

03/06/90

$03 / 08 / 90$

$03 / 08 / 90$ 
Roy $F$. Weston, Inc. - Iionville Laboratory

INORGANIC ANALYTICAL DATA PACRAGE FOR

WSRC-GRACE ROAD

DATE RECBIVED: $02 \not 23 / 90$

RFW LOT \& $\$ 9002 L 606$

\begin{tabular}{|c|c|c|c|c|c|c|}
\hline CLIENT ID /ANALYSIS & RFW & MTX & PREP & COLLECTION & EXIR/PREP & ẠNALYSIS \\
\hline $\begin{array}{l}\text { TOTAL ORGANIC CARBON } \\
\text { PH }\end{array}$ & $\begin{array}{l}013 \\
013\end{array}$ & $\begin{array}{l}\mathbf{s} \\
\mathbf{s}\end{array}$ & $\begin{array}{l}\text { 90LTZOO2 } \\
\text { 9OLPHO42 }\end{array}$ & $\begin{array}{l}02 / 21 / 90 \\
02 / 21 / 90\end{array}$ & $\begin{array}{l}03 / 02 / 90 \\
03 / 08 / 90\end{array}$ & $\begin{array}{l}03 / 02 / 90 \\
03 / 08 / 90\end{array}$ \\
\hline
\end{tabular}

GRS-08-03E

GROSS ALPHA

GROSS BETA

014

พ

$02 / 21 / 90$

014

$02 / 21 / 90$

LAB QC:

TOTAL ORGANIC CARBON MBI TOTAL ORGANIC CARBON TOTAL ORGANIC CARBON TOTAL . ORGANIC CARBON TOTAL ORGANIC CARBON
MB1 BS

MB1 BSD

MB2

MB2 BS . s 90LTz002

S 90LTZ002

s 90LTz002

s 90LTz002

s 90 TT2002
N/A

$N / A$

$N / A$

$N / A$

$N / A$
$03 / 02 / 90$

$03 / 02 / 90$

$03 / 02 / 90$

$03 / 02 / 90$

$03 / 02 / 90$
$03 / 02 / 90$

$03 / 02 / 90$

$03 / 02 / 90$

$03 / 02 / 90$

03/02/90 



\section{5}

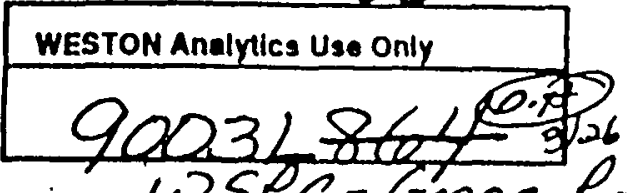

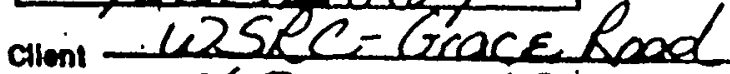

work order $0 / 30-28-10$

Dato Racid. 3/2,3/90 Dato pis $5 / 2 / 80$

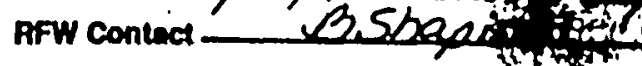

Clent Contact/phone

(1)

yole ow, green, w.lab, Bon

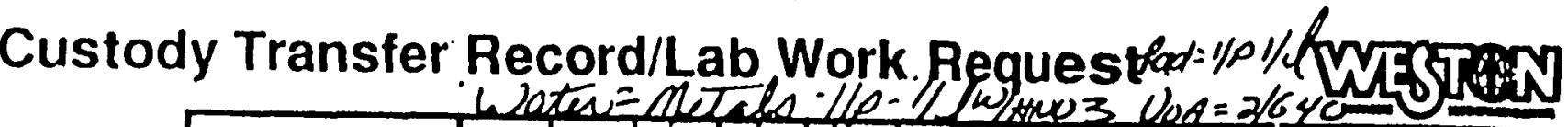

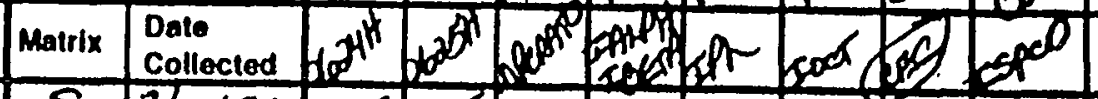

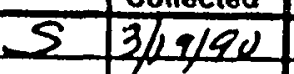

Reanind initers

Leating (iriproperty

\begin{tabular}{|c|c|c|c|c|c|c|c|c|}
\hline Relrigerator: & 1. & 6 & 4 & 4 & 4 & 4 & 4 & 4 \\
\hline mype Container & 16 & $7 / 6$ & 16 & 116 & $1 / 6$ & $\theta$ & $\pi / 2$ & 166 \\
\hline Volume & 25 & 250 & 125 & 250 & 500. & I & 125 & ketph \\
\hline Preservalive & - & - & - & - & - & - & - & - \\
\hline $\begin{array}{l}\text { NALYSES } \\
\text { OUESTED }\end{array}$ & & & & & & & & \\
\hline
\end{tabular}

\begin{tabular}{|c|c|c|}
\hline & $1613=02-01$ & \\
\hline & $+04-01$ & \\
\hline & $04-01 A$ & \\
\hline 4 & $03+0 k$ & \\
\hline 5 & 05201 & \\
\hline$\varphi$ & $0, a-01$ & $\angle$ Recrsenon \\
\hline & $11-a l$ & alph Bitak \\
\hline & $\alpha 1-a l$ & sublex to \\
\hline & 12001 & Ima ebelexe \\
\hline 10 & $13-01$ & pes D thomal \\
\hline L & $14-01$ & $3629190 \mathrm{KS}$ \\
\hline 12 & $13-O 1 E$ & \\
\hline$\angle 3$. & B.oE & \\
\hline 14 & $14-013$ & \\
\hline 13 & $E 14-010$ & \\
\hline
\end{tabular}

1

$1<1$

$\frac{1}{3 / 2060}$

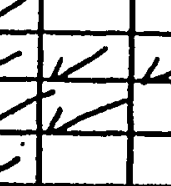

7

1019

\section{WESTON Analytlas \\ Use Only \\ Sarplantwero: \\ 1 sripged Hend. \\ Nonered \\ NORE \\ 26 \\ Nơ}

Seded

$Y$

NOTES:

beled

(N)

4 Propirly Preserved

kio

N

$1 / 2+1<$

2

\section{$3 / 24 / 90$}

$1 \frac{1}{3 / 2 / 50}$

$1<2$

$1 / 2$

4

4

$1<3$

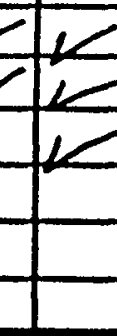

Spocial instructions: Metals = Tota RCeA

8. Eall O.On OL. Orum Uqulds

$R A D=$ Gross Alphavibeta

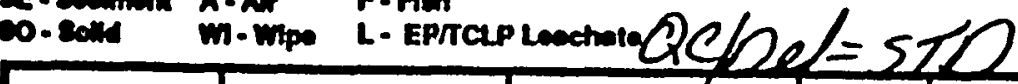

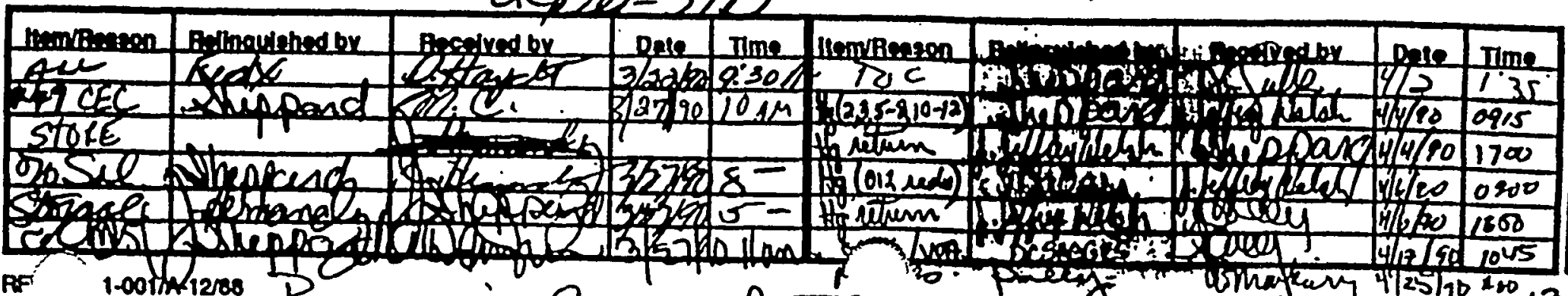

5 Recotved Within

elekfing ${ }^{\mathrm{N}} \mathrm{N}$

MOTES:

COC Tape Was:

1 Present on Outer

Package Y ND

2 Unbroken on Outer

Packing $Y$

3 Present on Samplo

4 Unbroken on semplo

NOTES: $Y$ N

COC Pocord Was:

1 Prosent Upon Racalot of Sempter (Y) N

Diecrepanctes Botween

Samplo Labole god COC

Rocord? $Y$. N

NOTES:ag Reques F fore

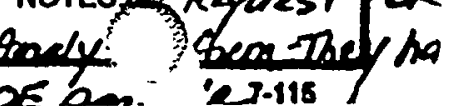


CLIENT: WSRC-GRACE ROAD

RFW" : 9003L865, BNA

W.O. \#: 0630-28-12
BAMPLES RECATVED: 0 03\%,22/9.0

\section{NARRATIVE}

The set of samples consisted of 11 soil samples coilected on $03 / 19,20,21 / 90$.

The samples were extracted on $03 / 27 / 90$ and analyzed according to criteria set forth in SW 846 method 8270 for TCL semivolatije target compounds on $: 04 / 02,03 / 90$.

The following is a summary of the QC results accompanying these $\because$ if sample results and a description of any problems encounterè $\because !$ during their analysis:

1. Non-target compounds were detected in these samples.

2. All surrogate recoveries are within EPA QC limits.

3. One of 22 blank and matrix spike recoveries is. outside EPA QC limits.

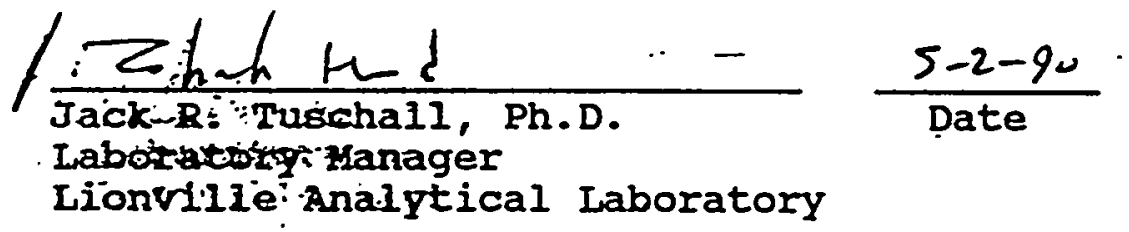

sjd:70103L8658.cn 



\section{DATA QUATTFIERS}

$U$ = compound was analyzed for but not detected. The associated mmerical value is the estimated sample quantitation limit. which is included and corrected for dilution and percent. moisture.

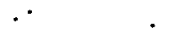

$J=$ Indicates an estimated value. Iris flag is used either when. estimating a concentration for tentatively identified compounds where a 1:1 respanse is assumed or when the mass spectral. data incicate the presence of a canpound that meets the identification ciciteria but the result is less than the specified detection limit but greater than zero: for example, if the linit of detection is $10 \mathrm{ug} / \mathrm{L}$ and a concentration of 3 $\mathrm{ug} / \mathrm{L}$ is calculated, it is reported as $3 \mathrm{~J}$.

$B=$ This flag is used when the analyte is found in the associated blank as well as in the sample. It indicates possible/probable blank contamination. this flag is also used for a IIC as well as for a positively identified TCL conpound.

$E=$ Indicates that the coupound was detected beyond the calibration range and was subsequently analyzed at a dilution.

$I=$ Interference.

$A=$ 'Aldol Condensation Product.

$X$ = Adaitional qualifiers used as required are explained in the case narrative.

$\mathrm{NQ}=$ Result qualitatively confinmed but not able to quantify.

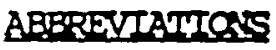

BS = Indicates blank spike in wich reagent grada water is spiked with tha CPP matrix spiking solutions and carried through all the eteps in the method. Spike recoveries are reported.

BSD = . Indicates blank spike cuplicate.

is = Iriklates matrix spike.

MSD = Indicates matrix spike dupliate. ”-

DL = Indicates that surogata recoveries were not obtatned because the extract bad to be diluted for analyais.

NA = Not applicable.

DF = Dilution factor.

NR - Not required. 
Roy F. Weston, Inc. - Lionville Laboratory

Semivolatiles by GC/MS, HSL IL Cllent: WSRC-GRACE ROAD

Work Order: 0630-28-12-0000

Cust ID: GRS-02-01

RFW: : 001

Sample

Information
GRS-02-01

001 MS

D.F. 1.00

Unita?
SOIL

GRS-04-01

1.00

$\mathrm{ug} / \mathrm{Kg}$

SOIL
GRS-04-01

002

1.00

002 REP

SOIL

1.00

GRS-04-01A

003

soII

1.00

1.00
$\mathrm{ug} / \mathrm{Kg}$

$\begin{array}{llll}73 & & 75 & \\ 65 & 73 & & \\ 67 & 68 & & \\ 76 & 81 & & \\ 69 & 78 & \end{array}$

$\begin{array}{llll}65 & 8 & 65 & 8 \\ 57 & & 67 & 8 \\ 63 & & 62 & \\ 79 & & 66 & \\ 74 & 8 & 59 & \\ 77 & & 63 & \end{array}$

$\begin{array}{llll}73 & & 75 & \\ 65 & 73 & & \\ 67 & 68 & & \\ 76 & 81 & & \\ 69 & 78 & \end{array}$

Surrogate

Nitrobenzene-d5
2-Fluorobipheny 1

p-Terphenyl-d14

Phenol-a5

2-Fluorophenol

2, 4,6-Tribromophenol

Phenol

b L ( 2-Chloroethyl)ether

2-Chlorophenol.

1, 3-DLchlorobenzene

1, 4-DLchlorobenzene

is Benzyl alcohol

ज 1,2-Dichlorobenzene

2-Methy lphenol

b1g (2-Chlorolsopropyl)ether
4-Methyl phenol

N-Nitroso-Di-n-propylamine

Hexachloroethani

Nitrobenzene

Isophorone

- 2-Nitropheñol

2, 4-Dimethyiphenol

Benzolo acid

ble (2-Chloroethoxy) methane

2,4-Dichlorophenol

1,2,4-Tr1chlorobenxene

Naphthalene

4-Chloroan111ne

Hexachlorobutadiene

4-Chloro-3-methylphenol

2-Hethylnaphthalene

Hexachlorocyclopentadieno

* outcilde of BPA CLP gC IImits.

$\begin{array}{lll}76 & 9 \\ 68 & 9 \\ 59 & 7 & 9 \\ 81 & 9 \\ 66 & 9\end{array}$

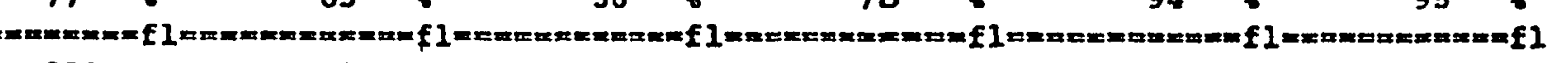

$390 \mathrm{U}$

$72350 \mathrm{U}$

$390 \mathrm{U} \quad 350 \mathrm{U}$

$69 \cdot 350 \quad 0$

390 U

$390 \mathrm{U}$

$390 \mathrm{v}$

$390 \mathrm{v}$

390 U

$390 \mathrm{U}$

$390 \mathrm{U}$

$390 \mathrm{v}$

$390 \mathrm{U}$

$390 \mathrm{U}$

$390 \mathrm{U}$

$390 \mathrm{U}$

390 U

$390 \mathrm{U}$

$1900 \mathrm{U}$

390 v

390 U

390 U

$390 \mathrm{v}$

390 U

390 U

390 v

$390 \mathrm{v}$

$390 \mathrm{U}$

390 U

60 \&

$390 \mathrm{U}$

390 0

$390 \mathrm{U}$

390 U

$390 \mathrm{U}$

83

390 U

390 U

$.390 \mathrm{v}$

$390 \mathrm{U}$

$390 \mathrm{v}$

$2000 \mathrm{U}$

$390 \mathrm{~V}$

390 v

73

390 U

390 U

390 U

$\begin{array}{ll}72 & 8 \\ 390 & 0\end{array}$

$\begin{array}{ll}390 & 0 \\ 390 & v\end{array}$

350 U

$350 \mathrm{U}$

350 U

350 U

350 v

$350 \quad \mathbf{U}$

$350 \quad \mathbf{U}$

$350 \quad \mathbf{U}$

350 U

$350 \quad \boldsymbol{U}$

350 v

$350 \quad \mathbf{U}$

$350 \mathrm{U}$

$1700 \mathrm{v}$

$350 \quad 0$

$350 \mathrm{U}$

$350 \quad$ U

$350 \mathrm{U}$

$350 \mathrm{U}$

$350 \mathrm{U}$

$350 \mathrm{U}$

$350 \quad \mathbf{U}$

$350 \mathrm{U}$

$350 \quad U$

350 v

350 U

$350 \quad 0$

350 v

$350 \quad 0$

350 U

$350 \quad 0$

$350 \quad 0$

$350 \quad v$

$350 \quad v$

350 v

$350 \quad 0$

350 v

$350 \quad U$

350 v

1700 U

350 v

350 v

$350 \mathrm{U}$

$350 \mathrm{U}$

$350 \mathrm{v}$

$350 \mathrm{U}$

$350 \mathrm{v}$

$350 \mathrm{U}$

350 U

$350^{\circ}$ U 390 v

350 U

$\begin{array}{llll}350 & \mathbf{U} & 390 & \mathbf{U} \\ \mathbf{3 5 0} & \mathbf{U} & 390 & \mathbf{U}\end{array}$

350 U 390 U

$\begin{array}{llll}350 & U & 390 & U\end{array}$

$350 \quad U \quad 390$ U

350 U 390 u

$\begin{array}{llll}350 & U & 390 & U\end{array}$

$350 \quad \mathbf{3} \quad 390 \quad$ U

350 U 390 v

$350 \quad U \quad 390$ U

350 U 390 U

350 U 390 U

$\begin{array}{llll}350 & \mathbf{3} & 390 & \mathrm{U}\end{array}$

$\begin{array}{llll}350 & v & 390 & v\end{array}$

$1700 \mathrm{U} \quad 1900 \mathrm{U}$

$\begin{array}{llll}350 & \boldsymbol{U} & 390 & \mathrm{U}\end{array}$

$\begin{array}{llll}350 & U & 390 & \end{array}$

$350 \quad U \quad 390 \quad U$

$350 \quad U \quad 390 \quad v$

350 v 390 v

$350 \cdot \mathbf{U} \quad \cdot 390 \quad \mathbf{v}$

$\begin{array}{llll}350 & \mathbf{U} & 390^{\circ} & \mathbf{U}\end{array}$

$\begin{array}{llll}350 & 0 & 390 & U\end{array}$ $r$ 
RFWII

001

001 MS

002

002 REP

003

004

2,4,6-Trichlorophenol

2,4,5-Trichlorophenol

2-Chloronaphthalene

2-N1troaniline

Dimethylphthalate

scenaphthylene

2,6-Dinitrotolueno

3-Nitroaniline

Acenaphthene

2,4-Dinitrophenol

4-Nitrophenol

Dibenzofuran

2,4-Dinitrotoluene

Dlethylphthalate

4-Chloropheny 1-phenylether

Eluorene

4-Nitroanillne

to 4,6-Dinitro-2-methylphenol

i N-Nitrosodiphenylamine (1)

Un 4-Bromophenyl-phenylether

Hexachlorobenzene

Pentachlorophenol

Phenanthrene

Anthracene

D1-n-Butylphthalate_. i

Fluoranthene

Pyrene

Butylbensylphthalate

3, $3^{\prime}-$-DLchlorobenzidine

Benzo (a) anthracene

Chryeene

blo (2-Ethylhoxy 1) phthalate

D1-n-octyl phthalate

Benzo (b) fluoxanthene

Benzo(k) fluoranthene

\section{Bonzo (a) pyrene}

Indeno $(1,2,3-c d)$ pyrene

Dibenzo $(a, h)$ anthracene

\begin{tabular}{rl}
390 & $\mathrm{U}$ \\
1900 & $\mathrm{U}$ \\
390 & $\mathrm{U}$ \\
1900 & $\mathrm{U}$ \\
390 & $\mathrm{U}$ \\
390 & $\mathrm{U}$ \\
390 & $\mathrm{U}$ \\
1900 & $\mathrm{U}$ \\
390 & $\mathrm{U}$ \\
1900 & $\mathrm{U}$ \\
1900 & $\mathrm{U}$ \\
390 & $\mathrm{U}$ \\
390 & $\mathrm{U}$ \\
390 & $\mathrm{U}$ \\
390 & $\mathrm{U}$ \\
390 & $\mathrm{U}$ \\
1900 & $\mathrm{U}$ \\
1900 & $\mathrm{U}$ \\
390 & $\mathrm{U}$ \\
390 & $\mathrm{U}$ \\
390 & $\mathrm{U}$ \\
1900 & $\mathrm{U}$ \\
390 & $\mathrm{U}$ \\
390 & $\mathrm{U}$ \\
390 & $\mathrm{U}$ \\
390 & $\mathrm{U}$ \\
390 & $\mathrm{U}$ \\
390 & $\mathrm{U}$ \\
780 & $\mathrm{U}$ \\
390 & $\mathrm{U}$ \\
390 & $\mathrm{U}$ \\
130 & $\mathrm{JB}$ \\
390 & $\mathrm{U}$ \\
390 & $\mathrm{U}$ \\
390 & $\mathrm{U}$ \\
390 & $\mathrm{U}$ \\
390 & $\mathrm{U}$ \\
390 & $\mathrm{U}$ \\
390 & $\mathrm{U}$ \\
\hline 1990 & \\
&
\end{tabular}

\begin{tabular}{|c|c|c|c|}
\hline 390 & U & 350 & $\mathbf{U}$ \\
\hline 2000 & v & 1700 & U \\
\hline 390 & $\mathbf{U}$ & .350 & v \\
\hline 2000 & $\mathbf{u}$ & 1700 & $\mathbf{U}$ \\
\hline 390 & $\mathbf{U}$ & 350 & u \\
\hline 390 & $\mathbf{U}$ & 350 & U \\
\hline 390 & $\mathbf{u}$. & 350 & $\mathbf{U}$ \\
\hline 2000 & U & 1700 & $\mathbf{v}$ \\
\hline 73 & 1 & 350 & v \\
\hline 2000 & $\mathbf{u}$ & 1700 & v \\
\hline 85 & 1 & 1700 & U \\
\hline 390 & $\mathbf{U}$ & 350 & U \\
\hline 71 & .t & 350 & 0 \\
\hline 390 & $\mathbf{U}$ & 350 & $\mathbf{0}$ \\
\hline 390 & $\mathbf{U}$ & 350 & 0 \\
\hline 390 & $\mathbf{U}$ & 350 & $\mathbf{U}$ \\
\hline 2000 & $\mathbf{v}$ & 1700 & U \\
\hline 2000 & $\mathbf{U}$ & 1700 & U \\
\hline 390 & $\mathbf{U}$ & 350 & U \\
\hline 390 & ס & 350 & U \\
\hline 390 & $\mathbf{0}$ & 350 & U \\
\hline 88 & 8 & 1700 & $\mathbf{u}$ \\
\hline 390 & $\mathbf{v}$ & 350 & $\mathbf{U}$ \\
\hline 390 & 0 & 350 & $\mathbf{U}$ \\
\hline 160 & $\mathbf{J}$ & 350 & $\mathbf{U}$ \\
\hline 390 & U & 350 & $\mathbf{u}$ \\
\hline 60 & 1 & 350 & $\mathbf{U}$ \\
\hline 390 & $\mathbf{U}$ & 350 & v \\
\hline 780 & $\mathbf{U}$ & 700 & $\mathbf{0}$ \\
\hline 390 & U & 350 & $\mathbf{U}$ \\
\hline 390 & U & 350 & $\mathbf{U}$ \\
\hline 260 & JB & 140 & JB \\
\hline 390 & $\mathbf{v}$ & 350 & $\mathbf{U}$ \\
\hline 390 & U & 350 & $\mathbf{U}$ \\
\hline 390 & $\mathbf{U}$ & 350 & $\mathbf{U}$ \\
\hline 390 & $\mathbf{U}$ & 350 & $\mathbf{U}$ \\
\hline 390 & $\mathbf{U}$ & 350 & $\mathbf{U}$ \\
\hline 390 & $\mathbf{v}$ & 350 & $\boldsymbol{U}$ \\
\hline 390 & U & 350 & $\mathbf{v}$ \\
\hline
\end{tabular}

$\begin{array}{rl}350 & \mathbf{U} \\ 1700 & \mathbf{U} \\ 350 & \mathbf{U} \\ 1700 & \mathbf{U} \\ 350 & \mathbf{U} \\ 350 & \mathbf{U} \\ 350 & \mathbf{U} \\ 1700 & \mathbf{U} \\ 350 & \mathbf{U} \\ 1700 & \mathbf{U} \\ 1700 & \mathbf{U} \\ 350 & \mathbf{U} \\ 350 & \mathbf{U} \\ 350 & \mathbf{U} \\ 350 & \mathbf{U} \\ 350 & \mathbf{U} \\ 1700 & \mathbf{U} \\ 1700 & \mathbf{U} \\ 350 & \mathbf{U} \\ 350 & \mathbf{U} \\ 350 & \mathbf{U} \\ 1700 & \mathbf{U} \\ 350 & \mathbf{U} \\ 350 & \mathbf{U} \\ 350 & \mathbf{U} \\ 350 & \mathbf{U} \\ 350 & \mathbf{U} \\ 350 & \mathbf{U} \\ 700 & \mathbf{U} \\ 350 & \mathbf{U} \\ 350 & \mathbf{U} \\ 75 & \mathbf{J} \\ 350 & \mathbf{U} \\ 350 & \mathbf{U} \\ 350 & \mathbf{U} \\ 350 & \mathbf{U} \\ 350 & \mathbf{U} \\ 350 & \mathbf{U} \\ 350 & \mathbf{U} \\ & \end{array}$

(1) - Cannot be separated from Dlphenylamine. "tw Outaide of BPA CLP DC IImitb. 
Roy F. Weston, Inc. - Llonv1lle Laboratory Semivolatiles by GC/MS, HSL List

Report Date: $04 / 24 / 9020203$

Cust ID: GRS-05-01 GRS-06-01 GRS-11-01 GRS-01-01 GRS-12-01 . GRS-13-01

Sample

Information

RFW":
Matrix:

D.F.8

Unita :

surrogate

Recovery

N1trobenzene-d5 2-Eluoroblphenyl

p-Terphenyl-d14
Phenol-d5

2-8luorophenol

2, 4, 6-Tribromophenol

Phenol

b1s (2-Chloroethyl)ether

2-Chlorophenol

1,3-Dichlorobenzene

$1 \quad$ 1,4-Dichlorobenzene

it Benzyl alcohol

ज̆ 1,2-Dichlorobenzene

2-Methylphenol

bls (2-Chlorol opropyl) ether

4-Kethylphenol.

N-N1troso-D1-n-propylamine

Hexachloroethane

Nitrobenzene

Irophorone

2-N1trophenol

2, 4-Dimethyl phenol

Benzolo acid

b1s (2-Chloroethoxy)methane

2,4-DLchlorophenol

1,2,4-Tr1chlorobenzene

Naphthalene

4-Chloroaniline

Hexachlorobutadlene

4-Chloro-3-methylphenol

2-Methylnaphthalene

Hexachlorocyclopentadiene

- Outbida of SPA CLP gC IImits.

$\begin{array}{cccc}005 & 006 & 007 & 008 \\ \text { soIL } & \text { sOIL } & \text { soIL } & \text { soIL } \\ 1.00 & 1.00 & 1.00 & 1.00 \\ \mathrm{ug} / \mathrm{kg} & \mathrm{ug} / \mathrm{kg} & \mathrm{ug} / \mathrm{Kg} & \mathrm{ug} / \mathrm{kg}\end{array}$

009

SOII.

1.00

ug/kg

SOII

\begin{tabular}{|c|c|c|c|}
\hline 86 & 1 & 78 & 8 \\
\hline 83 & 8 & 69 & 8 \\
\hline 68 & 8 & 69 & 8 \\
\hline 86 & \& & 78 & 8 \\
\hline 69 & 8 & 52 & 8 \\
\hline .98 & 8 & 59 & 8 \\
\hline
\end{tabular}

\begin{tabular}{|c|c|c|c|}
\hline 86 & 1 & 78 & 8 \\
\hline 83 & 8 & 69 & 8 \\
\hline 68 & 8 & 69 & 8 \\
\hline 86 & \& & 78 & 8 \\
\hline 69 & 8 & 52 & 8 \\
\hline .98 & 8 & 59 & 8 \\
\hline
\end{tabular}

$\begin{array}{ll}76 & 8 \\ 76 & 8 \\ 71 & 8 \\ 60 & 8 \\ 89\end{array}$

$\begin{array}{lllll}400 & U & 420 & U & 350 \\ 400 & U & 420 & U & 350\end{array}$

$400 \mathrm{U}$

$400 \mathrm{U}$

$400 \mathrm{U}$

$400 \mathrm{~J}$

$400 \mathrm{~V}$

$400 \mathrm{~V}$

$400 \mathrm{v}$

$400 \mathrm{v}$

$400 \mathrm{U}$

$400 \mathrm{U}$

$400 \mathrm{~J}$

$400 \mathrm{U}$

$400 \mathrm{U}$

$400 \mathrm{~V}$

$400 \mathrm{~V}$

2000 U

$400 \mathrm{U}$

$400 \mathrm{U}$

$400 \mathrm{U}$

$400 \mathrm{U}$

$400 \mathrm{U}$

$400 \mathrm{U}$

$400 \mathrm{U}$

$400 \mathrm{U}$

420
420
$U$

420 U

$420 \mathrm{U}$

$420 \mathrm{~V}$

$420 \mathrm{~V}$

$420 \mathrm{~V}$

$420 \mathrm{~V}$

$420 \mathrm{~V}$

$420 \mathrm{~V}$

$420 \mathrm{U}$

$420 \mathrm{U}$

$.420 \mathrm{U}$

$420 \mathrm{U}$

$420 \mathrm{U}$

$2100 \mathrm{~V}$

$420 \mathrm{U}$

$420 \mathrm{U}$

$420 \mathrm{U}$

$420 \mathrm{U}$

$420 U$

$420 \mathrm{U}$

$420 \mathrm{U}$

$420 \mathrm{U}$

$400 \mathrm{U} \cdot 420 \mathrm{U}$

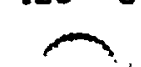

$\begin{array}{ll}70 & 8 \\ 69 & 8 \\ 82 & 8 \\ 71 & 8 \\ 56 & 8 \\ 43 & 8 \\ 350 & \mathrm{U}\end{array}$

\begin{tabular}{llll} 
ug/Kg & \multicolumn{2}{l}{ ug/Rg } \\
\hline 75 & 8 & 69 & \\
75 & 85 & 79 & \\
84 & 8 & 81 & \\
83 & 8 & 67 & \\
64 & 8 & 64 &
\end{tabular}

$\begin{array}{ll}350 & \mathbf{U} \\ 350 & \mathbf{U} \\ 350 & \mathbf{U} \\ 350 & \mathbf{D}\end{array}$

$\begin{array}{ll}350 & \mathrm{U} \\ 350 & 0\end{array}$

$350 \mathrm{U}$

350 v

$350 \mathrm{U}$

$350 \mathrm{v}$

350 v

350 U

350 U

350 v

350 U

350 v

$350 \quad \mathbf{v}$

$350 \mathrm{v}$

$1800 \mathrm{U}$

350 v

350 U

350 U

350 v

$350 \mathrm{v}$

$350 \quad 0$

350 U

350 U

$\begin{array}{llllll}350 & v & 350 & v & 380 & 0\end{array}$

$\begin{array}{llllll}350 & U & 350 & U & 380 & U\end{array}$

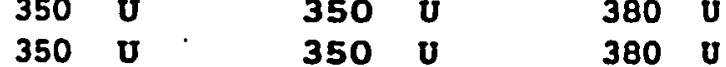

$\begin{array}{llllll}350 & \mathbf{N} & \mathbf{3 5 0} & \mathbf{U} & 380 & \mathbf{U}\end{array}$

$\begin{array}{llllll}350 & \mathbf{U} & \mathbf{3 5 0} & \mathbf{U} & \mathbf{3 8 0} & \mathbf{U} \\ \mathbf{3 5 0} & \mathbf{U} & \mathbf{3 5 0} & \mathbf{U} & 380 & \mathbf{U}\end{array}$

$\begin{array}{lllll}350 & U & 350 & v & 380\end{array}$

$\begin{array}{llllll}350 & \mathbf{v} & \mathbf{3 5 0} & \mathbf{U} & 380 & \mathbf{U} \\ 350 & \mathbf{v} & \mathbf{3 5 0} & \mathbf{U} & \mathbf{3 8 0} & \mathbf{v}\end{array}$

$\begin{array}{llllll}350 & 0 & 350 & 0 & 380 & 0\end{array}$

$\begin{array}{llllll}350 & \mathbf{v} & 350 & \mathbf{U} & 380 & 0 \\ 350 & \mathbf{U} & 350 & \mathbf{U} & 380 & \mathbf{U}\end{array}$

$\begin{array}{llllll}350 & 0 & 350 & v & 380 & v\end{array}$

$\begin{array}{llllll}350 & 0 & 350 & 0 & 380 & 0\end{array}$

$\begin{array}{llllll}350 & 0 & 350 & u & 380 & 0\end{array}$

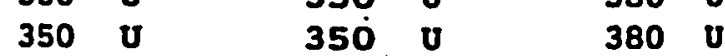

$\begin{array}{llllll}350 & U & 350 & U & 380 & u\end{array}$

$\begin{array}{llllll}350 & U & 350 & v & 380 & 0\end{array}$

250 JB $1800 \quad 0 \quad 1900 \quad U$

$\begin{array}{llllll}350 & \mathrm{U} & 350 & \mathrm{U} & 380 \mathrm{U}\end{array}$

$\begin{array}{llllll}350 & U & 350 & U & 380 & U\end{array}$

350 v 350

$350 \quad 350$

$\begin{array}{llll}350 & \mathbf{3} & \mathbf{3 5 0} & \mathrm{U}\end{array}$

$350 \quad \mathbf{U} \cdot 350 \cdot \mathbf{U}$

$\begin{array}{llll}350 & \mathbf{3} & 350 & 0\end{array}$

$\begin{array}{llll}350 & \mathbf{U} & \mathbf{3} 50 & \mathbf{U}\end{array}$

$350 \mathrm{U} \quad 350 . \mathrm{U}$

$350 \mathrm{U}$

380 U

$380 \mathrm{U}$

380 U

380 U

380 U

380 U

$380 \mathrm{U}$ 
RFW :

005.006

007

008

009

010

2,4,6-Tr1chlorophenol

2,4,5-Trichlorophenol

2-Chloronaphthalene

2-Nitroaniline

Dimethylphthalate

Acenaphthylene

2,6-Dinitrotoluene

3-Nitroaniline

Acenaphthene

2, 4-DInitrophenol

4-Nitrophenol

Dibenzofuran

2, 4-Dinitrotoluene

Dlethylphthalate

4-Chloropheny 1-phenylether

Fluorene

4-NLtroaniline

if 4,6-Dinitro-2-methy lphenol

$\omega$ N-Nitrosodiphenylamine (1)

4-Bromopheny 1-pheny lether

kexachlorobenzene

Pentachl, orophenol

Phenanthrene

Anthracene

D1-n-Buty lphthalate il

Fluoranthene

Pyrene

Butýlbenzylphthalate

3, 3'-Dlchlorobenzldine

Benzo (a) anthracene

Chrybene

bie (2-Bthylhexyl)phthalate

D1-n-octyl phthalate

Benzo(b) Eluoranthene

Benzo (k) fluoranthene

$\begin{array}{rlrl}400 & U & 420 & U \\ 2000 & U & 2100 & U \\ 400 & U & 420 & U \\ 2000 & U & 2100 & U \\ 400 & U & 420 & U \\ 400 & U & 420 & U \\ 400 & U & 420 & U\end{array}$

$\begin{array}{rl}350 & \mathbf{U} \\ 1800 & \mathbf{U} \\ 350 & \mathbf{U} \\ 1800 & \mathbf{U} \\ 350 & \mathbf{U} \\ 350 & \mathbf{U} \\ 350 & \mathbf{U} \\ 1800 & \mathbf{U} \\ 350 & \mathbf{U}\end{array}$

\begin{tabular}{|c|c|c|c|c|}
\hline 350 & $\mathbf{v}$ & 350 & $\mathbf{U}$ & 380 \\
\hline 1800 & $\boldsymbol{U}$ & 1800 & $\mathbf{v}$ & 1900 \\
\hline
\end{tabular}

$350 \mathrm{U} . \quad 350 \mathrm{U}^{\circ} \quad 380 \mathrm{U}$

$1800 \mathrm{U} \cdot 1800 \mathrm{U} \quad 1900 \mathrm{U}$

$\begin{array}{lllll}350 & U & 350 & 0 & 380\end{array}$

$\begin{array}{llllll}350 & U & 350 & U & 380 & U\end{array}$

$\begin{array}{lllll}350 & U & 350 & U & 380\end{array}$

$2000 \mathrm{U}$

4000

$2000 \mathrm{U}$

$2100 \mathrm{U}$

$1800 \mathrm{U}$

$350 \mathrm{U}$

$1800 \mathrm{v}$

$1800 \mathrm{v}$

$2000 \mathrm{v}$

2100 U

400

$2100 \mathrm{U}$

4000

4000

\begin{tabular}{l}
420 \\
420 \\
\hline
\end{tabular}

$420 \mathrm{U}$

$350 \mathrm{v}$

$350 \quad 0$

350 v

$350 \mathrm{v}$

$350 \mathrm{v}$

$400 \mathrm{U}$

4200

4200

$2100 \mathrm{v}$

$2100 \mathrm{v}$

20000

$400 \mathrm{U}$

$420 \mathrm{U}$

$420 \mathrm{U}$

$400 \mathrm{U}$

$420 \mathrm{~V}$

$2000 \mathrm{~V}$

$400 \mathrm{~V}$

$2100 \mathrm{U}$

$420 \mathrm{U}$

$420 \mathrm{U}$

$42 \mathrm{~J}$

$49 \mathrm{~J}$

$400 \mathrm{~V}$

$400 \mathrm{U}$

$420 \mathrm{U}$

$420 \mathrm{U}$

$400 \mathrm{U}$

$800 \mathrm{U}$

$420 U$

$830 \mathrm{U}$

$400 \mathrm{U}$

$400 \mathrm{U}$

$420 U$

$420 U$

$63 \mathrm{JB} \quad 210 \mathrm{JB}$

$400 \mathrm{U}$

$420 \mathrm{~V}$

$400 \mathrm{U}$

$400 \mathrm{U}$

$420 \mathrm{U}$

$420 \mathrm{~V}$

$400 \mathrm{v}$

Benzo (a) pyrene

$420 \mathrm{U}$

$420 \mathrm{U}$

$400 \mathrm{v}$

DLbenzo $(a, h)$ anthracene

$420 \mathrm{U}$

$1800 \mathrm{U}$

$1800 \mathrm{U}$

$350 \mathrm{U}$

$350 \mathrm{U}$

350 v

$1800 \mathrm{U}$

$350 \mathrm{v}$

350 v

350 v

3500

350 U

350 U

$700 \mathrm{U}$

350 U

350 U

$120 \mathrm{JB}$

350 U

$350 \mathrm{U}$

350 U

350 U

350 U

350 U

$350 \mathrm{v}$

1800 U

350 U

$1800 \mathrm{v}$

$1800 \mathrm{U}$

$350 \mathrm{v}$

350 v

$350 \mathrm{v}$

$350^{\circ}$

3500

$1800 \mathrm{v}$

$1800 \mathrm{v}$

350 v

350 v

3500

$1800 \mathrm{v}$

350. 0

3500

$53 \mathrm{~J}$

350 U

$350 \mathrm{U}$

350 U

$700 \mathrm{U}$

350 U

$350 \mathrm{U}$

54 JB

350 U

350 v

350 v

$350^{\circ} \mathrm{U}$

350 v

$350 \mathrm{v}$

350 U

$1900 \mathrm{U}$

$380 \mathrm{v}$

$1900 \mathrm{U}$

1900 U

380 v

380 U

380 U

$380 \mathrm{v}$

380 v

$1900 \mathrm{U}$

$1900 \mathrm{~V}$

$380 \mathrm{v}$

$380 \mathrm{v}$

$380 \mathrm{v}$

$1900 \mathrm{U}$

$380 \mathrm{v}$

380 U

$49 \mathrm{~J}$

380 U

380 U

$380 \mathrm{U}$

$770 \mathrm{v}$

$380 \mathrm{U}$

$380 \mathrm{v}$

$1500 \mathrm{~B}$

$380 \mathrm{U}$

380 U

$380 \mathrm{U}$

$380 \mathrm{v}$

$380^{\circ}$ v

$380 v$

(1) - Cannot be separated from Diphenylamine. * outalde of BPA CLP gC 1imita.

380 v 
Roy F. Weston, Inc. - Lionville Laboratory Semivolat1les by GC/MS, HSL Llat

$\begin{array}{lll}\text { Cugt ID: GRS-14-01 SBLK SBLR BS } & \text { SR }\end{array}$

\begin{tabular}{|c|c|c|c|c|}
\hline Sample & RFW I: & 011 & 90LE0366-MBL & 90LE0366-MB1 \\
\hline Information & $\begin{array}{r}\text { Matrix: } \\
\text { D.F. : } \\
\text { Unita: }\end{array}$ & $\begin{array}{l}\text { soIt } \\
1.00 \\
u g / \mathrm{kg}\end{array}$ & $\begin{array}{l}\text { soIL } \\
1.00 \\
u g / \mathrm{kg}\end{array}$ & $\begin{array}{l}\text { soIL } \\
1.00 \\
u g / \mathrm{kg}\end{array}$ \\
\hline
\end{tabular}

\begin{tabular}{lr}
\hline & Nitrobenzene-d5 \\
Surrogate & 2-Fluorobiphenyl \\
Recovery & p-Terphenyl-d14 \\
Phenol-d5 \\
2-Fluorophenol
\end{tabular}

2,4,6-Tribromophenol.

$\begin{array}{ll}66 & 8 \\ 64 & 8 \\ 77 & \\ 73 & \\ 58 & 8 \\ 50 & 8\end{array}$

\begin{tabular}{|c|c|c|c|}
\hline 70 & 8 & 70 & 8 \\
\hline 68 & 8 & 82 & 8 \\
\hline 84 & 8 & 81 & 8 \\
\hline 71 & 8 & 89 & 8 \\
\hline 67 & 8 & 94 & 8 \\
\hline 60 & 8 & 73 & 8 \\
\hline
\end{tabular}

\section{Phenol}

ble (2-Chloroethyl)ether

2-Chlorophenol.

1,3-D1chlorobenzene

1.4-D1chlorobenzene

क्ष Benzyl alcohol

in 1,2-D Lchlorobenzene

2-Methylphenol

ble (2-Chlorol sopropyl) ether

4-Methylphonol.

N-N1troso-D1-n-propylamine

Hexachloroethane

Nitrobenzene

Isophorone

2-Nitrophenol

2, 4-Dimathylphenol

Bensolc acid

bis (2-Chloroethoxy) methane

2, 4-Dlchlorophenol

1,2,4-Tr1chlorobenzene

Naphthalene

4-Chloroaniline

Bexachlorobutadiene

4-Chloro-3-methylphenol

2-Kethyl naphthalene

Bexachlorocyclopentadiene

* outgide of EPA CLP gC IImitB.

\begin{tabular}{|c|c|c|c|c|}
\hline 390 & $\mathbf{U}$ & 330 & U & 97 \\
\hline 390 & $\mathbf{U}$ & 330 & $\mathbf{U}$ & 330 \\
\hline 390 & $\mathbf{U}$ & 330 & $\mathbf{U}$ & 86 \\
\hline 390 & $\mathbf{U}$ & 330 & $\mathbf{v}$ & 330 \\
\hline 390 & $\mathbf{U}$ & 330 & $\boldsymbol{U}$ & 73 \\
\hline 390 & $\mathbf{U}$ & 330 . & $\mathbf{0}$ & 330 \\
\hline 390 & $\mathbf{U}$ & 330 & $\mathbf{U}$ & 330 \\
\hline 390 & $\mathbf{U}$ & 330 & $\mathbf{v}$ & 330 \\
\hline 390 & $\mathbf{U}$ & 330 & $\mathbf{v}$ & 330 \\
\hline 390 & $\mathbf{U}$ & 330 & $\mathbf{v}$ & 330 \\
\hline 390 & $\mathbf{U}$ & 330 & $\mathbf{U}$ & 90 \\
\hline 390 & $\mathbf{U}$ & 330 & $\mathbf{U}$ & 330 \\
\hline 390 & $\mathbf{U}$ & 330 & $\mathbf{v}$ & 330 \\
\hline 390 & $\mathbf{U}$ & 330 & $\mathbf{U}$ & 330 \\
\hline 390 & $\mathbf{U}$ & 330 & $\mathbf{U}$ & 330 \\
\hline 390 & $\mathbf{U}$ & 330 & $\mathbf{U}$ & 330 \\
\hline 000 & $\mathbf{U}$ & 1700 & $\mathbf{U}$ & 1700 \\
\hline 390 & $\mathbf{U}$ & 330 & $\mathbf{U}$ & 330 \\
\hline 390 & $\mathbf{U}$ & 330 & $\boldsymbol{U}$ & 330 \\
\hline 390 & U & 330 & $\mathbf{U}$ & 78 \\
\hline 390 & $\mathbf{U}$ & 330 & $\mathbf{U}$ & 330 \\
\hline 390 & $\mathbf{U}$ & 330 & $\mathbf{U}$ & 330 \\
\hline 390 & $\mathbf{U}$ & 330 & $\mathbf{U}$ & 330 \\
\hline 390 & $\mathbf{U}$ & 330 & $\mathbf{U}$ & 87 \\
\hline 390 & $\mathbf{U}$ & 330 & $\mathbf{0}$ & 330 \\
\hline 390 & $\mathbf{U}$ & 330 & $\mathbf{U}$ & 330 \\
\hline
\end{tabular}

0


RFi ch viumber: 90032865 Client: HSRC-GRACE Cust ID \& GRS-14-01 SBLR

$\underline{D}$ SBLR BS

\section{RFW \&}

011 9OLE0366-MB1 90LE0366-MB1

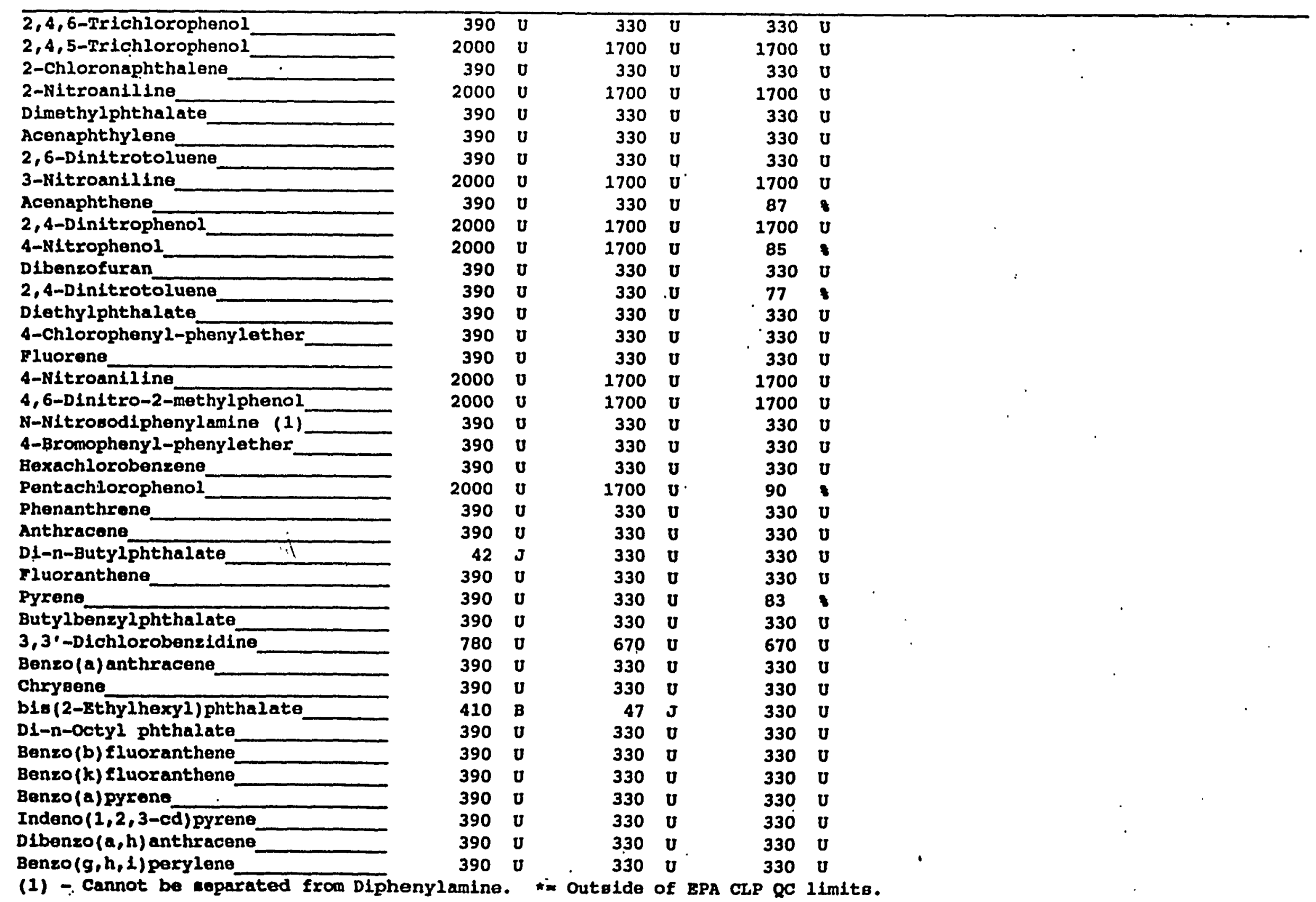

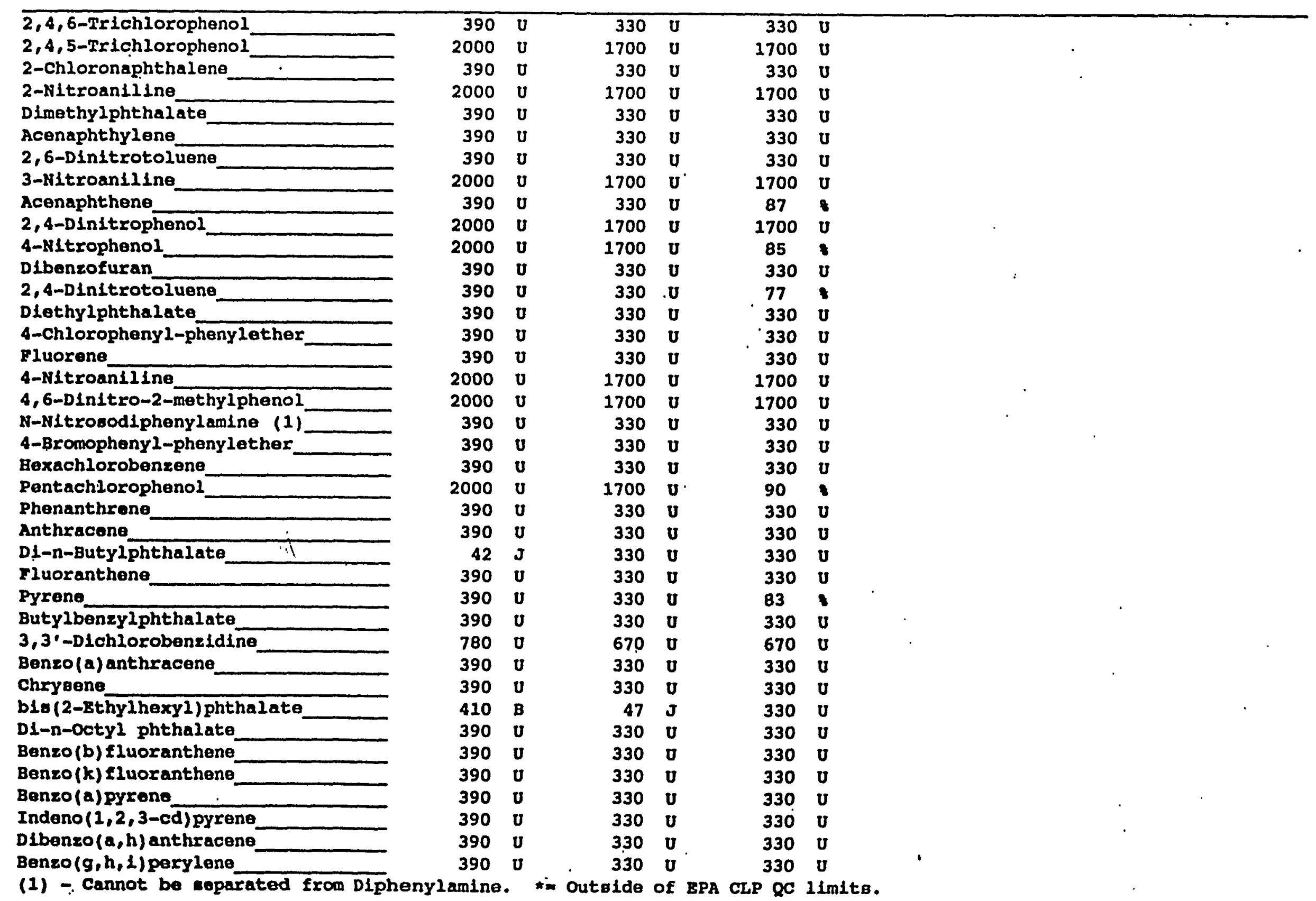


Lab Name: Roy F. Fegton, Inc. Work Order: 0630-28-12-0000

Client: WSRC-GRACE ROAD

Matrix:

SOII

Sample wt/vol:

$$
30.3(\mathrm{~g} / \mathrm{mL}) \mathrm{G}
$$

Lab Sample ID:

Lab File ID:

Date Received: 03/22/90

Level: (low/med) LOW

Date Extracted: $03 / 27 / 90$

Date Analyzed: $\underline{04 / 02 / 90}$

Extraction: (SepR/Cont/Sonc) SONC

GPC Cleanup: $(\Psi / N) \underline{I}$ pa:

Dilution Factor: 1.00

Number TICg found: 5

CONCENTRATION UNITS:

(ug/L or $\mathrm{ug} / \mathrm{Kg}$ ) ug/ $\mathrm{kg}$

\begin{tabular}{|c|c|c|c|c|}
\hline CAS NOMBER & COMPOUND NAME & $\mathbf{R T}$ & EST. CONC. & $\mathbf{Q}$ \\
\hline 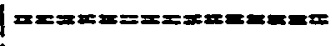 & 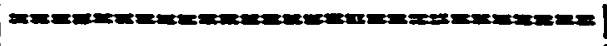 & $=m=n=m$ & 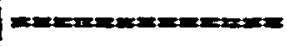 & $=0=2=$ \\
\hline 1. & ATDOT CONDENSATS & 6.33 & 2000 & JAB \\
\hline 2. & ATDOL CONDENSÄTE & 7.07 & 1000 & $\boldsymbol{J}$ \\
\hline 3. & UNRAOONN & 7.90 & 400 & JA \\
\hline 4. & ALDOL CONDENSATE & 8.10 & 1900 & JA \\
\hline 5 . & ORGANIC ACID & 19.87 & 500 & JB \\
\hline
\end{tabular}


Iab Namez Roy $F$. Weston, Inc. Work Order: 0630-28-12-0000

|GRS-04-0I

Client: WSRC-GRACE ROAD

Matrix:

Lab Sample ID: 9003L865-002

Sample wt/vols $\quad 30.1 \quad(\mathrm{~g} / \mathrm{mL}) \mathrm{G}$

Lab F11e ID:

Level: (low/med) LOW

Date Received: $03 / 22 / 90$

8 Molsture: not dec.

Date Extracted: $03 / 27 / 90$

Extraction: (SepF/Cont/Sonc) soNe

Date Analyzed: $\underline{04 / 02 / 90}$

GPC Cleanup: $\quad(Y / N) \underline{Y}$

pH: 7.0

Dilution Factor: 1.00

Number TICs found: 5

CONCENTRATION ONITS:

(ug/L or ug/Rg) uag/Rg

\begin{tabular}{|c|c|c|c|c|}
\hline CAS NUMBER & COKPOUND NAKR & RT & BST. CONC. & $\mathbf{Q}$ \\
\hline 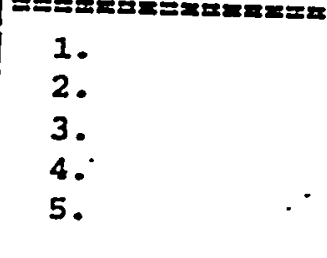 & 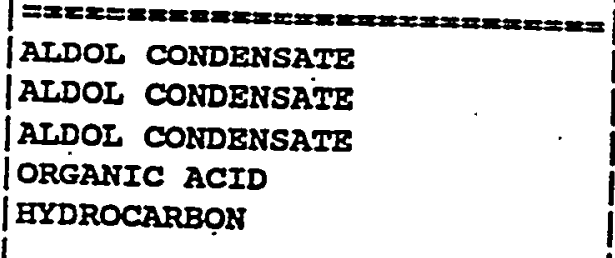 & \begin{tabular}{r|}
$\mid-x .22$ \\
$6.98 \mid$ \\
$8.05 \mid$ \\
19.85 \\
23.57
\end{tabular} & $\begin{array}{l}1000 \\
1000 \\
800 \\
500 \\
500 \\
300\end{array}$ & $\begin{array}{l}=2=\infty \\
J A B \\
J A \\
J A \\
J B \\
J\end{array}$ \\
\hline
\end{tabular}


Lab Name: Roy $F$. Feston, Inc. Work Ordex: 0630-28-12-0000

Client: WSRC-GRACE ROAD

Matrix:

SOII

Sample wt/vol: $30.1(\mathrm{~g} / \mathrm{mr}) \mathrm{G}$

Level: (low/med) LOW

\& Molstures not dec. 5 dec.

Extraction: (SepE/Cont/Sonc) SONC

GPC Cleanup: $\quad(X / N) \underline{Y}$

$\mathrm{pH}: \quad 7.0$
Lab Sample ID: 9003L865-002 REP

Lab File ID: $\quad \underline{\underline{1040207}}$

Date Received: $03 / 22 / 90$

Date Bxtracted: $03 / 27 / 90$

Date Analyzed: $\underline{04 / 02 / 90}$

Dilution Factor: 1.00

Number TICs found: $\underline{5}$

CONCENTRATION UNITS:

(ug/L or ug/ $\mathrm{kg}$ ) ug/Rg

\begin{tabular}{|c|c|c|c|c|}
\hline CAS NUMBER & COMPOUND NAMES & $\mathbf{R T}$ & EST. CONC. & $\mathbf{Q}$ \\
\hline 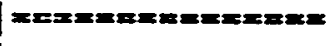 & 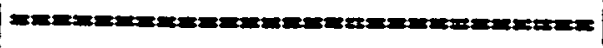 & $=m-m=m$ & 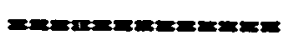 & $m=m=$ \\
\hline 1. & ATDOL CONDENSATB & 6.22 & 900 & JAB \\
\hline 2 . & ATDOL CONDENSATE & 6.98 & 1000 & JA \\
\hline 3. & ALDOL CONDENSATE & 8.05 & 800 & JA \\
\hline 4 & ORGANIC ACID & 19.87 & 600 & JB \\
\hline 5. & HYDROCARBON & 23.58 & 400 & $\boldsymbol{J}$ \\
\hline
\end{tabular}


Lab Name: Roy E. Feston, Inc. Hork Order: 0630-28-12-0000

Client: WSRC-GRACE ROAD

Matrix: SoIL

sample wt/rols 30.2 (g/mt) . G

Level: (low/med) IOW 5 dec.

8 Molsture: not dec. $\underline{-12}$

Extraction: (Sepr/cont/sonc) SoNC

GPC Cleanup: $(\Psi / N) \underline{Y}$ pH: 7.0

CONCENTRATION UNITS:

Number TICs found: $\underline{5}$ (ug/L or ug/Rg) ug/ $\mathrm{ug}$
Lab Sample ID: 9003L865-003

Lab Fila ID: $\quad$ L040208

Date Received: $03 / 22 / 90$

Date Bxtracted: 03/27/90

Date Analyzed: 04/02/90

Dilution Factor: 1.00

\begin{tabular}{|c|c|c|c|c|}
\hline CAS NOLBER & COKPOUND NAYB & $\mathbf{R T}$ & EST. CONC. & $\mathbf{Q}$ \\
\hline 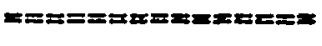 & 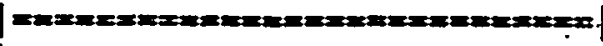 & 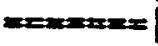 & | & $=$ \\
\hline 1. & ATDOI CONDENSATB & 6.23 & 300 & JAB \\
\hline 2. & ALDOL CONDENSATB & 6.98 & 500 & JA \\
\hline 3. & AIDOL CONDENSATB & 8.07 & 300 & JA \\
\hline 4. & ORGANIC ACID & 19.87 & 400 & $\mathbf{J B}$ \\
\hline 5. & HYDROCARBON & 23.58 & 200 & $\mathbf{3}$ \\
\hline
\end{tabular}


SEMIVOLATILE ORGANICS ANALYSIS SERET TENTATIVELY IDENTIFIED COMPOUNDS

Iab Name: Roy $\mathrm{F}$. Weston, Inc, Work Order: 0630-28-12-0000 GRS-03-01

in Client: MSRC-GRACE ROAD

Matrix:

SOIL

Sample wt/vol: $30.2(\mathrm{~g} / \mathrm{mr}) \mathrm{G}$

Level: (Low/med) LOW

\%olsture: not dec. 14 dec.

Extraction: (SepF/Cont/sonc) SONC

GPC Cleanup: $\quad(\Psi / N) \underline{Y}$

$\mathrm{pH}=7.0$
Lab Sample ID: 9003L865-004

Lab File ID: L040209

Date Received: $03 / 22 / 90$

Date Extracted: $\underline{03 / 27 / 90}$

Date Analyzed: $04 / 02 / 90$

Dilution Factor: 1.00

Number TICB found: 5

CONCENTRATION UNITS:

(ug/I or ug/Kg) ug/ $\mathrm{kg}$

\begin{tabular}{|c|c|c|c|c|}
\hline $\begin{array}{l}\text { CAS NURBER } \\
==x=x=x=x=m=m x=\end{array}$ & 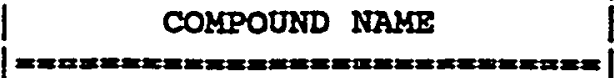 & 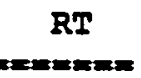 & BST. CONC. & $\underset{z=m=m}{Q}$ \\
\hline 1. & ALDOL CONDENSATE & 6.22 & 2000 & JAB \\
\hline 2. & ALDOL CONDENSATE & 6.98 & 2000 & JA \\
\hline 3. & ALDOL CONDENSATE & 7.38 & 500 & JA \\
\hline 4. & |ALDOL CONDENSATB & 8.05 & 1000 & JA \\
\hline 5 . & |ORGANIC ACID & 19.85 & 1600 & JB \\
\hline
\end{tabular}


Lab Name: Roy $F$. Weston. Inc. Work Order: 0630-28-12-0000 |GRS-05-01.

Cllent: WSRC-GRACE ROAD

Matrix:

Sample wt/vol:

SOIL

$30.2(\mathrm{~g} / \mathrm{mL}) \mathrm{G}$

Level: (low/med) LOW

Moisture: not dec. dec.

18 dae.

Extraction: (Sepr/Cont/Sonc) sowc

GPC Cleanup: $\quad(Y / N) \underline{Y}$

$\mathrm{pH}=\mathbf{7 . 0}$
Lab Sample ID: 9003L865-005

Lab File ID: ${ }^{\circ} \quad$ L040210

Date Received: $03 / 22 / 90$

Date Bxtracted: $03 / 27 / 90$

Date Analyzed: $\underline{04 / 02 / 90}$

Dilution Factor: 1.00

Number TICs found: 5

CONCENTRATION UIITS:

(ug/L or ug/Rg) ug/Rq

\begin{tabular}{|c|c|c|c|c|}
\hline $\begin{array}{l}\text { CAS NUMBER } \\
====m=m=m=m=m=1\end{array}$ & | & $=2$ & EST. CONC. & $\mathbf{Q}$ \\
\hline $\begin{array}{l}1 . \\
2 . \\
3 . \\
4 . \\
5\end{array}$ & $\begin{array}{l}\text { ALDOL CONDENSATB } \\
\text { ALDOL CONDENSATB } \\
\text { |ALDOI CONDENSATE } \\
\text { ORGANIC ACID } \\
\text { | EYDROCARBON }\end{array}$ & $\begin{array}{r}6.22 \\
6.98 \\
8.05 \\
19.85 \\
23.60\end{array}$ & $\begin{array}{l}1000 \\
1000 \\
1000 \\
700 \\
400\end{array}$ & $\begin{array}{l}\text { JAB } \\
\text { JA } \\
J A \\
J B\end{array}$ \\
\hline
\end{tabular}


$1 F$

SEMIVOLATIIS ORGANICS ANATISIS SEHEW

TENTATIVETY IDENTIEIED COMPOUNDS

Iab Name: Roy F. Wegton. Inc. Work Order: 0630-28-12-0000
CLIBNT SAKPLE NO.

GRS-06-01

\section{Client: FSRC-GRACE ROAD}

Matrix:

SOIL

Sample wt/vol: $30.1(\mathrm{~g} / \mathrm{mr}) . \underline{G}$

Level: (low/med) IOW

\& Nolsture: not dec: L_ 20 dec.

Extraction: (sepF/Cont/Sonc) soNe

GPC Cleanup: $\quad(\Psi / N) \underline{Y}$

Number TICs found: $\underline{5}$
Lab sample ID: 90032865-006

Lab File ID: I040211

Date Received: $03 / 22 / 90$

Date Bxtracted: $03 / 27 / 90$

Date Analyzed: $04 / 02 / 90$

Dilution Factor: 1.00

CONCENTRATION UNITS:

(ug/L or $u g / \mathrm{kg}$ ) $\underline{\mathrm{ug}} / \mathrm{kq}$

\begin{tabular}{|c|c|c|c|c|}
\hline CAS NUMBER & COMPOUND NAKIS & RT & BST. CONC. & $\mathbf{Q}$ \\
\hline 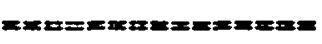 & | " & $=-m=-\infty=$ & 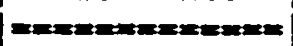 & 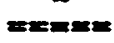 \\
\hline 1 & ALDOL CONDENSATE & 6.22 & 1000 & JAB \\
\hline 2. & ALDOL CONDENSATE & 6.98 & 1000 & JA \\
\hline 3. & |ALDOL CONDENSATE & 8.05 & 500 & JA \\
\hline 4. & PROPENYL BENZODIOXOLE & 12.10 & 2000 & $\mathbf{J}$ \\
\hline 5. & IORGANIC ACID & 19.85 & 800 & JB \\
\hline
\end{tabular}


Lab Name: Roy F. Westan, Inc. Mork Order: 0630-28-12-0000

Cllent: WSRC-GRACE ROAD

Natr 1x:

sorr

Sample wt/rol: $: 30.2(g / m r) G$

Level: (low/med) LOH 6 dec.

\& Molsture: not dec.

Extraction: (Sepr/cont/Sonc) sonc

GPC Cleanup: $\quad(Y / N) \underline{Y}$ $\mathrm{pH}: \quad 2.0$
Lab Sample ID: 9003L865-007

Lab Elle ID:

$\mathbf{1 0 4 0 2 1 2}$

Date Recelved: $03 / 22 / 90$

Date Extracted: $03 / 27 / 90$

Date Analyzed: 04/02/90

Dilution Factor: 1.00

Number TICs found: $\underline{5}$

CONCENTRATION ONITS:

(ug/L or ug/ $\mathrm{kg}$ ) ug/ $\mathrm{kg}$

\begin{tabular}{|c|c|c|c|c|}
\hline CAS NOMBER & COMPOUND NAMB & $\mathbf{R T}$ & EST. CONC. & $\boldsymbol{Q}$ \\
\hline 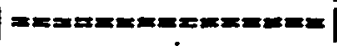 & 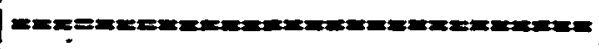 & $m=-m=m$ & 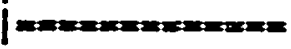 & $=-\infty=$ \\
\hline 1 & ATDOL CONDENSATE & 6.22 & 12000 & JAB \\
\hline 2. & ATDOL CONDENSATB & 6.98 & 800 & JA \\
\hline 3. & ORGANIC ACID & 19.85 & 400 & $\mathbf{J B}$ \\
\hline 4. & PETHARATHS & 27.22 & 200 & $\mathbf{J}$ \\
\hline 5 . & PETHATATE. & 27.40 & 200 & $\mathbf{J}$ \\
\hline
\end{tabular}


$1 F$

SEMIVOLATILE ORGANICS ANALYSIS SEBET

TENTATIVELY IDENTIPIED COMPOUNDS

Lab Name: Roy F. Weston, Inc. Work Order: 0630-28-12-0000
CLIBNT' SAKPLE NO. GRS-01-01

Client: HSRC-GRACE ROAD

Matrix:

SOIL

Sample wt/vol: $30.2(\mathrm{~g} / \mathrm{mL}) \mathrm{G}$

Level: (low/med) LOW

6 dec.

\& Molsture: not dec.

Extraction: (SepF/Cont/Sonc) SoNC $\mathrm{pE}: \quad 7.0$

GPC Cleanup: $\quad(Y / N) \underline{Y}$

Iab Sample ID: 9003L865-008

Iab Rile ID! ·

Date Received: $03 / 22 / 90$ 5

CONCENTRATION ONITS:

Number TICs found: 5

\begin{tabular}{|c|c|}
\hline CAS NUMBER & COMPOUND NAME \\
\hline 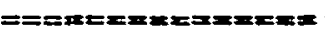 & 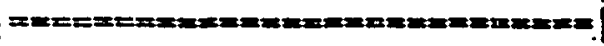 \\
\hline 1 & ALDOL CONDENSATB \\
\hline 2. & ATDOL CONDENSATE \\
\hline 3. & UNRAOWN \\
\hline 4. & UNRAOWN \\
\hline 5. & ORGANIC ACID \\
\hline
\end{tabular}

\begin{tabular}{|l|l|l|}
\hline$R T$ & EST. CONC. & 0 \\
\hline $6.35 \mid 1000$ & \\
$7.08 \mid 1000$ & JAB \\
$7.92 \mid 300$ & JA \\
$9.63 \mid 400$ & JA \\
$19.88 \mid 400$ & $J$ \\
& JB \\
\hline
\end{tabular}


Iab Name: Roy E. Wegton. Inc. Hork Order: 0630-28-12-0000

Cllent: WSRC-GRACE ROAD

Matrix:

SOIL

Lab Sample ID: 9003L865-010

Sample wt/vol:

$30.2(\mathrm{~g} / \mathrm{mL}) \mathrm{G}$

Lab File ID: s040307

Level: (low/med) IOW

Date Recelved: $\underline{0} 3 / 22 / 90$

8 Molsture: not dec. 14 dec.

Date Extracted: $\underline{03 / 27 / 90}$

Extraction: (SepF/Cont/Sonc) SoNC

Date Analyzed: $\underline{04 / 03 / 90}$

GPC Cleanup: $\quad(Y / N) \underline{Y}$ pH: 7.0

Dilution Factor: 1.00

Number TICg found: $\underline{5}$

CONCENTRATION UNITS:

(ug/I or ug/Rg) ug/ $\mathrm{kq}$

\begin{tabular}{|c|c|c|c|c|}
\hline CAS NUKBER & COMPOUND NAMIS & RT & EST. CONC. & $\mathbf{Q}$ \\
\hline mEm & 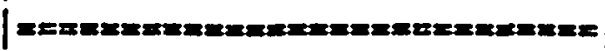 & 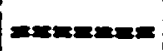 & 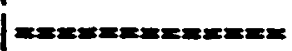 & $\Rightarrow=$ \\
\hline 1. & |AIDOL CONDENSATTE & 5.53 & 600 & JA \\
\hline 2. & ALDOL CONDENSATY & 5.85 & 1000 & JAB \\
\hline 3. & ATDOL CONDBNSATB & 6.53 & 12000 & JA \\
\hline 4. & ATDOI CONDENSATB & 7.57 & 1000 & $\mathbf{J A}$ \\
\hline 5. & PETEAT.ATB. & 24.92 & 500 & $\mathbf{J}$ \\
\hline
\end{tabular}


19

SEMIVOLATIIE ORGANICS ANALYSIS SERET TENTATIVELY IDENTIFIBD -COKPOUNDS

Lab Name: Roy F. Weston, Inc. Work Order: 0630-28-12-0000
CLIENT SAMPLE NO.

GRS-12-01

Client: WSRC-GRACE ROAD

Matrix: SoIr.

Lab Sample ID: 90032865-009

Sample wt/rol:

$30.1(\mathrm{~g} / \mathrm{mr}) \mathrm{G}$

Lab Eile ID: $\quad$ s040306

Level: (low/med) LOW

Date Receiveds $03 / 22 / 90$

\& Moisture: not dec. 5 dec.

Date Extracted: $03 / 27 / 90$

Extraction: (SepF/Cont/Sonc) SONC

Date Analyzed: $04 / 03 / 90$

GPC Cleanup: $(Y / N) \underline{Y}$

pEs 7.0

Dllution Factor: 1.00

CONCENTRATION UNITS:

Number TICs found: 4

(ug/L or ug/Rg) ug $/ \mathrm{Rq}$

\begin{tabular}{|c|c|c|c|c|}
\hline CAS NUMBER & COMPOUND NAME & $\mathbf{R T}$ & BST. CONC. & $\mathbf{Q}$ \\
\hline 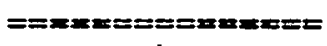 & 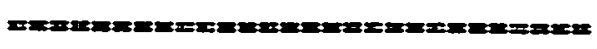 & x=men=m & 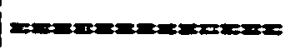 & $x=$ \\
\hline 1 & ALDOL CONDENSATE & 5.83 & 700 & JAB \\
\hline 2. & ATDOT CONDENSATB & 6.53 & 800 & JA \\
\hline 3. & ALDOL CONDENSATE & 7.55 & 200 & JA \\
\hline 4. & EYDROCARBON & 22.22 & 500 & $\boldsymbol{J}$ \\
\hline
\end{tabular}


17

SEMIVOLATILE ORGANICS ANATYSIS SHEAT TENTAT̈IVELY IDENTIEIBD COMPOUNDS

Iab Name: Roy F. Weaton, Inc. Fork Order: 0630-28-12-0000

CLIBNT SAMPLE No.

|GRS-14-01

Cllent: WSRC-GRACE ROAD

Matrix: .

SOIL

Sample wt/vol: $30.3(\mathrm{~g} / \mathrm{mr}) \mathrm{G}$

Level: (low/med) IOW

Moleture: not dec.

15. dec.

Extraction: (Sepr/Cont/sonc) sonc

GPC Cleanup: $\quad(Y / N) \underline{Y}$

Number TICs found: 5
Iab Sample ID: 9003L865-011

Iab Fila ID: $\quad$ s040308

Date Recelved: $03 / 22 / 90$

Date Extracteds 03/27/90

Date Analyzed: 04/03/90

Dilution Factor: 1.00

CONCBNTHATION UNITS (ug/I or ug/kg) ug/Rg

\begin{tabular}{|c|c|c|c|c|}
\hline CAS NUMBER & COMPOUND NAMB & RT & EST. CONC. & $\mathbf{Q}$ \\
\hline Im=m & 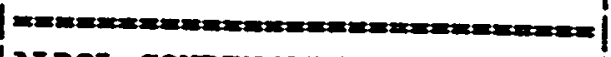 & $=-m=m=1$ & 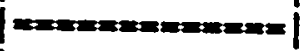 & $=-m=$ \\
\hline 1. & ALDOL CONDENSATE & 5.83 & 1000 & JAB \\
\hline 2 . & AIDOL CONDENSATE & 6.531 & 1000 & JA \\
\hline 3. & PETHAIATE & $25.07 j$ & 1000 & $\boldsymbol{J}$ \\
\hline 4. & PETHAIATE & 25.18 & 1000 & $\boldsymbol{J}$ \\
\hline 5. & | PHTHALATB. & 25.32 & 1000 & $\boldsymbol{J}$ \\
\hline
\end{tabular}


SEMIVOLATILE ORGANICS ANALYSIS SEEETT TENTATIVELY IDENTIFIED COMPOUNDS

Iab Names Roy F. Weston, Inc. Work Order:

Client: WSRC-GRACE ROAD

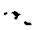

Matrix:

SOIL

Sample wt/vols $\quad 30.0 \quad(\mathrm{~g} / \mathrm{mL}) . \underline{G}$

Level: (low/med) LOW

\& Moisture: not dec. Extraction: (Seps/Cont/Sonc) SONC

GPC Cleanup: $\quad(Y / N) \underline{Y}$ pH: 7.0
Lab Sample ID: 90LE0366-KBI

Iab File ID: $\quad$ 040203

Date Received: $03 / 27 / 90$

Date Extracted: $03 / 27 / 90$

Date Analyzed: $04 / 02 / 90$

Dilution Factor: 1.00

CONCENTRATION UNITS:

Number TICs found: 4 (ug/L or ug/Rg) ug/Rg

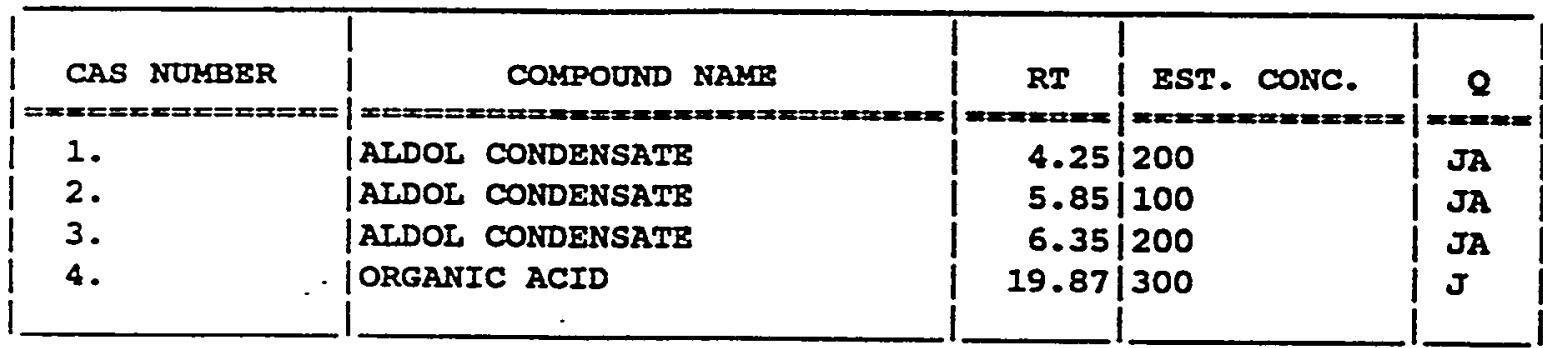


Roy F. Weston, Inc. - Lionville Laboratory VOA ANALYTICAL DATA PACKAGE FOR WSRC-GRACB ROAD

DATE RECEIVED: 03/22/90

RFH LOT : $: 9003 L 865$

\begin{tabular}{|c|c|c|c|c|c|c|}
\hline CLIENT ID & RFW & MTX & PREP & COLLECTION & EXIR/PRBP & ANALYSIS \\
\hline GRS-02-01 & 001 & $\mathbf{s}$ & 90LVพ051 & $03 / 19 / 90$ & $N / A$ & $03 / 27 / 90$ \\
\hline GRS-04-01 & 002 & $\mathbf{s}$ & 90LVพO51 & $03 / 19 / 90$ & $N / A$ & $03 / 27 / 90$ \\
\hline GRS-04-01A & 003 & s & 90LVพ051 & $03 / 19 / 90$ & $N / A$ & $03 / 27 / 90$ \\
\hline GRS-03-01 & 004 & $\mathbf{s}$ & 90LVพ051 & $03 / 19 / 90$ & $N / A$ & $03 / 27 / 90^{\circ}$ \\
\hline GRS-05-01 & 005 & s & 90LVพ051 & $03 / 20 / 90$ & $N / A$ & $03 / 27 / 90$ \\
\hline GRS-06-01 & 006 & $\mathbf{s}$ & 90LVH051 & $03 / 20 / 90$ & $N / A$ & $03 / 27 / 90$ \\
\hline GRS-11-01 & 007 & $s$ & 90LVF051 & $03 / 20 / 90$ & $N / A$ & $03 / 27 / 90$ \\
\hline GRS-01-01 & 008 & $\mathbf{s}$ & 90LVพ054 & $03 / 20 / 90$ & $\mathrm{~N} / \mathrm{A}$ & $03 / 31 / 90$ \\
\hline GRS-12-01 & 009 & $\mathbf{s}$ & 90LVพ051 & $03 / 20 / 90$ & $N / A$ & $03 / 27 / 90$ \\
\hline GRS-13-01 & 010 & $\mathbf{s}$ & 90IVF051 & $03 / 21 / 90$ & $N / A$ & $03 / 27 / 90$ \\
\hline GRS-14-01 & 011 & $\mathbf{s}$ & 90IVพ051 & $03 / 21 / 90$ & $N / A$ & $03 / 27 / 90$ \\
\hline GRS-13-01E & 012 & พ & $90 L V B 043$ & $03 / 21 / 90$ & $N / A$ & $03 / 29 / 90$ \\
\hline GRS-14-018 & .014 & พ & $902 V B 043$ & $03 / 21 / 90$ & $N / A$ & $03 / 29 / 90$ \\
\hline GRS-14-01C & 015 & พ & $90 L V B 043$ & $03 / 21 / 90$ & N/A & $03 / 29 / 90$ \\
\hline
\end{tabular}

LAB QC:

VBLR

VBLR

VBLR
MB1

MBI

MB1
S 90LVพ051

S 90LVW054

พ 9OLVBO43
N/A N/A

N/A N/A

$\mathrm{N} / \mathrm{A} \quad \mathrm{N} / \mathrm{A}$
$03 / 27 / 90$

$03 / 30 / 90$

$03 / 29 / 90$ 
B-174 
ROY F. HESTON, INC.

Lionville Laboratory

CLIENT: WSRC-GRACE ROAD

RFW \#: 9003L865

พ.0. \#: $0630-28-12$

SAMPLES RECEIVED: 03-22-90

\section{INORGANIC NARRATIVE}

The following is a summary of the quality control results and a description of any problems encountered during the analysis of this batch of samples:

1. All sample holding times as required by 40 CFR136 were met for water samples. Note: Holding times for soil samples have not been promulgated by the USEPA.

2. All preparation blanks were analyzed below the required detection limit.

3. All calibration verification checks were within the required control limits of 90-100\%. Calibration verification is performed using independent standards.

4. Matrix spike recoveries for total organic carbon were outside the 75-125\% guidance limits. This may be due to an interference present in the sample matrix.

5. Replicate results for total organic carbon were outside the $20 \%$ guidance limit. This may be due to sample inhomogeneity.

6. The analytical methods applied by the laboratory, unless otherwise requested, for all inorganic analyses are derived from the USEPA Method for Chemical Analysis of Water and Wastes (USEPA 600/4-79-020), and Standard Methods for the Examination of Water and Wastewater 16 ed. Methods for the analysis of solid samples are derived from Test Methods for Evaluating Solid waste (USEPA SW846).

NOTE: For solid samples, all results are reported on a dry weight basis.

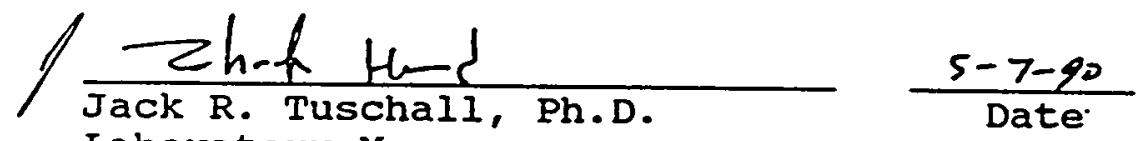

Laboratory Manager

Lionville Analytical Laboratory

pas. $21 \backslash i 3-865$ 


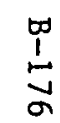




\section{ROY F. WESTON, INC. \\ GLOSSARY OF TERMS - INORGANIC REPORTS}

\section{DATA QUALIEIERS}

$U$ - Indicates that the parameter was not detected at or above the reported limit. The associated numerical value is the sample detection limit.

* - Indicates that the original sample result is greater than $4 \times$ the spike amount added. The USEPA-CLP has determined that spike results on samples where this occurs may be unreliable and, therefore, the control limits are not applicable.

\section{ABBREVIATIONS}

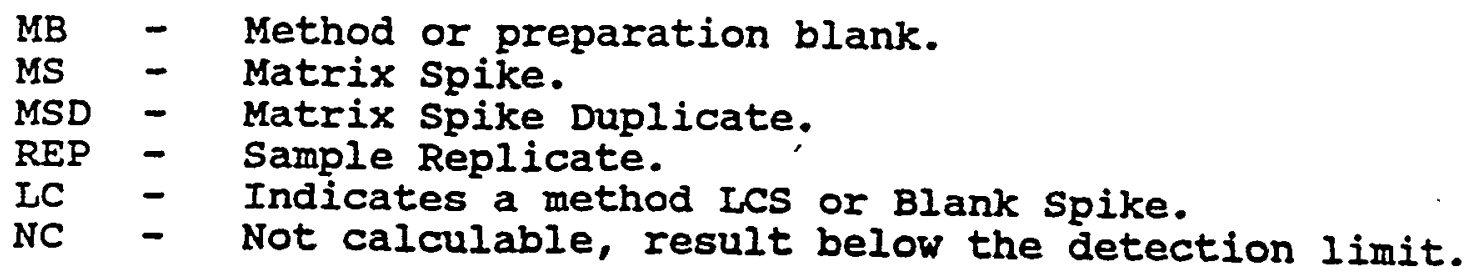

\section{LABORATORY CHRONOLOGY AND HOLPTIME REPORT}

The test code listed indicates the specific analysis or preparation procedure employed. The codes may be interpreted as follows:

$$
\begin{aligned}
& \text { MAAW - Metals prep test for AA digestion, water matrix. } \\
& \text { MAAS - Metals prep test for AA digestion, soil matrix. } \\
& \text { MICW - Metals prep test for ICP digestion, water matrix. } \\
& \text { MICS - Metals prep test for ICP digestion, soil matrix. } \\
& \text { M**TO- This type of code indicates a total metal analysis } \\
& \text { (eg. MAGTO indicates an analysis for total silver). }
\end{aligned}
$$

A suffix of $-R$ or $-S$ following these codes indicates $a$ replicate or spike analysis respectively. 
ROY F. WESTON IXC.

INORGANICS DATA SULARRY REPORT 05/01/90

CLIENT: WSRC-GRACE ROAD

WORK ORDER: 0630-28-12-0000

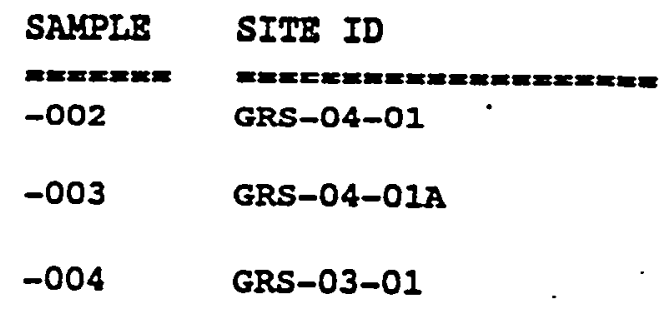

ANALYTB

* SOLIDS

* SOLIDS

CATION EXChANGE CAPACIT EXCHANGEABLE BASES EXCEANGEABIT ACIDS TOTAL ORGANIC CARBON PE

$\begin{array}{ll}-005 & \text { GRS-05-01 } \\ -006 & \text { GRS-06-01 } \\ -007 & \text { GRS-11-01 } \\ -008 & \text { GRS-01-01 } \\ -009 & \text { GRS-12-01. }\end{array}$

$-010 \quad$ GRS $-13-01$

-011 GRS-14-01
8 SOLIDS

\& SOLIDS

* SOLIDS

8 SOLIDS

t SOLIDS CATION EXCEANGB CAPACIT EXCEANGEABLR BASES EXCHANGEABIB ACIDS TOTAL ORGANIC CARBON PE

sorIDS

\& sorIDS

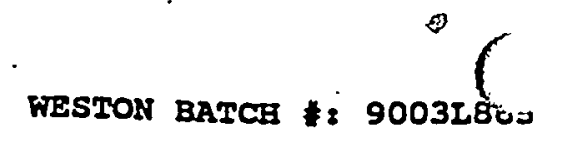

REPORTING

\begin{tabular}{|c|c|c|}
\hline $\begin{array}{l}\text { RESULT } \\
== \pm== \\
94.2\end{array}$ & ONITS . & $\begin{array}{l}\text { LIKIT. } \\
\quad 0.10\end{array}$ \\
\hline 81.8 & 8 & 0.10 \\
\hline 2.4 & $\operatorname{meq} / 100 \mathrm{~g}$ & 0.011 \\
\hline $\begin{array}{l}2.3 \\
2.3\end{array}$ & meq/ $/ 100 \mathrm{~g}$ & 0.011 \\
\hline $\begin{array}{c}470^{2.3} \\
6.2\end{array}$ & $\begin{array}{l}\text { MG/RG } \\
\text { PH ONITS }\end{array}$ & $\begin{array}{c}0.20 \\
-52.0 \\
0.10\end{array}$ \\
\hline
\end{tabular}

$91.6 \quad 0.10$

$88.6 \quad 0.10$

$90.4 \& 0.10$

$93.0 \quad 0.10$

$\begin{array}{clc}94.7 & & 0.10 \\ 1.8 & \text { meg/100g } & 0 \\ 1.4 & \mathrm{meg} / 100 \mathrm{~g} & .0 \\ 1.4 & \mathrm{meq} / 100 \mathrm{~g} & .0 .20 \\ 808 & \mathrm{MG} / \mathrm{RG} & 58.0 \\ 6.5 & \text { PH UNITS } & 0.10\end{array}$

$\begin{array}{ll}82.7 & 0.20\end{array}$

$85.3 \quad 0.10$ 
ROY F. WESTON INC.

INORGANICS METEOD BLANK DATA SULLARY PAGE 05/01/90.

CLIENT: WSRC-GRACE ROAD WORK ORDER: 0630-28-12-0000

\begin{tabular}{|c|c|c|}
\hline $\begin{array}{l}\text { SALPLB } \\
\text { =-2:= }\end{array}$ & SITE ID & AKALYTE \\
\hline BLANK10 & 90ITZ003-LBI & TOTAL ORGANIC CARBON \\
\hline BLANR2O & 90LTZ003-LB2 & TOTAL ORGANIC CARBON \\
\hline
\end{tabular}

WESTON BATCE $i^{\circ} 90032865$

\begin{tabular}{cccc} 
RESULT & UNITS & \multicolumn{1}{l}{$\begin{array}{l}\text { REPORTING } \\
\text { LIMIT . }\end{array}$} \\
\hline 20.0 u & KG/KG & .20 .0 \\
20.0 u & MG/KG & 20.0
\end{tabular}


ROY E. WESTON INC.

INORGANICS ACCURACY REPORT 05/01/90

CLIENT: WSRC-GRACE ROAD

FORK ORDER: 0630-28-12-0000

BLANR20 90LTZ003-MB2

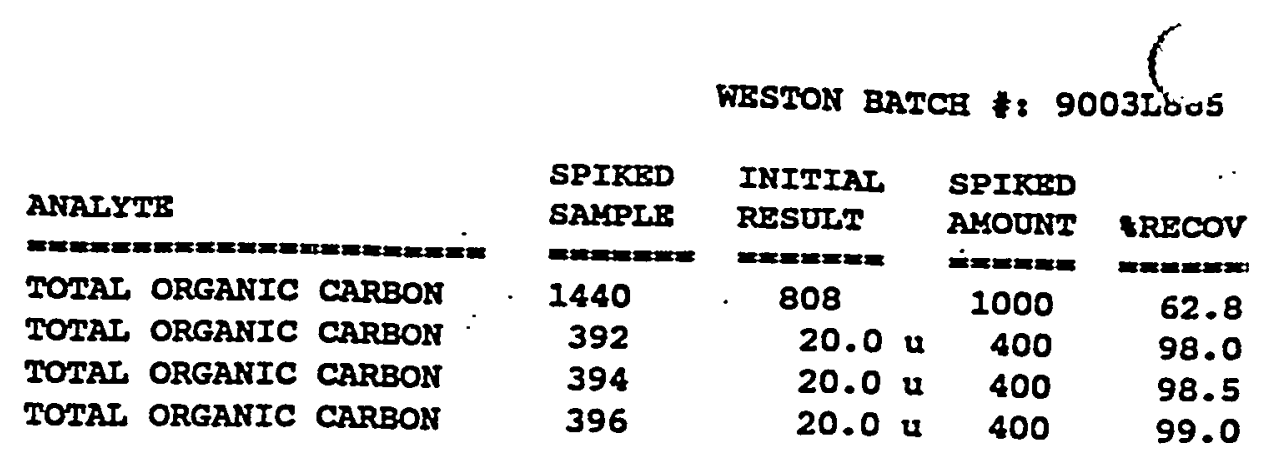


ROY P. WESTON INC.

INORGANICS DUPLICATE SPIKR REPORT 05/01/90

CLIENT: WSRC-GRACB ROAD WORK ORDER: 0630-28-12-0000

SAMPLE SITE ID

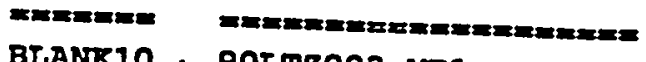

BLANK10 - 90LTZ003-MBI
ANÄTYTE

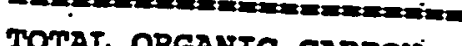

WESTON BATCH $\$ 290032865$

\begin{tabular}{lll} 
SPIKB 1 & SPIKE $\$ 2$ \\
IRECOV & IRECOV & IDIFP \\
\hline 98.0 & 98.5 & 0.50
\end{tabular}


ROY F. WESTON INC.

INORGANICS PRECISION RERORT 05/01/90

CLIENT: WSRC-GRACE ROAD

FORK ORDER: 0630-28-12-0000

\begin{tabular}{|c|c|}
\hline SAMPLE & SITE ID \\
\hline$m=x=m=$ & 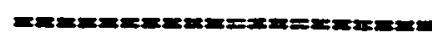 \\
\hline$-004 R R P$ & GRS-03-01 \\
\hline -009REP & GRS $-12-01$ \\
\hline
\end{tabular}

ANATYTB
CATION EXCEANGB CAPACIT
EXCEANGEABIE BASES
PA
CATION BXCHANGB CAPACIT
EXCBANGBABLE BASBS
EXCEANGEABLE ACIDS
TOTAL ORGANIC CARBON

HESTON BATCH \& 90032865

INITIAL RESULT

$m=2 \pi=m$

REPLICATE DIEP

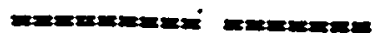

2.4

2.3

6.2

1.8

1.4

1.4

808

$$
0.00
$$

0.00

2.3

0.16

6.2

$1.6 \quad 11.8$

$1.2 \quad 15.4$

1.215 .4

540 
Roy F. Weston, Inc. - ILonville Laboratory INORGANIC ANALYTICAI DATA PACKAGE FOR WSRC-GRACE ROAD

DATE RECEIVED: $\quad 03 / 22 / 90$

REF LOT *:9003L865

CLIENT ID /ANALYSIS REW * MTX PREP \& COITECTION EXTR/PREP

ANAIYSIS

$\longrightarrow$

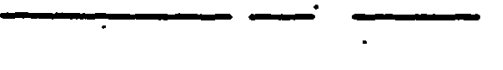

GRS-04-01

\section{SOLIDS}

GROSS ALPEA

GROSS BETA

GRS-04-01A

* SOLIDS

GRS-03-01

CATION. EXCEANGE CAPA CATION EXCHANGE CAPA CBC BASB

CEC BASE

CEC PREP

CEC TITRATION

TOTAL ORGANIC CARBON $\mathrm{PH}$

$\mathrm{PH}$

GRS-05-01

- SOLIDS

GRS-06-01

- SOLIDS

GROSS ALPEA

GROSS BETA

GRS-11-01

* solids

GRS-01-01

SOLIDS
002

002

002

003

004

004 REP

004

004 REP

004

004

004

004

004 REP s

$s$

s

90L\&S05I 03/19/90

$03 / 19 / 90$

$03 / 19 / 90$

s 90L8s051 03/19/90

$03 / 27 / 90$

$03 / 28 / 90$

$$
03 / 27 / 90 \quad 03 / 28 / 90
$$

$\begin{array}{lll}\text { s } & 90 L C E 003 & 03 / 19 / 90 \\ s & 90 L C E 003 & 03 / 19 / 90 \\ s & 90 L C E 003 & 03 / 19 / 90 \\ s & 90 L C B 003 & 03 / 19 / 90 \\ \text { s } & & 03 / 19 / 90 \\ \text { s } & 90 L C 8003 & 03 / 19 / 90 \\ \text { s } & 90 L T 2003 & 03 / 19 / 90 \\ \text { s } & 90 L \text { LEO54 } & 03 / 19 / 90 \\ \text { s } & 90 L \text { LEO54 } & 03 / 19 / 90\end{array}$

$04 / 27 / 90$

$04 / 27 / 90$

$04 / 26 / 90$

$04 / 26 / 90$

$04 / 25 / 90$

$04 / 03 / 90$

$03 / 28 / 90$

$03 / 28 / 90$

$04 / 27 / 90$

$04 / 27 / 90$

$04 / 27 / 90$

$04 / 27 / 90$

$04 / 25 / 90$

$04 / 26 / 90$

$04 / 03 / 90$

$03 / 28 / 90$

$03 / 28 / 90$

005

$S$ 90L:S051 03/20/90

$03 / 27 / 90$

$03 / 28 / 90$

006

006

006

s 90L\&S051

$03 / 20 / 90$

$03 / 20 / 90$

$03 / 27 / 90$

$03 / 28 / 90$

$03 / 20 / 90$

007

S 902\$5051

$03 / 20 / 90$

$03 / 27 / 90$

$03 / 28 / 90$

008

s 90Lะs051 03/20/90

$03 / 27 / 90$

$03 / 28 / 90$ 
Roy F. Weaton, Inc. - ILonville Laboratory

INORGANIC ANALYTICAL DATA PACRAGE FOR

WSRC-GRACB ROAD

DATE RECEIVED: $03 / 22 / 90$

RFW LOT :9003L865

CLIENT ID /ANALYSIS RFW * MTX PREP * COLLECTION EXTR/PREP

ANATYYSIS.

GRS-12-01

$\begin{array}{ll}\text { \& SOLIDS } & 009 \\ \text { CATION EXCHANGE CAPA } & 009 \\ \text { CATION EXCHANGE CAPA } & 009 \text { REP } \\ \text { CEC BASE } & 009 \\ \text { CEC BASB } & 009 \text { REP } \\ \text { CEC PREP } & 009 \\ \text { CEC TITRATION } & 009 \\ \text { CEC TITRATION } & 009 \mathrm{REP} \\ \text { TOTAI ORGANIC CARBON } & 009 \\ \text { TOTAI ORGANIC CARBON } & 009 \mathrm{RBP} \\ \text { TOTAI ORGANIC CARBON } & 009 \mathrm{MS} \\ \text { PE } & 009\end{array}$

90Lt5051

9OLCE003

9OLCE003

90 LCE003

90LCE003

$901 \mathrm{LEO03}$

$901 \mathrm{LB} 003$

$90 L T Z 003$

$901 T 2003$

$901 T 2003$

9OLPRO54

$03 / 20 / 90$

$03 / 20 / 90$

$03 / 20 / 90$

$03 / 20 / 90$

$03 / 20 / 90$

$03 / 20 / 90$

$03 / 20 / 90$

$03 / 20 / 90$

$03 / 20 / 90$

$.03 / 20 / 90$

$03 / 20 / 90$

$03 / 20 / 90$

$03 / 27 / 90$

$04 / 27 / 90$

$04 / 27 / 90$

$04 / 26 / 90$

$04 / 26 / 90$

$04 / 25 / 90$

$04 / 25 / 90$

$04 / 03 / 90$

$04 / 03 / 90$

$04 / 03 / 90$

$03 / 28 / 90$

GRS-13-01

8 SOLIDS

010

S 90LtS051

$03 / 21 / 90$

$03 / 27 / 90$

$03 / 28 / 90$

GRS-14-01

8 SOLIDS

011

s 90285051

$03 / 21 / 90$

$03 / 27 / 90$

$03 / 28 / 90$

GRS-13-OB

GROSS ALPEA

GROSS BETA

013

013

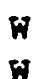

$03 / 21 / 90$

$03 / 21 / 90$
$03 / 28 / 90$

$04 / 27 / 90$

$04 / 27 / 90$

$04 / 27 / 90^{-}$

$04 / 27 / 90$

$04 / 25 / 90$

$04 / 26 / 90$

$04 / 26 / 90$

$04 / 03 / 90$

$04 / 03 / 90$

$04 / 03 / 90$

$03 / 28 / 90$

LAB QC:

TOTAL ORGANIC CARBON TOTAL ORGANIC CARBON TOTAL ORGANIC CARBON TOTAL ORGANIC CARBON TOTAL ORGANIC CARBON
KBI

MBI BS

MBI BSD

MB2

MB2 BS

\begin{abstract}
90LTZ003
90LTZ003

90LTZ003

90LTZ003

$902 T 2003$
\end{abstract}

$N / A$
$N / A$
$N / A$
$N / A$
$N / A$

$04 / 03 / 90$

$04 / 03 / 90$

$04 / 03 / 90$

$04 / 03 / 90$

$04 / 03 / 90$ 


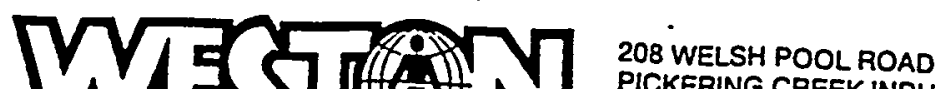 \\ PICKERING CREEK INDUSTRIAL PARK \\ LONVILLE PA 19353

\section{June 1990}

Mr. Howard Hickey

Westinghouse Savannah River Company
Building 740-3A

Aiken, SC 29808 Re: Analytical results for sample collected
At WSRC-Grace Road (RFW Batches 9002L606, 607, and 9003L865)

Dear Mr. Hickey:

Enclosed are the analytical results for WSRC release order 13. These results consist of an electronic data deliverable, and a marizing $Q A / Q C$ results. If we can provide any further information, please call either
myself or Ben Shapiro.

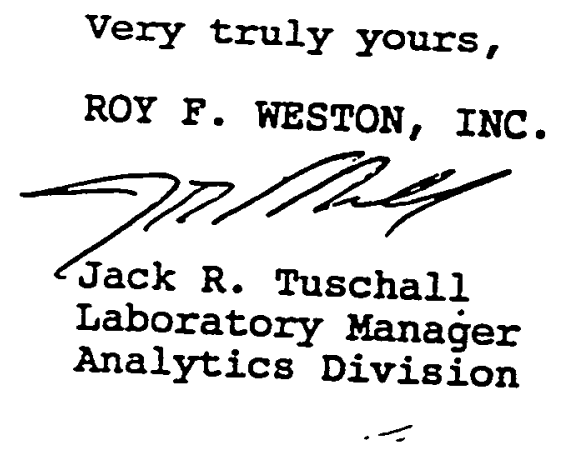

/clk

Enclosure:

cc: Michelle Wilson

Benjamin Shapiro

David Oliphant 
WESTON ANALYTICS

LIONVILIE, PA

DATA TRANSFER RECORD
SHEET 1 OF 1 DISK 1 OF $\frac{1}{1}$ IIMS. . WALIS $\bar{x}$ INITIAL $\mathbf{X}$ CORRECTED OTHER

CLIENT: WSRC-GRACE ROAD.

PROJECT MANAGER: BEN SHAPIRO

ELECTRONIC $\mathrm{X}$ HARD COPY

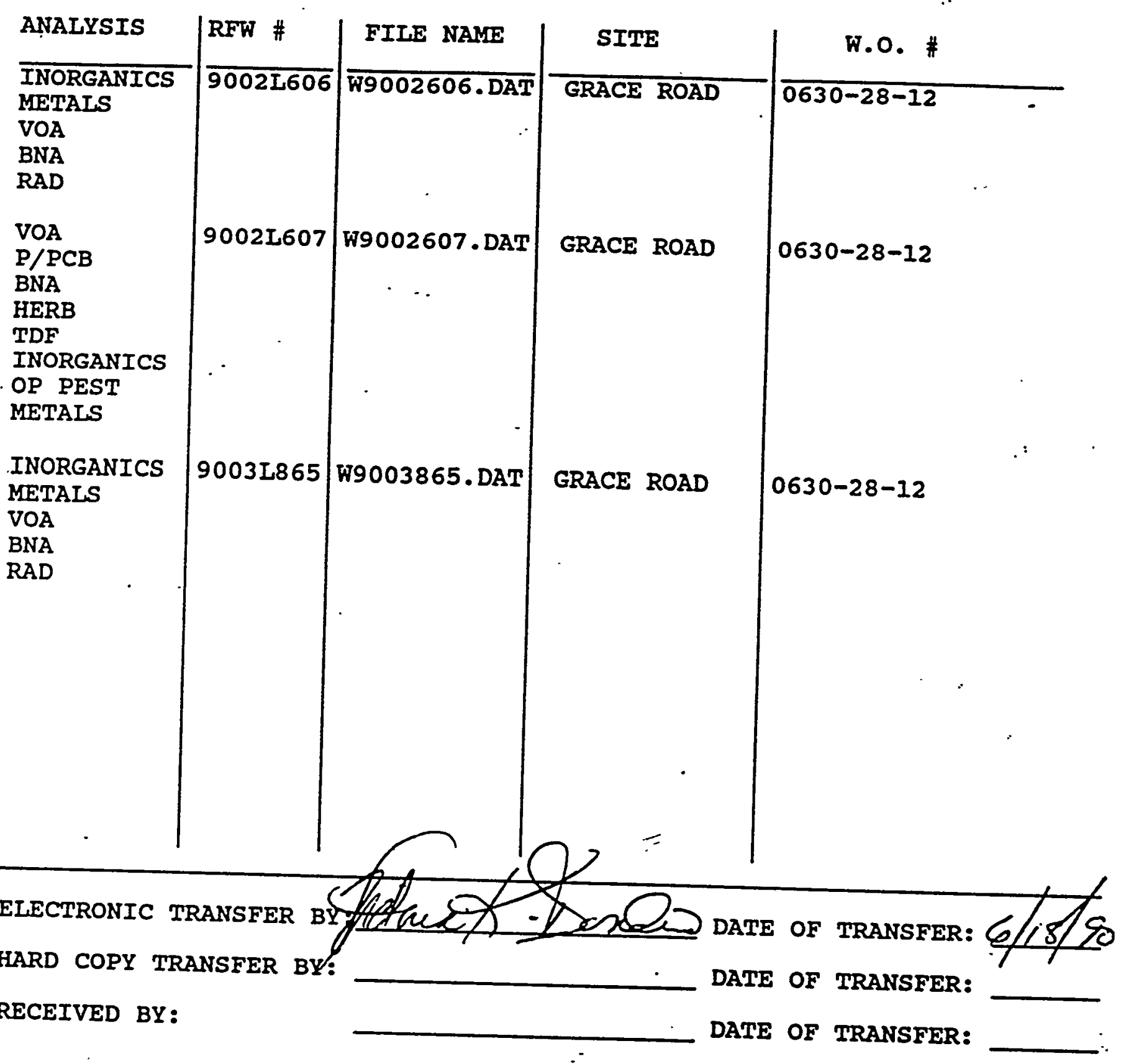


Roy F. Wegton, Inc. - Ilonville Laboratory BNA ANALYTICAC DATA PACKAGE FOR WSRC-GRACE ROAD

DATE RECEIVED: $03 / 22 / 90$

RFW IOT *:9003L865

\begin{tabular}{ll} 
CLIENT ID & RFW \\
\hline GRS-02-01 & 001 \\
GRS-02-01 & 001 KS \\
GRS-04-01 & 002 \\
GRS-04-01 & 002 REP \\
GRS-04-01A & 003 \\
GRS-03-01 & 004 \\
GRS-05-01 & 005 \\
GRS-06-01 & 006 \\
GRS-11-01 & 007 \\
GRS-01-01 & 008 \\
GRS-12-01 & 009 \\
GRS-13-01 & 010 \\
GRS-14-01 & 011
\end{tabular}

MITX PREP * COLLECTION EXTR/PREP

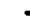

S 90250366

s 90L.80366

s $90 \mathrm{Lz} 0366$

s 90 LE0366

s 90280366

s 90 LE0366

S $90 \mathrm{LE0366}$

s $90 \mathrm{LE0366}$

s 90LE0366

s $90 \mathrm{LB} 0366$

s 90LE0366

S 90LE0366

S 90LE0366
$03 / 19 / 90$

$03 / 19 / 90$

$03 / 19 / 90$

$03 / 19 / 90$

$03 / 19 / 90$

$03 / 19 / 90$

$03 / 20 / 90$

$03 / 20 / 90$

$03 / 20 / 90$

$03 / 20 / 90$

$03 / 20 / 90$

$03 / 21 / 90$

$03 / 21 / 90$
$03 / 27 / 90$

$03 / 27 / 90$

$03 / 27 / 90$

$03 / 27 / 90$

$03 / 27 / 90$

$03 / 27 / 90$

$03 / 27 / 90$

$03 / 27 / 90$

$03 / 27 / 90$

$03 / 27 / 90$

$03 / 27 / 90$

$03 / 27 / 90$

$03 / 27 / 90$
ANALYSIS

$04 / 02 / 90$

$04 / 02 / 90$

$04 / 02 / 90$

$04 / 02 / 90$

$04 / 02 / 90$

$04 / 02 / 90$

$04 / 02 / 90$

$04 / 02 / 90$

$04 / 02 / 90$

$04 / 03 / 90$

$04 / 03 / 90$

$04 / 03 / 90$

$04 / 03 / 90$

\section{$\angle A B Q C$ :}

SBLK

SBLR

MB1

MBI BS

S $902 \mathrm{LO} 0366$

N/A

$03 / 27 / 90$

S 90L:0366

N/A

$03 / 27 / 90$

$04 / 02 / 90$

$04 / 02 / 90$ 


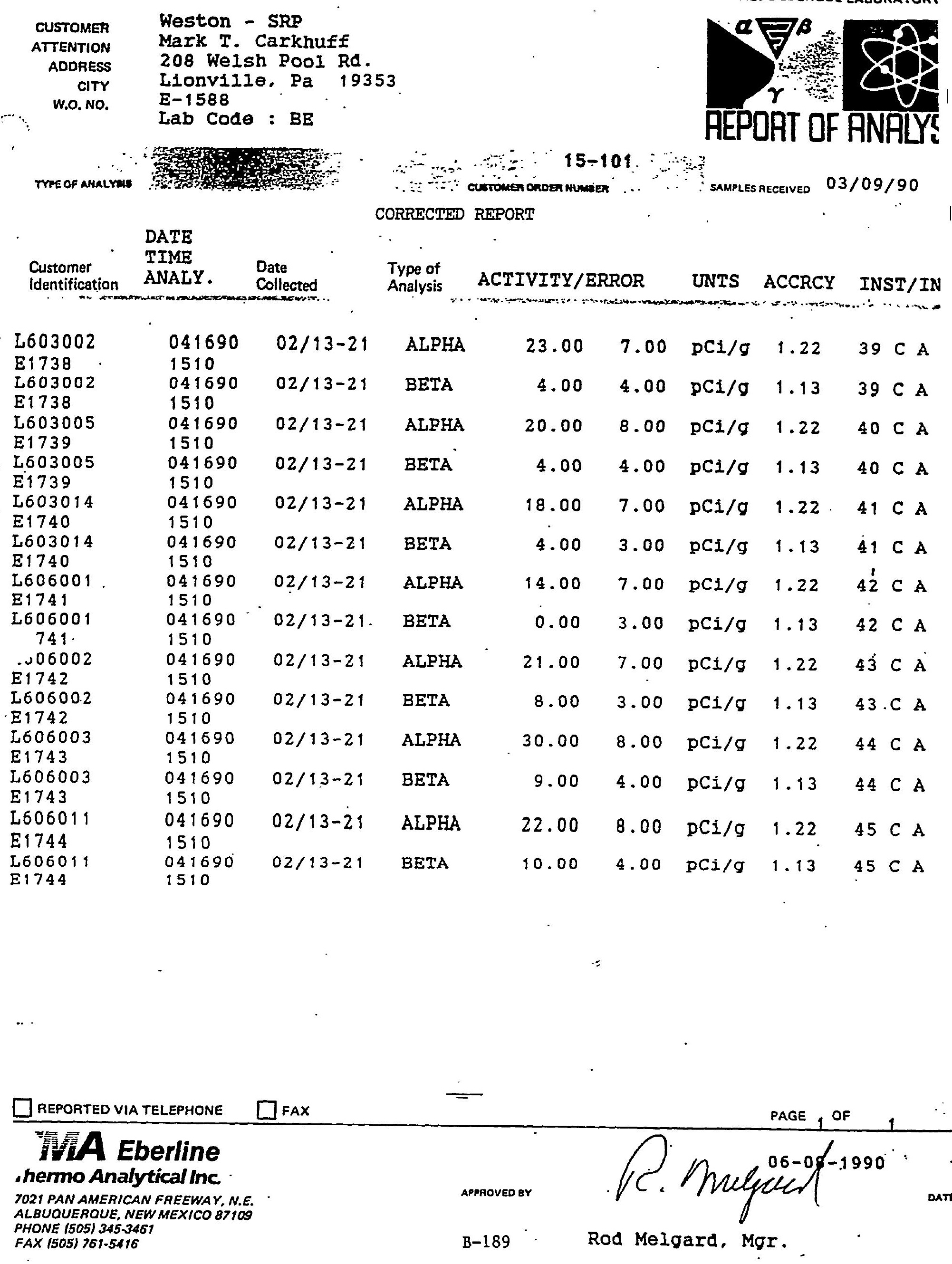




$\begin{aligned} \text { CUSTOMER } & \text { Weston - SRP } \\ \text { ATTENTION } & \text { Mark T. Carkhuff } \\ \text { ADDRESS } & 208 \text { Weish POOI Rd. } \\ \text { CITY } & \text { Lionvilie. Pa } 19353 \\ \text { W.O.NO. } & \text { E-1585 } \\ & \text { Lab Code : BE }\end{aligned}$

$15-101:=$

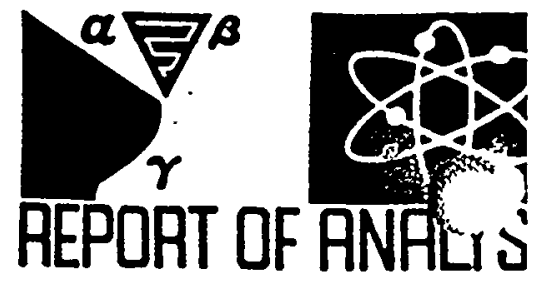

TYPE OF ANALYSIS

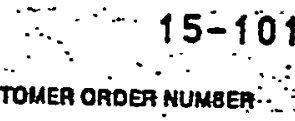

SAMPLES ReCEIVED $03 / 30 / 90$

DATE

$\begin{array}{ll}\text { Customer } & \text { TIME } \\ \text { Identification } & \text { ANALY. }\end{array}$

Date

Collected

042390

0820

042390

0820

042390

0820

042390

0820

041990

0755

041990

0755

9003865013

$\begin{array}{ll}9003865002 & 042390 \\ E 2072 & 0820 \\ 9003865002 & 042390 \\ E 2072 & 0820 \\ 9003865006 & 042390 \\ E 2073 & 0820 \\ 9003865006 & 042390 \\ E 2073 & 0820 \\ 9003865013 & 041990 \\ E 2074 & 0755 \\ 9003865013 & 041990 \\ E 2074 & 0755\end{array}$

$\begin{array}{llllllll}03 / 19-21 & \text { ALPHA } & 11.00 & 6.00 & \mathrm{pCi} / \mathrm{g} & 1.24 & 39 \mathrm{CA} \\ 03 / 19-21 & \text { BETA } & 13.00 & 6.00 & \mathrm{pCi} / \mathrm{g} & 1.12 & 39^{\circ} \mathrm{CA} \\ 03 / 19-21 & \text { ALPHA } & 5.00 & 3.00 & \mathrm{pCi} / \mathrm{g} & 1.24 & 41 \mathrm{CA} \\ 03 / 19-21 & \text { BETA } & 7.00 & 3.00 & \mathrm{pCi} / \mathrm{g} & 1.12 & 41 \mathrm{CA} \\ 03 / 19-21 & \text { ALPHA } & 0.00 & 1.00 & \mathrm{pCi} / 1 & 1.24 & 40 \mathrm{DDP} \\ 03 / 19-21 & \text { BETA } & 0.00 & 4.00 & \mathrm{pCi} / 2 & 1.12 & 40 \mathrm{DDP}\end{array}$

E2074

ACTIVITY/ERROR

UNTS

ACCRCY

INST/IN]

11.00 $\infty$ 
10C 0220900304900040WA9002L606 001

GRS 10010220900416901200 WA9002L606 001 ALPHAG9W

GRS 10010220900416901200 WA9002L606 001 BETAG 9WLT

GRS 1001 0220900315900002WA9002L606 001 CEC EX

GRS 10010220900315900002 WA9002L606 001RCEC EX

GRS 10010220900312900002 WA9002L606 001 CECB EX

GRS 10010220900312900002 WA9002L606 001RCECB EX

10010220900308900002 WA9002L606 001 CECT EX

10010220900308900002 WA9002L606 001RCECT EX

10010220900302900002 WA9002L606 001 TOC 9I

$10010220900308900042 \mathrm{WA} 9002 \mathrm{~L} 606001 \mathrm{PH} \quad 9 \mathrm{R}$

$10010220900323900226 \mathrm{WA} 9002 \mathrm{~L} 606001 \mathrm{AG}$ 22LT

10010220900330900225 WA9002L606 001 AS IBLT

$10010220900323900226 \mathrm{WA} 9002 \mathrm{~L} 606001 \mathrm{BA}$ 22LT

$10010220900323900226 \mathrm{WA} 9002 L 606001 \mathrm{CD} \quad 22 \mathrm{LT}$

$10010220900323900226 \mathrm{FA9002L606} 001 \mathrm{CR} \quad 22$

GRS $10010220900319900042 \mathrm{WA} 90024606001$ HGTOT 1DLT

GRS $10010220900405900225 \mathrm{WA} 90021606001 \mathrm{~PB}^{\circ} 1 \mathrm{~B}$

-GRS-1001-0220900401900225אĀ9002L606-001-SE-IBTT

GRS $10010220900228900032 \mathrm{WA} 9002 \mathrm{~L} 606001 \mathrm{MEC} 6 \mathrm{D} 82 \mathrm{~J}$

GRS 10010220900228900032 WA9002L606 001 PBFB 2J

GRS 10010220900228900032 WA9002L606 001 12DCD42J

GRS $10010220900228900032 \mathrm{WA} 9002 L 606001$ CH3CL 2JLT

10010220900228900032 WA9002L606 001 CH3BR 2JLT

GRS 10010220900228900032 WA9002L606 001 C2H5CL2JLT

GRS $1001.0220900228900032 \mathrm{WA} 9002 L 606001$ CH2CL22JB

GRS $10010220900228900032 \mathrm{FA9002L606} 001$ ACET 2JLT

GRS $10010220900228900032 \mathrm{WA9002L606} 001$ CS2 2JLT

GRS 10010220900228900032 WA9002L606 001 11DCE 2JLT

GRS $10010220900228900032 \mathrm{WA} 9002 \mathrm{~L} 606001$ 11DCLE2JLT

GRS 10010220900228900032 พื่9002L606 001 T12DCE2JLT

GRS 10010220900228900032 WA9002L606 001 CHCL3 2JLT

GRS 10010220900228900032 WA9002L606 001 12DCLE2JLT

GRS 10010220900228900032 WA9002L606 001 MEK 2JLT

GRS $10010220900228900032 \mathrm{WA} 9002 \mathrm{~L} 606001$ 111TCE2JLT

GRS 10010220900228900032 WA9002L606 001 CCL4 2JLT

GRS 10010220900228900032 WA9002L606 001 VINYLA2JLT

GRS 10010220900228900032 WA90021606 001 BRDCLM2JLT

GRS 10010220900228900032 WA9002L606 001 12DCLP2JLT

GRS 10010220900228900032 WA9002L606 001 13DCPC2JLT

GRS 10010220900228900032 WA9002L606 001 TRCLE 2JLT

GRS 10010220900228900032 WA9002L606 001 DBRCLM2JLT

GRS 10010220900228900032 WA9002L606 001 112TCE2JLT

GRS $10010220900228900032 \mathrm{WA} 9002 \mathrm{~L} 606001$ C6H6 2JLT

GRS 10010220900228900032 WA9002L606 001 T13DCP2JTLT

GRS $10010220900228900032 \mathrm{WA} 9002 \mathrm{~L} 606001$ CHBR3 2JLT

GRS 10010220900228900032 WA9002L606 001 4ME2PE2JLT
94.08

14. OPCIG

0.0 PCIG

$0.94 \mathrm{MEQ1}$

$0.92 \mathrm{MEQI}$

$0.34 \mathrm{MEQ1}$

$0.32 \mathrm{MEQ1}$

$0.60 \mathrm{MEQ} 1$

$0.60 \mathrm{MEQ1}$

513MGKG

$6.6 \mathrm{PH} \mathrm{U}$

1. 9 MGKG

1. $9 M$ MGKG

37. 3MGKG

$0.93 M G K G$

4.2MGKG

0.11 MGKG

1. 4 MGKG

0.97 MEKG

103PER

99PER

100PER

11UGKG

11UGKG

11UGKG

IIUGKG

14UGKG

11UGKG

5UGKG

5UGKG

SUGKG

5UGKG

5UGKG

SUGKG

11UGKG

5UGKG

5UGKG

11UGKG

5UGKG

5UGKG

5UGKG

5UGKG

5UGKG

5UGKG

5UGKG

5UGKG

5UGKG

IIUGKG
$10 \quad \mathrm{BT}$

10EBUNK

1OEBUNK

IONADKS

IONADKS

IONAALM

IONAALM

IONAJS

10NAJS

1ODODKS

$10 \mathrm{PHJH}$

1.00ICALM

1.00 AAAER

1.00 ICALM

1.00 ICALM

1.00 ICALM

$1.00 \mathrm{HGSO}$

1. 00AACMT

. OOAAMLR

$1.0000 M S J B D$

1.0000MSJBD

$1.0000 M S J B D$

1.0000 MSJBD

$1.0000 \mathrm{MSJBD}$

$1.0000 M S J B D$

1.0000 MSJBD

$1.0000 \mathrm{MSJBD}$

$1.0000 M S J B D$

1.0000 MSJBD

$1.0000 \mathrm{MSJBD}$

$1.0000 \mathrm{MSJBD}$

$1.0000 \mathrm{MSJBD}$

$1.0000 \mathrm{MSJBD}$

$1.0000 \mathrm{MSJBD}$

1.0000 MSJBD

$1.0000 \mathrm{MSJBD}$

$1.0000 \mathrm{MSJBD}$

$1.0000 \mathrm{MSJBD}$

$1.0000 M S J B D$

$1.0000 M S J B D$

$1.0000 \mathrm{MSJBD}$

$1.0000 M S J B D$

$1.0000 \mathrm{MSJBD}$

$1.0000 \mathrm{MSJBD}$

$1.0000 \mathrm{MSJBD}$

$1.0000 \mathrm{MSJBD}$

$1.0000 \mathrm{MSJBD}$

$1.0000 \mathrm{MSJBD}$
O\% SOLIDS

If SOLN

OGROSS ALPHA

ONONVOLATILE BETA IBETAN

OCATION EXCHANGE CAPAICEC $N$ OCATION EXCHANGE CAPAICEC R OEXCHANGEABLE BASES ICECBN OEXCHANGEABLE BASES ICECBR OEXCHANGEABLE ACIDS ICECTN OEXCHANGEABLE ACIDS ICECTR OTOTAL ORGANIC CARBONIOCT N OPH

OSILVER, TOTAL MAGTON

OARSENIC, TOTAL MASTON

OBARIUM, TOTAL MBATON

OCADMIUM, TOTAL MCDTON

OCHROMIUM, TOTAL MCRTON

OMERCURY, TOTAL MHGTON OLEAD, TOTAL

OSELENIUM, TOTAL

MPBTON

MSETON

54. 50OTOLUENE-D8

$0624 \mathrm{HN}$

OOROMOFLUOROBENZENE O624HN

$54.5001,2-D I C H Z O R O E T H A N E-D 0624 \mathrm{HN}$ OCHLOROMETHANE O624HN OBROMOMETHANE O624HN OVINYI CHLORIDE O624HN OCHLOROETHANE O624HN OMETHYLENE CHLORIDE O624HN OACETONE

OCARBON DISULEIDE

$0624 \mathrm{HN}$ $0624 \mathrm{HN}$ 011-DICHIOROETHENE 0624HN 01,1-DICHLOROETHANE O624HN 01,2-DICHLOROETHENE 10624HN OCHLOROFORM

$01,2-D T C H I$ 02-BUTANONE $0624 \mathrm{HN}$ $01,1,1-$ TRICHLOROETHANO $624 \mathrm{HN}$ OCARBON TETRACHLORIDEO $624 \mathrm{HN}$ OVINYI ACETATE O624HN OBROMODICHLOROMETHANEO $624 \mathrm{HN}$ 01,2-DICHLOROPROPANE $062.4 \mathrm{HN}$ OCIS-1, 3-DICHIOROPROPO624HN OTRICHLOROETHENE O624HN ODIBROMOCHLOROMETHANEO $624 \mathrm{HN}$ $01,1,2-T R I C H L O R O E T H A N O 624 \mathrm{HN}$ OBENZENE $0624 \mathrm{HN}$ OTRANS-1, 3-DICHLOROPRO624HN OBROMOEORM

$0694 \mathrm{HN}$ 04-METHYL-2-PENTANONEO624HN 
GRS 10010220900228900032 WA9002L606 001 2HXONE2JLT GRS $10010220900228900032 \mathrm{WA} 9002 \mathrm{~L} 606001$ TCLEE 2JLT GRS 10010220900228900032 WA9002L606 001 TCLEA 2JLT GRS 10010220900228900032 WA9002L606 001 MEC 6 H52JLT GRS 10010220900228900032 WA9002L606 001 CLC6H52JLT

10010220900228900032 WA 9002 L606 001 ETC6H52JLT 10010220900228900032 WA9002L606 001 STYR 2JLT 10010220900228900032 WA9002L606 001 XYLEN 2JLT 10010220900302900034 WA9002L606 001RMEC6D82J 10010220900302900034 WA9002L606 001RPBFB 2J 10010220900302900034 WA9002L606 001R12DCD42J 10010220900302900034 WA9002L606 001RCH3CL 2JLT $10010220900302900034 \mathrm{WA} 9002 \mathrm{~L} 606001 \mathrm{RCH} 3 \mathrm{BR}$ 2JLT 10010220900302900034 WA9002L606 001RC2H3CL2JLT 10010220900302900034 WA9002L606 001RC2H5CL2JLT 10010220900302900034 WA9002L606 001RCH2CL22JB 10010220900302900034 WA9002L606 001RACET 2JLT $10010220900302900034 \mathrm{WA} 9002 \mathrm{~L} 606$ 001RCS2 2JLT 10010220900302900034 WA9002L606 001R11DCE 2JLT 10010220900302900034 WA9002L606 001R11DCLE2JLT 10010220900302900034 WA9002L606 001RT12DCE2JLT 10010220900302900034 FA9002L606 001RCHCL3 2JLT 10010220900302900034 WA9002L606 001R12DCLE2JLT 10010220900302900034 WA9002L606 001RMEK 2JLT 10010220900302900034 WA9002L606 001R111TCE2JLT 10010220900302900034 WA9002L606 001RCCL4 2JLT 10010220900302900034 WA9002L606 001RVINYLA2JLT 10010220900302900034 WA9002L606 001RBRDCLM2JLT $10010220900302900034 \mathrm{WA} 9002 L 606$ 001R12DCLP2JLT 10010220900302900034 WA9002L606 001R13DCPC2JLT 10010220900302900034 WA9002L606 O01RTRCLE 2JLT 10010220900302900034 WA9002L606 001RDBRCLM2JLT 10010220900302900034 WA9002L606 001R112TCE2JLT 10010220900302900034 WA9002L606 001RC6H6 2JLT 10010220900302900034 WA9002L606 001RT13DCP2JLT 10010220900302900034 WA9002L606 001RCHBR3 2JLT 10010220900302900034 WA9002L606 O01R4ME2PE2JLT $10010220900302900034 \mathrm{WA} 9002 \mathrm{~L} 606$ 001R2HXONE2JLT 10010220900302900034 WA9002L606 O01RTCLEE 2JLT 10010220900302900034 WA9002L606 001RTCLEA 2JLT 10010220900302900034 WA9002L606 001RMEC6H52JLT 10010220900302900034 HA9002L606 001RCLC6H52JLT 10010220900302900034 FA9002L606 001RETC6H52JLT 10010220900302900034 HA9002L606 001RSTYR 2JLT $10010220900302900034 \mathrm{HA} 9002 \mathrm{~L} 606$ 001RXYLEN 2JLT $10010220900305900036 \mathrm{WA} 9002 L 606$. 001SMEC6D82J $10010220900305900036 \mathrm{WA9} 002 \mathrm{~L} 606 \quad 001 \mathrm{SPBEB} \quad 2 \mathrm{~J}$ 10010220900305900036 WA9002L606 001S12DCD42J
11 UGKG

5UGKG

SUGKG

5UGKG

SUGKG

5UGKG

SUGKG

SUGKG

105PER

$110 P E R$

87PER

12 UGKG

12UGKG

12UGKG

12 UGKG

BUGKG

12UGKG

6UGKG

6UGKG

6UGKG

6UGKG

6UGKG

6UGKG

12UGKG

6UGKG

6UGKG

12UGKG

6UGKG

6UGKG

6UGKG

6UGKG

6UGKG

6UGKG

6UGKG

6UGKG

6UGKG

12 UGKG

12UGKG

6UGKG

6UGKG

6UGKG

6UGKG

6UGKG

6UGKG

6UGKG

89PER

93PER

77PER
1. $0000 \mathrm{MSJBD}$ $1.0000 \mathrm{MSJBD}$

$1.0000 \mathrm{MSJBD}$

$1.0000 \mathrm{MSJBD}$

1. $0000 \mathrm{MSJBD}$

1. $0000 \mathrm{MSJBD}$

1.0000MSJBD

$1.0000 \mathrm{MSJBD}$

$1.0000 \mathrm{MSJT}$

$1.0000 \mathrm{MSJT}$

$1.0000 \mathrm{MSJT}$

1. $0000 \mathrm{MSJT}$

$1.0000 \mathrm{MSJT}$

$1.0000 \mathrm{MSJT}$

$1.0000 \mathrm{MSJT}$

$1.0000 \mathrm{MSJT}$

$1.0000 \mathrm{MSJT}$

1.0000MSJT

$1.0000 \mathrm{MSJT}$

$1.0000 \mathrm{MSJT}$

$1.0000 \mathrm{MSJT}$

$1.0000 \mathrm{MSJT}$

$1.0000 \mathrm{MSJT}$

$1.0000 \mathrm{MSJT}$

1. 0000MSJT

$1.0000 \mathrm{MSJT}$

$1.0000 \mathrm{MSJT}$

$1.0000 \mathrm{MSJT}$

1.0000MSJT

$1.0000 \mathrm{MSJT}$

$1.0000 \mathrm{MSJT}$

1. 0000MSJT

$1.0000 \mathrm{MSJT}$

$1.0000 \mathrm{MSJT}$

1.0000MSJT

$1.0000 \mathrm{MSJT}$

$1.0000 \mathrm{MSJT}$

$1.0000 M S J T$

$1.0000 \mathrm{MSJT}$

1.0000MSJT

$1.0000 \mathrm{MSJT}$

$1.0000 \mathrm{MSJT}$

$1.0000 \mathrm{MSJT}$

$1.0000 \mathrm{MSJT}$

$1.0000 \mathrm{MSJT}$

1.0000MSJBD

1. $0000 \mathrm{MSJBD}$

1. 0000MSJBD
02-HEXANONE

$0624 \mathrm{HN}$

OTETRACHLOROETHENE O624HN

$01,1,2,2$ TETRACHLOROEO62 $4 \mathrm{HN}$ OTOLUENE

$0624 \mathrm{HN}$

OCHLOROBENZENE O624HN

OETHYLBENZENE

OSTYRENE

OXYLENE (TOTAL) O624HN

58.000TOLUENE-D8 O624HR

58.000BROMOELUOROBENZENE O624HR

$58.0001,2-D I C H L O R O E T H A N E-D 0624 \mathrm{HR}$ OCHLOROMETHANE O624HR OBROMOMETHANE O624HR OVINYL CHLORIDE O624HR OCHLOROETHANE O624HR OMETHYLENE CHLORIDE 0624HR OACETONE

$0624 \mathrm{HR}$

OCARBON DISULFTDE O624HR

01,1-DICHLOROETHENE 0624HR 01,1-DICHLOROETHANE O624HR 01,2-DICHLOROETHENE (0624HR OCHIOROEORM O624HR 01,2-DICHLOROETHANE 0624HR 02-BUTANONE O624HR $01,1,1$-TRICHLOROETHANO $624 \mathrm{HR}$ OCARBON TETRACHLORIDEO624HR OVINYL ACETATE

O624HR OBROMODICHLOROMETHANEO 624 HR 01,2-DICHLOROPROPANE 0624HR OCIS-1, 3-DICHLOROPROPO624HR OTRICHLOROETHENE O624HR ODIBROMOCHLOROMETHANEO624HR 01, 1, 2-TRICHLOROETHANO 624 HR OBENZENE O624HR OTRANS-1, 3-DICHLOROPRO62 4HR OBROMOEORM O62 4HR 04-METHYL-2-PENTANONEO62 4HR 02-HEXANONE 0624HR OTETRACHLOROETHENE O624HR $01,1,2,2-T E T R A C H L O R O E O 624 \mathrm{HR}$ OTOLUENE $0624 \mathrm{HR}$ OCHLOROBENZENE O624HR OETHYLBENZENE O624HR OSTYRENE

OXYLENE (TOTAL) O624HR

54.500TOLUENE-D8

'54.500BROMOELUOROBENZENE O624HS 54.5001,2-DICHLOROETHANE, $-2624 \mathrm{HS}$ 
GRS 10、 0220900305900036WA9002L606 001SCH3CL 2JLT GRS $10010220900305900036 \mathrm{HA9002L} 606001 \mathrm{SCH} 3 \mathrm{BR}$ 2JLT GRS $10010220900305900036 \mathrm{WA} 0002 \mathrm{~L} 606001 \mathrm{SC} 2 \mathrm{H} 3 \mathrm{CL} 2 \mathrm{JLT}$ GRS $10010220900305900036 \mathrm{WA} 9002 \mathrm{~L} 606001 \mathrm{SC} 2 \mathrm{HSCL} 2 \mathrm{JLT}$ GRS $10010220900305900036 \mathrm{KA} 9002 \mathrm{~L} 606001$ SCH2CL22JB GRS $10010220900305900036 \mathrm{WA} 90022606001$ SACET 2JB GRS $10010220900305900036 \mathrm{WA} 9002 \mathrm{~L} 606001 \mathrm{SCS} 2$ 2JLT GRS $10010220900305900036 \mathrm{KA} 9002 \mathrm{~L} 606001 \mathrm{~S} 11 \mathrm{DCE} 2 \mathrm{~J}$ GRS $10010220900305900036 \mathrm{WA} 9002 \mathrm{~L} 606$ 001S11DCLE2JLT GRS 10010220900305900036 WA9002L606 001ST12DCE2JLT GRS $10010220900305900036 \mathrm{WA} 90021606001$ SCHCL 3 JLT

10010220900305900036 WA9002L606 001S12DCLE2JLT $10010220900305900036 \mathrm{WA} 9002 \mathrm{~L} 606$ 001SMEK 2JLT $10010220900305900036 \mathrm{WA} 9002 \mathrm{~L} 606$ 001SIIITCE2JLT $10010220900305900036 \mathrm{WA} 9002 \mathrm{~L} 606001$ SCCL4 2JLT 10010220900305900036 WA9002L606 001SVINYLA2JLT $10010220900305900036 \mathrm{KA} 9002 \mathrm{~L} 606$ 001SBRDCLM2JLT 10010220900305900036 WA9002L606 001S12DCLP2JLT $10010220900305900036 \mathrm{WA} 9002 \mathrm{~L} 606001$ S13DCPC2JLT $10010220900305900036 \mathrm{WA} 9002 \mathrm{~L} 606$ 001STRCLE 2J $10010220900305900036 \mathrm{WA} 9002 \mathrm{~L} 606$ 001SDBRCLM2JLT $10010220900305900036 \mathrm{WA} 9002 \mathrm{~L} 606$ 001S112TCE2JLT $10010220900305900036 \mathrm{WA} 9002 \mathrm{~L} 606001 \mathrm{SC} 6 \mathrm{H} 6 \quad 2 \mathrm{~J}$ 10010220900305900036 WA9002L606 001ST13DCP2JLT $10010220900305900036 \mathrm{WA} 9002 \mathrm{~L} 606$ 001SCHBR3 2JLT $10010220900305900036 \mathrm{WA} 9002 \mathrm{~L} 606$ 001S4ME2PE2JLT 1001. $0220900305900036 \mathrm{WA} 9002 \mathrm{~L} 606$ 001S2HXONE2JLT $10010220900305900036 \mathrm{WA} 9002 \mathrm{~L} 606$ 001STCLEE 2JLT 10010220900305900036 WA9002L606 001STCLEA 2JLT $10010220900305900036 \mathrm{WA} 9002 \mathrm{~L} 606$ 001SMEC6H52J $10010220900305900036 \mathrm{WA} 90021606$ 001SCLC6H52J 10010220900305900036 WA9002L606 001SETC6H52JLT 10.010220900305900036 WA9002L606 001SSTYR 2JLT 10010220900305900036 WA9002L606 001SXYLEN 2JLT 10010220900308900326 WA9002L606 001 NBD5 1X 10010220900308900326 WA9002L606 001 2FBP $1 \mathrm{X}$ 10010220900308900326 WA9002L606 001 PTERP $1 X$ 10010220900308900326 WA9002L606 001 PHEND51X 10010220900308900326 WA9002L606 001 2FP IX 10010220900308900326 HA9002L606 001 246TBP1X 10010220900308900326 WA9002L606 001 PHENOLIXLT 10010220900308900326 WA9002L606 001 B2CEE 1XLT 10010220900308900326 WA9002L606 001 2CLP 1XLT $10010220900308900326 \mathrm{FA} 90022606001$ 13DCLB1XIT 10010220900308900326 WA9002L606 001 14DCLB1XLT $10010220900308900326 \mathrm{WA} 9002 \mathrm{~L} 606001$ BZALC 1XLT $10010220900308900326 \mathrm{WA} 9002 L 606001$ 12DCLB1XLT $10010220900308900326 \mathrm{WA} 9002 \mathrm{~L} 606001$ 2MP 1XLT
$76 P E R$

SUGKG

SUGKG

SUGKG

SUGKG

11UGKG

SUGKG

5UGKG

11UGKG

5UGKG

5UGKG

5UGKG

107PER

5UGKG

5UGKG

86PER

SUGKG

5UGKG

'11UGKG

11UGKG

SUGKG

5UGKG

86PER

96PER

5UGKG

5UGKG

SUGKG

73PER

84PER

95PER

B3PER

B2PER

72PER

400 UGKG

400UGKG

400 UGKG

400 UGKG

400UGKG

400 UGKG

400UGKG

400 UGKG
1. $0000 \mathrm{MSJBD}$

1. $0000 \mathrm{MSJBD}$

1. $0000 \mathrm{MS}$ JBD

1. $0000 \mathrm{MS} \mathrm{JBD}$

$1.0000 M S J B D$

$1.0000 \mathrm{MSJBD}$

1. $0000 \mathrm{MSJBD}$

1. $0000 \mathrm{MSJBD}$

$1.0000 \mathrm{MSJBD}$

1. $0000 \mathrm{MSJBD}$

1. 0000MS JBD

1. $0000 \mathrm{MSJBD}$

1. $0000 \mathrm{MSJBD}$

$1.0000 \mathrm{MSJBD}$

1. $0000 \mathrm{MSJBD}$

1.0000MSJBD

$1.0000 \mathrm{MSJBD}$

1. $0000 \mathrm{MSJBD}$

1. $0000 \mathrm{MSJBD}$

$1.0000 \mathrm{MSJBD}$

$1.0000 \mathrm{MSJBD}$

1.0000MSJBD

$1.0000 \mathrm{MSJBD}$

1.0000MSJBD

$1.0000 \mathrm{MSJBD}$

1. 0 OOOMSJBD

1.0000MSJBD

$1.0000 \mathrm{MSJBD}$

1. $0000 \mathrm{MSJBD}$

1.0000MSJBD

$1.0000 \mathrm{MSJBD}$

1. $0000 \mathrm{MSJBD}$

1. 000OMSJBD

1.000OMSJBD

1.0000MSDAR

1. 0000MSDAR

1.0000MSDAR

1.0000MSDAR

1. 0000MSDAR

1. 0000MSDAR

1.0000MSDAR

1.000OMSDAR

1. 0000MSDAR

1.000OMSDAR

1.000OMSDAR

1. 0000MSDAR

1.000OMSDAR 1. 000OMSDAR
OCHLOROMETHANE

OBROMOMETHANE

$0624 \mathrm{HS}$

OVINYL CHLORIDE

(24HS

OCHLOROETHANE

$0624 \mathrm{HS}$

CHIORIDE 0624HS

OACETONE

OCARBON DISULFIDE

$0624 \mathrm{HS}$

$0624 \mathrm{HS}$

54.5001,1-DICHLOROETHENE 0624HS 01,1-DICHLOROETHANE 0624HS 01,2-DICHLOROETHENE (0624HS OCHLOROFORM

01,2-DICHLOROETHANE O624HS 02-BUTANONE O624HS $01,1,1$-TRICHLOROETHANO 624KS OCARBON TETRACHLORIDEO624HS OVINYI ACETATE O624HS OBROMODICHLOROMETHANEO624HS $01,2-D I C H L O R O P R O P A N E$ O624HS 0CIS-1, 3-DICHLORORROPO624HS

54.50OTRICHLOROETHENE O624HS ODIBROMOCHLOROMETHANEO624HS 01, 1, 2-TRICHLOROETHANO 624 HS
54.500BENZENE $0624 \mathrm{HS}$ 0.TRANS - 1, 3-DICHLOROPRO 62 4HS OBROMOFORM O624HS 04-METHYL-2-PENTANONEO624HS 02-HEXANONE O624HS OTETRACHLOROETHENE O624HS $01,1,2,2$-TETRACHLOROEO 624 HS 54.500TOLUENE

54.50OCHLOROBENZENE OETHYLBENZENE OSTYRENE O624HS OXYLENE (TOTAL) O624HS 1792.793NITROBENZENE-D5 0625HN 1792.7932-ELUOROBIPHENYL O625HN 1792.793P-TERPHENYL-D14 O625HN 3585.587 PHENOL-D5 $0625 \mathrm{HN}$ 3585.5872-ELUOROPHENOL O625HN $3585.5872,4,6$-TRIBROMOPHENOLO $625 \mathrm{HN}$ ORHENOL O625HN OBIS (2-CHLOROETHYL) ETO625HN 02-CHLOROPHENOY O625HN 01, 3-DICHIOROBENZENE 0625HN 01, 4-DICHLOROBENZENE 0625HN OBENZYL ALCOHOL O625HN 01,2-DICHLOROBENZENE OE?.5HN 02-METHYLPHENOL O625HN 
GRS $10010220900308900326 \mathrm{WA} 9002 \mathrm{~L} 606001$ B2CIPE $1 \times L T$ GRS $10010220900308900326 \mathrm{WA} 9002 \mathrm{~L} 606001$ 4MP 1XLT GRS $10010220900308900326 \mathrm{WA} 9002 \mathrm{~L} 606001$ NDNPA 1 1XLT GRS $10010220900308900326 \mathrm{WA} 9002 \mathrm{~L} 606001$ CL6ET 2XLT GRS $10010220900308900326 \mathrm{WA} 9002 \mathrm{~L} 606001 \mathrm{NB} \quad 1 \times \mathrm{LT}$ GRS $10010220900308900326 \mathrm{WA} 90022606001$ ISOPHR IXLT GRS $10010220900308900326 \mathrm{WA} 9002 \mathrm{~L} 6060012 \mathrm{NP} \quad 1 \times \mathrm{LT}$ GRS $10010220.900308900326 \mathrm{WA} 90022606001$ 24DMPN1XLT GRS $10010220900308900326 \mathrm{WA} 9002 \mathrm{~L} 606001$ BENZOA1XLT GRS $10010220900308900326 \mathrm{WA} 9002 L 606001$ B2CEXM1XLT GRS $10010220900308900326 \mathrm{KA} 9002 \mathrm{~L} 606001$ 24DCLP1XLT GRS $10010220900308900326 \mathrm{WA} 9002 \mathrm{~L} 606001 \quad 124 \mathrm{TCB} 1 \mathrm{XLT}$ GRS $10010220900308900326 \mathrm{WA} 9002 \mathrm{~L} 606001$ NAP 1XLT GRS $10010220900308900326 \mathrm{WA} 9002 \mathrm{~L} 606001$ 4CANILIXLT GRS $10010220900308900326 \mathrm{WA} 9002 \mathrm{~L} 606001$ HCBD $1 \mathrm{XLT}$ GRS $10010220900308900326 \mathrm{FA} 9002 \mathrm{~L} 606001$ 4CL3C 1XLT GRS $10010220900308900326 \mathrm{WA} 9002 \mathrm{~L} 606001$ 2MENAP1XLT GRS $10010220900308900326 \mathrm{WA} 90022606001$ CL6CP 1XLT GRS $10010220900308900326 \mathrm{KA} 9002 \mathrm{~L} 606001$ 246TCP1XLT GRS $10010220900308900326 \mathrm{FA} 9002 \mathrm{~L} 606001 \quad 245 \mathrm{TCP} 1 \mathrm{XLT}$ GRS $10010220900308900326 \mathrm{WA} 9002 \mathrm{~L} 606001$ 2CNAP 1XLT GRS $10010220900308900326 \mathrm{FA9002L606} 001$ 2NANIL1XLT GRS $10010220900308900326 \mathrm{WA} 90022606001$ DMP 1XLT क GRS $10010220900308900326 \mathrm{WA} 9002 \mathrm{~L} 606001$ ANAPYL1XLT $\div$ GRS $10010220900308900326 \mathrm{WA} 9002 \mathrm{~L} 606001$ 26DNT 1XLT
$10010220900308900326 \mathrm{WA9002L606001} \mathrm{3NANIL1XLT}$ $10010220900308900326 \mathrm{WA} 9002 \mathrm{~L} 606001$ ANAPNE1XLT $10010220900308900326 \mathrm{KA} 9002 \mathrm{~L} 606001$ 24DNP 1XLT $10010220900308900326 \mathrm{WA} 9002 \mathrm{~L} 606001$ 4NP 1XLT $10010220900308900326 \mathrm{WA} 9002 \mathrm{~L} 606001$ DIBZFU1XLT $10010220900308900326 \mathrm{WA} 9002 \mathrm{~L} 606001$ 24DNT 1XLT $10010220900308900326 \mathrm{WA} 9002 \mathrm{~L} 606001$ DEP 1XLT $10010220900308900326 \mathrm{WA} 9002 L 606001$ 4CLPPE1XLT $10010220900308900326 \mathrm{WA} 9002 \mathrm{~L} 606001$ FLRENE1XLT $10010220900308900326 \mathrm{WA} 9002 \mathrm{~L} 606001$ 4NANIL1XLT $10010220900308900326 \mathrm{WA} 9002 \mathrm{~L} 606001$ 46DN2C1XLT $10010220900308900326 \mathrm{WA} 9002 \mathrm{~L} 606001$ NNDPA $1 \mathrm{XLT}$ $10010220900308900326 \mathrm{WA} 9002 \mathrm{~L} 606001$ 4BRPPE1XLT $10010220900308900326 \mathrm{WA} 9002 \mathrm{~L} 606001$ PCP 1XLT $10010220900308900326 \mathrm{WA} 9002 \mathrm{~L} 606001$ PHANTR1XLT $10010220900308900326 \mathrm{FA9002L606} \mathrm{001} \mathrm{ANTRC} \mathrm{1XLT}$ $10010220900308900326 \mathrm{WA} 9002 \mathrm{~L} 606001$ DNBP 1XLT $10010220900308900326 \mathrm{WA} 90021606001$ FANT 1XLT $10010220900308900326 \mathrm{FA9002L606} \mathrm{001} \mathrm{PYR} \mathrm{1XLT}$ $10010220900308900326 \mathrm{KA} 90021606001$ BBZP 1XLT $10010220900308900326 \mathrm{WA} 9002 L 606001$ 33CL2B1XLT $10010220900308900326 \mathrm{WA} 90024606001$ CL6B2 1XLT $10010220900308900326 \mathrm{KA} 9002 \mathrm{~L} 606001$ BAANTR1XLT
40OUGKG

400 UGKG

40OUGKG

400UGKG

40OUGKG

400 UGKG

4 OOUGKG

400 UGKG

2000UGKG

400UGKG

400 UGKG

40 OUGKG

400 UGKG

40OUGKG

40 OUGKG

400UGKG

400 UGKG

40 OUGKG

400 UGKG

2000UGKG

400 UGKG

2000UGKG

400UGKG

400 UGKG

40OUGKG

2000UGKG

40 OUGKG

2000UGKG

2000UGKG

400 UGKG

40 OUGKG

400UGKG

400 UGKG

40 OUGKG

2000UGKG

2000UGKG

400 UGKG

400 UGKG

4 OOUGKG

2000UGKG

400UGKG

400 UGKG

40 OUGKG

400UGKG

400UGKG

400UGKG

800 UGKG

4RRUGKG
$1.0000 M S D A R$

$1.0000 M S D A R$

$1.0000 M S D A R$

$1.0000 M S D A R$

$1.0000 M S D A R$

$1.0000 M S D A R$

1.0000MSDAR

$1.0000 M S D A R$

1.0000MSDAR

1. $0000 \mathrm{MSDAR}$

$1.0000 M S D A R$

$1.0000 M S D A R$

1.0000MSDAR

1.000OMSDAR

$1.0000 \mathrm{MSDAR}$

1.0000MSDAR

1.0000MSDAR

$1.0000 \mathrm{MSDAR}$

$1.0000 \mathrm{MSDAR}$

1.0000MSDAR

1.0000MSDAR

1.0000MSDAR

$1.0000 M S D A R$

1.0000MSDAR

1.0000MSDAR

$1.0000 M S D A R$

1.0000MSDAR

1.0000MSDAR

1.0000MSDAR

1.0000MSDAR

1.0000MSDAR

$1.0000 \mathrm{MSDAR}$

1.0000MSDAR

1. 0000MSDAR

1.0000MSDAR

1. 0000MSDAR

1.0000MSDAR

1.0000MSDAR

1.0000MSDAR

$1.0000 M S D A R$

1. 0000MSDAR

1.0000MSDAR

1.0000MSDAR

1. 0000MSDAR

1.000OMSDAR

1.0000MSDAR

1.0000MSDAR

1.0000MSDAR
OBIS (2-CHLOROISORROPYO625HN 04-METHYLPHENOL O625HN ON-NITROSO-DI-N-PROPYO $625 \mathrm{HN}$ OHEXACHLOROETHANE O625HN ONITROBENZENE O625HN OISOP HORONE $\cdot$ O625HN 02-NITROPHENOL $\quad 0625 \mathrm{HN}$ 02,4-DIMETHYLPHENOL O625HN OBENZOIC ACID O625HN OBIS (2-CHLOROETHOXY) MO625HN $02,4-D I C H L O R O P H E N O L$ O625HN $01,2,4-T R I C H L O R O B E N Z E 0625 \mathrm{HN}$ ONAPHTHALENE O625HN O4-CHLOROANILINE O625HN OHEXACHLOROBUTADIENE O625HN 04-CHLORO-3-METHYLP HEO $625 \mathrm{HN}$ 02-METHYLNAPHTHALENE 0625HN OHEXACHLOROCYCLOPENTAO $625 \mathrm{HN}$ $02,4,6$-TRICHLOROPHENOO $625 \mathrm{HN}$ $02,4,5-T R I C H L O R O P H E N O 0625 \mathrm{HN}$ 02-CHLORONAPHTHALENE 0625HN 02-NITROANIIINE 0625HN ODIMETHYLPHTHALATE O625HN OACENAPHTHYLENE O625HN 02,6-DINITROTOLUENE O625HN 03-NITROANILINE 0625HN OACENAPHTHENE $0625 \mathrm{HN}$ 02,4-DINITROPHENOL $0625 \mathrm{HN}$ 04-NITROPHENOL 0625HN ODIBENZOFURAN $0625 \mathrm{HN}$ 02,4-DINITROTOLUENE 0625HN ODIETHYLPHTHALATE $0625 \mathrm{HN}$ 04-CHLOROP HENYL-PHENYO $625 \mathrm{HN}$ OFLUORENE 0625HN 04-NITROANIIINE $0625 \mathrm{HN}$ 04, 6-DINITRO-2-METHYLO625HN ON-NITROSODIPHENYLAMIO625HN 04-BROMOPHENYL-PHENYLO625HN OHEXACHLOROBENZENE O625HN OPENTACHLOROPHENOL $0625 \mathrm{HN}$ OPHENANTHRENE O625HN OANTHRACENE O625HN ODI-N-BUTYLPHTHALATE O625HN OELUORANTHENE $\because 0625 \mathrm{HN}$ OPYRENE. O625HN OBUTYLBENZYLPHTHALATEO $625 \mathrm{HN}$ 03, $3^{\prime}$-DICHLOROBENZIDIO625HN OBENZO (A) ANTHRACENE $25 \mathrm{HN}$ 
GRS 10. 0220900308900326WA9002L606 001 CHRY 1XLT GRS $10010220900308900326 \mathrm{WA} 9002 \mathrm{~L} 606001$ B2EHP 1X

GRS 10010220900308900326 WA9002L606 001 DNOP 1XLT GRS $10010220900308900326 \mathrm{WA} 9002 \mathrm{~L} 606001$ BF2ANT1XLT GRS $10010220900308900326 \mathrm{WA} 9002 \mathrm{~L} 606001$ BKFANT $1 X L T$ GRS $10010220900308900326 \mathrm{WA9002L606} 001$ BAPYR 1XLT GRS $10010220900308900326 \mathrm{WA9002L606001}$ ICDPYR1XLT GRS $10010220900308900326 \mathrm{WA} 90022606001$ DBAHA 1 XLT GRS $10010220900308900326 \mathrm{WA} 9002 \mathrm{~L} 606001$ BGHIPY1XLT GRS 10020220900304900040 WA9002L606 002

GRS 10020220900416901200 WA9002L606 002 ALPHAG9W GRS 10020220900416901200 WA9002L606 002 BETAG 9W GRS 10020220900315900002 WA9002L606 002 CEC EX GRS 10020220900312900002 WA9002L606 002 CECB EX GRS 10020220900308900002 WA9002L606 002 CECT EX GRS 10020220900302900002 WA9002L606 002 TOC $9 I$ GRS 10020220900308900042 WA9002L606 $002 \mathrm{PH}$ 9R GRS 10020220900228900032 FA9002L606 002 MEC6D82J GRS $10020220900228900032 \mathrm{WA} 9002 L 606002$ PBFB $2 \mathrm{~J}$ GRS 10020220900228900032 WA9002L606 002 12DCD42J

GRS $1002 \cdot 0220900228900032 \mathrm{WA} 9002 \mathrm{~L} 606002$ CH3CL 2JLT GRS 10020220900228900032 WA9002L606 002 CH3BR 2JLT GRS 10020220900228900032 WA9002L606 002 C2H3CL2JLT $10020220900228900032 \mathrm{WA} 9002 \mathrm{~L} 606002$ C2H5CL2JLT $10020220900228900032 \mathrm{WA} 9002 \mathrm{~L} 606002 \mathrm{CH} 2 \mathrm{CL} 22 \mathrm{JB}$ 10020220900228900032 WA9002L606 002 ACET 2JB GRS $10020220900228900032 \mathrm{WA9002L606.002} \mathrm{CS2} \mathrm{2JLT}$ GRS 10020220900228900032 WA9002L606 002 11DCE 2JLT GRS. 1002 O220900228900032WA9002L606 002 11DCLE2JLT GRS 10020220900228900032 WA9002L606 002 T12DCE2JLT GRS 10020220900228900032 WA9002L606 002 CHCL3 2JLT GRS 10020220900228900032 WA9002L606 002 12DCLE2JLT GRS $10020220900228900032 \mathrm{WA} 9002 \mathrm{~L} 606002 \mathrm{MEK}$ 2JLT GRS 10020220900228900032 WA9002L606 002 111TCE2JLT GRS $10020220900228900032 \mathrm{WA9002L606} 002$ CCL4 2JIT GRS 10020220900228900032 WA9002L606 002 VINYLA2JLT GRS 10020220900228900032 WA9002L606 002 BRDCLM2JIT GRS 10020220900228900032 WA9002L606 002 12DCLP2JIT GRS $10020220900228900032 \mathrm{WA} 9002 \mathrm{~L} 606002$ 13DCPC2JLT GRS 10020220900228900032 WA9002L606 002 TRCLE 2JLT GRS $10020220900228900032 \mathrm{WA} 9002 L 606002$ DBRCLM2JLT GRS 10020220900228900032 WA9002L606 002 112TCE2JLT GRS $10020220900228900032 \mathrm{FA} 9002 \mathrm{~L} 606002$ C6H6 2JLT GRS 10020220900228900032 WA9002L606 002 T13DCP2JLT GRS 1002.0220900228900032 WA9002L606 002 CHBR3 2JLT GRS 10020220900228900032 KA9002L606 002 4ME2PE2JLT GRS $10020220900228900032 \mathrm{WA} 9002 \mathrm{~L} 606002$ 2HXONE2JLT GRS $10020220900228900032 \% A 9002 L 606002$ TCLEE 2JLT
५ UUUGKG 1300 UGKG 400 UGKG 400 UGKG 400UGKG 400 UGKG 400 UGKG 400 UGKG 400 UGKG 88.38

21. OPCIG 8. OPCIG 5. 9ME்Q1 $0.26 \mathrm{MEQ} 1$ 5. 6MEQ1 258MGKG 4.7PH U 105PER

103PER

11OPER

12UGKG

12UGKG

12UGKG

12UGKG

28UGKG

53UGKG

6UGKG

6UGKG

6UGKG

6UGKG

6UGKG

6UGKG

12UGKG

6UGKG

6UGKG

12UGKG

6UGKG

6UGKG

6UGKG

6UGKG

6UGKG

6UGKG

6UGKG

6UGKG

6UGKG

12UGKG

- 12UGKG 6UGKG
1.0000MSDAR $1.0000 M S D A R$ 1.0000MSDAR 1.0000MSDAR 1.0000MSDAR 1. $0000 \mathrm{MSDAR}$ 1. $0000 M S D A R$ 1.0000MSDAR 1.0000MSDAR 10 BT 10EBUNK 10EBUNK 10NADKS 1ONAALM 1ONAJS 1ODODKS 10PHJH

$1.0000 \mathrm{MSJBD}$ $1.0000 \mathrm{MSJBD}$

$1.0000 \mathrm{MSJBD}$

$1.0000 \mathrm{MSJBD}$

$1.0000 M S J B D$

$1.0000 M S J B D$

$1.0000 M S J B D$

$1.0000 \mathrm{MSJBD}$

$1.0000 \mathrm{MSJBD}$

$1.0000 \mathrm{MSJBD}$

$1.0000 M S J B D$

$1.0000 \mathrm{MSJBD}$

$1.0000 \mathrm{MSJBD}$

$1.0000 M S J B D$

$1.0000 \mathrm{MSJBD}$

1.0000MSJBD

1.0000MSJED

$1.0000 \mathrm{MSJBD}$

1. $0000 \mathrm{MSJBD}$

1.0000MSJBD

1.0000MSJBD

$1.0000 M S J B D$

$1.0000 M S J B D$

$1.0000 M S J B D$

$1.0000 \mathrm{MSJBD}$

$1.0000 \mathrm{MSJBD}$

1. $0000 \mathrm{MSJBD}$

$1.0000 M S J B D$

$1.0000 \mathrm{MSJBD}$

1.0000MSJBD

1. $0000 \mathrm{MSJBD}$
OCHRYSENE

$0625 \mathrm{HN}$

OBIS (2-ETHYLHEXYL) PHTO625HN ODI-N-OCTYL PHTHALATEO625HN OBENZO (B) ELUORANTHENEO62.5HN OBENZO (K) ELUORANTHENEO 625HN OBENZO (A) PYRENE O625HN OINDENO $(1,2,3-C D)$ PYREO625HN ODIBENZO $(A, H)$ ANTHRACEO $625 \mathrm{HN}$ OBENZO (G, H, I) PERYLENEO 625HN O\& SOLIDS

OGROSS ALPHA IALPHN ONONVOLATILE BETA IBETAN OCATION EXCHANGE CAPAICEC N OEXCHANGEABLE BASES ICECBN OEXCHANGEABLE ACIDS ICECTN OTOTAL ORGANIC CARBONIOCT N $\mathrm{OPH}$

IPH N

61.000TOLUENE-D8 O624HN

61.000BROMOFLUOROBENZENE O624HN

61.0001,2-DICHLOROETHANE-D0624HN OCHLOROMETHANE OBROMOMETHANE O624HN OVINYL CHLORIDE O624HN OCHLOROETHANE O624HN OMETHYLENE CHLORIDE O624HN OACETONE O624HN OCARBON DISULFIDE O624HN 01,1-DICHLOROETHENE 0624HN 01,1-DICHLOROETHANE O624HN 01,2-DICHLOROETHENE 10624HN OCHLOROFORM O624HN 01,2-DICHLOROETHANE 0624HN 02-BUTANONE O624HN $01,1,1$-TRICHLOROETHANO $624 \mathrm{HN}$ OCARBON TETRACHLORIDEO624HN OVINYL ACETATE O624HN OBROMODICHLOROMETHANEO624HN 01,2-DICHLORORROPANE 0624HN OCIS-1, 3-DICHLOROPROPO624HN OTRICHLOROETHENE O624HN ODIBROMOCHLOROMETHANEO $624 \mathrm{HN}$ 01, 1,2-TRICHLOROETHANO $624 \mathrm{HN}$ OBENZENE O624HN OTRANS-1, 3-DICHLORORRO624HN OBROMOFORM O624HN 04-METHYL-2-PENTANONEO624HN 02-HEXANONE $0624 \mathrm{HN}$ OTETRACHLOROETHENE O624HN 
GRS 10020220900228900032 WA $9002 L 606002$ TCLEA 2JLT GRS $1002.0220900228900032 \mathrm{WA} 9002 \mathrm{~L} 606002$ MEC6H52JLT GRS 10020220900228900032 WA9002L606 002 CLC6H52JLT GRS 10020220900228900032 WA9002L606 002 ETC6H52JLT GRS $10020220900228900032 \mathrm{WA} 9002 \mathrm{~L} 606002$ STYR 2JLT GRS 10020220900228900032 WA9002L606 002 XYLEN 2JLT GRS 10020220900308900326 WA9002L606 002 NBDS $1 \mathrm{X}$ GRS $10020220900308900326 \mathrm{WA} 9002 \mathrm{~L} 606002$ 2FBP $1 \mathrm{X}$ GRS 10020220900308900326 WA9002L606 002 PTERP. IX GRS 10020220900308900326 WA9002L606 002 PHEND51X GRS $10020220900308900326 \mathrm{WA} 9002 \mathrm{~L} 606002 \quad 2 \mathrm{FP} \quad 1 \mathrm{X}$ GRS 10020220900308900326 WA9002L606 002 246TBP 1X GRS $10020220900308900326 \mathrm{WA} 9002 \mathrm{~L} 606002$ PHENOL1XLT GRS $10020220900308900326 \mathrm{WA} 9002 \mathrm{~L} 606002$ B2CEE 1XLT GRS 10020220900308900326 WA9002L606 002 2CLP 1XLT GRS $10020220900308900326 \mathrm{FA} 9002 \mathrm{~L} 606002$ 13DCLB1XLT GRS $10020220900308900326 \mathrm{WA} 9002 \mathrm{~L} 606002$ 14DCLBIXLT GRS $10020220900308900326 \mathrm{WA} 9002 \mathrm{~L} 606002$ BZALC 1XLT GRS $10020220900308900326 \mathrm{WA} 9002 \mathrm{~L} 606002$ 12DCLB1XLT GRS $10020220900308900326 \mathrm{WA} 9002 \mathrm{~L} 606002$ 2MP 1XLT GRS $10020220900308900326 \mathrm{WA} 9002 \mathrm{~L} 606002$ B2CIPE1XLT GRS $10020220900308900326 \mathrm{WA} 9002 \mathrm{~L} 606002 \quad 4 \mathrm{MP}$ 1XLT GRS $10020220900308900326 \mathrm{WA} 9002 \mathrm{~L} 606002$ NDNPA 1XLT 1 GRS $10020220900308900326 \mathrm{WA} 9002 \mathrm{~L} 606002$ CL6ET 1XLT $\vec{\circ}$ GRS GRS 10020220900308900326 WA9002L606 002 ISOPHRIXLT GRS $10020220900308900326 \mathrm{WA} 9002 \mathrm{~L} 606002$ 2NP 1XLT GRS 10020220900308900326 WA9002L606 002 24DMPN1XLT GRS $10020220900308900326 \mathrm{WA} 9002 \mathrm{~L} 606002$ BENZOA1XLT GRS $10020220900308900326 \mathrm{WA} 9002 \mathrm{~L} 606002$ B2CEXM1XLT GRS $10020220900308900326 \mathrm{WA} 9002 \mathrm{~L} 606002$ 24DCLPIXLT GRS $10020220900308900326 \mathrm{WA} 9002 \mathrm{~L} 606002 \quad 124 \mathrm{TCB} 1 \mathrm{KLT}$ GRS $10020220900308900326 \mathrm{FA} 9002 \mathrm{~L} 606002$ NAP 1XLT GRS $10020220900308900326 \mathrm{WA} 90022606002$ 4CANIL1XLT GRS $10020220900308900326 \mathrm{KA} 9002 \mathrm{~L} 606002 \mathrm{HCBD}$ 1XLT GRS $1002 \cdot 0220900308900326 \mathrm{WA} 9002 \mathrm{~L} 606002$ 4CL3C 1XLT GRS $10020220900308900326 \mathrm{WA} 9002 \mathrm{~L} 606002$ 2MENAP1XLT GRS $10020220900308900326 \mathrm{WA} 9002 \mathrm{~L} 606002$ CL6CP 1XLT GRS $10020220900308900326 \mathrm{WA} 9002 \mathrm{~L} 606002 \quad 246 \mathrm{TCP} 1 \mathrm{XLT}$ GRS $10020220900308900326 \mathrm{WA} 9002 \mathrm{~L} 606002$ 245TCP1XLT GRS $10020220900308900326 \mathrm{WA} 9002 \mathrm{~L} 606002$ 2CNAP 1XLT GRS 10020220900308900326 WA9002L606 002 2NANIL1XLT GRS $10020220900308900326 \mathrm{WA} 9002 \mathrm{~L} 606002$ DMP 1XLT GRS $10020220900308900326 \mathrm{WA} 9002 \mathrm{~L} 606002$ ANAPYL1XLT GRS $10020220900308900326 \mathrm{FA} 9002 \mathrm{~L} 606002$ 26DNT 1XLT GRS $10020220900308900326 \mathrm{WA} 9002 \mathrm{~L} 606.002$ 3NANIL1XLT GRS $10020220900308900326 \mathrm{WA} 9002 \mathrm{~L} 606002$ ANAPNE1XLT GRS $10020220900308900326 \mathrm{WA} 0002 \mathrm{~L} 606002$ 24DNP 1XLT
6UGKG

GUGKG

6UGKG

6UGKG

6UGKG

6UGKG

$78 P E R$

86PER

108PER

92PER

98PER

94 PER

45 OUGKG

450 UGKG

450UGKG

450UGKG

450UGKG

450UGKG

450 UGKG

450 UGKG

450UGKG

450UGKG

450UGKG

450UGKG

450UGKG

450UGKG

450UGKG

450UGKG

2200UGKG

450UGKG

450UGKG

450UGKG

450UGKG

45 UUGKG

450UGKG

450UGKG

450UGKG

450UGKG

450UGKG

2200UGKG

450UGKG

2200UGKG

450 UGKG

450UGKG

450UGKG

2200UGKG

450UGKG

2200UGKG
$1.0000 \mathrm{MSJBD}$ $1.0000 \mathrm{MSJBD}$ $1.0000 M S J B D$

$1.0000 M S J B D$

$1.0000 \mathrm{MSJBD}$

1.0000MSJBD

1.0000MSDAR

1.0000MSDAR

1.0000MSDAR

1.0000MSDAR

1.0000MSDAR

1.0000MSDAR

1.000OMSDAR

1.0000MSDAR

1.0000MSDAR

1.0000MSDAR

$1.0000 M S D A R$

$1.0000 \mathrm{MSDAR}$

1.0000MSDAR

1.0000MSDAR

1.0000MSDAR

1.0000MSDAR

1. $0000 M S D A R$

1.0000MSDAR

$1.0000 M S D A R$

1.0000MSDAR

1.0000MSDAR

1. $0000 \mathrm{MSDAR}$

1.0000MSDAR

1.0000MSDAR

1.0000MSDAR

1.000OMSDAR

1.0000MSDAR

1.000OMSDAR

1.0000MSDAR

1.0000MSDAR

1.0000MSDAR

1.0000MSDAR

1.0000MSDAR

1. $0000 \mathrm{MSDAR}$

1.0000MSDAR

1.0000MSDAR

1.0000MSDAR

1.0000MSDAR

1. $0000 \mathrm{MSDAR}$

1.0000MSDAR

1.0000MSDAR

1.0000MSDAR
01, 1,2,2-TETRACHLOROEO $624 \mathrm{HN}$ OTOLUENE

O624HN

OCHLOROBENZENE O624HN

OETHYLBENZENE O624HN

OSTYRENE

OXYLENE (TOTAL)

2018.019 NITROBENZENE-D5

2018.0192-ELUOROBIPHENYL

$0624 \mathrm{HN}$

$0625 \mathrm{HN}$

$0625 \mathrm{HN}$

$0625 \mathrm{HN}$

$0625 \mathrm{HN}$

4036.037 PHENOL-D5

$\begin{array}{lr}4036.0372-E L U O R O P H E N O L & \text { O625HN } \\ 4036.0372,4,6-T R I B R O M O P H E N O L O & 625 \mathrm{HN}\end{array}$

ORHENOL

OBIS (2-CHLOROETHYL) ETO 625HN

02-CHLOROPHENOL O625HN

$01,3-D I C H L O R O B E N Z E N E$ O625HN

01,4 -DICHLOROBENZENE O625HN OBENZYL ÁLCOHOL $0625 \mathrm{HN}$

$01,2-D I C H L O R O B E N Z E N E$ O625HN 02-METHYLPHENOL O625HN OBIS (2-CHLOROISOPROPYO $625 \mathrm{HN}$ 04-METHYLPHENOL O625HN ON-NITROSO-DI-N-PROPYO $625 \mathrm{HN}$ OHEXACHLOROETHANE O625HN ONITROBENZENE OISOPHORONE $\quad 0625 \mathrm{HN}$ 02-NITROPHENOL O625HN 02, 4-DIMETHYLPHENOL O625HN OBENZOIC ACID O625HN OBIS (2-CHLOROETHOXY) MO625HN 02, 4-DICHLOROPHENOL 0625HN 01,2, 4-TRICHLOROBENZEO 625HN ONAPHTHALENE $0625 \mathrm{HN}$ 04-CHLOROANILINE O625HN OHEXACHLOROBUTADIENE O625HN 04-CHLORO-3-METHYLPHEO 625HN 02-METHYLNAPHTHALENE O625HN OHEXACHLOROCYCLOPENTAO $625 \mathrm{HN}$ $02,4,6$ TRICHLOROPHENOO625HN $02,4,5$-TRICHLOROPHENOO625HN 02-CHLORONAPHTHALENE O625HN 02-NITROANILINE 0625HN ODIMETHYLPHTHALATE O625HN OACENAPHTHYLENE ' O625HN 02,6-DINITROTOLUENE O625HN 03-NITROANILINE $0625 \mathrm{HN}$ OACENAPHTHENE O625HN 02, 4-DINITROPHENOL, $625 \mathrm{HN}$ 
GRS 1L $0220900308900326 \mathrm{WA} 9002 \mathrm{~L} 606002$ 4NP 1XLT

GRS 10020220900308900326 WA9002L606 002 DIBZEU1XLT

GRS 10020220900308900326 WA9002L606 002 24DNT 1XLT

GRS $10020220900308900326 \mathrm{WA} 9002 \mathrm{~L} 606002$ DEP IXLT

GRS 10020220900308900326 WA9002L606 002 ACLPPEIXLT

GRS $10020220900308900326 \mathrm{WA} 9002 \mathrm{~L} 606002$ FLRENE1XLT

GRS $10020220900308900326 \mathrm{WA} 9002 L 606002$ 4NANILIXLT

GRS 10020220900308900326 WA9002L606 002 46DN2CIXLT

GRS 10020220900308900326 WA9002L606 002 NNDPA 1XLT

GRS $10020220900308900326 \mathrm{WA} 9002 \mathrm{~L} 606.002$ 4BRPPEIXLT

GRS $10020220900308900326 \mathrm{WA} 9002 \mathrm{~L} 606002$ CL6BZ 1XLT

GRS 10020220900308900326 WA9002L606 002 PCP 1XLT

GRS $10020220900308900326 \mathrm{WA} 9002 \mathrm{~L} 606002$ PHANTRIXLT

GRS 10020220900308900326 WA9002L606 002 ANTRC 1XLT

GRS 10020220900308900326 WA9002L606 002 DNBP 1XLT

GRS $10020220900308900326 \mathrm{WA} 9002 \mathrm{~L} 606 \quad 002$ FANT 1XLT

GRS 10020220900308900326 WA9002L606 002 PYR 1XLT

GRS $10020220900308900326 \mathrm{WA} 9002 L 606002$ BB2P. 1XLT

GRS 10020220900308900326 WA9002L606 002 33CL2B1XIT

GRS 10020220900308900326 WA9002L606 002 BAANTR1XLT

GRS 10020220900308900326 WA9002L606 002 CHRY 1XLT

GRS 10020220900308900326 WA9002L606 002 B2EHP 1X

GRS 10020220900308900326 WA9002L606 002 DNOP 1XLT

W GRS

$\stackrel{1}{1}$ GRS

10020220900308900326 WA9002L606 002 BE2ANT1XLT

10020220900308900326 WA9002L606 002 BKEANT 1XLT

$10020220900308900326 \mathrm{WA} 9002 \mathrm{~L} 606002$ BAPYR 1XLT

GRS $10020220900308900326 \mathrm{WA} 9002 \mathrm{~L} 606002$ ICDPYR $1 X L T$

GRS 10020220900308900326 WA9002L606 002 DBAHA 1XLT

GRS 10020220900308900326 WA9002L606 002 BGHIPY1XIT

GRS 10030220900304900040 WA9002L606 003

GRS 10030220900416901200 WA9002L606 003 ALPHAG9W

GRS $10030220900416901200 W A 002 L 606003$ BETAG 9W

GRS 10030220900315900002 WA9002L606 003 CEC EX

GRS 10030220900312900002 WA9002L606 003 CECB EX

GRS 10030220900308900002 WA9002L606 003 CECT EX

GRS 10030220900302900002 WA9002L606 003 TOC 9 I

GRS $10030220900308900042 W A 9002 L 606003$ PH 9R

GRS - 10030220900228900032 WA9002L606 003 MEC6D82J

GRS 10030220900228900032 WA9002L606 003 PBEB 2J

GRS $\quad 1003 \quad 0220900228900032$ WA9002L606 $003 \quad 12 D C D 42 J$

GRS 10030220900228900032 WA9002L606 003 CH3CL 2JLT

GRS 10030220900228900032 WA9002L606 003 CH3BR 2JLT

GRS 10030220900228900032 WA9002L606 003 C2H3CL2JIT

GRS 10030220900228900032 WA9002L606 003 C2H5CL2JLT

GRS 10030220900228900032 WA9002L606 003 CH2CL22JB

GRS 10030220900228900032 WA9002L606 003 ACET 2JB

GRS 10030220900228900032 WA9002L606 003 CS2 2JLT

GRS $10030220900228900032 \mathrm{HA9002L606} 003$ 11DCE 2JIT $2\llcorner$ UUGKG

45 OUGKG

45 OUGKG

450 UGKG

450 UGKG

450UGKG

2200 UGKG

2200 UGKG

45OUGKG

45 OUGKG

450 UGKG

2200 UGKG

450UGKG

450 UGKG

450UGKG

450UGKG

45OUGKG

450UGKG

900 UJKG

450UGKG

450UGKG

6900UGKG

45OUGKG

45OUGKG

45 OUGKG

45OUGKG

45 OUGKG

450UGKG

450UGKG

$88.6 \%$

30. OPCIG

9. OPCIG

4. 6MEQ1

$0.78 \mathrm{MEQ1}$

3. 8MEQ1

$2410 \mathrm{MGKG}$

$4.72 \mathrm{H} \mathrm{U}$

108PER

102RER

109PER

12UGKG

12UGKG

12UGKG

12 UGKG

33UGKG

23 UGKG

6UGKG

6UGKG
1. 0000MSDAR

$1.0000 M S D A R$

1.0000MSDAR

1.0000MSDAR

1. 00OOMSDAR

1. 000OMSDAR

1.0000MSDAR

1.000OMSDAR

1.000OMSDAR

1.0000MSDAR

1. 000OMSDAR

1.0000MSDAR

1. 0000MSDAR

1.0000MSDAR

1.0000MSDAR

1. 0000MSDAR

1.0000MSDAR

1.0000MSDAR

1. 0000MSDAR

1.0000MSDAR

1.0000MSDAR

1. 000OMSDAR

1. 0000MSDAR

1.0000MSDAR

1. 0000MSDAR

1.0000MSDAR

1. 0000MSDAR

1. 0000MSDAR

1.0000MSDAR

10 BT

10EBUNK

10EBUNK

10NADKS

1ONAALM

1ONAJS

10DODKS

1OPHJH

1. 0000MSJBD

1. $0000 \mathrm{MSJBD}$

1.0000MSJBD

1. $0000 \mathrm{MSJBD}$

1.000OMSJBD

$1.0000 \mathrm{MSJBD}$

1. 0000MSJBD

1. 0000MSJBD

1. $0000 \mathrm{MSJBD}$

1.0000MSJBD

1.0000MSJBD
04-NITROPHENOL

ODIBENZOFURAN

$0625 \mathrm{HN}$

2, 4-DINITROTOLUENE 0625HN ODIETHYIPHTHALATE O625HN 04-CHLOROPHENYL-PHENYO 625HN OFLUORENE O625HN 04-NITROANIIINE 0625HN 04,6-DINITRO-2-METHYLO625HN ON-NITROSODIPHENYIUAMI0625HN 04-BROMOPHENYL-PHENYLO625HN OHEXACHLOROBENZENE O625HN OPENTACHLOROPHENOL 0625HN OPHENANTHRENE 0625HN OANTHRACENE 0625HN ODI-N-BUTYLPHTHAIATE 0625HN OELUORANTHENE O625HN OPYRENE O625HN OBUTYLBENZYLPHTHALATEO $625 \mathrm{HN}$ $03,3^{\circ}-D I C H L O R O B E N Z I D I 0625 \mathrm{HN}$ OBENZO (A) ANTHRACENE O625HN OCHRYSENE $0625 \mathrm{HN}$ OBIS (2-ETHYLHEXYL) PHTO625KN ODI-N-OCTYL PHTHALATEO625HN OBENZO (B) FLUORANTHENEO 625HN OBENZO (K) ELUORANTHENEO 625HN OBENZO (A) PYRENE 0625HN O INDENO $(1,2,3-C D)$ PYREO $625 \mathrm{HN}$ ODIBENZO $(A, H)$ ANTHRACEO $625 \mathrm{HN}$ OBENZO (G, H, I) PERYLENEO 625HN 0 \% SOLIDS I\&SOLN OGROSS ALPHA IALPHN ONONVOLATILE BETA IBETAN OCATION EXCHANGE CAPAICEC N OEXCHANGEABLE BASES ICECBN OEXCHANGEABLE ACIDS ICECTN OTOTAL ORGANIC CARBONIOCT N $O P H$

59.00OTOLUENE-D8 59.00OBROMOEIUOROBENZENE O624KN 59.0001, 2-DICHLOROETHANE-DO624 HN OCHLOROMETHANE O624HN OBROMOMETHANE O624HN OYINYL CHLORIDE O624HN OCHLOROETHANE . 0624HN OMETHYLENE CHLORIDE 0624HN OACETONE O624 HN OCARBON DISULEIDE O624HN 01,1-DICHLOROETHENE 0624HN 
GRS 10030220900228900032 WA9002L606 003 11DCLE2JLT GRS 10030220900228900032 WA9002L606 003 T12DCE2JLT GRS 10030220900228900032 WA9002L606 003 CHCL3 2JLT GRS 10030220900228900032 WA9002L606 003 12DCLE2JLT GRS 10030220900228900032 WA9002L606 003 MEK 2JLT GRS 10030220900228900032 WA9002L606 003 111TCE2JLT GRS 10030220900228900032 WA9002L606 003 CCL4 2JLT GRS 10030220900228900032 WA9002L606 003 VINYLA2JLT GRS 10030220900228900032 WA9002L606 003 BRDCLM2JLT GRS 10030220900228900032 WA9002L606 003 12DCLP2JLT GRS 10030220900228900032 WA9002L606 003 13DCPC2JLT GRS 10030220900228900032 WA9002L606 003 TRCLE 2JLT GRS 10030220900228900032 WA9002L606 003 DBRCLM2JLT GRS 10030220900228900032 WA9002L606 003 112TCE2JLT GRS $10030220900228900032 \mathrm{FA9002L606003} \mathrm{C6H6} \mathrm{2JLT}$ GRS 10030220900228900032 WA9002L606 003 T13DCP2JLT GRS 10030220900228900032 WA9002L606 003 CHBR3 2JLT GRS 10030220900228900032 WA9002L606 003 4ME2PE2JLT GRS $10030220900228900032 \mathrm{WA} 9002 \mathrm{~L} 606003$ 2HXONE2JLT GRS $10030220900228900032 \mathrm{WA} 9002 \mathrm{~L} 606003$ TCLEE 2JLT GRS 10030220900228900032 WA9002L606 003 TCLEA 2JLT GRS 10030220900228900032 WA9002L606 003 MEC6H52JLT GRS 10030220900228900032 WA9002L606 003 CLC6H52JLT WO GRS $1 \frac{1}{6}$ GRS io GRS GRS GRS GRS GRS GRS GRS GRS GRS GRS GRS GRS GRS GRS GRS GRS GRS GRS GRS GRS GRS GRS GRS GRS
6UGKG

6UGKG

6UGKG

6UGKG

6UGKG

GUGKG

6UGKG

7OPER

81PER

93PER

BOPER

82PER

84PER

430UGKG 430 UGKG 430UGKG 430UGKG 430UGKG 430UGKG 430UGKG 430UGKE 430UGKG 430 UGKG 430 UGKG 430UGKG 430UGKG 430UGKG 430UGKG 430UGKG
$1.0000 M S J B D$ $1.0000 M S J B D$

$1.0000 \mathrm{MSJBD}$

$1.0000 M S J B D$

$1.0000 M S J B D$

$1.0000 \mathrm{MSJBD}$

$1.0000 \mathrm{MSJBD}$

$1.0000 M S J B D$

$1.0000 \mathrm{MSJBD}$

$1.0000 \mathrm{MSJBD}$

$1.0000 \mathrm{MSJBD}$

$1.0000 \mathrm{MSJBD}$

$1.0000 M S J B D$

$1.0000 \mathrm{MSJBD}$

$1.0000 \mathrm{MSJBD}$

$1.0000 \mathrm{MSJBD}$

$1.0000 \mathrm{MSJBD}$

$1.0000 \mathrm{MSJBD}$

$1.0000 M S J B D$

$1.0000 \mathrm{MSJBD}$

$1.0000 \mathrm{MSJBD}$

$1.0000 M S J B D$

$1.0000 M S J B D$

$1.0000 M S J B D$

$1.0000 \mathrm{MSJBD}$

$1.0000 \mathrm{MSJBD}$

1. 0000MSDAR

1.0000MSDAR

1. 0000MSDAR

1.0000MSDAR

1.0000MSDAR

1.0000MSDAR

1.0000MSDAR

$1.0000 \mathrm{MSDAR}$

1. 0000MSDAR

1.0000MSDAR

1.0000MSDAR

1. 0000MSDAR

$1.0000 \mathrm{MSDAR}$

1.0000MSDAR

1. $0000 \mathrm{MSDAR}$

1.0000MSDAR

1.0000MSDAR

1. $0000 \mathrm{MSDAR}$

1.000OMSDAR

1.000OMSDAR

1.0000MSDAR

1.0000MSDAR
01,1-DICHLOROETHANE O624HN 01,2-DICHLOROETHENE $10624 \mathrm{HN}$ OCHLOROEORM $0624 \mathrm{HN}$ 01,2-DICHLOROETHANE O624HN O2-BUTANONE $0624 \mathrm{HN}$ $01,1,1-T R I C H L O R O E T H A N O 624 \mathrm{HN}$ OCARBON TETRACHLORIDEO624HN OVINYL ACETATE O624HN OBROMODICHLOROMETHANEO624HN 01,2-DICHIORORROPANE O624HN OCIS-1, 3-DICHLOROPROPO $624 \mathrm{HN}$ OTRICHLOROETHENE O624HN ODIBROMOCHLOROMETHANEO $624 \mathrm{HN}$ $01,1,2$-TRICHLOROETHANO $624 \mathrm{HN}$ OBENZENE $0624 \mathrm{HN}$ OTRANS-1, 3-DICHLORORRO $624 \mathrm{HN}$ OBROMOFORM O624HN 04-METHYL-2-PENTANONEO624HN 02-HEXANONE O624HN OTETRACHLOROETHENE O624HN $01,1,2,2$-TETRACHLOROEO $624 \mathrm{HN}$ OTOLUENE $0624 \mathrm{HN}$ OCHLOROBENZENE O624HN OETHYLBENZENE O624HN OSTYRENE

OXYLENE (TOTAL)

1945.946NITROBENZENE-D5

1945.9462-FLUOROBIPHENYL $0624 \mathrm{HN}$ $0624 \mathrm{HN}$ $0625 \mathrm{HN}$ $0625 \mathrm{HN}$ 1945.946P-TERPHENYL-D14 O625HN 3891.893PHENOL-D5 O625HN 3891.8932-ELUOROPHENOL O625HN $3891.8932,4,6$-TRIBROMOPHENOLO625HN OPHENOL O625HN OBIS (2-CHLOROETHYL) ETO625HN 02-CHLOROPHENOL 0625HN 01,3-DICHLOROBENZENE O625HN 01, 4-DICHLOROBENZENE O625HN OBENZYL ALCOHOL $0625 \mathrm{HN}$ 01,2-DICHLOROBENZENE O625HN 02-METHYLPHENOL O625HN OBIS 12-CHLOROISORROP YO $625 \mathrm{HN}$ 04-METHYLPHENOL O625HN ON-NITROSO-DI-N-PROPYO625HN OHEXACHLOROETHANE O625HN ONITROBENZENE OISOPRORONE O625HN 02-NITROPHENOL O625HN 02, 4-DIMETHYLPHENOL $2625 \mathrm{HN}$ 
GRS IL $0220900308900326 \mathrm{FA} 9002 L 606003$ BENZOA1XLT GRS $1003 \cdot 0220900308900326 \mathrm{FA} 9002 \mathrm{~L} 606003$ B2CEXM1XLT GRS $10030220900308900326 \mathrm{FA} 9002 \mathrm{~L} 606003$ 24DCLP1XLT GRS $10030220900308900326 \mathrm{FA9002L606} 003 \quad 124 \mathrm{TCB} 1 \mathrm{XLT}$ GRS $10030220900308900326 \mathrm{FA9002L606} 003$ NAP · 1XLT GRS $10030220900308900326 \mathrm{WA} 9002 \mathrm{~L} 606003$ 4CANIL1XLT GRS $10030220900308900326 \mathrm{FA} 9002 \mathrm{~L} 606003 \mathrm{HCBD}$ 1XLT GRS $10030220900308900326 \mathrm{WA} 9002 \mathrm{~L} 606003$ 4CL3C 1XLT GRS $10030220900308900326 \mathrm{FA} 90021606003$ 2MENAP1XLT GRS 10030220900308900326 WA9002L606 003 CL6CP 1XLT GRS $10030220900308900326 \mathrm{FA} 9002 \mathrm{~L} 606003 \quad 246 \mathrm{TCP} 1 \mathrm{XLT}$ GRS $10030220900308900326 \mathrm{WA} 9002 \mathrm{~L} 606003245 \mathrm{TCP} 1 \mathrm{XLT}$ GRS 10030220900308900326 FA9002L606 003 2CNAP 1XLT GRS 10030220900308900326 WA9002L606 003 2NANIL1XLT GRS $10030220900308900326 \mathrm{FA} 9002 \mathrm{~L} 606003$ DMP 1XLT GRS $10030220900308900326 \mathrm{HA} 9002 \mathrm{~L} 606003$ ANAPYL1XLT GRS $10030220900308900326 \mathrm{WA} 9002 \mathrm{~L} 606003$ 26DNT 1XLT GRS 10030220900308900326 WA9002L606 003 3NANIL1XLT GRS $10030220900308900326 \mathrm{WA} 90021606003$ ANAPNE1XLT GRS $10030220900308900326 \mathrm{WA} 9002 \mathrm{~L} 606003$ 24DNP 1XLT GRS $10030.220900308900326 \mathrm{WA} 9002 \mathrm{~L} 606003$ 4NP 1XLT GRS $10030220900308900326 \mathrm{WA} 9002 \mathrm{~L} 606003$ DIB2EU1XLT GRS $10030220900308900326 \mathrm{FA} 9002 \mathrm{~L} 606003$ 24DNT 1XLT
10030220900308900326 WA9002L606 003 DEP 1XLT $10030220900308900326 \mathrm{WA} 9002 \mathrm{~L} 606003$ 4CLPPE1XLT 10030220900308900326 WA9002L606 003 FLRENE1XLT 10030220900308900326 WA9002L606 003 4NANIL1XLT $10030220900308900326 \mathrm{WA} 9002 \mathrm{~L} 606003$ 46DN2C1XLT $10030220900308900326 \mathrm{WA} 9002 \mathrm{~L} 606003$ NNDPA 1XLT $10030220900308900326 \mathrm{WA} 9002 L 606003$ 4BRPPE1XLT $10030220900308900326 \mathrm{FA} 9002 L 606003$ CL6BZ 1XLT 10030220900308900326 ผैA 9002 L606 003 PCP 1XLT $10030220900308900326 \mathrm{WA} 9002 \mathrm{~L} 606003$ PHANTR1XLT 10030220900308900326 KA9002L606 003 ANTRC 1XLT 10030220900308900326 WA9002L606 003 DNBP 1XLT $1003^{\prime} 0220900308900326$ WA9002L606 003 FANT 1XLT $10030220900308900326 \mathrm{WA} 9002 L 606003$ PYR 1XLT 10030220900308900326 WA9002L606 003 BBZP 1XLT $10030220900308900326 \mathrm{WA} 9002 \mathrm{~L} 606003$ 33CL2B1XLT $10030220900308900326 \mathrm{WA} 9002 \mathrm{~L} 606003$ BAANTR1XLT $10030220900308900326 \mathrm{WA} 9002 L 606003$ CHRY 1XLT $10030220900308900326 \mathrm{WA} 9002 L 606003$ B2EHP 1XB 10030220900308900326 KA9002L606 003 DNOP 1XLT $10030220900308900326 \mathrm{WA} 9002 \mathrm{~L} 606003$ BE2ANTIXLT $10030220900308900326 \mathrm{WA} 9002 \mathrm{~L} 606003$ BKFANT1XLT 10030220900308900326 WA9002L606.003 BAPYR 1XLT $10030220900308900326 \mathrm{WA} 9002 \mathrm{~L} 606003$ ICDPYRIXLT 10030220900308900326 FA9002L606 003 DBAHA 1XLT
2. UGKG 430UGKG 430 UGKG 430UGKG 430UGKG 43 OUGKG 430UGKG 430UGKG 430 UGKG 43 OUGKG 430UGKG 2200UGKG 430UGKG 2200 UGKG 430UGKG 430UGKG 43 OUGKG 2200UGKG 430UGKG 2200UGKG 2200UGKG 43 OUGKG 43 UUGKG 430UGKG 43 OUGKG 430UGKG 2200UGKG 2200 UGKG 430UGKG 430UGKG 430UGKG 2200UGKG 430UGKG 430UGKG 430UGKG 430UGKG 430UGKG 430UGKG 860 UGKG 430UGKG 43 OUGKG UGKG 430UGKG 43OUGKG 430 UGKG 430UGKG 4 3OUGKG 430UGKG
$1.0000 \mathrm{MSDAR}$ $1.0000 \mathrm{MSDAR}$ $1.0000 \mathrm{MSDAR}$

$1.0000 M S D A R$

$1.0000 M S D A R$

$1.0000 M S D A R$

$1.0000 \mathrm{MSDAR}$

$1.0000 M S D A R$

$1.0000 \mathrm{MSDAR}$

1. 0000MSDAR

1.0000MSDAR

1.0000MSDAR

1.0000MSDAR

1.0000MSDAR

1.0000MSDAR

1.0000MSDAR

1.0000MSDAR

1.0000MSDAR

1.0000MSDAR

1.0000MSDAR

1.0000MSDAR

$1.0000 \mathrm{MSDAR}$

1.0000MSDAR

1.0000MSDAR

1.0000MSDAR

1.0000MSDAR

1.0000MSDAR

1.0000MSDAR

1.0000MSDAR

1.0000MSDAR

1.0000MSDAR

1.0000MSDAR

1.0000MSDAR

1.0000MSDAR

1.0000MSDAR

1.0000MSDAR

1.0000MSDAR

1. 0000MSDAR

$1.0000 M S D A R$

1.0000MSDAR

1.0000MSDAR

1.0000MSDAR

1.0000MSDAR

1.0000MSDAR

1.0000MSDAR

1.0000MSDAR

1.0000MSDAR 1.0000MSDAR
OBENZOIC ACID (2-CHLOROETHOXY) MO625HN $02,4-D I C H L O R O P H E N O L$ O625HN $01,2,4-T R I C H L O R O B E N Z E 0625 \mathrm{HN}$ ONAPHTHALENE $0625 \mathrm{HN}$ 04-CHLOROANILINE O625HN OHEXACHLOROBUTADIENE $0625 \mathrm{HN}$ 04-CHLORO-3-METHYLPHEO 625HN 02-METHYLNAPHTHALENE 0625HN OHEXACHLOROCYCLOPENTAO $625 \mathrm{HN}$ $02,4,6$-TRICHLOROPHENOO625HN $02,4,5$-TRICHLOROPHENOO $625 \mathrm{HN}$ 02-CHLORONAPHTHALENE O625HN 02-NITROANILINE 0625HN ODIMETHYLPHTHALATE O625HN OACENAPHTHYLENE O625HN 02, 6-DINITROTOLUENE $0625 \mathrm{HN}$ 03-NITROANILINE $0625 \mathrm{HN}$ OACENAPHTHENE O625HN 02, 4-DINITROPHENOL O625HN O4-NITROPHENOL O625HN ODIBENZOFURAN $0625 \mathrm{HN}$ 02, 4-DINITROTOLUENE O625HN ODIETHYLPHTHALATE $0625 \mathrm{HN}$ 04-CHLOROPHENYL-P HENYO625HN OELUORENE O625HN 04-NITROANILINE O625HN 04, 6-DINITRO-2-METHYLO625HN ON-NITROSODIPHENYLAMIO $625 \mathrm{HN}$ 04-BROMOPHENYL-PHENYLO625HN OHEXACHLOROBENZENE O625HN OPENTACHLOROPHENOL O625HN OPHENANTHRENE O625HN OANTHRACENE $0625 \mathrm{HN}$ ODI-N-BUTYLPHTHALATE $0625 \mathrm{HN}$ OFLUORANTHENE O625HN OPYRENE - O625HN OBUTYLBENZYLPHTHALATEO $625 \mathrm{HN}$ $03,3^{\prime}$-DICHLOROBENZ IDIO625HN OBENZO (A) ANTHRACENE 0625HN OCHRYSENE O625HN OBIS (2-ETHYLHEXYL) PHTO625HN ODI-N-OCTYL PHTHALATEO $625 \mathrm{HN}$ OBENZO (B) FLUORANTHENEO $625 \mathrm{HN}$ OBENZO (K) FLUORANTHENEO625HN OBENZO (A) PYRENE O625HN OINDENO $(1,2,3-C D)$ PYREO625HN ODIBENZO (A, H) ANTHRACEO $625 \mathrm{HN}$ 
GRS $10030220900308900326 \mathrm{WA} 9002 \mathrm{~L} 606003$ BGHIPY1XLT GRS 10030220900309900326 WA9002L606 003 NBD5 $1 X$ GRS 10030220900309900326 WA9002L606 003 2FBP $1 \times$ GRS $10030220900309900326 \mathrm{WA} 002 \mathrm{~L} 606003$ PTERP $1 \mathrm{X}$ GRS 10030220900309900326 WA9002L606 003 PHEND51X GRS $10030220900309900326 \mathrm{WA} 9002 L 606003 \quad 2 \mathrm{FP} \quad 1 \mathrm{X}$ GRS 1003022090030,9900326 WA9002L606 $003 \quad 246$ TBP 1X GRS 10030220900309900326 WA9002L606 003 PHENOL1XLT GRS $10030220900309900326 \mathrm{WA} 9002 \mathrm{~L} 606003$ B2CEE 1XLT GRS 10030220900309900326 WA9002L606 003 2CLP 1XLT GRS $10030220900309900326 \mathrm{WA} 9002 \mathrm{~L} 606003$ 13DCLB1XLT GRS $\quad 10030220900309900326 \mathrm{WA} 9002 \mathrm{~L} 606003$ 14DCLB1XLT GRS $10030220900309900326 \mathrm{WA} 9002 L 606003$ BZALC 1XLT GRS $10030220900309900326 \mathrm{WA} 9002 \mathrm{~L} 606 \quad 003$ 12DCLB1XLT GRS $10030220900309900326 \mathrm{WA} 9002 \mathrm{~L} 606003$ 2MP 1XLT GRS 10030220900309900326 WA9002L606 003 B2CIPE1XLT GRS 10030220900309900326 WA9002L606 003 AMP 1XLT GRS 10030220900309900326 WA9002L606 003 NDNPA 1XLT GRS 10030220900309900326 WA9002L606 003 CL6ET 1XLT GRS $10030220900309900326 \mathrm{WA} 9002 \mathrm{~L} 606003 \mathrm{NB}$ 1XLT GRS 10030220900309900326 WA9002L606 003 ISOPHR1XLT GRS 10030220900309900326 WA9002L606 003 2NP 1XLT GRS 10030220900309900326 WA9002L606 $00324 D M P N 1 X I T$
$10030220900309900326 \mathrm{WA} 9002 L 606003$ BENZOA $1 X L T$ 10030220900309900326 WA9002L606 003 B2CEXM1XLT 10030220900309900326 WA9002L606 003 24DCLP 1XLT 1003.0220900309900326 WA9002L606 003 124TCB1XLT 10030220900309900326 WA90025606 003 NAP 1XLT 10030220900309900326 WA9002L606 003 4CANIL1XLT 10030220900309900326 WA9002L606 003 HCBD 1XLT $10030220900309900326 \mathrm{WA9002L} 606003$ 4CL3C 1XLT $10030220900309900326 \mathrm{WA} 9002 \mathrm{~L} 606003$ 2MENAP 1XLT 10030220900309900326 WA9002L606 003 CL6CP 1XLT $10030220900309900326 \mathrm{FA} 9002 L 606003$ 246TCP 1XLT $10030220900309900326 \mathrm{WA} 9002 \mathrm{~L} 606003$ 245TCP 1XLT 10030220900309900326 WA9002L606 003 2CNAP 1XLT $10030220900309900326 \mathrm{WA} 90022606003$ 2NANIL1XLT 10030220900309900326 WA9002L606 003 DMP 1XLT $10030220900309900326 \mathrm{WA} 9002 \mathrm{~L} 606003$ ANAPYL1XLT $10030220900309900326 \mathrm{WA} 9002 \mathrm{~L} 606003$ 26DNT 1XLT 10030220900309900326 WA9002L606 003 3NANILIXLT $10030220900309900326 \mathrm{WA} 9002 L 606003$ ANAPNE1XLT 10030220900309900326 WA9002L606 003 24DNP 1XLT $10030220900309900326 \mathrm{KA} 9002 \mathrm{~L} 606003$ 4NP 1XLT 10030220900309900326 WA9002L606 003 DIBZFU1XLT $10030220900309900326 \mathrm{WA9} 002 \mathrm{~L} 606003$ 24DNT 1XIT $10030220900309900326 \mathrm{FA} 9002 \mathrm{~L} 606003$ DEP 1XLT $10 Q 30220900309900326$ WA9002L606 003 4CLPPE1XLT
2200 UGKG

$2200 U G K G$

2200 UGKG

2200 UGKG

2200 UGKG

2200 UGKG

2200UGKG

2200 UGKG

2200 UGKG

2200UGKG

2200 UGKG

2200UGKG

2200UGKG

2200UGKG

2200 UGKG

2200 UUKG

11000 UGKG

2200 UGKG

22 OOUGKG

2200UGKG

2200UGKG

2200UGKG

2200UGKG

2200UGKG

2200UGKG

2200 UGKG

2200 UGKG

11000 UGKG

2200UGKG

11000 UGKG

2200 UGKG

2200UGKG

2200UGKG

1100 OUGKG

2200UGKG

1100 OUGKG

11000 UGKG

2200UGKG

2200UGKG

2200 UGKG

22مQUGKG
1.0000MSDAR 5 IMSDAR

51MSDAR

51MSDAR

51MSDAR

51MSDAR

51MSDAR

5IMSDAR

51MSDAR

$51 M S D A R$

5IMSDAR

51MSDAR

51MSDAR

51MSDAR

51MSDAR

51MSDAR

51MSDAR

51MSDAR

51MSDAR

51MSDAR

51MSDAR

51MSDAR

51MSDAR

51MSDAR

51MSDAR

51MSDAR

51MSDAR

51MSDAR

51MSDAR

51MSDAR

51MSDAR

51MSDAR

51MSDAR

51MSDAR

51MSDAR

51MSDAR

51MSDAR

51MSDAR

51MSDAR

51MSDAR

51MSDAR

51MSDAR

51MSDAR

51MSDAR

51MSDAR

51MSDAR

51MSDAR

51MSDAR
OBENZO (G, $\mathrm{H}, \mathrm{I})$ PERYLENEO 625HN

945.946NITROBENZENE-D5 O625HN

$1945.9462-$ FLUOROBIPHENYL $0625 \mathrm{HN}$

946P-TERPHENYL-D14

$3891.893 \mathrm{PHENOL}-\mathrm{DS} \quad 0625 \mathrm{HN}$

$3891.8932-$ FLUOROPHENOL ' O625HN

$3891.8932,4,6$-TRIBROMOPHENOLO625HN OPHENOL 0625HN OBIS (2-CHLOROETHYL) ETO625HN 02-CHLOROPHENOL $0625 \mathrm{HN}$ 01,3-DICHLOROBENZENE O625HN 01,4 -DICHLOROBENZENE 0625HN OBENZYL ALCOHOL O625HN 01,2-DICHLOROBENZENE $0625 \mathrm{HN}$ 02-METHYLPHENOL $\quad 0625 \mathrm{HN}$ OBIS (2-CHLOROISOPROP YO $625 \mathrm{HN}$ 04-METHYLPHENOL O625HN ON-NITROSO-DI-N-PROPYO625HN OHEXACHLOROETHANE O625HN ONITROBENZENE OISOPHORONE $\quad 0625 \mathrm{HN}$ 02-NITRORHENOL $0625 \mathrm{HN}$ 02, 4-DIMETHYLPHENOL 0625HN OBENZOIC ACID O625HN OBIS (2-CHLOROETHOXY) MO625HN 02,4-DICHLOROPHENOL 0625HN 01,2, 4-TRICHLOROBENZE0625HN ONAPHTHALENE $0625 \mathrm{HN}$ 04-CHLOROANILINE 0625HN OHEXACHLOROBUTADIENE $0625 \mathrm{HN}$ 04-CHLORO-3-METHYLPHEO $625 \mathrm{HN}$ 02-METHYLNAPHTHALENE 0625HN OHEXACHLOROCYCLOPENTAO625HN $02,4,6$-TRICHLOROPHENO0625HN $02,4,5$-TRICHIOROPHENOO625HN 02-CHLORONAPHTHALENE 0625HN 02-NITROANILINE 0625HN ODIMETHYLPHTHALATE O625HN OACENAPHTHYLENE $\quad 0625 \mathrm{HN}$ 02,6-DINITROTOLUENE 0625HN 03-NITROANILINE O625HN OACENAPHTHENE O625HN 02,4-DINITROPHENOL O625HN O4-NITRORHENOL O625HN ODIBENZOFURAN O625HN 02,4-DINITROTOLUENE 0625HN ODIETHYLPHTHALATE OGR5HN 04-CHLOROPHENYL-PHEP $625 \mathrm{HN}$ 
GRS 106 J220900309900326WA9002L606 003 FLRENE1XLT GRS 10030220900309900326 WA9002L606 003 4NANILIXLT GRS $10030220900309900326 \mathrm{WA} 9002 \mathrm{~L} 606003$ 46DN2C1XLT GRS $10030220900309900326 \mathrm{WA} 9002 \mathrm{~L} 606003$ NNDPA 1XLT GRS $10030220900309900326 \mathrm{WA9002L606} 003$ 4BRPPEIXLT GRS $10030220900309900326 \mathrm{WA} 9002 \mathrm{~L} 606003 \mathrm{CL} 6 \mathrm{BZ}$ 1XLT GRS $10030220900309900326 \mathrm{WA} 9002 \mathrm{~L} 606003$ PCP 1XLT GRS $10030220900309900326 \mathrm{WA} 9002 \mathrm{~L} 606003$ PHANTRIXLT GRS $10030220900309900326 \mathrm{WA} 9002 \mathrm{~L} 606003$ ANTRC 1XLT GRS 10030220900309900326 WA9002L606 003 DNBP 1XLT GRS $1.0030220900309900326 \mathrm{WA} 9002 L 606003$ EANT 1XLT GRS $10030220900309900326 \mathrm{WA} 9002 \mathrm{~L} 606003$ PYR 1XLT GRS $10030220900309900326 \mathrm{WA} 90022606003$ BBZP 1XLT GRS $10030220900309900326 \mathrm{WA} 9002 \mathrm{~L} 606003$ 33CL2B1XLT GRS $10030220900309900326 \mathrm{WA} 9002 \mathrm{~L} 606003$ BAANTRIXLT GRS $10030220900309900326 \mathrm{WA} 9002 \mathrm{~L} 606003$ CHRY 1XLT GRS $10030220900309900326 \mathrm{WA} 9002 L 606003$ B2EHP 1X

GRS 10030220900309900326 WA9002L606 003 DNOP 1XLT

GRS $10030220900309900326 \mathrm{KA} 9002 \mathrm{~L} 606003$ BF2ANT1XLT

GRS $10030220900309900326 \mathrm{WA} 9002 \mathrm{~L} 606003$ BKFANT1XLT

GRS $10030220900309900326 \mathrm{WA} 9002 \mathrm{~L} 606003$ BAPYR 1XLT

GRS

is GRS

O GRS

GRS

GRS

GRS

GRS

GRS

GRS

GRS

GRS

GRS

GRS

GRS

GRS

GRS

GRS

GRS

GRS

GRS

GRS

GRS

GRS

GRS

GRS

GRS
$10030220900309900326 \mathrm{KA} 9002 \mathrm{~L} 606003$ ICDPYR1XLT

$10030220900309900326 \mathrm{WA} 9002 \mathrm{~L} 606003$ DBAHA 1XLT

$10030220900309900326 \mathrm{WA} 9002 \mathrm{~L} 606003$ BGHIPY1XLT

$09010220900304900040 \mathrm{WA} 9002 \mathrm{~L} 606004$

$09010220900323900226 \mathrm{KA} 9002 \mathrm{~L} 606004$ AG 22LT

$09010220900330900225 \mathrm{KA} 9002 \mathrm{~L} 606004 \mathrm{AS}$ 1BLT

$09010220900323900226 \mathrm{WA} 9002 \mathrm{~L} 606004 \mathrm{BA} \quad 22 \mathrm{LT}$

$09010220900323900226 \mathrm{WA} 9002 \mathrm{~L} 606004 \mathrm{CD} \quad 2 \mathrm{LLT}$

09010220900323900226 WA9002L606 $004 \mathrm{CR} \quad 2 \mathrm{Z}$

$09010220900319900042 \mathrm{WA} 9002 \mathrm{~L} 606$ 004 HGTOT 1DLT

$09010220900405900225 \mathrm{HA} 9002 \mathrm{~L} 606004 \mathrm{~PB} \quad 1 \mathrm{~B}$

$09010220900401900225 \mathrm{FA} 9002 \mathrm{~L} 606004 \mathrm{SE}$ 1BLT

09010220900228900032 WA9002L606 004 MEC6D82J

09010220900228900032 WA9002L606 004 PBEB 2J

09010220900228900032 WA9002L606 004 12DCD42J

$09010220900228900032 \mathrm{WA} 9002 \mathrm{~L} 606004$ CH3CL 2JLT

09010220900228900032 WA9002L606 004 CH3BR 2JLT

09010220900228900032 WA9002L606 0.04 C2H3CL2JLT

09010220900228900032 WA9002L606 004 C2H5CL2JLT

09010220900228900032 WA $9002 L 606004$ CH2CL22JB

09010220900228900032 WA9002L606 004 ACET 2JB

09010220900228900032 WA9002L606 004 CS2 2JLT

09010220900228900032 HA9002L606 004 11DCE 2JLT

09010220900228900032 WA9002L606 004 11DCLE2JLT

09010220900228900032 WA9002L606 004 T12DCE2JLT

09010220900228900032 FA9002L606 004 CHCL3 2JLT

09010220900228900032 HA9002L606 004 12DCLE2JLT
22 UUUGKG

11000 UGKG

11000 UGKG

2200UGKG

2200 UGKG

2200UGKG

1100 OUGKG

2200 UGKG

2200UGKG

2200 UGKG

2200UGKG

2200 UGKG

2200UĠKG

430 OUGKG

220 OUGKG

2200UGKG

5900 UGKG

2200UGKG

2200UGKG

2200UGKG

2200 UGKG

2200UGKG

2200UGKG

2200UGKG

$93.8 \%$

2.1MGKG

1. $5 \mathrm{MGKG}$

41. 7MGKG

1. OMGKG

3. 9MGKG

$0.11 M G K G$

2. OMGKG

$0.77 \mathrm{MGKG}$

108PER

105PER

112PER

11UGKG

IIUGKG

11UGKG

11UGKG

12UGKG

17UGKG

5UGKG

5UGKG

5UGKG

5UGKG

5UGKG 5UGKG
51MSDAR

5IMSDAR

SIMSDAR

5IMSDAR

5IMSDAR

51MSDAR

5IMSDAR

5IMSDAR

5IMSDAR

5IMSDAR

5IMSDAR

5IMSDAR

51MSDAR

51MSDAR

51MSDAR

5IMSDAR

51MSDAR

51MSDAR

5IMSDAR

51MSDAR

5IMSDAR

5IMSDAR

5IMSDAR

5IMSDAR

$10 \mathrm{BT}$

1.00 ICALM

1.00AAAER

1.00 ICALM

1.00 ICALM

1. 00 ICALM

$1.00 \mathrm{HGSQ}$

1.00AACMT

1.00AAMLR

1.0000MSJBD

$1.0000 \mathrm{MSJBD}$

1.0000MSJBD

$1.0000 M S J B D$

$1.0000 \mathrm{MSJBD}$

$1.0000 \mathrm{MSJBD}$

$1.0000 \mathrm{MSJBD}$

$1.0000 \mathrm{MSJBD}$

$1.0000 M S J B D$

1.0000MSJBD

$1.0000 \mathrm{MSJBD}$

1.0000MSJBD

$1.0000 \mathrm{MSJBD}$

$1.0000 \mathrm{MSJBD}$

1.0000MSJBD
OFLUORENE

$0625 \mathrm{HN}$

04-NITROANIIINE O625HN

04, 6-DINITRO-2-METHYLO625HN ON-NITROSODIP HENYLAMIO $625 \mathrm{kN}$ 04-BROMOPHENYL-PHENYLO625HN OHEXACHLOROBENZENE O625HN OPENTACHLOROPHENOL $0625 \mathrm{HN}$ OPHENANTHRENE OANTHRACENE 0625HN ODI-N-BUTYLPHTHALATE O625HN OFLUORANTHENE O625HN OPYRENE $0625 \mathrm{HN}$ OBUTYLBENZYLPHTHALATEO625HN $03,3^{\circ}$-DICHLOROBENZIDIO625HN OBENZO (A) ANTHRACENE O625HN OCHRYSENE O625HN OBIS (2-ETHYLHEXYL) PHTO625HN ODI-N-OCTYL PHTHALATEO625HN OBENZO (B) ELUORANTHENE0625HN OBENZO (K) ELUORANTHENEO625HN OBENZO (A) PYRENE O625HN OINDENO $(1,2,3-C D)$ PYREO625HN ODIBENZO $(A, H)$ ANTHRACE0 $625 \mathrm{HN}$ OBENZO $(G, \mathrm{H}, \mathrm{I})$ PERYLENEO $625 \mathrm{HN}$ O\& SOLIDS

OSILVER, TOTAL

OARSENIC, TOTAL

OBARIUM, TOTAL

OCADMIUM, TOTAL

OCHROMIUM, TOTAL

OMERCURY, TOTAL

OLEAD, TOTAL

OSELENIUM, TOTAL

I8SOLN

MAGTON

MASTON

MBATON

MCDTON

MCRTON

MHGTON

MPBTON

MSETON

54. 50OTOLUENE-D8

$0624 \mathrm{HN}$

54.500BROMOELUOROBENZENE O624HN

54.5001,2-DICHLOROETHANE-DO624HN OCHLOROMETHANE O624HN OBROMOMETHANE O624HN OVINYL CHLORIDE O624HN OCHLOROETHANE OMETHYLENE CHLORIDE OACETONE

OCARBON DISULEIDE $0624 \mathrm{HN}$ 01,1-DICHLOROETHANE O624HN 01,2-DICHLOROETHENE 10624HN OCHLOROFORM 01,2-DICHLOROETHANE
, 


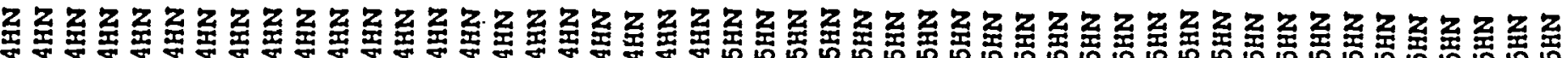

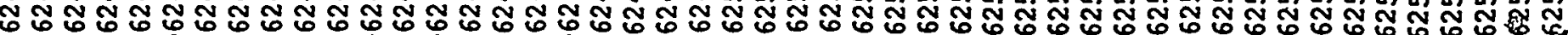

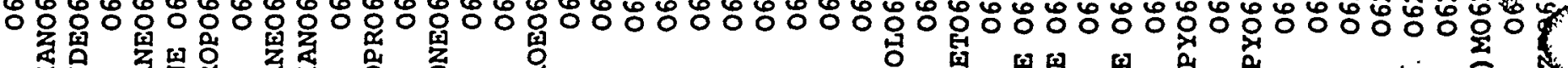
窟

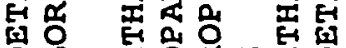

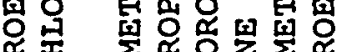

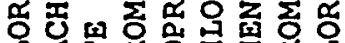

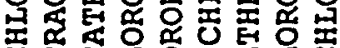

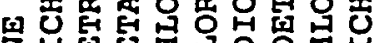

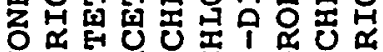

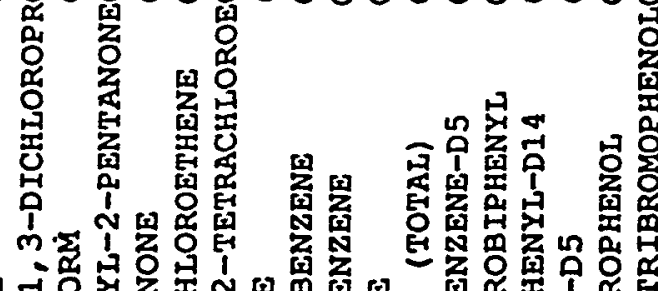

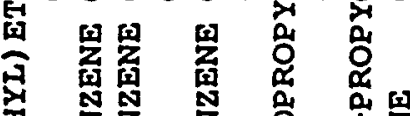

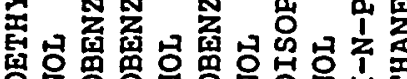

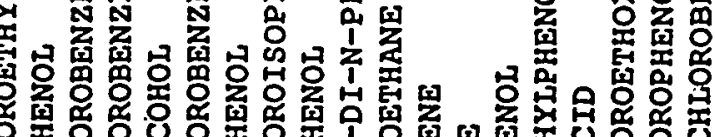

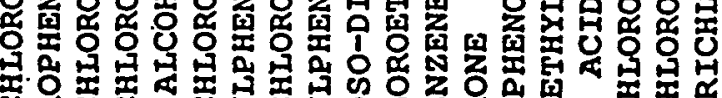

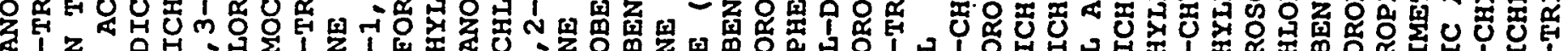
1

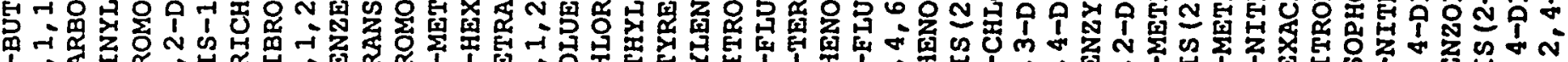

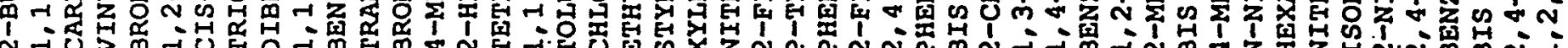

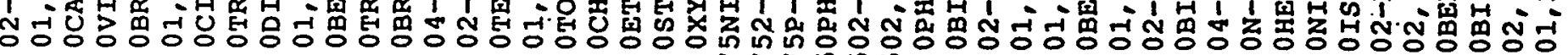
드는

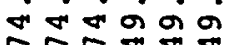

$5 \Sigma 5$ 药

नानलm m

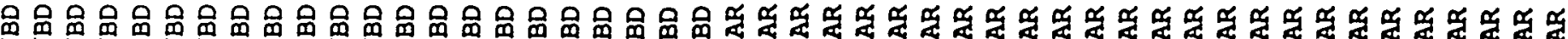
展5 55

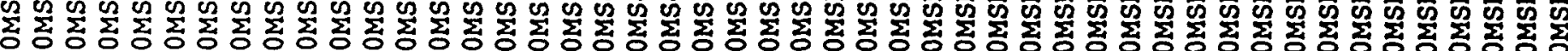

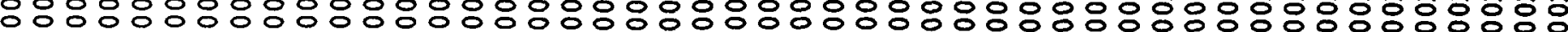

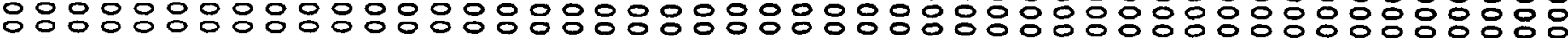

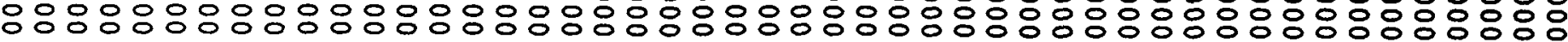
નન નન

U⿴囗十介

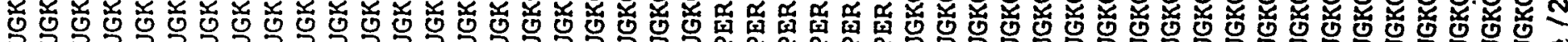

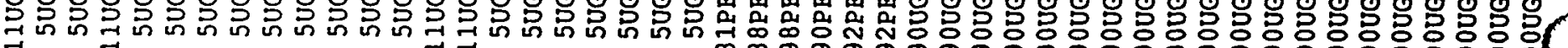

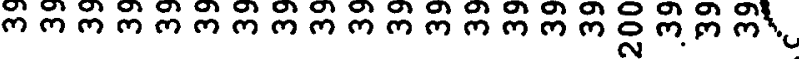

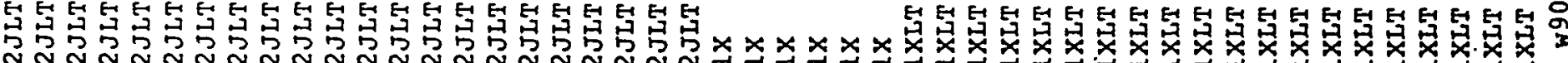

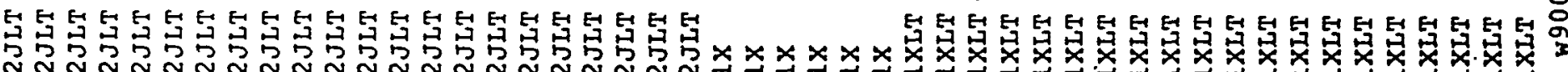
N

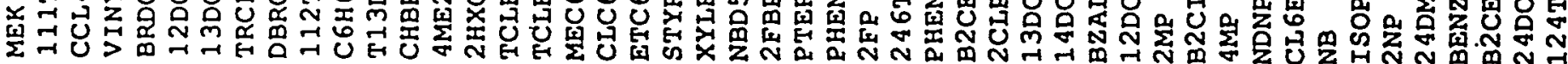
б б

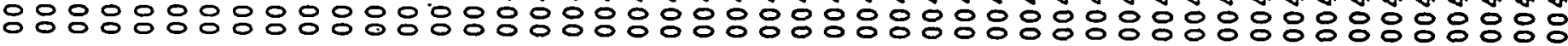
ம

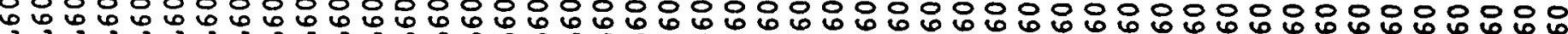

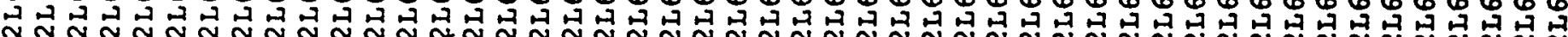
$\circ 000000000000000000000000 \%$

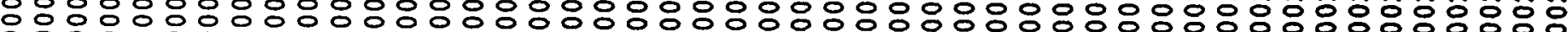
\%

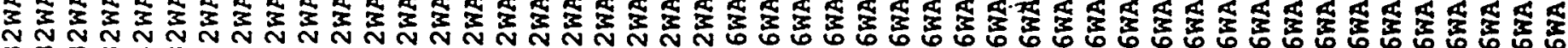

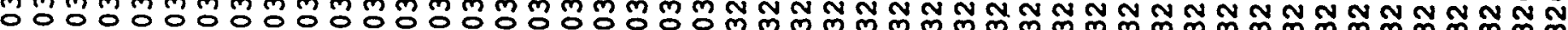

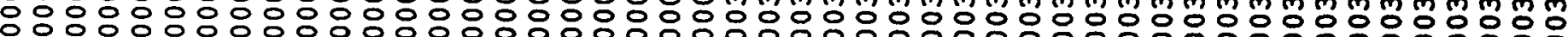

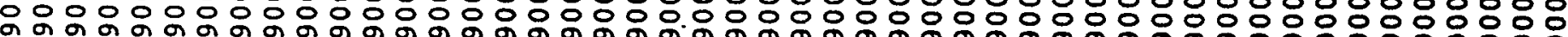
然 車

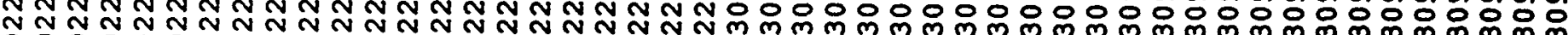

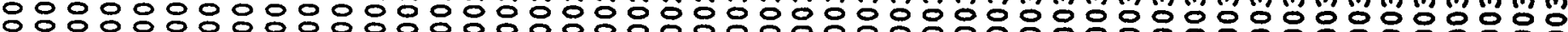

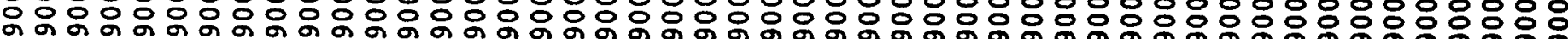

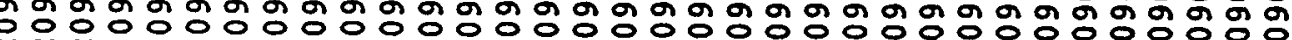

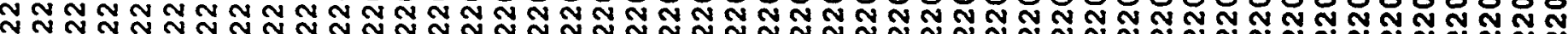

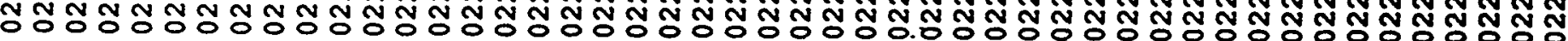

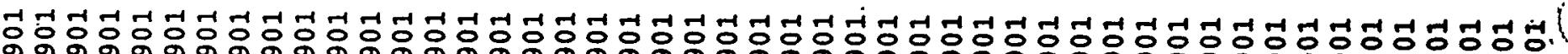

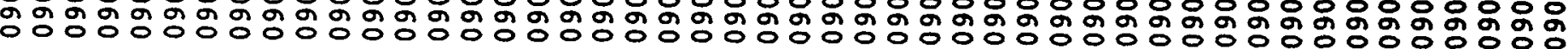


$090 \perp 0220900309900326 \mathrm{WA} 90024606004$ NAP 1XLT $09010220900309900326 \mathrm{WA} 90024606004$ 4CANILIXLT $09010220900309900326 \mathrm{WA} 90022606004$ HCBD 1XLT $09010220900309900326 \mathrm{WA} 9002 L 606004$ 4CL3C 1XLT 09010220900309900326 WA9002L606 004 2MENAPIXLT 09010220900309900326 WA9002L606 004 CL6CP 1XLT $0901022090030^{\prime} 9900326 \mathrm{WA} 9002 \mathrm{~L} 606004246 \mathrm{TCP} 1 \times L T$ 09010220900309900326 WA9002L606 004 245TCPIXLT 09010220900309900326 WA9002L606 004 2CNAP 1XLT 09010220900309900326 WA9002L606 004 2NANILIXLT $09010220900309900326 \mathrm{WA} 9002 L 606004$ DMP 1XLT $09010220900309900326 \mathrm{WA} 90024606004$ ANAPYLIXLT $09010220900309900326 \mathrm{WA} 9002 \mathrm{~L} 606004$ 26DNT 1XLT $09010220900309900326 \mathrm{WA} 9002 \mathrm{~L} 606004$ 3NANILIXLT 09010220900309900326 WA9002L606 004 ANAPNE1XLT $09010220900309900326 \mathrm{WA} 9002 \mathrm{~L} 606004$ 24DNP 1XLT 09010220900309900326 WA9002L606 004 4NP IXLT 09010220900309900326 WA9002L606 004 DIBZEU1XLT 09010220900309900326 WA9002L606 004 24DNT 1XLT 09010220900309900326 WA9002L606 004 DEP 1XLT 09010220900309900326 WA9002L606 004 ACIPPE1XLT 09010220900309900326 WA9002L606 004 ELRENEIXLT $09010220900309900326 \mathrm{WA} 9002 \mathrm{~L} 606004$ 4NANIL1XLT 09010220900309900326 WA9002L606 004 46DN2C1XLT 09010220900309900326 WA9002L606 004 NNDPA 1XLT 09010220900309900326 WA9002L606 004 4BRPPE1XLT $0901.0220900309900326 \mathrm{WA} 9002 \mathrm{~L} 606004$ CL6BZ 1XLT $09010220900309900326 \mathrm{WA} 9002 \mathrm{~L} 606004$ PCP 1XLT $09010220900309900326 \mathrm{WA} 9002 \mathrm{~L} 606004$ PHANTR1XLT $09010220900309900326 \mathrm{WA} 9002 \mathrm{~L} 606004$ ANTRC 1XLT $09010220900309900326 \mathrm{HA9002L606} 004$ DNBP 1XLT 09010220900309900326 WA'9002L606 004 FANT 1XLT 09010220900309900326 WA9002L606 004 PYR 1XLT $09010220900309900326 \mathrm{WA} 9002 \mathrm{~L} 606004$ BBZP 1XLT $09010220900309900326 \mathrm{WA} 9002 L 606004$ 33CL2B1XLT 09010220900309900326 WA9002L606 004 BAANTR1XIT 09010220900309900326 WA9002L606 004 CHRY 1XLT $09010220900309900326 \mathrm{WA} 9002$ L606 004 B2EHP $1 \mathrm{X}$ 09010220900309900326 WA9002L606 004 DNOP . 1XLT $09010220900309900326 \mathrm{WA} 9002 \mathrm{~L} 606004$ BF2ANT1XLT 09010220900309900326 WA9002L606 004 BKFANT1XLT $09010220900309900326 \mathrm{WA} 9002 L 606004$ BAPYR 1XLT 09010220900309900326 HA9002L606 004 ICDPYR1XLT 09010220900309900326 WA9002L606 004 DBAHA 1XLT 09010220900309900326 WA9002L606 004 BGHIPY1XLT 09020220900228900032 HA9002L606 005 MEC6D82J $09020220900228900032 W A 9002 L 606005$ PBEB $2 J$ 09020220900228900032 HA9002L606 005 12DCD42J
3 YUUGKG 390UGKG 390 UGKG 390 UGKG $390 U G K G$ 390 UGKG 39 OUGKG

2000 UGKG 390 UंGKG 2000 UGKG 390UGKG 39 OUGKG 39 OUGKG 2000 UGKG 390UGKG 2000 UGKG 2000 UGKG 390 UGKG 390 UGKG 39 OUGKG 390 UGKG 390UGKG 2000UGKG 2000UGKG 390 UGKG 390 UGKG 39OUGKG 2000 UGKG 390 UGKG 390 UGKG 390UGKG 390 UGKG 390UGKG 390 UGKG 790 UGKG 390UGKG 390UGKG

1000UGKG

390UGKG 390UGKG 390UGKG 390 UGKG 390UGKG 390UGKG 390UGKG 107PER 105PER 111PER
1.000 OMSDAR 1.000 OMSDAR 1.000 OMSDAR 1.000 OMSDAR 1.000 OMSDAR 1.000 OMSDAR 1.000 OMSDAR 1.000 OMSDAR 1.000 OMSDAR 1.000 OMSDAR 1.000 OMSDAR 1.000 OMSDAR 1.000 OMSDAR 1.000 OMSDAR 1.000 OMSDAR 1. 000 OMSDAR 1.000 OMSDAR 1.000 OMSDAR 1.000 OMSDAR 1.000 OMSDAR 1.000 OMSDAR 1.000 OMSDAR 1.000 OMSDAR 1.000 OMSDAR 1. $0000 \mathrm{MSDAR}$ 1.000 OMSDAR 1.000 OMSDAR 1.000 OMSDAR 1.000 OMSDAR 1.000 OMSDAR 1.000 OMSDAR 1.000 OMSDAR 1. 000 OMSDAR 1.000 OMSDAR 1.000 OMSDAR 1.000 OMSDAR 1.000 OMSDAR 1.000 OMSDAR 1.000 OMSDAR 1.000 OMSDAR 1.000 OMSDAR 1.000 OMSDAR 1.000 OMSDAR 1.000 OMSDAR 1.000 OMSDAR 1.000 OMSJBD 1.000 OMSJBD 1.000 OMSJBD
ONAPHTHALENE $0625 \mathrm{HN}$ 04-CHLOROANILINE 0625HN OHEXACHLOROBUTADIENE 0625HN 04-CHLORO-3-METHYLPHEO625HN 02-METHYLNAPHTHALENE 0625HN OHEXACHLOROCYCLOPENTAO625HN 02, 4, 6-TRICHLOROPHENO0625HN $02,4,5$-TRICHLOROPHENO0625HN 02-CHLORONAPHTHALENE 0625HN 02-NITROANILINE 0625HN ODIMETHYLPHTHALATE 0625HN OACENAPHTHYLENE 0625HN 02,6-DINITROTOLUENE 0625HN 03-NITROANILINE O625HN OACENAPHTHENE O625HN 02,4-DINITROPHENOL 0625HN 04-NITROPHENOL O625HN ODIBENZOFURAN O625HN 02,4-DINITROTOLUENE 0625HN ODIETHYLPHTHALATE $0625 \mathrm{HN}$ 04-CHLOROPHENYL-PHENYO625HN OELUORENE. O625HN 04-NITROANILINE 0625HN 04, 6-DINITRO-2-METHYLO625HN ON-NITROSODIPHENYLAMIO625HN 04-BROMOPHENYL-PHENYL0625HN OHEXACHLOROBENZENE 0625HN OPENTACHLOROPHENOL O625HN OPHENANTHRENE O625HN OANTHRACENE $0625 \mathrm{HN}$ ODI-N-BUTYLPHTHALATE 0625HN OFLUORANTHENE 0625HN OPYRENE $0625 \mathrm{HN}$ OBUTYLBENZYLPHTHALATEO625HN $03,3^{\prime}-D I C H L O R O B E N Z$ IDI0625HN OBENZO (A) ANTHRACENE O625HN OCHRYSENE 0625HN OBIS (2-ETHYLHEXYL) PHTO625HN ODI-N-OCTYL PHTHALATEO625HN OBENZO (B) ELUORANTHENEO625HN OBENZO (K) ELUORANTHENEO $625 \mathrm{HN}$ OBENZO (A) PYRENE O625HN OINDENO $(1,2,3-C D)$ PYREO $625 \mathrm{HN}$ ODIBENZO (A, H) ANTHRACEO $625 \mathrm{HN}$ OBENZO (G, H, I) PERYLENEO625HN 59.500TOLUUENE-D8 59.500BROMOELUOROBENZENE O684HN 59 . 5001, 2-DICHLOROETHANE-DO624HN 
GRS 09020220900228900032 WA9002L606 005 CH3CL 2JLT GRS 09020220900228900032 WA9002L606 005 CH3BR 2JLT GRS 09020220900228900032 WA9002L606 005 C2H3CL2JLT GRS 09020220900228900032 WA9002L606 005 C2H5CL2JLT GRS 09020220900228900032 WA $9002 L 606005$ CH2CL22JB GRS 09020220900228900032 WA $9002 L 606005$ ACET 2JB GRS 09020220900228900032 WA9002L606 005 CS2 2JLT GRS 09020220900228900032 WA9002L606 005 11DCE 2JLT GRS 09020220900228900032 WA9002L606 005 11DCLE2JLT GRS 09020220900228900032 WA $9002 L 606005$ T12DCE2JLT GRS $09020220900228900032 \mathrm{WA} 9002 \mathrm{~L} 606005$ CHCL3 2JLT GRS $09020220900228900032 \mathrm{WA} 9002 \mathrm{~L} 606005$ 12DCLE2JLT GRS 09020220900228900032 WA9002L606 005 MEK 2JLT GRS $09020220900228900032 \mathrm{WA} 9002 \mathrm{~L} 606 \quad 005$ 111TCE2JLT GRS 09020220900228900032 WA9002L606 005 CCL4 2JLT GRS $09020220900228900032 \% A 9002 L 606005$ VINYLA2JLT GRS 09020220900228900032 FA9002L606 005 BRDCLM2JLT GRS 09020220900228900032 WA9002L606 005 12DCLP2JLT GRS 09020220900228900032 WA9002L606 005 13DCPC2JLT GRS $09020220900228900032 \mathrm{WA} 9002 L 606005$ TRCLE 2JLT GRS 0902.0220900228900032 WA $9002 L 606005$ DBRCLM2JLT GRS 09020220900228900032 WA9002L606 005 112TCE2JLT
$09020220900228900032 \mathrm{WA} 002 \mathrm{~L} 606005 \mathrm{C} 6 \mathrm{H} 6$ 2JLT

09020220900228900032 WA 90022606005 T13DCP2JLT

$09020220900228900032 \mathrm{WA} 9002 \mathrm{~L} 606005$ CHBR3 2JLT 09020220900228900032 WA9002L606 005 4ME2PE2JLT 09020220900228900032 WA9002L606 005 2HXONE2JLT 09020220900228900032 WA9002L606 005 TCLEE 2JLT 09020220900228900032 WA9002L606 005 TCLEA 2JLT $09020220900228900032 \mathrm{WA} 9002 \mathrm{~L} 606005$ MEC6H52JLT 09020220900228900032 WA9002L606 005 CLC6H52JLT. 09020220900228900032 स $9002 L 606005$ ETC6H52JLT $09020220900228900032 \mathrm{WA} 9002 \mathrm{~L} 606005$ STYR 2JLT 09020220900228900032 WA9002L606 005 XYLEN 2JLT 09020220900309900326 WA9002L606 005 NBD5 $1 \mathrm{X}$ $09020220900309900326 \mathrm{WA} 9002 \mathrm{~L} 606005$ 2FBP $1 \mathrm{X}$ 09020220900309900326 WA9002L606 005 PTERP $1 \mathrm{X}$ 09020220900309900326 WA9002L606 005 PHEND51X $09020220900309900326 \mathrm{WA} 9002 \mathrm{~L} 606005$ 2EP $1 \mathrm{X}$ 09020220900309900326 WA9002L606 005 246TBP1X 09020220900309900326 WA9002L606 005 PHENOL1XLT $09020220900309900326 \mathrm{WA} 9002 \mathrm{~L} 606$ 005. B2CEE 1XLT $09020220900309900326 \mathrm{FA} 9002 \mathrm{~L} 606005$ 2CLP 1XLT $09020220900309900326 \mathrm{WA} 9002 \mathrm{~L} 606005$ 13DCLB1XLT $09020220900309900326 \mathrm{WA} 9002 \mathrm{~L} 606005$ 14DCLBIXLT $09020220900309900326 \mathrm{WA} 9002 \mathrm{~L} 606$ 005 BZALC 1XLT $09020220900309900326 \mathrm{KA} 9002 \mathrm{~L} 606005$ 12DCLB1XLT $09020220900309900326 \mathrm{WA} 9002 \mathrm{~L} 606005$ 2MP 1XLT
12UGKG

6UGKG

6UGKG

12UGKG

6UGKG

6UGKG

6UGKG

6UGKG

6UGKG

6UGKG

6UGKG

6UGKG

6UGKG

12UGKG

12UGKG

6UGKG

6UGKG

6UGKG

6UGKG

6UGKG

GUGKG

6UGKG

53PER

65PER

8OPER

67PER

64PER

64PER

440UGKG

44 OUGKG

44 OUGKG

44 OUGKG

4 OOUGKG

44 OUGKG

44 OUGKG
$1.0000 \mathrm{MSJBD}$ $1.0000 \mathrm{MSJBD}$ $1.0000 M S J B D$ $1.0000 M S J B D$ $1.0000 \mathrm{MSJBD}$ $1.0000 \mathrm{MSJBD}$ 1. $0000 \mathrm{MSJBD}$ 1. $0000 \mathrm{MSJBD}$ 1. $0000 \mathrm{MSJBD}$ $1.0000 M S J B D$ $1.0000 \mathrm{MSJBD}$ $1.0000 \mathrm{MSJBD}$ 1. $0000 \mathrm{MSJBD}$ 1. 000OMSJBD 1. $0000 \mathrm{MSJBD}$ 1.0000MSJBD 1.000OMSJBD 1. $0000 \mathrm{MSJBD}$ $1.0000 \mathrm{MSJBD}$ 1.0000MSJBD $1.0000 \mathrm{MSJBD}$ 1.000OMSJBD 1.0000MSJBD 1. $0000 \mathrm{MSJBD}$ 1.0000MSJBD 1. $0000 \mathrm{MSJBD}$ 1.0000MSJBD 1.0000MSJBD 1. 000 OMSJBD $1.0000 \mathrm{MSJBD}$ 1.0000MSJBD 1. 0000MSJBD $1.0000 \mathrm{MSJBD}$ 1.0000MSJBD 1. 0000MSDAR 1.0000MSDAR 1.000OMSDAR 1. 000OMSDAR 1.0000MSDAR 1. 0000MSDAR 1.0000MSDAR 1.000OMSDAR 1. 0000MSDAR 1.0000MSDAR 1.000OMSDAR 1. 0000MSDAR 1.0000MSDAR 1.000OMSDAR
OCHLOROMETHANE

OBROMOMETHANE

OVINYL CHLORIDE

$0624 \mathrm{HN}$ OCHLOROETHANE OACETONE

OCARBON DISULEIDE O624KN 01,2-DICHLOROETHENE (0624HN OCHLOROFORM O624HN 01,2-DICHLOROETHANE O624HN 02-BUTANONE O624HN $01,1,1-T R I C H L O R O E T H A N O 624 \mathrm{HN}$ OCARBON TETRACHLORIDEO $624 \mathrm{HN}$ OVINYL ACETATE O624HN OBROMODICHLOROMETHANEO624HN 01,2-DICHLOROPROPANE O624HN OCIS-1, 3-DICHLOROPROPO624HN OTRICHLOROETHENE O624HN ODIBROMOCHLOROMETHANEO $624 \mathrm{HN}$ $01,1,2-T R I C H L O R O E T H A N O 624 \mathrm{HN}$ OBENZENE O624HN OTRANS-1, 3-DICHLORORRO624HN OBROMOFORM O624HN 04-METHYL-2-PENTANONEO624HN 02-HEXANONE O624HN OTETRACHLOROETHENE O624HN $01,1,2,2-T E T R A C H L O R O E O 624 \mathrm{HN}$ OTOLUENE OCHIORO OETHYLBENZENE O624HN OSTYRENE O624HN OXYLENE (TOTAL) O624HN 1959.460NITROBENZENE-D5 O625HN 1959.4602-FLUOROBIPHENYL O625HN 1959.460P-TERPHENYL-D14 O625HN 3918.920 PHENOL-D5 O625HN 3918.9202-FLUOROPHENOL O625HN 3918 . 9202, 4, 6-TRIBROMORHENOLO $625 \mathrm{HN}$ OPHENOL 0625HN OBIS (2-CHLOROETHYL) ETO625HN 02-CHLOROPHENOL O625HN $01,3-D I C H L O R O B E N 2 E N E$ 062.5HN 01, 4-DICHLOROBENZENE 0625HN OBENZYL ALCOHOL O625HN 01,2-DICHLOROBENZENE 0625HN 02-METHYLPHENOL 
GRS 09L $0220900309900326 \mathrm{WA} 9002 \mathrm{~L} 606005$ B2CIPEIXLT GRS $09020220900309900326 \mathrm{WA} 9002 \mathrm{~L} 606005$ 4MP 1XLT GRS $09020220900309900326 \mathrm{WA} 9002 \mathrm{~L} 606005$ NDNPA 1XLT GRS $09020220900309900326 \mathrm{FA} 9002 \mathrm{~L} 606005$ CL6ET 1XLT GRS $09020220900309900326 \mathrm{WA} 9002 \mathrm{~L} 606005 \mathrm{NB}$ 1XLT GRS $09020220900309900326 \mathrm{WA} 9002 \mathrm{~L} 606005$ ISOPHRIXLT GRS $09020220900309900326 \mathrm{WA} 9002 \mathrm{~L} 606005$ 2NP 1XLT GRS 09020220900309900326 संA9002L606 005 24DMPN1XLT GRS $09020220900309900326 \mathrm{WA} 9002 \mathrm{~L} 606005$ BENZOAIXLT GRS $09020220900309900326 \mathrm{WA} 9002 \mathrm{~L} 606005$ B2CEXM1XLT GRS $09020220900309900326 \mathrm{WA} 9002 \mathrm{~L} 606005$ 24DCLP1XLT GRS $09020220900309900326 \mathrm{WA} 9002 \mathrm{~L} 606005 \quad 124 \mathrm{TCB} 1 \mathrm{XLT}$ GRS $09020220900309900326 \mathrm{WA} 9002 \mathrm{~L} 606005$ NAP 1XLT GRS 09020220900309900326 WA9002L606 005 4CANILIXLT GRS $09020220900309900326 \mathrm{WA} 9002 \mathrm{~L} 606005$ HCBD 1XLT GRS $09020220900309900326 \mathrm{WA} 9002 \mathrm{~L} 606005$ 4CL3C 1XLT GRS $09020220900309900326 \mathrm{WA} 9002 \mathrm{~L} 606005$ 2MENAP1XLT GRS $09020220900309900326 \mathrm{WA} 9002 \mathrm{~L} 606005$ CL6CP 1XLT GRS $09020220900309900326 \mathrm{WA} 9002 \mathrm{~L} 606005$ 246TCP1XLT GRS $09020220900309900326 \mathrm{WA} 9002 \mathrm{~L} 606005$ 245TCP1XLT GRS $09020220900309900326 \mathrm{WA} 9002 \mathrm{~L} 606005$ 2CNAP 1XLT GRS $09020220900309900326 \mathrm{WA} 90021606005$ 2NANIL1XLT GRS $09020220900309900326 \mathrm{FA} 90021606005$ DMP 1XLT

$09020220900309900326 \mathrm{WA} 9002 \pi 606005$ ANAPYL1XLT $09020220900309900326 \mathrm{WA} 90024606005$ 26DNT 1XLT $09020220900309900326 \mathrm{WA} 90021606005$ 3NANILIXLT $09020220900309900326 \mathrm{WA} 9002 L 606005$ ANAPNE1XLT 09020220900309900326 WA9002L606 005 24DNP 1XLT $09020220900309900326 \mathrm{WA} 9002 \mathrm{~L} 606005$ 4NP 1XLT 09020220900309900326 WA9002L606 005 DIBZEU1XLT $09020220900309900326 \mathrm{FA} 9002$ L606 005 24DNT 1XLT $09020220900309900326 \mathrm{WA} 9002 \mathrm{~L} 606005$ DEP 1XLT 09020220900309900326 WA9002L606 005 4CLPRE1XLT 09020220900309900326 WA9002L606 005 FLRENE1XLT 09020220900309900326 HA9002L606 005 4NANIL1XLT 09020220900309900326 WA9002L606 005 46DN2C1XLT 09020220900309900326 WA9002L606 005 NNDPA 1XLT $09020220900309900326 \mathrm{WA} 9002 L 606005$ 4BRPRE1XLT 09020220900309900326 WA9002L606 005 CL6BZ IXLT $09020220900309900326 \mathrm{WA} 9002 \mathrm{~L} 606005$ PCP 1XLT 09020220900309900326 WA9002L606 005 PHANTR1XLT 09020220900309900326 WA9002L606 005 ANTRC 1XLT 09020220900309900326 WA9002L606 005 DNBP 1XLT 09020220900309900326 WA9002L606 005 EANT 1XLT 09020220900309900326 WA9002L606 005 RYR 1XLT 09020220900309900326 WA9002L606 005 BBZP 1XLT $09020220900309900326 \% A 9002 L 606005$ 33CL2B1XLT $09020220900309900326 \mathrm{FA9002L606} \mathrm{005} \mathrm{BAANTR1XLT}$
1.0000MSDAR 1.0000MSDAR 1.0000MSDAR 1.0000MSDAR 1. 000OMSDAR 1.0000MSDAR 1.0000MSDAR 1. OOOOMSDAR 1.0000MSDAR $1.0000 \mathrm{MSDAR}$ 1. 000OMSDAR 1.0000MSDAR 1. 0000MSDAR 1.000OMSDAR 1.0000MSDAR 1. OOOOMSDAR 1.0000MSDAR 1. $0000 \mathrm{MSDAR}$ 1. OOOOMSDAR 1. 000OMSDAR 1.000OMSDAR 1. $0000 \mathrm{MSDAR}$ 1.0000MSDAR 1.0000MSDAR 1. 0000MSDAR 1.0000MSDAR 1. 000OMSDAR 1. 000OMSDAR 1. 000OMSDAR 1. OOOOMSDAR 1. 000OMSDAR 1.0000MSDAR 1. 0000MSDAR 1.0000MSDAR

1. 0000MSDAR 1. 0000MSDAR

1.0000MSDAR

1.0000MSDAR

1.0000MSDAR

1.0000MSDAR

1. 000OMSDAR

1. 0000MSDAR

1.0000MSDAR

1. OOOOMSDAR

1.0000MSDAR

1. 0000MSDAR

1. OOOOMSDAR

1.0000MSDAR
OBIS (2-CHLOROISOPROPYO625HN O4-METHYLPHENOL O625HN ON-NITROSO-DI-N-PROPYO625HN OHEXACHLOROETHANE. 0625HN ONITROBENZENE O625HN OISORHORONE O625HN 02-NITROPHENOL 0625HN 02,4 -DIMETHYLPHENOL 0625HN OBENZOIC ACID 0625HN OBIS (2-CHLOROETHOXY) MO625HN $02,4-D I C H L O R O P H E N O L$ 0625HN $01,2,4-T R I C H L O R O B E N Z E 0625 \mathrm{HN}$ ONAPHTHALENE O625HN 04-CHLOROÄNILINE 0625HN OHEXACHLOROBUTADIENE O625HN 04-CHLORO-3-METHYLPHEO 625HN 02-METHYLNAPHTHALENE 0625HN OHEXACHLOROCYCLOPENTAO 625HN $02,4,6$-TRICHLOROPHENOO $625 \mathrm{HN}$ $02,4,5-T R I C H L O R O P H E N O 0625 \mathrm{HN}$ 02-CHLORONAPHTHALENE 0625HN 02-NITROANIIINE 0625HN ODIMETHYLPHTHALATE $0625 \mathrm{HN}$ OACENAPHTHYLENE $0625 \mathrm{HN}$ 02,6-DINITROTOLUENE 0625HN 03-NITROANILINE $0625 \mathrm{HN}$ OACENAPHTHENE O625HN 02, 4-DINITROPHENOI 0625HN 04-NITROPHENOL O625HN ODIBENZOEURAN $0625 \mathrm{HN}$ 02, 4-DINITROTOLUENE 0625HN ODIETHYLPHTHALATE 0625HN 04-CHLOROPHENYL-PHENYO625HN OFLUORENE O625HN 04-NITROANILINE 0625HN 04, 6-DINITRO-2-METHYI0625HN ON-NITROSODIPHENYLAMIO625HN 04-BROMOPHENYL-PHENYLO $625 \mathrm{HN}$ OHEXACHLOROBENZENE O625HN ORENTACHLOROPHENOL O625HN OPHENANTHRENE O625HN OANTHRACENE 0625HN ODI-N-BUTYLPHTHALATE 0625HN OELUORANTHENE O625HN OPYRENE O625HN OBUTYLBENZYLPHTHALATEO $625 \mathrm{HN}$ $03,3^{\circ}-D I C H L O R O B E N 2 I D I O 625 \mathrm{HN}$ OBENZO (A) ANTHRACENE 0625HN 
GRS $09020220900309900326 \mathrm{WA} 9002 \mathrm{~L} 606005$ CHRY 1XLT GRS 09020220900309900326 WA9002L606 005 B2EHP 1X GRS $09020220900309900326 \mathrm{WA} 9002 \mathrm{~L} 606005$ DNOP 1XLT GRS $09020220900309900326 \mathrm{WA} 9002 \mathrm{~L} 606005$ BE2ANT 1 XLT GRS $09020220900309900326 \mathrm{WA} 9002 \mathrm{~L} 606005$ BKEANT 1 XLT GRS $09020220900309900326 \mathrm{WA} 90024606005$ BAPYR 1XLT GRS $09020220900309900326 \mathrm{WA} 9002 \mathrm{~L} 606005$ ICDPYRIXLT GRS 09020220900309900326 WA9002L606 005 DBAHA 1XLT GRS $09020220900309900326 \mathrm{WA} 9002 \mathrm{~L} 606005$ BGHIPY1XLT GRS 09030220900228900032 WA9002L606 006 MEC6D82J GRS 09030220900228900032 WA9002L606 006 PBFB $2 J$ GRS 09030220900228900032 WA9002L606 006 12DCD42J GRS 09030220900228900032 WA9002L606 006 CH3CL 2JLT GRS $09030220900228900032 \mathrm{WA} 9002 L 606006$ CH3BR 2JLT GRS $09030220900228900032 \mathrm{WA} 9002 L 606006$ C2H3CL2JLT GRS 09030220900228900032 WA9002L606 006 C2H5CL2JLT GRS $09030220900228900032 \mathrm{WA} 9002 \mathrm{~L} 606006$ CH2CL22JB GRS $09030220900228900032 \mathrm{WA} 9002 L 606006$ ACET. 2JB GRS $09030220900228900032 \mathrm{WA} 90022606006 \mathrm{CS} 2$ 2JLT GRS $09030220900228900032 \mathrm{WA} 9002 \mathrm{~L} 606006$ 11DCE 2JLT GRS 09030220900228900032 WA9002L606 006 11DCLE2JLT GRS $09030220900228900032 \mathrm{WA} 9002 \mathrm{~L} 606006$ T12DCE2JLT GRS $09030220900228900032 \mathrm{WA} 9002 L 606006$ CHCL3 2JLT to GRS

09030220900228900032 WA9002L606 006 12DCLE2JLT $09030220900228900032 \mathrm{WA} 9002 \mathrm{~L} 606006 \mathrm{MEK}$ 2JLT $09030220900228900032 \mathrm{WA} 9002 \mathrm{~L} 606006$ 111TCE2JLT 09030220900228900032 WA9002L606 006 CCL4 2JLT 09030220900228900032 WA9002L606 006 VINYLA2JLT $09030220900228900032 \mathrm{WA} 9002 \mathrm{~L} 606006$ BRDCLM2JLT 09030220900228900032 WA9002L606 006 12DCLP2JLT 09030220900228900032 WA9002L606 006 13DCPC2JLT 09030220900228900032 स $9002 L 606006$ TRCLE 2JLT 09030220900228900032 WA9002L606 006 DBRCLM2JLT 09030220900228900032 WA9002L606 006 112TCE2JLT 09030220900228900032 WA9002L606 006 C6H6 2JLT 09030220900228900032 WA9002L606 006 T13DCP2JLT $09030220900228900032 \mathrm{WA} 9002 L 606006$ CHBR3 2JLT 09030220900228900032 WA9002L606 006 4ME2PE2JLT 09030220900228900032 WA9002L606 006 2HXONE2JLT 09030220900228900032 WA9002L606 006 TCLEE 2JLT 09030220900228900032 WA9002L606 006 TCLEA 2JLT 09030220900228900032 WA9002L606 006 MEC6H52JLT 09030220900228900032 KA9002L606 006 CLC6H52JLT 09030220900228900032 WA9002L606 006 ETC6H52JLT 09030220900228900032 WA9002L606 006 STYR 2JLT 09030220900228900032 WA9002L606 006 XYLEN 2JLT $09030220900309900326 \mathrm{WA} 9002 \mathrm{~L} 606006$ NBDS IX $09 Q 30220900309900326 \mathrm{FA9002L606006} \mathrm{2FBP} \mathrm{iX}$
44 OUGKG

4800 UGKG

44 OUGKG

440 UGKG

440 UGKG

44 OUGKG

440 UGKG

440 UGKG

440 UGKG

108 PER

111PER

114PER

12 UGKG

12 UGKG

12UGKG

12UGKG

14UGKG

69UGKG

6UGKG

6UGKG

6UGKG

6UGKG

6UGKG

6UGKG

12UGKG

6UGKG

6UGKG

12UGKG

6UGKG

6UGKG

6UGKG

6UGKG

6UGKG

6UGKG

6UGKG

6UGKG

6UGKG

12UGKG

12UGKG

6UGKG

6UGKG

6UGKG

6UGKG

6UGKG

6UGKG

6UGKG

54PER
$.0000 M S D A R$

$1.0000 M S D A R$

1. $0000 M S D A R$

1.0000MSDAR

1. O00OMSDAR

1.0000MSDAR

1. 0000MSDAR

1. OOOOMSDAR

1.0000MSDAR

1.0000MSJBD

1. $0000 M S J B D$

1. 0000MSJBD

1. $0000 \mathrm{MSJBD}$

1.0000MSJBD

1. $0000 \mathrm{MSJBD}$

1. $0000 \mathrm{MSJBD}$

1. $0000 \mathrm{MSJBD}$

1.0000MSJBD

1. $0000 \mathrm{MSJBD}$

$1.0000 \mathrm{MSJBD}$

1. 0000MSJBD

1. 0000MSJBD

1. $0000 \mathrm{MSJBD}$

1. 0000MSJBD

$1.0000 \mathrm{MSJBD}$

1.0000MSJBD

1. 0000MSJBD

1.0000MSJBD

1. 0000MSJBD

1. $0000 \mathrm{MSJBD}$

1. $0000 \mathrm{MSJBD}$

1.0000MSJBD

1. $0000 M S J B D$

1. 0000MSJBD

1. 0000MSJBD

1. 0000 MSJBD

1.0000MSJBD

1. 0000MSJBD

1. 0000MSJBD

1. 0000MSJBD

1. O000MSJBD

$1.0000 \mathrm{MSJBD}$

1. $0000 \mathrm{MSJBD}$

1. $0000 M S J B D$

1. 0000MSJBD

1. 000OMSJBD

1. 0000MSDAR

1. 000OMSDAR
OCHRYSENE

$0625 \mathrm{HN}$

OBIS (2-ETHYLHEXYL) PHTO625HN ODI-N-OCTYL PHTHALATEO625HN OBENZO (B) FLUORANTHENE0625HN OBENZO (K) FLUORANTHENEO625HN OBENZO (A) PYRENE O625HN O INDENO $(1,2,3-C D)$ PYREO625HN ODIBENZO $(\mathrm{A}, \mathrm{H})$ ANTHRACEO $625 \mathrm{HN}$ OBENZO (G, H, I) RERYIENEO625HN 59.500TOLUENE-D8 O624HN 59.50OBROMOELUOROBENZENE O624HN 59.5001, 2-DICHLOROETHANE-DO624HN OCHLOROMETHANE O624HN OBROMOMETHANE O624HN OVINYL CHLORIDE O624HN OCHLOROETHANE O624HN OMETHYLENE CHLORIDE · 0624HN OACETONE 0624HN OCARBON DISULEIDE O624HN 01,1-DICHLOROETHENE 0624HN 01,1-DICHLOROETHANE 0624HN 01,2-DICHIOROETHENE 10624HN OCHLOROFORM 0624HN 01,2-DICHLOROETHANE 0624HN 02-BUTANONE O624HN $01,1,1-T R I C H L O R O E T H A N O 624 \mathrm{HN}$ OCARBON TETRACHLORIDEO624HN OVINYI ACETATE O624HN OBROMODICHLOROMETHANEO624HN 01, 2-DICHLOROPROPANE 0624HN OCIS-1, 3-DICHLOROPROPO62 4HN OTRICHLOROETHENE O624HN ODIBROMOCHLOROMETHANEO $624 \mathrm{HN}$ $01,1,2-T R I C H L O R O E T H A N O 624 \mathrm{HN}$ OBENZENE O624HN OTRANS-1, 3-DICHLORORRO62 4HN OBROMOFORM . 0624HN 04-METHYL-2-PENTANONEO624HN 02-HEXANONE O624HN OTETRACHLOROETHENE O624HN $01,1,2,2$-TETRACHLOROEO $624 \mathrm{HN}$ OTOLUENE 0624HN OCHLOROBENZENE O624HN OETHYIBENZENE $\quad 0624 \mathrm{HN}$ OSTYRENE O O624HN OXYLENE (TOTAL) 0624HN 1950.451NITROBENZENE-D5 O625HN 1950.4512-ELUOROBIPHENYL 
GRS 09u, 0220900309900326KA9002L606 006 PTERP IX GRS $09030220900309900326 \mathrm{WA} 9002 \mathrm{~L} 606006$ PHEND51X GRS $09030220900309900326 \mathrm{KA} 9002 \mathrm{~L} 606006$ 2FP 1X GRS $09030220900309900326 \mathrm{WA} 9002 \mathrm{~L} 606006$ 246TBP 1X GRS $09030220900309900326 \mathrm{KA} 9002 \mathrm{~L} 606006$ PHENOLIXLT GRS $09030220900309900326 \mathrm{WA} 9002 \mathrm{~L} 606006$ B2CEE IXLT GRS $09030220900309900326 \mathrm{KA} 9002 \mathrm{~L} 606006$ 2CLP 1XLT GRS 09030220900309900326 WंA9002L606 006 13DCLB1XLT GRS $09030220900309900326 \mathrm{WA} 9002 \mathrm{~L} 606006$ 14DCLB1XLT GRS $09030220900309900326 \mathrm{WA} 9002 \mathrm{~L} 606006$ B2ALC 1XLT GRS $09030220900309900326 \mathrm{WA} 9002 \mathrm{~L} 606006$ 12DCLB1XLT GRS $09030220900309900326 \mathrm{FA9002L606} 006$ 2MP IXLT GRS $09030220900309900326 \mathrm{FA} 9002 \mathrm{~L} 606006$ B2CIPEIXLT GRS $09030220900309900326 \mathrm{WA} 9002 \mathrm{~L} 606006$ 4MP IXLT GRS $09030220900309900326 \mathrm{KA} 9002 \mathrm{~L} 606006$ NDNPA 1XLT GRS $09030220900309900326 \mathrm{KA9002L606} 006$ CL6ET 1XLT GRS $09030220900309900326 \mathrm{WA9} 002 \mathrm{~L} 606006 \mathrm{NB}$ 1XLT GRS $09030220900309900326 \mathrm{WA} 9002 \mathrm{~L} 606006$ ISOPHRIXLT GRS $09030220900309900326 \mathrm{WA} 9002 \mathrm{~L} 606006$ 2NP 1XLT GRS $0903.0220900309900326 \mathrm{WA} 9002 \mathrm{~L} 606006$ 24DMPN1XLT GRS $09030220900309900326 \mathrm{WA} 9002 \mathrm{~L} 606006$ BENZOA1XLT GRS $09030220900309900326 \mathrm{WA} 9002 \mathrm{~L} 606006$ B2CEXM1XLT GRS $09030220900309900326 \mathrm{WA} 9002 \mathrm{~L} 606006$ 24DCLP 1XLT GRS $09030220900309900326 \mathrm{WA} 9002 \mathrm{~L} 606006 \quad 124 \mathrm{TCB} 1 \mathrm{XLT}$ NGRS $09030220900309900326 \mathrm{WA} 90022606006 \mathrm{NAP}$ 1XLT OGRS $09030220900309900326 \mathrm{WA} 9002 \mathrm{~L} 606006$ 4CANILIXLT GRS $09030220900309900326 \mathrm{WA} 9002 \mathrm{~L} 606006 \mathrm{HCBD}$ 1XLT GRS $0903.0220900309900326 \mathrm{WA} 9002 \mathrm{~L} 606006$ 4CL3C 1XLT GRS $09030220900309900326 \mathrm{WA} 9002 \mathrm{~L} 606006$ 2MENAP 1XLT GRS $09030220900309900326 \mathrm{WA9002L606} 006$ CL6CP 1XLT GRS $09030220900309900326 \mathrm{KA9002L606} 006$ 246TCP 1XLT GRS $09030220900309900326 \mathrm{WA} 9002 \mathrm{~L} 606006245 \mathrm{TCP} 1 \mathrm{XLT}$ GRS $09030220900309900326 \mathrm{WA} 9002 \mathrm{~L} 606006$ 2CNAP 1XLT GRS $09030220900309900326 \mathrm{WA} 9002 \mathrm{~L} 606006$ 2NANILIXLT GRS 09030220900309900326 WA9002L606 006 DMP 1XLT GRS $09030220900309900326 \mathrm{WA} 9002 \mathrm{~L} 606006$ ANAPYLIXLT GRS $09030220900309900326 \mathrm{WA} 9002 \mathrm{~L} 606006$ 26DNT 1XLT GRS $09030220900309900326 \mathrm{WA} 9002 L 606006$ 3NANILIXLT GRS $09030220900309900326 \mathrm{WA} 9002 \mathrm{~L} 606006$ ANAPNE1XLT GRS $09030220900309900326 \mathrm{WA} 9002 \mathrm{~L} 606006$ 24DNP 1XLT GRS $09030220900309900326 \mathrm{WA} 9002 \mathrm{~L} 606006$ 4NP 1XLT GRS $09030220900309900326 \mathrm{FA9002L606} 006$ DIBLFUIXLT GRS $09030220900309900326 \mathrm{WA} 9002 \mathrm{~L} 606006$ 24DNT 1XLT GRS $09030220900309900326 \mathrm{WA} 9002 \mathrm{~L} 606006$ DEP 1XLT GRS $09030220900309900326 \mathrm{WA} 9002 \mathrm{~L} 606006$ 4CLPPE1XLT GRS $09030220900309900326 \mathrm{HA9002L606} 006$ FLRENE1XIT GRS $09030220900309900326 \mathrm{FA} 9002 L 606006$ 4NANILIXLT GRS $09030220900309900326 \mathrm{HA9002L606} 006$ 46DN2C1XLT
1.0000MSDAR $1.0000 \mathrm{MSDAR}$ 1.0000MSDAR $1.0000 M S D A R$ 1.0000MSDAR 1.000OMSDAR $1.0000 \mathrm{MSDAR}$ $1.0000 \mathrm{MSDAR}$ $1.0000 M S D A R$ 1.0000MSDAR $1.0000 \mathrm{MSDAR}$ 1.0000MSDAR 1.0000MSDAR 1. $0000 \mathrm{MSDAR}$ 1.0000MSDAR 1.0000MSDAR $1.0000 \mathrm{MSDAR}$ 1. $0000 \mathrm{MSDAR}$ 1.0000MSDAR 1. O00OMSDAR 1.0000MSDAR 1.0000MSDAR 1.0000MSDAR 1.0000MSDAR 1.0000MSDAR 1.0000MSDAR 1.0000MSDAR $1.0000 M S D A R$ 1.0000MSDAR 1.0000MSDAR 1. $0000 \mathrm{MSDAR}$ 1.0000MSDAR 1.0000MSDAR 1. O00OMSDAR $1.0000 \mathrm{MSDAR}$ 1. O000MSDAR 1.0000MSDAR 1.0000MSDAR 1. O00OMSDAR $1.0000 \mathrm{MSDAR}$ $1.0000 \mathrm{MSDAR}$ 1. $0000 \mathrm{MSDAR}$ 1.0000MSDAR 1.0000MSDAR $1.0000 \mathrm{MSDAR}$ 1.0000MSDAR 1.0000MSDAR $1.0000 \mathrm{MSDAR}$
1950. 451P-TERP HENYL-D14

3900.902PHENOL-D5

$0625 \mathrm{HN}$

3900 . 9022-FLUOROPHENOL $0625 \mathrm{HN}$ $0625 \mathrm{HN}$

$3900.9022,4,6$-TRIBROMOPHENOLO625HN OPHENOL O625HN OBIS (2-CHLOROETHYL) ETO625HN 02-CHLOROPHENOL O625HN 01,3-DICHLOROBÉNZENE O625HN $01,4-D I C H L O R O B E N Z E N E$ O625HN OBENZYL ALCOHOL O625HN 01,2-DICHLOROBENZENE $0625 \mathrm{HN}$ 02-METHYLPHENOL O625HN 0BIS (2-CHLOROISOPROPYO 625HN O4-METHYLPHENOL $\quad 0625 \mathrm{HN}$ ON-NITROSO-DI-N-PROPYO $625 \mathrm{HN}$ OHEXACHLOROETHANE O625HN ONITROBENZENE $0625 \mathrm{HN}$ OISOPHORONE O625HN 02-NITROPHENOL O625HN 02, 4-DIMETHYLPHENOL O625HN OBENZOIC ACID O625HN OBIS (2-CHLOROETHOXY) MO625HN 02, 4-DICHIOROPHENOL O625HN $01,2,4-T R I C H L O R O B E N Z E O 625 \mathrm{HN}$ ONAPHTHALENE O625HN 04-CHLOROANILINE O625HN OHEXACHLOROBUTADIENE 0625HN 04-CHLORO-3-METHYLPHEO $25 \mathrm{HN}$ 02-METHYLNAPHTHALENE 0625HN OHEXACHLOROCYCLOPENTAO $625 \mathrm{HN}$ $02,4,6$-TRICHLOROP HENOO $625 \mathrm{HN}$ $02,4,5-T R I C H L O R O P H E N O 0625 \mathrm{HN}$ 02-CHLORONAPHTHALENE O625HN 02-NITROANILINE $0625 \mathrm{HN}$ ODIMETHYLPHTHALATE $0625 \mathrm{HN}$ OACENAPHTHYLENE O625HN 02,6-DINITROTOLUENE 0625HN 03-NITROANILINE 0625HN OACENAPHTHENE O625HN 02,4 -DINITROPHENOL $0625 \mathrm{HN}$ 04-NITROPHENOL O625HN ODIBENZOEURAN $0625 \mathrm{HN}$ $02,4-D I N I T R O T O L U E N E$ 0625HN ODIETHYLR HTHALATE . 0625HN 04-CHLOROPHENYL-PHENYO625HN OFLUORENE $0625 \mathrm{HN}$ 04-NITROANILINE 0625HN 04, 6-DINITRO-2-METHYLO625HN 


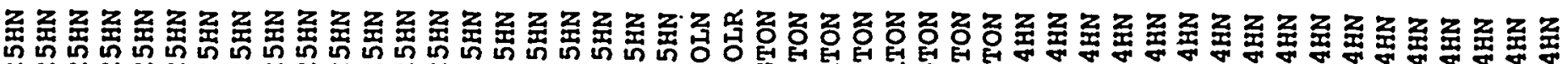
员

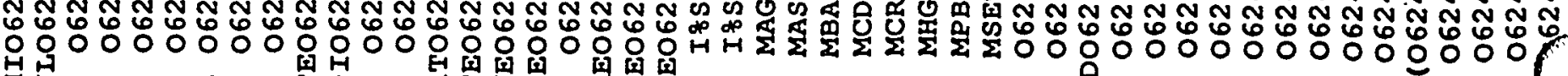
党

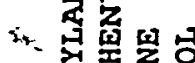

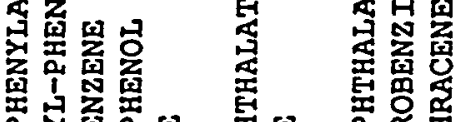

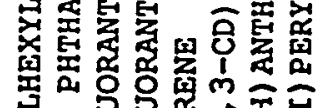

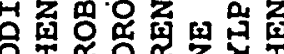

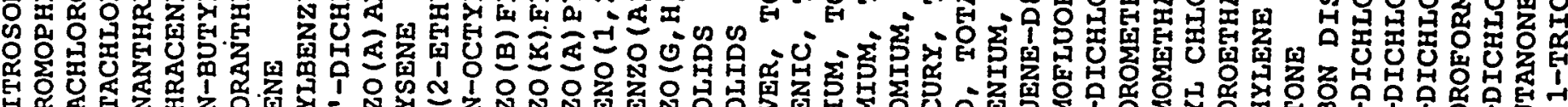

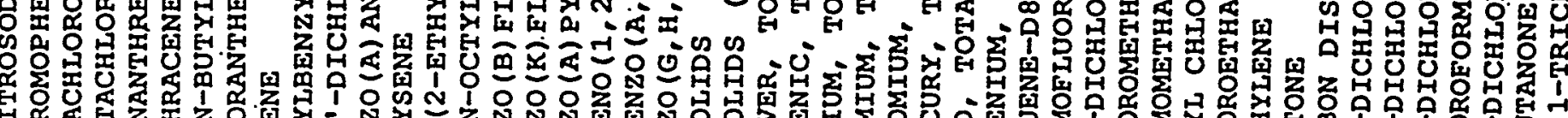

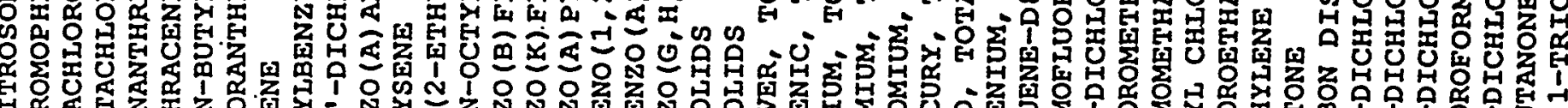
回回

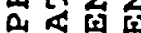
م

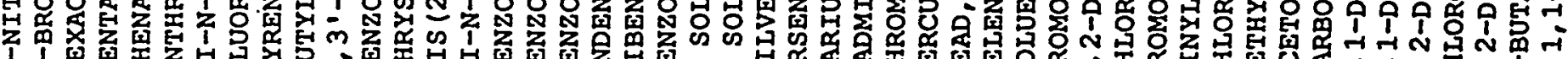

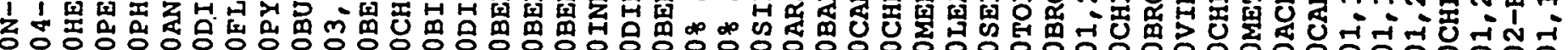

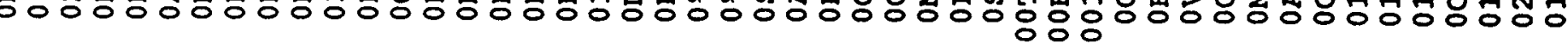
in in in

in

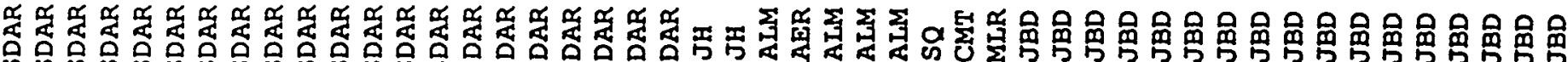

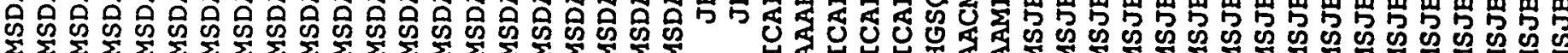

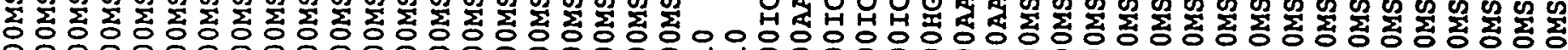

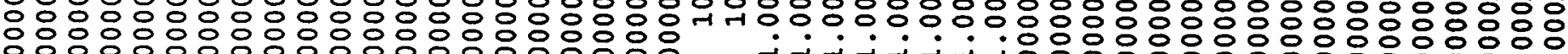

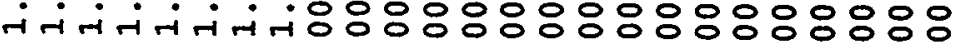

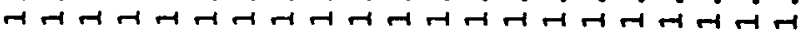

न

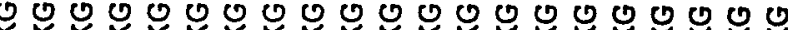

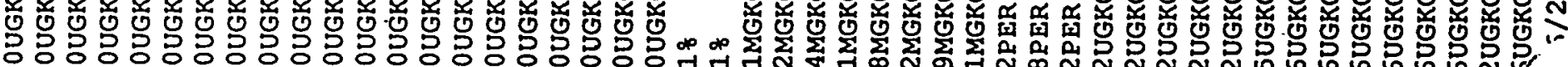

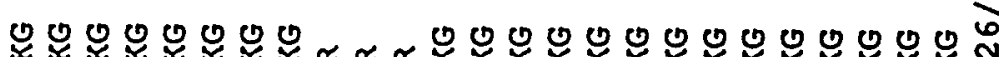

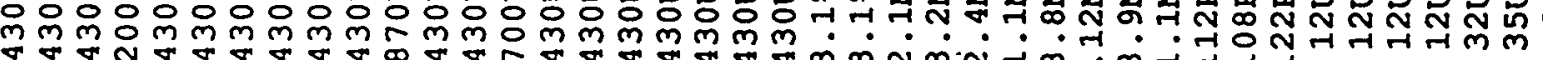

$N$

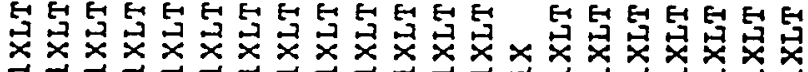

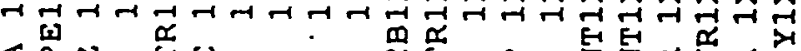

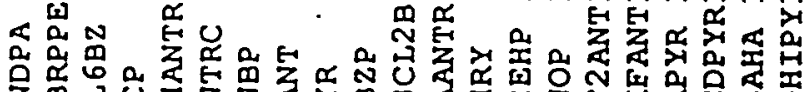

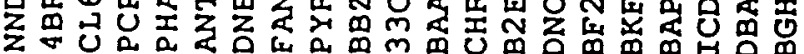
(0)

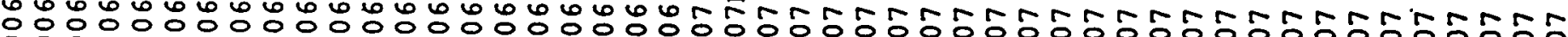

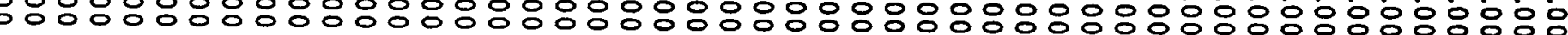
ம

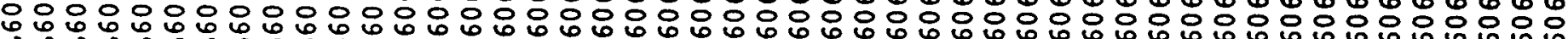

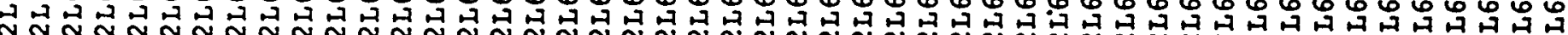
$000000000000000 \%$

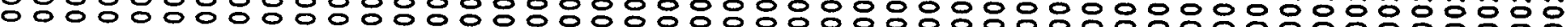
의의 응의 앵의

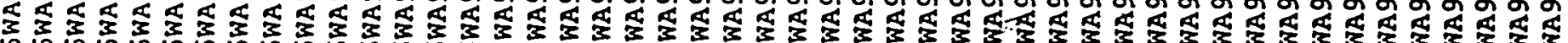

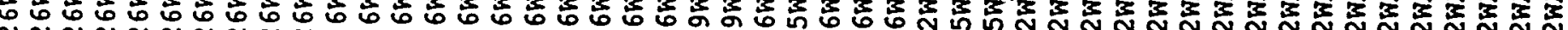

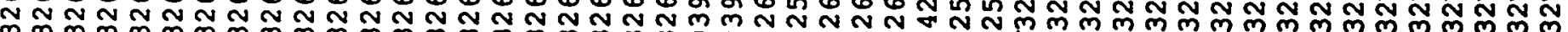

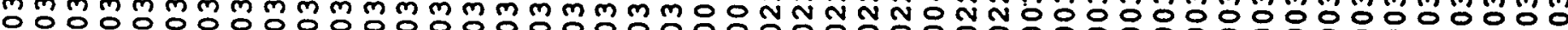

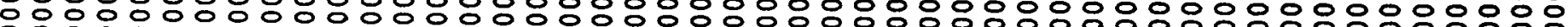
a h 作

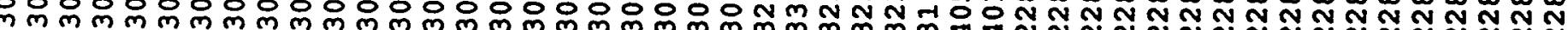

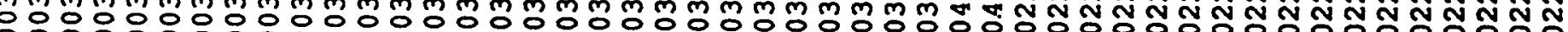

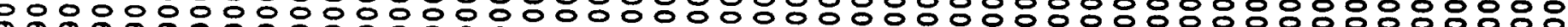

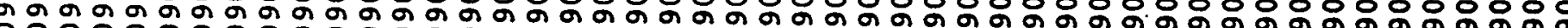

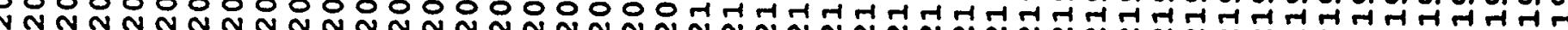

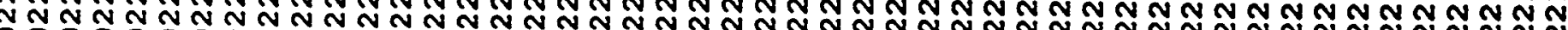

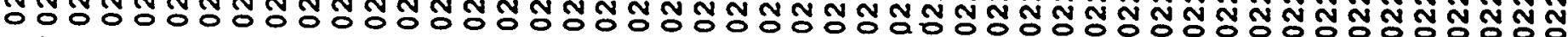

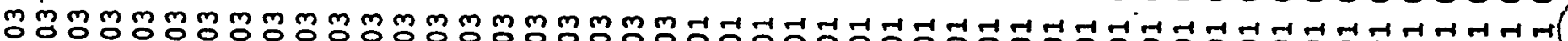

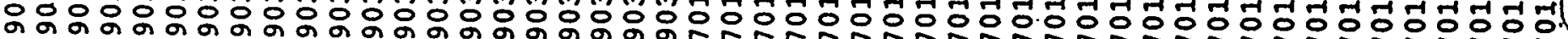

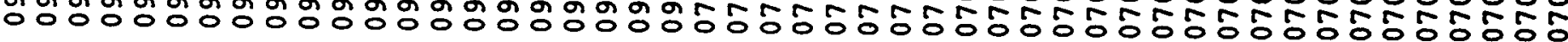


GRS 07 0221900228900032WA9002L606 007 CCL4 2JLT GRS $07010221900228900032 \% A 9002 L 606007$ VINYLA2JLT GRS $07010221900228900032 \mathrm{KA} 90021606007$ BRDCLM2JLT GRS $07010221900228900032 \mathrm{WA} 9002 \mathrm{~L} 606007$ 12DCLP2JLT GRS $07010221900228900032 \mathrm{WA} 9002 \mathrm{~L} 606007$ 13DCPC2JLT GRS 07010221900228900032 KA9002L606 007 TRCLE 2JLT GRS $07010221900228900032 \mathrm{WA9002L606} 007$ DBRCLM2JLT GRS 07010221900228900032 WA9002L606 007 112TCE2JLT GRS $07010221900228900032 \mathrm{FA9002L606} 007$ C6H6 2JLT GRS 07010221900228900032 FA9002L606 007 T13DCP2JLT GRS 07010221900228900032 WA9002L606 007 CHBR3 2JLT GRS 07010221900228900032 WA9002L606 007 4ME2PE2JLT GRS $07010221900228900032 \mathrm{WA} 9002 \mathrm{~L} 606007$ 2HXONE2JLT GRS 07010221900228900032 WA9002L606 007 TCLEE 2JLT GRS $07010221900228900032 \% A 90021606007$ TCLEA 2JLT GRS 07010221900228900032 WA9002L606 007 MEC6H52JLT GRS 07010221900228900032 WA9002L606 007 CLC6H52JLT GRS $07010221900228900032 \mathrm{FA} 00021606007$ ETE $6 \mathrm{H} 52 \mathrm{JLT}$ GRS 07010221900228900032 WA9002L606 007 STYR 2JLT GRS 07010221900228900032 WA9002L606 007 XYLEN 2JLT GRS $07010221900309900326 \mathrm{WA} 9002 \mathrm{~L} 606007$ NBD5 $1 \mathrm{X}$ GRS 07010221900309900326 WA9002L606 007 2FBP 1X GRS $07010221900309900326 \mathrm{WA} 9002 \mathrm{~L} 606007$ PTERP 1X 07010221900309900326 WA9002L606 007 PHEND51X $07010221900309900326 \mathrm{WA} 0002 \mathrm{~L} 606007$ 2EP $1 \mathrm{X}$ $07010221900309900326 \mathrm{WA} 0002 \mathrm{~L} 606007$ 246TBP 1X $07010221900309900326 \mathrm{WA} 9002 \mathrm{~L} 606007$ PHENOL1XLT $07010221900309900326 \mathrm{WA} 002 \mathrm{~L} 606007$ B2CEE 1XLT $07010221900309900326 \mathrm{WA} 002 \mathrm{~L} 606007$ 2CLP 1XLT $07010221900309900326 \mathrm{WA} 9002 \mathrm{~L} 606007$ 13DCLB1XLT 07010221900309900326 KA9002L606 007 1ADCLB1XLT 07010221900309900326 WA9002L606 007 BZALC 1XLT $07010221900309900326 \mathrm{WA} 9002 \mathrm{~L} 606007$ 12DCLB1XLT $07010221900309900326 \mathrm{WA} 9002 L 606007$ 2MP 1XLT $07010221900309900326 \mathrm{WA} 90024606007$ B2CIPE1XLT $07010221900309900326 \mathrm{WA} 002 \mathrm{~L} 606007$ 4MP 1XLT $07010221900309900326 \mathrm{WA} 002 \mathrm{~L} 606007$ NDNPA 1XLT 07010221900309900326 WA9002L606 007 CL6ET 1XLT $07010221900309900326 \mathrm{WA} 90022606007 \mathrm{NB}$ 1XLT $07010221900309900326 \mathrm{WA} 9002 \mathrm{~L} 606007$ ISOPHR1XLT $\begin{array}{llllll}07010221900309900326 \mathrm{WA} 9002 \mathrm{~L} 606 & 007 & \text { 2NP 1XLT }\end{array}$ $07010221900309900326 \mathrm{KA} 9002 \mathrm{~L} 606007$ 24DMPN1XLT 07010221900309900326 WA9002L606 007 BENZOA1XLT $07010221900309900326 \mathrm{WA} 90021606007$ B2CEXM1XLT $07010221900309900326 \mathrm{FA} 90022606007$ 24DCLP1XLT $07010221900309900326 \mathrm{FA9002L606} 007$ 124TCB1XLT $07010221900309900326 \mathrm{KA} 9002 \mathrm{~L} 606007$ NAP - 1XLT 07010221900309900326 FA9002L606 007 4CANIL1XLT
OUGKG

12UGKG

6UGKG

6UGKG

6UGKG

6UGKG

6UGKG

6UGKG

6UGKG

6UGKG

6UGKG

12UGKG

12UGKG

6UGKG

6UGKG

6UGKG

6UGKG

6UGKG

6UGKG

6UGKG

60PER

68PER

76PER

69PER

73PER

64PER

440 UGKG

440 UGKG

4 4 OUGKG

44 OUGKG

440 UGKG

44 OUGKG

44 OUGKG

44 OUGKG

44 OUGKG

44 OUGKG

440 UGKG

440 UGKG

44 OUGKG

44 OUGKG

440 UGKG

44 OUGKG

2200UGKG

44 OUGKG

44OUGKG

44 OUGKG

440 UGKG

44 OUGKG
1.0000MSJBD

$1.0000 \mathrm{MSJBD}$

$1.0000 \mathrm{MSJBD}$

$1.0000 \mathrm{MSJBD}$

$1.0000 \mathrm{MSJBD}$

$1.0000 \mathrm{MSJBD}$

$1.0000 \mathrm{MSJBD}$

$1.0000 \mathrm{MSJBD}$

$1.0000 \mathrm{MSJBD}$

$1.0000 \mathrm{MSJBD}$

$1.0000 \mathrm{MSJBD}$

$1.0000 \mathrm{MSJBD}$

$1.0000 \mathrm{MSJBD}$

$1.0000 \mathrm{MSJBD}$

$1.0000 \mathrm{MSJBD}$

$1.0000 \mathrm{MSJBD}$

$1.0000 \mathrm{MSJBD}$

$1.0000 \mathrm{MSJBD}$

$1.0000 \mathrm{MSJBD}$

$1.0000 \mathrm{MSJBD}$

$1.0000 \mathrm{MSDAR}$

1.0000MSDAR

1.0000MSDAR

1. $0000 \mathrm{MSDAR}$

$1.0000 M S D A R$

1.0000MSDAR

1.0000MSDAR

$1.0000 M S D A R$

1.0000MSDAR

1.0000MSDAR

1.0000MSDAR

1.0000MSDAR

1.0000MSDAR

1.0000MSDAR

1.0000MSDAR

1.0000MSDAR

1.0000MSDAR

1.0000MSDAR

$1.0000 \mathrm{MSDAR}$

1.0000MSDAR

1.0000MSDAR

$1.0000 M S D A R$

1.0000MSDAR

1.0000MSDAR

$1.0000 \mathrm{MSDAR}$

1.0000MSDAR

1.0000MSDAR

$1.0000 \mathrm{MSDAR}$
OCARBON TETRACHLOR̈IDEO624HN OVINYL ACETATE O624HN OBROMODICHLOROMETHANEO624HN 01,2-DICHLOROPROPANE O624HN 0CIS-1, 3-DICHLOROPROPO624HN OTRICHLOROETHENE 0624HN ODIBROMOCHLOROMETHANEO624HN 01, 1, 2-TRICHLOROETHANO624HN OBENZENE $0624 \mathrm{HN}$ OTRANS-1, 3-DICHLOROPRO624HN OBROMOFORM O624HN

04-METHYL-2-PENTANONEO624HN 02-HEXANONE $0624 \mathrm{HN}$ OTETRACHLOROETHENE 0624HN $01,1,2,2$-TETRACHLOROEO $624 \mathrm{HN}$ OTOLUENE

$0624 \mathrm{HN}$

OCHLOROBENZENE OETHYLBENZENE O624HN OSTYRENE

OXYLENE (TOTAL)

1972 . 974NITROBENZENE-D5

1972. 9742-FLUOROBIPHENYL

$0624 \mathrm{HN}$

$0624 \mathrm{HN}$

$0625 \mathrm{HN}$

$0625 \mathrm{HN}$

$0625 \mathrm{HN}$

3945.947PHENOL-D5 $0625 \mathrm{HN}$

3945.9472-FLUOROPHENOL O625HN

$3945.9472,4,6$-TRIBROMOPHENOLO625HN OPHENOL $0625 \mathrm{HN}$ OBIS (2-CHLOROETHYL) ETO625HN 02-CHLOROPHENOL O625HN 01,3-DICHLOROBENZENE 0625HN 01,4-DICHLOROBENZENE O625HN OBENZYL ALCOHOL O625HN 01,2-DICHLOROBENZENE O625HN 02-METHYLPHENOL O625HN OBIS (2-CHLOROISOR ROPYO625HN O4-METHYLPHENOL O625HN ON-NITROSO-DI-N-PROPYO 625HN OHEXACHLOROETHANE O625HN ONITROBENZENE OISOPHORONE

02-NITROPHENOL $0625 \mathrm{HN}$ O625HN OBENZOIC ACID (2-CHLOROETHOXY) MO625HN 02,4-DICHLOROPHENOI 0625HN 01, 2, 4-TRICHLOROBENZE0625HN ONAPHTHALENE 04-CHLOROANILINE 
GRS 07010221900309900326 WA9002L606 007 HCBD 1XLT GRS $07010221900309900326 \mathrm{WA} 9002 \mathrm{~L} 606007$ 4CL3C 1XLT GRS $07010221900309900326 \mathrm{WA} 9002 \mathrm{~L} 606007$ 2MENAP1XLT GRS $07010221900309900326 \mathrm{WA} 90022606007$ CL6CP 1XLT GRS $07010221900309900326 \mathrm{WA} 9002 \mathrm{~L} 606007 \quad 246 \mathrm{TCP} 1 \mathrm{XLT}$ GRS $07010221900309900326 \mathrm{WA} 9002 \mathrm{~L} 606007$ 245TCP1XLT GRS 07010221900309900326 WA9002L606 007 2CNAP 1XLT

$07010221900309900326 \mathrm{WA} 9002 \mathrm{~L} 606007$ 2NANIL1XLT 07010221900309900326 WA9002L606 007 DMP ' IXLT 07010221900309900326 WA9002L606 007 ANAPYL1XLT $07010221900309900326 \mathrm{WA} 9002 \mathrm{~L} 606007$ 26DNT IXLT 07010221900309900326 WA9002L606 007 3NANIL1XLT $07010221900309900326 \mathrm{WA} 9002 \mathrm{~L} 606007$ ANAPNE1XLT $07010221900309900326 \mathrm{WA} 9002 \mathrm{~L} 606007$ 24DNP 1XLT 07010221900309900326 WA9002L606 007 4NP IXLT 07010221900309900326 WA9002L606 007 DIBZEU1XLT $07010221900309900326 \mathrm{WA9002L606} 007$ 24DNT 1XLT 07010221900309900326 WA9002L606 007 DEP 1XLT 07010221900309900326 WA9002L606 007 4CLPPE1XLT 07010221900309900326 WA9002L606 007 FLRENE1XLT 07010221900309900326 WA9002L606 007 4NANIL1XLT 07010221900309900326 WA9002L606 007 46DN2C1XLT $07010221900309900326 \mathrm{WA} 9002 \mathrm{~L} 606007$ NNDPA 1XLT 07010221900309900326 WA9002L606 007 4BRPPE1XLT 07010221900309900326 WA9002L606 007 CL6BZ 1XLT 07010221900309900326 WA9002L606 007 PCP 1XLT 07010221900309900326 WA9002L606 007 PHANTR1XLT 07010221900309900326 WA9002L606 007 ANTRC 1XLT 07010221900309900326 WA9002L606 007 DNBP 1XLT 07010221900309900326 WA9002L606 007 FANT 1XLT 07010221900309900326 FA9002L606 007 PYR 1XLT $07010221900309900326 \mathrm{WA} 9002 \mathrm{~L} 606007$ BBZP 1XLT 07010221900309900326 WA9002L606 007 33CL2B1XLT $07010221900309900326 \mathrm{WA9} 002 \mathrm{~L} 606007$ BAANTRIXLT 070.10221900309900326 WA9002L606 007 CHRY IXLT $07010221900309900326 \mathrm{WA} 9002 L 606007$ B2EHP $1 X$ 07010221900309900326 WA9002L606 007 DNOP 1XLT 07010221900309900326 WA9002L606 007 BE2ANT1XLT 07010221900309900326 WA9002L606 007 BKFANT1XLT 07010221900309900326 WA9002L606 007 BAPYR 1XLT 07010221900309900326 WA9002L606 007 ICDPYRIXLT 07010221900309900326 WA9002L606 007. DBAHA 1XLT 07010221900309900326 WA9002L606 007 BGHIPY1XIT $0701 A 0221900308900039$ WA9002L606 008 . 0701A0221900323900226WA9002L606 008 AG $0701 A 0221900330900225$ FA9002L606.008 AS $0701 A 0221900323900226$ พA9002L606 008 BA $0701 \wedge 0221900323900226$ WA9002L606 $008 \mathrm{CD}$
440UGKG 44 OUGKG 440 UGKG 440 UUGKG 44 OUGKG 2200 UGKG 44 OUGKG 2200 UGKG 44 OUGKG 44 OUGKG $440 U G K G$ 2200 UUKG 44 OUGKG 2200 UGKG 2200 OUGKG 44 OUGKG 44 OUGKG 44 OUGKG 440 UGKG 44 OUGKG 2200 UGKG 2200 UGKG 44 OUGKG 440 UGKG 44 OUGKG 2200 UUKG 44 OUGKG 44 OUGKG 440 UGKG 44 OUGKG 44 OUGKG 44 OUGKG 880 UGKG 44 OUGKG 44 OUGKG

1400 UGKG 44 OUGKG 44 OUGKG 44 OUGKG 44 OUGKG 440 UJGKG 44 OUGKG 44 OUGKG $83.5 \%$

2. OMGKG 2ZLT $1 \mathrm{~B}$ 2ZLT 2ZLT $0.98 \mathrm{MGKG}$
1.0000MSDAR 1.0000MSDAR $1.0000 M S D A R$ 1. 000OMSDAR 1. $0000 \mathrm{MSDAR}$ 1. 0000MSDAR 1.0000MSDAR 1.000OMSDAR 1.0000MSDAR 1.0000MSDAR 1. 0000MSDAR 1.0000MSDAR 1. $0000 M S D A R$ 1. 0000MSDAR 1.0000MSDAR 1.000OMSDAR 1.0000MSDAR 1.0000MSDAR 1.0000MSDAR 1.0000MSDAR 1.0000MSDAR 1.0000MSDAR 1.0000MSDAR 1.0000MSDAR 1. 0000MSDAR 1.0000MSDAR 1. 0000MSDAR 1.0000MSDAR 1:0000MSDAR 1. 0000MSDAR 1.0000MSDAR 1.0000MSDAR 1.0000MSDAR 1.0000MSDAR 1.0000MSDAR 1. 0000MSDAR 1.0000MSDAR 1. 0000MSDAR 1.0000MSDAR 1.0000MSDAR 1. O0OOMSDAR 1.0000MSDAR 1. 0000MSDAR $10 \mathrm{JH}$

1. OOICAIM 1. 00AAAER 1. OOICAIM 1. OOICALM
OHEXACHLOROBUTADIENE $0625 \mathrm{HN}$ 04-CHLORO-3-METHYLPHEO625HN 02-METHYLNAPHTHALENE 0625HN OHEXACHLOROCYCLOPENTAO625HN $02,4,6$-TRICHLOROPHENOO625HN $02,4,5-T R E C H L O R O P H E N O 0625 \mathrm{HN}$ 02-CHLORONAPHTHALENE 0625HN 02-NITROANILINE 0625HN ODIMETHYLPHTHALATE $0625 \mathrm{HN}$ OACENAPHTHYLENE O625HN 02,6-DINITROTOLUENE 0625HN 03-NITROANILINE 0625HN OACENAPHTHENE O625HN 02,4 -DINITROPHENOL O625HN 04-NITROPHENOL O625HN ODIBENZOFURAN $0625 \mathrm{HN}$ 02, 4-DINITROTOLUENE O625HN ODIETHYLPHTHALATE O625HN 04-CHLOROP HENYL-PHENYO $25 \mathrm{HN}$ OFLUORENE ' O625HN 04-NITROANILINE 0625HN 04,6-DINITRO-2-METHYL0625HN ON-NITROSODIPHENYLAMIO625HN 04-BROMOPHENYL-PHENYLO625HN OHEXACHLOROBENZENE O625HN OPENTACHLOROPHENOL O625HN ORHENANTHRENE O625HN OANTHRACENE O625HN ODI-N-BUTYLPHTHALATE 0625HN OFIUORANTHENE O625HN OPYRENE O625HN OBUTYLBENZYLPHTHALATEO 625HN $03,3^{\circ}$-DICHLOROBENZIDIO $625 \mathrm{HN}$ OBENZO (A) ANTHRACENE O625HN .OCHRYSENE 0625HN OBIS (2-ETHYLHEXYL) PHTO625HN ODI-N-OCTYL PHTHALATEO625HN OBENZO (B) ELUORANTHENEO625HN OBENZO (K) ELUORANTHENEO625HN OBENZO (A) PYRENE O625HN OINDENO $(1,2,3-C D)$ PYREO625HN ODIBENZO (A, H) ANTHRACEO625HN OBENZO (G, H, I) PERYLENEO 625HN $0 \%$ SOIIDS "I\&SOLN OSIIVER, TOTAL MAGTON OARSENIC, TOTAL MASTON OBARIUM, TOTAL MBATON OCADMIUM, TOTAL 
(22 0701A0221900319900042WA9002L606 008 HGTOT IDLT $0701 A 0221900405900225$ WA9002L606 008 PB $1 \mathrm{~B}$ 0701A0221900401900225WA9002L606 008 SE 1BLT 0701A0221900301900032WA9002L606 008 MEC6D82J 0701 A0221900301900032WA9002L606 O0B PBFB 2J $0701 A 022.1900301900032$ WA9002L606 008 12DCD42J 0701A0221900301900032WA9002L606 008 CH3CL 2JLT 0701 A0221900301900032WA9002L606 008 CH3BR. 2JLT $0701 A 0221900301900032$ HA9002L606 008 C2H3CL2JLT 0701A0221900301900032WA9002L606 008 C2H5CL2JLT $0701 A 0221900301900032$ WA9002L606 008 CH2CL22JB 0701 A0221900301900032WA9002L606 008 ACET 2JB 0701A0221900301900032WA9002L606 008 CS2 2JLT 0701A0221900301900032WA9002L606 008 11DCE 2JLT 0701A0221900301900032WA9002L606 008 11DCLE2JLT $0701 A 0221900301900032$ WA9002L606 008 T12DCE2JLT 0701A0221900301900032WA9002L606 00B CHCL3 2JLT 0701A0221900301900032WA9002L606 008 12DCLE2JLT $0701 A 0221900301900032 W A 9002 L 606008$ MEK 2JLT 0701A0221900301900032WA9002L606 008 111TCE2JLT 0701A0221900301900032WA9002L606 008 CCL4 2JLT 0701A0221900301900032WA9002L606 008 VINYLA2JLT $0701 A 0221900301900032$ WA9002L606 008 BRDCLM2JLT $0701 A 0221900301900032 W A 9002 L 606008$ 12DCLP2JLT 0701A0221900301900032WA9002L606 008 13DCPC2JLT 0701A0221900301900032WA9002L606 008 TRCLE 2JLT $0701 A 0221900301900032$ WA9002L606 O0B DBRCLM2JLT $0701 A 0221900301900032$ WA9002L606 008 112TCE2JLT 0701A0221900301900032WA9002L606 00B C6H6 2JLT 0701A0221900301900032WA9002L606 008 T13DCP2JLT 0701A0221900301900032WA9002L606 008 CHBR3 2JLT 0701A0221900301900032WA9002L606 008 AME2PE2JLT 0701A0221900301900032WA9002L606 008 2HXONE2JLT 0701A0221900301900032WA9002L606 008 TCLEE 2JLT 0701A0221900301900032WA9002L606 008 TCLEA 2JLT $0701 A 0221900301900032$ WA9002L606 008 MEC6H52JLT 0701A0221900301900032WA9002L606 008 CLC6H52JLT 0701A0221900301900032WA9002L606 008 ETC6H52JLT 0701A0221900301900032WA9002L606 008 STYR 2JLT 0701A0221900301900032WA9002L606 008 XYLEN 2JLT $0701 A 0221900313900326$ WA9002L606 008 NBD5 IX $0701 A 0221900313900326 \mathrm{FA9002L606} 008$ 2FBP $1 \mathrm{X}$ 0701A0221900313900326WA9002L606 008 PTERP $1 \mathrm{X}$ 0701A0221900313900326HA9002L606 008 PHEND51X $0701 A 0221900313900326 \mathrm{WA} 9002 L 606008$ 2FP $1 X$ $0701 A 0221900313900326$ WA9002L606 008 246TBP $1 X$ $0701 A 0221900313900326$ FA9002L606 008 PHENOL1XLT
29. UMGKG

$0.12 \mathrm{MGKG}$

8. IMGKG

1. 1MGKG

112PER

106PER

112PER

12 UGKG

12 UGKG

12 UGKG

12 UGKG

24 UGKG

29 UGKG

6UGKG

6UGKG

6UGKG

6UGKG

6UGKG

6UGKG

12 UGKG

6UGKG

6UGKG

12 UGKG

6UGKG

6UGKG

6UGKG

6UGKG

6UGKG

6UGKG

6UGKG

6UGKG

6UGKG

12UGKG

12UGKG

6UGKG

6UGKG

6UGKG

6UGKG

6UGKG

6UGKG

6UGKG

6OPER

69PER

61PER

66PER

46PER

47PER

430 UGKG
1.00ICALM 1.00HGSQ

1.00AACMT

1. 00AAMLR

1. $0000 \mathrm{MSJBD}$

1. $0000 \mathrm{MSJBD}$

1. $0000 \mathrm{MSJBD}$

1. $0000 \mathrm{MSJBD}$

1. $0000 \mathrm{MSJBD}$

$1.0000 \mathrm{MSJBD}$

$1.0000 \mathrm{MSJBD}$

$1.0000 M S J B D$

1. $0000 \mathrm{MSJBD}$

1. $0000 \mathrm{MSJBD}$

1.0000MSJBD

1.0000MSJBD

1. $0000 \mathrm{MSJBD}$

1. 0000MSJBD

1. $0000 \mathrm{MSJBD}$

1. $0000 \mathrm{MSJBD}$

1. 0000MSJBD

1.0000MSJBD

1. $0000 \mathrm{MSJBD}$

1.0000MSJBD

1. $0000 \mathrm{MSJBD}$

1. $0000 \mathrm{MSJBD}$

$1.0000 \mathrm{MSJBD}$

1. $0000 \mathrm{MSJBD}$

1. $0000 \mathrm{MSJBD}$

1. 0000MSJBD

1. $0000 \mathrm{MSJBD}$

1. $0000 \mathrm{MSJBD}$

$1.0000 \mathrm{MSJBD}$

1. $0000 \mathrm{MSJBD}$

1. $0000 \mathrm{MSJBD}$

1.0000MSJBD

$1.0000 \mathrm{MSJBD}$

1. $0000 \mathrm{MSJBD}$

1. $0000 \mathrm{MSJBD}$

1. $0000 \mathrm{MSJBD}$

1. 0000MSJBD

1. 0000MSDAR

1. 0000MSDAR

1. 0000MSDAR

1. 0000MSDAR

1.0000MSDAR

1. 0000MSDAR 1. 000OMSDAR
OCHROMIUM, TOTAL OMERCURY, TOTAL

OLEAD, TOTAI

OSELENIUM, TOTAI

59.500TOLUENE-D8

MCRTON MHGTON MPBTON MSETON $0624 \mathrm{HN}$ 59.50OBROMOELUOROBENZENE O624HN

59:5001, 2-DICHIOROETHANE-DO624HN OCHLOROMETHANE O624HN OBROMOMETHANE O624HN OVINYI CHIORIDE 0624HN OCHLOROETHANE O624HN OMETHYLENE CHLORIDE O624HN OACETONE O624HN OCARBON DISULEIDE O624KN 01,1-DICHIOROETHENE 0624HN 01,1 -DICHI,OROETHANE 0624HN 01,2-DICHIOROETHENE (0624HN OCHLOROFORM 0624HN 01,2-DICHIOROETHANE 0624HN 02-BUTANONE O624HN 01, 1, 1-TRICHLOROETHANO 624HN OCARBON TETRACHLORIDEO624HN OVINYL ACETATE O624HN OBROMODICHLOROMETHANEO624HN 01,2-DICHLOROPROPANE 0624HN OCIS-1, 3-DICHLOROPROPO624HN OTRICHLOROETHENE 0624HN ODIBROMOCHLOROMETHANEO $624 H N$ 01, 1, 2-TRICHLOROETHANO $244 \mathrm{HN}$ OBENZENE OTRANS-1, 3-DICHLOROPRO $624 \mathrm{KN}$ OBROMOFORM O624HN 04-METHYL-2-PENTANONEO624HN 02-HEXANONE $0624 \mathrm{HN}$ OTETRACHIOROETHENE O624HN $01,1,2,2-T E T R A C H L O R O E O 624 \mathrm{HN}$ OTOLUENE OETHYLBENZENE O624HN OSTYRENE 0624HN OXYLENE (TOTAL) 0624HN 1927.929NITROBENZENE-D5 O625HN 1927.9292-ELUOROBIPHENYL O625HN 1927.929P-TERPHENYT-D14: 062.5HN 3855.857RHENOL-D5 O625HN 3855.8572-ELUOROPHENOL 0625HN 3855 .8572, 4, 6-TRIBROMORHENOLO 625HN ORHENOL 


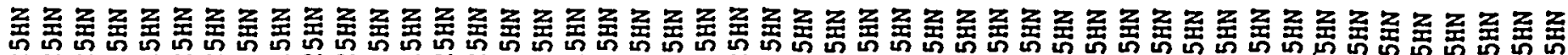

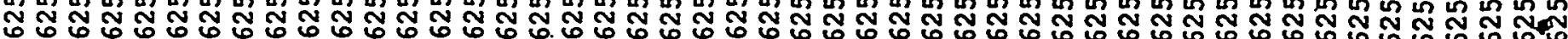

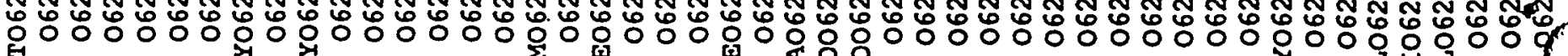

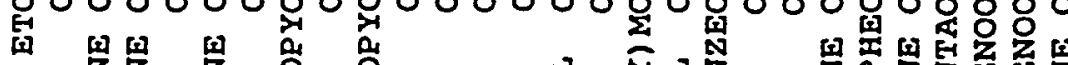

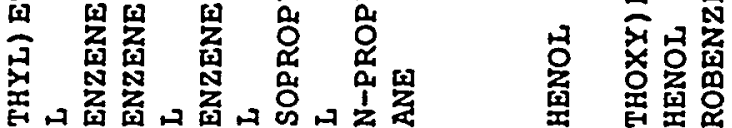

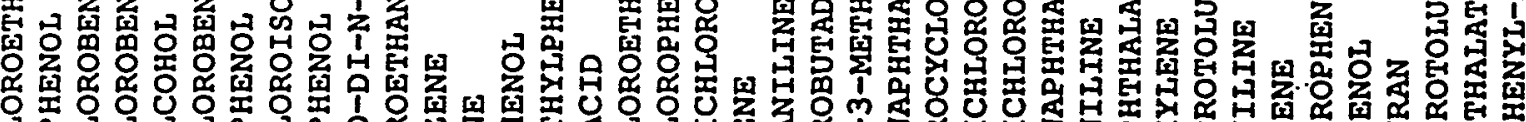

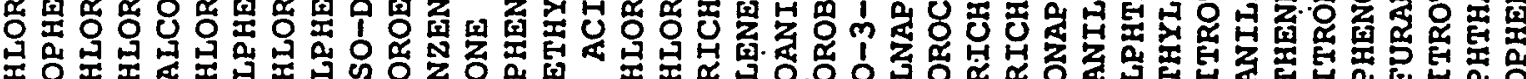

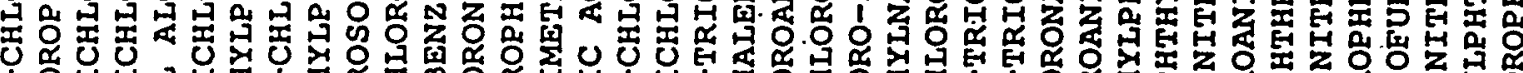

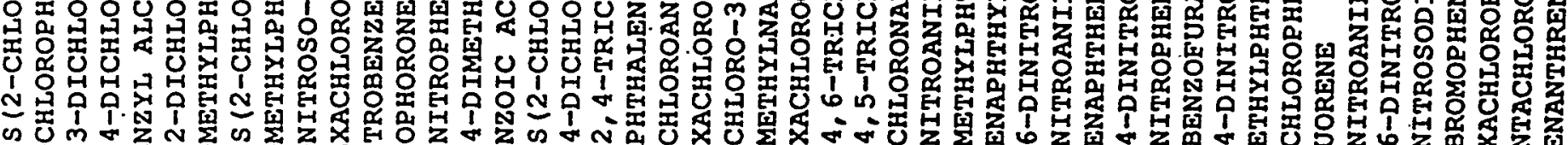

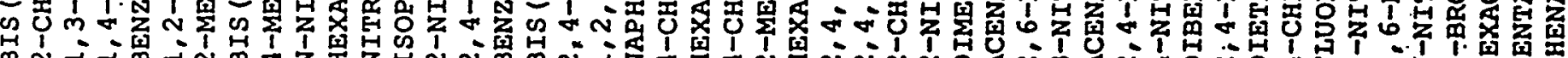

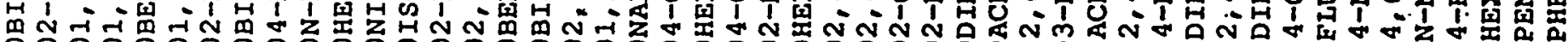

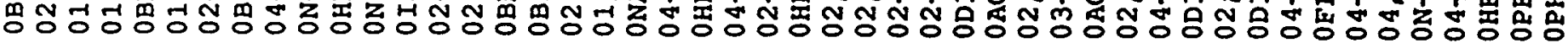

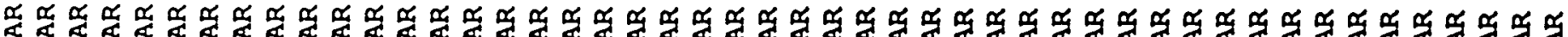

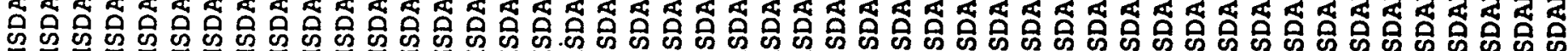

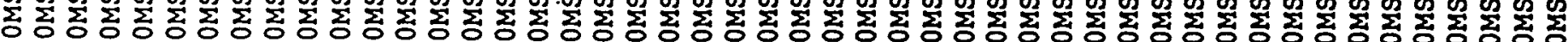

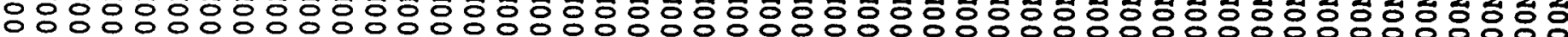

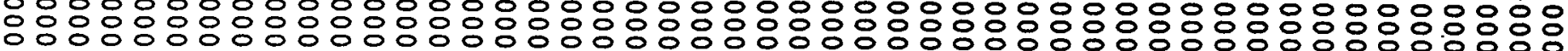
ન

O

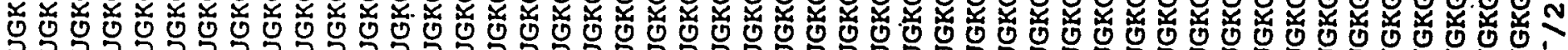

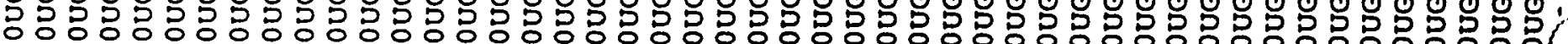

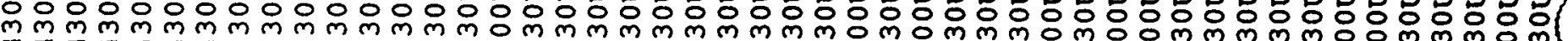

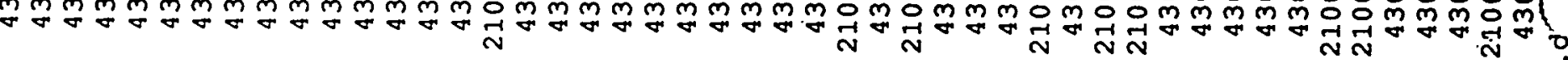

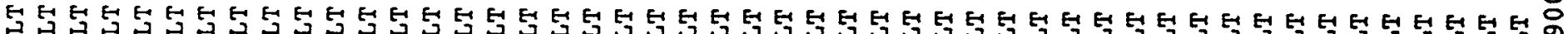

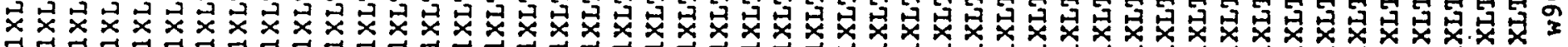

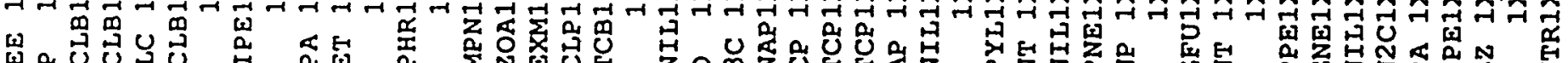

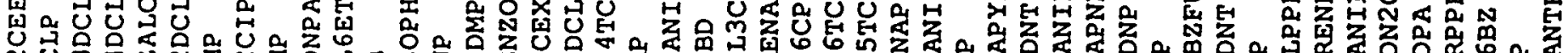

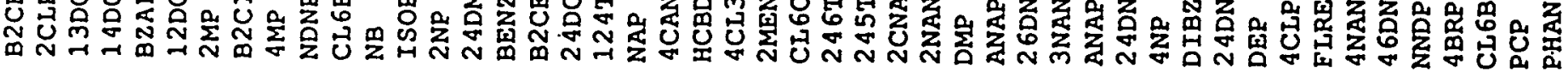

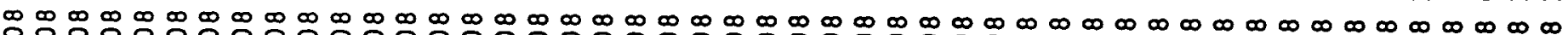

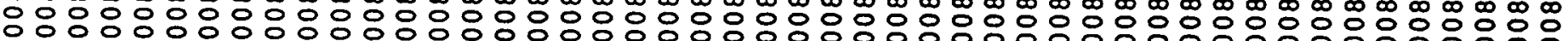
ம

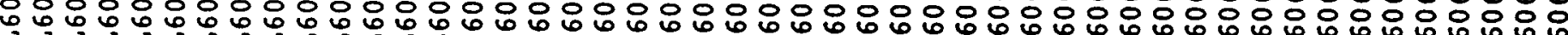

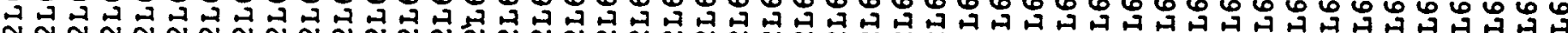

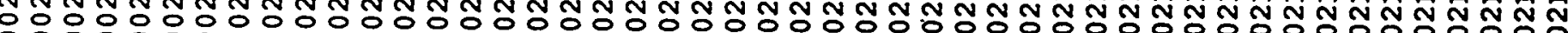

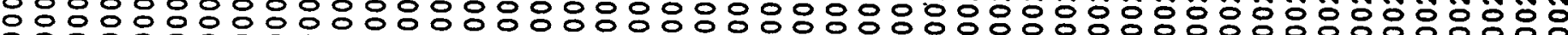


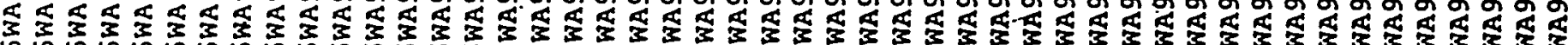
6 N

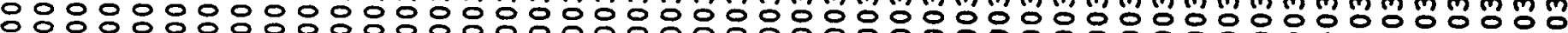

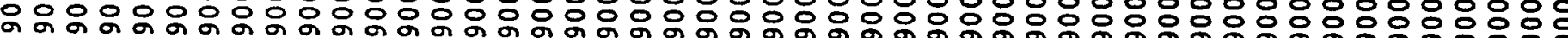

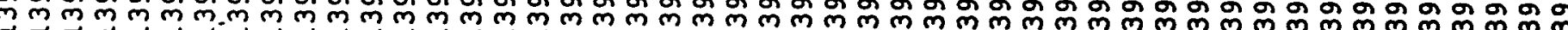

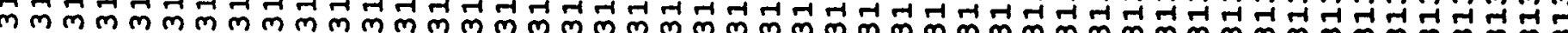

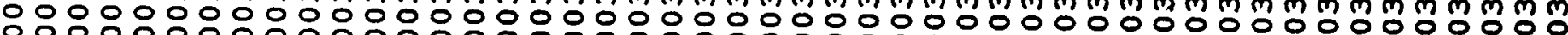

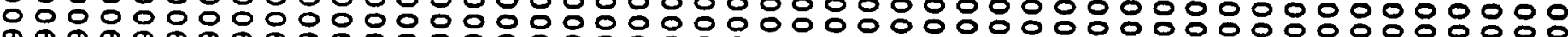

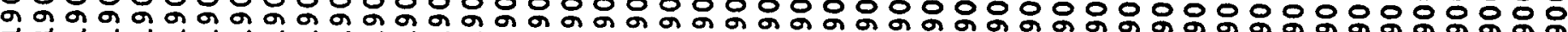

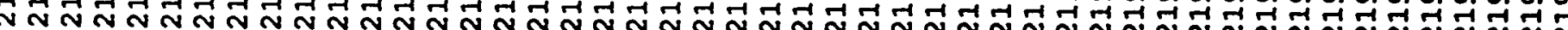

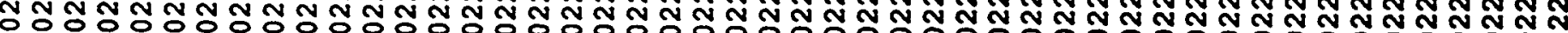

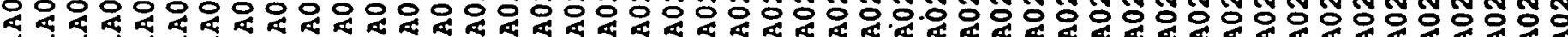

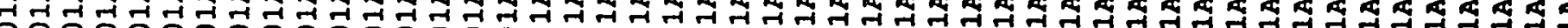

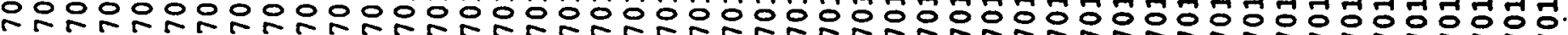

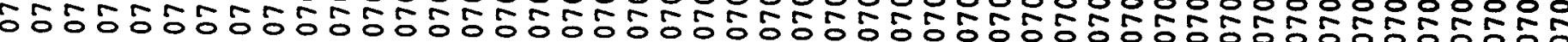


GRS $0 \%: 0221900313900326$ WA9002L606 008 ANTRC 1XLT GRS 0701A0221900313900326KA9002L606 008 DNBP 1XLT GRS 0701A0221900313900326\%A9002L606 00B FANT 1XLT GRS 0701A0221900313900326FA9002L606 008 PYR 1XLT GRS 0701A0221900313900326\%A9002L606 008 BBZP 1XLT GRS 0701A0221900313900326WA9002L606 008 33CL2B1XLT GRS 0701A0221900313900326WA9002L606 008 BAANTR1XLT GRS 0701A0221900313900326KA9002L606 00B CHRY 1XLT GRS 0701A0221900313900326WA9002L606 008 B2EHP $1 X$ GRS 0701A0221900313900326WA9002L606 008 DNOP 1XLT GRS 0701A0221900313900326WA9002L606 008 BE2ANT1XLT GRS 0701A0221900313900326WA9002L606 008 BKFANT1XLT GRS 0701A0221900313900326 FA9002L606 008 BAPYR 1XLT GRS 0701A0221900313900326WA9002L606 008 ICDPYRIXIT GRS 0701A0221900313900326WA9002L606 008 DBAHA 1XLT GRS 0701A0221900313900326 HA9002L606 008 BGHIPY1XIT GRS
07020221900301900032 WA9002L606 009 MEC6D82J 07020221900301900032 HA9002L606 009 PBFB 2J $07020221900301900032 \mathrm{WA} 9002 \mathrm{~L} 606009$ 12DCD42J 07020221900301900032 WA9002L606 009 CH3CL 2JLT 07020221900301900032 WA9002L606 009 CH3BR 2JLT 07020221900301900032 WA9002L606 009 C2H3CL2JLT $07020221900301900032 \mathrm{WA} 9002 \mathrm{~L} 606009$ C2H5CL2JLT 07020221900301900032 WA9002L606 009 ACET 2JB 07020221900301900032 WA9002L606 009 CS2 2JLT 07020221900301900032 WA9002L606 009 11DCE 2JLT 07020221900301900032 WA9002L606 009 11DCLE2JLT 07020221900301900032 WA9002L606 009 T12DCE2JLT 07020221900301900032 WA9002L606 009 CHCL3 2JLT 07020221900301900032 FA9002L606 009 12DCLE2JLT 07020221900301900032 W 9002 L606 009 MEK 2JLT 07020221900301900032 WA9002L606 009 111TCE2JLT 07020221900301900032 WA9002L606 009 CCL4 2JLT 07020221900301900032 WA9002I606 009 VINYLA2JLT 07020221900301900032 WA9002L606 009 BRDCLM2JLT 07020221900301900032 WA9002L606 009 13DCPC2JLT 07020221900301900032 FA90024606 009 TRCLE 2JLT 07020221900301900032 WA9002L606 009 DBRCLM2JLT 07020221900301900032 FA9002L606 009 112TCE2JLT 07020221900301900032 FA9002L606 009 C6H6 2JLT 07020221900301900032 \$A9002L606 009 T13DCP2JLT 07020221900301900032 $09002 L 606009$ CHBR3 2JLT 07020221900301900032 WA9002L606 009 4ME2PE2JLT 07020221900301900032 พA9002L606 009 2HXONE2JLT 07020221900301900032 WA9002L606 009 TCLEE 2JLT $07020221900301900032 \% A 9002 L 606009$ TCLEA 2JLT $07020221900301900032 \mathrm{WA} 9002 \mathrm{~L} 606009$ CH2CL22JB 07020221900301900032 WA9002L606 009 12DCLP2JLT
$=$ - JUGKG 430UGKG 430 UGKG 430 UGKG 430 UGKG 860 UGKG 430 UGKG 430 UGKG

1900 UGKG 430 UGKG 43 OUGKG 430 UGKG 430 UGKG 430 UGKG 430 UGKG 430UGKG 114PER 112PER 121PER

11UGKG

11UGKG

11UGKG

11 UGKG

$18 U G K G$

99UGKG

6UGKG

6UGKG

6UGKG

6UGKG

6UGKG

6UGKG

11 UGKG

6UGKG

6UGKG

11 UGKG

6UGKG

6UGKG

6UGKG

6UGKG

6UGKG

6UGKG

6UGKG

6UGKG

6UGKG

11 UGKG

11UGKG

6UGKKG 6UGRG
1. 0000MSDAR $1.0000 M S D A R$ 1. 0000MSDAR 1.0000MSDAR 1.0000MSDAR 1. 0000MSDAR 1. 0000MSDAR 1.0000MSDAR 1. 0000MSDAR

1.0000MSDAR

1.0000MSDAR

1. 0000MSDAR

1. OOOOMSDAR

1. 0000MSDAR

1. 0000MSDAR

1. 0000MSDAR

1. 0000MSJBD

1.0000MSJBD

1. 0000MSJBD

1. $0000 \mathrm{MSJBD}$

1. $0000 \mathrm{MSJBD}$

1.0000MSJBD

1. $0000 \mathrm{MSJBD}$

1.0000MS JBD

1.0000MSJBD

1. $0000 \mathrm{MSJBD}$

1. $0000 \mathrm{MSJBD}$

1. $0000 \mathrm{MSJBD}$

1. $0000 \mathrm{MSJBD}$

1.0000MSJBD

1. $0000 \mathrm{MSJBD}$

1. $0000 M S J B D$

1.0000MSJBD

1. $0000 \mathrm{MSJBD}$

1.0000MSJBD

1.0000MSJBD

1. $0000 \mathrm{MSJBD}$

1.0000MSJBD

1.0000MSJBD

1. $0000 \mathrm{MSJBD}$

1. $0000 \mathrm{MSJBD}$

1. $0000 \mathrm{MSJBD}$

1. $0000 \mathrm{MSJBD}$

1.0000MSJBD

1. OOOOMSJBD

1. 0000MSJBD

1. 0000MSJBD

1. $0000 \mathrm{MSJBD}$
OANTHRACENE O625HN ODI-N-BUTYIPHTHATATE O625HN OELUORANTHENE O625HN OPYRENE O625HN OBUTYLBENZYLPHTHALATEO $625 \mathrm{HN}$ $03,3^{\prime}$-DICHLOROBENZIDIO 625HN OBENZO (A) ANTHRACENE O625HN OCHRYSENE O625HN OBIS (2-ETHYLHEXYL) PHTO 625HN ODI-N-OCTYL PHTHALATEO625HN OBENZO (B) ELUORANTHENEO 625HN OBENZO (K) ELUORANTHENEO 625HN OBENZO (A) PYRENE O625HN OINDENO (1, 2, 3-CD) PYREO625HN ODIBENZO $(\mathrm{A}, \mathrm{H})$ ANTHRACEO $625 \mathrm{HN}$ OBENZO (G, H, I) PERYIENEO 625HN 56.00OTOLUENE-D8 $0624 \mathrm{HN}$ 56. 000BROMOELUOROBENZENE O624HN 56.0001,2-DICHLOROETHANE-DO624HN OCHLOROMETHANE . O624HN OBROMOMETHANE O624HN OVINYI CHLORIDE O624HN OCHLOROETHANE O624HN OMETHYLENE CHLORIDE O624HN OACETONE OCARBON DISULEIDE 01,1-DICHLOROETHENE O624HN $01,1-D I C H L O R O E T H A N E$ O624HN 01,2-DICHLOROETHENE $10624 \mathrm{HN}$ OCHLOROFORM 01,2-DICHLOROETHANE O624HN 02-BUTANONE O624HN $01,1,1-T R I C H L O R O E T H A N O 624 \mathrm{HN}$ OCARBON TETRACHLORIDEO $624 \mathrm{HN}$ OVINYI ACETATE O624HN OBROMODICHLOROMETHANEO $624 \mathrm{HN}$ $01,2-D I C H L O R O P R O P A N E$ O624HN OCIS-1, 3-DICHLOROPROPO 624 HN OTRICHLOROETHENE O624HN ODIBROMOCHLOROMETHANEO $624 \mathrm{HN}$ 01, 1, 2-TRICHLOROETHANO $624 \mathrm{kN}$ OBENZENE $0624 \mathrm{HN}$ OTRANS-1, 3-DICHLOROPRO 62 4HN OBROMOFORM . O624HN 04-METHYL-2-PENTANONEO $624 \mathrm{HN}$ 02-HEXANONE O624HN OTETRACHLOROETHENE O624HN $01,1,2,2$-TETRACHLOROEO $624 \mathrm{HN}$ 
GRS 07020221900301900032 WA9002L606 009 MEC6H52JLT GRS 07020221900301900032 WA9002L606 009 CLC6H52JLT GRS 07020221900301900032 WA9002L606 009 ETC6H52JLT GRS 07020221900301900032 WA9002L606 009 STYR 2JLT GRS 07020221900301900032 WA9002L606 009 XYLEN 2JLT GRS 07020221900309900326 WA9002L606 009 NBDS $1 \mathrm{X}$ GRS $07020221900309900326 \mathrm{WA} 9002 \mathrm{~L} 606009$ 2FBP $1 X$ GRS $07020221900309900326 \mathrm{WA} 9002 \mathrm{~L} 606009$ PTERP 1X GRS $07020221900309900326 \mathrm{WA} 9002 \mathrm{~L} 606009$ PHEND51X GRS $07020221900309900326 \mathrm{WA} 9002 \mathrm{~L} 606009$ 2FP $1 \mathrm{X}$ GRS 07020221900309900326 WA9002L606 009246 TBP IX GRS 07020221900309900326 WA9002L606 009 PHENOLIXLT GRS $07020221900309900326 \mathrm{WA} 9002 \mathrm{~L} 606009$ B2CEE 1XLT GRS $07020221900309900326 \mathrm{WA} 9002 \mathrm{~L} 606009$ 2CLP 1XLT GRS $07020221900309900326 \mathrm{WA} 9002 \mathrm{~L} 606009$ 13DCLB1XLT GRS $07020221900309900326 \mathrm{WA} 9002 \mathrm{~L} 606009$ 14DCLB1XLT GRS 07020221900309900326 WA9002L606 009 BZALC 1XLT GRS $07020221900309900326 \mathrm{WA} 9002 \mathrm{~L} 606009$ 12DCLB1XLT GRS $07020221900309900326 \mathrm{WA} 9002 L 606009$ 2MP 1XLT GRS $07020221900309900326 \mathrm{WA} 9002 \mathrm{~L} 606009$ B2CIPE1XLT GRS $07020221900309900326 \mathrm{WA} 9002 \mathrm{~L} 606009$ 4MP 1XLT GRS $07020221900309900326 \mathrm{KA} 9002 \mathrm{~L} 606009$ NDNRA 1XLT GRS $07020221900309900326 \mathrm{WA} 9002 \mathrm{~L} 606009$ CL6ET 1XLT GRS $07020221900309900326 \mathrm{WA} 9002 \mathrm{~L} 606009 \mathrm{NB}$ 1XLT $\stackrel{\sim}{\sim}$ GRS 07020221900309900326 WA9002L606 009 ISORHRIXLT

$07020221900309900326 \mathrm{WA} 9002 \mathrm{~L} 606009$ 2NP 1XLT 0702. 0221900309900326WA9002L606 009 24DMPN1XLT $0702.0221900309900326 \mathrm{WA} 9002 L 606009$ BENZOA1XLT $07020221900309900326 \mathrm{WA} 9002 \mathrm{~L} 606009$ B2CEXM1XLT $07020221900309900326 \mathrm{WA} 9002 \mathrm{~L} 606009$ 24DCLP1XLT $07020221900309900326 \mathrm{WA} 9002 \mathrm{~L} 606009$ 124TCB1XLT $07020221900309900326 \mathrm{WA} 9002 \mathrm{~L} 606009 \mathrm{NAP} \quad 1 \mathrm{XLT}$ $07020221900309900326 \mathrm{WA} 9002 \mathrm{~L} 606009$ 4CANIL1XLT $07020221900309900326 \mathrm{WA} 9002 \mathrm{~L} 606009$ HCBD 1XLT $07020221900309900326 \mathrm{WA9002L606} 009$ 4CL3C 1XLT $07020221900309900326 \mathrm{WA} 9002 \mathrm{~L} 606009$ 2MENAP 1XLT $07020221900309900326 \mathrm{WA} 9002 \mathrm{~L} 606009$ CL6CP 1XLT $07020221900309900326 \mathrm{WA} 002 \mathrm{~L} 606009246 \mathrm{TCP} 1 \mathrm{XLT}$ $07020221900309900326 \mathrm{WA} 9002 \mathrm{~L} 606009$ 245TCP 1 XLT $07020221900309900326 \mathrm{WA} 9002 \mathrm{~L} 606009$ 2CNAP IXLT $07020221900309900326 \mathrm{WA} 9002 \mathrm{~L} 606009$ 2NANIL1XLT $07020221900309900326 \mathrm{FA9002L606} 009$ DMP 1XLT $07020221900309900326 \mathrm{WA} 9002 \mathrm{~L} 606009$ ANAPYL1XLT $07020221900309900326 \mathrm{WA} 9002 \mathrm{~L} 606009$ 26DNT 1XLT $07020221900309900326 \mathrm{WA} 9002 L 606009$ 3NANIL1XLT $07020221900309900326 \mathrm{KA} 9002 \mathrm{~L} 606009$ ANAPNE1XLT $07020221900309900326 \mathrm{WA} 9002 \mathrm{~L} 60600924 \mathrm{DNP} 1 \mathrm{XLT}$ $07020221900309900326 \mathrm{FA9002L606} 009$ 4NP 1XLT
$49 P E R$

6OPER

71PER

STPER

55PER

48 PER

42 OUGKG

42 OUGKG

42 OUGKG

42 OUGKG

42 OUGKG

42 OUGKG

42 OUGKG

42 OUGKG

42 OUGKG

420 UGKG

420UGKG

42 OUGKG

42 OUGKG

420UGKG

42 OUGKG

420UGKG

2100UGKG

420UGKG

420UGKG

420UGKG

420UGKG

420UGKG

420UGKG

42 OUGKG

42 OUGKG

420UGKG

420UGKG

2100UGKG

420UGKG

2100UGKG

420UGKG

420UGKG

420UGKG

2100UGKG

420UGKG

2100UGKKG

2100UGKG
$1.0000 \mathrm{MSJBD}$ $1.0000 \mathrm{MSJBD}$ $1.0000 \mathrm{MSJBD}$ $1.0000 \mathrm{MSJBD}$ $1.0000 \mathrm{MSJBD}$

1.0000MSDAR

1.0000MSDAR

1.0000MSDAR

1. $0000 M S D A R$

1.0000MSDAR

1.0000MSDAR

1. $0000 \mathrm{MSDAR}$

1.0000MSDAR

1.0000MSDAR

$1.0000 M S D A R$

1.0000MSDAR

1.0000MSDAR

$1.0000 \mathrm{MSDAR}$

$1.0000 M S D A R$

1. $0000 \mathrm{MSDAR}$

1.0000MSDAR

1.0000MSDAR

1.0000MSDAR

$1.0000 \mathrm{MSDAR}$

1.0000MSDAR

1.0000MSDAR

1.0000MSDAR

1.0000MSDAR

1.0000MSDAR

1.0000MSDAR

1.0000MSDAR

1.0000MSDAR

1.0000MSDAR

1.0000MSDAR

1.0000MSDAR

1. O0OOMSDAR

$1.0000 \mathrm{MSDAR}$

1.0000MSDAR

1.0000MSDAR

$1.0000 \mathrm{MSDAR}$

$1.0000 \mathrm{MSDAR}$

1. 0000MSDAR

1.0000MSDAR

$1.0000 \mathrm{MSDAR}$

1.0000MSDAR

1.0000MSDAR

1.0000MSDAR

1. 0000MSDAR

\section{OTOLUENE}

OCHLOROBENZENE

OETHYLBENZENE

OSTÝRENE

OXYLENE (TOTAL)

1873.875 NITROBENZENE-D5

$1873.8752-$ ELUOROBIPHENYL

$1873.875 P$-TERPHENYL-D14

3747.749 PHENOL-D5

$3747.7492-$ ELUOROPHENOL

$3747.7492,4,6$-TRIBROMOPHENOLO625HN OPHENOL $0625 \mathrm{HN}$ OPHE (2-CHLOROETHYL) ETO625HN 02-CHLOROPHENOL 0625HN 01,3-DICHLOROBENZENE O625HN 01,4-DICHLOROBENZENE $0625 \mathrm{HN}$ OBENZYL ALCOHOL O625HN 01,2-DICHLOROBENZENE 0625HN 02-METHYLPHENOL $\quad 0625 \mathrm{HN}$ OBIS (2-CHLOROISOPROPYO $625 \mathrm{kN}$ 04-METHYLPHENOL O625HN ON-NITROSO-DI-N-PROP YO $625 \mathrm{HN}$ OHEXACHLOROETHANE $0625 \mathrm{HN}$ ONITROBENZENE OISORHORONE $0625 \mathrm{HN}$ 02-NITROPHENOL $\quad 0625 \mathrm{HN}$ 02,4-DIMETHYLPHENOL O625HN OBENZOIC ACID , O625HN OBIS (2-CHLOROETHOXY) MO625HN 02,4-DICHLOROPHENOL O625HN $01,2,4-T R I C H L O R O B E N Z E O 625 \mathrm{HN}$ ONAPHTHALENE O625HN 04-CHLOROANIIINE O625HN OHEXACHLOROBUTADIENE O625HN 04-CHLORO-3-METHYLPHEO625HN 02-METHYLNAPHTHALENE O625HN OHEXACHLOROCYCLOPENTAO $625 \mathrm{HN}$ $02,4,6$-TRICHLOROPHENOO $625 \mathrm{HN}$ $02,4,5$-TRICHLOROPHENOO625HN 02-CHLORONAPHTHALENE O625HN 02-NITROANILINE O625HN ODIMETHYLPHTHALATE O625HN OACENAPHTHYLENE $0625 \mathrm{HN}$ 02,6-DINITRÓTOLUENE. O625HN 03-NITROANILINE 0625HN OACENAPHTHENE 0625HN 02,4-DINITROPHENOL OE25HN O4-NITRORHENOL 
07020221900309900326 WA9002L606 009 DIBZFUIXLT GRS 07020221900309900326 WA9002L606 009 24DNT 1XLT GRS 07020221900309900326 WA9002L606 009 DEP IXIT GRS $07020221900309900326 \mathrm{WA} 9002 \mathrm{~L} 606 \quad 009$ 4CLPPEIXLT GRS 07020221900309900326 WA9002L606 009 ELRENE1XLT GRS 07020221900399900326 WA9002L606 009 4NANILIXLT GRS $07020221900309900326 \mathrm{WA} 9002 L 606 \quad 009$ 46DN2C1XLT GRS $07020221900309900326 \mathrm{WA9002L606} 009$ NNDPA 1XLT GRS $07020221900309900326 \mathrm{WA} 9002 \mathrm{~L} 606009$ 4BRPPEIXLT GRS $07020221900309900326 \mathrm{WA} 9002 L 606 \quad 009$ CL6B2 1XLT GRS $07020221900309900326 \mathrm{WA} 9002 \mathrm{~L} 606 \quad 009$ PCP IXLT GRS $07020221900309900326 \mathrm{WA} 9002 \mathrm{~L} 606009$ PHANTR1XLT GRS $07020221900309900326 \mathrm{WA} 9002 L 606 \quad 009$ ANTRC 1XLT GRS $07020221900309900326 \mathrm{WA} 90024606009$ DNBP 1XLT GRS 07020221900309900326 WA9002L606 009 EANT IXLT GRS 07020221900309900326 FA9002L606 009 PYR 1XLT GRS $07020221900309900326 \mathrm{FA} 9002 L 606 \quad 009$ BBRP 1XLT GRS 07020221900309900326 WA9002L606 009 33CL2B1XLT GRS 07020221900309900326 WA9002L606 009 BAANTRIXIT GRS 07020221900309900326 WA9002L606 009 CHRY IXLT GRS $07020221900309900326 \mathrm{WA} 9002 \mathrm{~L} 606 \quad 009$ B2EHP 1XLT GRS $07020221900309900326 \mathrm{FA9002L606} 009$ DNOP IXLT GRS $07020221900309900326 \mathrm{WA} 9002$ I606 009 BE2ANTIXLT W GRS 07020221900309900326 WA9002L606 009 BKFANT1XLT ${ }_{i}^{N}$ GRS 07020221900309900326 WA9002L606 009 BAP.YR 1XLT $v$ GRS $07020221900309900326 \mathrm{WA} 90021606009$ ICDPYR1XLT GRS 0702.0221900309900326 WA9002L606 009 DBAHA 1XLT GRS 0702.0221900309900326 WA9002L606 009 BGHIPY1XLT GRS $07030221900305900036 \mathrm{WA} 9002 L 606010 \mathrm{MEC} 6 \mathrm{D} 82 \mathrm{~J}$ GRS $07030221900305900036 \mathrm{WA} 9002 L 606010$ PBEB 2J GRS $07030221900305900036 \mathrm{KA9002L606} 010 \quad 12 D C D 42 \mathrm{~J}$ GRS - 07030221900305900036 FA9002L606 010 CH3CL 2JLT GRS 07030221900305900036 WA9002L606 010 CH3BR 2JLT GRS 07.030221900305900036 WA9002L606 010 C2H3CL2JLT GRS 07030221900305900036 WA9002L606 010 C2H5CL2JLT GRS 07030221900305900036 WA9002L606 010 CH2CL22JB GRS 07030221900305900036 WA9002L606 010 ACET 2JB GRS 07030221900305900036 WA9002L606 010 CS2 2JIT GRS $07030221900305900036 \mathrm{WA9002L606} 010$ 11DCE 2JLT GRS $07030221900305900036 \mathrm{WA} 90021606 \quad 010$ 11DCLE2JIT GRS 07030221900305900036 WA9002L606 010 T12DCE2JLT GRS 07030221900305900036 HA9002L606 010 CHCL3 2JLT GRS $07030221900305900036 \mathrm{KA9002L606} 010$ 12DCLE2JLT GRS 07030221900305900036 WA9002L606 010 MEK 2JLT GRS $07030221900305900036 \mathrm{FA} 9002$ L606 010 111TCE2JLT GRS 07030221900305900036 WA9002L606 010 CCL4 2JLT GRS 07030221900305900036 WA9002L606 010 VINYLA2JLT GRS 07030221900305900036 HA9002L606 010 BRDCLM2JLT
420UGKG

42 OUGKG

420 UGKG

420UGKG

420 UGKG

2100 UGKG

2100UGKG

42 UUGKG

42 OUGKG

420 UGKG

210 OUGKG

42 OUGKG

420 UGKG

42 OUGKG

420UGKG

42 OUGKG

420UGKG

830 UGKG

42 UUGKG

420 UGKG

420 UGKG

420 UGKG

420 UGKG

420 UGKG

42 OUGKG

420UGKG

42 UUGKG

42 OUGKG

89PER

97PER

85PER

12UGKG

12 UGKG

12 UGKG

12 UGKG

IOUGKG

81UGKG

6UGKG

6UGKG

6UGKG

6UGKG

6UGKG

6UGKG

12UGKG

6UGKG

6UGKG

12UGKG

6UGKG
1.0000MSDAR

1. 0000MSDAR

1.0000MSDAR

1.0000MSDAR

1. $0000 \mathrm{MSDAR}$

1. 0000MSDAR

1. 000OMSDAR

1. $0000 \mathrm{MSDAR}$

1. $0000 \mathrm{MSDAR}$

1.0000MSDAR

1. 0000MSDAR

1.0000MSDAR

1.0000MSDAR

1. $0000 \mathrm{MSDAR}$

1.0000MSDAR

1. O0OOMSDAR

1. 000OMSDAR

1.0000MSDAR

1. $0000 \mathrm{MSDAR}$

1.0000MSDAR

1. 0000MSDAR

1. $0000 \mathrm{MSDAR}$

1. 0000MSDAR

1. 000OMSDAR

1. 000OMSDAR

1. $0000 \mathrm{MSDAR}$

1. $0000 M S D A R$

1. OOOOMSDAR

1 . 000OMSJBD

1. $0000 \mathrm{MSJBD}$

1. 0000MSJBD

1. 0000MSJBD

1. $0000 \mathrm{MSJBD}$

1. 0000MSJBD

1. $0000 \mathrm{MSJBD}$

1. $0000 \mathrm{MSJBD}$

1. 0000MSJBD

1. $0000 \mathrm{MSJBD}$

1. $0000 \mathrm{MSJBD}$

1. 0000MSJBD

1. $0000 \mathrm{MSJBD}$

1. 000OMSJED

1.000OMSJBD

1. $0000 \mathrm{MSJBD}$

1.0000MSJBD

1.000OMSJBD

1. $0000 \mathrm{MSJBD}$

$1.0000 \mathrm{MSJBD}$
ODIBENZOFURAN

0625HN

02,4-DINITROTOLUENE 0625HN ODIETHYLPHTHALATE O625HN 04-CHIOROPHENYI-PHENYO 625HN OFLUORENE - 0625HN 04-NITROANILINE 0625HN 04, 6-DINITRO-2-METHYL0625HN ON-NITROSODIPHENYLAMIO625HN 04-BROMOPHENYL-PHENYLO625HN OHEXACHIOROBENZENE O625HN OPENTACHLOROPHENOL 0625HN OPHENANTHRENE O625HN OANTHRACENE O625HN ODI-N-BUTYLPHTHALATE 0625HN OEIUORANTHENE O625HN OPYRENE $0625 \mathrm{HN}$ OBUTYIBBENZYLPHTHALATEO $625 \mathrm{HN}$ 03; 3 -DICHLOROBENZ IDIO625HN OBENZO (A) ANTHRACENE O625HN OCHRY SENE 0625HN OBIS (2-ETHYLHEXYI) PHTO625HN ODI-N-OCTYL PHTHALATEO625HN OBENZO (B) FLUORANTHENEO $625 \mathrm{HN}$ OBENZO (K) ELUORANTHENE0625KN OBENZO (A) PYRENE O625HN OINDENO $(1,2,3-C D)$ PYRE0625HN ODIBENZO $(A, H)$ ANTHRACEO $625 \mathrm{HN}$ OBENZO (G, H, I) PERYLENEO $625 \mathrm{HN}$

57. 500TOLUENE-D8 $0624 \mathrm{HN}$ 57.50OBROMOELUOROBENZENE O624HN

57.5001,2-DICHLOROETHANE-DO624HN OCHLOROMETHANE 0624HN OBROMOMETHANE O624HN OVINYI CHLORIDE O624HN OCHLOROETHANE O624HN OMETHYLENE CHLORIDE O624HN OACETONE $0624 \mathrm{HN}$ OCARBON DISULFIDE O624HN 01,1-DICHLOROETHENE 0624HN 01,1-DICHLOROETHANE 062.4HN $01,2-D I C H L O R O E T H E N E$ (0624 HN OCHLOROEORM O624HN 01;2-DICHLOROETHANE 0624HN 02-BUTANONE - O624HN $01,1,1$-TRICHLOROETHANO624HN OCARBON TETRACHLORIDEO $624 \mathrm{HN}$ OVINYY ACETATE OGQ $4 \mathrm{HN}$ OBROMODICHLOROMETHANEO $624 \mathrm{HN}$ 

07030221900305900036 WA9002L606 010 13DCPC2JLT 07030221900305900036 WA9002L606 010 TRCLE 2JLT 07030221900305900036 WA9002L606 010 DBRCLM2JLT $07030221900305900036 \mathrm{WA} 9002 \mathrm{~L} 606010$ 112TCE2JLT 07030221900305900036 WA9002L606 010 C6HG 2JLT 07030221900305900036 WA9002L606 010 T13DCP2JLT $07030221900305900036 \mathrm{WA} 9002 \mathrm{~L} 606010$ CHBR3 2JLT 07030221900305900036 WA9002L606 010 4ME2PE2JLT $07030221900305900036 \mathrm{WA} 9002 \mathrm{~L} 606010$ 2HXONE2JLT $07030221900305900036 \mathrm{WA} 9002 \mathrm{~L} 606010$ TCLEE 2JLT 07030221900305900036 WA9002L606 010 TCLEA 2JLT $07030221900305900036 \mathrm{WA} 9002 \mathrm{~L} 606010$ MEC6H52JLT 07030221900305900036 WA9002L606 010 CLC6H52JLT 07030221900305900036 WA9002L606 010 ETC6H52JLT $07030221900305900036 \mathrm{WA} 9002 \mathrm{~L} 606010$ STYR 2JLT 07030221900305900036 WA9002L606 010 XYLEN $2 J$ $07030221900309900326 \mathrm{WA} 9002 \mathrm{~L} 606010$ NBD5 $1 \mathrm{X}$ 07030221900309900326 WA9002L606 010 2FBP $1 X$ 07030221900309900326 WA9002L606 010 PTERP $1 \mathrm{X}$ 07030221900309900326 WA9002L606 010 PHEND $51 \mathrm{X}$ 07030221900309900326 WA9002L606 010 2EP 1X 07030221900309900326 WA9002L606 010 246TBP $1 \mathrm{X}$ 07030221900309900326 WA9002L606 010 PHENOL1XLT $07030221900309900326 \mathrm{WA} 90024606010$ B2CEE 1XLT $07030221900309900326 \mathrm{WA} 9002 \mathrm{~L} 606010$ 2CLP 1XLT $07030221900309900326 \mathrm{WA} 9002 \mathrm{~L} 606010$ 13DCLB1XLT 07030221900309900326 WA9002L606 010 14DCLB1XLT $07030221900309900326 \mathrm{WA} 9002 \mathrm{~L} 606010$ BZALC 1XLT 07030221900309900326 WA9002L606 010 12DCLB1XLT $07030221900309900326 \mathrm{WA} 9002 \mathrm{~L} 606010$ 2MP 1XLT $07030221900309900326 \mathrm{WA} 9002 L 606010$ B2CIPE1XLT $07030221900309900326 \mathrm{WA} 9002 \mathrm{~L} 606 \quad 010$ 4MP 1XLT 07030221900309900326 WA9002L606 010 NDNPA $1 X L T$ 07030221900309900326 WA9002L606 010 CL6ET 1XLT $07030221900309900326 \mathrm{WA} 9002 L 606010 \mathrm{NB}$ 1XLT 07030221900309900326 WA9002L606 O10 ISOPHRIXLT $07030221900309900326 \mathrm{WA} 9002 \mathrm{~L} 606010$ 2NP IXLT 07030221900309900326 WA9002L606 010 24DMPN1XLT $07030221900309900326 \mathrm{WA} 9002 \mathrm{~L} 606010$ BENZOAIXLT $07030221900309900326 \mathrm{WA} 9002 \mathrm{~L} 606010$ B2CEXM1XLT 07030221900309900326 WA9002L606 010 24DCLP1XLT $07030221900309900326 \mathrm{FA} 9002 \mathrm{~L} 606010$ 124TCB1XLT $07030221900309900326 \mathrm{WA} 9002 \mathrm{~L} 606$ 010. NAP IXLT $07030221900309900326 \mathrm{FA} 9002 \mathrm{~L} 606010$ 4CANIL1XLT $07030221900309900326 \mathrm{WA9002L606} 010 \mathrm{HCBD} 1 \mathrm{XLT}$ $07030221900309900326 \mathrm{FA9002L606} 010$ 4CL3C IXLT $07030221900309900326 \mathrm{FA} 90022606010$ 2MENAP 1XLT
6UGKG

6UGKG

6UGKG

6UGKG

6UGKG

6UGKG

6UGKG

6UGKG

12 UGKG

12 UGKG

6UGKG

6UGKG

6UGKG

6UGKG

6UGKG

6UGKG

4UGKG

65PER

$76 \mathrm{PER}$

94PER

8OPER

82PER

65PER

42 OUGKG

42 OUGKG

42 OUGKG

42 OUGKG

42 OUGKG

42 OUGKG

42 OUGKG

42 OUGKG

42 UUGKG

42 OUGKG

42 UUGKG

42 OUGKG

42 OUGKG

42 OUGKG

42 OUGKG

42 OUGKG

2100 UGKG

42 OUGKG

420 UGKG

420 UGKG

42 OUGKG

420UGKG

420 UGKG

42 UUGKG

420UGKG
1. $0000 \mathrm{MSJBD}$

$1.0000 M S J B D$

1. $0000 M S J B D$

1. $0000 \mathrm{MSJBD}$

1. $0000 \mathrm{MSJBD}$

1. $0000 M S J B D$

1.000OMSJBD

$1.0000 \mathrm{MSJBD}$

1. $0000 \mathrm{MSJBD}$

$1.0000 \mathrm{MSJBD}$

$1.0000 M S J B D$

1. $0000 \mathrm{MSJBD}$

1.000OMSJBD

1. $0000 \mathrm{MSJBD}$

1. $0000 \mathrm{MSJBD}$

1.0000MSJBD

1. $0000 \mathrm{MSJBD}$

1.0000MSDAR

1.0000MSDAR

1. 0000MSDAR

1. 0000MSDAR

$1.0000 \mathrm{MSDAR}$

1. 000OMSDAR

1.0000MSDAR

1. 0000MSDAR

1. 0000MSDAR

1. 0000MSDAR

1. 000OMSDAR

1.0000MSDAR

1.000OMSDAR

1. 000OMSDAR

1. 0000MSDAR

1.0000MSDAR

1. 000OMSDAR

1.0000MSDAR

1. 0000MSDAR

1.0000MSDAR

1. 0000MSDAR

1. $0000 \mathrm{MSDAR}$

1.0000MSDAR

1.000OMSDAR

1. OOOOMSDAR

1.0000MSDAR

1. 0000MSDAR

1. 000OMSDAR

1.0000MSDAR

1. 0000MSDAR

1. $0000 \mathrm{MSDAR}$
01,2-DICHLOROPROPANE O624HN OCIS-1,3-DICHLOROPROPO624HN OTRICHLOROETHENE O624HN ODIBROMOCHLOROMETHANEO $624 \mathrm{HN}$ $01,1,2$-TRICHLOROETHANO $624 \mathrm{HN}$ OBENZENE $0624 \mathrm{HN}$ OTRANS-1, 3-DICHLOROPRO62 4HN OBROMOFORM O624HN 04-METHYL-2-PENTANONEO $624 \mathrm{HN}$ 02-HEXANONE O624HN OTETRACHLOROETHENE O624HN $01,1,2,2$-TETRACHIOROEO $624 \mathrm{HN}$ OTOLUENE OCHLOROBENZENE O624HN OETHYLBENZENE O624HN OSTYRENE O624HN OXYLENE (TOTAL) O624HN 1887.388 NITROBENZENE-D5 O625HN 1887.3882 -FLUOROBIPHENYL O625HN $1887.388 \mathrm{P}-\mathrm{TERPHENYL}-\mathrm{D} 14 \quad 0625 \mathrm{HN}$ 3774.776 PHENOL-D5 $^{\circ}$ 0625HN 3774.7762-FLUOROPHENOL O625HN $3774.7762,4,6$-TRIBROMOPHENOLO $625 \mathrm{HN}$ OPHENOL O625HN OBIS (2-CHLOROETHYL) ETO625HN 02-CHLOROPHENOL 0625HN 01,3-DICHLOROBENZENE 0625HN 01, 4-DICHLOROBENZENE 0625HN OBENZYI ALCOHOI O625HN 01,2-DICHLOROBENZENE O625HN 02-METHYLPHENOL O625HN OBIS (2-CHLOROISOPROP YO 625HN 04-METHYLPHENOL O625HN ON-NITROSO-DI-N-PROPYO625 $\mathrm{kN}$ OHEXACHLOROETHANE $0625 \mathrm{HN}$ ONITROBENZENE O625HN OISOPHORONE O625HN 02-NITROPHENOI O625HN 02,4-DIMETHYLPHENOL O625HN OBENZOIC ACID O625HN OBIS (2-CHLOROETHOXY) MO625HN 02, 4-DICHLOROPHENOL O625HN $01,2,4$-TRICHLOROBENZEO625HN ONAPHTHALENE $: 0625 \mathrm{HN}$ 04-CHLOROANILINE O625HN OHEXACHLOROBUTADIENE O625HN 04-CHLORO-3-METHYLPHEO $225 \mathrm{HN}$ 02-METHYLNAPHTHALENE $625 \mathrm{HN}$ 
$0 \%$ 0221900309900326\%A9002L606 010 CL6CP 1XLT $07030221900309900326 \mathrm{WA} 9002 \mathrm{~L} 606010$ 246TCP 1 XLT $07030221900309900326 \mathrm{FA9002L606} 010$ 245TCP $1 \times L T$ 07030221900309900326 WA9002L606 010 2CNAP 1XLT .07030221900309900326 WA9002L606 010 2NANIL1XLT 07030221900309900326 WA9002L606 010 DMP 1XLT $07030221900309900326 \mathrm{HA} 90021606010$ ANAPYL1XLT $07030221900309900326 \mathrm{HA} 002 \mathrm{~L} 606010$ 26DNT 1XLT $07030221900309900326 \mathrm{WA} 9002 \mathrm{~L} 606010$ 3NANIL1XLT $07030221900309900326 \mathrm{WA} 9002 \mathrm{~L} 606010$ ANAPNE1XLT $07030221900309900326 \mathrm{WA} 9002 \mathrm{~L} 606010$ 24DNP 1XLT $07030221900309900326 \mathrm{WA} 9002 \mathrm{~L} 606010$ ANP 1XLT $07030221900309900326 \mathrm{WA} 90024606010$ DIBZEU1XLT $07030221900309900326 \mathrm{WA} 9002 \mathrm{~L} 606010$ 24DNT 1XLT $07030221900309900326 \mathrm{WA} 9002 \mathrm{~L} 606010 \mathrm{DEP}$ IXLT $07030221900309900326 \mathrm{WA} 9002 \mathrm{~L} 606010$ 4CLPPE1XLT 07030221900309900326 WA9002L606 010 FLRENELXLT $07030221900309900326 \mathrm{WA} 9002 \mathrm{~L} 606010$ ANANILIXIT 07030221900309900326 WA9002L606 010 46DN2C1XLT 07030221900309900326 WA9002L606 010 NNDPA 1XLT $07030.221900309900326 \mathrm{WA} 9002 \mathrm{~L} 606010$ 4BRPPE1XLT 07030221900309900326 WA9002L606 010 CL6BZ 1XLT $07030221900309900326 \mathrm{WA} 9002 \mathrm{~L} 606010 \mathrm{PCP}$ 1XLT $070302219003099003264 A 9002 L 606010$ PHANTR1XLT 07030221900309900326 WA9002L606 010 ANTRC 1XLT 07030221900309900326 WA9002L606 010 DNBP 1XLT 0703. 0221900309900326WA9002L606 010 EANT IXIT 07030221900309900326 WA9002L606 010 PYR 1XIT $07030221900309900326 \mathrm{WA} 9002 \mathrm{~L} 606010$ BB2P 1XLT 07030221900309900326 WA9002L606 010 33CL2BIXLT 07030221900309900326 WA9002L606 010 BAANTR1XIT 07030221900309900326 WA90024606 010 CHRY IXLT 07030221900309900326 WA9002L606 010 B2EHP $1 X$

GRS 07030221900309900326 WA9002L606 010 DNOP 1XLT GRS 07030221900309900326 WA9002L606 010 BE2ANT1XIT GRS 07030221900309900326 WA9002L606 010 BKFANT1XLT GRS 07030221900309900326 WA9002L606 010 BAPYR 1XLT GRS 07030221900309900326 WA9002L606 010 ICDPYR1XLT GRS 07030221900309900326 WA9002L606 010 DBAHA 1XLT GRS $07030221900309900326 \mathrm{HA} 9002$ L606 010 BGHIPY1XLT GRS 08010221900308900039 KA9002L606 011

GRS 08010221900308900039 KA9002L606 011R

GRS 08010221900416901200 WA9002L606 011 ALPHAG9W GRS 08010221900416901200 WA90021606 011 BETAG 9W GRS 08010221900315900002 FA9002L606 011 CEC EX GRS 08010221900312900002 FA9002L606 011 CECB EX

$\begin{array}{lllllllll}\text { GRS } & 0801 & 0221900308900002 \% A 90021606 & 011 & \text { CECT } & \text { EX } \\ \text { GRS } & 0801 & 0221900302900002 \text { FA9002L606 } & 011 & \text { TOC } & \text { 9I }\end{array}$ GRS
4. UUGKG 420UGKG 2100 UGKG 420UGKG

2100 UGKG 420 UGKG 420 UGKG 420 UGKG

2100 UGKG 420UGKG 2100 UGKG 2100 UGKG 420UGKG 420UGKG 420UGKG 420 UGKG 420 UGKG 2100UGKG 2100UGKG 420UGKG 420UGKG 420 UGKG

2100 UGKG

420UGKG 42 OUGKG 420UGKG 420UGKG 420 UKKG 420UGKG 84 OUGKG 420 UGKG 42 OUGKG

420 OUGKG

420 UGKG

42 OUGKG 420UGKG 420UGKG 42 OUGKG 420UGKG 42 OUGKG

$82.8 \%$

$82.9 \%$

22 . OPCIG

10. OPCIG

7. TMEQL

$0.92 \mathrm{MEQ} 1$

$6.8 M E Q 1$ 873MGKG
1.0000MSDAR

1.0000MSDAR

1.0000MSDAR

1.0000MSDAR

1. $0000 \mathrm{MSDAR}$

1.0000MSDAR

1.0000MSDAR

1. $0000 \mathrm{MSDAR}$.

1.0000MSDAR

1.0000MSDAR

1. 0000MSDAR

1.0000MSDAR

1.0000MSDAR

1. 0000MSDAR

1. 0000MSDAR

1. $0000 \mathrm{MSDAR}$

$1.0000 \mathrm{MSDAR}$

1.0000MSDAR

1. $0000 \mathrm{MSDAR}$

1.0000MSDAR

1.0000MSDAR

1. $0000 M S D A R$

1.0000MSDAR

1. 0000MSDAR

1. $0000 \mathrm{MSDAR}$

1.0000MSDAR

1. 0000MSDAR

1. 0000MSDAR

1.0000MSDAR

1. 0000MSDAR

1. $0000 \mathrm{MSDAR}$

1.000OMSDAR

1. OOOOMSDAR

1.000OMSDAR

1.0000MSDAR

1. 0000MSDAR

1. 0000MSDAR

1. 0000MSDAR

1. 0000MSDAR

1.0000MSDAR

$10 \mathrm{JH}$

$10 \mathrm{JH}$

1OEBUNK

1 OEBUNK

IONADKS

IONAALM

1 ONAJS

10DODKS
OHEXACHLOROCYCLOPENTAO625HN $02,4,6$-TRICHLOROPHENOO625HN $02,4,5-T R I C H L O R O R H E N O 0625 \mathrm{HN}$ 02-CHLORONAPHTHALENE 0625HN 02-NITROANIIINE ${ }^{\circ}$ 0625HN ODIMETHYIPHTHALATE 0625HN OACENAPHTHYLENE O625HN 02,6-DINITROTOLUENE 0625HN 03-NITROANIIINE 0625HN OACENAPHTHENE 0625HN 02, 4-DINITROPHENOL 0625HN 04-NITROPHENOL O625HN ODIBENZOEUंRAN $0625 \mathrm{HN}$ 02,4-DINITROTOLUENE O625HN ODIETHYLPHTHALATE O625HN 04-CHLOROPHENYL-PHENYO625HN OELUORENE O625HN 04-NITROANIIINE 0625HN 04, 6-DINITRO-2-METHYLO625HN ON-NITROSODIPHENYLAMIO $625 \mathrm{HN}$ 04-BROMOPHENYL-PHENYLO625HN OHEXACHLOROBENZENE 0625HN OPENTACHLOROPHENOL O625HN OPHENANTHRENE O625HN OANTHRACENE O625HN ODI-N-BUTYLPHTHALATE O625HN OFLUORANTHENE O625HN OPYRENE O625HN OBUTYLBENZYIPHTHALATEO625HN $03,3^{\prime}$-DICHLOROBENZIDIO625HN OBENZO (A) ANTHRACENE O625HN OCHRYSENE O625HN OBIS (2-ETHYLHEXYL) PHTO625HN ODI-N-OCTYL PHTHALATEO625HN OBENZO (B) ELUORANTHENEO 625HN OBENZO (K) ELUORANTHENEO 625HN OBENZO (A) PYRENE O625HN O INDENO $(1,2,3-C D)$ PYREO $625 \mathrm{HN}$ ODIBENZO (A, H) ANTHRACEO $625 \mathrm{HN}$ OBENZO (G, H, I) PERYLENEO $625 \mathrm{HN}$ $0 \%$ SOLIDS I\&SOLN O\& SOLIDS (REP) I\&SOLR OGROSS ALPHA IALPHN ONONVOLATILE BETA IBETAN OCATION EXCHANGE CAPAICEC N OEXCHANGEABLE BASES ICECBN OEXCHANGEABLE ACIDS ICECTN OTOTAL ORGANIC CARBONIOCT N 
GRS 08010221900308900042 WA9002L606 011 PH $9 R$ $\begin{array}{llllllll}\text { GRS } & 0802 & 0221900308900039 W A 9002 L 606 & 012 & & \\ \text { GRS } & 0802 & 0221900315900002 \text { WA9002L606 } & 012 & \text { CEC } & \text { EX }\end{array}$ GRS 08020221900312900002 WA9002L606 012 CECB EX GRS 08020221900308900002 WA9002L606 012 CECT EX. GRS 08020221900302900002 WA9002L606 012 TOC 9I GRS 08020221900308900042 WA9002L606 $012 \mathrm{PH} \quad 9 R$ GRS 08020221900301900033 KA 9002L606 012 MEC6D82J GRS 08020221900301900033 WA9002L606 012 PBEB 2J GRS 08020221900301900033 WA9002L606 $012 \quad$ 12DCDA2J GRS $08020221900301900033 \mathrm{WA} 9002 \mathrm{~L} 606012 \mathrm{CH} 3 \mathrm{CL}$ 2JLT GRS 08020221900301900033 WA9002L606 012 CH3BR 2JLT GRS 08020221900301900033 WA9002L606 012 C2H3CL2JLT GRS 08020221900301900033 WA9002L606 012 C2H5CL2JLT GRS 08020221900301900033 WA9002L606 012 CH2CL22JB GRS 08020221900301900033 WA9002L606 012 ACET 2JB GRS 08020221900301900033 WA9002L606 012 CS2 2JLT GRS 08020221900301900033 WA9002L60.6 012 11DCE 2JLT GRS 08020221900301900033 WA9002L606 012 11DCLE2JLT GRS 08020221900301900033 \%A9002L606 012 T12DCE2JLT GRS 08020221900301900033 WA9002L606 012 CHCL3 2JLT GRS 08020221900301900033 WA9002L606 012 12DCLE2JLT GRS 08020221900301900033 WA9002L606 $012 \mathrm{MEK}$ 2JLT \% GRS 08020221900301900033 WA9002L606 012 111TCE2JLT $\stackrel{i}{\sim}$ GRS 08020221900301900033 WA9002L606 012 CCL4 2JLT $\leftarrow$ GRS 08020221900301900033 WA9002L606 012 VINYLA2JLT GRS. 08020221900301900033 WA9002L606 012 BRDCLM2JLT GRS $08020221900301900033 \mathrm{WA} 9002 \mathrm{~L} 606012$ 12DCLP2JLT GRS 08020221900301900033 WA9002L606 012 13DCPC2JLT GRS 08020221900301900033 WA9002L606 012 TRCLE 2JLT GRS 08020221900301900033 WA9002L606 012 DBRCLM2JLT GRS . 08020221900301900033 WA 90022606012 112TCE2JLT GRS 08020221900301900033 WA9002L606 012 C6H6 2JLT GRS $08020221900301900033 \mathrm{WA} 9002 \mathrm{~L} 606012$ T13DCP2JLT GRS 08020221900301900033 WA9002L606 012 CHBR3 2JLT GRS 08020221900301900033 WA9002L606 012 4ME2PE2JLT GRS 08020221900301900033 WA9002L606 012 2HXONE2JLT GRS 08020221900301900033 WA9002L606 012 TCLEE 2JLT GRS 08020221900301900033 WA9002L606 012 TCLEA 2JLT GRS 08020221900301900033 WA9002L606 012 MEC6H52JLT GRS 08020221900301900033 FA9002L606 012 CLC6H52JLT GRS $08020221900301900033 \mathrm{WA} 9002 \mathrm{~L} 606012$ ETC6H52JLT GRS 08020221900301900033 WA9002L606 012 STYR 2JLT GRS 08020221900301900033 FA9002L606 012 XYLEN 2JLT GRS $08020221900309900326 \mathrm{WA} 9002 \mathrm{~L} 606012$ NBD5 $1 \mathrm{X}$ .GRS $08020221900309900326 \mathrm{KA} 9002 \mathrm{~L} 606012$ 2FBP $1 \mathrm{X}$ GRS $0 B 020221900309900326$ WA9002L606 012 PTERP IX GRS 08020221900309900326 KA9002L606 012 PHEND51X
$7.9 \mathrm{PH} \mathrm{U}$

91.88

5. $5 \mathrm{MEQ1}$

$0.51 \mathrm{MEQ} 1$

5. OMEQ1

45 4 MGKG

4. $8 \mathrm{PH} \cdot \mathrm{U}$

108PER

$111 \mathrm{PER}$

10OPER

I2UGKG

12UGKG

12 UGKG

12UGKG

13 UGKG

4 9UGKG

6UGKG

6UGKG

6UGKG

6UGKG

6UGKG

6UGKG

12UGKG

6UGKG

6UGKG

12UGKG

GUGKG

6UGKG

6UGKG

6UGKG

6UGKG

6UGKG

6UGKG

6UGKG

6UGKG

12UGKG

12UGKG

6UGKG

6UGKG

6UGKG

6UGKG

6UGKG

6UGKG

6UGKG

65PER

77PER

- 94PER

80PER
10PHJH $10 \mathrm{JH}$ 1ONADKS IONAALM 1 IONAJS 1ODODKS $10 \mathrm{PHJH}$

1.0000MSJBD

$1.0000 M S J B D$

1.0000MSJBD

$1.0000 M S J B D$

$1.0000 M S J B D$

$1.0000 \mathrm{MSJBD}$

$1.0000 \mathrm{MSJBD}$

$1.0000 \mathrm{MSJBD}$

$1.0000 \mathrm{MSJBD}$

$1.0000 \mathrm{MSJBD}$

1. $0000 \mathrm{MSJBD}$

$1.0000 M S J B D$

$1.0000 M S J B D$

$1.0000 M S J B D$

$1.0000 \mathrm{MSJBD}$

$1.0000 \mathrm{MSJBD}$

$1.0000 \mathrm{MSJBD}$

$1.0000 \mathrm{MSJBD}$

$1.0000 \mathrm{MSJBD}$

$1.0000 \mathrm{MSJBD}$

$1.0000 \mathrm{MSJBD}$

1. $0000 \mathrm{MSJBD}$

$1.0000 M S J B D$

1. $0000 \mathrm{MSJBD}$

$1.0000 M S J B D$

1. $0000 \mathrm{MSJBD}$

$1.0000 \mathrm{MSJBD}$

$1.0000 \mathrm{MSJBD}$

$1.0000 \mathrm{MSJBD}$

$1.0000 \mathrm{MSJBD}$

1.0000MSJBD

$1.0000 M S J B D$

$1.0000 \mathrm{MSJBD}$

$1.0000 \mathrm{MSJBD}$

1. $0000 \mathrm{MSJBD}$

$1.0000 M S J B D$

$1.0000 \mathrm{MSJBD}$

1.0000MSDAR

1. 0000 MSDAR

$1.0000 M S D A R$

1.0000MSDAR

\section{$\mathrm{OPH}$}

08 SOLIDS

7 IRH N OCATION EXCHANGE CAPAICEC N OEXCHANGEABLE BASES ICECBN OEXCHANGEABLE ACIDS ICECTN OTOTAL ORGANIC CARBONIOCT N $\mathrm{OPH}$

58.000TOLUENE-D8

IPH N

58.000BROMOELUOROBENZENE O624HN

$58.0001,2-D I C H L O R O E T H A N E-D 0624 \mathrm{HN}$ OCHLOROMETHANE O624HN OBROMOMETHANE O624HN OVINYI CHLORIDE O624HN OCHLOROETHANE O624HN OMETHYLENE CHLORIDE O624HN OACETONE

OCARBON DISULEIDE O624HN

01,1-DICHLOROETHENE O624HN $01,1-D I C H L O R O E T H A N E$ O624HN 01,2-DICHLOROETHENE (0624HN OCHLOROFORM $0624 \mathrm{HN}$ 01,2-DICHLOROETHANE O624HN 02-BUTANONE O624HN $01,1,1$-TRICHLOROETHANO $624 \mathrm{HN}$ OCARBON TETRACHLORIDEO $624 \mathrm{HN}$ OVINYL ACETATE O624HN OBROMODICHLOROMETHANEO $624 \mathrm{HN}$ 01,2-DICHLOROPROPANE $0624 \mathrm{HN}$ OCIS-1, 3-DICHLOROPROPO624HN OTRICHLOROETHENE O624HN ODIBROMOCHLOROMETHANEO $624 \mathrm{HN}$ $01,1,2$-TRICHLOROETHANO $624 \mathrm{HN}$ OBENZENE O624HN OTRANS-1, 3-DICHLOROPRO624HN OBROMOFORM O624HN 04-METHYL-2-PENTANONEO624HN 02-HEXANONE O624HN OTETRACHLOROETHENE O624HN $01,1,2,2$-TETRACHLOROEO $624 \mathrm{HN}$ OTOLUENE O624HN OCHLOROBENZENE O62 $4 \mathrm{HN}$ OETHYLBENZENE O624HN OSTYRENE.$\quad 0624 \mathrm{HN}$ OXYLENE (TOTAL) O624HN 1869.370NITROBENZENE-D5 O625HN 1869.3702-ELUOROBIPHENYL O625HN 1869.370P-TERPHENYL-D14 0625HN 3738.740 OHENOL-D5 

$08020221900309900326 \mathrm{WA} 002 \mathrm{~L} 606012$ 246TBP $1 \mathrm{X}$ 08020221900309900326 HA9002L606 012 PHENOL1XLT $08020221900309900326 \mathrm{WA9002L606} 012$ B2CEE 1XLI $08020221900309900326 \mathrm{WA} 9002 \mathrm{~L} 606012$ 2CLP IXLT 08020221900309900326 WA9002L606 012 13DCLB1XLT $08020221900309900326 \mathrm{HA9002L606} 012$ 14DCLB1XLT $08020221900309900326 \mathrm{KA} 9002 \mathrm{~L} 606012$ BZALC IXLT $08020221900309900326 \mathrm{FA} 9002 \mathrm{~L} 606012$ 12DCLB1XLT $08020221900309900326 \mathrm{WA} 9002 \mathrm{~L} 606012$ 2MP IXLT $08020221900309900326 \mathrm{FAg} 002 \mathrm{~L} 606012$ B2CIPEIXLT $08020221900309900326 \mathrm{WA} 9002 \mathrm{~L} 606 \quad 012$ 4MP 1XLT 08020221900309900326 WA9002L606 012 NDNPA 1XLT $08020221900309900326 \mathrm{WA} 9002 \mathrm{~L} 606012$ CL6ET 1XLT $08020221900309900326 \mathrm{WA9} 002 \mathrm{~L} 606012 \mathrm{NB} \quad$ IXLT $08020221900309900326 \mathrm{FA} 9002 \mathrm{~L} 606012$ ISOPHR1XLT $08020221900309900326 \mathrm{WA} 9002 \mathrm{~L} 606012$ 2NP 1XLT 08020221900309900326 HA9002L606 012 24DMPNIXLT $08020221900309900326 \mathrm{FA} 9002$ L606 012 BENZOA1XLT 08020221900309900326 WA9002L606 012 B2CEXM1XLT $0802 \cdot 0221900309900326 \mathrm{WA9} 002 \mathrm{~L} 606012$ 24DCLP1XLT 08020221900309900326 WA9002L606 012124 TCB1XLT 08020221900309900326 WA9002L606 012 NAP 1XIT $08020221900309900326 \mathrm{WA} 9002 \mathrm{~L} 606012$ 4CANILIXLT $08020221900309900326 \mathrm{WAg} 002 \mathrm{~L} 606012 \mathrm{HCBD} 1 \mathrm{XLT}$ 08020221900309900326 WA9002L606 012 4CL3C 1XLT 08020221900309900326 WA9002L606 012 2MENAP 1XLT 08020221900309900326 WA9002L606 $012 \mathrm{CL} 6 \mathrm{CP}$ 1XLT 08020221900309900326 WA9002L606 012 246TCP 1XLT $08020221900309900326 \mathrm{WA} 002 \mathrm{~L} 606012$ 245TCP1XLT 08020221900309900326 WA9002L606 012 2CNAP 1XLT $08020221900309900326 \mathrm{WA} 9002 L 606012$ 2NANIL1XLT $08020221900309900326 \mathrm{WA} 0024606012$ DMP 1XLT $08020221900309900326 \mathrm{WA} 9002 \mathrm{~L} 606012$ ANAPYL1XLT $08020221900309900326 \mathrm{FAg} 002 \mathrm{~L} 606012$ 26DNT IXLT 08020221900309900326 WA9002L606 012 3NANIL1XLT $08020221900309900326 \mathrm{WA} 9002 \mathrm{~L} 606012$ ANAPNE1XLT $08020221900309900326 \mathrm{WA} 9002 \mathrm{~L} 606012$ 24DNP 1XLT 08020221900309900326 KA9002L606 012 4NP 1XLT 08020221900309900326 WA9002L606 012 DIBZFU1XIT 08020221900309900326 WA9002L606 012 24DNT 1XLT $08020221900309900326 \mathrm{FA9002L606} 012$ DEP 1XLT $08020221900309900326 \mathrm{WA9} 002 \mathrm{~L} 606012$ 4CLPRE1XLT $08020221900309900326 \mathrm{WA} 9002 \mathrm{~L} 606012$ FLRENE1XLT $08020221900309900326 \mathrm{HA} 9002 \mathrm{~L} 606012$ 4NANIL1XLT $08020221900309900326 \mathrm{WA} 9002 \mathrm{~L} 606012$ 46DN2CIXLT $08020221900309900326 \mathrm{WA} 90024606012$ NNDPA 1XLT 08020221900309900326 HA9002L606 012 4BRPPE1XLT $\triangle 1$ PER

75PER 42 OUGKG 420 UGKG 42 OUGKG 42 OUGKG 42 OUGKG 42 OUGKG 42 UUGKG 42 OUGKG 420 UGKG 42 OUG̣KG 42 OUGKG 42 OUGKG 42 OUGKG 42 OUGKG 420UGKG 420 UGKG

2100 UGKG 42 OUGKG 420 UGKG 42 OUGKG 42 OUGKG 42 OUGKG 42 OUGKG 42 OUGKG 42 OUGKG 42 OUGKG $4200 G K G$

2100 UGKG 420UGKG 210 OUGKG 420UGKG 420 UGKG 420UGKG 2100 UGKG $4200 G K G$ 2100 UGKG 2100 UGKG 420UGKG 420UGKG 42 UUGKG 420 UGKG 420UGKG 2100 UGKG 2100 UGKG 420UGKंG 420 UGKG
1. $0000 \mathrm{MSDAR}$

1. 0000MSDAR

1. $0000 M S D A R$

1. $0000 M S D A R$

1.0000MSDAR

1.0000MSDAR

1. 000OMSDAR

1. 0000MSDAR

1.0000MSDAR

1.0000MSDAR

1.0000MSDAR

1. 0000MSDAR

1.0000MSDAR

1.0000MSDAR

1. 0000MSDAR

1. 0000MSDAR

1.0000MSDAR

1.0000MSDAR

1. 0000MSDAR

1. 0000MSDAR

1.0000MSDAR

1. 0000MSDAR

1. 0000MSDAR

1.0000MSDAR

1.000OMSDAR

1. 0000MSDAR

1.000OMSDAR

$1.0000 M S D A R$

1. 0000MSDAR

1. 000OMSDAR

1.0000MSDAR

1. 0000MSDAR

1.000OMSDAR

1. $0000 \mathrm{MSDAR}$

1.000OMSDAR

1. 0000MSDAR

1. 0000MSDAR

1.0000MSDAR

1. 000OMSDAR

1. 000OMSDAR

1. 000OMSDAR

1. 000OMSDAR

1. 0000MSDAR

1.000OMSDAR

1. 0000MSDAR

1. O0OOMSDAR

1. O0OOMSDAR 1. $0000 \mathrm{MSDAR}$
3738.7402-FLUOROPHENOL $0625 \mathrm{HN}$ $3738.7402,4,6-T R I B R O M O P H E N O T 0625 \mathrm{HN}$ OPHENOT $0625 \mathrm{HN}$ OBIS (2-CHLOROETHYL) ETO625HN 02-CHLOROPHENOT 0625HN 01, 3-DICHLOROBENZENE 0625HN 01, 4-DICHIOROBENZENE 0625HN OBENZYY AICOHOI 0625HN 01,2-DICHLOROBENZENE 0625HN 02-METHYLPHENOL 0625HN OBIS 12-CHIOROISOPROPYO625HN 04-METHYLPHENOL 0625HN ON-NITROSO-DI-N-PROPYO625HN OHEXACHLOROETHANE O625HN ONITROBENZENE O625HN OISOPHORONE O625HN 02-NITROPHENOT, 0625HN 02,4-DIMETHYLPHENOL 0625HN OBENZOIC ACID 0625HN OBIS (2-CHIOROETHOXY) MO625HN 02, 4-DICHLOROPHENOL 0625HN $01,2,4-T R I C H L O R O B E N Z E 0625 \mathrm{HN}$ ONAPHTHALENE O625HN 04-CHLOROANILINE 0625HN OHEXACHLOROBUTADIENE O625HN 04-CHLORO-3-METHYLPHEO 625HN 02-METHYLNAPHTHALENE 0625HN OHEXACHLOROCYCLOPENTAO $625 \mathrm{HN}$ 02, 4, 6-TRICHLOROPHENOO625HN $02,4,5-T R I C H L O R O P H E N O O 625 \mathrm{HN}$ 02-CHLORONAPHTHALENE 0625HN 02-NITROANILINE 0625HN ODIMETHYLPHTHALATE O625HN OACENAPHTHYLENE 02,6-DINITROTOLUENE 0625HN 03-NITROANIIINE OACENAPHTHENE O625HN 02, 4-DINITROPHENOL 0625HN 04-NITROPHENOL O625HN ODIBENZOFURAN O625HN 02, 4-DINITROTOLUENE 0625HN ODIETHYLPHTHALATE O625HN 0.4-CHLOROPHENYI-PHENYO 625HN OFLUORENE $\because$ O625HN 04-NITROANILINE O625HN 04, 6-DINITRO-2-METHYLO625HN ON-NITROSODIPHENYLAMIO625HN 04-BROMOPHENYL-P HENYLO $625 \mathrm{HN}$ 
GRS $08020221900309900326 \mathrm{WA} 9002 \mathrm{~L} 606012$ CL6BZ 1XLT GRS 08020221900309900326 WA9002L606 012 PCP 1XLT GRS 08020221900309900326 WA9002L606 012 PHANTR1XLT GRS 08020221900309900326 WA9002L606 012 ANTRC 1 XLT GRS $08020221900309900326 \mathrm{WA} 9002 \mathrm{~L} 606012$ DNBP 1XLT GRS 08020221900309900326 WA9002L606 012 FANT 1XLT GRS 08020221900309900326 WA9002L606 012 PYR 1XLT GRS 08020221900309900326 WA9002L606 012 BBZP 1XLT GRS 08020221900309900326 WA9002L606 012 33CL2B1XLT GRS 08020221900309900326 WA9002L606 012 BAANTR1XLT GRS 08020221900309900326 WA9002L606 012 CHRY 1XLT GRS 08020221900309900326 WA9002L606 012 B2EHP $1 X$ GRS 08020221900309900326 WA9002L606 012 DNOP 1XLT GRS 08020221900309900326 WA9002L606 012 BF2ANT1XLT GRS 08020221900309900326 WA9002L606 012 BKFANTIXLT GRS 08020221900309900326 WA9002L606 012 BAPYR 1XLT GRS 08020221900309900326 WA9002L606 012 ICDPYR1XLT GRS 08020221900309900326 WA9002L606 012 DBAHA 1XLT GRS 08020221900309900326 WA9002L606 012 BGHIPY1XLT GRS 08030221900308900039 WA9002L606 013

GRS 08030221900315900002 WA9002L606 013 CEC EX GRS 08030221900312900002 WA9002L606 013 CECB EX GRS 08030221900308900002 WA9002L606 013 CECT EX 08030221900302900002 WA9002L606 013 TOC $9 I$ 08030221900308900042 WA9002L606 $013 \mathrm{PH} 9 \mathrm{R}$ 08030221900302900033 WA9002L606 013 MEC6D82J 08030221900302900033 WA9002L606 013 PBFB 2J 08030221900302900033 WA9002L606 013 12DCD42J 0803 O221900302900033WA9002L606 013 CH3CL 2JLT $08030221900302900033 W A 9002 L 606013$ CH3BR 2JLT 08030221900302900033 WA9002L606 013 C2H3CL2JLT $08030221900302900033 \% A 9002 L 606013$ C2H5CL2JLT 08030221900302900033 WA9002L606 013 CH2CL22JB 08030221900302900033 WA9002L606 013 ACET 2JB 08030221900302900033 WA9002L606 013 CS2 2JLT $0803 \cdot 0221900302900033 W A 9002 L 606013$ 11DCE 2JLT 08030221900302900033 WA9002L606 013 11DCLE2JLT 08030221900302900033 WA9002L606 013 T12DCE2JLT 08030221900302900033 W9002L606 013 CHCL3 2JLT 08030221900302900033 WA9002L606 013 12DCLE2JLT 08030221900302900033 WA9002L606 013 MEK 2JLT $08030221900302900033 \% A 9002 L 606013$ 111TCE2JLT 08030221900302900033 WA9002L606 013 CCL4 2JLT 08030221900302900033 WA9002L606 013 VINYLA2JLT 08030221900302900033 WA9002L606 013 BRDCLM2JLT 08030221900302900033 WA9002L606 013 12DCLP2JLT 08030221900302900033 WA9002L606 013 13DCPC2JLT 08030221900302900033 WA9002L606 013 TRCLE 2JLT
420 UGKG

2100 UGKG

420 UGKG

420 UGKG

420 UGKG

42 OUGKG

420 UGKG

42 OUGKG

830 UGKG

42 OUGKG

42 OUGKG

$6800 U G K G$

42 OUGKG

420 UGKG

42 OUGKG

42 OUGKG

42 OUGKG

420 UGKG

420 UGKG

$91.8 \%$

3. 4MEQ1

0 . $23 \mathrm{MEQ1}$

3. $2 \mathrm{MEQ1}$

292MGKG

5. OPH U

102PER

108PER

102PER

11 UGKG

11UGKG

11 UGKG

11 UGKG

15UGKG

61UGKG

6UGKG

6UGKG

6UGKG

6UGKG

6UGKG

6UGKG

11UGKG

6UGKG

6UGKG

11UGKG

6UGKG

6UGKG

6UGKG 6UGKG
1.000OMSDAR

1.0000MSDAR

1.0000MSDAR

1. $0000 M S D A R$

1. OOOOMSDAR

1. $0000 M S D A R$

1.0000MSDAR

1. $0000 M S D A R$

1. $0000 M S D A R$

1. $0000 M S D A R$

$1.0000 M S D A R$

1.000OMSDAR

1.000OMSDAR

1.000OMSDAR

1.0000MSDAR

1.0000MSDAR

1.0000MSDAR

1.0000MSDAR

1.000OMSDAR $10 \mathrm{JH}$ 10NADKS IONAALM 1 IONAJS 10DODKS $10 \mathrm{PHJH}$

$1.0000 \mathrm{MSJBD}$

$1.0000 \mathrm{MSJBD}$

$1.0000 \mathrm{MSJBD}$

1. 0000MSJBD

$1.0000 \mathrm{MSJBD}$

1. $0000 \mathrm{MSJBD}$

1.0000MSJBD

1. $0000 \mathrm{MSJBD}$

$1.0000 \mathrm{MSJBD}$

1. $0000 \mathrm{MSJBD}$

1. $0000 \mathrm{MSJBD}$

1. $0000 \mathrm{MSJBD}$

1.0000MSJBD

$1.0000 \mathrm{MSJBD}$

$1.0000 \mathrm{MSJBD}$

1. $0000 \mathrm{MSJBD}$

$1.0000 \mathrm{MSJBD}$

$1.0000 M S J B D$

1. $0000 \mathrm{MSJBD}$

$1.0000 \mathrm{MSJBD}$

1.0000MSJBD

1.0000MSJBD

$1.0000 \mathrm{MSJBD}$
OHEXACHLOROBENZENE OPENTACHLOROPHENOL OPHENANTHRENE

OANTHRACENE

$0625 \mathrm{HN}$

$0625 \mathrm{HN}$

$0625 \mathrm{HN}$

ODI-N-BUTYLPHTHATATE O625HN

OFLUORANTHENE $0625 \mathrm{HN}$

OPYRENE 0625HN

OBUTY.LBENZYLPHTHALATE0625HN

$03,3^{\prime}$-DICHLOROBENZ IDIO $625 \mathrm{HN}$ OBENZO (A) ANTHRACENE 0625HN OCHRYSENE

$0625 \mathrm{HN}$

OBIS (2-ETHYLHEXYL) PHTO625HN ODI-N-OCTYI PHTHALATEO625HN OBENZO (B) ELUORANTHENEO625HN OBENZO (K) FLUORANTHENE0625HN OBENZO (A) PYRENE O625HN OINDENO $(1,2,3-C D)$ PYREO $625 \mathrm{HN}$ ODIBENZO $(A, H)$ ANTHRACEO625HN OBENZO (G, H, I) PERYLENEO625HN O\& SOLIDS. I\&SOLN OCATION EXCHANGE CAPAICEC N OEXCHANGEABLE BASES ICECBN OEXCHANGEABLE ACIDS ICECTN OTOTAI ORGANIC CARBONIOCT N $\mathrm{OPH}$ IPH N 56.000TOLUENE-D8 56.000BROMOFLUOROBENZENE O624HN $56.0001,2-D I C H L O R O E T H A N E-D O 624 \mathrm{HN}$ OCHLOROMETHANE OBROMOMETHANE O624HN OVINYL CHLORIDE O624HN OCHLOROETHANE O624HN OMETHYLENE CHLORIDE O624HN OACETONE O624HN OCARBON DISULEIDE O624HN 01,1-DICHLOROETHENE O624HN 01,1-DICHLOROETHANE 0624HN 01,2-DICHLOROETHENE 10624HN OCHLOROFORM O624HN 01,2-DICHLOROETHANE O624HN 02-BUTANONE 0624HN $01,1,1$-TRICHLOROETHANO $624 \mathrm{HN}$ OCARBON TETRACHLORIDEO624HN OVINYL ACETATE $\because 0624 \mathrm{HN}$ OBROMODICHLOROMETHANEO624HN 01,2-DICHLOROPROPANE 0624HN OCIS-1, 3-DICHLOROPROPO624HN OTRICHIOROETHENE 
GRS 08 J221900302900033HA90021606 013 DBRCLM2JLT GRS 08030221900302900033 WA9002L606 0.13 112TCE2JLT GRS 0803.0221900302900033 HA9002L606 013 C6H6 2JLT GRS $08030221900302900033 \% A 9002 L 606013$ T13DCP2JLT GRS 08030221900302900033 HA9002L606 013 CHBR3 2JLT GRS 08030221900302900033 HA9002L606 013 4ME2PE2JLT GRS 08030221900302900033 WA9002L606 013 2HXONE2JLT GRS 08030221900302900033 WA9002L606 013 TCLEE 2JLT GRS 08030221900302900033 HA9002L606 013 TCLEA 2JLT GRS 08030221900302900033 WA9002L606 013 MEC6H52JLT GRS 08030221900302900033 WA9002L606 013 CLC6H52JLT GRS 08030221900302900033 HA9002L606 013 ETC6H52JLT GRS $08030221900302900033 W A 9002 \mathrm{~L} 606013$ STYR 2JLT GRS $0803 \cdot 0221900302900033$ WA9002L606 013 XYLEN 2JLT GRS 08030221900309900326 WA9002L606 013 NBD5 IX GRS 08030221900309900326 WA9002L606 013 2FBP $1 X$ GRS 08030221900309900326 WA9002L606 013 PTERP 1X GRS 08030221900309900326 WA9002L60.6 013 PHEND51X GRS 08030221900309900326 WA9002L606 $013 \quad 2 \mathrm{FP} \quad 1 \mathrm{X}$ GRS $08030221900309900326 \mathrm{WA} 9002 L 606013 \quad 246 T B P 1 X$ GRS 08030221900309900326 WA9002L606 013 PHENOLIXLT GRS 08030221900309900326 WA9002L606 013 B2CEE 1XLT GRS 08030221900309900326 WA9002L606 013 2CLP 1XLT

08030221900309900326 WA9002L606 013 13DCLBIXLT $08030221900309900326 \mathrm{WA} 9002 L 606013$ 14DCLB1XLT $08030221900309900326 \mathrm{WA} 9002 \mathrm{~L} 606013$ B2ALC 1XLT $08030221900309900326 \mathrm{WA} 9002 \mathrm{~L} 606013$ 12DCLB1XLT $08030221900309900326 \mathrm{WA} 9002 \mathrm{~L} 606013$ 2MP 1XLT $08030221900309900326 \mathrm{WA} 9002 \mathrm{~L} 606013$ B2CIPE1XLT $08030221900309900326 \mathrm{WA} 9002 \mathrm{~L} 606013$ 4MP 1XLT 08030221900309900326 WA9002L606 013 NDNPA 1XLT 08030221900309900326 WA9002L606 013 CL6ET 1XLT 08030221900309900326 WA9002L606 013 NB 1XLT $08030221900309900326 \mathrm{WA} 9002 \mathrm{~L} 606013$ ISORHR1XLT $08030221900309900326 \mathrm{WA} 002 \mathrm{~L} 606013$ 2NP 1XLT $08030221900309900326 \mathrm{HA9002L606} 013$ 24DMPN1XIT 08030221900309900326 WA9002L606 013 BENZOA 1 XLT 08030221900309900326 WA9002L606 013 B2CEXM1XLT 08030221900309900326 WA9002L606 013 24DCLP1XLT 08030221900309900326 HA9002L606 013 124TCB1XIT $08030221900309900326 \mathrm{FA9002L606} 013 \mathrm{NAP}$ 1XLT $08030221900309900326 \mathrm{WA9002L606} 013$ 4CANIL1XLT $08030221900309900326 \mathrm{WA} 002 \mathrm{~L} 606013 \mathrm{HCBD} 1 \mathrm{XLT}$ $08030221900309900326 \mathrm{FA9002L606} 013$ 4CL3C 1XLT $08030221900309900326 \mathrm{WA9002L606} 013$ 2MENAP IXLT $08030221900309900326 \mathrm{KA} 9002 \mathrm{~L} 606013 \mathrm{CL} 6 \mathrm{CP}$ IXLT 08030221900309900326 HA9002L606 013 246TCP 1XLT $08030221900309900326 \mathrm{WA} 9002 \mathrm{~L} 606013245 \mathrm{TCP} 1 \mathrm{XLT}$
UUGKG

6UGKG

6UGKG

6UGKG

6UGKG

IIUGKG

IIUGKG

6UGKG

6UGKG

6UGKG

6UGKG

6UGKG

6UG่KG

6UGKG

69PER

85PER

102PER

8OPER

69RER

47PER

42 UUGKG

42 OUGKG

420 UGKG

420 UGKG

420 UGKG

420 UUKG

42 OUGKG

420UGKG

42 OUGKG

420UGKG

420UGKG

42 OUGKG

420UGKG

42 UUGKG

42 OUGKG

420UGKG

2100 UGKG

420UGKG

42 OUGKG

420UGKG

42 OUGKG

420UGKG

420 UGKG

420UGKG

420UGKG

420UGKG

420UGKG

2100UGKG
I. $0000 \mathrm{MSJBD}$ $1.0000 \mathrm{MSJBD}$ 1. 0000MSJBD 1. $0000 \mathrm{MSJBD}$ 1. $0000 \mathrm{MSJBD}$ 1. 0000MSJBD 1. $0000 \mathrm{MSJBD}$ 1. $0000 \mathrm{MSJBD}$ 1. 0000MSJBD 1. $0000 \mathrm{MSJBD}$ 1. $0000 \mathrm{MSJBD}$ 1. $0000 \mathrm{MSJBD}$ 1. 0000MSJBD 1.0000MSJBD 1. 0000MSDAR 1. O000MSDAR 1.0000MSDAR 1. 0000MSDAR 1.0000MSDAR 1.0000MSDAR 1. 0000MSDAR 1.0000MSDAR 1.0000MSDAR 1. $0000 \mathrm{MSDAR}$ 1. 0000MSDAR 1.0000MSDAR 1. 0000MSDAR 1.0000MSDAR 1.0000MSDAR 1.0000MSDAR 1.000OMSDAR 1. 0000MSDAR 1.000OMSDAR 1. 000OMSDAR 1. 0000MSDAF 1.0000MSDAR 1.0000MSDAR 1.0000MSDAR 1. 0000MSDAR 1. 0000MSDAR 1.0000MSDAR 1.0000MSDAR 1. 0000MSDAR 1.0000MSDAR 1. 0000MSDAR 1. OOOOMSDAR 1. 0000MSDAR 1. 000OMSDAR
ODIBROMOCHIOROMETHANEO624HN $01,1,2-T R I C H L O R O E T H A N O 624 \mathrm{HN}$ OBENZENE $0624 \mathrm{HN}$ OTRANS-1, 3-DICHLOROPRO 624HN OBROMOFORM $0624 \mathrm{HN}$ O4-METHYL-2-PENTANONEO624HN 02-HEXANONE O O624HN OTETRACHLIOROETHENE 0624HN $01,1,2,2$-TETRACHLOROEO $624 \mathrm{HN}$ OTOLUENE OCHIOROBENZENE OETHYII OSTYRENE

OXYLENE (TOTAL)

1873.875NITROBENZENE-D5 1873.8752-FLUOROBIPHENYL 1873.875 P-TERPHENYL-D1 4 $0624 \mathrm{HN}$ $0624 \mathrm{HN}$ O625HN O625HN $0625 \mathrm{HN}$ 3747 . 749PHENOL-D5 $0625 \mathrm{HN}$ $3747.7492-$ ELUOROPHENOI O625HN $3747.7492,4,6$-TRIBROMOPHENOLO $625 \mathrm{HN}$ OPHENOL O625HN OBIS (2-CHLOROETHYL) ETO625HN 02-CHLOROPHENOL O625HN 01,3-DICHLOROBENZENE 0625HN 01, 4-DICHLOROBENZENE O625HN OBENZYL ALCOHOL O625HN 01,2-DICHLOROBENZENE 0625HN 02-METHYLPHENOL O625HN OBIS (2-CHIOROISOPROP YO 625HN 04-METHYLPHENOL O625HN ON-NITROSO-DI-N-PROPYO625HN OHEXACHLOROETHANE O625HN ONITROBENZENE O625HN OISOPHORONE $0625 \mathrm{HN}$ 02-NITROPHENOL $0625 \mathrm{HN}$ 02, 4-DIMETHYLPHENOL 0625HN OBENZOIC ACID O625HN OBIS (2-CHLOROETHOXY) MO625HN $02,4-D I C H L O R O P H E N O L$ O625HN $01,2,4$-TRICHLOROBENZEO $625 \mathrm{HN}$ ONAPHTHALENE $0625 \mathrm{HN}$ 04-CHLOROANILINE 0625HN OHEXACHLOROBUTADIENE 0625HN 04-CHLORO-3-METHYLPHEO625HN 02-METHYLNAPHTHALENE 0625HN OHEXACHLOROCYCLORENTAO $625 \mathrm{HN}$ $02,4,6-T R I C H L O R O P H E N O 0625 \mathrm{HN}$ $02,4,5-T R I C H L O R O P H E N O O 625 \mathrm{HN}$ 
GRS $08030221900309900326 \mathrm{WA} 9002 L 606013$ 2CNAP 1XLT GRS 08030221900309900326 WA9002L606 013 2NANIL1XLT GRS 08030221900309900326 WA9002L606 013 DMP 1XLT GRS $08030221900309900326 \mathrm{HA9002L606} 013$ ANAPYL1XLT GRS 08030221900309900326 WA9002L606 013 26DNT 1XLT GRS $08030221900309900326 \mathrm{WA} 9002 \mathrm{~L} 606013$ 3NANIL1XLT GRS $08030221900309900326 \mathrm{WA} 9002 \mathrm{~L} 606013$ ANAPNE $1 \times L T$ GRS $08030221900309900326 \mathrm{WA} 9002 \mathrm{~L} 606013$ 24DNP 1XLT GRS $08030221900309900326 \mathrm{WA} 9002 \mathrm{~L} 606013$ 4NP . 1XLT GRS 08030221900309900326 WA9002L606 013 DIBZEULXLT GRS $08030221900309900326 \mathrm{WA} 9002 \mathrm{~L} 606 \quad 013$ 24DNT 1XLT GRS 08030221900309900326 WA9002L606 01.3 DEP 1XLT GRS $08030221900309900326 \mathrm{WA} 9002 \mathrm{~L} 606013$ 4CLPPE IXLT GRS 08030221900309900326 WA9002L606 013 FLRENE1XLT GRS 08030221900309900326 WA9002L606 013 4NANIL1XLT GRS 08030221900309900326 WA9002L606 013 46DN2C1XLT GRS $08030221900309900326 \mathrm{WA} 9002 \mathrm{~L} 606013$ NNDRA 1XLT GRS $08030221900309900326 \mathrm{WA} 9002 \mathrm{~L} 606013$ 4BRPPE1XIT GRS $08030221900309900326 \mathrm{WA} 9002 \mathrm{~L} 606 \quad 013 \mathrm{CL} 6 \mathrm{BZ}$ 1XLT GRS 08030221900309900326 HA9002L606 013 PCP 1XLT GRS 08030221900309900326 WA9002L606 013 PHANTR1XLT GRS $08030221900309900326 \mathrm{WA} 90021606013$ ANTRC 1XLT GRS 08030221900309900326 WA9002L606 013 DNBP 1XLT GRS $08030221900309900326 \mathrm{WA9002L606} 013$ FANT 1XLT G GRS $08030221900309900326 \mathrm{WA} 90024606013$ PYR 1XLT $N$ GRS 08030221900309900326 WA9002L606 013 BBZP 1XLT $N$ GRS 08030221900309900326 WA9002L606 013 33CL2B1XLT GRS 08030221900309900326 WA9002L606 013 BAANTRIXLT GRS 08030221900309900326 HA9002L606 013 CHRY 1XLT GRS 08030221900309900326 WA9002L606 013 B2EHP $1 X$ GRS 08030221900309900326 WA9002L606 013. DNOP 1XIT GRS $08030221900309900326 \mathrm{WH} 9002 L 606013$ BE2ANT1XLT GRS $08030221900309900326 \mathrm{WA} 90024606013$ BKFANTIXLT GRS 08030221900309900326 WA9002L606 013 BAPYR 1XLT GRS 08030221900309900326 WA9002L606 013 ICDPYRIXLT GRS 0803. 0221900309900326WA9002L606 013 DBAHA 1XLT GRS 08030221900309900326 WA9002L606 013 BGHIPY1XLT GRS 0803E0221900327901200WA9002L606 014 ALPHAG9W GRS O803E0221900327901200WA9002L606 014 BETAG 9W GRS 0803E0221900323900219WA9002L606 014 AG 2ZLT GRS 0803E0221900330900218WA9002L606 014 AS 1BLT GRS 0803E0221900323900219WA9002L606 014 BA 2ZLT GRS 0803E0221900323900219WA9002L606 014 CD 2ZLT GRS 0803E0221900323900219FA9002L606 014 CR 2ZLT GRS 0803E0221900319900042WA9002L606 014 HGTOT IDLT GRS 0803E0221900405900218WA9002L606:014 PB 1BLT GRS 0803E0221900402900218WA9002L606 014 SE 1BLT GRS
420 UGKG

2100 UGKG 42 UUGKG 420UGKG 420 UGKG

2100 UGKG

42 OUGKG

2100 UGKG

2100 UGKG

420 UGKG

420 UGKG

42 OUGKG

42 UUGKG

42 OUGKG

2100 UGKG

2100 UGKG

42 OUGKG

420 UGKG

420UGKG

2100UGKG

420 UGKG

42 OUGKG

420 UGKG

420 UGKG

420UGKG

42 OUGKG

830 UGKG

420UGKG

42 OUGKG

3700UGKG

42 OUGKG

420 UGKG

42 OUGKG

420UGKG

42 OUGKG

420UGKG

420 UGKG

0.0 PPCIL 2.00

0.00 PCIL 3.00

10. OUGL

10.0 UGL

200UGL

5. OUGL

10. OUGL

0.20 UGL

3. OUGL

5. OUGİ

110RER
1. 000OMSDAR

1. 0000MSDAR

1.000OMSDAR

1. 0000MSDAR

1. 000OMSDAR

1. 000OMSDAR

1.0000MSDAR

1. OOOOMSDAR

1.0000MSDAR

1.0000MSDAR

1. 0000MSDAR

1. 0000MSDAR

1. 0000MSDAR

1. 0000MSDAR

1. $0000 \mathrm{MSDAR}$

1. 000OMSDAR

1. 0000MSDAR

1.0000MSDAR

1. $0000 \mathrm{MSDAR}$

1. 0000MSDAR

1. 0000MSDAR

1.0000MSDAR

1.000OMSDAR

1. 0000MSDAR

1. OOOOMSDAR

1. 0000MSDAR

1. $0000 \mathrm{MSDAR}$

1.0000MSDAR

1: 000OMSDAR

1.0000MSDAR

1. 0000MSDAR

1. 0000MSDAR

1. 0000MSDAR

1. 0000MSDAR

1. OOOOMSDAR

1.0000MSDAR

1. 0000MSDAR 1OEBUNK 10EBUNK

1. OOICALM

1. 00AAAER

1. OOICATM

1. OOICAIM

1.00ICALM

1. 0OHGSQ

1. 00AACMT

1. 00AASSQ 1. $0000 \mathrm{MSBB}$
02-CHLORONAPHTHALENE 0625HN 02-NITROANILINE $0625 \mathrm{HN}$ ODIMETHYLPHTHALATE $0625 \mathrm{HN}$ OACENAPHTHYLENE 0625HN 02,6-DINITROTOLUENE 0625HN 03-NITROANIIINE $0625 \mathrm{HN}$ OACENAPHTHENE O625HN $02,4-D I N I T R O P H E N O L$ O625HN 04-NITROPHENOL ODIBENZOFURAN 0625HN 02,4-DINITROTOLUENE 0625HN ODIETHYLPHTHALATE O625HN 04-C̈HLOROPHENYL-P HENYO625HN OFLUORENE O625HN 04-NITROANIIINE O625HN 04,6-DINITRO-2-METHYLO625HN ON-NITROSODIPHENYLAMIO625HN 04-BROMOPHENYL-PHENYLO625HN OHEXACHLOROBENZENE O625HN OPENTACHLOROPHENOL 0625HN OPHENANTHRENE O625HN OANTHRACENE O625HN ODI-N-BUTYIPHTHALATE O625HN OFLUORANTHENE O625HN ORYRENE O625HN OBUTYLBENZYIPHTHALATEO625HN $03,3^{\prime}$-DICHLOROBENZ ID IO $625 \mathrm{HN}$ OBENZO (A) ANTHRACENE O625HN OCHRYSENE $0625 \mathrm{HN}$ OBIS (2-ETHYLHEXYL) PHTO625HN ODI-N-OCTYL PHTHALATEO625HN OBENZO (B) FLUORANTHENEO625HN OBENZO (K) ELUORANTHENEO625HN OBENZO (A) PYRENE O625HN OINDENO $(1,2,3-C D)$ PYREO $625 \mathrm{HN}$ ODIBENZO (A, H) ANTHRACEO625HN OBENZO (G; H, I) PERYLENEO625HN OGROSS ALPHA IALPHN ONONVOLATILE BETA IBETAN OSILVER, TOTAL MAGTON OARSENIC, TOTAL MASTON OBARIUM, TOTAL MBATON OCÄDMIUM, TOTAL MCDTON OCHROMIUM, TOTAL : MCRTON OMERCURY, TOTAL MHGTON OLEAT, TOTAL MPBTON OSELENIUM, TOTAL MSETON 50.000TOLUENE-D8 : 
GRS 08น_N0221900302900035WA9002L606 014 PBEB 2J GRS 0803E0221900302900035WA9002L606 014 12DCD42J

GRS 0803E0221900302900035WA9002L606 014 CH3CL 2JLT

GRS 0803E0221900302900035WA9002L606 014 CH3BR 2JLT

GRS 0803E0221900302900035WA9002L606 014 C2H3CL2JLT

GRS 0803E0221900302900035WA9002L606 014 C2H5CL2JLT

GRS 0803E0221900302900035WA9002L606 014 CH2CL22JLT

GRS 0803E0221900302900035WA9002L606 014 ACET 2JB

GRS 0803E0221900302900035WA9002L606 014 CS2 . 2JLT

GRS 0803E0221900302900035WA9002L606 014 11DCE 2JLT

GRS 0803E0221900302900035WA9002L606 014 11DCLE2JLT

GRS 0803E0221900302900035WA9002L606 014 T12DCE்2JLT

GRS 0803E0221900302900035WA9002L606 014 CHCL3 2JLT

GRS 0803E0221900302900035WA9002L606 014 12DCLE2JLT

GRS 0803E0221900302900035WA9002L606 014 MEK 2JLT

GRS 0803E0221900302900035WA9002L606 014 111TCE2JLT

GRS 0803E0221900302900035WA9002L606 014 CCL4 2JLT

GRS 0803E0221900302900035WA9002I606 014 VINYIA2JIT

GRS 0803E0221900302900035WA9002L606 014 BRDCLM2JLT

GRS 0803E0221900302900035WA9002L606 014 12DCLP2JLT

GRS 0803E0221900302900035WA9002L606 014 13DCPC2JLT

GRS 0803E0221900302900035WA9002L606 014 TRCLE 2JLT

GRS 0803E0221900302900035WA9002L606 014 DBRCLM2JLT

চ̛GRS 0803E0221900302900035WA9002L606 014 112TCE2JLT

NGGS 0803E0221900302900035WA9002L606 014 C6H6 2JLT

wGRS 0803E0221900302900035WA9002L606 014 T13DCP2JLT

GRS 0803E0221900302900035HA9002L606 014 CHBR3 2JIT

GRS 0803E.0221900302900035WA9002L606 014 4ME2PE2JLT

GRS 0803E0221900302900035WA9002L606 014 2HXONE2JLT

GRS 0803E0221900302900035WA9002L606 014 TCLEE 2JLT

GRS 0803E0221900302900035WA9002L606 014 TCLEA 2JLT

GRS 0803E0221900302900035WA9002L606 014 MEC6H52JLT

GRS 0803E0221900302900035WA9002I606 014 CLC6H52JLT

GRS 0803E0221900302900035WA90021606 014 ETC6H52JLT

GRS 0803E0221900302900035WA9002L606 014 STYR 2JLT

GRS 0803E0221900302900035WA9002L606 014 XYLEN 2JLT

GRS 0001B0221900302900035WA9002L606 015 MEC6D82J

GRS 0001B0221900302900035WA9002I606 015 PBFB 2J

GRS 0001B0221900302900035WA9002L606 015 12DCD42J

GRS 0001B0221900302900035WA9002L606 015 CH3CL 2JLT

GRS 0001B0221900302900035WA9002L606 015 CH3BR 2JLT

GRS 0001B0221900302900035WA9002L606 015. C2H3CL2JLT

GRS 0001B0221900302900035WA9002L606 015 C2H5CL2JLT

GRS 0001B0221900302900035WA9002L606 015 CH2CL22JB

GRS 0001B0221900302900035WA9002L606 015 ACET 2JLT

GRS 0001B0221900302900035WA9002L606 015 CS2 2.JLT

GRS 0001B0221900302900035WA9002L606 015 11DCE 2JLT

GRS 000180221900302900035 FA9002L606 015 11DCLE2JLT
YSPER

98 PER

10UGL

1OUGL

1OUGL

10UGL

5UGL

11 UGL

5UGL

5UGL

5UGL

5UGL

5UG்

5UGL

1OUGL

5UGL

5UGL

1OUGL

5UGL.

5 UGI

5UGL

5UGL

5UGL

5UGL

5UGL

5UGI

5UGL

$10 \mathrm{UGI}$

1OUGL

5UGL

5UGI

5UGI

5UGL

5UGT

5UGL

5UGI

116 PER

99PER

104PER

10UGT

10UGL

10UGL

10UGL

5UGL

10UGL

5UGL

5UGi்

5UGL
$1.0000 \mathrm{MSBB}$

$1.0000 \mathrm{MSBB}$

$1.0000 \mathrm{MSBB}$

$1.0000 \mathrm{MSBB}$

$1.0000 \mathrm{MSBB}$

$1.0000 \mathrm{MSBB}$

$1.0000 \mathrm{MSBB}$

$1.0000 \mathrm{MSBB}$

$1.0000 \mathrm{MSBB}$

$1.0000 \mathrm{MSBB}$

$1.0000 \mathrm{MSBB}$

$1.0000 \mathrm{MSBB}$

$1.0000 \mathrm{MSBB}$

$1.0000 \mathrm{MSBB}$

$1.0000 \mathrm{MSBB}$

1. $0000 \mathrm{MSBB}$

$1.0000 \mathrm{MSBB}$

$1.0000 \mathrm{MSBB}$

$1.0000 \mathrm{MSBB}$

1. $0000 \mathrm{MSBB}$

$1.0000 \mathrm{MSBB}$

$1.0000 \mathrm{MSBB}$

$1.0000 \mathrm{MSBB}$

$1.0000 \mathrm{MSBB}$

1. $0000 \mathrm{MSBB}$

1. $0000 \mathrm{MSBB}$

1. $0000 \mathrm{MSBB}$

1. $0000 \mathrm{MSBB}$

$1.0000 \mathrm{MSBB}$

$1.0000 \mathrm{MSBB}$

$1.0000 \mathrm{MSBB}$

1. $0000 \mathrm{MSBB}$

1. $0000 \mathrm{MSBB}$

1. 0000MSBB

1. $0000 \mathrm{MSBB}$

$1.0000 \mathrm{MSBB}$

$1.0000 \mathrm{MSBB}$

1. $0000 \mathrm{MSBB}$

1. $0000 \mathrm{MSBB}$

$1.0000 \mathrm{MSBB}$

$1.0000 \mathrm{MSBB}$

1.0000MSBB

$1.0000 \mathrm{MSBB}$

$1.0000 \mathrm{MSBB}$

1. $0000 \mathrm{MSBB}$

1. $0000 \mathrm{MSBB}$

$1.0000 \mathrm{MSBB}$

$1.0000 \mathrm{MSBB}$
50.000BROMOELUOROBENZENE 0624HN $50.0001,2-D I C H L O R O E T H A N E-D O 624 \mathrm{HN}$ OCHLOROMETHANE O624HN OBROMOMETHANE O624HN OVINYI CHLORIDE O624HN OCHLOROETHANE O624HN OMETHYIENE CHLORIDE O624HN OACETONE OCARBON DISULFIDE O624HN 01,1-DICHLOROETHENE 0624HN 01,1-DICHLOROETHANE 0624HN 01,2-DICHIOROETHENE 10624HN OCHLOROFORM O624HN 01,2-DICHLOROETHANE O624HN 02-BUTANONE O624HN $0.1,1,1-T R I C H L O R O E T H A N O 624 H N$ OCARBON TETRACHLORIDEO624HN OVINYL ACETATE 0624HN OBROMODICHLOROMETHANEO $624 \mathrm{HN}$ 01,2-DICHLOROPROPANE 0624HN OCIS-1, 3-DICHLOROPROPO624HN OTRICHLOROETHENE $0624 \mathrm{HN}$ ODIBROMOCHLOROMETHANEO $624 \mathrm{HN}$ $01,1,2$-TRICHLOROETHANO $624 \mathrm{HN}$ OBENZENE O624HN OTRANS-1, 3-DICHLOROPRO624HN OBROMOEORM O624HN 04-METHYL-2-PENTANONEO624HN 02-HEXANONE 0624HN OTETRACHLOROETHENE O624HN $01,1,2,2$-TETRACHLOROEO $624 \mathrm{HN}$ OTOLUENE O624HN OCHLOROBENZENE O624HN OETHYLBENZENE O624HN OSTYRENE OXYLENE . (TOTAL) O624HN $50.000 T O L U E N E-D 8$ O624HN 50. 000BROMOELUOROBENZENE O624HN

50.0001, 2-DICHLOROETHANE-DO624 HN OCHLOROMETHANE O624HN OBROMOMETHANE O624HN OVINYL CHLORIDE $0624 \mathrm{HN}$ OCHLOROETHANE O624HN OMETHYLENE CHLORIDE O624HN OACETONE O624HN OCARBON DISULFIDE 0624HN 01,1-DICHLOROETHENE 0624HN 01,1-DICHLOROETHANE 0624HN 

0001B0221900302900035WA9002L606 015 CHCL3 2JLT 0001B0221900302900035WA9002L606 015 12DCLE2JLT $0001 B 0221900302900035$ HA9002L606 015 MEK 2JLT 0001B0221900302900035WA9002L606 015 111TCE2JLT 0001B0221900302900035WA9002L606 015 CCL4 2JLT $0001 B 0221900302900035$ WA9002L606 015 VINYLA2JLT $0001 B 0221900302900035 W A 90024606015$ BRDCLM2JIT $0001 B 0221900302900035$ WA 90021606015 12DCLP2JLT $0001 B 0221900302900035$ WA9002L606 015 13DCPC2JLT 0001B0221900302900035WA9002L606 015 TRCLE 2JLT $0001 B 0221900302900035$ WA9002L606 015 DBRCLM2JLT 0001B0221900302900035WA9002L606 015 112TCE2JLT $0001 B 0221900302900035$ WA9002L606 015 C6H6 2JLT 0001B0221900302900035WA9002L606 015 T13DCP2JLT $0001 B 0221900302900035$ WA9002L606 015 CHBR3 2JLT $0001 B 0221900302900035 \mathrm{FA} 9002 L 606015$ 4ME2PE2JLT $0001 B 0221900302900035$ WA9002L606 015 2HXONE2JLT 0001B0221900302900035WA9002L606 015 TCLEE 2JLT $0001 B 0221900302900035$ WA9002L606 015 TCLEA 2JLT 0001B0221900302900035WA9002L606 015 MEC6H52JLT $0001 B 0221900302900035$ WA9002L606 015 CLC6H52JLT 0001B0221900302900035WA9002L606 015 ETC6H52JLT 0001B0221900302900035WA9002L606 015 STYR 2JLT $0001 B 0221900302900035$ WA9002L606 015 XYLEN 2JLT $0001 C 0221900302900035$ WA9002L606 016 MEC6D82J $0001 C 0221900302900035$ WA9002L606 016 PBFB 2J $0001 C 0221900302900035$ WA9002L606 016 12DCD42J $0001 C 0221900302900035 \mathrm{WA} 9002 L 606016 \mathrm{CH} 3 \mathrm{CL}$ 2JLT $0001 C 0221900302900035 \% A 9002 L 606016 \mathrm{CH} 3 B R$ 2JLT $0001 C 0221900302900035 W A 9002 L 606016$ C2H3CL2JLT 0001C0221900302900035WA9002L606 016 C2H5CL2JLT $0001 C 0221900302900035$ WA9002L606 016 CH2CL22JLT $0001 C 0221900302900035$ AA9002L606 016 ACET 2JB $0001 C 0221900302900035$ WA9002L606 $016 \mathrm{CS} 2$ 2JLT $0001 C 0221900302900035 \% A 9002 L 606016$ 11DCE 2JLT $0001 C 0221900302900035$ WA9002L606 016 11DCLE2JLT $0001 C 0221900302900035 \mathrm{WA} 9002 \mathrm{~L} 606016$ T12DCE2JLT $0001 C 0221900302900035$ WA9002L606 016 CHCL3 2JLT 0001C0221900302900035WA9002L606 016 12DCLE2JLT $0001 C 0221900302900035$ HA9002L606 016 MEK 2JIT $0001 C 0221900302900035$ FA9002L606 016 111TCE2JLT $0001 C 0221900302900035$ FA9002L606 016 CCL4 2JIT $0001 C 0221900302900035$ FA9002L606 016 VINYLA2JLT 0001C0221900302900035FA9002L606 016 BRDCIM2JIT $0001 C 0221900302900035$ HA9002L606 016 12DCLP2JLT 0001C0221900302900035\%A9002L606 016 13DCPC2JLT 0001C0221900302900035WA9002L606 016 TRCLE 2JLT

\begin{tabular}{|c|c|}
\hline 5UGL & $1.0000 \mathrm{MSBB}$ \\
\hline 5UGL & $1.0000 \mathrm{MSBB}$ \\
\hline 5UGL & $1.0000 \mathrm{MSBB}$ \\
\hline 10UGL & $1.0000 \mathrm{MSBB}$ \\
\hline 5UGL & $1.0000 \mathrm{MSBB}$ \\
\hline 5UGL & $1.0000 \mathrm{MSBB}$ \\
\hline 10UGL & $1.0000 \mathrm{MSBB}$ \\
\hline 5UGL & $1.0000 \mathrm{MSBB}$ \\
\hline 5UGL & $1.0000 \mathrm{MSBB}$ \\
\hline 5UGL & $1.0000 \mathrm{MSBB}$ \\
\hline 5UGL & $1.0000 \mathrm{MSBB}$ \\
\hline 5UGL & $1.0000 \mathrm{MSBB}$ \\
\hline 5UGL & $1.0000 \mathrm{MSBB}$ \\
\hline 5UGL & $1.0000 \mathrm{MSBB}$ \\
\hline 5UGL & $1.0000 \mathrm{MSBB}$ \\
\hline 5UGL & $1.0000 \mathrm{MSBB}$ \\
\hline 10UGL & $1.0000 \mathrm{MSBB}$ \\
\hline 10UGL & $1.0000 \mathrm{MSBB}$ \\
\hline 5UGL & $1.0000 \mathrm{MSBB}$ \\
\hline 5UGL & $1.0000 \mathrm{MSBB}$ \\
\hline 5UGL & $1.0000 \mathrm{MSBB}$ \\
\hline 5UGL & $1.0000 \mathrm{MSBB}$ \\
\hline 5UGL & $1.0000 \mathrm{MSBB}$ \\
\hline 5UGL & $1.0000 \mathrm{MSBB}$ \\
\hline 5UGL & $1.0000 \mathrm{MSBB}$ \\
\hline 14PER & $1.0000 \mathrm{MSBB}$ \\
\hline O1PER & $1.0000 \mathrm{MSBB}$ \\
\hline 14PER & $1.0000 \mathrm{MSBB}$ \\
\hline 10UG் & $1.0000 \mathrm{MSBB}$ \\
\hline 10UGL & $1.0000 \mathrm{MSBB}$ \\
\hline 10UGL & $1.0000 \mathrm{MSBB}$ \\
\hline 10UGL & $1.0000 \mathrm{MSBB}$ \\
\hline 5UGI & $1.0000 \mathrm{MSBB}$ \\
\hline 45UGL & $1.0000 \mathrm{MSBB}$ \\
\hline 5UGL & $1.0000 \mathrm{MSBB}$ \\
\hline 5UGL & $1.0000 \mathrm{MSBB}$ \\
\hline 5UGL & $1.0000 \mathrm{MSBB}$ \\
\hline 5UGL & $1.0000 \mathrm{MSBB}$ \\
\hline 5UGL & $1.0000 \mathrm{MSBB}$ \\
\hline 5UGL & $1.0000 \mathrm{MSBB}$ \\
\hline 10 UGL & $1.0000 \mathrm{MSBB}$ \\
\hline 5UGL & $1.0000 \mathrm{MSBB}$ \\
\hline 5UGL & $1.0000 \mathrm{MSBB}$ \\
\hline 10UGL & 1. $0.000 \mathrm{MSBB}$ \\
\hline 5UGL & $1.0000 \mathrm{MSBB}$ \\
\hline 5UGL & $1.0000 \mathrm{MSBB}$ \\
\hline 5UGI & $1.0000 \mathrm{MSBB}$ \\
\hline 5UGL & $1.0000 \mathrm{MSBB}$ \\
\hline
\end{tabular}

01,2-DICHLOROETHENE $10624 \mathrm{HN}$ OCHLOROFORM O624HN $01,2-D I C H L O R O E T H A N E$ O624HN 02-BUTANONE O624HN $01,1,1-T R I C H L O R O E T H A N O 624 \mathrm{HN}$ OCARBON TETRACHLORIDEO $624 \mathrm{HN}$ OVINYL ACETATE O624HN OBROMODICHLOROMETHANEO624HN $01,2-D I C H L O R O P R O P A N E$ 0624HN OCIS-1, 3-DICHLOROPROPO62 4HN OTRICHLOROETHENE O624HN ODIBROMOCHLOROMETHANEO $624 \mathrm{HN}$ $01,1,2-T R I C H L O R O E T H A N O 624 \mathrm{HN}$ OBENZENE O624HN OTRANS-1, 3-DICHLORORRO624HN OBROMOFORM 0624HN 04-METHYL-2-PENTANONEO $624 \mathrm{HN}$ 02-HEXANONE $0624 \mathrm{HN}$ OTETRACHLOROETHENE O624HN $01,1,2,2$-TETRACHLOROEO62 $4 \mathrm{HN}$ OTOLUENE O624HN OCHLOROBENZENE $0624 \mathrm{HN}$ OETHYLBENZENE $0624 \mathrm{HN}$ OSTYRENE $0624 \mathrm{HN}$ OXYLENE (TOTAL) $0624 \mathrm{HN}$ 50.000TOLUENE-D8 $0624 \mathrm{HN}$ 50.000BROMOFLUOROBENZENE O624HN $50.0001,2$-DICHLOROETHANE-DO624HN OCHLOROMETHANE O624HN OBROMOMETHANE $0624 \mathrm{HN}$ OVINYI CHLORIDE O624HN OCHLOROETHANE O624HN OMETHYLENE CHLORIDE 0624HN OACETONE O624HN OCARBON DISULEIDE 0624HN 01,1-DICHLOROETHENE 0624HN 01,1-DICHLOROETHANE 0624HN $01,2-D I C H L O R O E T H E N E$ (0624HN OCHLOROFORM O624HN 01,2-DICHLOROETHANE O624HN 02-BUTANONE O624HN $01,1,1$-TRICHLOROETHANO 62 4HN OCARBON TETRACHLORIDEO $624 \mathrm{HN}$ OVINYI ACETATE $0624 \mathrm{HN}$ OBROMODICHLOROMETHANEO62 4HN $01,2-D I C H L O R O P R O P A N E$ O624HN OCIS-1, 3-DICHLOROPROPO $624 \mathrm{HN}$ OTRICHLOROETHENE 
$0001 C 0221900302900035$ WA9002L606 016 DBRCLM2JLT $0001 \mathrm{C} 0221900302900035 \mathrm{WA} 9002 \mathrm{~L} 606016112 \mathrm{TCE} 2 \mathrm{JLT}$ $0001 C 0221900302900035 \mathrm{WA} 9002 \mathrm{~L} 606016 \mathrm{C} 6 \mathrm{H} 6$ 2JLT $0001 C 0221900302900035$ WA9002L606 016 T13DCP2JLT $0001 C 0221900302900035 \mathrm{WA} 9002 \mathrm{~L} 606016$ CHBR3 2JLT $0001 C 0221900302900035 \mathrm{WA} 9002 \mathrm{~L} 606016$ 4ME2PE2JLT 0001 C0221900302900035FA9002L606 016 2HXONE2JLT $0001 C 0221900302900035 \mathrm{WA} 9002 \mathrm{~L} 606016$ TCLEE 2JLT $0001 C 0221900302900035$ WA9002L606 016 TCLEA 2JLT $0001 C 0221900302900035$ WA9002L606 016 MEC6H52JLT $0001 C 0221900302900035 \mathrm{WA} 9002 \mathrm{~L} 606016$ CLC6H52JLT

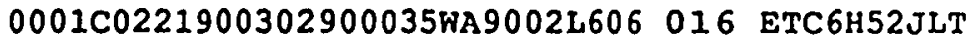
$0001 C 0221900302900035$ WA9002L606 016 STYR 2JLT $0001 C 0221900302900035 \mathrm{KA} 9002 \mathrm{~L} 606016$ XYLEN 2JLT 0319900042 WA90CO42A LC1 LHGTOT 1D 0319900042WA90C042A LC1 LHGTOT ID 0319900042WA90C0 42A LC2 LHGTOT 1D 0319900042WA90C042A LC2 LHGTOT ID 0319900042WA90C042A LC3 LHGTOT ID 0319900042WA90C042A LC3 LHGTOT ID 0319900042WA90C042A LC4 LHGTOT 1D 0319900042WA90CO42A LC4 LHGTOT ID 0319900042WA90C042A MB1 HGTOT 1DLT 0319900042 WA9OC042A MB2 HGTOT 1DLT $0319900042 \mathrm{WA} 90 \mathrm{C} 042 \mathrm{~A}$ MB3 HGTOT IDLT $0319900042 \mathrm{FA} 90 \mathrm{C} 042 \mathrm{~A}$ MB 4 HGTOT 1DLT $0319900042 \mathrm{WA} 90 \mathrm{CO} 42 \mathrm{~B}$ LCI LHGTOT ID 0319900042WA90CO 42B LC1 LHGTOT ID 0319900042 WA90CO $42 B$ LC2 LHGTOT 1D 0319900042WA90C042B LC2 LHGTOT ID 0319900042WA90C042B LC3 LHGTOT 1D 0319900042 WA90C0 42B LC3 LHGTOT ID 0319900042WA90C042B LC4 LHGTOT 1D $0319900042 \mathrm{WA90C042B}$ LC4 LHGTOT 1D 0319900042WA90C042B MBI HGTOT 1DLT 0319900042KA90CO42B MB2 HGTOT 1DLT 0319900042 WA 90 C042B MB3 HGTOT IDLT 0319900042WA9OC042B MB4 HGTOT 1DLT 0330900218 WA90L0218 LC1 LAS $1 B$ $0330900218 \mathrm{WA} 90 \mathrm{~L} 0218$ LC1 LAS 0330900218 WA90L0218 LC2 LAS

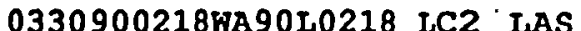
0330900218 WA90L0218 MB1. AS $0405900218 \mathrm{FA} 90 \mathrm{~L} 0218$ LC1 LPB 0405900218 \%90L0218 LC1 LPB 0405900218 \%A $90 \mathrm{~L} 0218$ LC2 LPB 0405900218 KA 90L0218 LC2 LPB 0405900218พA90L0218 MBI PB 1B

$\begin{array}{ll}\text { 5UGL } & 1.0000 \mathrm{MSBB} \\ \text { 5UGL } & 1.0000 \mathrm{MSBB}\end{array}$

5UGL $\quad 1.0000 \mathrm{MSBB}$

SUGL . $\quad 1.0000 \mathrm{MSBB}$

5UGL $\quad 1.0000 \mathrm{MSBB}$

10UGL $\quad 1.0000 \mathrm{MSBB}$

1OUGL $\quad 1.0000 \mathrm{MSBB}$

SUGL $\quad 1.0000 \mathrm{MSBB}$

5UGL $\quad 1.0000 \mathrm{MSBB}$

5UGL $1.0000 \mathrm{MSBB}$

5UGL $\quad 1.0000 \mathrm{MSBB}$

SUGL $\quad 1.0000 \mathrm{MSBB}$

5UGL $\quad 1.0000 \mathrm{MSBB}$

5UGI $\quad 1: 0000 \mathrm{MSBB}$

1.7UGL $1.00 \mathrm{HGSQ}$

86.2PER $1.00 \mathrm{HGSO}$

1.8UGL $1.00 \mathrm{HGSO}$

90.4PER $1.00 \mathrm{HGSQ}$

1.8UGL $\quad 1.00 \mathrm{HGSQ}$

90.4PER $1.00 \mathrm{HGSQ}$

1.9UGL $1.00 \mathrm{HGSQ}$

94.6PER $1.00 \mathrm{HGSQ}$

0.20 UGL $1.00 \mathrm{HGSQ}$

0.20 UGL $1.00 \mathrm{HGSQ}$

0.20 UGL $\quad 1.00 \mathrm{HGSO}$

$0.20 \mathrm{UGL} \quad 1.00 \mathrm{HGSQ}$

$0.86 \mathrm{MGKG} \quad 1.00 \mathrm{HGSQ}$

86.2PER $\quad 1.00 \mathrm{HGSO}$

$0.90 \mathrm{MGKG} \quad 1.00 \mathrm{HGSQ}$

90.4PER $\quad 1.00 \mathrm{HGSQ}$

$0.90 \mathrm{MGKG} \quad 1.00 \mathrm{HGSO}$

$90.4 \mathrm{PER} \quad 1.00 \mathrm{HGSQ}$

$0.95 \mathrm{MGKG} \quad 1.00 \mathrm{HGSO}$

94.6PER $1.00 \mathrm{HGSQ}$

$0.10 \mathrm{MGKG} \quad 1.00 \mathrm{HGSQ}$

$0.10 \mathrm{MGKG} \quad 1.00 \mathrm{HGSO}$

$0.10 \mathrm{MGKG} \quad 1.00 \mathrm{HGSQ}$

$0.10 \mathrm{MGKG} \quad 1.00 \mathrm{HGSQ}$

26.1UGL 1.00AAAER

87. ORER 1.00AAAER

27.2UGL 1.00AAAER

$\begin{array}{lll}1 \mathrm{~B} & 90.7 \mathrm{PER} & 1.00 \text { AAAER } \\ \text { 1BLT } & 10.0 \mathrm{UGL} & 1.00 \mathrm{AAAER}\end{array}$

$\begin{array}{lll}1 \mathrm{BLT} & 10.0 \mathrm{UGL} & 1.00 \mathrm{AAAER} \\ 1 \mathrm{~B} & 31.6 \mathrm{UGL} & 1.00 \mathrm{ICCMT}\end{array}$

$1 \mathrm{~B} \quad 105 \mathrm{PER} \quad 1.00 \mathrm{ICCMT}$

1B 31.3UGL 1.00 ICCMT

1B 104PER 1.00 ICCMT

1BLT 3.0UGL
ODIBROMOCHLOROMETHANEO624HN $01,1,2-T R I C H L O R O E T H A N O 624 \mathrm{HN}$ OBENZENE 0624HN OTRANS-1, 3-DICHLOROPRO62 4HN OBROMOEORM O624HN 04-METHYL-2-PENTANONEO624HN 02-HEXANONE O624HN OTETRACHLOROETHENE O624HN $01,1,2,2-T E T R A C H L O R O E 0624 \mathrm{HN}$ OTOLUENE $0624 \mathrm{HN}$ OCHLOROBENZENE OETHYLBENZENE O624HN OSTYRENE O624HN OXYLENE (TOTAL) O624HN OMERCURY, LCS MHGLCL

2. OMERCURY, LCS MHGLCL OMERCURY; LCS MHGLCI

2. OMERCURY, LCS MHGLCL OMERCURY, LCS MHGLCI

2. OMERCURY, LCS MHGLCI OMERCURY, LCS MHGLCL

2. OMERCURY; LCS MHGLCI OMERCURY, TOTAL MHGTON OMERCURY, TOTAL MHGTON OMERCURY, TOTAL MHGTON OMERCURY, TOTAL MHGTON OMERCURY, LCS MHGLCL

1. OMERCURY, LCS MHGLCL OMERCURY, LCS MHGLCL

1. OMERCURY, LCS MHGLCL OMERCURY, LCS MHGLCL

1. OMERCURY, LCS MHGLCL OMERCURY, LCS MHGLCL

1. OMERCURY, LCS MHGLCL OMERCURY, TOTAL MHGTON OMERCURY, TOTAL MHGTON OMERCURY, TOTAL MHGTON OMERCURY, TOTAL MHGTON OARSENIC, LCS MASLCL 30.0ARSENIC, LCS MASLCL OARSENIC, LCS MASLCL

30. OARSENIC, LCS MASLCL OARSENIC, TOTAL MASTON OLEAD, ICS - MPBLCL

30. OLEAD, LCS . MPBLCL OLEAD, LCS. MPBLCL

30 . OLEAD, ICS OLEAD, TOTAL : MPBTON 
0402900218 WA90L0218 LC1 LSE 0402900218 WA 9020218 LCL LSE 0402900218 WA90L0218 LC2 LSE 0402900218 KA 9020218 LC2 LSE 0402900218 WA9LO0218 MB1 SE 0323900219 WA90L0219 LC1 LAG 0323900219 WA90L0219 LC1 LAG 0323900219 WA90L0219 LC2 LAG 0323900219 WA90L0219 LC2 LAG 0323900219 WA90L0219 MB1 AG 0323900219 WA90L0219 LC1 LBA 0323900219 WA90L0219 LCI LBA 0323900219 WA90L0219 LC2 LBA 0323900219 WA90LP219 LC2 LBA 0323900219WA90L0219 MB1 BA 0323900219 WA90L0219 LC1 LCD 0323900219WA90L0219 LC1 LCD 0323900219 WA90L0219 LC2 LCD 0323900219 WA90L0219 LC2 LCD 0323900219WA90L0219 MB1 CD 0323900219 WA90L0219 LC1 ICR 0323900219 WA90L0219 LC1 LCR 0323900219WA90L0219 LC2 LCR 0323900219 WA90L0219 LC2 LCR 0323900219WA90L0219 MB1 CR 0330900225 WA90L0225 LC1 LAS 0330900225 WA 90 L 0225 LC1 LAS $0330900225 W A 90 L 0225$ LC2 LAS $0330900225 \mathrm{WA} 90 \mathrm{~L} 0225$ LC2 LAS 0330900225 WA90L0225 MB1 AS 0405900225 WA90L0225 LC1 LPB 0405900225 WA90L0225 LC1 LPB 0405900225 WA90L0225 LC2 LPB 0405900225 WA90L0225 LC2 LPB 0405900225 WA90L0225 MB1 PB $0401900225 W A 90 L 0225$ LC1 ISE 0401900225WA90L0225 LC1 LSE 0401900225WA90L0225 LC2 LSE 0401900225WA90L0225 LC2 LSE 0401900225WA90L0225 MB1 SE $0323900226 \mathrm{HA} 90 \mathrm{~L} 0226$ LC1 LAG $0323900226 \mathrm{KA} 90 \mathrm{~L} 0226$ LC1 LAG 0323900226 WA90L0226 LC2 LAG $0323900226 \mathrm{WA} 90 \mathrm{~L} 0226$ LC2 IAG $0323900226 \mathrm{WA} 90 \mathrm{~L} 0226 \mathrm{MB1}$ AG 0323900226 WA90L0226 LC1 LBA $0323900226 \mathrm{KA} 90 \mathrm{~L} 0226$ LC1 IBA 0323900226 WA90L0226 LC2 LBA

\begin{tabular}{|c|c|c|}
\hline $1 \mathrm{~B}$ & 33. 1UGL & $1.00 I C S S Q$ \\
\hline $1 B$ & $110 \mathrm{PER}$ & $1.00 I C S S Q$ \\
\hline $1 \mathrm{~B}$ & 34. OUGL & 1.00 ICSSQ \\
\hline $1 \mathrm{~B}$ & 113PER & $1.00 I \operatorname{CsS} Q$ \\
\hline IBLT & 5.0 UGL & $1.00 A A S S Q$ \\
\hline 22 & 633UGL & $1.00 I C A L M$ \\
\hline 22 & $127 \mathrm{PER}$ & $1.00 I C A L M$ \\
\hline $2 z$ & 596UGL & 1.00 ICALM \\
\hline $2 z$ & 119PER & $1.00 \mathrm{ICAL}$ \\
\hline $2 \mathrm{ZLT}$ & 10.0 UGL & $1.00 \mathrm{ICAL}$ \\
\hline $2 \mathrm{z}$ & 5100UGL & $1.00 \mathrm{ICAL}$ \\
\hline 22 & 102RER & $1.00 \mathrm{ICAL}$ \\
\hline 22 & 5040UGL & $1.00 \mathrm{ICAL}$ \\
\hline $2 \mathrm{Z}$ & 101PER & $1.00 \mathrm{ICAL}$ \\
\hline $2 \mathrm{ZLT}$ & 200UGL & $1.00 I C A L$ \\
\hline $2 z$ & 287UGL & 1.00ICAL \\
\hline $2 z$ & 115PER & $1.00 \mathrm{ICAL}$ \\
\hline $2 z$ & $278 U G L$ & $1.00 \mathrm{ICAL}$ \\
\hline $2 z$ & 111PER & 1.00ICAL \\
\hline $2 \mathrm{ZLT}$ & 5.0 UGL & 1.00ICAL \\
\hline 22 & 599UGL & $1.00 \mathrm{ICAI}$ \\
\hline $2 z$ & 120PER & 1.00ICAL \\
\hline $2 z$ & 580UGL & 1.00ICALM \\
\hline 27 & 116PER & $1.00 \mathrm{ICAL}$ \\
\hline 2ZLT & 10.0 UGL & $1.00 \mathrm{ICAL}$ \\
\hline $1 \mathrm{~B}$ & 5. 6MGKG & 1. O0AAAE \\
\hline $1 \mathrm{~B}$ & 94. OPER & 1. 00AAAE \\
\hline $1 B$ & 5. 9MGKG & 1.00AAAE \\
\hline $1 B$ & 97.7 PER & 1. 00AAAE \\
\hline 1BLT & 2. OMGKG & 1. OOAAAE \\
\hline $1 B$ & 6.8MGKG & $1.00 \mathrm{ICCM}$ \\
\hline $1 B$ & 113PER & $1.00 I \mathrm{CCMI}$ \\
\hline $1 B$ & 6.7MGKG & $1.00 \mathrm{ICCM}$ \\
\hline $1 B$ & 112PER & $1.00 \mathrm{ICCMT}$ \\
\hline 1BLT & $0.60 \mathrm{MGKG}$ & $1.00 \mathrm{AACM}$ \\
\hline $1 B$ & 6.7MGKG & $1.00 \mathrm{ICML}$ \\
\hline $1 B$ & $111 \mathrm{PER}$ & $1.00 \mathrm{ICML}$ \\
\hline $1 B$ & 6.8MGKG & $1.00 \mathrm{ICML}$ \\
\hline $1 B$ & 113PER & $1.00 \mathrm{ICML}$ \\
\hline 1BLT & 1. OMGKG & 1. 00AAML \\
\hline $2 z$ & 59.0MGKG & $1.00 \mathrm{ICAL}$ \\
\hline $2 z$ & 59. OPER & $1.00 \mathrm{ICAL}$ \\
\hline 22 & 107MGKG & $1.00 \mathrm{ICAL}$ \\
\hline $2 z$ & 107PER & 1.00ICAL \\
\hline 2ZLT & 2. OMGKG & $1.00 \mathrm{ICAL}$ \\
\hline 22 & 1020MGKG & $1.00 I C A L$ \\
\hline & 102PER & 1.00ICAL \\
\hline 22 & $1010 \mathrm{MGKG}$ & 1.00ICALN \\
\hline
\end{tabular}

OSELENIUM, LCS

30. OSELENIUM, LCS OSELENIUM, LCS

30. OSELENIUM, LCS OSELENIUM, TOTAL OSILVER, LCS

500SILVER, LCS OSIIVER, LCS

50OSILVER, LCS OSILVER, TOTAL OBARIUM, LCS

5000BARIUM, ICS OBARIUM, LCS

5000BARIUM, ICS OBARIUM, TOTAL OCADMIUM, LCS

250CADMIUM, ICS OCADMIUM, LCS

250CADMIUM, ICS OCADMIUM, TOTAL OCHROMIUM, LCS

500CHROMIUM, LCS OCHROMIUM, ICS

500 CHROMIUM, ICS OCHROMIUM, TOTAL OARSENIC, ICS

6. OARSENIC, ICS OARSENIC, ICS

6. OARSENIC, ICS OARSENIC, TOTAL OLEAD, LCS

6. OLEAD, ICS OLEAD, LCS

6. OLEAD, ICS OLEAD, TOTAL OSELENIUM, LCS

6. OSELENIUM, ICS OSELENIUM, LCS

6.0SELENIUM, ICS OSELENIUM, TOTAL OSILVER, LCS

100SIIUER, LCS OSILVER, LCS

100SILVER, ICS OSIIVER, TOTAL OBARIUM, LCS

1000BARIUM, ICS OBARIUM, LCS :
MSELCL MSELCL MSELCL MSELCL MSETON MAGLCL MAGLCL MAGLCI

MAGLCL

MAGTON

MBALCL

MBALCL

MBALCL

MBALCI

MBATON

MCDLCL

MCDLCL

MCDLCL

MCDLCI

MCDTON

MCRLCL

MCRLCL

MCRLCL

MCRICI

MCRTON

MASLCL

MASLCL

MASLCL

MASLCL

MASTON

MPBLCL

MPBLCL

MPBLCL

MPBLCL

MPBTON

MSELCL

MSELCL

MSELCI

MSELCL

MSETON

MAGLCI

MAGLCL

MAGLCL

MAGLCL

MAGTON MBALCL MEALLCL 
MBLK MBLK MBLK MBLK MBLK MBLK MBLK MBLK MBLK MBLK MBLK MBLK MBLK MBLK MBLK MBLK MBLK MBLK MBLK MBLK MBLK
0323900226 WA90L0226 LC2 LBA 0323900226 WA9020226 MB1 BA

22 $0323900226 \mathrm{WA} 90 \mathrm{~L} 0226$ LC1 LCD 0323900226 WA90L0226 LC1 LCD $0323900226 \mathrm{KA} 90 \mathrm{~L} 0226$ LC2 LCD $0323900226 \mathrm{KA} 90 \mathrm{~L} 0226$ LC2 LCD $0323900226 \mathrm{WA} 90 \mathrm{~L} 0226 \mathrm{MBI} C D$ 0323900226 WA90L0226 LC1 LCR $0323900226 \mathrm{WA} 90 \mathrm{~L} 0226$ LC1 LCR $0323900226 \mathrm{WA} 9010226$ LC2 LCR $0323900226 \mathrm{WA} 90 \mathrm{~L} 0226$ LC2 LCR $0323900226 \mathrm{WA} 90 \mathrm{~L} 0226 \mathrm{MB} 1 \mathrm{CR}$ 0308900326 WA90LE0286 MB1 NBD5 0308900326 WA90LE028 6 MB1 2FBP 0308900326 WA90LE0286 MB1 PHEND51X 0308900326 KA90LE0286 MB1 2FP IX $0308900326 \mathrm{WA} 90 \mathrm{LE} 0286$ MB1 246TBP 1X 0308900326 WA90LE028 6 MB1 PHENOL1XLT 0308900326 WA90LE0286 MB1 B2CEE IXLT 0308900326 KA90LE0286 MB1 2CLP 1XLT 0308900326 KA90LE028 6 MB1 13DCLB1XLT 0308900326 WA90LE0286 MB1 14DCLB1XLT $0308900326 \mathrm{WA} 90 \mathrm{LE} 0286$ MB1 BZALC 1XLT 0308900326 WA90LE0286 MB1 12DCLB1XLT 0308900326 WA90LE0286 MB1 2MP 1XLT 0308900326 WA9OLE028 6 MB1 B2CIPE1XLT 0308900326 WA90LE0286 MB1 4MP 1XLT 0308900326 WA90LE0286 MB1 NDNPA 1XLT 0308900326 WA90LE0286 MB1 CL6ET 1XLT $0308900326 \mathrm{KA} 902 \mathrm{~L} 0286 \mathrm{MB1} \mathrm{NB}$ 1XLT 0308900326 WA9 $9020286 \mathrm{MB1}$ ISOPHR $1 \times L T$ $0308900326 \mathrm{WA} 90 \mathrm{LE} 0286 \mathrm{MB1}$ 2NP 1XLT 0308900326 WA90LE028 6 MB1 24DMPN1XIT 0308900326 WA90LE0286 MB1 BENZOA1XLT 0308900326 WA90LE0286 MB1 B2CEXM1XLT 030890032 6WA90LE0286 MB1 24DCLP 1XLT 0308900326 WA90LE0286 MB1 124TCB1XLT 0308900326 WA90LE0286 MB1 NAP 1XLT 0308900326 WA90LE0286 MB1 4CANIL1XLT 0308900326 WA90LE0286 MB1 HCBD 1XLT 0308900326 WA90LE0286 MB1 4CL3C 1XLT 0308900326 WA90LE0286 MB1 2MENAP 1XLT 0308900326WA90LE0286 MB1 CL6CP 1XLT 0308900326 KA90LE0286 MB1 246TCP 1XLT 0308900326 FA90LE02B 6 MB1 245TCR 1XLT $0308900326 \mathrm{FA} 90 \mathrm{LE} 0286 \mathrm{MB1}$ 2CNAP 1XLT 0308900326 KA90LE0286 MBI 2NANILIXLT
IUIPER

40. OMGKG

53.1MGKG

106PER

53. OMGKG

106PER

1. OMGKG

112MGKG

112PER

111MGKG

111PER

2. OMGKG

S3PER

61PER

T PPER

70PER

66PER

77PER

370UGKG

370UGKG

370UGKG

370UGKG

370UGKG

370UGKG

370UGKG

370UGKG

370UGKG

370UGKG

37 OUGKG

370UGKG

370UGKG

370UGKG

370UGKG

370UGKG

1800UGKG

370UGKG

370UGKG

370UGKG

370UGKG

370UGKG

370UGKG

370UGKG

370UGKG

370UGKG

370UGKG

1800UGKG

370UGKG

1800 UGKG
1.00ICALM

1.00 ICAIM

1.00 ICALM

1.00 ICALM

1.00 ICALM

$1.00 I$ CALM

1.00 ICALM

1.00 ICALM

1.00ICALM

1.00 ICALM

1.00 ICALM

1.00 ICALM

1.0000 MSTAS

$1.0000 \mathrm{MSTAS}$

$1.0000 \mathrm{MSTAS}$

$1.0000 \mathrm{MSTAS}$

$1.0000 \mathrm{MSTAS}$

$1.0000 \mathrm{MSTAS}$

$1.0000 \mathrm{MSTAS}$

$1.0000 \mathrm{MSTAS}$

$1.0000 \mathrm{MSTAS}$

$1.0000 \mathrm{MSTAS}$

$1.0000 \mathrm{MSTAS}$

$1.0000 \mathrm{MSTAS}$

1.0000MSTAS

$1.0000 \mathrm{MSTAS}$

$1.0000 \mathrm{MSTAS}$

1.0000MSTAS

1.0000MSTAS

$1.0000 \mathrm{MSTAS}$

$1.0000 M S T A S$

$1.0000 \mathrm{MSTAS}$

$1.0000 \mathrm{MSTAS}$

$1.0000 M S T A S$

$1.0000 \mathrm{MSTAS}$

$1.0000 \mathrm{MSTAS}$

1.0000 MSTAS

$1.0000 \mathrm{MSTAS}$

$1.0000 \mathrm{MSTAS}$

1.0000 MSTAS

$1.0000 \mathrm{MSTAS}$

1.0000 MSTAS

$1.0000 \mathrm{MSTAS}$

$1.0000 \mathrm{MSTAS}$

$1.0000 \mathrm{MSTIAS}$

1.0000 MSTAS

1.0000MSTAS

1.0000MSTAS
1000BARIUM, LCS OBARIUM, TOTAL OCADMIUM, LCS

50.0CADMIUM, LCS OCADMIUM, LCS

50.0CADMIUM, LCS OCADMIUM, TOTAL OCHROMIUM, LCS

100CHROMIUM, LCS OCHROMIUM, LCS

100CHROMIUM, ICS OCHROMIUM, TOTAL

1666.667NITROBENZENE-D5

1666.6672-FLUOROBIPHENYL

1666.667P-TERPHENYL-D14

3333.334PHENOL-D5

3333.3342-FLUORORHENOL

$3333.3342 ; 4,6$-TRIBROMOPHENOLO625HN OPHENOL OBIS (2-CHLOROETHYL) ETO625HN 02-CHLORORHENOL O625HN $01,3-D I C H L O R O B E N Z E N E$ 0625HN 01,4 -DICHLOROBENZENE 0625HN OBENZYL ALCOHOL $0625 \mathrm{HN}$ $01,2-D I C H L O R O B E N Z E N E$ O625HN 02-METHYIPHENOL O625HN OBIS (2-CHLOROISOPROPYO625HN 04-METHYLPHENOL O625HN ON-NITROSO-DI-N-PROP YO625HN OHEXACHLOROETHANE 0625HN ONITROBENZENE $0625 \mathrm{HN}$ OISOPHORONE O625HN 02-NITRORHENOL $0625 \mathrm{HN}$ $02,4-D I M E T H Y L P H E N O L$ O625HN OBENZOIC ACID O625HN OBIS (2-CHLOROETHOXY) MO625HN $02,4-D I C H L O R O P H E N O L$ O625HN $01 ; 2,4-T R I C H L O R O B E N 2 E 0625 \mathrm{HN}$ ONAPHTHALENE O625HN 04-CHLOROANILINE O625HN OHEXACHLOROBUTADIENE O625HN 04-CHLORO-3-METHYLPHEO 625HN 02-METHYLNAPHTHALENE 0625HN OHEXACHLOROCYCLOPENTAO $625 \mathrm{HN}$ $02,4,6$-TRICHLOROPHENOO $625 \mathrm{HN}$ $02,4,5$-TRICHLOROPHENOO625HN 02-CHLORONAPHTHALENE O625 HN 02-NITROANILINE O625HN 
0308900326 WA9OLE0286 MBI DMP IXLT $0308900326 \mathrm{WA} 90 \mathrm{LE} 0286 \mathrm{MBI}$ ANAPYL1XLT 0308900326 WA90LE0286 MBI 26DNT 1XLT 0308900326 WA90LE0286 MB1 3NANILIXLT 0308900326 WA90LE0286 MBI ANAPNE 1 XLT $0308900326 \mathrm{WA} 90 \mathrm{LE} 0286 \mathrm{MB1}$ 24DNP 1XLT $0308900326 \mathrm{WA} 90 \mathrm{LE} 0286 \mathrm{MB} 1$ 4NP 1 XLT 0308900326 WA90LE0286 MB1 DIBZFU1XLT 0308900326 WA90LE0286 MB1 24DNT 1 XLT 0308900326 WA90LE0286 MBI DEP IXLT 0308900326 WA90LE0286 MBI 4CLPPE1XLT 0308900326 WA90LE0286 MB1 FLRENE 1 XLT 0308900326 WA90LE0286 MB1 4NANIL1XLT $0308900326 \mathrm{WA} 90 \mathrm{LE} 0286 \mathrm{MB1}$ 46DN2C1XLT 0308900326 WA90LE0286 MB1 NNDPA 1XLT 0308900326 WA90LE0286 MBI 4BRPPE1XLT 0308900326 WA90LE0286 MBI CL6BZ 1XLT 0308900326 WA90LE0286 MBI PCP 1XLT 0308900326 WA90LE0286 MB1 PHANTR1XLT 0308900326 WA90LE0286 MB1 ANTRC 1XLT 0308900326 KA 90 LE0286 MB1 DNBP IXLT 0308900326 WA90LE0286 MB1 FANT 1XLT 0308900326 WA90LE0286 MBI PYR IXLT 0308900326 WA90LE0286 MB1 BBZP 1XLT 0308900326 WA9 OLE0286 MB1 33CL2B1XLT 0308900326 WA90LE0286 MB1 BAANTRIXLT 0308900326WA90LE0286 MBI CHRY 1XLT 0308900326 WA90LE0286 MB1 B2EHP 1XLT 0308900326 WA90LE0286 MB1 DNOP 1XLT 0308900326 WA90LE0286 MB1 BF2ANT1XLT 0308900326 KA90LE0286 MB1 BKEANT1XLT 0308900326 WA90LE0286 MB1 BAPYR 1XLT 0308900326 WA90LE0286 MB1 ICDPYR1XLT 0308900326 WA9OLE0286 MB1 DBAHA 1XLT 0308900326 WA90LE0286 MB1 BGHIPY1XLT 0308900326 WA90LE0286 MB1SNBD5 $1 X$ 0308900326 WA90LE0286 MB1S2FBP 1X 0308900326 WA90LE0286 MB1SPTERP IX 0308900326 WA 90 LE028 6 MB1SPHEND $1 X$ 0308900326 WA9OLE0286 MB1S2FP 1X 0308900326 WA90LE0286 MB1S246TBP 1X 0308900326 WA9OLE0286 MB1SPHENOL1X 0308900326 WA90LE0286 MB1SB2CEE 1XLT 0308900326 WA90LE0286 MB1S2CLP 1X 0308900326 WA9OLE0286 MB1S13DCLB1X 0308900326 WA90LE0286. MB1S1,4DCLB1X 0308900326 WA90LE0286 MB1SBZALC 1XIT 0308900326 WA90LE0286 MB1S12DCLB1XLT
370 UGKG 370 UGKG

370 UGKG

1800 UGKG 370 UGKG

1800 UGKG

1800 UGKG

370 UGKG

370 UGKG

370 UGKG

37 OUGKG

370 UGKG

1800 UGKG

1800 UGKG

370 UGKG

37 OUGKG

370 UGKG

1800 UGKG

370UGKG

370 UGKG

370UGKG

370UGKG

370 UGKG

37 OUGKG

74 OUGKG

370 UGKG

37 OUGKG

370 UGKG

370UGKG

370 UGKG

37 OUGKG

37 OUGKG

370 UGKG

370 UGKG

370UGKG

49PER

6IPER

71PER

59PER

50PER

46PER

62PER

370UGKG

56PER

91 OUGKG

51PER

370UGKG 370 UGKG
1. 0000MSTAS

$1.0000 \mathrm{MSTAS}$

1.0000MSTAS

1. 0000MSTAS

1.0000MSTAS

1.0000MSTAS

1. $0000 \mathrm{MSTAS}$

$1.0000 \mathrm{MSTAS}$

1.0000MSTAS

1. 0000MSTAS

1.0000MSTAS

1.0000MSTAS

1.0000MSTAS

1.0000MSTAS

1.0000MSTAS

$1.0000 \mathrm{MSTAS}$

$1.0000 \mathrm{MSTAS}$

1. $0000 \mathrm{MSTAS}$

1.0000MSTAS

1. 0000MSTAS

1.0000MSTAS

1.0000MSTAS

1.0000MSTAS

1.0000MSTAS

1.0000MSTAS

1. 0000MSTAS

1.0000MSTAS

1. 0000MSTAS

1. 0000MSTAS

1.0000MSTAS

1.000OMSTAS

1. 0000MSTAS

$1.0000 \mathrm{MSTAS}$

1. 0000MSTAS

1.0000MSTAS

$1.0000 \mathrm{MSTAS}$

1. 0000MSTAS

1.0000MSTAS

1.0000MSTAS

1. 0000MSTAS

$1.0000 \mathrm{MSTAS}$

1.0000MSTAS

1.0000MSTAS

$1.0000 M S T A S$

1. 0000MSTAS

1.0000MSTAS

1.0000MSTAS 1. 0000MSTAS
ODIMETHYLPHTHALATE

OACENAP HTHYLENE

$0625 \mathrm{HN}$

02,6-DINITROTOLUENE 0625HN 03-NITROANILINE 0625HN OACENAPHTHENE O625HN

02,4-DINITROPHENOL 0625HN 04-NITROPHENOL O625HN ODIBENZOFURAN $0625 \mathrm{HN}$

02,4-DINITROTOLUENE 0625HN ODIETHYIPHTHALATE O625HN 04-CHLOROP HENYL-PHENYO 62 $5 \mathrm{HN}$ OFLUORENE O625HN 04-NITROANILINE 0625HN 04, 6-DINITRO-2-METHYLO625HN ON-NITROSODIPHENYLAMIO $625 \mathrm{HN}$ 04-BROMOPHENYL-PHENYI0625HN OHEXACHLOROBENZENE O625HN OPENTACHLOROPHENOI 0625HN OPHENANTHRENE 0625HN OANTHRACENE O625HN ODI-N-BUTYLPHTHALATE O625HN OFLUORANTHENE 0625HN OPYRENE O625HN OBUTYLBENZYLPHTHALATEO625HN $03,3^{\prime}$-DICHLOROBENZ IDIO 625HN OBENZO (A) ANTHRACENE O625HN OCHRYSENE 0625HN OBIS (2-ETHYLHEXYL) P HTO625HN ODI-N-OCTYL PHTHALATEO625HN OBENZO (B) FLUORANTHENEO625HN OBENZO (K) FLUORANTHENEO625HN OBENZO (A) PYRENE O625HN OINDENO (1,2, 3-CD) P YREO625HN ODIBENZO $(A, H)$ ANTHRACEO $625 \mathrm{HN}$ OBENZO (G, H, I) PERYLENEO $625 \mathrm{HN}$ 1666.667NITROBENZENE-D5 0625HS 1666.6672-FLUOROBIPHENYI O625HS 1666.667P-TERPHENYI-D14 0625HS 3333.334 PHENOL-D5 0625HS 3333.3342-FLUORORHENOL O625HS $3333.3342,4,6$-TRIBROMOPHENOLO625HS 3333.334 PHENOL $0625 \mathrm{HS}$ OBIS (2-CHLOROETHYL) ETO625HS 3333.3342-CHLOROPHENOL O625HS 01,3-DICHLOROBENZENE O625HS

$1666.6671,4$-DICHIOROBENZENE 0625HS OBENZYL ALCOHOL 0625HS 01,2-DICHLOROBENZENFE-2625 
0308900326WA90LE0286 MB1S2MP IXLT 0308900326 WA90LE0286 MB1SB2CIPE1XLT 0308900326 WA 90LE028 6 MBIS4MP. 1XLT 0308900326 WA90LE02B 6 MB1SNDNPA $1 X$ 0308900326 WA90LE0286 MB1SCL6ET IXLT $0308900326 \% A 90 L E 0286$ MBISNB 1XLT 0308900326 WA90LE0286 MB1SISOPHRIXLT 0308900326WA90LE0286 MB1S2NP 1XLT 0308900326 WA90LE028 6 MB1S2 4DMPN1XLT 0308900326 WA90LE028 6 MB1SBENZOA1XLT 0308900326 WA90LE0286 MB1SB2CEXM1XLT 0308900326 WA90LE0286 MB1S2 4DCLP1XLT 0308900326 WA90LE0286 MB1S124TCB1X 0308900326 WA9OLE0286 MB1SNAP 1XLT 0308900326 WA90LE028 6 MB1S4CANILIXLT 0308900326WA90LE0286 MB1SHCBD 1XLT 0308900326 WA9OLE0286 MB1S4CL3C $1 X$ 0308900326 WA 90LE028.6 MB1S2MENAP 1XLT 0308900326 WA90LE0286 MB1SCL6CP 1XLT 0308900326 WA90LE0286 MB1S246TCP1XLT 0308900326 WA90LE028 6 MB1S245TCP1XLT 0308900326 WA90LE0286 MB1S2CNAP 1XLT 0308900326 WA90LE0286 MB1S2NANIL1XLT 0308900326 WA90LE0286 MB1SDMP 1XLT $0308900326 \mathrm{WA} 90 \mathrm{LE} 0286$ MB1SANAPYL1XLT $0308900326 \mathrm{WA} 90 \mathrm{LE} 0286$ MB1S26DNT 1XLT 0308900326 WA90LE0286 MB1S3NANIL1XLT 0308900326 WA9OLE0286 MB1SANAPNE1X 0308900326WA90LE0286 MB1S24DNP 1XLT 0308900326 WA $90 L E 0286$ MA1S4NP $1 X$ $0308900326 \mathrm{KA} 90 \mathrm{LE} 0286$ MB1SDIBZFU1XLT 0308900326 WA 90 LE0286 MB1S24DNT $1 \mathrm{X}$ 0308900326WA90LE0286 MB1SDEP 1XLT 0308900326WA90LE0286 MB1S4CLPPEIXLT $0308900326 \mathrm{WA} 9$ 0LE0286 MB1SELRENE1XLT 0308900326 WA90LE0286 MB1S4NANILIXLT $0308900326 \mathrm{HA}$ OLLE0286 MB1S46DN2C1XLT 0308900326WA90LE0286 MBISNNDPA IXLT $0308900326 \mathrm{WA} 90 \mathrm{LE} 0286$ MB1S4BRPPEIXLT $0308900326 \mathrm{WA} 90 \mathrm{LE} 0286$ MB1SCL6BZ 1XLT 0308900326 WA90LE0286 MB1SPCP $1 \mathrm{X}$ $0308900326 \mathrm{WA} 90 \mathrm{LE} 0286$ MB1SPHANTR1XLT $0308900326 \mathrm{FA}$ OLLE0286 MB1SANTRC 1XLT 0308900326WA90LE0286 MB1SDNBP 1XLT $0308900326 \mathrm{WA} 90 \mathrm{LE} 0286 \mathrm{MBISFANT} 1 \mathrm{XLT}$ $0308900326 \mathrm{KA}$ OLE0286 MB1SPYR $1 X$ 0308900326\%A90LE0286 MBISBBZP IXLT 0308900326 FA90LE0286 MB1S33CL2B1XLT
ᄀ. UUGKG 370UGKG

37 OUGKG

49PER

37 UUGKG 370 UGKG

370 UGKG

370 UGKG

$370 U G K G$

1800 UGKG

370 UGKG

370 UGKG

62PER

37 UGKG

370 UGKG

370UGKG

53PER

37OUGKG

370UGKG

370UGKG

1800 UGKG

370UGKG

1800 UGKG

370UGKG

37 OUGKG

37 OUGKG

1800 UGKG

72PER

1800 UGKG

89PER

37 UUGKG

58PER

37 OUGKG

37 OUGKG

37 OUGKG

180 OUGKG

180 OUGKG

37 UUGKG

370UGKG

370UGKG

68PER

370 UGKG

370UGKG

370UGKG

370UGKG

92PER

370 UGKKG

740 UGKG
$1.0000 \mathrm{MSTAS}$

$1.0000 M S T A S$

$1.0000 \mathrm{MSTAS}$

$1.0000 \mathrm{MSTAS}$

1.0000 MSTAS

$1.0000 M S T A S$

$1.0000 \mathrm{MSTAS}$

1.0000 MSTAS

$1.0000 \mathrm{MSTAS}$

$1.0000 \mathrm{MSTAS}$

$1.0000 \mathrm{MSTAS}$

$1.0000 \mathrm{MSTAS}$

$1.0000 \mathrm{MSTAS}$

1.0000 MSTAS

$1.0000 \mathrm{MSTAS}$

$1.0000 M S T A S$

$1.0000 M S T A S$

$1.0000 \mathrm{MSTAS}$

$1.0000 M S T A S$

$1.0000 \mathrm{MSTAS}$

$1.0000 \mathrm{MSTAS}$

$1.0000 \mathrm{MSTAS}$

$1.0000 \mathrm{MSTAS}$

$1.0000 \mathrm{MSTAS}$

$1.0000 \mathrm{MSTAS}$

$1.0000 \mathrm{MSTAS}$

$1.0000 \mathrm{MSTAS}$

$1.0000 \mathrm{MSTAS}$

$1.0000 \mathrm{MSTAS}$

$1.0000 \mathrm{MSTAS}$

$1.0000 \mathrm{MSTAS}$

1.0000 MSTAS

$1.0000 \mathrm{MSTAS}$

$1.0000 \mathrm{MSTAS}$

$1.0000 \mathrm{MSTAS}$

$1.0000 \mathrm{MSTAS}$

1.0000 MSTAS

$1.0000 M S T A S$

$1.0000 \mathrm{MSTAS}$

$1.0000 \mathrm{MSTAS}$

$1.0000 \mathrm{MSTAS}$

$1.0000 \mathrm{MSTAS}$

$1.0000 \mathrm{MSTAS}$

1.0000 MSTAS

1.0000MSTAS

1.0000 MSTAS

1.0000 MSTAS
$1.0000 \mathrm{MSTAS}$
02-METHYLPHENOL

0625HS

OBIS (2-CHLOROISOPROP Y0625HS

04-METHYIPHENOI O625HS

$1666.667 \mathrm{~N}$-NITROSO-DI-N-PROPYO625HS OHEXACHLOROETHANE O625HS

ONITROBENZENE

OISOPHORONE O625HS

02-NITROPHENOL O625HS

02, 4-DIMETHYLPHENOI 0625HS OBENZOIC ACID O625HS OBIS (2-CHLOROETHOXY) MO625HS 02,4-DICHLOROPHENOL O625HS

$1666.6671,2,4$-TRICHLOROBENZEO625HS ONAPHTHALENE O625HS 04-CHLOROANILINE O625HS OHEXACHLOROBUTADIENE O625HS

3333. 3344-CHLORO-3-METHYLPHEO $625 \mathrm{HS}$ 02-METHYLNAPHTHALENE 0625HS OHEXACHLOROCYCLOPENTAO625HS $02,4,6$-TRICHLOROPHENOO625HS $02,4,5$-TRICHLOROPHENOO $625 \mathrm{HS}$ 02-CHLORONAPHTHALENE O625HS 02-NITROANILINE O625HS ODIMETHYLPHTHALATE O625HS OACENAPHTHYLENE O625HS 02,6-DINITROTOLUENE O625HS 03-NITROANIIINE O625HS 1666.667ACENAPHTHENE O625HS 02,4-DINITROPHENOL O625HS ODIBENZOFURAN O625HS

1666.6672,4-DINITROTOLUENE O625HS ODIETHYLPHTHALATE O625HS 04-CHLOROPHENYI-PHENYO625HS OELUORENE O625HS 04-NITROANILINE O625HS 04, 6-DINITRO-2-METHYLO625HS ON-NITROSODIPHENYLAMIO625HS 04-BROMOPHENYL-PHENYLO625HS OHEXACHLOROBENZENE O625HS 3333.334PENTACHLOROPHENOL O625HS ORHENANTHRENE O625HS OANTHRACENE O625HS ODI-N-BUTYLPHTHALATE O625HS OELUORANTHENE O625HS

1666. 667PYRENE O625HS 03, $3^{\prime}$-DICHLOROBENZIDIO625HS
3333.3344-NITROPHENOL O625HS OBUTYLBENZYLPHTHALATEO $\$ 25 \mathrm{HS}$ 
0308900326WA90LE0286 MB1SBAANTR 1XLT 0308900326 WA 90 LE0286 MB1SCHRY 1XLT 0308900326 WA90LE0286 MB1SB2EHP 1XLT 0308900326 WA9OLE0286 MB1SDNOP 1XLT 0308900326 WA 90 LE0286 MB1SBE2ANT 1 XLT $0308900326 \mathrm{WA} 90 \mathrm{LE} 0286$ MBISBKFANT 1 XLT $0308900326 \mathrm{WA} 90 \mathrm{LE} 0286$ MB1SBAPYR 1XLT 0308900326 WA9OLE0286 MB1SICDPYR $1 X L T$ 0308900326 WA $90 \mathrm{LE} 0286$ MBISDBAHA 1XLT 0308900326 WA 90 LE0286 MB1SBGHIPY1XLT 0302900002 WA90LTZ002 MB1 TOC 9ILT 0302900002 WA9OLT2002 MB1STOC 9I 0302900002 WA90LT2002 MB1TTOC 9I 0302900002 WA9OLTZ002 MB2 TOC 9ILT 0302900002 WA9OLTZ002 MB2STOC 9I 0302900035 WA $90 L V K 035$ MB1 MEC6D82J 0302900035 WA 90 LVK035 MB1 PBEB 2J 0302900035 WA9OLVK035 MB1 12DCD42J 0302900035 WA90LVK035 MB1 CH3CL 2JLT $0302900035 \mathrm{WA} 90 \mathrm{LVK} 035 \mathrm{MB} 1 \mathrm{CH} 3 \mathrm{BR}$ 2JLT 0302900035 WA9OLVK035 MB1 C2H3CL2JLT 0302900035 WA90LVK035 MB1 C2H5CL2JLT 0302900035 WA90LVK035 MB1 CH2CL22J $0302900035 \mathrm{WA} 90 \mathrm{LVK} 035 \mathrm{MB1}$ ACET 2JLT 0302900035 WA90LVK035 MB1 CS2 2JLT $0302900035 \mathrm{WA} 90 \mathrm{LVK} 035 \mathrm{MB1}$ 11DCE 2JLT $0302900035 \mathrm{WA} 90 \mathrm{LVK} 035$ MBI 11DCLE2JLT 0302900035 WA90LVK035 MB1 T12DCE2JLT $0302900035 \mathrm{WA} 90 \mathrm{LVK} 035 \mathrm{MB1}$ CHCL3 2JLT 0302900035 WA90LVK035 MB1 12DCLE2JLT 0302900035 WA9OLVK035 MBI MEK 2JLT 0302900035 WA 90 LVK035 MBI 111TCE2JLT 0302900035WA90LVK035 MB1 CCL4 2JLT $0302900035 \mathrm{WA} 90$ LVKỎ35 MBI VINYLA2JLT $0302900035 \mathrm{FA} 90 L V K 035$ MB1 BRDCLM2JLT 0302900035 WA90LVK035 MBI 12DCLP2JLT 0302900035FA90LVK035 MB1 13DCPC2JLT $0302900035 \mathrm{WA} 90 L V K 035$ MB1 TRCLE 2J 0302900035 WA90LVK035 MB1 DBRCLM2JLT 0302900035 WA90LVK035 MBI 112TCE2JLT $0302900035 \mathrm{WA} 90 \mathrm{LVK} 035 \mathrm{MB} 1 \mathrm{C} 6 \mathrm{H} 6$ 2JLT $0302900035 \mathrm{WA} 90 \mathrm{LVK} 035 \mathrm{MBI}$ T13DCP2JLT $0302900035 \mathrm{KA} 90 \mathrm{LVK} 035 \mathrm{MBI}$ CHBR3 2JLT $0302900035 \mathrm{WA} 90 \mathrm{LVK} 035 \mathrm{MB1}$ 4ME2PE2JLT 0302900035 WA9OLVKO35 MBI 2HXONE2JLT 0302900035WA90LVK035 MB1 TCLEE 2JLT 0302900035 WA90LVK035 MB1 TCLEA 2JLT 0302900035 WA90LVK035 MB1 MEC6H52JLT
37 OUGKG 370UGKG

37 OUGKG

370UGKG

37 UUGKG

370UGKG

37 UUGKG

370UGKG

37 OUGKG

370UGKG

20. OMGKG

1OOPER

101PER

20.0MGKG

1OOPER

104 PER

96PER

$104 \mathrm{PER}$

IOUGL

10UGL

1OUGL

1OUGL

7UGL

1OUGL

5UGI

5UGL

5UGL

5UGL

5UGI

5UGI

1OUGL

5UGL

SUGI

10UGL

5UGL

5UGL

5UGL

5UGL

5UGL

5UGL

5UGI

5UGL

5UGI

10UGL

10UGL

SUGL

5UGI

5UGL
$1.0000 \mathrm{MSTAS}$

$1.0000 \mathrm{MSTAS}$

$1.0000 \mathrm{MSTAS}$

$1.0000 \mathrm{MSTAS}$

$1.0000 \mathrm{MSTAS}$

$1.0000 \mathrm{MSTAS}$

1.0000MSTAS

1.0000MSTAS

$1.0000 \mathrm{MSTAS}$

1.0000MSTAS 10DODKS 10DODKS 10DODKS 10DODKS 10DODKS

$1.0000 \mathrm{MSB}$

$1.0000 \mathrm{MSB}$

$1.0000 \mathrm{MSB}$

$1.0000 \mathrm{MSB}$

$1.0000 \mathrm{MSB}$

$1.0000 \mathrm{MSB}$

$1.0000 \mathrm{MSB}$

$1.0000 \mathrm{MSB}$

$1.0000 \mathrm{MSB}$

$1.0000 \mathrm{MSB}$

$1.0000 \mathrm{MSB}$

$1.0000 \mathrm{MSB}$

$1.0000 \mathrm{MSB}$

$1.0000 \mathrm{MSB}$

$1.0000 \mathrm{MSB}$

$1.0000 \mathrm{MSB}$

$1.0000 \mathrm{MSB}$

$1.0000 \mathrm{MSB}$

$1.0000 \mathrm{MSB}$

$1.0000 \mathrm{MSB}$

$1.0000 \mathrm{MSB}$

$1.0000 \mathrm{MSB}$

$1.0000 \mathrm{MSB}$

$1.0000 \mathrm{MSB}$

$1.0000 \mathrm{MSB}$

$1.0000 \mathrm{MSB}$

$1.0000 \mathrm{MSB}$

$1.0000 \mathrm{MSB}$

1. $0000 \mathrm{MSB}$

$1.0000 \mathrm{MSB}$

$1.0000 \mathrm{MSB}$

$1.0000 \mathrm{MSB}$

$1.0000 \mathrm{MSB}$
OBENZO (A) ANTHRACEN 2 O625HS OCHRYSENE O625HS OBIS (2-ETHYLHEXYL) PHTO625HS ODI-N-OCTYL PHTHALATEO625HS OBENZO (B) FLUORANTHENEO625HS OBENZO (K) FLUORANTHENEO625HS OBENZO (A) PYRENE O625HS O INDENO $(1,2,3-C D)$ PYREO625HS ODIBENZO $(\mathrm{A}, \mathrm{H})$ ANTHRACEO625HS OBENZO (G, H, I) PERYLENEO625HS OTOTAL ORGANIC CARBONIOCT N 400TOTAL ORGANIC CARBONIOCT S 40OTOTAI ORGANIC CARBONIOCT T

OTOTAL ORGANIC CARBONIOCT N

400TOTAL ORGANIC CARBONIOCT S 50.000TOLUENE-D8 $0624 \mathrm{HN}$ 50.000BROMOELUOROBENZENE O624HN $50.0001,2-D I C H L O R O E T H A N E-D 0624 \mathrm{HN}$ OCHLOROMETHANE O624HN OBROMOMETHANE O624HN OVINYI CHLORIDE $0624 \mathrm{HN}$ OCHLOROETHANE O624HN OMETHYLENE CHLORIDE $0624 \mathrm{HN}$ OACETONE OCARBON DISULEIDE $0624 \mathrm{HN}$ 01,1-DICHLOROETHENE 0624HN 01,1-DICHLOROETHANE $0624 \mathrm{HN}$ 01,2-DICHLOROETHENE $10624 \mathrm{HN}$ OCHLOROFORM $0624 \mathrm{HN}$ 01,2-DICHLOROETHANE O624HN 02-BUTANONE 0624kN 01, 1, 1-TRICHLOROETHANO $624 \mathrm{HN}$ OCARBON TETRACHLORIDEO $624 \mathrm{HN}$ OVINYL ACETATE $0624 \mathrm{HN}$ OBROMODICHLOROMETHANEO624HN 01,2-DICHLOROPROPANE O624HN OCIS-1, 3-DICHLOROPROPO624HN OTRICHLOROETHENE O624HN ODIBROMOCHLOROMETHANEO624HN $01,1,2$-TRICHLOROETHANO $624 \mathrm{HN}$ OBENZENE $0624 \mathrm{HN}$ OTRANS-1, 3-DICHLOROPRO 624HN OBROMOFORM. O624HN O4-METHYL-2-PENTANONEO624HN 02-HEXANONE O624HN OTETRACHLOROETHENE O624HN 01, 1,2, 2-TETRACHLOROEO624HN OTOLUENE 
MBLK

MBLK

MBLK

MBLK

MBLK

MBLK

MBLK

MBLK

MBLK

MBLK

MBLK

MBLK

MBLK

MBLK

MBLK

MBLK

MBLK

MBLK

MBLK

MBLK

MBLK

MBLK

MBLK

MBLK

MBLK

MBLK

MBLK

MBLKK

MALK

MBLK

MBLK

MBLK

MBLK

MBLK

MBLK

MBLK

MBLK

MBLK

MBLK

MBLK

MBLK

MBLK

MBLK

MBLK

MBLK

MBLK
0302900035WA90LVK035 MB1 CLC6H52JLT 0302900035WA90LVK035 MB1 ETC6H52JLT 0302900035WA90LVK035 MBI STYR 2JLT 0302900035 WA90LVK035 MBI XYLEN 2JLT 0228900032 WA90LVW032 MBI MEC6D82J 0228900032 WA90LVW0 32 MB1 PBFB 2J 0228900032 WA9OLVW0 $32 \mathrm{MBI}$ 12DCD42J 0228900032WA90LVW032 MB1 CH3CL 2JLT 0228900032 WA90LVW032 MB1 CH3BR 2JLT 0228900032 WA9OLVW0 32 MBI C2H3CL2JLT 0228900032 WA90LVW032 MB1 C2H5CL2JLT 0228900032 WA90LVW032 MB1 CH2CL22J 0228900032 WA9OLVW0 32 MB1 ACET $2 \mathrm{~J}$ 0228900032 WA90LVW032 MB1 CS2 2JLT 0228900032WA90LVW032 MB1 11DCE 2JLT 0228900032 WA90LVW032 MB1 11DCLE2JLT 0228900032WA90LVW032 MB1 T12DCE2JLT 0228900032 WA90LVW032 MB1 CHCL3 2JLT 0228900032 WA90LVW032 MB1 12DCLE2JLT 0228900032 WA90LVW032 MB1 MEK 2JIT 0228900032 WA90LVW032 MB1 111TCE2JLT 0228900032 WA90LVW032 MB1 CCL4 2JLT 0228900032 WA90LVW032 MB1 VINYLA2JLT 0228900032 WA90LVW032 MB1 BRDCLM2JLT 0228900032 WA90LVW032 MB1 12DCLP2JLT 0228900032WA90LVW032 MB1 13DCPC2JLT 0228900032 WA90LVW032 MBI TRCLE 2JLT 0228900032 FA90LVW032 MB1 DBRCLM2JIT 0228900032WA90LVW032 MB1 112TCE2JLT 0228900032 WA90LVW032 MB1 C6H6 2JLT 0228900032 WA90LVW032 MB1 T13DCP2JLT 0228900032WA90LVW032 MBI CHBR3 2JLT 0228900032 WA9OLVW032 MB1 4ME2PE2JLT 0228900032 WA9OLVW032 MB1 2HXONE2JLT 0228900032 WA90LVW032 MB1 TCLEE 2JLT 0228900032 WA90IVW032 MB1 TCLEA 2JLT $0228900032 W A 90 L V W 032$ MB1 MEC6H52JLT 0228900032 WA90IVW032 MB1 CLC6H52JLT $0228900032 W A 90 L V W 032$ MBI ETC6H52JLT 0228900032 WA90LVW032 MB1 STYR 2JLT 0228900032 WA90LVW032 MB1 XYLEN 2JLT 0301900033 HA90LVW033 MB1 MEC6D82J $0301900033 \% A 90 L V W 033$ MB1 PBEB 2J 0301900033 WA90LVW033 MB1 12DCD42J 0301900033 FA90LVW033 MBI CH3CL 2JLT $0301900033 \% A 90 L V W 033$ MBI CH3BR 2JLT 0301900033 HA90LVW033 MB1 C2H3CL2JLT 0301900033 HA9OLVHO33 MB1 C2H5CL2JIT

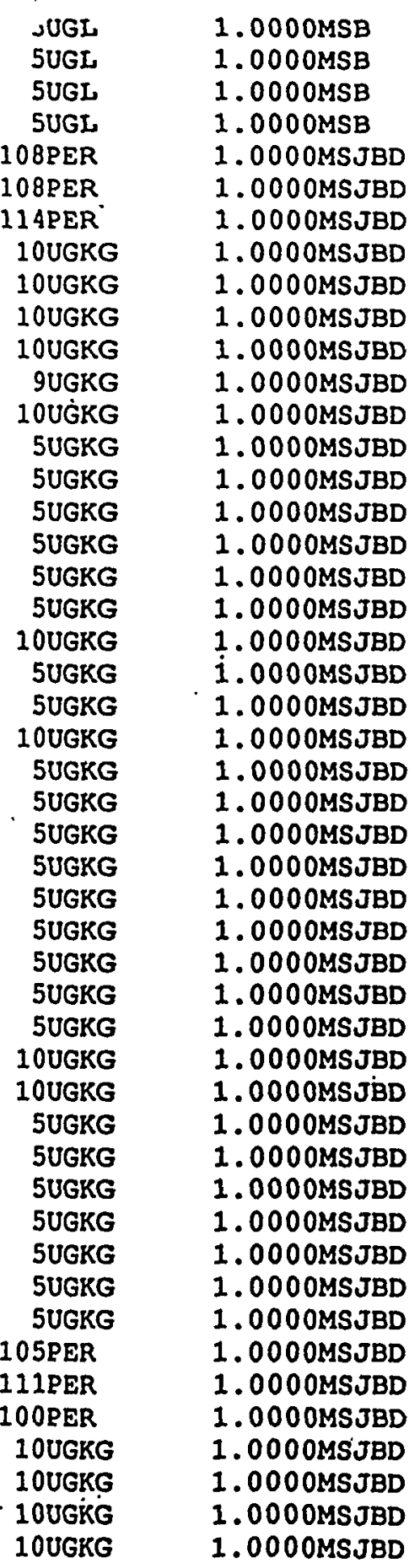
w9002606. dat $-6 / 26 / 90-41$
OCHLOROBENZENE

$0624 \mathrm{HN}$ OETHYLBENZENE

O624HN

OSTYRENE

OXYLENE (TOTAL)

$0624 \mathrm{HN}$

$0624 \mathrm{HN}$

50. 000TOLUENE-D8

$0624 \mathrm{HN}$

50.000BROMOELUOROBENZENE O624HN

$50.0001,2-D I C H L O R O E T H A N E-D O 624 \mathrm{HN}$ OCHLOROMETHANE OBROMOMETHANE O624HN OVINYL CHLORIDE O624HN OCHLOROETHANE O624HN OMETHYLENE CHLORIDE O624HN OACETONE OCARBON DISULFIDE 0624HN 01,1-DICHLOROETHENE 0624HN 01,1-DICHLOROETHANE 0624HN 01,2-DICHLOROETHENE $10624 \mathrm{HN}$ OCHLOROFORM $0624 \mathrm{HN}$ 01,2-DICHLOROETHANE O624HN 02-BUTANONE O624HN 01, 1, 1-TRICHLOROETHANO $624 \mathrm{HN}$ OCARBON TETRACHIORIDEO624HN OVINYL ACETATE O624HN OBROMODICHLOROMETHANEO624HN 01,2-DICHLOROPROPANE 0624HN OCIS-1, 3-DICHLOROPROPO624HN OTRICHLOROETHENE O624HN ODIBROMOCHLOROMETHANEO624HN 01,1,2-TRICHLOROETHANO624HN OBENZENE. $0624 \mathrm{HN}$ OTRANS-1, 3-DICHLOROPRO624HN OBROMOFORM O624HN 04-METHYL-2-PENTANONEO624HN 02-HEXANONE $0624 \mathrm{HN}$ OTETRACHLOROETHENE O624HN $01,1,2,2-T E T R A C H L O R O E 0624 \mathrm{HN}$ OTOLUENE OCHLOROBENZENE O624HN OETHYLBENZENE OSTYRENE O624HN OXYLENE (TOTAL) O624HN

50.000TOLUENE-D8 $0624 \mathrm{HN}$

50.000BROMOFLUOROBENZENE O624HN

50.0001,2-DICHLOROETHANE-D062 4HN OCHLOROMETHANE OBROMOMETHANE $0624 \mathrm{HN}$ OVINYL CHLORIDE $0624 \mathrm{HN}$ OCHLOROETHANE $0624 \mathrm{HN}$ 
0301900033 WA9OLVW0 33 MB1 CH2CL22J 0301900033 WA9OLVW0 33 MB1 ACET $2 \mathrm{~J}$ 0301900033 WA90LVW0 33 MBI CS2 2JLT 0301900033 WA90LVW033 MBI 11DCE 2JLT 0301900033 WA90LVW0 33 MBI 11DCLE2JLT 0301900033 WA9OLVWO 33 MB1 T12DCE2JLT 0301900033 WA90LVWO 33 MBI CHCL3 2JLT 0301900033 WA $90 L V W 033$ MB1 12DCLE2JLT 0301900033 WA90LVW033 MB1 MEK . 2JLT 0301900033 WA90LVW0 33 MB1 111TCE2JLT 0301900033WA90LVW033 MB1 CCL4 2JLT 0301900033 WA $90 L V W 033$ MB1 VINYLA2JLT 0301900033 WA90LVW033 MB1 BRDCLM2JLT 0301900033 WA9OLVW033 MB1 12DCLP2JLT 0301900033 WA90LVW033 MB1 13DCPC2JLT 0301900033 WA9LVVW033 MB1 TRCLE 2JLT 0301900033 WA9OLVW033 MB1 DBRCLM2JLT 0301900033WA90LVW033 MB1 112TCE2JLT 0301900033 WA90LVW033 MB1 C6H6 2JLT 0301900033WA90LVW033 MB1 T13DCP2JLT 0301900033 WA90LVW033 MB1 CHBR3 2JLT 0301900033 WA9OLVW033 MB1 4ME2PE2JLT 0301900033 WA90LVW033 MB1 2HXONE2JLT 0301900033WA90LVW033 MB1 TCLEE 2JLT 0301900033 WA90LVW033 MB1 TCLEA 2JLT 0301900033WA90LVW033 MB1 MEC6H52JLT 0301900033 WA9OLVW033 MB1 CLC6H52JLT 0301900033WA90LVW033 MB1 ETC6H52JLT 0301900033WA90LVW033 MB1 STYR 2JLT 0301900033 WA90LVW033 MB1 XYLEN 2JLT 0302900034 WA9 OLVW034 MBI MEC6D82J 0302900034WA90LVW034 MB1 PBFB 2J 0302900034 WA9OLVW034 MB1 12DCD42J 0302900034 FA9OLVW034 MB1 CH3CL 2JLT 0302900034WA90LVW034 MB1 CH3BR 2JLT $0302900034 \mathrm{WA} 90 \mathrm{LVW034} \mathrm{MB1}$ C2H3CL2JLT 0302900034FA90LVW034 MB1 C2H5CL2JLT 0302900034 WA9OLVW034 MB1 CH2CL22J 0302900034 WA9OLVW034 MB1 ACET 2JLT 0302900034 FA9OLVW034 MB1 CS2 2JLT $0302900034 \mathrm{KA} 90 \mathrm{LVW034}$ MBI 11DCE 2JLT 0302900034WA9OLVW034 MBI 11DCLE2JLT 0302900034 FA90LVW034 MB1 T12DCE2JLT 0302900034 WA90LVW034 MB1. CHCL3 2JLT 0302900034 WA9OLVW034 MB1 12DCLE2JLT $0302900034 \mathrm{FA} 90 \mathrm{LVW034}$. MBI MEK 2JLT 0302900034 WA9OLVW034 MB1 111TCE2JLT $0302900034 \mathrm{FA} 90 \mathrm{LVW034}$ MB1 CCL4 2JLT
I OUGKG

21 UGKG

5UGKG

5UGKG

5UGKG

5UGKG

5UGKG

SUGKG

1 OUGKG

SUGKG

5UGKG

1 OUGKG

5UGKG

5UGKG

5UGKG

5UGKG

5UGKG

5UGKG

5UGKG

5UGKG

5UGKG

10 UGKG

10UGKG

5UGKG

5UGKG

5UGKG

5UGKG

5UGKG

5UGKG

5UGKG

105PER

107PER

107PER

10UGKG

1 OUGKG

1 OUGKG

10UGKG

6UGKG

10UGKG

5UGKG

5UGKG

5UGKG

5UGKG

5UGKG

5UGKG

10UGKG

5UGKG

5UGKG
$1.0000 M S J B D$ 1. OOOOMSJBD

1. $0000 \mathrm{MSJBD}$

1. $0000 \mathrm{MSJBD}$

1. $0000 M S J B D$

1. $0000 \mathrm{MSJBD}$

1. $0000 \mathrm{MSJBD}$

1. $0000 \mathrm{MSJBD}$

1. $0000 \mathrm{MSJBD}$

1. $0000 M S J B D$

1. $0000 \mathrm{MSJBD}$

1. $0000 \mathrm{MSJBD}$

1. $0000 \mathrm{MSJBD}$

1. $0000 \mathrm{MSJBD}$

1. $0000 \mathrm{MSJBD}$

1. $0000 \mathrm{MSJBD}$

1. $0000 \mathrm{MSJBD}$

1. $0000 \mathrm{MSJBD}$

1. $0000 \mathrm{MSJBD}$

1. O0OOMSJBD

1. $0000 M S J B D$

1. $0000 \mathrm{MSJBD}$

1. $0000 \mathrm{MSJBD}$

1. $0000 \mathrm{MSJBD}$

1. $0000 \mathrm{MSJBD}$

1. $0000 \mathrm{MSJBD}$

1. $0000 \mathrm{MSJBD}$

1. $0000 \mathrm{MSJBD}$

1. OOOOMSJBD

1. $0000 \mathrm{MSJBD}$

1. 0000MSJBD

1. 000 OMSJBD

1. $0000 \mathrm{MSJBD}$

1. $0000 \mathrm{MSJBD}$

1. $0000 \mathrm{MSJBD}$

$1.0000 M S J B D$

1. 0000MSJBD

1. $0000 \mathrm{MSJBD}$

$1.0000 \mathrm{MSJBD}$

1. 0000MSJBD

1. $0000 M S J B D$

1. $0000 \mathrm{MSJBD}$

1. $0000 \mathrm{MSJBD}$

1. $0000 \mathrm{MSJBD}$

1.0000MSJBD

$1.0000 \mathrm{MSJBD}$

1. $0000 \mathrm{MSJBD}$

1. $0000 \mathrm{MSJBD}$
OMETHYLENE CHLORIDE 0624HN OACETONE O624HN OCARBON DISULEIDE O624HN 01,1-DICHLOROETHENE 0624HN 01,1-DICHLOROETHANE 0624HN 01,2-DICHLOROETHENE (0624HN OCHLOROEORM O624HN 01,2-DICHLOROETHANE 0624HN 02-BUTANONE O624HN $01,1,1-T R I C H L O R O E T H A N O 624 \mathrm{HN}$ OCARBON TETRACHLORIDEO $624 \mathrm{HN}$ OVINYL ACETATE O624HN OBROMODICHLOROMETHANEO62 4HN 01,2-DICHLOROPROPANE $0624 \mathrm{HN}$ 0CIS-1, 3-DICHLOROPROPO624 HN OTRICHLOROETHENE O624HN ODIBROMOCHLOROMETHANEO62 $4 \mathrm{HN}$ $01,1,2$-TRICHLOROETHANO $624 \mathrm{HN}$ OBENZENE O624HN 0TRANS-1, 3-DICHLOROPRO62 4HN OBROMOFORM O624HN 04-METHYL-2-PENTANONEO624HN 02-HEXANONE 0624HN OTETRACHLOROETHENE O624HN $01,1,2,2$ TETRACHLOROEO $624 \mathrm{HN}$ OTOLUENE O624HN OCHLOROBENZENE O624HN OETHYLBENZENE $0624 \mathrm{HN}$ OSTYRENE O624HN OXYLENE (TOTAL) O624HN 50.000TOLUENE-D8 $8624 \mathrm{HN}$ 50.000BROMOFLUOROBENZENE O624HN

$50.0001,2-D$ ICHLOROETHANE-DO624HN OCHLOROMETHANE 0624HN OBROMOMETHANE O624HN OVINYI CHLORIDE O624HN OCHLOROETHANE O624HN OMETHYLENE CHLORIDE O624HN OACETONE O624HN OCARBON DISULFIDE O624HN 01,1-DICHLOROETHENE 0624HN 01,1-DICHLOROETHANE 0624HN 01;2-DICHLOROETHENE (0624HN OCHLOROFORM . O624HN 01,2-DICHLOROOETHANE 0624HN 02-BUTANONE O624HN 01, 1, 1-TRICHLOROETHANO62 4HN OCARBON TETRACHLORIP $24 \mathrm{HN}$ 
MBLK

MBLK

MBLK

MBLK

MBLK

MBLK

MBLK

MBLK

MBLK

MBLK

MBLK

MBLK

MBLK

MBLK

MBLK

MBLK

MBLK

MBLK

MBLK

MBLK

MBLK

MBLK

MBLK

T MBLK

i MBLK

w MBLK

MBLK

MBLK

MBLK

MBLKK

MBLK

MBLK

MBLK

MBLK

MBLK

MBLK

MBLK

MBLK

MBLK

MBLK

MBLK

MBLK

MBLK

MBLK

MBLK

MBLK
0302900034 WA 90 LVW0 34 MB1 VINYLA2JLT 0302900034 WA90LVW034 MB1 BRDCLM2JLT 0302900034 WA90LVW034 MB1 12DCLP2JLT 0302900034 WA $90 \mathrm{LVW0} 34 \mathrm{MB1}$ 13DCPC2JLT 0302900034 WA90LVW034 MB1 TRCLE 2JLT 0302900034 WA $90 L V W 034$ MB1 DBRCLM2JLT 0302900034 WA90LVW034 MB1 112TCE2JLT 0302900034 WA90LVW034 MB1 C6H6 2JLT 0302900034 WA90LVW034 MB1 T13DCP2JLT 0302900034WA90LVW034 MB1 CHBR3 2JLT 0302900034WA90LVW034 MB1 4ME2PE2JLT 0302900034 WA9OLVW034 MB1 2HXONE2JLT 0302900034WA90LVW034 MBI TCLEE 2JLT 0302900034 WA90LVW034 MB1 TCLEA 2JLT $0302900034 \mathrm{WA} 90 \mathrm{LVW034}$ MB1 MEC6H52JLT 0302900034WA90LVW034 MB1 CLC6H52JLT 0302900034 WA9OLVW034 MB1 ETC6H52JLT 0302900034 WA9OLVW034 MB1 STYR 2JLT 0302900034 WA90LVW034 MB1 XYLEN 2JLT 0305900036WA90LVW036 MB1 MEC6DB2J 0305900036WA90LVW036 MB1 PBEB 2J 0305900036WA90LVW036 MB1 12DCD42J $0305900036 \mathrm{WA} 90 \mathrm{LVW} 036 \mathrm{MB1}$ CH3CL 2JLT 0305900036 WA9OLVW036 MB1 CH3BR 2JLT 0305900036 WA9OLVW036 MB1 C2H3CL2JLT 0305900036 KA90LVW036 MB1 C2H5CL2JLT 0305900036 WA9OLWW036 MB1 CH2CL22J 0305900036 WA90LVW036 MBI ACET 2J 0305900036 WA90LVW036 MB1 CS2 2JLT $0305900036 \mathrm{WA} 90 \mathrm{LVW036}$ MB1 11DCE 2JLT 0305900036 WA $90 L V W 036 \mathrm{MBI}$ 11DCLE2JLT 0305900036 WA $90 \mathrm{LVW036} \mathrm{MBI}$ T12DCE2JLT 0305900036 WA90LVW036 MB1 CHCL3 2JLT 0305900036 WA9OLVW036 MBI 12DCLE2JLT 0305900036 WA90LVW036 MB1 MEK 2JLT 0305900036 WA90LVW036 MB1 111TCE2JLT $0305900036 \mathrm{WA} 90 \mathrm{LVW036} \mathrm{MBI}$ CCL4 2JLT 0305900036WA90LVW036 MB1 VINYLA2JLT 0305900036 WA90LVW036 MB1 BRDCLM2JLT 0305900036 WA90LVW036 MBI 12DCLP2JLT 0305900036 WA90LVW036 MB1 13DCPC2JLT $0305900036 \mathrm{WA} 90 \mathrm{LVW0} 36 \mathrm{MB1}$ TRCLE 2JLT 0305900036 WA90LVW036 MB1 DBRCLM2JLT 0305900036WA90LVW036 MB1 112TCE2JLT 0305900036 WA90LVW036 MBI C6H6 2JLT $0305900036 \mathrm{WA} 90 \mathrm{LVW036} \mathrm{MB1}$ Tं13DCP2JLT 0305900036 WA90LVW036 MB1 CHBR3 2JLLT 0305900036 FA90LVW036 MB1 4ME2PE2JLT

\begin{tabular}{|c|c|}
\hline UUGKG & 1.0000 MSJBD \\
\hline 5UGKG & $1.0000 M S J B D$ \\
\hline SUGKG & $1.0000 M S J B D$ \\
\hline 5UGKG & $1.0000 M S J B D$ \\
\hline SUGKG & $1.0000 M S J B D$ \\
\hline 5UGḲG & $1.0000 \mathrm{MSJBD}$ \\
\hline 5UGKG & $1.0000 M S J B D$ \\
\hline 5UGKG & $1.0000 M S J B D$ \\
\hline 5UGKG & $1.0000 \mathrm{MSJBD}$ \\
\hline SUGKG & $1.0000 M S J B D$ \\
\hline 1 OUGKG & $1.0000 \mathrm{MSJBD}$ \\
\hline 10 UGKG & $1.0000 \mathrm{MSJBD}$ \\
\hline 5UGKG & 1.0000 MSJBD \\
\hline SUGKG & 1.0000 MSJBD \\
\hline 5UGKG & $1.0000 \mathrm{MSJBD}$ \\
\hline 5UGKG & $1.0000 \mathrm{MSJBD}$ \\
\hline 5UGKG & $1.0000 \mathrm{MSJBD}$ \\
\hline 5UGKG & 1.0000 MSJBD \\
\hline 5UGKG & 1.0000 MSJBD \\
\hline 92PER & 1.0000 MSJBD \\
\hline 97PER & 1.0000 MSJBD \\
\hline 84PER & 1.0000 MSJBD \\
\hline 10UGKG & $1.0000 \mathrm{MSJBD}$ \\
\hline 10UGKG & $1.0000 \mathrm{MSJBD}$ \\
\hline 10UGKG & $1.0000 \mathrm{MSJBD}$ \\
\hline 10UGKG & $1.0000 \mathrm{MSJBD}$ \\
\hline 8UGKG & $1.0000 \mathrm{MSJBD}$ \\
\hline 16 UGKG & $1.0000 \mathrm{MSJBD}$ \\
\hline SUGKG & 1.0000 MSJBD \\
\hline 5UGKG & $1.0000 \mathrm{MSJBD}$ \\
\hline 5UGKG & $1.0000 \mathrm{MSJBD}$ \\
\hline 5UGKG & $1.0000 \mathrm{MSJBD}$ \\
\hline 5UGKG & 1.0000MSJ.BD \\
\hline 5UGKG & 1.0000 MSJBD \\
\hline 10UGKG & 1.0000 MSJBD \\
\hline 5UGKG & $1.0000 M S J B D$ \\
\hline 5UGKG & $1.0000 \mathrm{MSJBD}$ \\
\hline 10UGKG & $1.0000 M S J B D$ \\
\hline 5UGKG & $1.0000 \mathrm{MSJBD}$ \\
\hline 5UGKG & 1.0000 MSJBD \\
\hline 5UGKG & 1.0000 MSJBD \\
\hline 5UGKG & $1.0000 \mathrm{MSJBD}$ \\
\hline 5UGKG & $1.0000 M S J B D$ \\
\hline 5UGKG & 1.0000 MSJBD \\
\hline 5UGKG & $1.0000 M S J B D$ \\
\hline 5UGḲG & 1.0000 MSJBD \\
\hline 5UGKG & $1.0000 \mathrm{MSJBD}$ \\
\hline 10UGKG & $1.0000 M S J B D$ \\
\hline
\end{tabular}
w9002606.dat $-6 / 26 / 90-43$
OVINYL ACETATE

$0624 \mathrm{HN}$

OBROMODICHLOROMETHANEO624HN 01,2-DICHLOROPROPANE O624HN OCIS-1,3-DICHLOROPROPO624HN OTRICHLOROETHENE O624HN ODIBROMOCHLOROMETHANEO624HN $01,1,2-T R I C H L O R O E T H A N O 624 \mathrm{HN}$ OBENZENE : $0624 \mathrm{HN}$ OTRANS-1, 3-DICHLORORRO624HN OBROMOFORM $0624 \mathrm{HN}$ 04-METHYL-2-PENTANONEO624HN 02-HEXANONE O624HN OTETRACHLOROETHENE O624HN $01,1,2,2$-TETRACHLOROEO $624 \mathrm{HN}$ OTOLUENE

$0624 \mathrm{HN}$ OCHLOROBENZENE O624HN OETHYLBENZENE O624HN OSTYRENE O624HN OXYLENE (TOTAL) O624HN 50.000TOLUENE-D8 O624HN 50.000BROMOFLUOROBENZENE O624HN

$50.0001,2-D I C H L O R O E T H A N E-D O 624 \mathrm{HN}$ OCHLOROMETHANE O624HN OBROMOMETHANE O624HN OVINYL CHLORIDE $0624 \mathrm{HN}$ OCHLOROETHANE O624HN OMETHYLENE CHLORIDE O624HN OACETONE O624HN OCARBON DISULETDE O624HN 01,1-DICHLOROETHENE O624HN 01,1-DICHLOROETHANE 0624HN 01,2-DICHLOROETHENE $10624 \mathrm{HN}$ OCHLOROFORM O624HN 01,2-DICHLOROETHANE O624HN 02-BUTANONE 0624HN $01,1,1$-TRICHLOROETHANO $624 \mathrm{HN}$ OCARBON TETRACHLORIDE0624HN OVINYL ACETATE O624HN OBROMODICHLOROMETHANEO624HN 01,2-DICHLOROPROPANE 0624HN OCIS-1, 3-DICHLOROPROPO624 HN OTRICHLOROETHENE O624HN ODIBROMOCHLOROMETHANEO624HN $01,1,2-T R I C H L O R O E T H A N O 624 \mathrm{HN}$ OTRANS-1, 3-DICHLORORRO624HN OBROMOEORM O624HN 04-METHYL-2-PENTANONEO624HN 
0305900036 WA90LVW036 MBI 2HXONE2JLT 0305900036 WA90LVW036 MB1 TCLEE 2JLT 0305900036 WA90LVW036 MB1 TCLEA 2JLT 0305900036 WA90LVW036 MB1 MEC6H52JLT 0305900036WA90LVW036 MB1 CLC6H52JLT 0305900036 WA90LVW036 MBI ETC6H52JLT 0305900036 WA90LVW036 MBI STYR 2JLT 0305900036 WA90LVW036 MB1 XYLEN 2JLT
$1.0000 \mathrm{MSJBD}$

$1.0000 \mathrm{MSJBD}$

1.0000 MSJBD

$1.0000 \mathrm{MSJBD}$

$1.0000 \mathrm{MSJBD}$

$1.0000 \mathrm{MSJBD}$

$1.0000 \mathrm{MSJBD}$

1.0000MSJBD
02-HEXANONE

$0624 \mathrm{HN}$ OTETRACHLOROETHENE O624HN $01,1,2,2$-TETRACHLOROEO $624 \mathrm{HN}$ OTOLUENE

$0624 \mathrm{HN}$ OCHLOROBENZENE O624HN OETHYLBENZENE O624HN

OSTYRENE

OXYLENE (TOTAL) 
GRS 02C 0319900327900051 WA9003L865 001 MEC6D82J

GRS $0201 \cdot 0319900327900051$ WA9003L865 001 PBFB $2 \mathrm{~J}$

GRS 02010319900327900051 HA9003L865 001 12DCD42J

GRS 02010319900327900051 WA9003L865 001 CH3CL 2JLT

GRS 02010319900327900051 WA9003L865 001 CH3BR 2JLT

GRS 02010319900327900051 WA9003L865 001 C2H3CL2JLT

GRS 02010319900327900051 WA9003L865 001 C2H5CL2JLT

GRS 02010319900327900051 WA9003L865 001 CH2CL22JB

GRS 02010319900327900051 WA9003L865 001 ACET - 2JLT

GRS 02010319900327900051 WA9003L865 001 CS2 2JLT

GRS 02010319900327900051 WA9003L865 001 11DCE. 2JLT

GRS 02010319900327900051 WA9003L865 001 11DCLE2JLT

GRS 02010319900327900051 WA9003L865 001 T12DCE2JLT

GRS 02010319900327900051 WA9003L865 001 CHCL3 2JLT

GRS 02010319900327900051 WA9003L865 001 12DCLE2JLT

GRS 02010319900327900051 WA9003L865 001 MEK 2JLT

GRS 02010319900327900051 WA9003L865 001 111TCE2JLT

GRS 02010319900327900051 WA9003L865 001 CCL4 2JLT

GRS 02010319900327900051 WA9003L865 001 VINYLA2JLT

GRS 02010319900327900051 WA9003L865 001 BRDCLM2JLT

GRS 02010319900327900051 WA9003L865 001 12DCLP2JLT

GRS 02010319900327900051 WA9003L865 001 13DCPC2JLT

GRS 02010319900327900051 WA9003L865 001 TRCLE 2JLT

I GRS 02010319900327900051 WA9003L865 001 DBRCLM2JLT

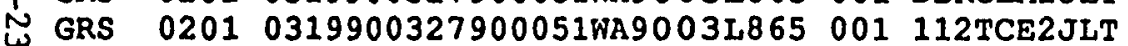

$w$ GRS 02010319900327900051 WA9003L865 001 C6H6 2JLT

GRS 02010319900327900051 WA9003L865 001 T13DCP2JIT

GRS 02010319900327900051 WA9003L865 001 CHBR3 2JLT

GRS 02010319900327900051 WA9003L865 001 4ME2PE2JLT

GRS 02010319900327900051 WA9003L865 001 2HXONE2JLT

GRS 02010319900327900051 WA9003L865 001 TCLEE 2JLT

GRS 02010319900327900051 WÀ9003L865 001 TCLEA 2JLT

GRS 02010319900327900051 WA9003L865 001 MEC6H52JLT

GRS 02010319900327900051 WA9003L865 001 CLC6H52JLT

GRS 02010319900327900051 WA9003L865 001 ETC6H52JLT

GRS 02010319900327900051 WA9003L865 001 STYR 2JLT

GRS 02010319900327900051 WA9003L865 001 XYLEN 2JLT

GRS 02010319900402900406 WA9003L865 001 NBD5 1X

GRS $02010319900402900406 \mathrm{WA9003L865} 001$ 2FBP $1 \mathrm{X}$

GRS 02010319900402900406 WA9003L865 001 PTERP $1 \mathrm{X}$

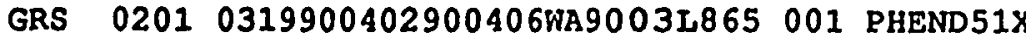

GRS $02010319900402900406 \mathrm{WA} 9003 L 865001$ 2FP $1 \mathrm{X}$

GRS 02010319900402900406 WA9003L865 001 246TBP1X

GRS $02010319900402900406 \mathrm{WA} 9003 L 865001$ PHENOL1XLT

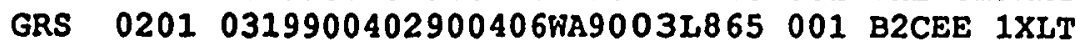

GRS $0201,0319900402900406 \mathrm{FA} 9003 \mathrm{~L} 865001$ 2CLP 1XLT

GRS $02010319900402900406 \mathrm{WA} 90032865001$ 13DCLB1XLT

GRS 02010319900402900406 FA9003L865 001 14DCLB1XLT $\checkmark 9 P E R$

$96 P E R$

BOPER

12 UGKG

12UGKG

12 UGKG

12 UGKG

11UGKG

12UGKG

6UGKG

6UGKG

6UGKG

6UGKKG

6UGKG

6UGKG

12UGKG

6UGKG

6UGKG

12UGKG

6UGKG

6UGKG

6UGKG

6UGKG

6UGKG

6UGKG

6UGKG

6UGKG

6UGKG

12 UGKG

12UGKG

6UGKG

6UGKG

6UGKG

6UGKG

6UGKG

6UGKG

6UGKG

65PER

57PER

63PER

79PER

74 PER

77RER

39OUGKG

390UGKG

390 UGKG

390UGKG

39 OUGKG
1. $0000 \mathrm{MSJBD}$

1. 0000MSJBD

$1.0000 \mathrm{MSJBD}$

1. $0000 \mathrm{MSJBD}$

1. $0000 \mathrm{MSJBD}$

1. $0000 \mathrm{MSJBD}$

1. $0000 \mathrm{MSJBD}$

1.0000MSJBD

1. $0000 \mathrm{MSJBD}$

1.0000MSJBD

1. $0000 \mathrm{MSJBD}$

1. $0000 \mathrm{MSJBD}$

1.0000MS JBD

$1.0000 \mathrm{MSJBD}$

1. $0000 \mathrm{MSJBD}$

1.0000MSJBD

1. $0000 \mathrm{MSJBD}$

1. $0000 \mathrm{MSJBD}$

1. $0000 \mathrm{MSJBD}$

1. $0000 \mathrm{MSJBD}$

1. $0000 \mathrm{MSJBD}$

1.0000MSJBD

1. $0000 \mathrm{MSJBD}$

1.0000MSJBD

$1.0000 \mathrm{MSJBD}$

1. $0000 \mathrm{MS}$ JBD

$1.0000 M S J B D$

1. $0000 \mathrm{MSJBD}$

1.0000MS JBD

1. $0000 \mathrm{MSJBD}$

1. $0000 \mathrm{MSJBD}$

1. $0000 \mathrm{MSJBD}$

1. $0000 \mathrm{MSJBD}$

1. $0000 \mathrm{MSJBD}$

1. $0000 \mathrm{MSJBD}$

1.0000MS JBD

1. $0000 \mathrm{MSJBD}$

1.0000MSCDT

1.0000MSCDT

$1.0000 \mathrm{MSCDT}$

1.0000MSCDT

1.0000MSCDT

1.0000MSCDT

1.0000MSCDT

1. $0000 \mathrm{MSCDT}$

1.0000MSCDT

1.0000MSCDT

1.0000MSCDT

61. 000TOLUENE-D8

$0624 \mathrm{HN}$

61.000BROMOEIUOROBENZENE O624HN

61.0001,2-DICHLOROETHANE-DO624HN OCHLOROMETHANE O624HN OBROMOMETHANE O624HN OVINYI CHIORIDE O624HN OCHIOROETHANE O624HN OMETHYIENE CHIORIDE O624HN OACETONE O624HN OCARBON DISULEIDE O624HN 01,1-DICHLOROETHENE 0624HN 01,1 -DICHLOROETHANE O624HN 01,2-DICHLOROETHENE (0624HN OCHLOROFORM 01,2-DICHLOROETHANE O624HN 02-BUTANONE O624HN $01,1,1$-TRICHLOROETHANO $24 \mathrm{HN}$ OCARBON TETRACHLORIDEO $624 \mathrm{HN}$ OVINYL ACETATE O624HN OBROMODICHLOROMETHANEO624HN 01,2-DICHLOROPROPANE O624HN OCIS-1, 3-DICHLOROPROPO624HN OTRICHLOROETHENE O624HN ODIBROMOCHLOROMETHANEO624HN $01,1,2-T R I C H L O R O E T H A N O 624 \mathrm{HN}$ OBENZENE O624HN OTRANS-1, 3-DICHLOROPRO624HN OBROMOFORM O624HN 04-METHYI-2-PENTANONEO624HN 02-HEXANONE - 0624HN OTETRACHLOROETHENE O624HN $01,1,2,2-T E T R A C H L O R O E O 624 \mathrm{HN}$ OTOLUENE OCHLOROBENZENE OETHYLBENZENE O624HN OSTYRENE

OXYLENE (TOTAL)

$0624 \mathrm{HN}$

1940.000NITROBENZENE-D5

1940.0002-ELUOROBIPHENYL

1940.000P-TERPHENYL-D14

$0624 \mathrm{HN}$

$0625 \mathrm{HN}$

3880.000 PHENOL-D5

$0625 \mathrm{HN}$

$0625 \mathrm{HN}$

$3880.0002,4,6$ O625HN OPHENOL '0625HN OBIS (2-CHLORंOETHYL) ETO625HN 02-CHLORORHENOL O625HN 01, 3-DICHLOROBENZENE O625HN 01, 4-DICHLOROBENZENE O625HN 
GRS 02010319900402900406 WA9003L865 001 B2ALC 1XLT GRS 02010319900402900406 WA9003L865 001 12DCLB1XLT GRS 02010319900402900406 WA9003L865 001 2MP 1XLT GRS $02010319900402900406 \mathrm{WA} 90032865001$ B2CIPE1XLT GRS $02010319900402900406 \mathrm{WA} 9003 \mathrm{~L} 865001$ 4MP 1 XLT GRS $02010319900402900406 \mathrm{WA} 9003$ L865 001 NDNPA 1XLT

02010319900402900406 WA9003L865 001 CL6ET 1XLT $0201031990040^{\circ} 2900406 \mathrm{WA} 90032865001 \mathrm{NB} \quad 1 \times \mathrm{LT}$ 02010319900402900406 WA9003L865 001 ISOPHR 1XLT $02010319900402900406 \mathrm{WA} 9003 \mathrm{~L} 8650012 \mathrm{NP} \quad 1 \times \mathrm{LT}$ 02010319900402900406 WA9003L865 001 24DMPN1XLT $02010319900402900406 \mathrm{WA} 9003$ L865 001 BENZOA1XLT $02010319900402900406 \mathrm{WA} 9003 \mathrm{~L} 865001$ B2CEXM1XLT 02010319900402900406 WA9003L865 001 24DCLP1XLT $02010319900402900406 \mathrm{WA} 9003 \mathrm{~L} 865001 \quad 124 \mathrm{TCB} 1 \mathrm{XLT}$ $02010319900402900406 \mathrm{WA} 9003 \mathrm{~L} 865001 \mathrm{NAP} 1 X L \mathrm{~T}$ $02010319900402900406 \mathrm{WA} 90032865001$ 4CANILIXLT $02010319900402900406 \mathrm{KA} 90034865001$ HCBD 1XLT 02010319900402900406 WA9003L865 001 4CL3C 1XLT $02010319900402900406 \mathrm{WA} 9003 \mathrm{~L} 865001$ 2MENAP1XLT $02010319900402900406 \mathrm{WA} 9003 \mathrm{~L} 865001$ CL6CP 1XLT $02010319900402900406 \mathrm{KA} 90032865001246 \mathrm{TCP} 1 \mathrm{XLT}$ 02010319900402900406 WA9003L865 001 245TCP1XLT $02010319900402900406 \mathrm{WA} 9003 \mathrm{~L} 865001$ 2CNAP 1XLT $02010319900402900406 \mathrm{WA} 9003 \mathrm{~L} 865001$ 2NANIL1XLT $02010319900402900406 \mathrm{WA} 90034865001$ DMP 1XLT 02010319900402900406 WA9003L865 001 ANAPYL1XLT 02010319900402900406 WA9003L865 001 26DNT 1XLT 02010319900402900406 WA9003L865 001 3NANIL1XLT $02010319900402900406 \mathrm{KA} 90034865001$ ANAPNE1XLT $02010319900402900406 \mathrm{KA} 9003 L 865001$ 24DNP 1XLT $02010319900402900406 \mathrm{WA} 9003 \mathrm{~L} 865001$ 4NP 1XLT $02010319900402900406 \mathrm{FA9003L865001}$ DIBZFU1XLT 02010319900402900406 WA9003L865 001 24DNT 1XLT $02010319900402900406 \mathrm{WA} 9003 L 865001$ DEP 1XLT $02010319900402900406 \mathrm{WA} 9003 \mathrm{~L} 865001$ 4CLPPE1XLT $02010319900402900406 \mathrm{WA} 9003 \mathrm{~L} 865001$ FLRENE1XLT $02010319900402900406 \mathrm{WA} 9003 \mathrm{~L} 865001$ 4NANIL1XLT $02010319900402900406 \mathrm{WA} 9003 \mathrm{~L} 865001$ 46DN2C1XLT $02010319900402900406 \mathrm{FA} 9003 \mathrm{~L} 865001$ NNDPA IXLT $02010319900402900406 \mathrm{KA} 9003 \mathrm{~L} 865001$ 4BRPPE1XLT $02010319900402900406 \mathrm{WA} 9003 L 865001$ CL6BZ 1XLT $02010319900402900406 \mathrm{WA} 9003 \mathrm{~L} 865001$ PCP 1XLT $02010319900402900406 \mathrm{KA} 9003 \mathrm{~L} 865001$ PHANTR1XLT $02010319900402900406 \mathrm{WA} 9003 L 865001$ ANTRC 1XLT $02010319900402900406 \mathrm{WA} 9003 \mathrm{~L} 865001$ DNBP 1XLT $02010319900402900406 \mathrm{KA} 9003 \mathrm{~L} 865$ 001 FANT 1XLT $02010319900402900406 \mathrm{WA9003L865001 \text {P.YR1XLT }}$
1. $0000 \mathrm{MSCDT}$ $1.0000 M S C D T$ $1.0000 \mathrm{MSCDT}$ $1.0000 \mathrm{MSCDT}$ $1.0000 \mathrm{MSCDT}$ $1.0000 \mathrm{MSCDT}$ $1.0000 \mathrm{MSCDT}$ $1.0000 \mathrm{MSCDT}$ 1. $0000 \mathrm{MSCDT}$ $1.0000 \mathrm{MSCDT}$ $1.0000 \mathrm{MSCDT}$ $1.0000 \mathrm{MSCDT}$ $1.0000 \mathrm{MSCDT}$ $1.0000 \mathrm{MSCDT}$ 1.0000MSCDT $1.0000 \mathrm{MSCDT}$ $1.0000 M S C D T$ $1.0000 \mathrm{MSCDT}$ $1.0000 M S C D T$ 1.0000MSCDT $1.0000 \mathrm{MSCDT}$ $1.0000 \mathrm{MSCDT}$ $1.0000 \mathrm{MSCDT}$ 1.0000MSCDT $1.0000 \mathrm{MSCDT}$ $1.0000 \mathrm{MSCDT}$ $1.0000 \mathrm{MSCDT}$ $1.0000 M S C D T$ 1.0000MSCDT $1.0000 \mathrm{MSCDT}$ 1.0000MSCDT $1.0000 \mathrm{MSCDT}$ 1.0000MSCDT 1.0000MSCDT $1.0000 \mathrm{MSCDT}$ $1.0000 \mathrm{MSCDT}$ $1.0000 \mathrm{MSCDT}$ $1.0000 \mathrm{MSCDT}$ $1.0000 \mathrm{MSCDT}$ $1.0000 \mathrm{MSCDT}$ 1.0000MSCDT 1.0000MSCDT $1.0000 \mathrm{MSCDT}$ 1. $0.000 \mathrm{MSCDT}$ 1.0000MSCDT $1.0000 \mathrm{MSCDT}$ 1.0000MSCDT 1.0000MSCDT
OBENZYL ALCOHOL $0625 \mathrm{HN}$ $01,2-D I C H L O R O B E N Z E N E$ O625HN 02-METHYLPHENOL O625HN OBIS 12-CHLOROISOPROPYO $625 \mathrm{HN}$ 0 4-METHYLRHENOL O625HN ON-NITROSO-DI-N-PROPYO625HN OHEXACHLOROETHANE O625HN ONITROBENZENE O625HN OISOPHORONE $\quad 0625 \mathrm{HN}$ 02-NITROPHENOL $0625 \mathrm{HN}$ 02,4 -DIMETHYLPHENOL $0625 \mathrm{HN}$ OBENZOIC ACID O625HN OBIS (2-CHLOROETHOXY) MO625HN $02,4-D I C H L O R O P H E N O L$ O625HN $01,2,4-T R I C H L O R O B E N Z E O 625 \mathrm{HN}$ ONAPHTHALENE O625HN 04-CHLOROANILINE O.625HN OHEXACHLOROBUTADIENE $0625 \mathrm{HN}$ 0 4-CHLORO-3-METHYLPHEO $625 \mathrm{HN}$ 02-METHYLNAPHTHALENE O625HN OHEXACHLOROCYCLOPENTAO625HN $02,4,6$-TRICHLOROPHENOO625HN $02,4,5-T R I C H L O R O P H E N O 0625 \mathrm{HN}$ 02-CHLORONAPHTHALENE 0625HN 02-NITROANILINE O625HN ODIMETHYLPHTHALATE $0625 \mathrm{HN}$ OACENAPHTHYLENE $0625 \mathrm{HN}$ 02,6-DINITROTOLUENE O625HN 03-NITROANILINE 0625HN OACENAPHTHENE O625HN 02,4-DINITROPHENOL O625HN 04-NITROPHENOL O625HN ODIBENZOFURAN $0625 \mathrm{HN}$ 02, 4-DINITROTOLUENE 0625HN ODIETHYLPHTHALATE O625HN 04-CHLOROPHENYL-PHENYO625HN OFLUORENE $0625 \mathrm{HN}$ 04-NITROANILINE $0625 \mathrm{HN}$ 04,6-DINITRO-2-METHYLO625HN ON-NITROSODIPHENYLAMIO625HN 04-BROMOPHENYL-PHENYLO625HN OHEXACHLOROBENZENE O625HN OPENTACHLOROPHENOL O625HN OPHENANTHRENE : $0625 \mathrm{HN}$ OANTHRACENE 0625HN ODI-N-BUTYLPHTHALATE O625HN OELUORANTHENE O625HN OPYRENE 
GRS 02U 0319900402900406 WA9003L865 001 BBZP 1XLT GRS $02010319900402900406 \mathrm{WA} 9003 \mathrm{~L} 865001$ 33CL2B1XLT GRS $02010319900402900406 \mathrm{FA9003L865} 001$ BAANTR1XLT GRS $02010319900402900406 \mathrm{KA} 0003 \mathrm{~L} 865001$ CHRY 1XLT GRS $02010319900402900406 \mathrm{WA} 9003 \mathrm{~L} 865001$ B2EHP 1XLT GRS 02010319900402900406 KA9003L865 001 DNOP 1XLT GRS $02010319900402900406 \mathrm{WA} 9003 \mathrm{~L} 865001$ BE2ANT1XLT GRS $02010319900402900406 \mathrm{HA} 9003 \mathrm{~L} 865$ 001 BKFANT1XLT GRS 02010319900402900406 WA9003L865 001 BAPYR 1XLT GRS $02010319900402900406 \mathrm{WA} 9003 \mathrm{~L} 865001$ ICDPYR1XLT GRS $02010319900402900406 \mathrm{WA} 9003 \mathrm{~L} 865001$ DBAHA 1XLT GRS $02010319900402900406 \mathrm{WA} 0003 \mathrm{~L} 865001$ BGHIPY1XLT GRS 02010319900402900406 WA9003L865 001SNBD5 $1 \mathrm{X}$ GRS 02010319900402900406 WA9003L865 001S2FBP $1 \mathrm{X}$ GRS 02010319900402900406 WA9003L865 001SPTERP 1X GRS 02010319900402900406 FA9003L865 001SPHEND51X GRS 02010319900402900406 WA9003L865 001S2FP 1X GRS 02010319900402900406 WA9003L865 001S246TBP1X GRS 02010319900402900406 WA9003L865 001SPHENOL1X GRS $02010319900402900406 \mathrm{WA} 90031865$ 001SB2CEE 1XLT GRS $02010319900402900406 \mathrm{WA} 9003 \mathrm{~L} 865$ 001S2CLP 1X GRS 02010319900402900406 WA9003L865 001S13DCLB1XLT GRS 02010319900402900406 WA9003L865 001S14DCLB1X

Ф GRS $02010319900402900406 \mathrm{WA} 9003 \mathrm{~L} 865$ 001SBZALC 1XLT

$\sim$ GRS $02010319900402900406 \mathrm{WA} 9003 \mathrm{~L} 865$ 001S12DCLB1XLT

$\underset{\sim}{\sim}$ GRS $02010319900402900406 \mathrm{WA} 9003 \mathrm{~L} 865$ 001S2MP 1XLT GRS $02010319900402900406 \mathrm{WA9003L865} 001$ SB2CIPE1XLT GRS 0201.0319900402900406 WA9003L865 001S4MP 1XLT GRS $02010319900402900406 \mathrm{WA} 9003 \mathrm{~L} 865$ 001SNDNPA $1 \mathrm{X}$ GRS $02010319900402900406 \mathrm{WA} 9003 \mathrm{~L} 865$ 001SCL6ET 1XLT GRS $02010319900402900406 \mathrm{KA9003L865}$ 001SNB 1XLT GRS $02010319900402900406 \mathrm{WA} 9003 L 865$ 001SISOPHR1X்T GRS $02010319900402900406 \mathrm{WA} 003 \mathrm{LB} 65$ 001S2NP 1XLT GRS $02010319900402900406 \mathrm{WA} 9003 L 865$ 001S24DMPN1XLT GRS 02010319900402900406 WA9003L865 001SBENZOA1XLT GRS 02010319900402900406 WA9003L865 001SB2CEXM1XLT GRS 02010319900402900406 WA9003L865 001S24DCLP1XLT GRS $02010319900402900406 \mathrm{WA} 003 \mathrm{~L} 8650015124 \mathrm{TCB} 1 \mathrm{X}$ GRS $02010319900402900406 \mathrm{WA} 9003 \mathrm{~L} 865$ 001SNAP 1XLT GRS $02010319900402900406 \mathrm{WA} 9003 \mathrm{~L} 865$ 001S4CANIL1XLT GRS $02010319900402900406 \mathrm{FA9003L865}$ 001SHCBD 1XLT GRS 02010319900402900406 WA9003L865 001S4CL3C IX GRS $02010319900402900406 \mathrm{WA} 0003 \mathrm{~L} 865$ 001S2MENAP1XLT GRS $02010319900402900406 \mathrm{FA9003L865}$ 001SCL6CP 1XLT GRS 02010319900402900406 WA9003L865 001S246TCP1XLT GRS $02010319900402900406 \mathrm{WA} 9003 \mathrm{~L} 8650015245 \mathrm{TCP} 1 \mathrm{XLT}$ GRS $02010319900402900406 \mathrm{FA} 9003 L 865$ 001S2CNAP 1XLT GRS $02010319900402900406 \mathrm{WA9003L865}$ 001S2NANIL1XLT
3YOUGKG 78 OUGKG 390UGKG 390UGKG 390UGKG 390UGKG 390UGKG 390UGKG 390UGKG 390UGKG 390UGKG 390UGKG 65PER 67PER 62PER 66PER 59PER 63PER

72PER

39OUGKG 69PER

39OUGKG 60RER

390UGKG 390UGKG 390UGKG 390UGKG 39 OUGKG 83PER

39OUGKG 390UGKG 390UGKG 390UGKG 390UGKG

2000UGKG 390UGKG 39OUGKG 73PER

39OUGKG 390UGKG 390UGKG 72PER

39OUGKG 390UGKG 390UGKG 2000UGKG 390 UGKG 2000 UGKG
$1.0000 \mathrm{MSCDT}$ $1.0000 M S C D T$ $1.0000 \mathrm{MSCDT}$ $1.0000 \mathrm{MSCDT}$ $1.0000 \mathrm{MSCDT}$ $1.0000 \mathrm{MSCDT}$ $1.0000 M S C D T$ $1.0000 \mathrm{MSCDT}$

$1.0000 \mathrm{MSCDT}$

$1.0000 \mathrm{MSCDT}$

$1.0000 \mathrm{MSCDT}$

$1.0000 \mathrm{MSCDT}$

$1.0000 \mathrm{MSCDT}$

$1.0000 \mathrm{MSCDT}$

$1.0000 \mathrm{MSCDT}$

$1.0000 \mathrm{MSCDT}$

$1.0000 \mathrm{MSCDT}$

$1.0000 \mathrm{MSCDT}$

$1.0000 \mathrm{MSCDT}$

$1.0000 \mathrm{MSCDT}$

$1.0000 \mathrm{MSCDI}$

$1.0000 \mathrm{MSCDT}$

$1.0000 \mathrm{MSCDT}$

$1.0000 \mathrm{MSCDT}$

$1.0000 \mathrm{MSCDT}$

$1.0000 \mathrm{MSCDT}$

$1.0000 \mathrm{MSCDT}$

1.0000MSCDT

1:0000MSCDT

$1.0000 \mathrm{MSCDT}$

$1.0000 \mathrm{MSCDT}$

$1.0000 \mathrm{MSCDT}$

$1.0000 \mathrm{MSCDT}$

$1.0000 \mathrm{MSCDT}$

$1.0000 \mathrm{MSCDT}$

$1.0000 \mathrm{MSCDT}$

$1.0000 \mathrm{MSCDT}$

$1.0000 \mathrm{MSCDT}$

$1.0000 \mathrm{MSCDT}$

$1.0000 \mathrm{MSCDT}$

$1.0000 \mathrm{MSCDT}$

$1.0000 \mathrm{MSCDT}$

$1.0000 \mathrm{MSCDT}$

$1.0000 \mathrm{MSCDT}$

$1.0000 \mathrm{MSCDT}$

$1.0000 \mathrm{MSCDT}$

$1.0000 \mathrm{MSCDT}$

$1.0000 \mathrm{MSCDT}$
OBUTYLBENZYLPHTHALATEO $625 \mathrm{HN}$ 03, $3^{\prime}$-DICHLOROBENZIDIO625HN OBENZO (A) ANTHRACENE O625HN OCHRYSENE 0625HN OBIS (2-ETHYLHEXYL) PHTO625HN ODI-N-OCTYL PHTHALATEO $625 \mathrm{HN}$ OBENZO (B) FLUORANTHENEO625 $\mathrm{HN}$ OBENZO (K) FLUORANTHENEO625HN OBENZO (A) PYRENE O625HN 0 INDENO $(1,2,3-C D)$ PYREO625HN ODIBENZO $(\mathrm{A}, \mathrm{H})$ ANTHRACEO $625 \mathrm{HN}$ OBENZO (G, H, I) PERYLENEO $625 \mathrm{HN}$

1955.00ONITROBENZENE-D5 . O625HS 1955.0002-FLUOROBIPHENYL O625HS 1955.000P-TERPHENYL-D14 O625HS 3910.001PHENOL-D5 0625HS 3910.0012-FLUOROPHENOL O625HS $3910.0012,4,6$-TRIBROMOPHENOLO625HS 3910.001 PHENOI $0625 \mathrm{HS}$ OBIS (2-CHLOROETHYL) ETO625HS

3910.0012-CHLOROPHENOL O625HS 01,3-DICHLOROBENZENE O625HS 1955.0001,4-DICHLOROBENZENE O625HS OBENZYL ALCOHOL O625HS 01,2-DICHLOROBENZENE O625KS 02-METHYLPHENOL O625HS OBIS (2-CHLOROISORROPYO625HS 04-METHYLPHENOL O625HS

1955.000N-NITROSO-DI-N-PROPYO625HS OHEXACHLOROETHANE O625HS ONITROBENZENE O625HS OISOPHORONE O625HS 02-NITROPHENOL O625HS 02, 4-DIMETHYLPHENOL O625HS OBENZOIC ACID O625HS OBIS (2-CHLOROETHOXY) MO625HS 02, 4-DICHLOROPHENOL O625HS

$1955.0001,2,4$-TRICHLOROBENZEO625HS ONAPHTHALENE O625HS 04-CHLOROANILINE O625HS OHEXACHLOROBUTADIENE O625HS

3910.0014-CHLORO-3-METHYLPHEO625HS 02-METHYLNAPHTHALENE 0625HS OHEXACHLOROCYCLOPENTAO625HS 02, 4, 6-TRICHLOROPHENOO625HS 02, 4, 5-TRICHLOROPHENOO625HS 02-CHLORONAPHTHALENE O625HS 02-NITROANILINE O625HS 
GRS $02010319900402900406 \mathrm{WA} 9003 \mathrm{~L} 865001$ SDMP 1XLT GRS $02010319900402900406 \mathrm{WA} 9003 \mathrm{~L} 865001$ SANAPYL1XLT GRS $02010319900402900406 \mathrm{WA} 9003 \mathrm{~L} 865001$ S26DNT 1XLT GRS $02010319900402900406 \mathrm{WA} 9003 \mathrm{~L} 865001$ S3NANIL1XLT GRS $02010319900402900406 \mathrm{WA} 9003 \mathrm{~L} 865001$ SANAPNE $1 X$ GRS $02010319900402900406 \mathrm{WA} 9003 \mathrm{~L} 865001$ S24DNP 1XLT GRS 02010319900402900406 WA9003L865 001S4NP $1 X$ GRS $02010319900402900406 \mathrm{WA} 9003 \mathrm{~L} 865001$ SDIBZFUIXLT GRS $02010319900402900406 \mathrm{WA} 9003 \mathrm{~L} 865$ 001S24DNT 1X GRS $02010319900402900406 \mathrm{WA} 9003 \mathrm{~L} 865001$ SDEP 1XLT GRS $02010319900402900406 \mathrm{WA} 9003 \mathrm{~L} 865001$ S4CLPPE1XLT GRS $02010319900402900406 \mathrm{WA} 90032865$ 001SFLRENE1XLT GRS $02010319900402900406 \mathrm{WA} 9003 \mathrm{~L} 865001$ S4NANIL1XLT

$02010319900402900406 \mathrm{WA} 9003 \mathrm{~L} 655001546 \mathrm{DN2C1XLT}$ 02010319900402900406 WA9003L865 001SNNDPA 1XLT $02010319900402900406 \mathrm{WA} 9003 \mathrm{~L} 86500154 \mathrm{BRPPE} 1 \mathrm{XLT}$ $02010319900402900406 \mathrm{WA} 9003 L 865001$ SCL6BZ 1XLT 02010319900402900406 WA9003L865 001SPCP 1X $02010319900402900406 \mathrm{WA} 90032865001$ SPHANTR1XLT 02010319900402900406 WA9003L865 001SANTRC 1XLT $02010319900402900406 \mathrm{WA} 9003 \mathrm{~L} 865001$ SDNBP 1XLT 02010319900402900406 WA9003L865 001SFANT 1XLT 02010319900402900406 WA9003L865 001SPYR $1 \mathrm{X}$ 02010319900402900406 WA9003L865 001SBBZP 1XLT $02010319900402900406 \mathrm{WA} 9003 \mathrm{~L} 865001$ S33CL2B1XLT 02010319900402900406 WA9003L865 001SBAANTR1XLT $02010319900402900406 \mathrm{WA} 9003 \mathrm{~L} 865$ 001SCHRY 1XLT 02010319900402900406 WA9003L865 001SB2EHP 1XLT 02010319900402900406 WA9003L865 001SDNOP 1XLT $02010319900402900406 \mathrm{WA} 9003 L 865001$ SBE2ANT1XLT 02010319900402900406 KA9003L865 001SBKFANT1XLT $02010319900402900406 \mathrm{WA} 90032865$ 001SBAPYR 1XLT $02010319900402900406 \mathrm{WA} 9003 \mathrm{~L} 865001$ SICDPYR1XLT $02010319900402900406 \mathrm{WA} 9003 \mathrm{~L} 865$ 001SDBAHA 1XLT 02010319900402900406 WA9003L865 001SBGHIPY1XLT 04010319900328900051 WA9003L865 002 04010319900423901200 WA9003L865 002 ALPHAG9W 04010319900423901200 WA9003L865 002 BETAG 9W 04010319900519900451 WA9003L865 002 AG 2ZLT 04010319900518900450 WA9003L865 002 AS 1BLT 04010319900519900451 WA9003L865 002 BA 2ZLT 04010319900519900451 WA9003L865 002 CD 22 04010319900519900451 KA9003L865 002 CR $\quad 2 \mathrm{z}$ 04010319900405900055 WA9003L865 002 HGTOT 1DLT 04010319900518900450 WA9003L865 002 PB $1 B$ 04010319900518900450 WA9003L865 $002 \mathrm{SE}$ 1BLT 04010319900327900051 WA9003L865 002 MEC6D82J 04010319900327900051 FA9003L865 002 PBEB 2J

102PER

390 UGKG 390 UGKG 390 UGKG 2000UGKG 73PER

2000 UGKG 85 PER

39OUGKG

$71 P E R$

390 UGKG

390UGKG

390 UGKG

2000UGKG

2000 UGKG

390 UGKG

390 UGKG

390UGKG

88PER

39OUGKG

390 UGKG

39 OUGKG

390 UGKG

60PER

390 UGKG

780 UGKG

390UGKG

39 OUGKG

390 UGKG

39 OUGKG

39 OUGKG

390 UGKG

390 UGKG

39OUGKG

39OUGKG

390 UGKG

$94.2 \%$

11. OPCIG

13. OPCIG

1. 9 MGKG

2. 1MGKG

37. 5MGKG

1. 2MGKG

5. 7MGKG

$0.11 M G K G$

$0.95 \mathrm{MGKG}$

1.1 MGKG

113PER
$1.0000 \mathrm{MSCDT}$

1.0000MSCDI

1.0000MSCDT

1.0000MSCD

$1.0000 \mathrm{MSCDT}$

$1.0000 \mathrm{MSCD}$

$1.0000 \mathrm{MSCDT}$

1.0000MSCDT

$1.0000 \mathrm{MSCDT}$

1. $0000 \mathrm{MSCDT}$

1.0000MSCDT

1. $0000 \mathrm{MSCDT}$

$1.0000 \mathrm{MSCDT}$

1.0000MSCDI

$1.0000 \mathrm{MSCDT}$

1.0000MSCDT

1.0000MSCDT

1.0000MSCDT

1.0000MSCDT

1.0000MSCDT

$1.0000 \mathrm{MSCDT}$

1.0000MSCDT

1. $0000 \mathrm{MSCDT}$

$1.0000 \mathrm{MSCDT}$

1. $0000 \mathrm{MSCD}$

1. $0000 \mathrm{MSCDT}$

1.0000MSCDT

1. $0000 \mathrm{MSCDT}$

1.0000MSCDT

1.0000MSCDT

1. $0000 \mathrm{MSCDT}$

1.0000MSCDT

1.0000MSCDT

1. $0000 \mathrm{MSCDT}$

1.0000MSCDT $10 \mathrm{JH}$

10 EBUNK

10EBUNK

1.00ICALM

1. 00AAAER

1. OOICALM

1.00ICAIM

1.00ICALM

$1.00 \mathrm{HGJJH}$

1. OOAAEJS

1.00AAMLR

$1.0000 M S J B D$

1.0000MSJBD
ODIMETHYLPHTHALATE

OACENAP HTHYLENE

$0625 \mathrm{HS}$

0625HS

02,6-DINITROTOLUENE 0625HS

03-NITROANIIINE O625HS

1955.000ACENAPHTHENE 0625HS

02,4-DINITROPHENOL 0625HS

3910.0014-NITROPHENOL O625HS ODIBENZOFURAN

1955.0002,4-DINITROTOLUENE O625HS ODIETHYLPHTHALATE 0625HS 04-CHLOROPHENYL-PHENYO625HS OFLUORENE O625HS 04-NITROANILINE 0625HS 04, 6-DINITRO-2-METHYLO625HS ON-NITROSODIPHENYLAMIO $625 \mathrm{HS}$ 04-BROMOPHENYL-PHENYLO625HS OHEXACHLOROBENZENE O625HS

3910.001PENTACHLOROPHENOL O625HS OPHENANTHRENE O625HS OANTHRACENE O625HS ODI-N-BUTYLPHTHALATE 0625HS OELUORANTHENE O625HS 1955. 000PYRENE O625HS OBUTYIBENZYLPHTHALATEO $625 \mathrm{HS}$ $03,3^{\prime}$-DICHLOROBENZIDIO625HS OBENZO (A) ANTHRACENE O625HS OCHRYSENE 0625HS OBIS (2-ETHYLHEXYL) PHTO625HS ODI-N-OCTYI PHTHALATEO625HS OBENZO (B) FLUOORANTHENE0625HS OBENZO (K) ELUORANTHENEO $625 \mathrm{HS}$ OBENZO (A) PYRENE 0625HS OINDENO $(1,2,3-C D)$ PYREO 625HS ODIBENZO (A, H) ANTHRACEO625HS OBENZO (G, H, I) PERYLENEO 625HS Of SOIIDS OGROSS AIPHA IALPHN ONONVOLATILE BETA IBETAN OSILVER, TOTAL MAGTON OARSENIC, TOTAL MASTON OBARIUM, TOTAL MBATON OCADMIUM, TOTAL MCDTON OCHROMIUM, TOTAI MCRTON OMERCURY, TOTAL . MHGTON OLEAD, TOTAL, MPBTON OSELENIUM, TOTAL MSETON '54.500TOLUENE-D8 8 OG24HN 54. 500BROMOELUOROBENZENE 
GRS $04 U_{2} 0319900327900051$ WA9003L865 002 12DCD42J

GRS 04010319900327900051 WA9003L865 002 CH3CL 2JLT

GRS 04010319900327900051 WA9003L865 002 CH3BR 2JLT

GRS 04010319900327900051 WA9003LB65 002 C2H3CL2JLT

GRS 04010319900327900051 WA9003L865 002 C2HSCL2JLT

GRS 04010319900327900051 WA9003L865 002 CH2CL22JB

GRS 04010319900327900051 WA9003L865 002 ACET 2JB

GRS 04010319900327900051 WA9003L865 002 CS2 2JLT

GRS 04010319900327900051 WA9003L865 002 11DCE 2JLT

GRS 04010319900327900051 WA9003L865 002 11DCLE2JLT

GRS 04010319900327900051 WA9003L865 002 T12DCE2JLT

GRS 04010319900327900051 WA9003L865 002 CHCL3 2JLT

GRS 04010319900327900051 WA9003L865 002 12DCLE2JLT

GRS 04010319900327900051 WA9003L865 002 MEK 2JLT

GRS 04010319900327900051 FA9003L865 002 111TCE2JLT

GRS 04010319900327900051 WA9003L865 002 CCL4 2JLT

GRS 04010319900327900051 WA9003L865 002 VINYLA2JLT

GRS 04010319900327900051 WA9003L865 002 BRDCLM2JLT

GRS 04010319900327900051 WA9003L865 002 12DCLP2JLT

GRS 04010319900327900051 WA9003L865 002 13DCPC2JLT

GRS 04010319900327900051 WA9003L865 002 TRCLE 2JLT

GRS 04010319900327900051 WA9003L865 002 DBRCLM2JLT

GRS 04010319900327900051 WA9003L865 002 112TCE2JLT

to GRS 04010319900327900051 WA9003L865 002 C6H 6 2JLT

$\tilde{\omega}$ GRS 04010319900327900051 WA9003L865 002 T13DCP2JLT

GRS 04010319900327900051 WA9003L865 002 CHBR3 2JLT

GRS 0401.0319900327900051 WA9003L865 002 4ME2PE2JLT

GRS 04010319900327900051 WA9003L865 002 2HXONE2JLT

GRS 04010319900327900051 WA9003L865 002 TCLEE 2JLT

GRS 04010319900327900051 WA9003L865 002 TCLEA 2JLT

GRS 04010319900327900051 KA9003L865 002 MEC6H52JLT

GRS - 04010319900327900051 WÄ9003L865 002 CLC6H52JLT

GRS 04010319900327900051 WA9003L865 002 ETC6H52JLT

GRS 04010319900327900051 WA9003L865 002 STYR 2JLT

GRS 04010319900327900051 WA9003L865 002 XYLEN 2JLT

GRS 04010319900402900406 WA9003L865 002 NBD5 $1 X$

GRS 04010319900402900406 WA9003L865 002 2FBP $1 \mathrm{x}$

GRS 04010319900402900406 WA9003L865 002 PTERP 1X

GRS 04010319900402900406 KA9003L865 002 PHEND51X

GRS 04010319900402900406 WA9003L865 002 2FP $1 \mathrm{X}$

GRS 04010319900402900406 KA9003L865 $002 \quad 246 \mathrm{TBP} 1 \mathrm{X}$

GRS $04010319900402900406 \mathrm{KA} 90032865002$ PHENOLIXLT

GRS 04010319900402900406 FA9003L865 002 B2CEE 1XLT

GRS $04010319900402900406 \mathrm{KA} 9003 L 865002$ 2CLP 1XLT

GRS $04010319900402900406 \mathrm{KA} 90032865002$ 13DCLB1XLT

GRS 04010319900402900406 FA9003L865 002 14DCLB1XLT

GRS $04010319900402900406 \mathrm{KA} 9003 L 865002$ BZALC 1XLT

GRS 0401 0319900402900406\%A9003L865 002 12DCLB1XLT
97PER

IIUGKG

IIUGKG

IIUGKG

11UGKG

17UGKG

12UGKG

SUGKG

5UंGKG

5UGKG

5UGKG

5UGKG

5UGKG

IIUGKG

5UGKG

SUGKG

IIUGKG

SUGKG

5UGKG

5UGKG

5UGKG

5UGKG

SUGKG

5UGKG

5UGKG

5UGKG

11UGKG

IIUGKG

5UGKG

5UGKG

SUGKG

5UGKG

5UGKG

SUGKG

SUGKG

$73 P E R$

65PER

67PER

76 PER

69PER

56PER

35 OUGKG

350UGKG

350UGKG

350UGKG

350UGKG

350UGKG

350UGKG
$1.0000 M S J B D$

$1.0000 M S J B D$

$1.0000 \mathrm{MSJBD}$

$1.0000 M S J B D$

$1.0000 M S J B D$

$1.0000 M S J B D$

$1.0000 \mathrm{MSJBD}$

$1.0000 M S J B D$

$1.0000 \mathrm{MSJBD}$

$1.0000 M S J B D$

$1.0000 \mathrm{MSJBD}$

$1.0000 M S J B D$

$1.0000 \mathrm{MSJBD}$

$1.0000 \mathrm{MSJBD}$

$1.0000 \mathrm{MSJBD}$

$1.0000 \mathrm{MSJBD}$

$1.0000 \mathrm{MSJBD}$

1.0000MSJBD

1.0000MSJBD

$1.0000 \mathrm{MSJBD}$

$1.0000 \mathrm{MSJBD}$

$1.0000 \mathrm{MSJBD}$

$1.0000 \mathrm{MSJBD}$

$1.0000 \mathrm{MSJBD}$

$1.0000 \mathrm{MSJBD}$

$1.0000 \mathrm{MSJBD}$

1.0000MSJBD

1. 0000 MSJBD

1.0000MSJBD

$1.0000 M S J B D$

1.0000 MSJBD

$1.0000 M S J B D$

1.0000MSJBD

$1.0000 M S J B D$

$1.0000 \mathrm{MSJBD}$

$1.0000 \mathrm{MSCDT}$

$1.0000 \mathrm{MSCDT}$

$1.0000 \mathrm{MSCDT}$

$1.0000 \mathrm{MSCDT}$

1.0000MSCDT

$1.0000 \mathrm{MSCDT}$

$1.0000 \mathrm{MSCDT}$

1.0000MSCDT

$1.0000 \mathrm{MSCDT}$

$1.0000 \mathrm{MSCDT}$

$1.0000 \mathrm{MSCDT}$

$1.0000 \mathrm{MSCDT}$

$1.0000 \mathrm{MSCDT}$
54.5001,2-DICHLOROETHANE-DO624HN OCHLOROMETHANE OBROMOMETHANE O624HN OVINYI CHLORIDE O624HN OCHLOROETHANE . 0624HN OMETHYLENE CHLORIDE O624HN OACETONE $0624 \mathrm{HN}$ OCARBON DISULFIDE O624HN 01,1-DICHLOROETHENE O624HN 01,1-DICHLOROETHANE O624HN 01,2-DICHLOROETHENE $10624 \mathrm{HN}$ OCHLOROFORM $0624 \mathrm{kN}$ 01,2-DICHLOROETHANE 0624HN 02-BUTANONE $0624 \mathrm{HN}$ $01,1,1$-TRICHLOROETHANO $624 \mathrm{HN}$ OCARBON TETRACHLORIDEO $624 \mathrm{HN}$ OVINYL ACETATE $0624 \mathrm{HN}$ OBROMODICHLOROMETHANEO $24 \mathrm{HN}$ 01,2-DICHLOROPROPANE 0624HN OCIS-1, 3-DICHLOROPROPO $624 \mathrm{HN}$ OTRICHIOROETHENE O624HN ODIBROMOCHLOROMETHANEO624HN 01,1,2-TRICHLOROETHANO624HN OBENZENE $0624 \mathrm{HN}$ OTRANS - 1, 3-DICHLOROPRO $624 \mathrm{HN}$ OBROMOFORM $0624 \mathrm{HN}$

04-METHYL-2-PENTANONEO624HN 02-HEXANONE O624HN OTETRACHLOROETHENE O624HN 01, 1, 2, 2-TETRACHLOROEO624HN OTOLUENE $0624 \mathrm{HN}$ OCHLOROBENZENE O624HN OETHYLBENZENE O624HN OSTYRENE

OXYLENE (TOTAL)

1745.000NITROBENZENE-D5 1745.0002-FLUOROBIPHENYL $0624 \mathrm{HN}$ $0624 \mathrm{HN}$ $0625 \mathrm{HN}$ $0625 \mathrm{HN}$ $0625 \mathrm{HN}$ 3489.999 PHENOL-D5 $\quad$ O625HN 3489.9992-FLUOROPHENOL O625HN $3489.9992,4,6$-TRIBROMOPHENOLO $625 \mathrm{HN}$ OPHENOL $0625 \mathrm{HN}$ OBIS (2-CHLOROETHYL) ETO625HN 02-CHLOROPHENOL O625HN 01,3-DICHLOROBENZENE O625HN 01,4-DICHLOROBENZENE 0625 HN OBENZYL ALCOHOL OGP5HN 01,2-DICHLOROBENZENE O625HN 
(X) 0319900402900406 WA9003L865 002 2MP IXLT 04010319900402900406 WA9003L865 002 4MP 1XLT $04010319900402900406 \mathrm{WA} 9003 \mathrm{~L} 865002$ NDNPA 1XLT 04010319900402900406 WA9003L865 002 CL6ET 1XLT 04010319900402900406 WA9003L865 $002 \mathrm{NB}$ 1XLT 04010319900402900406 WA9003L865 002 ISOPHRIXLT 04010319900402900406 WA9003L865 002 2NP 1XLT 04010319900402900406 WA90034865002 24DMPN1XLT $04010319900402900406 \mathrm{WA} 9003 L 865002$ BENZOA1XLT 04010319900402900406 WA9003L865 002 B2CEXM1XLT 04010319900402900406 WA9003L865 002 24DCLP1XLT 04010319900402900406 WA9003L865 $002 \quad 124 \mathrm{TCB} 1 \times L T$ 04010319900402900406 WA9003L865 002 NAP 1XLT 04010319900402900406 WA9003L865 002 4CANIL1XLT 04010319900402900406 WA9003L865 002 HCBD 1XLT $04010319900402900406 \mathrm{WA} 9003 L 865002$ 4CL3C 1XLT 04010319900402900406 พA9003L865 002 2MENAP 1XLT 04010319900402900406 WA9003L865 002 CL6CP 1XLT 04010319900402900406 HA9003L865 002 246TCP1XLT $04010319900402900406 \mathrm{WA} 9003 \mathrm{~L} 865002$ 245TCP1XLT 04010319900402900406 WA9003L865 002 2CNAP 1XLT 04010319900402900406 WA9003L865 002 2NANILIXLT 04010319900402900406 WA9003L865 002 DMP 1XLT 04010319900402900406 WA9003L865002 ANAPYLIXLT 04010319900402900406 WA9003L865 002 26DNT 1XLT $04010319900402900406 \mathrm{WA} 90032865002$ 3NANILIXLT 04010319900402900406 WA9003L865 002 ANAPNE1XLT 04010319900402900406 WA9003L865 002 24DNP 1XLT 04010319900402900406 WA9003L865 002 4NP 1XIT 04010319900402900406 FA9003L865 002 DIBZEU1XLT 04010319900402900406 WA9003L865 002 24DNT 1XLT 04010319900402900406 WA9003L865 002 DEP IXLT 04010319900402900406 WA9003L865 002 4CLPPE1XLT 04010319900402900406 WA9003L865 002 FLRENE1XLT $04010319900402900406 \mathrm{WA} 90032865002$ 4NANILIXIT 04010319900402900406 WA9003L865 002 46DN2C1XLT 04010319900402900406 WA9003L865 002 NNDPA 1XLT 04010319900402900406 WA9003L865 002 4BRPPEIXLT 04010319900402900406 WA9003L865 002 CL6BZ 1XLT 04010319900402900406 WA9003L865 002 PCP 1XLT 04010319900402900406 WA9003L865 002 PHANTR1XLT 04010319900402900406 WA9003L865 002 ANTRC 1XIT 04010319900402900406 WA9003L865 002. DNBP 1XLT 04010319900402900406 WA9003L865 002 FANT 1XLT 04010319900402900406 WA90031865.002 PYR 1XIT 04010319900402900406 FA9003L865 002 BBZP 1XLT 04010319900402900406 คA9003L865 002 33CL2B1XLT
350UGKG 350 UGKG 350UGKG 350 UUGKG 350UGKG 350UGKG 350UGKG 350UGKG 350UGKG

1700 UGKG

350UGKG

350UGKG

350UGKG

350 UGKG

350 UGKG

350UGKG

350UGKG

350UGKG

350UGKG

350UGKG

1700UGKG

350 UGKG

1700 UGKG

350UGKG

350UGKG

35 OUGKG

1700 UGKG

350UGKG

1700UGKG

1700UGKG

350UGKG

350UGKG

350UGKG

350UGKG

350UGKG

1700UGKG

1700UGKG

350UGKG

350UGKG

350UGKG

1700 UGKG 350UGKG 350UGKG 350UGKG 350UGKG 350UGKG 350UGKG 700UGKG
1.0000MSCDT $1.0000 \mathrm{MSCDT}$ $1.0000 \mathrm{MSCDT}$ 1.0000MSCDT $1.0000 \mathrm{MSCDT}$ $1.0000 \mathrm{MSCDT}$ 1.0000MSCDI $1.0000 \mathrm{MSCDT}$ $1.0000 \mathrm{MSCDT}$ 1.0000MSCDT 1.0000MSCDT $1.0000 \mathrm{MSCDT}$ 1.0000MSCDT $1.0000 \mathrm{MSCDT}$ $1.0000 \mathrm{MSCD}$ 1.0000MSCDT 1.0000MSCDT $1.0000 \mathrm{MSCDT}$ 1.0000MSCDT $1.0000 \mathrm{MSCDT}$ 1.0000MSCDT 1.0000MSCDT 1.0000MSCDT $1.0000 \mathrm{MSCDT}$ 1.0000MSCDT 1.0000MSCDT 1.0000MSCDT 1.0000MSCD' $1.0000 \mathrm{MSCDT}$ 1.0000MSCDT $1.0000 \mathrm{MSCDT}$ $1.0000 \mathrm{MSCDT}$ 1.0000MSCDT $1.0000 \mathrm{MSCDI}$ $1.0000 M S C D T$ $1.0000 M S C D T$ $1.0000 \mathrm{MSCDT}$ $1.0000 \mathrm{MSCDT}$ 1.0000MSCDT $1.0000 \mathrm{MSCDT}$ 1.0000MSCDT $1.0000 \mathrm{MSCDT}$ $1.0000 \mathrm{MSCDT}$ $1.0000 \mathrm{MSCDT}$ 1.0000MSCDT $1.0000 \mathrm{MSCDT}$ 1.0000MSCDT $1.0000 \mathrm{MSCDT}$
02-METHYLPHENOL $0625 \mathrm{HN}$ O2-METHYLPHENOL 04-METHYLPHENOL O625HN ON-NITROSO-DI-N-PROPYO $625 \mathrm{HN}$ OHEXACHLOROETHANE O625HN ONITROBENZENE O625HN OISOPHORONE O625HN 02-NTTROPHENOL 02, 4-D IMETHYLPHENOL O625HN OBENZOIC ACID 0625HN OBIS (2-CHLOROETHOXY) MO625HN $02,4-D I C H L O R O P H E N O L$ O625HN $01,2,4-T R I C H L O R O B E N Z E 0625 \mathrm{HN}$ ONAPHTHALENE O625HN 04-CHLOROANILINE 0625HN OHEXACHLOROBUTADIENE 0625HN 04-CHLORO-3-METHYLPHEO 625HN 02-METHYLNAPHTHALENE 0625HN OHEXACHLOROCYCLOPENTAO625HN $02,4,6$-TRICHLOROPHENOO625HN $02,4,5$-TRICHLOROPHENO0625HN 02-CHLORONAPHTHALENE O625HN 02-NITROANILINE O625 HN ODIMETHYLPHTHALATE O625HN OACENAPHTHYLENE 0625HN 02,6-DINITROTOLUENE 0625HN 03-NITROANILINE O625HN OACENAPHTHENE O625HN 02, 4-DINITROPHENOL O625HN O4-NITROPHENOL 0625HN ODIBENZOFURAN O625HN 02, 4-DINITROTOLUENE O625HN ODIETHYLPHTHALATE O625HN 04-CHLOROPHENYL-PHENYO625HN OFLUORENE O625HN 04-NITROANILINE $0625 \mathrm{HN}$ 04, 6-DINITRO-2-METHYLO625HN ON-NITROSODIPHENYLAMIO625HN 04-BROMOPHENYL-PHENYLO625HN OHEXACHLOROBENZENE 0625HN OPENTACHLOROPHENOL O625HN OPHENANTHRENE. O625HN OANTHRACENE 0625HN ODI-N-BUTYLPHTHALATE O625HN OFLUORANTHENE O625HN OPYRENE O625HN OBUTYLBENZYLPHTHAILATEO $625 \mathrm{HN}$ 03, $3^{\prime}$-DICHLOROBENZIP $625 \mathrm{HN}$ 
GRS 04L J319900402900406KA9003L865 002 BAANTRIXLT GRS $04010319900402900406 \mathrm{WA} 9003 \mathrm{LB} 65002$ CHRY 1XLT GRS $04010319900402900406 \mathrm{WA} 9003 \mathrm{~L} 865002$ B2EHP 1XLT GRS 04010319900402900406 WA9003L865 002 DNOP 1XLT GRS $04010319900402900406 \mathrm{WA} 9003 \mathrm{~L} 865002$ BE2ANT1XLT GRS $04010319900402900406 \mathrm{WA} 9003 \mathrm{~L} 865002$ BKEANT1XLT GRS 04010319900402900406 WA9003LB65 002 BAPYR 1XLT GRS $04010319900402900406 \mathrm{WA} 9003 L 865002$ ICDPYR1XLT GRS $04010319900402900406 \mathrm{WA} 003 \mathrm{LB} 65002$ DBAHA 1XLT GRS 04010319900402900406 WA9003L865 002 BGHIPY1XLT GRS 04010319900402900406 WA9003L865 002RNBD5 1X GRS 04010319900402900406 WA9003L865 002R2FBP $1 \mathrm{X}$ GRS 04010319900402900406 WA9003L865 002RPTERP 1X GRS 04010319900402900406 WA9003L865 002RPHEND51X GRS $0401 \quad 0319900402900406$ WA9003L865 002R2FP 1X GRS 04010319900402900406 WA9003L865 002R246TBP1X

GRS $04010319900402900406 \mathrm{WA} 9003 \mathrm{~L} 865$ 002RPHENOL1XLT GRS $04010319900402900406 \mathrm{WA} 90034865$ 002RB2CEE 1XLT GRS $04010319900402900406 \mathrm{WA} 9003 \mathrm{~L} 865$ 002R2CLP 1XLT GRS $04010319900402900406 \mathrm{WA} 9003 \mathrm{~L} 865$ 002R13DCLB1XLT GRS 04010.319900402900406 WA9003L865 002R14DCLB1XLT GRS $04010319900402900406 \mathrm{WA} 9003 L 865$ 002RBZALC 1XLT GRS $04010319900402900406 \mathrm{WA} 9003 \mathrm{~L} 865$ 002R12DCLB1XLT 04010319900402900406 WA9003L865 002R2MP 1XLT $04010319900402900406 \mathrm{WA} 9003 \mathrm{~L} 865$ 002RB2CIPE1XLT $04010319900402900406 \mathrm{WA} 9003 \mathrm{~L} 865$ 002R4MP 1XLT 0401. 0319900402900406WA9003L865 002RNDNPA 1XLT $04010319900402900406 \mathrm{WA} 9003 \mathrm{~L} 865$ 002RCL6ET 1XLT $04010319900402900406 \mathrm{WA} 9003 \mathrm{~L} 865$ 002RNB 1XLT 04010319900402900406 WA9003L865 002RISOPHR1XLT $04010319900402900406 \mathrm{WA} 9003 L 865$ 002R2NP 1XLT $04010319900402900406 \mathrm{WA} 9003 \mathrm{~L} 865$ 002R24DMPN1XLT 04010319900402900406 WA9003L865 002RBENZOA1XLT $04010319900402900406 \mathrm{WA} 003 \mathrm{~L} 865$ 002RB2CEXM1XLT $04010319900402900406 \mathrm{FA} 9003 L 865002 \mathrm{R} 24 \mathrm{DCLP} 1 X L \mathrm{~T}$ $04010319900402900406 \mathrm{WA} 9003 \mathrm{~L} 865$ 002R124TCB1XLT $04010319900402900406 \mathrm{WA} 9003 L 865$ 002RNAP 1XLT 04010319900402900406 KA9003L865 002R4CANIL1XLT $04010319900402900406 \mathrm{WA} 9003 L 865$ 002RHCBD 1XLT $04010319900402900406 \mathrm{WA} 9003 \mathrm{~L} 865$ 002R4CL3C 1XLT $04010319900402900406 \mathrm{WA} 9003 L 865$ 002R2MENAP1XLT $04010319900402900406 \mathrm{WA} 9003 \mathrm{~L} 865$ 002RCL6CP 1XLT $04010319900402900406 \mathrm{NA} 9003 \mathrm{~L} 865$ 002R246TCP 1XLT 04010319900402900406 KA9003L865 002R245TCP1XLT $04010319900402900406 \mathrm{WA} 9003 L 865$ 002R2CNAP 1XLT $04010319900402900406 \mathrm{KA} 9003 L 865$ 002R2NANIL1XLT $04010319900402900406 \mathrm{WA} 9003 \mathrm{~L} 865$ 002RDMP 1XLT 04010319900402900406 KA9003L865 002RANAPYL1XLT
35OUGKG

75PER

T3PER

68PER

81PER

78PER

78PER

350UGKG

350UGKG

350UGKG

350UGKG

350UGKG

350UGKG

350UGKG

350UGKG

350UGKG

350UGKG

35 OUGKG

350UGKG

350UGKG

350UGKG

350UGKG

350UGKG

1700UGKG

350UGKG

350UGKG

350UGKG

350UGKG

350UGKG

350UGKG

350UGKG

350UGKG

350UGKG

350UGKG

1700UGKG

350UGKG

1700 UGKG

350UGKG

350UGKG
$1.0000 \mathrm{MSCDT}$

1.0000MSCDT

1.0000MSCDT

$1.0000 \mathrm{MSCDT}$

$1.0000 \mathrm{MSCDT}$

$1.0000 \mathrm{MSCDT}$

$1.0000 \mathrm{MSCDT}$

$1.0000 \mathrm{MSCDT}$

$1.0000 \mathrm{MSCDT}$

$1.0000 \mathrm{MSCDT}$

$1.0000 \mathrm{MSCDT}$

$1.0000 \mathrm{MSCDT}$

$1.0000 M S C D T$

$1.0000 \mathrm{MSCDT}$

$1.0000 \mathrm{MSCDT}$

$1.0000 \mathrm{MSCDT}$

$1.0000 \mathrm{MSCDT}$

$1.0000 \mathrm{MSCDT}$

$1.0000 \mathrm{MSCDT}$

$1.0000 \mathrm{MSCDT}$

$1.0000 \mathrm{MSCDT}$

$1.0000 \mathrm{MSCDT}$

$1.0000 \mathrm{MSCDT}$

$1.0000 \mathrm{MSCDT}$

1.0000MSCDT

1.0000MSCDT

1.0000MSCDT

$1.0000 \mathrm{MSCDT}$

$1.0000 \mathrm{MSCDT}$

$1.0000 \mathrm{MSCDT}$

$1.0000 \mathrm{MSCDT}$

$1.0000 \mathrm{MSCDT}$

1.0000MSCDT

$1.0000 \mathrm{MSCDT}$

1. $0000 \mathrm{MSCDT}$

$1.0000 \mathrm{MSCDT}$

$1.0000 \mathrm{MSCDT}$

$1.0000 \mathrm{MSCDT}$

$1.0000 \mathrm{MSCDT}$

$1.0000 M S C D T$

1.0000MSCDT

$1.0000 \mathrm{MSCDT}$

$1.0000 \mathrm{MSCDT}$

$1.0000 \mathrm{MSCDT}$

$1.0000 \mathrm{MSCDT}$

$1.0000 M S C D T$

$1.0000 \mathrm{MSCDT}$ 1.000OMSCDT
OBENZO (A) ANTHRACENE $0625 \mathrm{HN}$ OCHRYSENE $0625 \mathrm{HN}$ OBIS (2-ETHYLHEXYL) PHTO625HN ODI-N-OCTYI PHTHALATEO625HN OBENZO (B) FLUORANTHENEO625HN OBENZO (K) FLUORANTHENEO625HN OBENZO (A) PYRENE O625HN OINDENO $(1,2,3-C D)$ PYREO $625 \mathrm{HN}$ ODIBENZO $(A, H)$ ANTHRACEO625HN OBENZO (G, H, I) PERYLENEO625HN 1745.000NITROBENZENE-D5 O625HR 1745.0002-ELUOROBIPHENYL O625HR 1745.000R-TERPHENYL-D14 O625HR 3489.999PHENOL-D5 O625HR 3489.9992-ELUOROPHENOL O625HR 3489.9992, 4, 6-TRIBROMOPHENOLO625HR OPHENOL OBIS (2-CHLOROETHYL) ETO625HR 02-CHLOROPHENOL O625HR 01,3-DICHLOROBENZENE O625HR 01, 4-DICHLOROBENZENE O625HR OBENZYL ALCOHOL O625HR 01;2-DICHIOROBENZENE O625HR 0.2-METHYLPHENOL O625HR OBIS (2-CHLOROISORROPYO625HR 04-METHYLPHENOL O625HR ON-NITROSO-DI-N-PROPYO625HR OHEXACHLOROETHANE O625HR ONITROBENZENE O625HR OISOPHORONE . $\quad$ O625HR 02-NITROPHENOL O625HR 02, 4-DIMETHYLPHENOI O625HR OBENZOIC ACID O625HR OBIS (2-CHLOROETHOXY) MO625HR 02, 4-DICHLOROPHENOL O625HR $01,2,4-T R I C H L O R O B E N Z E O 625 \mathrm{HR}$ ONAPHTHALENE O625HR 04-CHLOROANILINE O625HR OHEXACHLOROBUTADIENE 0625HR 04-CHLORO-3-METHYLPHEO 625HR 02-METHYLNAPHTHALENE O625HR OHEXACHLOROCYCLORENTAO625HR 02, 4, 6-TRICHLOROPHENOO625HR $02,4,5$-TRICHLOROPHENOO625HR 02-CHLORONAPHTHALENE O625HR 02-NITROANILINE O625HR ODIMETHYLPHTHALATE OOPHR OACENAPHTHYLENE O625HR 
GRS $04010319900402900406 \mathrm{WA} 9003 \mathrm{~L} 865$ 002R26DNT 1XLT GRS $04010319900402900406 \mathrm{WA} 9003 \mathrm{~L} 865$ 002R3NANILIXLT GRS $04010319900402900406 \mathrm{WA} 9003 \mathrm{~L} 865$ 002RANAPNE1XLT GRS $04010319900402900406 \mathrm{KA} 9003 \mathrm{~L} 865$ 002R24DNP 1XLT GRS $04010319900402900406 \mathrm{WA} 9003 L 865002 R 4 N P$ 1XLT GRS 04010319900402900406 KA9003L865 002RDIBZFU1XLT GRS $04010319900402900406 \mathrm{FA} 9003 \mathrm{~L} 865$ 002R24DNT 1XLT GRS $04010319900402900406 \mathrm{WA} 9003$ L865 002RDEP 1XLT GRS $04010319900402900406 \mathrm{WA} 9003 \mathrm{~L} 865$ 002R4CLPPE1XLT GRS $04010319900402900406 \mathrm{WA} 9003 \mathrm{~L} 865$ 002RFLRENE1XLT GRS $04010319900402900406 \mathrm{WA} 9003 \mathrm{~L} 865$ 002R4NANIL1XLT GRS $04010319900402900406 \mathrm{WA} 9003 \mathrm{~L} 865002 \mathrm{R} 46 \mathrm{DN} 2 \mathrm{C} 1 \mathrm{XLT}$ GRS $04010319900402900406 \mathrm{WA} 9003 \mathrm{~L} 865$ 002RNNDPA 1XLT GRS $04010319900402900406 \mathrm{WA} 9003 \mathrm{~L} 865$ 002R4BRPPE1XLT GRS $04010319900402900406 \mathrm{WA} 9003 \mathrm{~L} 865$ 002RCL6BZ 1XLT GRS 04010319900402900406 WA9003L865 002RPCP 1XLT GRS 04010319900402900406 WA9003L865 002RPHANTR1XLT GRS $04010319900402900406 \mathrm{WA} 9003 \mathrm{~L} 865$ 002RANTRC 1XLT GRS $04010319900402900406 \mathrm{WA} 90031865$ 002RDNBP 1XLT GRS $04010319900402900406 \mathrm{WA} 9003 \mathrm{~L} 865$ 002RFANT 1XLT GRS $04010319900402900406 \mathrm{WA} 9003 \mathrm{~L} 865$ 002RPYR 1XLT GRS $04010319900402900406 \mathrm{WA} 9003 \mathrm{~L} 865$ 002RBBZP 1XLT GRS $04010319900402900406 \mathrm{WA} 9003 \mathrm{~L} 865$ 002R33CL2B1XLT

$04010319900402900406 \mathrm{WA} 9003 \mathrm{~L} 865$ 002RBAANTR1XLT 04010319900402900406 WA9003L865 002RCHRY 1XLT $04010319900402900406 \mathrm{WA} 9003 \mathrm{~L} 865$ 002RB2EHP 1XLT $04010319900402900406 \mathrm{WA} 9003 L 865$ 002RDNOP 1XLT $04010319900402900406 \mathrm{WA} 9003 L 865$ 002RBF2ANT1XLT $04010319900402900406 \mathrm{WA} 9003 \mathrm{~L} 865$ 002RBKFANT1XLT $04010319900402900406 \mathrm{WA} 90034865$ 002RBAPYR 1XLT $04010319900402900406 \mathrm{WA} 90032865$ 002RICDPYR1XLT $04010319900402900406 \mathrm{WA} 9003 \mathrm{~L} 865$ 002RDBAHA 1XLT 04010319900402900406 WA9003L865 002RBGHIPY1XLT $0401 A 0319900328900051$ WA9003L865 003

04.01A0319900519900451WA9003L865 003 AG 2ZLT $0401 A 0319900518900450$ WA9003L865 003 AS 1BLT 0401A0319900519900451WA9003L865 003 BA 2ZLT 0401A0319900519900451KA9003L865 003 CD 22 $0401 A 0319900519900451$ WA9003L865 003 CR 22 0401A0319900405900055WA9003L865 003 HGTOT 1DLT 0401A0319900518900450WA9003L865 003 PB 1B $0401 A 0319900518900450$ WA9003L865 $003 \mathrm{SE}$ 1BLT 0401A0319900327900051WA9003L865 003 MEC6D82J 0401A0319900327900051KA9003L865 003 PBFB 2J $0401 A 0319900327900051$ WA9003L865 $003 \quad 12 \mathrm{DCD} 42 \mathrm{~J}$ 0401 0319900327900051WA9003L865 003 CH3CL 2JLT 0401A0319900327900051KA9003L865 003 CH3BR 2JLT 040110319900327900051 HA9003L865 003 C2H3CL2JLT
35 OUGKG 170 OUGKG 350 UGKG 1700 UGKG 170 OUGKG 350UGKG 350UGKंG

35 OUGKG 35 OUGKG 35 OUGKG 1700 UGKG 170 OUGKG

350UGKG

35 OUGKG

35 OUGKG

1700UGKG

350UGKG

350UGKG

350UGKG

350UGKG

350UGKG

350UGKG

70 OUGKG

35 OUGKG

350UGKG

35 OUGKG

350UGKG

350UGKG

350UGKG

350UGKG

35 OUGKG

35 OUGKG

35 OUGKG

$81.8 \%$

2. OMGKG

2. 3MGKG

40. 1MGKG

1. 2MGKG

5. 3MGKG

$0.12 \mathrm{MGKG}$

1. IMGKG

1.2MGKG

98PER

105PER

87PER

11UGKG

11UGKG 12UGKG
$1.0000 \mathrm{MSCDT}$

1. $0000 \mathrm{MSCDT}$

$1.0000 \mathrm{MSCD}$

1. $0000 \mathrm{MSCDT}$

1. $0000 \mathrm{MSCDT}$

$1.0000 \mathrm{MSCDT}$

1. $0000 \mathrm{MSCDT}$

$1.0000 M S C D T$

1. $0000 \mathrm{MSCDT}$

1. $0000 \mathrm{MSCDT}$

1.0000MSCD

1.0000MSCDT

1. $0000 \mathrm{MSCDT}$

$1.0000 M S C D T$

1.0000MSCDT

$1.0000 \mathrm{MSCDT}$

$1.0000 M S C D T$

$1.0000 M S C D T$

$1.0000 M S C D T$

$1.0000 \mathrm{MSCDT}$

1.0000MSCDT

$1.0000 \mathrm{MSCDT}$

$1.0000 \mathrm{MSCDT}$

$1.0000 \mathrm{MSCDT}$

$1.0000 \mathrm{MSCDT}$

$1.0000 M S C D T$

$1.0000 \mathrm{MSCDT}$

1. $0000 \mathrm{MSCDT}$

$1.0000 \mathrm{MSCDT}$

$1.0000 \mathrm{MSCDT}$

1.0000MSCDT

1.0000MSCDT

1. $0000 \mathrm{MSCDT}$ $10 \mathrm{JH}$

1. OOICALM

1.00AAAER

1. OOICALM

1. OOICALM

1.00 ICALM

$1.00 \mathrm{HGJJW}$

$1.00 \mathrm{AAEJS}$

$1.00 A A M L R$

1. $0000 \mathrm{MSJBD}$

1. $0.000 \mathrm{MSJBD}$

$1.0000 M S J B D$

$1.0000 \mathrm{MSJBD}$

$1.0000 M S J B D$ 1.0000MSJBD
02, 6-DINITROTOLUENE 03-NITROANILINE 0625HR OACENAPHTHENE O625HR ODIBENZOEURAN ' O625HR 02, 4-DINITROTOLUENE O625HR ODIETHYLPHTHALATE O625HR 04-CHLORORHENYL-PHENYO625HR OFLUORENE O625HR 04-NITROANILINE O625HR 04,6-DINITRO-2-METHYLO625HR ON-NITROSODIPHENYLAMIO625HR 04-BROMORHENYL-PHENYLO625HR OHEXACHLOROBENZENE O625HR OPENTACHLOROPHENOL O625HR OPHENANTHRENE O625HR OANTHRACENE O625HR ODI-N-BUTYLPHTHALATE O625HR OFLUORANTHENE O625HR OPYRENE O625HR OBUTYLBENZYLPHTHALATE0 $625 \mathrm{HR}$ $03,3^{\prime}$-DICHLOROBENZIDIO625HR OBEṄZO (A) ANTHRACENE O625HR OCHRYSENE. O625HR OBIS (2-ETHYLHEXYL) PHTO625HR ODI-N-OCTYL PHTHALATEO625HR OBENZO (B) FLUORANTHENEO625HR OBENZO (K) FLUORANTHENEO 625HR OBENZO (A) PYRENE O625HR O INDENO (1, 2, 3-CD) PYREO625HR ODIBENZO $(\mathrm{A}, \mathrm{H})$ ANTHRACEO625HR OBENZO (G, H, I) PERYLENEO625HR O\& SOLIDS OSILVER, TOTAL OARSENIC, TOTAL OBARIUM, TOTAI OCADMIUM, TOTAL OCHROMIUM, TOTAL OMERCURY, TOTAL OLEAD, TOTAL

OSELENIUM, TOTAL I\&SOLN MAGTON MASTON MBATON MCDTON MCRTON MHGTON MPBTON MSETON 53.000TOLUENE-D8 $0624 \mathrm{HN}$ 53.000BROMOELUOROBENZENE: 0624HN 53.0001,2-DICHLOROETHANE-DO624HN OCHLOROMETHANE O624HN OBROMOMETHANE O624HN OVINYL CHLORIDE $24 \mathrm{HN}$ 
LA0319900327900051WA9003L865 003 C2H5CL2JLT $0401 A 0319900327900051 W A 9003 \mathrm{~L} 865003 \mathrm{CH} 2 \mathrm{CL} 22 \mathrm{JB}$ $0401 A 0319900327900051$ WA9003L865 003 ACET 2JLT $0401 A 0319900327900051$ KA9003L865 003 CS2 2JLT 0401A0319900327900051WA9003L865 003 11DCE 2JLT 0401A0319900327900051WA9003L865 003 11DCLE2JLT 0401A0319900327900051WA9003L865 003 T12DCE2JLT 0401A0319900327900051WA9003L865 003 CHCL3 2JLT $0401 A 0319900327900051$ HA9003L865 003 12DCLE2JLT $0401 A 0319900327900051$ WA9003L865 003 MEK 2JLT $0401 A 0319900327900051$ WA9003L865 003 111TCE2JLT $0401 A 0319900327900051$ WA9003L865 003 CCL4 2JLT $0401 A 0319900327900051$ WA9003L865 003 VINYLA2JLT 0401A0319900327900051WA9003L865 003 BRDCLM2JLT $0401 A 0319900327900051$ WA9003L865 003 12DCLP2JLT 0401A0319900327900051WA9003L865 003 13DCPC2JLT 0401A0319900327900051WA9003L865 003 TRCLE 2JLT 0401A0319900327900051WA9003L865 003 DBRCLM2JLT 0401A0319900327900051WA9003L865 003 112TCE2JLT $0401 A 0319900327900051$ WA9003L865 003 C6H6 2JLT 0401A0319900327900051WA9003L865 003 T13DCP2JLT $0401 A 0319900327900051$ WA9003L865 003 CHBR3 2JLT 0401A0319900327900051WA9003L865 003 4ME2PE2JLT $0401 A 0319900327900051$ WA9003L865 003 2HXONE2JLT $0401 A 0319900327900051$ WA9003L865 003 TCLEE 2JLT 0401A0319900327900051WA9003L865 003 TCLEA 2JLT 0401A0319900327900051WA9003L865 003 MEC6H52JLT 0401A0319900327900051WA9003L865 003 CLC6H52JLT 0401A0319900327900051WA9003L865 003 ETC6H52JLT 0401A0319900327900051WA9003L865 003 STYR 2JLT $0401 A 0319900327900051$ HA9003L865 003 XYLEN 2JLT. $0401 A 0319900402900406$ W $9003 L 865003$ NBD5 $1 X$ $0401 A 0319900402900406$ AA9003L865 003 2FBP $1 X$ $0401 A 0319900402900406$ WA9003L865 003 PTERP $1 \mathrm{X}$ $0401 A 0319900402900406$ WA9003L865 003 PHEND51X $0401 A 0319900402900406$ HA9003L865 003 2EP 1X $0401 A 0319900402900406$ WA9003L865 003 246TBP1X 0401A0319900402900406WA9003L865 003 PHENOL1XLT 0401A0319900402900406\%A9003L865 003 B2CEE 1XLT 0401A0319900402900406WA9003L865 003 2CLP 1XLT 0401A0319900402900406\%A9003L865 003 13DCLB1XLT 0401A0319900402900406\%A9003L865 003 14DCLB1XLT 0401A0319900402900406WA9003L865 003 BZALC 1XLT $0401 A 0319900402900406$ KA9003L865 003 12DCLB1XLT 0401A0319900402900406\%A9003L865 003 2MP 1XLT $0401 A 0319900402900406$ WA9003L865 003 B2CIPEIXLT 0401A0319900402900406KA9003L865 003 4MP 1XLT 0401A0319900402900406\%A9003L865 003 NDNPA 1XIT

\begin{tabular}{|c|c|}
\hline IIUGKG & $1.0000 \mathrm{MSJBD}$ \\
\hline 7UGKG & $1.0000 \mathrm{MSJBD}$ \\
\hline IIUGKG & $1.0000 \mathrm{MSJBD}$ \\
\hline 5UGKG & 1.0000 MSJBD \\
\hline 5UGKG & $1.0000 \mathrm{MSJBD}$ \\
\hline 5UGKG & $1.0000 \mathrm{MSJBD}$ \\
\hline 5UGKG & $1.0000 \mathrm{MSJBD}$ \\
\hline 5UGKG & $1.0000 \mathrm{MSJBD}$ \\
\hline 5UGKG & 1.0000 MSJBD \\
\hline 11 UGKG & $1.0000 \mathrm{MSJBD}$ \\
\hline 5UGKG & $1.0000 \mathrm{MSJBD}$ \\
\hline 5UGKG & 1.0000MSJBD \\
\hline 11 UGKG & $1.0000 \mathrm{MSJBD}$ \\
\hline 5UGKG & $1.0000 \mathrm{MSJBD}$ \\
\hline 5UGKG & $1.0000 \mathrm{MSJBD}$ \\
\hline 5UGKG & $1.0000 \mathrm{MSJBD}$ \\
\hline 5UGKG & $1.0000 \mathrm{MSJBD}$ \\
\hline 5UGKG & $1.0000 \mathrm{MSJBD}$ \\
\hline 5UGKG & $1.0000 \mathrm{MSJBD}$ \\
\hline 5UGKG & 1.0000 MSJBD \\
\hline 5UGKG & $1.0000 \mathrm{MSJBD}$ \\
\hline 5UGKG & $1.0000 \mathrm{MSJBD}$ \\
\hline 11UGKG & 1.0000 MSJBD \\
\hline 11UGKG & $1.0000 \mathrm{MSJBD}$ \\
\hline 5UGKG & $1.0000 \mathrm{MSJBD}$ \\
\hline 5UGKG & $1.0000 \mathrm{MSJBD}$ \\
\hline 5UGKG & $1.0000 \mathrm{MSJBD}$ \\
\hline 5UGKG & $1.0000 \mathrm{MSJBD}$ \\
\hline 5UGKG & $1.0000 \mathrm{MSJBD}$ \\
\hline 5UGKG & $1.0000 \mathrm{MSJBD}$ \\
\hline 5UGKG & $1.0000 \mathrm{MSJBD}$ \\
\hline 76PER & $1.0000 \mathrm{MSCDT}$ \\
\hline 68PER & $1.0000 \mathrm{MSCDT}$ \\
\hline 59PER & $1.0000 \mathrm{MSCDT}$ \\
\hline 81PER & $1.0000 \mathrm{MSCDT}$ \\
\hline 66PER & $1.0000 \mathrm{MSCDT}$ \\
\hline 94RER & $1.0000 \mathrm{MSCDT}$ \\
\hline 350 UGKG & $1.0000 \mathrm{MSCDT}$ \\
\hline 35 OUGKG & $1.0000 M S C D T$ \\
\hline 350UGKG & $1.0000 \mathrm{MSCDT}$ \\
\hline 350UGKG & $1.0000 \mathrm{MSCDT}$ \\
\hline 350UGKG & $1.0000 \mathrm{MSCDT}$ \\
\hline & $1.0000 \mathrm{MSCDT}$ \\
\hline 350UGKG & $1.0000 \mathrm{MSCDT}$ \\
\hline 350 UGKG & $1.0000 \mathrm{MSCDT}$ \\
\hline & $1.0000 M S C D T$ \\
\hline JuGKe & $1.0000 \mathrm{MSCDT}$ \\
\hline SOUGKG & $1.0000 M S C D T$ \\
\hline
\end{tabular}
w9003865.dat $-6 / 26 / 90-9$
OCHLOROETHANE $0624 \mathrm{HN}$ OMETHYLENE CHLORIDE O624HN OACETONE

OCARBON DISULEIDE O624HN

01,1-DICHLOROETHENE 0624HN 01,1 -DICHLOROETHANE $0624 \mathrm{HN}$ 01,2-DICHLOROETHENE $10624 \mathrm{HN}$ OCHLOROFORM 01,2-DICHLOROETHANE O624HN 02-BUTANONE O624HN 01, 1, 1-TRICHLOROETHANO62 4HN OCARBON TETRACHLORIDEO $624 \mathrm{HN}$ OVINYL ACETATE O624HN OBROMODICHLOROMETHANEO62 $4 \mathrm{HN}$ 01,2-DICHLOROPROPANE $0624 \mathrm{HN}$ OCIS-1, 3-DICHLOROPROPO624HN OTRICHLOROETHENE O624HN ODIBROMOCHLOROMETHANEO $624 \mathrm{HN}$ 01, 1, 2-TRICHLOROETHANO $624 \mathrm{HN}$ OBENZENE $0624 \mathrm{HN}$ OTRANS-1, 3-DICHLOROPRO624HN OBROMOFORM O624HN 04-METHYL-2-PENTANONEO62 4HN 02-HEXANONE O624HN OTETRACHLOROETHENE O624HN $01,1,2,2$-TETRACHLOROEO $624 \mathrm{HN}$ OTOLUENE OCHLOROBENZENE O624HN OETHYLBENZENE O624HN OSTYRENE OXYLENE (TOTAL) 1745. 000NITROBENZENE-D5 1745.0002-FLUOROBIPHENYL 1745. 000P-TERPHENYL-D14 3489.999 PHENOL-D5

3.489.9992-FLUOROPHENOL $3489.9992,4,6$-TRIBROMOPHENOLO625HN OPHENOI

$0624 \mathrm{HN}$ $0624 \mathrm{HN}$ 0625HN $0625 \mathrm{HN}$ 0625HN 0625HN 02-CHLOROPHENOL (2) ETO625HN 01,3-DICHLOROBENZENE O625HN $01,4-D I C H L O R O B E N Z E N E$ O625HN OBENZYL ALCOHOL O625HN 01,2-DICHLOROBENZENE O625HN 02-METHYLPHENOL O625HN OBIS (2-CHLOROISORROPYO625HN O4-METHYLPHENOL O625HN ON-NITROSO-DI-N-PROPYO625HN 
GRS 0401A0319900402900406WA9003L865 003 CL6ET 1XLT GRS 040.1A0319900402900406WA9003L865 003 NB 1XLT $0401 A 0319900402900406$ WA9003L865 003 ISORHR1XLT 0401A0319900402900406WA9003L865003 2NP 1XLT 0401A0319900402900406WA9003L865 003 24DMPN1XLT $0401 A 0319900402900406$ WA $9003 L 865003$ BENZOA1XLT 0401A0319900402900406WA9003L865 003 B2CEXM1XLT 0401A0319900402900406WA9003L865003 24DCLP1XLT $0401 A 0319900402900406$ WA9003L865 003 124TCB1XLT $0401 A 0319900402900406 \mathrm{WA} 9003 \mathrm{~L} 865003$ NAP 1XLT $0401 A 0319900402900406$ WA9003L865 003 4CANIL1XLT $0401 A 0319900402900406 \mathrm{WA} 90031865003 \mathrm{HCBD} 1 \mathrm{XLT}$ 0401A0319900402900406WA9003L865 003 4CL3C 1XLT 0401A0319900402900406WA9003L865 003 2MENAP1XIT $0401 A 0319900402900406$ WA9003L865 003 CL6CP 1XLT 0401A0319900402900406WA9003L865 003 246TCP1XLT 0401A0319900402900406WA9003L865 003 245TCP1XLT $0401 A 0319900402900406 \mathrm{WA} 9003 L 865.003$ 2CNAP 1XLT 0401A0319900402900406WA9003L865 003 2NANILIXLT 0401A0319900402900406אA9003L865 003 DMP 1XLT $0401 A 0319900402900406$ WA9003L865 003 ANAPYL1XLT 0401A0319900402900406WA9003L865 003 26DNT 1XLT $0401 A 0319900402900406$ WA9003L865 003 3NANILIXLT 0401A0319900402900406WA9003L865 003 ANAPNE1XLT $0401 A 0319900402900406$ WA9003L865 003 24DNP 1XLT $0401 A 0319900402900406 \mathrm{WA} 9003 L 865003$ 4NP IXLT 0401A0319900402900406\%A9003L865 003 DIBZFU1XLT $0401 A 0319900402900406$ FA9003L865 003 24DNT 1XLT $0401 A 0319900402900406 \mathrm{WA} 90032865003$ DEP 1XLT 0401A0319900402900406WA9003L865 003 4CLPPE1XLT $0401 A 0319900402900406$ FA9003L865 003 FLRENEIXLT 0401A0319900402900406WA'9003L865 003 4NANIL1XLT $0401 A 0319900402900406 \mathrm{WA} 9003 L 865003$ 46DN2C1XLT $0401 A 0319900402900406 \mathrm{WA} 9003 \mathrm{~L} 865003$ NNDPA 1XLT 0401A0319900402900406WA9003L865 003 4BRPPE1XLT $0401 A 0319900402900406$ WA9003L865 003 CL6BZ 1XLT $0401 A 0319900402900406 \mathrm{WA} 9003 L 865003$ PCP 1XLT $0401 A 0319900402900406 \mathrm{WA} 9003 \mathrm{~L} 865003$ PHANTR1XLT $0401 A 0319900402900406$ WA9003L865 003 ANTRC 1XLT 0401A0319900402900406WA9003L865 003 DNBP 1XLT 0401A0319900402900406WA9003L865 003 FANT 1XLT 0401A0319900402900406WA9003L865 003 PYR 1XLT $0401 A 0319900402900406 \mathrm{FA} 9003 L 865003$ BBZP 1XLT 0401A0319900402900406 KA9003L865 003 33CL2B1XLT $0401 A 0319900402900406 \mathrm{HA9003L865} 003$ BAANTR1XLT 0401A0319900402900406WA9003L865.003 CHRY 1XLT $0401 A 0319900402900406 \mathrm{WA} 9003 \mathrm{~L} 865003$ B2EHP 1XLT $0401 A 0319900402900406$ WA9003L865 003 DNOP IXLT
1700 UGKG

35 OUGKG

350 UGKG

$350 U G K G$

35 OUGKG

35 OUGKG

350 UGKG

35 OUGKG

350 UGKG

350UGKG

35 OUGKG

170 OUGKG

350UGKG

1700UGKG

350 UGKG

350UGKG

350 UGKG

1700UGKG

350UGKG

170 OUGKG

170OUGKG

350UGKG

350 UGKG

350UGKG

350UGKG

350UGKG

1700UGKG

170 OUGKG

350UGKG

350UGKG

$350 U G K G$

170 OUGKG

350UGKG

350 UGKG

350UGKG

350 UGKG

35 OUGKG

350UGKG

700 UGKG

350UGKG

.350UGKG

350 UGKG 35QUGKG
1.0000MSCDT $1.0000 \mathrm{MSCDT}$ $1.0000 \mathrm{MSCDT}$ 1. $0000 \mathrm{MSCDT}$ $1.0000 M S C D T$ 1.0000MSCDT 1.0000MSCDT $1.0000 \mathrm{MSCDT}$ 1. $0000 \mathrm{MSCDT}$ 1. $0000 \mathrm{MSCDT}$ 1.0000MSCDT 1.0000MSCDT $1.0000 \mathrm{MSCDT}$ 1. $0000 \mathrm{MSCDT}$ 1. $0000 \mathrm{MSCDT}$ $1.0000 \mathrm{MSCDT}$ 1.0000MSCDT 1.0000MSCDT 1.0000MSCDT 1.0000MSCDT 1. 0000MSCDT 1.0000MSCDT $1.0000 \mathrm{MSCDT}$ 1.0000MSCDT 1. $0000 \mathrm{MSCDT}$ $1.0000 \mathrm{MSCDT}$ 1.0000MSCDT 1. $0000 \mathrm{MSCDT}$ 1.0000MSCDT 1.0000MSCDT 1.0000MSCDT 1.0000MSCDT 1.0000MSCDT 1.0000MSCDI 1. 0000MSCDT 1. $0000 \mathrm{MSCDT}$ 1.0000MSCDT 1.0000MSCDT 1. $0000 \mathrm{MSCDT}$ 1.0000MSCDT 1. $0000 \mathrm{MSCDT}$ 1.0000MSCDT 1.0000MSCDT 1. 0000MSCDT 1.0000MSCDT 1.0000MSCDT 1. 0000MSCDT 1.0000MSCDT
0625HN $0625 \mathrm{HN}$ OISOPHORONE $0625 \mathrm{HN}$ 02-NITROPHENOI 0625HN 02, 4-DIMETHYLPHENOL 0625HN OBENZOIC ACID O625HN OBIS (2-CHLOROETHOXY) MO625HN 02, 4-DICHLOROPHENOI 0625HN $01,2,4-T R I C H L O R O B E N Z$ EO 625HN ONAPHTHALENE O625HN 04-CHLOROANIIINE 0625HN OHEXACHLOROBUTADIENE O625HN 04-CHLORO-3-METHYLPHEO625HN 02-METHYLNAPHTHALENE 0625HN OHEXACHLOROCYCLOPENTAO $625 \mathrm{HN}$ $02,4,6$-TRICHLOROP HENOO625HN $02,4,5-T R I C H L O R O P H E N O O 625 \mathrm{HN}$ 02-CHLORONAPHTHALENE 0625HN 02-NITROANILINE 0625HN ODIMETHYLPHTHALATE 0625HN OACENAPHTHYLENE 0625HN 02,6-DINITROTOLUENE 0625HN 03-NITROANIIINE 0625HN OACENAPHTHENE 0625HN 02,4-DINITROPHENOL 0625HN 04-NITROPHENOL O625HN ODIBENZOEURAN 0625HN 02, 4-DINITROTOLUENE 0625HN ODIETHYLPHTHALATE O625HN 04-CHLOROPHENYI-PHENYO $625 \mathrm{HN}$ OFLUORENE O625HN 04-NITROANILINE O625HN 04, 6-DINITRO-2-METHYLO 625HN ON-NITROSODIPHENYLAMI0625HN 04-BROMOPHENYL-PHENYLO625HN OHEXACHLOROBENZENE O625HN OPENTACHLOROPHENOI 0625HN ORHENANTHRENE 0625HN OANTHRACENE O625HN ODI-N-BUTYLPHTHALATE 0625HN OFLUORANTHENE 0625HN OPYRENE O625HN OBUTYLBENZYLPHTHAIATEO625HN $03,3^{\prime}$-DICHLOROBENZ IDIO625HN OBENZO (A) ANTHRACENE 0625HN OCHRYSENE 0625HN OBIS (2-ETHYLHEXYL) PHTO625HN ODI-N-OCTYL PHTHALAPM $2625 \mathrm{HN}$ 
GRS 04 .0319900402900406WA9003L865 003 BE2ANTIXIT GRS 0401A0319900402900406WA9003L865 003 BKFANT1XLT GRS 0401A0319900402900406\%A9003L865 003 BAPYR 1XIT GRS 0401A0319900402900406WA9003L865 003 ICDPYRIXIT GRS 0401A0319900402900406WA9003L865 003 DBAHA IXLT GRS 0401A0319900402900406WA9003L865 003 BGHIPYIXLT GRS 03010319900427900003 WA9003L865 004 CEC EX GRS 03010319900427900003 WA9003L865 004RCEC EX GRS 03010319900427900003 WA9003L865 004 CECB EX GRS 03010319900427900003 WA9003L865 004RCECB EX GRS 03010319900426900003 WA9003L865 004 CECT EX GRS 03010319900403900003 WA9003L865 004 TOC $9 I$ GRS $03010319900328900054 W A 9003 L 865004$ PH $\quad 9 R$ GRS 03010319900328900054 WA9003L865 004RPH 9R GRS 03010319900327900051 WA9003L865 004 MEC6D82J GRS 03010319900327900051 WA9003L865 004 PBEB 2J GRS 03010319900327900051 WA9003L865 004 12DCD42J GRS 03010319900327900051 WA9003L865 004 CH3CL 2JLT GRS 03010319900327900051 WA9003L865 004 CH3BR 2JIT GRS 03010319900327900051 WA9003L865 004 C2H3CL2JLT GRS 03010319900327900051 WA9003L865 004 C2H5CL2JIT GRS 03010319900327900051 WA9003L865 004 CH2CL22JB GRS 03010319900327900051 WA9003L865 004 ACET 2JB $\begin{array}{llllll}0301 & 0319900327900051 W A 9003 L 865 & 004 & \text { ACET } & \text { 2JB } \\ 0301 & 0319900327900051 W A 9003 L 865 & 004 & \text { CS2 } & \text { 2JLT }\end{array}$ 03010319900327900051 WA9003L865 004 11DCE $2 \mathrm{JIT}$ 03010319900327900051 WA9003L865 004 11DCLE2JLT 03010319900327900051 WA9003L865 004 T12DCE2JLT 03010319900327900051 WA9003L865 004 CHCL3 2JLT 03010319900327900051 WA9003L865 004 12DCLE2JLT 03010319900327900051 WA9003L865 004 MEK 2JIT 03010319900327900051 WA9003L865 004 111TCE2JLT 03010319900327900051 WA9003L865 004 CCI4 2JLT 03010319900327900051 WA9003L865 004 VINYLA2 JLT 03010319900327900051 WA9003L865 004 BRDCLM2JLT 03010319900327900051 WA9003L865 004 12DCLP2JLT 03010319900327900051 WA9003L865 004 130CPC2JLT 03010319900327900051 HA9003L865 004 TRCLE 2JLT 03010319900327900051 WA9003L865 004 DBRCLM2JLT 03010319900327900051 WA9003L865 004 112TCE2JLT 03010319900327900051 WA9003L865 004 C6H6 2JLT 03010319900327900051 WA9003L865 004 T13DCP2JLT 03010319900327900051 WA9003L865 004 CHBR3 2JLT 03010319900327900051 WA9003L865 004 4ME2PE2JLT 03010319900327900051 WA9003L865 004 2HXONE2JLT 03010319900327900051 HA9003L865 004 TCLEE 2JLT 03010319900327900051 HA9003L865 004 TCLEA 2JLT 03010319900327900051 WA9003L865 004 MEC6H52JLT 03010319900327900051 HA9003L865 004 CLC6H52JLT
د-UUGKG

350UGKG

350UGKG

350 UGKG

350UGKG

350UGKG

2. $4 M E Q 1$

2. $4 \mathrm{MEQ1}$

2. 3MEQ1

2. $3 M E Q 1$

2. 3MEQ1

2470MGKG

$6.2 \mathrm{PH} . \mathrm{U}$

$6.2 \mathrm{PH} \mathrm{U}$

105PER

105PER

89RER

11 UGKG

11UGKG

11UGKG

11UGKG

28UGKG

28UGKG

6UGKG

6UGKG

6UGKG

6UGKG

6UGKG

6UGKG

IIUGKG

6UGKG

6UGKG

11UGKG

6UGKG

6UGKG

6UGKG

6UGKG

6UGKG

6UGKG

6UGKG

6UGKG

6UGKG

11UGKG

11UGKG

6UGKG

6UGKG

6UGKG 6UGKG
1.0000MSCDT

1. 0000MSCDT

1.0000MSCDT

$1.0000 \mathrm{MSCDT}$

1.0000MSCDT

1.0000MSCDT

IONADKS

10 NADKS

10NAALM

10NAALM

1ONADKS

10DODKS

10PHJH

10PHJH

1. 0000MSJBD

1. 0000MSJBD

1. 0000MSJBD

1. 0000MSJBD

1.0000MSJBD

1. $0000 \mathrm{MSJBD}$

1. $0000 \mathrm{MSJBD}$

$1.0000 \mathrm{MSJBD}$

1. 0000MSJBD

1. $0000 \mathrm{MSJBD}$

1. 0000MSJBD

1. $0000 \mathrm{MSJBD}$

1.0000MSJBD

1. 0000MSJBD

1 ..0000MSJBD

1.0000MSJBD

1. $0000 \mathrm{MSJBD}$

1. 0000MSJBD

1. 0000MSJBD

$1.0000 \mathrm{MSJBD}$

1. 0000MSJBD

1.0000MSJBD

1. $0000 \mathrm{MSJBD}$

1. $0000 \mathrm{MSJBD}$

1.0000MSJBD

1. $0000 \mathrm{MSJBD}$

1.0000MSJBD

1.0000MSJBD

1. $0000 \mathrm{MSJBD}$

1.0000MSJBD

1.0000MSJBD

1. 0000MSJBD

1. 0000MSJBD 1.0000MSJBD
OBENZO (B) ELUORANTHENE0625HN OBENZO (K) FLUORANTHENEO $625 \mathrm{HN}$ OBENZO (A) PYRENE O625HN OINDENO (1,2,3-CD) PYREO625HN ODIBENZO (A, H) ANTHRACEO625HN OBENZO (G, H, I) PERYLENEO625HN OCATION EXCHANGE CAPAICEC N OCATION EXCHANGE CAPAICEC $R$ OEXCHANGEABLE BASES ICECBN OEXCHANGEABLE BASES ICECBR OEXCHANGEABLE ACIDS ICECTN OTOTAL ORGANIC CARBONIOCT N OPH

OPH (REP)

IPH N

55.500BROMOFLUOROBENZENE $0624 \mathrm{HN}$ $55.5001,2-D I C H L O R O E T H A N E-D 0624 H N$ OCHIOROMETHANE O624HN OBROMOMETHANE O624HN OVINYI CHLORIDE $0624 \mathrm{HN}$ OCHIOROETHANE $0624 \mathrm{HN}$ OMETHYLENE CHLORIDE O624HN OACETONE O624HN OCARBON DISULFIDE O624HN 01, 1-DICHLOROETHENE 0624HN 01,1 -DICHLOROETHANE 0624HN $01,2-D I C H L O R O E T H E N E$ 10624HN OCHLOROFORM O624HN $01,2-D I C H L O R O E T H A N E$ 0624HN 02-BUTANONE O624HN $01,1,1$-TRICHLOROETHANO $624 \mathrm{HN}$ OCARBON TETRACHLORIDEO $624 \mathrm{HN}$ OVINYL ACETATE 0624HN OBROMODICHLOROMETHANEO $624 \mathrm{HN}$ $01,2-D I C H L O R O P R O P A N E$ O624HN OCIS-1, 3-DICHLOROPROPO624HN OTRICHLOR̈OETHENE O624HN ODIBROMOCHLOROMETHANEO $624 \mathrm{HN}$ $01,1,2-T R I C H L O R O E T H A N O 624 \mathrm{HN}$ OBENZENE O624HN OTRANS-1, 3-DICHLOROPRO 62 4HN OBROMOEORM 0624HN 04-METHYL-2-DENTANONEO $624 \mathrm{HN}$ 02-HEXANONE . . 0624HN OTETRACHLOROETHENE O624HN $01,1,2,2-T E T R A C H L O R O E O 624 \mathrm{HN}$ OTOLUENE $0624 \mathrm{HN}$ OCHLOROBENZENE 
3010319900327900051 HA9003L865 004 ETC6H52JLT 03010319900327900051 WA9003L865 004 STYR 2JLT 03010319900327900051 WA9003L865 004 XYLEN 2JLT 03010319900402900406 WA9003L865 004 NBD5 $1 X$ 03010319900402900406 WA9003L865 004 2FBP $1 X$ 03010319900402900406 WA 90032865004 PTERP $1 X$ 03010319900402900406 WA9003L865 004 PHEND51X 03010319900402900406 WA9003L865 004 2FP $1 X$ 03010319900402900406 WA9003L865 004 246TBP $1 X$ $030103199004029004064 A 90031865004$ PHENOLIXLT $0.3010319900402900406 \mathrm{WA} 9003 L 865004$ B2CEE 1XLT 03010319900402900406 WA9003L865 004 2CLP 1XLT $03010319900402900406 \mathrm{KA} 003 \mathrm{~L} 865004$ 13DCLB1XLT $03010319900402900406 \mathrm{WA} 9003 L 865004$ 14DCLB1XLT $03010319900402900406 \mathrm{FA} 9003 L 865004$ BZALC 1XLT $03010319900402900406 \mathrm{WA} 9003 L 865004$ 12DCLB1XLT 03010319900402900406 WA9003L865 004 2MP 1XLT 03010319900402900406 WA9003L865 004 B2CIPE1XLT 03010319900402900406 FA9003L865 004 4MP 1XLT 03010319900402900406 HA9003L865 004 NDNPA 1XLT 03010319900402900406 WA $9003 L 865004$ CL6ET 1XLT $03010319900402900406 \mathrm{WA} 9003 \mathrm{~L} 865004 \mathrm{NB}$ 1XLT $03010319900402900406 \mathrm{WA} 9003 \mathrm{~L} 865004$ ISOPHR1XLT 03010319900402900406 WA9003L865 004 2NP 1XLT 03010319900402900406 WA9003L865 004 24DMPN1XLT $03010319900402900406 \mathrm{WA} 9003 \mathrm{~L} 865004$ BENZOA1XLT $03010319900402900406 \mathrm{WA} 9003 L 865004$ B2CEXM1XLT 03010319900402900406 WA9003L865 004 24DCLP1XLT $03010319900402900406 \mathrm{WA} 9003 \mathrm{~L} 865004$ 124TCB1XLT $03010319900402900406 \mathrm{WA} 9003 \mathrm{~L} 865004 \mathrm{NAP}$ 1XLT 03010319900402900406 WA9003L865 004 4CANILIXLT $03010319900402900406 \mathrm{WA} 9003 \mathrm{~L} 865004$ HCBD 1XLT 03010319900402900406 WA9003L865 004 4CL3C 1XLT 03010319900402900406 WA9003L865 004 2MENAP1XLT 03010319900402900406 WA9003L865 004 CL6CP 1XLT $03010319900402900406 \mathrm{WA} 9003 \mathrm{~L} 865004$ 246TCP 1XLT $03010319900402900406 \mathrm{HA9003L865004}$ 245TCP1XLT $03010319900402900406 \mathrm{WA} 9003 \mathrm{~L} 865004$ 2CNAP 1XLT 03010319900402900406 HA9003L865 004 2NANIL1XLT $03010319900402900406 \mathrm{WA} 9003 \mathrm{~L} 865004$ DMP 1XLT $03010319900402900406 \mathrm{WA} 9003 \mathrm{~L} 865004$ ANAPYL1XLT $03010319900402900406 \mathrm{HA9003LB65} 004$ 26DNT 1XLT 03010319900402900406 WA9003L865 004 3NANILIXLT 03010319900402900406 WA9003L865 004. ANAPNEIXLT $03010319900402900406 \mathrm{WA} 9003 \mathrm{~L} 865004$ 24DNP 1XLT 03010319900402900406 WA9003L865.004 4NP 1XLT 03010319900402900406 WA9003L865 004 DIBZFU1XLT $03010319900402900406 \mathrm{FA9003L865004}$ 24DNT 1XLT
39 OUGKG

390UGKG

390 UGKG

390UǴKG

390UGKG

390UGKG

390 UGKG

390UGKG

390UGKG

390UGKG

390 UGKG

390UGKG

39 OUGKG

390UGKG

39 OUGKG

390UGKG

1900 UGKG

390UGKG

390UGKG

390UGKG

390UGKG

390UGKG

390 UGKG

390UGKG

390 UGKG

390 UGKG

390UGKG

1900 UGKG 390 UGKG

1900 UGKG

390UGKG 3900 UKG

390UGKG

1900 UGKG

390 UGKG

1900 UGKG

1900 UGKG

390 UGKG

32RUGKG
$1.0000 \mathrm{MSJBD}$ 1.0000MSJBD

1.0000MSJBD

$1.0000 M S C D T$

1. $0000 \mathrm{MSCDT}$

$1.0000 \mathrm{MSCDT}$

1.0000MSCDT

$1.0000 \mathrm{MSCDT}$

1.0000MSCDT

$1.0000 \mathrm{MSCD}$

$1.0000 \mathrm{MSCDT}$

1.0000MSCDT

1.0000MSCDT

1. $0000 \mathrm{MSCDT}$

$1.0000 \mathrm{MSCDT}$

1. $0000 \mathrm{MSCDT}$

$1.0000 \mathrm{MSCDT}$

$1.0000 \mathrm{MSCDT}$

$1.0000 \mathrm{MSCDT}$

$1.0000 \mathrm{MSCDT}$

1.0000MSCDT

1.0000MSCDT

1.0000MSCDT

1. $0000 \mathrm{MSCDT}$

1.0000MSCDT

1.0000MSCDT

1. $0000 \mathrm{MSCDT}$

$1.0000 \mathrm{MSCDT}$

1:0000MSCDT

1.0000MSCDT

$1.0000 \mathrm{MSCDT}$

1.0000MSCDT

1. $0000 \mathrm{MSCDT}$

1.000OMSCDT

1. 000OMSCDT

1.0000MSCDT

1. 0000MSCDT

1. 0000MSCDT

1.0000MSCDT

1.0000MSCDT

1. 0000MSCDT

1.0000MSCDT

1.0000MSCDT

1. 0000MSCDT

1. 0000MSCDT

1. $0000 \mathrm{MSCDT}$

1.0000MSCDT

1.0000MSCDT
OETHYLBENZENE

OSTYRENE

OXYLENE (TOTAL)

1930.000NITROBENZENE-D5

$1930.0002-F L U O R O B I P H E N Y L$

$1930.000 P-T E R P H E N Y L-D 14$

380.001PHENOL-D5

$3860.0012-F L U O R O P H E N O L$

$3860.0012,4,6$-TRIBROMOPHENOLO625HN

ORHENOL

02-CHLOROPHENOL O625HN

01,3-DICHLOROBENZENE O625HN

01,4-DICHLOROBENZENE 0625HN

OBENZYL ALCOHOL O625H

01,2-DICHLOROBENZENE O625HN

02-METHYLPHENOI O625HN

OBIS 12-CHLOROISORROPYO625HN

04-METHYLPHENOL O625HN ON-NITROSO-DI-N-PROPYO625HN OHEXACHLOROETHANE O625HN ONITROBENZENE O625HN OISOPHORONE $0625 \mathrm{HN}$ 02-NITROPHENOL O625HN 02,4-DIMETHYLPHENOI 0625HN OBENZOIC ACID O625HN OBIS (2-CHLOROETHOXY) MO625HN 02, 4-DICHLOROPHENOI $0625 \mathrm{HN}$ $01,2,4-T R I C H L O R O B E N Z E O 625 \mathrm{HN}$ ONAPHTHALENE O625HN 04-CHLOROANIIINE 0625HN OHEXACHLOROBUTADIENE 0625HN 04-CHLORO-3-METHYLPHEO 625HN 02-METHYLNAPHTHALENE 0625HN OHEXACHLOROCYCLOPENTAO $625 \mathrm{HN}$ $02,4,6$-TRICHLOROPHENOO625HN $02,4,5-T R I C H L O R O P H E N O 0625 \mathrm{HN}$ 02-CHLORONAPHTHALENE 0625HN 02-NITROANILINE O625HN ODIMETHYLPHTHALATE O625HN OACENAPHTHYLENE O625HN 02,6-DINITROTOLUENE O625HN 03-NITROANIIINE O625HN OACENAPHTHENE $:$ O625HN 02,4-DINITROPHENOL 0625HN O4-NITROPHENOL O625HN ODIBENZOFURAN 0625HN $02,4-D I N I T R O T O L U E N E-9625 \mathrm{HN}$ 
GRS 03L_ 0319900402900406\%A9003L865 004 DEP 1XLT GRS $0301.0319900402900406 \mathrm{FA} 9003 \mathrm{~L} 865004$ 4CLPPE1XLT GRS 03010319900402900406 WA9003L865 004 FLRENE1XLT

03010319900402900406 WA9003L865 004 4NANILIXLT 03010319900402900406 WA9003L865 004 46DN2CIXLT $03010319900402900406 \mathrm{WA} 9003 L 865004$ NNDPA IXLT $03010319900402900406 \mathrm{WA} 9003 \mathrm{~L} 865004$ 4BRPPE1XLT 03010319900402900406 WA9003L865 004 CL6BZ 1XLT $03010319900402900406 \mathrm{FA} 9003 \mathrm{LB65} 004 \mathrm{PCP} .1 \times L T$ 03010319900402900406 WA9003L865 004 PHANTRIXLT $03010319900402900406 \mathrm{WA} 9003 L 865004$ ANTRC 1XLT 03010319900402900406 WA9003L865 004 DNBP " IXLT 03010319900402900406 WA9003L865 004 FANT 1XLT 03010319900402900406 WA9003L865 004 PYR 1XLT $03010319900402900406 \mathrm{WA} 9003 \mathrm{~L} 865004$ BBZP 1XLT 03010319900402900406 WA9003L865 004 33CL2B1XLT 03010319900402900406 WA9003L865 004 BAANTR1XLT 03010319900402900406 WA9003L865 004 CHRY 1XLT 03010319900402900406 WA9003L865 004 B2EHP 1XLT 03010319900402900406 WA9003L865 004 DNOP 1XLT 03010319900402900406 WA $9003 L 865004$ BE2ANT1XLT $03010319900402900406 \mathrm{FA} 9003 \mathrm{~L} 865004$ BKFANT1XLT 03010319900402900406 WA9003L865 004 BAPYR 1XIT 03010319900402900406 WA9003L865 004 ICDPYR1XLT $03010319900402900406 \mathrm{WA} 9003 \mathrm{~L} 865004$ DBAHA 1XLT 03010319900402900406 WA9003L865 004 BGHIPY1XLT 05010320900328900051 WA9003L865 005

05010320900519900451 WA9003L865 005 AG 2ZLT 05010320900518900450 WA9003L865 005 AS 1BLT 05010320900519900451 WA9003L865 005 BA 2ZLT 05010320900519900451 HA9003L865 005 CD 27 05010320900519900451 พั9003L865 005 CR $2 Z$ $05010320900405900055 \% A 90034865$ 005 HGTOT IDLT 05010320900518900450 WA9003L865 005 PB 1B 05010320900518900450 WA9003L865 005 SE IBLT $0501 \cdot 0320900327900051$ WA9003L865 005 MEC6D82J 05010320900327900051 WA9003L865 005 PBEB $2 J$ 05010320900327900051 WA9003L865 005 12DCD42J 05010320900327900051 WA9003L865 005 CH3CL 2JLT 05010320900327900051 WA9003L865 005 CH3BR 2JLT 05010320900327900051 KA9003L865 005 C2H3CL2JLT 05010320900327900051 WA9003L865 005 C2H5CL2JLT 05010320900327900051 KA9003L865 005 CH2CL22JB 05010320900327900051 WA9003L865 005 ACET 2JLT 05010320900327900051 HA9003L865 005 CS2 2JLT 05010320900327900051 HA9003L865. 005 11DCE 2J̣LT 05010320900327900051 HA9003L865 005 11DCLE2JLT 05010320900327900051 AA9003L865 O05 T12DCE2JLT
ذ > UUGKG

39 OUGKG

390UGKG

1900 UGKG

1900 UGKG

390UGKG

390UGKG

39 OUGKG

1900 UGKG

390UGKG

39 OUGKG

390 UGKG

390UGKG

390 UGKG

390UGKG

77 OUGKG

390UGKG

390UGKG

39 OUGKG

390UGKG

390 UGKG

39 OUGKG

390 UGKG

$390 U G K G$

390 UGKG

390 UGKG

91.68

2. OMGKG

2. 1MGKG

39. 3MGKG

1. 2MGKG

8. OMGKG

$0.11 \mathrm{MGKG}$

2. 1MGKG

1. OMGKG

98RER

102PER

87PER

12 UGKG

12UGKG

12 UGKG

12 UGKG

32 UGKG

12UGKG

6UGKG

6UGKG

6UGK̈G 6UGKG
1.0000MSCDT

1. $0000 \mathrm{MSCDT}$

1.0000MSCDT

1. 0000MSCDT

1. $0000 \mathrm{MSCDT}$

1. 0000MSCDT

1.0000MSCDT

1.0000MSCDT

1.0000MSCDT

1.0000MSCDT

1.0000MSCDT

1.0000MSCDT

1.0000MSCDT

1.0000MSCDT

1.0000MSCDT

1.0000MSCDT

1. 0000MSCDT

1. 0000MSCDT

1.0000MSCDT

1.0000MSCDT

1. 0000MSCDT

1.0000MSCDT

1.0000MSCDT

1. $0000 \mathrm{MSCDT}$

1.0000MSCDT

1.0000MSCDT $10 \mathrm{JH}$

1. OOICALM

1. OOAAAER

1. OOICALM

1. 00ICALM

1. 00ICAIM

1. 0OHGJJW

1. OOAAEJS

1. OOAAMLR

1. 0000MSJBD

1.0000MSJBD

1. $0000 \mathrm{MSJBD}$

1. 0000MSJBD

1.0000MSJBD

1. $0000 \mathrm{MSJBD}$

1.0000MSJBD

1. 0000MSJBD

1.0000MSJBD

1. $0000 \mathrm{MSJBD}$

1. 000OMSJBD

1.0000MSJBD

1. 0000MSJBD
ODIETHYLPHTHALATE O625HN 04-CHLOROPHENYL-PHENYO $625 \mathrm{HN}$ OELUORENE 0625HN 04-NITROANIIINE 0625HN 04, 6-DINITRO-2-METHYLO625HN ON-NITROSODIPHENYIAMIO625HN 04-BROMOPHENYL-PHENYLO625HN OHEXACHLOROBENZENE O625HN OPENTACHLOROPHENOT O625HN OPHENANTHRENE $0625 \mathrm{HN}$ OANTHRACENE 0625HN ODI-N-BUTYLPHTHALATE 0625HN OELUORANTHENE O625HN OPYRENE - O625HN OBUTYLBENZYLPHTHALATEO625HN $03,3^{3}$-DICHLOROBENZIDI0625HN OBENZO (A) ANTHRACENE 0625HN OCHRYSENE $0625 \mathrm{HN}$ OBIS (2-ETHYIHEXYL) PHTO625HN ODI-N-OCTYL PHTHALATEO625HN OBENZO (B) FLUORANTHENEO $625 \mathrm{HN}$ OBENZO (K) ELUORANTHENEO625HN OBENZO (A) PYRENE O625HN OINDENO $(1,2,3-C D)$ P YREO625HN ODIBENZO (A, H) ANTHRACEO625HN OBENZO (G, H, I) PERYLENEO625HN O\& SOLIDS I\&SOLN OSILVER, TOTAL MAGTON OARSENIC, TOTAL MASTON OBARIUM, TOTAL MBATON OCADMIUM, TOTAL MCDTON OCHROMIUM, TOTAL MCRTON OMERCURY, TOTAL MHGTON OLEAD, TOTAL MPBTON OSELENIUM, TOTAL MSETON 59.500TOLUENE-D8 O624HN 59.50OBROMOELUOROBENZENE O624HN 59.5001,2-DICHLOROETHANE-DO624HN OCHLOROMETHANE O624HN OBROMOMETHANE O624HN OVINYL CHLORIDE OCHLOROETHANE O62.4HN OMETHYLENE CHLORIDE O624HN OACETONE O62.4HN OCARBON DISULFIDE O624HN 01,1-DICHLOROETHENE 0624HN 01,1-DICHLOROETHANE O624HN 01,2-DICHLOROETHENE $10624 \mathrm{HN}$ 
GRS 05010320900327900051 WA9003L865 005 CHCL3 2JLT GRS 05010320900327900051 WA9003L865 005 12DCLE2JLT GRS 05010320900327900051 WA9003L865 005 MEK 2JLT GRS 05010320900327900051 WA9003L865 005 111TCE2JLT GRS 05010320900327900051 WA9003L865 005 CCL4 2JLT GRS 05010320900327900051 WA9003L865 005 VINYLA2JLT GRS 05010320900327900051 WA9003L865 005 BRDCLM2JLT GRS 05010320900327900051 WA9003L865 005 12DCLP2JLT GRS 05010320900327900051 WA 0003 L 865005 13DCPC2JLT GRS 05010320900327900051 WA9003L865 005 TRCLE 2JLT GRS 05010320900327900051 WA9003L865 005 DBRCLM2JLT GRS 05010320900327900051 WA9003L865 005 112TCE2JLT GRS 05010320900327900051 WA9003L865 005 C6H6 2JLT GRS 05010320900327900051 WA9003L865 005 T13DCP2JLT GRS 05010320900327900051 WA9003L865 005 CHBR3 2JLT GRS 05010320900327900051 WA9003L865 005 4ME2PE2JLT GRS 05010320900327900051 WA9003L865 005 2HXONE2JLT GRS 05010320900327900051 WA9003L865 005 TCLEE 2JLT GRS 05010320900327900051 WA9003L865 005 TCLEA 2JLT GRS 05010320900327900051 WA9003L865 005 MEC6H52JLT GRS 05010320900327900051 WA9003L865 005 CLC6H52JLT GRS 05010320900327900051 WA9003L865 005 ETC6H52JLT 由 GRS $\stackrel{1}{\sim}$ GRS $\underset{\infty}{\infty}$ GRS GRS GRS GRS 05010320900327900051 WA 90032865005 STYR 2JLT 05010320900327900051 WA9003L865 005 XYLEN 2JLT $05010320900402900406 \mathrm{WA} 90031865005$ NBD5 $1 \mathrm{X}$ 05010320900402900406 WA9003L865 005 2FBP 1X 05010320900402900406 WA9003L865 005 PTERP 1X 05010320900402900406 WA9003L865 005 PHEND51X $05010320900402900406 \mathrm{WA} 9003 \mathrm{~L} 865005$ 2EP $1 \mathrm{X}$ $05010320900402900406 \mathrm{WA} 9003 \mathrm{~L} 865005$ 246TBP1X 05010320900402900406 FA9003L865 005 PHENOL1XLT 05010320900402900406 WA9003L865 005 B2CEE 1XLT $05010320900402900406 \mathrm{WA} 9003 L 865005$ 2CLP 1XLT $05010320900402900406 \mathrm{WA} 9003 \mathrm{~L} 865005$ 13DCLB1XLT $05010320900402900406 \mathrm{WA} 9003 \mathrm{~L} 865005$ 14DCLB1XLT 05010320900402900406 WA9003L865 005 BZALC 1XLT $05010320900402900406 \mathrm{KA} 9003 \mathrm{~L} 865005$ 12DCLB1XLT $05010320900402900406 \mathrm{WA9003L865005} \mathrm{2MP \quad 1XLT}$ 05010320900402900406 WA9003L865 005 B2CIPE1XLT 05010320900402900406 WA9003L865 005 4MP IXLT $05010320900402900406 \mathrm{WA} 9003 \mathrm{~L} 865005$ NDNPA 1XLT $05010320900402900406 \mathrm{FA9003L865} 005$ CL6ET 1XLT $05010320900402900406 \mathrm{KA} 9003 \mathrm{~L} 865005 \mathrm{NB}$ 1XLT 05010320900402900406 WA9003L865 005 ISOPHRIXLT 05010320900402900406 FA9003L865 005 2NP 1XLT $05010320900402900406 \mathrm{WA9003L865} 005$ 24DMPN1XLT $05010320900402900406 \mathrm{KA} 903 \mathrm{~L} 865005$ BENZOA1XLT 05010320900402900406 HA9003L865 005 B.2CEXM1XLT
6UGKG 6UGKG 6UGKG 6UGKG 6UGKG 6UGKG

86PER

S3PER

68PER

86PER

69PER

98PER

400UGKG

400 UGKG

400UGKG

400UGKG

400UGKG

400UGKG

400UGKG

400 UGKG

400 UGKG

400UGKG

400 UGKG

40 OUGKG

400UGKG

400UGKG

400 UGKG

400UGKG

2000UGKKG 400UGKG
$1.0000 \mathrm{MSJBD}$

$1.0000 \mathrm{MSJBD}$

$1.0000 \mathrm{MSJBD}$

$1.0000 M S J B D$

$1.0000 \mathrm{MSJBD}$

$1.0000 M S J B D$

$1.0000 \mathrm{MSJBD}$

$1.0000 \mathrm{MSJBD}$

$1.0000 M S J B D$

$1.0000 \mathrm{MSJBD}$

$1.0000 \mathrm{MSJBD}$

1.0000 MSJBD

$1.0000 \mathrm{MSJBD}$

$1.0000 \mathrm{MSJBD}$

$1.0000 M S J B D$

$1.0000 \mathrm{MSJBD}$

$1.0000 \mathrm{MSJBD}$

$1.0000 \mathrm{MSJBD}$

$1.0000 \mathrm{MSJBD}$

$1.0000 \mathrm{MSJBD}$

$1.0000 \mathrm{MSJBD}$

1.0000 MSJBD

1.0000MSJBD

$1.0000 \mathrm{MSJBD}$

$1.0000 \mathrm{MSCDT}$

1.0000MSCDT

$1.0000 \mathrm{MSCDT}$

$1.0000 M S C D T$

1.0000 MSCDT

1. $0000 \mathrm{MSCDT}$

1.0000MSCDT

$1.0000 \mathrm{MSCDT}$

$1.0000 \mathrm{MSCDT}$

1. 0000MSCDT

1.0000MSCDT

$1.0000 \mathrm{MSCDT}$

1.0000MSCDT

$1.0000 \mathrm{MSCDT}$

1.0000MSCDT

$1.0000 \mathrm{MSCDT}$

$1.0000 \mathrm{MSCDT}$

1. $0000 \mathrm{MSCDT}$

$1.0000 \mathrm{MSCDT}$

1.0000MSCDT

$1.0000 \mathrm{MSCDT}$

$1.0000 \mathrm{MSCDT}$

$1.0000 \mathrm{MSCDT}$

$1.0000 \mathrm{MSCDT}$
OCHLOROFORM

$0624 \mathrm{HN}$

01,2-DICHLOROETHANE 0624HN

02-BUTANONE

$0624 \mathrm{HN}$

$01,1,1$-TRICHLOROETHANO624HN OCARBON TETRACHLORIDEO624HN OVINYL ACETATE '. $0624 \mathrm{HN}$ OBROMODICHLOROMETHANEO $624 \mathrm{HN}$ 01,2-DICHLOROPROPANE $0624 \mathrm{HN}$ OCIS-1, 3-DICHLOROPROPO624HN OTRICHLOROETHENE O624HN ODIBROMOCHLOROMETHANEO $624 \mathrm{HN}$ 01, 1,2-TRICHLOROETHANO $624 \mathrm{HN}$ OBENZENE $0624 \mathrm{HN}$ OTRANS-1, 3-DICHLOROPRO624HN OBROMOFORM $0624 \mathrm{HN}$ 04-METHYL-2-PENTANONE0624HN 02-HEXANONE O624HN OTETRACHLOROETHENE O624HN $01,1,2,2-T E T R A C H L O R O E O 624 \mathrm{HN}$ OTOLUENE

OCHIOROBENZENE OETHYLBENZENE $0624 \mathrm{HN}$ OSTYRENE OXYLENE (TOTAL) 0624HN $2010.000 N I T R O B E N Z E N E-D 5 \quad 0625 \mathrm{HN}$ 2010.0002-FLUOROBIPHENYL O625HN 2010.000P-TERPHENYL-D14 0625HN 4020.000 PHENOL-D5 $0625 \mathrm{HN}$ 4020.0002-FLUOROPHENOL O625HN $4020.0002,4,6$-TRIBROMOPHENOLO $625 \mathrm{HN}$ OPHENOL O625HN OBIS (2-CHLOROETHYL) ETO625HN 02-CHLOROPHENOL O625HN 01,3-DICHLOROBENZENE O625HN 01, 4-DICHLOROBENZENE O625HN OBENZYI ALCOHOL O625HN 01,2-DICHLOROBENZENE $0625 \mathrm{HN}$ 02-METHYLPHENOL O625HN OBIS (2-CHLOROISOPROPYO $625 \mathrm{HN}$ 04-METHYLPHENOL O625HN ON-NITROSO-DI-N-PROPYO $625 \mathrm{HN}$ OHEXACHLOROETHANE O625HN ONITROBENZENE OISOPHORONE $\quad 0625 \mathrm{HN}$ 02-NITROPHENOL $\quad 0625 \mathrm{HN}$ 02,4-DIMETHYLPHENOL 0625HN OBENZOIC ACID $0625 \mathrm{HN}$ OBIS (2-CHLOROETHOXY Y $2625 \mathrm{HN}$ 
05 - $0320900402900406 \mathrm{FA9003L865005} \mathrm{24DCLP1XLT}$ $05010320900402900406 \mathrm{FA} 9003 \mathrm{~L} 865005$ 124TCB1XLT $05010320900402900406 \mathrm{FA} 9003 \mathrm{~L} 865005 \mathrm{NAP}$ 1XLT $05010320900402900406 \mathrm{HA} 90032865005$ 4CANIL1XLT

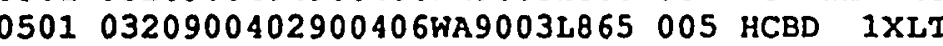
$05010320900402900406 \mathrm{WA} 9003 \mathrm{~L} 865005$ 4CL3C 1XLT $05010320900402900406 \mathrm{WA} 9003 \mathrm{~L} 865005$ 2MENAP1XLT $05010320900402900406 \mathrm{WA} 9003 \mathrm{~L} 865005$ CL6CP 1XLT $05010320900402900406 \mathrm{KA} 9003 \mathrm{~L} 865005$ 246TCP1XLT $05010320900402900406 \mathrm{WA} 9003 \mathrm{~L} 865005$ 245TCP1XLT $05010320900402900406 \mathrm{FA} 9003 \mathrm{~L} 865005$ 2CNAP 1XLT $05010320900402900406 \mathrm{FA} 9003 \mathrm{~L} 865005$ 2NANIL1XLT $05010320900402900406 \mathrm{FA} 90032865005$ DMP 1XLT $05010320900402900406 \mathrm{WA} 9003 \mathrm{~L} 865005$ ANAPYL1XLT $05010320900402900406 \mathrm{WA} 9003 \mathrm{~L} 865005$ 26DNT 1XLT

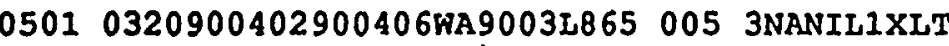
$05010320900402900406 \mathrm{FA} 90032865005$ ANAPNE1XLT $05010320900402900406 \mathrm{HA} 9003 \mathrm{~L} 865005$ 24DNP 1XLT $05010320900402900406 \mathrm{FA9003L865} 005$ 4NP 1XLT $05010320900402900406 \mathrm{FA} 9003 L 865005$ DIBZFU1XLT 05010320900402900406 WA9003L865 005 24DNT 1XLT $05010320900402900406 \mathrm{FA} 9003 \mathrm{~L} 865005$ DEP 1XLT $05010320900402900406 \mathrm{WA} 9003 \mathrm{~L} 865005$ 4CLPPE1XLT $05010320900402900406 \mathrm{WA} 9003$ L865 005 FLRENE1XLT $05010320900402900406 \mathrm{WA} 9003 \mathrm{~L} 865005$ 4NANILIXLT $05010320900402900406 \mathrm{WA} 9003 \mathrm{~L} 865005$ 46DN2C1XLT 05010320900402900406 WA9003L865 005 NNDPA 1XLT 05010320900402900406 WA9003L865 005 4BRPPE1XLT $05010320900402900406 \mathrm{FA} 9003 \mathrm{~L} 865005$ CL6BZ 1XLT 05010320900402900406 WA9003L865 005 PCP 1XLT $05010320900402900406 \mathrm{HA} 9003 \mathrm{~L} 865005$ PHANTR1XLT $05010320900402900406 \mathrm{WA} 9003 \mathrm{~L} 865005$ ANTRC 1XLT $05010320900402900406 \mathrm{WA} 9003 \mathrm{~L} 865005$ DNBP 1XLT $05010320900402900406 \mathrm{WA} 003 \mathrm{~L} 865005$ FANT 1XLT 05010320900402900406 WA9003L865 005 PYR 1XLT 05010320900402900406 WA9003L865 005 BBZP 1XLT $05010320900402900406 \mathrm{WA} 9003 \mathrm{~L} 865005$ 33CL2B1XLT 05010320900402900406 WA9003L865 005 BAANTR1XLT 05010320900402900406 WA9003L865 005 CHRY 1XLT $05010320900402900406 \mathrm{WA} 9003 L 865005$ B2EHP 1XLT 05010320900402900406 WA9003L865 005 DNOP 1XLT $05010320900402900406 \mathrm{WA} 9003 \mathrm{~L} 865005$ BF2ANT1XLT 05010320900402900406 WA9003L865 005 BKEANT1XLT $05010320900402900406 \mathrm{WA} 9003 \mathrm{~L} 865005$ BAPYR 1XLT $05010320900402900406 \mathrm{WA} 9003 L 865005$ ICDPYR1XLT $05010320900402900406 \mathrm{FA} 9003 \mathrm{~L} 865005$ DBAHA 1XLT $05010320900402900406 \mathrm{FA9003L865005}$ BGHIPY1XLT 06010320900328900051 KA9003L865 006
৭ UUGKG 40 OUGKG 40 OUGKG $\triangle 0$ OUGKG 40 OUGKG 40 OUGKG $\triangle$ OOUGKG 40 OUGKG 400 UGKG 200 OUGKG 40 OUGKG 2000 UGKG 40 OUGKKG 40 OUGKG 40 OUGKG 200 OUGKG 4 OOUGKG 2000UGKG 200 OUGKG 40 OUGKG 40 OUGKG 40 OUGKG 40 OUGKG 40 OUGKG 2000 UGKG 2000UGKG 40 OUGKG 40 OUGKG 40 OUGKG 200 OUGKG 40 OUGKG 400 UGKG 40 OUGKG 40 OUGKG 40 OUGKG 40 OUGKG 80 OUGKG 40 OUGKG 40 OUGKG 40 OUGKG 40 OUGKG 40 OUGKG 400 UGKG 40 OUGKG 40 OUGKG 40 OUGKG 40 OUGKG 88.68
1.0000MSCDT $1.0000 \mathrm{MSCDT}$ $1.0000 \mathrm{MSCDT}$ $1.0000 \mathrm{MSCDT}$ $1.0000 \mathrm{MSCDT}$

$1.0000 \mathrm{MSCDT}$

$1.0000 \mathrm{MSCDT}$

$1.0000 \mathrm{MSCDT}$

$1.0000 \mathrm{MSCDT}$

$1.0000 \mathrm{MSCDT}$

$1.0000 \mathrm{MSCDT}$

$1.0000 \mathrm{MSCDT}$

$1.0000 \mathrm{MSCDT}$

$1.0000 \mathrm{MSCDT}$

$1.0000 M S C D T$

$1.0000 \mathrm{MSCDT}$

$1.0000 \mathrm{MSCDT}$

$1.0000 \mathrm{MSCDT}$

$1.0000 \mathrm{MSCDT}$

$1.0000 \mathrm{MSCDT}$

$1.0000 \mathrm{MSCDT}$

$1.0000 \mathrm{MSCDT}$

$1.0000 \mathrm{MSCDT}$

1.0000MSCDT

$1.0000 \mathrm{MSCDT}$

$1.0000 \mathrm{MSCDT}$

1. $0000 \mathrm{MSCDT}$

$1.0000 \mathrm{MSCDT}$

1:0000MSCDT

1.0000MSCDT

$1.0000 \mathrm{MSCDT}$

1.0000MSCDT

$1.0000 \mathrm{MSCDT}$

1.0000MSCDT

$1.0000 \mathrm{MSCDT}$

$1.0000 \mathrm{MSCDT}$

$1.0000 M S C D T$

$1.0000 \mathrm{MSCDT}$

$1.0000 \mathrm{MSCDT}$

$1.0000 M S C D T$

$1.0000 \mathrm{MSCDT}$

$1.0000 \mathrm{MSCDT}$

1.0000MSCDT

$1.0000 \mathrm{MSCDT}$

$1.0000 M S C D T$

$1.0000 \mathrm{MSCDT}$

$1.0000 \mathrm{MSCDT}$ $10 \mathrm{JH}$
02,4-DICHLOROPHENOL O625HN $01,2,4$-TRICHLOROBENZEO625HN ONAPHTHALENE 0625HN 04-CHLOROANILINE O625HN OHEXACHLOROBUTADIENE $0625 \mathrm{HN}$ 04-CHLORO-3-METHYLPHEO625HN 02-METHYLNAPHTHALENE 0625HN OHEXACHLOROCYCLOPENTAO625HN $02,4,6$-TRICHLOROPHENOO625HN $02,4,5$-TRICHLOROPHENOO $625 \mathrm{HN}$ 02-CHLORONAPHTHALENE 0625HN 02-NITROANILINE 0625HN ODIMETHYLPHTHALATE O625HN OACENAPHTHYLENE 0625HN 02,6-DINITROTOLUENE 0625HN 03-NITROANILINE $0625 \mathrm{HN}$ OACENAPHTHENE O625HN 02,4-DINITRORHENOI O625HN 04-NITRORHENOL $0625 \mathrm{HN}$ ODIBENZOFURAN O625HN 02,4-DINITROTOLUENE 0625HN ODIETHYLP HTHALATE 0625HN 04-CHLOROPHENYL-PHENYO625HN OFLUORENE O625HN 04-NITROANILINE $0625 \mathrm{HN}$ 04, 6-DINITRO-2-METHYLO625HN ON-NITROSODIPHENYLAMIO $625 \mathrm{HN}$ 04-BROMOPHENYL-PHENYLO625HN OHEXACHLOROBENZ ENE O625HN OPENTACHLOROPHENOL O625HN OPHENANTHRENE 0625HN OANTHRACENE O625HN ODI-N-BUTYLPHTHALATE $0625 \mathrm{HN}$ OFLUORANTHENE O625HN OPYRENE 0625HN OBUTYLBENZYLPHTHALATEO625HN $03,3^{\prime}$-DICHLOROBENZIDIO $625 \mathrm{HN}$ OBENZO (A) ANTHRACENE O625HN OCHRYSENE O625HN OBIS (2-ETHYLHEXYL) PHTO625HN ODI-N-OCTYI PHTHALATEO $625 \mathrm{HN}$ OBENZO (B) FLUORANTHENEO625HN OBENZO (K) FLUORANTHENE0625HN OBENZO (A) PYRENE : O625HN OINDENO $(1,2,3-C D)$ PYRE0625 HN ODIBENZO (A, H) ANTHRACEO $625 \mathrm{HN}$ OBENZO (G, H, I) PERYLENEO $625 \mathrm{HN}$ O\& SOLIDS

I\& SOLN 
GRS 06010320900423901200 WA9003L865 006 ALPHAG9W GRS 060.20320900423901200 WA9003L865 006 BETAG 9W

GRS 06010320900519900451 WA9003L865 006 AG. 2ZLT

GRS 06010320900518900450 WA9003L865 006 AS 1BLT

GRS 06010320900519900451 WA9003L865 006 BA 2ZLT

GRS 06010320900519900451 WA9003L865 $006 \mathrm{CD} \quad 22$

GRS 06010320900519900451 WA9003L865 006 CR 22

GRS 06010320900405900055 WA9003L865 006 HGTOT 1D

GRS 06010320900518900450 WA $9003 \mathrm{~L} 865006 \mathrm{~PB} \quad 1 \mathrm{~B}$

GRS 06010320900518900450 WA9003L865 $006 \mathrm{SE}$ 1BLT

GRS 06010320900327900051 WA9003L865 006 MEC6D82J

GRS 06010320900327900051 WA9003L865 006 PBFB 2J

GRS 06010320900327900051 WA9003L865 006 12DCD 42J

GRS 06010320900327900051 WA9003L865 $006 \mathrm{CH} 3 \mathrm{CL}$ 2JLT

GRS $06010320900327900051 \mathrm{WA} 9003 \mathrm{~L} 865006$ CH3BR 2JLT

GRS $06010320900327900051 \mathrm{WA} 9003 \mathrm{~L} 865006$ C2H3CL2JLT

GRS 06010320900327900051 WA9003L865 006 C2H5CL2JLT

GRS 06010320900327900051 WA9003L865 006 CH2CL22JB

GRS 06010320900327900051 WA9003L865 006 ACET 2JLT

GRS $06010320900327900051 \mathrm{WA} 9003 \mathrm{~L} 865006$ CS2 2JLT

GRS 060103209003279.00051 WA9003L865 006 IIDCE 2JLT

GRS 06010320900327900051 WA9003L865 006 11DCLE2JLT

GRS 06010320900327900051 WA9003L865 006 T12DCE2JLT

GRS

GRS

G GRS

GRS

GRS

GRS

GRS

GRS

GRS .

GRS

GRS

GRS

GRS

GRS

GRS

GRS

GRS

GRS

GRS

GRS

GRS

GRS

GRS

GRS

GRS

06010320900327900051 WA9003L865 006 CHCL3 2JLT

06010320900327900051 WA9003L865 006 12DCLE2JLT

06010320900327900051 WA9003L865 006 MEK 2JLT

0601.0320900327900051 WA9003L865 006 111TCE2JLT

06010320900327900051 WA9003L865 006 CCL4 2JLT

06010320900327900051 WA9003L865 006 VINYLA2JLT

06010320900327900051 WA9003L865 006 BRDCLM2JLT

06010320900327900051 WA9003L865 006 12DCLP2JLT

06010320900327900051 WA9003L865 006 13DCPC2JLT

06010320900327900051 WA9003L865 006 TRCLE 2JLT

$06010320900327900051 \mathrm{WA} 90031865006$ DBRCLM2JLT

06010320900327900051 WA9003L865 006 112TCE2JLT

06010320900327900051 WA9003L865 006 C6H6 2JLT

06010320900327900051 WA9003L865 006 T13DCP2JLT

06010320900327900051 WA9003L865 006 CHBR3 2JLT

06010320900327900051 WA9003L865 006 4ME2PE2JIT

$06010320900327900051 \mathrm{KA} 9003 \mathrm{~L} 865006$ 2HXONE2JLT

06010320900327900051 HA9003L865 006 TCLEE 2JLT

06010320900327900051 WA9003L865 006 TCLEA 2JLT

06010320900327900051 WA9003L865 006 MEC6H52JLT

06010320900327900051 HA9003L865 006 CLC6H52JLT

06010320900327900051 KA9003L865. 006 ETC6H52JLT

06010320900327900051 HA9003L865 006 STYR 2JLT

06010320900327900051 HA9003L865 006 XYLEN 2JLT

5. OPCIG

7. OPCIG

1.9MGKG

2. OMGKG

37. 7 MGKG

1.2MGKG

14.9MGKG

$0.14 M G K G$

5. $1 \mathrm{MGKG}$

1. OMGKG

102PER

107PER

91PER

11UGKG

11UGKG

11UGKG

11UGKG

6UGKG

11UGKG

6UGKG

6UGKG

6UGKG

6UGKG

6UGKG

6UGKG

11UGKG

6UGKG

6UGKG

11UGKG

6UGKG

6UGKG

6UGKG

6UGKG

6UGKG

6UGKG

6UGKG

6UGKG

6UGKG

11UGKG

11UGKG

6UGKG

6UGKG

6UGKG

6UGKG

6UGKG

6UGKG

6UGKG

0601. 0320900402900406\%A9003L865 006 NBD5 $1 \times$
10 EBUNK

10EBUNK

1. OOICALM

1. O0AAAER

1. OOICALM

1. OOICALM

1. OOICALM

$1.00 \mathrm{HGJJW}$

1. OOAAEJS

1. 00AAMLR

1. $0000 \mathrm{MSJBD}$

1. $0000 \mathrm{MSJBD}$

1.0000MSJBD

1. $0000 \mathrm{MSJBD}$

1. $0000 \mathrm{MSJBD}$

1. $0000 \mathrm{MSJBD}$

1.0000MSJBD

1. $0000 \mathrm{MSJBD}$

1. $0000 \mathrm{MSJBD}$

$1.0000 \mathrm{MSJBD}$

1.000OMSJBD

1. $0000 \mathrm{MSJBD}$

1. 0000MSJBD

1. $0000 \mathrm{MSJBD}$

1. $0000 \mathrm{MSJBD}$

1. 0000MSJBD

1. $0000 \mathrm{MSJBD}$

1.0000MSJBD

1. $0000 \mathrm{MSJBD}$

1. $0000 \mathrm{MSJBD}$

1.0000MSJBD

1. 0000MSJBD

1. $0000 \mathrm{MSJBD}$

1. 0000MSJBD

1.0000MSJBD

1. $0000 \mathrm{MSJBD}$

1.0000MSJBD

1. $0000 \mathrm{MSJBD}$

1. $0000 \mathrm{MSJBD}$

1.0000MSJBD

1. $0000 \mathrm{MSJBD}$

1.0000MSJBD

1.0000MSJBD

1. $0000 M S J B D$

1.0000MSJBD

1. $0000 \mathrm{MSJBD}$

1.0000MSJBD

1.0000MSCDT
OGROSS ALPHA

ONONVOLATILE BETA

OSILVER, TOTAL

OARSENIC, TOTAL

OBARIUM, TOTAL

OCADMIUM, TOTAL

OCHROMIUM, TOTAL

OMERCURY, TOTAL

OLEAD, TOTAL

OSELENIUM, TOTAL

56.500TOLUENE-D8

OCHLOROMETHANE
OCHANE-DO624HN
OBROMOMETHN

OBROMOMETHANE O624HN

OVINYI CHLORIDE O624HN

OCHLOROETHANE O624HN

OMETHYLENE CHLORIDE O624HN

OACETONE

OCARBON DISULEIDE

01,1-DICHLOROETHENE 0624HN

$01,1-D I C H L O R O E T H A N E$ 0624HN

$01 ; 2-D I C H L O R O E T H E N E$ (O624HN

OCHLOROEORM

OETHANE O624HN

01, 1, 1-TRICHLOROETHANO 624HN

OCARBON TETRACHLORIDEO $624 \mathrm{HN}$

OVINYL ACETATE O624HN

OBROMODICHLOROMETHANEO624HN

01,2-DICHLOROPROPANE 0624HN

OCIS-1, 3-DICHLOROPROPO62 4HN

OTRICHLOROETHENE O624HN

ODIBROMOCHLOROMETHANEO624HN

$01,1,2$-TRICHLOROETHANO $624 \mathrm{HN}$ OBENZENE

$0624 \mathrm{HN}$

OTRANS-1,3-DICHIOROPRO 624HN

OBROMOFORM O624HN

04-METHYI-2-PENTANONEO624HN

02-HEXANONE O624HN

OTETRACHLOROETHENE O624HN

$01,1,2,2-T E T R A C H L O R O E O 624 \mathrm{HN}$

OTOLUENE

$0624 \mathrm{HN}$

OCHLOROBENZENE . O624HN

OETHYIBENZENE O624HN

OSTYRENE $0624 \mathrm{HN}$

OXYLENE (TOTAL)

2075 . 000NITROBENZENE-D5

O $24 \mathrm{HN}$ 
J320900402900406WA9003L865 006 2FBP 1X 06010320900402900406 WA9003L865 006 PTERP 1X 06010320900402900406 HA9003L865 006 PHEND51X $06010320900402900406 \mathrm{FA} 90032865006$ 2FP $1 X$ $06010320900402900406 \mathrm{FA9003L865} 006246 \mathrm{TBP} 1 \mathrm{X}$ 06010320900402900406 WA9003L865 006 PHENOL1XLT 06010320900402900406 HA9003L865 006 B2CEE 1XLT $06010320900402900406 \mathrm{WA} 9003 \mathrm{~L} 865006$ 2CLP 1XLT $06010320900402900406 \mathrm{WA} 9003 \mathrm{~L} 865006$ 13DCLB1XLT $06010320900402900406 \mathrm{HA} 9003 \mathrm{~L} 865006$ 14DCLB1XLT 06010320900402900406 WA9003L865 006 BZALC 1XLT $06010320900402900406 \mathrm{WA} 9003 L 865006$ 12DCLB1XLT $06010320900402900406 \mathrm{WA} 90032865006$ 2MP 1XLT 06010320900402900406 WA9003L865 006 B2CIPE1XLT 06010320900402900406 WA9003L865 006 4MP 1XLT $06010320900402900406 \mathrm{WA} 9003 L 865006$ NDNPA 1XLT 06010320900402900406 WA9003L865 006 CL6ET 1XLT $06010320900402900406 \mathrm{WA} 90031865006 \mathrm{NB}$. 1XLT 06010320900402900406 WA9003L865 006 ISOPHRIXLT 06010320900402900406 WA9003L865 006 2NP 1XLT 06010320900402900406 WA9003L865 006 24DMPN1XLT 06010320900402900406 FA9003L865 006 BENZOA1XLT $06010320900402900406 \mathrm{HA} 90032865006$ B2CEXM1XLT $06010320900402900406 \mathrm{WA} 90032865$ 006 24DCLP1XIT 06010320900402900406 WA9003L865 006 124TCB1XLT $06010320900402900406 \mathrm{WA} 9003 \mathrm{~L} 865006 \mathrm{NAP}$ 1XLT $0601.0320900402900406 \mathrm{WA} 9003 L 865006$ 4CANIIIXLT $06010320900402900406 \mathrm{HA} 9003 L 865006$ HCBD 1XLT 06010320900402900406 WA9003L865 006 4CL3C 1XLT 06010320900402900406 WA9003L865 006 2MENAP1XLT 06010320900402900406 WA9003L865 006 CL6CP 1XLT $06010320900402900406 \mathrm{WA} 9003 \mathrm{~L} 865006$ 246TCP1XIT 06010320900402900406 WA9003L865 006 245TCP1XLT $06010320900402900406 \mathrm{WA} 9003 L 865006$ 2CNAP 1XLT 06010320900402900406 WA9003L865 006 2NANIIIXIT $06010320900402900406 \mathrm{WA} 9003 L 865006$ DMP 1XLT 06010320900402900406 WA9003L865 006 ANAPYIIXLT 06010320900402900406 HA9003L865 006 26DNT 1XLT 06010320900402900406 FA9003L865 006 3NANILIXLT $06010320900402900406 \mathrm{FA} 90032865006$ ANAPNE1XIT 06010320900402900406 HA9003L865 006 24DNP 1XLT 06010320900402900406 WA9003L865 006 4NP 1XLT 06010320900402900406 HA9003L865 006 DIBZEU1XLT 06010320900402900406 HA9003L865 006 24DNT IXLT $06010320900402900406 \mathrm{WA} 9003 L 865006$ DEP 1XLT 06010320900402900406 WA9003L865 006 4CLPREIXLT 06010320900402900406 HA9003L865 006 FLRENE1XLT 06010320900402900406 HA9003L865 006 4NANILIXLT
420UGKG

2100 UGKG

420UGKG

42 OUGKG

420UGKG

42 OUGKG

42 OUGKG

420 UGKG

420 UGKG

420 UGKG

42 OUGKG

420UGKG

2100UGKG

420UGKG

2100UGKG

420 UGKG

420UGKG

42 OUGKG

2100 UGKG

420UGKG

2100 UGKG

2100UGKG

420UGKG

420 UGKG

420UGKG

42 OUGKG

420 UGKG

2100 UGKG
1.0000MSCDT

1.0000MSCDT

$1.0000 M S C D T$

$1.0000 \mathrm{MSCDT}$

1.0000MSCDT

$1.0000 M S C D T$

$1.0000 \mathrm{MSCDT}$

1.0000MSCDT

$1.0000 \mathrm{MSCDT}$

1.0000MSCDT

1.0000MSCDT

1.0000MSCDT

1.0000MSCDT

$1.0000 \mathrm{MSCDT}$

1.0000MSCDT

1.0000MSCDT

1.0000MSCDT

1.0000MSCDT

1.0000MSCDT

1.0000MSCDT

1.0000MSCDT

$1.0000 \mathrm{MSCDT}$

1.0000MSCDT

1. $0000 \mathrm{MSCDT}$

$1.0000 \mathrm{MSCDI}$

1.0000MSCDT

1. 0000MSCDT

$1.0000 \mathrm{MSCDT}$

1.0000MSCDT

1. $0000 \mathrm{MSCDT}$

1.0000MSCDT

$1.0000 \mathrm{MSCDT}$

1. 0000MSCDT

1.0000MSCDT

1. 0000MSCDT

1.0000MSCDT

1.0000MSCDT

1.0000MSCDT

$1.0000 M S C D T$

1.0000MSCDT

1. $0000 \mathrm{MSCDT}$

1.0000MSCDT

1.0000MSCDI

1.0000MSCDT

1.0000MSCDT

1. 0000MSCDT

1.0000MSCDT

1.0000MSCDT
075 ,0002-EIUOROBIPHENYI

2075. 000P-TERPHENYL-D14

4150 . 000RHENOI-D5

625HN $0625 \mathrm{HN}$ $0625 \mathrm{HN}$ 150.0002-FLUOROPHENOI 0625HN $4150.0002,4,6$-TRIBROMORHENOLO $625 \mathrm{HN}$ OPHENOT OBIS (2-CHLOROETHYL) ETO625HN 02-CHLOROPHENOL 0625HN 01,3-DICHLOROBENZENE O625HN 01, 4-DICHLOROBENZENE 0625HN OBENZYL ALCOHOL $0625 \mathrm{HN}$ 01,2-DICHLOROBENZENE 0625HN 02-METHYIPHENOL O625HN OBIS (2-CHLOROISOPROPYO625HN 04-METHYLPHENOI 0625HN ON-NITROSO-DI-N-PROPYO625HN OHEXACHLOROETHANE O625HN ONITROBENZENE $0625 \mathrm{HN}$ OISORHORONE $0625 \mathrm{HN}$ 02-NITROPHENOI O625HN 02,4-DIMETHYLPHENOL O625HN OBENZOIC ACID 0625HN OBIS (2-CHLOROETHOXY) MO625HN 02, 4-DICHLOROPHENOL O625HN $01,2,4-T R I C H L O R O B E N Z E 0625 \mathrm{HN}$ ONAPHTHALENE $0625 \mathrm{HN}$ 04-CHLOROANILINE O625HN OHEXACHLOROBUTADIENE 0625HN 04-CHLORO-3-METHYLPHEO 625HN 02-METHYLNAPHTHALENE 0625HN OHEXACHLOROCYCLOPENTAO 625HN $02,4,6-T R I C H L O R O P H E N O O 625 \mathrm{HN}$ $02,4,5$-TRICHLOROPHENOO625HN 02-CHLORONAPHTHALENE O625HN 02-NITROANILINE O625HN ODIMETHYLPHTHALATE O625HN OACENAPHTHYLENE O625HN 02;6-DINITROTOLUENE 0625HN 03-NITROANIIINE 0625HN OACENAPHTHENE 0625HN 02, 4-DINITROPHENOL O625HN 04-NITROPHENOI O625HN ODIBENZOEURAN O625HN 02, 4-DINITROTOLUENE . 0625HN ODIETHYLPHTHALATE 0625HN 04-CHLORORHENYL-PHENYO $625 \mathrm{HN}$ OFLUORENE O6P5HN 04-NITROANIIINE 0625HN 


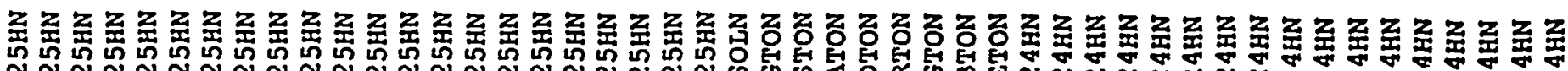

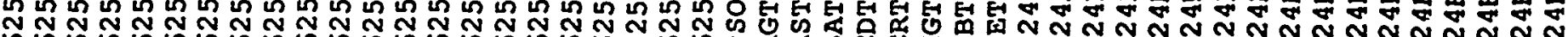

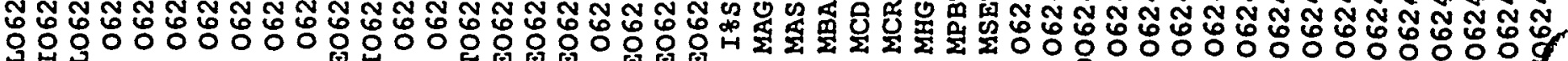
罢

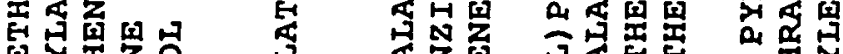

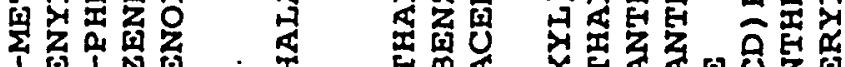

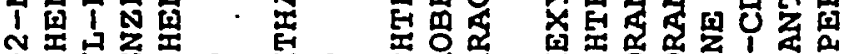

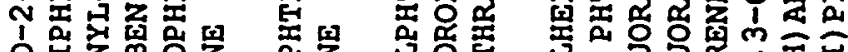

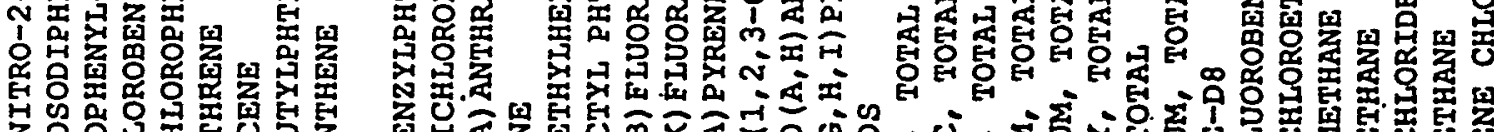

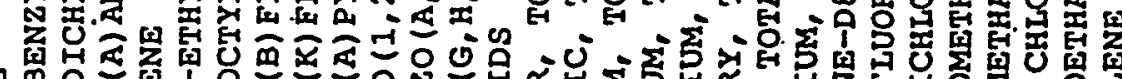

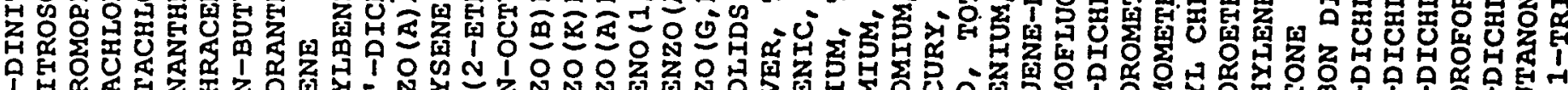

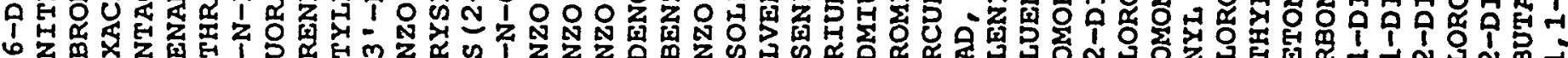

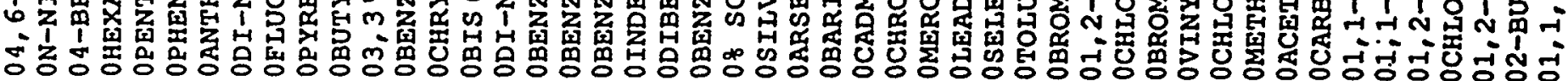
응요용

$m$ m

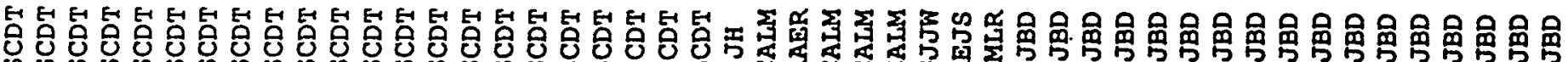

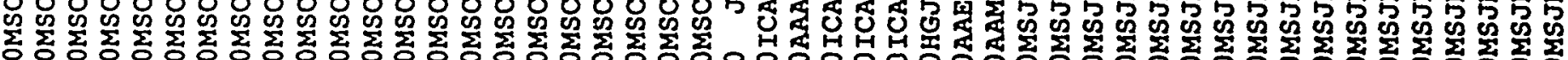
立

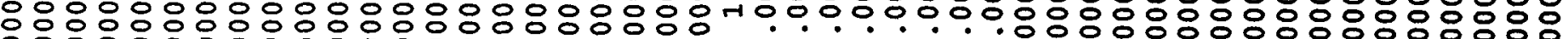

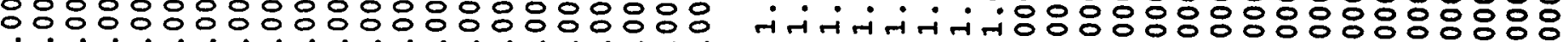

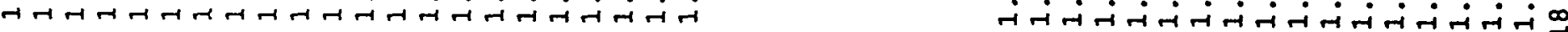

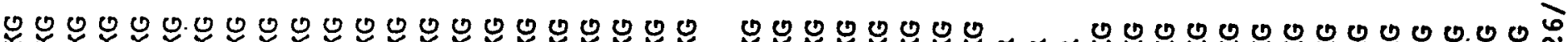

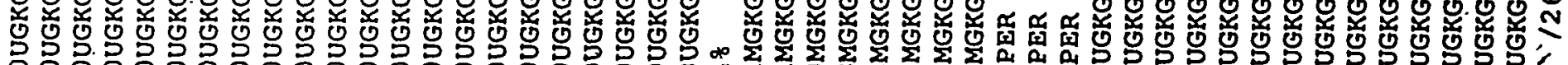

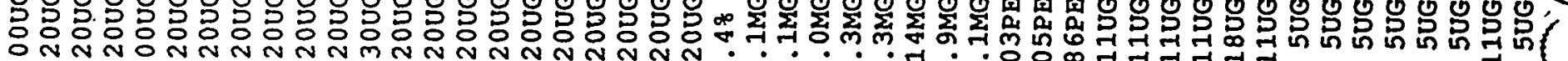

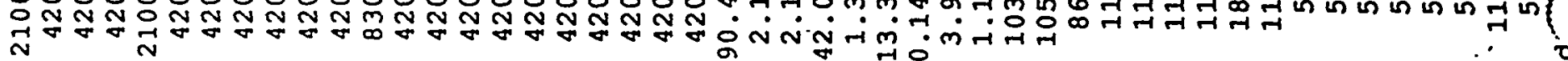

HEG G

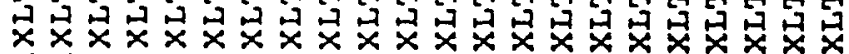

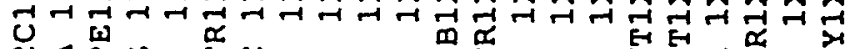

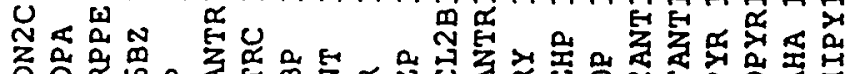

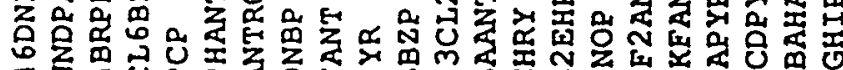

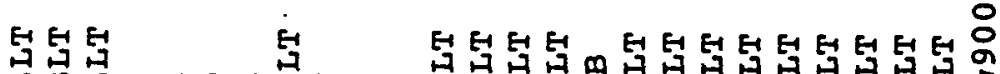

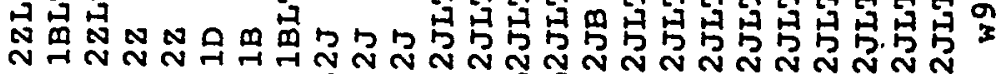

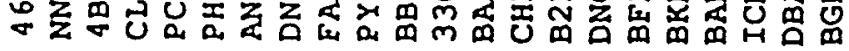

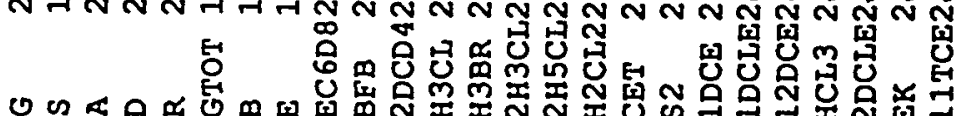

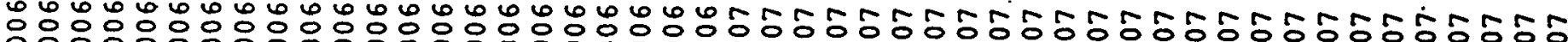

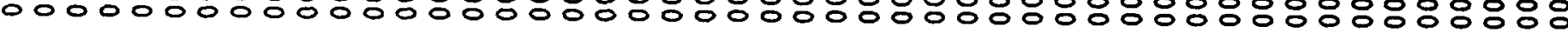

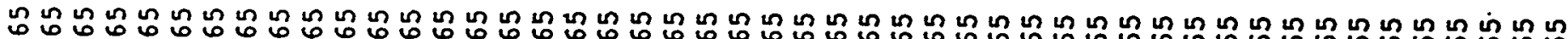
क 牙承 m

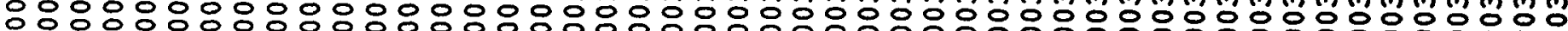

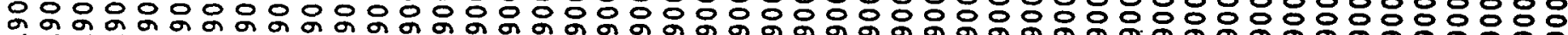

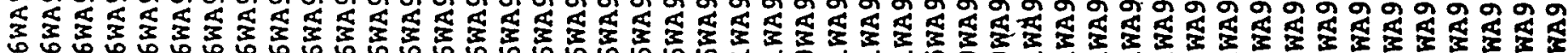

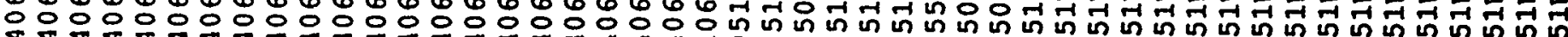
$00000500050000000 \%$

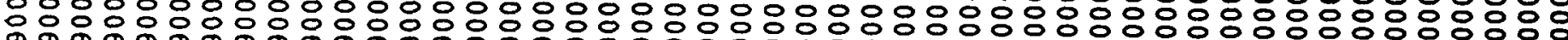

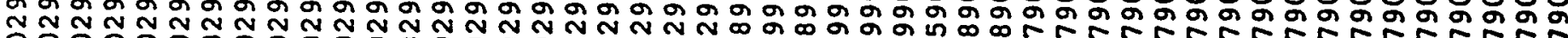

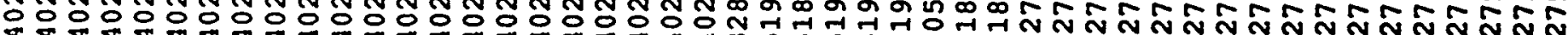
가규

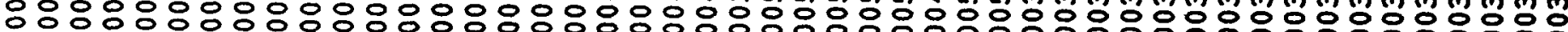

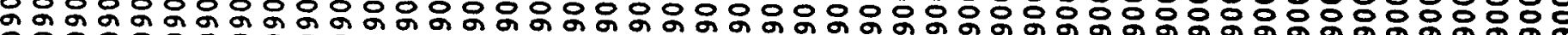

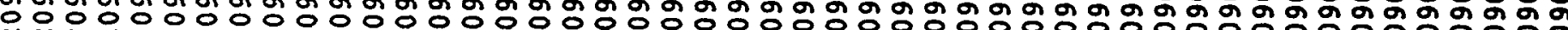
N

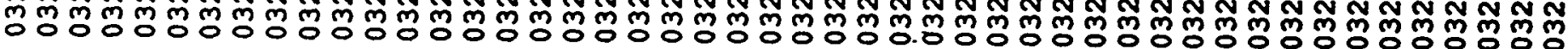

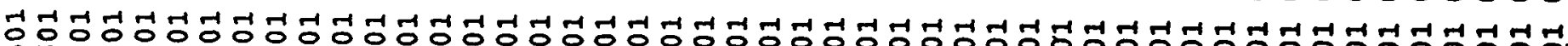

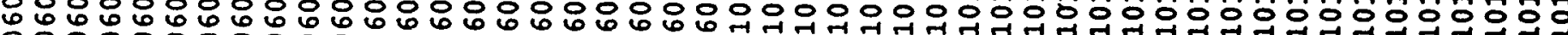

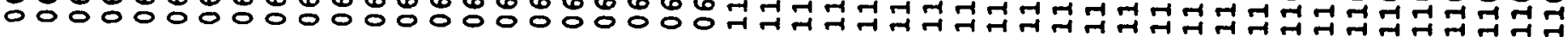

कृ

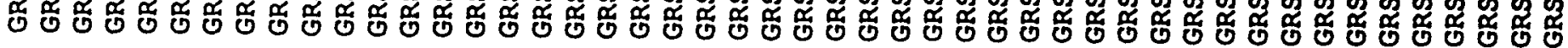


GRS 11L U320900327900051WA9003L865 007 CCL4 2JLT GRS 11010320900327900051 WA9003L865 007 VINYLA2JLT GRS 11010320900327900051 WA9003L865 007 BRDCLM2JLT GRS 11010320900327900051 WA9003L865 007 12DCLP2JLT GRS 11010320900327900051 WA9003L865 007 13DCPC2JLT GRS 11010320900327900051 WA9003L865 007 TRCLE 2JLT GRS 11010320900327900051 WA9003L865 007 DBRCLM2JLT GRS 11010320900327900051 WA9003L865 007 112TCE2JLT GRS 11010320900327900051 WA9003L865 007 C6H6 2JLT GRS 11010320900327900051 WA 9003 L865 007 T13DCP2JLT GRS 11010320900327900051 WA9003L865 007 CHBR3 2JLT GRS 11010320900327900051 WA9003L865 007 4ME2PE2JLT GRS 11010320900327900051 WA9003L865 007 2HXONE2JLT GRS 11010320900327900051 WA9003L865 007 TCLEE 2JLT GRS 11010320900327900051 WA9003L865 007 TCLEA 2JLT GRS 11010320900327900051 WA9003L865 007 MEC6H52JLT GRS 11010320900327900051 WA9003L865 007 CLC6H52JLT GRS 11010320900327900051 WA9003L865 007 ETC6H52JLT GRS $11010320900327900051 \mathrm{WA} 9003 L 865007$ STYR 2JLT GRS 11010320900327900051 WA9003L865 007 XYLEN 2JLT GRS 110103209004029.00406 WA9003L865 007 NBD5 1X 11010320900402900406 WA9003L865 007 2FBP $1 \mathrm{X}$ 11010320900402900406 WA9003L865 007 PTERP 1X $11010320900402900406 \mathrm{WA} 9003 \mathrm{~L} 865007$ PHEND51X 11010320900402900406 WA9003L865 $007 \quad$ FP $1 \mathrm{X}$ 11010320900402900406 WA9003L865 $007246 \mathrm{TBP} 1 \mathrm{X}$ 1101. 0320900402900406WA9003L865 007 PHENOL1XLT $11010320900402900406 \mathrm{WA} 9003 \mathrm{~L} 865007$ B2CEE 1XLT 11010320900402900406 WA9003L865 007 2CLP 1XLT $11010320900402900406 \mathrm{WA} 9003 \mathrm{~L} 865007$ 13DCLB1XLT $11010320900402900406 \mathrm{WA} 9003 \mathrm{~L} 865007$ 14DCLB1XLT 11010320900402900406 WA9003L865 007 BZALC 1XLT $11010320900402900406 \mathrm{WA} 9003 \mathrm{~L} 865007$ 12DCLB1XL' 11010320900402900406 WA9003L865 007 2MP 1XLT 11010320900402900406 WA9003L865 007 B2CIPE1XLT 11010320900402900406 WA9003L865 007 4MP 1XLT 11010320900402900406 WA9003L865 007 NDNPA 1XLT 11010320900402900406 WA9003L865 007 CL6ET 1XLT $11010320900402900406 \mathrm{WA} 9003 \mathrm{~L} 865007 \mathrm{NB}$ 1XLT $11010320900402900406 \mathrm{WA} 9003 \mathrm{~L} 865007$ ISOPHRIXLT $11010320900402900406 \mathrm{WA} 9003 \mathrm{~L} 865007$ 2NP 1XLT 11010320900402900406 WA9003L865 007 24DMPN1XLT $11010320900402900406 \mathrm{WA} 9003 \mathrm{~L} 865007$ BENZOA1XLT $11010320900402900406 \mathrm{WA} 9003 \mathrm{~L} 865007$ B2CEXM1XLT $11010320900402900406 \mathrm{FA9003L865007}$ 24DCLP1XLT $11010320900402900406 \mathrm{WA} 9003 \mathrm{~L} 865007 \quad 124 \mathrm{TCB} 1 \mathrm{XLT}$ $11010320900402900406 \mathrm{FA} 9003 \mathrm{~L} 865007$ NAP 1XLT 11010320900402900406 WA9003L865 007 4CANIL1XLT
JUGKG

11UGKG

5UGKG

5UGKG

5UGKG

SUGKG

5UGKG

SUGKG

5UGKG

SUGKG

SUGKG

11UGKG

11UGKG

5UGKG

5UGKG

SUGKG

SUGKG

SUGKG

5UGKG

SUGKG

76 PER

76 PER

71PER

82PER

60PER

89PER

350UGKG

350UGKG

350UGKG

350UGKG

350UGKG

350UGKG

350UGKG

350UGKG

350UGKG

350UGKG

350UGKG

350UGKG

350UGKG

350UGKG

350UGKG

350UGKG

1800UGKG

350UGKG

350UGKG

350UGKG

350UGKG 350UGKG
$1.0000 M S J B D$

$1.0000 \mathrm{MSJBD}$

$1.0000 \mathrm{MSJBD}$

$1.0000 M S J B D$

$1.0000 \mathrm{MSJBD}$

1.0000MSJBD

$1.0000 M S J B D$

$1.0000 M S J B D$

$1.0000 \mathrm{MSJBD}$

$1.0000 \mathrm{MSJBD}$

$1.0000 \mathrm{MSJBD}$

$1.0000 \mathrm{MSJBD}$

$1.0000 \mathrm{MSJBD}$

$1.0000 \mathrm{MSJBD}$

$1.0000 M S J B D$

$1.0000 \mathrm{MSJBD}$

1.0000MSJBD

$1.0000 \mathrm{MSJBD}$

$1.0000 \mathrm{MSJBD}$

$1.0000 \mathrm{MSJBD}$

$1.0000 \mathrm{MSCDT}$

$1.0000 \mathrm{MSCDT}$

$1.0000 \mathrm{MSCDT}$

$1.0000 \mathrm{MSCDT}$

1.0000MSCDT

$1.0000 M S C D T$

$1.0000 \mathrm{MSCDT}$

$1.0000 \mathrm{MSCDT}$

$1.0000 \mathrm{MSCDT}$

$1.0000 \mathrm{MSCDT}$

$1.0000 \mathrm{MSCDT}$

$1.0000 M S C D T$

$1.0000 \mathrm{MSCDT}$

$1.0000 \mathrm{MSCDT}$

$1.0000 \mathrm{MSCDT}$

$1.0000 \mathrm{MSCDT}$

$1.0000 \mathrm{MSCDT}$

$1.0000 \mathrm{MSCDT}$

$1.0000 \mathrm{MSCDT}$

$1.0000 \mathrm{MSCDT}$

$1.0000 \mathrm{MSCDT}$

$1.0000 \mathrm{MSCDT}$

$1.0000 \mathrm{MSCDT}$

$1.0000 \mathrm{MSCDT}$

$1.0000 \mathrm{MSCDT}$

$1.0000 M S C D T$

$1.0000 \mathrm{MSCDT}$ $1.0000 \mathrm{MSCDT}$
OCARBON TETRACHLORIDEO624HN OVINYI ACETATE O624HN OBROMODICHLOROMETHANEO624HN 01,2-DICHLOROPROPANE 0624HN OCIS-1, 3-DICHLOROPROPO624HN OTRICHLOROETHENE O624HN ODIBROMOCHLOROMETHANEO624HN 01, 1,2-TRICHLOROETHANO62 4HN OBENZENE $0624 \mathrm{HN}$ OTRANS-1, 3-DICHLOROPRO624HN O.BROMOFORM 0624HN 0 4-METHYL-2-PENTANONEO $624 \mathrm{HN}$ 02-HEXANONE 0624HN OTETRACHLOROETHENE 0624HN $01,1,2,2$-TETRACHLOROEO $624 \mathrm{HN}$ OTOLUENE OCHOR OETHYLBENZENE $\quad 0624 \mathrm{HN}$ OSTYRENE

OXYLENE (TOTAL)

1755.00 ONITROBENZENE-D5

$0624 \mathrm{HN}$ $0624 \mathrm{HN}$ 3510.001PHENOL-D5 0625 3510.0012-FLUOROPHENOL O625HN $3510.0012,4,6$-TRIBROMOPHENOLO625HN ORHENOI $0625 \mathrm{HN}$ OBIS (2-CHLOROETHYL) ETO625HN 02-CHLOROPHENOL 0625HN 01,3-DICHLOROBENZENE 0625HN $01,4-D I C H L O R O B E N 2 E N E$ 0625HN OBENZYL ALCOHOL O625HN 01,2-DICHLOROBENZENE O625HN 02-METHYLPHENOL 0625HN OBIS (2-CHLOROISOPROPYO625HN 04-METHYLPHENOL O625HN ON-NITROSO-DI-N-PROPYO $625 \mathrm{HN}$ OHEXACHLOROETHANE O625HN ONITROBENZENE O625HN OISOPHORONE O625HN 02-NITROPHENOL $062.5 \mathrm{HN}$ 02, 4-DIMETHYLPHENOL O625HN OBENZOIC ACID O625HN OBIS (2-CHLOROETHOXY) MO625 HN 02, 4-DICHLOROPHENOL 0625HN $01,2,4-T R I C H L O R O B E N 2 E 0625 \mathrm{HN}$ ONAPHTHALENE $0625 \mathrm{HN}$ 04-CHLOROANILIINE O625HN 
GRS 11010320900402900406 WA9003L865 007 HCBD 1XLT GRS $11010320900402900406 \mathrm{HA} 9003 \mathrm{~L} 865007$ 4CL3C 1XLT GRS 11010320900402900406 WA9003L865 007 2MENAPIXLT GRS 11010320900402900406 WA9003L865 007 CL6CP 1XLT GRS $11010320900402900406 \mathrm{WA} 9003 \mathrm{~L} 865007246 \mathrm{TCP} 1 \times \mathrm{LT}$ GRS $11010320900402900406 \mathrm{WA} 9003 \mathrm{~L} 865007$ 245TCP1XLT GRS 11010320900402900406 WA9003L865 007 2CNAP 1XLT GRS $11010320900402900406 \mathrm{WA} 9003 \mathrm{~L} 865007$ 2NANILIXLT GRS 11010320900402900406 WA9003L865 007 DMP 1XLT GRS 11010320900402900406 WA9003L865 007 ANAPYL1XLT GRS 11010320900402900406 WA9003L865 007 26DNT 1XLT GRS 11010320900402900406 WA9003L865 007 3NANILIXLT GRS 11010320900402900406 WA9003L865 007 ANAPNE1XLT GRS 11010320900402900406 WA9003L865 007 24DNP 1XLT GRS 11010320900402900406 WA9003L865 007 4NP 1XLT GRS 11010320900402900406 WA9003L865 007 DIBZFUIXLT GRS 11010320900402900406 WA9003L865 007 24DNT 1XLT GRS 11010320900402900406 WA9003L865 007 DEP 1XLT GRS $11010320900402900406 \mathrm{WA} 9003 L 865007$ 4CLPPEIXLT GRS 11010320900402900406 WA9003L865 007 FLRENE1XLT GRS 11010320900402900406 WA9003L865 007 4NANILIXLT GRS 11010320900402900406 WA9003L865 007 46DN2C1XLT GRS 11010320900402900406 WA9003L865 007 NNDPA 1XLT GRS $11010320900402900406 \mathrm{WA} 9003 L 865007$ 4BRPPEIXLT $\sim$ GRS $11010320900402900406 \mathrm{WA} 9003 \mathrm{~L} 865007$ CL6BZ 1XLT I GRS 11010320900402900406 WA9003L865 007 PCP 1XLT GRS $11010320900402900406 \mathrm{WA} 9003 \mathrm{~L} 865007$ PHANTRIXLT GRS 11010320900402900406 WA9003L865 007 ANTRC 1XLT GRS 11010320900402900406 WA9003L865 007 DNBP 1XLT GRS $11010320900402900406 \mathrm{WA} 90032865007$ FANT 1XLT GRS $11010320900402900406 \mathrm{WA} 9003 \mathrm{~L} 865007$ PYR 1XLT. GRS $11010320900402900406 \hbar$ Aे $9003 \mathrm{~L} 865007 \mathrm{BBZP}$ 1XLT GRS $11010320900402900406 \mathrm{WA} 9003 \mathrm{~L} 865007$ 33CL2B1XLT GRS $11010320900402900406 \mathrm{FA} 9003 \mathrm{~L} 865007$ BAANTR1XLT GRS 11010320900402900406 WA9003L865 007 CHRY 1XLT GRS 11010320900402900406 WA9003L865 007 B2EHP 1XLT GRS 11010320900402900406 WA9003L865 007 DNOP 1XLT GRS $11010320900402900406 \mathrm{WA} 90032865007$ BF2ANT1XLT GRS 11010320900402900406 WA9003L865 007 BKFANT1XLT GRS 11010320900402900406 सA9003L865 007 BAPYR 1XLT GRS $11010320900402900406 \mathrm{WA} 9003 \mathrm{~L} 865007$ ICDPYR1XLT GRS $11010320900402900406 \mathrm{WA} 9003$ L865 007 DBAHA 1XLT GRS $11010320900402900406 \mathrm{WA} 9003 L 865007$ BGHIPY1XLT GRS 01010320900328900051 พA9003L865 008 GRS $01010320900519900451 \mathrm{WA} 9003 \mathrm{~L} 865008$ AG GRS 01010320900518900450 WA9003L865 008 AS GRS 01010320900519900451 WA9003L865 008 BA GRS 01010320900519900451 WA9003L865 008 CD
350UGKG

350UGKG

350UGKG

350UGKG

350UGKG

1800 UGKG

350 UGKG

1800 UGKG

350UGKG

35 OUGKG

350UGKG

1800 UGKG

350UGKG

1800 UGKG

1800 UGKG

350UGKG

350UGKG

350UGKG

350UGKG

350UGKG

1800 UGKG

1800 UGKG

350UGKG

350UGKG

350UGKG

1800 UGKG

350UGKG

350UGKG

350UGKG

35 OUGKG

350UGKG

350UGKG

70 OUGKG

350UGKG

35 OUGKG

350UGKG

350UGKG

350UGKG

35 OUGKG

350 UGKG

350UGKG

350UGKG

350UGKG

93.08

2ZLT

1BLT

22LT

1. 9 MGKG

1. 8MGKG

38. IMGKG

1. 8MGKG
1.0000MSCDT

$1.0000 M S C D T$

$1.0000 \mathrm{MSCDT}$

$1.0000 M S C D T$

$1.0000 \mathrm{MSCDT}$

$1.0000 \mathrm{MSCDT}$

1. $0000 \mathrm{MSCDT}$

$1.0000 \mathrm{MSCDT}$

$1.0000 \mathrm{MSCDT}$

$1.0000 \mathrm{MSCDT}$

1.0000MSCDT

$1.0000 \mathrm{MSCDT}$

$1.0000 \mathrm{MSCDT}$

1.0000MSCDT

$1.0000 \mathrm{MSCDT}$

1. $0000 \mathrm{MSCDT}$

$1.0000 \mathrm{MSCDT}$

$1.0000 \mathrm{MSCDT}$

$1.0000 \mathrm{MSCDT}$

$1.0000 \mathrm{MSCDT}$

$1.0000 \mathrm{MSCDT}$

$1.0000 \mathrm{MSCDT}$

$1.0000 \mathrm{MSCDT}$

$1.0000 \mathrm{MSCDT}$

$1.0000 \mathrm{MSCDT}$

1.0000MSCDT

$1.0000 \mathrm{MSCDT}$

$1.0000 \mathrm{MSCDT}$

$1.0000 \mathrm{MSCDT}$

$1.0000 \mathrm{MSCDT}$

$1.0000 \mathrm{MSCDT}$

$1.0000 M S C D T$

$1.0000 \mathrm{MSCDT}$

$1.0000 \mathrm{MSCDT}$

$1.0000 \mathrm{MSCDT}$

1.0000MSCDT

$1.0000 \mathrm{MSCDT}$

$1.0000 \mathrm{MSCDT}$

1.0000MSCDT

$1.0000 M S C D T$

$1.0000 \mathrm{MSCDT}$

$1.0000 \mathrm{MSCDT}$

$1.0000 \mathrm{MSCDT}$

$10 \mathrm{JH}$

1. OOICAIM

1.00AAAER

1.00 ICALM

1. OOICAIM
OHEXACHLOROBUTADIENE O625HN 0 4-CHLORO-3-METHYLPHEO 625HN 02-METHYLNAPHTHALENE 0625HN OHEXACHLOROCYCLOPENTAO625HN 02, 4, 6-TRICHLOROPHENOO625HN 02, 4, 5-TRICHLOROPHENOO625HN 02-CHLORONAPHTHALENE 0625HN 02-NITROANILINE 0625HN ODIMETHYLPHTHALATE O625HN OACENAP HTHYLENE O625HN 02,6-DINITROTOLUENE 0625HN 03-NITROANILINE 0625HN OACENAP HTHENE O625HN 02,4-DINITROPHENOL O625HN 04-NITROPHENOL O625HN ODIBENZOFURAN $0625 \mathrm{HN}$ 02,4-DINITROTOLUENE O625HN ODIETHYLPHTHALATE O625HN 04-CHLOROPHENYL-PHENY0625HN OELUORENE. 04-NITROANILINE $\quad 0625 \mathrm{HN}$ 04, 6-DINITRO-2-METHYLO625HN ON-NITROSODIPHENYLAMIO $625 \mathrm{HN}$ 04-BROMOPHENYL-PHENYL0625HN OHEXACHLOROBENZENE 0625HN OPENTACHLORORHENOL O625HN OPHENANTHRENE OANTHRACENE O625HN ODI-N-BUTYLPHTHALATE $0625 \mathrm{HN}$ OFLUORANTHENE 0625HN OPYRENE $0625 \mathrm{HN}$ OBUTYLBENZYLPHTHALATEO625HN 03, $3^{\prime}$-DICHLOROBENZ IDIO625HN OBENZO (A) ANTHRACENE O625HN OCHRYSENE O625HN OBIS (2-ETHYLHEXYL) PHTO625HN ODI-N-OC'TYL PHTHALATE0625HN OBENZO (B) FLUORANTHENEO625HN OBENZO (K) ELUORANTHENEO $625 \mathrm{HN}$ OBENZO (A) PYRENE O625HN OINDENO $(1,2,3-C D)$ PYREO625HN ODIBENZO $(\mathrm{A}, \mathrm{H})$ ANTHRACEO $25 \mathrm{HN}$ OBENZO (G, H, I) PERYLENEO $625 \mathrm{HN}$ O\% SOLIDS . If SOLN OSILVER, TOTAL MAGTON OARSENIC, TOTAL MASTON OBARIUM, TOTAL MBATON OCADMIUM, TOTAL 


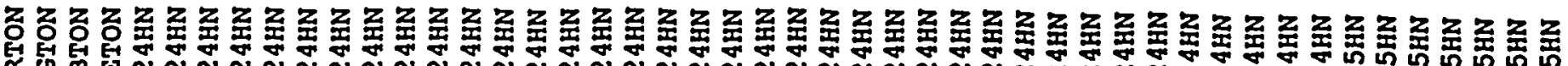
d

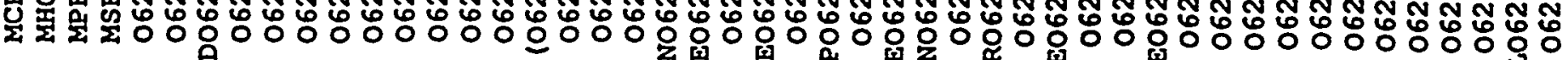

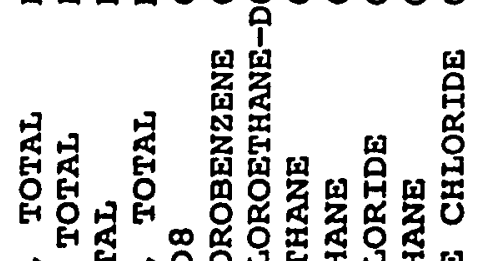

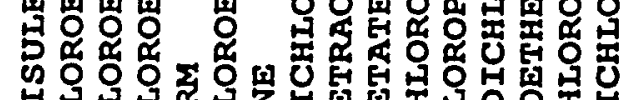

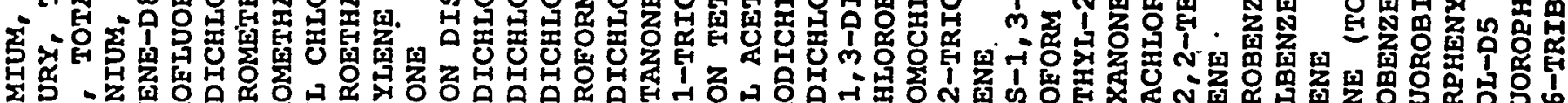

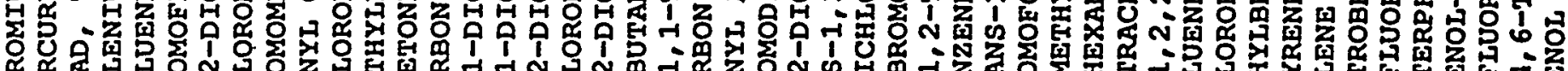

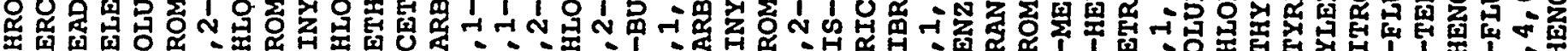

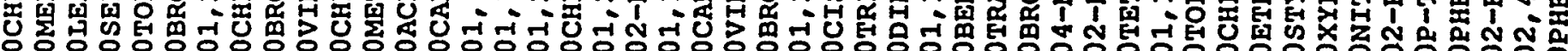
웅ㅇㅇㅇ 응ํํ

N $\mathrm{N}$ (1)

กิ ถึก

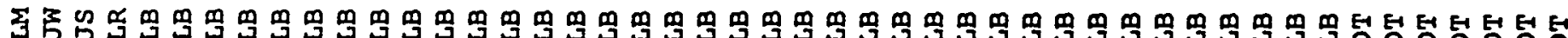

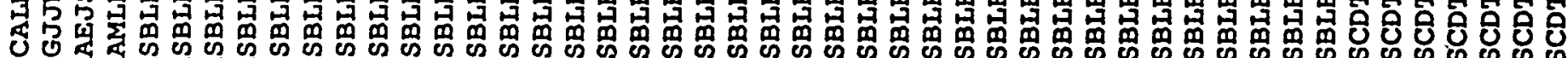

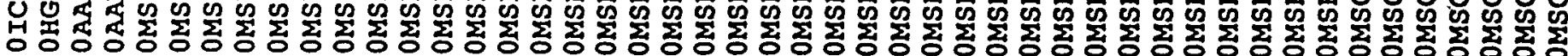

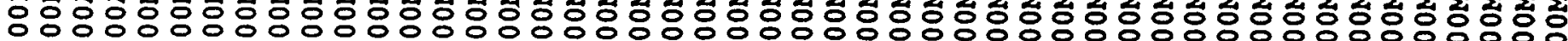

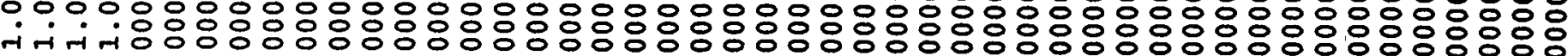

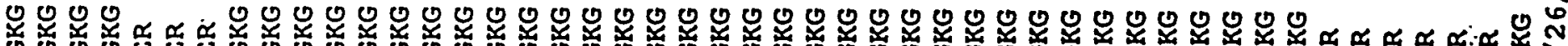

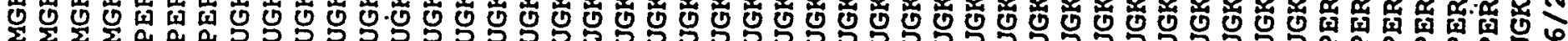

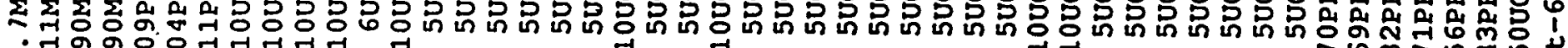
गं:

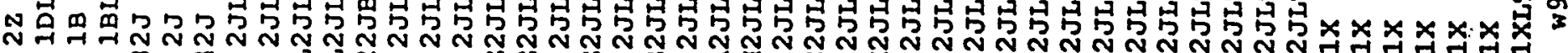

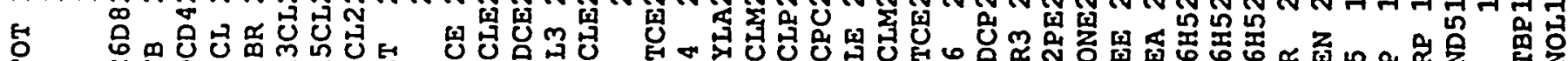

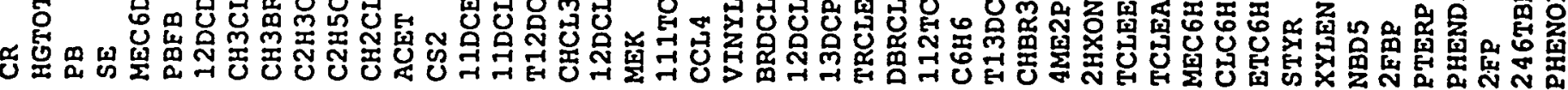
N

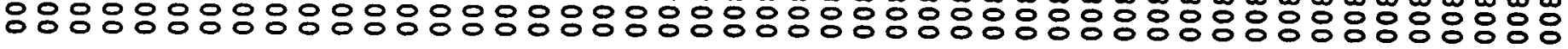

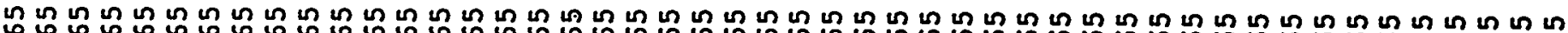
必 m

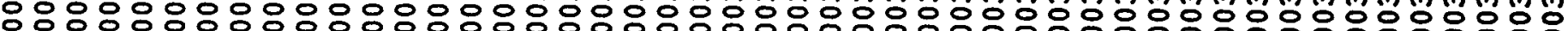

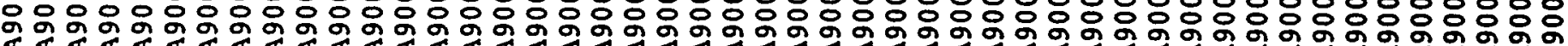

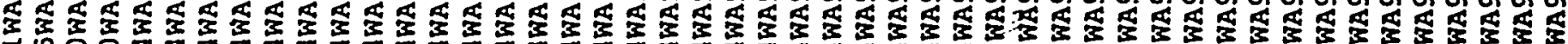

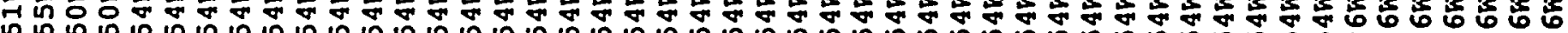

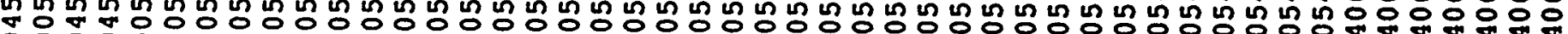

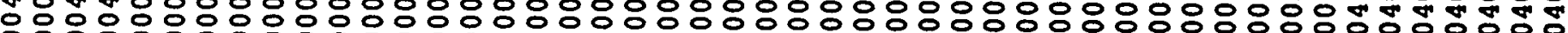

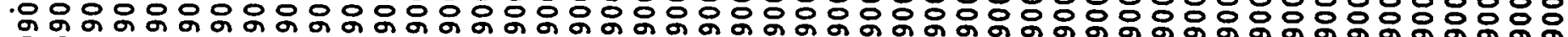

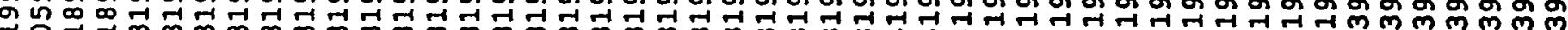

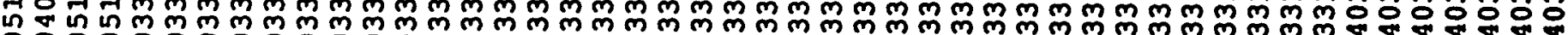

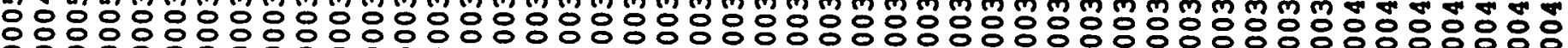

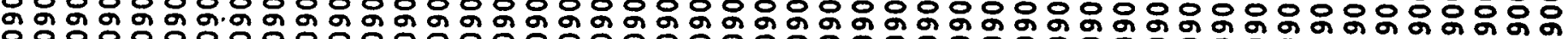
N $N \mathcal{N} N$ N $N$ N N

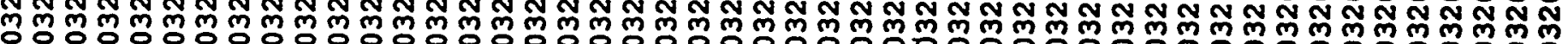

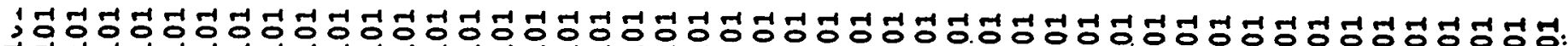

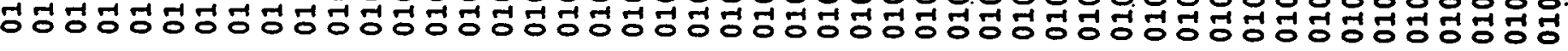

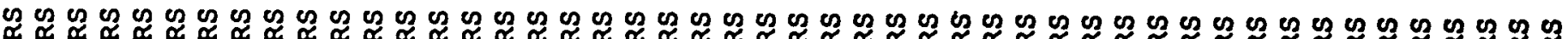

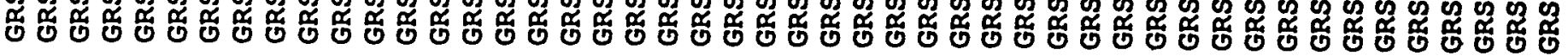




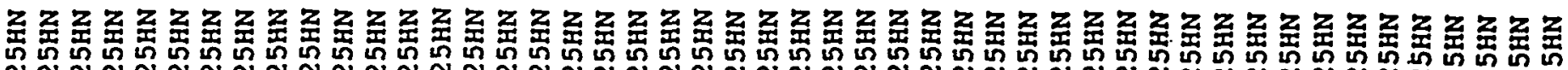
ง

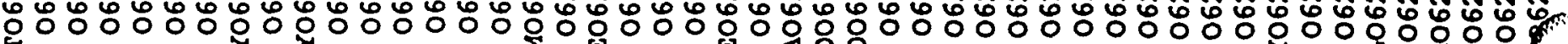

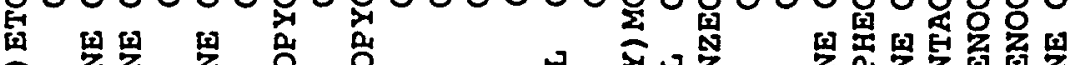

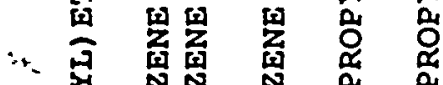

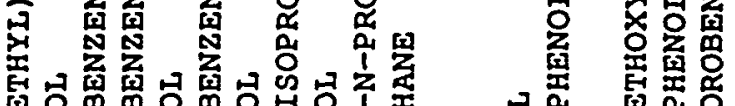

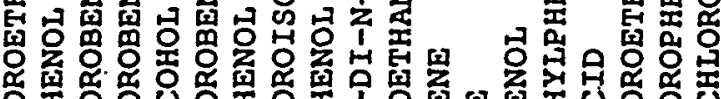

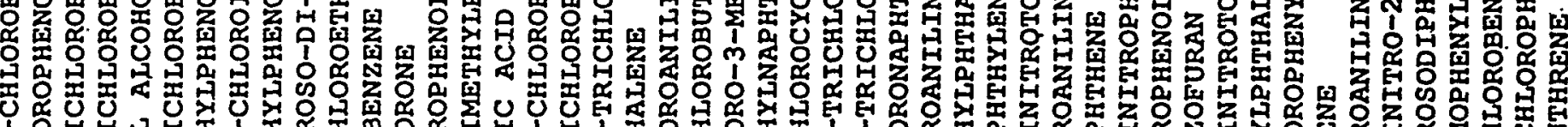
10

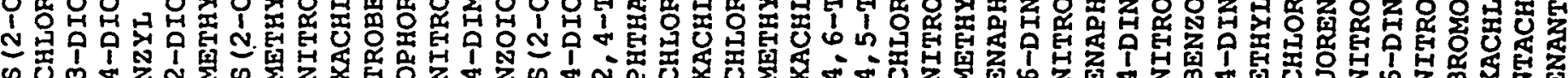

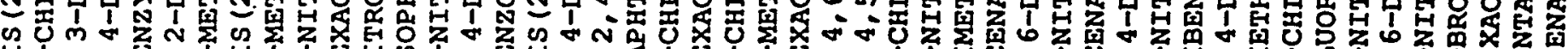

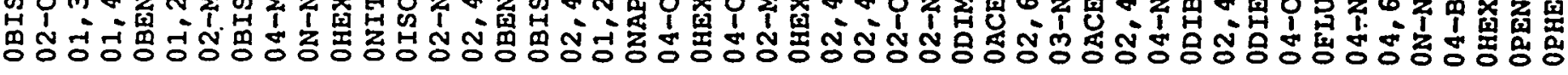

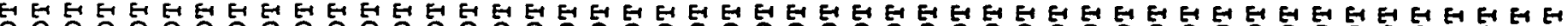

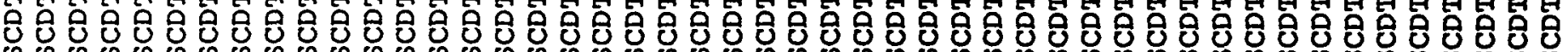

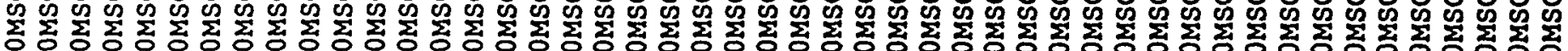

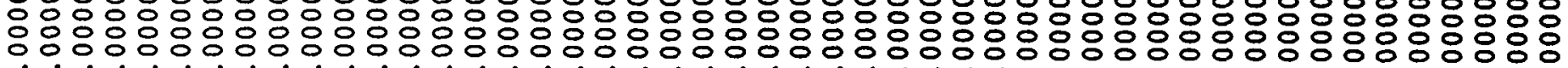
-

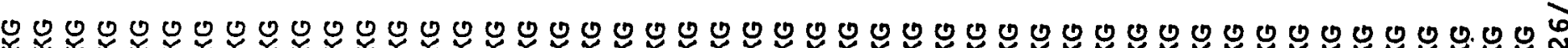

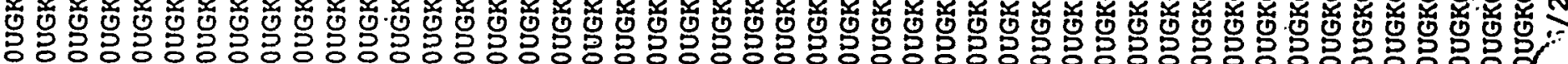
in

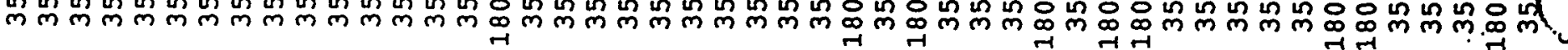

EFEG

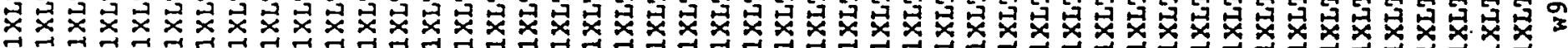

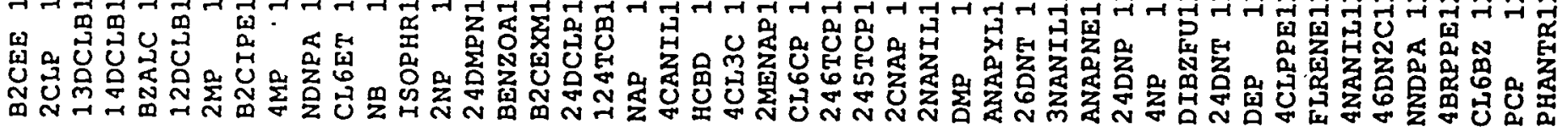
巾

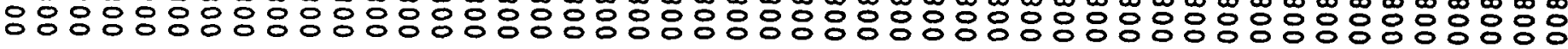
n i m

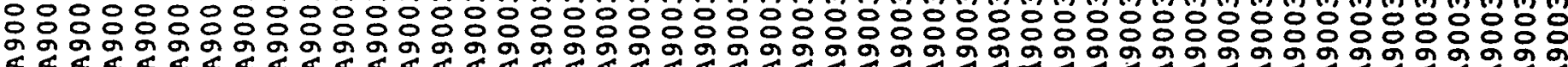

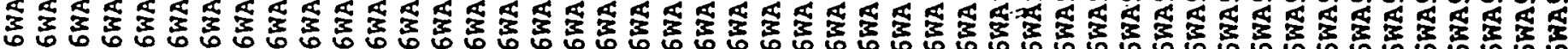
6.6006

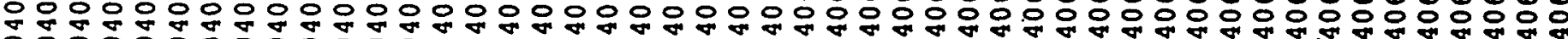

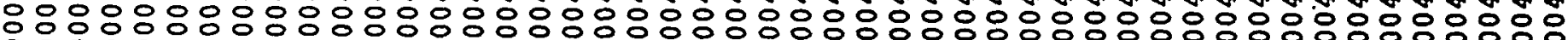

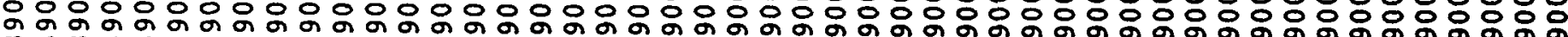

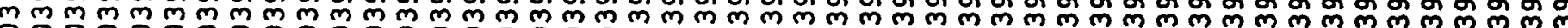

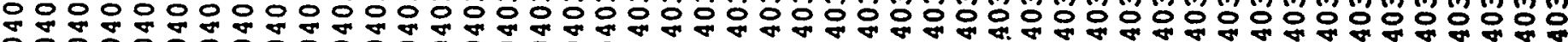

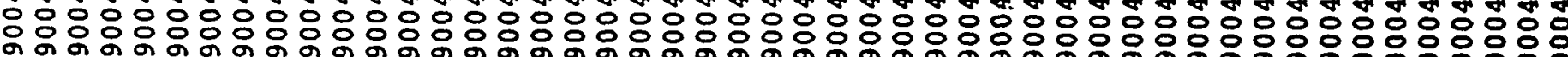

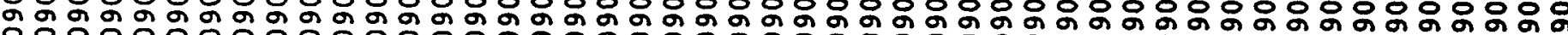

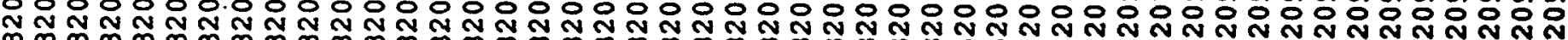

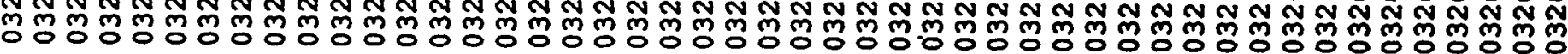

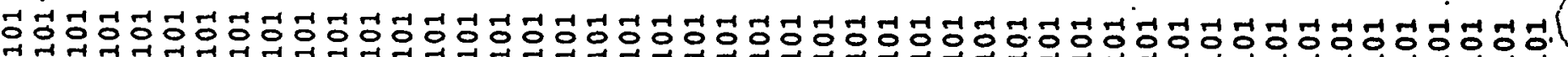

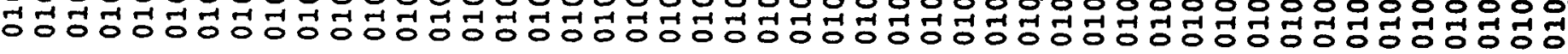

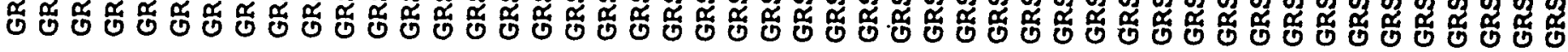


GRS $01 U 10320900403900406$ WA9003L865 008 ANTRC 1XLT GRS 01010320900403900406 HA9003L865 008 DNBP 1XLT GRS 01010320900403900406 HA9003L865 008 EANT 1XLT GRS 01010320900403900406 FA9003L865 008 PYR IXLT GRS 01010320900403900406 WA9003L865 008 BBZP IXLT GRS 01010320900403900406 WA9003L865 008 33CL2B1XLT GRS $01010320900403900406 \mathrm{WA} 003 L 865008$ BAANTR1XLT GRS 01010320900403900406 WA9003L865 008 CHRY 1XLT GRS 01010320900403900406 WA9003L865 008 B2EHP 1XLT GRS $01010320900403900406 \mathrm{WA} 003 \mathrm{~L} 865008$ DNOP 1XLT GRS $01010320900403900406 \mathrm{WA} 90031865008$ BE2ANT1XLT GRS 01010320900403900406 FA9003L865 008 BKEANT1XLT GRS 01010320900403900406 WA9003L865 008 BAPYR 1XLT GRS 01010320900403900406 HA9003L865 008 ICDPYRIXLT GRS $01010320900403900406 \mathrm{WA} 9003 \mathrm{~L} 865008$ DBAHA 1XLT GRS 01010320900403900406 WA9.003L865 008 BGHIPYIXIT GRS 12010320900328900051 HA9003L865 009

GRS 12010320900427900003 WA90031 865009 CEC EX GRS 12010320900427900003 WA9003L865 009RCEC EX GRS 12010320900427900003 WA9003L865 009 CECB EX GRS 12010320900427900003 FA9003L865 009RCECB EX GRS 12010320900426900003 HA9003L865 009 CECT EX GRS 12010320900426900003 WA9003L865 009RCECT EX 12010320900403900003 WA9003L865 009 TOC 9I

12010320900403900003 WA9003L865 009RTOC

12010320900403900003 WA9003L865 009STOC $9 \mathrm{I}$

12010320900328900054 WA9003L865 $009 \mathrm{PH} 9 \mathrm{R}$

12010320900327900051 HA9003L865 009 MEC6D82J

12010320900327900051 WA9003L865 009 PBEB $2 \mathrm{~J}$

12010320900327900051 WA9003L865 009 12DCD42J

12010320900327900051 NA9003L865 009 CH3CL 2JLT

12010320900327900051 HA9003L865 009 CH3BR 2JLT

12010320900327900051 WA9003L865 009 C2H3CL2JLT

12010320900327900051 WA9003L865 009 C2H5CL2JLT

$12010320900327900051 \mathrm{HAg} 003 \mathrm{~L} 865009$ CH2CI22JB

12010320900327900051 WA9003L865 009 ACET 2JB

12010320900327900051 WA9003L865 009 CS2 2JLT

12010320900327900051 WA9003L865 009 11DCE 2JLT

12010320900327900051 WA9003L865 009 11DCLE2JLT

12010320900327900051 HA9003L865 009 T12DCE2JLT

12010320900327900051 WA9003L865 009 CHCL3 2JLT

12010320900327900051 WA9003L865 009 12DCLE2JLT

12010320900327900051 HA9003I865 009 MEK $2 J 1 T$

12010320900327900051 WA9003L865 009 111TCE2JLT

12010320900327900051 HA9003L865 009 CCL4 2JLT

12010320900327900051 HA9003L865 009 VंINYLA2JIT

12010320900327900051 HA9003L865 009 BRDCLM2JLT
35OUGKG

350UGKG

35 OUGKG

350UGKG

350UGKG

350UGKG

350UGKG

350UGKG

$94.7 \%$

1. 8MEQ1

1. 6MEQ1

1. $4 M E Q 1$

1. 2MEQ1

1. 4MEQ1

1. 2MEQ1

$808 M G K G$

5.40MGKG

62.8PER

$6.5 P H ~ U$

104PER

113PER

91PER

1 OUGKG

IOUGKG

IOUGKG

1 OUGKG

10UGKG

1OUGKG

5UGKG

5UGKG

5UGKG

5UGKG

5UGKG

5UGKG

IOUGKG

5UGKG

5UGKG

1OUGKG

5UGKG

5UGKG
$1.0000 \mathrm{MSCDT}$ $1.0000 \mathrm{MSCDT}$

1.0000MSCDT

1.0000MSCDT

$1.0000 \mathrm{MSCDT}$

1. $0000 \mathrm{MSCDT}$

1.0000MSCDT

1.0000MSCDT

1.0000MSCDT

1.0000MSCDT

1. $0000 \mathrm{MSCDT}$

1.0000MSCDT

1.0000MSCDT

1. $0000 \mathrm{MSCDT}$

1.0000MSCDT

1.0000MSCDT $10 \mathrm{JH}$ 10NADKS IONADKS 1ONAALM IONAALM 10NADKS 1ONADKS 10DODKS 10DODKS 10DODKS 10PHJH

1. $0000 \mathrm{MSJBD}$

1:0000MSJBD

1.0000MSJBD

1. $0000 \mathrm{MSJBD}$

$1.0000 \mathrm{MSJBD}$

$1.0000 \mathrm{MSJBD}$

1. $0000 \mathrm{MSJBD}$

1.0000MSJBD

1. $0000 \mathrm{MSJBD}$

1. $0000 \mathrm{MSJBD}$

1.0000MSJBD

1. $0000 M S J B D$

1.000OMSJBD

1.000OMSJBD

1. 000 OMSJBD

1. $0000 \mathrm{MSJBD}$

1. 000 OMSJBD

1. $0000 \mathrm{MSJBD}$

1. 0000MSJBD

1. $0000 \mathrm{MSJBD}$

1. 000OMSJBD
OANTHRACENE

ODI-N-BUTYIPHTHATATE 0625HN OFLUORANTHENE O625HN OPYRENE O625HN OBUTYLBENZYLPHTHALATE0625HN $03,3^{\prime}$-DICHLOROBENZIDI0625HN OBENZO (A) ANTHRACENE O625HN OCHRYSENE $0625 \mathrm{HN}$ OBIS (2-ETHYLHEXYL) PHTO625HN ODI-N-OCTYL PHTHAIATEO625HN OBENZO (B) ELUORANTHENEO $625 \mathrm{HN}$ OBENZO (K) ELUORANTHENEO625HN OBENZO (A) PYRENE O625HN OINDENO $(1,2,3-C D)$ PYREO625HN ODIBENZO ( $A, H)$ ANTHEACEO625HN OBENZO (G, H, I) PERYIENEO625HN $0 \%$ SOLIDS IFSOLN OCATION EXCHANGE CAPAICEC N OCATION EXCHANGE CAPAICEC $R$ OEXCHANGEABLE BASES ICECBN OEXCHANGEABLE BASES ICECBR OEXCHANGEABLE ACIDS ICECTN OEXCHANGEABLE ACIDS ICECTR OTOTAL ORGANIC CARBONIOCT $N$ OTOTAL ORGANIC CARBONIOCT $R$

1000TOTAL ORGANIC CARBONIOCT S OPH

IPH N

51.500 TOLUENE-DB $0624 \mathrm{HN}$

51.500BROMOELUOROBENZENE 0624HN

51.5001,2-DICHLOROETHANE-DO624HN OCHLOROMETHANE O624HN OBROMOMETHANE O624HN OVINYL CHIORIDE O624HN OCHLOROETHANE O624HN OMETHYLENE CHLORIDE O624HN OACETONE. O624HN OCARBON DISULFIDE O624HN 01,1-DICHLOROETHENE 0624HN 01,1-DICHLOROETHANE 0624HN 01,2-DICHLOROETHENE (0624HN OCHLOROFORM O624HN 01,2-DICHLOROETHANE 0624HN 02-BUTANONE O624HN $01,1,1$-TRICHLOROETHÁNO 624HN OCARBON TETRACHLORIDEO $624 \mathrm{HN}$ OVINYL ACETATE O624HN OBROMODICHLOROMETHANEO624HN 01,2-DICHLOROPROPANE O624HN 

TRCLE 2JLT $12010320900327900051 \mathrm{WA} 90032865009$ DBRCLM2JLT $12010320900327900051 \mathrm{WA} 9003 \mathrm{~L} 865009$ 112TCE2JLT 12010320900327900051 WA9003L865 009 C6H6 2JLT 12010320900327900051 WA9003L865 009 T13DCP2JLT 12010320900327900051 WA9003L865 009 CHBR3 2JLT 12010320900327900051 WA9003L865 009 AME2PE2JLT 12010320900327900051 WA9003L865 009 2HXONE2JLT 12010320900327900051 WA9003L865 009 TCLEE 2JLT 12010320900327900051 WA9003L865 009 TCLEA 2JLT 12010320900327900051 KA9003L865 009 MEC6H52JLT 12010320900327900051 WA9003L865 009 CLC6H52JLT 12010320900327900051 WA9003L865 009 ETC6H52JLT 12010320900327900051 WA9003L865 009 STYR 2JLT 12010320900327900051 WA9003L865 009 XYLEN 2JLT $12010320900403900406 \mathrm{KA9003L865} 009$ NBD5 1X $12010320900403900406 \mathrm{WA} 90034865009$ 2FBP $1 \mathrm{X}$ 12010320900403900406 WA9003L865 009 PTERP 1X 12010320900403900406 WA9003L865 009 PHEND51X 12010320900403900406 WA9003L865 009 2FP $1 \mathrm{X}$ 12010320900403900406 WA9003L865 009 246TBP1X $12010320900403900406 \% A 9003 \mathrm{~L} 865009$ PHENOL1XLT $12010320900403900406 \mathrm{WA} 9003 \mathrm{~L} 865$ 009 B2CEE 1XLT $12010320900403900406 \mathrm{FA9003L865009}$ 2CLP 1XLT $12010320900403900406 \mathrm{WA} 9003 \mathrm{~L} 865009$ 13DCLB1XLT 12010320900403900406 WA9003L865 009 14DCLB1XLT $12010320900403900406 \mathrm{WA} 9003 \mathrm{~L} 865009$ BZALC 1XLT 12010320900403900406 WA9003L865 009 12DCLB1XLT 12010320900403900406 WA9003L865 009 2MP 1XLT $12010320900403900406 \mathrm{FA} 90034865009$ B2CIPE1XLT 12010320900403900406 พÀ9003L865 009 4MP 12010320900403900406 WA9003L865 009 NDNPA 1XLT $12010320900403900406 \mathrm{FA} 90032865009$ CL6ET 1XLT 12010320900403900406 WA9003L865 009 NB 1XLT $12010320900403900406 \mathrm{WA} 9003$ L865 009 ISOPHRIXLT 12010320900403900406 WA9003L865 009 2NP 1XLT 12010320900403900406 WA9003L865 009 24DMPNIXLT 12010320900403900406 HA9003L865 009 BENZOA1XLT 12010320900403900406 WA9003L865 009 B2CEXMIXLT $12010320900403900406 \mathrm{WA9} 003 L 865009$ 24DCLPIXLT $12010320900403900406 \mathrm{WA} 9003 \mathrm{~L} 865009$ 124TCB1XLT $12010320900403900406 \mathrm{WA9} 003 \mathrm{~L} 865009 \mathrm{NAP}$ 1XLT 12010320900403900406 WA9003L865 009. 4CANILIXLT 12010320900403900406 WA9003L865 009 HCBD 1XLT 12010320900403900406 KA9003L865.009 4CL3C 1XLT $12010320900403900406 \mathrm{WA} 9003 \mathrm{~L} 865$ 009 2MENAP 1XLT 12Q1 0320900403900406\%A9003L865 009 CL6CP 1XLT
5UGKG

75PER

84 PER

83PER

64PER

52PER

35 OUGKG

350UGKG

350UGKG

350UGKG

35 OUGKG

350UGKG

350 UGKG

350UGKG

350UGKG

350UGKG

350UGKG

350UGKG

350UGKG

350UGKG

350UGKG

35 OUGKG

180 OUGKG

35OUGKG

350 UGKG

350 UGKG

350 UGKG

350 UGKG

350UGKG

350 UKKG

350UGKG 350 UGKG
1. $0000 \mathrm{MSJBD}$ 1. $0000 \mathrm{MSJBD}$ 1. $0000 M S J B D$ 1. $0000 \mathrm{MSJBD}$ 1. $0000 \mathrm{MSJBD}$ 1. $0000 \mathrm{MSJBD}$ 1. $0000 \mathrm{MSJBD}$ 1. $0000 \mathrm{MSJBD}$ 1. $0000 \mathrm{MSJBD}$ $1.0000 M S J B D$ 1. $0000 \mathrm{MSJBD}$ 1. 0000MSJBD 1. 0000MSJBD 1. $0000 \mathrm{MSJBD}$ 1.0000MSJBD 1. $0000 \mathrm{MSJBD}$ 1. 0000MSBKR 1. 0000MSBKR 1. 0000MSBKR 1. 0000MSBKR 1. 0000MSBKR 1.0000MSBKR 1. $0000 \mathrm{MSBKR}$ 1. 0000MSBKR 1. 0000MSBKR 1. 0000MSBKR 1. 0000MSBKR 1. 0000MSBKR 1: 0000MSBKR 1. 0000MSBKR 1. 0000MSBKR 1. 0000MSBKR 1. 0000MSBKR 1. 0000MSBKR 1. 0000MSBKR 1. 0000MSBKR $1.0000 \mathrm{MSBKR}$ 1. 000OMSBKR 1. O0OOMSBKR 1. OOOOMSBKR 1. 0000MSBKR 1. 000OMSBKR 1. 0000MSBKR 1. 0000MSBKR 1. 0000MSBKR 1. 0000MSBKR

1. 0000MSBKR 1. $0000 \mathrm{MSBKR}$
OCIS-1, 3-DICHLOROPROPO624HN OTRICHLOROETHENE O624HN ODIBROMOCHIOROMETHANEO $624 \mathrm{HN}$ 01, 1, 2-TRICHLOROETHANO $624 \mathrm{HN}$ OBENZENE. O624HN OTRANS-1, 3-DICHLOROPRO624HN OBROMOFORM O624HN 04-METHYL-2-PENTANONEO624HN 02-HEXANONE 0624HN OTETRACHLOROETHENE O624HN $01,1,2,2-T E T R A C H L O R O E O 624 \mathrm{HN}$ OTOLUENE OCHLOROBENZENE O624HN OETHYLBENZENE O624HN OSTYRENE O624HN OXYLENE (TOTAL) 0624HN $1750.000 N I T R O B E N Z E N E-D 5$ O625HN $1750.0002-F L U O R O B I P H E N Y L$ O625HN 1750.000P-TERPHENYL-D14 O625HN 3500.000RHENOI-D5 0625HN 3500.0002-ELUOROPHENOL O625HN $3500.0002,4,6$-TRIBROMOPHENOLO625HN OPHENOL $0625 \mathrm{HN}$ OBIS (2-CHLOROETHYL) ETO625HN 02-CHLOROPHENOL O625HN 01,3-DICHLOROBENZENE O625HN 01, 4-DICHLOROBENZENE O625HN OBENZYL ALCOHOL O625HN 01,2-DICHLOROBENZENE 0625HN 02-METHYLPHENOL O625HN OBIS (2-CHLOROISOPROP YO625HN 04-METHYLPHENOL $0625 \mathrm{HN}$ ON-NITROSO-DI-N-PROPYO625HN OHEXACHLOROETHANE $0625 \mathrm{HN}$ ONITROBENZENE $\quad 0625 \mathrm{HN}$ OISOPHORONE $\quad 0625 \mathrm{HN}$ 02-NITROPHENOL $0625 \mathrm{HN}$ 02, 4-DIMETHYLPHENOL $0625 \mathrm{HN}$ OBENZOIC ACID O625HN OBIS (2-CHLOROETHOXY) MO625HN 02,4-DICHLOROPHENOL $0625 \mathrm{HN}$ 01,2,4-TRICHLOROBENZEO625HN ONAPHTHALENE $0625 \mathrm{HN}$ 04-CHLOROANILINE O625HN OHEXACHLOROBUTADIENE O625HN 04-CHLORO-3-METHYLPHEO 625HN 02-METHYLNAPHTHALENE 0625HN OHEXACHLOROCYCLOPEY $625 \mathrm{HN}$ 
GRS 12U $0320900403900406 \mathrm{FA} 9003 \mathrm{~L} 865009$ 246TCP 1XLT

GRS $1201.0320900403900406 \mathrm{WA} 9003 \mathrm{~L} 865009$ 245TCP 1XLT

GRS $12010320900403900406 \mathrm{WA} 0003 \mathrm{~L} 865$ 009 2CNAP 1XLT

GRS $12010320900403900406 \mathrm{WA} 9003 \mathrm{~L} 865009$ 2NANIL1XLT

GRS $12010320900403900406 \mathrm{WA} 9003 \mathrm{~L} 865009$ DMP 1XLT

GRS 12010320900403900406 WA9003L865 009 ANAPYL1XLT

GRS $12010320900403900406 \mathrm{WA} 9003 \mathrm{~L} 865009$ 26DNT 1XLT

GRS 12010320900403900406 W่A9003L865 009 3NANIL1XLT

GRS $12010320900403900406 \mathrm{WA} 90032865009$ ANAPNE1XLT

GRS $12010320900403900406 \mathrm{WA} 9003 \mathrm{~L} 865009$ 24DNP 1XLT

GRS $12010320900403900406 \mathrm{WA} 9003 \mathrm{~L} 865009$ 4NP 1XLT

GRS $12010320900403900406 \mathrm{WA} 90032865009$ DIBZFU1XLT

GRS $12010320900403900406 \mathrm{WA} 9003 L 865009$ 24DNT 1XLT

GRS $12010320900403900406 \mathrm{WA} 9003 \mathrm{~L} 865009$ DEP 1XLT

GRS 12010320900403900406 WA9003L865 009 4CLPPE1XLT

GRS $12010320900403900406 \mathrm{WA} 90032865009$ FLRENE1XLT

GRS 12010320900403900406 WA9003L865 009 4NANIL1XLT

GRS $12010320900403900406 \mathrm{WA} 9003 \mathrm{~L} 865009$ 46DN2C1XLT

GRS $12010320900403900406 \mathrm{WA} 9003 \mathrm{~L} 865009$ NNDPA 1XLT

GRS 12010320900403900406 WA9003L865 009 4BRPRE1XLT

GRS $12010320900403900406 \mathrm{FA} 9003 \mathrm{~L} 865009 \mathrm{CL} 6 \mathrm{BZ}$ 1XLT

GRS $12010320900403900406 \mathrm{WA} 9003 \mathrm{~L} 865009$ PCP 1XLT

12010320900403900406 WA9003L865 009 PHANTR1XLT $12010320900403900406 \mathrm{WA} 90032865009$ ANTRC 1XLT 12010320900403900406 WA9003L865 009 DNBP 1XLT $12010320900403900406 \mathrm{WA} 9003 \mathrm{~L} 865009$ FANT 1XLT $12010320900403900406 \mathrm{WA} 9003 \mathrm{~L} 865009$ PYR 1XLT 12010320900403900406 WA9003L865 009 BBZP 1XLT $12010320900403900406 \mathrm{WA} 9003 \mathrm{~L} 865009$ 33CL2B1XLT $12010320900403900406 \mathrm{FA9003L865009}$ BAANTR1XLT 12010320900403900406 WA9003L865 009 CHRY 1XLT 12010320900403900406 WA 90031865009 B2EHP 1XLT 12010320900403900406 WA90031865 009 DNOP 1XLT 12010320900403900406 KA9003L865 009 BF2ANT1XLT 12010320900403900406 WA9003L865 009 BKFANT1XLT 1201.0320900403900406WA9003L865 009 BAPYR 1XLT $12010320900403900406 \mathrm{WA} 9003 \mathrm{~L} 865009$ ICDPYR1XLT $12010320900403900406 \mathrm{WA} 9003 \mathrm{~L} 865009$ DBAHA $1 X \mathrm{KT}$ 12010320900403900406 WA9003L865 009 BGHIPY1XLT 13010321900328900051 WA9003L865 010

$13010321900519900451 \mathrm{FAg} 0032865010 \mathrm{AG}$. 13010321900518900450 WA9003L865 010 AS 13010321900519900451 WA9003L865 $010 \mathrm{BA}$ 2ZLT 13010321900519900451 WA9003L865 $010 \mathrm{CD}$ 22LT 13010321900519900451 WA9003L865 $010 \mathrm{CR}$ 1301 $0321900519900451 \mathrm{Ag} 003 \mathrm{~L}$ $13010321900518900450 \mathrm{WA} 90032865010 \mathrm{~PB}$ 1B 22 $13010321900518900450 \mathrm{WA} 9003 \mathrm{~L} 865010 \mathrm{SE}$
350UGKG 1800 UGKG 350UGKG

1800UGKG

350UGKG

350UGKG

350UGKG

1800 UGKG

350UGKG

1800 UGKG

1800 UGKG

350UGKG

350UGKG

350UGKG

350UGKG

350UGKG

1800 UGKG

1800 UGKG

350UGKG

350UGKG

350UGKG

1800UGKG

350UGKG

350UGKG

350UGKG

350UGKG

350UGKG

350UGKG

700UGKG

350UGKG

350UGKG

350UGKG

350UGKG

350UGKG

350UGKG

350UGKG

350UGKG

350UGKG

350UGKG

82.78

2. OMGKG

2. 3MGKG

40. 3MGKG

1. 2MGKG

22.9MGKG

$0.15 M G K G$

48.1MGKG

1. 3MGKG
$1.0000 \mathrm{MSBKR}$ $1.0000 \mathrm{MSBKR}$ $1.0000 \mathrm{MSBKR}$

$1.0000 \mathrm{MSBKR}$

$1.0000 \mathrm{MSBKR}$

$1.0000 M S B K R$

1.0000MSBKR

$1.0000 \mathrm{MSBKR}$

$1.0000 \mathrm{MSBKR}$

$1.0000 \mathrm{MSBKR}$

1. $0000 \mathrm{MSBKR}$

$1.0000 \mathrm{MSBKR}$

$1.0000 \mathrm{MSBKR}$

$1.0000 M S B K R$

1.0000MSBKR

$1.0000 \mathrm{MSBKR}$

$1.0000 \mathrm{MSBKR}$

1.0000MSBKR

$1.0000 \mathrm{MSBKR}$

$1.0000 \mathrm{MSBKR}$

$1.0000 \mathrm{MSBKR}$

$1.0000 \mathrm{MSBKR}$

$1.0000 \mathrm{MSBKR}$

$1.0000 \mathrm{MSBKR}$

$1.0000 \mathrm{MSBKR}$

$1.0000 \mathrm{MSBKR}$

$1.0000 \mathrm{MSBKR}$

$1.0000 \mathrm{MSBKR}$

$1.0000 \mathrm{MSBKR}$

1. $0000 \mathrm{MSBKR}$

$1.0000 \mathrm{MSBKR}$

$1.0000 \mathrm{MSBKR}$

1. $0000 M S B K R$

$1.0000 \mathrm{MSBKR}$

$1.0000 \mathrm{MSBKR}$

$1.0000 \mathrm{MSBKR}$

$1.0000 \mathrm{MSBKR}$

1.0000MSBKR

1. 0000MSBKR $10 \mathrm{JH}$

1. OOICALM

1.00AAAER

1.00 ICALM

1.00 ICALM

1.00ICALM

1.00 HGJJW

10.01AAEJS

1. 00AAAER
$02,4,6$-TRICHLOROPHENOO625HN $02,4,5-T R I C H L O R O P H E N O 0625 \mathrm{HN}$ 02-CHLORONAPHTHALENE $0625 \mathrm{HN}$ 02-NITROANILINE 0625HN ODIMETHYLPHTHALATE O625HN OACENAPHTHYLENE $0625 \mathrm{HN}$ 02,6-DINITROTOLUENE 0625HN 03-NITROANIIINE $0625 \mathrm{HN}$ OACENAPHTHENE $0625 \mathrm{HN}$ 02,4-DINITROPHENOI $0625 \mathrm{HN}$ 04-NITROPHENOI $\quad 0625 \mathrm{HN}$ ODIBENZOFURAN O625HN

02, 4-DINITROTOLUENE O625HN ODIETHYLPHTHALATE O625HN 04-CHLOROPHENYL-PHENYO625HN OELUORENE 04-NITROANILINE 0625HN 04, 6-DINITRO-2-METHYLO625HN ON-NITROSODIPHENYLAMIO $625 \mathrm{HN}$ 04-BROMOPHENYL-P HENYLO625HN OHEXACHLOROBENZENE O625HN OPENTACHLOROPHENOI O625HN OPHENANTHRENE OANTHRACENE O625HN ODI-N-BUTYLPHTHALATE $0625 \mathrm{HN}$ OFLUORANTHENE O625HN OPYRENE O625HN OBUTYLBENZYLPHTHALATEO625HN $03,3^{\prime}$-DICHLOROBENZ IDIO625HN OBENZO (A) ANTHRACENE O625HN OCHRYSENE 0625HN OBIS (2-ETHYLHEXYL) PHTO625HN ODI-N-OCTYL PHTHALATEO625HN OBENZO (B) FLUORANTHENEO625HN OBENZO (K) FLUORANTHENEO625HN OBENZO (A) PYRENE O625HN O INDENO (1,2,3-CD) PYREO625HN ODIBENZO (A, H) ANTHRACEO625HN OBENZO $(G, H, I)$ PERYLENEO $625 \mathrm{HN}$ Of SOLIDS OSILVER, TOTAL OARSENIC, TOTAL I\& SOLN OBARIUM, TOTAL OCADMIUM, TOTAL OCHROMIUM, TOTAL OMERCURY, TOTAL OLEAD, TOTAL OSELENIUM, TOTAL
MASTON MBATON MCDTON MCRTON MHGTON MPBTON MSETON 
GRS 13010321900327900051 WA9003L865 010 MEC6D82J GRS 13010321900327900051 WA9003L865 010 PBEB 2J

GRS 13010321900327900051 WA9003L865 $010 \quad 12 D C D 42 \mathrm{~J}$

GRS 13010321900327900051 WA9003L865 010 CH3CL 2JLT GRS 13010321900327900051 WA9003L865 010 CH3BR 2JLT GRS 13010321900327900051 WA9003L865 010 C2H3CL2JLT GRS 13010321900327900051 WA9003L865 010 C2H5CL2JLT GRS 13010321900327900051 WA9003L865 010 CH2CL22JB GRS 13010321900327900051 WA9003L865 010 ACET 2JB GRS $1301 \quad 0321900327900051$ WA9003L865 010 CS2 $2 \mathrm{JLT}$ GRS $\quad 13010321900327900051$ WA9003L865 010 11DCE 2JLT GRS 13010321900327900051 WA9003L865 010 11DCLE2JLT GRS 13010321900327900051 WA9003L865 010 T12DCE2JLT GRS 13010321900327900051 WA9003L865 010 CHCL3 2JLT GRS 13010321900327900051 WA9003L865 010 12DCLE2JLT GRS $\quad 13010321900327900051$ HA9003L865 010 MEK 2JLT GRS 13010321900327900051 WA9003L865 010 111TCE2JLT GRS 13010321900327900051 WA9003L865 010 CCL4 2JIT GRS $\quad 13010321900327900051$ WA9003L865 010 VINYLA2JLT GRS 13010321900327900051 WA9003L865 010 BRDCLM2JLT GRS 13010321900327900051 WA9003L865 010 12DCLP2JLT GRS 13010321900327900051 WA9003L865 010 13DCPC2JLT GRS 13010321900327900051 WA9003L865 010 TRCLE 2JLT W GRS 13010321900327900051 WA9003L865 010 DBRCLM2JLT N GRS 13010321900327900051 WA9003L865 010 112TCE2JLT GRS 13010321900327900051 WA9003L865 010 C6H6 2JLT GRS 13010321900327900051 WA9003L865 010 T13DCP2JLT GRS 13010321900327900051 WA9003L865 010 CHBR3 2JLT GRS 13010321900327900051 WA9003L865 010 4ME2PE2JLT GRS 13010321900327900051 WA9003L865 010 2HXONE2JLT GRS 13010321900327900051 HA9003L865 010 TCLEE 2J GRS 13010321900327900051 WA90031865 010 TCLEA 2JIT GRS 13010321900327900051 WA9003L865 010 MEC6H52JLT GRS 13010321900327900051 WA9003L865 010 CLC6H52JLT GRS 13010321900327900051 WA9003L865 010 ETC6H52JLT GRS 13010321900327900051 HA9003L865 010 STYR 2JLT GRS 13010321900327900051 WA9003L865 010 XYLEN 2JLT GRS 13010321900403900406 WA9003L865 010 NBD5 $1 X$ GRS 13010321900403900406 WA9003L865 010 2FBP $1 X$ GRS 13010321900403900406 WA9003L865 010 PTERP 1X GRS 13010321900403900406 WA9003L865 010 PHEND51X GRS 13010321900403900406 HA9003L865 010 2EP $1 X$ GRS 13010321900403900406 WA9003L865 010 246TBP 1X GRS 13010321900403900406 WA9003L865 010 PHENOLIXLT GRS 13010321900403900406 WA9003L865 010 B2CEE 1XIT GRS 13010321900403900406 HA9003L865 010 2CLP 1XIT GRS 13010321900403900406 FA9003L865 010 13DCLBIXIT GRS 13010321900403900406 FA9003L865 010 14DCLB1XLT
31 UGKG

19 UGKG

6UGKG

6UGKG

6UGKG

6UGKG

6UGKG

6UGKG

12 UGKG

6UGKG

6UGKG

12 UGKG

6UGKG

6UGKG

6UGKG

6UGKG

6UGKG

6UGKG

6UGKG

6UGKG

6UGKG

12 UGKG

12UGKG

7UGKG

6UGKG

6UGKG

6UGKG

6UGKG

6UGKG

6UGKG

69PER

65PER

7 9PER

8IPER

67PER

64PER

38 OUGKG

38 OUGKG

38 OUGKG

380UGKG 3 \&OUGKG
1. $0000 \mathrm{MSJBD}$ 1.0000MSJBD

1. $0000 \mathrm{MSJBD}$

1.0000MSJBD

$1.0000 \mathrm{MSJBD}$

1. $0000 \mathrm{MSJBD}$

1.0000MSJBD

1. $0000 \mathrm{MSJBD}$

1. $0000 \mathrm{MSJBD}$

1.0000MSJBD

1. $0000 \mathrm{MSJBD}$

1. $0000 \mathrm{MSJBD}$

1. 0000MSJBD

1. $0000 \mathrm{MSJBD}$

1. $0000 \mathrm{MSJBD}$

1.0000MSJBD

1. $0000 \mathrm{MSJBD}$

$1.0000 \mathrm{MSJBD}$

1.0000MSJBD

1. $0000 \mathrm{MSJBD}$

1.0000MSJBD

1. $0000 \mathrm{MSJBD}$

1. $0000 \mathrm{MSJBD}$

$1.0000 \mathrm{MSJBD}$

1. $0000 \mathrm{MSJBD}$

1. $0000 \mathrm{MSJBD}$

1.0000MSJBD

1. $0000 \mathrm{MSJBD}$

$1.0000 \mathrm{MSJBD}$

1.0000MSJBD

1. $0000 \mathrm{MSJBD}$

1.0000MSJBD

1.0000MSJBD

1. 0000MSJBD

1.0000MSJBD

1. $0000 \mathrm{MSJBD}$

1.0000MSJBD

1.0000MSBKR

1. O0OOMSBKR

1.0000MSBKR

1.0000MSBKR

1. 000OMSBKR

1.0000MSBKR

1. $0000 \mathrm{MSBKR}$

1.0000MSBKR

1. OOOOMSBKR

1. $0000 \mathrm{MSBKR}$

1. $0000 \mathrm{MSBKR}$
59. OOOTOLUENE-D8

$0624 \mathrm{HN}$

000BOMOEIUOROBENZENE 0624HN

$59.0001,2-D$ ICHLOROETHANE-DO624HN OCHLOROMETHANE O624HN OBROMOMETHANE O624HN OVINYL CHLORIDE ' $\quad 0624 \mathrm{HN}$ OCHLOROETHANE O624HN OMETHYLENE CHLORIDE O624HN OACETONE O624HN OCARBON DISULETDE O624HN 01,1-DICHLOROETHENE 0624HN 01,1-DICHLOROETHANE $0624 \mathrm{HN}$ 01,2-DICHLOROETHENE $10624 \mathrm{HN}$ OCHLOROEORM $0624 \mathrm{HN}$ 01,2-DICHLOROETHANE O624HN 02-BUTANONE $0624 \mathrm{HN}$ $01,1,1$-TRICHLOROETHANO $624 \mathrm{HN}$ OCARBON TETRACHLORIDEO624HN OVINYI ACETATE O624HN OBROMODICHLOROMETHANEO $624 \mathrm{HN}$ 01,2-DICHIOROPROPANE 0624HN OCIS-1, 3-DICHLOROPROPO $624 \mathrm{HN}$ OTRICHLOROETHENE O624HN ODIBROMOCHLOROMETHANEO $624 \mathrm{HN}$ 01, 1, 2-TRICHIOROETHANO $624 \mathrm{HN}$ OBENZENE $0624 \mathrm{HN}$ OTRANS-1, 3-DICHLOROPRO624HN OBROMOFORM O624HN 04-METHYL-2-PENTANONEO624HN 02-HEXANONE O624HN OTETRACHLOROETHENE O624HN $01,1,2,2-T E T R A C H L O R O E O 624 \mathrm{HN}$ OTOLUENE OCHLOROBENZENE $0624 \mathrm{HN}$ 0624HN OETHYLBENZENE OSTYRENE

OXYLENE (TOTAL)

1925. O0ONITROBENZENE-D5 $0624 \mathrm{HN}$ $0624 \mathrm{HN}$ $0625 \mathrm{HN}$ 1925.0002-FLUOROBIPHENYL O625HN 1925.0002-TERPHENYL-D14 O625HN 3850 . 000PHENOL-D5 3850.0002-FLUOROPHENOL O625HN $3850.0002,4,6$-TRIBROMOPHENOLO625HN OPHENOL $0625 \mathrm{HN}$ OBIS (2-CHLOROETHYL) ETO625HN 02-CHLOROPHENOL O625HN 01,3-DICHLOROBENZENE O625HN $01,4-D I C H L O R O B E N Z E N F 25 \mathrm{HN}$ 
GRS 130+0321900403900406 FA9003L865 010, BZALC 1XLT GRS $13010321900403900406 \mathrm{WA} 9003 L 865010$ 12DCLB1XLT GRS $13010321900403900406 \mathrm{FA9003L865} 010$ 2MP 1 XLT GRS $13010321900403900406 \mathrm{WA} 9003 \mathrm{~L} 865010$ B2CIPE1XLT GRS $13010321900403900406 \mathrm{WA} 0003 \mathrm{~L} 865010$ 4MP 1XLT GRS $13010321900403900406 \mathrm{WA} 9003 L 865010$ NDNPA 1XLT GRS 13010321900403900406 W A9003L865 010 CL6ET 1XLT GRS $13010321900403900406 \mathrm{FA} 9003 \mathrm{~L} 865010 \mathrm{NB}$ 1XLT GRS $13010321900403900406 \mathrm{WA} 9003 \mathrm{~L} 865010$ ISOPHRIXLT GRS $13010321900403900406 \mathrm{WA} 9003 L 865010$ 2NP 1XLT GRS $1.3010321900403900406 \mathrm{WA} 003 \mathrm{~L} 865010$ 24DMPN1XLT GRS $13010321900403900406 \mathrm{WA} 9003 L 865010$ BENZOAIXLT GRS $13010321900403900406 \mathrm{FA9003L865} 010$ B2CEXM1XLT GRS $13010321900403900406 \mathrm{WA} 9003 \mathrm{~L} 865010$ 24DCLP1XLT GRS $13010321900403900406 \mathrm{WA} 9003 \mathrm{~L} 865010$ 124TCB1XLT GRS $13010321900403900406 \mathrm{WA} 9003 \mathrm{~L} 865010$ NAP 1XLT GRS $13010321900403900406 \mathrm{WA} 9003 \mathrm{~L} 865010$ 4CANIL1XLT GRS $13010321900403900406 \mathrm{WA} 003 \mathrm{~L} 865010 \mathrm{HCBD}$ 1XLT GRS $13010321900403900406 \mathrm{WA} 9003 L 865010$ 4CL3C 1XLT GRS $13010321900403900406 \mathrm{WA} 9003 \mathrm{~L} 865010$ 2MENAP1XLT GRS 13010321900403900406 WA9003L865 010 CL6CP 1XLT GRS $13010321900403900406 \mathrm{WA} 9003 \mathrm{~L} 865010 \quad 246 \mathrm{TCP} 1 \mathrm{XLT}$ GRS $13010321900403900406 \mathrm{WA} 9003 \mathrm{~L} 865010$ 245TCP1XLT 1 GRS 13010321900403900406 WA9003L865 010 2CNAP 1XLT $\sim$ GRS $13010321900403900406 \mathrm{WA} 9003 \mathrm{~L} 865010$ 2NANILIXLT 2 GRS 13010321900403900406 WA9003L865 010 DMP 1XLT GRS 13010321900403900406 WA9003L865 010 ANAPYLIXLT GRS $1301.0321900403900406 \mathrm{WA} 90034865010$ 26DNT 1XLT GRS 13010321900403900406 WA9003L865 010 3NANILIXLT GRS 13010321900403900406 WA9003L865 010 ANAPNE1XLT GRS $13010321900403900406 \mathrm{WA} 9003 L 865010$ 24DNP 1XLT GRS $13010321900403900406 \mathrm{WA} 9003 L 865010$ 4NP 1XLT GRS $13010321900403900406 \mathrm{WA} 9003 \mathrm{~L} 865010$ DIBZFU1XLT GRS $13010321900403900406 \mathrm{FA} 9003 L 865010$ 24DNT 1XLT GRS 13010321900403900406 WA9003L865 010 DEP 1XLT GRS $13010321900403900406 \mathrm{WA} 0034865010$ 4CLPPE1XLT GRS $13010321900403900406 \mathrm{WA} 9003 L 865010$ ELRENE1XLT GRS 13010321900403900406 WA9003L865 010 4NANILIXLT GRS $13010321900403900406 \mathrm{WA} 9003 \mathrm{~L} 865010$ 46DN2C1XLT GRS $13010321900403900406 \mathrm{WA} 9003 L 865010$ NNDPA 1XLT GRS $13010321900403900406 \mathrm{WA} 9003 \mathrm{~L} 865010$ 4BRPPE1XLT GRS $13010321900403900406 \mathrm{WA} 9003 L 865010$ CL6BZ 1XLT GRS $13010321900403900406 \mathrm{FA} 9003 L 865010$ PCP 1XLT GRS $13010321900403900406 \mathrm{FA} 9003 \mathrm{~L} 865010$ PHANTR1XLT GRS $13010321900403900406 \mathrm{WA} 9003 L 865010$ ANTRC 1XIT GRS 13010321900403900406 FA9003L865 010 DNBP 1XLT GRS $13010321900403900406 \mathrm{FA9003L865} 010$ FANT 1XLT GRS
380UGKG 38OUGKG 38OUGKG 380UGKG 380 UGKG 380 UGKG 380UGKG 380UGKG 380 UGKG 380UGKG 380UGKG

1900UGKG 380UGKG 380UGKG 380UGKG 380UGKG 380UGKG 380UGKG 380 UGKG 380UGKG 380UGKG 380UGKG 1900UGKG 380UGKG

1900 UGKG

380UGKG 380UGKG 380UGKG 1900UGKG 380UGKG 1900 UGKG 1900 UGKG 380UGKG 380UGKG 380UGKG 380UGKG 380UGKG 1900UGKG 1900UGKG 380UGKG 380UGKG 380UGKG 1900UGKG 380UGKG 380UGKG 380UGKG 380UGKG 380 UGKG
1.0000MSBKR

1.0000MSBKR

$1.0000 M S B K R$

$1.0000 \mathrm{MSBKR}$

$1.0000 \mathrm{MSBKR}$

$1.0000 M S B K R$

$1.0000 \mathrm{MSBKR}$

$1.0000 M S B K R$

1.0000MSBKR

$1.0000 M S B K R$

1.0000MSBKR

$1.0000 \mathrm{MSBKR}$

1.0000 MSBKR

$1.0000 \mathrm{MSBKR}$

$1.0000 \mathrm{MSBKR}$

$1.0000 M S B K R$

$1.0000 \mathrm{MSBKR}$

$1.0000 \mathrm{MSBKR}$

$1.0000 \mathrm{MSBKR}$

$1.0000 \mathrm{MSBKR}$

$1.0000 \mathrm{MSBKR}$

1.0000MSBKR

$1.0000 \mathrm{MSBKR}$

$1.0000 \mathrm{MSBKR}$

1.0000MSBRR

1.0000 MSBKR

1.0000MSBKR

$1.0000 \mathrm{MSBKR}$

1. 0000MSBKR

$1.0000 \mathrm{MSBKR}$

1.0000MSBKR

1. $0000 \mathrm{MSBKR}$

1.0000MSBKR

$1.0000 \mathrm{MSBKR}$

1. $0000 \mathrm{MSBKR}$

$1.0000 \mathrm{MSBKR}$

1.0000MSBKR

$1.0000 \mathrm{MSBKR}$

1.0000MSBKR

1.0000 MSBKR

$1.0000 \mathrm{MSBKR}$

1.0000MSBKR

1.0000MSBKR

1.0000MSBKR

$1.0000 \mathrm{MSBKR}$

1.0000MSBKR

$1.0000 \mathrm{MSBKR}$ 1.0000MSBKR
OBENZYL ALCOHOL 0625HN 01,2-DICHLOROBENZENE O625HN 02-METHYLPHENOL $\quad 0625 \mathrm{HN}$ OBIS 12-CHLOROISOPROPYO625HN 04-METHYLPHENOL O625HN ON-NITROSO-DI-N-PROPYO625HN OHEXACHLOROETHANE O625HN ONITROBENZENE $\quad 0625 \mathrm{HN}$

OISOPHORONE

02-NITROPHENOL $0625 \mathrm{HN}$ $2,4-D T M E T H Y L P H E N O I$ O625HN OBENZOIC ACID 0625HN OBIS (2-CHLOROETHOXY) MO625HN $02,4-D I C H L O R O P H E N O L$ 0625H 01,2, 4-TRICHLOROBENZE0625HN ONAPHTHALENE O625HN 04-CHLOROANILINE 0625HN OHEXACHLOROBUTADIENE O6.25HN 04-CHLORO-3-METHYLPHEO625HN 02-METHYLNAPHTHALENE 0625HN OHEXACHLOROCYCLOPENTAO625HN $02,4,6$-TRICHLOROP HENOO625HN 02, 4, 5-TRICHLOROPHENOO625HN 02-CHLORONAPHTHALENE 0625HN 02-NITROANILINE 0625HN ODIMETHYLPHTHALATE O625HN OACENAPHTHYLENE $0625 \mathrm{HN}$ 02,6-DINITROTOLUENE 0625HN 03-NITROANILINE 0625HN OACENAPHTHENE O625HN 02,4-DINITROPHENOL O625HN 04-NITROPHENOL O625HN ODIBENZOFURAN $0625 \mathrm{HN}$ 02,4-DINITROTOLUENE 0625HN ODIETHYLPHTHALATE O625HN 04-CHLOROPHENYL-PHENYO625HN OFLUORENE $0625 \mathrm{kN}$ O4-NITROANILINE 0625HN 04, 6-DINITRO-2-METHYLO625HN ON-NITROSODIPHENYLAMIO625HN 04-BROMOPHENYL-PHENYLO625HN OHEXACHLOROBENZENE O625HN OPENTACHLOROPHENOL $0625 \mathrm{HN}$ OPHENANTHRENE OANTHRACENE 0625HN ODI-N-BUTYLPHTHALATE O625HN OFLUORANTHENE O625HN ORYRENE 


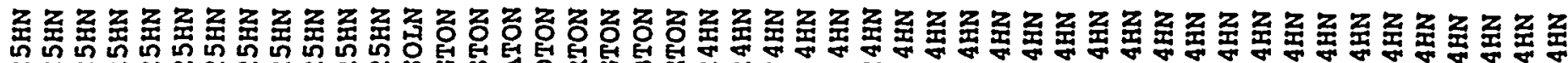

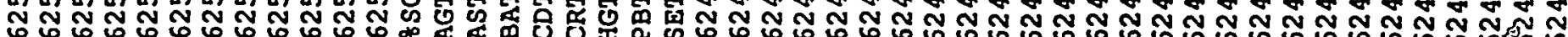

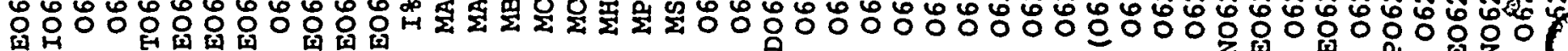

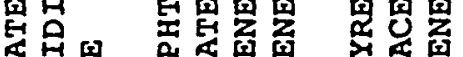

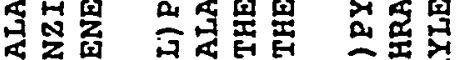

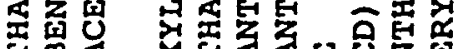

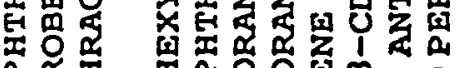
a

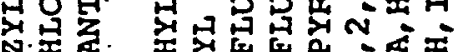

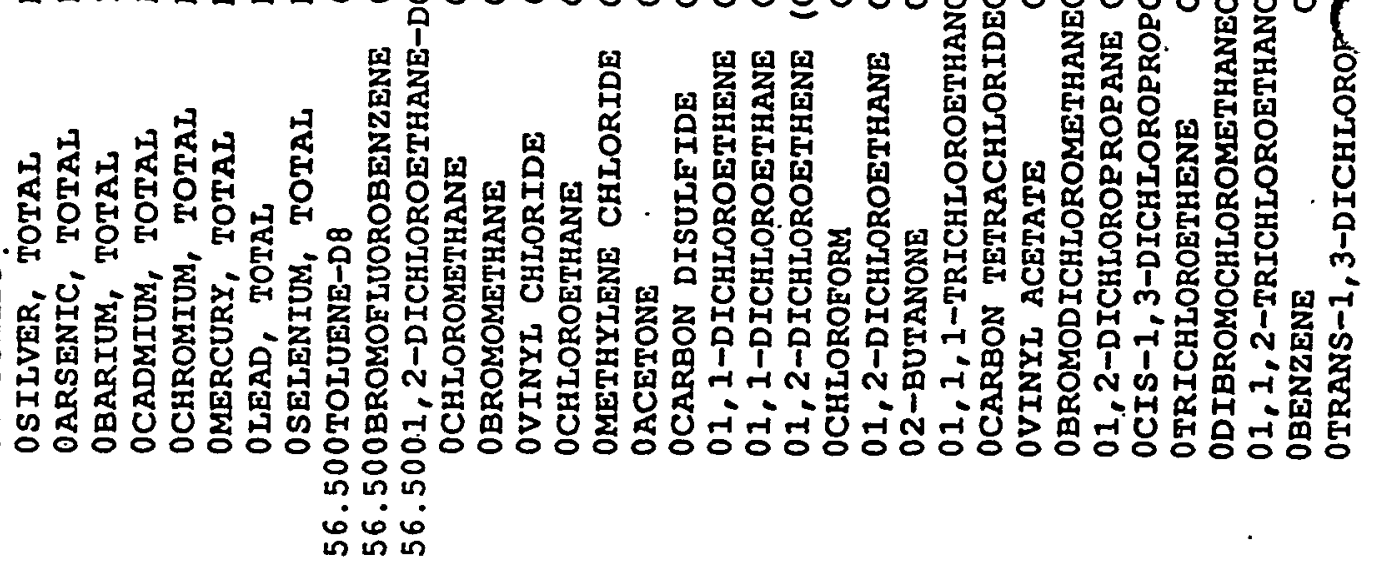

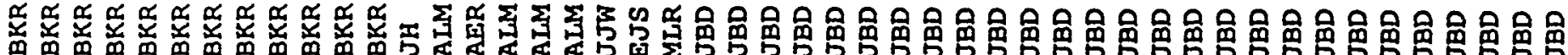

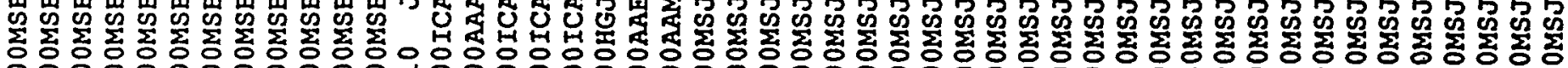

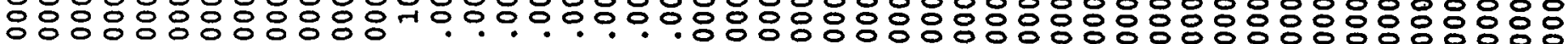

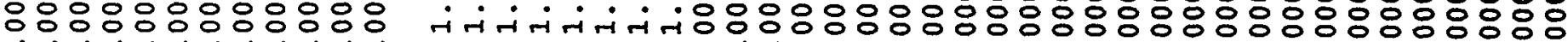

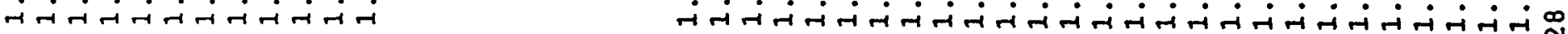

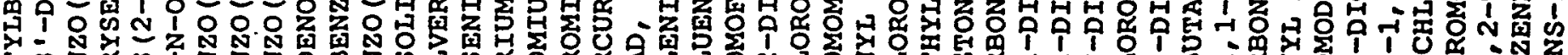

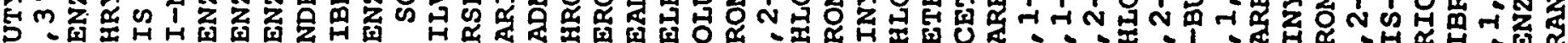

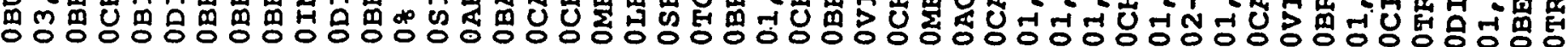

0

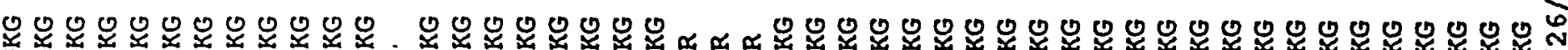

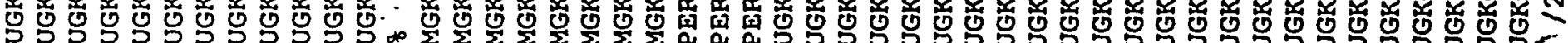

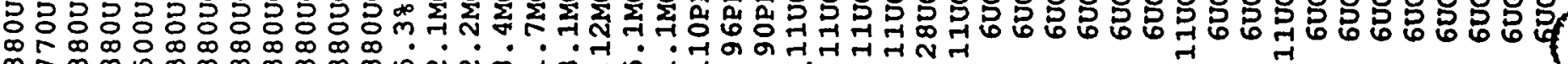
minm nm m m m m m in

H

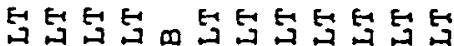

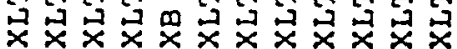

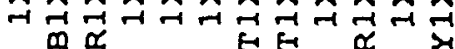

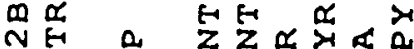

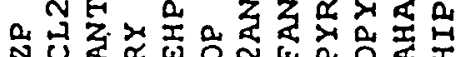

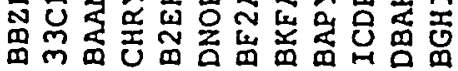
EE

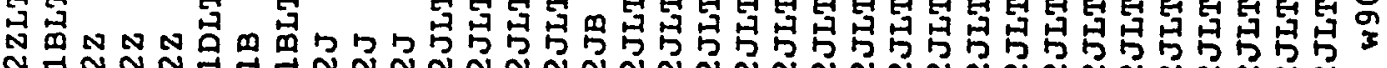
N

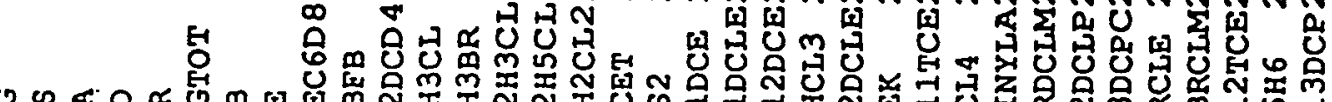

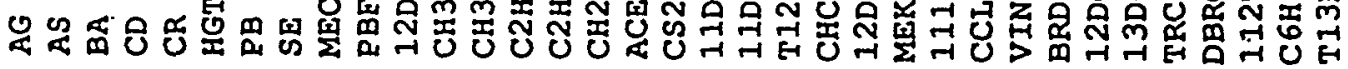

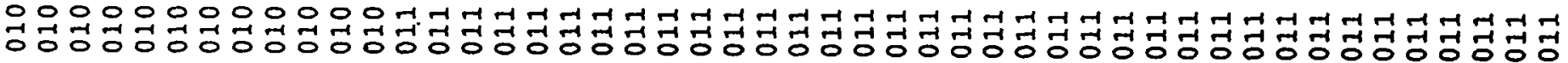
చู

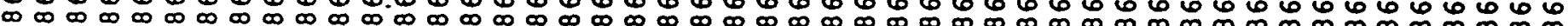

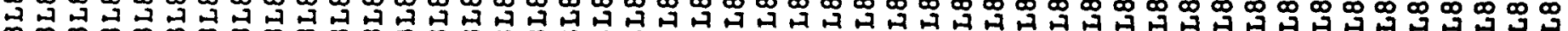
m m m m m m m m m m m m m m m m

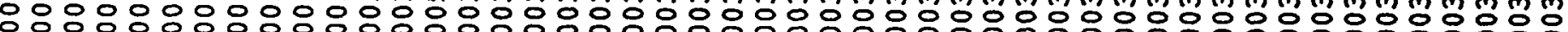
응ㅇㅇㅇㅇㅇㅇㅇㅇㅇㅇㅇㅇㅇㅇㅇㅇㅇㅇㅇㅇㅇㅇㅇㅇㅇㅇㅇㅇㅇㅇㅇㅇㅇㅇㅇㅇㅇㅇㅇㅇㅇㅇㅇㅇㅇㅇㅇㅇㅇㅇㅇㅇㅇㅇㅇㅇㅇㅇㅇㅇㅇㅇㅇㅇㅇㅇㅇㅇㅇㅇㅇㅇㅇㅇㅇㅇㅇㅇㅇㅇㅇ

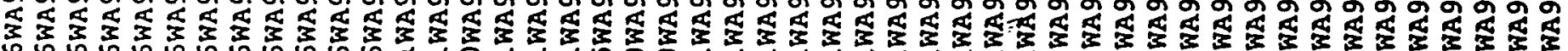

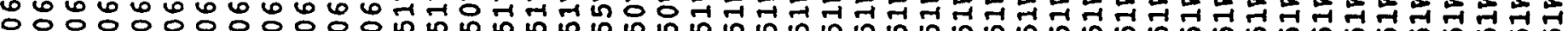
웅ㅇㅇㅇㅇㅇㅇㅇㅇㅇㅇㅇㅇㅇㅇㅇ요의

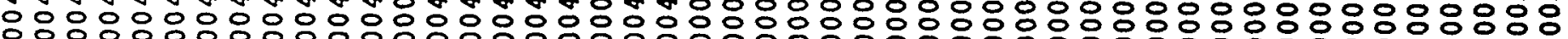

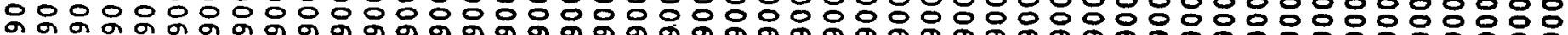
\%

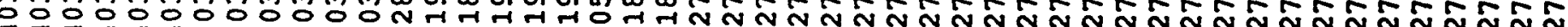

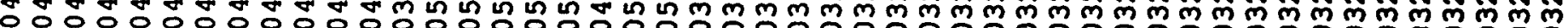

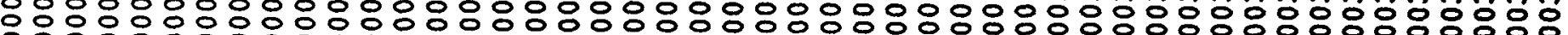

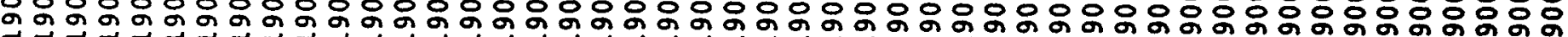

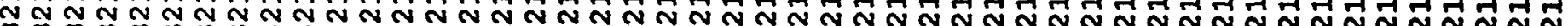

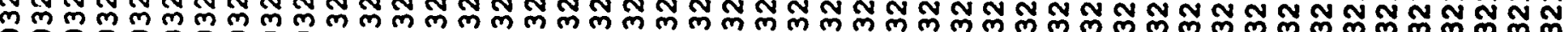

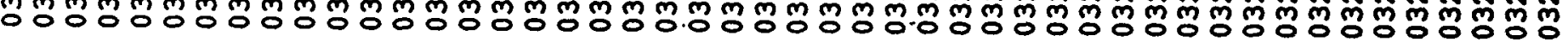

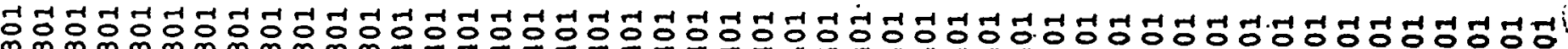

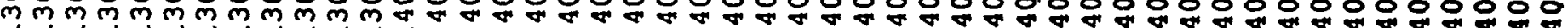

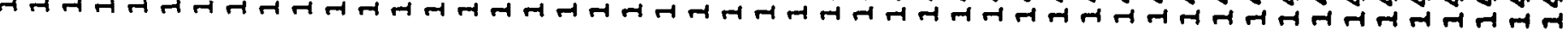


GRS 14L 0321900327900051WA9003L865 011 CHBR3 2JLT GRS 14010321900327900051 WA9003L865 011 4ME2PE2JLT GRS 14010321900327900051 WA9003L865 011 2HXONE2JLT GRS 14010321900327900051 WA9003L865 011 TCLEE 2J GRS 14010321900327900051 WA9003L865 011 TCLEA 2JLT GRS 14010321900327900051 WA9003L865 011 MEC6H52JLT GRS 14010321900327900051 WA9003L865 011 CLC6H52JLT GRS 14010321900327900051 WA9003L865 011 ETC6H52JLT GRS 14010321900327900051 WA9003L865 011 STYR 2JLT GRS 14010321900327900051 WA9003L865 011 XYLEN 2JLT GRS 14010321900403900406 WA9003L865 011 NBDS $1 \mathrm{X}$ GRS 14010321900403900406 WA9003L865 011 2FBP 1X GRS 14010321900403900406 WA9003L865 011 PTERP 1X GRS 14010321900403900406 WA9003L865 011 PHEND51X GRS 14010321900403900406 WA9003L865 011 2FP 1X GRS 14010321900403900406 WA9003L865 $011246 \mathrm{TBP} 1 \mathrm{X}$ 14010321900403900406 WA9003L865 011 PHENOLIXIT $14010321900403900406 \mathrm{WA} 90032865011$ B2CEE 1XLT $14010321900403900406 \mathrm{WA} 9003 \mathrm{~L} 865011$ 2CLP 1XLT $14010321900403900406 \mathrm{WA9003L865} 011$ 13DCLB1XLT 14010321900403900406 WA9003L865 011 14DCLB1XLT 14010321900403900406 WA9003L865 011 BZALC 1XLT $14010321900403900406 \mathrm{WA} 9003 L 865011$ 12DCLB1XLT 14010321900403900406 WA9003L865 011 2MP 1XLT 14010321900403900406 WA9003L865 011 B2CIPE1XLT 14010321900403900406 WA9003L865 011 4MP 1XLT 14010321900403900406 WA9003L865 011 NDNPA 1XLT 14010321900403900406 WA9003L865 011 CL6ET 1XLT 14010321900403900406 WA9003L865 011 NB 1XLT 14010321900403900406 WA9003L865 011 ISOPHR1XLT 14010321900403900406 KA9003L865 011 2NP 1XLT 14010321900403900406 WA $9003 L 865011$ 24DMPN1XLT $14010321900403900406 \mathrm{WA} 9003 L 865011$ BENZOA1XLT 14010321900403900406 WA9003L865 011 B2CEXM1XLT 14010321900403900406 WA9003L865 011 24DCLP1XLT $14010321900403900406 \mathrm{WA} 9003 \mathrm{~L} 865011124 \mathrm{TCB} 1 \mathrm{XLT}$ $14010321900403900406 \mathrm{WA} 90034865011$ NAP 1XLT 14010321900403900406 WA9003L865 011 4CANIL1XLT 14010321900403900406 WA9003L865 011 HCBD 1XLT 14010321900403900406 WA9003L865 011 4CL3C 1XLT 14010321900403900406 FA9003L865 011 2MENAP1XLT 14010321900403900406 WA9003L865 011 CL6CP 1XLT $14010321900403900406 \mathrm{WA} 9003 \mathrm{~L} 865011$ 246TCP1XLT 14010321900403900406 WA9003L865 011245 TCP1XLT $14010321900403900406 \mathrm{WA} 9003 \mathrm{~L} 865011$ 2CNAP 1XLT 14010321900403900406 \%A9003L865 011 2NANIL1XLT 14010321900403900406 FA9003L865 011 DMP 1XLT 14010321900403900406 WA9003L865 011 ANAPYL1XLT
UUGKG

11UGKG

11UGKG

6UGKG

6UGKG

6UGKG

6UGKG

6UGKG

6UGKG

6UGKG

66PER

64PER

77PER

73PER

58PER

50PER

390 UGKG

390 UGKG

390UGKG

390UGKG

390UGKG

390 UGKG

390UGKG

390UGKG

390UGKG

390UGKG

390 UGKG

390UGKG

390 UGKG

390UGKG

390UGKG

390UGKG

2000UGKG

390UGKG

390UGKG

390UGKG

390UGKG

390UGKG

390 UGKG

390 UGKG

390UGKG

390UGKG

390UGKG

2000UGKG

39OUGKG

2000UGKG

390UGKG

390UGKG
1. $0000 \mathrm{MSJBD}$ 1.0000MSJBD 1.0000MSJBD 1. 0000MSJBD 1.0000MSJBD $1.0000 \mathrm{MSJBD}$ 1. $0000 \mathrm{MSJBD}$ 1.0000MSJBD 1.0000MSJBD $1.0000 \mathrm{MSJBD}$ $1.0000 \mathrm{MSBKR}$ 1. 0000MSBKR 1. 0000MSBKR 1.0000MSBKR 1. 0000MSBKR 1.0000MSBKR 1. $0000 \mathrm{MSBKR}$ 1. $0000 \mathrm{MSBKR}$ 1.0000MSBKR 1. 0000MSBKR 1.0000MSBKR 1.0000MSBKR 1. 0000MSBKR 1. 0000MSBKR 1.0000MSBKR 1. OOOOMSBKR 1. 0000MSBKR 1. 0000MSBKR 1. $0000 \mathrm{MSBKR}$ 1.0000MSBKR 1. 0000MSBKR 1.0000MSBKR 1.000OMSBKR 1. 0000MSBKR 1.0000MSBKR 1.0000MSBKR 1. 0000MSBKR 1.0000MSBKR 1.0000MSBKR 1. 0000MSBKR 1.0000MSBKR 1. 0000MSBKR 1.0000MSBKR 1.0000MSBKR 1. 0000MSBKR 1. 0000MSBKR 1.0000MSBKR 1. O000MSBKR
OBROMOEORM

0624HN

04-METHYL-2-PENTANONE0624HN 02-HEXANONE 0624HN OTETRACHLOROETHENE O624HN $01,1,2,2-T E T R A C H L O R O E O 624 \mathrm{HN}$ OTOLUENE

OCHLOROBENZENE $0624 \mathrm{HN}$ $0624 \mathrm{HN}$ OSTYRENE

OXYLENE (TOTAL)

1950 . 00.0NITROBENZENE-D5 1950.0002-ELUOROBIPHENYI 1950.000P-TERPHENYL-D14

3900.000PHENOL-D5 $0624 \mathrm{HN}$ $0624 \mathrm{HN}$ $0624 \mathrm{HN}$ $0625 \mathrm{HN}$ O625HN $0625 \mathrm{HN}$ $0625 \mathrm{HN}$ 3900.0002-EIUOROPHENOL O625HN $3900.0002,4,6-T R I B R O M O P H E N O L 0625 \mathrm{HN}$ ORHENOI, $0625 \mathrm{HN}$ OBIS (2-CHIOROETHYL) ETO625HN 02-CHLOROPHENOL 0625HN 01, 3-DICHLOROBENZENE 0625 HN 01,4-DICHLOROBENZENE 0625HN OBENZYT ALCOHOT 01,2-DICHLOROBENZENE 0625HN 02-METHYIPHENOL $0625 \mathrm{HN}$ OBIS (2-CHLOROISOPROPYO625HN 04-METHYLPHENOL O625HN ON-NITROSO-DI-N-PROPYO625HN OHEXACHLOROETHANE 0625HN ONITROBENZENE OISOPHORONE O625HN 02-NITROPHENOI O625HN 02, 4-DIMETHYLPHENOI 0625HN OBENZOIC ACID O625HN OBIS (2-CHLOROETHOXY) MO625HN 02, 4-DICHLOROPHENOL 0625HN $01,2,4-T R I C H L O R O B E N Z E O 625 \mathrm{HN}$ ONAPHTHALENE O625HN 04-CHLOROANILINE 0625HN OHEXACHLOROBUTADIENE 0625HN 04-CHLORO-3-METHYLPHEO 625HN 02-METHYLNAPHTHALENE 0625HN OHEXACHLOROCYCLOPENTAO625HN $02,4,6-T R I C H L O R O P H E N O 0625 \mathrm{HN}$ $02,4,5-T R I C H L O R O P H E N O 0625 \mathrm{HN}$ 02-CHLORONAPHTHALENE O625HN 02-NITROANILINE 0625HN ODIMETHYLPHTHALATE O625HN OACENAPHTHYLENE $0625 \mathrm{HN}$ 
GRS 14010321900403900406 WA9003L865 01126 DNT 1XLT GRS 14010321900403900406 WA9003L865 011 3NANIL1XLT GRS 14010321900403900406 WA9003L865 011 ANAPNE1XLT GRS 14010321900403900406 WA9003L865 011 24DNP 1XLT GRS 14010321900403900406 WA9003L865 011 4NP 1XLT GRS 14010321900403900406 WA9003L865 011 DIBZFU1XLT GRS 14010321900403900406 WA9003L865 011 24DNT 1XLT GRS $14010321900403900406 \mathrm{WA} 9003 \mathrm{~L} 865011$ DEP 1XLT GRS 14010321900403900406 WA9003L865 011 4CLPPEIXLT GRS 14010321900403900406 WA9003L865 011 FLRENE1XLT GRS 14010321900403900406 WA9003L865 011 4NANIL1XLT GRS 14010321900403900406 WA9003L865 011 46DN2C1XLT GRS 14010321900403900406 WA9003L865 011 NNDPA 1XLT GRS 14010321900403900406 KA9003L865 011 4BRPRE1XLT GRS 14010321900403900406 WA9003L865 011 CL6BZ 1XLT GRS 14010321900403900406 WA9003L865 011 PCP 1XLT GRS 14010321900403900406 WA9003L865 011 PHANTR1XLT GRS $14010321900403900406 \mathrm{WA9003L865} 011$ ANTRC 1XLT GRS 14010321900403900406 WA9003L865 011 DNBP 1XLT GRS 14010321900403900406 WA9003L865 011 FANT 1XIT GRS 14010321900403900406 WA9003L865 011 PYR 1XLT GRS 14010321900403900406 WA9003L865 011 BBZP 1XLT GRS 14010321900403900406 WA9003L865 011 33CL2B1XLT GRS $14010321900403900406 \mathrm{KA} 9003 \mathrm{~L} 865011$ BAANTR1XLT $\sim$ GRS 14010321900403900406 WA9003L865 011 CHRY 1XLT If GRS $14010321900403900406 \mathrm{WA} 90034865011$ B2EHP $1 \times B$ GRS . 14010321900403900406 WA9003L865 011 DNOP 1XLT GRS $14010321900403900406 \mathrm{KA9003L865} 011$ BF2ANT1XLT GRS 14010321900403900406 WA9003I865 011 BKFANT1XLT GRS. 14010321900403900406 WA9003L865 011 BAPYR 1XLT GRS 14010321900403900406 WA9003L865 011 ICDPYR1XLT GRS $14010321900403900406 \mathrm{WA} 9003 \mathrm{~L} 865011$ DBAHA 1XIT GRS 14010321900403900406 WA9003L865 011 BGHIPY1XLT GRS 1301E0321900402900337WA9003L865 012 AG 2ZLT GRS 1301E0321900402900336WA9003L865 012 AS 1BLT GRS 1301E0321900402900337WA9003L865 $012 \mathrm{BA}$ 2ZLT GRS 1301E0321900402900337WA9003L865 012 CD 2ZLT GRS 1301E0321900402900337WA9003L865 $012 \mathrm{CR}$ 2ZLT GRS 1301E0321900408900056WA9003L865 012 HGTOT IDLT GRS 1301E0321900405900336WA9003L865 012 PB 1BLT GRS 1301E0321900404900336WA9003L865 012 SE 1BLT GRS 1301E0321900329900043WA9003L865 012 MEC6D82J GRS 1301E0321900329900043WA9003L865 012 PBEB 2J GRS 1301E0321900329900043WA9003L865 012. 12DCD42J GRS 1301E0321900329900043WA9003L865 012 CH3CL 2JLT GRS 1301E0321900329900043WA9003L865.012 CH3BR 2JLT GRS 1301E0321900329900043WA9003L865 012 C2H3CI2JLT GRS 1301E0321900329900043WA9003L865 012 C2H5CL2JLT
$390 U G K G$

2000UGKG

390 UGKG

2000 UGKG

$2000 U G K G$

390 UGKG

390 UGKG

390 UGKG

390 UGKG

390UGKG

2000 UGKG

2000 UGKG

390 UGKG

390 UGKG

39 OUGKG

2000UGKG

390 UGKG

390 UGKG

390UGKG

390UGKG

390 UGKG

390 UGKG

78 OUGKG

390 UGKG

390 UGKG

41 OUGKG

390 UGKG

390 UUKG

390 UGKG

390 UGKG

390 UGKG

390UGKG

390UGKG

10 . OUGL

10. OUGL

200 UGL

5 . OUGL

10. OUGL

0.20 UGL

3. OUGL

5. OUGI

103PER

106PER

77PER

1OUGL

10UGL.

10UGL'

10UGL
1. $0000 \mathrm{MSBKR}$

1. $0000 \mathrm{MSBKR}$

1. $0000 \mathrm{MSBKR}$

1. $0000 \mathrm{MSBKR}$

1. $0000 \mathrm{MSBKR}$

1. 000OMSBKR

1. 000OMSBKR

i. $0000 \mathrm{MSBKR}$

1. $0000 \mathrm{MSBKR}$

1.0000MSBKR

1.000OMSBKR

1. 0000MSBKR

1. 0000MSBKR

1. $0000 \mathrm{MSBKR}$

1.0000MSBKR

1. 0000MSBKR

1. 0000MSBKR

1. 0000MSBKR

1. 0000MSBKR

1. 0000MSBKR

1. 0000MSBKR

1. 0000MSBKR

1. $0000 \mathrm{MSBKR}$

1.000OMSBKR

1. 0000MSBKR

1.0000MSBKR

1. 0000MSBKR

1. 0000MSBKR

1. 0000MSBKR

1. 0000MSBKR

1. 0000MSBKR

$1.0000 \mathrm{MSBKR}$

1. 0000MSBKR

1. OOICALM

1. OOAAMLR

1. OOICALM

1. O0ICALM

1.00ICAIM

1. 0OHGJJW

1. 00AACMT

1. OOAAMLR

1. 0000MSMAZ

1. 0000MSMAZ

1. $0000 \mathrm{MSMAZ}$

1.0000MSMAZ

1. 000OMSMAZ

1. 00 OOMSMAZ

$1.0000 \mathrm{MSMAZ}$
02, 6-DINITROTOLUENE 03-NITROANILINE

OACENAP HTHENE

02, 4-DINITROPHENOL

04-NITROPHENOL

ODIBENZOEURAN

04-CHLOROPHENYI-PHENYO $25 \mathrm{HN}$

OFLUORENE 0625HN

04-NITROANILINE 0625HN

04, 6-D INITRO-2-METHYLO625HN

ON-NITROSODIPHENYLAMIO625HN

04-BROMOPHENYL-P HENYLO625HN

OHEXACHLOROBENZENE 0625HN

OPENTACHLOROPHENOL O625HN

OPHENANTHRENE $0625 \mathrm{HN}$

OANTHRACENE 0625HN

ODI-N-BUTYIPHTHALATE O625HN

OFLUORANTHENE 0625HN

ORYRENE 0625HN

OBUTYLBENZYLPHTHALATEO625HN

$03,3^{\prime}$-DICHLOROBENZIDI0625HN OBENZO (A) ANTHRACENE O625HN OCHRYSENE O625HN

OBIS (2-ETHYLHEXYL) PHTO625HN ODI-N-OCTYL PHTHALATEO625HN OBENZO (B) FLUORANTHENEO $625 \mathrm{HN}$ OBENZO (K) ELUORANTHENEO625HN OBENZO (A) PYRENE O625HN OINDENO ( $1,2,3-C D)$ PYREO625HN ODIBENZO $(\mathrm{A}, \mathrm{H})$ ANTHRACEO $625 \mathrm{HN}$ OBENZO (G, H, I) PERYLENEO $625 \mathrm{HN}$ OSILVER, TOTAL MAGTON OARSENIC, TOTAL MASTON OBARIUM, TOTAL MBATON OCADMIUM, TOTAL MCDTON OCHROMIUM, TOTAL MCRTON OMERCURY, TOTAL MHGTON OLEAD, TOTAL MPBTON OSELENIUM, TOTAL MSETON 50.000TOLUENE-D8 50.000BROMOELUOROBENZENE O624HN 50.0001, 2-DICHLOROETHANE-DO624HN OCHLOROMETHANE O624HN OBROMOMETHANE O624HN OVINYI CHIORIDE O624HN OCHLOROETHANE $-3624 \mathrm{HN}$ 
GRS 13L_-0321900329900043WA9003L865 012 CH2CL22JLT GRS 1301E0321900329900043WA9003L865 012 ACET 2JLT GRS 1301E0321900329900043WA9003L865 012 CS2. 2JLT GRS 1301E0321900329900043WA9003L865 012 11DCE 2JLT GRS 1301E0321900329900043WA9003L865 012 11DCLE2JLT GRS 1301E0321900329900043WA9003L865 012 T12DCE2JLT GRS 1301E0321900329900043WA9003L865 012 CHCL3 2JLT GRS 1301E0321900329900043WA9003L865 012 12DCLE2JLT GRS 1301E0321900329900043WA9003L865 012 MEK 2JLT GRS 1301E0321900329900043WA9003L865 012 111TCE2JLT GRS 1301E0321900329900043WA9003L865 012 CCL4 2JLT GRS 1301E0321900329900043WA9003L865 012 VINYLA2JLT GRS 1301E0321900329900043WA9003L865 012 BRDCLM2JLT GRS 1301E0321900329900043WA9003L865 012 12DCLP2JLT GRS 1301E0321900329900043WA9003L865 012 13DCPC2JLT GRS 1301E0321900329900043WA9003L865 012 TRCLE 2JLT GRS 1301E0321900329900043WA9003L865 012 DBRCLM2JLT GRS 1301E0321900329900043FA9003L865 012 112TCE2JLT GRS 1301E0321900329900043WA9003L865 012 C6H6 2JLT GRS 1301E0321900329900043WA9003L865 012 T13DCP2JLT GRS 1301E03219003299.00043WA9003L865 012 CHBR3 2JLT GRS 1301E0321900329900043WA9003L865 012 4ME2PE2JLT GRS 1301E0321900329900043WA9003L865 012 2HXONE2JLT If GRS 1301E0321900329900043WA9003L865 012 TCLEE 2JLT o GRS 1301E0321900329900043WA9003L865 012 TCLEA 2JLT जे GRS 1301E0321900329900043WA9003L865 012 MEC6H52JLT GRS 1301E0321900329900043WA9003L865 012 CLC6H52JLT GRS 1301E0321900329900043WA9003L865 012 ETC6H52JLT GRS 1301E0321900329900043WA9003L865 012 STYR 2JLT GRS 1301E0321900329900043WA9003L865 012 XYLEN 2JLT GRS 1301E0321900419901200WA9003L865 013 ALPHAG9W GRS 1301E0321900419901200WA9003L865 013 BETAG 9W GRS 1401B0321900329900043WA9003L865 014 MEC6D82J GRS 1401B0321900329900043WA9003L865 014 PBEB 2J GRS 1401B0321900329900043WA9003L865 014 12DCD42J GRS 1401B0321900329900043WA9003L865 014 CH3CL 2JLT GRS 1401B0321900329900043WA9003L865 014 CH3BR 2JLT GRS 1401B0321900329900043WA9003L865 014 C2H3CL2JLT GRS 1401B0321900329900043WA9003L865 014 C2H5CL2JLT GRS 1401B0321900329900043WA9003L865 014 CH2CL22JLT GRS 1401B0321900329900043WA9003L865 014 ACET 2JLT GRS 1401B0321900329900043WA9003L865 014 CS2 2JLT GRS 1401B0321900329900043KA9003L865 014 11DCE 2JLT GRS 1401B0321900329900043WA9003L865 014 11DCLE2JLT GRS 1401B0321900329900043WA9003L865 014 T12DCE2JLT GRS 1401B0321900329900043WA9003L865 014 CHCL3 2JLT GRS 1401B0321900329900043WA9003L865 014 12DCLE2JLT GRS 140.1B0321900329900043WA9003L865 014 MEK 2JLT
SUGL

1OUGL

SUGL

SUGL

SUGL

SUGL

5UGL

SUGL

IOUGL

5UGL

5UGL

1 OUGI

5UGL

SUGL

5UGL

SUGL

SUGL

5UGL

SUGL

5UGL

5UGL

1OUGL

1OUGL

5UGL

5UGL

5UGL

5UGL

5UGL

5UGL

5UGL

0.00 PCIL1.00

0.00 OPIL 4.00

94 PER

9 8PER

95PER

IOUGL

1OUGL

1OUGL

1 OUGL

5UGL

1OUGL

5UGL

5UGL

5UGL

5UGL

5UGL

5UGi

IOUGL
$1.0000 \mathrm{MSMAZ}$

$1.0000 \mathrm{MSMAZ}$

$1.0000 \mathrm{MSMAZ}$

$1.0000 \mathrm{MSMAZ}$

$1.0000 \mathrm{MSMAZ}$

$1.0000 \mathrm{MSMAZ}$

$1.0000 \mathrm{MSMAZ}$

$1.0000 \mathrm{MSMAZ}$

$1.0000 \mathrm{MSMAZ}$

$1.0000 \mathrm{MSMAZ}$

$1.0000 \mathrm{MSMAZ}$

$1.0000 \mathrm{MSMAZ}$

1.0000MSMAZ

$1.0000 \mathrm{MSMAZ}$

$1.0000 \mathrm{MSMAZ}$

$1.0000 \mathrm{MSMAZ}$

$1.0000 \mathrm{MSMAZ}$

$1.0000 \mathrm{MSMAZ}$

$1.0000 \mathrm{MSMAZ}$

$1.0000 \mathrm{MSMAZ}$

$1.0000 \mathrm{MSMAZ}$

$1.0000 \mathrm{MSMAZ}$

1. $0000 \mathrm{MSMAZ}$

$1.0000 \mathrm{MSMAZ}$

$1.0000 M S M A 2$

$1.0000 \mathrm{MSMA2}$

$1.0000 \mathrm{MSMAZ}$

$1.0000 \mathrm{MSMAZ}$

$1.0000 \mathrm{MSMAZ}$

$1.0000 \mathrm{MSMAZ}$ 10EBUNK 10EBUNK $1.0000 \mathrm{MSMAZ}$ 1.0000MSMAZ $1.0000 \mathrm{MSMAZ}$ $1.0000 \mathrm{MSMAZ}$ $1.0000 \mathrm{MSMAZ}$ $1.0000 \mathrm{MSMAZ}$ 1.0000MSMAZ 1.0000MSMAZ $1.0000 \mathrm{MSMAZ}$ $1.0000 M S M A 2$ $1.0000 \mathrm{MSMAZ}$ $1.0000 \mathrm{MSMAZ}$ 1.000OMSMAZ $1.0000 \mathrm{MSMAZ}$ $1.0000 \mathrm{MSMAZ}$ $1.0000 \mathrm{MSMA}$
OMETHYLENE CHLORIDE OACETONE

OCARBON DISULEIDE $0624 \mathrm{HN}$ $0624 \mathrm{HN}$ 01,1-DICHLOROETHENE 0624HN 01,1-DICHLOROETHANE 0624HN 01,2-DICHLOROETHENE $10624 \mathrm{HN}$ OCHLOROEORM O624HN 01,2-DICHLOROETHANE 0624HN 02-BUTANONE 0624HN $01,1,1-T R I C H L O R O E T H A N O 624 \mathrm{HN}$ OCARBON TETRACHLORIDEO $624 \mathrm{HN}$ OVINYL ACETATE O624HN OBROMODICHILOROMETHANEO624HN 01,2-DICHLOROPROPANE O624HN 0CIS-1, 3-DICHIOROPROPO624HN OTRICHLOROETHENE 0624HN OD.IBROMOCHLOROMETHANEO624HN $01,1,2-T R I C H L O R O E T H A N O 624 \mathrm{HN}$ OBENZENE $0624 \mathrm{HN}$ OTRANS-1, 3-DICHLOROPRO $624 \mathrm{HN}$ OBROMOFORM O624HN 04-METHYL-2-PENTANONEO624HN 02-HEXANONE 0624 HN OTETRACHLOROETHENE O624HN $01,1,2,2$-TETRACHLOROEO $624 \mathrm{HN}$ OTOLUENE OCHLOROBENZENE O624HN OETHYLBENZENE O624HN OSTYRENE OXYLENE (TOTAL) OGROSS ALPHA ONONVOLATILE BETA

$0624 \mathrm{HN}$ $0624 \mathrm{HN}$ IALPHN IBETAN 50. 000TOLUENE-D8 $0624 \mathrm{HN}$ 50.000BROMOFLUOROBENZENE O624HN $50.0001,2-D I C H L O R O E T H A N E-D O 624 \mathrm{kN}$ OCHLOROMETHANE O624HN OBROMOMETHANE O624HN OVINYL CHLORIDE $0624 \mathrm{HN}$ OCHLOROETHANE O624HN OMETHYLENE CHLORIDE $0624 \mathrm{HN}$ OACETONE O624HN OCARBON DISULFIDE O624HN 01,1-DICHLOROETHENE 0624HN 01,1-DICHLOROETHANE' O624HN 01,2-DICHLOROETHENE $10624 \mathrm{HN}$ OCHLOROFORM O624HN 01,2-DICHLOROETHANE O\$24HN 02-BUTANONE.$\quad 0624 \mathrm{HN}$ 
GRS 1401B0321900329900043WA9003L865 014111 TCE2JLT GRS 1401B0321900329900043WA9003L865 014 CCL4 2JLT GRS 1401B0321900329900043WA9003L865 014 VINYLA2JLT GRS 1401B0321900329900043WA9003LB65 014 BRDCLM2JLT GRS 1401B0321900329900043WA9003L865 014 12DCLP2JLT GRS 1401B0321900329900043WA9003L865 014 13DCPC2JLT GRS 1401B0321900329900043WA9003L865 014 TRCLE 2JLT GRS 1401B0321900329900043WA9003L865 014 DBRCLM2JLT GRS 1401B0321900329900043WA9003L865 014 112TCE2JLT GRS 1401B0321900329900043WA9003L865 014 C6H6. 2JLT GRS 1401B0321900329900043WA9003L865 014 T13DCP2JLT GRS 1401B0321900329900043WA9003L865 014 CHBR3 2JLT GRS 1401B0321900329900043WA9003L865 014 4ME2PE2JLT GRS 1401B0321900329900043WA9003L865 014 2HXONE2JLT GRS 1401B0321900329900043WA9003L865 014 TCLEE 2JLT GRS 1401B0321900329900043WA9003L865 014 TCLEA 2JLT GRS 1401B0321900329900043WA9003L865 014 MEC6H52JLT GRS 1401B0321900329900043WA9003L865 014 CLC6H52JLT GRS $140180321900329900043 \mathrm{WA} 9003 \mathrm{~L} 865014$ ETC6H52JLT GRS 1401B0321900329900043WA9003L865 014 STYR 2JLT GRS 1401B0321900329900043\%A9003L865 014 XYLEN 2JLT GRS 1401C0321900329900043WA9003L865 015 MEC6D82J GRS 1401C0321900329900043WA9003L865 015 PBFB 2J GRS 1401C0321900329900043WA9003L865 015 12DCD42J

1 GRS 1401C0321900329900043WA9003L865 015 CH3CL 2JLT \% GRS 1401C0321900329900043\%A9003L865 015 CH3BR 2JLT GRS 1401C0321900329900043WA9003L865 015 C2H3CL2JLT GRS 1401C0321900329900043WA9003L865 015 C2H5CL2JLT GRS 1401C0'321900329900043WA9003L865 015 CH2CL22JLT GRS 1401C0321900329900043WA9003L865 015 ACET 2JLT GRS 1401C0321900329900043WA9003L865 015 CS2 2JLT GRS . 1401C0321900329900043WA'9003L865 015 11DCE 2JLT GRS 1401C0321900329900043WA9003L865 015 11DCLE2JLT GRS 1401C0321900329900043WA9003L865 015 T12DCE2JLT GRS 1401C0321900329900043WA9003L865 015 CHCL3 2JLT GRS 1401C0321900329900043\%A9003L865 015 12DCLE2JLT GRS 1401C0321900329900043WA9003L865 015 MEK 2JLT GRS 1401C0321900329900043WA9003L865 015 111TCE2JLT GRS 1401C0321900329900043WA9003L865 015 CCL4 2JLT GRS 1401C0321900329900043WA9003L865 015 VINYLA2JLT GRS 1401C0321900329900043WA9003L865 015 BRDCLM2JLT GRS 1401C0321900329900043WA9003L865 015 12DCLP2JLT GRS 1401C0321900329900043WA9003L865 015 13DCPC2JLT GRS 1401C0321900329900043\%A9003L865 015 TRCLE 2JLT GRS 1401C0321900329900043WA9003L865 015 DBRCLM2JLT GRS 1401C0321900329900043\%A9003L865 015 I12TCE2JLT GRS 1401C0321900329900043WA9003L865 015 C6H6 2JLT GRS 1401C0321900329900043WA9003LB65 015 T13DCP2JLT
SUGL

5UGL

1OUGL

SUGL

SUGL

5UGL

SUGL

SUGL

SUGL

$5 U G L$

SUGL

SUGL

IOUGL

1OUGL

5UGI

5UGL

5UGL

5UGL

5UGI

5UGL

5UGL

101PER

107PER

93PER

1OUGL

10UGL

10UGL

1OUGL

5UGi

10UGL

5UGI

SUGL

5UGL

5 UGL

5UGL

5UGI

10UGI

5UGI

5UGI

IOUGL

5UGL

5UGL

5UGL

5UGL

5UGL

5UGL

5UGË
$1.0000 \mathrm{MSMAZ}$

$1.0000 \mathrm{MSMAZ}$

1. $0000 \mathrm{MSMAZ}$

1. $0000 \mathrm{MSMAZ}$

$1.0000 M S M A Z$

$1.0000 \mathrm{MSMAZ}$

$1.0000 \mathrm{MSMAZ}$

$1.0000 \mathrm{MSMAZ}$

$1.0000 \mathrm{MSMAZ}$

$1.0000 \mathrm{MSMAZ}$

$1.0000 \mathrm{MSMAZ}$

$1.0000 \mathrm{MSMAZ}$

$1.0000 \mathrm{MSMAZ}$

1. $0000 \mathrm{MSMAZ}$

$1.0000 \mathrm{MSMAZ}$

$1.0000 \mathrm{MSMAZ}$

$1.0000 \mathrm{MSMAZ}$

$1.0000 \mathrm{MSMAZ}$

$1.0000 M S M A Z$

$1.0000 \mathrm{MSMAZ}$

1.0000MSMAZ

$1.0000 \mathrm{MSMAZ}$

1. $0000 \mathrm{MSMAZ}$

$1.0000 M S M A Z$

$1.0000 \mathrm{MSMAZ}$

$1.0000 \mathrm{MSMAZ}$

$1.0000 \mathrm{MSMAZ}$

$1.0000 \mathrm{MSMAZ}$

$1.0000 \mathrm{MSMAZ}$

$1.0000 \mathrm{MSMAZ}$

$1.0000 \mathrm{MSMAZ}$

$1.0000 M S M A Z$

$1.0000 \mathrm{MSMAZ}$

1. $0000 \mathrm{MSMAZ}$

1.0000MSMAZ

$1.0000 \mathrm{MSMAZ}$

1.0000MSMAZ

1.0000MSMAZ

$1.0000 \mathrm{MSMAZ}$

$1.0000 \mathrm{MSMAZ}$

$1.0000 M S M A Z$

$1.0000 \mathrm{MSMAZ}$

1. $0000 M S M A Z$

$1.0000 \mathrm{MSMAZ}$

$1.0000 \mathrm{MSMAZ}$

$1.0000 \mathrm{MSMAZ}$

$1.0000 \mathrm{MSMAZ}$

$1.0000 \mathrm{MSMAZ}$
$01,1,1$-TRICHLOROETHANO $624 \mathrm{HN}$ OCARBON TETRACHLORIDE0624HN OVINYL ACETATE $0624 \mathrm{HN}$ OBROMODICHLOROMETHANEO624HN 01,2-DICHLOROPROPANE O624HN OCIS-1, 3-DICHLOROPROPO624HN OTRICHLOROETHENE O624HN ODIBROMOCHLOROMETHANEO624HN 01,1,2-TRICHLOROETHANO $624 \mathrm{HN}$ OBENZENE $0624 \mathrm{HN}$ OTRANS-1, 3-DICHLOROPRO624HN OBROMOFORM $0624 \mathrm{HN}$ 04-METHYL-2-PENTANONE0624HN 02-HEXANONE O624HN OTETRACHLOROETHENE O624HN 01,1,2, 2-TETRACHLOROEO $624 \mathrm{HN}$ OTOLUENE $0624 \mathrm{HN}$ OCHLOROBENZENE O624HN OETHYLBENZENE $\quad 0624 \mathrm{HN}$ OSTYRENE $0624 \mathrm{HN}$ OXYLENE (TOTAL) O624HN 50.000TOLUENE-D8 O624HN 50.000BROMOFLUOROBENZENE O624HN $50.0001,2-D I C H L O R O E T H A N E-D 0624 \mathrm{HN}$ OCHLOROMETHANE O624HN OBROMOMETHANE O624HN OVINYL CHLORIDE O624HN OCHLOROETHANE O624HN OMETHYLENE CHLORIDE O624HN OACETONE OCARBON DISULFIDE 01,1-DICHLOROETHENE 01,1-DICHLOROETHANE 01,2-DICHLOROETHENE OCHLOROFORM 01,2-DICHLOROETHANE O624HN 02-BUTANÓNE O624HN 01, 1, 1-TRICHLOROETHANO 624HN OCARBON TETRACHLORIDEO624HN OVINYL ACETATE O624HN OBROMODICHLOROMETHANEO $624 \mathrm{HN}$ 01,2-DICHLOROPROPANE O624HN OCIS-1, 3-DICHLOROPROPO624HN OTRICHLOROETHENE $\because 0624 \mathrm{HN}$ ODIBROMOCHLOROMETHANEO $624 \mathrm{HN}$ 01, 1, 2-TRICHLOROETHANO $624 \mathrm{HN}$ OBENZENE $0624 \mathrm{HN}$ OTRANS-1, 3-DICHLORO $624 \mathrm{HN}$ 
GRS 14: $0321900329900043 \omega A 9003 L 865015$ CHBR3 2JLT GRS 1401C0321900329900043WA9003L865 015 4ME2PE2JLT GRS 1401C0321900329900043HA9003L865 015 2HXONE2JLT GRS 1401C0321900329900043WA9003L865 015 TCLEE 2JLT GRS 1401C0321900329900043WA9003L865 015̣ TCLEA 2JLT GRS 1401C0321900329900043WA9003L865 015 MEC6H52JLT GRS 1401C0321900329900043\%A9003L865 015 CLC6H52JLT GRS 1401C0321900329900043WA9003L865 015 ETC6H52JLT GRS 1401C0321900329900043WA9003L865 015 STYR 2JLT GRS 1401C0321900329900043WA9003L865 015 XYLEN 2JLT
0405900055 FA90C055B LC1 LHGTOT 1D 0405900055 WA90C055B LC1 LHGTOT 1D 0405900055WA90C055B LC2 LHGTOT 1D 0405900055 $\mathrm{FA90C055B}$ LC2 LHGTOT 1D 0405900055WA90C055B LC3 LHGTOT ID 0405900055WA90C055B LC3 LHGTOT 1D 0405900055WA90C055B. LC4 LHGTOT ID 0405900055WA90C055B LC4 LHGTOT 1D 0405900055WA90C055B MB1 HGTOT 1DLT $0405900055 \mathrm{WA} 90 \mathrm{C055B}$ MB2 HGTOT 1DLT 0405900055WA90C055B MB3 HGTOT 1DLT 0405900055 KA90C055B MB4 HGTOT 1DLT 0408900056 FA90C056A LCI LHGTOT 1D 0408900056WA90C056A LC1 LHGTOT ID 0408900056WA90C056A LC2 LHGTOT 1D 0408900056WA90C056A LC2 LHGTOT 1D 0408900056 WA90C056A LC3 LHGTOT 1D 0408900056WA90C056A LC3 LHGTOT 1D 0408900056WA90C056A MB1 HGTOT 1DLT 0408900056 FA90C056A MB2 HGTOT 1DLT 0408900056WA90C056A MB3 HGTOT 1DLT 0402900336 WैA 9010296 LC1 LAS $0402900336 \mathrm{WA} 90 \mathrm{~L} 0296$ LC1 LAS 0402900336 WA90L0296 LC2 LAS $0402900336 \mathrm{FA} 90 \mathrm{~L} 0296$ LC2 LAS 0402900336WA90L0296 MB1 AS 0405900336 WA90L0296 LC1 LPB $0405900336 \mathrm{WA} 90 \mathrm{~L} 0296$ LC1 LPB 0405900336 WA90L0296 LC2 LPB 0405900336 WA90L0296 LC2 LPB 0405900336 FA90L0296 MBI PB 0404900336 WA90L0296 IC1 LSE 0404900336\%A90L0296 LC1 LSE 0404900336 FA9 $0 L 0296$ LC2 LSE 0404900336 WA90L0296 LC2 LSE 0404900336КA90L0296 MB1 SE 0402900337WA90L0297 LC1 LAG 0402900337 FA90L0297 LC1 LLAG
jUGI

1OUGI

1OUGL

SUGL

SUGI

SUGL

SUGL

5UGL

SUGL

5UGL

$0.96 \mathrm{MGKG}$

96.2PER

1.1MGKG

105PER

1. OMGKG

102PER

1. 1MGKG

108PER

$0.10 \mathrm{MGKG}$

$0.10 \mathrm{MGKG}$

$0.10 \mathrm{MGKG}$

$0.10 \mathrm{MGKG}$

2. OUGL

102PER

2.3UGL

113PER

2. 3UGL

113PER

0.20 UGL

0.20 UGL

0.20 UGL

27. 7UGL

$92.3 P E R$

28.9UGL

96.3PER

10. OUGL

32.4 UGL

108PER

32. 9UGL

11OPER

3. OUGL

30. 4UGL

101PER

31.5UGL

105PER

5. OUGL

$586 U G L$

117PER
$1.0000 \mathrm{MSMAZ}$

1.0000 MSMAZ

1.0000 MSMAZ

$1.0000 \mathrm{MSMAZ}$

$1.0000 M S M A Z$

$1.0000 \mathrm{MSMAZ}$

1. 0000 MSMAZ

$1.0000 \mathrm{MSMAZ}$

$1.0000 \mathrm{MSMAZ}$

1.0000MSMAZ

$1.00 \mathrm{HGJJW}$

$1.00 \mathrm{HGJJW}$

$1.00 \mathrm{HGJJW}$

$1.00 \mathrm{HGJJW}$

$1.00 \mathrm{HGJJW}$

$1.00 \mathrm{HGJJW}$

$1.00 \mathrm{HGJJW}$

$1.00 \mathrm{HGJJW}$

$1.00 \mathrm{HGJJW}$

$1.00 \mathrm{HGJJW}$

$1.00 \mathrm{HGJJW}$

$1.00 \mathrm{HGJJW}$

$1.00 \mathrm{HGJJW}$

$1.00 \mathrm{HGJJW}$

$1.00 \mathrm{HGJJW}$

$1.00 \mathrm{HGJJW}$

$1.00 \mathrm{HGJJW}$

1.00 HGJJW

$1.00 \mathrm{HGJJW}$

$1.00 \mathrm{HGJJW}$

$1.00 \mathrm{HGJJW}$

1.00AAMLR

1.00AAMLR

1.00 AAMLR

1. 00AAMLR

1.00AAMLR

1. O0ICCMT

1.00 ICCMT

1.00 ICCMT

1.00 ICCMT

1.00AACMT

1. OOICMLR

1.00ICMLR

1. OOICMLR

1.00 ICMLR

1. 00AAMLR

1 .00ICAIM 1. O0ICALM
OBROMOFORM

$0624 \mathrm{HN}$

04-METHYL-2-PENTANONEO624HN 02-HEXANONE O624HN OTETRACHLOROETHENE O624HN $01,1,2,2-T E T R A C H L O R O E O 624 \mathrm{HN}$ OTOLUENE OCHLOROBENZENE O624HN OETHYYLBENZENE O624HN OSTYRENE OXYLENE (TOTAL) OMERCURY, LCS

1. OMERCURY, LCS OMERCURY， LCS

1. OMERCURY, LCS OMERCURY, ICS

1. OMERCURY, LCS OMERCURY, LCS

1. OMERCURY, LCS OMERCURY, TOTAI OMERCURY, TOTAL OMERCURY, TOTAL OMERCURY, TOTAL OMERCURY, LCS

2. OMERCURY, LCS OMERCURY, LCS

2. OMERCURY, LCS OMERCURY, LCS

2. OMERCURY, ICS OMERCURY, TOTAL OMERCURY, TOTAL OMERCURY, TOTAL OARSENIC, LCS

30. OARSENIC, LCS OARSENIC, LCS

30. OARSENIC, LCS OARSENIC, TOTAL OLEAD, LCS

30. OLEAD, ICS OLEAD, LCS

30. OLEAD, LCS OLEAD, TOTAL OSELENIUM, LCS

30. 0.SELENIUM, LCS OSELENIUM, LCS

30. OSELENIUM, LCS OSELENIUM, TOTAL OSILVER, LCS 50OSILVER, LCS.
$0624 \mathrm{HN}$ $0624 \mathrm{HN}$ MHGLCL MHGLCL MHGLCL MHGLCL MHGLCL MHGLCL MHGLCL MHGLCL MHGTON MHGTON MHGTON MHGTON MHGLCL MHGLCL MHGLCI MHGLCI MHGLCL MHGLCL MHGTON MHGTON MHGTON MASLCL MASLCL MASLCL MASLCL MASTON MPBLCL MPBLCL MPBLCI MPBLCL MPBTON MSELCL MSELCL MSELCL MSELCL MSETON MAGLCL MAGLCL 


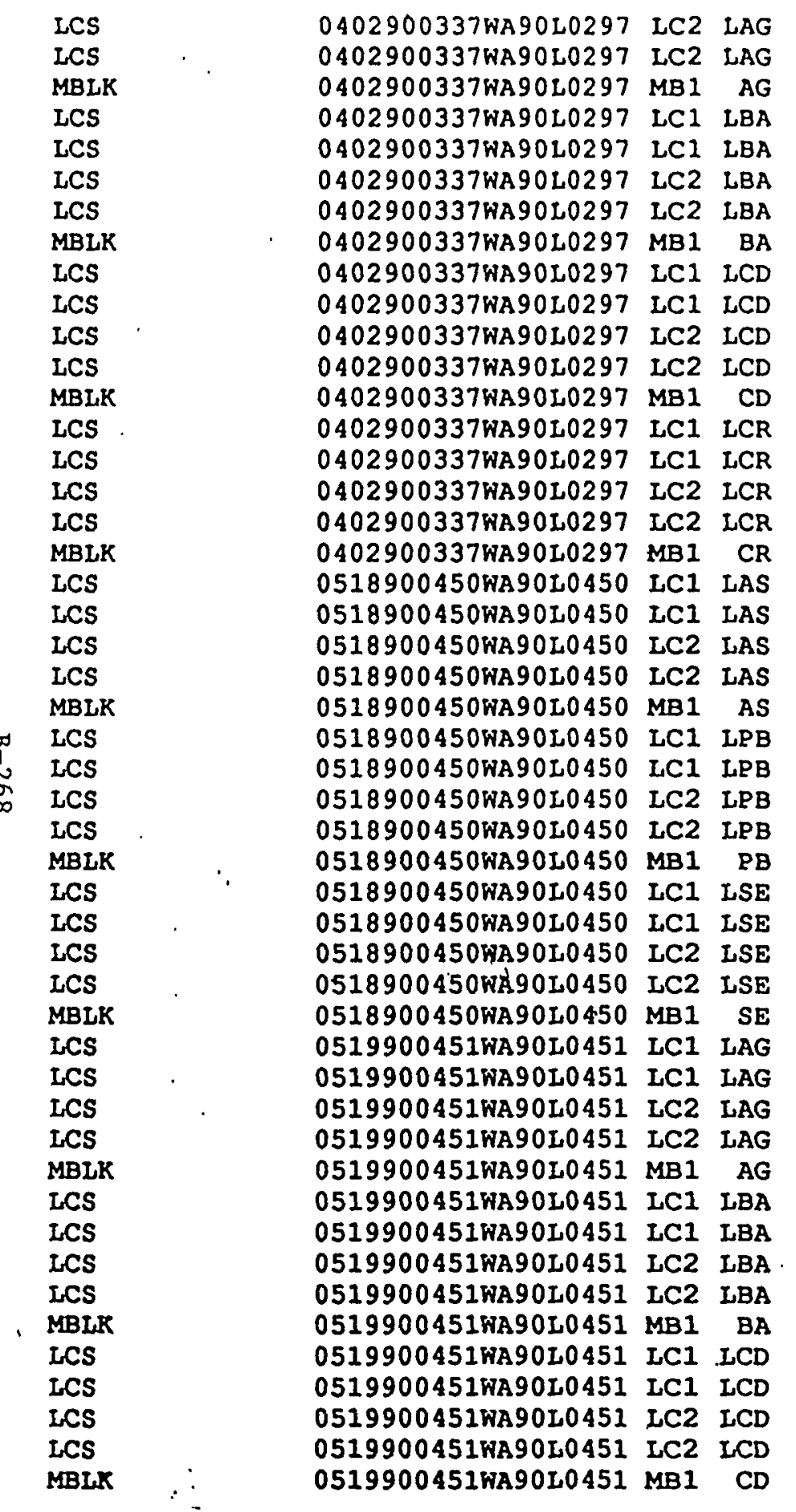

\begin{tabular}{|c|c|c|}
\hline 22 & 568 UGL & 1.00 ICALM \\
\hline $2 z$ & 114PER & 1.00 ICALM \\
\hline $2 \mathrm{ZLT}$ & 10.0 UGL & 1.00 ICALM \\
\hline 22 & 5120UGL & 1.00 ICALM \\
\hline $2 z$ & 102PER & 1.00 ICALM \\
\hline $2 z$ & 5100UGL & 1.00 ICALM \\
\hline 22 & 102PER & 1.00 ICALM \\
\hline $2 \mathrm{ZLT}$ & 200 UGL & 1.00 ICALM \\
\hline $2 z$ & 312UGL & 1.00 ICALM \\
\hline 22 & 125PER & 1.00 ICALM \\
\hline 22 & 296 UGL & 1.00 ICALM \\
\hline $2 z$ & $118 \mathrm{QER}$ & $1.00 \mathrm{ICALM}$ \\
\hline 2ZLT & 5. OUGL & 1.00ICALM \\
\hline 22 & 588 UGL & $1.00 \mathrm{ICALM}$ \\
\hline $2 z$ & 118PER & 1.00ICALM \\
\hline $2 z$ & 581UGL & 1. OOICALM \\
\hline 22 & 116PER & 1.00 ICALM \\
\hline 2ZLT & 10. OUGL & $1.00 I \mathrm{CALM}$ \\
\hline $1 B$ & 5. 3MGKG & 1. O0AAAER \\
\hline $1 B$ & 88. OPER & 1.00AAAER \\
\hline $1 B$ & 5. 8MGKG & 1.00AAAER \\
\hline $1 B$ & 97. 3PER & 1. 00AAAER \\
\hline 1BLT & 2. OMGKG & 1. OOAAAER \\
\hline IB & 5. 5MGKG & 1.00 ICEJS \\
\hline $1 B$ & 91. OPER & 1.00ICEJS \\
\hline $1 B$ & 5. 8MGKG & 1.00 ICEJS \\
\hline $1 \mathrm{~B}$ & 96. OPER & 1.00ICEJS \\
\hline 1BLT & $0.60 M G K G$ & 1. OOAAEJS \\
\hline $1 \mathrm{~B}$ & $7.0 M G K G$ & 1.00ICMLR \\
\hline IB & 117PER & 1.00ICMLR \\
\hline $1 B$ & $7.1 \mathrm{MGKG}$ & 1.00ICMLR \\
\hline $1 B$ & 119PER & 1.00ICMLR \\
\hline 1BLT & 1. OMGKG & 1. OOAAMLR \\
\hline 27 & 100MGKG & 1.00ICALM \\
\hline $2 \mathrm{z}$ & 10OPER & 1.00ICALM \\
\hline $2 z$ & 103MGKG & 1.00ICAIM \\
\hline $2 z$ & 103PER & 1.00ICALM \\
\hline 2ZLT & 4. OMGKG & 1.00ICALM \\
\hline 22 & 1020MGKG & 1.00ICALM \\
\hline 22 & 102PER & 1.00ICALM \\
\hline $2 \mathrm{z}$ & 1030MGKG & 1.00ICALM \\
\hline 22 & 103PER & 1. OOICALM \\
\hline 2ZLT & 5. OMGKG & $1.00 \mathrm{ICALM}$ \\
\hline $2 z$ & 46.7MGKG & 1.00 ICAIM \\
\hline $2 z$ & 93.3PER & 1.00ICALM \\
\hline $2 \mathrm{Z}$ & 46.5MGKG & $1.00 \mathrm{ICALM}$ \\
\hline $2 z$ & 93. OPER & $1.00 \mathrm{ICALM}$ \\
\hline 2ZLT & 2.0MGKG & 1.00ICALM \\
\hline
\end{tabular}

OSILVER, LCS

5OOSILVER, LCS

OSILVER, TOTAL

OBARIUM, LCS

5000 BARIUM, LCS

OBARIUM, ICS

5000BARIUM, LCS OBARIUM, TOTAL OCADMIUM, LCS

250CADMIUM, LCS OCADMIUM, LCS

250CADMIUM, LCS OCADMIUM, TOTAL OCHROMIUM, LCS

500CHROMIUM, LCS OCHROMIUM, LCS

50OCHROMIUM, LCS OCHROMIUM, TOTAL OARSENIC, LCS

6. OARSENIC, LCS OARSENIC, LCS

6.0ARSENIC, LCS OARSENIC, TOTAL OLEAD, LCS

6. OLEAD, LCS OLEAD, ICS

6. OLEAD, LCS OLEAD, TOTAL OSELENIUM, LCS

6. OSELENIUM, LCS OSELENIUM, LCS

6. OSELENIUM, LCS OSELENIUM, TOTAL OSILVER, LCS

100SILVER, LCS OSILVER, ICS

1OOSILVER, LCS OSILVER, TOTAL OBARIUM, LCS

1000BARIUM, LCS OBARIUM, LCS

1000BARIUM, LCS OBARIUM, TOTAL OCADMIUM, LCS

50. OCADMIUM, LCS OCADMIUM, LCS

50. OCADMIUM, LCS OCADMIUM, TOTAL

MAGLCL

MAGLCL

MAGTON

MBALCL

MBALCL

MBALCL

MBALCL

MBATON

MCDLCL

MCDLCL

MCDLCL

MCDLCL

MCDTON

MCRLCL

MCRLCL

MCRLCL

MCRLCL

MCRTON

MASLCL

MASLCL

MASICI

MASLCL

MASTON

MPBLCL

MPBLCL

MPBLCI

MPBLCL

MPBTON

MSELCL

MSELCL

MSELCI

MSELCL

MSETON

MAGLCL

MAGLCL

MAGLCL

MAGLCL

MAGTON

MBALCL

MBALCL

MBALCL

MBALCL

MBATON

MCDICL

MCDLCL

MCDLCL

MCDLCL w9003865.r $5 / 26 / 90-34$ 
0519900451 WA90L0451 LCI LCR 0519900451 WA90L0451 LCI LCR 0519900451 HA90L0451 LC2 LCR 0519900451 KA90L0451 LC2 LCR 0519900451WA90L0451 MBI CR 0402900406 WA90LE0366 MB1 NBD5 $1 X$ 0402900406 WA90LE0366 MB1 2FBP $1 X$ 0402900406 KA 90LE0366 MB1 PTERP IX 0402900406 WA90LE0366 MB1 PHEND51X 0402900406 WA90LE0366 MB1 2FP 1X 0402900406 WA90LE0366 MBI 246TBP $1 X$ $0402900406 \mathrm{WA} 90 \mathrm{LE} 0366 \mathrm{MB} 1$ PHENOL 1 XLT 0402900406 WA90LE0366 MB1 B2CEE 1XLT 0402900406 WA90LE0366 MB1 2CLP 1XLT 0402900406 WA90LE0366 MB1 13DCLB1XLT 0402900406 FA90LE0366 MB1 14DCLB1XLT 0402900406 WA90LE0366 MB1 BZALC 1XLT $0402900406 \mathrm{FA90LE0366} \mathrm{MB1} \mathrm{120CLB1XLT}$ 0402900406 FA90LE0366 MB1 2MP 1XLT 0402900406 WA90LE0366 MB1 B2CIPE1XLT 0402900406 FA90LE0366 MB1 4MP 1XLT 0402900406 WA90LE0366 MB1 NDNPA 1XLT 0402900406 WA90LE0366 MB1 CL6ET 1XLT $0402900406 \mathrm{WA} 90 \mathrm{LE} 0366 \mathrm{MB1}$ NB 1XLT 0402900406 WA90LE0366 MB1 ISOPHR1XLT $0402900406 \mathrm{WA} 90 \mathrm{LE} 0366 \mathrm{MB1}$ 2NP 1XLT $0402900406 \mathrm{WA} 90 \mathrm{LE} 0366 \mathrm{MB1}$ 24DMPN1XLT 0402900406 WA90LE0366 MB1 BENZOA1XLT 0402900406 WA90LE0366 MB1 B2CEXM1XLT 0402900406 WA90LE0366 MB1 24DCLP1XLT 0402900406 WA90LE0366 MB1 124TCB1XLT 0402900406 WAO 0 LE0366 MB1 NAP 1XLT 0402900406 WA90LE0366 MB1 . 4CANIL1XLT 0402900406 WA90LE0366 MB1 HCBD 1XLT 0402900406 WA90LE0366 MB1 4CL3C 1XLT 0402900406 WA90LE0366 MB1 2MENAP 1XLT 0402900406 WA90LE0366 MBI CL6CP 1XLT 0402900406WA90LE0366 MB1 246TCP IXLT 0402900406 WA9OLE0366 MB1 245TCP1XLT 0402900406 WA9OLE0366 MB1 2CNAP 1XLT 0402900406 WA90LE0366 MBI 2NANILIXIT 0402900406 WA90LE0366 MB1 DMP IXLT 0402900406 WA90IE0366 MB1 ANAPYL1XIT $0402900406 \mathrm{WA} 90 \mathrm{LE0366} \mathrm{MB1}$ 26DNT 1XLT 0402900406 KA90LE0366 MBI 3NANIL1XLT 0402900406 WA90LE0366 MB1 ANAPNE1XIT 0402900406WA9OLE0366 MBI 24DNP 1XLT 0402900406 HA90LE0366 MBI 4NR 1XIT

\begin{tabular}{|c|c|}
\hline $99.9 M G K G$ & $1.00 I C A L M$ \\
\hline $99.9 P E R$ & 1.00ICAIM \\
\hline 102MGKG & 1.00ICALM \\
\hline 102PER & 1.00ICALM \\
\hline 4. OMGKG & 1.00ICALM \\
\hline 70PER & $1.0000 \mathrm{MSCDT}$ \\
\hline 68PER & $1.0000 \mathrm{MSCDT}$ \\
\hline 84 PER & $1.0000 \mathrm{MSCDT}$ \\
\hline 71PER & $1.0000 \mathrm{MSCDT}$ \\
\hline 67PER & $1.0000 \mathrm{MSCDT}$ \\
\hline 60PER & $1.0000 \mathrm{MSCDT}$ \\
\hline 330 UGKG & $1.0000 \mathrm{MSCDT}$ \\
\hline 330 UGKG & $1.0000 \mathrm{MSCDT}$ \\
\hline 330 UGKG & $1.0000 \mathrm{MSCDT}$ \\
\hline 330 UGKG & $1.0000 \mathrm{MSCDT}$ \\
\hline 330 UGKG & $1.0000 \mathrm{MSCDT}$ \\
\hline 330 UGKG & $1.0000 M S C D T$ \\
\hline 330UGKG & $1.0000 \mathrm{MSCDT}$ \\
\hline 330 UGKG & $1.0000 \mathrm{MSCDT}$ \\
\hline 330 UGKG & 1.0000MSCDT \\
\hline 330 UGKG & $1.0000 \mathrm{MSCDT}$ \\
\hline 330 UGKG & $1.0000 \mathrm{MSCDT}$ \\
\hline 330UGKG & $1.0000 \mathrm{MSCDT}$ \\
\hline 330 UGKG & $1.0000 \mathrm{MSCDT}$ \\
\hline 330UGKG & $1.0000 \mathrm{MSCDT}$ \\
\hline 330UGKG & $1.0000 \mathrm{MSCDT}$ \\
\hline 330UGKG & $1.0000 \mathrm{MSCDT}$ \\
\hline 1700 UGKG & 1. $.0000 \mathrm{MSCDT}$ \\
\hline 330UGKG & $1: 0000 \mathrm{MSCDT}$ \\
\hline 330UGKG & $1.0000 \mathrm{MSCDT}$ \\
\hline 330 UGKG & $1.0000 \mathrm{MSCDT}$ \\
\hline 330 UGKG & $1.0000 \mathrm{MSCDT}$ \\
\hline 330UGKG & $1.0000 \mathrm{MSCDT}$ \\
\hline 330UGKG & $1.0000 \mathrm{MSCDT}$ \\
\hline 330 UGKG & $1.0000 \mathrm{MSCDT}$ \\
\hline 330UGKG & $1.0000 \mathrm{MSCDT}$ \\
\hline 330UGKG & $1.0000 \mathrm{MSCDT}$ \\
\hline 330UGKG & $1.0000 \mathrm{MSCDT}$ \\
\hline 1700UGKG & $1.0000 \mathrm{MSCDT}$ \\
\hline 330UGKG & $1.0000 \mathrm{MSCDT}$ \\
\hline 1700UGKG & $1.0000 \mathrm{MSCDT}$ \\
\hline 330UGKG & $1.0000 \mathrm{MSCDT}$ \\
\hline 330UGKG & $1.0000 \mathrm{MSCDT}$ \\
\hline 330UGKG & $1.0000 \mathrm{MSCDT}$ \\
\hline 1700UGKG & $1.0000 \mathrm{MSCDT}$ \\
\hline .330UGKG & $1.0000 M S C D T$ \\
\hline 1700 UGKG & $1.0000 \mathrm{MSCDT}$ \\
\hline 1700 UGKG & 1.0000MSCDT \\
\hline
\end{tabular}
w9003865. dat $-6 / 26 / 90-35$
OCHROMIUM, ICS

100CHROMIUM, ICS

OCHROMIUM, ICS

100CHROMIUM, ICS

OCHROMIUM, TOTAI

1665.000NITROBENZENE-D5

1665.0002-ELUOROBIPHENYL

1665.000P-TERPHENYL-D14

3330 . 000PHENOL-D5

3330 . 0002-EIUOROPHENOI

$3330.0002,4,6$-TRIBROMOPHENOLO $625 \mathrm{HN}$ OPHENOL $0625 \mathrm{HN}$ OBIS (2-CHLOROETHYI) ETO625HN 02-CHLOROPHENOL O625HN 01, 3-DICHLOROBENZENE O625HN $01,4-D I C H L O R O B E N Z E N E$ O625HN OBENZYL ALCOHOL O625HN 01,2-DICHLOROBENZENE 0625HN 02-METHYLPHENOL $0625 \mathrm{HN}$ OBIS (2-CHLOROISORROPYO $625 \mathrm{HN}$ 04-METHYLPHENOL O625HN ON-NITROSO-DI-N-PROPYO625HN OHEXACHLOROETHANE O625HN ONITROBENZENE O625HN OISOPHORONE 02-NITROPHENOI $0625 \mathrm{HN}$ 02,4-DIMETHYIPHENOI 0625HN OBENZOIC ACID O625HN OBIS (2-CHLOROETHOXY) MO625HN $02,4-D I C H L O R O P H E N O I$ O625HN $01,2,4-T R I C H L O R O B E N Z E 0625 \mathrm{HN}$ ONAP HTHALENE O625HN 04-CHLOROANILINE O625HN OHEXACHLOROBUTADIENE O625HN 04-CHLORO-3-METHYLPHEO625HN 02-METHYINAPHTHALENE 0625HN OHEXACHLOROCYCLOPENTAO625HN $02,4,6-T R I C H L O R O P H E N O 0625 \mathrm{HN}$ $02,4,5-T R I C H L O R O P H E N O 0625 \mathrm{HN}$ 02-CHLORONAPHTHALENE O625HN 02-NITROANILINE O625HN ODIMETHYLPHTHALATE O625HN OACENAPHTHYLENE $0625 \mathrm{HN}$ 02, 6-DINITROTOLUENE' - 0625HN 03-NITROANILINE O625HN OACENAPHTHENE $0625 \mathrm{HN}$ 02, 4-DINITRORHENOI O625HN 04-NITRORHENOL 
0402900406WA90LE0366 MB1 DIBZFU1XLT 0402900406 WA90LE0366 MB1 24DNT 1XLT 0402900406 KA $90 L E 0366$ MBI DEP 1XLT 0402900406WA90LE0366 MB1 4CLPPE1XLT 0402900406 WA $90 L E 0366$ MB1 FLRENE 1XLT $0402900406 \mathrm{WA} 90 \mathrm{LE} 0366 \mathrm{MB1}$ 4NANIL1XLT 0402900406 WA90LE0366 MB1 46DN2C1XLT $0402900406 \mathrm{KA} 90 \mathrm{LE} 0366 \mathrm{MB1}$ NNDPA 1 XLT 0402900406 WA90LE0366 MB1 4BRPPE1XLT $0402900406 \mathrm{WA} 90 \mathrm{LE} 0366$ MB1 CL6BZ 1XLT $0402900406 \mathrm{WA} 90 \mathrm{LE} 0366 \mathrm{MB1}$ PCP 1 XLT 0402900406 WA 90 LE0366 MB1 PHANTR1XLT 0402900406 WA9OLE0366 MB1 ANTRC 1XLT $0402900406 \mathrm{WA} 90 \mathrm{LE} 0366$ MB1 DNBP 1XLT 0402900406 WA9OLE0366 MB1 FANT 1XLT 0402900406 WA90LE0366 MB1 PYR 1XLT $0402900406 \mathrm{KA} 90 \mathrm{LE} 0366 \mathrm{MB1}$ BB2P 1XLT $0402900406 \mathrm{WA} 90 \mathrm{LE} 0366 \mathrm{MBI}$ 33CL2B1XLT 0402900406 WA90LE0366 MB1 BAANTR1XLT $0402900406 \mathrm{KA} 90 \mathrm{LE} 0366 \mathrm{MBI}$ CHRY 1XLT 0402900406 WA90LE0366 MB1 B2EHP 1XLT 0402900406 KA90LE0366 MBI DNOP 1XLT $0402900406 \mathrm{WA} 90 \mathrm{LE} 0366 \mathrm{MB1}$ BE2ANT1XLT 0402900406 WA90LE0366 MB1 BKEANT1XLT 0402900406 WA9OLE0366 MBI BAPYR 1XLT 0402900406 WA90LE0366 MBI ICDPYRIXLT 0402900406 WA90LE0366 MB1 DBAHA 1XLT 0402900406 WA90LE0366 MB1 BGHIPY1XLT 0402900406 WA9OLE0366 MBISNBDS $1 \mathrm{X}$ 0402900406 WA90LE0366 MB1S2FBP $1 \mathrm{X}$ 0402900406 WA90LE0366 MB1SPTERP 1X 0402900406 Wึม 90 LE0366 MB1SPHEND51X 0402900406 WA90LE0366 MB1S2FP 1X 0402900406 WA90LE0366 MB1S246TBP1X 0402900406 WA90LE0366 MB1SPHENOL1X $0402900406 \mathrm{WA} 90 \mathrm{LE} 0366$ MB1SB2CEE 1XLT 0402900406 WA90LE0366 MB1S2CLP 1X 0402900406 WA90LE0366 MB1S13DCLB1XLT 0402900406 WA90LE0366 MB1S14DCLB1X 0402900406 WA90LE0366 MB1SBZALC 1XLT 0402900406 WA90LE0366 MB1S12DCLBIXLT 0402900406 KA $90 L E 0366$ MBIS2MP 1XLT 0402900406 WA90LE0366 MB1SB2CIPE1XLT 0402900406WA90LE0366 MB1S4MP 1XIT 0402900406 WA90LE0366 MB1SNDNPA $1 X$ 0402900406 WA90LE0366. MB1SCL6ET 1XLT 0402900406 WA90LE0366 MB1SNB 1XLT $0402900406 \mathrm{WA} 90 L E 0366$ MB1SISOPHR1XLT
330UGKG

330UGKG

330UGKG

330UGKG

330UGKG

1700UGKG

170 OUGKG

330UGKG

330UGKG

330UGKG

1700 UGKG

330UGKG

330UGKG

33OUGKG

330UGKG

330UGKG

330UGKG

670UGKG

330UGKG

330UGKG

330UGKG

33OUGKG

330UGKG

330UGKG

33OUGKG

330UGKG

33OUGKG

330UGKG

70PER

82PER

8IRER

89PER

$94 \mathrm{PER}$

73PER

97PER

330UGKG

86RER

330UGKG

73PER

33OUGKG

330UंGKG

33OUGKG

330UGKG

330UGKG

9OPER

330UGKG

330UGKG

330UGKG
1.0000MSCDT $1.0000 M S C D T$ $1.0000 \mathrm{MSCDT}$

$1.0000 M S C D T$

$1.0000 \mathrm{MSCDT}$

$1.0000 \mathrm{MSCDT}$

$1.0000 \mathrm{MSCDI}$

$1.0000 \mathrm{MSCDT}$

1.0000MSCDT

$1.0000 \mathrm{MSCDT}$

$1.0000 \mathrm{MSCDT}$

$1.0000 \mathrm{MSCDT}$

$1.0000 \mathrm{MSCDT}$

$1.0000 \mathrm{MSCDT}$

$1.0000 \mathrm{MSCDT}$

$1.0000 \mathrm{MSCDT}$

$1.0000 \mathrm{MSCDT}$

$1.0000 \mathrm{MSCDT}$

$1.0000 \mathrm{MSCDT}$

1.0000MSCDT

$1.0000 \mathrm{MSCDT}$

$1.0000 \mathrm{MSCDT}$

$1.0000 \mathrm{MSCDT}$

$1.0000 \mathrm{MSCDI}$

$1.0000 \mathrm{MSCDT}$

$1.0000 \mathrm{MSCDT}$

$1.0000 \mathrm{MSCDT}$

1.0000MSCDT

1.0000MSDAR

1.0000MSDAR

1.0000MSDAR

1.0000MSDAR

1.0000MSDAR

1.0000MSDAR

1.0000MSDAR

$1.0000 \mathrm{MSDAR}$

1.0000MSDAR

1.0000MSDAR

1.0000MSDAR

1.0000MSDAR

1. 0000MSDAR

1.0000MSDAR

1.0000MSDAR

1. O00OMSDAR

1.0000MSDAR

1. 0000MSDAR

1.0000MSDAR

1.0000MSDAR
ODIBENZOFURAN

$0625 \mathrm{HN}$

02,4-DINITROTOLUENE O625HN ODIETHYLPHTHALATE O625HN 04-CHLOROPHENYL-PHENYO625HN OFLUORENE $0625 \mathrm{HN}$ 04-NITROANILINE O625HN 04,6-DINITRO-2-METHYL0625HN ON-NITROSODIPHENYLAMIO625HN 04-BROMOPHENYL-PHENYLO625HN OHEXACHLOROBENZENE O625HN OPENTACHLOROPHENOL O625HN OPHENANTHRENE O625HN OANTHRACENE O625HN ODI-N-BUTYLPHTHALATE O625HN OFLUORANTHENE O625HN OPYRENE $0625 \mathrm{HN}$ OBUTYLBENZYLPHTHALATEO625HN 03, 3'-DICHLOROBENZIDIO625HN OBENZO (A) ANTHRACENE O625HN OCHRYSENE - 0625HN OBIS (2-ETHY்LHEXYL) PHTO625HN ODI-N-OCTYL PHTHALATEO625HN OBENZO (B) FLUORANTHENEO $625 \mathrm{HN}$ OBENZO (K) FLUORANTHENEO $625 \mathrm{HN}$ OBENZO (A) PYRENE O625HN OINDENO $(1,2,3-C D)$ PYREO625HN ODIBENZO $(\mathrm{A}, \mathrm{H})$ ANTHRACEO $625 \mathrm{HN}$ OBENZO (G, H, I) PERYLENEO625HN

1665.000NITROBENZENE-D5 O625HS 1665.0002-ELUOROBIPHENYL O625HS 1665.000P-TERPHENYL-D14 O625HS 3330.000 PHENOL-D5 O625HS $3330.0002-$ ELUOROPHENOL O625HS $3330.0002,4,6$-TRIBROMOPHENOLO625HS 3330.000 PHENOT 0625HS 3330.0002 IS (2-CHLOROETHYL) ETO625HS 01, 3-DICHLOROBENZENE O625HS 1665.0001,4-DICHLOROBENZENE O625HS OBENZYL ALCOHOL O625HS 01,2-DICHLOROBENZENE O625HS 02-METHYLPHENOI O625HS OBIS (2-CHLOROISOPROPYO625HS O4-METHYLPHENOL O625HS

1665.000N-NITROSO-DI-N-PROPYO625HS OHEXACHLOROETHANE O625HS ONITROBENZENE O625HS OISORHORONE : P25HS 
0402900406KA90LE0366 MB1S2NP IXLT 0402900406WA90LE0366 MB1S24DMPN1XLT 0402900406 WA90LE0366 MB1SBENZOA1XLT $0402900406 \mathrm{WA} 90 \mathrm{LE} 0366$ MB1SB2CEXM1XLT $0402900406 \mathrm{WA} 90 \mathrm{LE} 0366$ MB1S24DCLP1XLT 0402900406 WA90LE0366 MB1S124TCB1X $0402900406 \mathrm{WA} 90 \mathrm{LE} 0366 \mathrm{MBISNAP}$ 1XLT $0402900406 \mathrm{WA} 90 \mathrm{LE} 0366$ MB1S4CANIL1XLT 0402900406 WA90LE0366 MB1SHCBD $1 \mathrm{XLT}$ 0402900406 WA90LE0366 MB1S4CL3C 1X $0402900406 \mathrm{WA} 90 \mathrm{LE} 0366$ MB1S2MENAP1XLT $0402900406 \mathrm{FA} 90 \mathrm{LE} 0366$ MB1SCL6CP 1XLT $0402900406 \mathrm{WA} 90 \mathrm{LE} 0366$ MB1S246TCP1XLT $0402900406 \mathrm{WA} 90 \mathrm{LE} 0366$ MB1S245TCP1XLT 0402900406 WA90LE0366 MB1S2CNAP 1XIT 0402900406 WA90LE0366 MB1S2NANIL1XIT $0402900406 \mathrm{WA} 90 \mathrm{LE} 0366$ MB1SDMP 1XLT 0402900406\%A9OLE0366 MB1SANAPYL1XLT 0402900406 WA90LE0366 MB1S26DNT 1XLT 0402900406 WA90LE0366 MB1S3NANIL1XLT 0402900406 WA90LE0366 MB1SANAPNE1X 0402900406 WA9OLE0366 MB1S24DNP 1XLT 0402900406WA90LE0366 MB1S4NP 1X 0402900406 WA90LE0366 MB1SDIBZFU1XLT 0402900406 WA90LE0366 MB1S24DNT 1X 0402900406 WA9OLE0366 MB1SDEP 1XLT $0402900406 \mathrm{WA} 90 \mathrm{LE} 0366$ MB1S4CLPPE1XLT 0402900406 WA9OLE0366 MBISFLRENE1XLT 0402900406 WA90LE0366 MB1S4NANIL1XLT 0402900406 WA90LE0366 MB1S46DN2C1XIT 0402900406FA90LE0366 MB1SNNDPA 1XLT 0402900406WA 90LE0366 MB1S4BRPPE1XLT 0402900406 WA9OLEO366 MB1SCL6BZ 1XLT 0402900406 WA90LE0366 MB1SPCP IX 0402900406KA90LE0366 MB1SPHANTRIXLT $0402900406 \mathrm{WA} 90 \mathrm{LE} 0366$ MB1SANTRC 1XLT 0402900406 WA9OLE0366 MB1SDNBP 1XLT 0402900406 WA90LE0366 MB1SEANT 1XIT $0402900406 \mathrm{WA} 0$ OLE0366 MB1SPYR 1X 0402900406WA90LE0366 MB1SBBZP 1XLT 0402900406 WA90LE0366 MB1S33CL2B1XLT 0402900406\%A90LE0366 MB1SBAANTR1XLT 0402900406 WA90LE0366 MB1SCHRY 1XLT 0402900406 WA90LE0366 MB1SB2EHP 1XLT 0402900406 WA90LE0366 MB1SDNOP 1XLT 0402900406\%A90IE0366 MB1SBF2ANT1XLT $0402900406 \mathrm{FA} 90 \mathrm{LE} 0366$ MB1SBKFANT1XLT 0402900406WA90LE0366 MB1SBAPYR 1XLT
juUUGKG 330UGKG 1700UGKG 330UGKG

330UGKG 78PER

330UGKG

330UGKG

330UGKG

87PER

330UGKG 330UGKG 330UGKKG 1700UGKG 330UGKG

1700UGKG 330UGKG 330UGKG

330UGKG

1700UGKG 87PER

1700UGKG 85PER

330UGKG

77PER

330UGKG

330UGKG

330UGKG 1700 UGKG 1700UGKG 330UGKG 330UGKG

330UGKG 9OPER

330UGKG

330UGKG

330UGKG

330UGKG

83PER

330UGKG

670UĠKG

330UGKG

330UGKG

330UGKG

330UGKG

330UGKG

330UGKG

330UGKG
1.0000MSDAR $1.0000 M S D A R$ $1.0000 M S D A R$ 1.0000MSDAR $1.0000 \mathrm{MSDAR}$ 1.0000MSDAR 1.0000MSDAR $1.0000 M S D A R$ 1.0000MSDAR

$1.0000 \mathrm{MSDAR}$

1.0000MSDAR

1.0000MSDAR

1.0000MSDAR

1.0000MSDAR

1.0000MSDAR

1.0000MSDAR

1.0000MSDAR

1.0000MSDAR

1.0000MSDAR

1.0000MSDAR

1.0000MSDAR

1.0000MSDAR

1.0000MSDAR

1.0000MSDAR

1.0000MSDAR

1.0000MSDAR

1.0000MSDAR

1.0000MSDAR

1.0000MSDAR

1.0000MSDAR

1. 0000MSDAR

1.0000MSDAR

$1.0000 M S D A R$

1. 0000MSDAR

1.0000MSDAR

$1.0000 M S D A R$

1.000OMSDAR

1.0000MSDAR

1.0000MSDAR

1.0000MSDAR

1.0000MSDAR

1.0000MSDAR

1.0000MSDAR

1.0000MSDAR

1.0000MSD́AR

1.0000MSDAR

1.0000MSDAR 1.000OMSDAR
02-NITROPHENOL

02,4-DIMETHYLPHENOL 0625HS OBENZOIC ACID $0625 \mathrm{HS}$ OBIS (2-CHLOROETHOXY) MO625HS 02, 4-DICHLOROPHENOL 0625HS $1665,0001,2,4$-TRICHLOROBENZEO625HS ONAPHTHALENE O625HS 04-CHLOROANILINE O625HS OHEXACHLOROBUTADIENE O625HS

$3330.0004-C H L O R O-3-M E T H Y L P H E 0625 \mathrm{HS}$ 02-METHYLNAPHTHALENE 0625HS OHEXACHLOROCYCIOPENTAO625HS $02,4,6$-TRICHLOROPHENOO625HS $02,4,5$-TRICHLOROPHENOO625HS 02-CHLORONAPHTHALENE O625HS 02-NITROANIIINE O625HS ODIMETHYLPHTHALATE O625HS OACENAPHTHYLENE O625HS 02,6-DINITROTOLUENE 0625HS 03-NITROANIIINE O625HS 1665.000ACENAPHTHENE O625HS 02, 4-DINITROPHENOL O625HS 3330.0004-NITRORHENOL O625HS ODIBENZOEURAN O625HS

1665.0002,4-DINITROTOLUENE O625HS ODIETHYLPHTHALATE O625HS 04-CHLOROPHENYL-PHENYO625HS OELUORENE O625HS 04-NITROANILINE 0625HS 04, 6-DINITRO-2-METHYLO625HS ON-NITROSODIPHENYLAMIO625HS 04-BROMOPHENYL-PHENYLO625HS OHEXACHLOROBENZENE O625HS 3330.000 PENTACHLOROPHENOL O625HS ORHENANTHRENE O625HS OANTHRACENE O625HS ODI-N-BUTYLPHTHALATE O625HS OFLUORANTHENE O625HS

1665.000PYRENE 0625HS OBUTYLBENZYLPHTHALATEO625HS $03,3^{\prime}$-DICHLOROBENZIDIO625HS OBENZO (A) ANTHRACENE O625HS OCHRYSENE O625HS OBIS (2-ETHYLHEXYL) PHTO625HS ODI-N-OCTYL PHTHALATEO625HS OBENZO (B) ELUORANTHENEO625HS OBENZO (K) ELUORANTHENEO625HS OBENZO (A) PYRENE O625HS 
SBLK

SBLK SBIK

MBIK
0402900406WA90LE0366 MB1SICDPYRIXLT 0402900406 WA90LE0366 MB1SDBAHA 1XLT 0402900406 WA90LE0366 MBISBGHIPY 1 XLT 0403900003 WA90LTZ003 MBI TOC 9ILT 0403900003 WA90LTZ003 MBISTOC $9 I$ 0403900003 WAOLTZ003 MBITTOC $9 I$ 0403900003 WA90LTZ003 MB2 TOC 9ILT 0403900003 WA9OLTZ003 MB2STOC $9 I$ 0329900043 WA90LVB0 43 MB1 MEC6D82J 0329900043 WA90LVBO43 MB1 PBFB 2J 0329900043 WA90LVBO43 MB1 12DCD42J 0329900043 WA90LVBO $43 \mathrm{MBI} \mathrm{CH} 3 \mathrm{CL}$ 2JLT 0329900043 WA90LVB0 43 MB1 CH3BR 2JLT 0329900043 WA9OLVBO43 MB1 C2H3CL2JLT 0329900043 FA90LVB0 43 MB1 C2H5CL2JLT 0329900043 WA90LVBO 43 MB1 CH2CL22JLT 0329900043 HA90LVB043 MB1 ACET 2JLT 0329900043 WA90LVB043 MB1 CS2 2JLT 0329900043 KA9OLVB0 43 MB1 11DCE 2JLT 0329900043 WA90LVB043 MB1 11DCLE2JLT 0329900043 WA9OLVBO 43 MB1 T12DCE2JLT $0329900043 W A 90 L V B 043$ MB1 CHCL3 2JLT 0329900043 WA90LVB0 43 MB1 12DCLE2JLT 0329900043 HA9OLVB0 43 MB1 MEK 2JLT 0329900043 WA90LVB043 MB1 111TCE2JLT 0329900043 WA90LVB043 MB1 CCL4 2JLT 0329900043 WA9OLVB0 43 MB1 VINYLA2JLT $0329900043 W A 90 L V B 043$ MB1 BRDCLM2JLT 0329900043 WA90IVB043 MB1 12DCLP2JLT $0329900043 W A 90 L V B 043$ MB1 13DCPC2JIT 0329900043 WA9OLVB0 43 MB1 TRCLE 2JIT $0329900043 W A ' 90 L V B 043$ MB1 DBRCLM2JLT 0329900043 WA90LVB0 43 MB1 112TCE2JLT 0329900043 WA90LVB043 MB1 C6H6 2JLT 0329900043WA90LVB043 MB1 T13DCP2JIT 0329900043 WA90LVB0 43 MB1 CHBR3 2JLT 0329900043 WA9 OLVB043 MB1 4ME2PE2JLT 0329900043 WA9OLVB0 43 MB1 2HXONE2JLT 0329900043 FA9OLVB043 MB1 TCLEE 2JLT 0329900043 FA90LVB043 MB1 TCLEA 2JLT $0329900043 \% A 90 L V B 043$ MB1 MEC6R52JLT $0329900043 \mathrm{HA9} 0 \mathrm{LVB} 043 \mathrm{MB1}$ CLC6H52JLT $0329900043 W A 90 L V B 043$ MB1 ETC6H52JLT 0329900043 HA9OLVB043 MB1 STYR 2JLT $0329900043 \% A 90 L V B 043$ MB1 XYLEN 2JLT 0327900051 WA90LVF051 MBI MEC6D82J 0327900051 KA90LVW051 MBI PBEB $2 J$ 0327900051 FA90LVH051 MB1 12DCD42J
330 UGKG 330UGKG 330 UGKG 20. OMGKG 98. OPER 98. 5PER 20. OMGKG 99. OPER 102PER 109PER

$77 \mathrm{PER}$

1OUGL

1OUGL

10 UGL

1OUGL

5UGI

1 OUGI

5UGL

SUGL

5UGL

5UGL

5UGL

5UGL

10 UGL

5UGI

5UGL

10UGL

5UGL

5UGI

5UGL

5UGL

5UGL

5UGL

5UGL

5UGL

5UGL

10UGL

10UGL

5UGL.

5UGL

5UGI

5UGI.

5UGL

5UGI

5UGL

92PER.

93PER

84PER
1. $0000 M S D A R$

1. $0000 \mathrm{MSDAR}$

1. OOOOMSDAR

1ODODKS

10DODKS

10DODKS

1ODODKS

10DODKS

1. $0000 \mathrm{MSMAZ}$

1. $0000 \mathrm{MSMAZ}$

1. $0000 \mathrm{MSMAZ}$

1. OOOOMSMAZ

1. $0000 \mathrm{MSMAZ}$

1. $0000 \mathrm{MSMAZ}$

1. $0000 \mathrm{MSMAZ}$

1. O0OOMSMAZ

1. $0000 \mathrm{MSMAZ}$

1. 0000MSMAZ

1. $0000 \mathrm{MSMAZ}$

1. 0000MSMAZ

1. $0000 \mathrm{MSMAZ}$

1. OOOOMSMAZ

1. $0000 \mathrm{MSMAZ}$

1. $0000 \mathrm{MSMAZ}$

1. $0000 \mathrm{MSMAZ}$

1.0000MSMAZ

1. $0000 \mathrm{MSMAZ}$

1. $0000 \mathrm{MSMAZ}$

1. $0000 \mathrm{MSMAZ}$

1. $0000 \mathrm{MSMAZ}$

1. $0000 \mathrm{MSMAZ}$

1. $0000 \mathrm{MSMAZ}$

1. $0000 \mathrm{MSMAZ}$

1. 0000MSMAZ

1. 0000MSMAZ

1. O0OOMSMAZ

1. $0000 \mathrm{MSMAZ}$

1. $0000 \mathrm{MSMAZ}$

1. $0000 \mathrm{MSMAZ}$

1. 000OMSMAZ

1. 0000MSMAZ

1. O000MSMAZ

1. $0000 \mathrm{MSMAZ}$

1. $0000 \mathrm{MSMAZ}$

1. O000MSMAZ

1. $0000 \mathrm{MSJBD}$

1. 0000MSJBD

1. $0000 \mathrm{MSJBD}$
O INDENO (1, 2, 3-CD) PYREO 625HS ODIBENZO $(A, H)$ ANTHRACEO625HS OBENZO (G, H, I) PERYLENEO 625HS OTOTAL ORGANIC CARBONIOCT N 400TOTAL ORGANIC CARBONIOCT S 400TOTAL ORGANIC CARBONIOCT T OTOTAL ORGANIC CARBONIOCT N 400TOTAL ORGANIC CARBONIOCT S 50.000TOLUENE-D8 $0624 \mathrm{HN}$ 50.000BROMOFLUOROBENZENE O624HN $50.0001,2-D I C H L O R O E T H A N E-D 0624 \mathrm{HN}$ OCHLOROMETHANE OBROMOMETHANE O624HN OVINYL CHLORIDE $0624 \mathrm{HN}$ OCHLOROETHANE 0624HN OMETHYLENE CHLORIDE $0624 \mathrm{HN}$ OACETONE O624HN OCARBON DISULFIDE O624HN 01,1-DICHLOROETHENE 0624HN 01,1-DICHLOROETHANE 0624HN 01,2-DICHLOROETHENE 10624HN OCHLOROEORM O624HN 01,2-DICHLOROETHANE O624HN 02-BUTANONE $0624 \mathrm{HN}$ $01,1,1$-TRICHLOROETHANO $24 \mathrm{HN}$ OCARBON TETRACHLORIDEO624HN OVINYI ACETATE O624HN OBROMODICHLOROMETHANEO $624 \mathrm{HN}$ 01,2-DICHLORORROPANE $0624 \mathrm{HN}$ OCIS-1, 3-DICHLOROPROPO $624 \mathrm{HN}$ OTRICHLOROETHENE O624HN ODIBROMOCHIOROMETHANEO $624 \mathrm{HN}$ $01,1,2-T R I C H L O R O E T H A N O 624 \mathrm{HN}$ OBENZENE : O624HN OTRANS-1, 3-DICHLOROPRO62 4HN OBROMOFORM O624HN 04-METHYL-2-PENTANONE0624HN 02-HEXANONE' 0624KN OTETRACHLOROETHENE O624HN $01,1,2,2$-TETRACHLOROEO $624 \mathrm{HN}$ OTOLUENE O624HN OCHLOROBENZENE O62 4HN OETHYLBENZENE O624HN OSTYRENE $\quad 0624 \mathrm{HN}$ OXYLENE (TOTAL) 0624HN 5Q.000TOLUENE-D8 $0624 \mathrm{HN}$ 50.000BROMOELUOROBENZENE O62 $4 \mathrm{HN}$ $50.0001,2-D I C H L O R O E T H A N E, 624 \mathrm{HN}$ 
0327900051WA90LVW051 MB1 CH3CL 2JLT 0327900051 WA9OLVW051 MBI CH3BR 2JLT 0327900051 KA $90 \mathrm{LVW051} \mathrm{MB1}$ C2H3CL2JLT 0327900051 WA9OLVF051 MB1 C2H5CL2JLT 0327900051 WA $90 \mathrm{LVW} 051 \mathrm{MB1}$ CH2CL22J 0327900051 WA9OLVW051 MB1 ACET 2JLT 0327900051 WA90LVW051 MB1 CS2 2JLT 0327900051WA9OLVW051 MB1 11DCE 2JLT 0327900051WA9OLVW051 MB1 11DCLE2JLT 0327900051WA9OLVW051 MB1 T12DCE2JLT 0327900051 WA9OLVW051 MB1 CHCL3 2JLT 0327900051WA90LVW051 MB1 12DCLE2JLT 0327900051 WA9OLVW051 MBI MEK 2JLT 0327900051 WA90LVW051 MB1 111TCE2JLT 0327900051WA90LVW051 MB1 CCL4 2JLT 0327900051 WA9OLVW051 MB1 VINYLA2JLT 0327900051WA90LVW051 MB1 BRDCLM2JLT 0327900051 WA 90 LVW051 MB1 12DCLP2JLT 0327900051 KA90LVW051 MB1 13DCPC2JLT 0327900051WA9OLVW051 MB1 TRCLE 2JLT 0327900051WA9OLVW051 MB1 DBRCLM2JLT 0327900051 WA9OLVW051 MB1 112TCE2JLT 0327900051 WA90LVW051 MB1 C6H6 2JLT 0327900051 WA9OLVW051 MB1 T13DCP2JLT 0327900051 WA90LVW051 MB1 CHBR3 2JLT 0327900051WA90LVW051 MB1 4ME2PE2JLT 0327900051WA9OLVW051 MB1 2HXONE2JLT 0327900051WA90LVW051 MB1 TCLEE 2JLT 0327900051 WA90LVW051 MB1 TCLEA 2JLT 0327900051 WA90LVW051 MB1 MEC6H52JLT 0327900051WA90LVW051 MB1 CLC6H52JLT 0327900051 พิ่90LVW051 MBI ETC6H52JLT 0327900051WA90LVW051 MB1 STYR 2JLT 0327900051WA90LVW051 MB1 XYLEN 2JLT 0330900054WA90LVW054 MB1 MEC6D82J 0330900054 WA90LVW054 MB1 PBFB 2J 0330900054 WA9OLVW054 MB1 12DCD42J 0330900054 WA90LVW054 MB1 CH3CL 2JLT 0330900054 WA90LVW054 MB1 CH3BR 2JLT 0330900054WA9OLVW054 MB1 C2H3CL2JLT 0330900054 WA9OLVW054 MBI C2H5CL2JLT 0330900054WA90LVW054 MB1 CH2CL22JLT 0330900054 KA9OLVW054 MB1 ACET 2JLT 0330900054 FA9OLVW054 MB1 CS2 2JLT 0330900054WA90LVW054 MB1 11DCE 2JLT 0330900054 WA9OLVF054 MB1 11DCLE2JLT 0330900054 FA9 OLVF054 MB1 T12DCE2JLT 0330900054KA90LVW054 MB1 CHCL3 2JLT
LUUGKG

1 OUGKG

1OUGKG

1OUGKG

7UGKG

10UGKG

5UGKG

SUGKG

5UGKG

SUGKG

SUGKG

5UGKG

IOUGKKG

5UGKG

5UGKG

1OUGKG

5UGKG

5UGKG

5UGKG

5UGKG

5UGKG

SUGKG

5UGKG

5UGKG

5UGKG

IOUGKG

10UGKG

5UGKG

5UGKG

5UGKG

5UGKG

5UGKG

5UGKG

SUGKG

96PER

100PER

94PER

1OUGKG

1OUGKG

10UGKG

10UGKG

5UGKG

1OUGKG

5UGKG

SUGKG

5UGKG

5UGKG 5UGKG
1.0000MSJED

$1.0000 M S J B D$

$1.0000 \mathrm{MSJBD}$

$1.0000 M S J B D$

$1.0000 \mathrm{MSJBD}$

$1.0000 \mathrm{MSJBD}$

$1.0000 \mathrm{MSJBD}$

$1.0000 \mathrm{MSJBD}$

$1.0000 \mathrm{MSJBD}$

$1.0000 \mathrm{MSJBD}$

1.0000MSJBD

1.0000MSJBD

$1.0000 M S J B D$

$1.0000 \mathrm{MSJBD}$

$1.0000 M S J B D$

$1.0000 \mathrm{MSJBD}$

$1.0000 \mathrm{MSJBD}$

$1.0000 \mathrm{MSJBD}$

$1.0000 \mathrm{MSJBD}$

1. $0000 \mathrm{MSJBD}$

$1.0000 \mathrm{MSJBD}$

$1.0000 \mathrm{MSJBD}$

$1.0000 \mathrm{MSJBD}$

$1.0000 \mathrm{MSJBD}$

$1.0000 \mathrm{MSJBD}$

1.000OMSJBD

$1.0000 \mathrm{MSJBD}$

$1.0000 M S J B D$

1 :0000MSJBD

$1.0000 \mathrm{MSJBD}$

$1.0000 \mathrm{MSJBD}$

$1.0000 \mathrm{MSJBD}$

$1.0000 \mathrm{MSJBD}$

$1.0000 \mathrm{MSJBD}$

$1.0000 \mathrm{MSBLB}$

$1.0000 \mathrm{MSBLB}$

$1.0000 M S B L B$

$1.0000 \mathrm{MSBLB}$

$1.0000 \mathrm{MSBLB}$

$1.0000 \mathrm{MSBLB}$

$1.0000 \mathrm{MSBLB}$

1.0000MSBLB

$1.0000 \mathrm{MSBLB}$

$1.0000 M S B L B$

1.0000 MSBLB

$1.0000 \mathrm{MSBLB}$

$1.0000 M S B L B$ $1.0000 \mathrm{MSBLB}$
OCHLOROMETHANE

$0624 \mathrm{HN}$ OBROMOMETHANE

O624HN

OVINYL CHLORIDE

$0624 \mathrm{HN}$

OCHLOROETHANE

$0624 \mathrm{HN}$

OMETHYLENE CHLORIDE 0624HN

OACETONE

$0624 \mathrm{HN}$

OCARBON DISULFIDE - O624HN

01,1-DICHLOROETHENE 0624HN

01,1-DICHLOROETHANE 0624HN 01,2-DICHLOROETHENE $10624 \mathrm{HN}$ OCHLOROFORM

$0624 \mathrm{HN}$

01,2-DICHLOROETHANE 0624HN

02-BUTANONE O624HN

01, 1, 1-TRICHLOROETHANO624HN OCARBON TETRACHLORIDEO624HN OVINYL ACETATE O624HN OBROMODICHLOROMETHANEO $624 \mathrm{HN}$ 01,2-DICHLOROPROPANE 0624HN OCIS-1, 3-DICHLOROPROPO624HN OTRICHLOROETHENE O624HN ODIBROMOCHLOROMETHANEO624HN $01,1,2$-TRICHLOROETHANO $624 \mathrm{HN}$ OBENZENE O624HN OTRANS-1, 3-DICHLOROPRO 62 4HN OBROMOFORM O624HN 04-METHYL-2-PENTANONEO624HN 02-HEXANONE 0624HN OTETRACHLOROETHENE O624HN $01,1,2,2$ TETRACHLOROEO $624 \mathrm{HN}$ OTOLUENE O624HN OCHLOROBENZENE O624HN OETHYLBENZENE O624HN OSTYRENE O624HN OXYLENE (TOTAL) O624KN 50.000 TOLUENE-DB $0624 \mathrm{HN}$ 50.000BROMOFLUOROBENZENE O624HN 50.000:1,2-DICHLOROETHANE-DO624HN OCHLOROMETHANE O624HN OBROMOMETHANE O624HN OVINYL CHLORIDE $0624 \mathrm{HN}$ OCHLOROETHANE O624 HN OMETHYLENE CHLORIDE O624HN OACETONE O624HN OCARBON DISULEIDE $: 0624 \mathrm{HN}$ 01,.1-DICHLOROETHENE 0624HN 01,1-DICHLOROETHANE 0624HN 01,2-DICHLOROETHENE (0624HN OCHLOROEORM

$0624 \mathrm{HN}$ 
0330900054 WA 90 LVW054 MBI 12DCLE2JLT 0330900054 WA9OLVW054 MBI MEK 2JLT 0330900054 WA9OLVW054 MB1 111TCE2JLT 0330900054 WA9OLVWO54 MBI CCL4 2JLT 0330900054 WA9OLVW054 MB1 VINYLA2JLT 0330900054 WA $90 L V W 054$ MB1 BRDCLM2JLT 0330900054 WA9OLVW054 MBI 12DCLP2JLT 0330900054 WA9OLVW054 MBI 13DCPC2JLT 0330900054WA90LVW054 MB1 TRCLE 2JLT 0330900054WA9OLVW054 MB1 DBRCLM2JLT 0330900054 WA $90 \mathrm{LVW} 054$ MB1 112TCE2JLT 0330900054 WA90LVW054 MB1 C6H6 2JLT 0330900054WA90LVF054 MB1 TI3DCP2JLT 0330900054WA90LVW054 MB1 CHBR3 2JLT 0330900054WA90LVW054 MB1 4ME2PE2JLT 0330900054WA90LVW054 MB1 2HXONE2JLT 0330900054WA90LVW054 MB1 TCLEE 2JLT 0330900054WA90LVW054. MB1 TCLEA 2JLT 0330900054WA90LVW054 MB1 MEC6H52JLT 0330900054 KA $0 L V W 054$ MB1 CLC6H52JLT 0330900054WA9OLVW054 MB1 ETC6H52JLT 0330900054 KA9OLVW054 MB1 STYR 2JLT 0330900054FA90LVW054 MB1 XYLEN 2JLT
$1.0000 \mathrm{MSBLB}$
$1.0000 \mathrm{MSBLB}$
$1.0000 \mathrm{MSBLB}$
$1.0000 \mathrm{MSBLB}$
$1.0000 \mathrm{MSBLB}$
$1.0000 \mathrm{MSBLB}$
$1.0000 \mathrm{MSBLB}$
1. $0000 \mathrm{MSBLB}$
$1.0000 \mathrm{MSBLB}$
$1.0000 \mathrm{MSBLB}$
$1.0000 \mathrm{MSBLB}$
$1.0000 M S B L B$
$1.0000 \mathrm{MSBLB}$
$1.0000 \mathrm{MSBLB}$
$1.0000 \mathrm{MSBLB}$
$1.0000 M S B L B$
$1.0000 \mathrm{MSBLB}$
1. $0000 \mathrm{MSBLB}$
1. $0000 \mathrm{MSBLB}$
1. $0000 \mathrm{MSBLB}$
1.0000MSBLB
$1.0000 \mathrm{MSBLB}$
$1.0000 \mathrm{MSBLB}$

01,2-DICHLOROETHANE 0624HN 02-BUTANONE 0624HN 01,1,1-TRICHLOROETHANO624HN OCARBON TETRACHLORIDE0624HN OVINYL ACETATE 0624HN OBROMODICHLOROMETHANEO $624 \mathrm{HN}$ 01,2-DICHLOROPROPANE 0624HN OCIS-1, 3-DICHLOROPROPO624HN OTRICHLOROETHENE O624HN ODIBROMOCHLOROMETHANEO624HN 01,1,2-TRICHLOROETHANO $624 \mathrm{HN}$ OBENZENE $0624 \mathrm{HN}$ OTRANS-1, 3-DICHLOROPRO624HN OBROMOFORM $0624 \mathrm{HN}$

0 4-METHYL-2-PENTANONEO624HN 02-HEXANONE O624HN OTETRACHLOROETHENE O624HN $01,1,2,2$-TETRACHLOROEO624HN OTOLUENE $0624 \mathrm{HN}$ OCHLOROBENZENE OETHYLBENZENE OSTYRENE OXYLENE (TOTAL) O624HN 
MESIINGHOUSE,

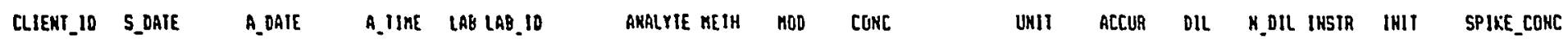

\begin{tabular}{|c|c|c|c|c|c|c|c|c|c|c|}
\hline $\begin{array}{l}n 386001 \\
\text { GR510030 }\end{array}$ & 022090 & 030190 & 1200 & II & 38600185 & AXAEXE & 27 & 14 & 390 & \\
\hline GRS1003D & 022090 & 030190 & 1200 & กI & 38BOOIRE & ANAPLY & 21 & LI & 390 & UEKG \\
\hline ERSIO03D & 022090 & 030190 & 1200 & וI & $386001 R E$ & ANTRC & 21 & 11 & 390 & UEKG \\
\hline GRS1003D & 022090 & 030190 & 1200 & MI & 38BO01RE & BEMLOA & 21 & LI & 2000 & K6 \\
\hline 6R51003D & 022090 & 030190 & 1200 & MI & $386001 R E$ & BAAKIR & 21 & 11 & 390 & UGKE \\
\hline GRS10030 & 022090 & 030190 & 1200 & NI & 38SOOIRE & BKFAKI & 21 & 41 & 390 & \\
\hline ERSS1003D & 022090 & 030190 & 1200 & If & 38600IRE & BEHIPY & 27 & LI & 390 & \\
\hline GRS10030 & 022090 & 030198 & 1200 & nI & 38BOOIRE & BAPYA & 21 & 11 & 390 & \\
\hline 6RS1003D & 022090 & 030190 & 1200 & זו & 386001RE & BZALC & 27 & 1! & 390 & 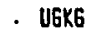 \\
\hline 6RS10030 & 022090 & 030190 & 1200 & MI & 386001RE & B2CEXY & 27 & Li & 390 & \\
\hline 6RS10030 & 022090 & 030190 & 1200 & HI, & J8BOOIRE & B2CEE & 27 & $4 T$ & 390 & \\
\hline GRSI003D & 022090 & 030190 & 1200 & II & 386001RE & 82CIPE & 27 & LT & 390 & \\
\hline GR510030 & 022090 & 030190 & 1200 & hit & 38600IRE & B2EFP & 27 & & 1000 & ak \\
\hline GRS10030 & 022090 & 030190 & $1200^{\circ}$ & $\boldsymbol{n}$ & 38SOOIRE & 4BRPPE & 27 & $4 T$ & 390 & \\
\hline 6RS1003B & 022090 & 030190 & 1200 & nT & JBGODIRE & BBIP. & 27 & LI & 390 & \\
\hline GRS10030 & 022090 & 030190 & 1200 & MI & 38SOOLRE & ACAMIL & 27 & LIT & 390 & \\
\hline GRS1003D & 022090 & 030190 & 1200 & nT & 386001RE & 2CNAP & 27 & LT & 390 & \\
\hline GRS10030 & 022090 & 030190 & 1200 & II & 386001RE & 2 QLP & 27 & LT & 390 & 6tik \\
\hline GRS1003D & 022090 & 030190 & 1200 & $\pi$ & 386001RE & 4CLPPE & 27 & LT & 390 & \\
\hline GRS10030 & 022090 & 030190 & 1200 & MI & 38GO0IRE & CHAY & 27 & LI & 390 & \\
\hline GRS1003D & 022090 & 030190 & 1200 & MT & 38SOOIRE & DBAHA & 27 & LT & 390 & \\
\hline 6RS1003D & 022090 & 030190 & 1200 & II & 38600IRE & DIBLFU & 27 & $\mathrm{LT}$ & 390 & \\
\hline 6RS1003D & 022090 & 030190 & 1200 & หI & 38600IRE & 120CLB & 27 & LI & 390 & 1 \\
\hline GRS10031 & 022090 & 030190 & 1200 & MI & 38600IRE & $130 \mathrm{CLB}$ & 27 & LI & 3990 & t \\
\hline GRS10030 & 022090 & 030190 & 1200 & ถा & 386001RE & 14000 & 27 & $\mathrm{LT}$ & 390 & \\
\hline GRS10031 & 022090 & 030190 & 1200 & MI & J日6OOIRE & OHBP & 27 & LT & 390 & \\
\hline 6R51003D & 022090 & 030190 & 1200 & MI & 388001RE & $33 C L 2 B$ & 27 & LT & 780 & \\
\hline GRS10033 & .022090 & 030180 & 1200 & $\mathrm{MI}$ & J8600IRE & 21DCLP & 27 & 4. & 390 & \\
\hline 6RS1003S & 022090 & 030190 & 1200 & MI & 38600IRE & $260 C L P$ & 27 & LT & 390 & \\
\hline 6R510031 & 0220993 & 030190 & 1200 & MT & 38600IRE & DEP & 27 & LI & 390 & \\
\hline 6RS1003D & 022090 & 030190 & 1299 & หT & 38600IRE & 240KPH & 27 & LT & 390 & \\
\hline 6RS10031 & 022090 & 030190 & $1200^{\circ}$ & $n \pi$ & J8SOOIRE & DKP & 27 & LI & 390 & \\
\hline 6R510038 & 022090 & 030190 & 1200 & III & J8600IRE & 240NP & 27 & LT & 2000 & \\
\hline 6RS10030 & 022090 & 030190 & 1200 & $n$ & 386001RE & $210 \mathrm{TH}$ & 27 & LI & 390 & \\
\hline 68510031 & 022090 & 030190 & 1200 & $\pi$ & 38600IRE & 2604T & 27 & 49 & 390 & \\
\hline ERS10031 & 022080 & 030190 & $1200^{\circ}$. & $n i$ & 38600IRE & DXOP & 27 & 47 & 390 & \\
\hline 6RS1003D & 022090 & 030190 & 1200 & nT & 38600IRE & FАКT & 27 & LT & 390 & \\
\hline GRS10030 & 022090 & 030190 & 1200 & $\mathrm{MT}$ & 3B6OOLRE & FLRENE & 27 & LI & 390 & \\
\hline 6R51003D & 022090 & 030190 & 1200 & MI & .386001RE & CL682 & 27 & LT & 390 & \\
\hline GRS1003D & 022090 & 030190 & 1200 & MT & 3BGOOLRE & HCBD & 27 & LI & 390 & \\
\hline 6R51003D & 022090 & 030190 & 1200 & זו & 386001RE & CL6CP & 27 & LI & 390 & \\
\hline GRS10031 & 022090 & 030190 & 1200 & nI & 38600IRE & CLLET & 27 & LI & 390 & U6X \\
\hline GRS1003D & 022090 & 030190 & 1200 & nT & 386OOIRE & ICOPYR & 27 & 47 & 390 & UEKC \\
\hline GRS10030 & 022090 & 030190 & 1200 & $n \pi$ & 386001RE & I5OPHR & 27 & LI & 390 & \\
\hline GRS10030 & 022090 & 030190 & 1200 & ถT & 386001RE & 2KEKAP & 27 & 4 & 390 & \\
\hline GRS10031 & 022090 & 030190 & 1200 & nT & 38600IRE & 2KP & 27 & LT & 390 & \\
\hline GRSIOOJI & 022090 & 030190 & 1200 & $n$ & JB6OOIRE & $\operatorname{MiP}$ & 27 & LI & 390 & UGKE \\
\hline 6RS10035 & 022090 & 030190 & 1200 & nT & 386001RE & MAP & 27 & LT & 390 & UGKE \\
\hline 68510030 & 022090 & 030190 & 1200 & nT & $386001 R E$ & 2KAMIL & 27 & LI & 2000 & \\
\hline GAS1003! & 022090 & 030190 & 1200 & $n$ & 386001RE & ЗKAHIL & 27 & LI & 2000 & Wokt \\
\hline
\end{tabular}

.

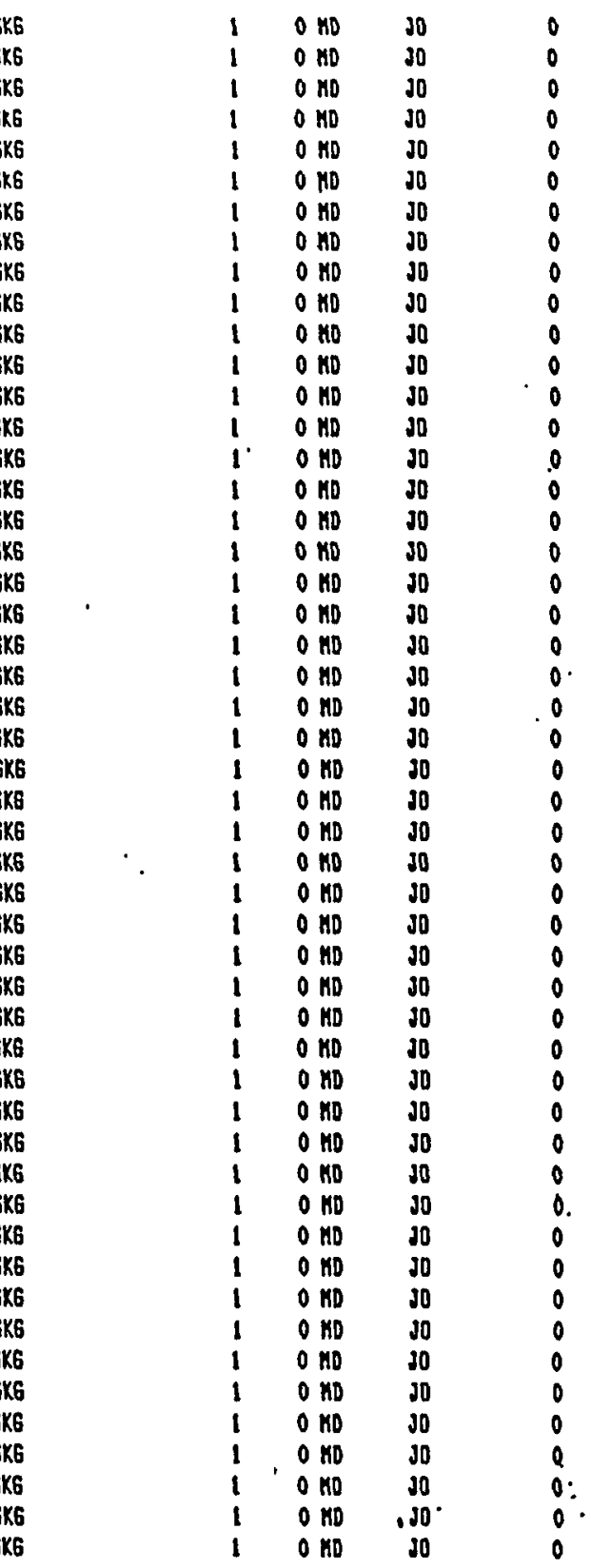




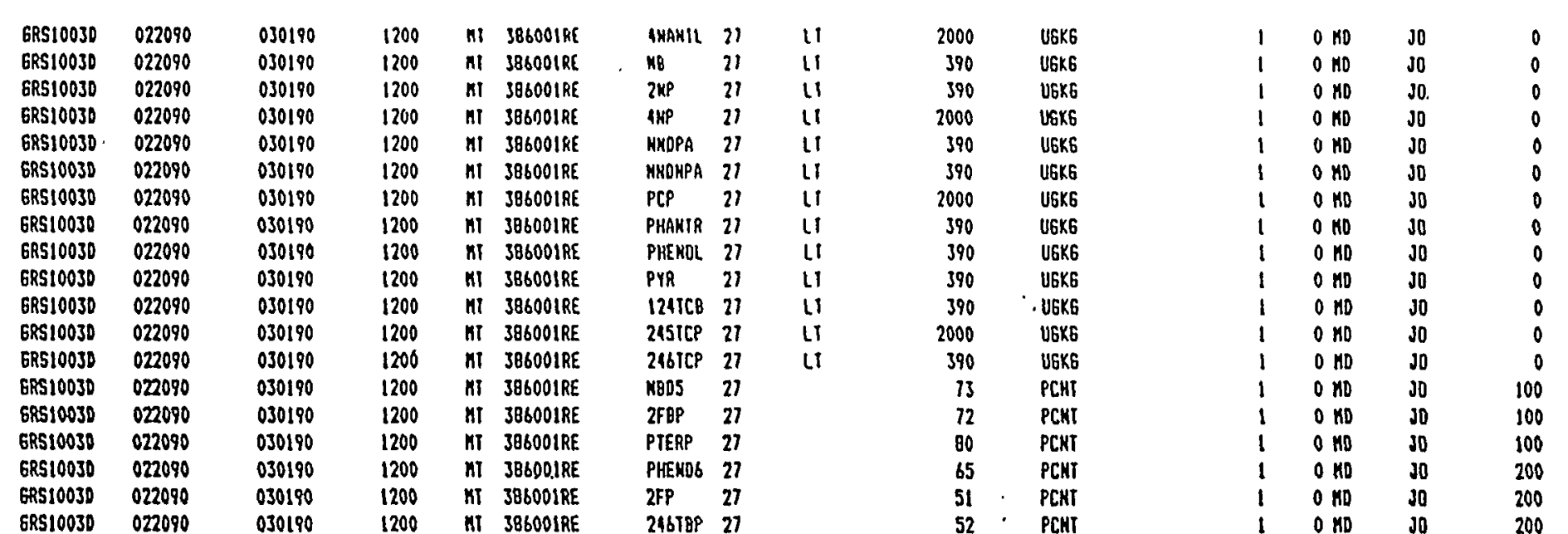

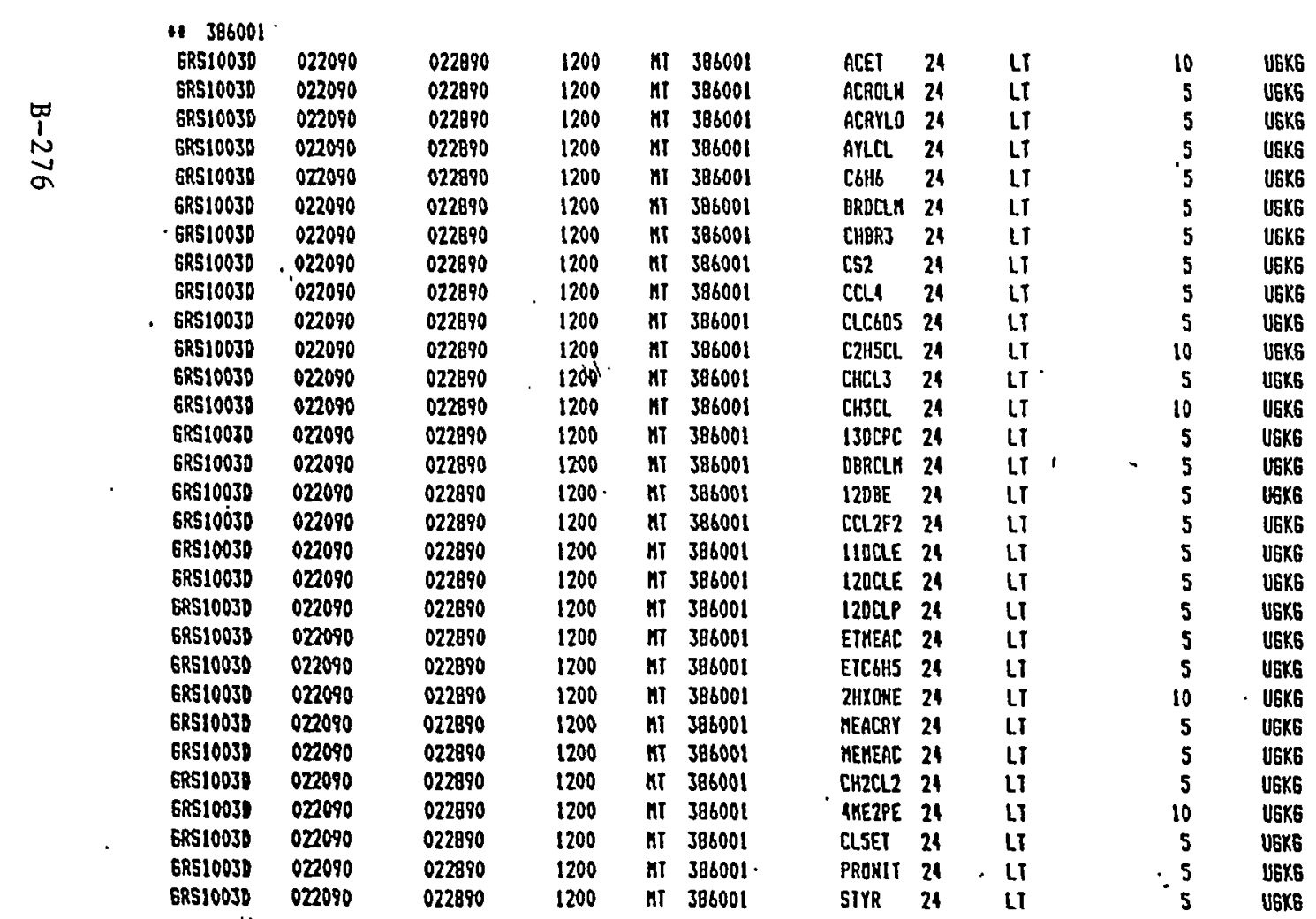

\begin{tabular}{|c|c|c|c|c|}
\hline UEK6 & 1 & $O \mathrm{KE}$ & 6W & 0 \\
\hline UGKG & 1 & $O M E$ & 6V & 0 \\
\hline UGKG & 1 & $O$ KE & 6W & 0 \\
\hline UGK6 & 1 & $O \mathrm{ME}$. & 6X & 0 \\
\hline UGK6 & 1 & $O \mathrm{KE}$ & 6U & 0 \\
\hline JEK6 & 1 & $O \mathrm{ME}$ & 6u & 0 \\
\hline UGKG & 1 & $O \mathrm{KE}$ & 64 & 0 \\
\hline UEKG & 1 & $O$ KE & GN & 0 \\
\hline UGKG & 1 & $O \mathrm{KE}$ & 6V & 0 \\
\hline UEKE & 1 & $O \mathrm{ME}$ & 6H & 0 \\
\hline UGK. & 1 & $O \mathrm{KE}$ & 6W & 0 \\
\hline I6K6 & 1 & $O \mathrm{ME}$ & 6Y & 0 \\
\hline UGK6 & 1 & $O$ KE & 64 & 0 \\
\hline UEKG & 1 & $0 \mathrm{KE}$ & 6K & 0 \\
\hline UGKG & 1 & $O \mathrm{KE}$ & 6N & 0 \\
\hline $46 \times 6$ & 1 & $0 \mathrm{ME}$ & 6V & 0 \\
\hline UGKG & 1 & $O \mathrm{KE}$ & GW & 0 \\
\hline UGKG & 1 & $O \mathrm{ME}$ & 6W & 0 \\
\hline J6K6 & 1 & $O \mathrm{KE}$ & 6V & 0 \\
\hline I6KG & 1 & $O$ KE & 6N & 0 \\
\hline JGKG & 1 & $O \mathrm{KE}$ & GN & 0 \\
\hline IGKG & 1 & $O M E$ & 6N & 0 \\
\hline UGKG & 1 & $O$ KE & 8H & 0 \\
\hline JGKG & 1 & $O \mathrm{NE}$ & GW & 0 \\
\hline JGK6 & 1 & $O$ ME & 6W & 0 \\
\hline IGKG & 1 & $O \mathrm{KE}$ & 6H & 0 \\
\hline JGKG & 1 & $O \mathrm{ME}$ & 6N & 0 \\
\hline USK & 1 & $O \mathrm{ME}$ & 6I & 0 \\
\hline UEK6 & 1 & $O \mathrm{KE}$ & , $6 \mathrm{~W}$ & 0 \\
\hline $56 K$ & 1 & $O \mathrm{ME}$ & 6u & 0 \\
\hline
\end{tabular}
$?$ 
Promo. 3

XESIINGHOUSE S

CLIEKI_ID S_DATE A_DAIE A.IIKE LAB LAE.10 AHALYIE MEIH MOD COKC

\begin{tabular}{|c|c|c|c|c|c|c|c|c|c|c|}
\hline $6 R 510030$ & 022090 & 022890 & 1200 & II & 386001 & RICLEA & 21 & LI & 5 & USK6 \\
\hline 6RS10030 & 022090 & 022890 & 1200 & nI & 386001 & ICLEA & 21 & 4 & 5 & U6K6 \\
\hline 6RS10030 & 022090 & 022890 & 1200 & II & 386001 & MECBHS & 21 & 41 & 5 & UEKE \\
\hline GRS10030 & 023090 & 022890 & 1200 & ז & 386001 & IIJOCP & 21 & 41 & 5 & USKG \\
\hline 6RS10030 & 022090 & 022890 & 1200 & MI & 386001 & IIIICE & 24 & 21 & 5 & USKG \\
\hline 6R510030 & 022090 & 022890 & 1200 & $\mathrm{MT}$ & 386001 & IIZTCE & 21 & LI & 5 & UEKG \\
\hline 6RS10030 & 022090 & 022890 & 1200 & กו & 388001 & CCL.JF & 24 & LT & 5 & UEXK \\
\hline 6R51003D & 022090 & 022890 & 1200 & nT & 386001 & $123 \mathrm{CPR}$ & 21 & 41 & 5 & U6k6 \\
\hline 6RS10030 & 022090 & 022890 & 1200 & וע & 388001 & VIMYLA & 24 & 41 & 5 & UEKG \\
\hline GRS1003D & 022090 & 022890 & 1200 & $\mathrm{nI}$ & 386001 & IYLEX & 21 & LI & 5 & UEKG \\
\hline 6RS1003D & 022090 & 022890 & 1200 & HI & 386001 & $120 \mathrm{CO}$ & 24 & & 101 & PCHI \\
\hline 6RS1003D & 022090 & 022890 & 1200 & nז & 386001 & MEC6DB & 24 & & 104 & PCHT \\
\hline 6RS1003D & 022090 & 022890 & 1200 & עI & 386001 & PBFB & 24 & & 100 & PCHI \\
\hline
\end{tabular}

11 386002

6R508020 $022190 \quad 030290$

GR508020 $022190 \quad 030290$

GR508020 022190

GRS08020 $022190 \quad 030290$

6R508020 022190 030290

GR508020 - 022190

030290

$\begin{array}{lll}65508020 & 022190 & 030290 \\ 68509020 & 022190 & 030290\end{array}$

6R508020 022190

GR508020 $022190 \quad 030290$

SR508020 $022190 \quad 030290$

GRSOBO20 $022190 \quad 030290$

6R508020 $022190 \quad 030290$

GR508020 $\cdot 022190 \quad 030290$

ER508020 $022190 \quad 030290$

GRSOB020 $022190 \quad 030290$

GR508020 $022190 \quad 030290$

$\begin{array}{lll}62508020 & 022190 & 030290\end{array}$

$032190 \quad 030290$

$\begin{array}{lll}022190 & 030290\end{array}$

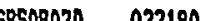

68508020022190

69508020 $022190 \quad 030290$

6R508020 $022190 \quad 030290$

GRSOBO21 O22190

GR508020 $022190 \quad 030290$

GF508021022190 030290

6R508020 022190 030290

GRSOBO20 $022190 \quad 030280$

$\begin{array}{lll}69508020 & 022190 & 030290\end{array}$

6R508021 $022190 \quad 030290$

GR508020 $022190 \quad 030290$

$\begin{array}{lll}58509020 & 022190 & 030290\end{array}$

$\begin{array}{lll}68508022 & 022190 & 030290\end{array}$

GRSO8020 $022190 \quad 030290$

$\begin{array}{lll}1200^{\circ} & n T & 386002 \\ 1200 & n T & 386002 \\ 1200 & n T & 386002 \\ 1200 & n T & 386002 \\ 1200 & n T & 386002 \\ 1200 & n T & 386002 \\ 1200 & n T & 386002 \\ 1200 & n T & 386002 \\ 1200 & n T & 386002 \\ 1200 & n T & 386002 \\ 1200 & n T & 386002 \\ 1200 & n T & 386002 \\ 1200 & n T & 386002 \\ 1200 & n T & 386002 \\ 1200 & n T & 386002 \\ 1200 & n T & 386002 \\ 1200 & n T & 386002 \\ 1200 & n T & 386002 \\ 1200 & n T & 386002 \\ 1200 & n T & 386002 \\ 1200 & n T & 386002 \\ 1200 . & n T & 386002 \\ 1200 & n T & 386002 \\ 1200 & n T & 386002 \\ 1200 & n T & 386002 \\ 1200 & n T & 386002 \\ 1200 & n T & 386002 \\ 1200 & n T & 386002 \\ 1200 & n T & 386002 \\ 1200 & n T & 386002 \\ 1200 & n T & 386002 \\ 1200 & n T & 386002 \\ 1200 & n T & 386002 \\ 1200 & n T & 386002 \\ 1200 & n T & 386002 \\ 1200 & n T & 386002\end{array}$

AKAPKE 27 LT

AKAPLY 27 LT

AXIFC $27 \quad 27$

BEMZOA $27 \quad$ LT

BAAKIR 27

BKFANT 27 LT

BEHIPY $27 \quad$ LI

$\begin{array}{lll}\text { antC } 27 & 27\end{array}$

$\begin{array}{lll}\text { A2CEX } & 27 & \text { LT } \\ \text { BCEE } & 27 & \text { LI }\end{array}$

BXCIPE 27 LT

B2EHP 27

ABAPPE 27 LT

BB2P 27

ICANIL 27

TCMAP 27

$\begin{array}{lll}2 \mathrm{CLP} & 27 & \text { LT }\end{array}$

ICLPPE 27

CHAY 27 LI

DBAHA 27 LI

DIBLFU 21

120CLB 27

130CLB 27

110CLB 27

\begin{tabular}{lll} 
DKBP & 27 \\
\hline$J C L$ & 27
\end{tabular}

J3CL.2B 27

240CLP 27

$260 \mathrm{CP} 27$

DEP 27 LT

LIT

24010N $27 \quad$ LT

$240 \mathrm{KP} 27 \quad \mathrm{LT}$

200NT 21 LI

$\begin{array}{lllll}\text { 260NT } & 27 & \text { LT } & & .380 \\ \text { BHOP } & 27 & \text { LT } & 380\end{array}$
UGKE

6.K6

380 - U6KG

$1900 \cdot$ UGKG

380 UGK6

$380 \quad$ UEKG

380 UEKG

$380 \quad$ U6K6

380 U6K6

3000 . UGKE

380 UGKG

UEKS

UGK6

UEKG

UEKG

UGKG

USKE

UEKG

UEK6

UGK6

U6K6

U6KG

UEK6

UGKG

UGKG

UGKG

U6x6

UGK6

UEKE

UGKG

\begin{tabular}{|c|c|c|}
\hline $0 \mathrm{KE}$ & 6N & 0 \\
\hline $0 \mathrm{ME}$ & 64 & 0 \\
\hline 0 KE & 6N. & 0 \\
\hline $0 \mathrm{ME}$ & $64^{\circ}$ & 0 \\
\hline $0 \mathrm{ME}$ & 6N & 0 \\
\hline$O \mathrm{ME}$ & SN & 0 \\
\hline$O \mathrm{ME}$ & 6H & 0 \\
\hline $0 \mathrm{KE}$ & 6II & 0 \\
\hline $0 \mathrm{ME}$ & 6II & 0 \\
\hline $0 \mathrm{ME}$ & 64 & 0 \\
\hline $0 \mathrm{ME}$ & 6II & 50 \\
\hline$O \mathrm{ME}$ & 6X & 50 \\
\hline$O \mathrm{KE}$ & 64 & 50 \\
\hline
\end{tabular}

\begin{tabular}{|c|c|c|}
\hline 0 ND & jo & 0 \\
\hline 0 MD & JO & 0 \\
\hline 0 KD & JO & 0 \\
\hline 0 No & JO & 0 \\
\hline 0 No & JO & 0 \\
\hline 0 KO & jo & 0 \\
\hline ס ס 0 & so & 0 \\
\hline$O$ MD & jo & 0 \\
\hline 0 KD & Jo & 0 \\
\hline 0 No & 10 & 0 \\
\hline 0 MD & Jo & 0 \\
\hline$O$ KD & J0 & 0 \\
\hline 0 ND & Jo & 0 \\
\hline 0 KD & Jo & 0 \\
\hline 0 MD & Jo & 0 \\
\hline$O$ KO & 50 & 0 \\
\hline O KO & 50 & 0 \\
\hline 0 KD & Jo & 0 \\
\hline 0 ND & 30 & 0 \\
\hline$O$ KD & Jo & 0 \\
\hline 0 KD & so & 0 \\
\hline$O$ KO & Jo & 0 \\
\hline$O$ ND & JO & 0 \\
\hline$O$ KD & 10 & 0 \\
\hline 0 KO & JO & 0 \\
\hline 0 KO & jo & 0 \\
\hline 0 & Jo & 0 \\
\hline 0 NO & so & 0 \\
\hline 0 ND & so & 0 \\
\hline 0 KO & 30 & 0 \\
\hline 0 KO & so & 0 \\
\hline 0 KD & JO & 0 \\
\hline 0 KD & Jo & 0 \\
\hline 0 KO & 39 & 0 \\
\hline 0 AD & 30 & 0 \\
\hline 0 & so & \\
\hline
\end{tabular}




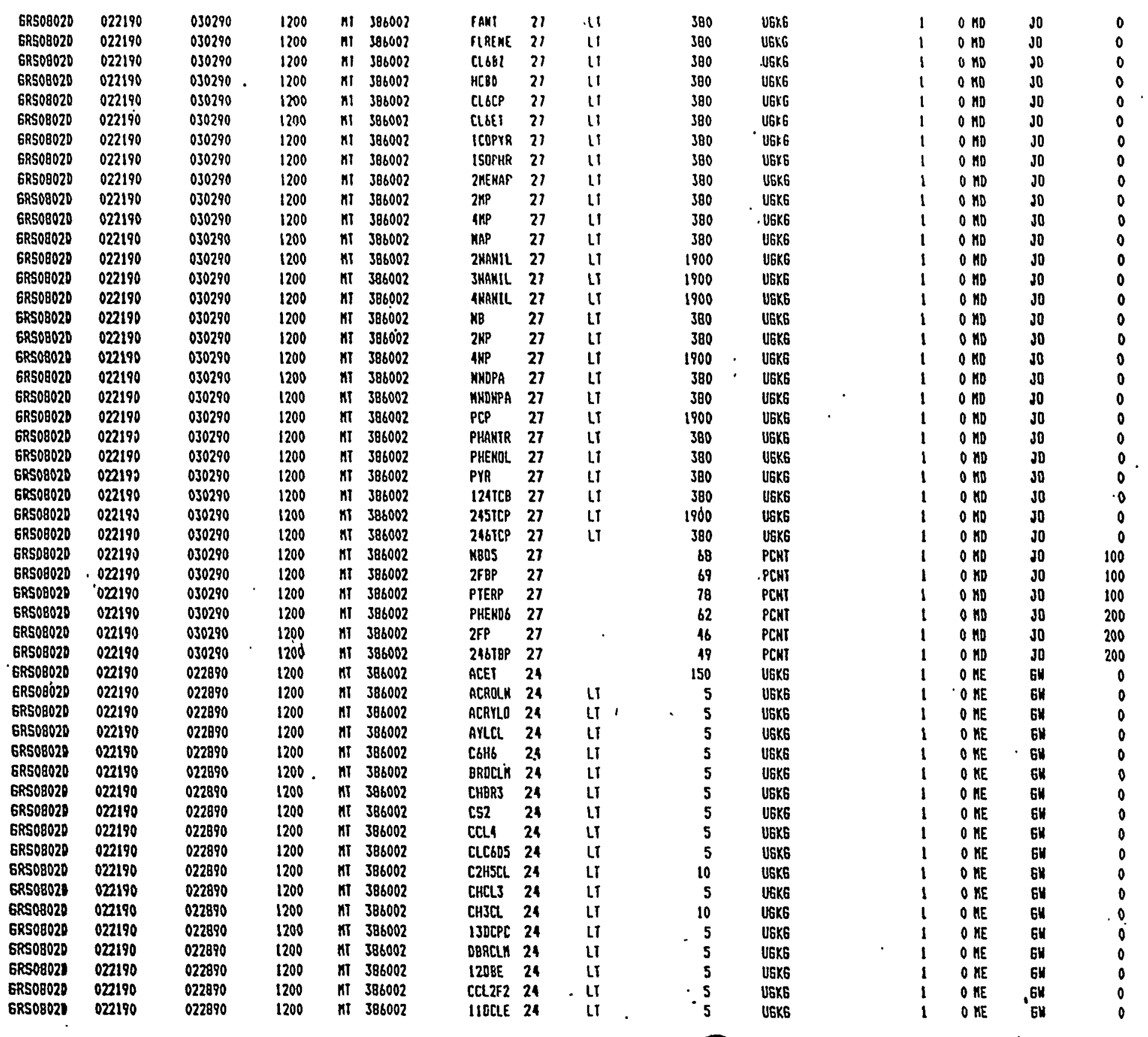


$p^{p-} \cdot p^{-}$

YestinghOUSE

A.date A. IIME LAB LAR. 10

ANAL YIE KETH

nOD CONC

UHIT ACCUR DIL NDIL INSTR IHIT SFIKE_CONC

GR508020 $022190 \quad 022890$

022890
022890

1200 in 386002

IZOCLE 21

I2DCLP 24

EIMEAC 24

022190

022890

300 II 396002

EICGHS 21

022890

1200 का 386002

2HTOME 21

IHOCAY 24

50508020

1200 nT 386002

MEACAY 29

6RS08020 022190

022890

1200 nI 386002

MEMEAC 21

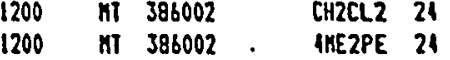

GROBO20 $022190-02289$

$\begin{array}{llllll}\text { GRS08020 } & 022190 & 022890 & 1200 & \text { KI } & 386002\end{array}$

CLSET 24

GR508020 022190

1200 if 386002

PROMIT 24

GRSOBO20 022190

1200 nI 386002

$\begin{array}{llllll}6 R S 08020 & 022190 & 022890 & 1200 & \text { nT } 386002\end{array}$

6RS0802D $022190 \quad 022890$

6R508020 $022190 \quad 022890$

GRSOBO20 $022190 \quad 022890$

1200 in 386002

SIYR 24

TICLEA 24

TCLEA 24

REC6H5 24

II3DCP 24

IIITCE 24

GR508020 022190

IIT 386002

IIITCE 24

00909020.022190

022890

1200 Mi 386002

CLIJ 24

I23CPR 24

VITYLA 24

GRSOB020 022190 022890

1200 IIT 386002

IILEW 21

I20CD4 21

GRS0802D $022190 \quad 022890$

1200 रा 386002

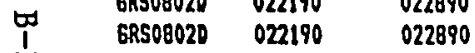

1200 ni 386002

MEC608 24

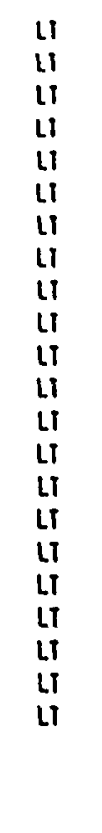

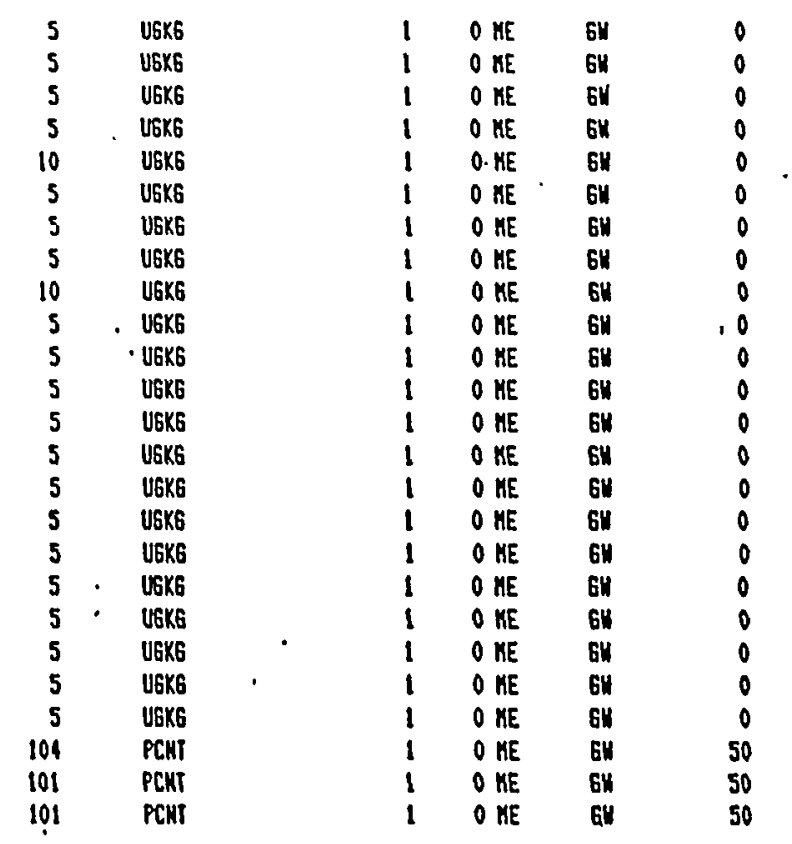




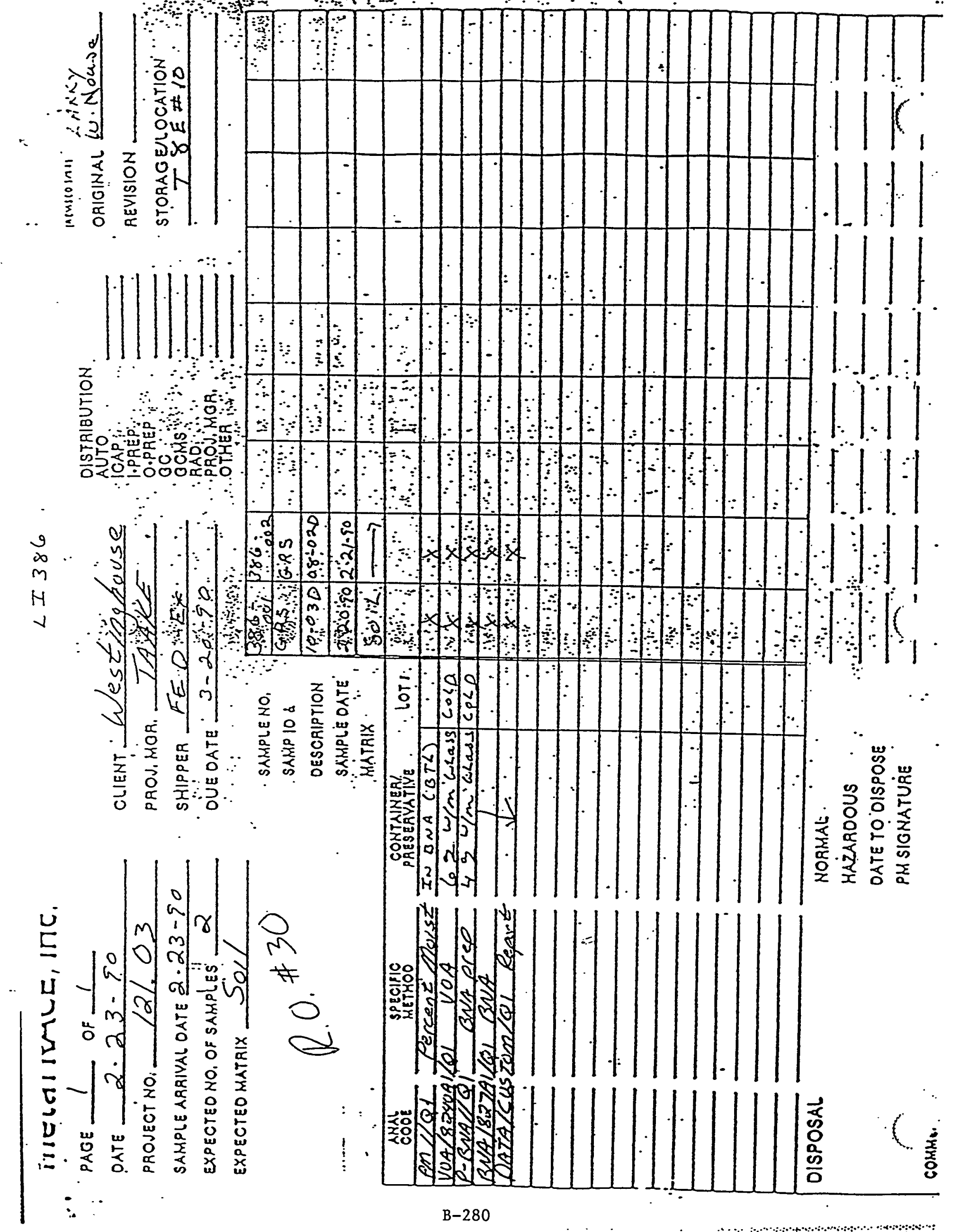


$\because$ VITRINE

Chain of Custody. Record

Page $\frac{i}{0}$ of $\frac{1}{68178}$

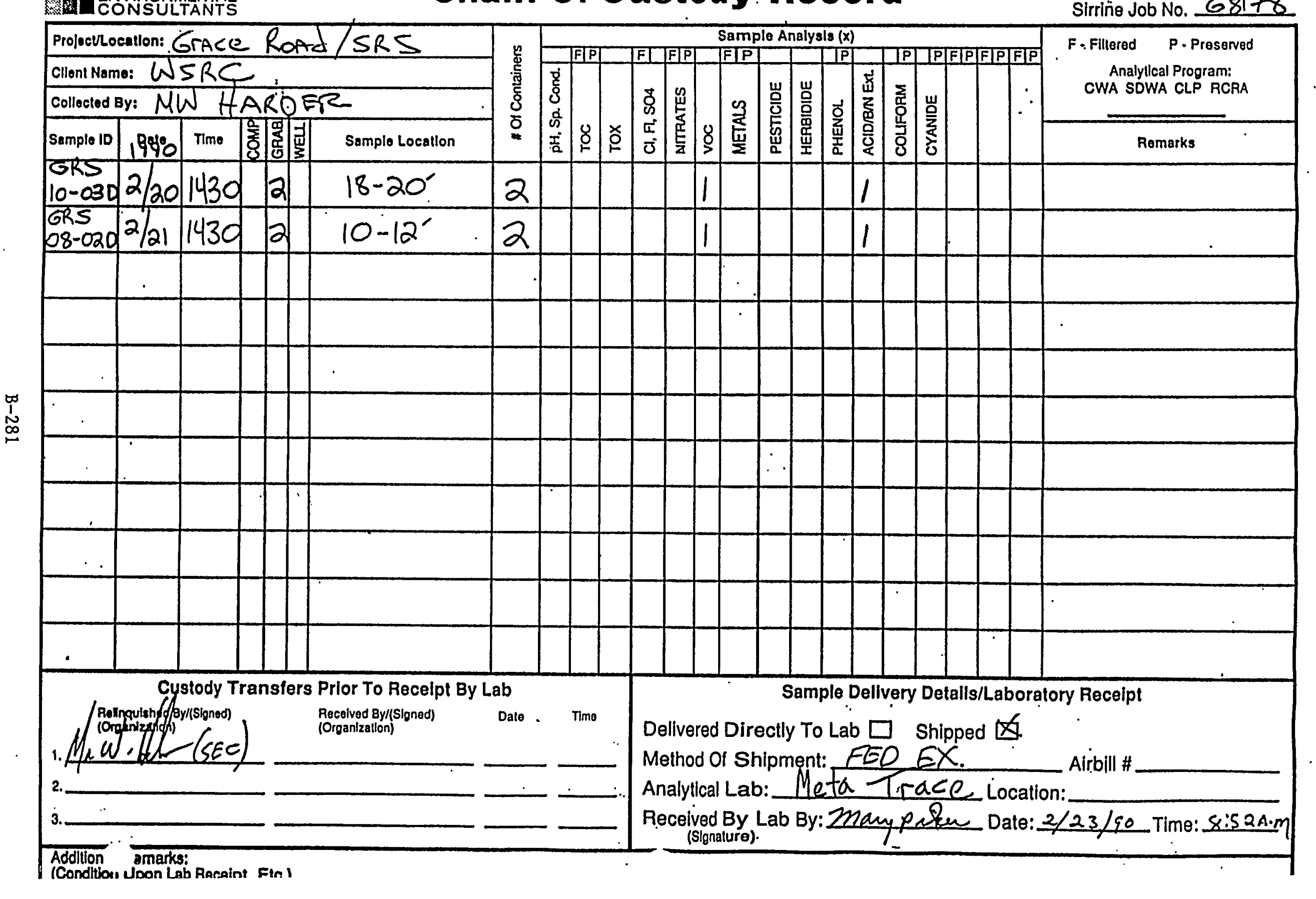


SIRRINE ENVIRONMENTAL CONSULTANTS

Post Omice Böx 24000

Greenville, Sóuth Carolina 29616

\section{REQUEST FOR ANALYSIS}

(This form must accompany samples.

Do Nol send under separale cover)
PROJECT NAME

PRO. MGR. / JOB NO.

PROFIT CENTER NO.

BILLTO

\section{P. O. NUMBER}

\section{\begin{tabular}{|l|l|l|}
\hline SAMTPLE NO. SAMPLE TYPE & SAMIPLE YOLUME \\
\hline
\end{tabular}}

RS - 10-030

GRS-08-020

Seil

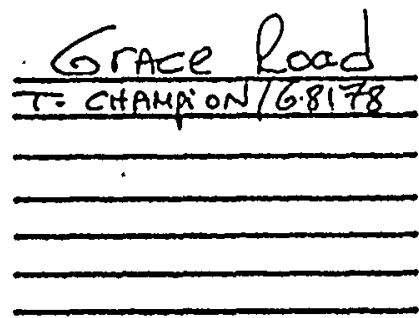

DATE SAMPLES SHIPPED

LAB DESTINATION

LAB CONTACT

REPORT TO

page lofl

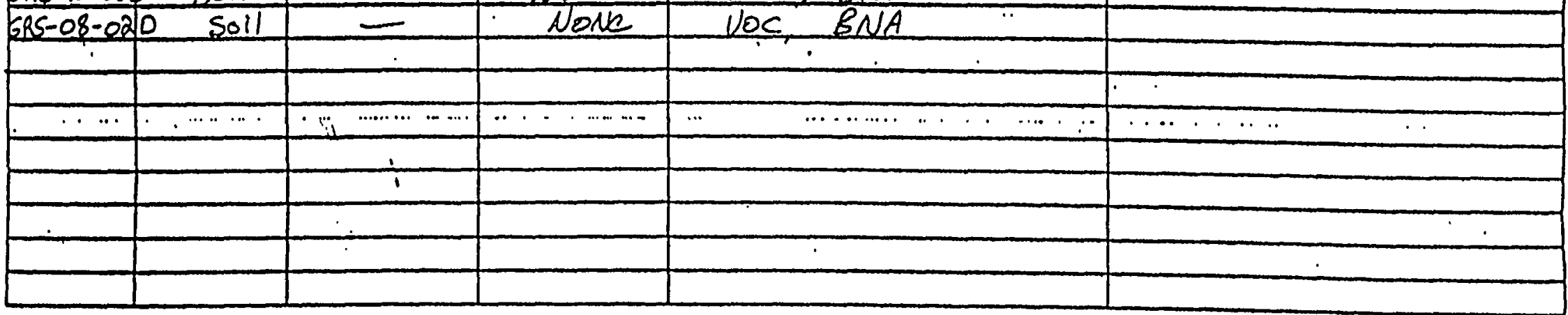

TURNAROUND TIME REQUIRED: (Turnaround time musl be arranged with lab prlor to shipment)
NORMAL
RUSH
(Subject to rush service charge)

POSSIBLE HAZARD IDENTIFICATION: (Please indicate if samples are hazardous materials and/or suspecled lo contain high levels of chemical compounds.)

FLAMMABLE POISON SKIN IRRITANT TOXIC

OTHER (Please speciry)

SAMPLE DISPOSAL: (Please indicate disposition of sample following añiysts-Lab will charge for packing, shippling and disposal.) RETURN TO CLIENT DISPOSAL BY LAB FO? 3 USE ONLY 


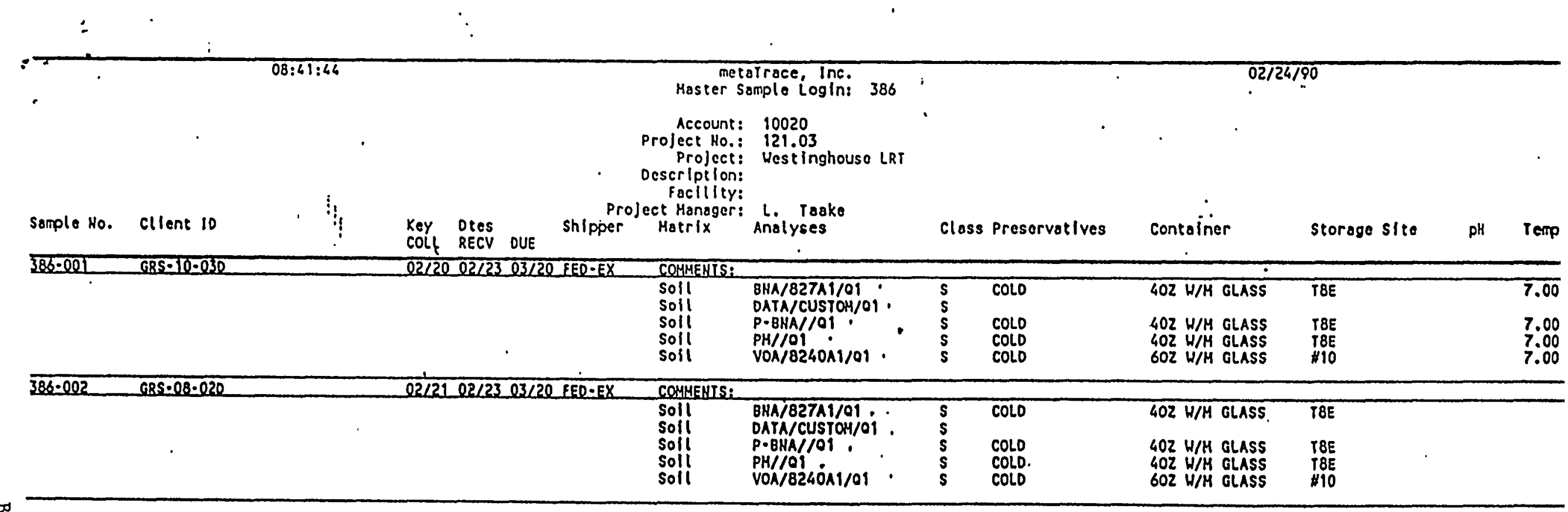

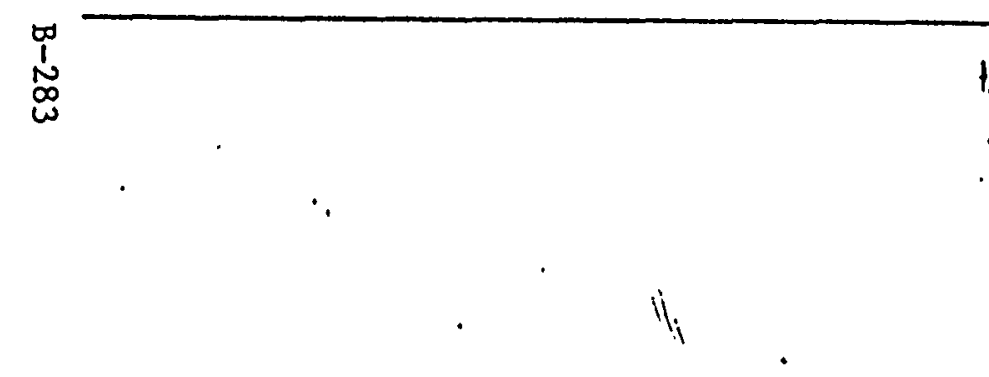



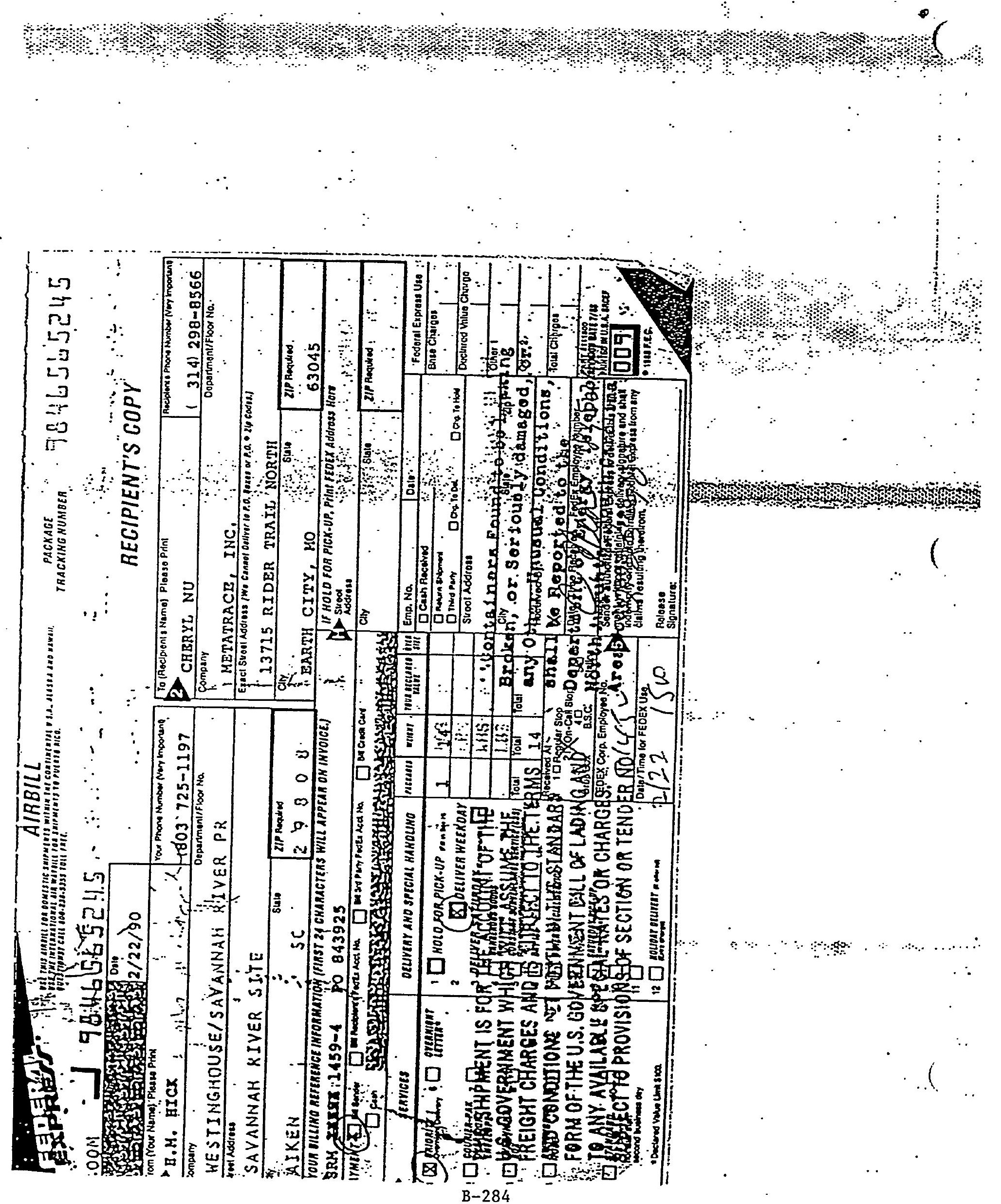
per.... 1

CLIEXT_IO S_DATE A.DAIE A.IIKE LAB LAB_IO GMALYTE HETH nOO CONE

UNII

ACCUR DIL N_DIL INSIR INIT SPINE_CONC

14 38600IRE

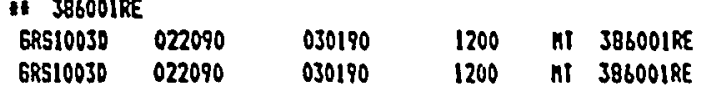

6R510030 $022090 \quad 030190 \quad 1200$ KI 386001RE

GRS10030 $022090 \quad 030190 \quad 1200$ II 386001 RE

6R51003D $022090 \quad 030190 \quad 1200$ nI 386001RE

GRS10030 $022090 \quad 030190 \quad 1200 \quad \mathrm{nt}$ 386001RE

6R510030 022090 030190 1200 nI 386001RE

GRS10030 $022090 \quad 030190 \quad 1200$ ni 386001RE

GRS100J0 $022090 \quad 030190 \quad 1200$ nI 38600IRE

6PS10030 $022090 \quad 030190$

1200 NI 386001 RE

$\begin{array}{lllll}\text { GRS10030 } 022090 & 030190 & 1200 & \text { NI } & \text { 38BOOLIRE }\end{array}$

$\begin{array}{lllll}\text { GRS } 10030 & 022090 & 030190 & 1200 & \text { IIT } \\ 386001 P E\end{array}$

$\begin{array}{lllll}\text { 6RS10030 } & .022090 & 030190 & 1200 & \text { NT } \\ \text { JBS6001RE }\end{array}$

GRS10030 $022090 \quad 030190 \quad 1200$. NT 386001 IIE

$\begin{array}{llllll}68510030 & 022090 & 030190 & 1200 & \text { ni } 386001 R E\end{array}$

GRS10030 $022090 \quad 030190 \quad 1200$ in J8 J800IRE

GRS10033 $022090-030190$

1200 AI 386001RE

$\begin{array}{lllll}\text { 6R510030 } 022090 & 030190 & 1200 & \text { HI } & 386001 \text { RE }\end{array}$

c8510030 $022090-030190$

6R510030 $022090 \quad 030190$

GR510030 022090 030190

1200 hi JB600IRE

1200 VT 38600IRE

1200 HI 3860018

GRS10030 $022090-030190$

1200 iI 386001 RE

6R510030 022090

030190

ERS10031 $022090 \quad 030190$

SRS100JS $022090 \quad 030190$

GR510033 $022090 \quad 030190$

GRS10030 $022090 \quad 030190$

NI 386001RE

1200 Ni 38600IRE

1200 AI 38600IRE

1200 II 386001 RE

1200 nI 386001RE

ER510039 $\cdot .022090$

030190

1200 जI JBSOOIRE

GRS10030 022090

$\begin{array}{llll}\text { 6RS10031 } & 022090 & & 030190 \\ \text { GRS10030 } & 022090 & . & 030190\end{array}$

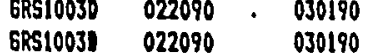

$\begin{array}{lll}\text { 6R510030 } & 022090 & 030190\end{array}$

6R510030 $022090 \quad 030190$

6R510039 $022090 \quad 030190$

6R510031 $022090 \quad 030190$

$\begin{array}{lll}\text { ERS10030 } & 022090 & 030190 \\ \text { GRS10030 } & 022090 & 030190\end{array}$

$\begin{array}{lll}\text { GAS1003D } & 022090 & 030190 \\ \text { GRS10031 } & 022090 & 030190\end{array}$

GRS10030 $022090 \quad 030190$

6RS10030 $022090 \quad 030190$

ERS1003D $022090 \quad 030190$

GRS10031 $022090 \quad 030190$

GRS10030 $022090 \quad 030190$

$\begin{array}{lll}68510030 & 022090 & 030190\end{array}$

GRS10030 $022090 \quad 030190$

6R510030 $022090 \quad 030190$

GRS10030 $022090 \quad 030190$

1200 IIT 386001RE

1200 HI 38600tRE

1200 hi 386001RE

1200 MI J86001RE

1200 nT 3BSOOIRE

1200 . IT 38600IRE

1200 II J880IRE

1200 HT 38BO0IRE

1200 MI .38600LRE

1200 MT 38600IRE

1200 II 386001 RE

1200 iT 38600LRE

$\begin{array}{lll}1200 & \text { HI } & 386001 \mathrm{RE} \\ 1200 & \text { hI } & 386001 R E\end{array}$

1200 IIT 386001RE

1200 IIT 38600IRE

1200 nT 39800IRE

030190

1200 NT 386001RE

GRS1003: 022090

1200 MI J86001RE

AKAFXE $27 \quad$ LI $\quad 390$

AMAPLY $27 \quad 11 \quad 390$

AMIRC $27 \quad$ LI $\quad 390$

$\begin{array}{lll}\text { BEMrOA } 27 & 41\end{array}$

BAAHIR $27 \quad$ LI $\quad 390$ UGKG

$\begin{array}{llll}\text { BKFANT } 27 & 11 & 390 & \end{array}$

BEHIPY 27 LI 390 U6KG

BAPYR 27 LI 390 UEKG

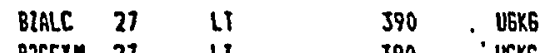

$\begin{array}{llll}\text { BCEEXI } & 27 & \text { LT } & \\ \text { BCEE } & 27 & 41 & 390\end{array}$

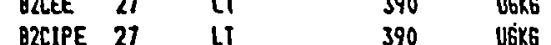

B2EFP $27 \quad 9000$ UGKG

4BRPPE 27 LT 390 W6KG

$\begin{array}{lllll}\text { BBLP } & 27 & \text { LT } & 390 & \text { US KG }\end{array}$

$\begin{array}{llll}\text { LIT } & 390 & \text { UEKG }\end{array}$

2017 $27 \quad 390 \div$ U65k6

4CLPPE 27 LT 390 UFK

CHAY 27 LI 390 U6K6

DBAHA 27 LI 390 UGKG

DIBIFU 27 LT $390 \quad$ UEKG

120CLR 27 LI 390 USKG

130CLB 27 LT 390 UEKG

$\begin{array}{llll}\text { 110C.B } 27 \text { LT } & 390 & \text { UGKG }\end{array}$

OHEP 27 ' LT 390 UEKG

$\begin{array}{lllll}33 C 28 & 27 & \text { LT } & 780 & \text { UEKG } \\ 240 C L P & 27 & \text { LI } & 390 & \text { UEKB }\end{array}$

$\begin{array}{llll}263 C L P & 27 & 17 & 390\end{array}$

$\begin{array}{lllll}\text { DEP } 27 & 27 & 390 & \text { UGKG }\end{array}$

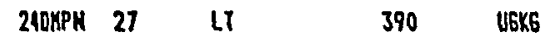

$\begin{array}{llll}\text { DMP } 27 & \text { LT } & 390 & \text { UGKG }\end{array}$

$\begin{array}{llll}240 \mathrm{NP} & 27 & \text { LT } & 2000 \\ & & \end{array}$

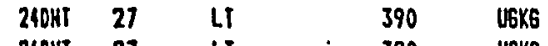

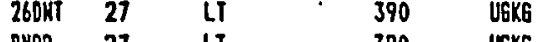

$\begin{array}{llll}\text { FAIT } 27 & 390 & \text { USKG }\end{array}$

FLAENE 27 LI $\quad 390$ UGKG

$\begin{array}{llll}\text { CL681 } 27 & \text { LT } & 390 & \text { UGKG }\end{array}$

$\begin{array}{lllll}\text { HCBO } & 27 & \text { LT } & 390 & \text { UEKG }\end{array}$

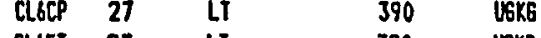

$\begin{array}{lll}27 & 390 & \text { Uak6 }\end{array}$

- ISTHR 27 LI

ISOHR 27

$\begin{array}{ll}2 \text { MRE } & 27 \\ 21 & 27\end{array}$

4

$\begin{array}{ll}\text { IHP } & 27 \\ \text { MAP } & 27\end{array}$

2 MANIL 27
3HAIL 27

उHANIL 27

$L T$
LT
LT
LT
LT

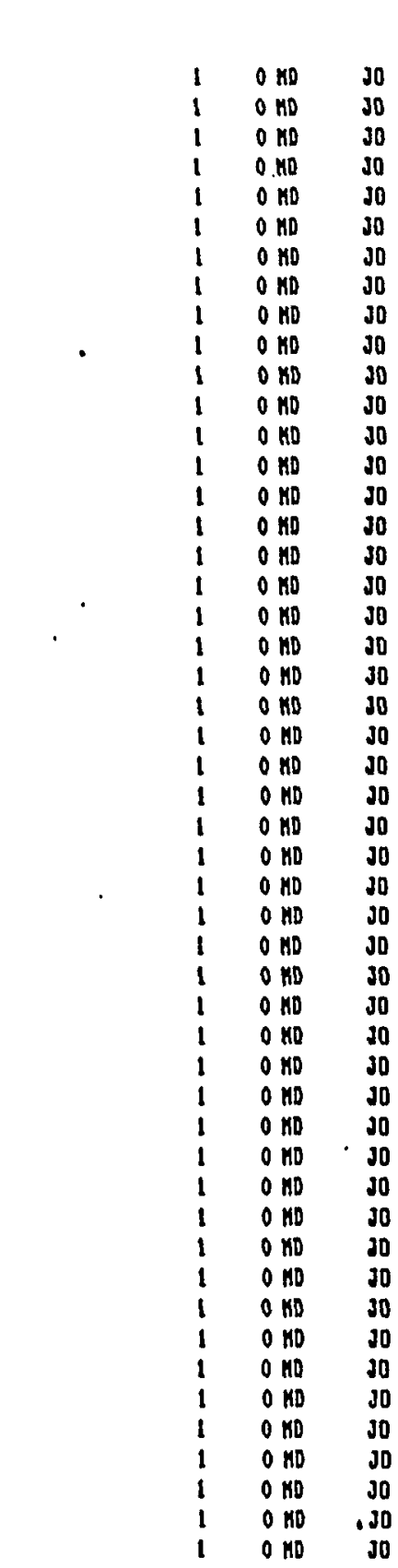


Page No.

WESTINGHOUSE 121-03

\begin{tabular}{|c|c|c|c|c|c|c|c|c|c|c|c|}
\hline CLIEMI_ID & 5_DAlE & A. OAIE & A.lline & $1 A 81 A B .10$ & AKALYIE ME IH & noo & CONC & UNII & ACCUR & DIL & N.OIL INSIR \\
\hline
\end{tabular}

\begin{tabular}{|c|c|c|c|c|c|c|c|c|c|c|}
\hline 6R5100JD & 022090 & 030190 & 1200 & mi & sob001kE & IXANIL & 21 & LI & 2000 & UGKG \\
\hline GRS10030 & 022080 & 030190 & 1200 & mI & 38600 IRE & NB & 21 & LI & 390 & U6KG \\
\hline GRS10030 & 022090 & 030190 & 1200 & nI & 38600IRE & $2 n$ & 21 & LI & 390 & UGKG \\
\hline GR510030 & 022090 & 030190 & 1200 & nI & JBSOOIRE & thep & 21 & 41 & 2000 & UGKE \\
\hline GRS10030 & 022090 & 030180 & 1200 & $n$ & 38600IRE & KKOPA & 21 & LI & 390 & UGKG \\
\hline GRS10030 & 022090 & 030190 & 1200 & nt & 3Q600IRE & NKONPA & 21 & $L I$ & 390 & UEK6 \\
\hline 6RS10030 & 022090 & 030190 & 1200 & $n i$ & J日6OOIRE & PCP & 27 & LI & 2000 & UGKG \\
\hline GRS10030 & 022090 & 030190 & 1200 & $\mathrm{nI}$ & 38600IRE & PHANIR & 21 & LI & 390 & UEKG \\
\hline 6RS10030 & 022090 & 030190 & 1200 & nT & 3ESO0IRE & PHENOL & 27 & LI & 390 & UGKG \\
\hline ERS1003D & 022090 & 030190 & 1200 & Mr & 38600IRE ${ }^{\circ}$ & PYR & 27 & LI & 390 & UGKG \\
\hline GRS10030 & 022090 & 030190 & 1200 & Tו & J86001RE & $124 \mathrm{TCB}$ & 27 & LI & 390 & . UEKG \\
\hline GRS1003D & 022090 & 030190 & 1200 & nT & 38600IRE & $245 T C P$ & 21 & LI & 2000 & UGK6 \\
\hline 6RS10030 & 022090 & 030190 & 1200 & ii & 38600LRE & $216 T C P$ & 27 & LI & 390 & UEKG \\
\hline ERS10030 & 022090 & 030190 & 1200 & nit & 38600IRE & NBDS & 27 & & 73 & PCHI \\
\hline 6RS10030 & 022090 & 030190 & 1200 & ИT & 38G00IRE & $2 F B P$ & 27 & & 72 & PCHI \\
\hline GRS10030 & 022090 & 030180 & 1200 & ni & 38600IRE & PIERP & 27 & & 80 & PCNI \\
\hline 6R510030 & 022090 & 030190 & 1200 & $n$ & J8600iRE & PHENOG & 27 & & 65 & PCHI \\
\hline SRS10030 & 022090 & 030190 & 1200 & Mr & 38600IRE & $2 F P$ & 27 & & $5 !$ & PCNT \\
\hline GRS10030 & 022090 & 030190 & 1200 & nT & 38600IRE & $246 T B P$ & 27 & & 52 & PCUT \\
\hline \multicolumn{11}{|l|}{ If 386001} \\
\hline GR510030 & 022090 & 022890 & 1200 & II & 386001 & ACET & 24 & LT & 10 & UEKG \\
\hline ER51003D & 022090 & 022890 & 1200 & MT & 386001 & ACROLK & 24 & LT & 5 & UGKG \\
\hline 6R51003D & 022090 & 022890 & 1200 & nit & 386001 & ACAYLO & 24 & LT & 5 & UEK6 \\
\hline GR510030 & 022090 & 022890 & 1200 & nT & 386001 & ArLCL & 24 & LT & 5 & UGKG \\
\hline GR510030 & 022090 & 022890 & 1200 & $n$ & 386001 & C6H6 & 24 & LT & 5 & UGKG \\
\hline GRS10030 & 022090 & 022890 & 1200 & $\mathrm{n}$ & 386001 & BROCLN & 24 & LT & 5 & UGKG \\
\hline GR510030 & 022090 & 022890 & 1200 & זT & 386001 & CHBRJ & 24 & LT & 5 & USKG \\
\hline GRS10030 & 022090 & 022890 & 1200 & KI & 386001 & CS2 & 24 & LT & 5 & UGKG \\
\hline 6R510030 & 022080 & 022890 & 1200 & II & 386001 & CCL4 & 24 & LI & 5 & UEKG \\
\hline GRS10030 & 022090 & 022890 & 1200 & $n \pi$ & 386001 & CLCGDS & 24 & 41 & 5 & UEKG \\
\hline ERS10030 & 022090 & 022890 & 1200 & MT & 386001 & C2HSCL & 24 & LT & 10 & UGKG \\
\hline GR5100030 & 022080 & 022890 & $1200^{\circ}$ & KI & 386001 & CHCL3 & 24 & LT & 5 & UEKG \\
\hline GRS10030 & 022090 & 022890 & 1200 & nI & 386001 & CHJCL & 24 & LT & 10 & UGK6 \\
\hline 6RS1003D & 022090 & 022890 & 1200 & KT & 386001 & 130CPC & 24 & LT & 5 & UEKG \\
\hline GRS1003: & 022090 & 022890 & 1200 & MT & 386001 & DBRCLK & 24 & LT & 5 & UEKG \\
\hline 6R51003: & 022090 & 022890 & $1200^{\circ}$ & MT & 386001 & I2OBE & 24 & L.T & 5 & UGKG \\
\hline 6RS10030 & 022090 & 022890 & 1200 & זות & 386001 & CCL2F2 & 24 & LI & 5 & UEKG \\
\hline GR510030 & 022090 & 022890 & 1200 & nT & 386001 & HIDCLE & 24 & LT & 5 & UGKG \\
\hline GRS1003D & 022090 & 022890 & 1200 & $n \pi$ & 386001 & 120CLE & 24 & LT & 5 & UGK6 \\
\hline GRS1003D & 022090 & 022890 & 1200 & nT & 386001 & $120 \mathrm{CP}$ & 24 & LT & 5 & UEKE \\
\hline GRS10030 & 022090 & 022890 & 1200 & $n$ & 386001 & ETMEAC & 24 & LT & 5 & UEKE \\
\hline GR510030 & 022090 & 022890 & 1200 & nT & 386001 & ETC6H5 & 24 & LI & 5 & UEKG \\
\hline GRS10030 & 022090 & 022890 & 1200 & ni & 386001 & 2HLONE & 24 & LI & 10 & UEKG \\
\hline 6RS10030 & 022090 & 022890 & 1200 & IT & 386001 & MEACRY & 24 & LT & $\mathbf{s}$ & UEKE \\
\hline 6R510030 & 022090 & 022890 & 1200 & nI & 386001 & NEMEAC & 24 & LT & 5 & UEKG \\
\hline ERS1003D & 022090 & 022890 & 1200 & $n T$ & 386001 & $\mathrm{CH}_{2} \mathrm{CL}_{2}$ & 24 & LT & $\mathbf{S}$ & UGKG \\
\hline 6RS10030 & 022090 & 022890 & 1200 & nT & 386001 & 4KE2PE & 24 & LT & 10 & UEKG \\
\hline 68510030 & 022090 & 022890 & 1200 & nit & 386001 & CLSEI & 24 & LT & 5 & UEKE \\
\hline 6RS1003: & 022090 & 022890 & 1200 & MT & 386001 & PRONIT & 24 & LI & $\cdot 5$ & UEKG \\
\hline ERS1003: & 022090 & 022890 & 1200 & nT & 386001 & SIYR & 24 & LI & 5 & UEKE \\
\hline
\end{tabular}

\begin{tabular}{|c|c|c|}
\hline 0 NO & jo & 0 \\
\hline 0 NO & 30 & 0 \\
\hline 0 KO & J0 & 0 \\
\hline 0 KO & Jo & 0 \\
\hline 0 KD & J0 & 0 \\
\hline$O \mathrm{HO}$ & J0 & 0 \\
\hline 0 ND & J0 & 0 \\
\hline 0 KO & 30 & 0 \\
\hline 0 KD & JD & 0 \\
\hline $0 \mathrm{NO}$ & 30 & 0 \\
\hline$O$ ND & Jo & 0 \\
\hline 0 no & J0 & 0 \\
\hline$O$ KD & Jo & 0 \\
\hline O KD & J0 & 100 \\
\hline 0 No & Jo & 100 \\
\hline $0 \mathrm{HD}$ & JO & 100 \\
\hline$O$ ND & Jo & 200 \\
\hline 0 & JO & 200 \\
\hline $0 \mathrm{MO}$ & 30 & 200 \\
\hline
\end{tabular}

$\underset{\infty}{\infty}$


p.r. 13

UESIIKEHDUSE , . .

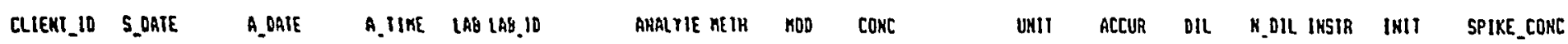

\begin{tabular}{|c|c|c|c|c|c|c|c|c|c|c|c|c|c|}
\hline GRS10030 & 022090 & 022890 & 1200 & nI & 386001 & 2ICLEA & 26 & II & 5 & UEKG & 1 & $O$ KE & 6N \\
\hline 6R5iooso & 022090 & 022890 & 1200 & KI & 386001 & ILLEA & 24 & LI & $s$ & UEKG & 1 & $O \mathrm{KE}$ & 64 \\
\hline 6RS10030 & 022090 & 022890 & 1200 & AI & 386001 & MECBHS & 21 & 41 & 5 & UEK6 & 1 & $O$ KE & 64 \\
\hline ERS1003D & 022090 & 022890 & 1200 & KI & 386001 & $1130 C P$ & 24 & II & 5 & 46K6 & 1 & $O \mathrm{KE}$ & 64 \\
\hline ERS10030 & 022090 & 022890 & 1200 & KI & 386001 & IIIICE & 21 & 11 & 5 & USK6 & 1 & $O \mathrm{KE}$ & EV \\
\hline ERS10030 & 022090 & 022890 & 1200 & HI & 386001 & II2ICE & 21 & Li & 5 & UEKG & 1 & $O \mathrm{HE}$ & 6W \\
\hline SRS1003D & 022090 & 022890 & 1200 & $n$ & 386001 & CCLSF & 24 & 11 & $s$ & UEKG & 1 & $O \mathrm{KE}$ & EN \\
\hline 6RS10030 & 022090 & 022890 & 1200 & II & 386001 & 123CPR & 21 & LI & 5 & UEKG & 1 & $O \mathrm{HE}$ & 64 \\
\hline 6R510030 & 022090 & 022890 & 1200 & $\mathrm{nI}$ & 386001 & VINYLA & 21 & LI & 5 & UEKG & 1 & $O \mathrm{ME}$ & 64 \\
\hline ERS10030 & 022090 & 022890 & 1200 & ถा & 388001 & IYLEN & 21 & uI & 5 & . UEKG & I & $O$ HE & 6H \\
\hline GR510030 & 022090 & 022890 & 1200 & XI & 386001 & $120 C 01$ & 24 & $\cdot$ & 101 & - PCHI & 1 & $O \mathrm{KE}$ & 6N \\
\hline 6RS10030 & 022090 & 022890 & 1200 & nI & 386001 & MECBDB & 24 & & 104 & PCHT & 1 & $O \mathrm{KE}$ & 64 \\
\hline ERS10030 & 022090 & 022890 & 1200 & nt & 386001 & PQFB & 24 & & 100 & PCHT & 1 & $O M E$ & 6H \\
\hline
\end{tabular}

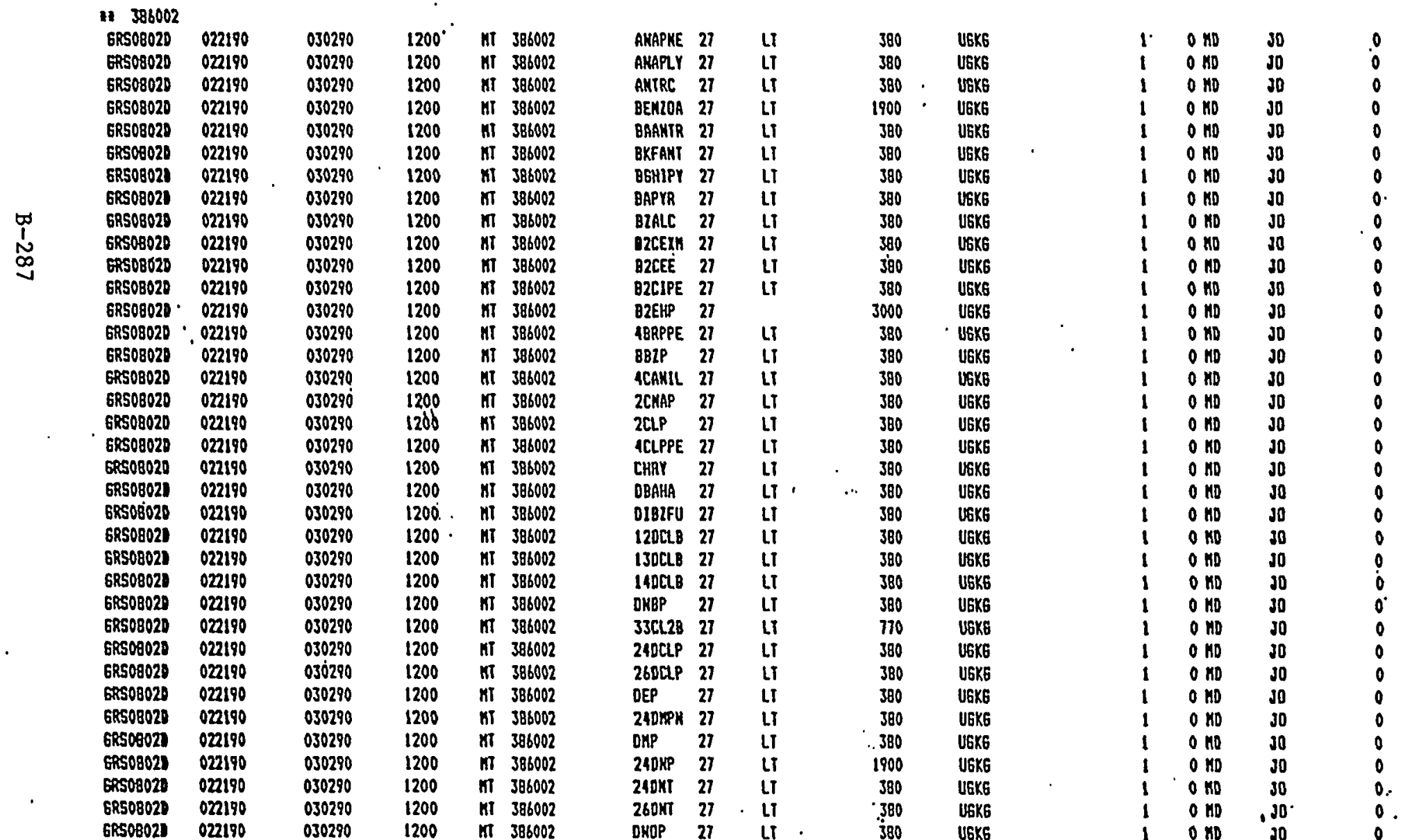

$\therefore: \because$ 


\begin{tabular}{|c|c|c|c|c|c|c|c|c|c|c|}
\hline CLIENI_10 & 5.DATE & A. DAIE & A. IIKE & LAB & LAG. 10 & AHALYIE & $\mathrm{HCIH}$ & MOD & COHC & US11 \\
\hline 6R508020 & 022190 & 030290 & 1200 & $n$ & 586002 & IANI & 21 & 11 & 380 & UEKG \\
\hline ERSOBO2D & 022190 & 030290 & 1200 & $n !$ & 386002 & FLAEKE & 21 & 11 & 380 & UGKG \\
\hline GR5OBO20 & 022190 & 030290 & 1200 & $n$ & 386002 & Clsel & 21 & LI & 380 & 86K6 \\
\hline 6R508020 & 022190 & 030290 & 1200 & $n$ & 386002 & HCBO & 21 & $\lfloor 1$ & 380 & U6K6 \\
\hline 6R508020 & 022190 & 030290 & 1200 & $n i$ & 386002 & CLSCP & 21 & $\$ 1$ & $3 B O$ & U6K6 \\
\hline 6R50802D & 022190 & 030290 & 1200 & $n 1$ & 386002 & CLGET & 21 & LI & 380 & UEKG \\
\hline GRS08020 & 022190 & 030290 & 1200 & nI & 386002 & ICDPYR & 21 & 41 & 380 & UGKG \\
\hline ER508020 & 022190 & 030290 & 1200 & КT & 386002 & ISOPHR & 21 & 11 & 380 & U6K6 \\
\hline 6RS0B02D & 022190 & 030290 & 1200 & nI & 386002 & 2MENAP & 21 & 41 & 380 & UGKG \\
\hline 6R50B02D & 022190 & 030290 & 1200 & $n i$ & 386002 & $2 \mathrm{NP}$ & 21 & 41 & 380 & UEKG \\
\hline 6RS08020 & 022190 & 030290 & 1200 & $m$ & 386002 & AhP & 27 & 11 & 380 & UGKG \\
\hline GRS08020 & 022190 & 030290 & 1200 & ז & 386002 & KAP & 27 & LI & 380 & UGKG \\
\hline 6R508020 & 022190 & 030290 & 1200 & MT & 386002 & 2HAMIL & 21 & LI & 1900 & UEKG \\
\hline 6RS0602B & 022190 & 030290 & 1200 & $n$ & 386002 & JHANIL & 27 & LI & 1900 & UGKG \\
\hline GRS08020 & 022190 & 030290 & 1200 & II & 388002 & AKAKIL & 27 & $\mathrm{LT}$ & 1900 & UGKG \\
\hline GRS08020 & 022190 & 030290 & 1200 & $\boldsymbol{n}$ & 3860002 & KB & 27 & LI & 380 & UEKE \\
\hline 6RS08020 & 022190 & 030290 & 1200 & ni & 386002 & $2 \mathrm{KP}$ & 27 & LI & 380 & UGKG \\
\hline 6RS08020 & 022190 & 030290 & 1200 & $n \pi$ & 386002 & ANP & 27 & LII & 1900 & UGKG \\
\hline 6R508020 & 022190 & 030290 & 1200 & $n$ & 386002 & MNDPA & 27 & LI & 380 & UGKG \\
\hline GRS08020 & 022190 & 030290 & 1200 & MI & 386002 & MKDKPA & 27 & LI & 380 & UEX6 \\
\hline 6R50B02D & 022190 & 030290 & 1200 & $\mathbf{n}$ & 386002 & PCP & 27 & LI & 1900 & UGKG \\
\hline 6R50802D & 022190 & 030290 & 1200 & $n T$ & 386002 & PHAMTR & 27 & LI & 380 & UGK6 \\
\hline 6R508020 & 022190 & 030290 & 1200 & nI & 396002 & PHEKDL & 27 & LI & 380 & UGKG \\
\hline 68508020 & 022190 & 030290 & 1200 & หI & 386002 & PYR & 27 & LI & 380 & UEKE \\
\hline 6RS08020 & 022190 & 030290 & 1200 & $\pi$ & 386002 & 124TCB & 27 & LI & 380 & UGKG \\
\hline 68508020 & 022190 & 030290 & 1200 & $\mathrm{nI}$ & 386002 & $2451 C P$ & 27 & LI & 1900 & UGKE \\
\hline 6R508020 & 022190 & 030290 & 1200 & hi & 386002 & $246 T C P$ & 27 & LT & 380 & UGKG \\
\hline GRSOBO2D & 022190 & 030290 & 1200 & $n$ & 386002 & MBOS & 27 & & 68 & PCHT \\
\hline 6R50802D & 022190 & 030290 & 1200 & MT & 386002 & 2FBP & 27 & & 69 & PCHT \\
\hline GRSOBO2D & 022190 & 030290 & 1200 & nI & 386002 & PIERP & 27 & & 78 & PCHT \\
\hline 6R508020 & 022190 & 030290 & 1200 & KT & 386002 & PHENDG & 27 & & 62 & РСКT \\
\hline ERSO8020 & 022190 & 030290 & 1200 & MT & 386002 & $2 \mathrm{FP}$ & 27 & $\cdot$ & 16 & PCAT \\
\hline 6R50802D & 022190 & 030290 & 1200 & $n$ & 386002 & $24678 P$ & 27 & & 19 & PCHT \\
\hline GRSOBO2D & 022190 & 022890 & 1200 & MI & 386002 & ACET & 24 & & 150 & UEKB \\
\hline 6R508020 & 022190 & 022890 & 1200 & nI & 386002 & ACROL.K & 24 & LI & 5 & UGKG \\
\hline GRSOBO2D & 022190 & 022890 & 1200 & MI & 386002 & ACRYLO & 24 & LI 1 & 5 & U6K6 \\
\hline 6R508020 & 022190 & 022890 & $1200^{\circ}$ & MI & 386002 & AYLCL & 24 & L.T & 5 & UEX6 \\
\hline GRS08020 & 022190 & 022890 & 1200 & nI & 386002 & CBHS & 24 & LT & 5 & UGKK \\
\hline GR50802D & 022190 & 022890 & 1200 & $n I$ & 386002 & BRDCAK & 24 & LI & 5 & UEKE \\
\hline 6RSOBO2D & 022190 & 022890 & 1200 & nI & 386002 & CHBR3 & 24 & LT & 5 & U6XG \\
\hline GRS08020 & 022190 & 022890 & 1200 & MI & 386002 & CS2 & 24 & LI & 5 & UGKG \\
\hline GRS08020 & 022190 & 022890 & 1200 & $n$ & 386002 & CCL4 & 24 & LT & 5 & U6KG \\
\hline GRSOBO2D & 022190 & 022890 & 1200 & KT & 386002 & ClcoDs & 24 & LI & 5 & UGKG \\
\hline $6 R 508020$ & 022190 & 022890 & 1200 & $\mathrm{nI}$ & 386002 & C2H5CL & 24 & LT & 10 & U6K6 \\
\hline 6R508028 & 022190 & 022890 & 1200 & KT & 385002 & CHCL3 & 24 & $\mathrm{LT}$ & 5 & UEKG \\
\hline GRSOBO20 & 022190 & 022890 & 1200 & nI & 388002 & CH3CL & 24 & LT & 10 & UEKG \\
\hline 6R50802D & 022190 & 022890 & 1200 & $\mathbf{m}$ & 386002 & - $13 D C P C$ & 24 & LT & 5 & UEKE \\
\hline 6RSOBO2D & 022190 & 022890 & 1200 & ит & 386002 & DBRCLX & 24 & LI & 5 & UEK6 \\
\hline GRS08021 & 022190 & 022890 & 1200 & KT & 586002 & 120BE & 24 & LT & 5 & UGKS \\
\hline 6PSO8020 & 022190 & 022890 & 1200 & $n$ & $398002^{\circ}$ & CCL2F2 & 24 & LT & 5 & U66KG \\
\hline 6R508021 & 022190 & 022890 & 1200 & $n t$ & 386002 & IIOCLE & 24 & LI & 5 & UEKG \\
\hline
\end{tabular}

\begin{tabular}{|c|c|c|}
\hline 0 KD & so & 0 \\
\hline O ND & Jo & 0 \\
\hline $0 \mathrm{HD}$ & jo & 0 \\
\hline 0 & Jo & 0 \\
\hline 0 KD & Jo & 0 \\
\hline $0 \mathrm{MD}$ & 30 & 0 \\
\hline 0 KO & Jo & 0 \\
\hline 0 HD & Jo & 0 \\
\hline 0 No & Jo & 0 \\
\hline $0 \mathrm{KD}$ & Jo & 0 \\
\hline$O$ NO & so & 0 \\
\hline 0 NO & Jo & 0 \\
\hline$O$ KD & JD & 0 \\
\hline 0 No & so & 0 \\
\hline$O$ HD & Jo & 0 \\
\hline$O$ NO & 30 & 0 \\
\hline$O$ KO & 20 & 0 \\
\hline 0 Yo & 10 & 0 \\
\hline 0 KD & Jo & 0 \\
\hline 0 ND & Jo & 0 \\
\hline O HO & 30 & 0 \\
\hline$O$ KD & Jo & 0 \\
\hline$O$ ND & 30 & 0 \\
\hline$O$ NO & Jo & 0 \\
\hline$O$ KD & Jo & 0 \\
\hline$O$ HO & 30 & 0 \\
\hline 0 KD & Jo & 0 \\
\hline$O$ HD & 30 & 100 \\
\hline$O \mathrm{KD}$ & JO & 100 \\
\hline 0 KD & 30 & 100 \\
\hline$O$ HD & Jo & 200 \\
\hline$O$ HD & Jo & 200 \\
\hline$O$ KD & 30 & 200 \\
\hline$O \mathrm{ME}$ & 64 & 0 \\
\hline $0 \mathrm{KE}$ & 64 & 0 \\
\hline$O \mathrm{KE}$ & BV & 0 \\
\hline 0 NE & 64 & 0 \\
\hline$O \mathrm{KE}$ & 6N & 0 \\
\hline$O \mathrm{KE}$ & 6H & 0 \\
\hline$O \mathrm{ME}$ & 6W & 0 \\
\hline $0 \mathrm{KE}$ & 6H & 0 \\
\hline $0 \mathrm{ME}$ & 64 & 0 \\
\hline$O \mathrm{KE}$ & 64 & 0 \\
\hline$O \mathrm{KE}$ & 6W & 0 \\
\hline $0 \mathrm{KE}$ & 64 & 0 \\
\hline $0 \mathrm{ME}$ & .6W & $\delta$ \\
\hline$O \mathrm{ME}$ & 64 & 0 \\
\hline$O$ KE & 6X & 0 \\
\hline$O \mathrm{KE}$ & 6N & 0 \\
\hline $0 \mathrm{ME}$ & .6N & 0 \\
\hline $0 \mathrm{ME}$ & 6N & 0 \\
\hline
\end{tabular}


$17=5$

WESIIHGHOUSE

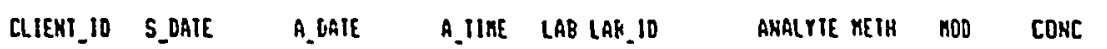

UNIT ACCUR DIL N_DIL INSIR IHII SPIKE,COANC

$\begin{array}{ll}\text { GRS08020 } & 022190 \\ \text { GRS08020 } & 022190\end{array}$

$022890 \quad 1200 \quad$ MI 386002

022890

1200 nI 386002

120CEE 21

120CLP 21

EIMEAC 21 it

GRSOBO2D 022190

1200 nI 386002

6850802002219

$022890 \quad 1200$ ni 386002

GR508020 022190

1200 NI 386002

GRSO8020 022190

$022890 \quad 1200$ ni 386002

6R5OBO20 022190

EICSHS 21

2HXONE 21

NEACRY 21

$\begin{array}{lll}1200 & \text { nI } & 386002 \\ 1200 & \text { nI } & 386002\end{array}$

HEREAC 21

CH2CL2 21

6R508020 022190

022890

1200 ni 388002

GRSOBO20 022190

022890

1200 nI 386002

CLSET 2

GR50BO20 022190

022890

300 ni 386002

PRONIT 24

GRSO8020 022190

$22890 \quad 1200$ NI 386002

ERSOBO20 022190

200 ni 386002

6R508020 022190

1200 Mi 386002

GRS08020 022190

022890 1200

6R5OBO20 022190

1200 nI 386002

6RSOB028 022190

GR508020 . 022190

$022890 \quad 1200$ MT 386002

$\begin{array}{llll}222890 & 1200 & \text { nT } & 386002 \\ 022890 & 1200 & \text { nT } 386002\end{array}$

6R508028 022190

$\begin{array}{lll}22890 & 1200 & \text { nT } 386002\end{array}$

$6 R 508020 \quad 022190$

022890

$\begin{array}{lll}1200 & \text { nT } & 386002 \\ 1200 & \text { nI } & 386002\end{array}$

SIYR 24
2ICLEA

2ICLEA 24

TCLEA 24

MECEHS 24

IIJOCP 24

IIITCE 24

II2ICE 24

CCLJF 24

I23CPR 24

VIAYLA 24

IYLEK 24

120CD4 24

GR508020 022190

1200

iา 386002

022890

1200
1200

NI $\begin{aligned} & 386002 \\ & \text { nI } 386002\end{aligned}$

MECGDB 24

PBFB 24

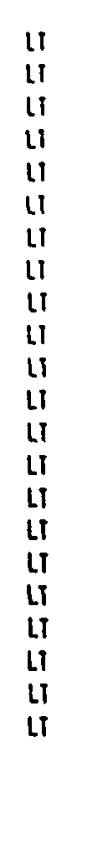

$\begin{array}{rr}5 & \text { UEKG } \\ 5 & \text { UGKG } \\ 5 & \text { UGKG } \\ 5 & \text { USKG } \\ 10 & \text { UGKG } \\ 5 & \text { USKG } \\ 5 & \text { UEKG } \\ 5 & \text { UGKG } \\ 10 & \text { UGKG } \\ 5 & \text { UGKG } \\ 5 & \text { UGKG } \\ 5 & \text { UGKG } \\ 5 & \text { UGKG } \\ 5 & \text { UEKG } \\ 5 & \text { UGKG } \\ 5 & \text { UEKG } \\ 5 & \text { UEKG } \\ 5 & \text { USKG } \\ 5 & \text { UGKG } \\ 5 & \text { UGKG } \\ 5 & \text { UGKG } \\ 5 & \text { UEKG } \\ 104 & \text { PCKT } \\ 101 & \text { PCNT } \\ 101 & \text { PCKT }\end{array}$

\begin{tabular}{|c|c|c|}
\hline $0 \mathrm{KE}$ & 64 & 0 \\
\hline $0 \mathrm{ME}$ & 61 & 0 \\
\hline 0 ME & 6H. & 0 \\
\hline$O$ KE & 64 & 0 \\
\hline $0 \mathrm{ME}$ & 64 & 0 \\
\hline O XE & 6II & 0 \\
\hline $0 \mathrm{ME}$ & GW & 0 \\
\hline$O$ ME & 6U & 0 \\
\hline $0 \mathrm{KE}$ & 64 & 0 \\
\hline $0 \mathrm{ME}$ & 6I & 0 \\
\hline$O \mathrm{ME}$ & GU & 0 \\
\hline 0 KE & 6U & 0 \\
\hline$O$ KE & 64 & 0 \\
\hline $0 \mathrm{ME}$ & 64 & 0 \\
\hline O ME & 6N & 0 \\
\hline$O \mathrm{ME}$ & GN & 0 \\
\hline$O \mathrm{KE}$ & 6N & 0 \\
\hline$O \mathrm{HE}$ & EU & 0 \\
\hline$O \mathrm{ME}$ & 6II & 0 \\
\hline$O \mathrm{ME}$ & 64 & 0 \\
\hline$O \mathrm{KE}$ & 6N & 0 \\
\hline$O \mathrm{ME}$ & 6II & 0 \\
\hline$O$ KE & GH & 50 \\
\hline O ME & 6II & 50 \\
\hline$O \mathrm{KE}$ & GN & \\
\hline
\end{tabular}

.



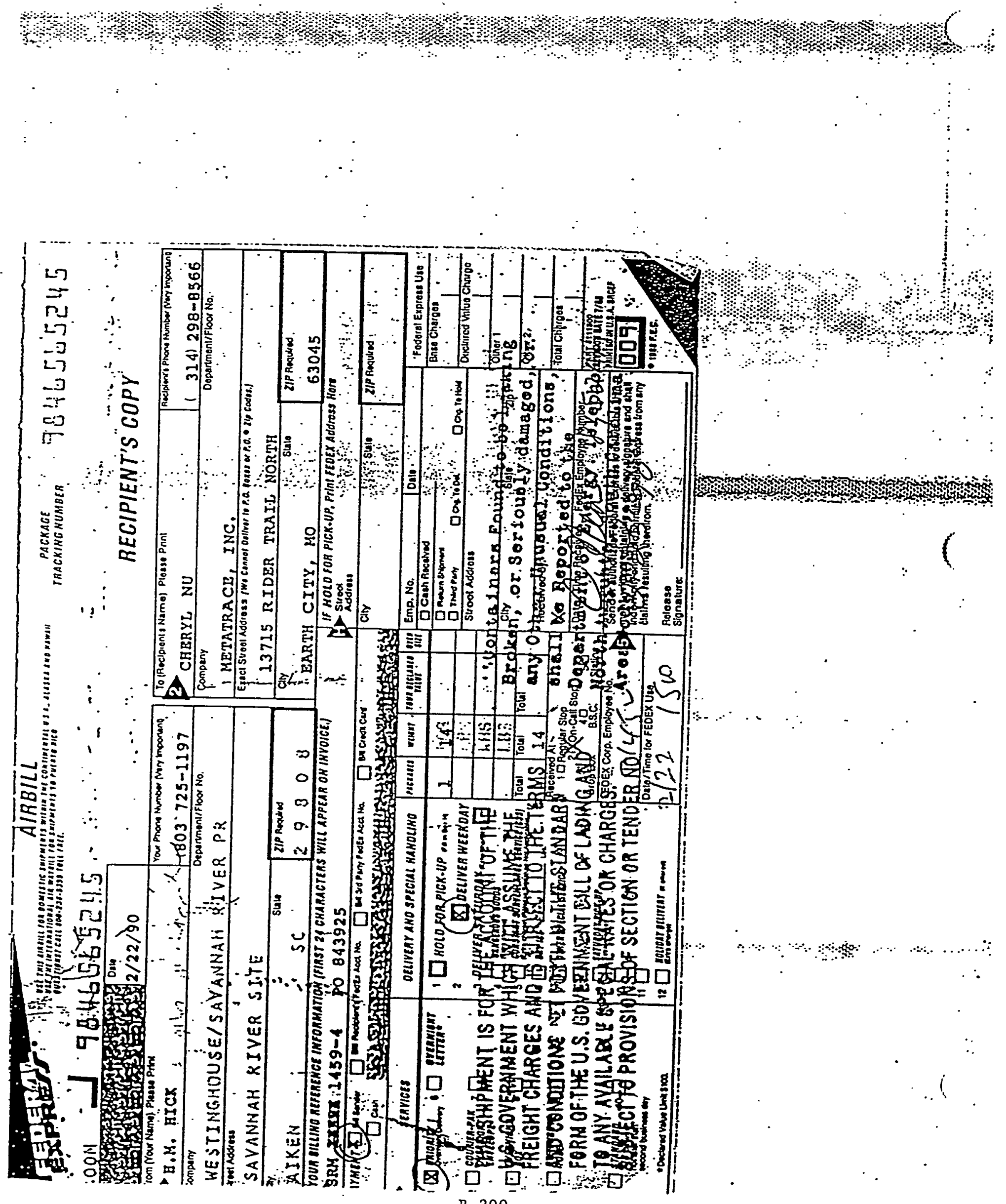
IITLIITMLE, IITC,

$\because \cdots$ PAGE 1 OF 1

PATE $2 \cdot 23-50$

PROJECTNONO $12 / .03$

SAMPLE ARRIYAL OATE $2.23-90$

EXPECTEONO. OF SAMPLES $\frac{2}{2}$

EXPECTEO MATRIX SOLL

$$
10 . \# 30
$$

n..........

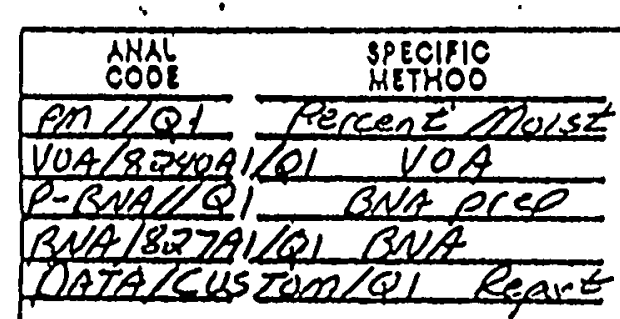

pataLUSTEDLQL Reart

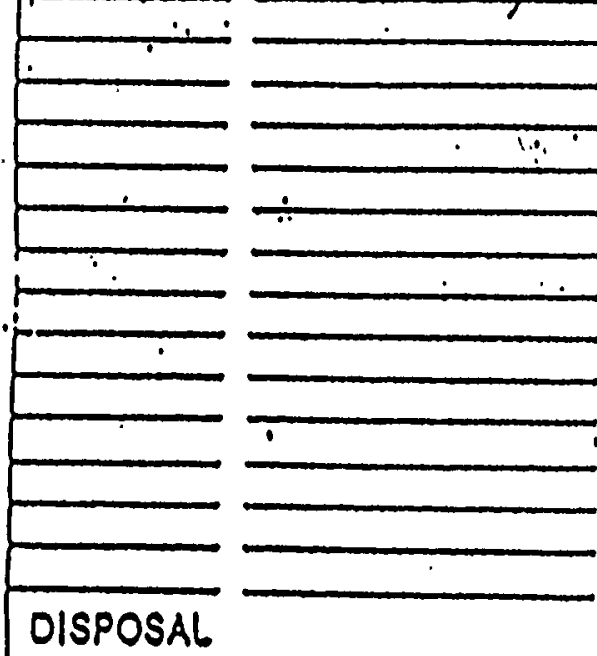

COMME

\section{$\angle I 386$}

.. OlstribuTION.

anent Westinghavise AUTO.

ICAPi. PROJ.HOA TAZYKE - : OPREP

SHIPPER FE.DHE C. OC

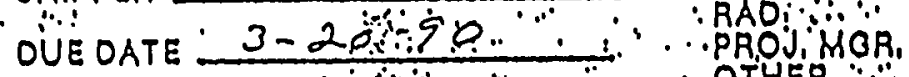

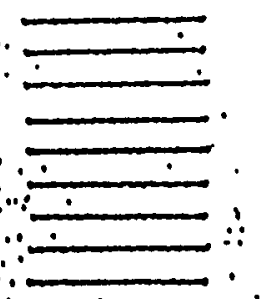

. .OTHER menscoinil isiriay ORIGINAL lu. Noune REVISION

\section{STORAGELOCATION} T 8 E 10 $\because \therefore \quad . \quad \therefore$.

SAMPLE NO, $38660 \%$ SASTP 10 \&

DESCRIPTION

SXMPLE OATE HiTRIX Gif 5 : G:RS

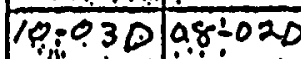

\begin{tabular}{ll}
\hline zitio: 90 & 2.21 .90 \\
\hline
\end{tabular}

$50.1+\div$

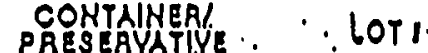

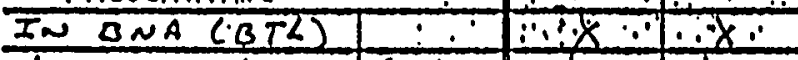

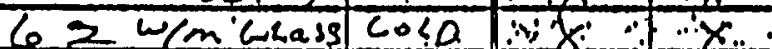

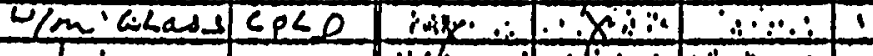

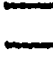

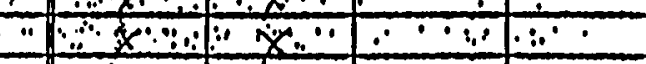

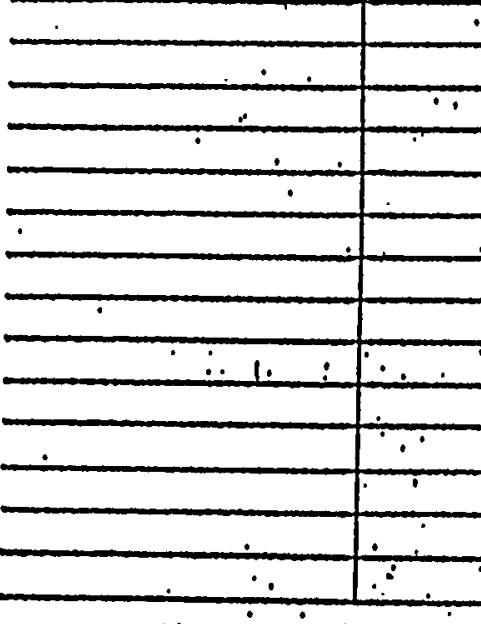

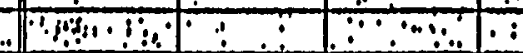

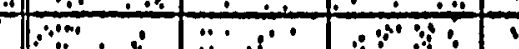

NORHAL:

HAZAROOUS
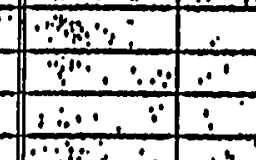

OATE TOOISPOSE

PM SIGNATURE

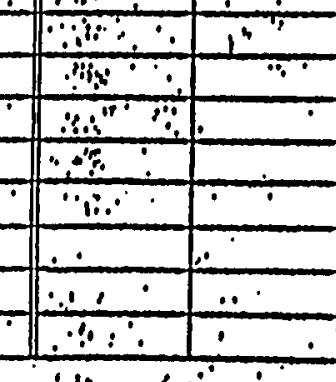

$\therefore 0^{\prime \prime} \cdot \therefore$

$\therefore, \therefore \therefore$ $\because \therefore \div \therefore$
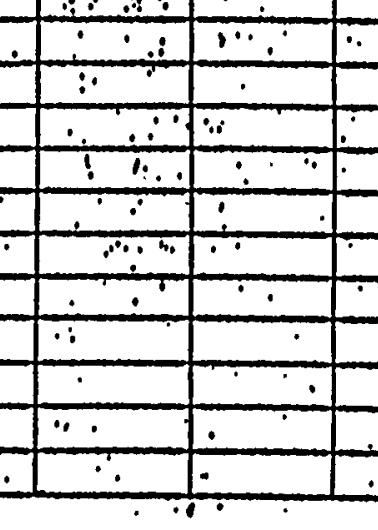

\begin{tabular}{l|l}
\hline$\cdots$ & \\
\hline$\cdot$ & $:$ \\
\hline &. \\
\hline & 1 \\
\hline
\end{tabular}

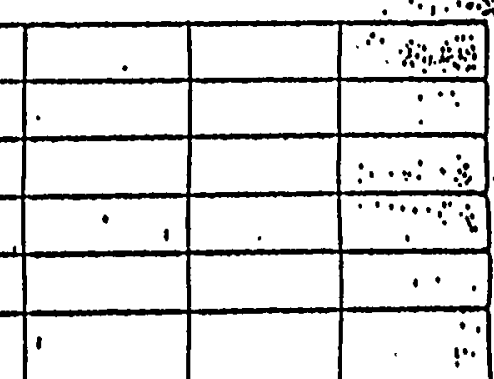

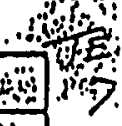




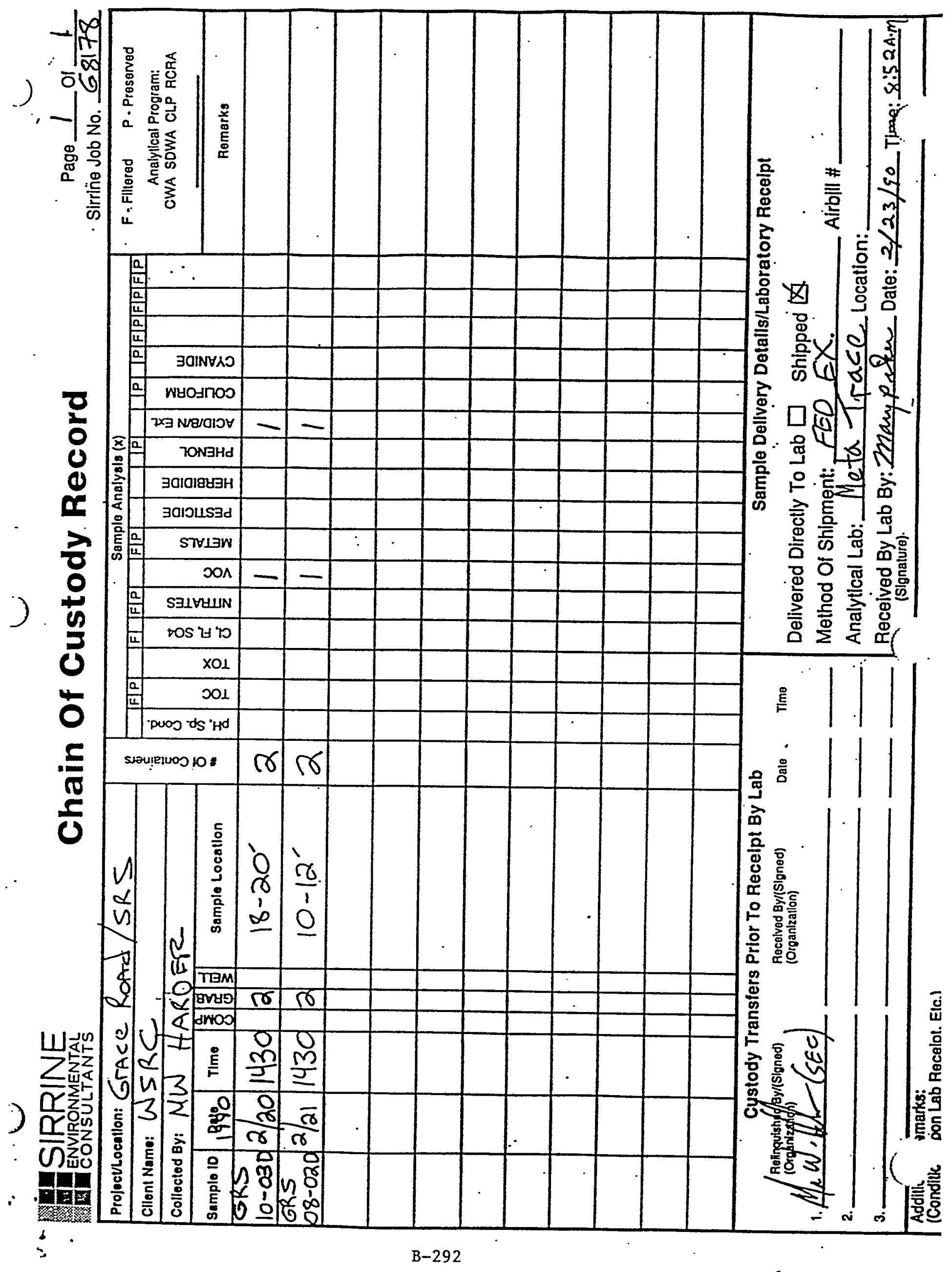




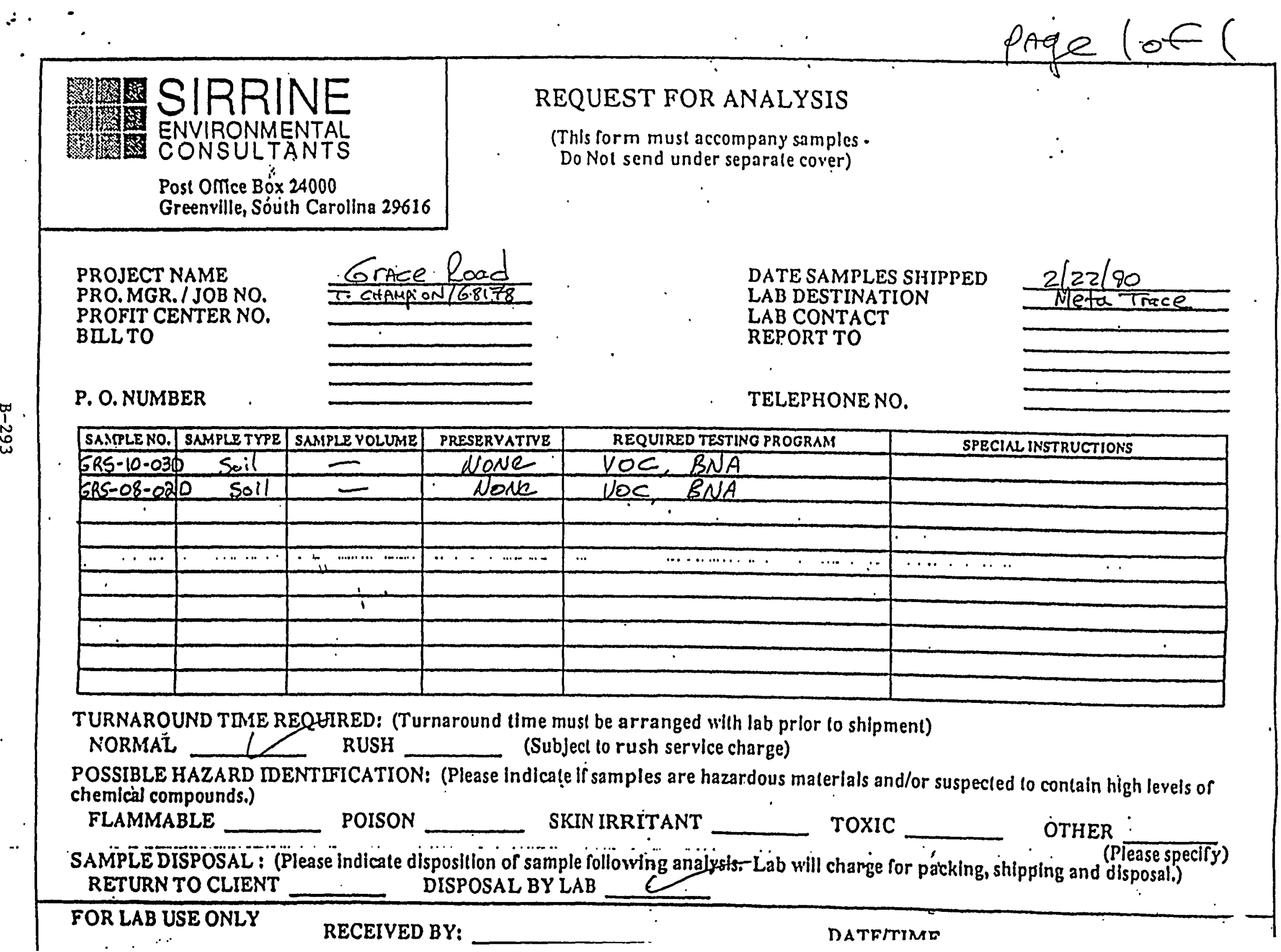




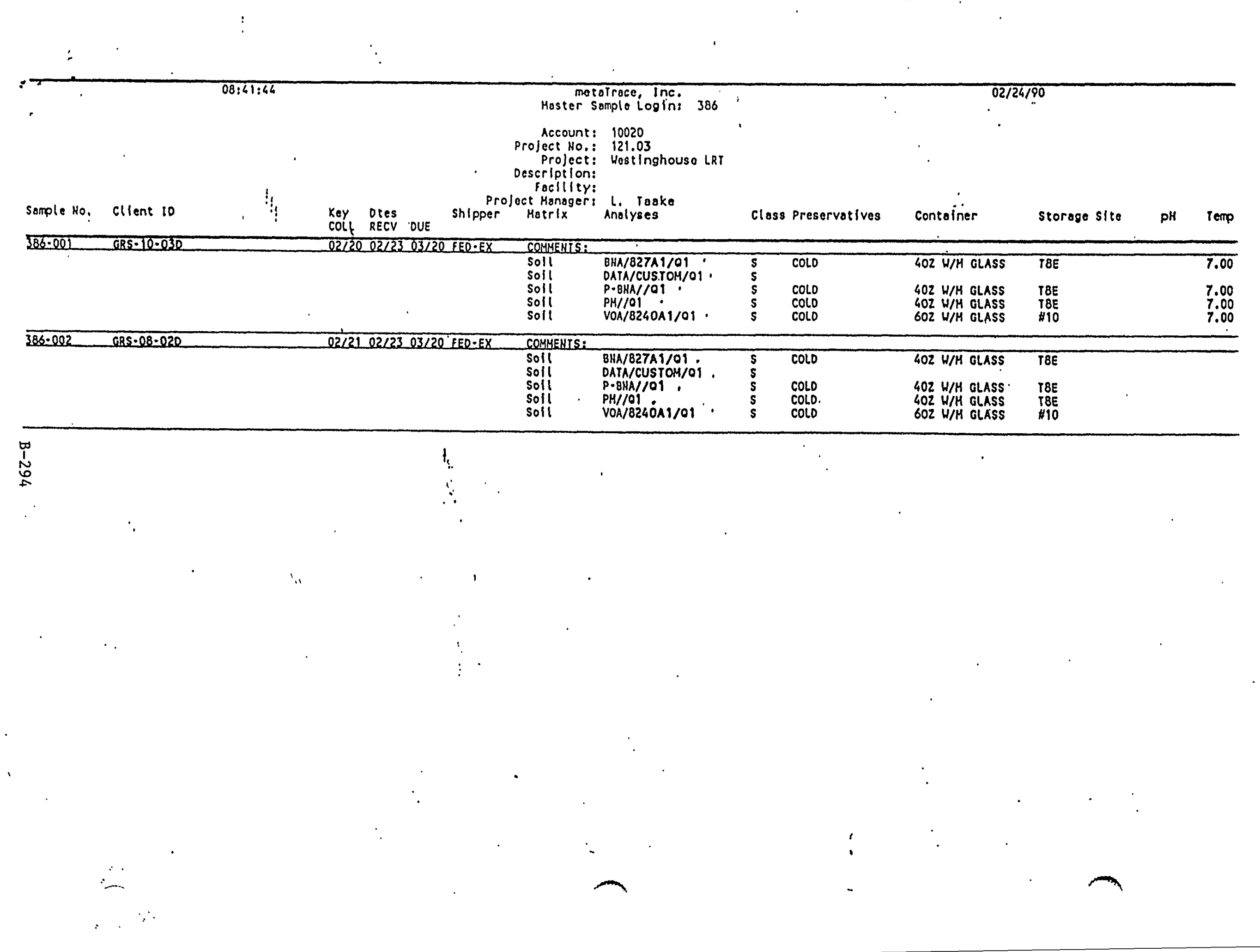




\section{APPENDIX C}

\section{RISK ASSESSMENT METHODS}

Summary of Calculations for the Future Resident Scenarios

- Dermal Contact with Surface Soil

- Soil Ingestion

- Inhalation

Parameter Values and Results of the Lead Uptake/Biokinetic Model 
Grace Road Site

RFI/RI Report
WSRC-RP-95-93, Rev. 1

November 1995 


\section{Dermal Contact with Surface Soil:}

Équation 1:

Absorbed Dose (mg/kg-day) $=$ CS $\times$ CF $\times$ SA $\times$ AF $\times$ ABS $\times$ EF $\times$ ED $B W \times A T \times A D$

where:

$\mathrm{CS}=$ Chemical Concentration in Soil (mg/kg)

$\mathrm{CF}=$ Conversion Factor (1.0E-06 kg/mg)

$\mathrm{SA}=$ Skin Surface Area Available for Contact ( $\mathrm{cm}^{2} /$ event)

$\mathrm{AF}=$ Soil to Skin Adherence Factor $\left(\mathrm{mg} / \mathrm{cm}^{2}\right)$

$\mathrm{ABS}=$ Absorption Factor (unitless)

$\mathrm{EF}=$ Exposure Frequency (events/year)

$\mathrm{ED}=$ Exposure Duration (years)

$\mathrm{BW}=$ Body Weight $(\mathrm{kg})$

AT $=$ Averaging Time (365 days/year)

$\mathrm{AD}=$ Averaging Duration (years) (equal to ED for non-carcinogens and 70 years for carcinogens)

Equation 2 (below) modifies Equation 1 for the hypothetical future residential scenario to apportion intake between children and adults based upon their different exposure parameters (see Table C-3). For carcinogens, the two components (adult and child) of Equation 2 were summed to yield an age-adjusted intake value. For non-carcinogens, the intake values for the adult plus child and the child only were considered separately in order to calculate both an age-adjusted value and a value for young children (0-6 years old), the likely period of highest dosage. The intake values for young children were calculated from the portion of Equation 2 that contains only child exposure parameters. 
Grace Road Site

RFI/RI Report
WSRC-RP-95-93, Rev. 1

November 1995

Equation 2:

Absorbed Dose for Arsenic (mg/kg-day)

$$
=\frac{\mathrm{CS} \times \mathrm{CF} \times \mathrm{SA}_{2} \times \mathrm{AF} \times \mathrm{ABS} \times \mathrm{EF}_{\mathrm{q}} \times \mathrm{ED}_{\mathrm{q}}}{\mathrm{BWa} \times \mathrm{AT} \times \mathrm{AD}}+
$$

$\mathrm{CS} \times \mathrm{CF} \times \mathrm{SA} A_{C} \times A F \times A B S \times E F_{C} \times E D_{c}$

$B W c \times A T \times A D$

Where:
$\mathrm{CS}=$ Chemical Concentration in Soil (mg/kg)
$\mathrm{CF}=$ Conversion Factor (1.0E-06 kg/mg)
$\mathrm{SA}_{\mathrm{a}}=\quad$ Adult Skin Surface Area Available for Contact ( $\mathrm{cm}^{2} /$ event)
$S A_{c}=$ Child Skin Surface Area Available for Contact (cm $2 /$ event)
$\mathrm{AF}=$ Soil to Skin Adherence Factor $\left(\mathrm{mg} / \mathrm{cm}^{2}\right)$
$\mathrm{ABS}=$ Absorption Factor (unitless)
$\mathrm{EF}_{\mathrm{a}}=$ Adult Exposure Frequency (events/year)
$E F_{c}=$ Child.Exposure Frequency (events/year)
$\mathrm{ED}_{\mathrm{a}}=$ Adult Exposure Duration (years)
$E D_{\mathrm{C}}=$ Child Exposure Duration (years)
$\mathrm{BWa}=$ Adult Body Weight $(\mathrm{kg})$
$\mathrm{BWc}=$ Child Body Weight $(\mathrm{kg})$
AT . = Averaging Time (365 days/year)
$\mathrm{AD}=$ Averaging Duration (years) (equal to $\mathrm{ED}$ for noncarcinogens and 70 years for carcinogens)

Absorbed Dose $=3: 2 \mathrm{mg} / \mathrm{kg} \times 1.0 \mathrm{E}-06 \mathrm{~kg} / \mathrm{mg} \times 5000 \mathrm{~cm}^{2} /$ event $\times 0.2 \mathrm{mg} / \mathrm{cm}^{2} \times 0.001 \times 350$ events $/ \mathrm{yr} \times 24 \mathrm{y}$ $70 \mathrm{~kg} \times 365 \mathrm{~d} / \mathrm{y} \times 70 \mathrm{y}$

$+3.2 \mathrm{mg} / \mathrm{kg} \times 1.0 \mathrm{E}-06 \mathrm{~kg} / \mathrm{mg} \times 1800 \mathrm{~cm}^{2} /$ event $\times 0.2 \mathrm{mg} / \mathrm{cm}^{2} \times 0.001 \times 350$ events $/ \mathrm{y} \times 6 \mathrm{y}$ $15 \mathrm{~kg} \times 365 \mathrm{~d} / \mathrm{y} \times 70 \mathrm{y}$

Absorbed Dose $(\mathrm{mg} / \mathrm{kg}$-day) $=\quad 2.0 \mathrm{E}-08+6.0 \mathrm{E}-09$

Absorbed Dose (mg/kg-day) $=2.6 \mathrm{E}-08$ 
Grace Road Site

WSRC-RP-95-93, Rev. 1

RFI/RI Report

November 1995

Risk for Arsenic

$=\quad$ Absorbed Dose $x$ Slope Factor

Risk for Arsenic

$=2.6 \mathrm{E}-08 \mathrm{mg} / \mathrm{kg}$-day $\times 1.8(\mathrm{mg} / \mathrm{kg}-\text { day })^{-1}$

Risk for Arsenic

$=\quad 4.7 \mathrm{E}-08$

Non-carcinogenic Risk Calculation:

Absorbed Dose for

$\operatorname{Arsenic}\left(\mathrm{mg} / \mathrm{kg}\right.$-day) $=\quad \mathrm{CS} \times \mathrm{CF} \times \mathrm{SA}_{\mathrm{a}} \times \mathrm{AF} \times \mathrm{ABS} \times \mathrm{EF}_{\mathrm{a}} \times \mathrm{ED}_{\mathrm{a}}+$ $B W a \times A T \times A D$

$\mathrm{CS} \times \mathrm{CF} \times \mathrm{SA}_{\mathcal{C}} \times \mathrm{AF} \times \mathrm{ABS} \times \mathrm{EF}_{\mathcal{C}} \times \mathrm{ED}_{\mathcal{C}}$

$B W c \times A T \times A D$

$=3.2 \mathrm{mg} / \mathrm{kg} \times 1.0 \mathrm{E}-06 \mathrm{~kg} / \mathrm{mg} \times 5000 \mathrm{~cm}^{2} /$ event $\times 0.2 \mathrm{mg} / \mathrm{cm}^{2} \times 0.001 \times 350$ events $/ \mathrm{y} \times 24 \mathrm{y}+$ $70 \mathrm{~kg} \times 365 \mathrm{~d} / \mathrm{y} \times 24 \mathrm{y}$

$3.2 \mathrm{mg} / \mathrm{kg} \times 1.0 \mathrm{E}-06 \mathrm{~kg} / \mathrm{mg} \times 1800 \mathrm{~cm}^{2} /$ event $\times 0.2 \mathrm{mg} / \mathrm{cm}^{2} \times 0.001 \times 350$ events $/ \mathrm{y} \times 6 \mathrm{y}$

$15 \mathrm{~kg} \times 365 \mathrm{~d} / \mathrm{y} \times 6 \mathrm{y}$

\begin{tabular}{|c|c|c|}
\hline Absorbed Dose for Arsenic & $=$ & $4.0 \mathrm{E}-08+7.4 \mathrm{E}-08$ \\
\hline Absorbed Dose for Arsenic & $=$ & $1.14 \mathrm{E}-07$ \\
\hline Hazard Quotient(HQ) & $=$ & Adsorbed Dose/Reference Dose \\
\hline Hazard Quotient(HQ) & $=$ & $1.14 \mathrm{E}-07 \mathrm{mg} / \mathrm{kg}$-day $/(2.9 \mathrm{E}-04 \mathrm{mg} / \mathrm{kg}$-day $)$ \\
\hline HQ for Adult and Child & $=$ & $3.9 \mathrm{E}-04$ \\
\hline HQ for Child Only & $=$ & $7.4 \mathrm{E}-08 / 2.9 \mathrm{E}-04$ \\
\hline HQ for Child Only & $=$ & $2.6 \mathrm{E}-0.4$ \\
\hline
\end{tabular}


Grace Road Site

RFI/RI Report
WSRC-RP-95-93, Rev. 1

November 1995

\section{Soil Ingestion}

Equation 3 was used to calculate intake from soil ingestion for the hypothetical residential scenario intake between children and adults, based upon their different exposure parameters (see Table 3A) and as recommended by EPA (1989 and 1991a).

For carcinogens, the two components (adult and child) were summed to yield an age-adjusted intake value. For noncarcinogens, the intake values for the adult plus child and the child only were considered separately in order to calculate both an age-adjusted value and a value for young children ( $0-6$ years old), the period of highest soil ingestion. the intake values for young children were calculated using only the child component of Equation 3.

Equation 3:

Intake for Arsenic $=$ CS $\times$ AIRO $\times$ CF $\times$ FI $\times E F \times E D_{a}+C S \times C I R O \times C F \times F I \times E F \times E D_{C}$ (mg/kg-day)

BWa $\times$ AT $\times A D$

$\mathrm{BW} \times \mathrm{AT} \times \mathrm{AD}$

Where:

$\mathrm{CS}=$ Chemical Concentration in Soil $(\mathrm{mg} / \mathrm{kg})$

$\mathrm{CF}=$ Conversion Factor (1.0E-06 kg/mg)

AIRO $=\quad$ Adult Ingestion Rate (mg soil/day)

$\mathrm{CIRO}=\quad$ Child Ingestion Rate (mg soil/day)

$\mathrm{FI}=$ Ingested from Contaminated Source (unitless)

$\mathrm{EF}=$ Exposure Frequency (events/year)

$E D_{\mathrm{a}}=$ Adult Exposure Duration (years)

$E D_{c}=$ Child Exposure Duration (years)

$\mathrm{BW}_{\mathrm{a}}=$ Adult Body Weight $(\mathrm{kg})$

$\mathrm{BWc}=$ Child Body Weight $(\mathrm{kg})$

AT $=$ Averaging Time (365 days/year)

$\mathrm{AD}=$ Averaging Duration (years) (equal to ED for noncarcinogens and 70 years for carcinogens)

Intake (mg/kg-day) $=3.2 \mathrm{mg} / \mathrm{kg} \times 100 \mathrm{mg} / \mathrm{d} \times 1.0 \mathrm{E}-06 \mathrm{~kg} / \mathrm{mg} \times 1 \times 350$ events $/ \mathrm{y} \times 24 \mathrm{y}+$ $70 \mathrm{~kg} \times 365 \mathrm{~d} / \mathrm{y} \times 70 \mathrm{y}$

$3.2 \mathrm{mg} / \mathrm{kg} \times 200 \mathrm{mg} / \mathrm{d} \times 1.0 \mathrm{E}-06 \mathrm{~kg} / \mathrm{mg} \times 1 \times 350$ events $/ \mathrm{y} \times 6 \mathrm{y}$ $15 \mathrm{~kg} \cdot \dot{\mathrm{x}} 365 \mathrm{~d} / \mathrm{y} \times 70 \mathrm{y}$ 
Grace Road Site

RFI/RI Report
WSRC-RP-95-93, Rev. 1

November 1995

$\begin{array}{lll}\text { Intake }(\mathrm{mg} / \mathrm{kg}-\text { day }) & = & 1.5 \mathrm{E}-06+3.60 \mathrm{E}-06 \\ \text { Intake }(\mathrm{mg} / \mathrm{kg} \text {-day }) & =5.0 \mathrm{E}-06 \\ & \\ \text { Risk for Arsenic } & =\quad \text { Intake } \times \text { Slope Factor } \\ \text { Risk for Arsenic } & =5.0 \mathrm{E}-06 \mathrm{mg} / \mathrm{kg} \text {-day } \times 1.75(\mathrm{mg} / \mathrm{kg} \text {-day)-1 } \\ \text { Risk for Arsenic } & = & 8.8 \mathrm{E}-06\end{array}$

Non-carcinogenic Risk Calculation:

Intake for Arsenic $=$ CS $\times$ AIRO $\times$ CF $\times$ FI $\times E F \times E D_{a}+$ CS $\times$ CIRO $\times C F \times F I \times E F \times E D_{\mathcal{C}}$ (mg/kg-day) $\mathrm{BWa} \times \mathrm{AT} \times \mathrm{AD}$ $B W c \times A T \times A D$

Intake (mg/kg-day) . = $3.2 \mathrm{mg} / \mathrm{kg} \times 100 \mathrm{mg} / \mathrm{d} \times 1.0 \mathrm{E}-06 \mathrm{~kg} / \mathrm{mg} \times 1 \times 350$ events $/ \mathrm{y} \times 24 \mathrm{y}+$ $70 \mathrm{~kg} \times 365 \mathrm{~d} / \mathrm{y} \times 24 \mathrm{y}$

$3.2 \mathrm{mg} / \mathrm{kg} \times 200 \mathrm{mg} / \mathrm{d} \times 1.0 \mathrm{E}-06 \mathrm{~kg} / \mathrm{mg} \times 1 \times 350$ events $/ \mathrm{y} \times 6 \mathrm{y}$ $15 \mathrm{~kg} \times 365 \mathrm{~d} / \mathrm{y} \times 6 \mathrm{y}$

Intake $(\mathrm{mg} / \mathrm{kg}$-day $)=4.4 \mathrm{E}-06+4.18 \mathrm{E}-05$
Intake $(\mathrm{mg} / \mathrm{kg}$-day $)=\quad 4.6 \mathrm{E}-05$

Hazard Quotient(HQ) $=\quad$ Intake Dose/Reference Dose

Hazard Quotient(HQ) $=4.6 \mathrm{E}-05 \mathrm{mg} / \mathrm{kg}-$ day $/(3 \mathrm{E}-04 \mathrm{mg} / \mathrm{kg}$-day $)$

HQ for Adult and Child = 0.15

HQ for Child Only $=4.18 \mathrm{E}-05 / 3 \mathrm{E}-04$

$\mathrm{HQ}$ for Child Only $=0.14$ 
Grace Road Site

RFI/RI Report
WSRC-RP-95-93, Rev. 1

November 1995

\section{Equation For Exposure to Air:}

This equation used to calculate intakes from inhalation of contaminated airbome soil particulates was obtained from EPA risk assessment guidance (EPA, 1989). Equation 4 was used to determine the inhalation of particulates between hypothetical future adult and child residents based on their different exposure parameters. The two components of Equation 4 were summed for the chemical carcinogens. For noncarcinogens, the intake values for the adult plus child and the child only were considered separately in order to calculate both an age-adjusted value and a value for young children (0-6 years old) only. Parameter values are shown in Table 3A.

Equation 4:

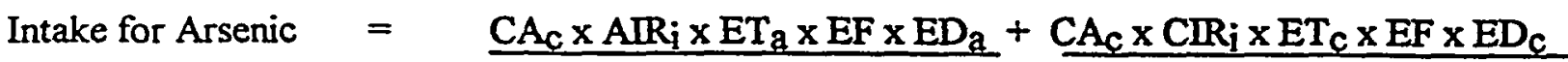
(mg/kg-day) $\mathrm{BWa} \times \mathrm{AT} \times \mathrm{AD}$

BWC $\times$ AT $\times A D$

Where:

$\mathrm{CA}_{\mathcal{C}}=$ Chemical Concentration in Air $\left(\mathrm{mg} / \mathrm{m}^{3}\right)$

$A R_{i}=$ Adult Inhalation Rate ( $m^{3}$ hour)

$\mathrm{CIR}_{\mathrm{i}}=$ Child Inhalation Rate $\left(\mathrm{m}^{3} /\right.$ hour $)$

$E T_{\mathrm{a}}=$ Adult Exposure Time (hr/d)

$E T_{c}=$ Child Exposure Time (hr/d)

$\cdot \mathrm{EF}=$ Exposure Frequency (d/year)

$E D_{a}=$ Adult Exposure Duration (years)

$E D_{c}=$ Child Exposure Duration (years)

$\mathrm{BW}_{\mathbf{a}}=\quad$ Adult Body Weight $(\mathrm{kg})$

$\mathrm{BWC}=$ Child Body Weight $(\mathrm{kg})$

$A T=$ Averaging Time (365 days/year)

$A D=$ Averaging Duration (years) (equal to ED for noncarcinogens and 70 years for carcinogens)

\section{Suspension of Particulates:}

Suspension of Particulates was modeled following Milham et al. (1975), who studied resuspension of plutonium from an old field that was converted to agriculture specifically for the 
purposes of the study. Inhalable concentration of contaminants associated with suspended particulate matter was calculated as follows:

where:

$$
\mathrm{CA}=\mathrm{SD} \times \mathrm{CS} \times \mathrm{SF} \times \mathrm{R}_{\mathrm{f}}
$$

$$
\begin{aligned}
& \mathrm{CA}=\text { contaminant concentration in air }\left(\mathrm{mg} / \mathrm{m}^{3}\right) \\
& \mathrm{SD}=\text { soil density }\left(\mathrm{kg} / \mathrm{m}^{3}\right), \\
& \mathrm{CS}=\text { contaminant concentration in soil }(\mathrm{mg} / \mathrm{kg}) \\
& \mathrm{SF}=\text { surface factor }(0.01) ; \text { used to calculate soil volume to a depth of } 1 \mathrm{~cm}, \\
& \mathrm{R}_{\mathrm{f}}=\quad \text { resuspension factor, represents the amount of material which may become } \\
& \quad \text { airborne (unitless). }
\end{aligned}
$$

A site-specific value of 5.0E-08 was used for $\mathrm{R}_{\mathrm{f}}$, based on the Milham et al. (1975) study. Milham obtained resuspension factors of 2.4E-07 next to an operating tractor, $1.03 \mathrm{E}-06$ at $7.6 \mathrm{~m}$ from the tractor, and 5.0E-08 at the edge of the field. Since none of the scenarios considered in the risk evaluation involve the high degree of soil disturbance associated with agriculture, the value of 5.0E-08 is chosen as a conservative, site-specific value.

For Arsenic, $\mathrm{CA}$ is calculated below:

$$
\begin{aligned}
& \mathrm{CA}=1.6 \mathrm{E}+03 \mathrm{~kg} / \mathrm{m}^{3} \times 3.2 \mathrm{mg} / \mathrm{kg} \times 0.01 \times 5.0 \mathrm{E}-08 \\
& \mathrm{CA}=2.56 \mathrm{E}-06 \mathrm{mg} / \mathrm{m}^{3} \\
& \text { Intake }=\frac{2.56 \mathrm{E}-06 \mathrm{mg} / \mathrm{m}^{3} \times 0.83 \mathrm{~m}^{3} / \mathrm{hr} \times 24 \mathrm{hr} / \mathrm{d} \times 350 \text { events } / \mathrm{y} \times 24 \mathrm{y}+}{70 \mathrm{~kg} \times 365 \mathrm{~d} / \mathrm{y} \times 70 \mathrm{y}}
\end{aligned}
$$

\section{$\underline{2.56 \mathrm{E}-06 \mathrm{mg} / \mathrm{m}^{3} \times 1.0 \mathrm{~m}^{3} / \mathrm{hr} \times 24 \mathrm{hr} / \mathrm{d} \times 350 \text { events } / \mathrm{y}} \times 6 \mathrm{y}$}

$15 \mathrm{~kg} \times 365 \mathrm{~d} / \mathrm{y} \times 70 \mathrm{y}$

$$
\begin{array}{lll}
\text { Intake }(\mathrm{mg} / \mathrm{kg}-\text { day }) & =2.4 \mathrm{E}-07+3.37 \mathrm{E}-07 \\
\text { Intake }(\mathrm{mg} / \mathrm{kg} \text {-day } & =5.8 \mathrm{E}-07
\end{array}
$$

Risk for Arsenic = Intake $\mathrm{x}$ Slope Factor

Risk for Arsenic $\quad=\quad 5.8 \mathrm{E}-07 \mathrm{mg} / \mathrm{kg}$-day $\times 5.0 \mathrm{E}+01(\mathrm{mg} / \mathrm{kg} \text {-day })^{-1}$

Risk for Arsenic $\quad=\quad 2.9 \mathrm{E}-05$ 
Grace Road Site

WSRC-RP-95-93, Rev. 1

RFI/RI Report

November 1995

Non-carcinogenic Risk Calculation:

Intake $=\quad 2.56 \mathrm{E}-06 \mathrm{mg} / \mathrm{m}^{3} \times 0.83 \mathrm{~m}^{3} / \mathrm{hr} \times 24 \mathrm{hr} / \mathrm{d} \times 350$ days $/ \mathrm{y} \times 24 \mathrm{y}+$ $70 \mathrm{~kg} \mathrm{x} \mathrm{365d/y} \mathrm{x} \mathrm{24y}$

$2.56 \mathrm{E}-06 \mathrm{mg} / \mathrm{m}^{3} \times 1.0 \mathrm{~m}^{3} / \mathrm{hr} \times 24 \mathrm{hr} / \mathrm{d} \times 350$ days $/ \mathrm{y} \times 6 \mathrm{y}$

$15 \mathrm{~kg} \times 365 \mathrm{~d} / \mathrm{y} \times 6 \mathrm{y}$

Intake $(\mathrm{mg} / \mathrm{kg}$-day $)=7.07 \mathrm{E}-07+3.9 \mathrm{E}-06$
Intake $(\mathrm{mg} / \mathrm{kg}-$ day $)=4.6 \mathrm{E}-06$

Hazard Quotient(HQ) $=$ Intake Dose/Reference Dose

Hazard Quotient(HQ) $=4.6 \mathrm{E}-06 \mathrm{mg} / \mathrm{kg}$-day/(8.3E-05 mg/kg-day)

HQ for Adult and Child $=\quad 0.055$

HQ for Child Only $=\quad 3.9 E-06 /(8.3 E-05)$

HQ for Child Only $=\quad .047$

Total Risk for Arsenic is shown in Table C-1:

\section{Table C-1}

Carcinógenic/Non-Carcinogenic Results for $3.2 \mathrm{mg} / \mathrm{kg}$ Arsenic

\begin{tabular}{|c|c|c|c|}
\hline \multirow{2}{*}{ Pathway } & \multirow{2}{*}{$\begin{array}{l}\text { Carcinogenic Risk } \\
\text { Adult and Child } \\
\text { (Unitless) }\end{array}$} & \multicolumn{2}{|c|}{ Non-Carcinogenic Risk } \\
\hline & & $\begin{array}{c}\text { Adult and Child } \\
\text { (Unitless) } \\
\end{array}$ & $\begin{array}{l}\text { Child only } \\
\text { (Unitless) }\end{array}$ \\
\hline $\begin{array}{l}\text { Dermal Contact } \\
\text { Ingestion } \\
\text { Inhalation } \\
\end{array}$ & $\begin{array}{r}4.7 \mathrm{E}-08 \\
8.8 \mathrm{E}-06 \\
2.9 \mathrm{E}-05 \\
\end{array}$ & $\begin{array}{l}3.9 \mathrm{E}-04 \\
1.5 \mathrm{E}-01 \\
5.5 \mathrm{E}-02 \\
\end{array}$ & $\begin{array}{l}2.6 \mathrm{E}-04 \\
1.4 \mathrm{E}-01 \\
4.7 \mathrm{E}-02 \\
\end{array}$ \\
\hline Total Risk & $3.8 \mathrm{E}-05$ & $2.0 \mathrm{E}-01$ & $1.9 \mathrm{E}-01$ \\
\hline
\end{tabular}


Grace Road Site

RFI/RI Report
WSRC-RP-95-93, Rev. 1

November 1995

Table C-2

TOXICITY VALUES FOR ARSENIC: CARCINOGENIC EFFECTS GRACE ROAD SITE

\begin{tabular}{|c|c|c|c|c|c|}
\hline \multirow{2}{*}{$\begin{array}{l}\text { Weight of Evidence } \\
\text { Classification }\end{array}$} & \multicolumn{2}{|c|}{ Slope Factor } & Tral \\
\cline { 2 - 5 } & $\begin{array}{c}\text { Onhalation } \\
(\mathbf{m g} / \mathbf{k g - d})^{-1}\end{array}$ & $\begin{array}{c}\text { Chemical } \\
(\mathbf{m g} / \mathbf{k g}-\mathbf{d})^{-1}\end{array}$ & $\begin{array}{c}\text { Type of Cancer: } \\
\text { Organ/Target } \\
\text { Species }\end{array}$ & Source \\
\hline A & 1.75 & 50 & $\begin{array}{c}\text { Oral } \\
\text { Inhalation }\end{array}$ & $\begin{array}{c}\text { Tumors: Respiratory } \\
\text { System/Humans } \\
\text { Cancer: Lung/Human }\end{array}$ & $\begin{array}{c}\text { IRIS } \\
\text { HEAST }\end{array}$ \\
\hline
\end{tabular}

ORAL TOXICITY VALUE ADJUSTED FOR AN ABSORBED DOSE (FOR USE IN CALCULATING RISK FROM DERMAL EXPOSURE)

\begin{tabular}{|c|c|c|c|}
\hline $\begin{array}{c}\text { Percent (\%) GI } \\
\text { Absorption }\end{array}$ & $\begin{array}{c}\text { Adjusted Slope Factor } \\
(\mathbf{m g} / \mathbf{k g}-\mathbf{d})^{-1}\end{array}$ & $\begin{array}{c}\text { Adjusted Reference Dose } \\
(\mathrm{mg} / \mathbf{k g}-\mathrm{d})^{-1}\end{array}$ & Reference \\
\hline $95 \%$ & 1.8 & $2.9 \mathrm{E}-04$ & ATSDR, 1991 \\
\hline
\end{tabular}

- TOXICITY VALUES FOR ARSENIC: NONCARCINOGENIC EFFECTS GRACE ROAD SITE

\begin{tabular}{|c|c|c|c|c|}
\hline $\begin{array}{c}\text { Oral RfD } \\
(\mathbf{m g} / \mathbf{k g}-\mathrm{d})\end{array}$ & $\begin{array}{c}\text { Inhalation RfC } \\
(\mathrm{mg} / \mathrm{kg}-\mathrm{d})\end{array}$ & Chemical Route & Critical Effects & Source \\
\hline $3 \mathrm{E}-04$ & $8.3 \mathrm{E}-05$ & Ora//nhalation: & $\begin{array}{c}\text { Hyperpigmentation } \\
\text { Keratosis }\end{array}$ & IRIS/ORNL \\
\hline
\end{tabular}


Grace Road Site

RFI/RI Report

WSRC-RP-95-93, Rev. 1

November 1995

TABLE C-3

VALUES ASSUMED FOR RME HUMAN EXPOSURE PARAMETERS (SOILS)

\begin{tabular}{|c|c|c|c|}
\hline Exposure Parameter & UNTT & $\begin{array}{l}\text { FUTURH ON-UNIT } \\
\text { RESDENT ADULT }\end{array}$ & $\begin{array}{l}\text { FUTURE ON-UNIT } \\
\text { RESWENT CHILD }\end{array}$ \\
\hline Body Weight (BW) & kg & 70 & 15 \\
\hline Exposure Duration (ED) & yr & 24 & 6 \\
\hline Exposure Frequency (EF) & $\mathrm{d} / \mathrm{yr}$ & 350 & 350 \\
\hline Exposure Time (ET) & $\mathrm{hr} / \mathrm{d}$ & 24 & 24 \\
\hline $\begin{array}{l}\text { Adherence Factor of } \\
\text { Soil to Skin (AF) }\end{array}$ & $\mathrm{mg} / \mathrm{cm}^{2}$ & 0.2 & 0.2 \\
\hline $\begin{array}{l}\text { Skin Surface Area Available } \\
\text { for Contact (SA) }\end{array}$ & $\mathrm{cm}^{2}$ & 5,000 & 1,800 \\
\hline Ingestion Rate $\left(\mathbb{I R}_{0}\right)$ & $\mathrm{mg} / \mathrm{d}$ & 100 & 200 \\
\hline Inhalation Rate ( $\left.\mathbf{I R}_{\mathbf{i}}\right)$ & $\mathrm{m}^{3} / \mathrm{hr}$ & 0.83 & 1.0 \\
\hline $\begin{array}{l}\text { Fraction Ingested from } \\
\text { Contaminated Source (FI) }\end{array}$ & Unitless & 1.0 & 1.0 \\
\hline Absorption Factor (ABS) & Unitless & 0.001 & 0.001 \\
\hline
\end{tabular}

\section{LEAD UPTAKE/BIOKINETIC MODEL RESULTS}

The output of the U. S. EPA integrated exposure uptake/biokinetic model for lead in children (EPA, 1994a) was used for the Grace Road Unit where the soil lead concentration was high, $48.1 \mathrm{mg} / \mathrm{kg}$. The site-specific and default parameter values employed in the model are presented in Table C-4. 



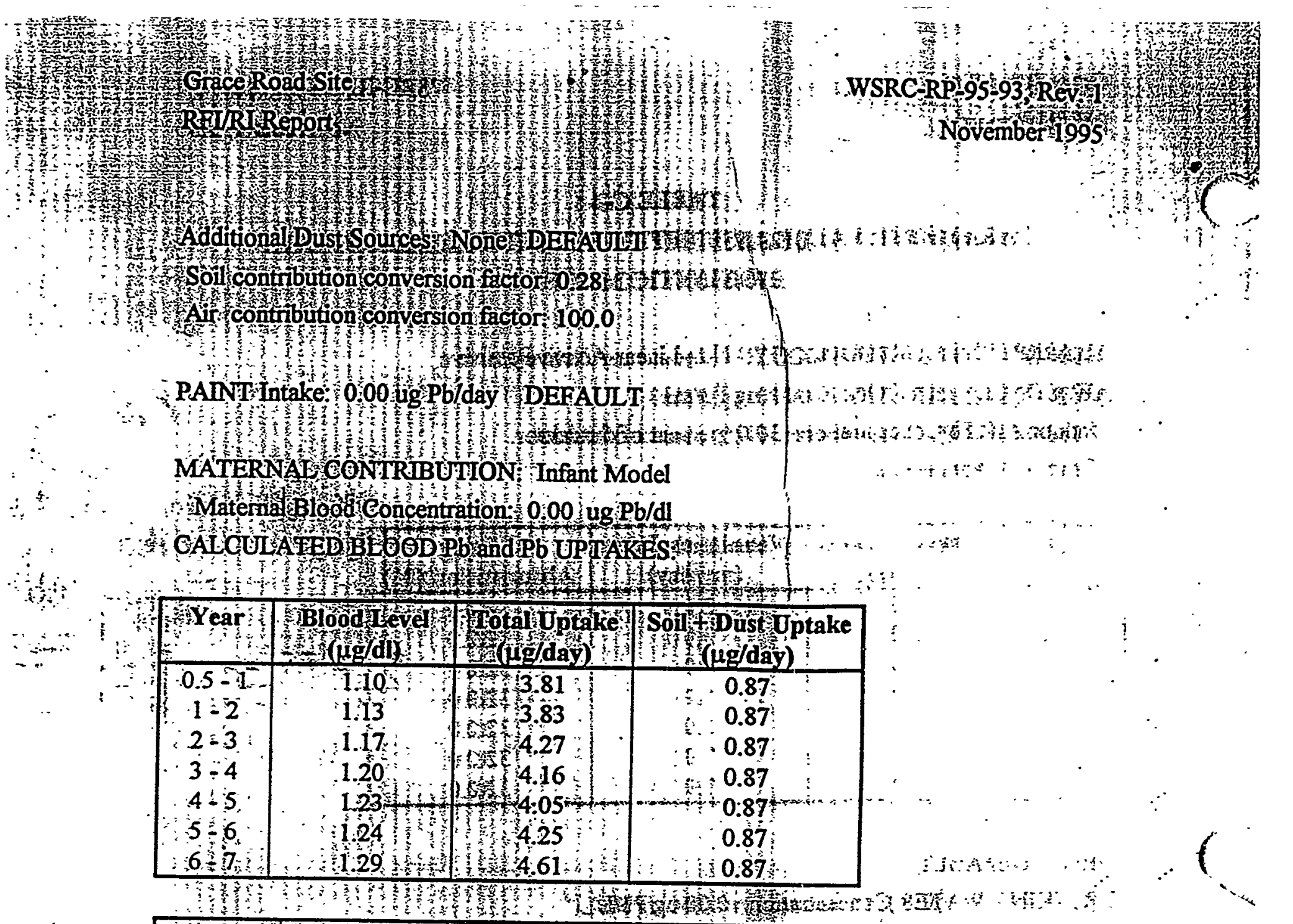

\begin{tabular}{|c|c|c|c|c|}
\hline - Yeấ & $\begin{array}{r}\text { Diet Uptake } \\
(\mu \mathrm{g} / \text { day })\end{array}$ & $\begin{array}{l}\text { Watér Uptake } \\
(\mu \mathrm{d} / \mathrm{day})\end{array}$ & 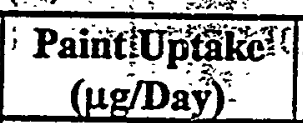 & $\begin{array}{l}\text { Air Uptâke } \\
(\mu g / \text { day }):\end{array}$ \\
\hline $0.5-1$ & $2: 94$ & $\because 0.00$ & 0.00 & 0.00 \\
\hline $1-2$ & 2.96 & 0.00 & $0.00::$ & 0.00 \\
\hline $2-3$ & 3.40 & 0.00 & 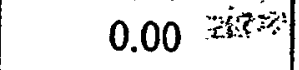 & $0.00^{\circ}$ \\
\hline $3-4$ & 3.29 & 0.00 & 0.00 & 0.00 \\
\hline $4-5$ & 3.18 & 0.00 & 0,00 & 0.00 \\
\hline $5-6$ & 3.38 & 0.00 & 0,00 & 0.00 \\
\hline $6-7$ & $3.74=$ & 0.00 & $0.00^{\circ}$ & 0.00 \\
\hline
\end{tabular}

